This document was too large to scan as a whole document, therefore it required breaking into smaller sections.

Document number: $S D-S N F-D R-003$

Section 1 of 3

Title:Ohulti-Canister Qverpack Design Report

Date: 6/9.197 Revision: A000

Originator: Smith $\mathrm{K \varepsilon}$

Co: hose nt

Recipient:

Co:

References: $E D T-620106$ 


\begin{tabular}{|c|c|}
\hline $\begin{array}{l}\text { 2. To: (Receiving organization) } \\
\text { Distribution }\end{array}$ & $\begin{array}{l}\text { 3. From: (originating Organization) } \\
\text { SNF Storage Projects }\end{array}$ \\
\hline $\begin{array}{l}\text { 5. Proj./Prog./Dept./Div.: } \\
\text { SNF Project/W-442 }\end{array}$ & $\begin{array}{l}\text { 6. Design Authority/ Design Agent/Cog. } \\
\text { Engr.: } \\
\text { L. H. Goldmann }\end{array}$ \\
\hline
\end{tabular}

8. Originator Remarks:

The attached document is being submitted for release.

Strikeouts to dually numbered pages made in order to

facilitate release process. Kes $5 / 28 / 97$
11. Receiver Remarks:
11A. Design Basel ine Document?
[X] Yes
[] No
4. Related EDT No.:
$\mathrm{N} / \mathrm{A}$
7. Purchase Order No.:
E56158
9. Equip./Component No.:
$N / A$
10. System/Bldg./Facil ity:
K Basins $/ 212 \mathrm{H}$
12. Major Assm. Dwg. No.:
$\mathrm{N} / \mathrm{A}$
13. Permit/Permit Application No.: $N / A$
14. Required Response Date: $N / A$

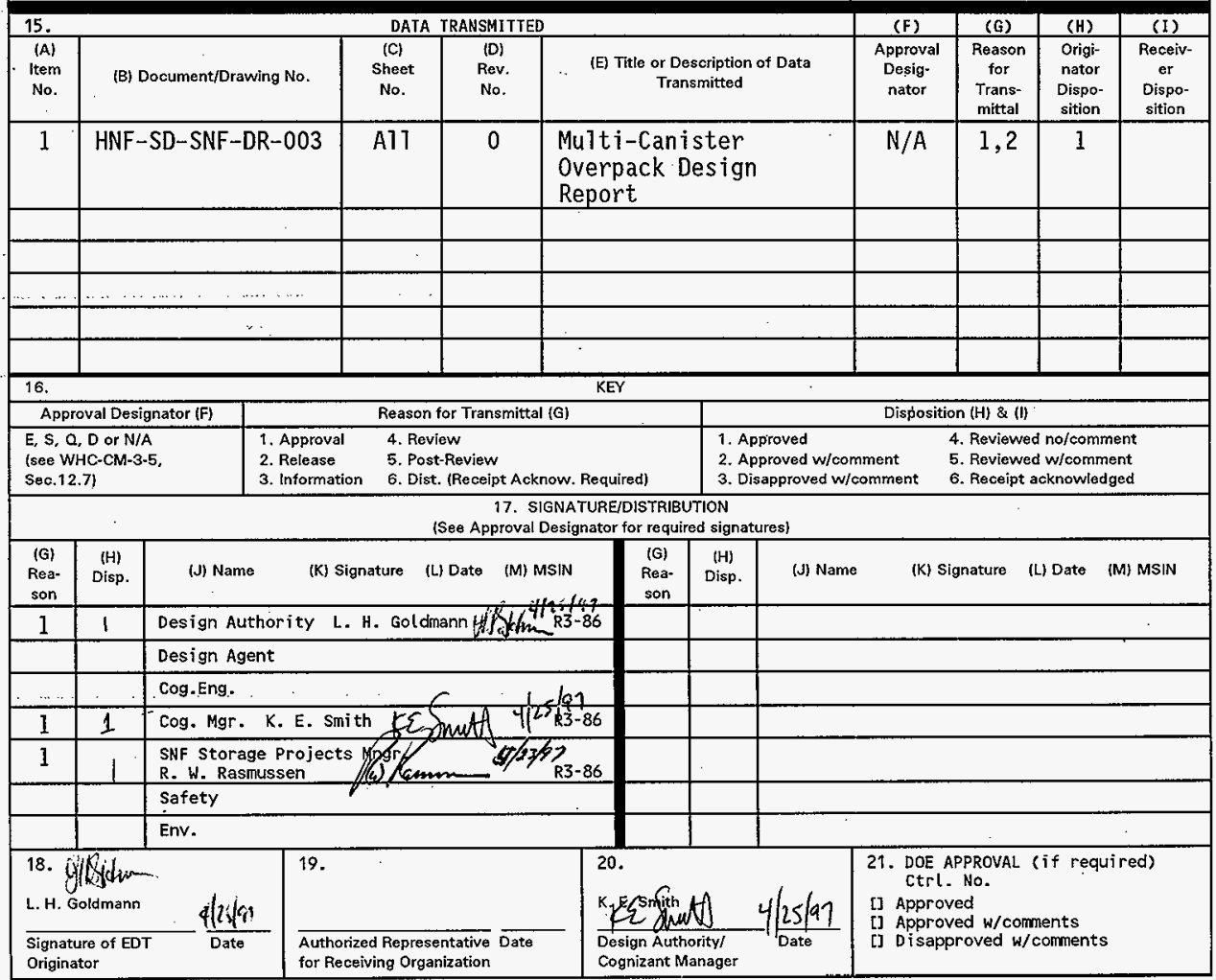


HNF-SD-SNF-DR-003, Rev. 0

Page 1 of 4

\title{
Multi-Canister Overpack Design Report
}

\author{
K.E. Smith
}

DESH, Richl and, WA 99352

U.S. Department of Energy Contract DE-AC06-96RL13200

$\begin{array}{lll}\text { EDT/ECN: } & \text { EDT-620106 } & \text { UC: } 600 \\ \text { Org Code: } & 2 T 340 & \text { Charge Code: LE008 } \\ \text { B\&R Code: } & \text { EW7040000 } & \text { Total Pages: } 960\end{array}$

Key Words: Spent Fuel, Canister, Packaging, Container

Abstract: This design report documents the final design for the MultiCanister Overpack.

IRADEMARK DISCLAIMER. Reference herein to any specific comercial product, process, or service by trade name, trademark, manufacturer, or otherwise, does not necessarily constitute or imply its endorsement, recommendation, or favoring by the United States Government or any agency thereof or its contractors or subcontractors.

Printed in the United States of America. To obtain copies of this document, contact: Document Control Services, P.0. Box 950, Mailstop H6-08, Richland WA 99352, Phone (509) 372-2420; Fax (509) 376-4989.
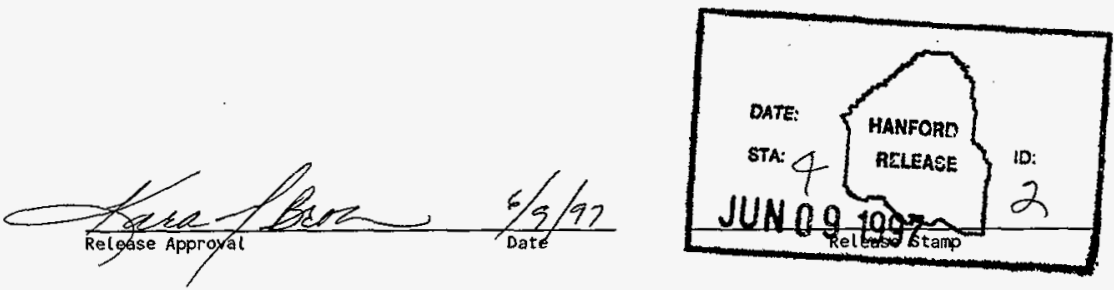

Approved for Public Release 
From: Tom Anderson To: Lars Meyer

Date: $4 / 23 / 97$ Time: $14: 04: 35$

SUPPORTING DOCUMENT OCPVERSHEET HNF-SD-SNF-DR-003, Rev. 0

PAGE 2 of 4

ISP

Industrial Screen Products, Inc.

P.O. Box $10673 \cdot$ Zephyr Cove, NV 89448

Phone: (800) 663-2702 - Fax: (800) 663-8060

Date: Wednesday, April 23, 1997

To: $\quad$ Duke Engineering \& Services Hanford, Inc.

Attn: Lars Meyer

Fax: $\quad 5009-376-3252$

Sub: Authorization

Duke Engineering is authorized to publish ISP drawing \# 5873 in the MCO Final Design Report.

Best regards,

Thomas A Andean 
SUPPORTING DOCUMENT

COVERSHEET PAGE 3 of 4

HNF-SD-SNF-DR-003, REV.
2770THEBOULEVARD

R.O. BOX 9889

COLUMBIA, SC29209 USA

TELEPHONE: (803) 783.1880

TELEFAX: (803) $783-4279$
N0.5093 P. 1

\section{Helicoflex}

Page 1 / 1
From: Michel LEFRANÇOIS

Sales \& Marketing Manager

Copy :

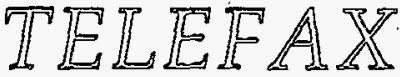

To: Lars MEYER

Company: DUKE ENGINEERING

Address: HANDFORD

Country/state: WA

Telefax \#: 5093763252

Columbia, April 24, 1997

\section{Subject: DRAWING DUPLICATION}

Ret.: MCO Cask Seal / Holding device for Helicoflex Seal H-305236 Rev NC / Re ML-2/14/97 Dear Sir,

Re our telephone conversation of 4/22/97, we hereby allow you to duplicate the sketch referenced above.

Yours Sincerely,

Michel Lefrançois

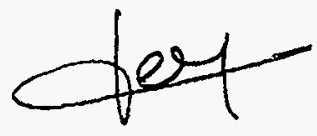




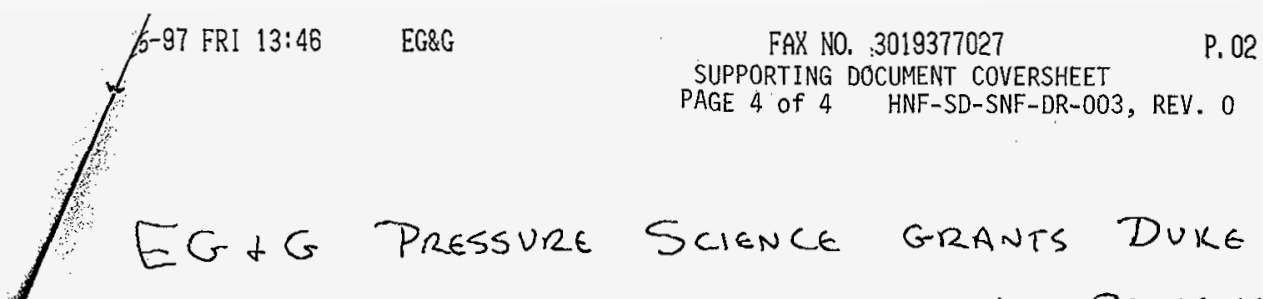
ENGINGERING Services Hanford and parsons. PERMISSION TO USE PART DRAWING NUMBERS $13503 \cdots ; 13632$ AND 13634 in ANY RePORT 3 OR PUBLICATIONS NeCESsARY.

JefF LaYer

ENGINEERING MGR: SEALS

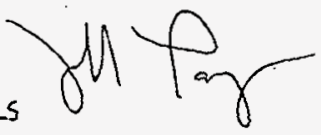
$E G+G$ pressure science 11642 OLD BALTIMORE PIKE BeLTSVILLE MD.

2797

SIGNGD: JEfF LAMER<smiles>CC(=O)NC(=O)C(C)C</smiles> 
HNF-SD-SNF-DR-003, Rev. 0

\section{MULTI-CANISTER OVERPACK DESIGN REPORT}

Prepared for Duke Engineering \& Services, Hanford, Inc.

Contract No. KH-8009-8

Document No. HNF-SD-SNF-DR-003, Revision 0

April 1997

Prepared by: Parsons Infrastructure \& Technology Group, Inc. 


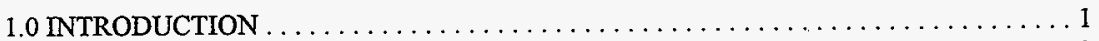

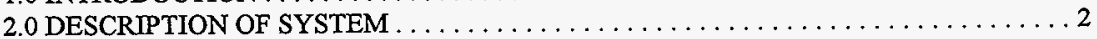

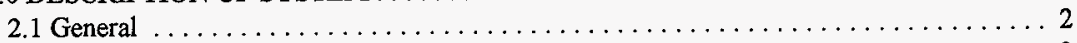

2.2 Interfaces . . . . . . . . . . . . . . . . . . . . . . . . . . . . . 2

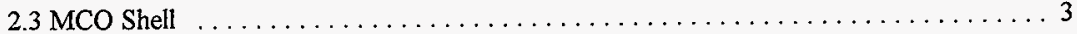

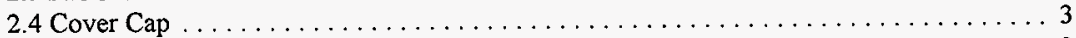

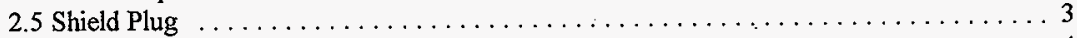

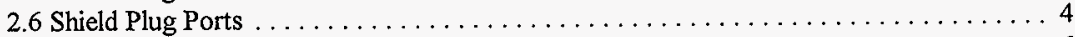

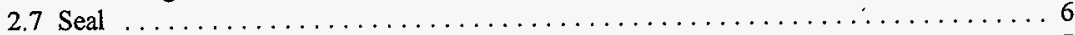

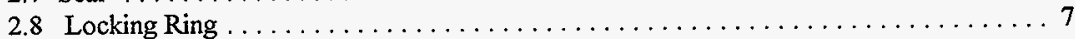

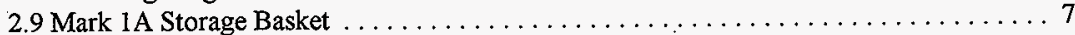

2.10 Mark 1A Scrap Basket . . . . . . . . . . . . . . . . . . . . . . . 8

2.11 Mark IV Storage Basket $\ldots \ldots \ldots \ldots \ldots \ldots \ldots \ldots \ldots \ldots \ldots \ldots \ldots \ldots$

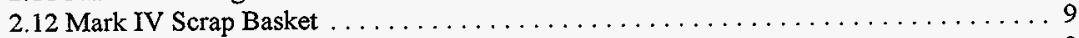

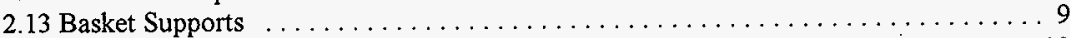

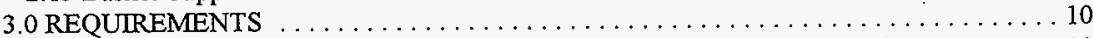

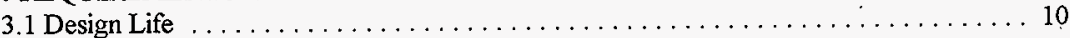

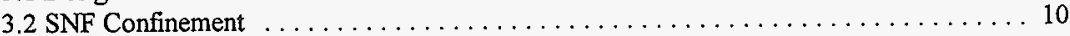

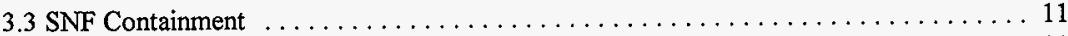

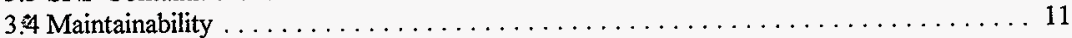

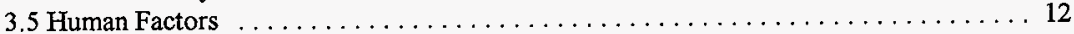

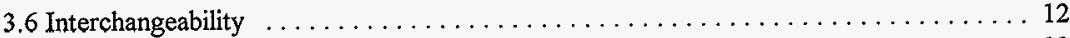

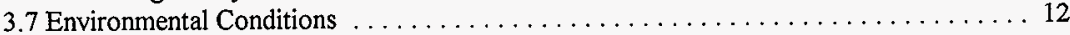

3.8 Transportability . . . . . . . . . . . . . . . . . . . . . . . . 13

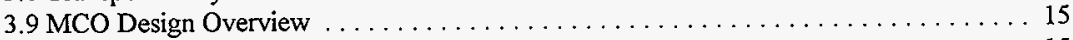

3.9 .1 Code Requirements . . . . . . . . . . . . . . . . . . . . . . . 15

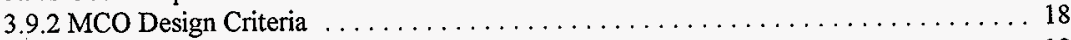

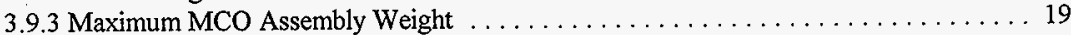

3.9 .4 Height of the MCO . . . . . . . . . . . . . . . . . . . . . . . . 19

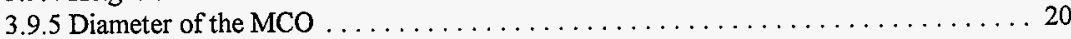

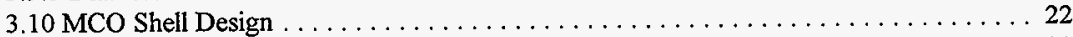

3.11 MCO Closure Design . . . . . . . . . . . . . . . . . . . . . . . 22

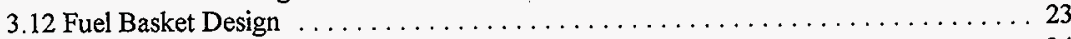

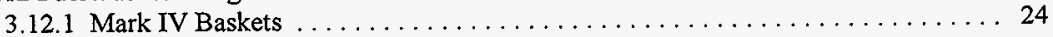

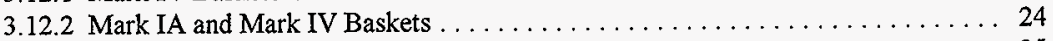

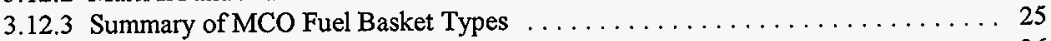

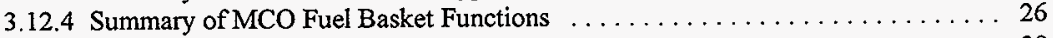

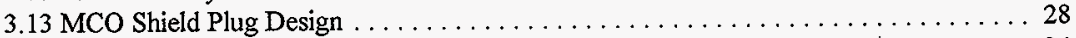

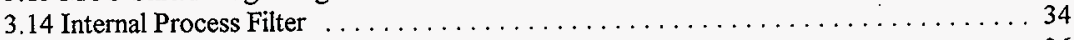

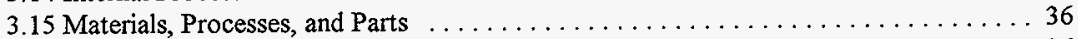

$3.16 \mathrm{MCO}$ Corrosion Control . . . . . . . . . .................... 36 
HNF-SD-SNF-DR-003, Rev. 0

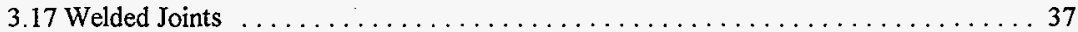

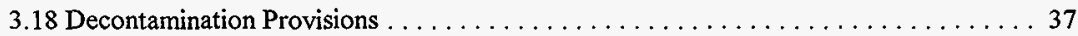

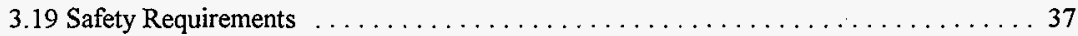

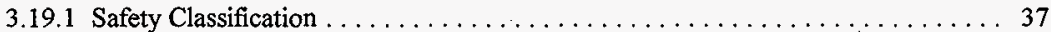

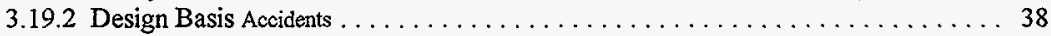

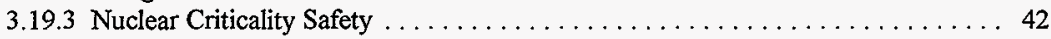

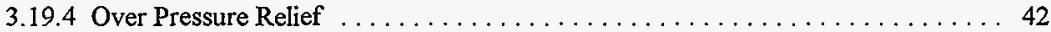

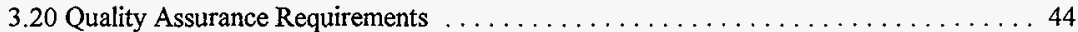

4.0 COMPLIANCE MATRIX . . . . . . . . . . . . . . . . . . . . . 46

5.0 SUMMARY OF COMPLIANCE WITH REQUIREMENTS $\ldots \ldots \ldots \ldots \ldots \ldots \ldots 53$

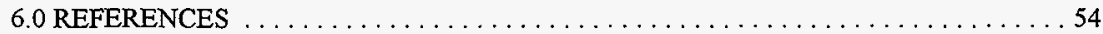

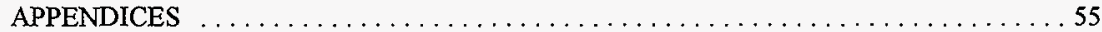


HNF-SD-SNF-DR-003, Rev. 0

\subsection{INTRODUCTION}

The Multi-Canister Overpack (MCO) is a storage/process vessel which will be used to stabilize and store the spent fuel currently stored in the Hanford $\mathrm{K}$ Basins. The spent fuel will be placed in one of four fuel basket designs which will be stacked inside the $\mathrm{MCO}$. The fuel stabilization process will include 1) fuel basket stacking within the $\mathrm{MCO}, 2$ ) transporting of the fuel inside the $\mathrm{MCO}$ to an initial processing facility, 3) cold vacuum drying of the fuel within the $\mathrm{MCO}, 4$ ) transporting of the $\mathrm{MCO} /$ fuel to the Canister Storage Building (CSB) where the MCOs will be placed inside storage tubes while awaiting final processing, 5) transporting of the $\mathrm{MCO} /$ fuel to a hot vacuum drying facility for final processing, 6) transporting of the $\mathrm{MCO} /$ fuel back to the CSB for storage for a period of up to 75 years.

This design report is limited to the features and functions of the MCO and fuel handling/storage baskets relative to the requirements set forth in the Performance Specification for the Spent Nuclear Fuel Multi-Canister Overpack (HNF-S-0426) Rev 3. The design is a modified version of the preliminary design supplied with the Performance Specification. Per the Statement of Work, no changes were made to the preliminary design developed by Duke Engineering Services Hanford, Inc., that were not required to either meet the specification requirements or changes requested by the buyer in the form of a revised Performance Specification.

The Performance Specification limits the evaluation to shielding, structural, and some functional considerations. Criticality, thermal/fluid and interface considerations were not part of the evaluation; however, features were included in the design to accommodate evaluations performed by the buyer and indicated in the Performance Specification. Components such as the relief valve, the filters, as well as lifting interfaces, are the responsibility of the buyer.

As specified in the Performance Specification, the MCO is designed to the "intent" of Section III of the ASME Boiler and Pressure Vessel Code. The welded pressure boundary is designed to Subsection NB of the ASME code with a few exceptions. The outer vessel is not required to meet the code for all accident cases, specifically when it is in the transfer cask. The final closure weld attaching the cover cap is not a full Code weld since it cannot be subjected to a full volumetric inspection. Likewise, a number of other features including the Mark IV baskets are exempt from the code per specification. The exceptions are applied to components that are not required for criticality or containment safety. Where geometry control is required for criticality safety per the Performance Specification, Section III, subsection NG design requirements of the code are applied. In actuality, the need to maintain geometry control requires more restrictive criteria that is met, than that imposed by subsection NG. 
HNF-SD-SNF-DR-003, Rev, 0

\subsection{DESCRIPTION OF SYSTEM}

\subsection{General}

The $\mathrm{MCO}$ is designed to facilitate the removal, processing and storage of the spent nuclear fuel currently stored in the K-Basins, East and West. The stainless steel canister is approximately 24 inches in diameter and 160 -inches long. The shell is fabricated from $1 / 2$ inch $304 \mathrm{~L}$ material. The bottom closure is a forging which is welded to the shell. The top closure consists of a shield plug with four processing ports and a locking ring with jacking bolts to preload the metal seal under the shield plug.

The fuel is placed in one of four types of baskets in the fuel pool. Each basket is then loaded into the $\mathrm{MCO}$ which is inside the transfer cask. Once all of the baskets are loaded in the MCO, a shield plug with process tube is placed in the end of the $\mathrm{MCO}$. This shield plug provides shielding for the workers when the transfer cask containing the $\mathrm{MCO}$ is lifted from the pool and dewatered. After being removed from the pool, the locking ring is installed and the jacking bolts are tightened to preload the metal main closure seal. The MCO is dewatered and vacuumed dried through the process ports. Covers for the process ports may be installed or removed as needed per operating procedures. The MCO is to be transferred to the Canister Storage Building (CSB), in the closed cask. The MCO is then transferred to a storage tube where it is stacked two per storage tube.

At a later time, the MCOs are taken out of the storage tube. Using the process ports on the shield plug, the MCO undergoes Hot Conditioning as a final stabilization of the fuel. At the completion of the fuel stabilization, the cover cap may be placed on the MCO. A temporary lifting device consisting of a threaded pipe or bar with a lifting eye is threaded in to the center opening above the rupture disk for handling purposes. The cover cap is welded to the shell providing a complete welded closure. The $\mathrm{MCO}$ is then placed back into the storage tubes for storage.

Details of all the $\mathrm{MCO}$ components including baskets can be found on the drawings in Appendix 1.

\section{$2.2 \quad$ Interfaces}

The MCO as described above must interface with many exterior components and environments. These include:
A.
Transfer Cask
B. Mark 1A Storage Basket
C. Mark 1A Scrap Basket
D. Mark IV Storage Basket
E. Mark IV Scrap Basket 
HNF-SD-SNF-DR-003, Rev. 0

$\begin{array}{ll}\text { F. } & \text { Fuel Basket Lifting Grapple } \\ \text { G. } & \text { Cold Vacuum Drying System } \\ \text { H. } & \text { Remote operators for Process Ports/valves } \\ \text { I. } & \text { Remote operators for Process Port covers } \\ \text { J. } & \text { Jacking bolts tightening equipment } \\ \text { K. } & \text { Transfer equipment at CSB } \\ \text { L. } & \text { CSB storage tubes } \\ \text { M. } & \text { Hot processing equipment } \\ \text { N. } & \text { Closure Cap installation equipment }\end{array}$

The design insures proper interface with the above components by compliance with the Performance Specification and the interface drawing provided by the Buyer (SK-1-80096).

\subsection{MCO Shell}

The MCO shell provides both confinement and containment of the fuel both during handling and storage. It also provides support for the shield plug and the baskets for stacking, lifting and handling including the expected drops. The shell is constructed out of $304 \mathrm{~L}$ stainless steel. The main section of the shell is fabricated from SA-312 304L 80s pipe. The bottom of the shell is a SA-182 304L machined forging. The top of the shell is another SA-182 $304 \mathrm{~L}$ or $304 \mathrm{LN}$ forging that is machined with a double lead buttress thread and a seal surface for the shield plug to rest on to complete the containment boundary. The nominal wall thickness of the shell is $0.5 \mathrm{in}$.

\subsection{Cover Cap}

The cover cap is a SA-182 304L forging in the shape of a dome that is placed on top of the MCO after the completion of hot conditioning. The purpose of the cover cap is to provide for a welded closure that can meet the leaktightness criteria for containment. The closure weld is a full penetration weld just below the top of the locking ring on the collar of the MCO shell. This weld is a field weld that cannot be volumetrically inspected due to the configuration and contents of the $\mathrm{MCO}$. The exterior of the closure cap is forged and machined to have the same lifting ring configuration as the locking ring so that the containers can be handled after installation of the cover cap.

\subsection{Shield Plug}

The shield plug is a multi functional component of the MCO. It provides a mechanical confinement and containment boundary until the cover cap is welded in place. It also provides for axial shielding to allow personnel access to the top of the MCO for closing the package as well as performing the drying and processing functions. The shield plug also retains the main seal which seals between the 
HNF-SD-SNF-DR-003, Rev. 0

MCO collar and the shield plug. The shield plug has four ports which connect to four penetrations. The first penetration connects to an internal HEPA filter bank that allows for filtered release of gases from the $\mathrm{MCO}$, either through a pressure relief device, or through one of the process ports during vacuum drying or hot processing. The filter bank is protected by a guard plate which will insure that the filters are not crushed during the postulated accident events. The guard plate assembly also protects a short process tube that serves as backup process port as well as a pressure relief port. The guard plate assembly traps air when the shield plug is lowered into the pool and keeps the filter bank dry. The other penetration connects to the long process tube which reaches to the bottom of the $\mathrm{MCO}$ aiding in the removal of water during the dewatering and drying process.

The shield plug and guard plate assembly also mates with the fuel baskets to provide top stability. The coupling of the fuel baskets to the shield plug assembly prevents potential migration of fuel rubble inside the center exclusion cavity, which is required for criticality safety. The machined ports in the shield plug act as valve bodies for the process ports and the relief devices. Each of these ports can also be covered with cover plates that have either orifices in them to control_the pressure relief flow rate or are blank to allow sealing of the $\mathrm{MCO}$ as needed. All of the shield plug port covers are the same, except for the one that connects to the center long process tube. The process tube cover plate has five bolts versus the four bolt pattern for the other ports. This insures proper connections during processing.

\subsection{Shield Plug Ports}

The four shield plug ports, as described above, serve different and unique functions for the handling, processing and safety in the storing of the fuel. One port has the capability of receiving several different types of pressure relief devices. The other three ports have a unique process plug in them. This process plug is a hollow cross-drilled threaded plug that seals when fully engaged and allows flow when the plug is turned out, until the cross drilled holes are exposed and the seal is disengaged. The seal is a special metal seal that has the capability of resealing over five times when the plug is torqued into place. One of the plugs connects to a short process tube which has a rupture disk built into it. This allows the one port to serve as a backup process port as well as the primary over pressure protection port. The rupture disk is designed to fail at the design pressure of the MCO which is 150 psig at $200^{\circ} \mathrm{C}$ and withstand a minimum of 75 psig at $375^{\circ} \mathrm{C}$.

Port number 1 uses a passageway one quarter of an inch in diameter or larger leading to the internal HEPA filter bank. This port is a multi-purpose port and serves the following needs:

1. This port serves as the process pressure relief of the $\mathrm{MCO}$ during the period of time when the $\mathrm{MCO}$ is producing gases intemally. This relief path allows the excess gasses to leave the $\mathrm{MCO}$ at a pressure which is above surrounding atmospheric pressure but is still well below the safety class pressure relief rupture disk setting for the MCO. This port has a Process 
Pressure Relief Valve (PPRV) installed at the port. This valve will reseat once the MCO internal pressure has bled down to the reset pressure for the PPRV. This mode of operation for the MCO would be likely during staging at the Canister Storage Building (CSB). This mode of operation allows the MCO to transfer internal pressure to the surrounding atmosphere effectively in one direction only, i.e. out of the $\mathrm{MCO}$.

2. This port may also serve as a receptacle for a process rupture disk to relieve pressure from the inside of the MCO. After HCS, a rupture disk is installed in this port to relieve the pressure. Once actuated this rupture disk allows the $\mathrm{MCO}$ to transfer in both directions with its surrounding atmosphere. The relief setting for this process rupture disk would be at a pressure well below the actuation setting for the safety class rupture disk.

3. As a possible option, this port also serves as a receptacle for an external HEPA filter holder. This port, when configured with a external HEPA, allows the MCO to be staged or stored at roughly Canister Storage Building (CSB) storage tube pressure. Using this option allows the $\mathrm{MCO}$ to communicate both ways with the surrounding atmosphere in the tube.

The application of a restrictive orifice cover over the process pressure relief, to limit the flow out of the MCO, to the equivalent of a $1 / 4$ inch orifice, is possible, if needed, with the implementation of this scenario. The orifice plate, if needed, is planned to be used during staging and after HCS is complete. Prevention of disk pieces and debris from getting into the orifice is considered in the design. It is also possible for the installed appliance to also serve as the restrictive orifice. Thus a cover, installed for orifice reasons, may not be needed in this case.

Path configuration of Port Number 1

The PPRV may be installed in the shield plug port at the factory or poolside at the basins before the shield plug is inserted into the fueled MCO in the load out pool water. The PPRV stays installed through staging phase at the CSB. If the PPRV adequately limits the flow for the needs of the processes, CVD, and CSB, then the PPRV will need a cover only for the physical protection of the shield plug installed PPRV appliance when the $\mathrm{MCO}$ is in the cask and when there is a danger of a heavy fixtures such as the cask lifting fixture being dropped on the appliance in the shield plug port. This cover would likely have a large hole to allow venting that would not limit gas flow release rates but still physically protect the appliance. This cover is the same as the orifice cover, but with a larger hole in the plate so there is no appreciable gas flow restriction.

The MCO may have a rupture disk, plug, or external HEPA filter installed in this port at any time as directed by operating procedures. The multi-functions of Port 1 is accomplished by making a threaded connection that will receive either a $3 / 4$ NPS threaded HEPA filter, plug, rupture disk or a bushing that will allow adaptation to a $1 / 8$ inch relief valve. The seals used are the similar to those 
HNF-SD-SNF-DR-003, Rev. 0

on the process ports. This seal is readily adaptable to straight pipe thread, captured for remote installation and replaceable without damage to the seal surface. The seal to the relief valve may not need as high a quality seal since using standard tapered pipe threads probably will give the same leakage rate as the relief valve. In accordance with the specification, Port 1 connects up to a oneinch in penetration leading to the internal HEPA filter bank.

Port 2 is a process plug, as described above, that connects with a one-inch penetration to the filter bank.

Port 3 connects with a 0.54 -inch (minimum diameter) to the center long process tube. It has a process plug. The flange cover, as indicated earlier, has a five bolt cover flange and sealing surface that can mate to the operator tool.

Port 4 connects to the short process tube through a one-inch diameter connection. The process plug it contains differs from the others by having a one-inch ASME certified rupture disk built into the upper portion instead of being solid. The rupture disk is replaceable by replacing the process plug. The cover flange has a four hole pattern that can contain either a solid flange for sealing or a flange with a $1 / 4$ inch orifice in it. All the cover plates have capture bolts which allow for remote removal and installation of the covers. The cover plates use a metal C-seal that requires a relatively low sealing pressure and has the capability to "snap" on to the cover plates by being slightly eccentric to facilitate remote handling. The rupture disk is sandwiched between the top of the plug (the actuator and discharge vents)and the bottom of the plug. These two parts are welded together in such a manner that the rupture disk is also welded to the bottom of the plug (see Appendix 1). This pressure boundary weld can not be fully volumetrically inspected and hence is an exception to Subsection NB, requirements Reference [2]. The weld has a large margin of safety as shown in Appendix 10.

The metal seal that is used for the process plugs is an Inconel C-seal plated with gold, produced by $E G \& G$ pressure seals. This seal has been demonstrated to reseal over five times. The seal plating allows it to seal at the high temperatures specified $\left(375^{\circ} \mathrm{C}\right)$ and still meet the reseal requirements.

\subsection{Seal}

The primary seal for the MCO seals the MCO shell to the shield plug. It is a Helicoflex seal comprised of a high strength alloy spring covered with a 300 series stainless steel jacket covered by a silver outer jacket. The seal is held in place with five stainless steel clips that are screwed to the shield plug. This seal, when properly preloaded (recommended minimum value of 1700 pounds per inch of seal), will maintain a leak tight condition. The analysis in Appendix 4 uses a preload value of 1850 pounds/inch which was specified originally for the seal and consistent with the published literature (Appendix 13). Recent data from the vendor shows seating loads as low as 1700 pounds 
per inch are acceptable, providing for a larger margin of safety. The seal is similar to the standard mechanical seals approved by the NRC for dry fuel storage applications.

\section{$2.8 \quad$ Locking Ring}

The preload on the seal is maintained by the use of a locking ring and jacking bolt arrangement. The locking ring is a stainless steel forging that is threaded into the buttress threads on the MCO collar after the MCO is removed from the pool. This ring serves two functions. It provides a grapple interface for handling the $\mathrm{MCO}$ and it provides support for the jacking bolts that preload the shield plug and the seal. Eighteen SA-193 B8M 1-1/2 inch set screws are threaded into the locking ring. The screws serve as jacking bolts to preload the seal and were sized to maintain the appropriate preload throughout the operating temperature range while not allowing any of the joint components to yield. Component yielding, during a thermal transient, could result in unacceptable preload losses. The set screws are to be torqued to $189 \mathrm{ft}-1 \mathrm{bs}$ with an accuracy of plus or minus $3 \%$. See Appendix 4 for details.

\subsection{Mark 1A Storage Basket}

The Mark $1 \mathrm{~A}$ storage basket is designed to hold 48 Mark 1A fuel assemblies, which are basically intact. The fuel assemblies, when fabricated, had the dimensions shown in Table 1. The Mark $1 \mathrm{~A}$ fuel differs from the Mark IV fuel in that it is shorter and smaller in diameter. The fuel is more reactive which imposes certain criticality control requirements on the Mark 1A basket. The controlling item is the need to maintain the center six-inch pipe within two inches of center for criticality control and to ensure that no fuel particles greater than $2 \mathrm{~mm}$ can get inside the pipe during all imposed load conditions.

The basket consists of a center six-inch pipe welded to a bottom support plate that also has six support rods at its periphery. The center pipe and six support rods, which are parallel to the center pipe, make up the main structural members of the basket. The basket also has a sheet metal shroud enveloping the six rods and an aluminum spacing plate in the bottom to help position the fuel during handling. The spacer plate has 48 holes to receive the fuel. Between the bottom plate and the spacer plate is a layer of expanded metal to keep the fuel from resting directly on the plate. The expanded metal layer prevents gas flow blockage through the 1/4-inch diameter holes in the bottom support plate. Neither the expanded metal nor the spacer plate are connected to the support plate. This is done to minimize the inertia loading to the bottom plate during the side drop accident condition. The six support rods on the periphery of the plate are trapezoidal in shape and the lengths are toleranced so that they share the load with the center tube during axial loading. This is done to ensure that the bottom plate is not deformed significantly and can still support the center pipe in position during a horizontal drop following a vertical drop. All of the structural members of the Mark 1A basket are designed to meet ASME Section III Subsection NG requirements, supplemented by criticality 
control deformation limits from the Performance Specification. See Appendix 7 for more details. As can be seen in the Appendix 1 drawings, the top of the center pipe has an end piece that extends up into the next basket above, or into the centering device on the guardplate on the shield plug assembly. This allows the center pipe to be supported at both ends during the side drop loading. Also the end fits snugly into the basket above so that in the worst orientation a $2 \mathrm{~mm}$ particle can not enter the center of the pipe. The top of the center pipe interfaces with a lifting grapple. The load path down through the center pipe, through the bottom plate, complies with ANSI N14.6 Reference [5]. The basket is lifted by an internal grove in the end piece of the center pipe. The lifting device is the responsibility of the buyer.

All welded portions of the Mark 1A storage basket is fabricated from 304L austenitic stainless steel. The spring pins used in assembly are made from 302 or 304 stainless steel, and conform to ASME B 18.8.2-1994.

Table 1. Fuel Dimensions

\begin{tabular}{c|cc}
\hline & Mark IV & Mark 1A \\
\hline Length cm (in) Max & $66.3(26.1)$ & $53.1(20.91)$ \\
& & \\
Diameter cm (in) & $6.15(2.42)$ & $6.10(2.40)$ \\
\hline
\end{tabular}

\subsection{Mark 1A Scrap Basket}

The Mark 1A scrap basket is similar in design to the Mark 1A storage basket. All of the structural components are identical. The same requirements for criticality control and ASME Code apply. The major difference is that the shroud extends the full length of the support rods and there is no aluminum positioning plate or expanded metal on the support plate. There are also six dividers that create compartments in the basket cavity. These sheetmetal dividers aid in the loading of the damaged fuel. To ensure adequate gas flow through the basket there are flow deflectors on the exterior of the shroud to force the gas flow through the holes in the bottom plate. The lifting of the basket is by the same means as the storage basket and complies with ANSI N 14.6 Reference [5].

\subsection{Mark IV Storage Basket}

The Mark IV storage basket is designed to hold up to 54 intact Mark IV fuel assemblies. Unlike the Mark 1A basket the Mark IV basket has no deformation limits for criticality control and hence has no requirements except for self support and meeting the lifting requirements of ANSI N14.6. To meet these requirements only minor modifications of the design supplied by the buyer was required. 
The design has a 2.75 -inch diameter center tube that is connected to a spacer plate consisting of a plate with 54 holes for the fuel with $3 / 8 \mathrm{X}^{1 / 4}$ inch bars to support the fuel at the bottom of the plate. The basket above is supported by the center tube and six 1-1/4 inch diameter round bars. The basket also has a 11 inch high non structural shroud to help in handling the fuel. The entire basket is made from $304 \mathrm{~L}$ stainless steel.

Like the Mark 1A baskets, the Mark IV basket is lifted from a groove on the interior of the center tube. The center tube extends above the support rods and up into the basket above. This coupling aids in the basket stability and facilitates the insertion of the process tube down the center of the basket.

\subsection{Mark IV Scrap Basket}

The Mark IV scrap basket is designed to handle damaged Mark IV fuel. The scrap basket design includes a 2.75 -inch diameter center tube that connects to $\mathrm{a} / / 4$ inch thick plate perforated with $1 / 4$-inch diameter holes. The basket has a full height shroud connected to six divider plates. The shroud and divider plates provide structural support to the baskets above as well as to the bottom plate. All components are welded together and are fabricated from 304L material. The scrap basket is required to meet the same criteria as that of the storage basket. Like the Mark 1A scrap basket the Mark IV scrap basket has flow deflectors on the exterior of the shroud to direct the processing gas flow through the bottom plate.

\subsection{Basket Supports}

The baskets are supported by a spider at the bottom of the MCO that keeps the baskets approximately 1.5 inches away from the bottom plate. This plenum, combined with a low point machined in the bottom forging aids in the removal of water by the long process tube and the distribution of process gases during drying and processing. The baskets are centered by a process tube guide cone which is in the center of the spider. The one-inch NPS process tube is inserted down the center of the baskets to remove the water and circulate process gases. The process tube, especially for the Mark IV baskets, provide some centering support for the baskets during non-axial load conditions.

At the top, as part of the shield plug/guardplate assembly, a MCO basket Stabilizer Extension mates with the center tube extensions for both basket designs to provide a locking mechanism to stabilize the baskets during non axial loadings. As with the bottom components the stabilizer is constructed of $304 \mathrm{~L}$ stainless. The stabilizer is designed to accommodate six Mark 1A baskets or five Mark IV baskets while maintaining a minimum space of one inch nominal and will maintain coupling in the worst tolerance undersize condition. 


\subsection{REQUIREMENTS}

This section establishes the essential requirements needed to define $\mathrm{MCO}$ performance, physical and quality characteristics, environmental conditions, and transportability. The italicized text below each requirement describes how the design complies.

\subsection{Design Life}

The MCO shall maintain fuel elements and fuel fragments in a critically safe array throughout its design life of 40 years both before and after being subjected to the design basis accidents described in Section 1 of Reference 1. The MCO shall not knowingly have design features that would prevent it's design life from being extended to a total of 75 years. Design life of the pressure relief valve shall be six years.

\section{Refer to Section 4, Item 1.}

The $M C O$ is constructed from austenitic stainless steel with high resistance to corrosion from all aspects of the environment the system is expected to see over the expected lifetime. There are no components making up the system that have any known mechanism that will cause the system not to sustain the require design lifetime. None of the design basis accidents described is known to prevent the MCO from completing the expected lifetime or extending it to 75 years. The relief valve is specified by DESH to having an expected design life greater than the required 6 years based on the expected usage.

\subsection{SNF Confinement}

The MCO shall confine its contents during all normal operations and after being subjected to the design basis accidents described in Section 4.19.2 of Reference 6.1. The MCO shall be designed to facilitate confinement while process connections are being made and in conjunction with process piping during process operations. This confinement requirement does not apply to a pressure relief discharge path during actuation of any $\mathrm{MCO}, \mathrm{CVD}$ or $\mathrm{HCS}$ pressure relief device.

\section{Refer to Section 4, Item 2.}

The MCO is designed to confine its contents during all normal operation and during the design basis accidents described in Reference [6.1]. The confinement is met by a 304L stainless steel shell that is closed with a mechanical seal between the shield plug and the shell. The vessel is designed to and fabricated in accordance with the intent of the ASME Code Section III Division 1 Subsection NB. Reference [6.2]. Exceptions to the code are listed in Appendix 18. The process plugs are designed to couple with an operator tool that will allow them to be operated within a sealed environment providing the confinement requested. See Appendix 1. 


\subsection{SNF Containment}

The MCO shall maintain its containment capabilities during and after being subjected to the design basis accidents described in Section 4.19.2 of Performance Specification Rev. 3, except for the cask drops as noted in Section 4.19.2 of the Performance Specification Rev. 3. During Hanford on-site transportation, process operations, and staging at CSB prior to hot conditioning, the total gaseous leakage across the MCO pressure boundary, including process connection seals but excluding controlled flow through any port, shall not exceed $1 \times 10^{-4} \mathrm{SCC} / \mathrm{sec}$. This gaseous leakage rate is based on a clean seal and a clean sealing surface at the final mechanical closure boundary. The MCO, when sealed by welding after hot conditioning, shall be capable of not exceeding a maximum total leak rate of $1 \times 10^{-7} \mathrm{SCC} / \mathrm{sec}$.

\section{Refer to Section 4, Item 3.}

The containment is provided by the same shell and seal system provided above. The port operators valves and pressure relief devices are designed to meet the $1 \times 10^{-4} \mathrm{scc} / \mathrm{sec}$ requirements. To provide additional containment all ports are designed to have covers with metal seals. These seals are capable, as is the main seal, of meeting the $1 \times 10^{-4}$ scc/sec criteria. The shell is designed and analyzed for the design basis to comply with Reference [6.2]. The shell is not required to meet Reference [6.2] when it is in the cask for the horizontal and corner loadings. This due to the cask being designed with a collar that provides a ring/ point load to the shell. The shell being fabricated from material with high elongation has the potential of deforming with out breaching over this feature but would have localized stresses above the allowables.

During fabrication the shell is leak tested to $1 \times 10^{-7} \mathrm{SCC} / \mathrm{sec}$ to verify its containment capability. The closure cap can not be leak tested after installation due to the configuration. The closure weld can not be volumetrically inspected, hence it can not fully comply with a Reference [6.2]. This is an exception to the code compliance and is fairly standard with commercial sealed dry fuel storage canisters. The design here differs, since the NRC typically requires redundant closure welds. The $M C O$ could provide redundant seals if the port covers were in place prior to the installation of the cover cap.

\subsection{Maintainability}

The MCO shall be designed to minimize the need for preventative maintenance throughout its design life. The MCO shall be designed to allow replacement of the externally mounted pressure relief devices/HEPA filter.

Refer to Section 4, Item 4.

As can be seen in the detailed drawing in Appendix 1 and discussed in Appendix 2 the MCO is designed entirely out of austenitic stainless steel which provides for basically a maintenance free package during its expected lifetime for the expected environment. The process plugs, covers and relief devices are designed for remote operation to facilitate maintenance on those components. 
package during its expected lifetime for the expected environment. The process plugs, covers and relief devices are designed for remote operation to facilitate maintenance on those components.

\subsection{Human Factors}

The MCO components shall be designed to facilitate handling and assembly with the use of appropriate handling equipment. The MCO design shall also enable handling while wearing protective clothing used in radiation zones (e.g. coveralls, gloves, booties, mask, breathing apparatus, etc.).

\section{Refer to Section 4, Item 5.}

The MCO components can all be handled with remote equipment and personnel in protective clothing. Small components such as seals and bolts are captured or fastened to the larger components that can be handled with the aid of hoists, cranes, etc. The MCO shell can be handled by threading in the locking ring and hoisting from the locking ring. The shield plug can be handled by attaching a lifting device to the bolts holes provided in the top section. The baskets can be handled by their lifting device provided by others. The cover plate seals are snapped into place by the used of elliptical seals. The main seal is held into place by small clips.

\subsection{Interchangeability}

To the maximum extent possible (design goal), $\mathrm{MCO}$ components with like functions shall be interchangeable (i.e., any set of like baskets can be loaded into any MCO shell, any MCO shield plug and locking ring can be used to close and seal any MCO shell, etc.).

The MCO shell, shield plug, lifting ring, cover cap, and the baskets shall have unique identification numbers for tracking and accountability purposes.

\section{Refer to Section 4, Item 6.}

All components are dimensioned as shown in Appendix 1 so that after welding and final machining they are all interchangeable. The drawings and the fabrication specification provide for the Buyer supplied numbering and marking system that provides for the required tracking and accountability.

\subsection{Environmental Conditions}

The MCO shall be capable of performing its mission while subjected to the environmental conditions listed in Table 3.1.

Refer to Section 4, Item 7. 
The selection of materials in Appendix 2 and the applicable stress values used for the materials in the analysis performed in Appendices 4-12 insure that the MCO and the fuel baskets are fully functional for the environmental conditions stated above. All containment seals are also selected for these environments, as are the process plug seals.

\subsection{Transportability}

After fabrication, $\mathrm{MCO}$ components shall be transportable by highway from the fabricator facility to the location within the Hanford site where they will be warehoused until requested for the packaging and removal of SNF.

\section{Refer to Section 4, Item 8.}

The dimensions of the MCO parts, as shown in Appendix l permit transport from the fabricator facility to the warehouse site within Hanford. Suggested packaging of the components for highway transport is provided in the fabrication specification. 
HNF-SD-SNF-DR-003, Rev. 0

Table 3.1. External Environmental Conditions (as seen by MCO)

\begin{tabular}{|c|c|}
\hline Parameter & Condition \\
\hline \multicolumn{2}{|l|}{ Hanford Site: } \\
\hline Temperature (Air) & $\begin{array}{l}\text { Range: }-33 \text { to } 46^{\circ} \mathrm{C}\left(-27 \text { to } 115^{\circ} \mathrm{F}\right) \\
\text { Rate of Increase: } 14^{\circ} \mathrm{C}\left(26^{\circ} \mathrm{F}\right) \text { in } 20 \text { minutes } \\
\text { Rate of decrease: } 13^{\circ} \mathrm{C}\left(24^{\circ} \mathrm{F}\right) \text { in } 1 \text { hour }\end{array}$ \\
\hline Relative Humidity & $\begin{array}{l}\text { Range: } 5 \text { to } 100 \% \\
\text { Rate of Change: Negligible }\end{array}$ \\
\hline \multicolumn{2}{|l|}{ K Basin Storage Pool: } \\
\hline Temperature (Water) & $\begin{array}{l}\text { Current Range: } 6^{\circ} \mathrm{C} \text { to } 38^{\circ} \mathrm{C}\left(43^{\circ} \mathrm{F} \text { to } 100^{\circ} \mathrm{F}\right) \\
\text { Maximum Allowable: } 38^{\circ} \mathrm{C}\left(100^{\circ} \mathrm{F}\right) \text { [see 3.3.1.3] }\end{array}$ \\
\hline $\mathrm{pH}$ & $\begin{array}{lc}\text { Current Range: } & \mathrm{KE}: 5.5 \text { to } 7.5 \\
& \mathrm{KW}: 5.5 \text { to } 7.5 \\
\text { Allowable Range: } & \mathrm{KE} \text { and } \mathrm{KW}: 5.0 \text { to } 9.5\end{array}$ \\
\hline Electrical Conductivity & 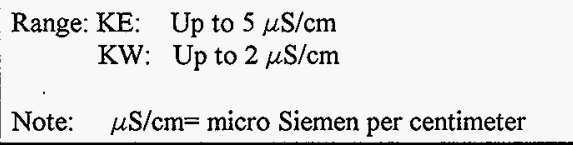 \\
\hline Chloride Content & less than $1 \mathrm{ppm}$ \\
\hline Nitrate Content & less than $1 \mathrm{ppm}$ \\
\hline Sulfate Content & less than $1 \mathrm{ppm}$ \\
\hline Phosphate Content & less than $1 \mathrm{ppm}$ \\
\hline Fluoride Content & $.25 \mathrm{ppm}$ \\
\hline Sodium Content & $1 \mathrm{ppm}$ \\
\hline Calcium Content & $2 \mathrm{ppm}$ \\
\hline Iron Content & $1 \mathrm{ppm}$ \\
\hline \multicolumn{2}{|c|}{ Cold Vacuum Drying Facility: } \\
\hline Temperature & $10^{\circ} \mathrm{C}$ to $75^{\circ} \mathrm{C}$ \\
\hline Transportation: & \\
\hline
\end{tabular}


HNF-SD-SNF-DR-003, Rev. 0

\begin{tabular}{|l|l|}
\hline \multicolumn{1}{|c|}{ Parameter } & \multicolumn{1}{|c|}{ Condition } \\
\hline Transportation: & $0^{\circ} \mathrm{C}$ to $75^{\circ} \mathrm{C}$ \\
\hline Temperature & \multicolumn{2}{|l|}{} \\
\hline CBS (Staging): & $10^{\circ} \mathrm{C}$ to $132^{\circ} \mathrm{C}$ \\
\hline Temperature (Tube gas) & Refer to Chapter 4 of HNF-SD-TP-RTP-004, Rev. 0 \\
\hline Temperature Cycling & Refer to Chapter 8 of HNF-SD-TP-RTP-004, Rev. 0 \\
\hline Relative Humidity & \multicolumn{2}{|l}{} \\
\hline Hot Conditioning System: & $25^{\circ} \mathrm{C}$ to $375^{\circ} \mathrm{C}$ \\
\hline Temperature & $10^{\circ}$ to $132^{\circ} \mathrm{C}$ \\
\hline CBS (Storage): & Refer to Chapter 4 of HNF-SD-TP-RTP-004, Rev. 0 \\
\hline Temperature (Tube) & Refer to Chapter 8 of HNF-SD-TP-RTP-004, Rev. 0 \\
\hline Temperature Cycling &
\end{tabular}

\subsection{MCO Design Overview}

\subsubsection{Code Requirements}

The MCO shall be designed in accordance with DOE Order 6430.1A, General Design Criteria (DOE 1989). Safety Class (SC) and Safety Significant Components (SS), providing fuel containment, confinement, and criticality control, shall be constructed to meet the intent of ASME Boiler and Pressure Vessel Code, Section III, to the extent specified in this Performance Specification, Rules for Construction of Nuclear Power Plant Components, Subsection NB (ASME 1995) under the component safety group as guided by the NUREG/CR 3854, UCRL-53544, Fabrication Criteria for Shipping Containers, 1984. The Nuclear Regulatory Commission (NRC) positions in Regulatory Guides 1.84 and 1.85 on ASME Section III code cases shall be reviewed prior to using such code cases for safety class applications for the MCO. All deviation from Subsection NB shall be documented and justified. (Appendix C, ASME Code Notes and Exceptions, is provided as a guide to the relevant ASME code. Appendix C contains a list of potential exceptions to the ASME Boiler and Pressure Vessel Code (Subsection NB) that may be applied for MCO construction; others may need to be added as the design evolves.).

Refer to Section 4, Item 9. 
The applicable sections of the ASME code are applied to the various components of the MCO in accordance with the Safety Analysis Manual HNF-CM-4-46 as shown below.

\begin{tabular}{|c|c|c|c|}
\hline System/Component & Function & $\begin{array}{c}\text { SSC } \\
\text { Designation }\end{array}$ & Failure Consequences \\
\hline \multicolumn{4}{|c|}{ MCO Components } \\
\hline Shell & Contain/Protect SNF & SC & $\begin{array}{l}\text { Release of radioactive contents which } \\
\text { could exceed offsite exposure limits; loss } \\
\text { of contingency protection against nuclear } \\
\text { criticality accident }\end{array}$ \\
\hline Shield Plug & $\begin{array}{l}\text { Contain SNF, protect } \\
\text { personnel }\end{array}$ & SC & $\begin{array}{l}\text { Release of radioactive contents which } \\
\text { could exceed offsite exposure limits }\end{array}$ \\
\hline L\&L Ring + Bolts & $\begin{array}{l}\text { Maintain pressure on } \\
\text { main seal, allows for } \\
\text { lift of loaded MCO }\end{array}$ & SC & $\begin{array}{l}\text { Release of radioactive contents which } \\
\text { could exceed offsite exposure limits }\end{array}$ \\
\hline Cover Cap & $\begin{array}{l}\text { Seal MCO after hot } \\
\text { conditioning }\end{array}$ & SC & $\begin{array}{l}\text { Release of radioactive contents which } \\
\text { could exceed offsite exposure limits }\end{array}$ \\
\hline IA Baskets & $\begin{array}{l}\text { Maintain MK IA SNF } \\
\text { elements and scrap in a } \\
\text { Critically Safe } \\
\text { Configuration }\end{array}$ & SC & $\begin{array}{l}\text { Loss of double-contingency protection } \\
\text { against nuclear criticality accident }\end{array}$ \\
\hline IV Baskets & $\begin{array}{l}\text { Hold MK IV SNF } \\
\text { elements and scrap }\end{array}$ & GS & No release consequences \\
\hline Primary Rupture Disk & $\begin{array}{l}\text { Protect MCO pressure } \\
\text { boundary }\end{array}$ & SC & $\begin{array}{l}\text { Overpressurization of } \mathrm{MCO} \text { resulting in an } \\
\text { uncontrolled release which could exceed } \\
\text { offsite exposure limits }\end{array}$ \\
\hline Plug Valves & $\begin{array}{l}\text { Process ports to } \\
\text { accommodate gas } \\
\text { flows in support of } \\
\text { MCO processing }\end{array}$ & SS & $\begin{array}{l}\text { Inability to process the } \mathrm{MCO} \text {, release of } \\
\text { radioactive materials into the environment } \\
\text { which exceed exposure limits }\end{array}$ \\
\hline Process Internal Filter & $\begin{array}{l}\text { Maintain most } \\
\text { radioactive solid } \\
\text { materials within the } \\
\text { MCO }\end{array}$ & SS & $\begin{array}{l}\text { Release of radioactive materials from the } \\
\mathrm{MCO} \text {, pressure buildup within the } \mathrm{MCO} \text {, } \\
\text { loss of defense in depth protection for } \\
\text { release of radioactive materiais }\end{array}$ \\
\hline
\end{tabular}


HNF-SD-SNF-DR-003, Rev. 0

\begin{tabular}{|c|c|c|c|}
\hline System/Component & Function & $\begin{array}{c}\text { SSC } \\
\text { Designation }\end{array}$ & Failure Consequences \\
\hline External HEPA & $\begin{array}{l}\text { Allows MCO to be } \\
\text { stored in a vented } \\
\text { configuration at the } \\
\text { surrounding } \\
\text { atmospheric pressure }\end{array}$ & SS & $\begin{array}{l}\text { Release of radioactive contaminants into } \\
\text { the storage tube, pressure buildup within } \\
\text { the } \mathrm{MCO} \text {, loss of defense-in-depth } \\
\text { protection for release of radioactive } \\
\text { materials }\end{array}$ \\
\hline Long Process Tube & $\begin{array}{l}\text { Bulk water removal, } \\
\text { introduction of gases } \\
\text { during processing, and } \\
\text { reflooding, if necessary }\end{array}$ & SS & $\begin{array}{l}\text { Inability to remove water from } \mathrm{MCO} \text {, } \\
\text { inability to introduce gases to process } \\
\mathrm{MCO} \text {, prevents processing which puts the } \\
\mathrm{MCO} \text { into a safe configuration }\end{array}$ \\
\hline Short Process Tube & $\begin{array}{l}\text { Possible water removal } \\
\text { prior to shipping to } \\
\text { CVD, connects to } \\
\text { ruprure disk as vent } \\
\text { path, backup process } \\
\text { exit }\end{array}$ & SC & $\begin{array}{l}\text { Rupture disk failure to relieve internal } \\
\text { MCO pressure, inability to remove water } \\
\text { prior to shipping to CVD }\end{array}$ \\
\hline $2 \mathrm{~mm}$ Process Tube Screens & $\begin{array}{l}\text { Keep particles }<2 \mathrm{~mm} \\
\text { in diameter in the MCO }\end{array}$ & SS & $\begin{array}{l}\text { Particles larger than } 2 \mathrm{~mm} \text { may leave the } \\
\mathrm{MCO}\end{array}$ \\
\hline Seal & $\begin{array}{l}\text { Seals MCO shield plug } \\
\text { to shell }\end{array}$ & SS & $\begin{array}{l}\text { Release of radioactive materials from the } \\
\text { MCO, pressure buildup within the } \mathrm{MCO} \text {, } \\
\text { loss of defense - depth protection for } \\
\text { release of radioactive materials }\end{array}$ \\
\hline Orifice Plates & $\begin{array}{l}\text { Regulate gas flow from } \\
\text { the } \mathrm{MCO}\end{array}$ & SS & $\begin{array}{l}\text { Unregulated gas release rate from the } \\
\mathrm{MCO}\end{array}$ \\
\hline Process Relief Valve & $\begin{array}{l}\text { Allows gases to leave } \\
\text { MCO at a pressure } \\
\text { below safety class } \\
\text { pressure retief device } \\
\text { setting }\end{array}$ & SS & $\begin{array}{l}\text { Pressure buildup within the } \mathrm{MCO} \text { resulting } \\
\text { in venting through rupture disk }\end{array}$ \\
\hline Guard Plate on Shield Plug & $\begin{array}{l}\text { Protects internal } \mathrm{MCO} \\
\text { process filter, short } \\
\text { process tube and } 2 \mathrm{~mm} \\
\text { screen }\end{array}$ & SS & $\begin{array}{l}\text { Potential damage to filter, short process } \\
\text { tube, and screen }\end{array}$ \\
\hline Basket Grapple Receptacle & $\begin{array}{l}\text { Provides an interface } \\
\text { between the baskets } \\
\text { and the MCO Loading } \\
\text { System }\end{array}$ & SS & $\begin{array}{l}\text { Fuel basket drop resulting in spilled fuel } \\
\text { within the basin }\end{array}$ \\
\hline
\end{tabular}


The Sections of the ASME Code Section III Division 1, Subsection NB, Reference [6.2] and Subsection NG. Reference [6.3] are applied to the containment boundary and Mark $1 \mathrm{~A}$ baskets, respectively. The containment boundary is an SC item, since its failure would result in a potential release. The Mark $1 A$ baskets require SC consideration since the geometric control is required for criticality control. The MCO and its fuel baskets are designed to the intent of the Code where applicable and are not Code stamped components. The significant exceptions to the Code for the containment vessel is the lack of any volumetric NDE of the final closure weld where the cover cap is welded to the MCO collar. The Mark $1 A$ baskets are assembled with spring pins that are unobtainable with all the Code material requirements. SS items are designed and fabricated to applicable sections of the ASME Code as set by the Performance Specification. GS items (Mark IV baskets) are evaluated to the applicable code specified by the Performance Specification (ANSI N14.6).

\subsubsection{MCO Design Criteria}

The MCO design shall implement the following criteria:

- No ASME Code stamp required

- Design pressure: 150 psig

- Design temperature: $375^{\circ} \mathrm{C}$

- Processing operating pressure: full vacuum internal with $25 \mathrm{psig}$ external pressure, at $75^{\circ} \mathrm{C}$

- Processing operating pressure: full vacuum internal with 0 psig external pressure, up to $375^{\circ} \mathrm{C}$

- Processing operating pressure: $75 \mathrm{psig}$ internal with 0 psig external pressure up to $375^{\circ} \mathrm{C}$

- Post processing maximum steady state pressure and temperature: $150 \mathrm{psig}$ internal, up to $132^{\circ} \mathrm{C}$

- Design thermal transient under normal HCS conditions (excluding cover cap weld) of $100^{\circ} \mathrm{C} / \mathrm{h}$ applicable from 20 to $350^{\circ} \mathrm{C}$ over a maximum of five (5) full thermal cycles

- The MCO assembly must be designed to accommodate 1.0 inch differential thermal expansion, in axial direction, between the basket stack and the MCO shell and maintain basket nesting and engagement of the top basket with the shield plug

- Maximum allowed radial temperature gradient between the outside of the MCO shell and the center of the $\mathrm{MCO}$ shield plug of $100^{\circ} \mathrm{C}$, and a design radial temperature difference within the $\mathrm{MCO}$ shell wall of $5^{\circ} \mathrm{C}$

The axial temperature distribution is as follows and as shown in the temperature distribution profile (Figure 1). Figure 1 illustrates the estimated wall temperatures along the MCO length during the heat up and cool down cycles of the hot conditioning process. 
HNF-SD-SNF-DR-003, Rev. 0

The curves presented on the figure are defined as follows:

$\begin{array}{ll}\text { TPLUG - } & \text { average temperature of MCO closure plug } \\ \text { T3 - } & \text { inlet air temperature } \\ \text { TA270 - } & \text { temperature of MCO sidewall at bottom } \\ \text { TB70 - } & \text { temperature of MCO sidewall approximately } 17 \text { inches above the bottom } \\ \text { TC70 - } & \text { temperature of MCO sidewall approximately } 45 \text { inches above the bottom } \\ \text { TD70 - } & \text { temperature of MCO sidewall approximately } 73 \text { inches above the bottom } \\ \text { TE70 - } & \text { temperature of MCO sidewall approximately } 101 \text { inches above the bottom } \\ \text { TF70 - } & \text { temperature of MCO sidewall approximately } 129 \text { inches above the bottom } \\ \text { TG70 - } & \text { temperature of MCO sidewall approximately } 132 \text { inches above the bottom } \\ \text { TG74 - } & \text { temperature of MCO sidewall approximately } 145 \text { inches above the bottom }\end{array}$

\section{Refer to Section 4, Item 10-22.}

The design pressure of 150 psig and a design temperature of $375^{\circ} \mathrm{C}$ is used for evaluation of the $M C O$ for all normal conditions and design basis conditions inn Appendices 4-12. Appendix 12 demonstrates the $M C O$ 's compliance with the applicable code requirements for the conditions stated above.

\subsubsection{Maximum MCO Assembly Weight}

The gross weight of MCO (including baskets ) containing 288 Mark IA fuel assemblies should not exceed $16,175 \mathrm{lbs}$ or $17,487 \mathrm{lbs}$ flooded. These weights are based on a $288 \mathrm{Mark}$ IA SNF assembly fuel load with a SNF weight of $11,436 \mathrm{lbs}$. The gross weight of a MCO containing $270 \mathrm{Mark}$ IV fuel assemblies shall not exceed $19,142 \mathrm{lbs}$ dry or $20,357 \mathrm{lbs}$ flooded. This is based on a $270 \mathrm{Mark}$ IV SNF assembly fuel load with a SNF weight of $14,950 \mathrm{lbs}$. Weights as quoted are design goals and subject to changes as the design evolves.

\section{Refer to Section 4, Item 23.}

Detailed weight calculations for each component of the $\mathrm{MCO}$ are provided in Appendix 3. The summary of the expected weights is shown that the nominal MCO, without cover, with Mark $1 \mathrm{~A}$ fuel dry weighs approximately $17,248 \mathrm{lbs}$ and flooded weighs approximately $18,552 \mathrm{lbs}$. The MCO with the Mark IV fuel weighs approximately 19,378 lbs dry and approximately 20,657 lbs flooded.

\subsubsection{Height of the $\mathrm{MCO}$}

The maximum height of the MCO shall not exceed 160 inches (without final cover cap) at a temperature of $25^{\circ} \mathrm{C}$. This includes any connections or devices integral to the MCO in facilitating connections to external process equipment and in providing pressure relief. When the final cover cap is welded in place, the maximum height shall not exceed 165.60 inches. 
HNF-SD-SNF-DR-003, Rev. 0

\section{Refer to Section 4, Item 24.}

The maximum height of the MCO with the cover cap off is 160 inches. The maximum height of the $\mathrm{MCO}$ with the cover cap in place is 165.4 inches, as shown in Appendix 1.

\subsubsection{Diameter of the MCO}

The nominal outside diameter of the $\mathrm{MCO}$ is 24 inches. In no case, including post-accident conditions, is the $\mathrm{MCO}$ inside circumference below the bottom of the shield plug allowed to exceed 73.04 inches ( 23.25 inches ${ }^{*} \pi$ ). The $\mathrm{MCO}$ shell is allowed to have a 25.31 inch maximum as-built $\mathrm{OD}$ above the 148 inch elevation measured from the MCO bottom. These dimensional limits are applicable during normal operations and post accident conditions.

\section{Refer to Section 4, Item 25.}

The inside circumference below the bottom of the shield plug is maintained to be less than 73.04 inches as shown in Appendix 5 and Appendix 11. These evaluations include the post-level D events as defined in the Performance Specification. The only event that has the potential of any significant distortion of the circumference of the shell is the side drop in the cask and is localized in the area of the collar in the cask. The distortion would be inward (localized denting) and hence the circumference requirement would not be violated. 
Figure 1. MCO Axial Temperature Distribution

\section{for Hot Conditioaing}

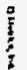

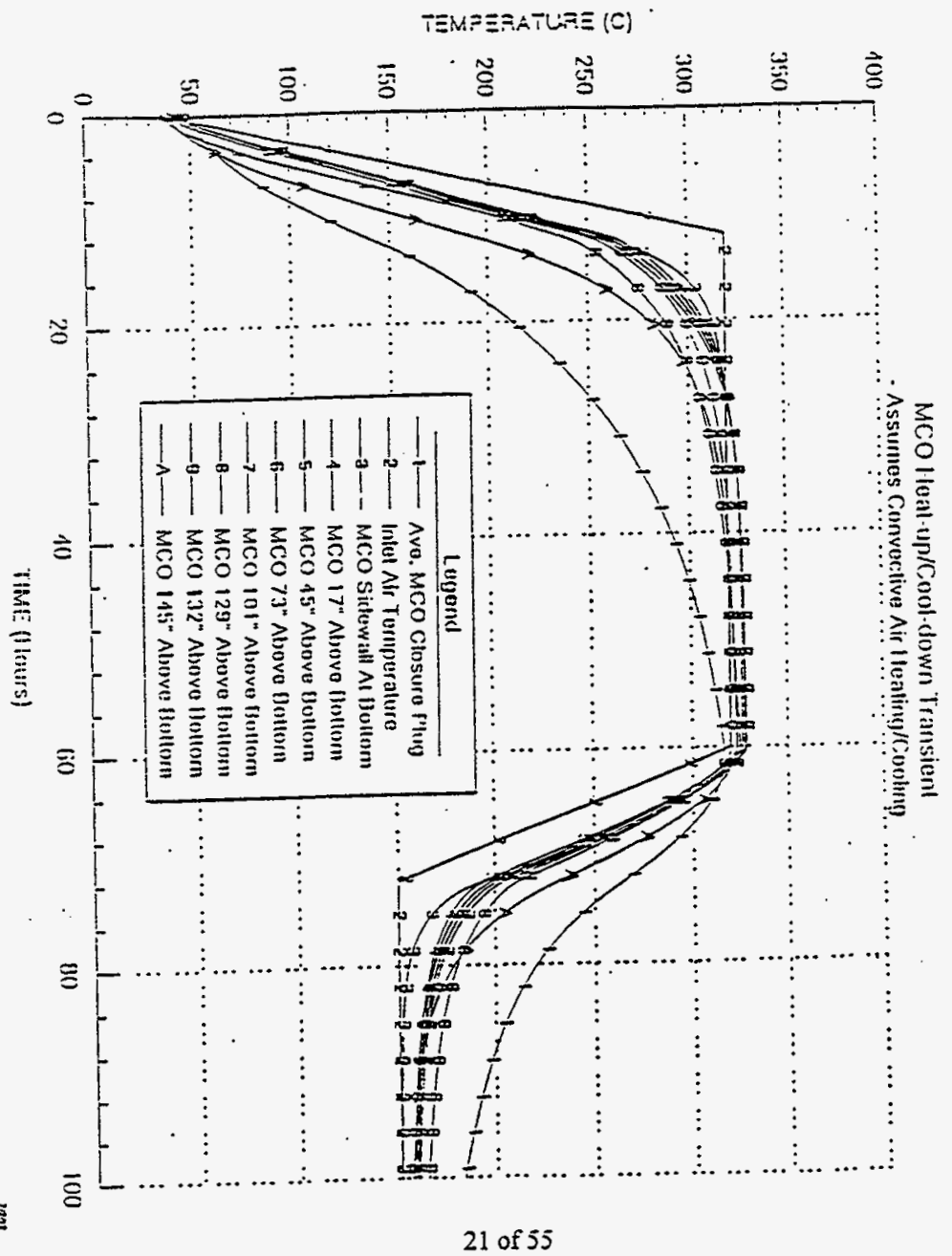




\subsection{MCO Shell Design}

The MCO shell is a cylindrical vessel that provides access to its cavity through its top end and receives a shield plug at its top end for closing. The $\mathrm{MCO}$ shell has a bottom assembly that provides a permanent sealed closure on the shell bottom end. The MCO bottom assembly is nominally flat and must include an internal liquid collection sump at the MCO centerline. The MCO must be designed with a 1.49 inch nominal distance between the inside of the MCO bottom assembly and the bottom of the lowest basket. The MCO must permit or allow loading and stacking of the fuel baskets within its cavity. The empty shell must be designed to load into and out of the transport cask.

Refer to Section 4, Item 18.

As shown in Appendix 1 the MCO shell assembly consists of a forged bottom closure with a low point in the center for facilitating the removal of water, a cylindrical 0.5 inch thick wall shell and a forging collar at the top that mates with the shield plug and locking ring. The top closure permits full access to the inside cavity for stacking of the fuel baskets. All materials are $304 \mathrm{~L}$ stainless steel. At the bottom of the cavity, there are 6 basket support plates in the form of a spider supporting the bottom basket and maintain a 1.49 in nominal distance between the basket and the bottom head. The empty shell can be loaded into the cask by assembling the locking ring in place and grappling the lift ring on the locking ring similar to what is done in handling the loaded MCOs. The MCO may be uprighted with standard rigging (slings etc.) although a turning fixture may facilitate the operation. The MCO should not be handled or lifted without the lifting ring in place. The use of other fixtures to lift the MCO increases the potential of damaging and misshaping the collar area so that insertion of the locking ring would be very difficult.

\subsection{MCO Closure Design}

The MCO shall be designed with a mechanical closure configuration. The closure shall rely on a mechanical crushable seal to maintain the containment and confinement requirement at the final closure interface. The closure system shall utilize the shield plug/shell interface as the closure boundary where the crushable seal shall be located. The shield plug shall be held in place via a locking ring threaded into the MCO shell. The locking ring shall contain screws that will be tightened to force the shield plug down against the crushable seal while pushing up on the locking ring.

The MCO shall be designed to incorporate a final welded closure cap over the shield plug. The cap shall be welded to the MCO shell. The cap shall be capable/configured for lifting the MCO with the same equipment described in Section 4.13 of the Performance Specification (Reference 6.1). The cap shall be capable of withstanding the pressure rating of $150 \mathrm{psi}$ at $132^{\circ} \mathrm{C}$ and shall meet the drop 
criteria for the drop into the CSB tubes per Table 3 of the Performance Specification. The closure cap shall be capable of being fitted with a replaceable rupture disk set at 150 psig.

Refer to Section 4, Item 19.

The MCO closure consists of a shield plug, locking ring and main seal as shown in Appendix 1. The shield plug assembly rests on a seal ledge on the inside of the MCO collar. The shield plug has a groove in the mating surface to this ledge which holds the main seal and prevents over crushing of the seal. The shield plug is held in place by the locking ring assembly which threads into the collar with a double lead buttress thread. Once the locking ring is installed, the eighteen 1-1/2 inch diameter set screws are torqued, generating a force between the shield plug and the locking ring. The force seats the metal main seal and maintains a minimum sealing load on the seal during all loading conditions. The evaluation of the set screws and their required torquing is shown in Appendix 4. The locking ring is designed so that, with an 1/8" gap between its bottom surface and the shield plug, the top surface of the locking ring is $1 / 8^{\prime \prime}$ below the top surface of the shield plug. The main seal is a Helicoflex seal. The details of the seal can be found in Appendix 13.

The closure cap consists of a $304 \mathrm{~L}$ stainless steel forging that mates with the MCO collar. That closure weld is a full thickness weld that is not 100\% volumetric examined. Analysis of the weld and the lifting ring which is attached to the cover cap is found in Appendix 6 . The lifting ring on the cover cap is the same diameter and thickness as the lift ring on the locking ring. Analysis is performed combining the lifting loads with the 150 psig internal pressure at $132^{\circ} \mathrm{C}$.

\subsection{Fuel Basket Design}

The Mark IA fuel storage and scrap baskets shall meet the intent of ASME Boiler and Pressure Vessel Code, Section III, Subsection NG (ASME 1995) under the component safety group as guided by the NUREG/CR 3854, UCRL-53544, Fabrication Criteria for Shipping Containers, 1984. (Explanation: Mark IA fuel has a higher $\mathrm{U}_{235}$ enrichment than Mark IV fuel. The Mark IA basket structural integrity is required for criticality control whereas basket structural integrity is not required for Mark IV fuel. Therefore, ASME Section III, Subsection NG requirements shall be applied to the construction of the Mark IA fuel storage and scrap baskets.)

The design shall meet Service Level A requirements for normal operating loads and Service Level D for accident conditions under ASME Boiler and Pressure Vessel Code, Section III, Subsection NG. During accident conditions the baskets designed for Mark IA fuel and Mark IA fuel fragments/scrap shall maintain the criticality control features defined in Section 4.19.3 of Reference 6.1.

Refer to Section 4, Item 20. 
The Mark $L A$ baskets are designed to the intent of the ASME Boiler and Pressure Vessel Code, Section III, Subsection NG (ASME 1995), Reference [6.37. The evaluation of the baskets for the Service Level $A$ and load conditions defined in the Performance Specification Reference [6.1] are found in Appendix 7. The combined requirement of sequential loading from the vertical and horizontal drop events is addressed by conforming to both the ASME Code, Service Level D requirements and the Performance Specification criticality requirements. This was necessary to ensure that the center tubes do not move more than two inches radially and that the basket axial deformations are small enough to prevent the baskets from becoming disconnected from one another permitting fuel particles to enter the center tube.

\subsubsection{Mark IV Baskets}

For clarification, the Mark IV fuel storage and scrap baskets do not have to be designed to meet the intent of the ASME Code. (Explanation: Mark IV fuel has a lower $U_{235}$ enrichment than Mark IA fuel. Analyses indicate that the Mark IV fuel cannot achieve criticality in an MCO under normal operating conditions or accident scenarios. It follows that basket structural integrity for Mark IV criticality control is not required as is the case for Mark IA fuel and scrap. Therefore, ASME Code, Section III, Subsection NG requirements are not required for the design or construction of the Mark IV fuel storage and scrap baskets.)

Refer to Section 4, Item 20.

For consistency with the Mark $1 \mathrm{~A}$ structural evaluations, the Mark IV baskets were evaluated to ASME Code, Section III, Subsection NG, allowables for the Level A service Loadings. No Level D loadings were considered for the Mark IV baskets.

\subsubsection{Mark IA and Mark IV Baskets}

For the handling of both loaded and unloaded Mark IA and Mark IV baskets, the design shall meet the safety factors required by the American National Standards Institute (ANSI) N14.6-1986 for noncritical lifts. (Design and qualification of the basket grapple interface will be performed by the Cask/Transportation subproject and will not be the responsibility of the MCO Design Agent). The ANSI safety factors apply from $5^{\circ} \mathrm{C}$ through $100^{\circ} \mathrm{C}$. Currently anticipated SNF basket code deviations are listed in Appendix C, ASME Code Notes and Exceptions. This list is subject to revision.

Materials of construction for the Mark IV and Mark IA storage and scrap baskets shall be 304L stainless steel or a material having equal or greater corrosion resistance properties. Other materials may be used if shown suitable. All baskets will be annular open-top containers with a maximum $O D$ of 22.625 inches at $25^{\circ} \mathrm{C}$. All baskets will be able to support the fuel at $1.0 \mathrm{~g}$ while at $375^{\circ} \mathrm{C}$. The basket grapple interface for all baskets shall be a $1 / 8$-inch deep by one-inch long radial groove 
beginning $17 / 8$-inch from the top end of the basket center tube. Basket sizing shall accommodate a $1 / 2$ " clearance between the top of the fuel elements and the bottom plate of the basket above. Refer to Section 4, Item 20.

Both the Mark $1 \mathrm{~A}$ and the Mark IV baskets were evaluated for lifting in accordance with ANSIN14.6 for non critical lifts. The baskets were also evaluated for the ability to support the fuel and the baskets above at $375^{\circ} \mathrm{C}$. The evaluations can be found in Appendix 7 for the Mark $1 \mathrm{~A}$ baskets and Appendix 8 for the Mark IV Storage basket and Appendix 9 for the Mark IV Scrap basket. The adequacy of the lifting groove per the Performance Specification is evaluated by the Buyer. The lifting configuration to interface with lifting equipment supplied by others, although not per the Performance Specification as stated above is per the technical direction given by the Buyer.

\subsubsection{Summary of MCO Fuel Basket Types}

The MCO fuel baskets are categorized into two major configurations: 1) intact fuel element baskets, and, 2) scrap fuel (fragment) baskets. Fuel baskets must also maintain criticality control for the higher enriched (Mark IA) fuel. These basic requirements lead to four different basket types as follows:

- Type (1) must have the ability to hold 48 Mark IA (higher enriched) intact-fuel elements and must have a criticality control exclusion void per Section 4.19 .3 of Reference 1 built into the basket.

- Type (2) must have the ability to hold 54 Mark IV intact-fuel elements, and does not need the exclusion void.

- Type (3) will hold Mark IA (higher enriched) scrap fuel (fragments), and must have a criticality control exclusion void per Section 4.19 .3 of Reference 1 built into the basket.

- Type (4) will hold Mark IV scrap fuel (fragments), and does not need the exclusion void.

Note: SPR fuel will be loaded into Mark IA scrap baskets.

\section{Refer to Section 4, Item 20.}

Appendix 1 shows that the Mark $1 \mathrm{~A}$ storage basket can hold 48 Mark $1 \mathrm{~A}$ intact-fuel elements and has the criticality exclusion void required. The Mark IV storage basket has the ability to hold 54 Mark $I V$ intact-fuel elements. The Mark $1 \mathrm{~A}$ scrap basket has the same criticality exclusion void as the Mark $1 A$ storage basket. The Mark IV scrap basket can hold fuel fragments as required. 
HNF-SD-SNF-DR-003, Rev. 0

\subsubsection{Summary of MCO Fuel Basket Functions Summary of MCO Fuel Basket Functions}

All basket designs shall incorporate a center support tube for axial support during lifting and for protection for the long process tabe.

All baskets have a center support tube for axial support during lifting and for process tube protection.

Each basket shall be capable of being loaded, in the upright position, by the Fuel Retrieval System equipment in the $\mathrm{K}$ Basin pool.

All baskets have the same open spacing and access defined in the preliminary design provided by Buyer.

The baskets must be stackable inside the $\mathrm{MCO}$ with the basket centerline coincident with the $\mathrm{MCO}$ centerline. While stacked inside the $\mathrm{MCO}$, the baskets must provide for insertion of a long process tube down the $\mathrm{MCO}$ centerline for water draining and gas transport, as needed.

All baskets have the same stackable features that allow them to be stacked with the centering coincident and have internal guides to provide for insertion of the long process tube. As described in Appendix 1, the interfaces between baskets are such that the tube, once started, will not hang up during the insertion operation.

The loaded baskets shall be capable of being easily and safely handled in the basin water, reliably loaded and nested into the $\mathrm{MCO} /$ cask assembly in the $\mathrm{K}$ Basins load out pits, and engaged with the shield plug shield/guard plate (to be designed) and axial stabilizer. Basket design shall account for differential thermal expansion when subjected to processing temperatures inside the $\mathrm{MCO}$.

The loaded baskets can be easily and safely handled in the basin water. Lead-ins and alignment mechanisms allow the baskets to be loaded and nested in the MCO in the K Basins. The shield plug assembly has an axial stabilizer that engages with the top basket and allows for a differential thermal expansion of a $100 \circ \mathrm{C}$.

The baskets shall drain free and not capture or retain excessive water to accomplish the bulk water removal step by the CVD System.

The baskets are designed to drain freely and there are no cavities that will retain excessive water.

The baskets shall support heat transfer into and out of the fuel while in gaseous and vacuum environments inside the $\mathrm{MCO}$. The primary heat transfer mode is radiation and conduction during 
the static (storage/staging) state. Forced flow (convective) cooling of the fuel in the $\mathrm{MCO}$ baskets (particularly the scrap baskets) is a very large and essential component of heat transfer during the HCS process, and must be facilitated by the basket design. The baskets shall support gas flows needed to properly dry and condition intact fuel and scrap fuel during the vacuum drying and hot conditioning processes.

The baskets are designed with similar air flow capability as the Buyer supplied preliminary design. Review by the Buyer indicates acceptability to criteria not presented in the Performance Specification.

The baskets shall be compatible with the fuel and $M C O$ containment materials during the expected temperatures, pressures, and atmospheres inside the MCO during handling, shipping, storage and processing.

The baskets are fabricated from austenitic stainless steel which is fully compatible with the MCO containment which is fabricated from austenetic stainless steel. All major parts of both components are fabricated from $304 \mathrm{~L}$ stainless steel.

The baskets shall maintain their structural integrity (with specified exceptions) during expected internal MCO environmental conditions, normal MCO handling situations, and after accidents (Mark IA storage and scrap baskets only). This structural integrity is required to maintain criticality safety of the MCO when loaded with Mark IA baskets.

As shown in Appendices 7-9 the baskets will maintain their structural integrity environmental and normal handling conditions. Appendix 9 shows that the Mark 1 A baskets will maintain the required structural integrity after the Design Basis Accident loadings specified in the Performance Specification. Reference [6.1].

The baskets shall be sufficiently strong to preserve the processing ability of the MCO for the bulk water removal, vacuum drying, and hot conditioning processes during normal MCO handling, various internal MCO environments, and after MCO DBA accidents of Section 4.19.2 of Reference 6.1 .

Appendix 7 shows that the Mark $1 \mathrm{~A}$ baskets will retain their geometric configuration so that processing capabilities are not compromised during normal handling and after the Design Basis Accident Loadings specified. The Mark IV baskets which have no structural integrity code criteria per the Performance Specification Reference [6.1] maintains its processing capability by the use of a one-inch XXS pipe for the long processing tube. The XXS processing tube design provides considerable resistance to crushing or shear which would reduce the processing capability. 
The baskets shall not introduce any additional gas producing materials into the $\mathrm{MCO}$ which significantly increases the pressure of the $\mathrm{MCO}$ during storage.

The baskets are made entirely out of austenitic stainless steel and have no known gas producing mechanisms in the environments specified.

The baskets shall not introduce any materials that will appreciably accelerate corrosion of or significantly alter the properties of the $\mathrm{MCO}$ containment boundary.

Both the baskets and the MCO containment boundary are made from austenitic stainless steel, therefore the baskets will not accelerate the corrosion of or significantly alter the properties of the MCO containment.

The baskets' bottom structural plate shall have a minimum weight not less than 50 lbs. (Note: Scrap baskets may include the weight of the gussets with the bottom plate to meet this requirement.)

As can be seen in Appendix 3 the bottom plates of the Mark IA baskets and the Mark IV storage basket weigh more than 50 pounds. The Mark $N$ scrap basket combined with the gussets or dividers in the baskets weighs more than 50 pounds.

\subsection{MCO Shield Plug Design}

The MCO shield plug will be a solid cylinder designed to mate with the open end of the MCO shell. The MCO shield plug also mates with the end effector on the top SNF fuel basket. The MCO assembly must have a one-inch minimum free space between the bottom of the guard plate and the top of the SNF elements or fragments at $72^{\circ} \mathrm{F}$. The shield plug will only provide worker shielding on the top of the $\mathrm{MCO}$. The shield plug shall feature an integrally machined axisymmetric lifting ring with a 12 ton lifting capacity when gripped with six equally spaced $1.97^{\prime \prime}$ tangential length by $0.66^{\prime \prime}$ radial contact length grippers. The ring will facilitate handling of the MCO package when unloading from the transport cask, CSB storage tubes, and process cells with the MHM.

Refer to Section 4, Item 21.

The shield plug assembly which consists of the shield plug , guard plate, internal filters, process valves and pressure relief devices has a basket stabilizer extension which centers the top basket. The assembly has a minimum of one inch clearance at $72^{\circ} \mathrm{F}$ above the fuel or fragments. The shield plug is held in place with a locking ring that has a lifting ring with a 12 ton capacity when gripped with six equally spaced grippers as specified. The demonstration of this capacity is shown in Appendix 11. 
The MCO lifting ring design and cover cap lifting rim area must exhibit the safety factors (for noncritical lifts) required by the American National Standards Institute (ANSI) N14.6-1986: "Special Lifting Devices for Shipping Containers Weighing 10,000 Pounds $(4500 \mathrm{~kg}$ ) or more." This standard requires that any handling or lift features required to perform non-critical lifts shall be capable of demonstrating a safety factor of three (3) on material yield and five (5) on material ultimate strength. ANSI N14.6 does not apply to the MCO shell.

The lifting ring area of both cover cap and the lifting ring complies with the factors of safety for non-critical lifts as specified in ANSI N14.6 as shown in Appendices 6 and 11, respectively.

The $\mathrm{MCO}$ shielding design shall meet as low as reasonably achievable requirements in accordance with 10 CFR 835, Occupational Radiation Protection (CFR 1993), Subpart K, DOE Order 5480.11, Radiation Protection for Occupational Workers (DOE 1988), Paragraph 9a, HSRCM-1, Hanford Site Radiological Control Manual (RL 1994), Sections 111 and 311, HNF-IP-1043, HNF Occupational ALARA Program (HNF 1995), Section 8.0, and NRC Regulatory Guide 8.8, Information Relative to Ensuring that Occupational Radiation Exposures at Nuclear Power Stations will be As Low As Reasonably Achievable, Revision 3, Section C.2.b, "Radiation Shields and Geometry", and Section C.2.f, "Isolation and Decontamination."

The MCO shield plug will shield workers against photons and neutrons emanating from the inside of the MCO. This shielding shall maintain an average dose across the top of the shield plug of 30 $\mathrm{mrem} / \mathrm{hr}$ on contact (two inches) for the average MCO fuel inventory shown in Table 1 of Reference 6.1 . The $30 \mathrm{mrem} / \mathrm{hr}$ limit includes radiation streaming between the MCO shield plug and $\mathrm{MCO}$ shell and streaming around penetrations. Streaming emanating from between the MCO and cask is not included. Streaming shall be minimized. For the worst case $\mathrm{MCO}$ specified in Reference 6.1, Section 3.1.3.1, Radioactive Source Term, the average dose across the top of the shield plug on contact (two inches) shall not exceed $100 \mathrm{mrem} / \mathrm{hr}$. 
The MCO provides more than the specified shielding as demonstrated in Appendix 16. A summary of the shielding analysis is provide below.

\begin{tabular}{|l|l|}
\hline Source Term & $\begin{array}{l}\text { Cleaned and reloaded Mark IV fuel elements: } \\
5.43 \mathrm{MTU} \text {; } 11 \text { energy bins; } 8.29 \times 10^{\prime 5} \\
\text { photons/sec }\end{array}$ \\
\hline Model Geometry & $\begin{array}{l}27 \mathrm{cmss} \text { lid; } 4 \text { penetrations; I"central and } \\
\text { lateral holes thru lid; } 4 \mathrm{~cm} \text { SS plate under } \\
\text { filters; collar in place. }\end{array}$ \\
\hline $\begin{array}{l}\text { Detector Geometry } \\
\text { average (Peak Load) }\end{array}$ & $\begin{array}{l}\text { Tissue equivalent plastic in } 3 \text { thicknesses and } 8 \\
\text { rings }\end{array}$ \\
\hline $\begin{array}{l}\text { Requirement-30 mrem/h contact, areal average } \\
\text { (Nominal Load) }\end{array}$ & $<2 \mathrm{mrem} / \mathrm{h}$ photons and neutrons. \\
\hline
\end{tabular}

The shield plug will provide access to the interior of the $\mathrm{MCO}$ via a minimum of three penetrations. The penetrations will connect to four ports counterbored into the top of the shield plug. A description of the ports, penetrations and associated equipment interface follows.

The shield plug has four penetrations with four ports as shown in Appendix 1.

Port \#1. Connects to a penetration that leads to the internal HEPA filter bank.

- Up to 1" diameter drilled penetration

- Contains resetting pressure relief device with built in $1 / 4$ " or less, restrictive orifice

- Capable of replacing relief valve with a non-safety class rupture disk, and

- Capable of replacing relief device with an external HEPA filter

One penetration is one inch in diameter that connects to the internal HEPA filter bank. The port consists of a $3 / 4$ NPSM female thread that can receive either a standard HEPA filter with similar thread (Appendix 15) or a rupture disk with mating threads (Appendix 12) or a bushing that adapts to a $1 / 8$-inch relief valve that is specified by specified by the buyer. The recommended seals for this port is metal $C$-seals that are retained on the components so that the parts can be replaced. The port as required can hove a cover flange that is either 
a blind flange or a flange with a $1 / 4$-inch diameter hole in it. This cover has metal $C$-seals that provide a seal. The covers and attachment bolts are evaluated in Appendix 10. These pressure relief devices are non ASME Code devices per the Performance Specification.

Port \#2. Connects to a penetration that leads to the internal HEPA filter bank.

- 1" diameter drilled penetration, and

- Port contains a process valve

Another port connects to a one-inch diameter penetration to the HEPA filter bank. The port is accessed by a process valve that may be covered with blind flange, as stated above. The process plug consists of a $1-7 / 8$ inch threaded plug that is drilled in the center and then cross drilled below the top of the threads. As the plug is unthreaded, it rises exposing the cross arilled holes and allowing flow. The top has a hex head that can mate with a valve operator. The seal is maintained with a $C$-seal at the top of the threads that has been proven to reseal in the excess of five times. The torque required to seat the seal is evaluated in Appendix 10 3 and the seal data is provided in Appendix 14. The valve operator can use the same sealing surface as the blind flanges to seal to the shield plug before operating the valve.

Port \#3. Connects to the long process tube which has a $2 \mathrm{~mm}$ screen at the end.

- Approximately $.54^{\prime \prime} \mathrm{DD}(1 / 2 "$ sch. 80 pipe) minimum diameter drilled penetration

- Port contains a process valve

- Requires differentiation in connection for valve operator

The center long process tube connects to a process valve on the exterior of the shield plug via $0.609^{\prime \prime}$ diameter penetration. The process valve is the same as discussed above however the cover flanges are different. The cover flange is a five bolt flange compared to the four bolt flanges used to cover the other ports.

Port \#4. Connects to the short process tube which has a $2 \mathrm{~mm}$ screen at the end.

- 1" diameter drilled penetration

- Port contains a safety class rupture disk that will be incorporated into a process valve head

- Provides backup port to Port \#2

The port that connects to the short process tube which is covered by a $2 \mathrm{~mm}$ screen does so through a one inch diameter penetration. The process valve is similar to the ones described 
above. The plug is drilled all the way through the center and has a rupture disk between the center bore and the exterior. Details can be found in Appendix 1. The rupture disk is the primary pressure relief device and is set at the design pressure. This is an exception to the ASME Code, Section III Division 1 Subsection NB, since the rupture disk cannot be used as the sole only Code relief device. The cover flanges have a four bolt pattern.

The connections leading to the long or short process tubes shall be designed to be easily differentiated by a worker looking at either the top or bottom of the shield plug. The design of the penetrations, ports, and valve mechanism shall implement the following criteria:

The seal connections for the valve operator have different bolt patterns.

- Process valves shall be capable of normal operation and achieve sealing criteria in Section 4.3 in Reference 1 for five complete cycles.

The process valves can reseal in the excess of 5 times as documented in Appendix 14.

- Provisions for pressurizing the MCO interior with an inert gas

The MCO can be pressurized by the gas of choice through any of the process valves.

- Provisions for purging gas from the MCO interior

Gas may be purged through the use of any two of the process valves. The use of the long process tube would be more efficient.

- Penetrations, connections, and seals shall be leakage rate testable in accordance with ANSI N14.5, Leakage Tests on Packages for Shipment of Radioactive Materials (ANSI 1987).

The entire MCO when assembled can be leak tested in accordance with ANSI N1+.5 by filling it through one of the process ports with a detectable gas and then placing it in a chamber where a vacuum could be pulled and the gas could be detected. Similarly a pressure drop or pressure rise could be used, provided that sensitive enough instrumentation were used to detect the $1 \times 10^{-1} \mathrm{SCC} / \mathrm{sec}$ required leak rate.

- Provisions to make or break all connections while continuing to maintain SNF containment, with minimal spread of contamination. 
By the use of the cover flange sealing surface a valve operator tool designed by others could be used to make and break all connections while maintaining containment.

All penetrations to be sealable to the containment leak rate criteria after the process connection is terminated.

As documented in Appendix 14, the process valve seals are resealable to the containment leak rate criteria.

- Connections shall be such as to facilitate their decontamination as per Section 4.18 of Reference 6.1.

The connections are angled to prevent the trapping of debris and are of a smooth finish to facilitate decontamination.

- Ports, penetrations, and connections shall be accessible to the operator from the top face of the MCO.

All ports, penetrations and connections are accessible to the operator from the top per Appendix 1.

- Penetrations and connection connections shall not appreciably reduce or impair MCO shielding.

The shielding requirements are met with consideration of the process valves and penetrations as shown in Appendix 16.

- Provisions for removal or reinstallation of sealing mechanisms as required to cover process connections, pressure relief devices, and a low flow HEPA filter; these sealing mechanisms cannot extend above the top of the shield plug, including fasteners.

The pressure relief and external HEPA filters are designed to be replaced and the same quality seal can be regained. The sealing mechanisms are compatible with remote operating equipment.

- Provision to bleed down, in a controlled way into the process piping, internal MCO pressure after process connections are made. 
The process valve can be used to bleed down the into the process piping, $M C O$ internal pressure with a correctly designed valve operator and pressure control device.

- Penetrations and connections shall be designed to facilitate remote operation via long handled tools, via a manipulator; and via manual means.

The penetrations and connections are designed to facilitate remote operating as well as manual means. Features such as captured bolts on the cover flanges, standard hex head operators, seals with ability to be retained insure this.

- Ports for pressure relief devices shall contain orifice plates as required (1/4 inch or less) to limit relief flow.

Where required cover plates with $1 / \mathrm{s}$ inch orifices can be installed.

- Seal material used in the resetting pressure relief valve shall maintain its properties for up to six years at up to $132^{\circ} \mathrm{C}$ and in a radiation environment of $40 \mathrm{R} / \mathrm{hr}$ (Total integrated dose of $2.1 \times 10^{6} \mathrm{R}$ ).

The pressure relief valve is specified by the Buyer.

- Process connections shall be designed with a hex cap on the process valve for the operator to engage.

As shown in Appendix I, the process valves have a hex cap for the operator to engage.

- The bottom side of the shield plug shall incorporate a feature (guardplate) that will keep the internal filter elements dry during insertion of the shield plug into the $\mathrm{MCO}$ at the K-Basin pool.

As part of the shield plug, there is guard plate that protects the filters as shown in see Appendix $I$ and Appendix 5 . This provides for an air pocket that keeps the filters dry when the shield plug is inserted into the MCO under water.

\subsection{Internal Process Filter}

The MCO shall have internal process filters to support the vacuum drying and hot conditioning outflows from the MCO. These filters shall meet the requirements of HNF-S-0556, MCO Internal 
HEPA Filter Specification, and be installed between the shield plug bottom and the guardplate. The internal process filters and short process tube shall be protected by a guardplate capable of withstanding the drop accelerations in Table 3 . The filter assembly installed with the shield plug shall be capable of withstanding the drop accelerations in Table 3 and still maintain flow capability. (Note: MCO Design Agent is responsible only for the structural attachment of the filter assembly to the shield plug). The filter assembly weight shall not exceed $50 \mathrm{lbs}$.

\section{Refer to Section 4, Item 22.}

The Internal HEPA Filter Specification is HNF-S-0556 is provided in Appendix 15. Appendix 10 provides verification that the structural attachment will support a filter assembly weighing up to 50 pounds for the maximum loading of $101 \mathrm{~g}$ 's. 


\subsection{Materials, Processes, and Parts}

The MCO shell shall be fabricated from type $304 \mathrm{~L}$ stainless steel. All components welded to the MCO shell must be made of type 304L stainless steel (the same material as the MCO shell). A mechanically attached shield plug and any components thereof must be made from any combination of the following: carbon steel, plated carbon steel, and/or austenitic stainless steel. All materials shall be ASME/ASTM certified materials. Ferritic steel materials used for the MCO pressure boundary shall meet the criteria of NUREG/CR-3826. See Appendix B, Mechanical Properties of Materials. Provision shall be made to preclude metal-to-metal galling in threaded MCO components. Nickel or other suitable plating may be used to reduce potential for corrosion. Thermal and chemical compatibility of materials must be shown suitable.

Refer to Section 4, Item 26.

The MCO shell, shield plug assembly and locking ring are fabricated from $304 \mathrm{~L}$ stainless steel to insure compatibility. The process plugs are fabricated from 300 series stainless steel. No Ferritic materials are used in the design. All materials are specified as either ASME (SA) or ASTM (A) materials, as shown in Appendix 1. Metal to metal galling is minimized by requiring the use of lubricants on all threaded surfaces and allowing them to be used on other closely fitting surfaces where operationally they would be permitted, such as between the radial surfaces of the locking ring and the shield plug. Harder materials such as XM-19 and 316 is used for the threaded fasteners. Also the use of $304 \mathrm{LN}$ and $304 \mathrm{~N}$ materials are permitted for the components to minimize the opportunity for galling.

\subsection{MCO Corrosion Control}

Specifications generated for the $\mathrm{MCO}$ and $\mathrm{MCO}$ components shall require cleanliness during fabrication, handling, and storage - before and during use. Standards such as ASTM A 380-94, "Standard Practice for Cleaning and Descaling Stainless Steel Parts, Equipment, and Systems" (ASTM 1996a), and ASME NQA-1, Quality Assurance Requirements for Nuclear Facility Applications (ASME 1994), shall be invoked for cleanliness control. Appendix D, MCO Corrosion Conditions, describes the corrosion environment encountered by the MCO during various phases of its operation. The MCO shall be designed and constructed to provide full service life under these corrosion conditions. The mechanical seal required for final closure shall be of a material best suited for this application.

Refer to Section 4, Item 27.

Appendix $D$ of the Performance Specification demonstrates the acceptability of austenitic stainless steel specifically $304 \mathrm{~L}$ for the environment that he MCO will experience. All components of the $M C O$ are fabricated from austenitic stainless steel and hence there is no significant corrosion 
impact on the design life of the MCO. Cleanliness requirements as noted are included in the fabrication specification.

\subsection{Welded Joints}

All MCO fabricator pressure boundary welds shall be made in accordance with ASME Code, Section III, Division I, NB-3350. All welds shall be sufficiently smooth to enable easy decontamination. Butt welds to be ground flush to within .03 inches of base metal. Weld joint designs shall avoid potential contamination traps to the greatest extent practicable. All MCO pressure boundary welds and welds bearing the weight of the fully loaded MCO must be designed for and pass $100 \%$ volumetric examination (x-rays or ultrasonic) per ASME requirements. Exceptions for field welds only shall be documented and justified using the guidelines listed in Appendix C: ASME Code Notes and Exceptions.

Refer to Section 4, Item 28.

All MCO pressure boundary welds are designed and produced to ASME Section III Division I, Subsection NB except the field closure weld which is a full thickness weld that can not be $100 \%$ volumetrically examined. The acceptance of this weld is that it provides closure to a stabilized system and the quality is insured by welding qualification and the use of liquid penetrant inspection. The margins of safety for the weld is provided in Appendices 5 and 11.

\subsection{Decontamination Provisions}

MCO exposed surfaces shall facilitate their decontamination. All exposed surfaces shall be smooth without cracks or crevices. Blind or hidden corners or joints in areas potentially exposed to contamination that can not be readily accessed by hand held spray devices shall be minimized.

Refer to Section 4, Item 29.

All exposed surfaces are smooth with out cracks or crevices to facilitate decontamination. Access to all surfaces is cvailable as can be seen in Appendix 1.

\subsection{Safety Requirements}

Refer to Section 4, Item 30.

\subsubsection{Safety Classification}

MCO components shall be classified by safety class in accordance with the requirements of HNF-CM-4-46, Safety Analysis Mamual (HNF 1995), Section 9.0. MCO components providing fuel containment and criticality control shall be Safety Class items and comply with the requirements 
of HNF-CM-4-46. All other MCO components except the external HEPA filter shall be Safety Significant items. The external HEPA filter shall be a General Service (undesignated) item. Safety Class items are:

- The MCO Shell

- Mark IA SNF storage and scrap baskets

- Mark IA Scrap baskets

- $\quad$ Rupture Disk used in Port \#4

- MCO Shield Plug Assembly (excluding all port components, except the rupture disk)

The various components of the $M C O$ are classified into safety classes in agreement to the above in section 3.9.1. The applicable code requirements are then applied. The containment boundary components are designed and fabricated to ASME Code, Section III, Division 1, Subsection NB and the Mark $1 \mathrm{~A}$ baskets for criticality control purposes are designed and fabricated to Subsection NG.

\subsubsection{Design Basis Accidents}

All Safety Class items shall maintain containment, confinement, and subcriticality during and after the Design Basis Accidents (DBAs) listed below. All Safety Significant items, whose failure could result in the failure of the Safety Class items above, shall also be designed to withstand the DBAs listed below.

NOTE: The following design basis accident loadings are required to meet the Service Level $D$ requirements of the ASME B \& PV Code, Section III, Subsection NB for Safety Class items which provide fuel containment, confinement, and criticality control. Items required to prevent failure of the Safety Class items are also required to meet the same Service Level D requirements. (Service Level D allowable stresses may be exceeded for horizontal and corner drops while the MCO is in the cask. However, the criticality control measures in 4.19 .3 of Reference 6.1 shall be maintained.)

- Design Basis Fire -- Temperatures resulting from exposure to a design basis accidental fire on the outside of the transportation cask. [From 10 CFR 71.73 (3)] This basis fire shall result in exposure of the outside of the cask for not less than 30 minutes to a heat flux not less than that of a radiation environment of $800^{\circ} \mathrm{C}$ with an emissivity coefficient of at least 0.9 . For purposes of calculation the surface 
absorptivity must be either that value which the shipping cask may be expected to possess if exposed to a fire or 0.8 , whichever is greater. (This fire shall raise the MCO shell temperature to $122^{\circ} \mathrm{C}$ for 180 minutes after the fire. The $375^{\circ} \mathrm{C}$ design temperature to accommodate the hot conditioning process bounds the temperature associated with this fire.) (Note: No analyses required by the MCO Design Agent.)

- Design Basis Earthquake -- The design ground acceleration at the CSB is $.35 \mathrm{~g}$. Although this will be amplified due to the position of the MCOs within the facility, the design basis accident drop accelerations bound any imposed earthquake accelerations from the $\mathrm{K}$ Basins, $\mathrm{CSB}, \mathrm{CVD}$, and $\mathrm{HCS}$. (Note: No analyses required by the MCO Design Agent.)

- Design Basis Tomado -- The CSB, CVD, and HCS incorporate preventive and mitigative features regarding radionuclide releases from MCOs due to tornadoes (as determined necessary by Probabalistic Risk Assessments). (Note: No analyses required by the MCO Design Agent.)

- Design Basis Hydrogen Deflagration -- The MCO shall maintain confinement during a design basis hydrogen deflagration event (Service Level $D$ event) beginning at atmospheric pressure inside the $\mathrm{MCO}$ at $75^{\circ} \mathrm{C}$. (Note: No analyses required by the MCO Design Agent.)

- Design Basis Drops -- The following design basis accident drops have been determined to create accelerations listed in Table 3 that must be survived while maintaining confinement, containment (except for cask drops) and subcriticality. Accelerations to be used for the design basis are listed in Table 3. Temperature range for these drops is 25 to $132^{\circ} \mathrm{C}$ and pressure range is 0 to $150 \mathrm{psig}$.

- A two foot vertical drop of a sealed MCO package onto flat reinforced concrete. The MCO lands on the bottom end and there is no credible possibility of a side slap down secondary impact of the MCO. 
HNF-SD-SNF-DR-003, Rev. 0

Table 3. MCO and Component Accelerations (g's) Resulting From Design Basis Accident Drops

\begin{tabular}{|c|c|c|c|c|c|}
\hline \multicolumn{2}{|c|}{ COMPONENT } & \multirow{2}{*}{$\begin{array}{c}\text { g's "PISTON DROP" } \\
\text { INTO CASK* } \\
\begin{array}{c}\text { N/A } \\
25 \\
\text { N/A }\end{array}\end{array}$} & \multirow{2}{*}{$\frac{\begin{array}{c}\mathrm{N} / \mathrm{A} \\
54 \\
\mathrm{~N} / \mathrm{A}\end{array}}{\text { g's TWO (2) FOOT DROP }}$} & \multirow{2}{*}{$\begin{array}{c}\text { g's DROP INTO CSB TUBE } \\
\text { N/A } \\
35 \text { (Spec) } \\
\text { Lower MCO } \\
28 \text { (Calculation) } \\
\text { Upper MCO } \\
\text { N/A }\end{array}$} & \multirow{2}{*}{$\begin{array}{l}\text { g's DROPPED } \\
\text { WITH CASK (1) } \\
\begin{array}{c}101 \\
27 \\
\\
\text { Lid Up 33.5 } \\
\text { lid down 27.4 }\end{array}\end{array}$} \\
\hline Loaded MCO & $\begin{array}{l}\text { Ilorizontal } \\
\text { Vertical } \\
\text { Corner }\end{array}$ & & & & \\
\hline $\begin{array}{l}\text { Mark IA Basket } \\
\text { Within MCO }\end{array}$ & $\begin{array}{l}\text { Horizontal } \\
\text { Vertical } \\
\text { Corner }\end{array}$ & $\begin{array}{c}\text { N/A } \\
25 \\
\text { Bottom Basket } \\
25 \\
\text { Other l3askets } \\
\text { N/A }\end{array}$ & $\begin{array}{c}N / A \\
54\end{array}$ & $\begin{array}{c}N / A \\
35(S P ' E C)\end{array}$ & $\begin{array}{c}101 \\
27 \\
\text { Botton Baskel } \\
27 \\
\text { Other Baskets } \\
\text { Lid down: lop bskt 27.4; other 27.4 } \\
\text { L.id up: bottom bskt 33.5; others 33.5 }\end{array}$ \\
\hline $\begin{array}{l}\text { Mark IV Basket } \\
\text { Within MCO }\end{array}$ & $\begin{array}{l}\text { Horizontal } \\
\text { Vertical } \\
\text { Corner }\end{array}$ & $\begin{array}{c}\text { N/A } \\
25 \\
\text { Bottom Basket } \\
25 \\
\text { Oiher Baskets } \\
\text { N/A }\end{array}$ & $\begin{array}{c}N / A \\
54\end{array}$ & $\begin{array}{c}N / A \\
35 \text { (Spec) }\end{array}$ & $\begin{array}{c}101 \\
27 \\
\text { Boltont Basket } \\
27 \\
\text { Other Baskets } \\
\text { Lid down: top bskt } 27.4 \text {; others } 27.4 \\
\text { l.id up:boltom bskt } 33.35 \text {; ollhers } 33.5\end{array}$ \\
\hline
\end{tabular}

* $g$ 's computed assuming the MCO is slowed by piston-like cushioning effect from air being squeezed through the 0.25 inch diametral clearance between the cask and the $\mathrm{MCO}$ and by the cushioning effect of adding water to the bottom of the cask. (Alternate means of limiting the acceleration to $<35 \mathrm{~g}$ are being investigated.)

(1) Reference SARP Tables B7.21 and B7.24

Angle of impact for $C / G$ drop in cask is 10.5 degrees off vertical. 
- A drop (worst case orientation) of the MCO package inside the sealed transportation cask. For an end drop scenario, a secondary side slap down shall be considered. The $\mathrm{MCO}$ is physically constrained by the cask walls and remains in the cask. Note: For all drops when the $\mathrm{MCO}$ is in the cask, the $\mathrm{MCO}$ does not need to maintain a leak tight seal. The MCO shall be able to retain all particulate greater than 2 $\mathrm{mm}$ in size or greater after the deformation occurs.

- A vertical drop of the MCO package into the transport cask. Drop heights not to exceed 21.5 feet. "Piston effect" shall be included.

- Vertical drops of MCO package into a CSB storage tube with and without another $\mathrm{MCO}$ already within the tube. The tubes will contain impact limiters as required to reduce impact acceleration on the $\mathrm{MCO}$ and internals. Each MCO acceleration is limited to $35 \mathrm{~g}$ within the CSB tubes.

For all accelerations the fuel shall be modeled with the properties of stainless steel except for the scrap baskets which shall have hydrostatic properties when externally loaded. In cases where one component is dropped onto another (e.g. $\mathrm{MCO}$ onto an $\mathrm{MCO}$ in the storage tube, and $\mathrm{MCO}$ impacting the inside top or bottom of the cask), the eccentricity of the drop is negligible and does not require consideration.

Refer to Section 4, Item 31.

In accordance with the requirements above, the loads provided in Table 3 are evaluated in two ways. The loads that are applied to the MCO shell are evaluated in Appendix 5. These are all the loads that are applied to the containment boundary and have acceptance criteria coming from subsection NB. As stated above, the horizontal loading and corner drop loads when the MCO is in the cask are not evaluated. The cask provides the containment in these load conditions. Additionally there is a high probability of localized denting of the MCO shell where it interacts with the ring inside the cask. The localized denting in the side wall will give stresses beyond those acceptable for Service Level $D$. However, due to the high elongation of the material and the limited amount of distortion (thickness of the ring), no expected breach of containment by the MCO is expected. Since this is an inward denting the circumference criteria for criticality is also not expected to be violated. For all other load conditions the criteria of Subsection NB Reference [6.2] was met. The shell is also evaluated to demonstrate that the circumference restriction for criticality control purposes is not violated for any of the load conditions.

The second set of evaluations for the loadings in Table 3 was performed for the Mark $1 \mathrm{~A}$ baskets. In addition to meeting the criteria of Subsection $N G$ [6.37 the criteria of maintaining geometry for criticality control in sequential loadings of first the vertical loading and then the horizontal and/or corner loadings was imposed. This evaluation is performed in Appendix 7. Both the criteria for Subsection NG and the criticality control geometry was met for all load conditions. 
The evaluations demonstrates that the center tube does not move radially more than two inches. It is also demonstrated that the baskets do not deflect sufficiently to be come uncoupled allowing the fuel particles bigger than $2 \mathrm{~mm}$ to enter into the center void. The Mark IV baskets were not evaluated since there was no criteria.

\subsubsection{Nuclear Criticality Safety}

The MCO design shall achieve and maintain a critically safe array throughout the MCO design life. A criticality safety value of 0.95 for Keff shall be used for the $\mathrm{MCO}$ design, functions, and related activities. Per criticality analyses performed by the Buyer, this will be satisfied for MCOs containing MKIA fuel by a nominal 6.625 inch diameter void space at the longitudinal centerline of the MCO. This void space shall be maintained clear of fuel and fuel fragments larger than $2 \mathrm{~mm}$ spheres. The void space centerline shall not deviate more than two inches from the MCO centerline. The MCO shall maintain these conditions during and after being subjected to the design basis accidents described in Section 1.19.2 of Reference 6.1. MCOs containing MKIV fuel do not require this void space. Additionally, the MCO (for all fuel types) shall be capable of withstanding the effects of the DBAs outlined in Section 1.19 .2 of Reference 6.1 with the maximum inside circumference not exceeding the limits allowed in Section 1.9.5 of Reference 6.1.

Refer to Section 4, Item 32.

As stated above the $M C O$ shell is evaluated for all loadings specified in Table 3. The evaluation shows that none of the criticality criteria stated above is violated. Details can be found in Appendix 5. The Mark $1 A$ baskets are evaluated for all applicable loads in Table 3 and are demonstrated not to violate any of the criticality control criteria stated above. The details of the Mark $1 \mathrm{~A}$ baskets can be found in Appendix 7.

\subsubsection{Over Pressure Summary of MCO Fuel Basket Functions Relief}

The MCO shall relieve internal pressure in excess of its design pressure specified in Section 4.9.2, of Reference 6.1, MCO Design Criteria, both during and after being subjected to the events described in Section 4.19.2, of Reference 6.1, Design Basis Accidents. The MCO shall provide pressure relief devices imbedded in the shield plug to facilitate the configurations listed in Table 4. The pressure relief devices shall be capable of reset or replacement after performing their function. The pressure relief devices shall be selected to minimize service requirements during normal conditions. The rupture disk shall be covered with removable $1 / 4$-inch orifice plates to limit relief flow to levels acceptable to the CSB design as required. The process pressure relief valve shall be sized to accommodate a flow equal to or less than a $1 / 4$-inch diameter equivalent or the valve flow can be clamped to a $1 / 4$-inch diameter equivalent or less through an orifice or other type of device/physical feature. Selection of the process pressure relief valve shall be performed by the Buyer.

\section{Refer to Section 4, Item 33.}


Table 4. MCO Pressure Relief Configurations

(See Note 1 for Definition of Configuration Codes)

\begin{tabular}{|l|c|c|}
\hline \multicolumn{1}{|c|}{ Activity } & $\begin{array}{c}\text { MCO Process } \\
\text { Relief } \\
\text { Configuration }\end{array}$ & $\begin{array}{c}\text { MCO Safety } \\
\text { Relief } \\
\text { Configuration }\end{array}$ \\
\hline \hline Basin MCO/Cask loadout & $\mathrm{D}$ & $\mathrm{D}$ \\
\hline $\begin{array}{l}\text { Transport from Basin to Cold Vacuum Drying } \\
\text { Station }\end{array}$ & $\mathrm{D}$ & $\mathrm{D}$ \\
\hline Cold Vacuum Drying Station Configuration & Note 2) & $\mathrm{C}(150)$ \\
\hline $\begin{array}{l}\text { Transport from Cold Vacuum Drying to the Canister } \\
\text { Storage Building }\end{array}$ & $\mathrm{B}(<150)$ & $\mathrm{C}(150)$ \\
\hline $\begin{array}{l}\text { Canister Storage Building - MCO/Cask Receipt and } \\
\text { transfer to service pit }\end{array}$ & $\mathrm{B}(<150)$ & $\mathrm{C}(150)$ \\
\hline $\begin{array}{l}\text { Canister Storage Building - MHM transfer from } \\
\text { service pit to staging tube }\end{array}$ & $\mathrm{B}(<150)$ & $\mathrm{C}(150)$ \\
\hline Canister Storage Building - Staging & $\mathrm{B}(<150)$ & $\mathrm{C}(150)$ \\
\hline $\begin{array}{l}\text { Canister Storage Building - MHM transfer from } \\
\text { staging to Hot Conditioning station }\end{array}$ & $\mathrm{B}(<150)$ & $\mathrm{C}(150)$ \\
\hline Hot Conditioning & $\mathrm{Note} 3)$ & $\mathrm{C}(150)$ \\
\hline $\begin{array}{l}\text { Canister Storage Building - MHM transfer from Hot } \\
\text { Conditioning to a storage tube }\end{array}$ & $\mathrm{C}(<150)$ & $\mathrm{C}(150)$ \\
\hline Canister Storage Building - Interim Storage & $\mathrm{C}(<150)$ & $\mathrm{C}(150)$ \\
\hline
\end{tabular}

Notes: 1. MCO Relief Configurations:
A - Sealed, no relief
$B$ - Active Pressure Relief Valve, with $1 / 4$-inch or less orifice plate (Relief Setting, psig)
C - Active Rupture Disk, with $1 / 4$-inch or less orifice plate
(Relief Setting, psig)
D. Active Vent

Process relief path includes filtration to reduce economic consequences of potential relief (the internal filter described in Section 4.14 of Reference 6.1, may be used for this function). Safety relief path is unfiltered to preclude potential for relief path flow restrictions. 
2. Filtered process relief path provided by the Cold Vacuum Drying offgas system via a process relief valve set to relieve at $25 \mathrm{psig}$ (provided by CVD).

3. Filtered process relief path provided by the Hot Conditioning offgas system via process relief valve set to relieve at approximately $10 \mathrm{psig}$ (provided by HCS).

Over pressure protection is provided for the MCO primarily by the use of a ASME Code rupture disk set at the design pressure of 150 psig. This device is located in the process valve that accesses the short process tube. It can be covered with a flange with at/4-inch diameter hole in it to provide the required flow restriction. The rupture disk can be replaced by replacing the process valve plug. The plug could then be refurbished with a new rupture disk if desired and decontamination levels permitting.

The other over pressure device is a pressure relief valve to be selected by the Buyer that will connected directly to the HEPA filter bank. This relief valve can be replaced by either an external HEPA filter or a rupture disk. Per the Specification non of these devices need to meet ASME Code criteria. In accordance with the ASME Code if they do not meet Code criteria they can not be counted on. Their presence however partially offsets the Code requirement that prevents a rupture disk from being the only pressure relief device for Subsection NB vessel. Appendix 12 provides details on the rupture disk.

\subsection{Quality Assurance Requirements}

All quality assurance activities shall be in accordance with 10 CFR 71, Packaging and Transportation of Radioactive Materials (CFR 1995), Subpart H.

A graded approach to quality assurance requirements shall be implemented in accordance with the following criteria:

- For Safety Class structures, systems and components, a comprehensive $10 \mathrm{CFR} 71$, Subpart $\mathrm{H}$, program shall be applied in addition to the quality requirements from applicable industry codes and standards.

- For Safety Significant structures, systems and components, select quality requirements that would enhance the reliability of the structures, systems and components performing their safety function (if any) shall be applied in addition to the quality requirements from applicable industry codes and standards.

- For other structures, systems and components, the quality requirements from applicable industry codes and standards shall be applied. 
The HNF Occurrence Reporting System shall be implemented as outlined in HNF-CM-1-5, Standard Operating Practices (HNF 1996), Section 7.1 for the design and fabrication of the MCO. The MCO fabrication specification shall require suppliers to report defects and noncompliances in items or services.

\section{Refer to Section 4, Item 33.}

$A$ graded quality assurance approach has been implemented in the design and fabrication requirements for the $M C O$ and its fuel baskets. Per DESH direction, a QA program viewed by $D E S H$ as equivalent to a comprehensive $10 \mathrm{CFR} 71$, Subpart $H$ program is required for the fabrication of the safety class items that being the containment boundary items plus the Mark $1 \mathrm{~A}$ baskets. These components, except as noted in Appendix 18 , shall be in full compliance with the applicable codes which are ASME Code, Section III, Division 1, Subsection NB for the containment boundary and ASME Code, Section III, Division 1, Subsection NG for the Mark $1 \mathrm{~A}$ baskets. Mark $I V$ baskets can be fabricated to a lesser quality assurance program where materials are still validated to the specification and the welding is qualified to a procedures and inspected. This combined with the acceptance dimensional checks verify that the baskets will function as required and have the necessary strength to comply with ANSI N14.6 as specified. For all items documentation is required to demonstrate compliance with the requirements. 


\begin{tabular}{|c|c|c|c|}
\hline $\begin{array}{l}\text { Design/Interface } \\
\text { Parameter }\end{array}$ & Requirement & Source(s) & $\begin{array}{l}\text { How Design Complies with P.S. } \\
\text { Requirement }\end{array}$ \\
\hline 1. Design Life & $\begin{array}{l}\text { Maintain fuel } \\
\text { elements and fuel } \\
\text { fragments for } 40 \\
\text { years. }\end{array}$ & $\begin{array}{l}\text { P.S. } 4.1 \text {, } \\
\text { Rev. } 3\end{array}$ & $\begin{array}{l}\text { Material and design have no } \\
\text { known aspects that will prevent } \\
\text { compliance. Materials of } \\
\text { construction were selected to } \\
\text { resist corrosion. }\end{array}$ \\
\hline 2. SNF Confinement & $\begin{array}{l}\text { Confine contents } \\
\text { during all normal } \\
\text { operations and } \\
\text { DBAs. }\end{array}$ & $\begin{array}{l}\text { P.S. } 4.2 \text {, } \\
\text { Rev. } 3\end{array}$ & $\begin{array}{l}\text { Confinement is demonstrated } \\
\text { Appendices } 4,5 \text { and } 11 \text {. }\end{array}$ \\
\hline 3. SNF Containment & $\begin{array}{l}\text { Total gaseous leaks } \\
\text { shall not exceed } 1 \mathrm{x} \\
10^{-4} \mathrm{SCC} / \mathrm{sec} \text {. When } \\
\text { sealed by welding } \\
\text { after hot } \\
\text { conditioning, shall be } \\
\text { capable of not } \\
\text { exceeding a } \\
\text { maximum total leak } \\
\text { rate of } 1 \times 10^{-7} \\
\mathrm{SCC} / \mathrm{sec} \text {. }\end{array}$ & $\begin{array}{l}\text { P.S. } 4.3 \\
\text { Rev. } 3\end{array}$ & $\begin{array}{l}\text { All seals and closure are } \\
\text { designed with capabilities of } \\
\text { leaktightness better than } 1 \times 10^{-4} \\
\text { SCC/sec. } \\
\text { The welded portion of the } \\
\text { containment boundary is tested } \\
\text { to demonstrated leaktightness } \\
\text { better than } 1 \times 10^{-7} \mathrm{SCC} / \mathrm{sec} \text {. The } \\
\text { field closure weld is liquid } \\
\text { penetrant inspected providing a } \\
\text { redundant seal to the mechanical } \\
\text { seal. }\end{array}$ \\
\hline 4. Maintainability & $\begin{array}{l}\text { Designed to } \\
\text { minimize the need } \\
\text { for preventive } \\
\text { maintenance, and } \\
\text { allow replacement of } \\
\text { the externally } \\
\text { mounted pressure } \\
\text { relief devices/HEPA } \\
\text { filter. }\end{array}$ & $\begin{array}{l}\text { P.S. } 4.4 \text {, } \\
\text { Rev. } 3\end{array}$ & $\begin{array}{l}\text { No preventive maintenance is } \\
\text { expected. The relief devices are } \\
\text { designed to be replaced as } \\
\text { needed. }\end{array}$ \\
\hline
\end{tabular}




\begin{tabular}{|c|c|c|c|}
\hline 5. Human Factors & $\begin{array}{l}\text { Components shall be } \\
\text { designed to facilitate } \\
\text { handling and } \\
\text { assembly with the } \\
\text { use of appropriate } \\
\text { handling equipment } \\
\text { and also enable } \\
\text { handling while } \\
\text { wearing protective } \\
\text { clothing used in } \\
\text { radiation zones. }\end{array}$ & $\begin{array}{l}\text { P.S. } 4.5 \text {, } \\
\text { Rev. } 3\end{array}$ & $\begin{array}{l}\text { The components can be easily } \\
\text { handled and assembled with the } \\
\text { appropriate handling equipment. }\end{array}$ \\
\hline 6. Interchangability & $\begin{array}{l}\text { To the maximum } \\
\text { extent possible. (i.e., } \\
\text { any set of like } \\
\text { baskets can be loaded } \\
\text { into any MCO shell, } \\
\text { any MCO shield plug } \\
\text { and locking ring can } \\
\text { be used to close and } \\
\text { seal any MCO shell, } \\
\text { etc.). }\end{array}$ & $\begin{array}{l}\text { P.S. } 4.6 \text {, } \\
\text { Rev. } 3\end{array}$ & $\begin{array}{l}\text { All major components, MCO } \\
\text { shell assembly, Shield Plug } \\
\text { Assembly, Process valve plugs, } \\
\text { cover caps, locking rings and all } \\
\text { baskets are designed to be fully } \\
\text { interchangeable. }\end{array}$ \\
\hline $\begin{array}{l}\text { 7. Environmental } \\
\text { Conditions }\end{array}$ & $\begin{array}{l}\text { Capable of } \\
\text { performing its } \\
\text { mission while } \\
\text { subjected to the } \\
\text { environmental } \\
\text { conditions listed in } \\
\text { Table } 3.1 \text {. }\end{array}$ & $\begin{array}{l}\text { P.S. } 4.7 \\
\text { Rev. } 3\end{array}$ & $\begin{array}{l}\text { The MCO materials are fully } \\
\text { compatible with the } \\
\text { environmental conditions } \\
\text { specified. }\end{array}$ \\
\hline 8. Transportability & $\begin{array}{l}\text { Transportable by } \\
\text { highway from the } \\
\text { fabricator facility to } \\
\text { the location within } \\
\text { the Hanford site. }\end{array}$ & $\begin{array}{l}\text { P.S. } 4.8, \\
\text { Rev. } 3\end{array}$ & $\begin{array}{l}\text { The dimensions of the } \\
\text { components shown in Appendix } \\
1 \text { makes them all transport } \\
\text { compatible. }\end{array}$ \\
\hline
\end{tabular}




\begin{tabular}{|c|c|c|c|}
\hline $\begin{array}{l}\text { 9. Code } \\
\text { Requirements }\end{array}$ & $\begin{array}{l}\text { DOE Order } 6430.1 \mathrm{~A} ; \\
\text { intent of ASME } \\
\text { Boiler and Pressure } \\
\text { Vessel Code, Section } \\
\text { III, Subsection NB; } \\
\text { NUREG/CR } 3854 \text {, } \\
\text { UCRL-53544. }\end{array}$ & $\begin{array}{l}\text { P.S. } 4.9 .1 \\
\text { Rev. } 3\end{array}$ & $\begin{array}{l}\text { The components meet the intent } \\
\text { of the ASME Code requirements } \\
\text { as specified. Subsection NB for } \\
\text { Containment and Subsection NG } \\
\text { for criticality sensitive baskets } \\
\text { (Mark 1A) }\end{array}$ \\
\hline 10. Design Pressure & 150 psig & $\begin{array}{l}\text { P.S. } 4.9 .2 \text {, } \\
\text { Rev. } 3\end{array}$ & $\begin{array}{l}\text { The MCO containment is } \\
\text { designed for a } 150 \text { psig design } \\
\text { pressure. }\end{array}$ \\
\hline $\begin{array}{l}\text { 11. Design } \\
\text { Temperature }\end{array}$ & $375^{\circ} \mathrm{C}$ & $\begin{array}{l}\text { P.S. } 4.9 .2 \\
\text { Rev. } 3\end{array}$ & $\begin{array}{l}\text { The MCO containment and } \\
\text { components are designed for } \\
\text { processing temperatures up to } \\
375^{\circ} \mathrm{C} \text {. See Appendix } 11\end{array}$ \\
\hline $\begin{array}{l}\text { 12. Processing } \\
\text { Operating Pressure }\end{array}$ & $\begin{array}{l}\text { Full internal 25.psig } \\
\text { external @ } 75^{\circ} \mathrm{C}\end{array}$ & $\begin{array}{l}\text { P.S. } 4.9 .2 \text {, } \\
\text { Rev. } 3\end{array}$ & $\begin{array}{l}\text { The load conditions evaluated } \\
\text { bound this condition. See } \\
\text { Appendix } 11 \text {. }\end{array}$ \\
\hline $\begin{array}{l}\text { 13. Processing } \\
\text { Operating Pressure }\end{array}$ & $\begin{array}{l}\text { Full vacuum internal } \\
0 \text { psig external @ } \\
375^{\circ} \mathrm{C}\end{array}$ & $\begin{array}{l}\text { P.S. } 4.9 .2 \text {, } \\
\text { Rev. } 3\end{array}$ & $\begin{array}{l}\text { The load conditions evaluated } \\
\text { bound this condition. See } \\
\text { Appendix } 11 \text {. }\end{array}$ \\
\hline $\begin{array}{l}\text { 14. Processing } \\
\text { Operating Pressure }\end{array}$ & $\begin{array}{l}75 \text { psig internal, } 0 \\
\text { psig external, @ } \\
375^{\circ} \mathrm{C}\end{array}$ & $\begin{array}{l}\text { P.S. } 4.9 .2 \text {, } \\
\text { Rev. } 3\end{array}$ & $\begin{array}{l}\text { The design pressure and pressure } \\
\text { bound this condition. See } \\
\text { Appendix } 11 \text {. }\end{array}$ \\
\hline $\begin{array}{l}\text { 15. Post Processing } \\
\text { Operating Pressure } \\
\text { and Temperature }\end{array}$ & $\begin{array}{l}150 \text { psig internal, @ } \\
132^{\circ} \mathrm{C}\end{array}$ & $\begin{array}{l}\text { P.S. } 4.9 .2 \text {, } \\
\text { Rev. } 3\end{array}$ & $\begin{array}{l}\text { The design pressure and } \\
\text { temperature bound this } \\
\text { condition. See Appendix } 11 \text {. }\end{array}$ \\
\hline $\begin{array}{l}\text { 16. Design Thermal } \\
\text { Transient Under } \\
\text { Normal Conditions }\end{array}$ & $\begin{array}{l}100^{\circ} \mathrm{C} / \mathrm{h} \text { from } 20^{\circ} \mathrm{C} \text { to } \\
350^{\circ} \mathrm{C}, 5 \text { thermal } \\
\text { cycles. }\end{array}$ & $\begin{array}{l}\text { P.S. 4.9.2., } \\
\text { Rev. } 3\end{array}$ & $\begin{array}{l}\text { This load condition is evaluated } \\
\text { in Appendix } 11 .\end{array}$ \\
\hline $\begin{array}{l}\text { 17. Maximum temp } \\
\text { gradient between } \\
\text { MCO shell and } \\
\text { center of shield plug. }\end{array}$ & $\begin{array}{l}100^{\circ} \mathrm{C} \text {, thermal } \\
\text { expansion } 1 \text { inch in } \\
\text { axial direction }\end{array}$ & $\begin{array}{l}\text { P.S. } 4.9 .2 \text {, } \\
\text { Rev. } 3\end{array}$ & $\begin{array}{l}\text { This load condition is evaluated } \\
\text { in Appendix } 11 .\end{array}$ \\
\hline
\end{tabular}


HNF-SD-SNF-DR-003, Rev. 0

\begin{tabular}{|c|c|c|c|}
\hline $\begin{array}{l}\text { 18. MCO Shell } \\
\text { Design }\end{array}$ & $\begin{array}{l}1.49 \text { inch Minimum } \\
\text { between inside of } \\
\text { MCO bottom and } \\
\text { bottom of lowest } \\
\text { basket. }\end{array}$ & $\begin{array}{l}\text { P.S. } 4.10 \text {, } \\
\text { Rev. } 3\end{array}$ & See Appendix 1 for compliance. \\
\hline $\begin{array}{l}\text { 19. MCO Closure } \\
\text { Design }\end{array}$ & $\begin{array}{l}\text { Final welded closure } \\
\text { cap. Mechanical } \\
\text { closure prior to } \\
\text { welding cover cap in } \\
\text { place. }\end{array}$ & $\begin{array}{l}\text { P.S. } 4.11 \text {, } \\
\text { Rev. } 3\end{array}$ & See Appendix 1 for compliance \\
\hline $\begin{array}{l}\text { 20. Fuel Basket } \\
\text { Design }\end{array}$ & $\begin{array}{l}\text { MKIA } 304 \mathrm{~L} \text { or a } \\
\text { material of equal or } \\
\text { greater corrosion } \\
\text { resistance properties. } \\
\text { Service Level A } \\
\text { requirements and } \\
\text { Service Level D. } \\
\text { ASME Boiler and } \\
\text { Pressure Vessel } \\
\text { Code, Section III, } \\
\text { Subsection NG; } \\
\text { NUREG/CR } 3854 \text {, } \\
\text { UCRI-53544; ANSI } \\
\text { N14.6-1986. } \\
\text { Capacity = } 48 \\
\text { elements. MKIV - } \\
\text { N14.6 for lifting } \\
\text { capacity } 54 \text { elements. } \\
\text { MKIA Scrap - Same } \\
\text { as MKIA except } \\
\text { capacity is N/A. } \\
\text { MKIV Scrap - Same } \\
\text { as MKIV except } \\
\text { capacity is N/A. }\end{array}$ & $\begin{array}{l}\text { P.S. } 4.12 \text {, } \\
\text { Rev. } 3\end{array}$ & $\begin{array}{l}\text { See Appendix } 1 \text { for compliance } \\
\text { with design requirements. } \\
\text { Appendix } 7 \text { demonstrates the } \\
\text { Mark IA baskets capabilities to } \\
\text { comply with the load } \\
\text { requirements and applicable } \\
\text { Code requirements. } \\
\text { Appendices } 8 \text { and } 9 \text { demonstrate } \\
\text { the capabilities of the Mark IV } \\
\text { basket to meet the required load } \\
\text { conditions including lifting. The } \\
\text { capacity of the Mark IV fuel } \\
\text { basket is } 54 \text { elements and the } \\
\text { capacity of the Mark } 1 \mathrm{~A} \text { basket } \\
\text { is } 48 \text { elements. }\end{array}$ \\
\hline
\end{tabular}


HNF-SD-SNF-DR-003, Rev. 0

\begin{tabular}{|c|c|c|c|}
\hline $\begin{array}{l}\text { 21. MCO Shield } \\
\text { Plug Design }\end{array}$ & $\begin{array}{l}\text { Designed to mate } \\
\text { with open end of the } \\
\text { MCO shell and also } \\
\text { mates with the end } \\
\text { effector on the top } \\
\text { SNF fuel basket. } \\
\text { One inch minimum } \\
\text { free space between } \\
\text { the bottom of the } \\
\text { shield plug assembly } \\
\text { and the top of the } \\
\text { SNF elements or } \\
\text { fragments. Feature } \\
\text { an integrally } \\
\text { machined } \\
\text { axisymmetric lifting } \\
\text { ring with a } 12 \text { ton } \\
\text { lifting capacity. } \\
\text { ANSI N14.6-1986; } \\
\text { 10 CFR } 835 \text {, Subpart } \\
\text { K; DOE Order } \\
5480.11, \text { Paragraph } \\
9 \text { a; HSRCM-1, } \\
\text { Sections } 111 \text { and } \\
311 ; \text { HNF-IP-1043, } \\
\text { Section } 8.0 \text {; and } \\
\text { NRC Regulatory } \\
\text { Guide } 8.8, \text { Section } \\
\text { C.2.b, and C. } 2 . f . \\
\text { a }\end{array}$ & $\begin{array}{l}\text { P.S. } 4.13 \text {, } \\
\text { Rev. } 3\end{array}$ & $\begin{array}{l}\text { The MCO Shield Plug assembly } \\
\text { closes the fully open MCO, } \\
\text { provides shielding, protects the } \\
\text { HEPA filter bank, stabilizes the } \\
\text { top fuel basket, allows for } \\
\text { penetrations to the filter bank the } \\
\text { process tube and the process } \\
\text { tubes. The features of the shield } \\
\text { plug assembly and the clearances } \\
\text { between the fuel and the } \\
\text { assembly can be seen in } \\
\text { Appendix } 1 \text {. The shield plug is } \\
\text { held in place with jacking bolts } \\
\text { (set screws) between it and the } \\
\text { locking ring. The locking ring } \\
\text { has a intregal lifting ring that has } \\
\text { in excess of a twelve ton capacity } \\
\text { cornplying with ANSI N14.6 for } \\
\text { non-critical lifts. }\end{array}$ \\
\hline $\begin{array}{l}\text { 22. Internal Process } \\
\text { Filter }\end{array}$ & $\begin{array}{l}4 \text { internal process } \\
\text { filters with up to } 20 \\
\mathrm{ft}^{2} \text { of filter media for } \\
\text { the assembly. Less } \\
\text { than } 3 \text { micro meters } \\
\text { in pore size. Capable } \\
\text { of withstanding a } 100 \\
\mathrm{G} \text { drop without } \\
\text { damage. }\end{array}$ & $\begin{array}{l}\text { P.S. } 4.14, \\
\text { Rev. } 3\end{array}$ & $\begin{array}{l}\text { The internal process filters are } \\
\text { specified by the buyer. Details } \\
\text { can be found in Appendix } 15 \text {. } \\
\text { The attachment capability of the } \\
\text { manifold to withstand a } 100 \mathrm{~g} \\
\text { loading. (Actually the maximum } \\
\text { loading is the } 101 \mathrm{~g} \text { horizontal } \\
\text { loading is shown in Appendix } \\
10 \text {. }\end{array}$ \\
\hline $\begin{array}{l}\text { 23. Design Goal } \\
\text { MCO Weight }\end{array}$ & $\begin{array}{l}\text { MCO with MKIA } \\
\text { fuel (dry) } 16175 \text { Ibs., } \\
\text { with MKIV fuel } \\
\text { (dry) } 19142 \text { lbs. }\end{array}$ & $\begin{array}{l}\text { P.S. 4.9.3, } \\
\text { Rev. } 3\end{array}$ & $\begin{array}{l}\text { The nominal weight with shield } \\
\text { plug and no cover, MK } 1 \mathrm{~A} \text { fuel } \\
\text { (dry is } 17,248 \mathrm{lbs} \text { and with } \\
\text { MKIV fuel (dry) is } 19,378 \mathrm{lbs} \text {. }\end{array}$ \\
\hline
\end{tabular}


HNF-SD-SNF-DR-003, Rev. 0

\begin{tabular}{|l|l|l|l|}
\hline 24. MCO Height & $\begin{array}{l}160 \text { inches with out } \\
\text { cap. 165.60 inches } \\
\text { with cap. }\end{array}$ & $\begin{array}{l}\text { P.S. 4.9.4, } \\
\text { Rev. 2 }\end{array}$ & $\begin{array}{l}\text { As seen in Appendix 1. } \\
\text { Maximum height without cap is } \\
160 \text { inches. } \\
\text { Maximum Height with cap is } \\
165.4 \text { inches. }\end{array}$ \\
\hline 25. MCO Diameter & $\begin{array}{l}\text { Nominal OD is 24". } \\
\text { Above bottom shield } \\
\text { plug is 25.31". }\end{array}$ & $\begin{array}{l}\text { P.S. 4.9.5, } \\
\text { Rev.3 }\end{array}$ & $\begin{array}{l}\text { The nominal OD is 24 inches. } \\
\text { Above the 148 inch elevation the } \\
\text { maximum OD is 25.31 inches. } \\
\text { Below 148 inches, the maximum } \\
\text { circumference is less than 73.04. }\end{array}$ \\
\hline 26. Materials, \\
Processes, and Parts & $\begin{array}{l}\text { MCO shell shall be } \\
\text { fabricated from type } \\
\text { 304L stainless steel. } \\
\text { All materials shall be } \\
\text { ASME/ASTM } \\
\text { certified materials. } \\
\text { Ferritic steel } \\
\text { materials shall meet } \\
\text { the criteria of } \\
\text { NUREG/CR-3826. }\end{array}$ & $\begin{array}{l}\text { P.S. 4.15, } \\
\text { Rev. 3 }\end{array}$ & $\begin{array}{l}\text { All welded components of the } \\
\text { MCO including the shell are } \\
\text { fabricated from 304L stainless } \\
\text { steel. No Ferritic materials are } \\
\text { used. All materials are } \\
\text { designated ASME (SA) or } \\
\text { ASTM (A) as shown in } \\
\text { Appendix 1. }\end{array}$ \\
\hline 27. MCO Corrosion \\
Control & $\begin{array}{l}\text { Cleanliness during } \\
\text { fabrication, handling, } \\
\text { and storage. ASTM } \\
\text { A 380-94 and ASME } \\
\text { NQA-1. A } \\
\text { mechanical seal is } \\
\text { required for final } \\
\text { closure. }\end{array}$ & $\begin{array}{l}\text { P.S. 4.16, } \\
\text { Rev.3 }\end{array}$ & $\begin{array}{l}\text { Cleanliness is specified during } \\
\text { fabrication handling, } \\
\text { transportation and storage. This } \\
\text { is covered in the fabrication } \\
\text { specification and the warehouse } \\
\text { plan Appendix 17. A } \\
\text { mechanical closure is used for } \\
\text { final closure. }\end{array}$ \\
\hline
\end{tabular}


HNF-SD-SNF-DR-003, Rev, 0

\begin{tabular}{|c|c|c|c|}
\hline 28. Welded Joints & $\begin{array}{l}\text { ASME Section III, } \\
\text { Division } 1, \text { NB-3350. } \\
\text { Butt welds to be } \\
\text { ground flush to } \\
\text { within .03 inches of } \\
\text { base metal. All } \\
\text { MCO pressure } \\
\text { boundary welds and } \\
\text { welds bearing the } \\
\text { weight of the fully } \\
\text { loaded MCO must be } \\
\text { designed for and pass } \\
100 \% \text { volumetric } \\
\text { examination per } \\
\text { ASME requirements. }\end{array}$ & $\begin{array}{l}\text { P.S. } 4.17 \text {, } \\
\text { Rev. } 3\end{array}$ & $\begin{array}{l}\text { All pressure boundary welds are } \\
\text { designed as ASME Section III } \\
\text { Division } 1, \mathrm{NB}-3350 \text { welds. The } \\
\text { final closure weld produced in } \\
\text { the field does not fully comply } \\
\text { since it can not be volumetrically } \\
\text { inspected. All welds are flush } \\
\text { within } 0.03 \text { inches. }\end{array}$ \\
\hline $\begin{array}{l}\text { 29. Decontamination } \\
\text { Provisions }\end{array}$ & $\begin{array}{l}\text { All exposed surfaces } \\
\text { shall be smooth } \\
\text { without cracks or } \\
\text { crevices. }\end{array}$ & $\begin{array}{l}\text { P.S. } 4.18, \\
\text { Rev. } 3\end{array}$ & $\begin{array}{l}\text { As shown in Appendix } 1 \text { all } \\
\text { exposed surfaces are smooth and } \\
\text { without cracks or crevices. }\end{array}$ \\
\hline $\begin{array}{l}\text { 30. Safety } \\
\text { Requirements }\end{array}$ & $\begin{array}{l}\text { HNF-CM-4-46, } \\
\text { Section } 9.0 ; 10 \text { CFR } \\
71.73 \text { (3); Service } \\
\text { Level D requirements } \\
\text { of the ASME B \& } \\
\text { PV Code, Section III, } \\
\text { Subsection NB. }\end{array}$ & $\begin{array}{l}\text { P.S. } 4.19 .1 \text {, } \\
\text { Rev. } 3\end{array}$ & $\begin{array}{l}\text { The components are classified in } \\
\text { accordance with HNF-CM-4-46 } \\
\text { and the applicable sections of the } \\
\text { ASME Code and Service Level } \\
\text { conditions are complied with. }\end{array}$ \\
\hline $\begin{array}{l}\text { Earthquake } \\
\text { Drops }\end{array}$ & $\begin{array}{l}\text { Temperature increase } \\
\text { of } 122^{\circ} \mathrm{C} \text { for } 180 \\
\text { minutes after the } \\
\text { flow. } \\
\text { Acceleration of CSB } \\
\text { of } 35 \mathrm{~g} \text {. } \\
\text { See Table } 3 \text { in } \\
\text { Section } 3.19 .2 \text {. }\end{array}$ & $\begin{array}{l}\text { P.S. 4.19.2, } \\
\text { Rev. } 3\end{array}$ & $\begin{array}{l}\text { The conditions resulting from the } \\
\text { fire are bounded by other cases } \\
\text { analyzed for. } \\
\text { The loadings for the drop } \\
\text { conditions bound the earthquake } \\
\text { conditions. The MCO is shown } \\
\text { to meet the drop load conditions } \\
\text { in Appendix } 5 \text {. The Mark A } \\
\text { baskets are shown to with stand } \\
\text { the loadings from Table } 3 \text { of the } \\
\text { Performance Specification in } \\
\text { Appendix } 7 \text {. Mark IV baskets } \\
\text { are not evaluated since there is } \\
\text { no criteria for them. }\end{array}$ \\
\hline
\end{tabular}




\begin{tabular}{|l|l|l|l|}
\hline 32. Criticality Safety & $\begin{array}{l}\text { 6.625" void space in } \\
\text { center of MCO for } \\
\text { MKIA baskets. }\end{array}$ & $\begin{array}{l}\text { Appendix } 7 \text { demonstrates that } \\
\text { the void space is maintained } \\
\text { even after sequential drops of } \\
\text { vertical and then horizontal. } \\
\text { Appendix 5 demonstrates that } \\
\text { the circumferential requirements } \\
\text { are met. }\end{array}$ \\
\hline $\begin{array}{l}\text { 33. Overpressure } \\
\text { Relief }\end{array}$ & $\begin{array}{l}\text { MCO shail relieve } \\
\text { internal pressure. }\end{array}$ & $\begin{array}{l}\text { P.S. } 4.19 .4, \\
\text { Rev. } 3\end{array}$ & $\begin{array}{l}\text { Relief device features have been } \\
\text { incorporated in the design. See } \\
\text { Appendix 1. }\end{array}$ \\
\hline 34. Q.A. & Subpart H. & $\begin{array}{l}\text { P.S. } 5.0, \\
\text { Rev. } 3\end{array}$ & $\begin{array}{l}\text { The fabrication specification } \\
\text { requires a full 10 CFR 71 } \\
\text { Subpart H program for the } \\
\text { fabrication of the safety class } \\
\text { items the pressure boundary } \\
\text { components and the criticality } \\
\text { control sensitive components, } \\
\text { (Mark 1A baskets) }\end{array}$ \\
\hline
\end{tabular}

\subsection{SUMMARY OF COMPLIANCE WITH REQUIREMENTS}

The design of the MCO and fuel baskets is in full compliance with the requirements of the Performance Specification, Reference 6.1. The compliance is demonstrated in the design drawings shown in Appendix 1 and in the evaluation of the design to the specified requirements in Appendices 2-17. 
HNF-SD-SNF-DR-003, Rev. 0

\subsection{REFERENCES}

6.1 HNF-S-0426, Rev 3, Performance Specification for the Spent Nuclear Fuel MultiCanister Overpack February 7, 1997

6.2 ASME Code, Section III, Division 1, Subsection NB, 1995 Edition, with 1995 Addenda

6.3 ASME Code, Section III, Division 1, Subsection NG, 1995 Edition with 1995 Addenda

6.4 HNF-SD-SNF-TI-015 Rev. 0, Spent Nuclear Fuel Project Technical Data book August 11, 1995

6.5 ANSI N 14.6, "Special Lifting Devices for Shipping Containers Weighing 10,000 Pounds $(4500 \mathrm{~kg}$ ) or More", 1986. 


\section{APPENDICES}

Appendix 1 MCO Drawings

Appendix 2 Material Evaluation

Appendix 3 Weight Summary

Calculation - 01

Appendix 4 MCO Closure Bolt Preload Modeling and Response

Calculation -02

Appendix 5 MCO Structural Drop Analysis

Calculation - 03

Appendix 6 Stress Analysis of the Lifting Cap and Canister Collar

Calculation - 04

Appendix 7 Stress Analysis of the Mark 1A Storage and Scrap Baskets

Calculation - 05

Appendix 8 Stress Analysis of the Mark IV Storage Baskets

Calculation - 06

Appendix 9 Stress Analysis of the Mark IV Scrap Basket

Calculation - 07

Appendix 10 Stress Analysis of Shield Plug Interface Components

Calculation - 08

Appendix 11 MCO Thermal Stress Evaluation

Calculation - 09

Appendix 12 Rupture Disk Data

Appendix 13 Main Seal Data

Appendix 14 Seal Data for Process Valve, Covers, and Filters

Appendix 15 HEPA Filter Data

Appendix 16 K-Basin MCO Shield Plug Thickness Technical Evaluation

Appendix 17 Warehouse Plan

Appendix 18 Exception Report

Appendix 19 Fabrication Specification for Multi-Canister Overpack 


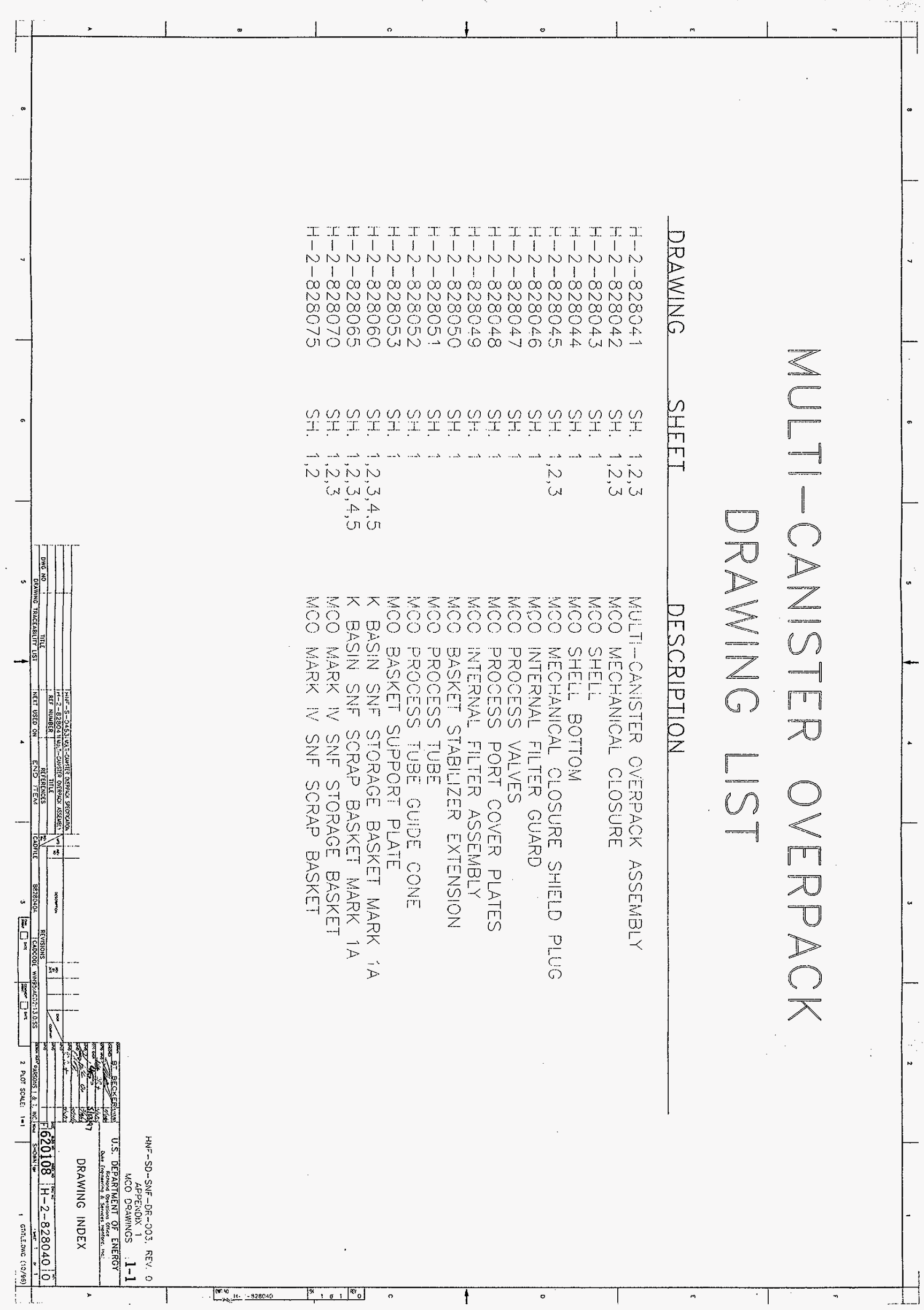




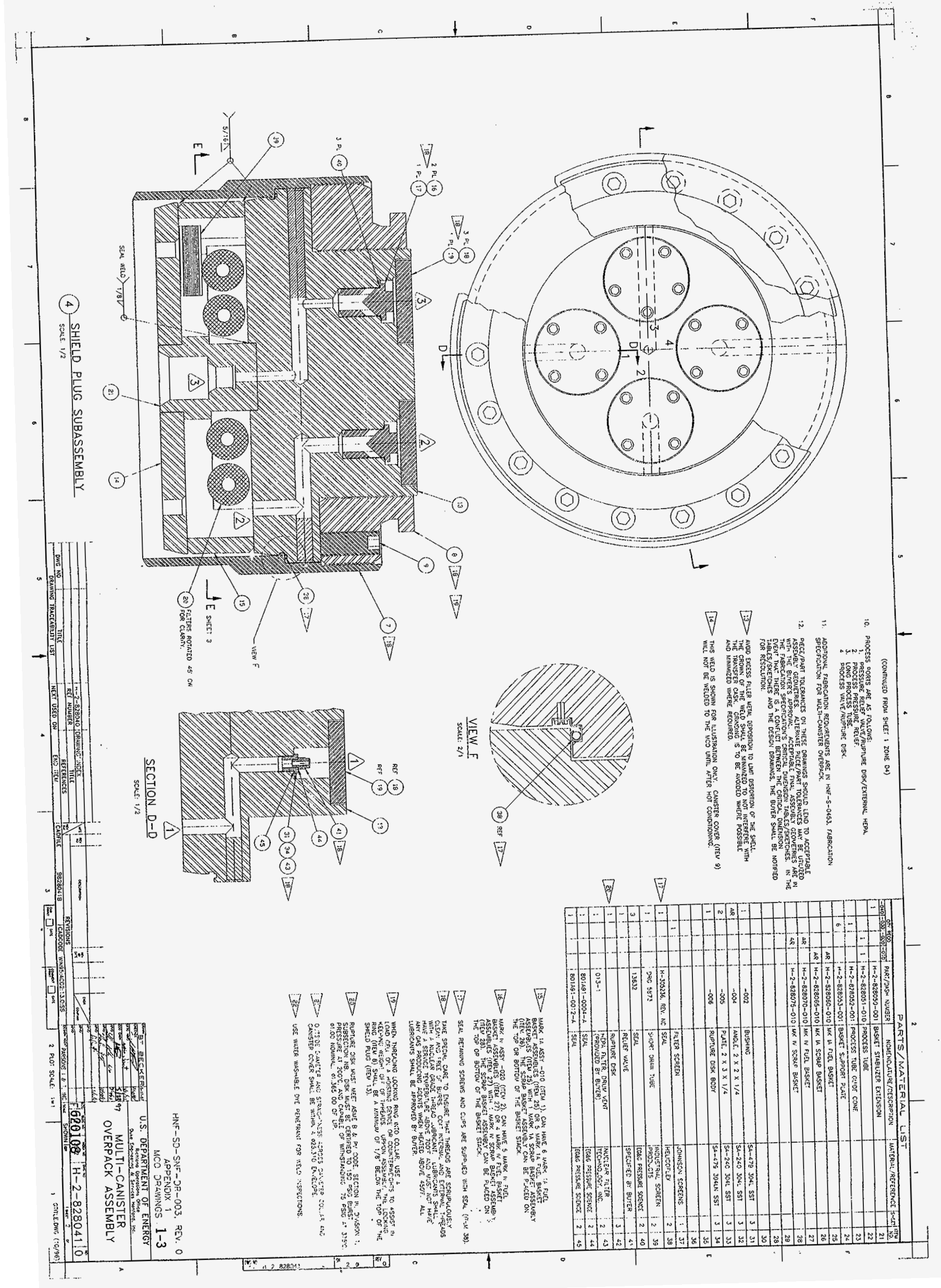




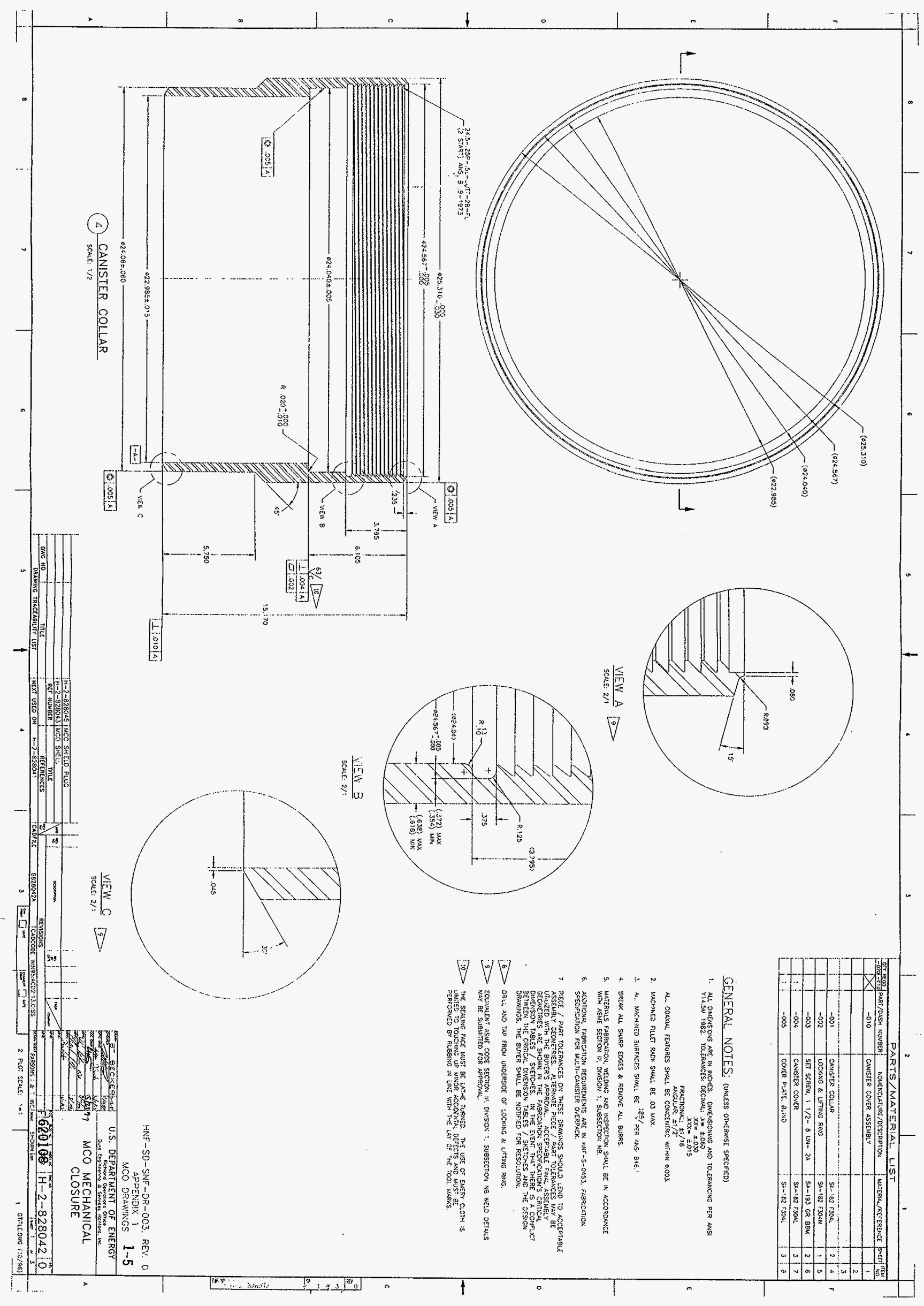




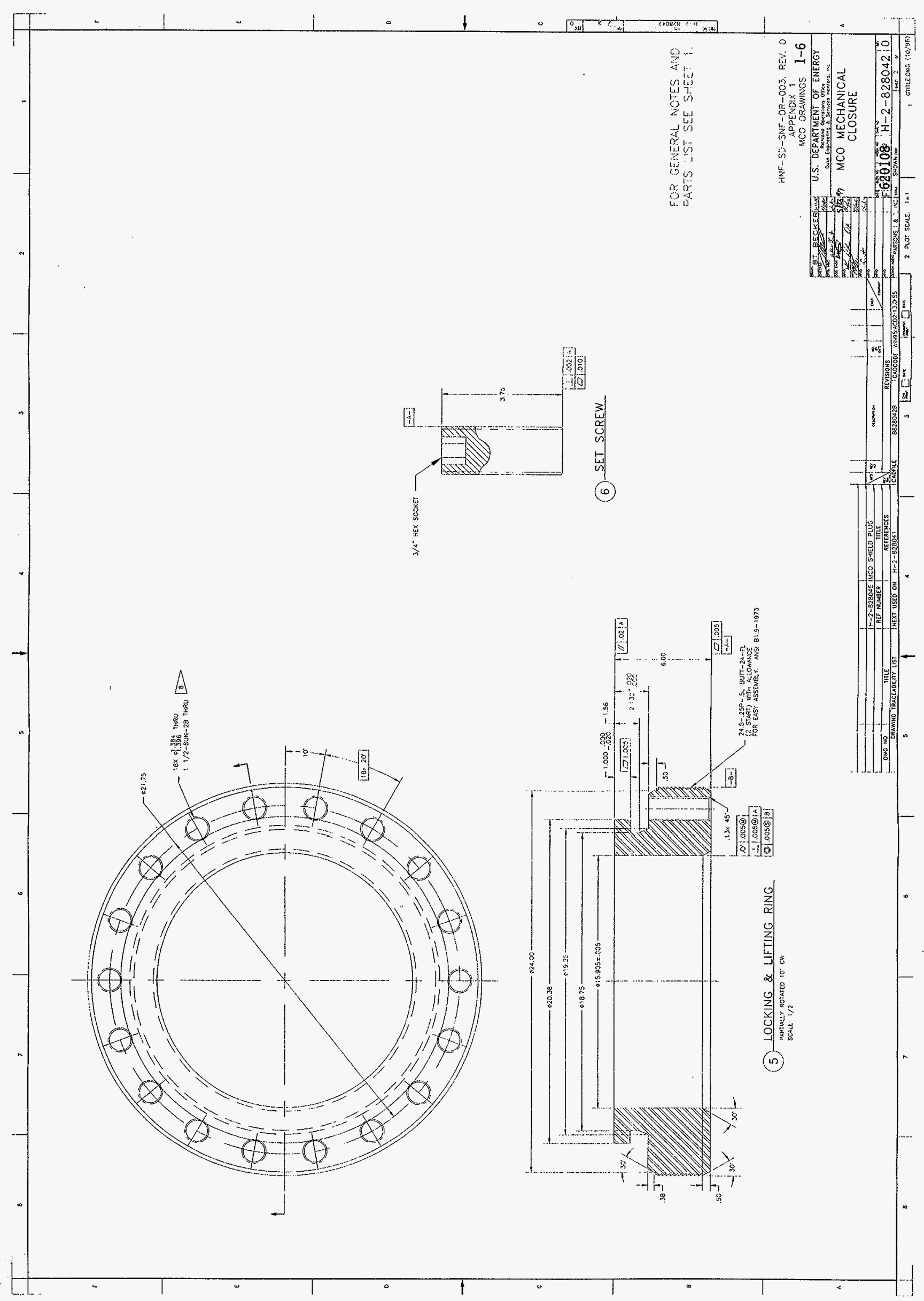




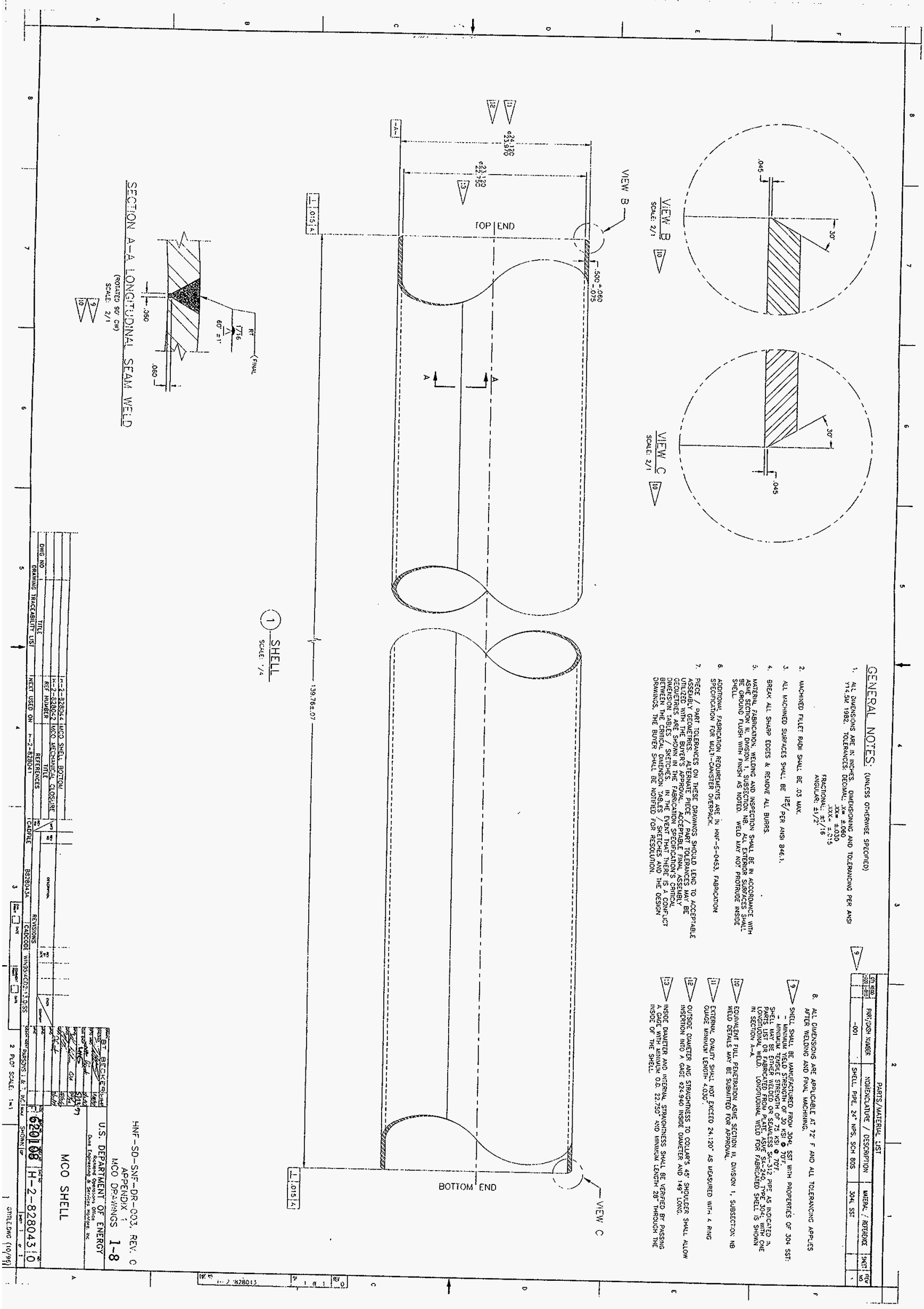




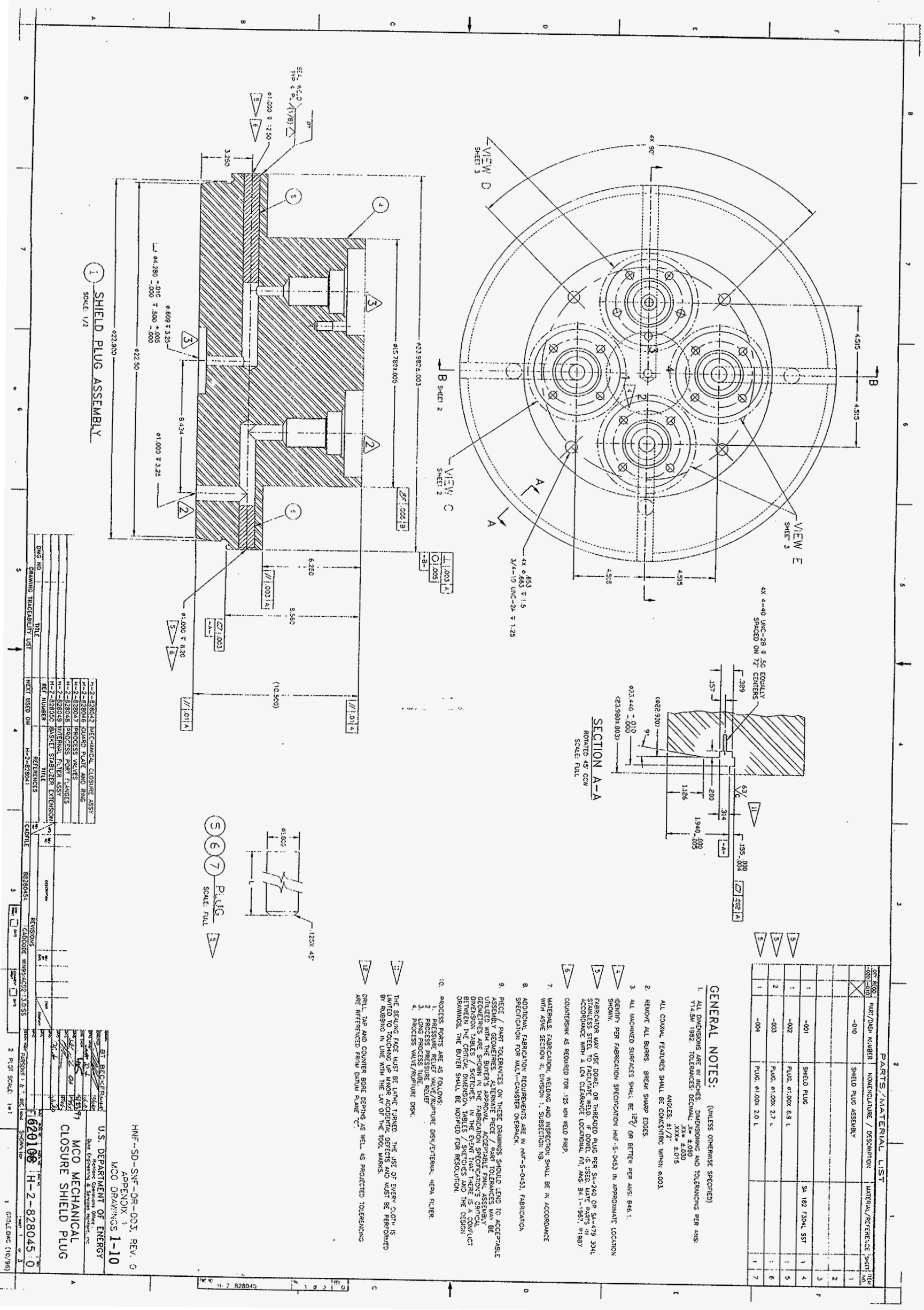




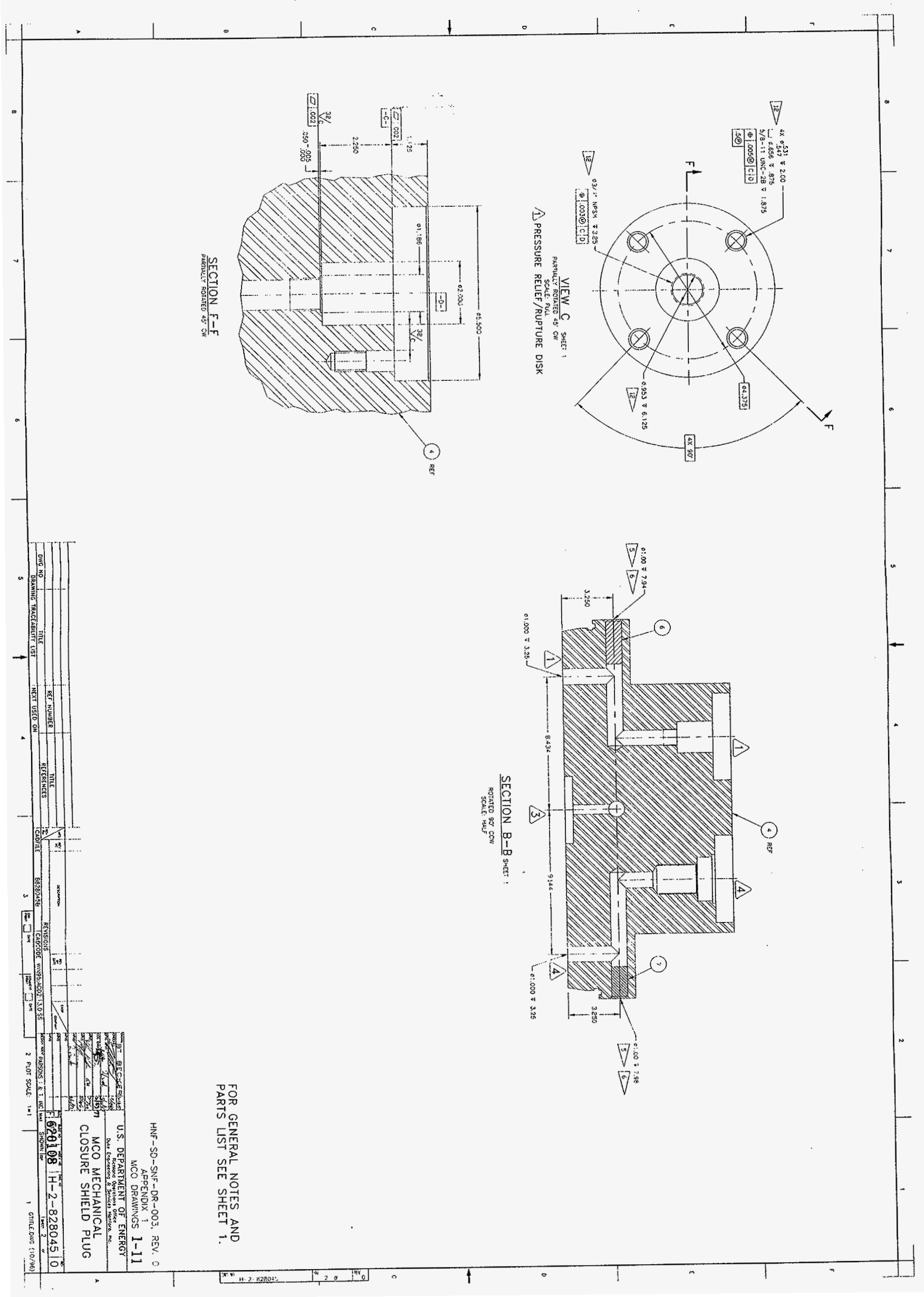




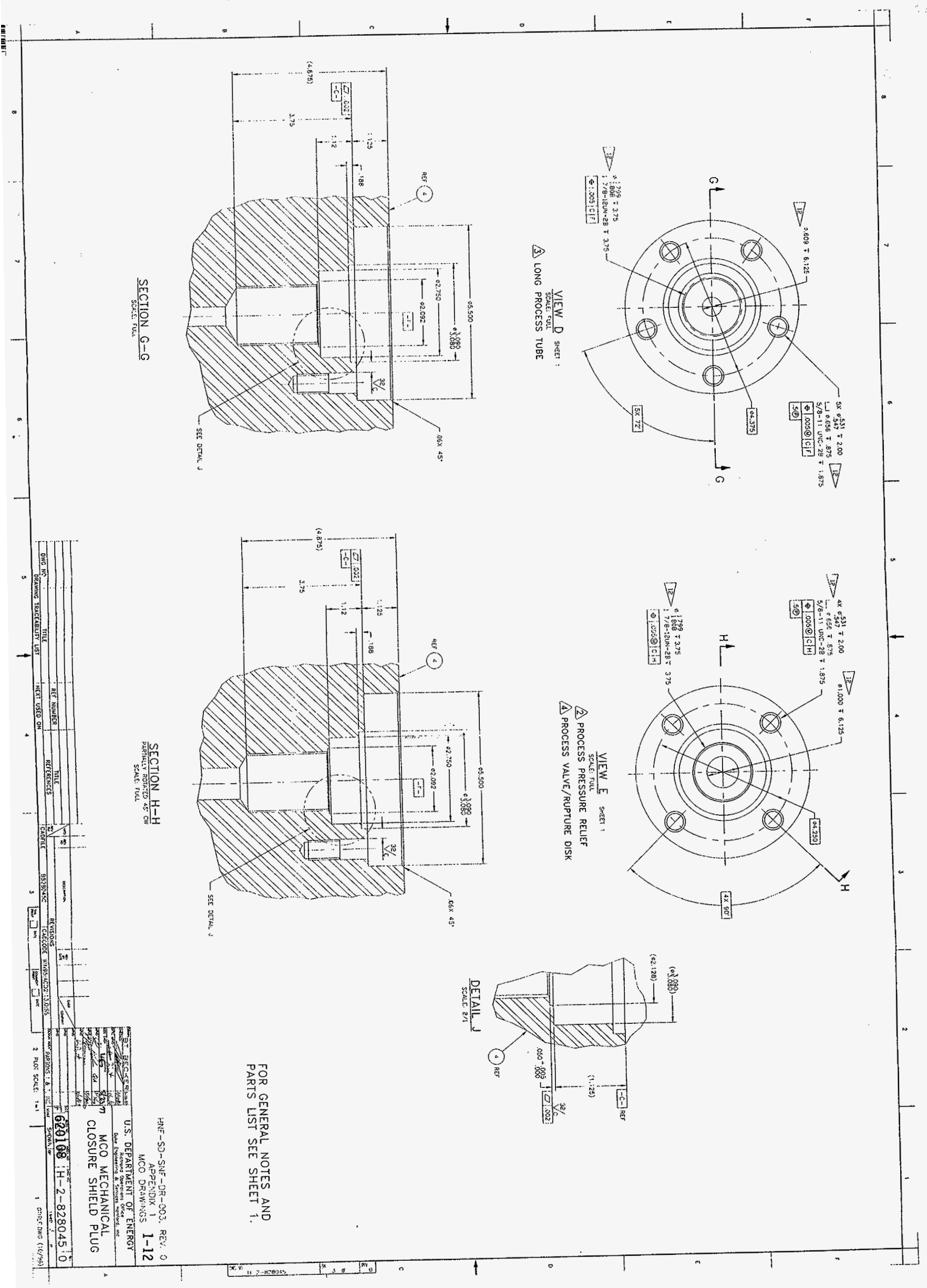




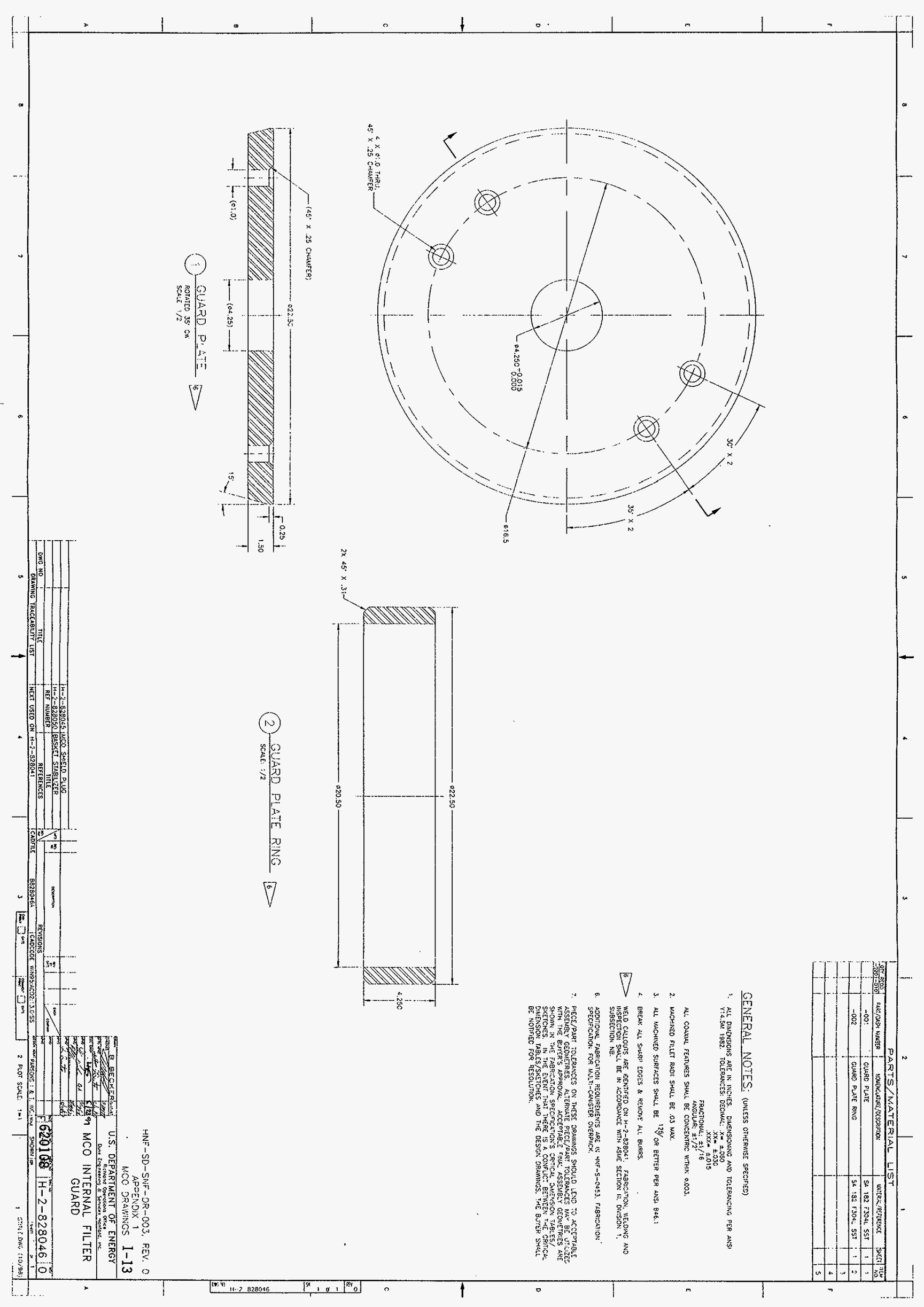




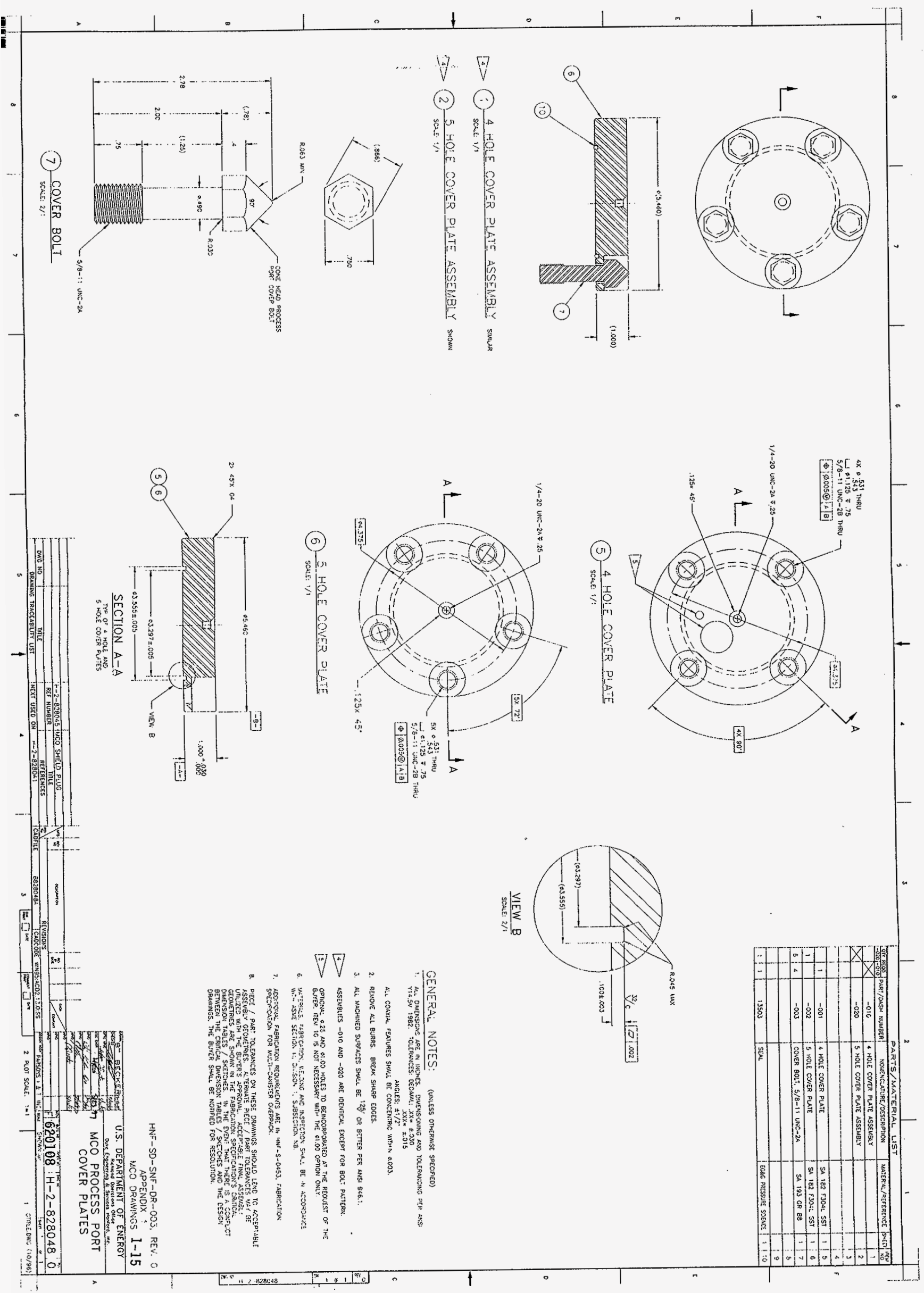




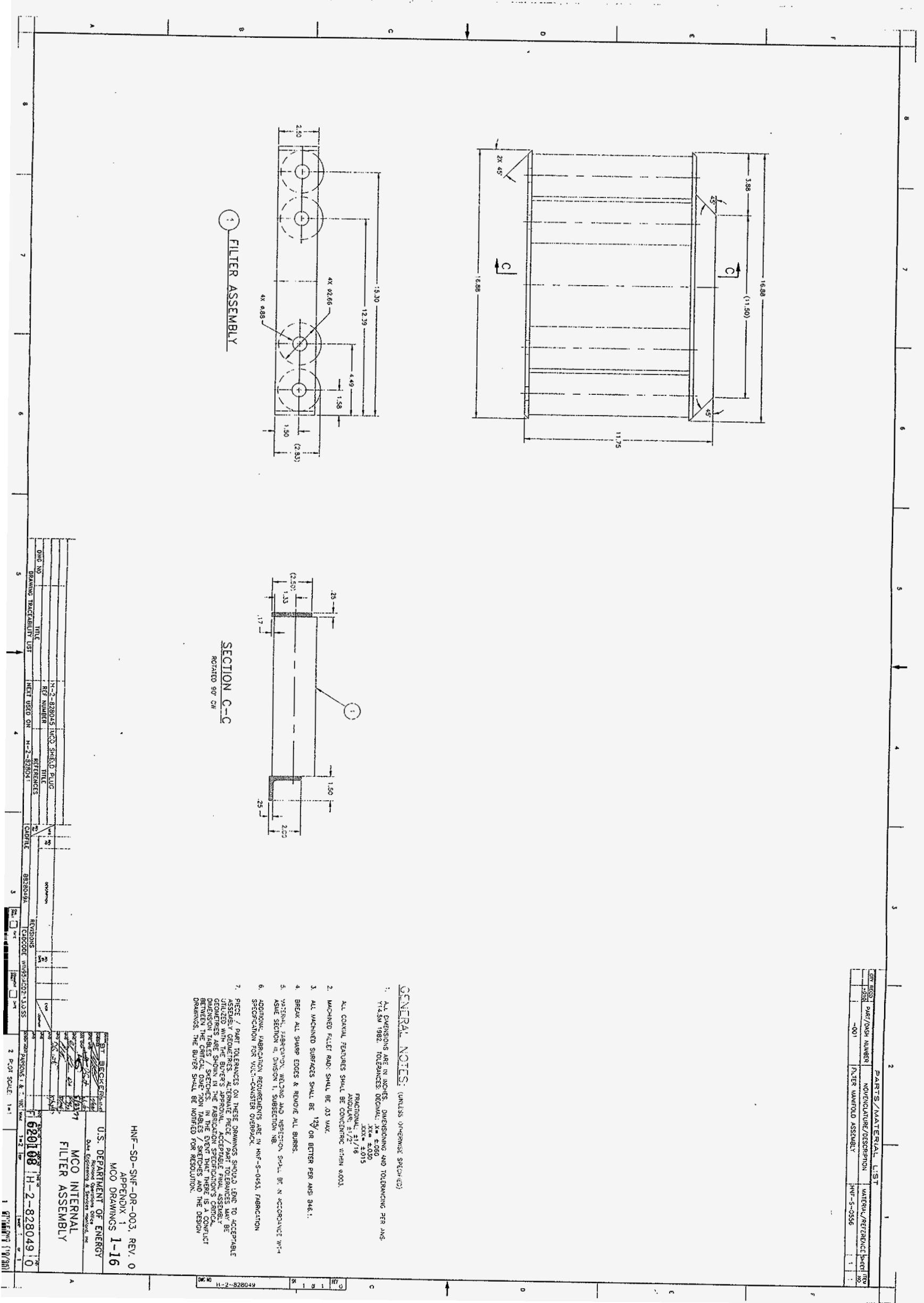



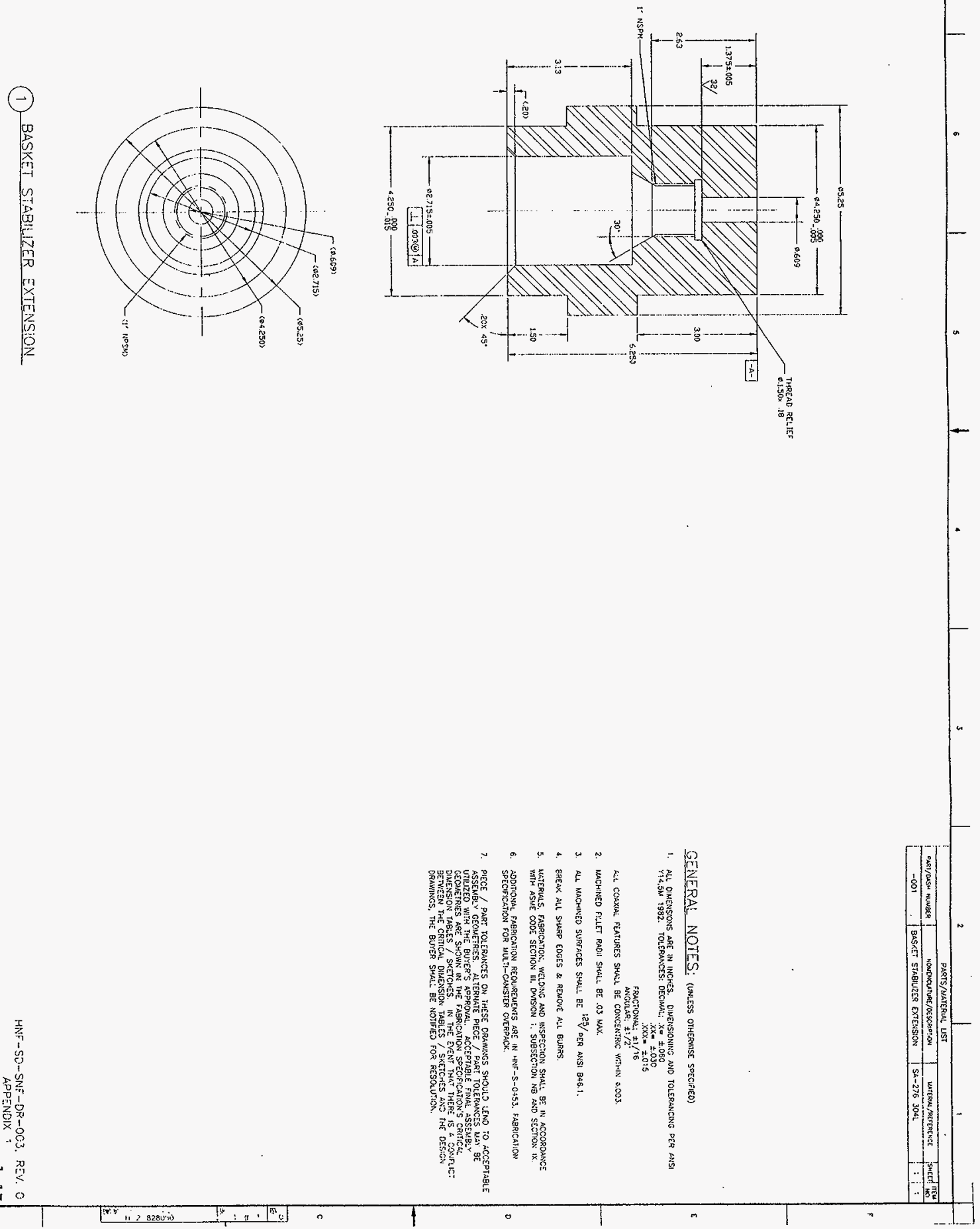


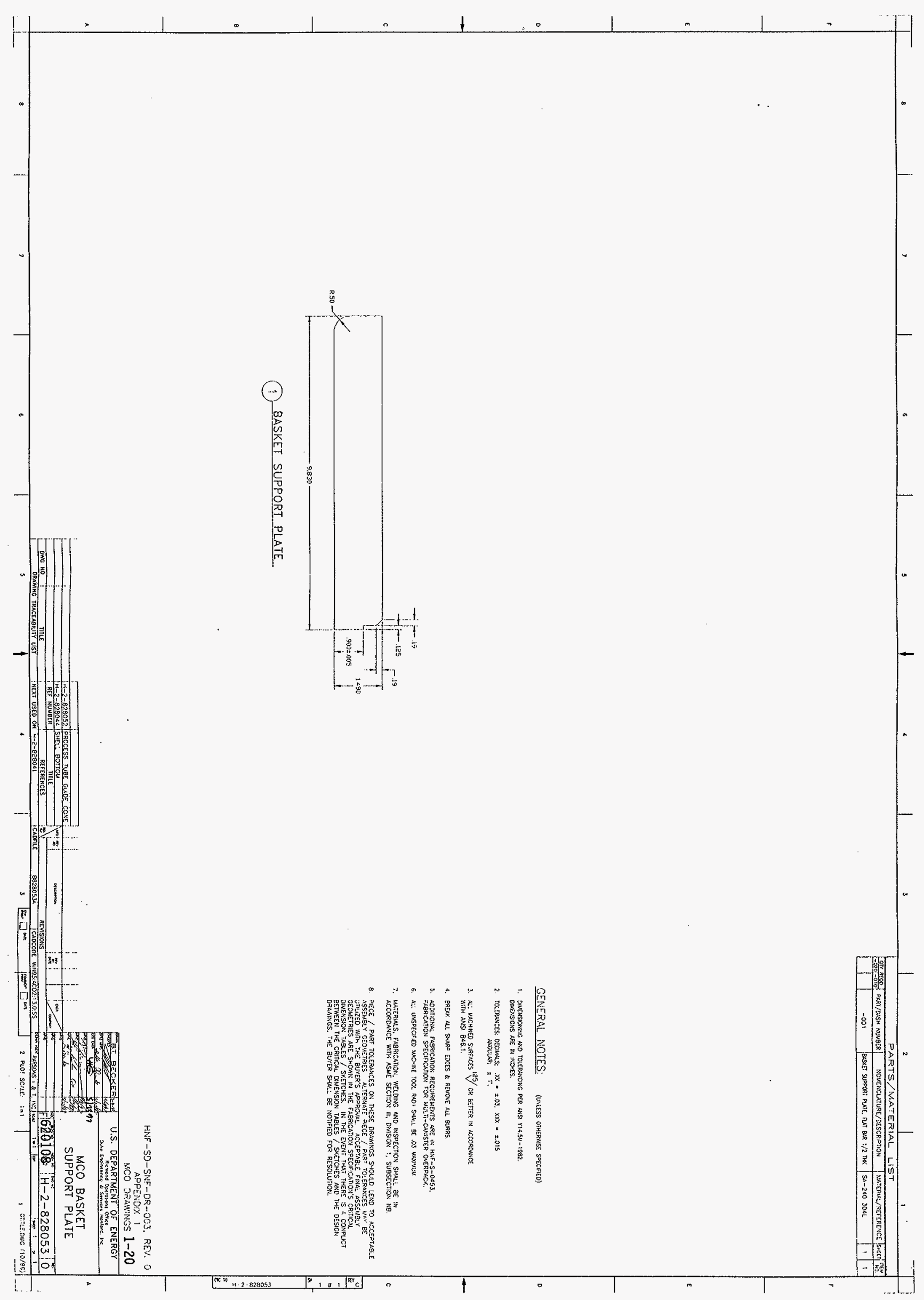




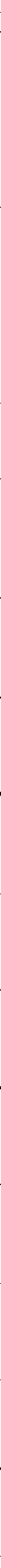



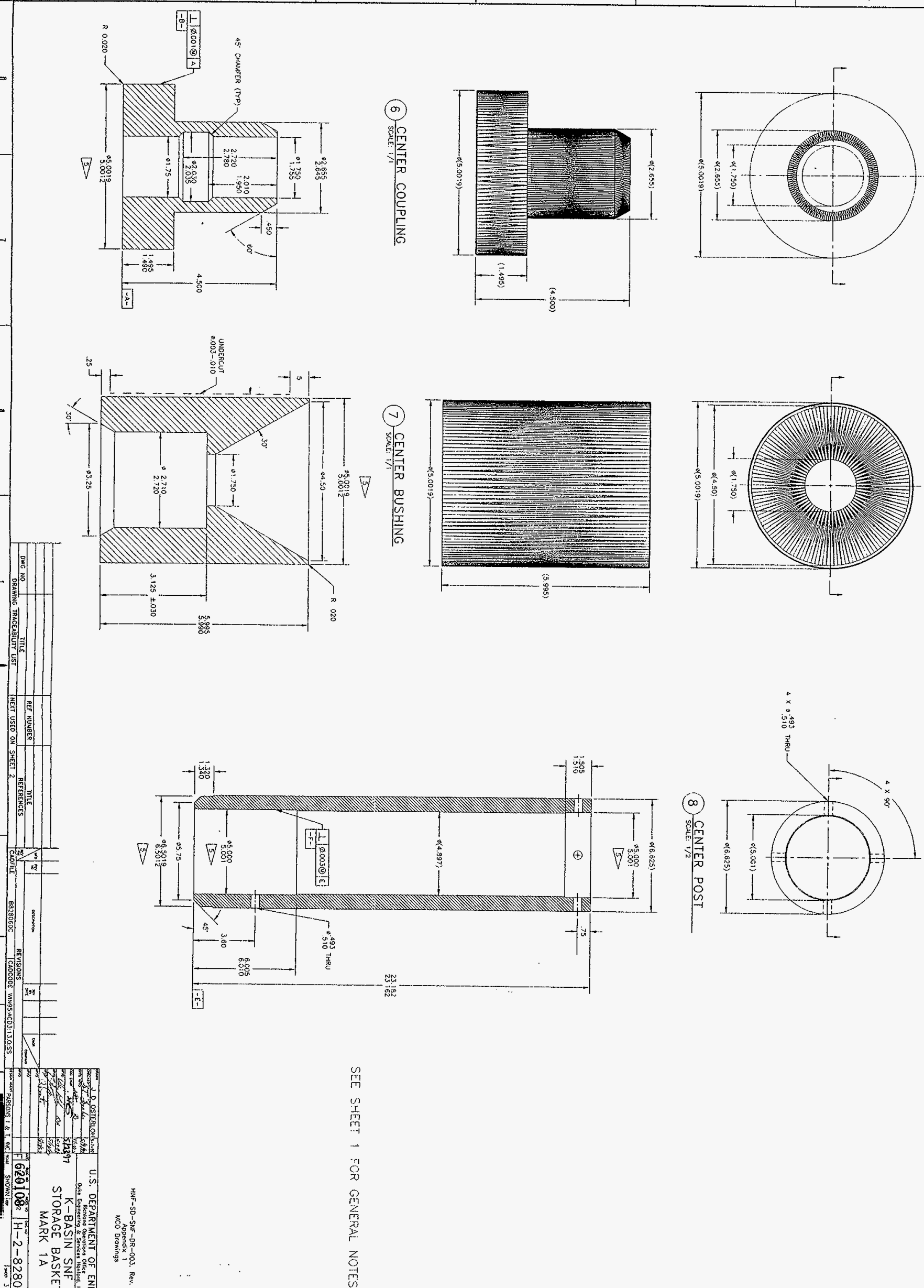


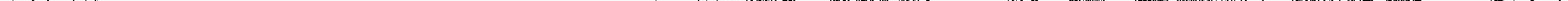




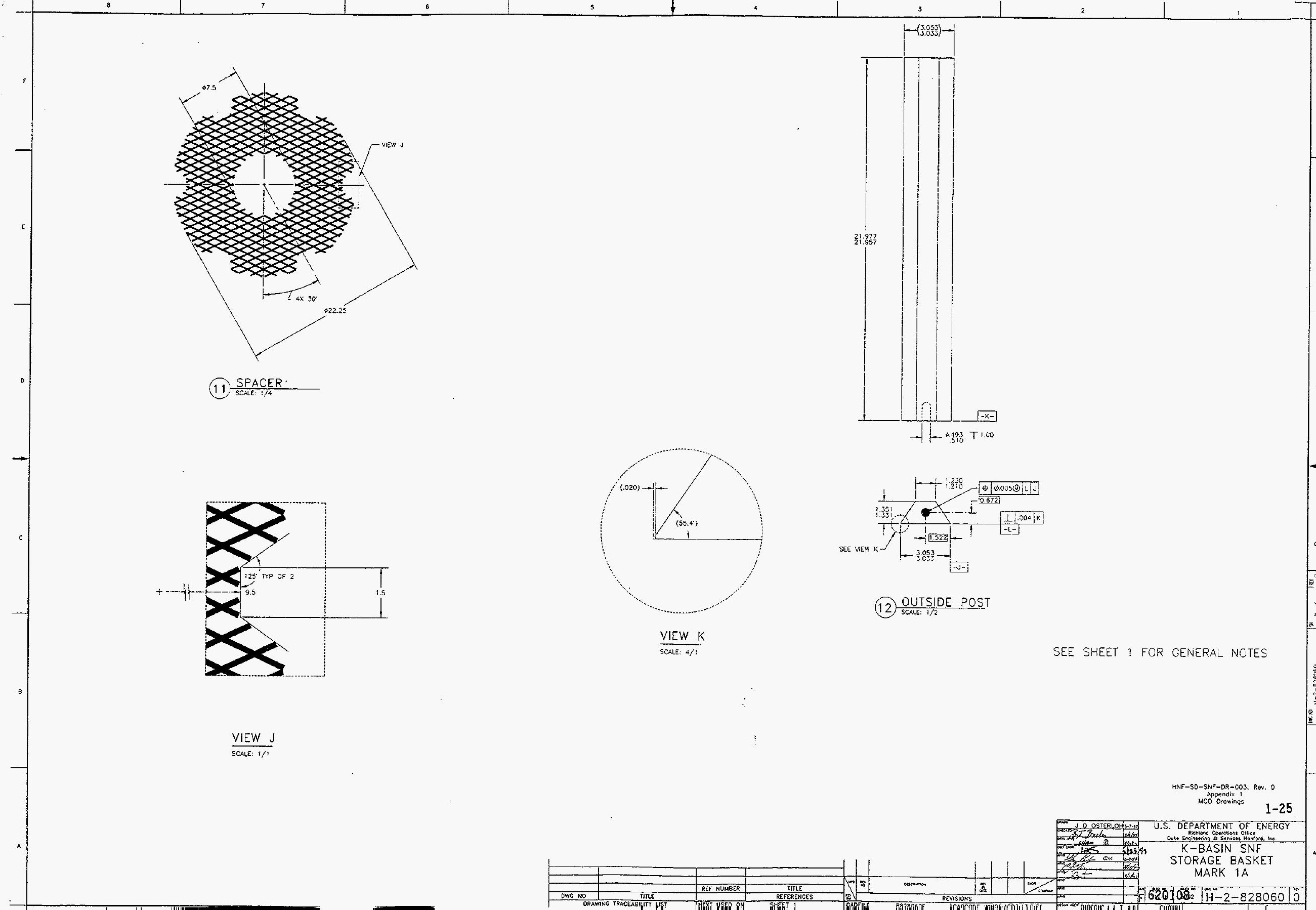




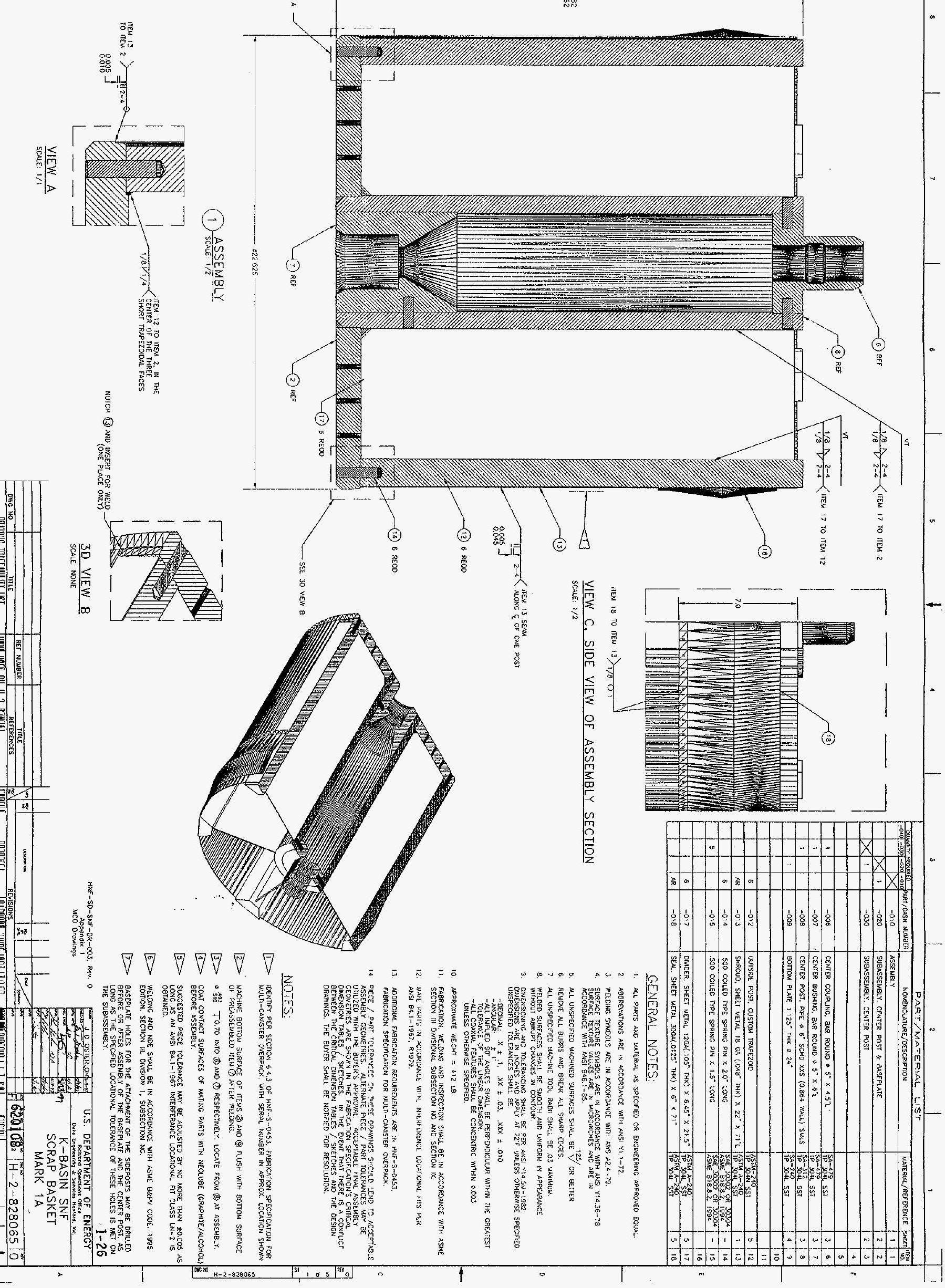




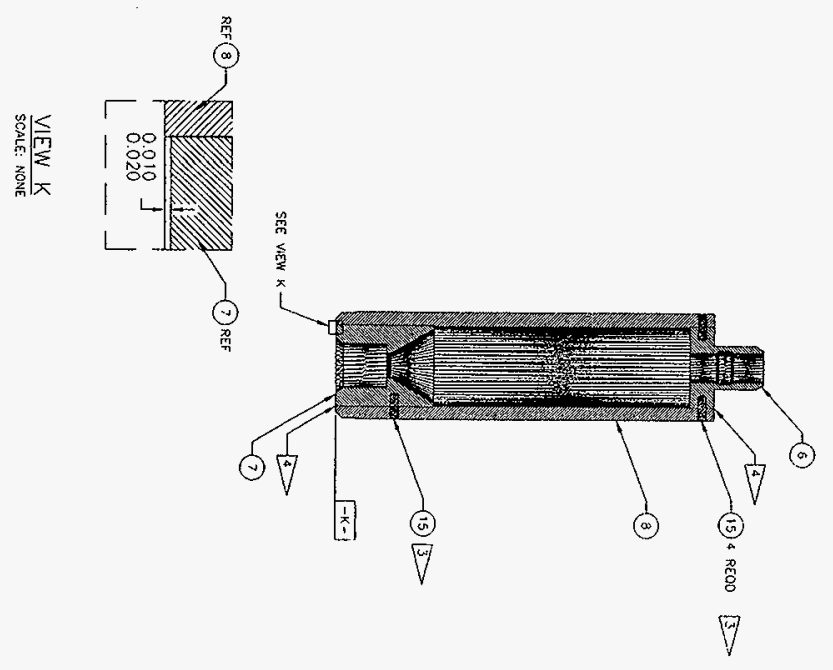

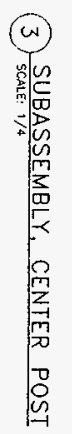
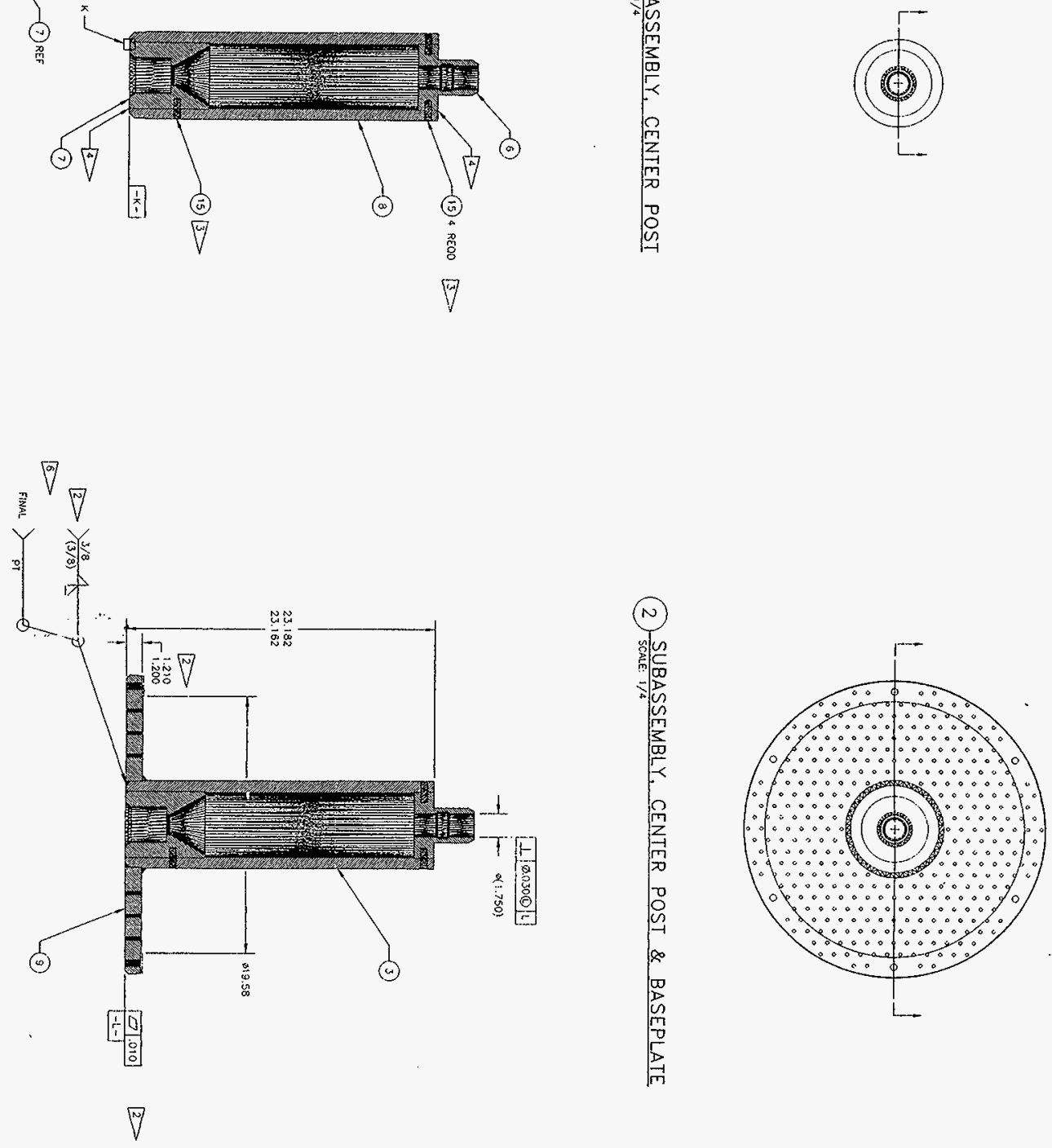


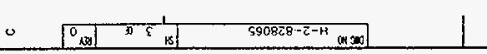
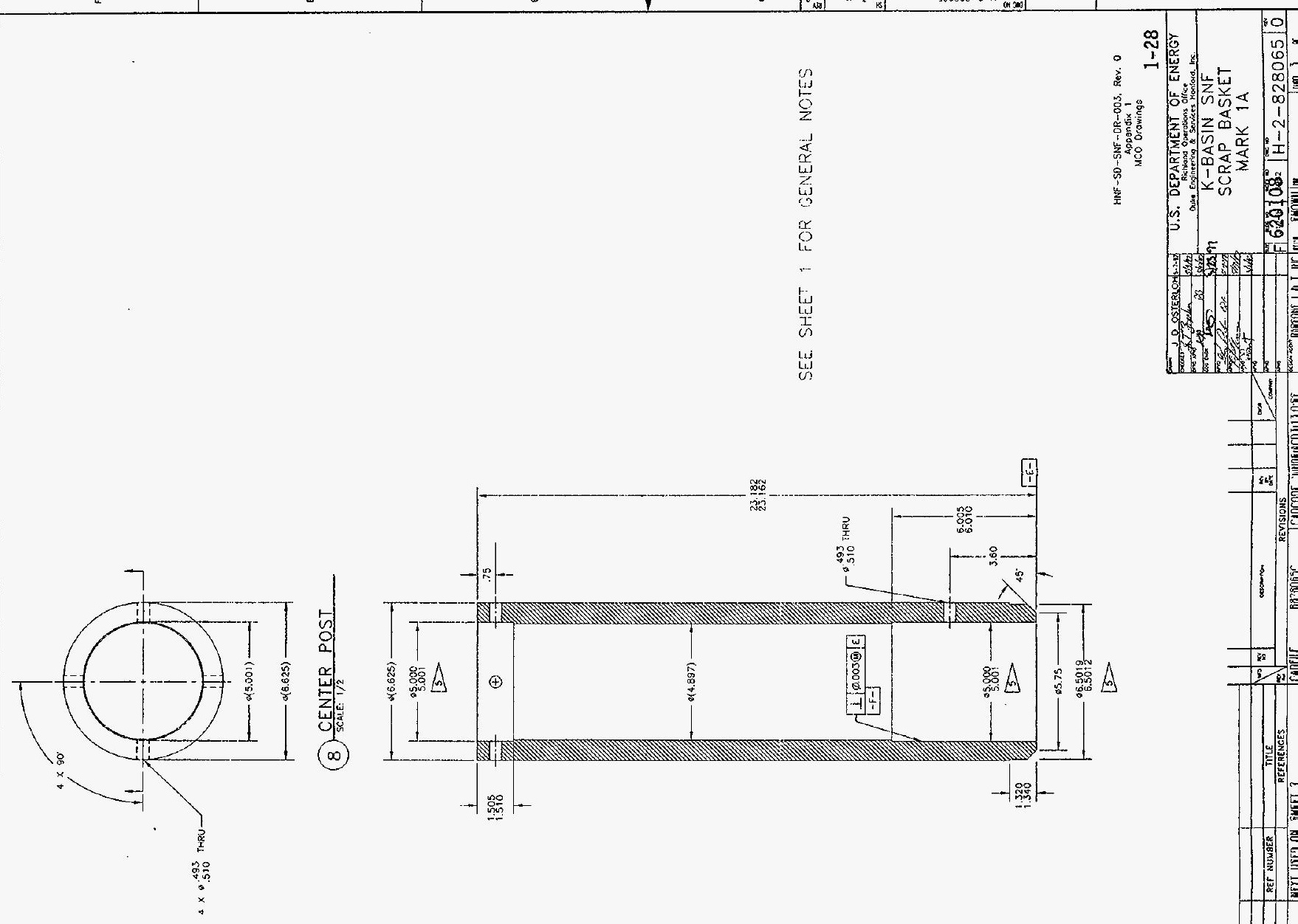

悉的
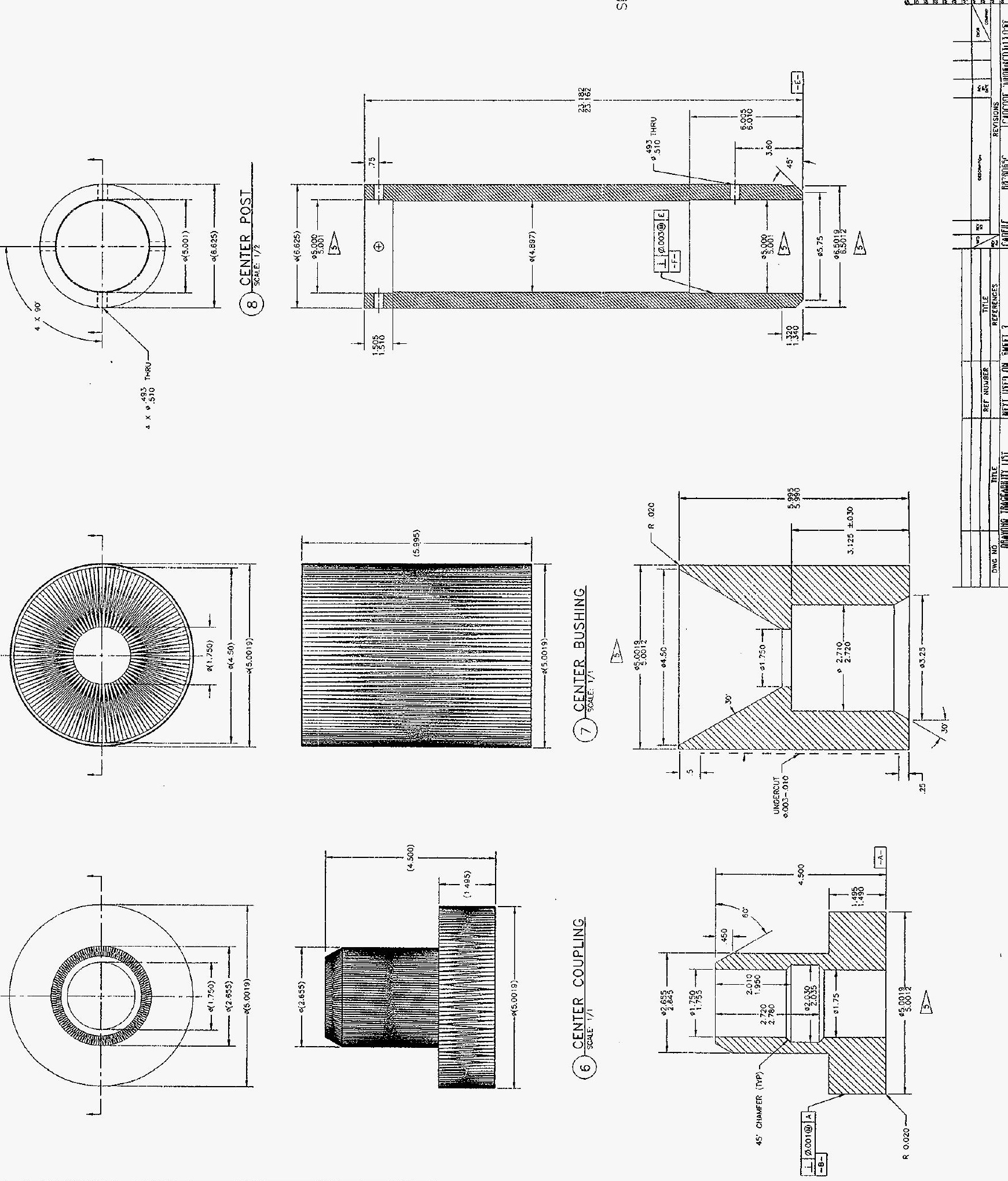


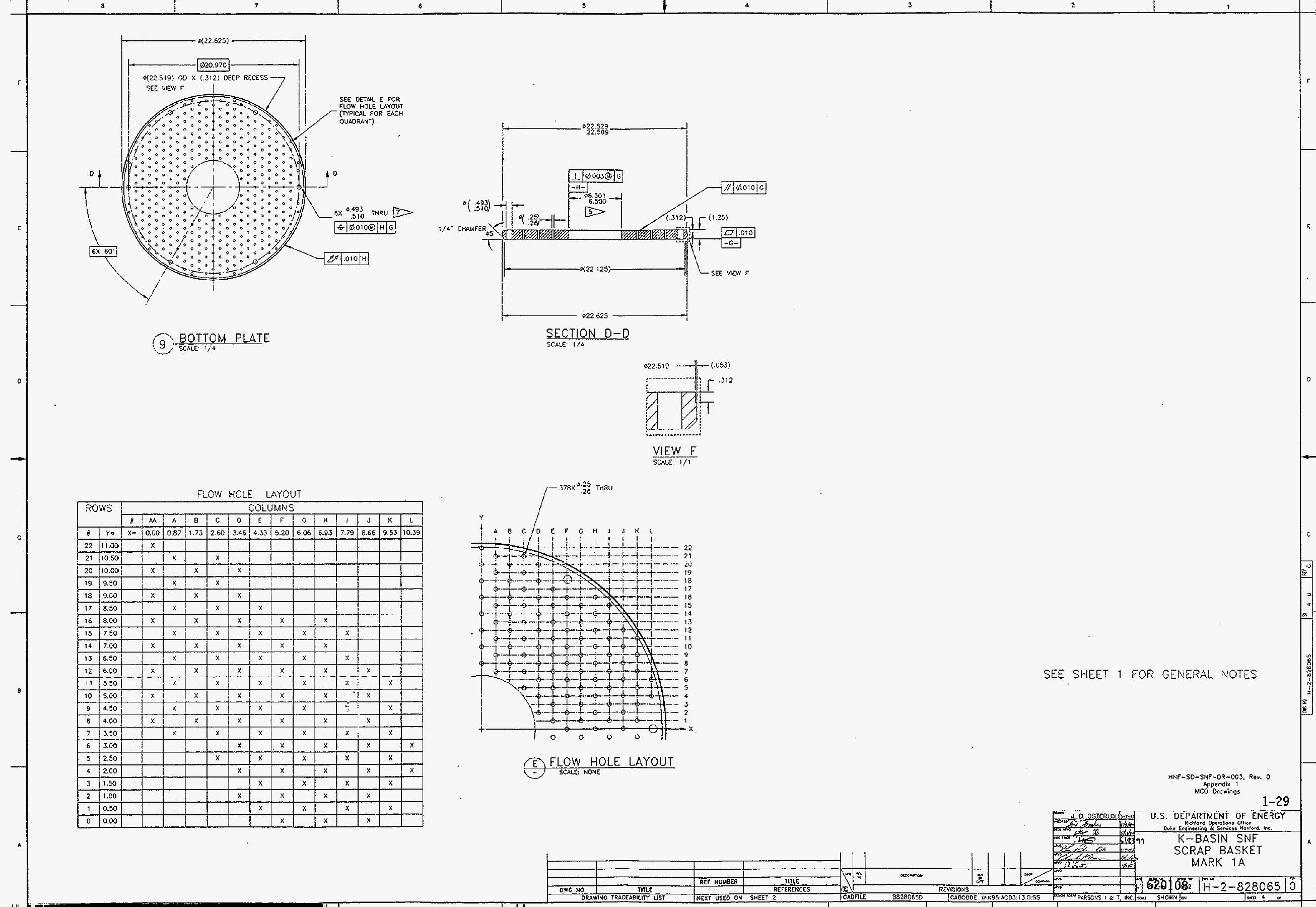



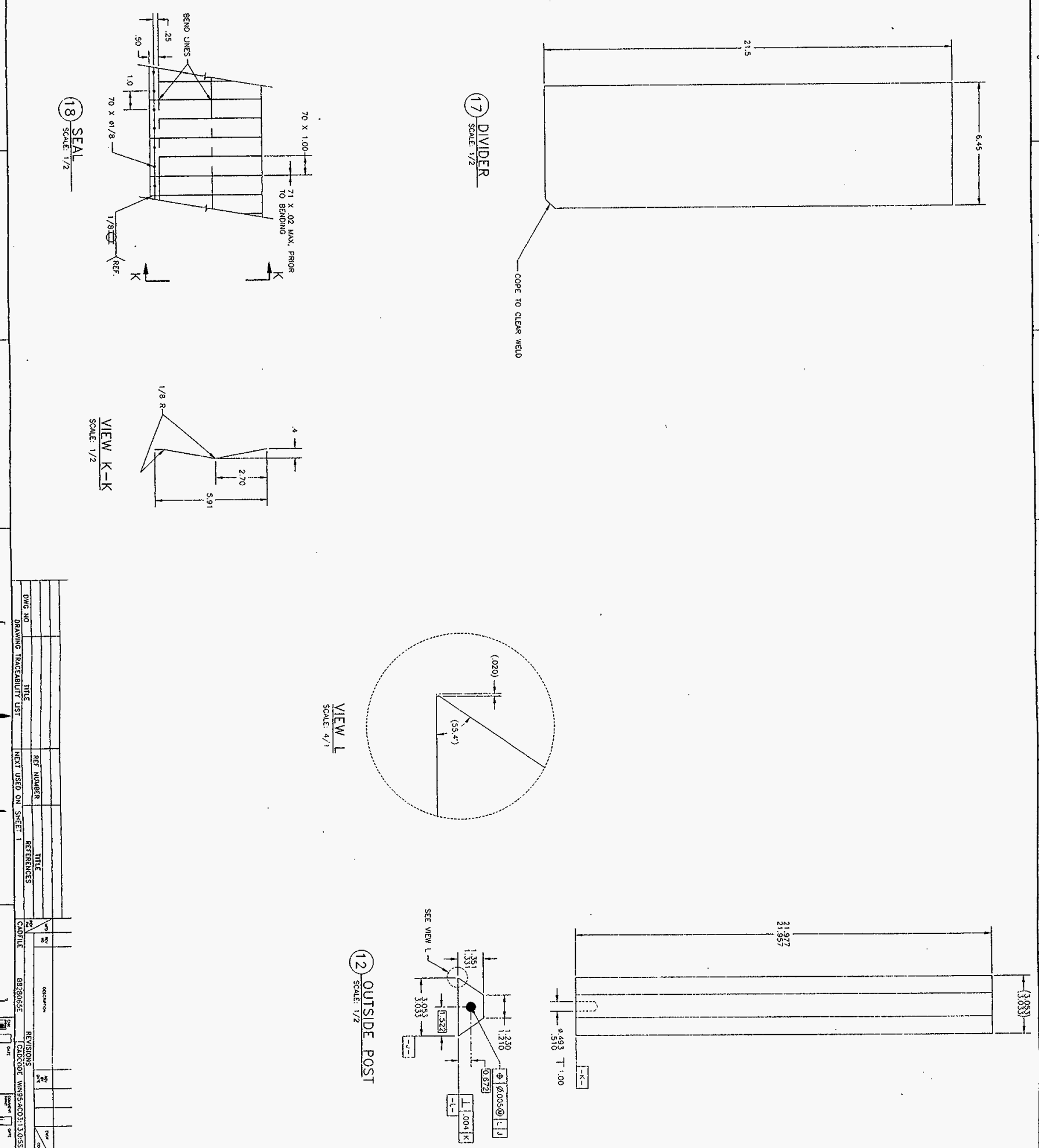

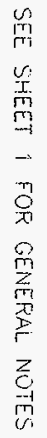



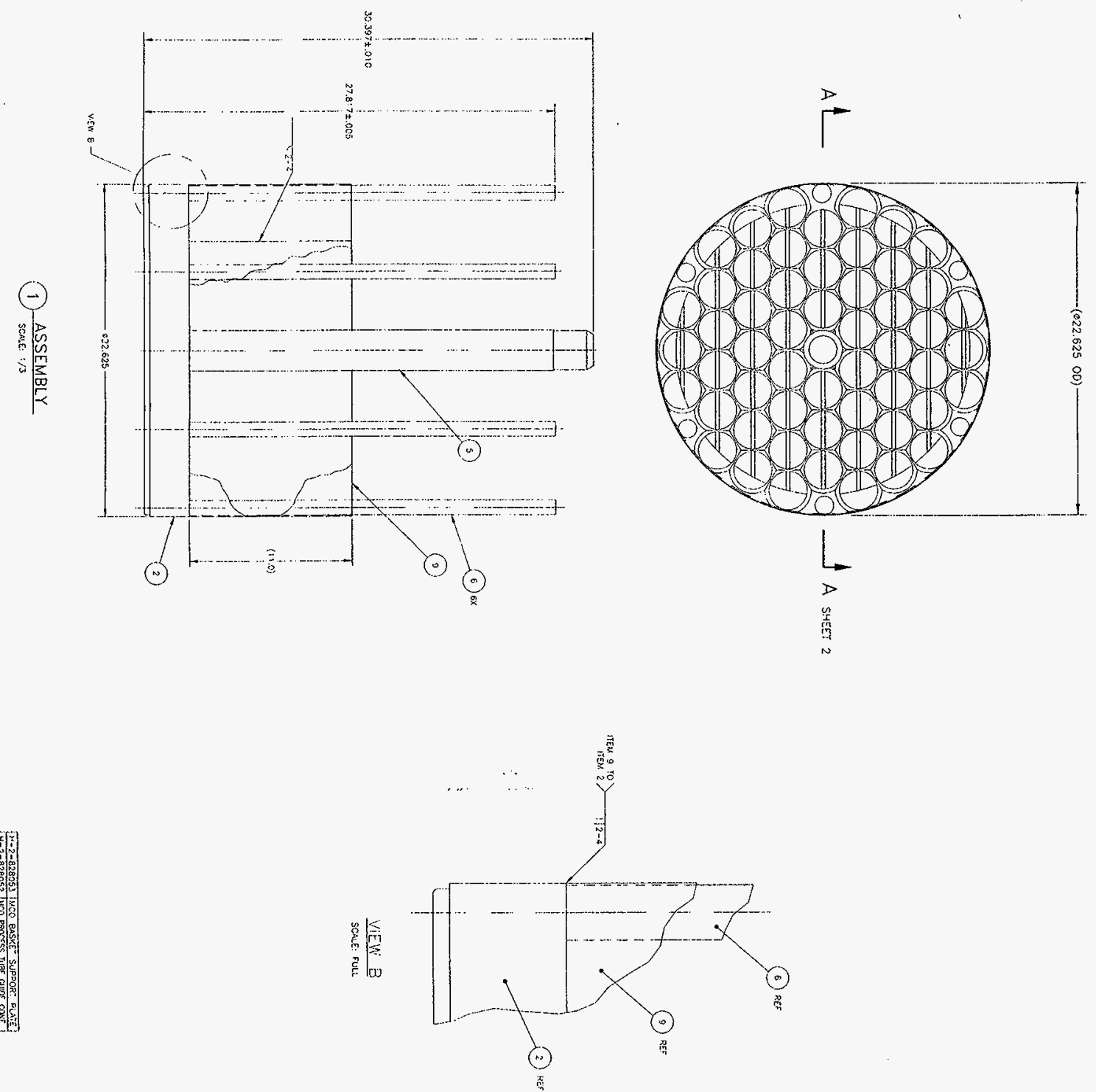

-
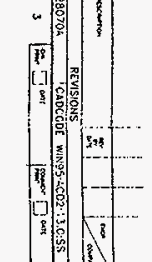

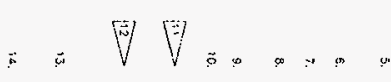

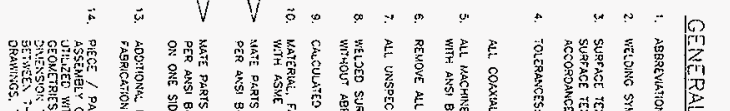

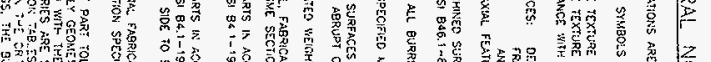

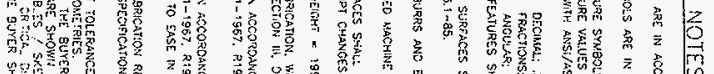

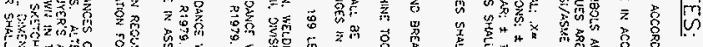

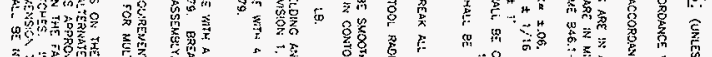

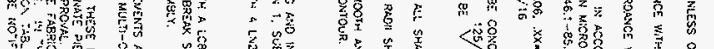

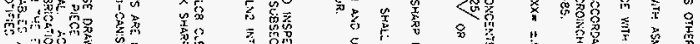

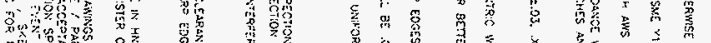

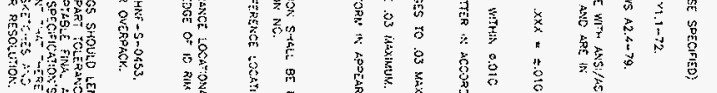

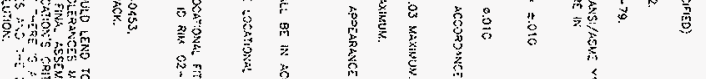

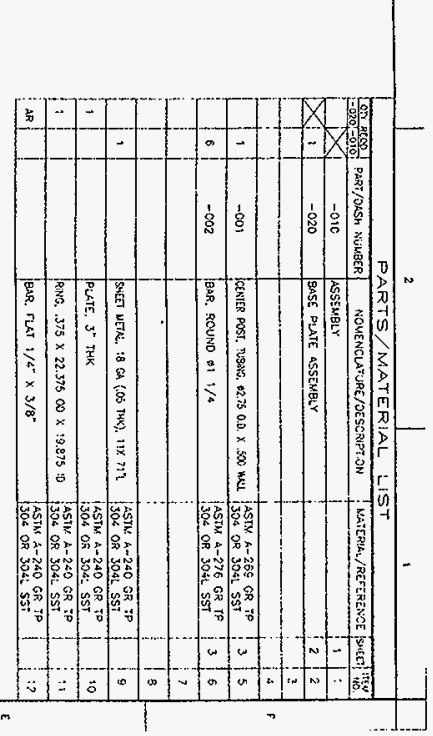




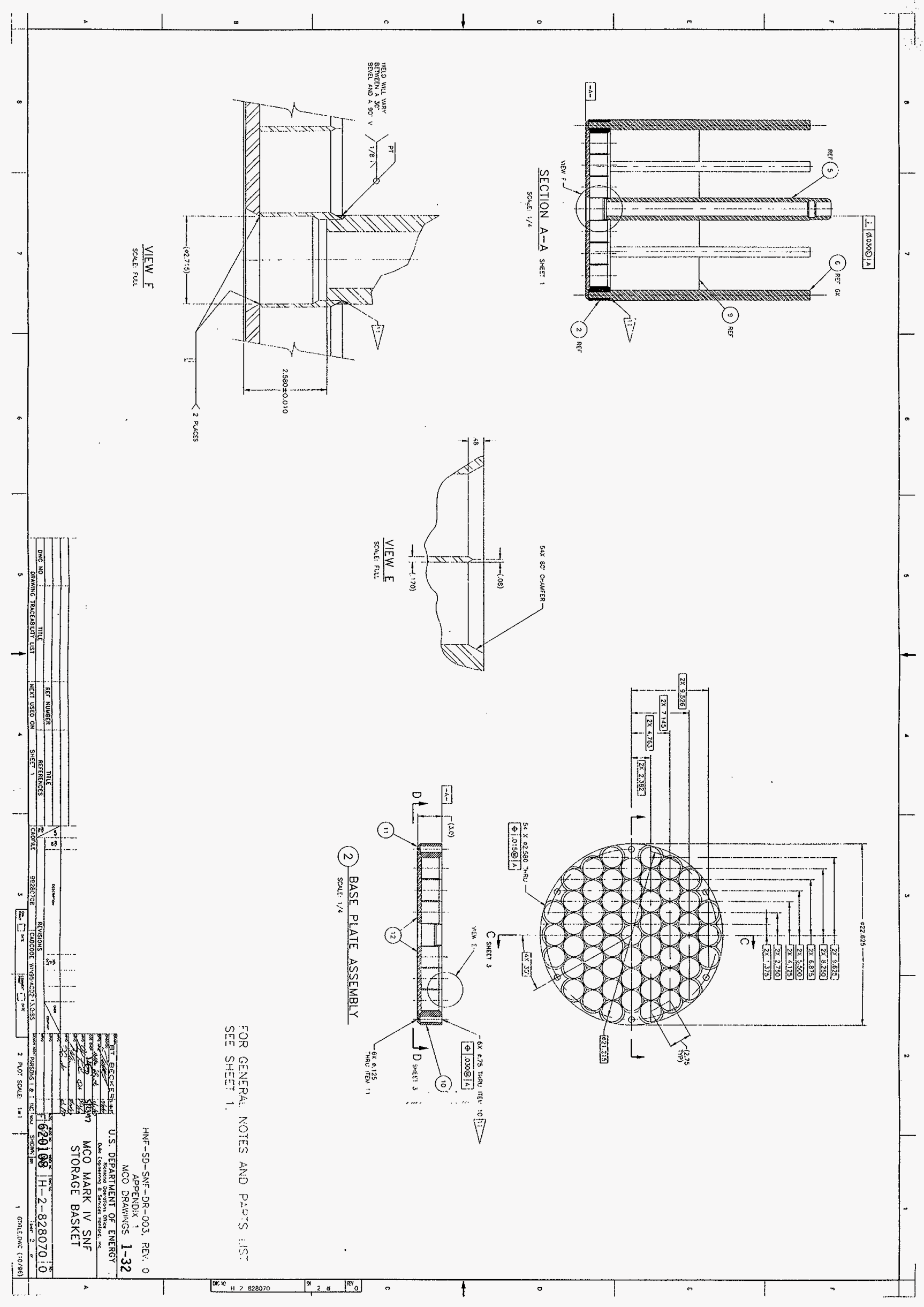




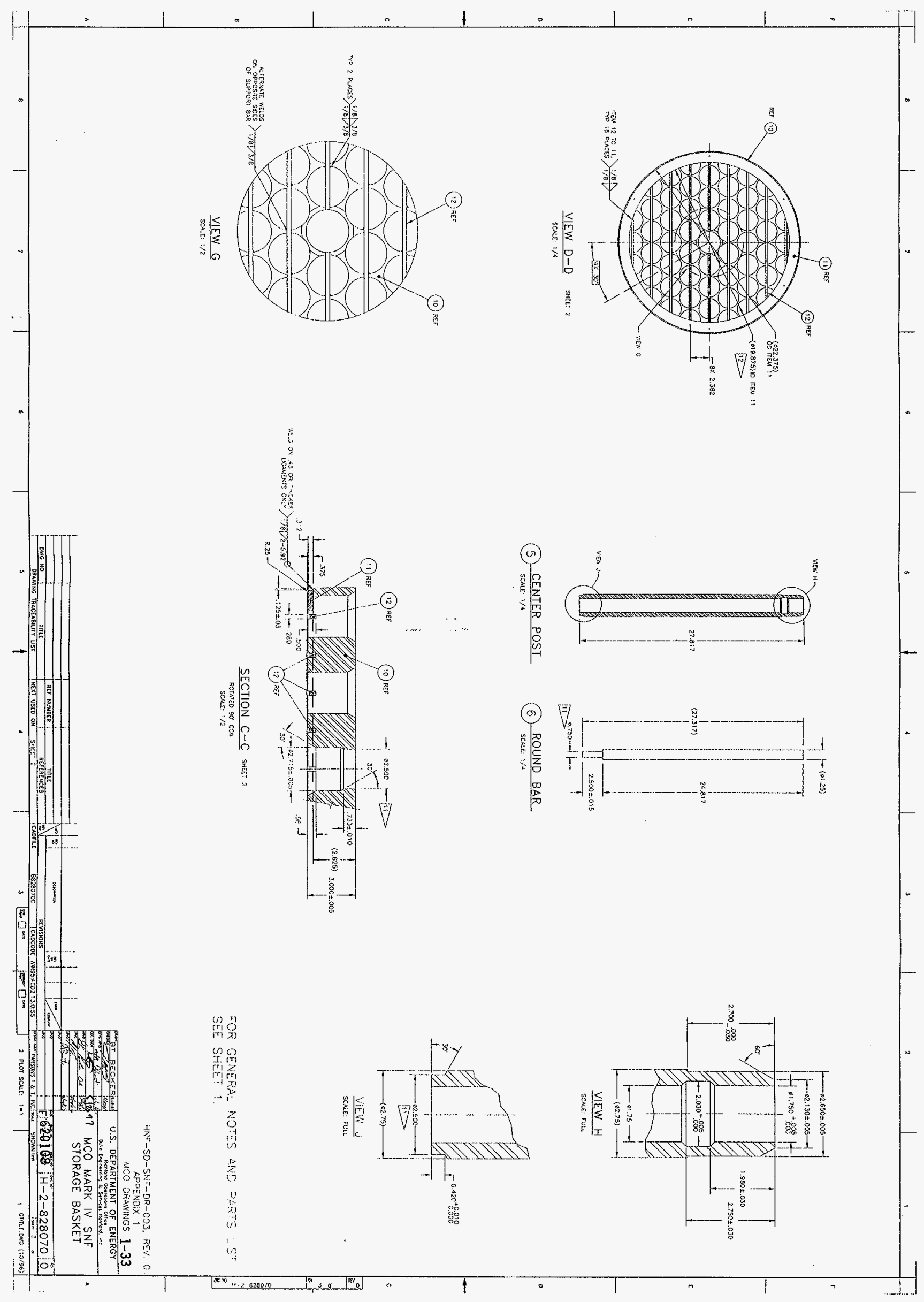




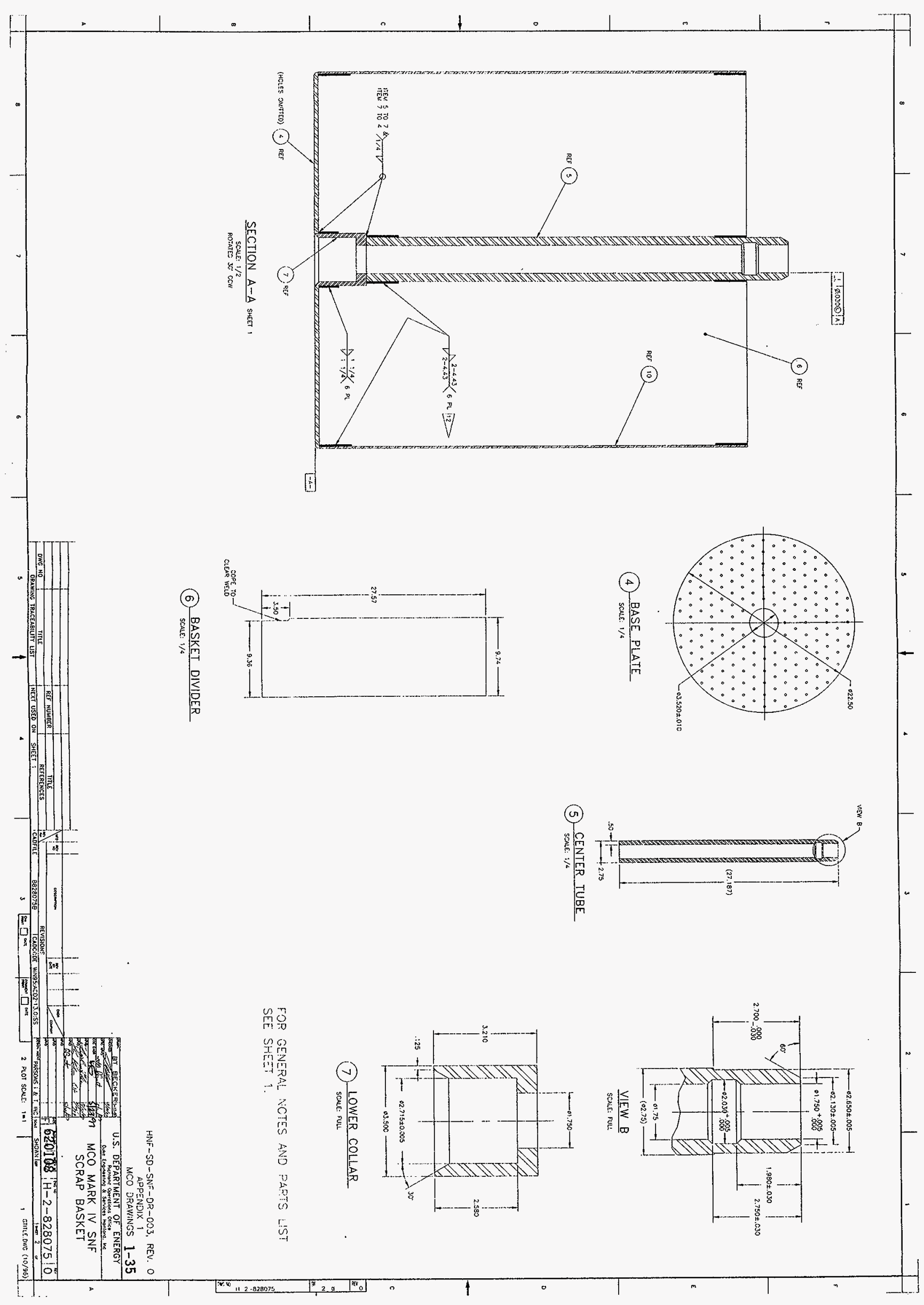


Document No. HNF-SD-SNF-DR-003,

Rev. 0, Appendix 2

\title{
MULTI-CANISTER OVERPACK DESIGN REPORT
}

\author{
MATERIAL EVALUATION
}


Document No. HNF-SD-SNF-DR-003,

Rev. 0, Appendix 2

\section{MCO MECHANICAL CLOSURE SHIELD PLUG AND LOCKING RING MATERIAL EVALUATION}

The MCO Mechanical Closure Shield Plug and locking ring were proposed to be fabricated from ferritic steel. Only SA-508 Grade 4N, Class 3 (formerly Class 4b) is suitable for use under the requirements of the ASME BPVC, Section III, Subsection NB. The Applicable Design Specification, "HNF-S-0426", originally required the application of Regulatory Guide 7.12 and NUREG/CR-3826 to ferritic steel components when greater than 4" thick. The NUREG/CR3826 requirements are augmented by Regulatory Guide 7.12 such that testing for Nil Ductility Transition (NDT) temperature in accordance with ASTM E208 must be done and a very low NDT temperature of -140 degrees Fahrenheit (for 12-inch thick sections) must be demonstrated. Additionally, the Lowest Service Metal Temperature (LSMT) must be taken as -20 degrees Fahrenheit, regardless of the design parameters. Industry experience indicates that these material requirements are not readily obtained. One large forging manufacturer was contacted to obtain a rough order of magnitude cost estimate and lead time. They stated that they would not bid on such a forging based on recent experience in trying to meet these specific requirements for the U.S. Navy. After extensive efforts to meet the requirements, the manufacturer gave up and subcontracted a foreign manufacturer to supply the material. The cost was high and the experience was such that they would not consider attempting manufacture themselves or supplying the material via their previous overseas supplier. The Regulatory Guide effectively excludes the use of ferritic steels for heavy sections from a practical standpoint of current domestic steel making practices.

The approach in the revised specification is to shed the additional requirements of the Regulatory Guide and use NUREG/CR-3826 as a free standing material requirement. The LSMT for the MCO has been changed to 32 degrees Fahrenheit in Revision 3 of the Performance Specification. Recognizing that the thick sections of the MCO would be subjected to low design stresses, the NUREG approach was reviewed for applicability. The fracture arrest criterion based upon extrapolation of the Pellini fracture toughness reference curve at 0.2 of the yield strength would permit the use of some of the ferritic steels discussed in the NUREG with a reasonable level of assurance that the failure criterion of the material is not exceeded. However, the basis for using such a comparatively liberal criterion must be addressed in light of the Regulatory Guide position. Several potential concerns may be raised. The use of the Pellini curve has several inherent assumptions which may not be suitable for thicker materials or at least difficult to apply with certainty. Other methodologies in the NUREG could be attempted, however, the cost of each is clearly heavily influenced by material examination requirements such as volumetric examination. Such examination could provide additional margin to the methodology based on the extrapolation of the Pellini curve or used for the methodology based on ASME Section XI. Such examination on thick sections is difficult and would not likely produce meaningful results, particularly in a forged component. If possible, such examination would be very expensive. 
Document No. HNF-SD-SNF-DR-003,

Rev. 0, Appendix 2

The NUREG/CR-3826 approach for protecting against brittle fracture is dependent on the users level of acceptable risk and clearly is subject to critical review by the package reviewer.

Another possible approach could be to use the ASME Section III, Subsection NB requirements for material testing. This may also be subject to critical review since the ASME Code was developed for reactor components. As such, it does not address the service conditions which an onsite storage package might be subjected such as impact loading at low temperatures.

Regulatory Guide 7.12 and NUREG/CR-3826 form the most justified and accepted set of criteria for heavy sections of ferritic steel for a storage or shipping container for spent nuclear fuel.

The latest revisions of the Performance Specification increased the operating temperatures to be considered and eliminated the requirement of Regulatory Guide 7.12. The raising of the temperature to $0^{\circ} \mathrm{C}$ has little influence on the selection of ferritic materials.

Based on the lack of readily available material which meets the Regulatory Guide 7.12 and NUREG/CR-3826 requirements for ferritic steels, a comparison to austenitic stainless steels is warranted. The appropriate base cost components for comparison include forged material cost, rough machining cost, and finish machining cost. NDT temperature testing is only required for the ferritic steel. The additional costs associated with the ferritic steel components when compared to austenitic stainless steels are Post Weld Heat Treatment (PWHT) after plug welding and/or inlay for the seal surfaces and thermal spray or other coating to protect the shield plug from rapid oxidation and hydrogen generation when wet.

Considering the additional material testing and fabrication costs as well as uncertainty in obtaining material with suitable properties, austenitic stainless steel will be used.

The advantages of going with the austenitic stainless is not only in the area of brittle fracture but in the area of corrosion and material compatibility. The austenitic stainless is compatible with the $\mathrm{MCO}$ shell which is already required to be type $304 \mathrm{~L}$ as well as the fuel baskets. The stainless steel has less fewer service related corrosion problems as discussed in Appendix D of the Performance Specification. Another significant advantage of austenitic stainless steel for this application is the reduced maintenance requirements throughout the operations during the life of the package. This also is important during storage prior to handling. Fewer requirements for the protection of threaded and seal surfaces will be required than if a ferritic material was used.

Selection of materials with the same coefficient of thermal expansion eliminates differential thermal expansion and subsequent thermal stresses. This design feature is particularly important during the thermal cycles associated with the Hot Processing operations. 
Some of the disadvantages of the material is the reduced strength. The lower allowable strength of the material results is less margin beyond that inherent in the code allowable stresses.

However, austenitic stainless steels are inherently tough and plastically deform, absorbing a great deal of energy, before catastrophic failure. Another potential disadvantage of the use of threaded austenitic materials in combination is there tendency to gall when moved under pressure. Proper handling and lubrication practices can prevent galling.

The MCO is constructed mainly out of austenitic stainless steels for the purpose of high corrosion resistance, good cold temperature behavior and high assurance of complying with the required design life in an economical manner. Also due to the large temperature cycles that the package is expected to see, all of the materials should be of the same basic type to preclude differential thermal expansion, which would challenge maintenance of the sealing system. This results in the design of austenitic materials being mated in threaded connections. Historically some difficulties have resulted in this situation due to galling of the materials together. This can result in excessive high torque values and/or insufficient preload.

This condition can be prevented by using smoother surface texture, use of coarse threads, slower wrenching speeds and most importantly good thread lubrication. (Fastener Standards, sixth edition) (An Introduction to the Design and Behavior of Bolted Joints, John H. Bickford, second edition) There are few restrictions on the use of lubricants on any of the threaded fasteners used on the MCO since none of the fasteners goes into the pool at any time. Also all of the fasteners except the process port valves are outside the pressure boundary. The lubricants must still be functional after being heated to $375^{\circ} \mathrm{C}$ and not having the capability to offgas after being heated to a minimum of $250^{\circ} \mathrm{C}$ (hot conditioning). With this in mind high quality lubricants will be used on all threaded surfaces. The lubricant should be a high grade nuclear grade lubricant such as Nickel Never Seize or Fel-Pro Nickel 5000 Never Seize. If concerns exist about some components being placed in the pool, lubricants such as NeoLube (graphite based) may be used. However the surfaces in all cases should be relubricated after being removed from the water if possible. Even some non threaded surfaces such as the vertical radial interfaces between the shield plug and the locking ring may be lubricated to facilitate assembly. Experience has shown that properly lubricated and properly assembled threads will not have a galling problem. Design of bolted connections where austenitic materials are used typically include a minimum differential hardness. This may be achieved by varying the cold work that the parts are subjected to during fabrication or by specifying different materials which inherently have differing hardness. Many of the components of the mechanical closures may be fabricated from harder materials such as the $\mathrm{N}$ grades of type 304 and $304 \mathrm{~L}$ to provide harder surfaces to further reduce the potential of galling. The bolts are designated to be fabricated from type 316 and XM-19 to also provide harder surfaces to minimize the potential for galling. 
Besides the various grades of stainless steel used in the design of the MCO and the fuel baskets aluminum is the only other major material used. The aluminum is used for the fuel rack insert on the Mark 1A fuel basket. Its only purpose is to provide for a positioning grid during the fuel loading. The major requirement is for material not to interfere with the processing of the fuel. The aluminum selected will not lose sufficient strength that it will block any of the gas flow passages in the baskets. A detailed evaluation of the specific alloy chosen in HNF-SD-SNF-ER018 titled "Evaluation of Cast Carbon Steel and Aluminum for Rack Insert in MCO Mark 1A Fuel Basket". The alloy selected is ASTM B26 356.0-T6. Thermal expansion is taken into consideration in the tolerancing of the components. Review of the fuel rack indicates that it is composed of relatively thin ligaments of a weak material when compared to the stainless steel shroud welded to the base plate. Hence the rack would deform before any significant deformation of the shroud would occur. Also it should be noted that after the loading of the fuel that neither the shroud nor the fuel rack have any significant role in the safety of the storage of the fuel.

Other metals that are used in the design in small amounts are the soft metals used on the Inconel and stainless steel seals. There coatings are inert metals such as silver that will have no adverse reactions with the stainless steel during the life of the $\mathrm{MCO}$. 


\begin{tabular}{|l|l|ll|}
\hline PRARSDNS & CALCULATION PACKAGE & $\begin{array}{l}\text { FILE NO: } \\
\text { DOC NO: }\end{array}$ & $\begin{array}{l}\text { KH-8009-8-01 } \\
\text { HNF-SD-SNF-DR-003, } \\
\text { Rev. 0, Appendix 3 } \\
\text { 1of 27 }\end{array}$ \\
\hline $\begin{array}{l}\text { PROJECT NAME: } \\
\text { MCO Final Design }\end{array}$ & \begin{tabular}{l} 
PAGE \\
DLIENT: \\
\hline Duke Engineering \& Services Hanford, inc.
\end{tabular} \\
\hline
\end{tabular}

CALCULATION TITLE:

Weight Summary

PROBLEM STATEMENT OR OBJECTIVE OF CALCULATION:

Calculate the nominal and maximum weight of the Multi-Canister Overpack.

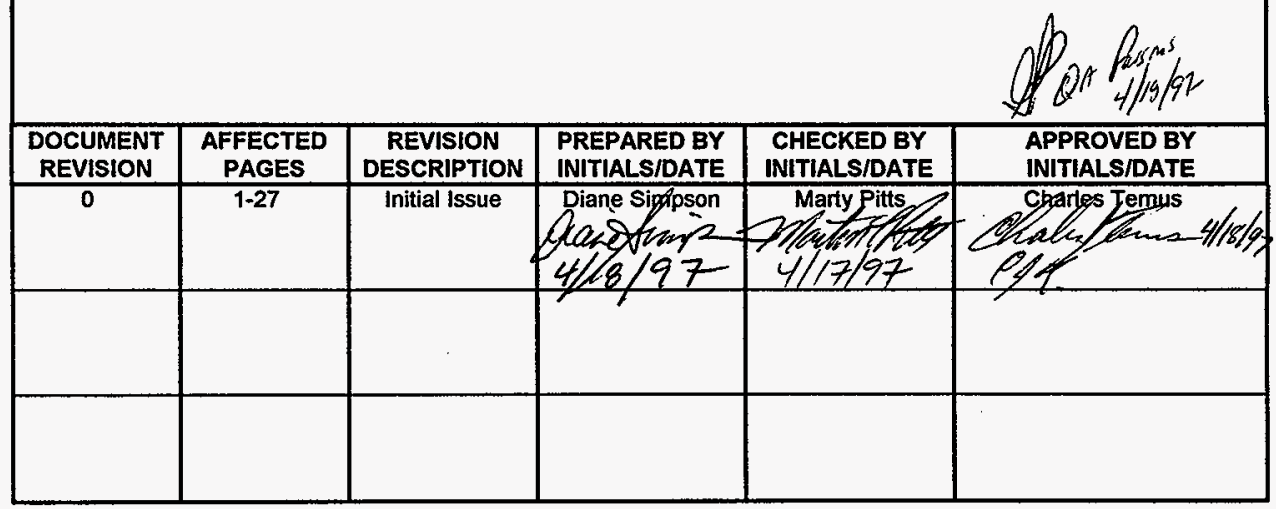


CLIENT: Duke Engineering \& Services Hanford

FILE NO: KH8009-8-01

PROJECT: MCO Final Design DOC. NO.: HNF-SD-SNF-DR-003, Rev. 0, Appendix 3

\section{TABLE OF CONTENTS}

1. INTRODUCTION 4

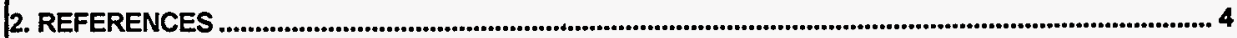

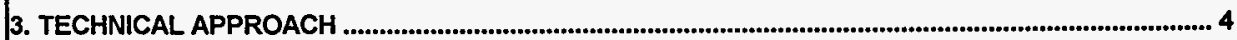


CLIENT: Duke Engineering \& Services Hanford

FILE NO: KH8009-8-01

PROJECT: MCO Final Design DOC. NO.: HNF-SD-SNF-DR-003, Rev. 0, Appendix 3

\section{List of Figures}

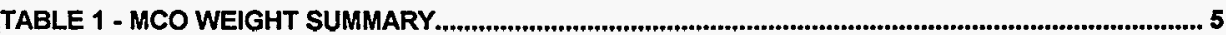

TABLE 2 - MCO ASSEMBLED WITH CANISTER COVER $\ldots$

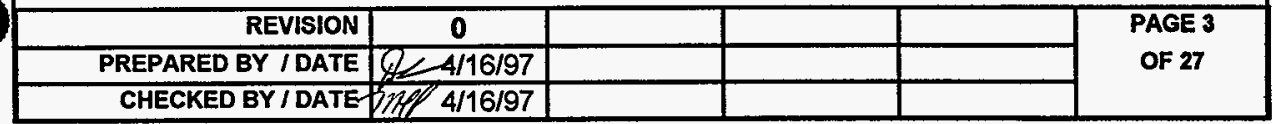




\section{INTRODUCTION}

This calculation package provides the calculated weights of fully assembled and loaded MCO's (with and without water), and the calculated weight of individual MCO componenets.

\section{REFERENCES}

1. DE\&S, 1997, Performance Specification for the Spent Nuclear Fuel Multi-Canister Overpack, HNF-S-0426, Rev. 3, Duke Engineering and Services Hanford, Richland, Washington.

2. Oberg, E., Jones, F. D., Horton, H. L., and Ryffel, H.H., 1996, Machinery's Handbook, 25th Edition, Industrial Press, inc., New York, New York.

3. Spent Nuclear Fuels, Spent Nuclear Fuel Project Technical Databook, WHC-SD-SNF-TI-015, Rev. 0, Westinghouse Hanford Company, Richland, Washington.

4. Department of Defense, United States of America, MIL-HDK-5G, 1 November 1994, Military Handbook, Metallic Materials and Elements for Aerospace Vehicle Structures Volume 1 of 2 Volumes.

5. Beer, F. P., Johnston, E. R. Jr., 1984, Vector Mechanics for Engineers Statics and Dynamics, Fourth Edition, McGraw-Hill Book Company, New York, New York.

6. MCO Drawing Package.

\section{TECHNICAL APPROACH}

The weight for the Multi-Canister Overpack (MCO) was determined by multiplying the volume of a component by the density of Stainless Steel $\left(0.286 \mathrm{Ib} / \mathrm{in}^{3}\right.$, Reference 3). Table 1 represents a summary of the weight calculation for the MCO under different load conditions. Table 2 represents the weight of an fully loaded MCO, dry, with the canister cover. Attached as an Appendix are weight tables for each individual MCO Component.

\begin{tabular}{|r|c|l|l|l|}
\hline REVISION & 0 & & & \\
\hline PREPARED BY IDATE & S $4446 / 97$ & & & \\
\hline CHECKED BYIDATE & 4/16/97 & & & \\
\hline
\end{tabular}


CLIENT: Duke Engineering \& Services Hanford

PROJECT: MCO Final Design

FILE NO: KH8009-8-01

DOC. NO.: HNF-SD-SNF-DR-003, Rev. 0, Appendix 3

Table 1 - MCO Weight Summary

\begin{tabular}{|c|c|c|c|}
\hline Item & Condition & Nominal Weight (lbs.) & Maximum Weight (lbs) \\
\hline MK1A Storage Basket & Empty & 397.17 & 397.17 \\
\hline MK1A Scrap Basket & Empty & 412.33 & 412.33 \\
\hline MK1A Storage Basket & Loaded 48 Fuel Assemblies & 2303.25 & 2303.25 \\
\hline MKIV Storage Basket & Empty & 199.44 & 199.44 \\
\hline MKIV Scrap Basket & Empty & 164.73 & 164.73 \\
\hline MKIV Storage Basket & Loaded 54 Fuel Assemblies & 3189.96 & 3189.96 \\
\hline MCO & $\begin{array}{l}\text { Empty, without upper shield } \\
\text { plug, dry }\end{array}$ & 1921.76 & 2114.12 \\
\hline MCO & $\begin{array}{l}\text { Empty, with upper shield plug, } \\
\text { dry }\end{array}$ & 3428.37 & 3691.92 \\
\hline MCO & $\begin{array}{l}\text { Loaded - Six Loaded MK1A } \\
\text { Storage Baskets, without upper } \\
\text { shield plug, filled with water }\end{array}$ & 17110.25 & 17302.61 \\
\hline MCO & $\begin{array}{l}\text { Loaded - Six loaded MK1A } \\
\text { Storage Baskets, with upper } \\
\text { shield plug, filled with water }\end{array}$ & 18551.86 & 18880.41 \\
\hline MCO & $\begin{array}{l}\text { Loaded - Six loaded MKTA } \\
\text { Storage Baskets, with upper } \\
\text { shield plug, dry }\end{array}$ & 17247.86 & 17511.41 \\
\hline MCO & $\begin{array}{l}\text { Loaded - Five loaded MKIV } \\
\text { Storage Baskets, without upper } \\
\text { shield plug, filled with water }\end{array}$ & 19216.56 & 19408.92 \\
\hline MCO & $\begin{array}{l}\text { Loaded - Five loaded MKIV } \\
\text { Storage Baskets, with upper } \\
\text { shield plug, filled with water }\end{array}$ & 20657.17 & 20986.72 \\
\hline MCO & $\begin{array}{l}\text { Loaded - Five loaded MKIV } \\
\text { Storage Baskets, with upper } \\
\text { shield plug, dry }\end{array}$ & 19378.17 & 19641.72 \\
\hline
\end{tabular}

\begin{tabular}{|l|l|l|l|}
\hline & & \\
\hline & & & \\
\hline & & \\
\hline
\end{tabular}


CLIENT: Duke Engineering \& Services Hanford

PROJECT: MCO Final Design DOC. NO.: HNF-SD-SNF-DR-003, Rev. 0, Appendix 3

FILE NO: KH8009-8-01

Table 2 - MCO Assembled with Canister Cover

\begin{tabular}{|l|l|c|c|}
\hline \multicolumn{1}{|c|}{ Item } & \multicolumn{1}{|c|}{ Condition } & Nominal Weight (bs.) & Maximum Weight (lbs) \\
\hline Canister Cover & N/A & 488.40 & 573.87 \\
\hline MCO & $\begin{array}{l}\text { Loaded - Six loaded } \\
\text { MK1A Storage Baskets, } \\
\text { with upper shield plug, } \\
\text { dry }\end{array}$ & 17736.26 & 18025.28 \\
\hline MCO & $\begin{array}{l}\text { Loaded - Five loaded } \\
\text { MKIV Storage Baskets, } \\
\text { with upper shield plug, } \\
\text { dry }\end{array}$ & 19866.57 & 20155.59 \\
\hline
\end{tabular}

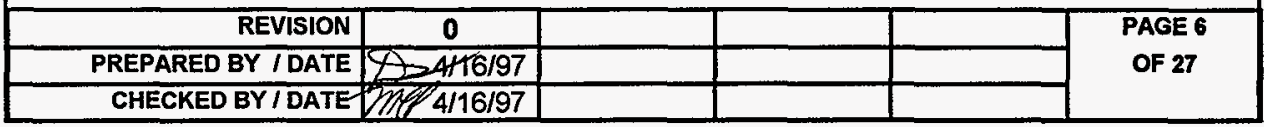


CLIENT: Duke Engineering \& Services Hanford

PROJECT: MCO Final Design

FILE NO: KH8009-8-01

DOC. NO.: HNF-SD-SNF-DR-003, Rev. 0, Appendix 3

\section{Appendix}

MCO Components Weight Summaries 


\begin{tabular}{|c|c|c|c|c|c|c|c|c|c|c|c|}
\hline \multicolumn{12}{|c|}{ Mechanical closurge Detall - Canister collar } \\
\hline & & & & & & & & & & & \\
\hline & & \multirow{2}{*}{$0.0, \max$ (in.) } & \multirow{2}{*}{ 1.0. nom. (In.) } & \multirow{2}{*}{ I.D. min. (in.) } & \multirow{2}{*}{ Helght nom. (in.) } & \multirow{2}{*}{ Helght max. (in.) } & \multirow{2}{*}{ Volume nom. $\left(\mathrm{in}^{5}\right)$} & \multirow{2}{*}{ Voiume $\max .\left(\ln ^{2}\right)$} & \multirow{2}{*}{ Density, 304L $\left(\mathrm{lb} / \mathrm{In}^{3}\right)$} & \multirow{2}{*}{ Welght nom. (bss.) } & \multirow{2}{*}{ Welght max. (bs).) } \\
\hline Part 1 & $\frac{0.0 . \text { nom. (In.) }}{25.310}$ & & & & & & & & & & \\
\hline Faft 2 & 25.310 & 25.310 & 24.567 & 24.567 & 0.375 & 0.390 & 10.915 & 11.351 & 0.286 & 3.12 & 3.25 \\
\hline Part 3 & 25.310 & 25.310 & 24.040 & 24.035 & 2.310 & 2.340 & 113.708 & 115.627 & 0.286 & 32.52 & 33.07 \\
\hline Part 4 & 25.310 & 25.310 & 24.045 & 24.030 & 3.315 & 3.360 & 162.553 & 166.663 & 0.286 & 46.49 & 47.67 \\
\hline Pan 5 & 25.310 & 25.310 & 24.045 & 24,030 & 0.633 & 0.663 & 15.520 & 16.443 & 0.286 & 4.44 & 4.70 \\
\hline Pait 6 & 24.045 & 24.060 & 22.985 & 22.970 & 9.065 & 9.095 & 354.927 & 366.180 & 0.286 & 101.51 & 104.73 \\
\hline Part 7 & 24.045 & 24.060 & 23.075 & 23.030 & 0.280 & 0.309 & 5.026 & 5.886 & 0.288 & 1.44 & 1.68 \\
\hline \multirow[t]{3}{*}{ Pant 8} & 23.075 & 23.090 & 22.985 & 22.970 & 0.280 & 0.309 & 0.912 & 1.341 & 0.286 & 0.26 & 0.38 \\
\hline & & & & & & & & & & & \\
\hline & & & & & & & & & & & \\
\hline & O.D. nom. (in.) & $0.0, m[n .(\ln )$ & B.D. nom. (in.) & 1.D. max. (in.) & Height nom. (ln.) & Holght min. (ln.) & Volume nom. $\left(\mathrm{n}^{3}\right)$ & Volume min. (in) & & & \\
\hline Top bevel & 25.310 & 25.280 & 24.567 & 24.567 & 0.100 & 0.088 & 7.455 & 7.228 & 0.286 & -0.42 & -0.35 \\
\hline \multirow[t]{3}{*}{ Threads } & 24.5000 & 24.500 & 24.040 & 24.070 & 3.310 & 3.235 & 29.023 & 26.532 & 0.286 & -8.30 & -7.59 \\
\hline & & & & & & & & & Tohat Welaht & 209.53 & 216.26 \\
\hline & & & & & & & & & & & \\
\hline & O.D. nom. (in.) & $0.0 . m x$. (nn.) & 1.D. nom. (ln.) & 1.0. $m \ln .(\ln )$. & Helght nom. (nol & Helaht max. (in) & Volume nom. $\left(\mathrm{ln}^{3}\right)$ & \multicolumn{2}{|l|}{ Volume max. $\left(\mathbf{n}^{3}\right)$} & & \\
\hline \multirow[t]{3}{*}{ Bottom Weld } & 24.045 & 24.05 & 23.075 & 23.03 & 0.280 & 0.309 & 5.026 & 5.886 & 0.286 & 1.44 & 1.68 \\
\hline & & & & & & & & & & 210.97 & 217.94 \\
\hline & & & & & & & & & & & \\
\hline Revision & & & $\mathbf{0}$ & & & & & & & & \\
\hline \multicolumn{2}{|c|}{ Prepared by/Date } & $Z$ & $-4 / 16 / 97$ & & & & & & & & \\
\hline \multicolumn{2}{|c|}{ Checked by/Date } & 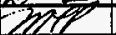 & $4 / \sqrt{717}$ & & & & & & & & \\
\hline
\end{tabular}




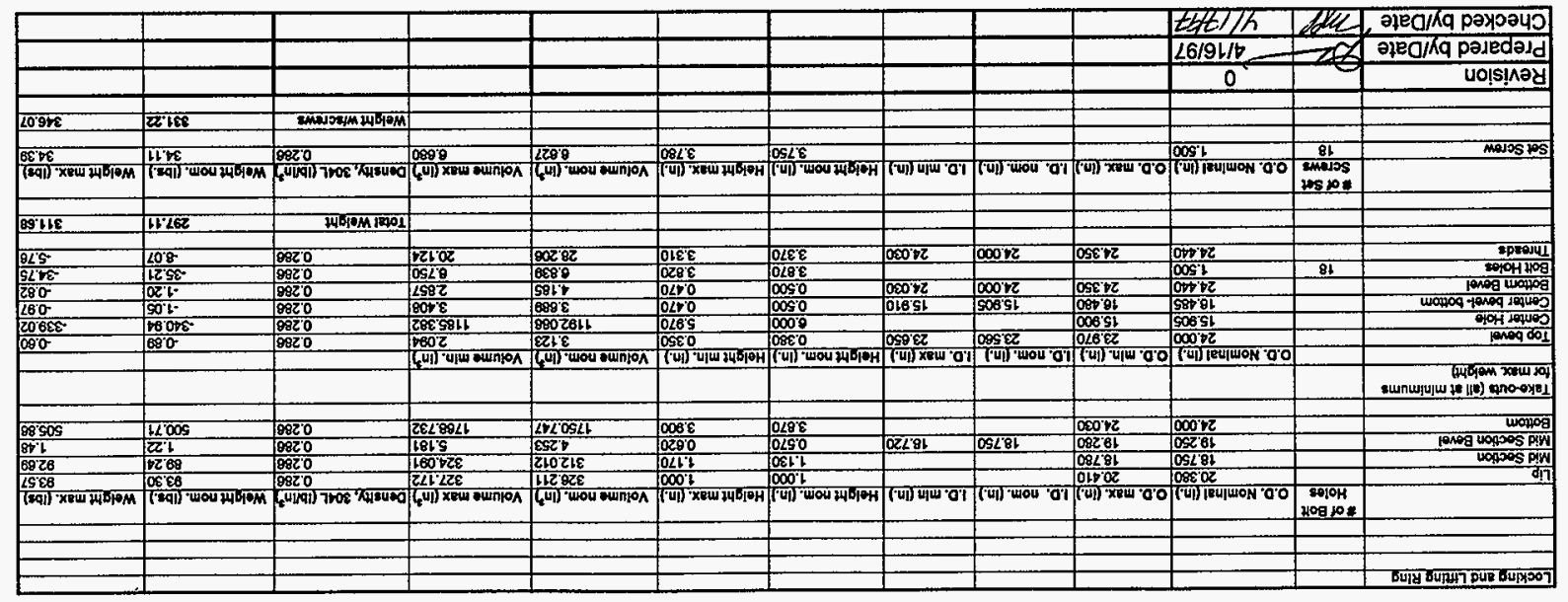




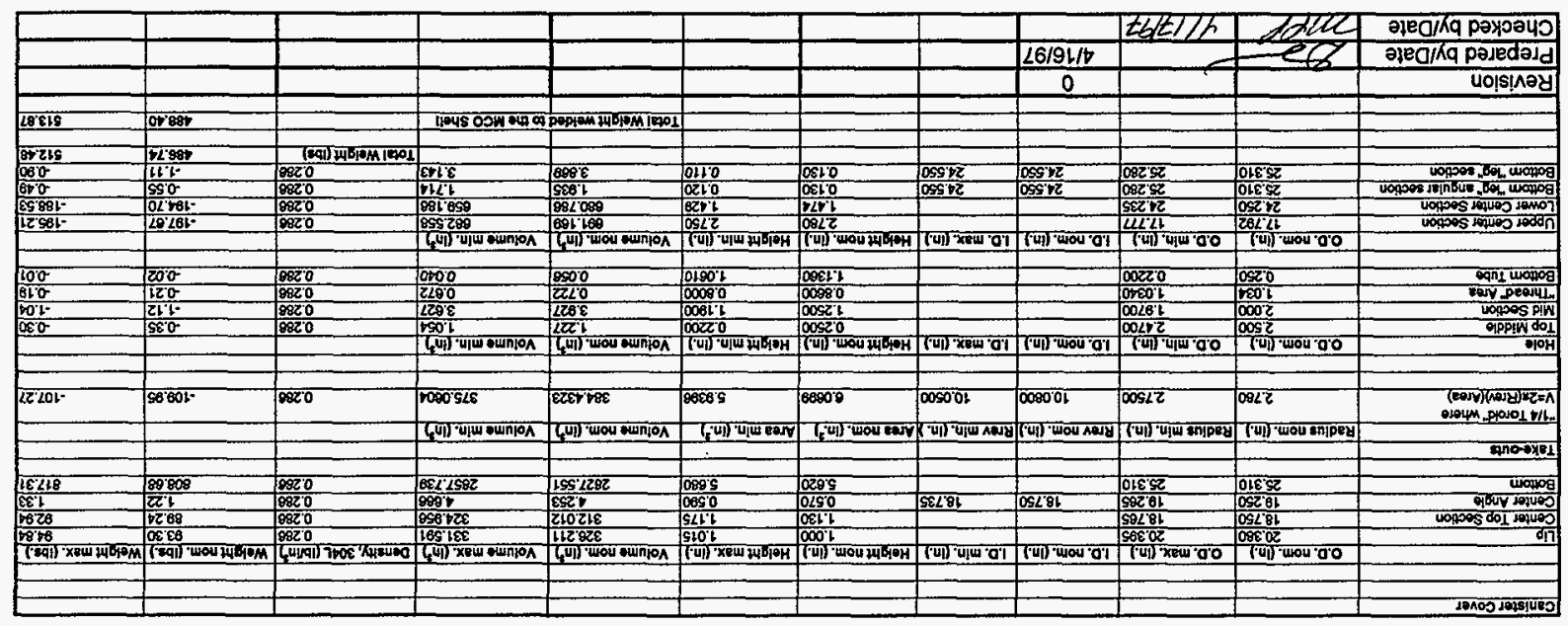




\begin{tabular}{|c|c|c|c|c|c|c|c|c|c|c|c|}
\hline \multicolumn{12}{|l|}{ MCo Sholl } \\
\hline & & & & & & & & & & & \\
\hline MCO shell (welded & $\frac{0.0 . \text { nom. }^{\prime}(\mathrm{ln} .)}{24.045}$ & 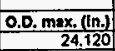 & $\frac{1.0, \mathrm{mom}_{1}(\mathrm{ln}, \mathrm{n}}{23.045}$ & $\frac{1.0 \cdot \min .(1 \mathrm{n})}{23.000}$ & \begin{tabular}{|c|c|} 
Helght nom. (in.) \\
39.760
\end{tabular} & $\frac{\text { Helght } \max .(\mathrm{n} .)}{139.830}$ & \begin{tabular}{|r} 
Volume nom. $\left(\mathrm{ln}^{3}\right)$ \\
5168.940 \\
\end{tabular} & \begin{tabular}{|l} 
Volume max. $\left(\ln ^{5}\right)$ \\
5795.802
\end{tabular} & $\begin{array}{r}\text { Density, } 304 \mathrm{~L}\left(\mathrm{lb}, \mathrm{n}^{2}\right) \\
0.286 \\
\end{array}$ & \begin{tabular}{|r|} 
Welght nom. (bbs.) \\
1478.32 \\
\end{tabular} & $\frac{\text { Welght max. (bs.) }}{1657.60}$ \\
\hline & & & & & & & & & Total Welght & 1478.32 & 1657.60 \\
\hline & & & & & & & & & Tor Weloht walded & 147832 & \\
\hline & & & & & & & & & & & \\
\hline Revision & & & 0 & & & & & & & & \\
\hline Prepared by/ & IDate & 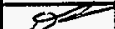 & $4 / 16 / 97$ & & & & & & & & \\
\hline Checked by/l & $\overline{\text { Date }}$ & -309 & $47 / 797$ & & & & & & & & \\
\hline
\end{tabular}


$\bigcirc^{\frac{0}{i^{1}}}$

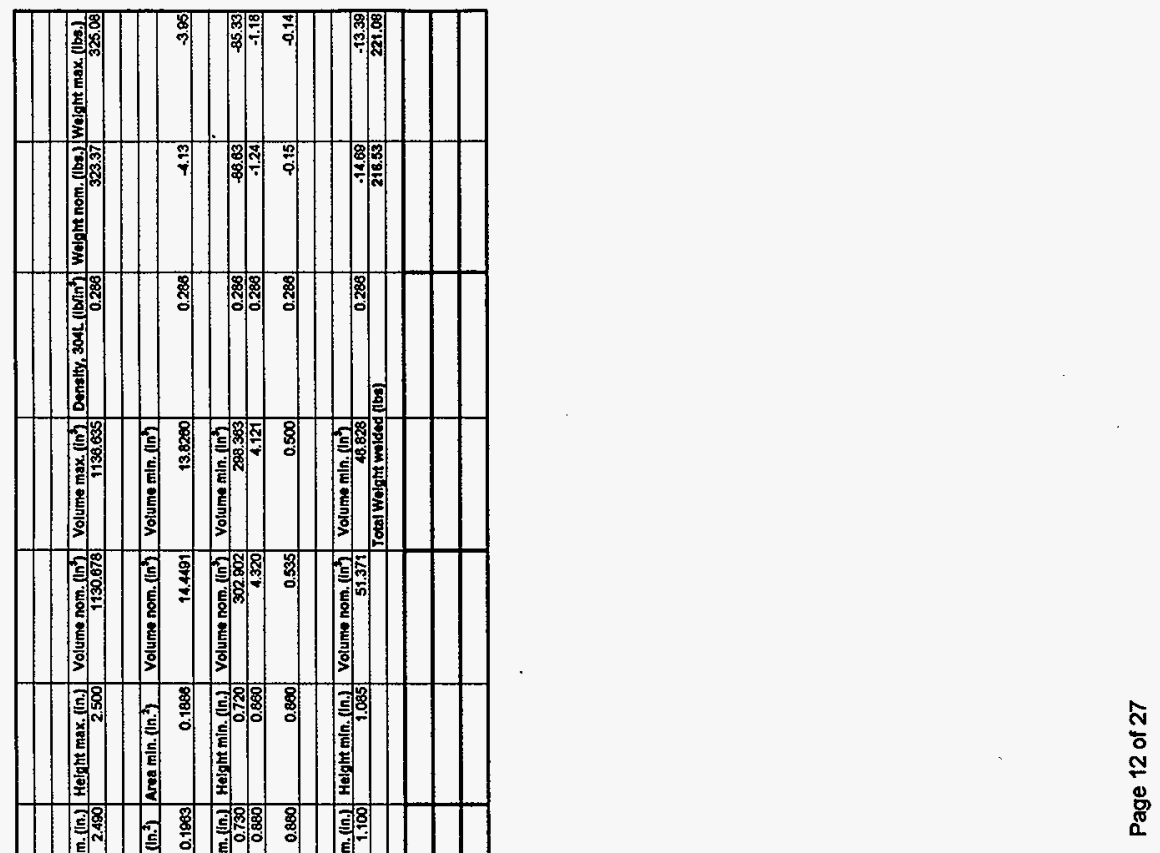

$0^{\frac{1}{2}}$ 


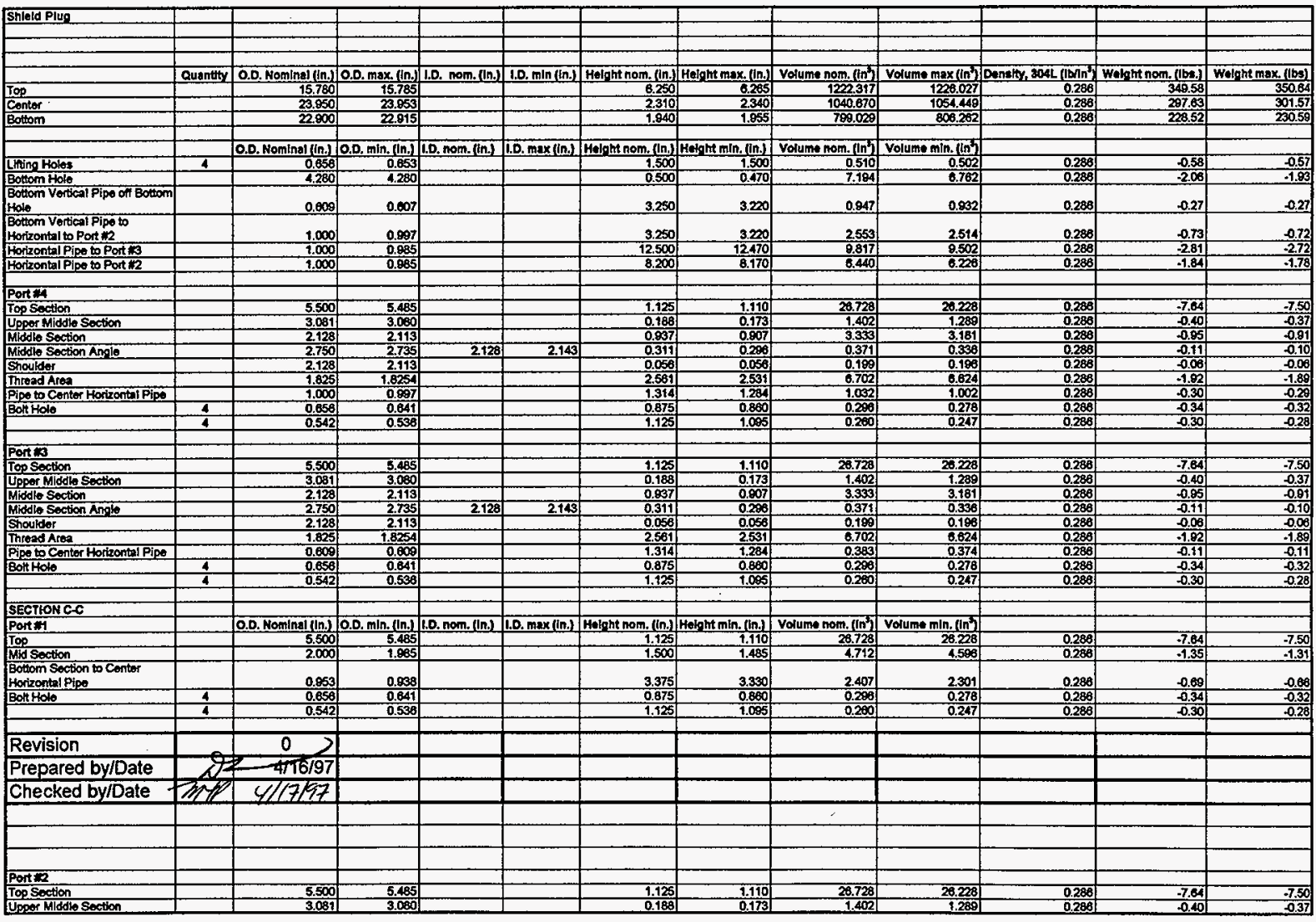

\subsection{5




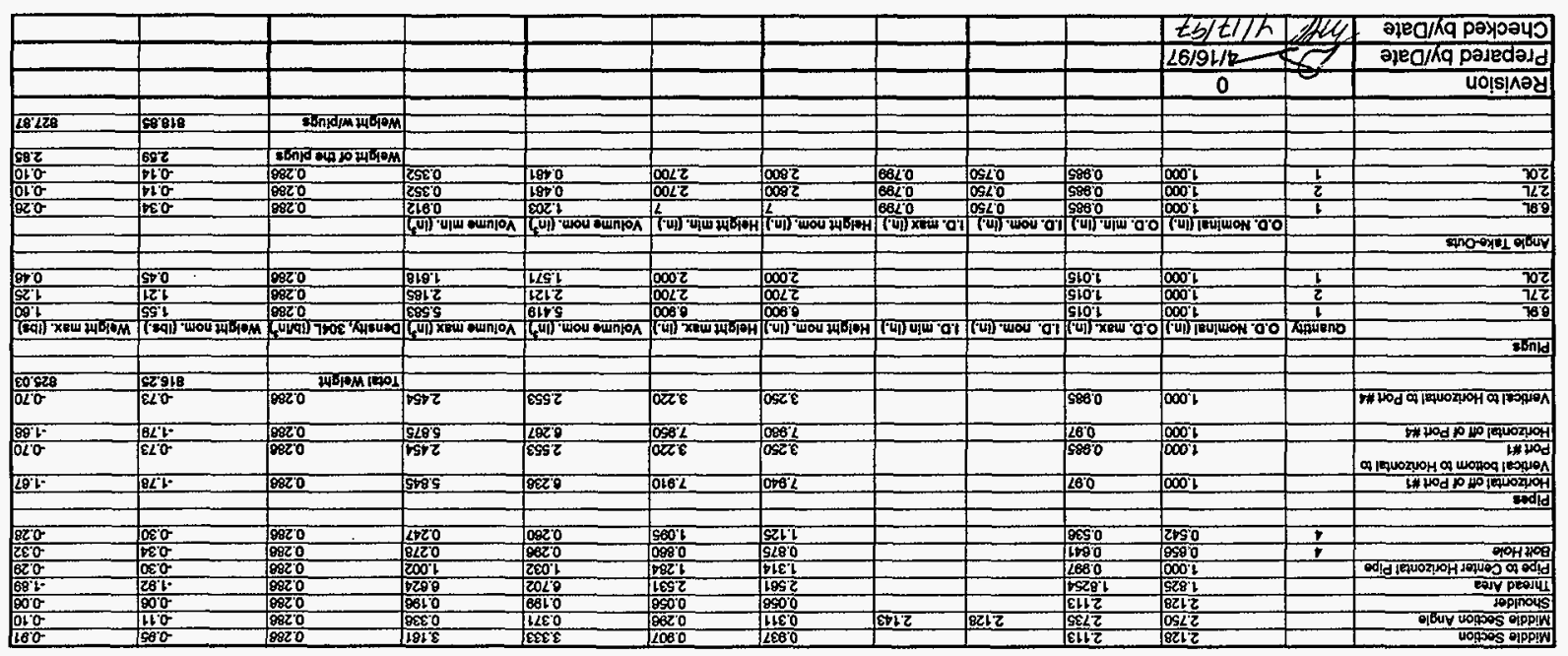

exipueddy 


\begin{tabular}{|c|c|c|c|c|c|c|c|c|c|c|c|c|}
\hline Imtomal Fllior Guard Plato & & & & & & & & & & & & \\
\hline & & & & & & & & & & & & \\
\hline & & & & & & & & & & & & \\
\hline Guand Plate & of Holte & O.D. Nomins (in) & 0,0, mex $(\ln )$ & LD. nom (in.) & L.D. $\mathrm{mln}\left(\mathrm{l} \mathrm{L}_{\mathrm{L}}\right.$ & Hoteht nom. (tn.) & Holght max (ln. & Volume nom. (ln & Volume max $\left(\mathrm{In}^{3}\right.$ & 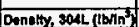 & Wotght nom (lbe) & Wolaht max (lbet) \\
\hline Plato & Eof Holles & 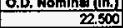 & $\frac{0.0 . \operatorname{mex}(\mathrm{ln}, \mathrm{n}}{22.530}$ & & & $\frac{1.500}{1.500}$ & $\frac{1.630}{1.530}$ & $\begin{array}{r}80.412 \\
56.412\end{array}$ & 609.963 & 0.286 & 170.57 & 174.45 \\
\hline & & & & & & & & & & & & \\
\hline $\begin{array}{l}\text { Takoouts (dit if milimums for } \\
\text { max, woight) }\end{array}$ & & & & & & & & & & & & \\
\hline & & O.D. Hominal (in) & $0.0 . \mathrm{m} / \mathrm{n}$ (In) & L. nom (in.) & L.D. $\max (i n)$ & Hetent nom. (in.) & Helght min. (ln.) & Volume nom. (lin') & Volume $\min \left(\mathrm{ln}^{3}\right)$ & & & \\
\hline Conter Hoto & & 4.250 & 4.250 & & & 1.500 & 1.470 & 21.279 & 20.854 & 0.266 & 6.09 & 5.96 \\
\hline side Angto & & 2.500 & 22,470 & 21.830 & 21.936 & 1.250 & 1.190 & 16.579 & 11.091 & 0.286 & -4.17 & 3.17 \\
\hline & & O.D. Noming (in.) & O.D. $\min (i \mathrm{n})$ & (D. nom (n.) & LD. max (in) & Hetght nom. (in.) & Heloht $m \ln$. (ln & Volume nom. $\left(\mathrm{tn}^{3}\right)$ & Volume min. $\left(\ln ^{2}\right)$ & & & \\
\hline Hotes & 4 & $\frac{1.000}{1.000}$ & 1.000 & & & 1.500 & 1.470 & 1.178 & $\begin{array}{r}1.155 \\
1.13\end{array}$ & 0.286 & -1.35 & -1.32 \\
\hline Chamfore & 4 & 1,500 & 1.440 & 1.000 & 1.000 & 0.250 & 0.220 & 0.123 & 0.093 & 0.236 & -0.16 & -0.11 \\
\hline & & & & & & & & & & Welopt of the pists & 15.23 & 163.69 \\
\hline & & & & & & & & & & & & \\
\hline Guturd Pitu Ring & & & & & & & 101 & 90 & Yotumener $10^{3}$ & & & \\
\hline Rho & & $\frac{0.0 . \text { Nomind (tn.) }}{22.000}$ & $\frac{0 . D \cdot \max (1 \mathrm{n})}{22.630}$ & 1.D. nom. (n.) & 1.0. min (in.) & $\frac{\text { Hotght nom. (ln) }}{4.250}$ & 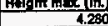 & $\frac{\text { Volume nom. (in) }}{1669.833}$ & $\frac{10}{1701.761}$ & 0.286 & 483.20 & 488.70 \\
\hline & & & & & & & & & & & & \\
\hline 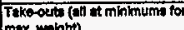 & & & & & & & & & & & & \\
\hline & & O.D. Noning $(\ln )$ & 0.0. min (In) & LD. nom. (lin) & L.D. $\max (\mathrm{In})$ & Helght nom. (in.) & Halght min (in) & Volum nom. $(\ln 2)$ & Volitme $\min \left(\mathrm{ln}^{3}\right)$ & & & \\
\hline Conter Hols & & 20.500 & 20.470 & & & 4.250 & 4.220 & 1402.770 & $13,8.795$ & 0.286 & +0.19 & -397.20 \\
\hline Top and Bottom STo Anoles & & 2.500 & 22.470 & 21.880 & 21.970 & 0.310 & 0.230 & 8.699 & 4.836 & 0.286 & -1.92 & -1.40 \\
\hline & & & & & & & & & & Worghete the Ring & $\infty .6$ & 0,11 \\
\hline & & & & & & & & & & Totel Wolght & 250.01 & 232.00 \\
\hline & & & & & & & & & & & & \\
\hline Revision & & 0 & & & & & & & & & & \\
\hline Prepared by/Date & & $4 / 16 / 97$ & & & & & & & & & & \\
\hline Checked by/Date & 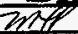 & $4 / 17 / 97$ & & & & & & & & & & \\
\hline
\end{tabular}




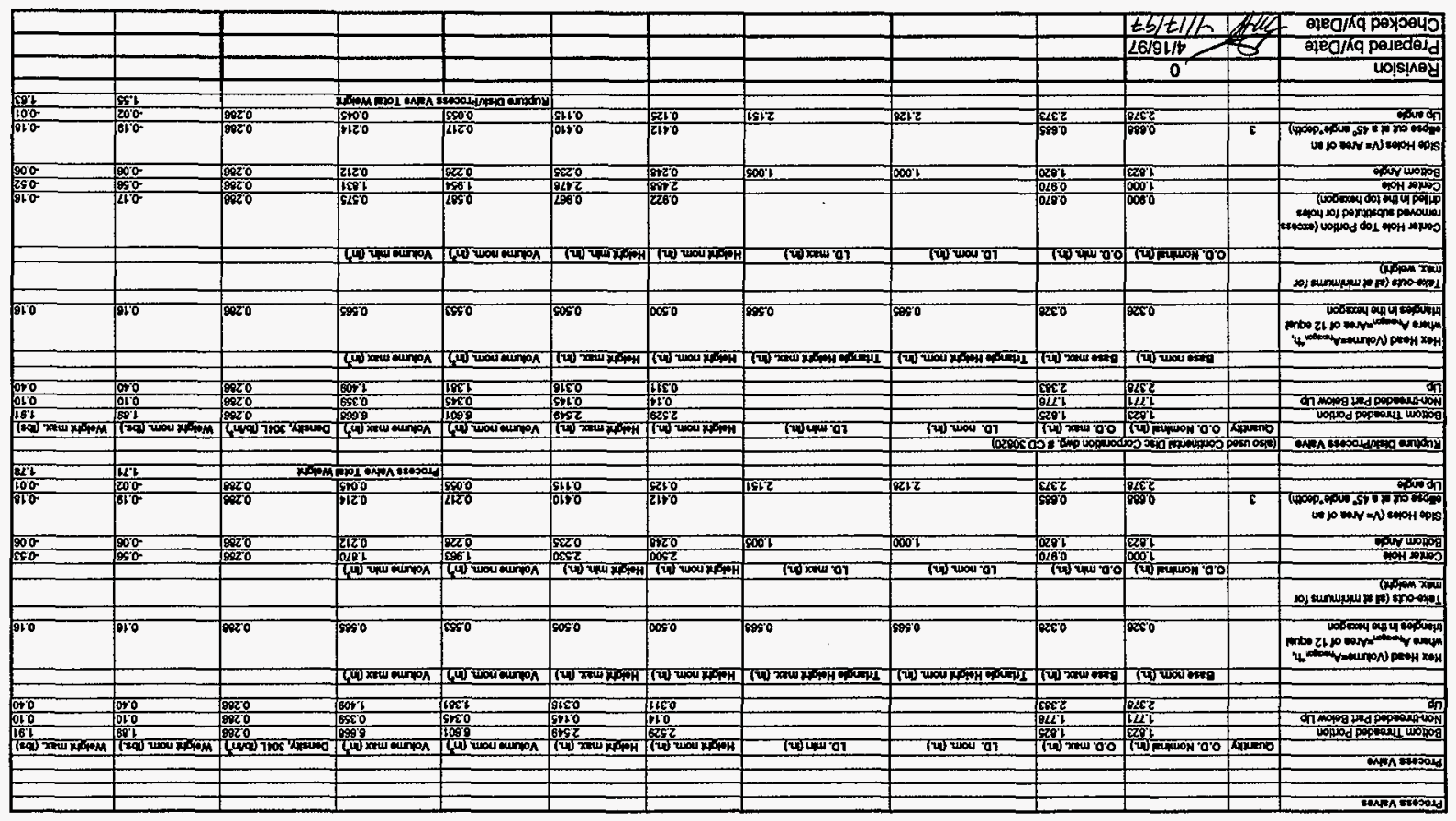



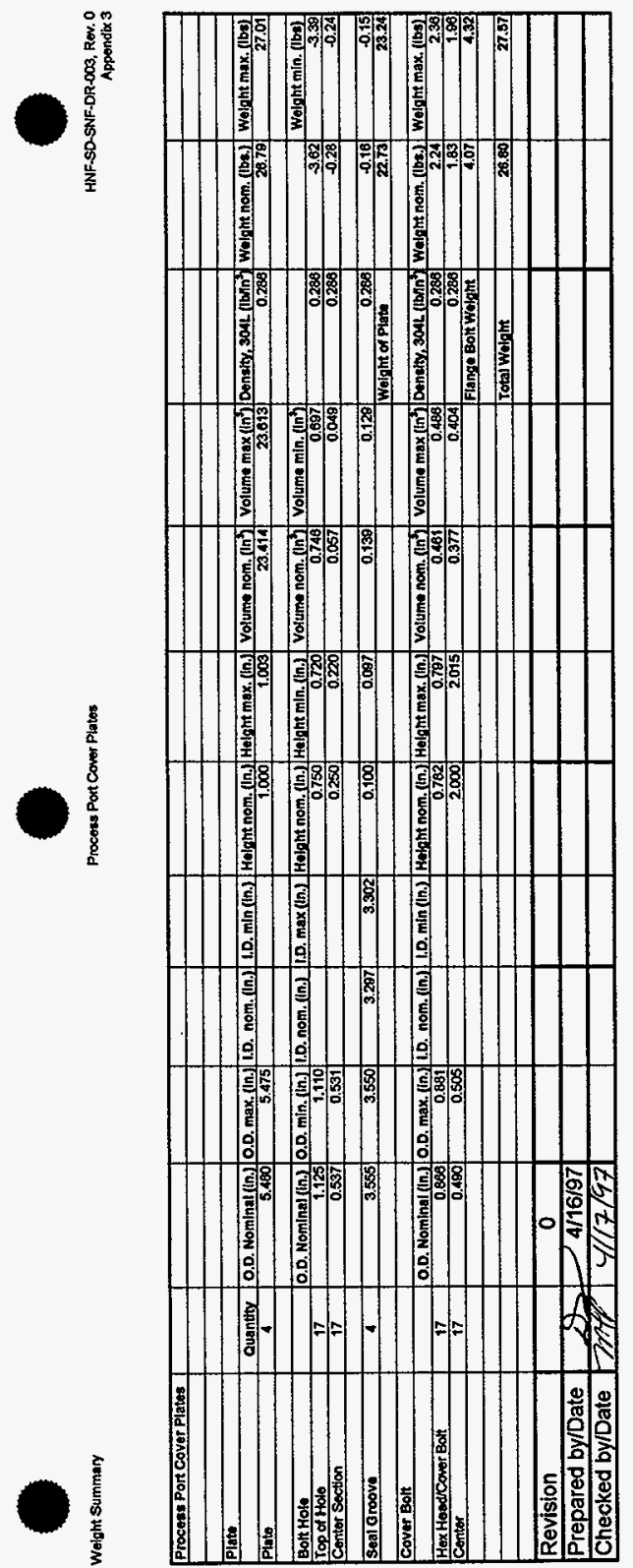


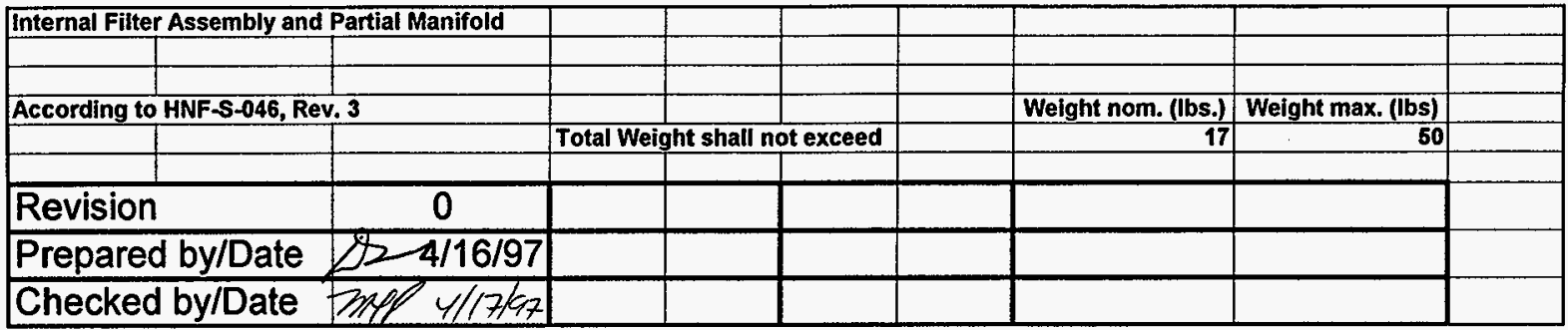




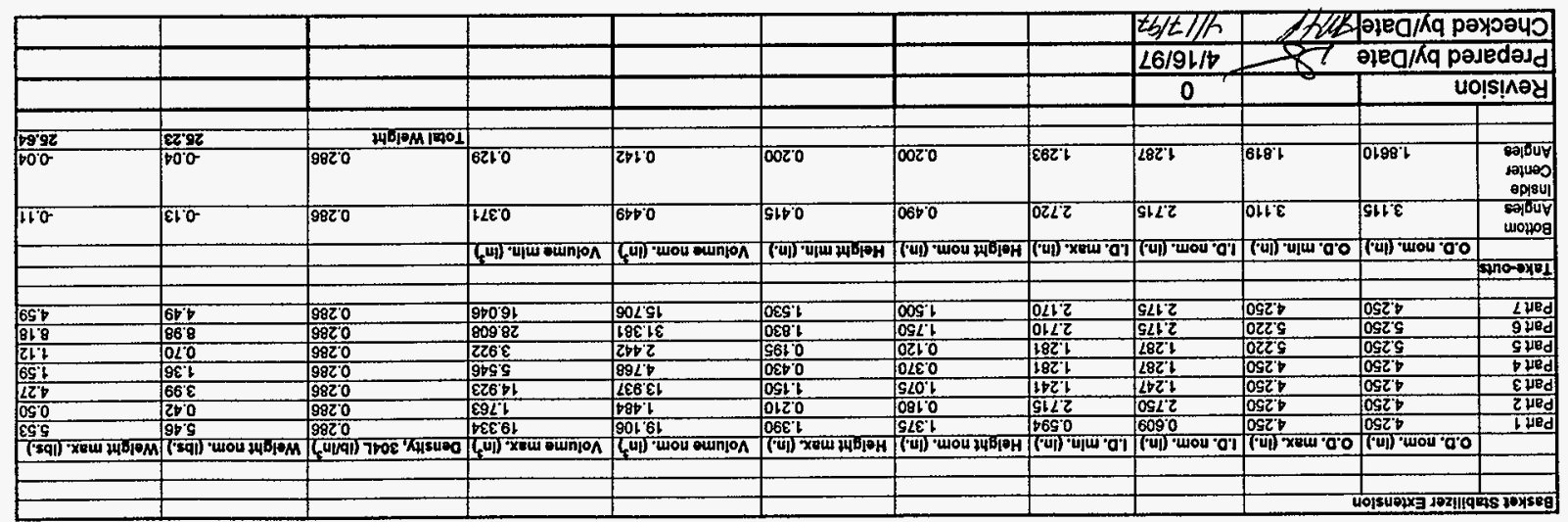


$\bullet$

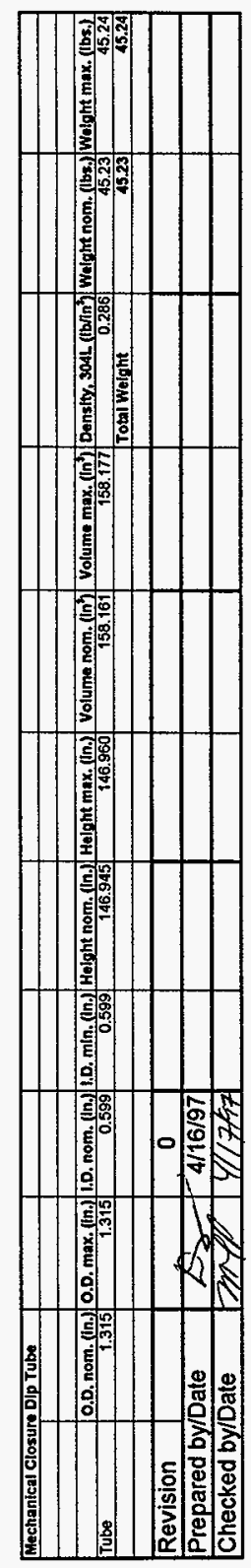

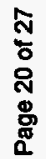

$\mathrm{O}^{\mathrm{i}}$ 


\begin{tabular}{|c|c|c|c|c|c|c|c|c|c|c|c|}
\hline \multirow{2}{*}{\multicolumn{12}{|c|}{ Dip Tube Gulde Cone }} \\
\hline & & & & & & & & & & & \\
\hline & O.D. nom. (ln.) & O.D. max. (ln.) & 1.0. nom. (In.) & I.D. $m\left[n_{\text {. }(\ln .)}\right.$ & Helght nom, (In.) & Helght max. (n.) & Volume nom. (ing) & Volume max. $\left(\ln ^{5}\right)$ & Density, 304L (1b/nn & Welght nom. (1bs.) & Welght max. (lbs.) \\
\hline Top and Midd & 2.650 & $\begin{array}{r}2.655 \\
\end{array}$ & 1.375 & 1.360 & 2.570 & 2.590 & 10.359 & 10.577 & 0.286 & 2.96 & 3.02 \\
\hline Bottom & 2.750 & 2.765 & 1.375 & 1.360 & 0.600 & 0.595 & 2.673 & 2.708 & 0.286 & 0.76 & 0.77 \\
\hline & & & & & & & & & & & \\
\hline \multicolumn{12}{|l|}{ Take-outs } \\
\hline & O.D. nom. (in.) & $0.0 . \min .(\ln )$. & 1.D. nom. (in.) & 1.0.max. (in.) & Helght nom. (In.) & Helght min. (in.) & Volume nom. (in) & Volume mln. $(\ln )^{3}$ & & & \\
\hline $\begin{array}{l}\text { Top Inside } \\
\text { Anqle }\end{array}$ & 1.725 & 1.710 & 1.375 & 1.390 & 4.891 & 0.488 & 2.084 & 0.190 & 0.286 & -0.60 & -0.05 \\
\hline $\begin{array}{l}\text { Top Outside } \\
\text { Anple }\end{array}$ & 2.6500 & 2.655 & 2.130 & 2.145 & 0.714 & 0.709 & 0.697 & 0.682 & 0.286 & -0.20 & -0.19 \\
\hline & & & & & & & & & Total Wolght & 2.93 & 3.55 \\
\hline & & & & & & & & & & & \\
\hline Revision & & & 0 & & & & & & & & \\
\hline \multicolumn{12}{|c|}{ Prepared by/Date } \\
\hline Checked & by/Date & 794 & 411717 & & & & & & & & \\
\hline
\end{tabular}




\begin{tabular}{|c|c|c|c|c|c|c|c|c|c|c|c|}
\hline \multicolumn{12}{|l|}{ Basket Support Piato } \\
\hline & & & & & & & & & & & \\
\hline & Length nom. (ln) & Length $\max$ (in.) & Wath nom. (ln.) & Woth mex (In,) & Hejent nom (in) & Helant max (In,) & Volume nom. (lB') & Volume max $\left(\mathrm{n}^{2}\right)$ & Denelty, 304L (bBAn & Wolont nom. (lbe) & Wolgh max (bs.) \\
\hline Support Plate & $\begin{array}{r}10.180 \\
\end{array}$ & 10.195 & $\begin{array}{r}1.490 \\
\end{array}$ & $\begin{array}{r}1.506 \\
\end{array}$ & 0.500 & 0.530 & 7.584 & 8.132 & 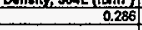 & 217 & 2.33 \\
\hline \multicolumn{12}{|l|}{ Take-oute" } \\
\hline & Lenth nom. (In.) & Leneth min. (lk.) & Wloth nom (In) & Whath nin find & Holfobt nom, (in.) & Hoight min (in.) & Volume nom. (in') & Volume min, $\left(1 \mathrm{n}^{2}\right)$ & & & \\
\hline Angula section & 0.190 & $\begin{array}{r}0.160 \\
\end{array}$ & 0.990 & 0.160 & 0.500 & 0.470 & 0.009 & 0.006 & 0.286 & 0.00 & 0.00 \\
\hline Rectargular Section & 0.125 & 0.110 & 0.590 & 0.380 & 0.500 & 0.470 & 0.018 & 0.015 & 0.286 & -0.01 & 0.00 \\
\hline \multirow{4}{*}{ 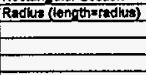 } & 0.500 & $0.53(\operatorname{man} x)$ & 1.000 & 0.940 & 0.500 & $0.47(m h), 0.53(\max )$ & 0.027 & -0.013 & 0.286 & -0.01 & 0.00 \\
\hline & & & & & & & & & Total Wolgm & 216 & 232 \\
\hline & & & & & & & & & & & \\
\hline & & & & & & & & & Weleded Wolght & 217 & 2.35 \\
\hline Revision & & & 0 & & & & & & & & \\
\hline \multicolumn{12}{|c|}{ Prepared by/Date } \\
\hline Checked by/D & ate & 18 & $4 / 17 / 97$ & & & & & & & & \\
\hline
\end{tabular}




\begin{tabular}{|c|c|c|c|c|c|}
\hline \multicolumn{5}{|c|}{ Mark 1A Storage Basket } & \\
\hline & Ouantity & Volume $\left(\mathrm{in}^{3}\right)$ & Densily $\left(1 \mathrm{~b} / \mathrm{in}^{3}\right)$ & Weiaht (lbs) & \\
\hline Bottom Plate & 1 & 432.7366 & 0.286 & 123.76 & \\
\hline Center Post & 1 & 352.4711 & 0.286 & 100.81 & \\
\hline Bottom Bushing & 1 & 71.5469 & 0.286 & 20.46 & \\
\hline Top Guide & 1 & 31.9856 & 0.286 & 9.15 & \\
\hline Support Rods & 6 & 62.9898 & 0.286 & 108.09 & \\
\hline Rod Pins & $\frac{6}{6}$ & 1.0460 & 0.286 & 1.79 & \\
\hline Fuel Rack & 1 & 202.0600 & 0.1000 & 20.21 & \\
\hline Shroud & 1 & 45.0940 & 0.286 & 12.90 & \\
\hline \multirow[t]{3}{*}{ Spacer } & 1 & $\bar{N} / A$ & N/A & Negligible & \\
\hline & & & & & \\
\hline & & & Total Weight (lbs) & 397.17 & \\
\hline & & & & & \\
\hline Revision & & & 0 & & \\
\hline \multicolumn{2}{|c|}{ Prepared by/Date } & & $4 / 16 / 97$ & & \\
\hline \multicolumn{2}{|c|}{ Checked by/Date } & & $17 / 97$ & & \\
\hline
\end{tabular}




\begin{tabular}{|c|c|c|c|c|c|}
\hline Mark 1A Scrap Bask & & & & & \\
\hline & & & & & \\
\hline & Quantity & Volume $\left(\mathrm{in}^{3}\right)$ & Density $\left(\mathrm{lb} / \mathrm{in}^{3}\right)$ & Weiaht (lbs) & \\
\hline Bottom Plate & 1 & 432.7366 & 0.286 & $\frac{123.76}{123 .)}$ & \\
\hline Bottom Plate Welds & 1 & 1.5180 & 0.286 & 0.43 & \\
\hline Bottom Plate Welds & 1 & 1.3800 & 0.286 & 0.39 & \\
\hline Center Post & 1 & 352.4711 & 0.286 & 100.81 & \\
\hline Bottom Bushing & 1 & 71.5469 & 0.286 & 20.46 & \\
\hline Top Guide & 1 & 31.9856 & 0.286 & 9.15 & \\
\hline Center Post Peg & 5 & 0.2950 & 0.286 & 0.42 & \\
\hline Side Post & 6 & 62.9820 & 0.286 & 108.08 & \\
\hline Side Post Pegs & 6 & 0.3930 & 0.286 & 0.67 & \\
\hline Shroud & 1 & 74.6400 & 0.286 & 21.35 & \\
\hline Seal & 1 & 5.3160 & 0.286 & 1.52 & \\
\hline Dividers & 6 & 14.7320 & 0.286 & 25.28 & \\
\hline & & & Total Weight (lbs) & 412.33 & \\
\hline & & & & & \\
\hline Revision & & & 0 & & \\
\hline Prepared by/[ & & & $4 / 16 / 97$ & & \\
\hline Checked by/[ & & & $4 / 17 / 97$ & & \\
\hline
\end{tabular}




\begin{tabular}{|c|c|c|c|c|c|}
\hline \multicolumn{5}{|c|}{ Mark IV Storage Basket } & \\
\hline & & & & & \\
\hline & Quantity & Volume $\left(\mathrm{in}^{3}\right)$ & Density $\left(\mathrm{b} / \mathrm{in}^{3}\right)$ & Weight (lbs.) & \\
\hline 3 Inch Plate & 1 & 340.2820 & 0.286 & 97.32 & \\
\hline Ring & 1 & 31.1090 & 0.286 & 8.90 & \\
\hline Round Bar & 1 & 190.3510 & 0.286 & 54.44 & \\
\hline Center Post & 1. & 91.2240 & 0.286 & 26.09 & \\
\hline Sheet Metal & 1 & 37.4880 & 0.286 & 10.72 & \\
\hline \multirow[t]{3}{*}{ Flat Bar } & 1 & 6.8770 & 0.286 & 1.97 & \\
\hline & & & & & \\
\hline & & & Total Weight (lbs) & 199.44 & \\
\hline Revision & & & 0 & & \\
\hline \multicolumn{2}{|c|}{ Prepared by/Date } & & $4 / 16 / 97$ & & \\
\hline \multicolumn{2}{|c|}{ Checked by/Date } & & $4 / 17 / 97$ & & \\
\hline
\end{tabular}




\begin{tabular}{|c|c|c|c|c|c|}
\hline Mark IV Scrap Basket & & & & & \\
\hline & & & & & \\
\hline & Quantity & Volume $\left(\mathrm{in}^{3}\right)$ & Density $\left(\mathrm{tb} / \mathrm{in}^{3}\right)$ & Weight (lbs.) & \\
\hline Base Plate & 1 & 94.8590 & 0.286 & 27.13 & \\
\hline Center Tube & 1 & 91.3600 & 0.286 & 26.13 & \\
\hline Basket Divider & 1 & 171.1380 & 0.286 & 48.95 & \\
\hline Lower Collar & 1 & 10.1780 & 0.286 & 2.91 & \\
\hline Sheet Metal Item 10 & 1 & 199.0580 & 0.286 & 56.93 & \\
\hline Sheet Metal Item 11 & 1 & 6.4330 & 0.286 & 1.84 & \\
\hline Sheet Metal item 12 & 1 & 2.9510 & 0.286 & 0.84 & \\
\hline & & & & & \\
\hline & & & Total Weight (lbs) & 164.73 & \\
\hline & & & & & \\
\hline Revision & & & 0 & & \\
\hline Prepared by/L & & & $4 / 16 / 97$ & & \\
\hline Checked by/D & & 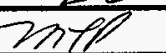 & $4 / 17 / 97$ & & \\
\hline
\end{tabular}


CLIENT: Duke Engineering \& Services Hanford

FILE NO: KH8009-8-01

PROJECT: MCO Final Design DOC. NO.: HNF-SD-SNF-DR-003, Rev. 0, Appendix 3

This page intentionally left blank. 


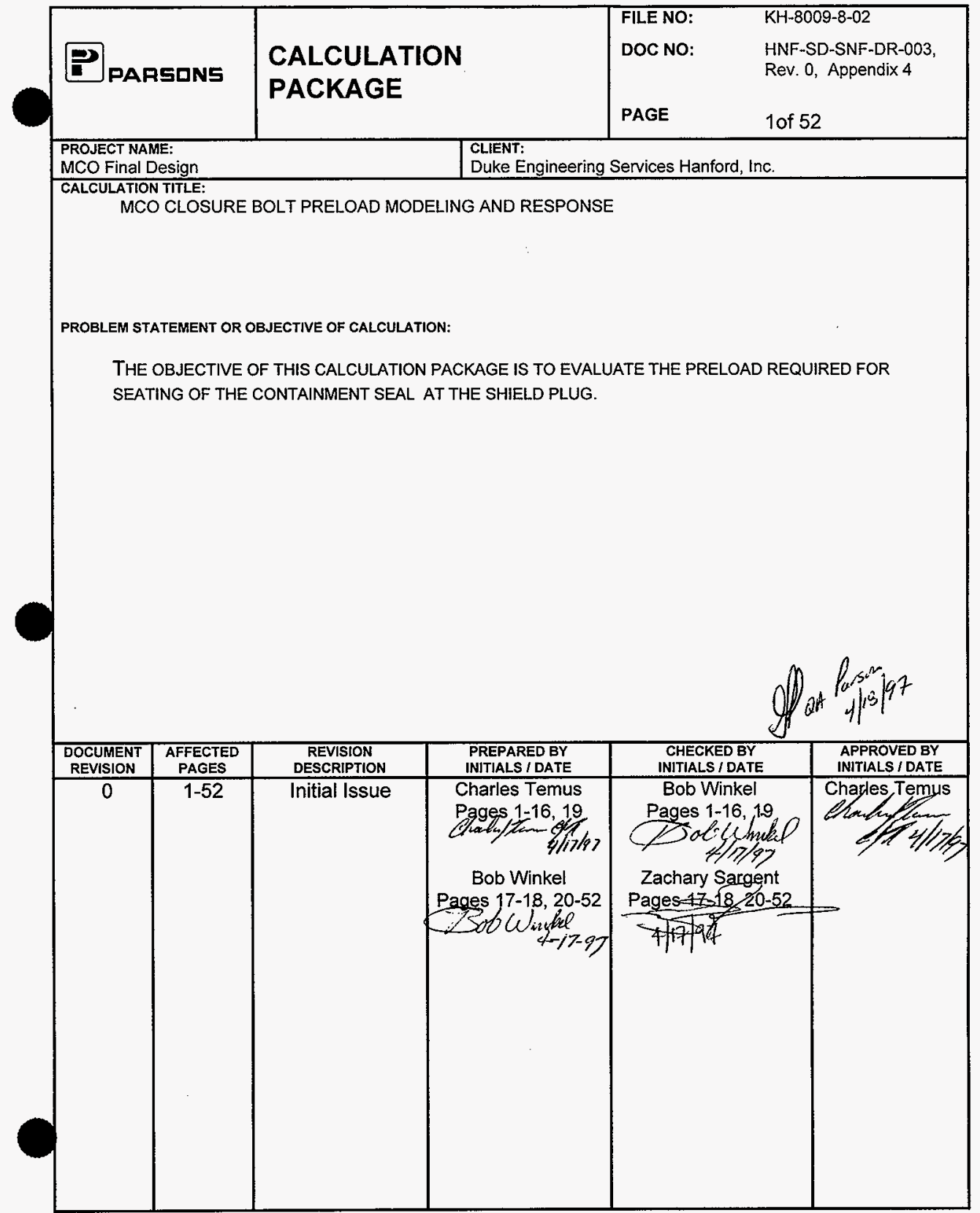


CLIENT: Duke Engineering Services Hanford

FILE NO: $\quad \mathrm{KH}-8009-8-02$

PROJECT: MCO Final Design Doc. No. HNF-SD-DR-003, Rev.0 Appendix 4 SNF

KES $5 / 23 / 97$

1. INTRODUCTION

2. REFERENCES

3. ASSUMPTIONS

4. MATERIAL PROPERTIES

5. METHOD OF ANALYSIS

5.1 Analysis Procedure

6. GEOMETRY

7. MATERIAL PROPERTIES

8. ACCEPTANCE CRITERIA

9. LOAD COMBINATIONS

10. ANALYSIS

11. SUMMARY

12. BOLT PRELOAD MODELING AND RESPONSE

12.1 Jacking Bolt / Seal Model Development

12.2 ANSYS Preload/Pressure Response

13. BOLT PRELOAD UNCERTAINTY 
CLIENT: Duke Engineering Services Hanford

FILE NO: $\mathrm{KH}-8009-8-02$

PROJECT: MCO Final Design Doc. No. HNF-SD-DR-003, Rev.0 Appendix 4 SNF KES $5 / 23 / 97$

\section{LIST OF TABLES}

TABLE 1: MATERIAL PROPERTIES FOR SA-182 AND SA-193

\section{APPENDICES}

APPENDIX A: COMPUTER RUN OUTPUT SHEETS \& INPUT FILE LISTINGS 
CLIENT: Duke Engineering Services Hanford

PROJECT: MCO Final Design

Foc. No. HNF-SD-DR-003, Rev.0 Appendix 4 $S N F$

kES $5 / 23 / 97$

\section{INTRODUCTION}

The MCO assembly is a single purpose Spent Nuclear Fuel (SNF) container that is capable of maintaining subcriticality at all times and maintain SNF containment and confinement after being closed and sealed. The MCO assembly consists of a shell, a shield plug and one to six SNF baskets. After the MCO is loaded with fuel, the shield plug is installed with the containment seal, the locking ring screwed down by means of double lead buttress threads, and the jacking bolts (hereinto refered as the 'bolts') are inserted in the locking ring. The bolts are then torqued to achieve the necessary preload to seat the seal.

The screws and the connecting components - the locking ring and the shield plug - are evaluated for the loads that are applied to the bolts. These loads consist of the preload, the torque uncertainty and the applied pressure loads.

\section{REFERENCES}

1. Bickford, John H., An Introduction to the Design and Behavior of Bolted Joints, $2^{\text {nd }}$ Edition, Marcel Dekker, Inc., New York, NY 1990.

2. Helicoflex Specification H-305236, Revision NC.

3. Helicoflex High Performance Sealing Data Brochure.

4. Industrial Fasteners Institute, Fastener Standards, $6^{\text {th }}$ Edition, Nova Machine Products Corp., Middleburg Heights, OH, 1988.

5. ASME Boiler and Pressure Vessel Code, Section II - Materials, Part D - Properties, 1995 Edition with 1995 Addenda.

6. ASME Boiler and Pressure Vessel Code, Section III, Division 1, Subsection NB Class 1 Components, 1995 Edition with 1995 Addenda and 1995 Appendix F.

\section{ASSUMPTIONS}

1. A design pressure $P$ of 150 psig is uniformly applied simultaneously with a design temperature of $375^{\circ} \mathrm{C}$. This is conservative relative to the maximum pressure/temperature combinations of $150 \mathrm{psi} / 200^{\circ} \mathrm{C}$ and $75 \mathrm{psi} / 375^{\circ} \mathrm{C}$.

2. All drop loads are carried through the shield plug to the shell.

Old ${ }^{4}$


CLIENT: Duke Engineering Services Hanford

FILE NO: $\mathrm{KH}-8009-8-02$

PROJECT: MCO Final Design

Doc. No. HNF-SD-DR-003, Rev.0 Appendix 4

SNF KES 5/23/97

3. Helicoflex preload seal requirement $P_{s s}$ of $1850 \mathrm{lb}$./in [2] Value has been modified to $1700 \mathrm{lb} / \mathrm{in}$ in the latest transmittal from Helicoflex. The use of $1850 \mathrm{lb} / \mathrm{in}$ is conservative for all calculations.

4. Diameter of the seal is $D_{s}=23.420-0.186=23.234$ inches [2]

5. Maximum design temperature is $375^{\circ} \mathrm{C}\left(707^{\circ} \mathrm{F}\right)$

\section{MATERIAL PROPERTIES}

The shield plug and locking ring are fabricated from Type $304 \mathrm{~L}$ stainless steel. The MCO shell and collar are fabricated from Type 304L stainless steel with the minimum yield and tensile strengths of Type 304 stainless steel ( 30 and $75 \mathrm{ksi}$, respectively) The bolts are fabricated from SA-193 Grade B8M.

\section{METHOD OF ANALYSIS}

The minimum preload for the bolts are determined by considering the pressure and the required compressive load to maintain the seal. Once the minimum preload is determined, the maximum preload is calculated based on the uncertainties of the torquing equipment, lubrication and friction of the bolts.

\subsection{Analysis Procedure}

a) Determine preload required to seat the seal.

b) Determine minimum compressive load required to maintain seal.

c) Calculate torque to seat the seal considering tool scatter, operator error and conversion error. Assume no relaxation or unloading due to the external load.

d) Check bolt load for preload plus external load.

e) Check maximum bolt load with preload with maximum scatter, plus external load, plus thermal load. Consider scatter from tool, operator, control, and external load.

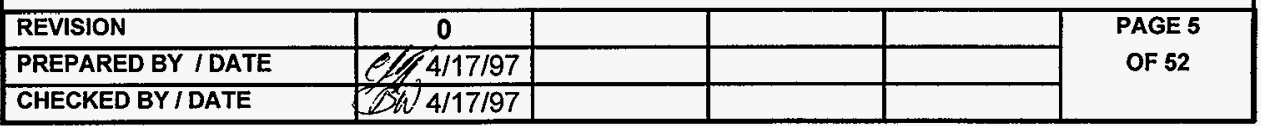


CLIENT: Duke Engineering Services Hanford

PROJECT: MCO Final Design Doc. No. HNF-SD-DR-003, Rev.0 Appendix 4

SNF KES 5/23/97

f) Check that minimum seal compression is maintained for a preload with maximum negative scatter, external pressure and thermal load. Also consider scatter, operator, control, relaxation and external load.

g) If minimum seal is not maintained, increase torque and recalculate.

h) Check maximum stress in the bolt, under the bolt and in the thread wall. Verify thread adequacy.

\section{GEOMETRY}

The geometry for this calculation is per Drawing H-2-828042 (MCO Mechanical Closure) and Drawing H-2-828045 (MCO Mechanical Closure Shield Plug).

Assuming the use of $181-1 / 2-8 \mathrm{UN}-2 \mathrm{~A}$ bolts, then:

The bolt diameter is

$$
D_{b}=1.50 \mathrm{in}^{2}
$$

Geometry of the bolt is per Reference 4, Section A, Table 1.

The stress area is

$$
A_{s}=1.49 \mathrm{in}^{2}
$$

and the thread root area is

$$
A_{r}=1.41 \mathrm{in}^{2}
$$

The thread stripping area for $2 A$ external thread, per inch of engagement is

$$
\mathrm{As}_{\mathrm{s}}=2.57 \mathrm{in}^{2} / \mathrm{in} \text {. }
$$

The thread stripping area for $2 \mathrm{~A}$ internal thread, per inch of engagement is

$$
A s_{n}=3.50 \mathrm{in}^{2} / \mathrm{in} \text {. }
$$

\begin{tabular}{|c|}
\hline 0 \\
\hline $\begin{array}{l}1 / 1 / 17 / 97 \\
1 / 17 / 97\end{array}$ \\
\hline
\end{tabular}

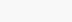


CLIENT: Duke Engineering Services Hanford

FILE NO: $\mathrm{KH}-8009-8-02$

PROJECT: MCO Final Design Doc. No. HNF-SD-DR-003, Rev.0 Appendix 4

SNF KES 5/23/97

\section{MATERIAL PROPERTIES}

The shield plug and locking ring are fabricated from SA-182 F304L forgings. The bolts are fabricated from SA-193 Grade B8M (S31600) material. The material properties are taken from [5] and are summarized in Table 1 . The loading temperature is $375^{\circ} \mathrm{C}\left(707^{\circ} \mathrm{F}\right)$.

Table 1: Material Properties for SA-182 and SA-193

\begin{tabular}{|c|c|c|c|c|}
\cline { 2 - 5 } \multicolumn{1}{c|}{} & \multicolumn{2}{c|}{ SA-182 $\mathrm{F304L}(\mathrm{psi})$} & \multicolumn{2}{c|}{ SA-193 B8M (S31600) (psi) } \\
\hline Component & $70^{\circ} \mathrm{F}$ & $707^{\circ} \mathrm{F}$ & $70^{\circ} \mathrm{F}$ & $707^{\circ} \mathrm{F}$ \\
\hline$E$ & $28.3 \times 10^{6}$ & $24.8 \times 10^{6}$ & $28.3 \times 10^{6}$ & $24.8 \times 10^{6}$ \\
\hline$S_{M}$ & -- & 13,500 & -- & - \\
\hline$S_{Y}$ & 25,000 & 14,900 & 30,000 & 18,058 \\
\hline$S_{U}$ & -- & 56,200 & 75,000 & -- \\
\hline$v$ & 0.3 & 0.3 & 0.3 & 0.3 \\
\hline$S_{M}$ & -- & -- & 10,000 & 5,993 \\
\hline
\end{tabular}

And from Table TM-1 of [5], the thermal expansion -from $70^{\circ} \mathrm{F}$ to $707^{\circ} \mathrm{F}-$ of the bolt is

$$
\begin{aligned}
& \Delta T=707-70=637^{\circ} \mathrm{F} \\
& \alpha_{B O L T}=9.76 \times 10^{-6} \mathrm{in} . / \mathrm{in} . /^{\circ} \mathrm{F} @ 707^{\circ} \mathrm{F} \\
& \alpha_{\mathrm{BOLT70}}=8.42 \times 10^{-6} \mathrm{in} . / \mathrm{in} . /^{\circ} \mathrm{F} @ 70^{\circ} \mathrm{F}
\end{aligned}
$$

therefore

$$
\varepsilon_{\mathrm{BOLT}}=\left(\alpha_{\mathrm{BOLT}}\right)(\Delta \mathrm{T})=6.217 \times 10^{-3} \mathrm{in} . / \mathrm{in} .
$$


CLIENT: Duke Engineering Services Hanford

FILE NO: KH-8009-8-02

PROJECT: MCO Final Design

Doc. No. HNF-SD-DR-003, Rev.0 Appendix 4

The thermal expansion for the shield plug is

$$
\begin{aligned}
& \Delta T=707-70=637^{\circ} \mathrm{F} \\
& \alpha_{\text {PLUG }}=9.69 \times 10^{-6} \mathrm{in} . / \mathrm{in} . /^{\circ} \mathrm{F} @ 707^{\circ} \mathrm{F} \\
& \alpha_{\text {PLUG70 }}=8.46 \times 10^{-6} \mathrm{in} . / \mathrm{in} . /^{\circ} \mathrm{F} @ 70^{\circ} \mathrm{F}
\end{aligned}
$$

therefore

$$
\varepsilon_{\text {PLUG }}=\left(\alpha_{\text {PLUG }}\right)(\Delta T)=6.173 \times 10^{-3} \text { in./in. }
$$

\section{ACCEPTANCE CRITERIA}

The minimum preload must be maintained at or above the preload required to maintain the seal. From Reference 3 , this minimum preload is $343 \mathrm{lb}$./in. for a $4.7 \mathrm{~mm}$ cross-sectional diameter with a silver coating.

Given $\quad P_{S M I N}=60 \mathrm{~N} \cdot \mathrm{mm}=342.6 \mathrm{lb} . / \mathrm{in} .=343 \mathrm{lb} . / \mathrm{in}$.

For all in-service combinations, including temperature cycling, the stress levels in the shield plug under the bolts, in the collar threads and in the locking ring threads should not exceed yield in order to avoid potential seal load losses. Plastic deformation of any of the components is permitted if the tight seal criteria of Appendix E of the ASME Code, Section III is met.

\section{LOAD COMBINATIONS}

Since the drop loads are taken directly through the shield plug to the shell, only those loads generated from the pressure and the preload required to maintain the seal will be transmitted through the bolts.

\begin{tabular}{|l|c|l|l|l|}
\hline REVISION & 0 & & & \\
\hline PREPARED BY IDATE & $4 / 17 / 97$ & & & \\
\hline CHECKED BY I DATE & SU $4 / 17 / 97$ & & & \\
\hline
\end{tabular}


CLIENT: Duke Engineering Services Hanford

FILE NO: $\mathrm{KH}-8009-8-02$

PROJECT: MCO Final Design

Doc. No. HNF-SD-DR-003, Rev.0 Appendix 4

SNF KES 5/23/97

\section{ANALYSIS}

The load generated by the internal design pressure is

$$
\mathrm{P}_{\mathrm{L}}=\frac{P D_{S}^{2}}{4}(\pi)=\frac{(150)(23.234)^{2}}{4}(\pi)=6.36 \times 10^{4} \mathrm{lb}
$$

The minimum load necessary to seat the seal is

$$
\begin{aligned}
& P_{M I N}=\left(D_{S}\right)\left(P_{S S}\right)(\pi)=(23.234 \mathrm{in} .)(1850 \mathrm{lb} . / \mathrm{in} .)(\pi) \\
& P_{M I N}=135,000 \mathrm{lb} . \text { and the minimum seal load to maintain the seal is } \\
& S L=\left(D_{S}\right)(\pi)\left(P_{S M I N}\right)=(23.234 \mathrm{in} .)(343 \mathrm{lb} . / \mathrm{in} .)(\pi) \\
& S L=2.501 \times 10^{4} \mathrm{lb} .
\end{aligned}
$$

The minimum total bolt load at closure temperature becomes

$$
\begin{aligned}
& L P_{M I N}=P_{L}+S L=6.36 \times 10^{4} \mathrm{lb}+2.501 \times 10^{4} \mathrm{lb} . \\
& L P_{M I N}=8.86 \times 10^{4} \mathrm{lb} .
\end{aligned}
$$

Checking the compliance with Subsection NB, NB-3231 [6], which states that the design mechanical loads calculated based on the equations of Appendix $E$ shall produce stresses less than the allowables from Section II, Part D, Table 4. The two equations address the forces required to seat the seal and the forces required to maintain the seal.

To seat the seal, the above equation shows $P_{\text {MiN }}=135,000 \mathrm{lb}$.

The force required to maintain the seal in a tight condition equals the pressure load $\left(P_{L}\right)$ plus the minimum sealing load $\left(L P_{\text {MIN }}\right)$. Since the seal seating load is larger, it controls.

Based on 18 bolts, the stress in the bolt is

$$
\begin{aligned}
& \sigma_{\mathrm{BOLT}}=\frac{\mathrm{P}_{\mathrm{MN}}}{18\left(\mathrm{~A}_{\mathrm{S}}\right)}=\frac{135,000 \mathrm{lb}}{18 \times\left(1.49 \mathrm{in}^{2}\right)} \\
& \sigma_{\mathrm{BOLT}}=5.035 \times 10^{3} \mathrm{psi}
\end{aligned}
$$

\section{REVISION}

PREPARED BY IDATE

\begin{tabular}{|c|l|l|l|}
\hline 0 & & & \\
\hline $1 / 4 / 17 / 97$ & & & \\
\hline $304 / 17 / 97$ & & & \\
\hline
\end{tabular}


CLIENT: Duke Engineering Services Hanford

FILE NO: $\quad \mathrm{KH}-8009-8-02$

PROJECT: MCO Final Design

Doc. No. HNF-SD-DR-003, Rev.0 Appendix 4

SNF KCES $5 / 23 / 97$

This is less than the bolt allowable at seating temperature $\left(\mathrm{S}_{\mathrm{M}} @ 70^{\circ} \mathrm{F}=1 \times 10^{4} \mathrm{psi}\right)$. It is also less than the allowable at maximum operating temperature $\left(S_{M} @ 707^{\circ} \mathrm{F}=5.993 \times 10^{3}\right.$ psi). Therefore the value of $\sigma_{\mathrm{BOLT}}$ is acceptable, and the margin of safety is

$$
\begin{aligned}
\mathrm{MS}_{\mathrm{BOLT}} & =\frac{5.993 \times 10^{3}}{\sigma_{B O L T}}-1 \\
\mathrm{MS}_{\mathrm{BOLT}} & =0.19
\end{aligned}
$$

Assuming the bolts are lubricated with a good grade lubricant, such as Never Seize, the variance due to the nut factor will be minimized (See [1], Table 5.1).

The variance in the applied preload can come from different sources. A suggested way of handling the variance in the preload due to the variance in torquing the bolts is set forth in [1].

Five variables are considered for determining the accuracy of the preload [1]. They are Tool Accuracy, Operator Accuracy, Control Accuracy, Short-term Relaxation and External Loads.

Tool Accuracy is the accuracy the tool reports when torquing. This involves repeatability as well as variance from the set value. Table 21.2 of [1] shows values of various torquing devices. Air impact wrenches have a value of $-100 \%$ to $+150 \%$ which is far beyond the tolerable range that the bolts can handle. Since this will be a remote operation with a gang of torque devices - which may be run hydraulically or pneumatically - some type of accurate control will be assumed to be built in. For calculational purposes, the value of $\pm 3 \%$ is used (reported accuracy of the tool to be used by the buyer). Hence

$$
V_{\text {TOOL }}=0.03
$$

Operator Accuracy relates to the accuracy determined by the set-up, calibration and application of the remote equipment to be used. It is assumed that built-in controls and checks will provide an accuracy of $\pm 2 \%$. Hence

$$
V_{O P}=0.02
$$

Control Accuracy is defined as the accuracy of what is controlled (i.e. torque..) and its ability to produce what is desired (i.e. bolt tension). This includes all the variables from the lubricants, bolt alignments, tool types and procedures. NUREG 6007 recommends a control accuracy value of $\pm 30 \%$ be applied. Assuming the bolts are torqued several times before the closure torque is applied, the bolts are carefully lubricated and a torquing

\begin{tabular}{|l|c|l|l|l|l|}
\hline REVISION & 0 & & & & PAGE 10 \\
OF 52 \\
PREPARED BY IDATE & CHEC $4 / 17 / 97$ & & & & \\
\hline
\end{tabular}




\section{PreAsDNS}

CLIENT: Duke Engineering Services Hanford

PROJECT: MCO Final Design
FILE NO: KH-8009-8-02

Doc. No. HNF-SD-DR-003, Rev.0 Appendix 4

procedure is developed with the appropriate equipment which will minimize scatter, a value of $\pm 15 \%$ is used. This value is based on what is expected by the buyer from testing similar bolts. Hence

$$
V_{c}=0.15
$$

The forth variable being considered is that of short-term relaxation. For simplicity, only the relaxation due to embedment is considered and is assumed to be $10 \%$. Since relaxation will only reduce the preload, there is no positive scatter. Hence

$$
V_{\text {STR }}=0.10 \quad \text { and } \quad V_{+S T R}=0
$$

The last variable considered is the effect of external forces such as applied loads and the effect of the joint due to the relative stiffness of the bolt to the parts being clamped. Since the jacking bolts are short and stiff relative to the joint, this variable is small. The following values are based on the results of applying an external (pressure) load to a finite element of the joint discussed in Section 12, hence

$$
V_{E X}=0 \quad \text { and } \quad V_{+E X}=0.061
$$

All the above variables can be combined to give the overall scatter.

For initial seating of the seal, there is no relaxation and no effects from the external loads. Therefore

$$
\begin{aligned}
& V_{\mathrm{TN}}=\sqrt{V_{T O O L}^{2}+V_{O P}^{2}+V_{C}^{2}} \\
& V_{\mathrm{TN}}=0.154
\end{aligned}
$$

The overall scatter of the load in the bolt and in sealing load is :

Negative scatter:

$$
\begin{aligned}
& V_{T N}=\sqrt{V_{T O O L}^{2}+V_{O P}^{2}+V_{C}^{2}+V_{S T R}^{2}+V_{E X}^{2}} \\
& V_{T N}=-0.206
\end{aligned}
$$

\begin{tabular}{|l|c|l|l|l|}
\hline REVISION & 0 & & & \\
\hline PREPARED BY I DATE & O $4 / 17 / 97$ & & & \\
\hline CHECKED BYI DATE & $34 / 4 / 17 / 97$ & & & \\
\hline
\end{tabular}


PROJECT: MCO Final Design

Doc. No. HNF-SD-DR-003, Rev.0 Appendix 4

Positive scatter:

$$
\begin{aligned}
& V_{\mathrm{TP}}=\sqrt{V_{T O O L}^{2}+V_{O P}^{2}+V_{C}^{2}+V_{+S T R}^{2}+V_{+E X}^{2}} \\
& \mathrm{~V}_{\mathrm{TP}}=0.166
\end{aligned}
$$

The nominal preload is then

$$
\begin{aligned}
& \mathrm{P}_{\text {NOM }}=\frac{P_{M N}}{\left(1-V_{T N I}\right)} \\
& \mathrm{P}_{\text {NOM }}=1.597 \times 10^{5} \mathrm{lb}
\end{aligned}
$$

And the maximum preload to seat the seal is

$$
\begin{aligned}
& P_{\text {MAX SS }}=P_{\text {NOM }}+\left(1+V_{T P}\right) \\
& P_{\text {MAX SS }}=1.862 \times 10^{5} \mathrm{lb} .
\end{aligned}
$$

With a nut factor $F=0.17$, the nominal torque to seat the seal becomes

$$
\begin{aligned}
& \mathrm{T}_{\text {NOM }}=\frac{F D_{B} P_{\text {NOM }}}{18} \\
& \mathrm{~T}_{\text {NOM }}=188.496 \mathrm{ft}-\mathrm{lb}
\end{aligned}
$$

Ensuring that the preload required to seat the seal is greater or less than the preload required to maintain the seal with the pressure load applied:

$$
\begin{aligned}
& \mathrm{P}_{\mathrm{REQ}}=\frac{L P_{M N N}}{\left(1+V_{T N}\right)} . \\
& \mathrm{P}_{\mathrm{REQ}}=1.115 \times 10^{5} \mathrm{lb} .
\end{aligned}
$$

The preload to seat the seal $\left(P_{\text {MAX ss }}\right)$ is greater than the preload required $\left(P_{\text {REQ }}\right)$ to maintain the seal with the pressure load applied, therefore the nominal preload used for calculating the stresses in the bolt and joint will be defined as needed to seat the seal. Therefore

\begin{tabular}{|l|c|l|l|l|}
\hline REVISION & 0 & & & \\
\hline PREPARED BY IDATE & O $/ 2 / 17 / 97$ & & & \\
\hline CHECKED BY I DATE & $\square / 4 / 17 / 97$ & & & \\
\hline
\end{tabular}


CLIENT: Duke Engineering Services Hanford

FILE NO: $\mathrm{KH}-8009-8-02$

PROJECT: MCO Final Design Doc. No. HNF-SD-DR-003, Rev.0 Appendix 4 SNF kES S/23/9?

$$
P_{\text {REQ }}=P_{\text {NOM }}=1.597 \times 10^{5} \mathrm{lb}
$$

The maximum preload in the bolt then becomes

$$
\begin{aligned}
& P_{\text {MAXB }}=P_{\text {REQ }}\left(1+V_{T P}\right) \\
& P_{\text {MAX B }}=1.862 \times 10^{5} \mathrm{lb}
\end{aligned}
$$

Checking joint heat up to $707^{\circ} \mathrm{F}$ :

Since the coefficient of thermal expansion is greater for the bolt, it will increase the preload as the MCO heats up. Therefore the increase will have to be added to the maximum preload. The only portion of the shield plug and locking ring that does not expand the same as the collar is the bolt.

The change in the total load from the differential strain can be calculated for the bolt.

$$
\begin{aligned}
& \Delta T=637^{\circ} \mathrm{F} \\
& P_{T H}=\left(A_{S}\right)\left(\left(\alpha_{B O L T}\right)(\Delta T)-\left(\alpha_{P L U G}\right)(\Delta T)\right)\left(24.8 \times 10^{6}\right)(18) \\
& P_{T H}=2.966 \times 10^{4} \mathrm{lb}
\end{aligned}
$$

The maximum load, including thermal strain, becomes

$$
\begin{aligned}
& P_{\text {MAX T }}=P_{\text {MAX }}+P_{T H} \\
& P_{\text {MAX T }}=2.158 \times 10^{5} \mathrm{lb}
\end{aligned}
$$

Then, the minimum force to maintain the seal becomes:

Consider the effect of cooling the joint to $32^{\circ} \mathrm{F}$ :

$$
\begin{aligned}
& \Delta t c=(70-32)=38^{\circ} \mathrm{F} \\
& \sigma_{B T C}=\left(\alpha_{\text {PLUG } 70}-\alpha_{\text {BOLT70 }}\right)(\Delta t c)\left(28.3 \times 10^{6}\right) \\
& \sigma_{\text {BTC }}=43.016 \mathrm{psi}
\end{aligned}
$$

Adding this minimal stress to the maximum expected bolt stress has no effect on the bolt to meet allowables.

\begin{tabular}{|l|c|l|l|l|}
\hline REVISION & 0 & & & \\
\hline PREPARED BY IDATE & $4 / 17 / 97$ & & & \\
\hline CHECKED BY / DATE & $S 4 / 4 / 17 / 97$ & & & \\
\hline
\end{tabular}


CLIENT: Duke Engineering Services Hanford

FILE NO: $\mathrm{KH}-8009-8-02$

PROJECT: MCO Final Design Doc. No. HNF-SD-DR-003, Rev.0 Appendix 4 SNF KES 5/23/97

$$
\begin{aligned}
& L P_{\text {THMIN }}=L P_{\text {MIN }}+\sigma_{\text {BTC }}\left(A_{S}\right)(18) \\
& L P_{\text {THMIN }}=8.975 \times 10^{4} \mathrm{lb}
\end{aligned}
$$

This is still less than the force required to seat the seal, hence the seating force controls.

The maximum bolt stress becomes

$$
\sigma_{\mathrm{BMAX}}=\frac{P_{\text {MAXT }}}{18\left(A_{S}\right)}=8.047 \times 10^{3} \mathrm{psi}
$$

The maximum load which would occur is compared to the allowable at the maximum operating pressure.

$$
\begin{aligned}
& \sigma_{B M A X A L L O W E D}=2 S_{M}=2(5993)=11986 \mathrm{psi} \\
& \sigma_{B M A X}<\sigma_{B M A X} \text { ALLOWED }
\end{aligned}
$$

Compressive capacity of the bolt

The allowable for all stresses in service per NB-3232.1 is $2 S_{M}$ at $707^{\circ} \mathrm{F}[6]$

$$
\begin{aligned}
& C_{B O L T}=A_{S}(2(5993)) \\
& C_{B O L T}=1.786 \times 10^{4} \mathrm{lb} .
\end{aligned}
$$

The compression capacity of the shield plug under the bolt is limited to the $304 \mathrm{~L}$ yield strength:

$$
\begin{aligned}
& C_{\text {PLUG }}=A_{R}(14900) \\
& C_{\text {PLUG }}=2.101 \times 10^{4} \mathrm{lb} .
\end{aligned}
$$

\section{Thread Engagement}

The allowable stress in the threads is governed by NB-3227.2 [6] which allow the thread stress to be equal to or less than $0.6 \mathrm{~S}_{M}$.

If the thread engagement length $L_{T H}=3.5$ in then the thread capacity is:

For the internal thread:

$$
T H_{C l}=\left(A s_{n}\right)\left(L_{T H}\right)(0.6)(13500)
$$

\section{REVISION}

PREPARED BY IDATE CHECKED BYI DATE

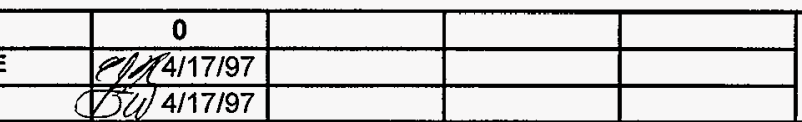


CLIENT: Duke Engineering Services Hanford

FILE NO: $\quad \mathrm{KH}-8009-8-02$

PROJECT: MCO Final Design

Doc. No. HNF-SQ-DR-003, Rev.0 Appendix 4

$$
\mathrm{TH}_{\mathrm{Cl}}=9.922 \times 10^{4} \mathrm{lb} \text {. }
$$

For the external thread:

$$
\begin{aligned}
& \mathrm{TH}_{\mathrm{CE}}=\left(A s_{S}\right)\left(\mathrm{L}_{\mathrm{TH}}\right)(0.6)(5993) \\
& \mathrm{TH}_{\mathrm{CE}}=3.234 \times 10^{4} \mathrm{lb} .
\end{aligned}
$$

The $2 S_{M}$ in-service stress limit of the bolt controls the serviceability of the joint. Hence the margin of safety is

$$
\mathrm{MS}_{\mathrm{JOINT}}=\frac{2 \times 5993}{\sigma_{B M A X}}-1=0.49
$$

The margin of safety for design of the bolts $\left(M S_{B O L T}=0.19\right)$ is smaller and controls the design of the joint.

The stress in the thinnest wall of the shell due to preload (tension only) is:

$$
\begin{aligned}
& T_{\text {WALL }}=0.354 \text { in }=\text { Thickness of the wall (Drawing H-2-828042) } \\
& D T_{\text {WALL }}=24.567 \text { in = Diameter at the thinnest part of shell } \\
& A T_{\text {WALL }}=(\pi)\left(D T_{\text {WALL }}\right)\left(\frac{T_{\text {WALL }}}{18}\right)=1.518 \text { in }^{2}
\end{aligned}
$$

and the stress in the thread at the thinnest part of the wall is based on adding the total pressure load to the shell area in addition to what is allocated to the max bolt load is:

$$
\begin{aligned}
\sigma_{\text {TWALL }} & =\frac{P_{L}+P_{\text {MAXX }}}{18\left(A T_{\text {WALL }}\right)} \\
\sigma_{\text {TWALL }} & =10.226 \times 10^{3} \mathrm{psi}
\end{aligned}
$$

This is less than both $S_{M}=13,500$ psi and $S_{Y}=14,900$ psi for the shell $\left(S_{M}\right.$ and $S_{Y}$ are conservative since the shell and collar are fabricated out of type $304 \mathrm{~L}$ stainless steel with minimum yield and tensile strengths of $30 \mathrm{ksi}$ and $75 \mathrm{ksi}$, respectively) and therefore is acceptable. Bending and combination of stresses are addressed in the detailed model and analysis of the MCO collar and buttress thread analysis (File No. KH-8009-8-04).

\begin{tabular}{|l|c|l|l|l|}
\hline REVISION & 0 & & & \\
\hline PREPARED BY IDATE & L $4 / 17 / 97$ & & & \\
\hline CHECKED BY I DATE & W 4/17/97 & & & \\
\hline
\end{tabular}


CLIENT: Duke Engineering Services Hanford

PROJECT: MCO Final Design Doc. No. HNF-SD-DR-003, Rev.0 Appendix 4 SNF KES $5 / 23 / 97$

\section{SUMMARY}

The joint and bolt are adequate to provide a minimum amount of preload required to both seat the seal and also maintain a tight seal during all loading events. The bolts meet both [6] and the Performance Specification criteria which specify that none of the joint components yield during the various conditions and that no leakage is to occur. This conclusion is based on the above calculation which takes a reasonable amount of uncertainty into consideration for the actual preloading based on torquing of the jacking bolts.

Post evaluation note: A subsequent detailed analysis of the thread region, reported in Section 10 of Appendix 6, predicts that bending stresses will exceed yield for the upper bound preload. However, associated preload losses are very small and the required seal load is maintained. 


\section{BOLT PRELOAD MODELING AND RESPONSE}

In order to evaluate the changes in bolt and MCO closure seal loading, the two-dimensional, axisymmetric model of the MCO shell (Appendix 5, Section 12), shield plug, locking ring and bolt circle is modified to focus on the jacking bolt and seal response. The following summarizes the model details and the bolt seal response to maximum and minimum preload conditions.

\subsection{Jacking Bolt / Seal Model Development}

In order to meet the seal manufacturer's specifications [3], a minimum seal preload of $135,000 \mathrm{lb}$. must be applied to seat the seal. This is the minimum initial bolt preload value. Accounting for uncertainties in the preload application, the maximum preload is estimated to be $215,800 \mathrm{lb}$.

The bolt preload is applied using CONTAC12 gap elements between the bolt tip and the top of the shield plug. To achieve the desired preload, and appropriate gap element interference is iteratively selected. As a two-dimensional model, the bolts are modeled as an equivalent ring, having the same area as the $181-1 / 2$ inch bolts. The stress area of the equivalent bolt is $1.49 \mathrm{in}^{2}$. Therefore, the area of the equivalent ring is:

$$
A_{\text {ring }}=18\left(1.49 \mathrm{in}^{2}\right)=26.82 \mathrm{in}^{2}
$$

Using a bolt circle diameter of 21.75 inches, the bolt ring thickness is:

$$
\mathrm{T}_{\mathrm{BR}}=\frac{A_{\text {ring }}}{\pi(21.75)}=0.393 \text { inches }
$$

A spring constant is selected for the ANSYS gap elements to model the seal response. The HelicoFlex seal response is nonlinear, but per page 24 of the brochure [3], the response is close to linear, once the seating load, $Y_{2}$, is achieved. The seal elastic spring constant is estimated as

$$
\mathrm{k}_{\mathrm{s}}=\frac{Y_{C}-Y_{2}}{e_{C}-e_{2}}=\frac{5710-1827}{0.0433-0.0315}=329,100 \mathrm{lb} . / \mathrm{in} / \mathrm{in}
$$

For the full cross-section

$$
\begin{aligned}
& K_{S}=\pi k_{s} D_{\text {seal }} \quad \text { where } D_{\text {seal }}=\text { Seal Diameter }=23.44 \text { inches } \\
& K_{S}=2.42 \times 10^{6} \mathrm{lb} / \mathrm{in}
\end{aligned}
$$

\begin{tabular}{|l|c|l|l|l|}
\hline REVISION & 0 & & & \\
\hline PREPARED BY I DATE COK 4/17/97 & & & \\
\hline CHECKED BY I DATE & ZZ 4/17/97 & & & \\
\hline
\end{tabular}


CLIENT: Duke Engineering Services Hanford

PROJECT: MCO Final Design Doc. No. HNF-SD-DR-003, Rev.0 Appendix 4 SNF KES S/23/97

The seal spring element is input as initially closed and is placed adjacent to and parallel to the gap elements under the seal stop. The seal stop gap is iteratively selected to very narrowly close when the minimum preload of $135,000 \mathrm{lb}$. is achieved.

\subsection{ANSYS Preload/Pressure Response}

Both minimum and maximum preloads are evaluated with the ANSYS computer analysis. Two load steps are utilized for each run: 1) preload alone, and 2) preload plus design pressure of 150 psig. The ANSYS input and output files are MINBOLT.inp \& MXBOLT.inp and MINBOLT.out \& MXBOLT.out, respectively. The bolt stress and seal load results are summarized in Table 2.

Table 2: Bolt Stress and Seal load Results

\begin{tabular}{|l|c|c|c|c|c|c|}
\cline { 2 - 7 } \multicolumn{1}{c|}{} & \multicolumn{4}{c|}{ Bolt Stress (psi) } & \multicolumn{3}{c|}{ Seal Load (lb) } \\
\hline ANSYS Run & Preload & $\begin{array}{c}\text { Preload } \\
+ \text { Pressure }\end{array}$ & $\%$ Change & Preload & $\begin{array}{l}\text { Preload + } \\
\text { Pressure }\end{array}$ & $\%$ Change \\
\hline Minbolt & 4918 & 6724 & +36.7 & 133,000 & 120,730 & -9.2 \\
\hline Maxbolt & 7005 & 7429 & +6.1 & 135,200 & 134,300 & -0.6 \\
\hline
\end{tabular}

The change in the maximum bolt stress due to the addition of the pressure load is consistent with the values used in determining maximum bolt stress.

\begin{tabular}{|l|c|l|l|l|}
\hline REVISION & 0 & & & \\
\hline PREPARED BY I DATE & J $2 / 4 / 17 / 97$ & & & \\
\hline CHECKED BY I DATE & 76 $6 / 17 / 97$ & & & \\
\hline
\end{tabular}


CLIENT: Duke Engineering Services Hanford

FILE NO: KH-8009-8-02

PROJECT: MCO Final Design

Doc. No. HNF-SQ-DR-003, Rev.0 Appendix 4

SNF KES S/23/97

\section{BOLT PRELOAD UNCERTAINTY}

The analysis uses five different uncertainties associated with developing the preload of the bolts/set screws by torquing. These uncertainties are used in the analysis to demonstrate some of the different parameters that influence obtaining the correct preload on the bolts. The values used are not bounding values in any sense. It is left up to the user to develop procedures and tooling that will deliver the required preload in a repetitive manner. Some of the things, other than tooling and technique, that can be done to obtain repetitive and accurate preloading is to use a good quality lubricant and procedures that get uniform and repetitive application on the bolts. Pretorquing of the bolts is also important so that the threads of both the bolts and the locking ring are slightly work hardened and any manufacturing imperfections are smoothed over so that the relationship between preload and torque is more consistent. This can easily be done by using the bolt and locking ring of each individual assembly in the hydrostatic testing of the unit rather than a test assembly.

It is highly recommended that a test program be undertaken to develop the relationship between torque and preload for the specific lubricant, equipment, procedures, and environment for the MCO. A basic program will minimize the uncertainty and potential problems when the units are put into production. The test program will also help in qualifying the seals by ensuring the proper preload, since the seals are not to be tested during production.

\begin{tabular}{|c|c|c|c|}
\hline REVISION & \multicolumn{3}{|l|}{0} \\
\hline PREPARED BY / DATE & $41 / 4 / 17 / 97$ & & \\
\hline CHECKED BY I DATE & $\sqrt{126} 4 / 17 / 97$ & & \\
\hline
\end{tabular}


CLIENT: Duke Engineering Services Hanford

FILE NO: $\mathrm{KH}-8009-8-02$

PROJECT: MCO Final Design Doc. No. HNF-SQ-DR-003, Rev.0 Appendix 4 SNF UES $5 / 23 / 91$

\section{APPENDIX A:}

\section{Computer Run Output Sheets}

\&

Input File Listings

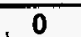


CLIENT: Duke Engineering Services Hanford

FILE NO: $\mathrm{KH}-8009-8-02$

PROJECT: MCO Final Design Doc. No. HNF-SD-DR-003, Rev.0 Appendix 4 SNF KES 5/23/91

\section{COMPUTER RUN COVER SHEET}

Project Number:

Computer Code:

Software Version:

Computer System:

Computer Run File Number:

Unique Computer Run Filename:

Run Description:

Creation Date / Time:
$\mathrm{KH}-8009-8$

ANSYS $\otimes-P C$

$5.0 \mathrm{~A}$

MS-DOS, Pentium® Processor

$\mathrm{KH}-8009-8-02$

MINBOLT.inp

Analysis of MCO Closure Response, Minimum Bolt Load.

26 February 1997 10:46:12 AM
Mabu uines

Prepared By: Bob V. Winkel
Date
Checked By: Zachary G. Sargent
4).79.7

\section{Date}

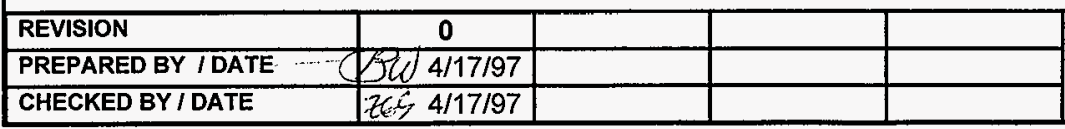


CLIENT: Duke Engineering Services Hanford

FILE NO: $\mathrm{KH}-8009-8-02$

PROJECT: MCO Final Design Doc. No. HNF-SD-DR-003, Rev.0 Appendix 4 SNF VES S/23197

\title{
LISTING OF MINBOLT.INP FILE
}

IBATCH,LIST

/FILENAM,minbolt

/PREP7

IIITLE,MCO DESIGN- 132 DEGREES C, 150 psi PRESSURE,

TREF,70

TUNIF,270

ETAN $=0.05$

! Tangent modulus

\author{
ICOM ** ELEMENT TYPES \#\#* \\ ET, 1,42,.,1 ! Shell \\ ET, $2,42,, 1$ ! Shield Plug \\ ET, 3,42,.,1 ! Lifting \& Locking Ring \\ ET,4,12 ! Gap Elements Between Shield Plug \& Shell
}

KEYOPT, $4,7,1$

ET, $5,42,, 1$ ! Bolt

ICOM ** REAL CONSTANTS FOR GAP ELEMENTS

R,4,-90,1.0e9,-0.06,3.0 I Shell/Shield Plug, Initially Open .06"

R,5,0,1.0e9, .0076 ! Gap Elements Under Bolt, Min. Preload Interference

$R, 6,0,1.009,-.009,2.0 \quad$ ! Sealing Stop, initially open, gap adjusted for max. stiffness

$R, 7,0,1.0 \mathrm{e} 9,0,1.0 \quad$ ! Bottom MCO Plate, closed

$r, 8,0,2.42 \mathrm{e} 7,2.0 \quad$ ! Seal Spring, max. stiffness (unloading stiffness)

ICOM

MP,DENS, 1,490/1728 ! 304L SS

MP,NUXY, 1,0.3

MP,DENS, $5,490 / 1728 \quad$ ! SA193 Grade B8M

MP,NUXY $, 5,0.3$

/COM DEFINING TEMPERATURES FOR MPDATA

MPTEMP, $1,70,100,200,300,400,500$

MPTEMP $, 7,600,650,700,750$

ICOM DEFINING ELASTIC MODULI FOR 304L \& SA-193 \#

MPDATA,EX, $1,1,28.3 e+06,28.1 e+06,27.6 e+06,27.0 e+06,26.5 e+06,25.8 e+06$

MPDATA,EX, $1,7,25.3 e+06,25.1 e+06,24.8 e+06,24.5 e+06$

ICOM ISA-193

MPDATA,EX,5,1,28.3e+06,28.1e+06,27.6e+06,27.0e+06,26.5e+06,25.8e+06

MPDATA,EX,5,7,25.3e+06,25.1e+06,24.8e+06,24.5e+06

ICOM INSTANTANEOUS COEFFICIENTS OF THERMAL EXPANSION, 304L \& SA-193

MPDATA,ALPX, 1,1,8.46e-06,8.63e-06,9.08e-06,9.46e-06,9.80e-06,10.10e-06

MPDATA,ALPX, 1,7,10.38e-06,10.50e-06,10.60e-06,10.70e-06

ICOM ! SA-193

MPDATA,ALPX,5,1,8.42e-06,8.59e-06,9.09e-06,9.56e-06,9.95e-06,10.25e-06

MPDATA,ALPX,5,7,10.51e-06,10.64e-06,10.76e-06,10.87e-06

ICOM SHELL GEOMETRY

IR=11.5 ! Internal Shell Radius @ Bottom

OR=12.000 ! Shell Outside Radius @ Bottom

IR2 $=12.02 \quad$ ! Inside Radius at Collar Sealing Surface

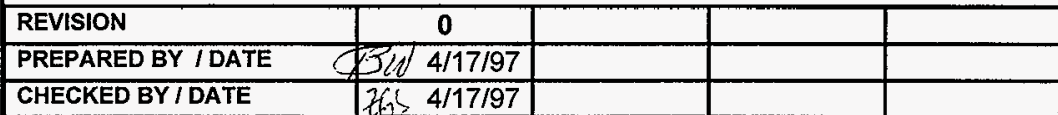


CLIENT: Duke Engineering Services Hanford

PROJECT: MCO Final Design Doc. No. HNF-SD-DR-003, Rev.0 Appendix 4

$\mathrm{OR} 2=12.625$

! Outside Radius at Collar Sealing Surface

$\operatorname{IR} 3=12.25$ ! Inside Radius at Collar-Lifting Ring Weld

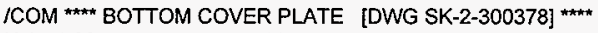

$\mathrm{N}, 1,-1.32$ ! Row 1

$\mathrm{N}, 2,1.25,-1.32$

$N, 3,2.13,-1.32$

$\mathrm{N}, 10,11,423,-1.32$

FILL

$\mathrm{N}, 41,0.00,-0.44$

! Row 3

$N, 42,1.25,-0.44$

$\mathrm{N}, 43,2.13,0.44$

$\mathrm{N}, 50, \mathrm{IR}, 0.44$

FILL, 43,50

$\mathrm{N}, 52, \mathrm{OR}, 0.44$

F!LL, 50,52

FILL, $1,41,1,21,1,10 \quad$ ! Middle Row

FILL, $10,50,1,30$

$\mathrm{N}, 32,12,-0.32$

FILL, 30,32

FILL, 10,32,1,11

$\mathrm{N}, 53, \mathrm{IR}, 1.17$

$\mathrm{N}, 55, \mathrm{OR}, 1.17$ ! Shell Stub Weld

FILL, 53,55

ICOM ** SHELL [DWGS SK-2-300379 \& SK-2-300461]

$\mathrm{N}, 65, \mathrm{IR}, 6.68$

$\mathrm{N}, 67, \mathrm{OR}, 6.68$

FILL.

FILL, 53,65,3,3,3,1

/COM SINGLE ROW SHELL *

$\mathrm{N}, 100, I R, 7.18$

! Inside

$\mathrm{N}, 140, \mathrm{IR}, 71.68$

$\mathrm{N}, 180, \mathrm{IR}, 136.68$

N,101,OR,7.18 IOutside

$N, 141, \mathrm{OR}, 71.68$

$\mathrm{N}, 181, \mathrm{OR}, 136.68$

FILL, $100,140,20,2,2,1,2.0$

FILL, $140,180,19,2,2,1,5$

/COM *** DOUBLE ROW SHELL ***

N,190,IR,137.18 ITransition to Double Row

$\mathrm{N}, 192, \mathrm{OR}, 137.18$

FILL

ICOM *** BASE OF CASK THROAT--ELEVATION: 138 INCHES ***

$\mathrm{N}, 217, \mathrm{IR}, 142.68$

! Transition to Double Row

$N, 219, O R, 142.68$

FILL

FILL, 190,217,8,3,3,1 IVertical Fill

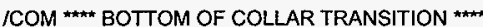

\begin{tabular}{|l|c|l|l|l|}
\hline REVISION & 0 & & & \\
\hline PREPARED BY I DATE & & & & \\
\hline CHECKED BY I DATE & $\mathrm{f} / 1 / 17 / 97$ & & & \\
\hline
\end{tabular}

PAGE 23

OF 52 
CLIENT: Duke Engineering Services Hanford

PROJECT: MCO Final Design

Doc. No. HNF-SD-DR-003, Rev.0 Appendix 4

$\mathrm{N}, 235, \mathrm{IR}, 146.06$

N,237,OR, 146.06

FILL

$\mathrm{N}, 238, \mathrm{IR}, 146.68$

N,240,OR, 146.68

FILL

FILL,217,235,5,3,3,1 ! Vertical Fi!l

/COM TOP OF COLLAR TRANSITION

$\mathrm{N}, 241, \mathrm{IR}, 147.31$

N,243,OR,147.31

FILL

NGEN, $2,3,241,243,1,0.75$

! End of Transition to Large O.D \&

! Assumed Location of Shield Plug Taper

! Horizontal Fill

! Start of Transition to Large O.D \&

! Assumed Location of Shield Plug Taper

/COM ${ }^{* \star \star}$ COLLAR SEALING SURFACE ****

$N, 247, I R, 149.63$

$N, 249, I R 2,149.63$

FIL.L.

! Inside Radius of Sealing Surface

! Outside Radius at Sealing Surface

! Horizontal Fill

ICOM THICK WALL AT COLLAR TRANSITION

NGEN,2, 10,240,249,3

$N, 255$, OR2, 147.31

! Nodes 250-259 Coincident w/240-249 (by 3)

$N, 261$, OR2, 149.63

! Outside Surface

$N, 258$, OR2, 148.06

! Outside Surface

$\mathrm{N}, 980, \mathrm{IR}, 149.38$

N,981,11.755, 149.38

N,982,IR2,149.38

N,983,12.317, 149.38

N,984,OR2,149.38

N,990,OR2,146.68

FILL,240,990,1,251

NGEN, $2,5,980,984,1,-0.66$

FILL, 246,258,1,257

FILL, 253,255,1, 1,3,3

FILL,237,990,1,991

/COM ${ }^{\star \star \star \star}$ COLLAR AT BOTTOM EDGE OF PLUG (.155" above Sealing Surface) ${ }^{\star \star \star \star}$

NGEN, $2,3,259_{1},, 0.245 \quad$ ! Nodes 262

ICOM * COLLAR AT TOP EDGE OF PLUG (2" above bottom Edge)

NGEN, $2,9,262, \ldots, 2.00 \quad$ ! Nodes 271

FILL,262,271,2

ICOM

$\mathrm{N}, 274, \mathrm{IR} 3,152.00$

$\mathrm{N}, 1000, \mathrm{IR} 2,152.00$

/COM TOP TO COLLAR (WELD CLOSURE) **

$\mathrm{N}, 295, \mathrm{IR} 3,156.00$

FILL, 274,295

NGEN, 3, 1,259,295,3,(OR2-IR2)/2

NGEN,3,1,274,295,3,(OR2-IR3)/2

ICOM LOCKING \& LIFTING RING GEOMETRY

RING1=7.94

RING2 $=9.375$

\begin{tabular}{|l|c|l|l|l|}
\hline REVISION & 0 & & & \\
\hline PREPARED BY I DATE & KU 4/17/97 & & & \\
\hline CHECKED BY I DATE & Jh $4 / 17 / 97$ & & & \\
\hline
\end{tabular}

PAGE 24

OF 52 
CLIENT: Duke Engineering Services Hanford

PROJECT: MCO Final Design

FILE NO: $\quad \mathrm{KH}-8009-8-02$ Doc. No. HNF-SD-DR-003, Rev.0 Appendix 4 SNF KES 5/23/97

RING3 $=9.625$

RING4 $=10.19$

RING5 $=12.23$

LOCAL, $11,0,152.00$ ! Local System $z=0$ at Base of Ring

CSYS, 11

/COM *** TOP EDGE

$\mathrm{N}, 401, \mathrm{RING} 1,6.13$

CSYS,O

$\mathrm{N}, 404,9.375,158.13$

FILL,401,404,.,1

$\mathrm{N}, 406, \mathrm{RING} 4,158.13$

FILL,404,406,,1

! Top Edge

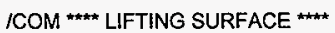

CSYS, 11

$\mathrm{N}, 421$, RING1,5.13

$\mathrm{N}, 424, \mathrm{RING} 2,5.13$

FILL, 421,424

$\mathrm{N}, 426, \mathrm{RING} 4,5.13$

FILL, 424,426

FILL,401,421,1,10,6,1

N,431, RING1,6.13-1.56

$\mathrm{N}, 434, \mathrm{RING2,6.13-1.56}$

FILL

ICOM *** BOLTING SURFACE ****

$\mathrm{N}, 441$, RING1,4

N,444,RING3,4

FILL

N,445,10.875-.197,4 ! Inside Edge of Bolt Hole

$\mathrm{N}, 447,10.875+.197,4 \quad$ ! Outside Edge of Bolt Hole

FILL

$\mathrm{N}, 910,10,875-.197,4$

$\mathrm{N}, 911,10.9375+.197,4$

N,448,RING5,4 IO.D of Ring

CSYS, 0 ! Bolt Extension

N,924,10.875-197,152.00 ! Double Nodes @ Bolt for Gap elements

$\mathrm{N}, 925,10.875+.197,152.00$

FILL, $910,924,6,2$

FILL, $911,925,6,2$

N,525,10.875-.197, 151.874 ! Bottom of Bolt Extension

$N, 527,10.875+.197,151.874$

FILL

/COM **** BOTTOM OF LIFTING/LOCKING RING ***

CSYS, 11

NGEN $, 2,70,441,448,1,-4$ ! Bottom Surface of Lifting/Locking Ring

FILL $, 441,511,6,10,8,1 \quad$ ! Fill in Lifting/Locking Ring

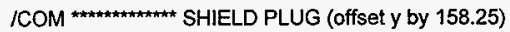

LOCAL, 20,0,,158.13

TYPE, 2

PLUGR1 $=11.975$

PLUGR2 $=11.45$

PLUGR3=11.25

\begin{tabular}{|c|c|c|c|}
\hline REVISION & 0 & & \\
\hline PREPARED BY / DATE & $(7 \bar{y} / 4 / 17 / 97$ & & \\
\hline CHECKED BY I DATE & 764 4/17/97 & & \\
\hline
\end{tabular}

PAGE 25

OF 52 
CLIENT: Duke Engineering Services Hanford

PROJECT: MCO Final Design Doc. No. HNF-SD-DR-003, Rev.0 Appendix 4

PLUGR4=7.89

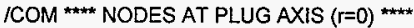

$\mathrm{N}, 601$

N, $602,0,-1$

$\mathrm{N}, 603,0,-1.994$

$\mathrm{N}, 606,0,-4,994$

FILL, 603,606,2,604

$\mathrm{N}, 607,0,-6.25$

$\mathrm{N}, 610,0,-8.25$

FILL, $607,610,2,608$

$\mathrm{N}, 611,0,-8.75$

$\mathrm{N}, 613,0,-10.5$

FILL,611,613

ICOM NODAL GENERATION

NGEN, $2,20,601,613,1,0.8825$

NGEN, $2,20,621,633,1,0.8825$

NGEN, $2,20,642,653,1,0.6875$

NGEN $, 2,20,662,673,1,0.6875$

NGEN, 2,20,683,693, 1, 0.4235

NGEN, $2,10,706,713,1,0.9515$

! Id Large Opening

I Id Medium Opening

! Id Small Opening

! Center of Opening

! Od Small Opening

$\mathrm{N}, 736,5.4665,-4.994$

FILL, 730,736,5,731

$\mathrm{N}, 737,5.4665,-6.25$

N, $740,5.4665,-8.25$

FILL, 737,740,2,738

N.741,5.4665,-8.75

$\mathrm{N}, 743,5.4665,-10.5$

FILL, 741,743

$N, 748,5.89,-1.0$

NGEN, $2,20,730,743,1,0.4235$

FILL, 748,750

$\mathrm{N}, 766,7.265,0$

NGEN, $2,20,748,763,1,1.375$

FILL, 766,768

NGEN, $3,20,766,768,1,0.3125$

$N, 789,7.5775,-1.56$

$\mathrm{N}, 796,7.5775,-5.56$

FILL, 789,796,6

NGEN, $2,20,789,796,1,0.3125$

NGEN, $3,20,777,783,1,0.3125$

/COM * UNDER LOCKING RING *

$\mathrm{N}, 824,8.5017,-6.25$

$\mathrm{N}, 827,8.5017,-8.25$

FILL

$\mathrm{N}, 828,8.5017,-8.75$

$\mathrm{N}, 830,8.5017,-10.5$

FILL

NGEN, $3,7,824,830,1,0.5616$

NGEN, $2,7,838,844,1,0.625$

NGEN, $2,7,845,851,1,0.625$

$\mathrm{N}, 859,10.875+.197,-6.25$

! Under Bolt

REVISION

PREPARED BY I DATE

CHECKED BY IDATE

Under Boit 


\section{Pingans}

CLIENT: Duke Engineering Services Hanford

PROJECT: MCO Final Design
FILE NO: KH-8009-8-02
Doc. No. HNF-SD-DR-003, Rev.0 Appendix 4

\footnotetext{
$\mathrm{N}, 860,10.875+.197,-6.917$

$\mathrm{N}, 861,10.875+.197,-7.584$

$\mathrm{N}, 862, \mathrm{PLUGR} 2,-8.25$

$\mathrm{N}, 863, \mathrm{PLUGR} 2,-8.75$

$\mathrm{N}, 865$, PLUGR3,-10.5

FILL,863,865, 1

$\mathrm{N}, 866, P L U G R 1-0.288,-6.25$

N,869,PLUGR1-0.288,-8.25

FILL,866,869,2

N,870,PLUGR1-0.288,-8.476

NGEN, $2,5,866,870,1,0.288$
}

/COM REFINING LIFTING EAR

CSYS, 0

$\mathrm{N}, 877,9.53,158.13$

$\mathrm{N}, 889,9.53,157.63$

$\mathrm{N}, 901,9.53,157.13$

FILL,403,404,1,876

FILL,413,414,1,888

FILL,423,424,1,900

FILL, $877,405,1,878$

FILL,405,406,2,879,1

FILL, $889,415,1,890$

FILL, 415,416,2,891,1

FILL,404,414,1,881

FILL, $877,889,1,882$

FILL, $878,890,1,883$

FILL, 405,415,1,884

FILL, $879,891,1,885$

FILL,880,892,1,886

FILL,406,416, 1,887

FILL,889,901,1,894

FILL,414,424,1,893

FILL,901,425, 1,902

FILL,890,902,1,895

FILL,415,425, 1,896

FILL,425,426,2,903,1

FILL,891,903,1,897

FILL,892,904,1,898

FILL,416,426,1,899

FILL,424,434,1,907

FILL,433,434,1,908

FILL,423,433,1,905

FILL,905,907

ICOM FILTER GUARD PLATE

PLATE $1=0.273$

PLATE2 $=0.6575$

PLATE $3=1.357$

PLATE4 $=10.25$

PLATE5 $=11.25$

N, 1200,PLATE4, 146.78

N,1202,PLATE5, 146.78

FILL

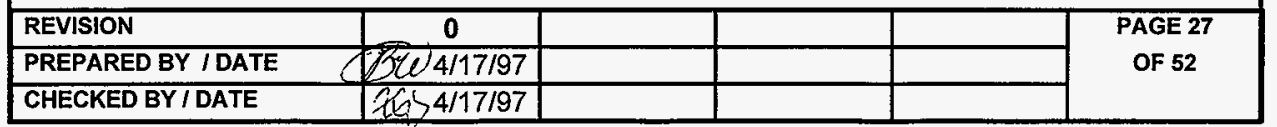


CLIENT: Duke Engineering Services Hanford

FILE NO: KH-8009-8-02

PROJECT: MCO Final Design Doc. No. HNF-SD-DR-003, Rev.0 Appendix 4 SNF KE $5 / 23 / 91$

NGEN,5,3,1200,1202,,,- -0.85

NGEN,2,3,1212,1214,,,-0.25

N.1221,PLATE4,141.88

N.1222, 10.75,141.88

$N, 1223,10.915,141.88$

FILL, 1215, 1221,1,1218

FILL, 1223, 1217,1,1220

FILL, 1216,1222, 1,1219

N, 1237,6.4375, 143.38

FILL,1212,1237,3,1225,4

N. 1249, 3.578, 143.38

FILL, 1237,1249,2,1241,4

NGEN, $2,1,1225,1249,4,,-0.25$

NGEN,2,2,1226, 1250,4, ,-1.25

FILL, 1226, 1228,1,1227, ,7,4

$N, 1253,2.625,145.255$

$N, 1254,2.625,145.005$

$\mathrm{N}, 1256,2.625,143.38$

FILL, 1254,1256

$\mathrm{N}, 1257,2.625,143.13$

$\mathrm{N}, 1259,2.625,141.88$

FILL, 1257,1259

NGEN, $2,10,1253,1259,1,-0.5$

NGEN, $2,10,1263,1269,1,-0.768$

$\mathrm{N}, 1283,0.6575,145.255$

$\mathrm{N}, 1284,0.6575,145.005$

$\mathrm{N}, 1260,2.125,147.63$

$\mathrm{N}, 1270,1,357,147.63$

$\mathrm{N}, 1280,0.6575,147.63$

$\mathrm{N}, 1290,0.273,147.63$

NGEN, $3,1,1260,1290,10,-0.5625$

ICOM NODES AT BOTTOM GAP ELEMENTS

NGEN,2,2000,1,10,1, -1.00

1 COM **** COUPLING NODES ****

/COM BETWEEN LIFTING/LOCKING RING \& SHELL

CP, 1,UY,508,277

! Start Threads

$C P, 2, U Y, 498,280$

$C P, 3, U Y, 488,283$

$\mathrm{CP}, 4, \mathrm{UY}, 478,286$

CP, $5, U Y, 468,289$

$C P, 6, U Y, 458,292$

ICOM BETWEEN BOLT \& LOCKING RING \#

$\mathrm{CP}, 7, \mathrm{UY}, 445,910$

$C P, 8, U X, 445,910$

$\mathrm{CP}, 9, \mathrm{UY}, \mathbf{4 4 7 , 9 1 1}$

$\mathrm{CP}, 10, \mathrm{UX}, 447,911$

*DO, $, 1,1,7$

CP, $10+1, U Y, 445+10^{*}[, 910+2 *]$

*ENDDO

${ }^{\star} \mathrm{DO}, \mathrm{I}, 1,7$

CP,17+1,UY,447+10*I,911+2*I

*ENDDO

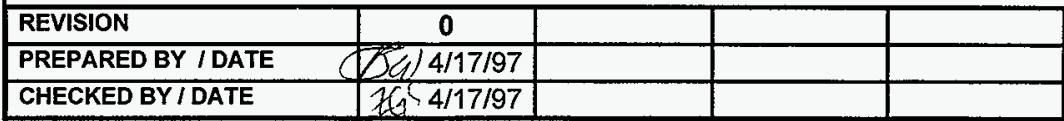

PAGE 28

OF 52 
CLIENT: Duke Engineering Services Hanford

PROJECT: MCO Final Design

FILE NO: $\quad \mathrm{KH}-8009-8-02$

Doc. No. HNF-SD-DR-003, Rev.0 Appendix 4 SNF kes 5/23/97

*DO,I,1,7

$\mathrm{CP}, 24+1, \mathrm{UX}, 445+10^{*} \mid, 910+2 * !$

*ENDDO

*DO,I,1,7

$C P, 31+1, U X, 447+10^{*}|, 911+2 *|$

*ENDDO

NALL

EALL

ICOM \# ELEMENT GENERATION FOR SHELL \#

TYPE, 1

MAT, 1

ICOM BOTTOM OF SHELL \#\#*

$E, 1,2,22,21$

$E, 2,3,23,22$

EGEN,8,1,-1

$\mathrm{E}, 10,11,30$

$E, 21,22,42,41$

$E, 22,23,43,42$

EGEN, 10,1,-1

$E, 11,31,30$

$E, 11,32,31$

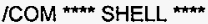

$E, 50,51,54,53$

EGEN, 2,1,-1

EGEN $, 5,3,-2$

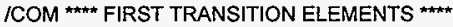

$E, 65,66,100$

$E, 100,66,101$

$E, 67,101,66$

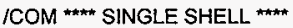

$E, 100,101,103,102$

EGEN,40,2,-1

ICOM SECOND TRANSITION ELEMENTS

E, 190,180,191

E, 180,181,191

$E, 181,192,191$

/COM TOP SHELL (DOUBLE ELEMENT)

E, 190,191,194,193

EGEN,2,1,-1

EGEN,18,3,-2

$E, 244,245,986,985$

EGEN, 2,1,-1

$E, 256,257,988,987$

$E, 257,258,989,988$

$E, 985,986,981,980$

EGEN,4,1,-1

$E, 980,981,248,247$

EGEN,2,1,-1

$E, 982,983,260,249$

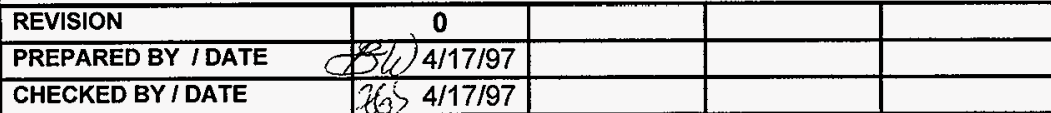

PAGE 29

OF 52 
CLIENT: Duke Engineering Services Hanford

PROJECT: MCO Final Design Doc. No. HNF-SD-DR-003, Rev.0 Appendix 4

$E, 983,984,261,260$

ICOM COLLAR TRANSITION \& THREADED REGIONS

$E, 237,991,251,240$

E,991,990,251

$E, 240,251,254,253$

$E, 251,990,255,254$

$E, 253,254,257,256$

EGEN, $2,1,-1$

$E, 259,260,263,262$

EGEN,2,1,-1

EGEN, 12,3,-2

$E, 271,274,1000$

ICOM MERGE COINCIDENT NODES FOR SHELL \#

ESEL,S,TYPE, 1

NSLE

NUMMRG,NODE,

EALL

NALL

$/_{\text {COM }}^{* \star *}$ END OF SHELL/COLLAR ELEMENT GENERATION *

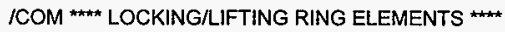

TYPE, 3

MAT,1

$E, 411,412,402,401$

EGEN, $2,1,-1$

EGEN,2,10,-2

$E, 413,888,876,403$

E, $881,404,876$

$E, 888,881,876$

E, $888,414,881$

E, 881,882,877,404

$E, 414,889,882,881$

$E, 882,883,878,877$

$E, 889,890,883,882$

$E, 883,884,405,878$

$E, 890,416,884,883$

$E, 884,885,879,405$

$E, 415,891,885,884$

$E, 885,886,880,879$

$E, 891,892,886,885$

$E, 886,887,406,880$

$E, 892,416,887,886$

$E, 423,900,888,413$

$E, 893,414,888$

$E, 900,893,888$

$E, 900,424,893$

$E, 893,894,889,414$

E.424,901,894,893

$E, 894,895,890,889$

$E, 901,902,895,894$

$E, 895,896,415,890$

$E, 902,425,896,895$

$\mathrm{E}, 896,897,891,415$

E,425,903,897,896

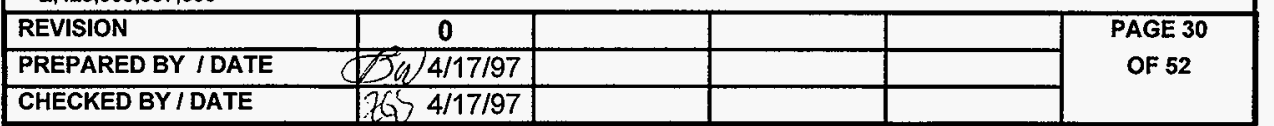


CLIENT: Duke Engineering Services Hanford

PROJECT: MCO Final Design

FILE NO: $\quad \mathrm{KH}-8009-8-02$ Doc. No. HNF-SD-DR-003, Rev.0 Appendix 4

$E, 897,898,892,891$

E, $903,904,898,897$

$E, 898,899,416,892$

$E, 904,426,899,898$

E,431,432,422,421

$E, 905,423,422$

E,432,905,422

$E, 432,433,905$

E, $905,906,900,423$

E,433,908,906,905

E,906,907,424,900

E, $908,434,907,906$

E, 441,442,432,431

EGEN,2, 1,-1

$E, 443,908,433$

E,443,444,434,908

E,451,452,442,441

EGEN, $3,1,-1$

EGEN, $7,10,-3$

E,454,912,910,444

$E, 464,914,912,454$

E,474,916,914,464

E, $484,918,916,474$

E.494,920,918,484

E,504,922,920,494

E,514,924,922,504

$E, 458,448,911,913$

E,468,458,913,915

E,478,468,915,917

$E, 488,478,917,919$

E,498,488,919,921

E, $508,498,921,923$

E, $518,508,923,925$

ICOM **** BOLT ****

TYPE, 5

MAT, 5

$E, 455,456,446,445$

EGEN,8,10,-1

$E, 456,457,447,446$

EGEN,8, 10,-1

ICOM END OF LOCKING/LIFTING RING

ICOM \$** SHIELD PLUG ELEMENTS

TYPE, 2

MAT, 1

$E, 602,622,621,601$

EGEN,11,1,-1

EGEN,2,20,-11

$E, 613,1290,612$

$E, 1290,1280,632,612$

E, $1280,633,632$

$\mathrm{E}, 633,1270,632$

$E, 1270,652,632$

$E, 1270,653,652$

$E, 643,663,662,642$

\begin{tabular}{|l|c|l|l|l|}
\hline REVISION & 0 & & & \\
\hline PREPARED BY IDATE & Yy/ 4/17/97 & & & \\
\hline CHECKED BYIDATE & $\mathbb{f} 6 / 4 / 17 / 97$ & & & \\
\hline
\end{tabular}


CLIENT: Duke Engineering Services Hanford

PROJECT: MCO Final Design Doc. No. HNF-SD-DR-003, Rev.0 Appendix 4

EGEN,10,1,-1

EGEN,2,20,-10

$E, 673,693,692,672$

EGEN,2,20,-10

$E, 653,1260,652$

$\mathrm{E}, 1260,672,652$

$\mathrm{E}, 1260,673,672$

$E, 707,717,716,706$

EGEN,7,1,-1

$E, 717,737,736,716$

EGEN,7,1,-1

$E, 731,751,750,730$

EGEN, 13,1,-1

$E, 749,769,768,748$

EGEN,15,1,-1

$E, 767,787,786,766$

EGEN, 17,1,-1

EGEN,2,20,-17

$\mathrm{E}, 818,825,824,817$

EGEN,6,1,-1

EGEN, $5,7,-6$

$E, 853,860,859,852$

EGEN, $6,1,-1$

$E, 860,867,866,859$

EGEN,3,1,-1

$E, 867,872,871,866$

EGEN, $4,1,-1$

/COM END OF SHIELD PLUG

/COM FILTER GUARD PLATE

$E, 1200,1201,858,851$

$E, 1201,1202,865,858$

$E, 1203,1204,1201,1200$

EGEN,2,1,-1

EGEN,6,3,-2

$E, 1221,1222,1219,1218$

E, 1222,1223,1220,1219

$E, 1226,1215,1212,1225$

E, 1227,1218,1215,1226

E, 1228,1221,1218,1227

$E, 1230,1226,1225,1229$

EGEN, $3,1,-1$

EGEN, $6,4,-3$

$E, 1257,1250,1249,1256$

EGEN,3,1,-1

$E, 1264,1254,1253,1263$

EGEN, $6,1,-1$

$E, 1271,1261,1260,1270$

EGEN, $9,1,-1$

$E, 1281,1271,1270,1280$

EGEN, $4,1,-1$

$E, 1291,1281,1280,1290$

EGEN,2,1,-1

/COM CONTACT ELEMENTS

ICOM BETWEEN LOCKING RING \& SHIELD PLUG *

\begin{tabular}{|l|c|l|l|l|}
\hline REVISION & 0 & & & \\
\hline PREPARED BY I DATE & V & & & \\
\hline CHECKED B $4 / 17 / 97$ & & & \\
\hline
\end{tabular}


CLIENT: Duke Engineering Services Hanford

PROJECT: MCO Final Design Doc. No. HNF-SD-DR-003, Rev.0 Appendix 4 SNF UES 5/23/97

TYPE, 4

REAL, 4

$E, 806,401$

$E, 807,411$

$E, 808,421$

$E, 809,431$

$E, 810,441$

$E, 811,451$

$E, 812,461$

$E, 813,471$

$E, 814,481$

$E, 815,491$

$E, 816,501$

$E, 817,511$

/COM **** BETWEEN SHIELD PLUG \& BOTTOM OF BOLT

REAL,5

$\mathrm{n}, 3000,10.875-.197,151.88$

$\mathrm{E}, 3000,525$

$E, 852,526$

$\mathrm{E}, 859,527$

/COM BETWEEN SHIELD PLUG \& SHELL (ABOVE SEAL)

REAL, 4

$E, 271,871$

$E, 268,872$

$E, 265,873$

$E, 262,874$

/COM BETWEEN SHIELD PLUG \& SHELL (BELOW SEAL)

$E, 863,980$

$/$ COM *** BETWEEN SHIELD PLUG AND SEAL LIP

TYPE, 4

REAL, 6

$E, 248,870$

$E, 249,875$

/COM **** BOTTOM GAP ELEMENTS

TYPE, 4

REAL, 7

$E, 2001,1$

EGEN,10,1,-1

NALL

EALL

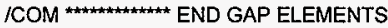

ICOM

CSYS, 0

NSEL,S,LOC,X,O

NSEL,R,LOC,Y,-1.33,158.14

$D, A L L, U X, 0$

NALL

EALL

NSEL,S,NODE,,2001,2010

D,ALL,ALL, 0

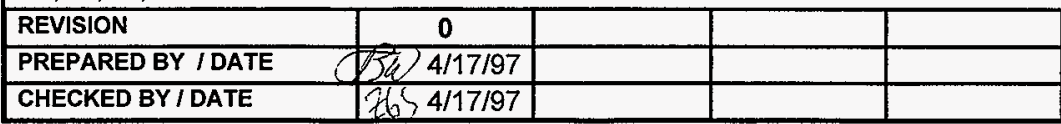


CLIENT: Duke Engineering Services Hanford

PROJECT: MCO Final Design

FILE NO: KH-8009-8-02

Doc. No. HNF-SD-DR-003, Rev.0 Appendix 4

EALL

NALL

/com fine tune seal \& bolt regions

nmodif,869,11.72,149.88

nmodif, $870,11.72,149.65$

type, 2

emodif, $420,1,3000,846,853,852$

e, $845,846,3000,3000$

type, 4

real, 8

e,248,869 ! seal spring

FINI

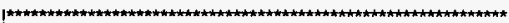

ICOM SOLUTION PHASE

ISOLUTION

time, 1 ! bolt preload only

Iswrite, 1

time,2 ! Preload + Pressure

ICOM 150 PSI INTERNAL PRESSURE ***

NSEL, S, LOC, X,0,1.26 ! Bottom Plate

NSEL,R,LOC,Y, $-0.45,-0.43$

SF,ALL,PRES, 150

NALL

EALL

NSEL, S,LOC, X,1.24,2.14

NSEL,R,LOC,Y,-0.45, 0.45

SF,ALL,PRES, 150

NALL

EALL

NSEL,S,LOC,X,2.12,11.51

NSEL, R,LOC,Y, $0.43,0.45$

SF,ALL, PRES, 150

NALL

EALL

NSEL, S, LOC,X,11.49,11.51 ! Inside Shell

NSEL, R,LOC, Y, $0.43,149.64$

SF,ALL,PRES, 150

NALL

EALL

NSEL, S,LOC,X,11.49,11.76 ! Edge Shell to Seal

NSEL, R,LOC, Y, 149.62,149.64

SF,ALL,PRES, 150

NALL

EALL

NSEL,S,LOC,X,11.67,11.69 ! Seal

NSEL, R,LOC, Y, 149.64, 149.89

SF,ALL,PRES, 150

NALL

EALL

NSEL, S,LOC,X,11.44,11.69 ! Shield Plug (above seal)

NSEL, R,LOC,Y, 149.87, 149.89

SF,ALL,PRES, 150

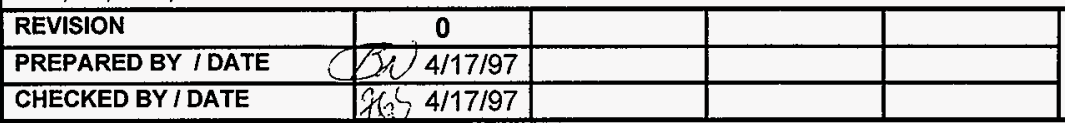


CLIENT: Duke Engineering Services Hanford

NALL

EALL

NSEL,S,LOC,X,11.44,11.46 ! Side of Shield Plug

NSEL, R,LOC,Y,149.37,149.89

SF,ALL,PRES, 150

NALL

EALL

NSEL,S,LOC,X,11.24,11.46 ! Shield Plug Taper

NSEL,R,LOC,Y,147.62,149.39

SF,ALL,PRES, 150

NALL

EALL

NSEL,S,LOC,X,11.24,11.26 ! Guard Plate Ring

NSEL,R,LOC,Y,143.12,147.64

SF,ALL,PRES, 150

NALL

EALL

NSEL,S,LOC,X,10.914,11.26 ! Guard Plate Taper

NSEL,R,LOC,Y,141.87,143.14

SF,ALL,PRES, 150

NALL

EALL

NSEL,S,LOC,X,1.34,10.92 ! Guard Plate Bottom

NSEL,R,LOC,Y,141.87,141.89

SF,ALL,PRES, 150

NALL

EALL

nsubst, 5

Iswrite, 2

Issolve, 1,2

FINI

/Post1

! Obtain Bolt/Seal Response

set, 1

Ipath, 525,527 ! Bolt Cross Section

prsect

Ipath,870,875 ISeal Stop Cross Section

prsect

etable,Force,smisc, 1 ! Seal Normal Force

esel,s,real, 8

pretab

eall

set, 2

Ipath, 525,527

prsect

ipath, 870,875 ! Seal Stop Cross Section

prsect

etable,Force,smisc, 1 ! Seal Normal Force

esel,s,real, ,8

pretab

fini

lexit

0

PREPARED BY I DATE

CHECKED BYIDATE

! Bolt Cross Section

ISeal Nomal Force


PROJECT: MCO Final Design
FILE NO: KH-8009-8-02

Doc. No. HNF-SD-DR-003, Rev.0 Appendix 4 SNF KES 5/23/97

\section{COMPUTER RUN COVER SHEET}

Project Number:

Computer Code:

Software Version:

Computer System:

Computer Run File Number:

Unique Computer Run Filename:

Run Description:

Run Date / Time:

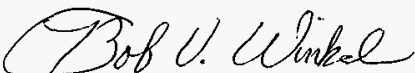

Prepared By: Bob V. Winkel
KH-8009-8

ANSYS®-PC

$5.0 \mathrm{~A}$

MS-DOS, Pentium® Processor

$\mathrm{KH}-8009-8-02$

MINBOLT.out

Analysis of MCO Closure Response, Minimum Bolt Load.

26 February $1997 \quad 3: 39: 46$ PM

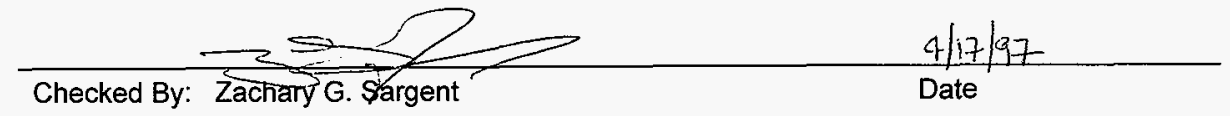


CLIENT: Duke Engineering Services Hanford

FILE NO: $\quad \mathrm{KH}-8009-8-02$

PROJECT: MCO Final Design Doc. No. HNF-SD-DR-003, Rev.0 Appendix 4 SNF KES Sl2367

\section{COMPUTER RUN COVER SHEET}

Project Number:

Computer Code:

Software Version:

Computer System:

Computer Run File Number:

Unique Computer Run Filename:

Run Description:

Creation Date / Time:
$\mathrm{KH}-8009-8$

ANSYS®-PC

$5.0 \mathrm{~A}$

MS-DOS, Pentium(B) Processor

$\mathrm{KH}-8009-8-02$

MXBOLT.inp

Analysis of MCO Closure Response, Maximum

Bolt Load.

26 February $1997 \quad 3: 09: 58$ PM

Prepared By: Bob V. Winkel

Date

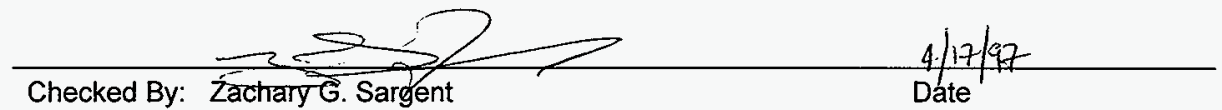

\begin{tabular}{|c|c|}
\hline & 0 \\
\hline$C$ & $5 \times 1 \longdiv { 4 / 1 7 / 9 7 }$ \\
\hline & $76,4 / 17 / 97$ \\
\hline
\end{tabular}


CLIENT: Duke Engineering Services Hanford

PROJECT: MCO Final Design

FILE NO: $\mathrm{KH}-8009-8-02$

\section{MCOFinal Design} Doc. No. HNF-SD-DR-003, Rev.0 Appendix 4 SNF KSS S/23/97

\section{LISTING OF MINBOLT.INP FILE}

/BATCH,LIST

/FILENAM, minbolt

IPREP7

IITLE,MCO DESIGN- 132 DEGREES C, 150 psi PRESSURE,

TREF,70

TUNIF,270

$E T A N=0.05$

! Tangent modulus

\begin{tabular}{|c|c|}
\hline /COM ELEI & TYPES \\
\hline $\mathrm{ET}, 1,42,,, 1$ & ! Sheil \\
\hline $\mathrm{ET}, 2,42_{1,}, 1$ & ! Shield Plug \\
\hline$E T, 3,42, \ldots 1$ & ! Lifting \& Locking Ring \\
\hline$E T, 4,12$ & ! Gap Elements Between Shield Plug \& Shell \\
\hline KEYOPT, $4,7,1$ & \\
\hline$E T, 5,42,,, 1$ & ! Bolt \\
\hline
\end{tabular}

ICOM REAL CONSTANTS FOR GAP ELEMENTS

R,4,-90,1.0e9,-0.06,3.0 I Shell/Shield Plug, Initially Open .06"

R,5,0,1.0e9,.0021 ! Gap Elements Under Bolt, Min. Preload Interference

$\mathrm{R}, 6,0,1.0 \mathrm{e} 9,-.006,2.0$ ! Sealing Stop, initially open, gap adjusted for max. stiffness

$R, 7,0,1.0 e 9,0,1.0 \quad$ ! Bottom MCO Plate, closed

$r, 8,0,2.42 e 7, .006$ ! Seal Spring, max. stiffness (unloading stiffness)

ICOM

MP,DENS, $1,490 / 1728 \quad$ ! 304 L SS

MP,NUXY, $1,0.3$

MP,DENS,5,490/1728 I SA193 Grade B8M

MP,NUXY, $5,0.3$

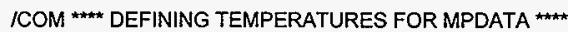

MPTEMP $, 1,70,100,200,300,400,500$

MPTEMP, $7,600,650,700,750$

ICOM DEFINING ELASTIC MODULI FOR 304L \& SA-193 *\#**

MPDATA,EX,1,1,28.3e+06,28.1e+06,27.6e+06,27.0e+06,26.5e+06,25.8e+06

MPDATA,EX, 1,7,25.3e+06,25.1e+06,24.8e+06,24.5e+06

ICOM ISA-193

MPDATA,EX,5,1,28.3e+06,28.1e+06,27.6e+06,27.0e+06,26.5e+06,25.8e+06

MPDATA,EX,5,7,25.3e+06,25.1e+06,24.8e+06,24.5e+06

ICOM INSTANTANEOUS COEFFICIENTS OF THERMAL EXPANSION, 304L \& SA-193

MPDATA,ALPX, 1,1,8.46e-06,8.63e-06,9.08e-06,9.46e-06,9.80e-06,10.10e-06

MPDATA,ALPX, 1,7,10.38e-06,10.50e-06,10.60e-06,10.70e-06

ICOM ISA-193

MPDATA,ALPX,5,1,8.42e-06,8.59e-06,9.09e-06,9.56e-06,9.95e-06,10.25e-06

MPDATA,ALPX, $5,7,10.51 \mathrm{e}-06,10.64 \mathrm{e}-06,10.76 \mathrm{e}-06,10.87 \mathrm{e}-06$

ICOM SHELL GEOMETRY

IR=11.5 ! Internal Shell Radius @ Bottom

OR=12.000 ! Shell Outside Radius @ Bottom

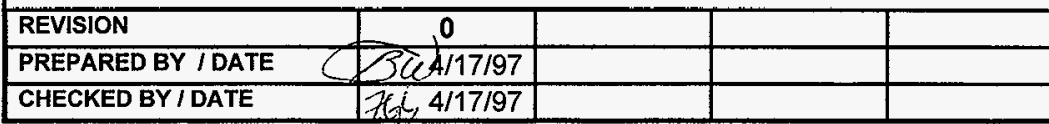


CLIENT: Duke Engineering Services Hanford

\section{ra}

PROJECT: MCO Final Design
FILE NO: $\mathrm{KH}-8009-8-02$

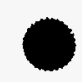
IR2 $=12.02$
$\mathrm{OR} 2=12.625$
IR3 $=12.25$
! Inside Radius at Collar Sealing Surface ! Outside Radius at Collar Sealing Surface
I Inside Radius at Collar-Lifting Ring Weld

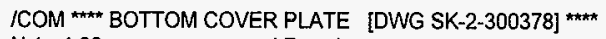

$N, 1,-1.32$

! Row 1

$\mathrm{N}, 2,1.25,-1.32$

$\mathrm{N}, 3,2.13,-1.32$

$N, 10,11.423,-1.32$

FILL

$\mathrm{N}, 41,0.00,-0.44$

$\mathrm{N}, 42,1.25,-0.44$

! Row 3

$N, 43,2.13,0.44$

$\mathrm{N}, 50, \mathrm{IR}, 0.44$

FILL, 43,50

N,52,OR, 0.44

FILL,50,52

FILL, 1,41,1,21, 1,10

! Middle Row

FILL, 10,50,1,30

$N, 32,12,-0.32$

FILL, 30,32

FILL, 10,32, 1, 11

N,53,IR, 1.17

N,55,OR, 1.17

FILL,53,55

! Shell Stub Weld

ICOM $^{\star \star \star}$ SHELL [DWGS SK-2-300379 \& SK-2-300461]

$\mathrm{N}, 65, \mathrm{IR}, 6.68$

N,67,OR,6.68

- FILL

FILL, $53,65,3,3,3,1$

ICOM *** SINGLE ROW SHELL

$N, 100, I R, 7.18$

! Inside

$N, 140, ! R, 71.68$

$\mathrm{N}, 180, \mathrm{IR}, 136.68$

$\mathrm{N}, 101, \mathrm{OR}, 7.18$

! Outside

$N, 141, O R, 71.68$

$\mathrm{N}, 181, \mathrm{OR}, 136.68$

FILL, 100,140,20, 2,2,1,2.0

FILL, $140,180,19,2,2,1,5$

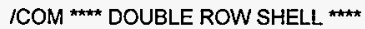

N, 190,IR,137.18 ! Transition to Double Row

$N, 192, O R, 137.18$

FILL

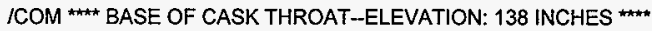

$\mathrm{N}, 217, \mathrm{IR}, 142.68$

! Transition to Double Row

N,219,OR, 142.68

FILL

FILL, $190,217,8,3,3,1 \quad$ ! Vertical Fill

\begin{tabular}{|c|c|c|c|c|}
\hline & & & & \\
\hline & & & & \\
\hline $76 / 17 / 97$ & $4 / 17 / 97$ & & & \\
\hline
\end{tabular}


CLIENT: Duke Engineering Services Hanford

PROJECT: MCO Final Design

Doc. No. HNF-SD-DR-003, Rev.0 Appendix 4

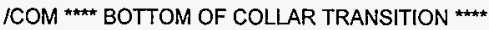 \\ $\mathrm{N}, 235, \mathrm{IR}, 146.06$ \\ ! Start of Transition to Large O.D \& \\ $N, 237, O R, 146.06$ \\ ! Assumed Location of Shield Plug Taper}

FILL

$\mathrm{N}, 238, \mathrm{IR}, 146.68$

$\mathrm{N}, 240, \mathrm{OR}, 146.68$

FILL

! Horizontal Fill

FILL,217,235,5,3,3,1 ! Vertical Fill

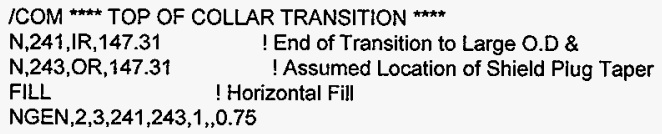

FILL ! Horizontal Fill

NGEN,2,3,241,243,1,0.75

/COM *** COLLAR SEALING SURFACE

$\mathrm{N}, 247, \mathrm{IR}, 149.63$

$N, 249, I R 2,149.63$

FILL

! Inside Radius of Sealing Surface

t Outside Radius at Sealing Surface

! Horizontal Fill

/COM *** THICK WALL AT COLLAR TRANSITION ***

NGEN, 2,10,240,249,3

! Nodes 250-259 Coincident w/240-249 (by 3)

$\mathrm{N}, 255, \mathrm{OR} 2,147.31$

Outside Surface

$\mathrm{N}, 261, \mathrm{OR} 2,149.63$

! Outside Surface

$\mathrm{N}, \mathbf{2 5 8}, \mathrm{OR} 2, \mathbf{1 4 8 . 0 6}$

$\mathrm{N}, 980, \mathrm{IR}, 149.38$

$\mathrm{N}, 981,11.755,149.38$

$\mathrm{N}, 982, \mathrm{IR} 2,149.38$

$\mathrm{N}, 983,12.317,149.38$

$N, 984, O R 2,149.38$

$\mathrm{N}, 990, \mathrm{OR} 2,146.68$

FILL, 240,990,1,251

NGEN $, 2,5,980,984,1,-0.66$

FILL,246,258, 1,257

FILL,253,255,1,1,3,3

FILL,237,990,1,991

/COM COLLAR AT BOTTOM EDGE OF PLUG (.155" above Sealing Surface)

NGEN, $2,3,259, \ldots, 0.245 \quad$ ! Nodes 262

/COM **** COLLAR AT TOP EDGE OF PLUG (2" above bottom Edge)

NGEN $, 2,9,262, \ldots, 2.00 \quad$ ! Nodes 271

FILL,262,271,2

ICOM *** COLLAR AT BASE OF THREADS

$\mathrm{N}, 274, \mathrm{IR} 3,152.00$

$\mathrm{N}, 1000, \mathrm{IR} 2,152.00$

ICOM

N,295, IR3,156.00

FILL,274,295

NGEN,3,1,259,295,3,(OR2-IR2)/2

NGEN, 3,1,274,295,3,(OR2-IR3)/2

ICOM LOCKING \& LIFTING RING GEOMETRY

RING1=7.94

\begin{tabular}{|l|c|l|l|l|}
\hline REVISION & 0 & & & \\
\hline PREPARED BY IDATE & Q $/$ I 4/17/97 & & & \\
\hline CHECKED BYI DATE & $X_{6}$ 4/17/97 & & & \\
\hline
\end{tabular}


CLIENT: Duke Engineering Services Hanford

PROJECT: MCO Final Design
FILE NO: KH-8009-8-02

RING2 $=9.375$

RING3 $=9.625$

RING4 $=10.19$

RING5 $=12.23$

LOCAL, $11,0,152.00$

! Local System $z=0$ at Base of Ring

CSYS, 11

ICOM TOP EDGE $\cdots$

$\mathrm{N}, 401, \mathrm{RING} 1,6.13$

CSYS,O

$\mathrm{N}, 404,9.375,158.13$

FILL, 401,404,,1

$\mathrm{N}, 406, \mathrm{RING} 4,158.13$

FILL,404,406,,1 ! Top Edge

ICOM LIFTING SURFACE

CSYS, 11

N,421,RING1,5.13

N,424,RING2,5.13

FILL, 421,424

N,426,RING4,5.13

FILL, 424,426

FILL,401,421,1,10,6,1

N,431,RING1,6.13-1.56

N,434,RING2,6.13-1.56

FILL

ICOM BOLTING SURFACE

$\mathrm{N}, 441, \mathrm{RING} 1,4$

$\mathrm{N}, 444, \mathrm{RING} 3,4$

FILL

N,445,10.875-.197,4 ! Inside Edge of Bolt Hole

N,447,10.875+.197,4 ! Outside Edge of Bolt Hole

FILL.

$\mathrm{N}, 910,10.875-.197,4$

$\mathrm{N}, 911,10.9375+.197,4$

$\mathrm{N}, 448, \mathrm{RING5,4}$

CSYS, 0

! O.D of Ring

! Bolt Extension

$\mathrm{N}, 924,10.875-.197,152.00$

! Double Nodes @ Bolt for Gap elements

$\mathrm{N}, 925,10.875+.197,152.00$

FILL, $910,924,6,2$

FILL,911,925,6,2

$\mathrm{N}, 525,10.875-.197,151.874$ ! Bottom of Bolt Extension

$\mathrm{N}, 527,10.875+.197,151.874$

FILL

ICOM BOTTOM OF LIFTING/LOCKING RING

CSYS, 11

NGEN, $2,70,441,448,1,-4$

FILLL,441,511,6, $10,8,1$

! Bottom Surface of Lifting/Locking Ring

! Fill in Lifting/Locking Ring

/COM SHIELD PLUG (offset y by 158.25)

LOCAL, $20,0,158.13$

TYPE, 2

PLUGR $1=11.975$

PLUGR2 $=11.45$

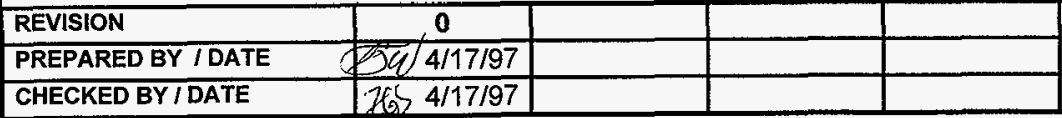


CLIENT: Duke Engineering Services Hanford

PLUGR3 $=11.25$

PLUGR4 $=7.89$

ICOM NoDES AT PLUG AXIS $(r=0)$

$\mathrm{N}, 601$

$\mathrm{N}, 602,0,-1$

$\mathrm{N}, 603,0,-1.994$

$\mathrm{N}, 606,0,-4.994$

FILL, 603,606,2,604

$\mathrm{N}, 607,0,-6.25$

$\mathrm{N}, 610,0,-8.25$

FILL,607,610,2,608

$\mathrm{N}, 611,0,-8.75$

$\mathrm{N}, 613,0,-10.5$

FILL,611,613

/COM NODAL GENERATION *

NGEN,2,20,601,613,1,0.8825

NGEN, 2,20,621,633,1,0.8825

NGEN $, 2,20,642,653,1,0.6875$

NGEN, $2,20,662,673,1,0.6875$

NGEN, 2,20,683,693,1,0.4235

NGEN $, 2,10,706,713,1,0.9515$

! Id Large Opening

! Id Medium Opening

! id Small Opening

! Center of Opening

! Od Small Opening

$N, 730,5.4665,-1.994$

$N, 736,5.4665,-4.994$

FILL, $730,736,5,731$

$\mathrm{N}, 737,5.4665,-6.25$

$\mathrm{N}, 740,5.4665,-8.25$

FILL, $737,740,2,738$

N, $741,5.4665,-8.75$

$N, 743,5.4665,-10.5$

FILL, 741,743

$\mathrm{N}, 748,5.89,-1.0$

NGEN,2,20,730,743,1,0.4235

FILL, 748,750

$N, 766,7.265,0$

NGEN, $2,20,748,763,1,1.375$

FILL, 766,768

NGEN,3,20,766,768,1,0.3125

$N, 789,7.5775,-1.56$

$N, 796,7.5775,-5.56$

FILL, $789,796,6$

NGEN $, 2,20,789,796,1,0,3125$

NGEN, $3,20,777,783,1,0.3125$

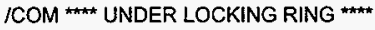

$\mathrm{N}, 824,8.5017,-6.25$

$\mathrm{N}, 827,8.5017,-8.25$

FILL

$\mathrm{N}, 828,8.5017,-8.75$

$\mathrm{N}, 830,8.5017,-10.5$

FILL

NGEN, $3,7,824,830,1,0.5616$

NGEN, $2,7,838,844,1,0.625$

NGEN, $2,7,845,851,1,0.625$

\begin{tabular}{|c|c|}
\hline REVISION & 0 \\
\hline PREPARED BY I DATE & $( 1 0 ) \longdiv { 4 / 1 7 / 9 7 }$ \\
\hline CHECKED BY / DATE & $x(9>4 / 17 / 97$ \\
\hline
\end{tabular}


CLIENT: Duke Engineering Services Hanford

$\mathrm{N}, 859,10.875+.197,-6.25$

$\mathrm{N}, 860,10.875+.197,-6.917$

$\mathrm{N}, 861,10.875+.197,-7.584$

$\mathrm{N}, 862$, PLUGR2,-8.25

$\mathrm{N}, 863$, PLUGR2,-8.75

N,865,PLUGR3,-10.5

FILL,863,865,1

N,866,PLUGR1-0.288,-6.25

N,869,PLUGR1-0.288,-8.25

FILL,866,869,2

N,870,PLUGR1-0.288,-8.476

NGEN, $2,5,866,870,1,0.288$

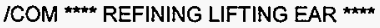

CSYS, 0

$\mathrm{N}, 877,9.53,158.13$

$\mathrm{N}, 889,9.53,157.63$

$\mathrm{N}, 901,9.53,157.13$

FILL, $403,404,1,876$

FILL,413,414, 1,888

FILL,423,424,1,900

FILL,877,405,1,878

FILL,405,406,2,879,1

FILL,889,415,1,890

FILL,415,416,2,891,1

FILL, 404,414, 1,881

FILL, $877,889,1,882$

FILL, $878,890,1,883$

FILL, $405,415,1,884$

FILL, 879,891, 1,885

FILL, 880,892, 1,886

FILL,406,416,1,887

FILL,889,901,1,894

FILL, $414,424,1,893$

FILL, $901,425,1,902$

FILL,890,902, 1,895

FILL, $415,425,1,896$

FILL,425,426,2,903,1

FILL,891,903,1,897

FILL,892,904,1,898

FILL,416,426, 1,899

FILL,424,434,1,907

FILL,433,434,1,908

FILL,423,433,1,905

FILL,905,907

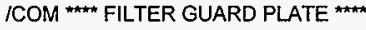

PLATE1 $=0.273$

PLATE2 $=0.6575$

PLATE3 $=1.357$

PLATE4 $=10.25$

PLATE5 $=11.25$

$N, 1200$, PLATE4, 146.78

$N, 1202, P L A T E 5,146.78$

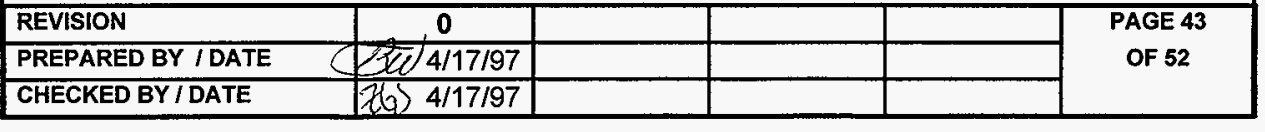


CLIENT: Duke Engineering Services Hanford

PROJECT: MCO Final Design Doc. No. HNF-SD-DR-003, Rev.0 Appendix 4

FILL

NGEN $, 5,3,1200,1202, \ldots-0.85$

NGEN, $2,3,1212,1214,-0.25$

N, 1221,PLATE4,141.88

$\mathrm{N}, 1222,10.75,141.88$

$\mathrm{N}, 1223,10.915,141.88$

FILL, $1215,1221,1,1218$

FILL, $1223,1217,1,1220$

FILL, $1216,1222,1,1219$

$\mathrm{N}, 1237,6.4375,143.38$

FILL, 1212,1237,3,1225,4

$\mathrm{N}, 1249,3.578,143.38$

FILL, $1237,1249,2,1241,4$

NGEN, $2,1,1225,1249,4,-0.25$

NGEN, 2,2,1226,1250,4,,-1.25

FILL, 1226, 1228, 1, 1227, ,7,4

$N, 1253,2.625,145.255$

$\mathrm{N}, 1254,2.625,145.005$

$\mathrm{N}, 1256,2.625,143.38$

FILL, 1254,1256

$\mathrm{N}, 1257,2.625,143.13$

$\mathrm{N}, 1259,2.625,141.88$

FILL, 1257,1259

NGEN, $2,10,1253,1259,1,-0.5$

NGEN,2,10,1263,1269,1,-0.768

$\mathrm{N}, 1283,0.6575,145.255$

$\mathrm{N}, 1284,0.6575,145.005$

$\mathrm{N}, 1260,2.125,147.63$

$\mathrm{N}, 1270,1.357,147.63$

$\mathrm{N}, 1280,0.6575,147.63$

$\mathrm{N}, 1290,0.273,147.63$

NGEN, $3,1,1260,1290,10,-0.5625$

ICOM **** NODES AT BOTTOM GAP ELEMENTS ***

NGEN,2,2000,1,10,1,-1.00

ICOM COUPLING NODES *

ICOM ** BETWEEN LIFTING/LOCKING RING \& SHELL ***

CP, 1,UY, 508,277 ! Start Threads

CP, $2, U Y, 498,280$

$C P, 3, \cup Y, 488,283$

$C P, 4, U Y, 478,286$

$C P, 5, U Y, 468,289$

$\mathrm{CP}, 6, \mathrm{UY}, 458,292$

/COM BETWEEN BOLT \& LOCKING RING ***

$\mathrm{CP}, 7, \mathrm{UY}, 445,910$

$C P, 8, U X, 445,910$

$\mathrm{CP}, 9, \mathrm{UY}, 447,911$

$C P, 10, U X, 447,911$

*DO, $1,1,7$

$\mathrm{CP}, 10+1, \mathrm{UY}, 445+10^{*}|, 910+2 *|$

"ENDDO

${ }^{*} D O, 1,1,7$

$C P, 17+1, U Y, 447+10^{*} \mid, 911+2 * 1$

\begin{tabular}{|l|c|c|c|c|c|}
\hline REVISION & 0 & & & & PAGE 44 \\
OF 52 \\
PREPARED BY IDATE
\end{tabular}


CLIENT: Duke Engineering Services Hanford

PROJECT: MCO Final Design
PARSDNS

Doc. No. HNF-SD-DR-003, Rev.0 Appendix 4 SNF KES S/23197

\section{*ENDDO}

${ }^{*} \mathrm{DO}, 1,1,7$

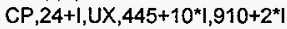

*ENDDO

*DO,I,1,7

$\mathrm{CP}, 31+1, \mathrm{UX}, 447+10^{*} 1,911+2^{*} \mid$

*ENDDO

NALL

EALL

ICOM ${ }^{* \star *}$ ELEMENT GENERATION FOR SHELL ****

TYPE, 1

MAT, 1

ICOM BOTTOM OF SHELL

$E, 1,2,22,21$

$E, 2,3,23,22$

EGEN,8,1,-1

$E, 10,11,30$

$E, 21,22,42,41$

$E, 22,23,43,42$

EGEN, 10,1,-1

$E, 11,31,30$

$E, 11,32,31$

ICOM **** SHELL ****

$E, 50,51,54,53$

EGEN,2, $1,-1$

EGEN, 5,3,-2

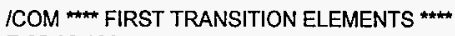

$E, 65,66,100$

$\mathrm{E}, 100,66,101$

E, $67,101,66$

ICOM SINGLE SHELL ***

$\mathrm{E}, 100,101,103,102$

EGEN,40,2,-1

ICOM SECOND TRANSITION ELEMENTS **

E,190,180,191

$E, 180,181,191$

E,181,192,191

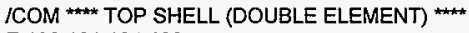

$E, 190,191,194,193$

EGEN,2,1,-1

EGEN, 18,3,-2

$E, 244,245,986,985$

EGEN, $2,1,-1$

E,256,257,988,987

$E, 257,258,989,988$

E, $985,986,981,980$

EGEN,4,1,-1

E, $980,981,248,247$

EGEN,2,1,-1

\begin{tabular}{|l|c|l|l|l|}
\hline REVISION & 0 & & & \\
\hline PREPARED BY I DATE & S & & & \\
\hline CHECKED BY I DATE & $\mathcal{2} 6 / 17 / 97$ & & & \\
\hline
\end{tabular}


CLIENT: Duke Engineering Services Hanford

PROJECT: MCO Final Design Doc. No. HNF-SD-DR-003, Rev.0 Appendix 4

$E, 982,983,260,249$

$E, 983,984,261,260$

/COM COLLAR TRANSITION \& THREADED REGIONS

$E, 237,991,251,240$

$\mathrm{E}, 991,990,251$

$E, 240,251,254,253$

$E, 251,990,255,254$

$E, 253,254,257,256$

EGEN,2,1,-1

$E, 259,260,263,262$

EGEN,2,1,-1

EGEN, 12,3,-2

$E, 271,274,1000$

ICOM MERGE COINCIDENT NODES FOR SHELL

ESEL,S,TYPE, 1

NSLE

NUMMRG,NODE,

EALL

NALL

/COM ${ }^{\star \star \star \star}$ END OF SHELL/COLLAR ELEMENT GENERATION ***

/COM *** LOCKING/LIFTING RING ELEMENTS ${ }^{\star \star \star \star ~}$

TYPE, 3

MAT, 1

$E, 411,412,402,401$

EGEN,2,1,-1

EGEN,2, 10,-2

$E, 413,888,876,403$

$E, 881,404,876$

E, 888,881,876

E,888,414,881

$E, 881,882,877,404$

E,414,889,882,881

$E, 882,883,878,877$

$E, 889,890,883,882$

E,883,884,405,878

E,890,415,884,883

$E, 884,885,879,405$

$E, 415,891,885,884$

E, 885,886,880,879

E,891,892,886,885

$E, 886,887,406,880$

$E, 892,416,887,886$

$E, 423,900,888,413$

E,893,414,888

E, $900,893,888$

E, $900,424,893$

E,893,894,889,414

$E, 424,901,894,893$

$E, 894,895,890,889$

$E, 901,902,895,894$

E,895,896,415,890

E,902,425,896,895

E,896,897,891,415

\begin{tabular}{|l|c|l|l|l|l|}
\hline REVISION & 0 & & & & PAGE 46 \\
PREPARED BY IDATE 52 \\
\cline { 1 - 5 } CHECKED BYIDATE & OF $4 / 17 / 97$ & & & & \\
\hline
\end{tabular}


CLIENT: Duke Engineering Services Hanford

\begin{abstract}
$E, 425,903,897,896$
$E, 897,898,892,891$

$\mathrm{E}, 903,904,898,897$

$E, 898,899,416,892$

$E, 904,426,899,898$

$E, 431,432,422,421$

$\mathrm{E}, 905,423,422$

$E, 432,905,422$

$E, 432,433,905$

$E, 905,906,900,423$

$\mathrm{E}, 433,908,906,905$

$E, 906,907,424,900$

$\mathrm{E}, 908,434,907,906$

$E, 441,442,432,431$

EGEN,2,1,-1

$E, 443,908,433$

$\mathrm{E}, 443,444,434,908$

$E, 451,452,442,441$

EGEN,3,1,-1

EGEN,7,10,-3

$E, 454,912,910,444$

$E, 464,914,912,454$

$E, 474,916,914,464$

$E, 484,918,916,474$

E,494,920,918,484

$\mathrm{E}, 504,922,920,494$

E,514,924,922,504

$E, 458,448,911,913$

$E, 468,458,913,915$

$E, 478,468,915,917$

$E, 488,478,917,919$

$E, 498,488,919,921$

$E, 508,498,921,923$

$E, 518,508,923,925$
\end{abstract}

ICOM *** BOLT *

TYPE,5

MAT, 5

$E, 455,456,446,445$

EGEN,8,10,-1

$E, 456,457,447,446$

EGEN,8,10,-1

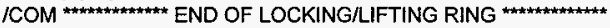

ICOM ${ }^{\star \star n \star}$ SHIELD PLUG ELEMENTS

TYPE,2

MAT, 1

$E, 602,622,621,601$

EGEN,11,1,-1

EGEN, 2,20,-11

$E, 613,1290,612$

$E, 1290,1280,632,612$

$E, 1280,633,632$

$E, 633,1270,632$

$E, 1270,652,632$

$E, 1270,653,652$

\begin{tabular}{|l|c|l|l|l|}
\hline REVISION & 0 & & & \\
\hline PREPARED BY IDATE & SUd 4/17/97 & & & \\
\hline CHECKED BY I DATE & XKS 4/17/97 & & & \\
\hline
\end{tabular}


CLIENT: Duke Engineering Services Hanford

PROJECT: MCO Final Design Doc. No. HNF-SD-DR-003, Rev.0 Appendix 4 SNE KES 5/23/91

$E, 643,663,662,642$

EGEN,10,1,-1

EGEN,2,20,-10

$\mathrm{E}, 673,693,692,672$

EGEN,2,20,-10

$E, 653,1260,652$

$E, 1260,672,652$

$E, 1260,673,672$

E, $707,717,716,706$

EGEN,7,1,-1

$E, 717,737,736,716$

EGEN,7,1,-1

$E, 731,751,750,730$

EGEN, 13,1,-1

E, $749,769,768,748$

EGEN, 15, 1,-1

$E, 767,787,786,766$

EGEN, 17,1,-1

EGEN, $2,20,-17$

$E, 818,825,824,817$

EGEN, 6,1,-1

EGEN,5,7,-6

$E, 853,860,859,852$

EGEN,6, 1,-1

$E, 860,867,866,859$

EGEN,3,1,-1

$E, 867,872,871,866$

EGEN,4,1,-1

/COM END OF SHIELD PLUG

ICOM FILTER GUARD PLATE

E, 1200,1201,858,851

$E, 1201,1202,865,858$

E,1203,1204,1201,1200

EGEN $, 2,1,-1$

EGEN, $6,3,-2$

$E, 1221,1222,1219,1218$

$E, 1222,1223,1220,1219$

E, 1226,1215,1212,1225

E, $1227,1218,1215,1226$

E, 1228,1221,1218,1227

$E, 1230,1226,1225,1229$

EGEN, $3,1,-1$

EGEN,6,4,-3

$E, 1257,1250,1249,1256$

EGEN, 3,1,-1

$E, 1264,1254,1253,1263$

EGEN,6,1,-1

$E, 1271,1261,1260,1270$

EGEN,9,1,-1

$E, 1281,1271,1270,1280$

EGEN,4,1,-1

$E, 1291,1281,1280,1290$

EGEN,2,1,-1

ICOM CONTACT ELEMENTS

\begin{tabular}{|l|c|l|l|l|}
\hline REVISION & 0 & & & \\
\hline PREPARED BY I DATE & $\mathbb{4} / 4 / 17 / 97$ & & & \\
\hline CHECKED BYIDATE & $\not 6 / 3>4 / 17 / 97$ & & & \\
\hline
\end{tabular}


CLIENT: Duke Engineering Services Hanford

PROJECT: MCO Final Design

ICOM ${ }^{\star \star \star \star}$ BETWEEN LOCKING RING \& SHIELD PLUG *
TYPE,4

Doc. No. HNF-SQ-DR-003, Rev.0 Appendix 4 SNF KES 5/23/91

REAL, 4

$E, 806,401$

$E, 807,411$

$E, 808,421$

$E, 809,431$

$E, 810,441$

$E, 811,451$

$E, 812,461$

$E, 813,471$

$E, 814,481$

$E, 815,491$

$E, 816,501$

$E, 817,511$

ICOM BETWEEN SHIELD PLUG \& BOTTOM OF BOLT

REAL, 5

$\mathrm{n}, 3000,10.875-.197,151.88$

$\mathrm{E}, 3000,525$

$E, 852,526$

$E, 859,527$

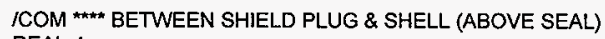

REAL, 4

$\mathrm{E}, 271,871$

$\mathrm{E}, 268,872$

$\mathrm{E}, 265,873$

$\mathrm{E}, 262,874$

/COM BETWEEN SHIELD PLUG \& SHELL (BELOW SEAL)

$E, 863,980$

/COM BETWEEN SHIELD PLUG AND SEAL LIP

TYPE, 4

REAL, 6

$E, 248,870$

$\mathrm{E}, 249,875$

$/$ COM BOTTOM GAP ELEMENTS

TYPE, 4

REAL, 7

$\mathrm{E}, 2001,1$

EGEN,10,1,-1

NALL

EALL

/COM END GAP ELEMENTS

ICOM BOUNDARY CONDITIONS

CSYS, 0

NSEL, S,LOC, $X, 0$

NSEL,R,LOC,Y,-1.33,158.14

$D, A L L, U X, 0$

NALL

EALL

NSEL,S,NODE,,2001,2010

\begin{tabular}{|l|c|l|l|l|}
\hline REVISION & 0 & & & \\
\hline PREPARED BY I DATE & Y & & \\
\hline CHECKED BY I DATE & $76 / 17 / 97$ & & & \\
\hline
\end{tabular}


CLIENT: Duke Engineering Services Hanford

PROJECT: MCO Final Design
FILE NO: KH-8009-8-02

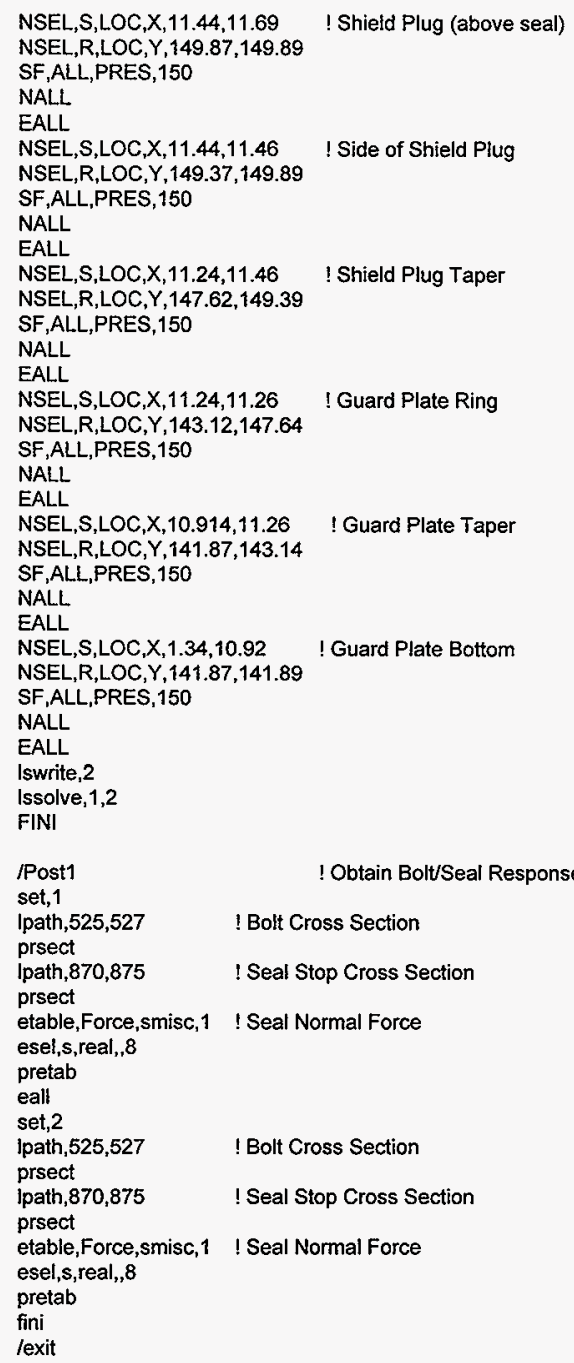


CLIENT: Duke Engineering Services Hanford

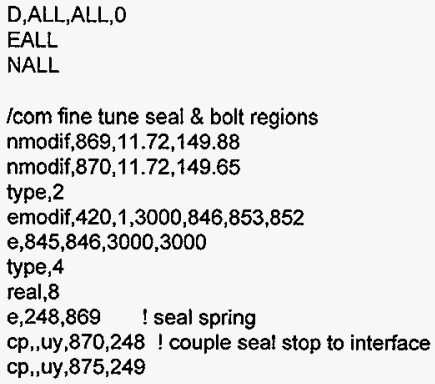

EALL

NALL

lcom fine tune seal \& bolt regions

nmodif,869,11.72,149.88

nmodif,870,11.72,149.65

type, 2

emodif,420,1,3000,846,853,852

$e, 845,846,3000,3000$

type, 4

real, 8

e,248,869 ! seal spring

cp, uy, 870,248 ! couple seal stop to interface

cp, ,uy, 875,249

FIN]

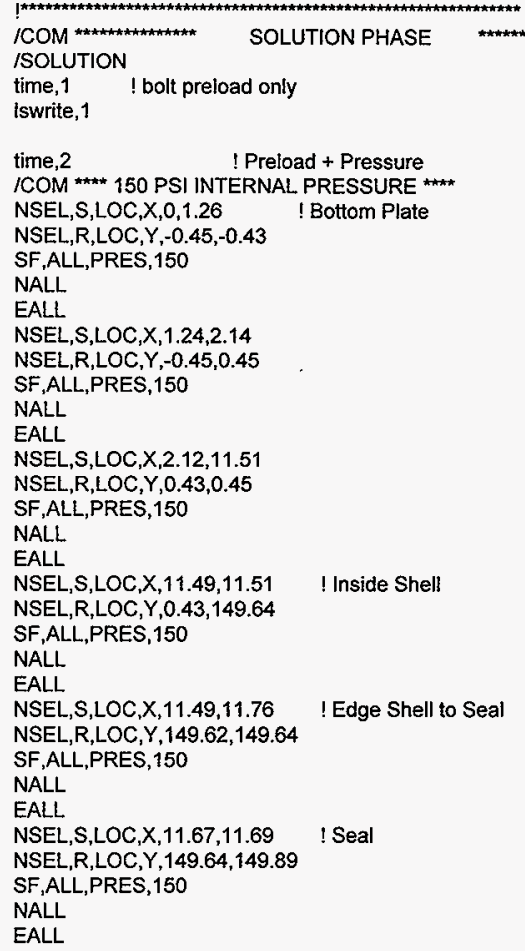

\begin{tabular}{|c|c|c|c|}
\hline 0 & & & \\
\hline $34 / 4 / 17 / 97$ & & & \\
\hline $36 / 4 / 17 / 97$ & & & \\
\hline
\end{tabular}


CLIENT: PROJECT: MCO Final Design D Duke Engineering Services Hanford
FILE NO: $\quad \mathrm{KH}-8009-8-02$

\section{COMPUTER RUN COVER SHEET}

Project Number:

Computer Code:

Software Version:

Computer System:

Computer Run File Number:

Unique Computer Run Filename:

Run Description:

Run Date / Time:
$\mathrm{KH}-8009-8$

ANSYSß-PC

$5.0 \mathrm{~A}$

MS-DOS, Pentium® Processor

KH-8009-8-02

MXBOLT.out

Analysis of MCO Closure Response, Maximum Bolt Load.

26 February $1997 \quad 3: 25: 14$ PM

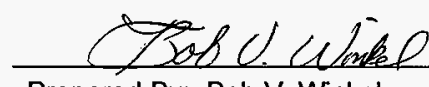

Prepared By: Bob V. Winkel st-17-97

Date

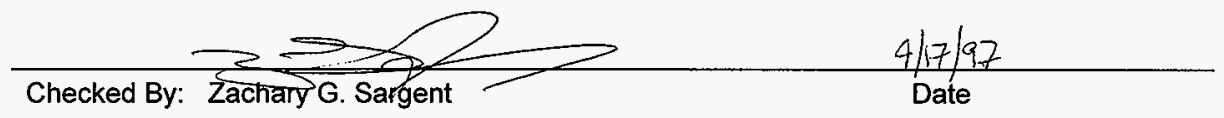

$\frac{0}{5 \operatorname{se}^{4 / 17 / 97}}$

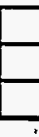




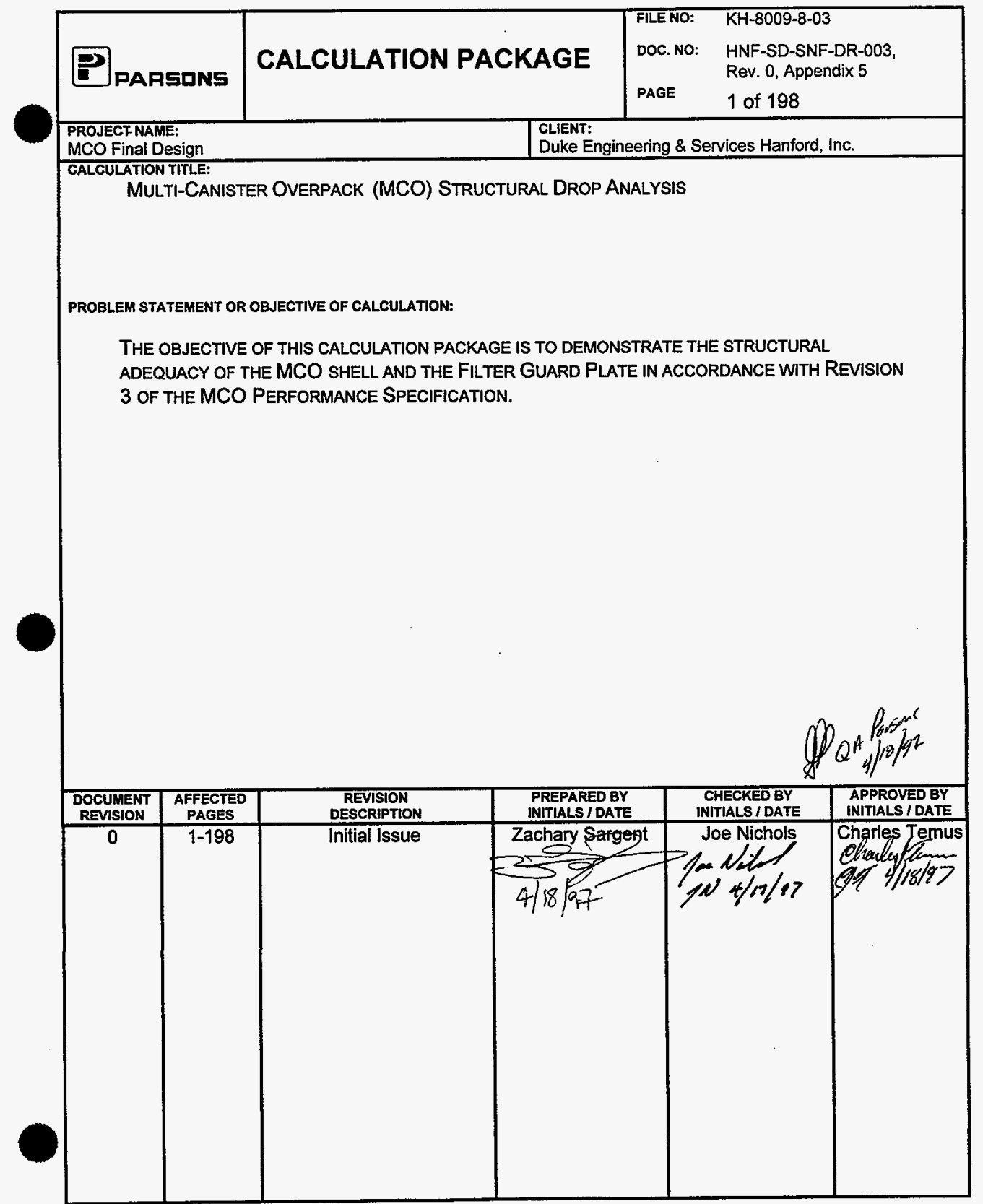


CLIENT: Duke Engineering \& Services Hanford

FILE NO: KH-8009-8-03

PROJECT: MCO Final Design DOC. NO.: HNF-SD-SNF-DR-003, Rev. 0, Appendix 5

\section{CONTENTS}

1. INTRODUCTION

2. REFERENCES

3. ASSUMPTIONS

4. MATERIAL PROPERTIES

5. ACCEPTANCE CRITERIA

6. LOAD CONDITIONS \& COMBINATIONS

7. FILTER GUARD PLATE

7.1 Introduction

7.2 Method of Analysis

7.3 Assumptions

7.4 Geometry

7.5 Material Properties

7.6 Acceptance Criteria

7.7 Load Combinations

7.8 Analysis

7.8.1 Support Ring

7.8.2 Weld Sizing

8. CLOSURE SEAL LEAKAGE

9. BARE BOTTOM END DROP

\subsection{Introduction}

9.2 Geometry

9.3 Assumptions

9.4 Analysis

9.4.1 Internal Pressure

9.4.2 Hydrostatic Pressure 
CLIENT: Duke Engineering \& Services Hanford

PROJECT: MCO Final Design

FILE NO: $\quad \mathrm{KH}-8009-8-03$

10. TOP END DROP

10.1 Introduction

10.2 Geometry

10.3 Assumptions

10.4 Analysis

10.4.1 Internal Pressure

10.4.2 Hydrostatic Pressure

11. CSB TUBE DROP

11.1 Introduction

11.2 Geometry

11.3 Assumptions

11.4 Analysis

12. ANSYSC ANALYSIS

12.1 Boundary Conditions

12.2 Loading

12.2.1 Bare Bottom End Drop

12.2.2 Top End Drops

12.2.3 CSB Tube Drops

13. RESULTS

\subsection{Buckling}

13.1.1 End Drop

13.2 Minimum Shell Thickness 
CLIENT: Duke Engineering \& Services Hanford

PROJECT: MCO Final Design DOC. NO.: HNF-SD-SNF-DR-003, Rev. 0 , Appendix 5

\section{LIST OF TABLES}

Page

TABLE 1: ASME CODE MATERIAL PROPERTIES FOR TYPE 304 STAINLESS STEEL 10

TABLE 2: ASME CODE MATERIAL PROPERTIES FOR TYPE 304L STAINLESS STEEL 11

TABLE 3: LEVEL D ALLOWABLE STRESSES - ELASTIC ANALYSIS - TYPE 304 STAINLESS STEEL

TABLE 4: LEVEL D ALLOWABLE STRESSES - ELASTIC ANALYSIS - TYPE 304L STAINLESS STEEL

TABLE 5: ANSYS MODEL STRESS REPORT SECTIONS

TABLE 6: CSB TUBE DROPS AND BOTTOM END DROP CALCULATED MAX. SHELL CIRCUMFERENCE

TABLE 7: TOP END DROPS CALCULATED MAX. SHELL CIRCUMFERENCE

TABLE 8: TOP END DROP (28G) WITHOUT CAP SUMMARY OF MAXIMUM STRESS INTENSITIES

TABLE 9: TOP END DROP (28G) WITH CAP SUMMARY OF MAXIMUM STRESS INTENSITIES

TABLE 10: BARE BOTTOM END DROP (54G) SUMMARY OF MAXIMUM STRESS INTENSITIES

TABLE 11: BARE BOTTOM END DROP W/ FINE MESH SUMMARY OF MAXIMUM STRESS INTENSITIES

TABLE 12: 28G CSB TUBE DROP WITH CAP SUMMARY OF MAXIMUM STRESS INTENSITIES

TABLE 13: 28G CSB TUBE DROP W/O CAP SUMMARY OF MAXIMUM STRESS INTENSITIES

TABLE 14: SUMMARY OF GUARD PLATE MAXIMUM DEFLECTIONS

\begin{tabular}{|l|l}
\hline 0 & \\
\hline $4 / 17 / 97$ & \\
\hline $4 / 17 / 97$ & \\
\hline
\end{tabular}


CLIENT: Duke Engineering \& Services Hanford

FILE NO: KH-8009-8-03

PROJECT: MCO Final Design DOC. NO.: HNF-SD-SNF-DR-003, Rev. 0, Appendix 5

\section{LIST OF FIGURES}

FIGURE 1: MCO HALF-SYMMETRY ASSEMBLY WITHOUT LIFTING CAP

Page

FIGURE 2: DETAIL OF UPPER MCO ASSEMBLY WITHOUT LIFTING CAP

FIGURE 3: MCO HALF-SYMMETRY ASSEMBLY WITH MODIFIED LIFTING CAP

FIGURE 4: DETAIL OF UPPER MCO ASSEMBLY WITH MODIFIED LIFTING CAP

45

FIGURE 5: MODEL WITH COUPLED NODES AT JACKING BOLT AND BUTTRESS THREADS

FIGURE 6: BARE BOTTOM END DROP LOWER BOUNDARY CONDITIONS W/ GAP

ELEMENTS

FIGURE 7: BARE BOTTOM END DROP UPPER BOUNDARY CONDITIONS

FIGURE 8: BARE BOTTOM END DROP PRESSURE DISTRIBUTION (TNTERNAL \&

HYDROSTATIC)

FIGURE 9: BARE BOTTOM END DROP STRESS INTENSITIES

FIGURE 10: BARE BOTTOM END DROP UPPER ASSEMBLY STRESS INTENSITIES

FIGURE 11: BARE BOTTOM END DROP LOWER ASSEMBLY STRESS INTENSITIES

FIGURE 12: BARE BOTTOM END DROP LOWER ASSEMBLY RADIAL DISPLACEMENTS

FIGURE 13: BARE BOTTOM END DROP W/ FINE MESH, LOWER STRESS INTENSITIES

FIGURE 14: BARE BOTTOM END DROP W/ FINE MESH, CLOSE-UP

FIGURE 15: BARE BOTTOM END DROP W/ FINE MESH, RADIAL DISPLACEMENTS

FIGURE 16: TOP END DROP LOWER MCO BOUNDARY CONDITIONS

FIGURE 17: TOP END DROP UPPER MCO BOUNDARY CONDITIONS W/ GAP ELEMENTS

FIGURE 18: TOP END DROP PRESSURE DISTRIBUTION (HYDROSTATIC \& INTERNAL)

FIGURE 19: TOP END DROP UPPER MCO ASSEMBLY STRESS INTENSITIES

FIGURE 20: TOP END DROP UPPER MCO ASSEMBLY RADIAL DISPLACEMENTS

FIGURE 21: CSB TUBE DROP (NO CAP) PRESSURE DISTRIBUTION

FIGURE 22: CSB TUBE DROP (NO CAP) UPPER MCO ASSEMBLY STRESS INTENSITIES

FIGURE 23: CSB TUBE DROP (NO CAP) LOWER MCO ASSEMBLY STRESS INTENSITIES

FIGURE 24: CSB TUBE DROP (NO CAP) LOWER MCO ASSEMBLY RADIAL

DISPLACEMENTS

FIGURE 25: TOP END DROP WITH MODIFIED CAP AND UPPER BOUNDARY CONDITIONS

FIGURE 26: TOP END DROP WITH MODIFIED CAP AND LOWER BOUNDARY CONDITIONS

FIGURE 27: TOP END DROP WITH MODIFIED CAP AND UPPER STRESS INTENSITIES

FIGURE 28: TOP END DROP, MODIFIED CAP @ CLOSURE WELD, STRESS INTENSITIES

FIGURE 29: TOP END DROP WITH MODIFIED CAP LOWER STRESS INTENSITIES

FIGURE 30: TOP END DROP WITH MODIFIED CAP AND RADIAL DISPLACEMENTS

46

47

48

49

50

51

52

53

54

55

56

57

58

59

60

61

62

63

64

65

66

67

68

69

70

71

FIGURE 31: CSB TUBE DROP WITH MODIFIED CAP AND UPPER BOUNDARY CONDITIONS

FIGURE 32: CSB TUBE DROP WITH MODIFIED CAP AND LOWER BOUNDARY

CONDITIONS

\begin{tabular}{|l|c|l|l|l|l|}
\hline REVISION & 0 & & & \\
\cline { 1 - 3 } PREPARED BY IDATE & PAGE 5 & \\
OF 198
\end{tabular}


CLIENT: Duke Engineering \& Services Hanford

PROJECT: MCO Final Design DOC. NO.: HNF-SD-SNF-DR-003, Rev. 0, Appendix 5

FIGURE 33: CSB TUBE DROP WITH MODIFIED CAP AND EQUIVALENT PRESSURE DISTRIBUTION

FIGURE 34: CSB TUBE DROP WITH MODIFIED CAP AND UPPER STRESS INTENSITIES 76

FIGURE 35: CSB TUBE DROP, MODIFIED CAP @ CLOSURE WELD STRESS INTENSITIES 77

FIGURE 36: CSB TUBE DROP WITH MODIFIED CAP AND LOWER STRESS INTENSITIES 78

FIGURE 37: CSB TUBE DROP WITH MODIFIED CAP AND RADIAL DISPLACEMENTS

\section{APPENDICES}

APPENDIX A: COMPUTER RUN OUTPUT SHEETS \& INPUT FILE LISTINGS 


\section{国 PAasons}

CLIENT: Duke Engineering \& Services Hanford

FILE NO: KH-8009-8-03

PROJECT: MCO Final Design

DOC. NO.: HNF-SD-SNF-DR-003, Rev. 0, Appendix 5

\section{INTRODUCTION}

The MCO assembly is a single purpose Spent Nuclear Fuel (SNF) container that is capable of maintaining subcriticality at all times and maintain SNF containment and confinement after being closed and sealed. The MCO assembly consists of a shell, a shield plug and one to six SNF baskets.

This calculation documents the evaluation of the MCO shell and the filter guard plate under different drop loads. The loads considered are:

- $54 \mathrm{~g}$ Bare Bottom End Drop, 150 psi internal pressure, $132^{\circ} \mathrm{C}\left(270^{\circ} \mathrm{F}\right)$ temperature.

- $28 \mathrm{~g}$ Top End Drop without lifting cap, $150 \mathrm{psi}$ internal pressure, $132^{\circ} \mathrm{C}\left(270^{\circ} \mathrm{F}\right)$ temperature.

- $28 \mathrm{~g}$ Top End Drop with modified lifting cap, 150 psi internal pressure, $132^{\circ} \mathrm{C}$ $\left(270^{\circ} \mathrm{F}\right)$ temperature.

- $28 \mathrm{~g}$ CSB tube drop of a fully loaded MCO onto another MCO without modified lifting cap.

- $28 \mathrm{~g} \mathrm{CSB}$ Tube Drop of a fully loaded MCO onto another MCO with modified lifting cap.

The evaluations are performed based on the criteria of the ASME Code. A combination of hand calculations and ANSYS@ analysis is used.

\section{REFERENCES}

1. "Performance Specification for the Spent Nuclear Fuel Multi-Canister Overpack," Specification HNF-S-0426, Revision 3, February 1997.

2. ASME Boiler and Pressure Vessel Code, Section II - Materials, Part D - Properties, 1995 Edition with 1995 Addenda.

3. ASME Boiler and Pressure Vessel Code, Section III, Subsection NB - Class 1 Components, 1995 Edition with 1995 Addenda and 1995 Appendix F.

4. Roark, Raymond J., \& Young, Warren C., "Formulas for Stress and Strain", $6^{\text {th }}$ Edition, McGraw-Hill Book Company, New York, 1989. 
CLIENT: Duke Engineering \& Services Hanford

FILE NO: $\quad \mathrm{KH}-8009-8-03$

PROJECT: MCO Final Design

DOC. NO.: HNF-SD-SNF-DR-003, Rev. 0, Appendix 5

5. Swanson Analysis Systems, Inc., ANSYS@ Engineering Analysis System User's Manual, Volumes I, II, and III, Version 5.0A, 23 December 1992.

6. Parsons I \& T, File KH-8009-8-05, "Stress Analysis of the Mark IA Storage and Scrap Basket", March 1997.

7. ASME Boiler and Pressure Vessel Code, Supplement No. 11, Code Cases for Nuclear Components, 1995 Edition.

8. Appendix 1 of the MCO Design Report, MCO Drawings.

9. Appendix 13 of the MCO Design Report, Main Seal Data.

10. Manual of Steel Construction, American Institute of Steel Construction, 8th Edition, 1980.

\section{ASSUMPTIONS}

1. Pressure is applied uniformly.

2. The maximum weight of a fully loaded Mark IV basket is $3189.96 \mathrm{lbs}$. The following analyses are conservative using $3200 \mathrm{lbs}$ for each basket.

3. Maximum shell diameter at the collar is increased from 25.27 to 25.31 inches. Calculations performed in this analysis are based on 25.27 inches and therefore are conservative.

4. Others as noted.

\section{MATERIAL PROPERTIES}

The MCO shell, shield plug and filter guard plate are fabricated from Type $304 \mathrm{~L}$ stainless steel. For the shell, Type 304L is used with the tensile and yield properties of Type 304 stainless steel ( 75 and $30 \mathrm{ksi}$, respectively). For this analysis, allowable stress values are taken from Section II, Part D of the Code (See [2]) and are listed in Table 1.

For the shield plug, locking ring, lifting cap and canister collar, Type $304 \mathrm{~L}$ stainless steel may be replaced with Type $304 \mathrm{~N}$ or $304 \mathrm{LN}$ at the discretion of the designer. The effects of thermal conductivity, thermal expansion, minimum yield strength and minimum tensile strength have been evaluated and it is the conclusion of the preparer that by using $304 \mathrm{~N}$ 
the minimum tensile and yield strengths are increased to $80 \mathrm{ksi}$ and $35 \mathrm{ksi}$, respectively, for each material, thereby decreasing the appropriate stress ratios of this calculation.

\section{ACCEPTANCE CRITERIA}

This calculation considers only drop loads. Criteria for the evaluated drop loads must meet Section III, Subsection NB of the ASME Code with Appendix F [3] and Section 4.9 .5 of the MCO Performance Specification [1].

The acceptance criteria of Appendix F (F-1331.1) of the ASME Code states that when an elastic analysis is used " the general primary membrane stress intensity $P_{M}$ shall not exceed the lesser of $2.4 \mathrm{~S}_{M}$ and $0.7 \mathrm{~S}_{\mathrm{U}}$ for materials included in Section II, Part D, Subpart I, Tables $2 \mathrm{~A}$ and $2 \mathrm{~B}$ for austenitic and high alloy steels, or $0.7 \mathrm{~S}_{U}$ for ferritic materials included in Table $2 A$ " and "the local primary membrane stress intensity $P_{L}$ shall not exceed $150 \%$ of the limit for general primary membrane stress intensity $P_{M}$."

At the maximum drop temperature of $132^{\circ} \mathrm{C}\left(270^{\circ} \mathrm{F}\right)$, the allowables $P_{M}$ and $P_{L}$ are summarized in Tables 3 \& 4 .

For Type 304:

$$
\begin{aligned}
& 2.4 S_{M}=(2.4) \times(20.0)=48.0 \mathrm{ksi} \\
& 0.7 S_{U}=(0.7) \times(67.5)=47.25 \mathrm{ksi} \\
& 2.4 S_{M}>0.7 S_{U} \\
& \Rightarrow \text { use } P_{M} \leq 47.25 \mathrm{ksi}
\end{aligned}
$$

For Type 304L:

$$
\begin{aligned}
2.4 S_{M} & =(2.4) \times(16.7)=40.08 \mathrm{ksi} \\
0.7 S_{U} & =(0.7) \times(62.5)=43.75 \mathrm{ksi} \\
& 2.4 S_{M}<0.7 S_{U}
\end{aligned}
$$

$$
\Rightarrow \text { use } P_{M} \leq 40.08 \mathrm{ksi}
$$

$\mid$\begin{tabular}{c|}
0 \\
$\mid 2 / 4 / 4 / 17 / 97$ \\
$\mid \mathbb{A} 4 / 17 / 97$
\end{tabular}


CLIENT: Duke Engineering \& Services Hanford

Table 1: ASME Code Material Properties for Type 304 Stainless Steel

\begin{tabular}{|c|c|c|c|c|c|c|}
\hline \multicolumn{2}{|c|}{ Temperature } & \multirow{2}{*}{$\begin{array}{c}E^{1} \\
\left(\times 10^{6} p s i\right) \\
\end{array}$} & \multirow{2}{*}{$\begin{array}{c}\alpha^{2} \\
\left(\times 10^{-6} \text { in./in. } /^{\circ} \mathrm{F}\right) \\
\end{array}$} & \multirow{2}{*}{$\begin{array}{l}\mathrm{sy}^{3} \\
\text { (ksi) }\end{array}$} & \multirow{2}{*}{$\begin{array}{l}s_{m^{4}} \\
(k s i) \\
\end{array}$} & \multirow{2}{*}{$\begin{array}{l}\mathrm{s}_{\mathrm{u}}^{5} \\
\text { (ksi) }\end{array}$} \\
\hline${ }^{\circ} \mathrm{F}$ & ${ }^{\circ} \mathrm{C}$ & & & & & \\
\hline-20 & -29 & - & - & 30.0 & 20.0 & 75.0 \\
\hline 70 & 21 & 28.3 & - & 30.0 & 20.0 & 75.0 \\
\hline 100 & 38 & $\underline{28.16}$ & 8.55 & 30.0 & 20.0 & 75.0 \\
\hline 200 & 93 & 27.6 & 8.79 & 25.1 & 20.0 & 71.0 \\
\hline 270 & 132 & 27.2 & 8.94 & 23.3 & 20.0 & 67.5 \\
\hline 300 & 149 & 27.0 & 9.00 & 22.5 & 20.0 & 66.0 \\
\hline 392 & 200 & $\underline{26.5}$ & 9.18 & 20.9 & 18.8 & $\underline{64.5}$ \\
\hline 400 & 204 & 26.5 & 9.19 & 20.8 & 18.7 & 64.4 \\
\hline 500 & 260 & 25.8 & 9.37 & 19.4 & 17.5 & 63.5 \\
\hline 600 & 316 & 25.3 & 9.53 & 18.3 & 16.4 & 63.5 \\
\hline 700 & 371 & 24.8 & 9.69 & 17.7 & 16.0 & 63.5 \\
\hline 707 & 375 & 24.8 & 9.70 & 17.6 & 16.0 & 63.5 \\
\hline 800 & 427 & 24.1 & 9.82 & 16.9 & 15.2 & 62.7 \\
\hline
\end{tabular}

${ }^{1}$ Table TM-1, Material Group G, P. 614

${ }^{2}$ Table TE-1, P 590-591

${ }^{3}$ Table Y-1, P. 530-531

${ }^{4}$ Table 2A, P. 326

${ }^{5}$ Table U, P. 441

${ }^{6}$ Underlined values determined by linear interpolation, all others taken from ASME Code, Section II, Part D. 


\section{PAASENS}

CLIENT: Duke Engineering \& Services Hanford

FILE NO: KH-8009-8-03

PROJECT: MCO Final Design

DOC. NO: HNF-SD-SNF-DR-003, Rev. 0, Appendix 5

Table 2: ASME Code Material properties for Type 304L Stainless Steel

\begin{tabular}{|c|c|c|c|c|c|c|}
\hline \multicolumn{2}{|c|}{ Temperature } & \multirow{2}{*}{$\begin{array}{c}E^{7} \\
\left(\times 10^{6} \mathrm{psi}\right) \\
\end{array}$} & \multirow{2}{*}{$\begin{array}{c}\alpha^{8} \\
\left(\times 10^{-6} \text { in./in./ } /{ }^{\circ} \mathrm{F}\right) \\
\end{array}$} & \multirow{2}{*}{$\begin{array}{l}\mathrm{S}_{\mathrm{y}}{ }^{9} \\
\text { (ksi) }\end{array}$} & \multirow{2}{*}{$\begin{array}{l}s_{m} 10 \\
(k s i)\end{array}$} & \multirow{2}{*}{$\begin{array}{l}s_{u^{11}} \\
(k s i)\end{array}$} \\
\hline${ }^{\circ} \mathrm{F}$ & ${ }^{\circ} \mathrm{C}$ & & & & & \\
\hline-20 & -29 & & - & 25.0 & 16.7 & 70.0 \\
\hline 70 & 21 & 28.3 & - & 25.0 & 16.7 & 70.0 \\
\hline 100 & 38 & $28.1^{12}$ & 8.55 & 25.0 & 16.7 & 70.0 \\
\hline 200 & 93 & 27.6 & 8.79 & 21.3 & 16.7 & 66.2 \\
\hline 270 & 132 & 27.2 & 8.94 & 19.8 & 16.7 & 62.5 \\
\hline 300 & 149 & 27.0 & 9.00 & 19.1 & 16.7 & 60.9 \\
\hline 392 & 200 & $\underline{26.5}$ & 9.18 & 17.6 & 15.9 & 58.7 \\
\hline 400 & 204 & 26.5 & 9.19 & 17.5 & 15.8 & 58.5 \\
\hline 500 & 260 & 25.8 & 9.37 & 16.3 & 14.8 & 57.8 \\
\hline 600 & 316 & 25.3 & 9.53 & 15.5 & 14.0 & 57.0 \\
\hline 700 & 371 & 24.8 & 9.69 & 14.9 & 13.5 & 56.2 \\
\hline 707 & 375 & 24.8 & 9.70 & 14.9 & $\underline{13.5}$ & 56.2 \\
\hline 800 & 427 & 24.1 & 9.82 & 14.4 & 13.0 & 55.5 \\
\hline
\end{tabular}

Furthermore, the criteria of Section 4.9.5 of the MCO Performance Specification states that

"In no case, including post accident conditions, is the MCO inside circumference below the bottom of the shield plug allowed to exceed 73.04 inches $(23.25$ in. ID $\times \pi)$ ". Uitimately the MCO shell inside diameter is not to exceed 23.25 inches.

7 Table TM-1, Material Group G, P. 614

${ }^{8}$ Table TE-1, P.590-591

${ }^{9}$ Table Y-1, P. 524

${ }^{10}$ Table 2A, P. 322

${ }^{11}$ Table U, P. 441

${ }^{12}$ Underlined values determined by linear interpolation, all others taken from ASME Code, Section II, Part D.

\begin{tabular}{|c|l|l|l|}
\hline 0 & & & \\
\hline Xy 4/17/97 & & & \\
\hline
\end{tabular}


CLIENT: Duke Engineering \& Services Hanford

FILE NO: $\quad \mathrm{KH}-8009-8-03$

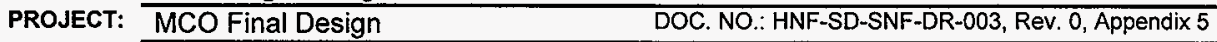

Although the MCO Performance Specification gives limits for Type 304L stainless steel, the values of Table 1 are used since the MCO drops must meet ASME Code requirements.

Table 3: Level D Allowable Stresses - Elastic Analysis - Type 304 Stainless Steel

\begin{tabular}{||c|c|c|c|c|c||}
\hline \hline \multicolumn{2}{||c|}{ Temperature } & $S_{U}(\mathrm{ksi})$ & \multicolumn{3}{c||}{ Level D Stress Limits ${ }^{1}(\mathrm{ksi})$} \\
\hline${ }^{\circ} \mathrm{F}$ & ${ }^{\circ} \mathrm{C}$ & $($ Table 1) & $\begin{array}{c}\mathrm{P}_{\mathrm{m}} \\
\left(0.7 \mathrm{~S}_{\mathrm{U}}\right)\end{array}$ & $\begin{array}{c}\mathrm{P}_{\mathrm{L}} \\
\left(1.5 \mathrm{P}_{\mathrm{m}}\right)\end{array}$ & $\begin{array}{c}\mathrm{P}_{\mathrm{M}}\left(\text { or } \mathrm{P}_{\mathrm{L}}\right)+\mathrm{P}_{\mathrm{B}} \\
\left(1.5 \mathrm{P}_{\mathrm{m}}\right)\end{array}$ \\
\hline 270 & 132 & 67.50 & 47.25 & 70.88 & 70.88 \\
\hline
\end{tabular}

Table 4: Level D Allowable Stresses - Elastic analysis - Type 304L Stainless Steel

\begin{tabular}{|l|c|c|c|c|c|}
\hline \multicolumn{2}{|c|}{ Temperature } & $\mathrm{S}_{\mathrm{M}}(\mathrm{ksi})$ & \multicolumn{3}{c|}{ Level D Stress Limits $^{1}(\mathrm{ksi})$} \\
\hline${ }^{\circ} \mathrm{F}$ & ${ }^{\circ} \mathrm{C}$ & $($ Table 2) & $\begin{array}{c}\mathrm{P}_{\mathrm{m}} \\
\left(2.4 \mathrm{~S}_{\mathrm{M}}\right)\end{array}$ & $\begin{array}{c}\mathrm{P}_{\mathrm{L}} \\
\left(1.5 \mathrm{P}_{\mathrm{m}}\right)\end{array}$ & $\begin{array}{c}\mathrm{P}_{\mathrm{M}}\left(\text { or } \mathrm{P}_{\mathrm{L}}\right)+\mathrm{P}_{\mathrm{B}} \\
\left(1.5 \mathrm{P}_{\mathrm{m}}\right)\end{array}$ \\
\hline 270 & 132 & 16.7 & 40.08 & 60.12 & 60.12 \\
\hline
\end{tabular}

\section{LOAD CONDITIONS \& COMBINATIONS}

The MCO assembly is evaluated for the following conditions:

1) $54 \mathrm{~g}$ Bare Bottom End Drop with $150 \mathrm{psi}$ internal pressure and a temperature of $132{ }^{\circ} \mathrm{C}$ $\left(270^{\circ} \mathrm{F}\right)$.

2) $28 \mathrm{~g}$ Top End Drop with $150 \mathrm{psi}$ internal pressure, a temperature of $132^{\circ} \mathrm{C}\left(270^{\circ} \mathrm{F}\right)$ without lifting cap.

3) $28 \mathrm{~g}$ Top End Drop with $150 \mathrm{psi}$ internal pressure, a temperature of $132^{\circ} \mathrm{C}\left(270^{\circ} \mathrm{F}\right)$ with modified lifting cap.

${ }^{1}$ Level D Stress Limits taken from Appendix F, F-1331, 1995 ASME Code.

${ }^{1}$ Level D Stress Limits taken from Appendix F, F-1331, 1995 ASME Code.

\begin{tabular}{|l|c|l|l|l|}
\hline REVISION & 0 & & & \\
\hline PREPARED BY IDATE & YKS 4/17/97 & & & \\
\hline CHECKED BY I DATE & ZN-4/17/97 & & & \\
\hline
\end{tabular}

PAGE 12

OF 198 


\section{E.paAsans}

CLIENT: Duke Engineering \& Services Hanford

PROJECT: MCO Final Design

FILE NO: KH-8009-8-03

In each of the above condition, the MCO assembly is fully loaded with five Mark IV baskets weighing each a maximum of $3,200 \mathrm{lbs}$ ( for a total weight of $16,000 \mathrm{lbs}$ ).

The $54 \mathrm{~g}$ Bare bottom End Drop binds the following cases :

- $25 \mathrm{~g}$ vertical "Piston Drop" into cask

- $27 \mathrm{~g}$ vertical drop with cask

- $35 \mathrm{~g}$ vertical drop into CSB tube

4) Drop of a MCO onto another MCO in the CSB tube. The bottom MCO receives the equivalent of $28 \mathrm{~g} \times$ the fully loaded top MCO onto its shield plug.

5) Drop of a MCO onto another MCO in the CSB tube. The bottom MCO receives the equivalent of $28 \mathrm{~g} \times$ the fully loaded top MCO onto its modified lifting cap.

\section{FILTER GUARD PLATE}

\subsection{Introduction}

The guard plate on the MCO shield plug is evaluated for its ability to protect the internal filters in a top down drop load. The plate has to withstand a $28 \mathrm{~g}$ load of the entire payload of the MCO including baskets. Conservatively, no credit is taken for the baskets to support any of the fuel. Service level D stress allowables are used for the acceptance criteria. The load level of $28 \mathrm{~g}$ 's binds the loading case of $27.4 \mathrm{~g}$ 's (lid down) in Table 3 of the MCO Performance Specification.

\subsection{Method of Analysis}

A hand calculation is performed for the buckling of the guard plate support ring. A hand calculation is also performed to size the weld between the ring and the shield plug and the weld between the plate and its supporting ring.

\subsection{Assumptions}

1. The fuel loads the plate uniformly

2. The baskets provide no support to any of the fuel

कर


CLIENT: Duke Engineering \& Services Hanford

FILE NO: KH-8009-8-03

PROJECT: MCO Final Design $\quad$ DOC. NO.: HNF-SD-SNF-DR-003, Rev. 0, Appendix 5

3. Temperature at impact is $132^{\circ} \mathrm{C}\left(270^{\circ} \mathrm{F}\right)$

4. Maximum weight comes from 5 Mark IV baskets; each basket weighing a maximum of $3,200 \mathrm{lbs}$.

\subsection{Geometry}

The support ring is a 1 inch thick ring 4.25 inches high and 22.50 in. OD. Drawing H-2828046, Rev C. "MCO Internal Filter Shield Plate" is used for reference and dimensions.

\subsection{Material Properties}

The guard plate and its support ring are fabricated from SA-240 Type 304L stainless steel.
At $132^{\circ} \mathrm{C}\left(270^{\circ} \mathrm{F}\right)$ :
$E=27.2 \times 10^{6} \mathrm{psi}$
$\mathrm{S}_{\mathrm{M}}=16.7 \mathrm{ksi}$
$v=0.3$
$S_{Y}=19.8 \mathrm{ksi}$
$\mathrm{S}_{\mathrm{u}}=62.5 \mathrm{ksi}$

\subsection{Acceptance Criteria}

The guard plate must not deflect more than one inch as it needs to protect the filters. The support ring must also be checked for buckling.

\subsection{Load Combinations}

The only load considered for this calculation is an acceleration of the fuel at $\mathbf{2 8} \mathrm{g}$ 's for buckling check of the ring. For the welds, an acceleration of $101 \mathrm{~g}$ 's is used as it provides a worst case acceleration for a side load.

\subsection{Analysis}

\subsubsection{Support Ring}

For the support ring, the assumption is that the entire load is taken on the ring.

The area of the ring is:

0

$4 / 17 / 97$

$-4 / 17 / 97$

PAGE 14 OF 198 


$$
\mathrm{A}_{\mathrm{r}}=\pi \times\left[\frac{22.5^{2}}{4}-\frac{20.5^{2}}{4}\right]=67.54 \mathrm{in}^{2}
$$

and $F=(5$ baskets $)(3200 \mathrm{lbs})(28 \mathrm{~g})=448,000 \mathrm{lbs}$

The stress in the ring is:

$$
\sigma=\frac{F}{A_{r}}=\frac{448,000 \mathrm{lbs}}{67.54 \mathrm{in}^{2}}=6633.1 \mathrm{psi} \text { or } 6633 \mathrm{psi}
$$

The stress $\sigma$ is well below the allowables of Service Level $D$ (See Table 2).

Assuming a uniform axial load $p$, the change in the height dimension can be found by:

From Roark's [4], Table 28, Case 1 a.

$$
\begin{aligned}
& \frac{R}{t}=\frac{10.25 \mathrm{in}}{1.00 \mathrm{in}}=10.25 \\
& \Delta y=\frac{p y}{E t}
\end{aligned}
$$

where $\quad p=$ Unit Load $=\frac{448000 \mathrm{lbs}}{(\pi) \times\left(\frac{20.5+22.5}{2}\right)}=6633 \mathrm{lbs} / \mathrm{in}$

$$
y=\text { Ring Height }=4.25 \text { inches }
$$

$$
\Delta y=\frac{(6633 \mathrm{lbs} / \mathrm{in}) \times(4.25 \mathrm{in})}{\left(27.2 \times 10^{6} \mathrm{psi}\right) \times(1.00 \mathrm{in})}=0.001 \text { inches }
$$

Because of its geometry and height, buckling of the ring is not a concern as shown above. 
CLIENT: Duke Engineering \& Services Hanford

PROJECT: MCO Final Design

FILE NO: KH-8009-8-03

DOC. NO.: HNF-SD-SNF-DR-003, Rev. 0, Appendix 5

\subsubsection{Weld Sizing}

Welding of the support ring to the shield plug and the guard plate to the support ring requires the use of structural welds. The size of the welds is determined as follows:

From Appendix 3, Calculation $\mathrm{KH}-8009-8-01$, the nominal weights of the support ring and the guard plate are given as follows:

$$
\begin{aligned}
& W_{\text {RING }}=81.12 \mathrm{lbs} \\
& W_{\text {PLATE }}=155.20 \mathrm{lbs}
\end{aligned}
$$

The center of gravity, from the bottom of the shield plug, of those two components is:

$$
\begin{aligned}
& C G=\frac{\left(W_{P L A T E}\right)\left(X_{P L A T E}\right)+\left(W_{R I N G}\right)\left(X_{R I N G}\right)}{W_{P L A T E}+W_{R I N G}} \\
& C G=\frac{(155.20 l b s)\left(4.25 \mathrm{in}+\frac{1.50 \mathrm{in}}{2}\right)+(81.12 \mathrm{lbs})\left(\frac{4.25 \mathrm{in}}{2}\right)}{(155.20 \mathrm{lbs})+(81.12 \mathrm{lbs})}
\end{aligned}
$$

$$
C G=4.01 \text { inches }
$$

The required length of weld is

$$
\mathrm{L}_{w}=\pi \mathrm{D}=70.7 \text { inches }
$$

and the weld section modulus is

$$
\begin{aligned}
& \mathrm{S}_{\mathrm{w}}=\frac{\pi(D)^{2}}{4}=\frac{\pi(22.50 \mathrm{in})^{2}}{4} \\
& \mathrm{~S}_{\mathrm{w}}=398 \mathrm{in}^{2}
\end{aligned}
$$

The rules and stress limits which must be satisfied for welds for any Level $A$ through $D$ Service are those given in Table NF-3324.5(a)-1 multiplied by the appropriate base material stress limit factor given in Table NF-3523(b)-1 for components supports. However, per Table NF-3523(b)-1, for Service Level D, the stress limit factors must be obtained from Appendix F (F-1334) of the Code.

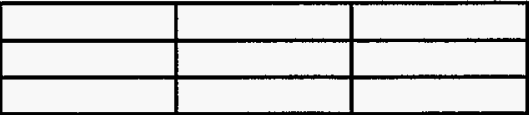




\section{P. Parsons}

CLIENT: Duke Engineering \& Services Hanford

FILE NO: KH-8009-8-03

PROJECT: MCO Final Design DOC. NO.: HNF-SD-SNF-DR-003, Rev. 0, Appendix 5

From Table NF-3324.5(a)-1:

For the base metal (Type 304L) $S_{U} @ 707^{\circ} \mathrm{F}=56.2 \mathrm{ksi}$ (from Table 1 above)

therefore $S_{U}$ for weld metal is $60 \mathrm{ksi}$.

The stress limits then become

For the base metal $F_{W}=0.40\left(S_{Y, \text { Base Metal }}\right)=0.40(14.9 \mathrm{ksi})=5.96 \mathrm{ksi}$

For the weld metal $F_{W}=0.30\left(S_{u, \text { weld }}\right)=0.30(60 \mathrm{ksi})=18.0 \mathrm{ksi}$

And from Appendix F, F-1334 of the Code "the allowable stress presented for Level A Service Condition may be increased using the following factors: the smaller of 2 or $1.167 \frac{S_{U}}{S_{Y}}$ if $S_{U}>1.2 S_{Y}$ or 1.4 , if $S_{U} \leq 1.2 S_{Y}$, where $S_{Y}$ is the yield strength, in $k s i$, and $S_{U}$ is the ultimate tensile strength, in ksi, both at temperature."

$1.2 S_{Y}=1.2(14.9)=17.88>S_{U}$, therefore

$1.167 \frac{S_{U}}{S_{y}}=1.167 \frac{56.2 \mathrm{ksi}}{14.9 \mathrm{ksi}}=4.38>2$

Using a factor of $2, F_{W}$ then becomes:

For the base metal $F_{W}=2(18.0 \mathrm{ksi})=36.0 \mathrm{ksi}$

For the weld metal $F_{W}=2(5.96 \mathrm{ksi}) 11.9 \mathrm{ksi}$

A $101 \mathrm{~g}$ side drop is the worst possible case and therefore is considered.

$$
\begin{aligned}
& f_{W 1}=\frac{(101 g)\left(W_{R N G}+W_{P L A T E}\right)}{L_{W}}=\frac{(101 g)(81.12 \mathrm{lbs}+155.20 \mathrm{lbs})}{70.7 \mathrm{in}} \\
& f_{W 1}=338 \mathrm{lb} / \mathrm{in} . \\
& f_{W 2}=\frac{(101 g)\left(W_{R N G}+W_{P L A T E}\right)(C G)}{S_{W}}=\frac{(101 g)(81.12 \mathrm{lbs}+155.20 \mathrm{lbs})(4.01 \mathrm{in})}{398 \mathrm{in}^{2}} \\
& f_{W 2}=240 \mathrm{lb} / \mathrm{in} .
\end{aligned}
$$

\begin{tabular}{|c|l|l|l|}
\hline 0 & & & \\
\hline $\lg >4 / 17 / 97$ & & & \\
\hline $\operatorname{Ln} 4 / 17 / 97$ & & & \\
\hline
\end{tabular}




$$
\begin{aligned}
& f_{W}=\sqrt{\left(f_{W 1}\right)^{2}+\left(f_{W 2}\right)^{2}}=\sqrt{(338 l b / i n)^{2}+(240 l b / i n)^{2}} \\
& f_{W}=415 \mathrm{lb} / \text { in }
\end{aligned}
$$

The minimum weld size required is

$$
\begin{aligned}
& \text { For the Throat }=\frac{415 \sqrt{2}}{11900}=0.0943 \mathrm{in} \\
& \text { For the Base }=\frac{415}{36000}=0.011 \mathrm{in}
\end{aligned}
$$

However, the minimum weld size per AISC Specification [10], Table1.17.2A is 5/16 inch. Therefore,

$$
\text { Weld }_{\text {MIN }}=0.3125 \mathrm{in} \text {. }
$$

And the stresses in the 5/16 inch weld are then:

$$
\text { For the Throat }=\frac{415 \sqrt{2}}{0.312 \sin }=1.88 \mathrm{ksi}
$$

For the Base $=\frac{415}{0.3125 \mathrm{in}}=1.33 \mathrm{ksi}$

\section{CLOSURE SEAL LEAKAGE}

A drop of one MCO onto another MCO inside the CSB Tube is defined by the MCO Performance Specification as a $28 \mathrm{~g}$ load. This drop load is reacted at the top of the bottom MCO shield plug, which, in turn, is reacted through the closure seal / seal stop at the bottom of the shield plug. Section 13, Table 7 indicates that the seal stop does not exceed yield, but rather comes close to the allowable yield strength $(22.7 \mathrm{ksi}$ calculated v. $19.8 \mathrm{ksi}$ allowed). This section evaluates the consequences when such seal stop exceeds minimum material yield strength. The seal stop is relatively narrow at 0.270 inches.

Given: $\quad W_{M C O}=$ Weight of fully loaded $M C O=19,374 \mathrm{lb}$.

\begin{tabular}{|l}
\hline REVISION \\
\hline PREPARED BY IDATE \\
\hline CHECKED BYIDATE
\end{tabular}

\begin{tabular}{|l|l|l|}
\hline & & \\
\hline & & \\
\hline & & \\
\hline
\end{tabular}


CLIENT: Duke Engineering \& Services Hanford

$$
\begin{aligned}
& G=G-l o a d=28 \\
& \sigma_{Y}=304 \mathrm{~L} \text { minimum yield strength @ } 132^{\circ} \mathrm{C}=19.8 \mathrm{ksi} \\
& O_{S P}=\text { Shield plug outside diameter }=23.950 \mathrm{in} . \\
& t_{S S}=\text { Seal stop width }=0.270 \mathrm{in} . \\
& A_{S S}=\text { Seal stop area }=20.09 \mathrm{in}^{2} \\
& F_{S}=\text { Minimum seal load }=135,000 \mathrm{lb} .[9] \\
& h_{S S}=\text { Seal stop height }=0.155 \mathrm{in} . \\
& k_{S}=\text { Seal spring constant }=2.42 \times 10^{6} \mathrm{psi}
\end{aligned}
$$

The seal stop stress for a $28 \mathrm{~g}$ drop is

$$
\sigma_{\text {SS }}=\frac{\left(W_{M C O}\right)(G)}{A_{S S}}=2.70 \times 10^{4} \mathrm{psi}
$$

Estimating the plastic strain by conservatively assuming a strain hardening slope of $\times 10^{5}[6]$ psi gives:

$$
\begin{aligned}
& \varepsilon_{\mathrm{P}}=\frac{\sigma_{S S}-\sigma_{y}}{1.6 \times 10^{5} p s i} \\
& \varepsilon_{\mathrm{P}}=0.045 \mathrm{in} / \mathrm{in} \\
& \delta_{\mathrm{P}}=\left(\varepsilon_{\mathrm{P}}\right)\left(\mathrm{h}_{\mathrm{SS}}\right) \\
& \delta_{\mathrm{P}}=6.97 \times 10^{-3} \mathrm{in} .
\end{aligned}
$$

Stress, psi

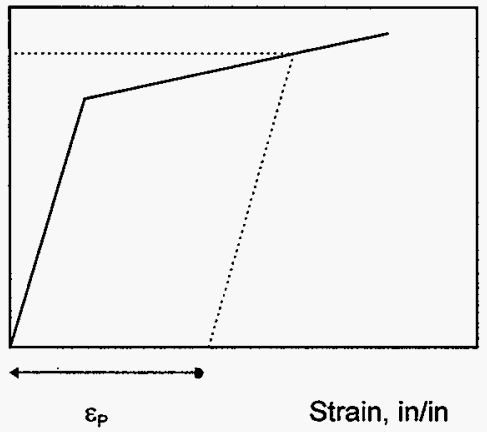

The seal load change due to plastic deformation in the stop is

$$
\begin{aligned}
& \delta \mathrm{F}=\left(\mathrm{k}_{\mathrm{s}}\right)\left(\delta_{\mathrm{p}}\right) \\
& \delta \mathrm{F}=1.125 \times 10^{3} \mathrm{Jb}
\end{aligned}
$$

$\lg 5$

\begin{tabular}{|l|l|l|l|}
\hline 0 & & & \\
\hline $4 / 17 / 97$ & & & \\
\hline $4 / 17 / 97$ & & & \\
\hline
\end{tabular}




\section{P. PARSENS}

CLIENT: Duke Engineering \& Services Hanford

PROJECT: MCO Final Design

FILE NO: $\quad \mathrm{KH}-8009-8-03$

The seal load change due to plastic deformation is very small relative to a permissible drop of $110,000 \mathrm{lb}(135,000-25,000=110,000 \mathrm{lb}$. See Calculation KH-8009-8-02), and therefore seal leakage is not expected.

\section{BARE BOTTOM END DROP}

\subsection{Introduction}

The MCO shell and its contents are evaluated for a two foot $54 \mathrm{~g}$ vertical drop onto a flat reinforced concrete surface. The MCO lands on the bottom end and there is no credible possibility of a side slap down secondary impact of the MCO.

The drop is performed at $132^{\circ} \mathrm{C}\left(270^{\circ} \mathrm{F}\right)$ with an internal pressure of $150 \mathrm{psi}$.

\section{$9.2 \quad$ Geometry}

The MCO geometry used in this calculation is shown in Figure 1. The MCO consists of a cylindrical shell, bottom plate, shield plug, locking ring and jacking bolts. The shell has a diameter which ranges from 24.00 inches to 25.27 inches. The shell diameter is increased to 25.27 inches at beginning of the canister collar to accommodate the shield plug and its locking ring. The MCO cylindrical shell has an inside diameter of 23.00 inches and a length of 139.76 inches. The bottom of the shell is a solid plate 0.880 inches thick at the center and 1.76 inches at the edges.

\subsection{Assumptions}

In the following analysis, it is assumed that the MCO is fully loaded with five Mark IV baskets, two of which are scrap baskets. The fuel is modeled with the properties of stainless steel except for the scrap baskets which have hydrostatic properties when externally loaded.

\section{$9.4 \quad$ Analysis}

\subsubsection{Internal Pressure}

The inside diameter of the MCO shell is 23.00 inches and its outer diameter is 24.00 inches. The wall thickness is therefore 0.5 inch. The stress through the shell due to the pressure load is then

\begin{tabular}{|l|c|l|l|l|}
\hline REVISION & 0 & & & \\
\hline PREPARED BY IDATE & $76) 4 / 17 / 97$ & & & \\
\hline CHECKED BYI DATE & / $4 / 17 / 97$ & & & \\
\hline
\end{tabular}




$$
\sigma_{p}=\frac{p R}{t}
$$

where $\quad p=$ internal pressure $=150 \mathrm{psig}$

$$
R=\text { Mean Radius }=(24.00+23.00) / 4=11.75 \text { in } .
$$

$T=$ thickness of MCO shell $=0.5 \mathrm{in}$.

Therefore

$$
\sigma_{P}=\frac{(150)(11.75)}{0.50}=3525 \text { psi or } 3.53 \mathrm{ksi}
$$

\subsubsection{Hydrostatic Pressure}

No credit is taken for the baskets to support the fuel and the top and bottom scrap baskets' fuel is modeled with hydrostatic properties. Upon impact it is assumed that the bottom basket will be crushed. The values of $\mathrm{K}$ and $\gamma$ are obtained from [6]. The pressure acting on the top of the bottom basket is equivalent to the weight of the four baskets above it and is:

Given:

$$
\begin{aligned}
& A=\text { MCO internal area }=\pi \times \frac{(23.00)^{2}}{4}=415.48 \mathrm{in}^{2} \\
& W_{B}=\text { Maximum weight of a Mark IV basket }=3200 \mathrm{lbs} \\
& G=g \text {-load }=54 \\
& K=\text { Pressure Coefficient }=0.3 \\
& \gamma=\text { Density of fuel }=0.217 \mathrm{lb} / \mathrm{in}^{3} \\
& h=\text { height of basket } \\
& \quad P=\frac{(4 \text { baskets }) \times(3200 \mathrm{lbs})}{415.48 \mathrm{in}^{2}} \times(54 \mathrm{~g}) \times(0.3)=623.86 \mathrm{psi}
\end{aligned}
$$

\begin{tabular}{|c|l|l|l|}
\hline 0 & & & \\
\hline$x / 4>4 / 17 / 97$ & & & \\
\hline$/ 24 / 17 / 97$ & & & \\
\hline
\end{tabular}




\section{PAASONS}

CLIENT: Duke Engineering \& Services Hanford

PROJECT: MCO Final Design

FILE NO: KH-8009-8-03

DOC. NO.: HNF-SD-SNF-DR-003, Rev. 0, Appendix 5

The fuel exerts against the wall of the shell a horizontal pressure equal to the vertical pressure. At any level, the vertical pressure equals the weight of a $1-\mathrm{ft}^{2}$ column of fuel above that level. Hence, the horizontal pressure $P_{h}$ at any level is

$$
\mathrm{P}_{\mathrm{h}}=(\mathrm{K})(\mathrm{G})(\Delta \mathrm{h})(\gamma)+\mathrm{P}
$$

The pressure distribution is triangular.

For $h=26.431$ inches, the additional pressure is

$$
P_{\text {add }}=\left(0.217 \mathrm{lb} / \mathrm{in}^{3}\right)(54 \mathrm{~g})(0.3)(26.431 \mathrm{in})=92.90 \mathrm{psi}
$$

and the maximum pressure (the base of the triangular distribution) is

$$
P=92.90+623.86=716.76 p s i
$$

This pressure is additional to the internal 150 psi stated above. In accordance with 4.9 .5 of the MCO Performance Specification, the shell's maximum circumference is not to exceed 73.04 inches $(23.25 \times \pi)$. At 23.25 inches, the maximum radial displacement allowed is 0.25 inch diametrically, or 0.125 inch radially. For a pressure at a point measured 18.58 inches above the inside bottom plate of the MCO, the radial displacement of the circumference is:

From Roark's [4], Table 28, Cases 1b \& 1d. Deformation in thin-walled pressure vessel, with a uniform radial pressure and an additional varying linearly radial pressure. A combination of Case $1 \mathrm{~b}$ and $1 \mathrm{~d}$ is used.

$$
\Delta R_{1 b}=\frac{q R^{2}}{E t} \quad \Delta R_{1 d}=\frac{q_{0} R^{2} y}{E t l} \text { and } q=\frac{q_{o} y}{l}
$$

where $q=$ unit pressure

$R=$ Radius of Curvature $=11.50$ inches

$\mathrm{E}=$ Young's Modulus $=27.2 \times 10^{6} \mathrm{psi}$

$\mathrm{t}=$ Wall thickness of the pressure vessel $=0.50$ inches

$y=$ Vertical position coordinate $=18.58$ inches

$I=$ Shell length $=139.76$ inches

Solving for $\mathrm{q}$ in Case $1 \mathrm{~b}$ :

$$
q=150 \mathrm{psi}
$$




\section{Dipasans}

CLIENT: Duke Engineering \& Services Hanford

FILE NO: $\quad \mathrm{KH}-8009-8-03$

PROJECT: MCO Final Design

DOC. NO.: HNF-SD-SNF-DR-003, Rev. 0, Appendix 5

therefore $\Delta R_{1 b}=\frac{(150 \mathrm{psi}) \times(11.5)^{2}}{\left(27.2 \times 10^{6} \mathrm{psi}\right) \times(0.5 \mathrm{in})}=0.00146$ inches

Solving for $q_{0}$ in Case $1 d$ :

$$
\begin{aligned}
& q=p+P+P_{\text {add }}=150+623.86+\left(0.217 \mathrm{lb} / \mathrm{in}^{3}\right)(54 \mathrm{~g})(0.3)(26.431 \mathrm{in}-18.580 \mathrm{in}) \\
& q=801.14 \mathrm{psi}
\end{aligned}
$$$$
\mathrm{q}_{0}=\frac{q l}{y}=\frac{(801.14)(139.76)}{18.58}=6026 \mathrm{lb} \text {. }
$$

therefore $\Delta \mathrm{R}_{1 \mathrm{~d}}=\frac{(6026 \mathrm{psi})(11.50)^{2}(18.58 \mathrm{in})}{\left(27.2 \times 10^{6} \mathrm{psi}\right)(0.50 \mathrm{in})(139.76 \mathrm{in})}=0.00779$ inches

Adding the two deflections gives, $\Delta R_{\text {total }}=0.00146+0.00779=0.00925$ inches

This radial displacement is well below the maximum of 0.125 inches

\section{TOP END DROP}

\subsection{Introduction}

The MCO shell and its contents are evaluated for two $28 \mathrm{~g}$ vertical drops. Reference [1] specifies a $25 \mathrm{~g}$ drop. The following $28 \mathrm{~g}$ drops bind the specified case.

The drops are performed at $132^{\circ} \mathrm{C}\left(270^{\circ} \mathrm{F}\right)$ with an internal pressure of $150 \mathrm{psi}$. One drop of the MCO is without the lifting cap and another drop is with the modified lifting cap.

\section{$10.2 \quad$ Geometry}

The MCO geometry used in this calculation is shown in Figures 1, 2, 3 and 4. Figures 1 and 2 refer to the MCO geometry without the lifting cap. Figures 3 and 4 refer to the MCO geometry with the lifting cap. The MCO consists of a cylindrical shell, bottom plate, shield plug, locking ring, jacking bolts and lifting cap. The shell has a diameter which ranges from 24.00 inches to 25.27 inches. The shell diameter is increased to 25.27 inches at beginning of the canister collar to accommodate the shield plug and its locking ring. The MCO shell has an inside diameter of 23.00 inches and a length of 139.76 inches. The bottom of the shell is a solid plate 0.880 inches thick at the center and 1.76 inches at the edges.

2654




\section{$10.3 \quad$ Assumptions}

In the following analysis, it is assumed that the MCO is fully loaded with five Mark IV baskets, two of which are scrap baskets. The fuel is modeled with the properties of stainless steel except for the scrap baskets which have hydrostatic properties when externally loaded.

\section{$10.4 \quad$ Analysis}

\subsubsection{Internal Pressure}

The inside diameter of the MCO shell is 23.00 inches and its outer diameter is 24.00 inches. The wall thickness is therefore $0.5 \mathrm{inch}$. The stress through the shell due to the pressure load is then

$$
\sigma_{P}=\frac{p R}{t}
$$

where $\quad p=$ internal pressure $=150 \mathrm{psig}$

$$
\begin{aligned}
& R=\text { Mean } \text { Radius }=(24.00+23.00) / 4=11.75 \mathrm{in} . \\
& T=\text { thickness of MCO shell }=0.5 \mathrm{in} .
\end{aligned}
$$

Therefore

$$
\sigma_{P}=\frac{(150)(11.75)}{0.50}=3525 \mathrm{psi} \text { or } 3.53 \mathrm{ksi}
$$

\subsubsection{Hydrostatic Pressure}

No credit is taken for the baskets to support the fuel and the top and bottom scrap baskets' fuel is modeled with hydrostatic properties. Upon impact it is assumed that the top basket will be crushed. The values of $K$ and $\gamma$ are obtained from [6]. The pressure acting on the bottom of the top basket is equivalent to the weight of the four baskets above it and is:

Given:

$$
A=\text { MCO internal area }=\pi \times \frac{(23.00)^{2}}{4}=415.48 \mathrm{in}^{2}
$$

36

0
$16 \sqrt{4 / 17 / 97}$
- $4 / 17 / 97$




\section{$P_{\text {Pansons }}$}

CLIENT: Duke Engineering \& Services Hanford

FILE NO: $\quad \mathrm{KH}-8009-8-03$

PROJECT: MCO Final Design

DOC. NO.: HNF-SD-SNF-DR-003, Rev. 0, Appendix 5

$$
\begin{aligned}
& W_{B}=\text { Maximum weight of a Mark IV basket }=3200 \mathrm{lbs} \\
& G=g-l o a d ~=28 \\
& K=\text { Pressure Coefficient }=0.3 \\
& \gamma=\text { Density of fuel }=0.217 \mathrm{lb} / \mathrm{in}^{3} \\
& \mathrm{~h}=\text { height of basket } \\
& \quad P=\frac{(4 \text { baskets }) \times(3200 \mathrm{lbs})}{415.48 \mathrm{in}^{2}} \times(28 \mathrm{~g}) \times(0.3)=258.79 \mathrm{psi}
\end{aligned}
$$

The fuel exerts against the wall of the shell a horizontal pressure equal to the vertical pressure. At any level, the vertical pressure equals the weight of a $1-\mathrm{ft}^{2}$ column of fuel above that level. Hence, the horizontal pressure $P_{h}$ at any level is

$$
P_{h}=(K)(G)(\Delta h)(\gamma)+P
$$

The pressure distribution is triangular.

For $h=27.24$ inches, the additional pressure would be

$$
P_{\text {add }}=\left(0.217 \mathrm{lb} / \mathrm{in}^{3}\right)(28 \mathrm{~g})(0.3)(27.24 \mathrm{in})=49.65 \mathrm{psi}
$$

and the maximum pressure (the base of the triangular distribution) would be

$$
P=49.65+258.79=308.44 \text { psi }
$$

This pressure is additional to the internal 150 psi pressure stated above. In accordance with 4.9.5 of the MCO Performance Specification, the shell's maximum circumference is not to exceed 73.04 inches $(23.25 \times \pi)$. At 23.25 inches, the maximum radial displacement is 0.25 inch diametrically, or 0.125 inch radially. For a pressure at a point measured 24.14 inches below the filter guard plate, the radial displacement of the circumference is:

From Roark's [4], Table 28, Cases $1 \mathrm{~b} \&$ 1d. Deformation in thin-walled pressure vessel, with a uniform radial pressure and an additional varying linearly radial pressure. $A$ combination of Case $1 \mathrm{~b}$ and $1 \mathrm{~d}$ is used.

$$
\Delta R_{1 b}=\frac{q R^{2}}{E t} \quad \Delta R_{1 d}=\frac{q_{0} R^{2} y}{E t l} \text { and } q=\frac{q_{0} y}{l}
$$

where $q=$ unit pressure

$R=$ Radius of Curvature $=11.50$ inches 


\section{E pARSENS}

CLIENT: Duke Engineering \& Services Hanford

FILE NO: KH-8009-8-03

PROJECT: MCO Final Design

DOC. NO.: HNF-SD-SNF-DR-003, Rev. 0, Appendix 5

$E=$ Young's Modulus $=27.2 \times 10^{6} \mathrm{psi}$

$t=$ Wall thickness of the pressure vessel $=0.50$ inches

$y=$ Vertical position coordinate $=24.14$ inches

$I=$ Shell length $=139.76$ inches

Solving for $q$ in Case $1 b$ :

$$
q=150 \mathrm{psi}
$$

therefore $\Delta R_{1 b}=\frac{(150 \mathrm{psi}) \times(11.5)^{2}}{\left(27.2 \times 10^{6} \mathrm{psi}\right) \times(0.5 \mathrm{in})}=0.00146$ inches

Solving for $q_{0}$ in Case $1 d$ :

$$
\begin{aligned}
& q=p+P+P_{\text {add }}=150+258.79+\left(0.217 \mathrm{lb} / \mathrm{in}^{3}\right)(28 \mathrm{~g})(0.3)(27.24 \mathrm{in}-24.14 \mathrm{in}) \\
& q=414.44 \mathrm{psi} \\
& q_{0}=\frac{q l}{y}=\frac{(414.44)(139.76)}{24.14}=2399 \mathrm{lb} .
\end{aligned}
$$

therefore $\Delta \mathrm{R}_{1 \mathrm{~d}}=\frac{(2399 \mathrm{psi})(11.50)^{2}(24.14 \mathrm{in})}{\left(27.2 \times 10^{6} \mathrm{psi}\right)(0.50 \mathrm{in})(139.76 \mathrm{in})}=0.00403$ inches

Adding the two deflections gives, $\Delta R_{\text {total }}=0.00146+0.00403=0.00549$ inches

This radial displacement is well below the maximum of 0.125 inches

\section{CSB TUBE DROP}

\subsection{Introduction}

The MCO is evaluated for a $28 \mathrm{~g}$ drop in the CSB tube. This simulates the vertical drop of one MCO onto another MCO. The top of the lower MCO being impacted receives a $28 \mathrm{~g}$ vertical load and is evaluated with and without the lifting cap on. This evaluation of the CSB Tube drop concentrates on the buckling of the shell and its impact.

\begin{tabular}{|l|c|l|l|l|}
\hline REVISION & 0 & & & \\
\hline PREPARED BY IDATE & $\not 6 / 4 / 17 / 97$ & & & \\
\hline CHECKED BYIDATE & / $4-4 / 17 / 97$ & & & \\
\hline
\end{tabular}




\section{QDansans}

CLIENT: Duke Engineering \& Services Hanford

FILE NO: $\quad \mathrm{KH}-8009-8-03$

PROJECT: MCO Final Design

DOC. NO.: HNF-SD-SNF-DR-003, Rev. 0, Appendix 5

The drop is performed at $132^{\circ} \mathrm{C}\left(270^{\circ} \mathrm{F}\right)$ with an internal pressure of 150 psi.

\section{$11.2 \quad$ Geometry}

The MCO geometry used in this calculation is shown in Figures 1, 2, 3, and 4. Figures 1 and 2 refer to the MCO without lifting cap and Figure 3 and 4 refer to the MCO with lifting cap. The MCO consists of a cylindrical shell, bottom plate, shield plug, locking ring, jacking bolts and lifting cap. The shell has a diameter which ranges from 24.00 inches to 25.27 inches. The shell diameter is increased to 25.27 inches at beginning of the canister collar to accommodate the shield plug and its locking ring. The MCO shell has an inside diameter of 23.00 inches and a length of 139.76 inches. The bottom of the shell is a solid plate 0.880 inches thick at the center and 1.76 inches at the edges.

\subsection{Assumptions}

In the following analysis, it is assumed that the MCO impacting the lower MCO is fully loaded with five Mark IV baskets. The lower MCO is restricted by the CSB Tube.

\subsection{Analysis}

As stated above, this load case analyzes the drop of a fully loaded MCO onto another one. The lower MCO is of concern since the shell is subject to buckling. The top of the MCO being impacted receives the equivalent of a $28 \mathrm{~g}$ load from the upper MCO. The upper MCO lands on the shield plug or the lifting cap of the lower MCO, depending on the evaluation.

The equivalent pressure received by the top of the lower MCO shield plug is calculated by taking the weight of a fully loaded, dry MCO, multiplied by the g-load and divided by the area of the shield plug or the lifting cap.

Where the MCO being impacted does not have the lifting cap, it is assumed that all the weight hits the shield plug first, and not the locking ring. From Appendix $A$ of [1], the weight of a fully loaded MCO, dry, with upper shield plug and five loaded Mark IV baskets is 19,374 Ibs. The area of the shield plug does not include the process ports, therefore it is

$$
\mathrm{A}_{\mathrm{SP}}=39.54 \mathrm{in}^{2}
$$

The equivalent pressure applied is 
CLIENT: Duke Engineering \& Services Hanford

$$
P_{E Q}=(28)(19,374)=542,472 \mathrm{lbs}
$$

therefore $\frac{P_{E Q}}{A_{S P}}=1371958$ or $13,720 \mathrm{psi}$

Where the MCO being impacted has the lifting cap, it is assumed that all the weight is distributed evenly. That distribution is represented as an equivalent pressure acting on top of the lifting cap. The area of the cap is:

$$
A_{\text {CAP }}=326.21 \mathrm{in}^{2}
$$

The equivalent pressure applied is

$$
P_{E Q}=(28)(19,374)=542,472 \text { lbs }
$$

therefore $\frac{P_{E Q}}{A_{S P}}=1662.95 \mathrm{psi}$

The bottom shell of the MCO sees a stress of $P_{E O} / A_{\text {SHELL }}$ equal to

$$
\begin{aligned}
& A_{\text {SHELL }}=(\pi) \frac{(24.00)^{2}-(23.00)^{2}}{4}=36.91 \mathrm{in}^{2} \\
& \sigma_{\text {SHELL }}=\frac{P_{E Q}}{A_{\text {SHELL }}}=14697 \mathrm{psi}
\end{aligned}
$$

Buckling of the shell is evaluated using the ANSYS program.

\section{ANSYSC ANALYSIS}

In addition to the hand calculations described in Sections 8 and 9 , an evaluation of the MCO assembly are performed using the finite element model shown in Figures 1 and 3. The model is axisymmetric (2-D) and was developed with PLANE42 elements with 2 degrees of freedom at each node.

\begin{tabular}{|l|l|l|l|}
\hline 0 & & & \\
\hline $4 / 17 / 97$ & & & \\
\hline $4 / 17 / 97$ & & & \\
\hline
\end{tabular}




\section{QDARSDNS}

CLIENT: Duke Engineering \& Services Hanford

FILE NO: KH-8009-8-03

PROJECT: MCO Final Design

DOC. NO.: HNF-SD-SNF-DR-003, Rev. 0, Appendix 5

\subsection{Boundary Conditions}

Figures 1 and 3 represent the axysimmetric models used in the analyses. Symmetry boundary conditions are applied at the edges of the model. One should note that nodes between the jacking bolts and the locking ring, and nodes between locking ring threads and shell threads are coupled (See Figure 5). The gap elements between the shield plug and the bottom of the jacking bolts have a very small positive interference to represent the preload on the bolts.

In the Bare Bottom End Drop ( $54 \mathrm{~g} \mathrm{load),} \mathrm{gap} \mathrm{elements} \mathrm{are} \mathrm{used} \mathrm{at} \mathrm{the} \mathrm{bottom} \mathrm{of} \mathrm{the} \mathrm{model}$ in order to anchor the model. In the Top End Drops ( $28 \mathrm{~g} \mathrm{load),} \mathrm{with} \mathrm{and} \mathrm{without} \mathrm{lifting} \mathrm{cap,}$ gap elements are used at the top of the model in order to anchor the model. The "free" end of the gap elements are restrained in both $X$ - and $Y$-directions (Figures $6,14,22 \& 29$ ). In the CSB Tube Drop ( $28 \mathrm{~g}$ equivalent), the load is applied at the top of the shield plug or at the top of the lifting cap, and gap elements at the bottom of the model are also restrained in both $\mathrm{X}$ - and $\mathrm{Y}$-directions.

\subsection{Loading}

\subsubsection{Bare Bottom End Drop}

The Bare Bottom End Drop consists of two models. The first model has a coarse mesh at the bottom (shell to bottom plate interface) and the second has a finer mesh to show that refining the mesh at that section of the model does not change the results. In both cases the geometry is identical. Figure 8 shows the hydrostatic pressure with $150 \mathrm{psi}$ internal pressure for the Bare Bottom End Drop. The pressure exerted on the bottom plate of the MCO is equivalent to the weight of five loaded Mark IV baskets distributed over the area of the plate. It is

$$
\begin{aligned}
& \mathrm{A}_{\mathrm{p}}=\text { MCO Bottom Plate area }=\pi \times \frac{(23.00)^{2}}{4}=415.48 \mathrm{in}^{2} \\
& \mathrm{~W}_{\mathrm{B}}=\text { Maximum weight of a Mark IV basket }=3200 \mathrm{lbs} \\
& \mathrm{P}_{\mathrm{bp}}=\frac{5\left(W_{B}\right)(54 \mathrm{~g})}{A_{p}}=\frac{5 \times(3,200 \mathrm{lbs}) \times(54 \mathrm{~g})}{415.48 \mathrm{in}^{2}}=2079.5 \mathrm{psi}
\end{aligned}
$$

The hydrostatic pressure is modeled and input in accordance with the above hand calculations (See Section 8 ). Tables 10 and 11 are summaries of maximum stress intensities at different sections of the MCO assembly. The area of interest in this model is

\begin{tabular}{|l|c|l|l|l|}
\hline REVISION & 0 & & & \\
\hline PREPARED BY I DATE & $\not K>4 / 17 / 97$ & & & \\
\hline CHECKED BY IDATE & $/ 44 / 17 / 97$ & & & \\
\hline
\end{tabular}




\section{P parsons}

CLIENT: Duke Engineering \& Services Hanford

PROJECT: MCO Final Design

FILE NO: KH-8009-8-03

where and how the hydrostatic pressure affects the MCO shell. Figure 10 shows the stress intensities at the process tube guide and guard plate interface. Figure 11(coarse mesh) and Figures $13 \& 14$ (fine mesh) show the stress intensities in the bottom of the MCO shell where the hydrostatic pressure is applied. Figure 12 (coarse mesh) and Figure 15 (fine mesh) represent the radial displacements in the shell where hydrostatic pressure is applied.

\subsubsection{Top End Drops}

Figure 15 shows the internal pressure distribution for both hydrostatic and $150 \mathrm{psi}$ internal pressure for the $28 \mathrm{~g}$ Top End Drop without lifting cap. The internal pressure distribution is the same for the $28 \mathrm{~g}$ Top End Drop with lifting cap. The pressure exerted on the filter guard plate is equivalent to the weight of five loaded Mark IV baskets distributed over the area of the plate. It is

$$
\begin{aligned}
& \mathrm{A}_{\mathrm{gp}}=\text { Filter Guard Plate Area }=\pi \times\left[\frac{(22.50)^{2}}{4}-\frac{(4.28)^{2}}{4}\right]=383.22 \mathrm{in}^{2} \\
& \mathrm{~W}_{\mathrm{B}}=\text { Maximum weight of a Mark IV basket }=3200 \mathrm{lbs} \\
& \mathrm{P}_{\mathrm{bp}}=\frac{5\left(W_{B}\right)(28 g)}{A_{g p}}=\frac{5 \times(3,200 \mathrm{lbs}) \times(28 g)}{383.22 \mathrm{in}^{2}}=1169.04 \mathrm{psi}
\end{aligned}
$$

The hydrostatic pressure is modeled and input in accordance with the above hand calculations (See Section 9). Slight differences in the results may exist because the model applies the pressure normal to the face of the element instead of applying the pressure at the nodes of the element. It is assumed that the fuel does not exert a pressure between the shield plug and the shell.

The area of interest in this model is where and how the hydrostatic pressure affects the MCO shell. Figure 16 shows the stress intensities in the top of the MCO shell (below guard plate) where the hydrostatic pressure is applied. Figures $17 \& 27$ represent the radial displacements in the shell where hydrostatic pressure is applied, for a Top End Drop without lifting cap and for a Top End Drop with lifting cap, respectively. Table 4 is a summary of the maximum stress intensities at different sections of the MCO assembly.

\begin{tabular}{|l|l|l|l|}
\hline & & \\
\hline & & & \\
\hline
\end{tabular}




\section{EDARSDNS}

CLIENT: Duke Engineering \& Services Hanford

PROJECT: MCO Final Design

FILE NO: $\quad \mathrm{KH}-8009-8-03$

\subsubsection{CSB Tube Drops}

The CSB Tube Drops were evaluated without internal pressure for more conservative results. Adding internal pressure lessens the compressive stresses in the shell, yielding non-conservative results.

Figure 18 represents the pressure distribution on the shield plug, for the pressure equivalent to that of a fully loaded MCO $\times 28 \mathrm{~g}$. Figures 19 and 20 show the stress intensities for the top and bottom MCO assembly without lifting cap, respectively. Figure 21 represents the radial displacements in the shell of the $\mathrm{MCO}$ assembly without lifting cap. The equivalent pressure is given in Section 11.4.

Figures 28 \& 29 show the upper an lower boundary conditions applied for the model with lifting cap. Figure 30 represents the equivalent pressure distribution on the lifting cap (See Section 11.4 for equivalent pressure). Figures 31 through 33 show stress intensities of the model and Figure 34 show the radial displacements of the model, again with lifting cap.

\section{RESULTS}

The PLANE42 elements used for these ANSYSC analyses report stresses mainly in the shell and in the lifting cap where the pressure distribution is rather great. These analyses show that ASME Code allowables (Subsection NB and Appendix F) are not violated. The radial displacements due to the load cases and the pressure distribution are well within the allowables given by the MCO Performance Specification. Stresses are classified as membrane plus bending stresses: $P_{m}+P_{B}$.

The results are summarized in the following tables. Table 5 is a compilation where the stress report sections are obtained using the LPATH/PRSECT command. In Tables 6 and 7 , the maximum radial displacement is the interior nodal radial displacement. The stress ratios in Tables 8 through 13 are calculated as the ratio of the maximum stress intensity to the allowed stress for that type of stress intensity.

For the CSB Tube Drop with lifting cap, the corresponding ANSYS input and output files are CSBMC.inp and CSBMC.out, respectively.

For the CSB Tube Drop without lifting cap, the corresponding ANSYS input and output files are CSB.inp and CSB.out, respectively.

(2)

0

\section{DOC. NO.: HNF-SD-SNF-DR-003, Rev. 0, Appendix 5}


CLIENT: Duke Engineering \& Services Hanford

PROJECT: MCO Final Design

FILE NO: KH-8009-8-03

For the Top End Drop with lifting cap, the corresponding ANSYS input and output files are TEDMC.inp and TEDMC.out, respectively.

For the Top End Drop without lifting cap, the corresponding ANSYS input and output files are TED.inp and TED.out, respectively.

For the Bare Bottom End Drop, coarse mesh, the corresponding ANSYS input and output files are BED.inp and BED.out, respectively.

For the Bare Bottom End Drop, fine mesh, the corresponding ANSYS input and output files are FINE.inp and FINE.out, respectively.

\begin{tabular}{|l|l|l|l|}
\hline 0 & & & \\
\hline $4 / 17 / 97$ & & & \\
\hline $4 / 17 / 97$ & & & \\
\hline
\end{tabular}


CLIENT: Duke Engineering \& Services Hanford

FILE NO: KH-8009-8-03

PROJECT: MCO Final Design $\quad$ DOC. NO.: HNF-SD-SNF-DR-003, Rev. 0, Appendix 5

Table 5: ANSYS Model Stress Report Sections

\begin{tabular}{|c|c|c|c|}
\hline Component & Section & Inside Node & Outside Node \\
\hline \multirow[t]{5}{*}{ Bottom Plate } & 1 & 1 & 41 \\
\hline & 2 & 4 & 44 \\
\hline & 3 & 6 & 46 \\
\hline & 4 & 9 & 49 \\
\hline & 5 & 10 & 50 \\
\hline \multirow[t]{4}{*}{ Lower Shell } & 6 & 50 & 52 \\
\hline & 7 & 53 & 55 \\
\hline & 8 & 62 & 64 \\
\hline & 9 & 65 & 67 \\
\hline \multirow[t]{9}{*}{ Middle Shell } & 10 & 100 & 101 \\
\hline & 11 & 116 & 117 \\
\hline & 12 & 122 & 123 \\
\hline & 13 & 130 & 132 \\
\hline & 14 & 134 & 135 \\
\hline & 15 & 150 & 152 \\
\hline & 16 & 156 & 157 \\
\hline & 17 & 170 & 171 \\
\hline & 18 & 180 & 181 \\
\hline \multirow[t]{6}{*}{ Upper Shell } & 19 & 202 & 204 \\
\hline & 20 & 235 & 237 \\
\hline & 21 & 985 & 989 \\
\hline & 22 & 262 & 264 \\
\hline & 23 & 277 & 279 \\
\hline & 24 & 292 & 294 \\
\hline \multirow[t]{9}{*}{ Shield Plug } & 25 & 601 & 641 \\
\hline & 26 & 601 & 613 \\
\hline & 27 & 603 & 703 \\
\hline & 28 & 606 & 706 \\
\hline & 29 & 766 & 806 \\
\hline & 30 & 748 & 808 \\
\hline & 31 & 862 & 873 \\
\hline & 32 & 870 & 874 \\
\hline & 33 & 870 & 875 \\
\hline
\end{tabular}


CLIENT: Duke Engineering \& Services Hanford

FILE NO: KH-8009-8-03

PROJECT: MCO Final Design DOC. NO.: HNF-SD-SNF-DR-003, Rev. 0, Appendix 5

Table 5: ANSYS Model Stress Report Sections, Cont'd

\begin{tabular}{|c|c|c|c|}
\hline Component & Section & Inside Node & Outside Node \\
\hline \multirow[t]{2}{*}{ Support Ring } & 34 & 1200 & 1202 \\
\hline & 35 & 1209 & 1211 \\
\hline \multirow[t]{3}{*}{ Guard Plate } & 36 & 1229 & 1232 \\
\hline & 37 & 1237 & 1240 \\
\hline & 38 & 1245 & 1248 \\
\hline \multirow[t]{2}{*}{ Process Tube Adapter } & 39 & 1284 & 1263 \\
\hline & 40 & 1261 & 1291 \\
\hline \multirow[t]{3}{*}{ Locking Ring } & 41 & 431 & 434 \\
\hline & 42 & 406 & 426 \\
\hline & 43 & 404 & 424 \\
\hline \multirow[t]{12}{*}{ Lifting Cap } & 44 & 1120 & 1097 \\
\hline & 45 & 1120 & 1168 \\
\hline & 46 & 1174 & 1149 \\
\hline & 47 & 1176 & 1152 \\
\hline & 48 & 1177 & 1155 \\
\hline & 49 & 1178 & 1158 \\
\hline & 50 & 1179 & 1161 \\
\hline & 51 & 1162 & 1164 \\
\hline & 52 & 295 & 297 \\
\hline & 53 & 1021 & 1027 \\
\hline & 54 & 1071 & 1077 \\
\hline & 55 & 1138 & 1169 \\
\hline
\end{tabular}

\begin{tabular}{|l|c|l|l|l|}
\hline REVISION & 0 & & & \\
\hline PREPARED BY IDATE & X6/ 4/17/97 & & & \\
\hline CHECKED BY I DATE & $/ N$ 4/17/97 & & & \\
\hline
\end{tabular}




\section{国 pansans}

CLIENT: Duke Engineering \& Services Hanford PROJECT: MCO Final Design

FILE NO: KH-8009-8-03

DOC. NO.: HNF-SD-SNF-DR-003, Rev. 0, Appendix 5

Table 6: CSB Tube Drops and Bottom End Drop Calculated Max. Shell Circumference

\begin{tabular}{|c|c|c|c|c|}
\hline Load Case & $\begin{array}{c}28 \mathrm{~g} \text { CSB Tube } \\
\text { Drop } \\
\text { Without Lifting } \\
\text { Cap }\end{array}$ & $\begin{array}{c}28 g \text { CSB Tube } \\
\text { Drop } \\
\text { With Lifting } \\
\text { Cap }\end{array}$ & $\begin{array}{l}54 \mathrm{~g} \text { Bottom } \\
\text { End Drop } \\
\text { Coarse Mesh }\end{array}$ & $\begin{array}{l}54 \mathrm{~g} \text { Bottom } \\
\text { End Drop } \\
\text { Fine Mesh }\end{array}$ \\
\hline Max. Radial Displacement & 0.02374 in. & 0.02374 in. & 0.02393 in. & 0.02393 in. \\
\hline Undeformed Shell Diameter & $23.00 \mathrm{in}$. & $23.00 \mathrm{in.}$ & $23.00 \mathrm{in}$. & 23.00 in. \\
\hline Max. Deformed Shell Diameter & 23.0475 in. & $23.0475 \mathrm{in}$. & $23.0479 \mathrm{in.}$ & 23.0479 in. \\
\hline Max. Calculated Circumference & 72.405 in. & 72.405 in. & 72.407 in. & $72.407 \mathrm{in.}$ \\
\hline Allowed Circumference & 73.04 in. & 73.04 in. & 73.04 in. & 73.04 in. \\
\hline
\end{tabular}

Table 7: Top End Drops Calculated Max. Shell Circumference

\begin{tabular}{||l|c|c||}
\hline \hline Load Case & $\begin{array}{c}\text { 28g Top End Drop } \\
\text { Without Lifting Cap }\end{array}$ & $\begin{array}{c}28 \mathrm{~g} \text { Top End Drop } \\
\text { With Lifting Cap }\end{array}$ \\
\hline Maximum Radial Displacement & 0.02376 inches & 0.02847 inches \\
\hline Undeformed Shell Diameter & 23.00 inches & 23.00 inches \\
\hline Maximum Deformed Shell Diameter & 23.048 inches & 23.0569 inches \\
\hline Maximum Calculated Circumference & 72.406 inches & 72.436 inches \\
\hline Allowed Circumference & 73.04 inches & 73.04 inches \\
\hline
\end{tabular}

From Tables 6 and 7, it is apparent that the maximum internal deformed shell circumference will not exceed the allowed value of 73.04 inches [1]. 


\section{Parsons}

CLIENT: Duke Engineering \& Services Hanford

FILE NO: KH-8009-8-03

PROJECT: MCO Final Design

DOC. NO.: HNF-SD-SNF-DR-003, Rev. 0, Appendix 5

For the following tables the lower shell, middle shell and upper shell have the properties of Type 304 stainless steel. The bottom plate, shield plug and locking ring have the properties of Type $304 \mathrm{~L}$ stainless steel.

Therefore, in calculating the stress ratios, the following apply:

Type 304: $\quad \mathrm{S}_{\mathrm{M}}=20.0 \mathrm{ksi} @ 132^{\circ} \mathrm{C}\left(270^{\circ} \mathrm{F}\right)$

Type 304L: $\quad S_{M}=16.7 \mathrm{ksi} @ 132^{\circ} \mathrm{C}\left(270^{\circ} \mathrm{F}\right)$

Table 8: Top End Drop (28g) without Cap Summary of Maximum Stress Intensities

\begin{tabular}{|c|c|c|c|c|}
\hline \multirow[b]{2}{*}{ COMPONENT } & \multicolumn{2}{|c|}{$\begin{array}{c}\text { Membrane } \\
\text { Stress Intensities }\end{array}$} & \multicolumn{2}{|c|}{$\begin{array}{l}\text { Membrane + Bending } \\
\text { Stress Intensities }\end{array}$} \\
\hline & Max SI & SR & $\operatorname{Max} S I$ & SR \\
\hline Bottom Plate & $3.19 \mathrm{ksi}$ & 0.07 & $8.65 \mathrm{ksi}$ & 0.12 \\
\hline Lower Shell (304) & $3.40 \mathrm{ksi}$ & 0.07 & $8.36 \mathrm{ksi}$ & 0.12 \\
\hline Middle Shell (304) & $3.77 \mathrm{ksi}$ & 0.08 & $3.85 \mathrm{ksi}$ & 0.05 \\
\hline Upper Shell (304) & $11.13 \mathrm{ksi}$ & 0.24 & $11.85 \mathrm{ksi}$ & 0.17 \\
\hline Support Ring & $7.43 \mathrm{ksi}$ & 0.16 & $17.03 \mathrm{ksi}$ & 0.24 \\
\hline Tube Adapter & $10.27 \mathrm{ksi}$ & 0.22 & $16.55 \mathrm{ksi}$ & 0.23 \\
\hline Shield Plug & $7.18 \mathrm{ksi}$ & 0.16 & $8.42 \mathrm{ksi}$ & 0.12 \\
\hline Guard Plate & $4.48 \mathrm{ksi}$ & 0.09 & $15.04 \mathrm{ksi}$ & 0.21 \\
\hline
\end{tabular}

Note:

$\mathrm{SR}=\frac{S I}{0.7 S_{U}} \quad$ or $\quad \mathrm{SR}=\frac{S I}{1.5 P_{M}}$ for Type 304 stainless steel

$\mathrm{SR}=\frac{S I}{2.4 S_{M}} \quad$ or $\quad \mathrm{SR}=\frac{S I}{1.5 S_{M}}$ for Type $304 \mathrm{~L}$ stainless steel 
CLIENT: Duke Engineering \& Services Hanford

PROJECT: MCO Final Design

FILE NO: $\quad \mathrm{KH}-8009-8-03$

DOC. NO.: HNF-SD-SNF-DR-003, Rev. 0, Appendix 5

Table 9: Top End Drop (28g) with Cap Summary of Maximum Stress Intensities

\begin{tabular}{|c|c|c|c|c||}
\hline & \multicolumn{2}{|c|}{$\begin{array}{c}\text { Membrane } \\
\text { Stress Intensities }\end{array}$} & Max SI \\
\hline & Max SI & SR & $8.65 \mathrm{ksi}$ & SR \\
\hline COMPONENT & $3.19 \mathrm{ksi}$ & 0.07 & $8.36 \mathrm{ksi}$ & 0.12 \\
\hline Bottom Plate & $3.80 \mathrm{ksi}$ & 0.08 & $18.44 \mathrm{ksi}$ & 0.12 \\
\hline Lower Shell (304) & $17.93 \mathrm{ksi}$ & 0.38 & $19.43 \mathrm{ksi}$ & 0.26 \\
\hline Middle Shell (304) & $17.52 \mathrm{ksi}$ & 0.37 & $19.8 \mathrm{ksi}$ & 0.27 \\
\hline Upper Shell (304) & $7.85 \mathrm{ksi}$ & 0.17 & $17.18 \mathrm{ksi}$ & 0.28 \\
\hline Support Ring & $10.09 \mathrm{ksi}$ & 0.21 & $4.72 \mathrm{ksi}$ & 0.24 \\
\hline Tube Adapter & $3.69 \mathrm{ksi}$ & 0.08 & $23.35 \mathrm{ksi}$ & 0.06 \\
\hline Shield Plug & $22.77 \mathrm{ksi}$ & 0.48 & $15.77 \mathrm{ksi}$ & 0.33 \\
\hline Lifting Cap & $4.42 \mathrm{ksi}$ & 0.09 & $26.48 \mathrm{ksi}$ & 0.22 \\
\hline Guard Plate & $21.45 \mathrm{ksi}$ & 0.45 & & 0.37 \\
\hline Weld & & & & \\
\hline
\end{tabular}




\section{P.}

CLIENT: Duke Engineering \& Services Hanford

FILE NO: $\quad \mathrm{KH}-8009-8-03$

PROJECT: MCO Final Design

DOC. NO.: HNF-SD-SNF-DR-003, Rev. 0, Appendix 5

\section{Table 10: Bare Bottom End Drop (54g) Summary of Maximum Stress Intensities}

\begin{tabular}{|c|c|c|c|c|}
\hline & \multicolumn{2}{|c|}{$\begin{array}{c}\text { Membrane } \\
\text { Stress Intensities }\end{array}$} & SR & Max SI \\
\hline COMPONENT & Max SI & 0.08 & $8.04 \mathrm{ksi}$ & SR \\
\hline Bottom Plate & $3.50 \mathrm{ksi}$ & 0.19 & $22.13 \mathrm{ksi}$ & 0.13 \\
\hline Lower Shell (304) & $9.02 \mathrm{ksi}$ & 0.45 & $21.90 \mathrm{ksi}$ & 0.31 \\
\hline Middle Shell (304) & $21.37 \mathrm{ksi}$ & 0.11 & $8.98 \mathrm{ksi}$ & 0.31 \\
\hline Upper Shell (304) & $5.33 \mathrm{ksi}$ & 0.01 & $1.13 \mathrm{ksi}$ & 0.13 \\
\hline Support Ring & $0.60 \mathrm{ksi}$ & 0.02 & $1.83 \mathrm{ksi}$ & 0.02 \\
\hline Tube Adapter & $0.90 \mathrm{ksi}$ & 0.17 & $8.15 \mathrm{ksi}$ & 0.03 \\
\hline Shield Plug & $6.83 \mathrm{ksi}$ & 0.01 & $1.44 \mathrm{ksi}$ & 0.14 \\
\hline Guard Plate & $0.50 \mathrm{ksi}$ & & & 0.02 \\
\hline
\end{tabular}

Table 11: Bare bottom End Drop w/ Fine Mesh Summary of Maximum Stress Intensities

\begin{tabular}{|c|c|c|c|c||}
\hline & \multicolumn{2}{|c|}{$\begin{array}{c}\text { Membrane } \\
\text { Stress Intensities }\end{array}$} & \multicolumn{2}{c|}{$\begin{array}{c}\text { Membrane + Bending } \\
\text { Stress Intensities }\end{array}$} \\
\hline COMPONENT & Max Si & SR & Max SI & SR \\
\hline Bottom Plate & $3.45 \mathrm{ksi}$ & 0.08 & $8.02 \mathrm{ksi}$ & 0.13 \\
\hline Lower Shell (304) & $9.35 \mathrm{ksi}$ & 0.19 & $22.33 \mathrm{ksi}$ & 0.32 \\
\hline Middle Shell (304) & $21.49 \mathrm{ksi}$ & 0.45 & $21.89 \mathrm{ksi}$ & 0.31 \\
\hline Upper Shell (304) & $5.25 \mathrm{ksi}$ & 0.11 & $8.91 \mathrm{ksi}$ & 0.14 \\
\hline Support Ring & $0.60 \mathrm{ksi}$ & 0.01 & $1.42 \mathrm{ksi}$ & 0.02 \\
\hline Tube Adapter & $0.90 \mathrm{ksi}$ & 0.02 & $1.83 \mathrm{ksi}$ & 0.03 \\
\hline Shield Piug & $6.61 \mathrm{ksi}$ & 0.16 & $8.06 \mathrm{ksi}$ & 0.13 \\
\hline Guard Plate & $0.50 \mathrm{ksi}$ & 0.01 & $1.45 \mathrm{ksi}$ & 0.02 \\
\hline
\end{tabular}


CLIENT: Duke Engineering \& Services Hanford

FILE NO: $\quad \mathrm{KH}-8009-8-03$

PROJECT: MCO Final Design DOC. NO.: HNF-SD-SNF-DR-003, Rev. 0, Appendix 5

Table 12: $28 \mathrm{~g}$ CSB Tube Drop with Cap Summary of Maximum Stress Intensities

\begin{tabular}{|c|c|c|c|c||}
\hline & \multicolumn{2}{|c|}{ Stress Intensities } & \multicolumn{2}{c|}{$\begin{array}{c}\text { Membrane + Bending } \\
\text { Stress Intensities }\end{array}$} \\
\hline COMPONENT & Max SI & SR & Max SI & SR \\
\hline Bottom Plate & $9.83 \mathrm{ksi}$ & 0.21 & $13.59 \mathrm{ksi}$ & 0.19 \\
\hline Lower Shell (304) & $14.74 \mathrm{ksi}$ & 0.31 & $17.24 \mathrm{ksi}$ & 0.24 \\
\hline Middle Shell (304) & $14.48 \mathrm{ksi}$ & 0.31 & $14.64 \mathrm{ksi}$ & 0.21 \\
\hline Upper Shell (304) & $14.65 \mathrm{ksi}$ & 0.31 & $14.88 \mathrm{ksi}$ & 0.21 \\
\hline Shield Plug & $11.79 \mathrm{ksi}$ & 0.25 & $13.80 \mathrm{ksi}$ & 0.19 \\
\hline Lifting Cap & $22.83 \mathrm{ksi}$ & 0.48 & $22.90 \mathrm{ksi}$ & 0.32 \\
\hline Weld & $21.55 \mathrm{ksi}$ & 0.46 & $26.20 \mathrm{ksi}$ & 0.37 \\
\hline
\end{tabular}


CLIENT: Duke Engineering \& Services Hanford

FILE NO: $\quad \mathrm{KH}-8009-8-03$

PROJECT: MCO Final Design

DOC. NO.: HNF-SD-SNF-DR-003, Rev. 0, Appendix 5

\section{Table 13: $28 \mathrm{~g}$ CSB Tube Drop w/o Cap Summary of Maximum Stress} Intensities

\begin{tabular}{|c|c|c|c|c|}
\hline & \multicolumn{2}{|c|}{$\begin{array}{c}\text { Membrane } \\
\text { Stress Intensities }\end{array}$} & \multicolumn{2}{c|}{$\begin{array}{c}\text { Membrane + Bending } \\
\text { Stress Intensities }\end{array}$} \\
\hline COMPONENT & Max SI & SR & Max SI & SR \\
\hline Bottom Plate & $9.85 \mathrm{ksi}$ & 0.21 & $13.62 \mathrm{ksi}$ & 0.19 \\
\hline Lower Shell (304) & $14.78 \mathrm{ksi}$ & 0.31 & $17.29 \mathrm{ksi}$ & 0.24 \\
\hline Middle Shell (304) & $14.55 \mathrm{ksi}$ & 0.31 & $14.68 \mathrm{ksi}$ & 0.21 \\
\hline Upper Shell (304) & $14.63 \mathrm{ksi}$ & 0.31 & $14.80 \mathrm{ksi}$ & 0.21 \\
\hline Shield Plug & $22.57 \mathrm{ksi}$ & 0.48 & $22.73 \mathrm{ksi}$ & 0.32 \\
\hline
\end{tabular}

Table 14: Summary of Guard Plate Maximum Deflections

\begin{tabular}{|l|c|}
\hline \multicolumn{1}{|c|}{ Load Case } & Maximum Plate Deflection (in.) \\
\hline 28g CSB Tube Drop with Cap & 0.173 \\
\hline 28g CSB Tube Drop without Cap & 0.170 \\
\hline 54g Bare Bottom End Drop & 0.205 \\
\hline 28g Top End Drop with Cap & 0.0182 \\
\hline 28g Top End Drop without Cap & 0.0273 \\
\hline
\end{tabular}

Tables 10 and 11 represent the maximum stress intensities at different sections of the model. However, Table 11 has the maximum stress intensities for the model with a finer mesh at the lower shell. As one can see the stresses are very comparable and meet allowables.. Figures 13 through 15 depict the Bare Bottom End Drop with the finer mesh and its resulting radial displacements.

\begin{tabular}{c}
0 \\
\hline yC) $4 / 17 / 97$ \\
\hline $1 / 4 / 17 / 97$
\end{tabular}


CLIENT: Duke Engineering \& Services Hanford

FILE NO: KH-8009-8-03

PROJECT: MCO Final Design

DOC. NO.: HNF-SD-SNF-DR-003, Rev. 0, Appendix 5

\subsection{Buckling}

\subsubsection{End Drop}

The maximum compressive membrane stress is checked according to Paragraph NB3133.6 of [3].

The weight of the MCO assembly, $W_{s}$, is $3424.17 \mathrm{lbs}$ or $3425 \mathrm{lbs}$ [1]. The cross-sectional of the shell is

$$
\begin{aligned}
& A_{s}=\pi \times\left(\frac{D_{O}^{2}}{4}-\frac{D_{1}^{2}}{4}\right)=\pi \times\left(\frac{24.00^{2}}{4}-\frac{23.00^{2}}{4}\right) \\
& A_{s}=36.91 \mathrm{in}^{2}
\end{aligned}
$$

The maximum compressive stress in the shell due to an end drop is:

$$
\begin{aligned}
& \sigma_{a}=\frac{A \times W_{s}}{A_{s}}=\frac{54 \mathrm{~g} \times(3425 \mathrm{lbs})}{36.91 \mathrm{in}^{2}} \\
& \sigma_{a}=5010.84 \mathrm{psi}
\end{aligned}
$$

Note that this value is greater than the maximum axial compressive stress $\left(\sigma_{Y}\right)$ in the shell for both top (3162 psi) and bottom end drops (4683 psi). The difference is the result of the applied internal pressure.

Per Paragraph NB-3133.6 of [3], the parameter $A$ is:

$$
\begin{aligned}
& A=\frac{0.125}{\frac{R_{C}}{T}} \quad \text { where } T=\text { Thickness of Shell and } R_{C}=\text { Inside Radius of Shell } \\
& A=\frac{0.125}{\left(\frac{11.50}{0.50}\right)}=0.0054
\end{aligned}
$$

The corresponding value of $\mathrm{B}$, obtained from $\mathrm{HA}-1$ of [2], at $132^{\circ} \mathrm{C}\left(270^{\circ} \mathrm{F}\right)$ is $11,500 \mathrm{psi}$ (interpolated value). Per paragraph F-1331.5 of [3], the allowable compressive stress is equal to $150 \%$ of $B$, or $17,250 \mathrm{psi}$. Since this value is greater than the computed compressive stress, the MCO shell meets the buckling criterion for an end drop. 
CLIENT: Duke Engineering \& Services Hanford

PROJECT: MCO Final Design

FILE NO: KH-8009-8-03

DOC. NO.: HNF-SD-SNF-DR-003, Rev. 0, Appendix 5

As stated above, the maximum allowed compressive stress is $17,250 \mathrm{psi}$. Tables 12 and 13 of Section 12 report maximum membrane stresses of $14.74 \mathrm{ksi}$ and $14.78 \mathrm{ksi}$ with and without the lifting cap, respectively. Since these stresses do not exceed the maximum allowed, the MCO shell meets the buckling criterion for a CSB Tube drop.

\subsection{Minimum Shell Thickness}

The minimum shell thickness permitted is

$$
\begin{aligned}
& \sigma_{M}=17250 \mathrm{psi} \\
& D_{O}=24.00 \text { inches } \\
& P_{\text {max }}=542,472 \mathrm{lbs} \\
& A_{\max }=\frac{542,472 \mathrm{lbs}}{17388 \mathrm{psi}}=31.4 \mathrm{in}^{2}
\end{aligned}
$$

therefore for a 24.00 inch outside diameter, the maximum permissible inside diameter is

$$
\mathrm{D}_{1, \text { MAX }}=\sqrt{\left(D_{o}^{2}\right)-\frac{4 A_{M A X}}{\pi}}=23.15 \text { in }
$$

and the minimum permissible thickness is

$$
\frac{D_{0}-D_{1}}{2}=0.425 \text { inches }
$$

\begin{tabular}{|c|c|c|c|}
\hline 0 & & & \\
\hline $264 / 17 / 97$ & & & \\
\hline $2 / 4 / 17 / 97$ & & & \\
\hline
\end{tabular}


CLIENT: Duke Engineering \& Services Hanford

PROJECT: MCO Final Design
FILE NO: $\quad \mathrm{KH}-8009-8-03$

DOC. NO.: HNF-SD-SNF-DR-003, Rev. 0, Appendix 5

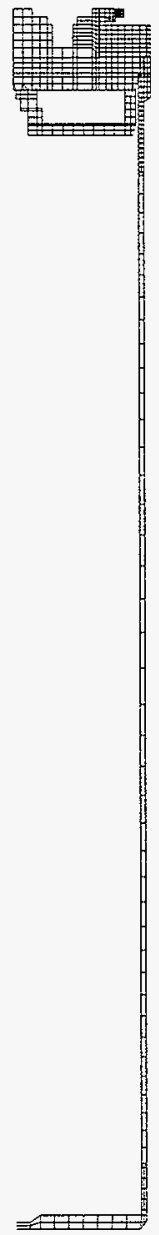

Figure 1: MCO Half-Symmetry Assembly without Lifting Cap 
CLIENT: Duke Engineering \& Services Hanford

FILE NO: $\quad \mathrm{KH}-8009-8-03$

PROJECT: MCO Final Design DOC. NO.: HNF-SD-SNF-DR-003, Rev. 0, Appendix 5

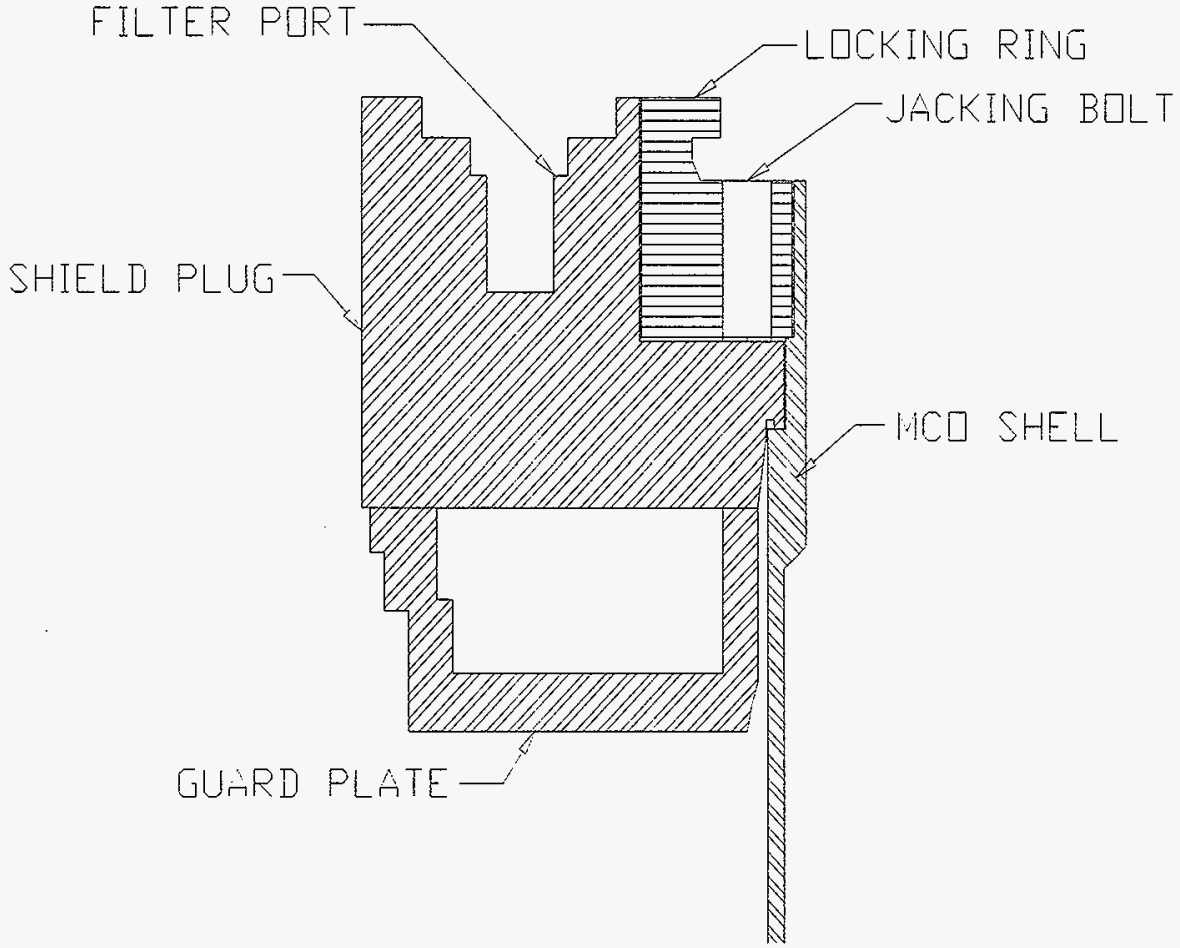

Figure 2: Detail of Upper MCO Assembly without Lifting Cap

10


CLIENT: Duke Engineering \& Services Hanford

PROJECT: MCO Final Design DOC. NO.: HNF-SD-SNF-DR-003, Rev. 0, Appendix 5

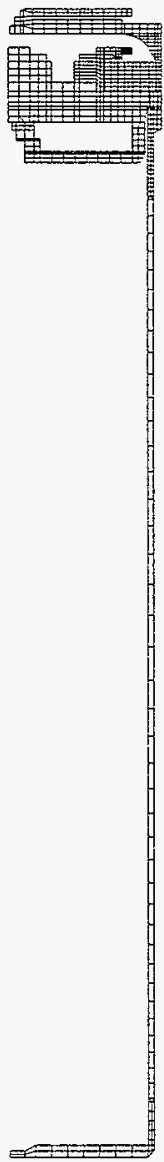

Figure 3: MCO Half-Symmetry Assembly with modified Lifting Cap 


\section{E paAsRNS}

CLIENT: Duke Engineering \& Services Hanford

FILE NO: $\quad \mathrm{KH}-8009-8-03$

PROJECT: MCO Final Design DOC. NO.: HNF-SD-SNF-DR-003, Rev. 0, Appendix 5

\section{RUPTURE DISK LDCMTION}

SHIELD PLUG

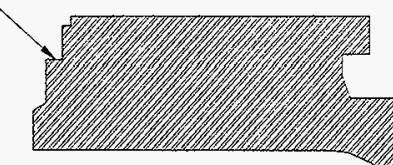
- LIFTING CiP - LDCKING RING

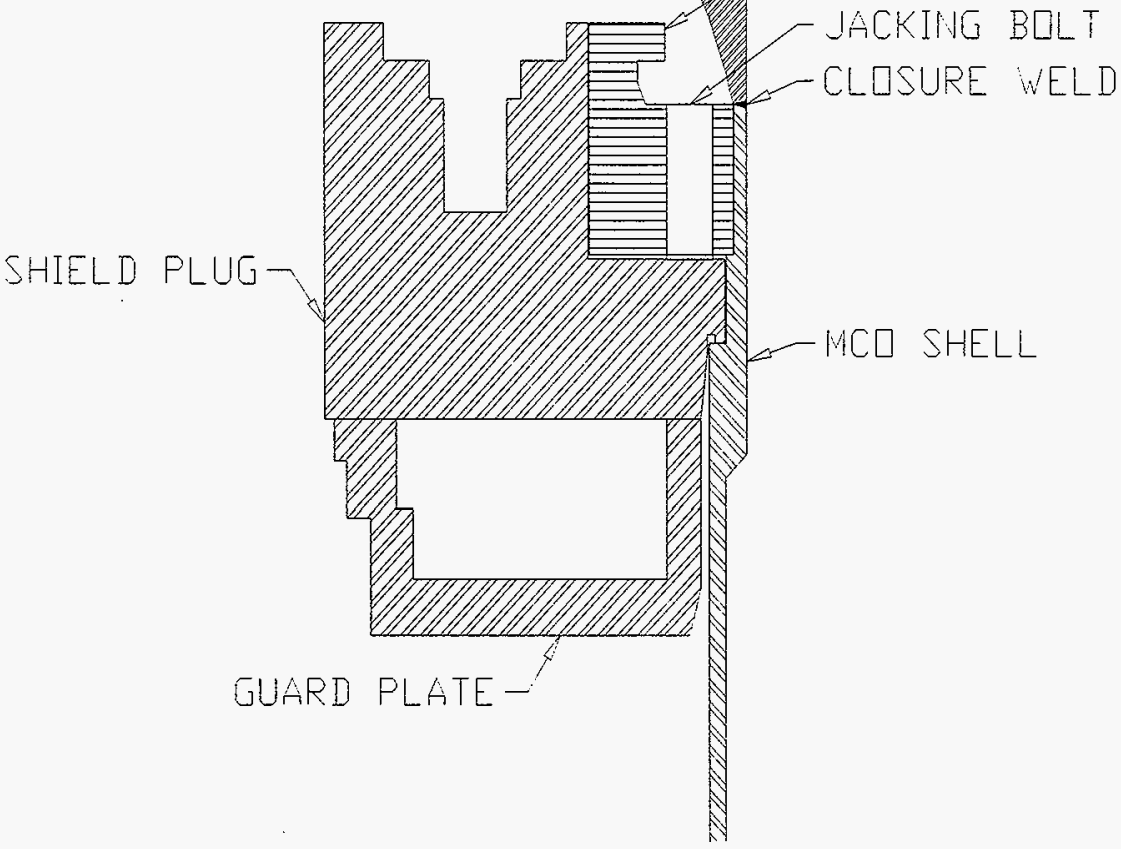

Figure 4: Detail of Upper MCO Assembly with Modified Lifting Cap

\begin{tabular}{|c|l|l|l|}
\hline 0 & & & \\
\hline$x 64 / 17 / 97$ & & & \\
\hline $12 / 4 / 17 / 97$ & & & \\
\hline
\end{tabular}


CLIENT: Duke Engineering \& Services Hanford

FILE NO: $\quad \mathrm{KH}-8009-8-03$

PROJECT: MCO Final Design $\quad$ DOC. NO.: HNF-SD-SNF-DR-003, Rev. 0, Appendix 5

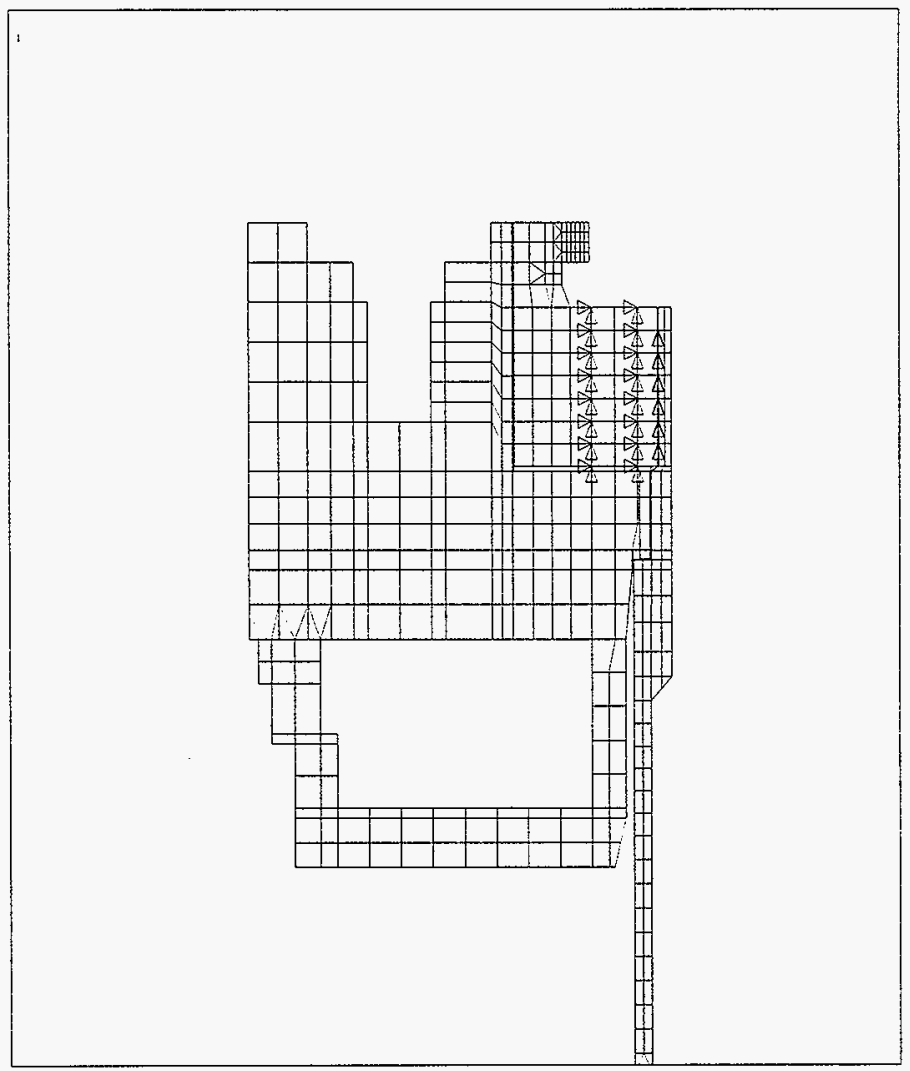

alls is $50 \div 56$

FEE 201997

$10: 58: 11$

PLDT 110. 1

ELEHENTS

TIPE NUII

$C P$

$Z Y=1$

$* 15 T=13.263$

* $\times F=6.141$

*YF $=150.146$

PRECISE HIDDEN

Figure 5: Model with Coupled Nodes at Jacking Bolt and Buttress Threads

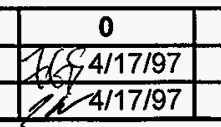


CLIENT: Duke Engineering \& Services Hanford

FILE NO: KH-8009-8-03

PROJECT: MCO Final Design DOC. NO.: HNF-SD-SNF-DR-003, Rev. 0, Appendix 5

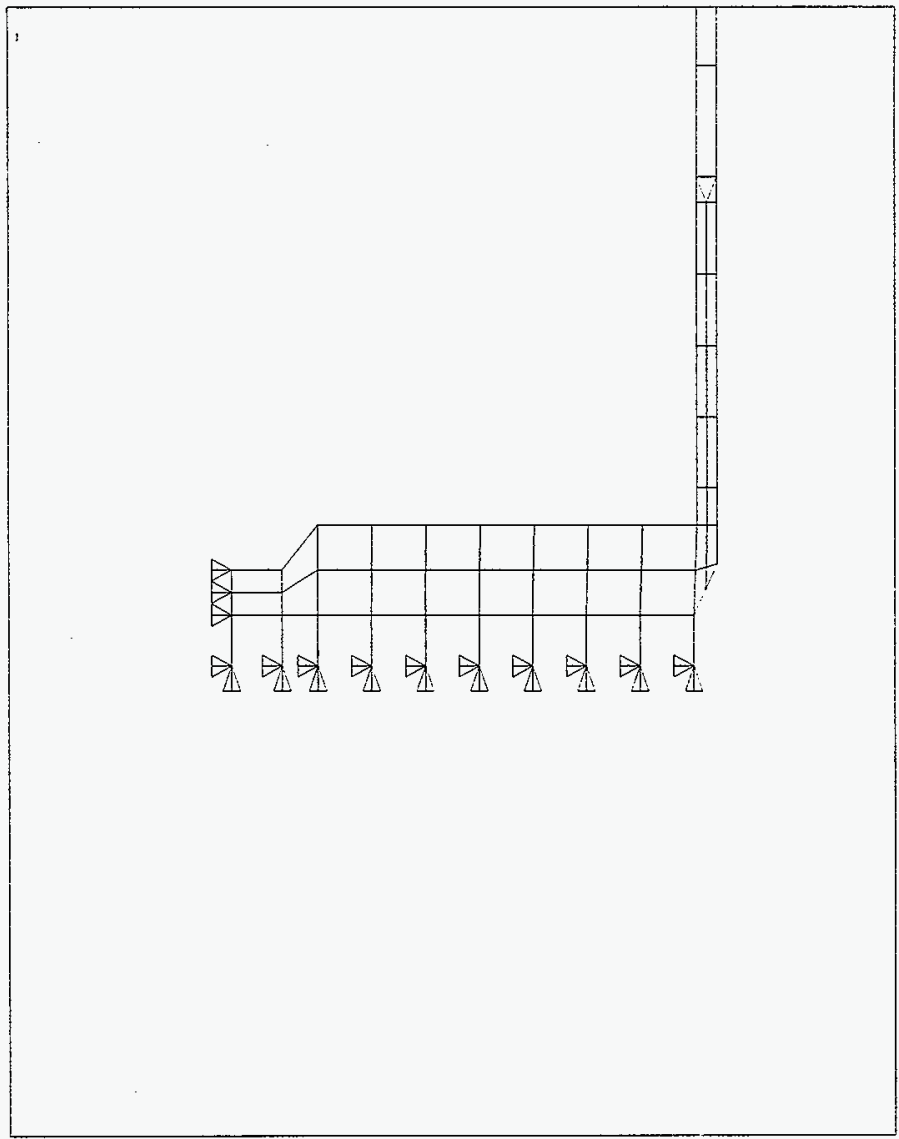

Ailsis 5.0 \& 56

FEE $20199^{-}$

11:19:39

PLOT HO. I

ELENENTS

TYPE HUM

U

IV $=1$

米IST $=10.935$

*.F $=5.432$

$\because F=-0.505665$

PRECISE HIDDEN

Figure 6: Bare Bottom End Drop Lower Boundary Conditions w/ Gap Elements 
CLIENT: Duke Engineering \& Services Hanford

PROJECT: MCO Final Design DOC. NO.: HNF-SD-SNF-DR-003, Rev. 0, Appendix 5

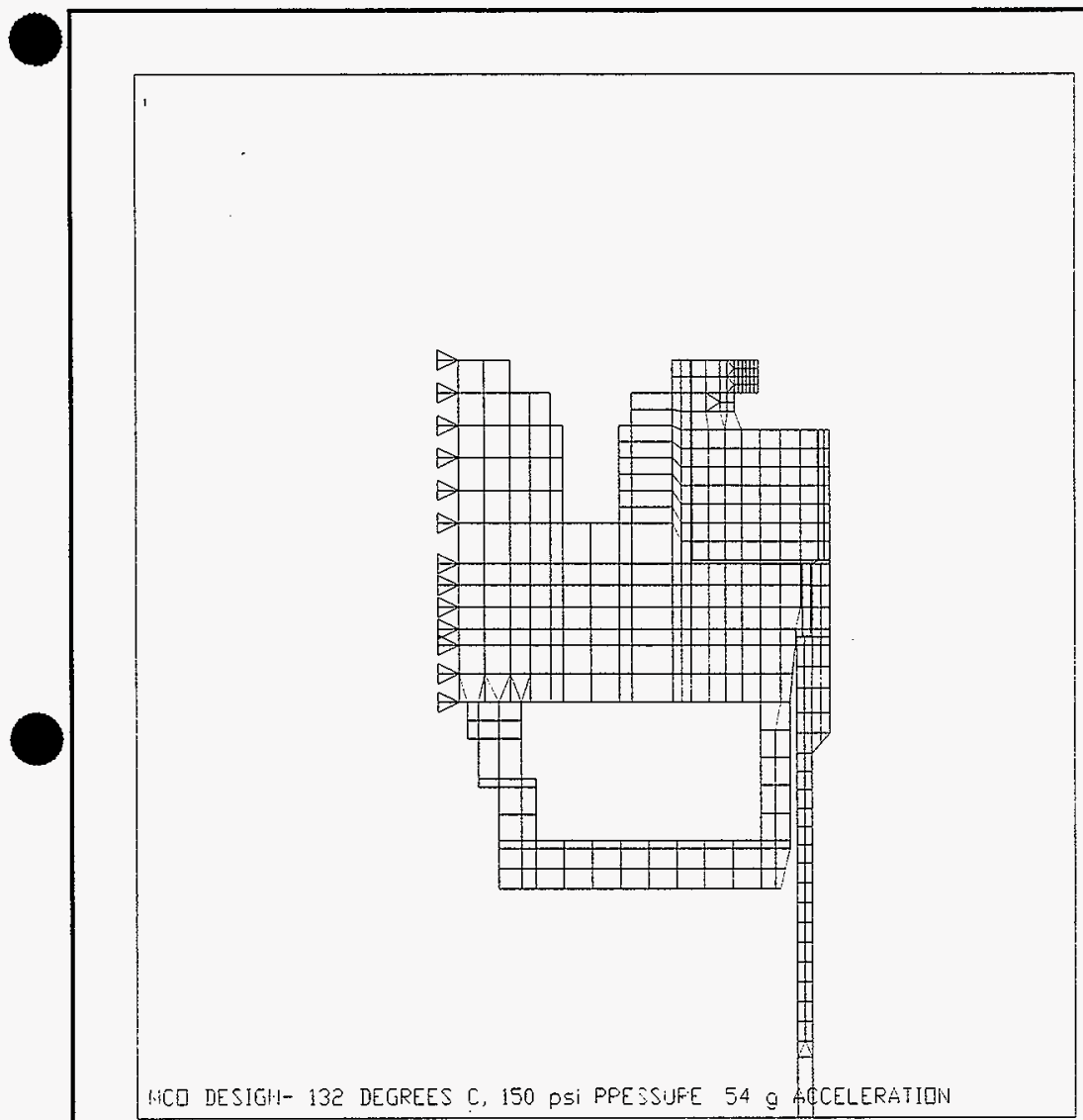

indsis 5.0 क 56

FEE 21 199-

11:0อ:อ2

PLIT ND. I

ELELENTS

TYPE NUH

U

$Z \vee=1$

*DIST $=15.9 \div 1$

$* X F=4.984$

*YF $=$ I50.81:

PRECISE HIDDEN

MCQ DESIGH- 132 DEGREES C, 150 pSI PPESSUfFE 54 g hLCELERAFIDN

Figure 7: Bare Bottom End Drop Upper Boundary Conditions

\begin{tabular}{|l|l|l|}
\hline & & \\
\hline & & \\
\hline
\end{tabular}


CLIENT: Duke Engineering \& Services Hanford

PROJECT: MCO Final Design DOC. NO.: HNF-SD-SNF-DR-003, Rev. 0, Appendix 5

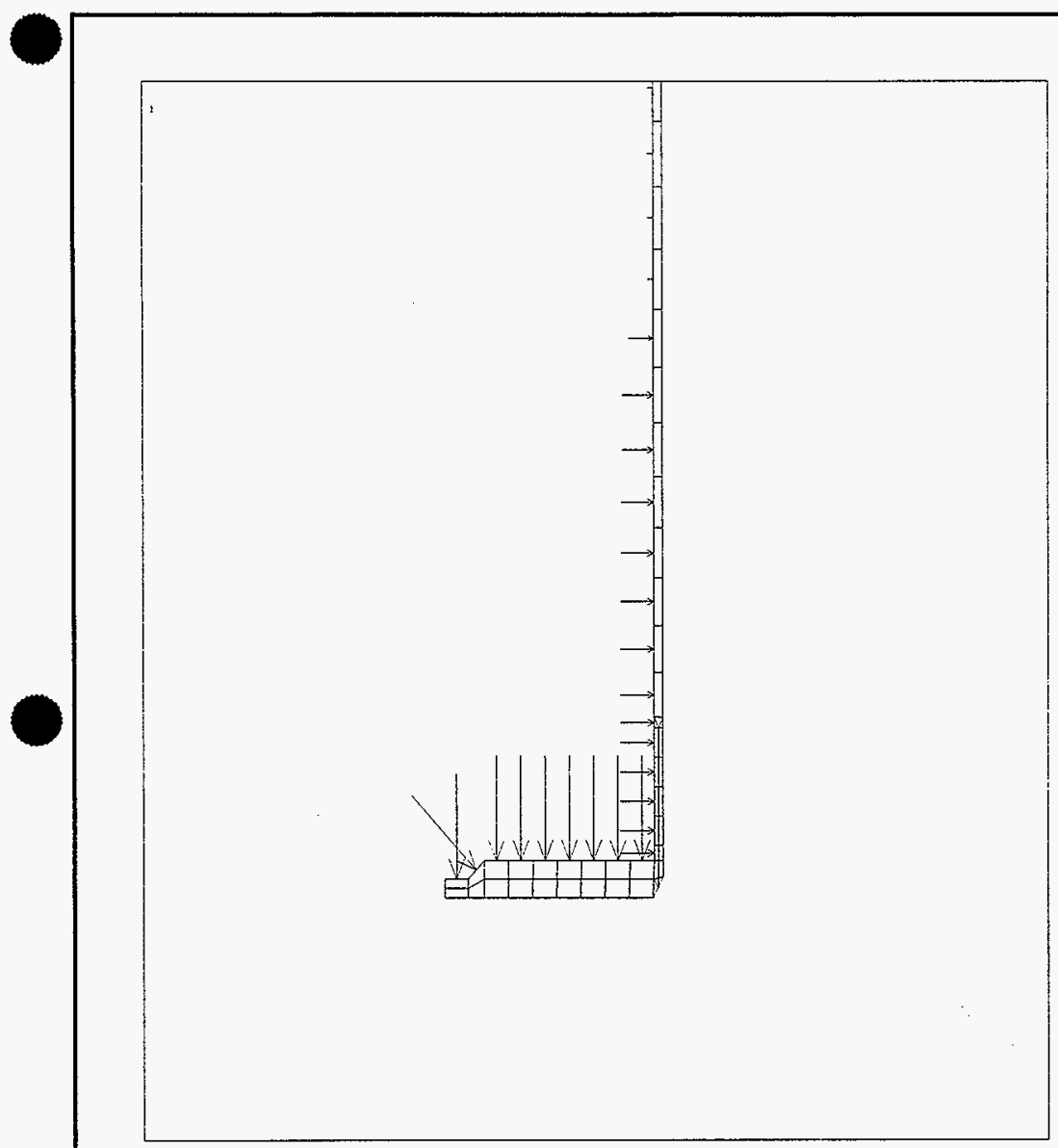

2ANSYS $5.0 \div 56$

FEB $20199^{\circ}$

$11: 25: 54$

PLOT HID. 2

ELEMENTS

TYPE NUI:

PRES

$Z V=1$

$*$ DIST $=24.881$

$* \times F \quad=8.306$

*YF $=12.119$

PRECISE HIDDEH: VSCA=2

Figure 8: Bare Bottom End Drop Pressure Distribution (Internal \& Hydrostatic)

\begin{tabular}{|l|l|l|l|}
\hline 0 & & & \\
\hline $4 / 17 / 97$ & & & \\
\hline $4 / 17 / 97$ & & & \\
\hline
\end{tabular}


CLIENT: Duke Engineering \& Services Hanford

PROJECT: MCO Final Design DOC. NO.: HNF-SD-SNF-DR-003, Rev. 0, Appendix 5

FILE NO: KH-8009-8-03

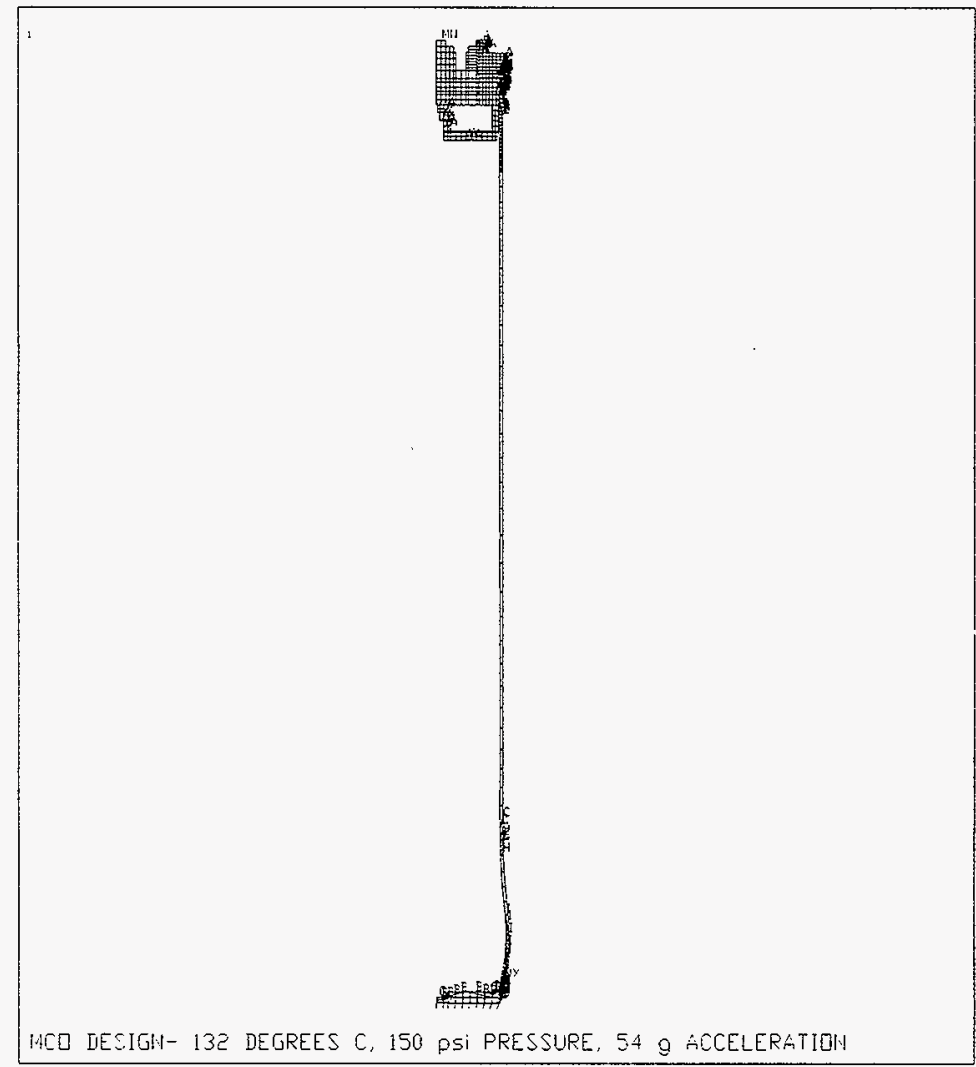

ANSIS 5.0 - 56

Hint 12 199:

$14: 02: 45$

PLOT ND. 2

NODÄL SOLUTION

STEP $=1$

SUB $=1$

TIME =1

SINT

(AंVG)

DWX $=0.247015$

SHN $=4.684$

SHX $=22715$

A $=1266$

B $=3790$

C $=6313$

D $=8837$

$E \quad=11360$

$\mathrm{F} \quad=13883$

G $=16407$

$\mathrm{H}=18930$

I $=21454$

Figure 9: Bare Bottom End Drop Stress Intensities

\begin{tabular}{|c|}
\hline 0 \\
\hline $\cos 4 / 17 / 97$ \\
\hline /4 $4 / 17 / 97$ \\
\hline
\end{tabular}


CLIENT: Duke Engineering \& Services Hanford

FILE NO: KH-8009-8-03

PROJECT: MCO Final Design DOC. NO.: HNF-SD-SNF-DR-003, Rev. 0, Appendix 5

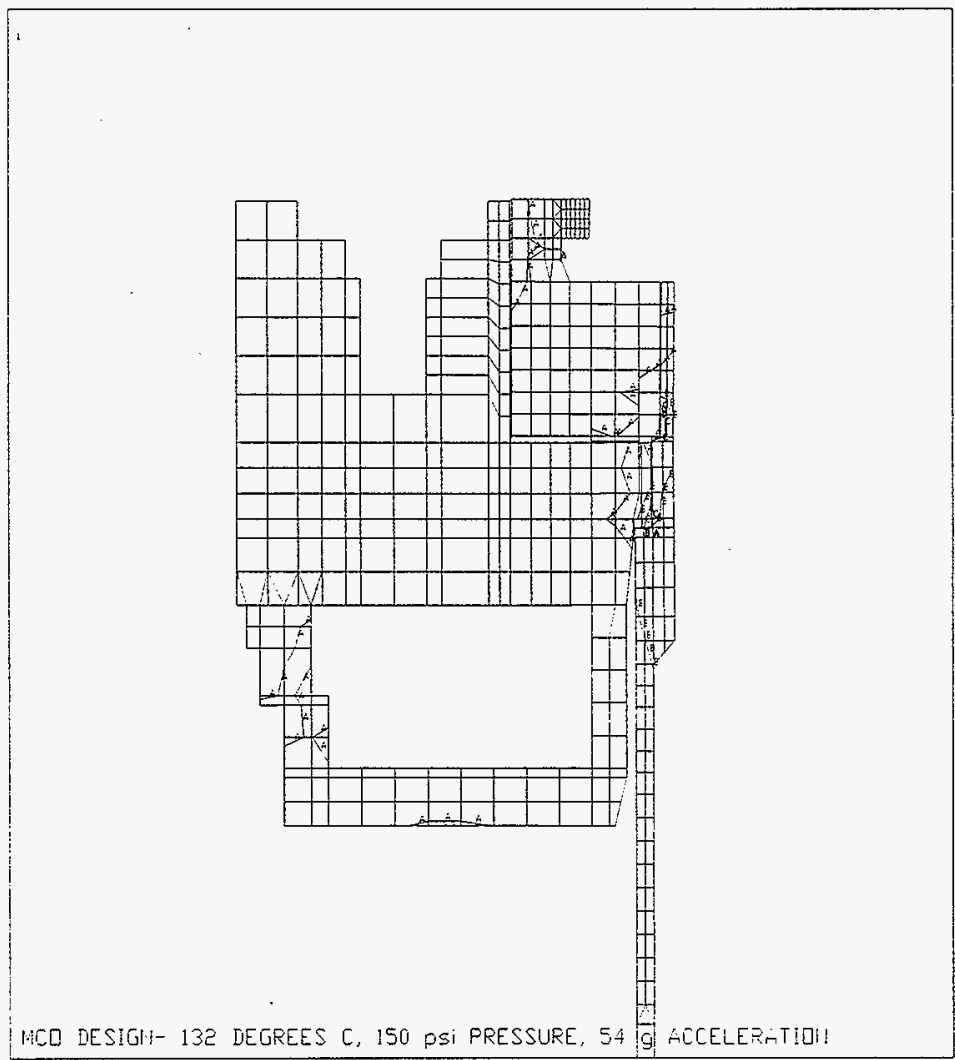

ANSYS 5.0 i 56

MAR 12 199'

$14: 03: 29$

PLOT WO. 3

NODḦL SOLUTION

STEP $=1$

SUR $=1$

TIME $=1$

SINT

(AVG)

DMX $=0.247015$

SINI $=4.684$

$54 x=22715$

$A \quad=1266$

$B=3790$

ᄃ $=6313$

D $=8837$

$\Sigma=11360$

$F \quad=13883$

G $=16407$

$H \quad=18930$

I $=21454$

Figure 10: Bare Bottom End Drop Upper Assembly Stress Intensities

265

0


CLIENT: Duke Engineering \& Services Hanford

FILE NO: $\quad \mathrm{KH}-8009-8-03$

PROJECT: MCO Final Design DOC. NO.: HNF-SD-SNF-DR-003, Rev. 0, Appendix 5

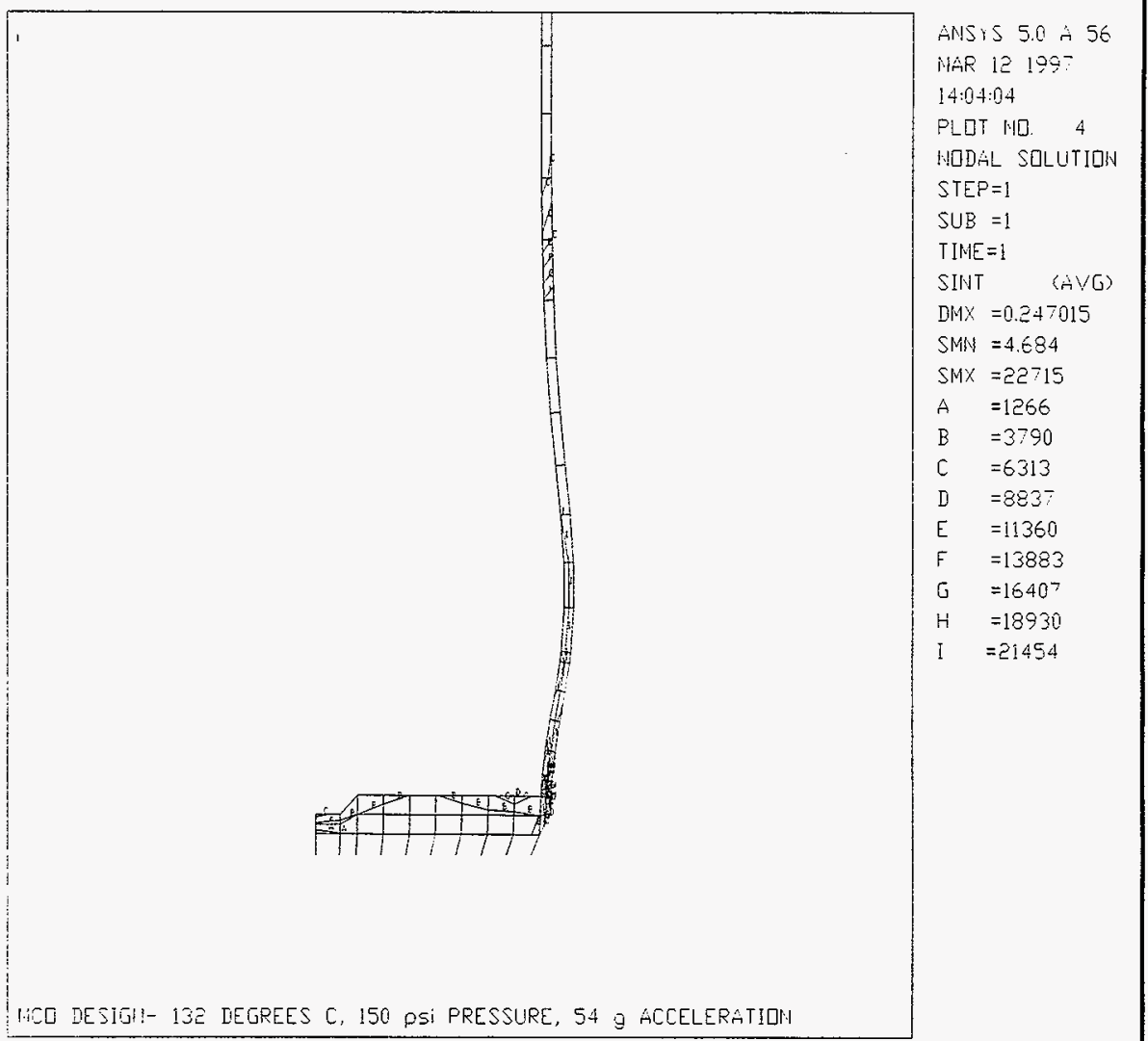

Figure 11: Bare Bottom End Drop Lower Assembly Stress Intensities

\begin{tabular}{|c|c|c|c|c|} 
& 0 & & & \\
\hline $2654 / 17 / 97$ & & & \\
\hline I/ $/ 17 / 97$ & & & \\
\hline
\end{tabular}


CLIENT: Duke Engineering \& Services Hanford

PROJECT: MCO Final Design DOC. NO.: HNF-SD-SNF-DR-003, Rev. 0, Appendix 5

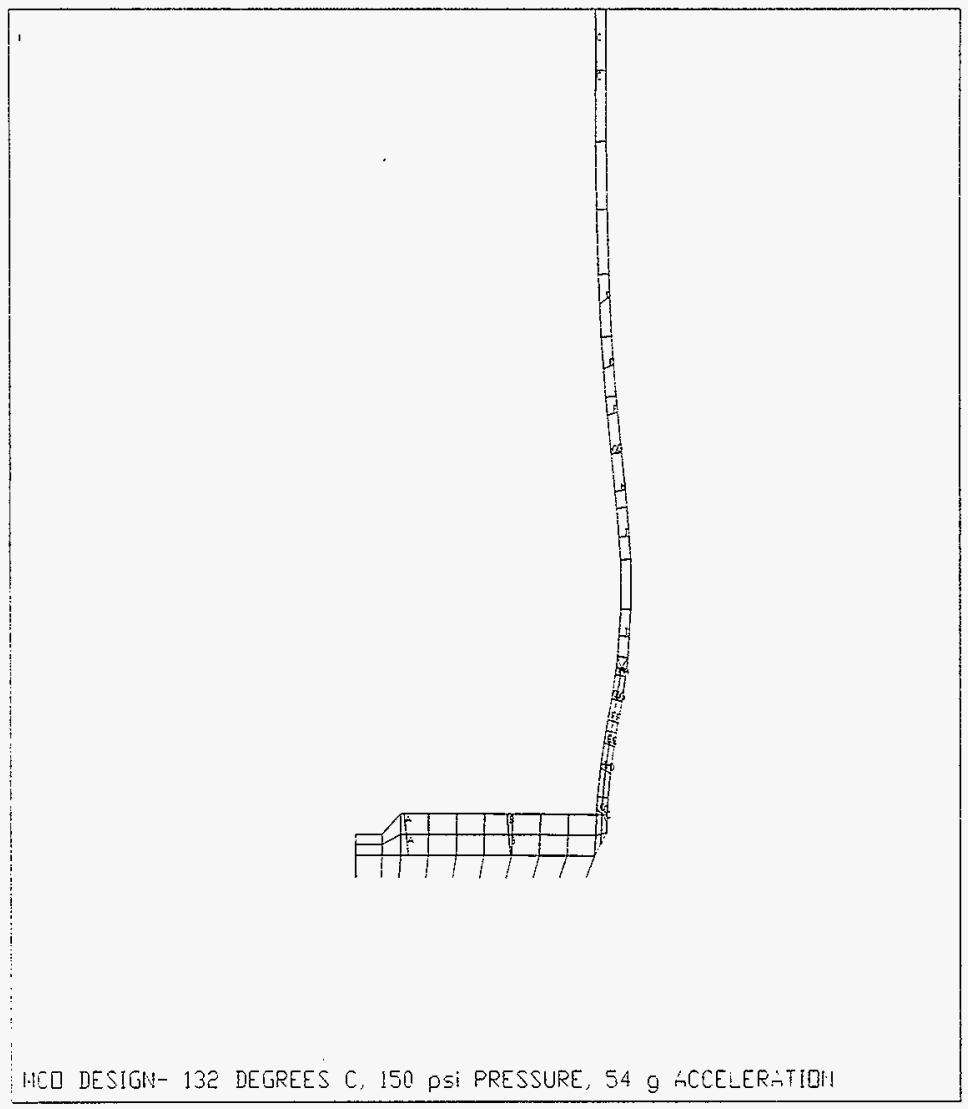

INSIS $5.0-56$

MinR 12 199:

$1359: 28$

PLDT HIJ 1

NODAL SOLUTION

STEP $=$ !

SUB $=1$

TIME $=$ :

Ux

RSYS $=0$

DiN $\times=0.247015$

SMY $=0.083828$

$A \quad=0.004657$

$B \quad=0.013971$

C $=0.023286$

D $=0.0326$

E $\quad=0.041914$

$\mathrm{F} \quad=0.051228$

G $=0.060543$

H $=0.069857$

I $\quad=0.079171$

Figure 12: Bare Bottom End Drop Lower Assembly Radial Displacements 
CLIENT: Duke Engineering \& Services Hanford

PROJECT: MCO Final Design DOC. NO.: HNF-SD-SNF-DR-003, Rev. 0, Appendix 5

FILE NO: KH-8009-8-03

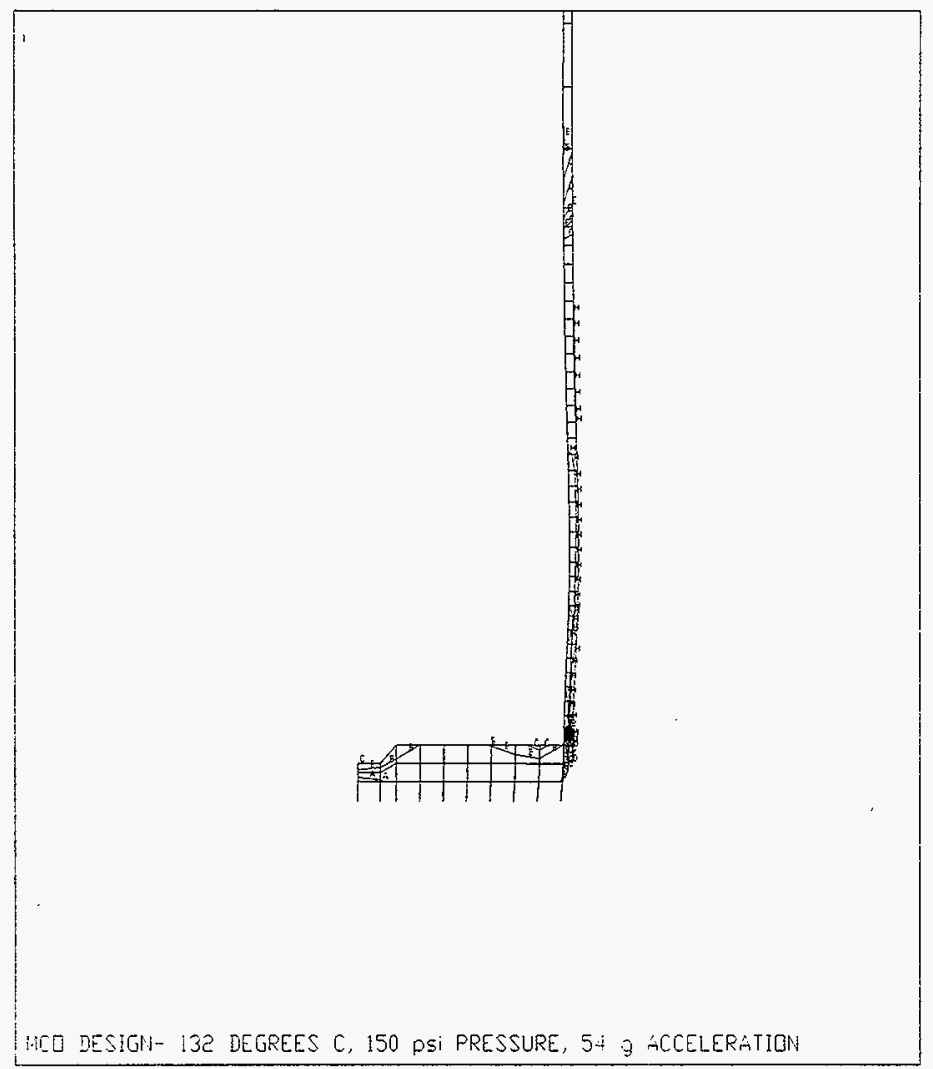

ANSYS 5.0 in 56

APR : 1997

$1 \mathrm{i}: 48: 21$

PLOT NO

NDDÁL SOLUTION

STEP $=1$

SUB $=50$

TIME $=1$

SINT

DMX $=0.229642$

SMIN $=4,621$

$S 14 X=25639$

ZV $=1$

$*$ DIST $=25.449$

$* X F \quad=6.173$

*YF $=10.368$

PRECISE HIDDEN

is $=1429$

$B=42 ;$

C $=7125$

D $=9974$

E $=12822$

$F \quad=15670$

C. $=18519$

$H=21367$

I $=24215$

Figure 13: Bare Bottom End Drop w/ Fine Mesh, Lower Stress Intensities

\begin{tabular}{c|c}
0 \\
\hline $2654 / 17 / 97$ \\
\hline$/ 24 / 17 / 97$
\end{tabular}


CLIENT: Duke Engineering \& Services Hanford

PAASDNS

PROJECT: MCO Final Design

FILE NO: $\quad \mathrm{KH}-8009-8-03$

DOC. NO.: HNF-SD-SNF-DR-003, Rev. 0, Appendix 5

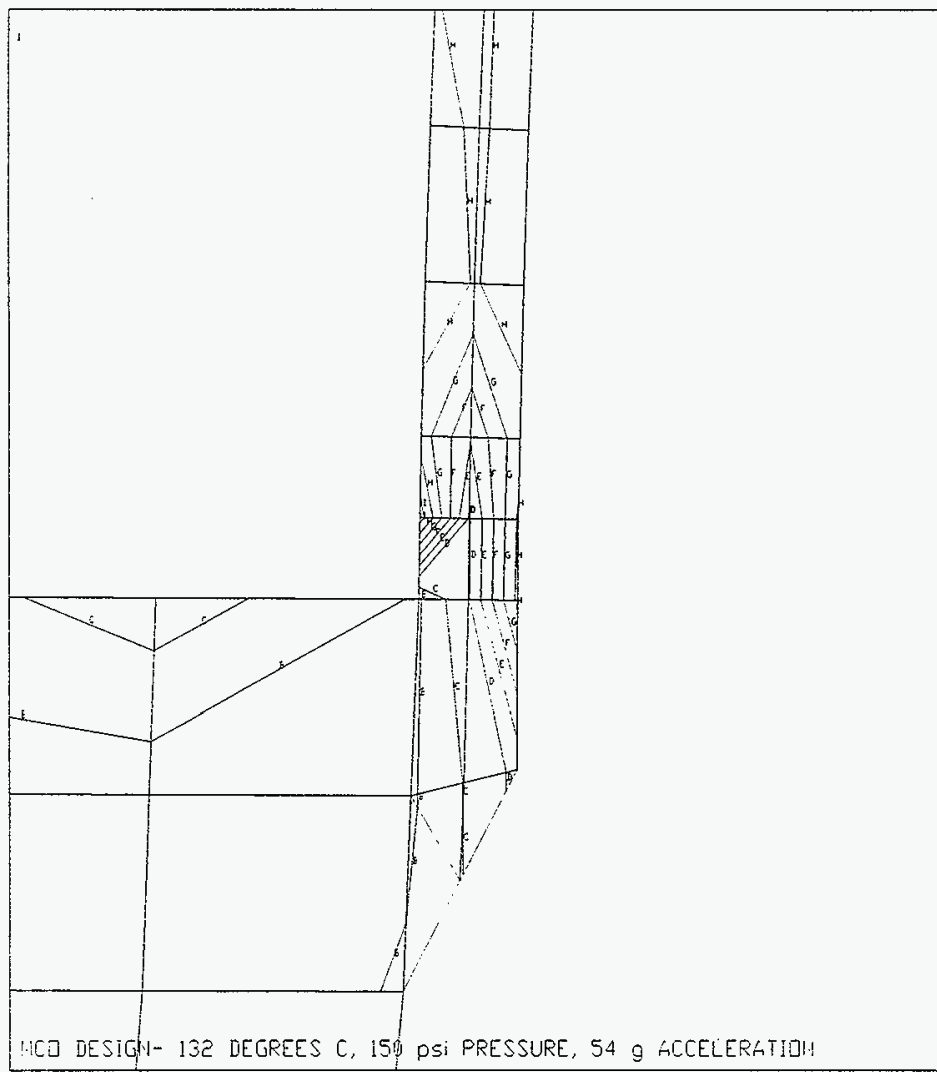

AIISYS 5.0 \& 56

APR 71997

11:50:20

PLOT NO. 2

NODAL SELUTION

STEP $=1$

SUE $=50$

TIME $=1$

SINT

(A) $\mathrm{G}$ )

DMX $=0.229642$

SHN $=4.621$

$S M X=25639$

$Z V=1$

$\approx D I S T=2.386$

*XF $=11.89$

*YF $=0.699034$

FRECISE HIDDEN

$\therefore \quad=1429$

B . $=4277$

$C \quad=7125$

i) $=9974$

$E \quad=12822$

$F \quad=15670$

G $\quad=18519$

$\mathrm{H} \quad=21367$

I $=24215$

Figure 14: Bare Bottom End Drop w/ Fine Mesh, Close-Up 
CLIENT: Duke Engineering \& Services Hanford

PROJECT: MCO Final Design DOC. NO.: HNF-SD-SNF-DR-003, Rev. 0, Appendix 5

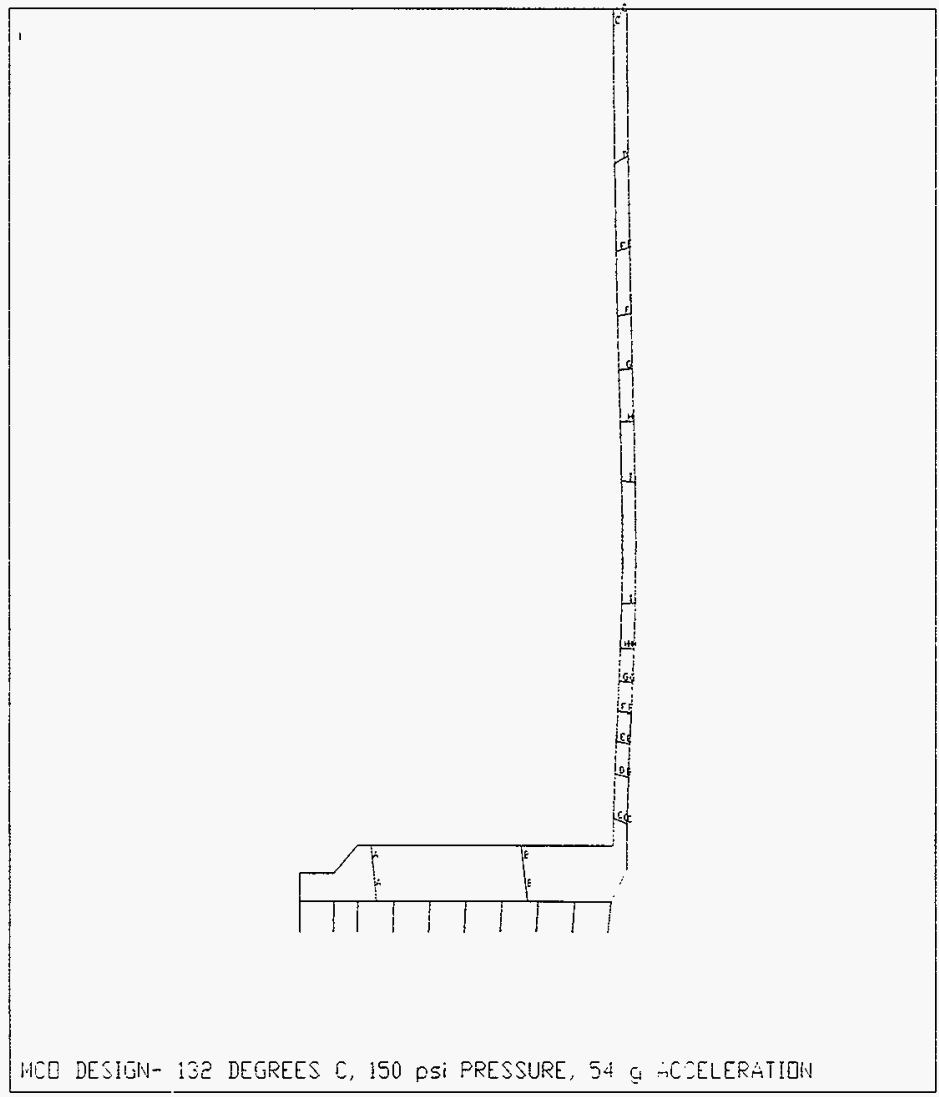

Aillsis 5.0 - 56

$A P R=199^{-}$

11:53:23

PLOT IID. 3

NDDAL SDLUTID!

$S T E P=1$

SUB $=50$

TIME $=1$

U.

RSIS $=0$

DMX $=0.229642$

sil. $x=0.090 \div 17$

$Z \forall=1$

$*$ DIST $=17.148$

$* F \quad=6.407$

$* Y \% \quad=9.776$

PRECISE HIDDEH

EDGE

$\dot{A} \quad=0.00504$

B $=0.01512$

C $=0.025100$

D $=0.035279$

E $\quad=0.045359$

$F \quad=0.055438$

G $\quad=0.065518$

$H \quad=0.075598$

I $=0.085678$

Figure 15: Bare Bottom End Drop w/ Fine Mesh, Radial Displacements 
CLIENT: Duke Engineering \& Services Hanford

\section{PROJECT: MCO Final Design DOC. NO.: HNF-SD-SNF-DR-003, Rev. 0, Appendix 5}

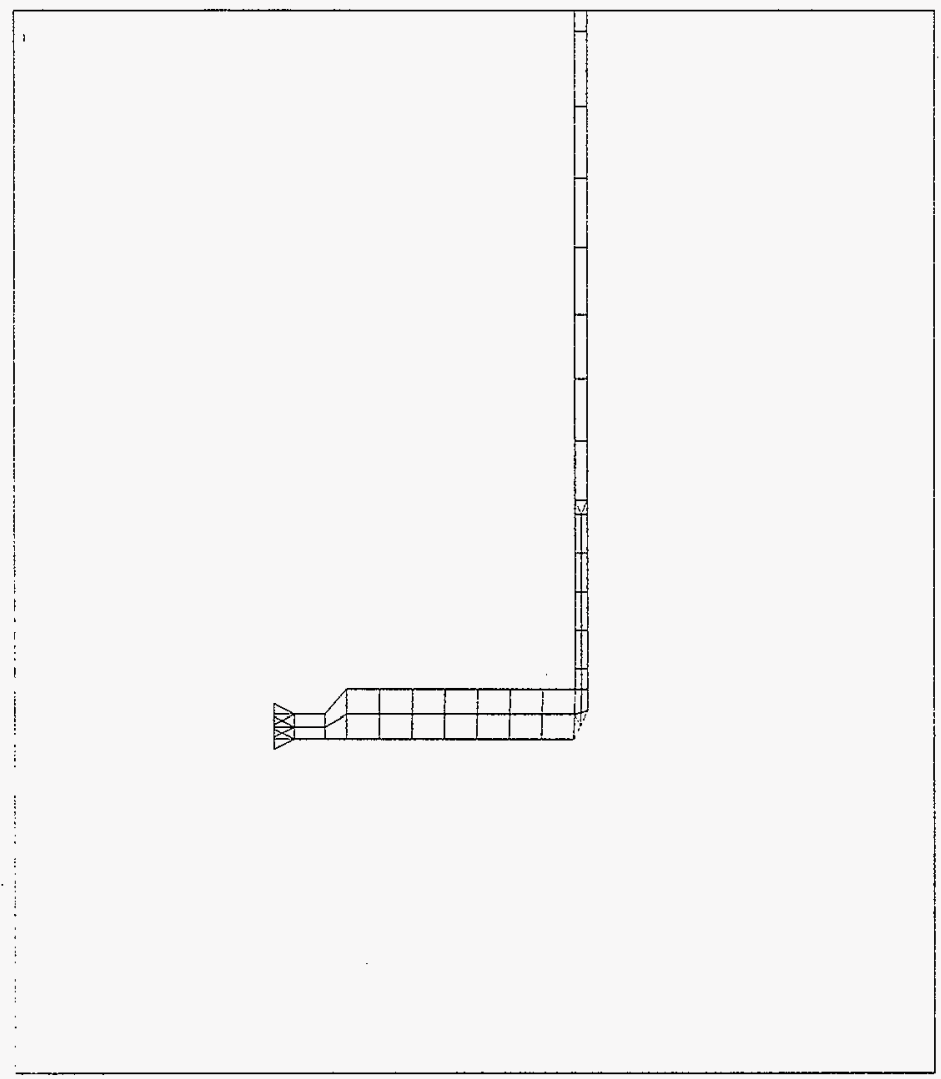

-NASis $5.0 \therefore 56$

FEB 2001997

10:56:40

PLOT NO. 3

ELEMENTS

TYPE NUN

U

$Z \sqrt{Z V}=1$

$*$ DIST $=18.829$

$\% \mathrm{XF}=7.356$

*YF $=5.622$

PRECISE HIDDEN

Figure 16: Top End Drop Lower MCO Boundary Conditions 
CLIENT: Duke Engineering \& Services Hanford PROJECT: MCO Final Design DOC. NO.: HNF-SD-SNF-DR-003, Rev. 0, Appendix 5

FILE NO: $\quad \mathrm{KH}-8009-8-03$

PROJECT: MCO Final Design

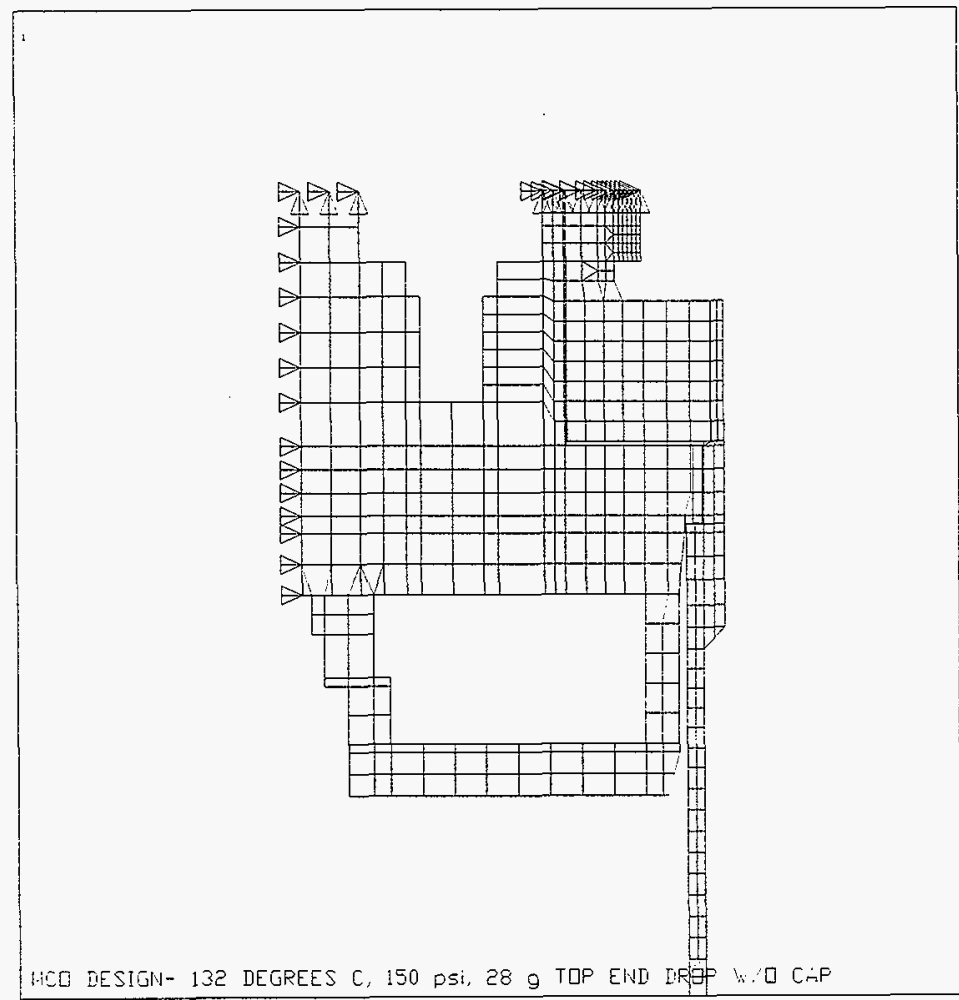

MNSYS $5.0 \div 56$

FEB 21 190-

$0 \div 31: 06$

PLOT NO. 3

ELEHENTS

TYPE NUW

$U$

$Z Y=1$

$\because D I S T=14.052$

$* X F \quad=5.568$

*YF $=150.16$ ?

PRECISE HIDDEN

Figure 17: Top End Drop Upper MCO Boundary Conditions w/ Gap Elements 
CLIENT: Duke Engineering \& Services Hanford

FILE NO: KH-8009-8-03

PROJECT: MCO Final Design DOC. NO.: HNF-SD-SNF-DR-003, Rev. 0, Appendix 5

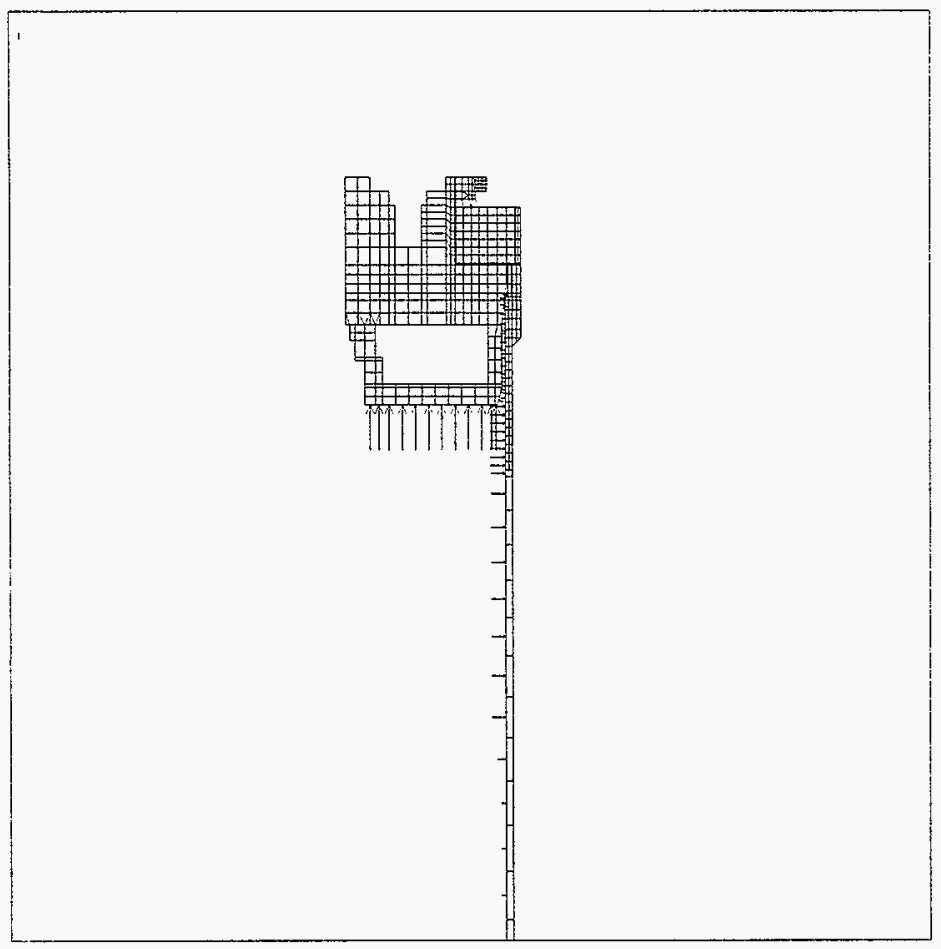

INSSIS $5.0 \div 56$

FEB 20 190-

10:06:3-

PLOT ND. ₹

ELEHENTS

TYPE NU!:

PRES

$Z \because \quad=1$

$*$ DIST $=33.253$

*XF $=8.906$

*YF $=136.592$

PRECISE HIDDEN

Figure 18: Top End Drop Pressure Distribution (Hydrostatic \& Internal) 
CLIENT: Duke Engineering \& Services Hanford

FILE NO: $\quad \mathrm{KH}-8009-8-03$

PROJECT: MCO Final Design DOC. NO:: HNF-SD-SNF-DR-003, Rev. 0, Appendix 5

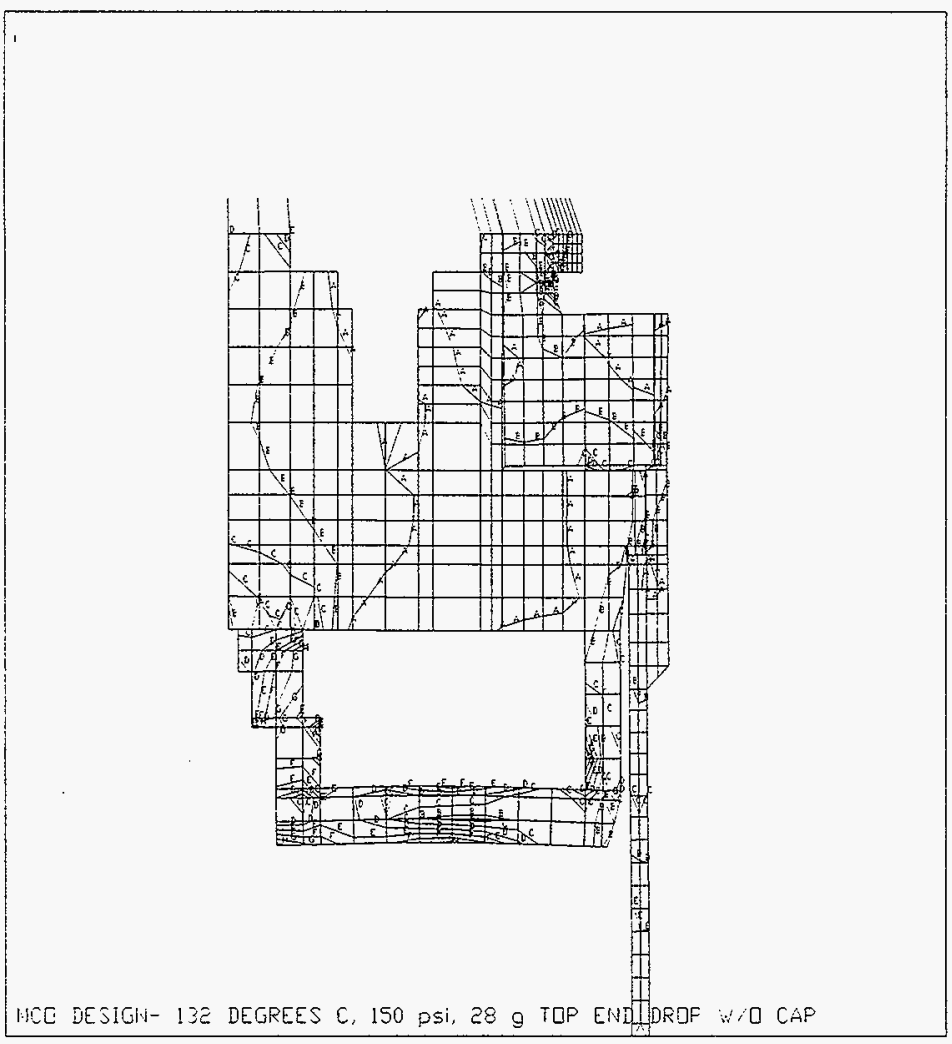

RNSIS 5.0 in 56 HAR $12199^{\circ}$

$15: 12: 55$

PLOT HO. I NODAL SOLUTIOH STEF $=1$

SUR $=1$

TIN $=1$

SINT (A)

$\mathrm{DHX}=0.303709$

SM $=319.7-75$

$\sin x=20756$

$\mathrm{A} \quad=1455$

B $\quad=3: 26$

C $=5997$

D $=8267$

$E \quad=10538$

$F \quad=12809$

G. $=15080$

$\mathrm{H}=1 \div 350$

$1=19621$

Figure 19: Top End Drop Upper MCO Assembly Stress Intensities

\begin{tabular}{|c|c|l|l|}
\hline 0 & & & \\
\hline x) 4/17/97 & & & \\
\hline 1e 4/17/97 & & & \\
\hline
\end{tabular}


E PAFD日N5

CLIENT: Duke Engineering \& Services Hanford

PROJECT: MCO Final Design DOC. NO.: HNF-SD-SNF-DR-003, Rev. 0, Appendix 5

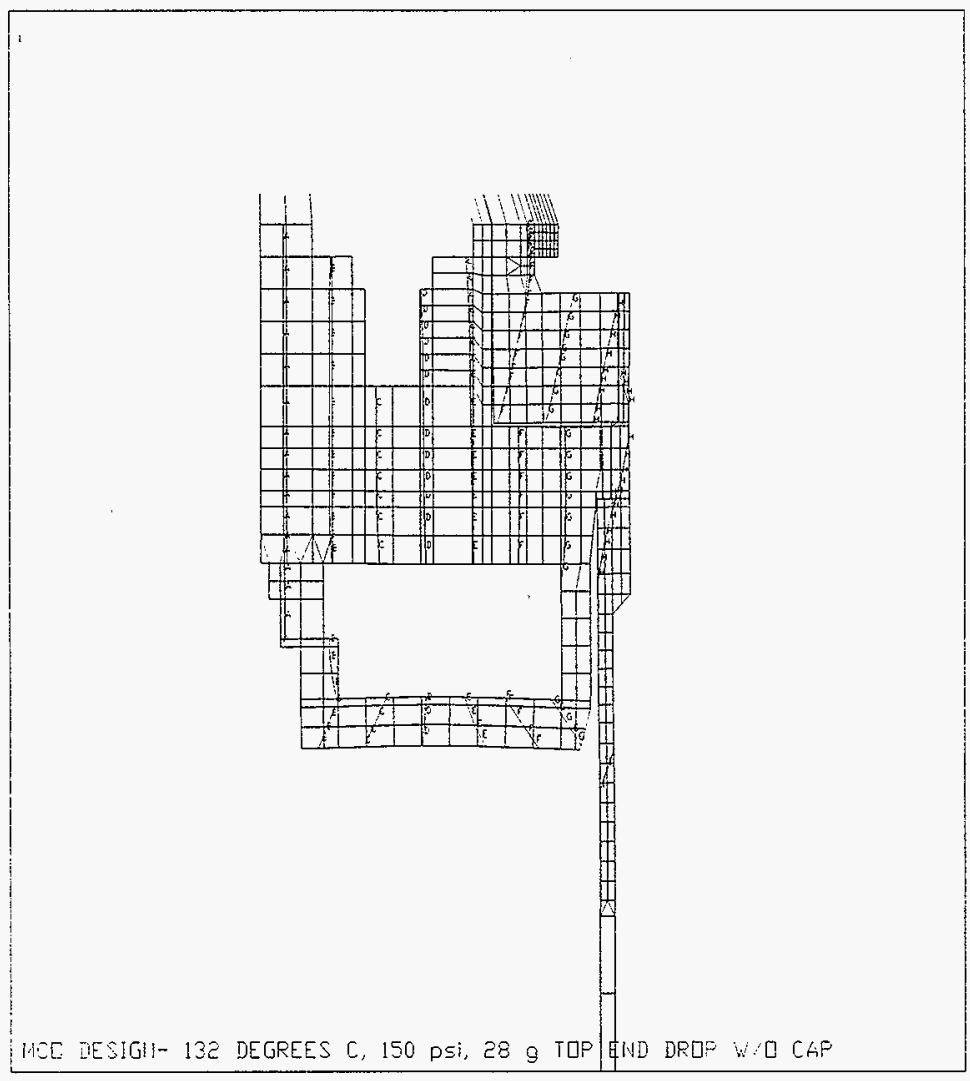

AIISIS $5.0 ; 56$

HAR 12 199:

$15: 15: 21$

PLOT HD. 2

MUDAL SELUTION

STEP $=1$

SUB $=1$

THNE $=1$

UX

RSIS $=0$

$D H X=0.303709$

SinX $=0.027078$

A $=0.001504$

$B=0.004513$

C $\quad=0.007522$

b) $=0.01053$

$E \quad=0.013539$

$F \quad=0.016548$

G $=0.019556$

$H=0.022565$

I $=0.0255^{-4}$

Figure 20: Top End Drop Upper MCO Assembly radial Displacements

\begin{tabular}{|c|}
0 \\
\hline 26s $4 / 17 / 97$ \\
\hline fet $4 / 17 / 97$ \\
\hline
\end{tabular}


CLIENT: Duke Engineering \& Services Hanford

FILE NO: KH-8009-8-03

PROJECT: MCO Final Design

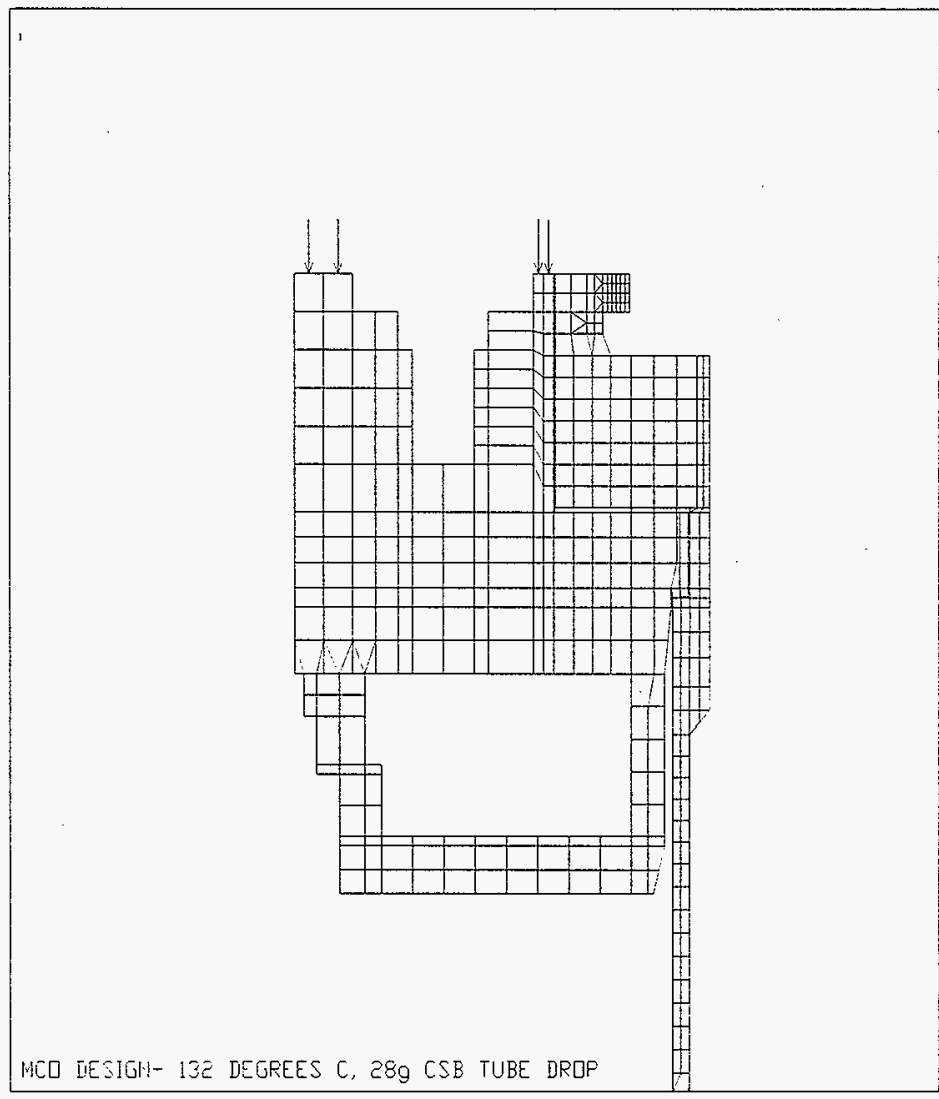

filsis 5.0 in 56

WHR 3 1997

20:11:4-

PLOT NO. I

ELEMEINTS

TYPE INUN

PRES

$Z \because=1$

*DIST $=14.122$

* $X F \quad=5.458$

*YF $=150.817$

PRECISE HIDDEN

Figure 21: CSB Tube Drop (no cap) Pressure Distribution 
CLIENT: Duke Engineering \& Services Hanford

FILE NO: $\quad \mathrm{KH}-8009-8-03$

PROJECT: MCO Final Design DOC. NO.: HNF-SD-SNF-DR-003, Rev. 0, Appendix 5

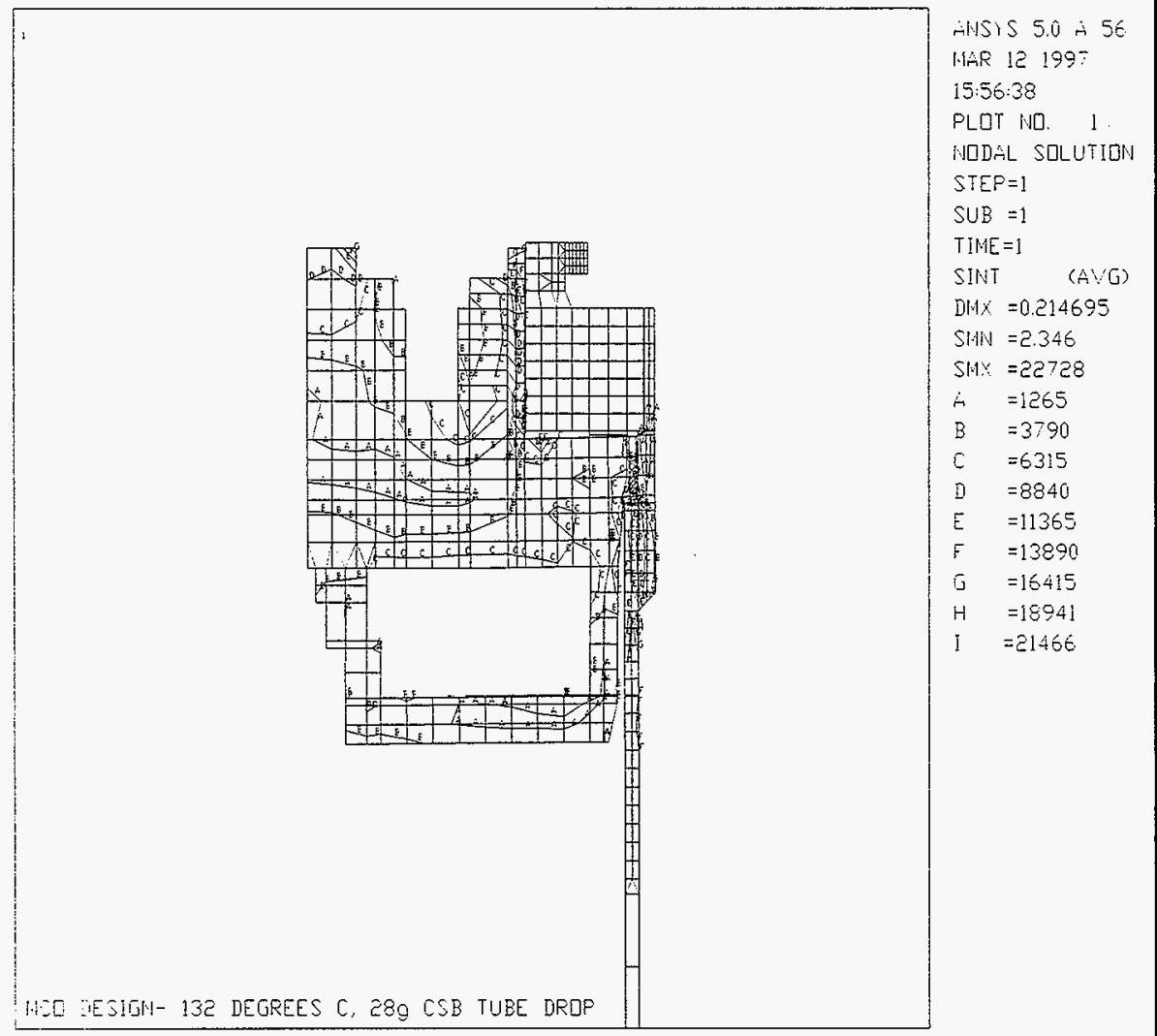

Figure 22: CSB Tube Drop (no cap) Upper MCO Assembly Stress Intensities

\begin{tabular}{|c|c|c|c|}
\hline 0 & & & \\
\hline $\operatorname{las} 4 / 17 / 97$ & & & \\
\hline $\ln 4 / 17 / 97$ & & & \\
\hline
\end{tabular}




\section{DaASENS}

CLIENT: Duke Engineering \& Services Hanford

FILE NO: $\mathrm{KH}-8009-8-03$

PROJECT: MCO Final Design DOC. NO.: HNF-SD-SNF-DR-003, Rev. 0, Appendix 5

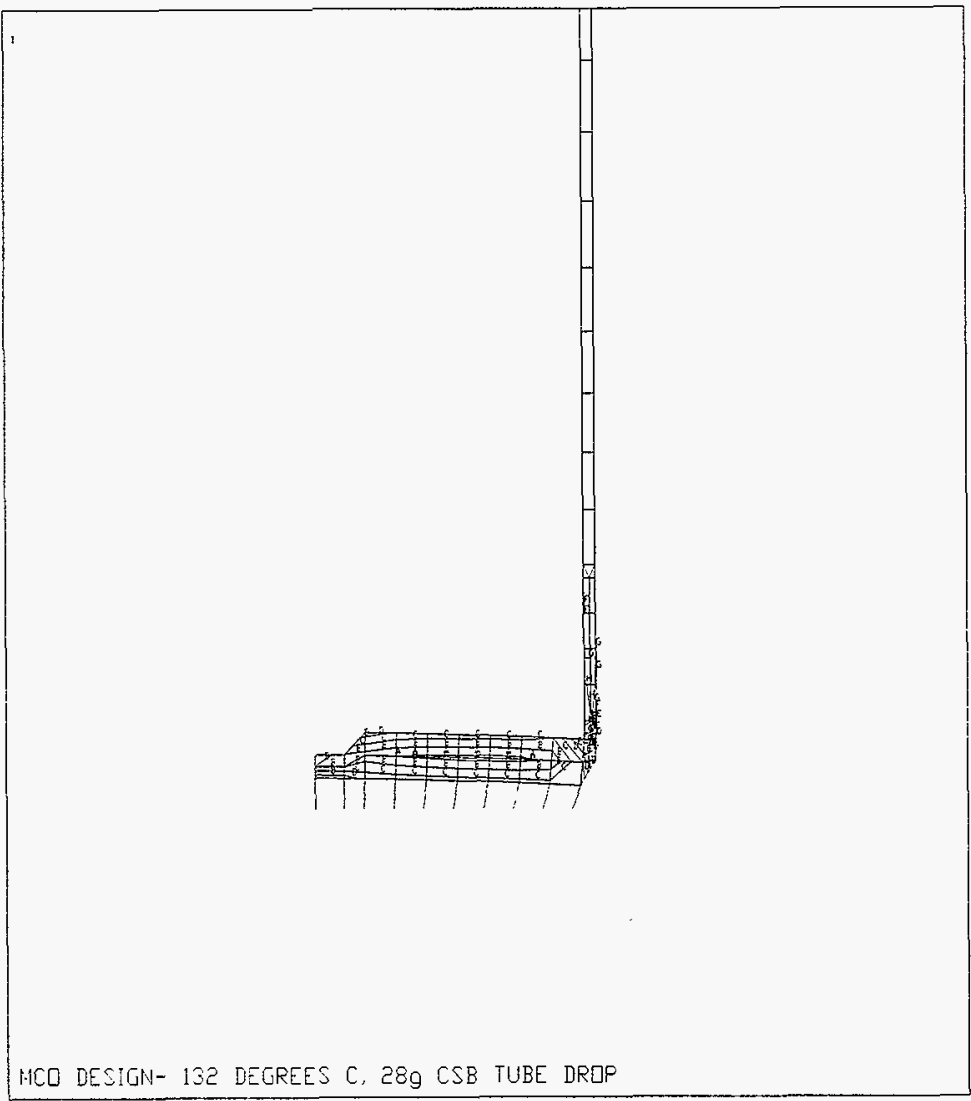

AIJSIS $5.0 \therefore 56$

I.IAR $12199^{-}$

15:57:28

PLOT NO. 2

NIDAAL SOLUTIOH

STEF $=1$

SUE $=1$

TIME $=1$

SINT

(Ai: G)

$D M X=0.214695$

SWN $=2.346$

SHX $=22728$

A $=1265$

$\bar{B}=3790$

C $=6315$

D $=8840$

$E=11365$

$F \quad=13890$

() $\mathbf{1 6 4}$ !5

$H=18941$

$I=214 E 6$

Figure 23: CSB Tube Drop (no cap) Lower MCO Assembly Stress Intensities

\begin{tabular}{|c|c|}
\hline 0 \\
$2(24 / 17 / 97$
\end{tabular}


CLIENT: Duke Engineering \& Services Hanford

(2)

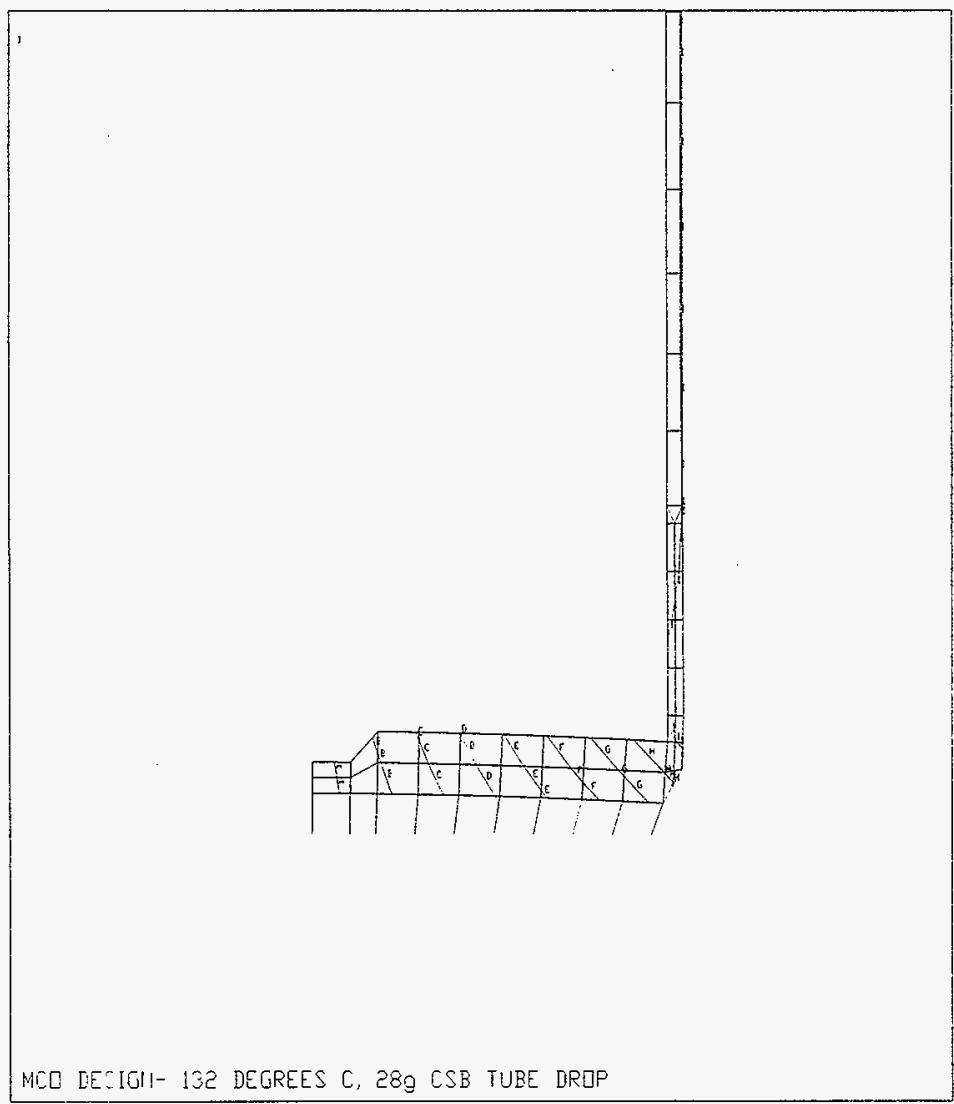

जils is $50 \therefore 56$

HAR 12 199?

15:50:34

FLOT ND. 3

HODAL SOLUTION

STEP $=1$

SUB $=1$

TIME $=1$

UY,

RSIS $=0$

DHX $=0.214695$

SH: $: 0.025684$

$A \quad=0.001427$

B $=0.004281$

C $=0.007134$

D $\quad=0.009988$

E $=0.012842$

$F \quad=0.015696$

5 $=0.01855$

$\mathrm{H}=0.021403$

I $=0.02425$ ?

Figure 24: CSB Tube Drop (no cap) Lower MCO Assembly Radial Displacements

\begin{tabular}{c}
0 \\
\hline 4/17/97 \\
\hline $1 / 17 / 97$
\end{tabular}


Duke Engineering \& Services HanSDNS PROJECT: MCO Final Design DOC. NO.: HNF-SD-SNF-DR-003, Rev. 0, Appendix 5

FILE NO: KH-8009-8-03

MCOFinal Design

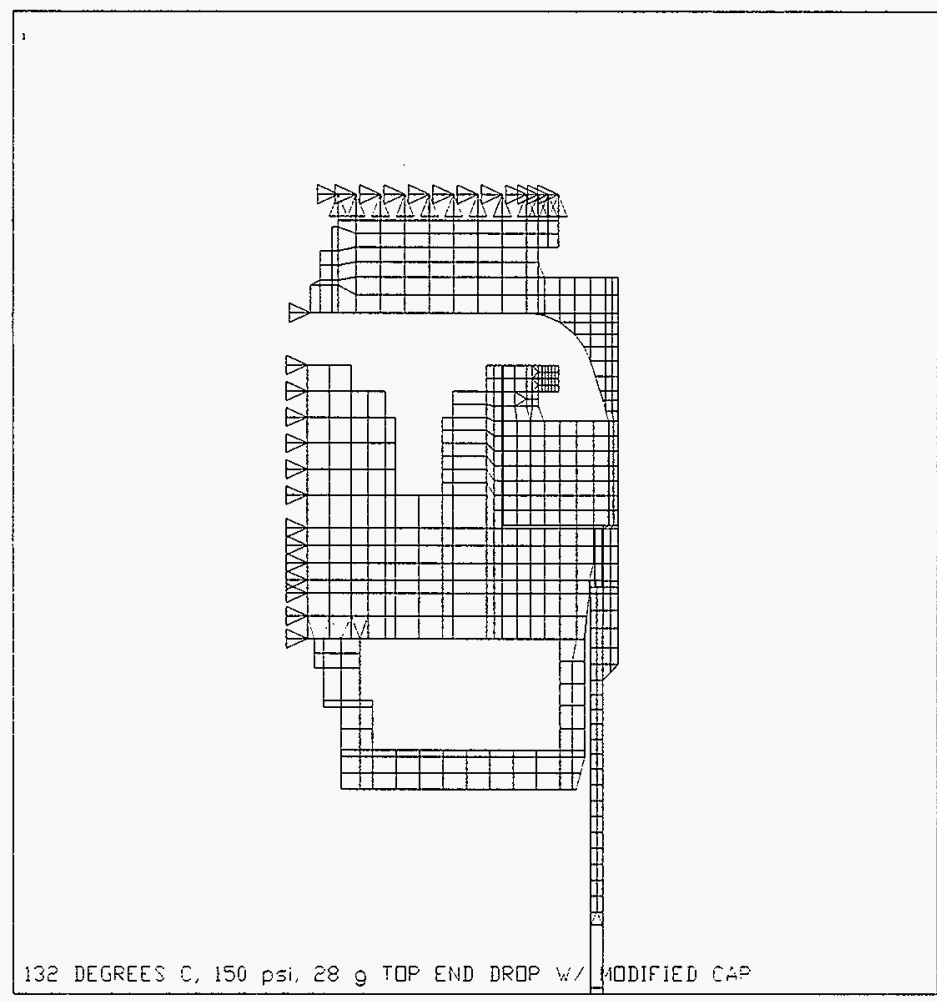

ANSYS 5.0 in 56

HiR 13 199-

09:25:40

PLOT NG. 5

ELEMENTS

TYPE NUM

U

$Z \because=1$

*DIST $=18.725$

*XF $=6.809$

*YF $=152.7 \in 9$

PRECISE HIDDEN

Figure 25: Top End Drop with Modified Cap and Upper Boundary Conditions

\begin{tabular}{|c|c|c|c|}
\hline 0 & & & \\
\hline $2 G 54 / 17 / 97$ & & & \\
\hline$/ N / 4 / 17 / 97$ & & & \\
\hline
\end{tabular}


CLIENT: Duke Engineering \& Services Hanford

\section{PROJECT: MCO Final Design $\quad$ DOC. NO.: HNF-SD-SNF-DR-003, Rev. 0, Appendix 5}

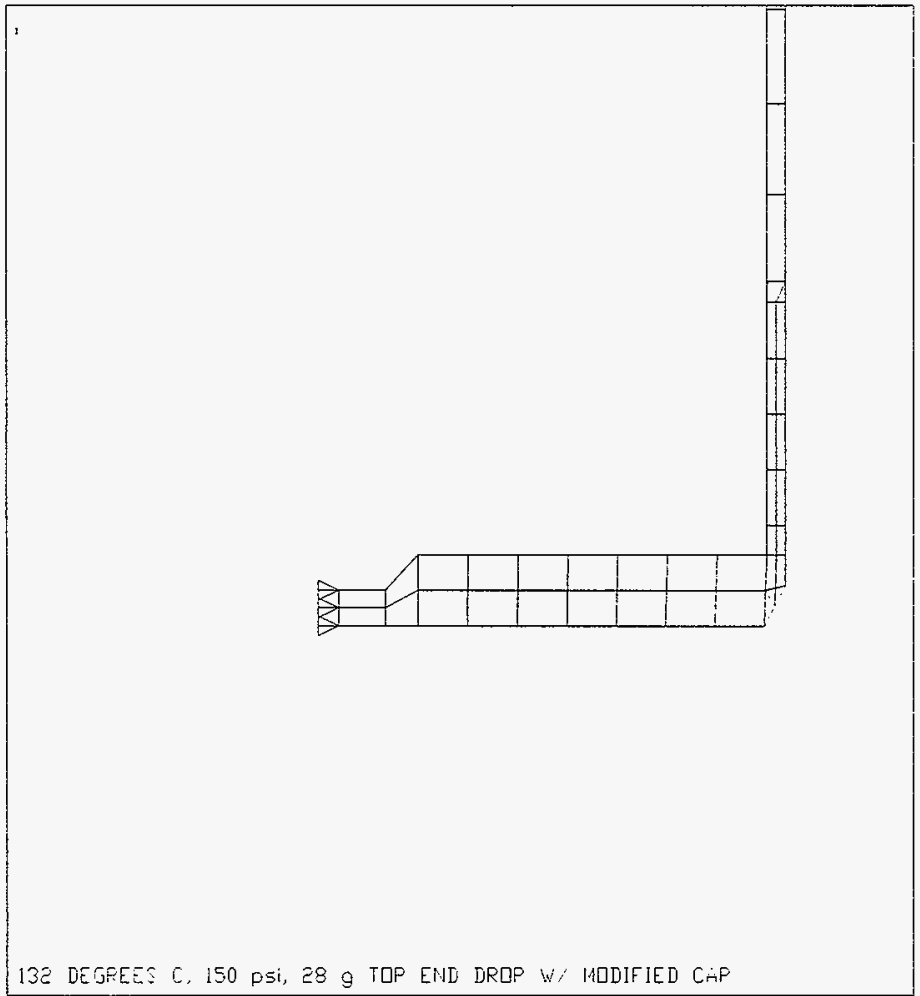

ANSIS 50 A 56

HIIRR 13 199:

09:26:17

PLOT HIO. 6

ELENENTS

TYPE NUI

U

$7=1$

*DIST $=12.153$

$\approx \times F \quad=3.252$

*YF $=1.716$

PRECISE HIDOEN

Figure 26: Top End Drop with Modified Cap and Lower Boundary Conditions

\begin{tabular}{|c|c|l|l|l|} 
& 0 & & & \\
\hline $\operatorname{Zg}, 4 / 17 / 97$ & & & \\
\hline $\operatorname{le} 4 / 17 / 97$ & & & \\
\hline
\end{tabular}


CLIENT: Duke Engineering \& Services Hanford FILE NO: KH-8009-8-03

PROJECT: MCO Final Design DOC. NO.: HNF-SD-SNF-DR-003, Rev. 0, Appendix 5

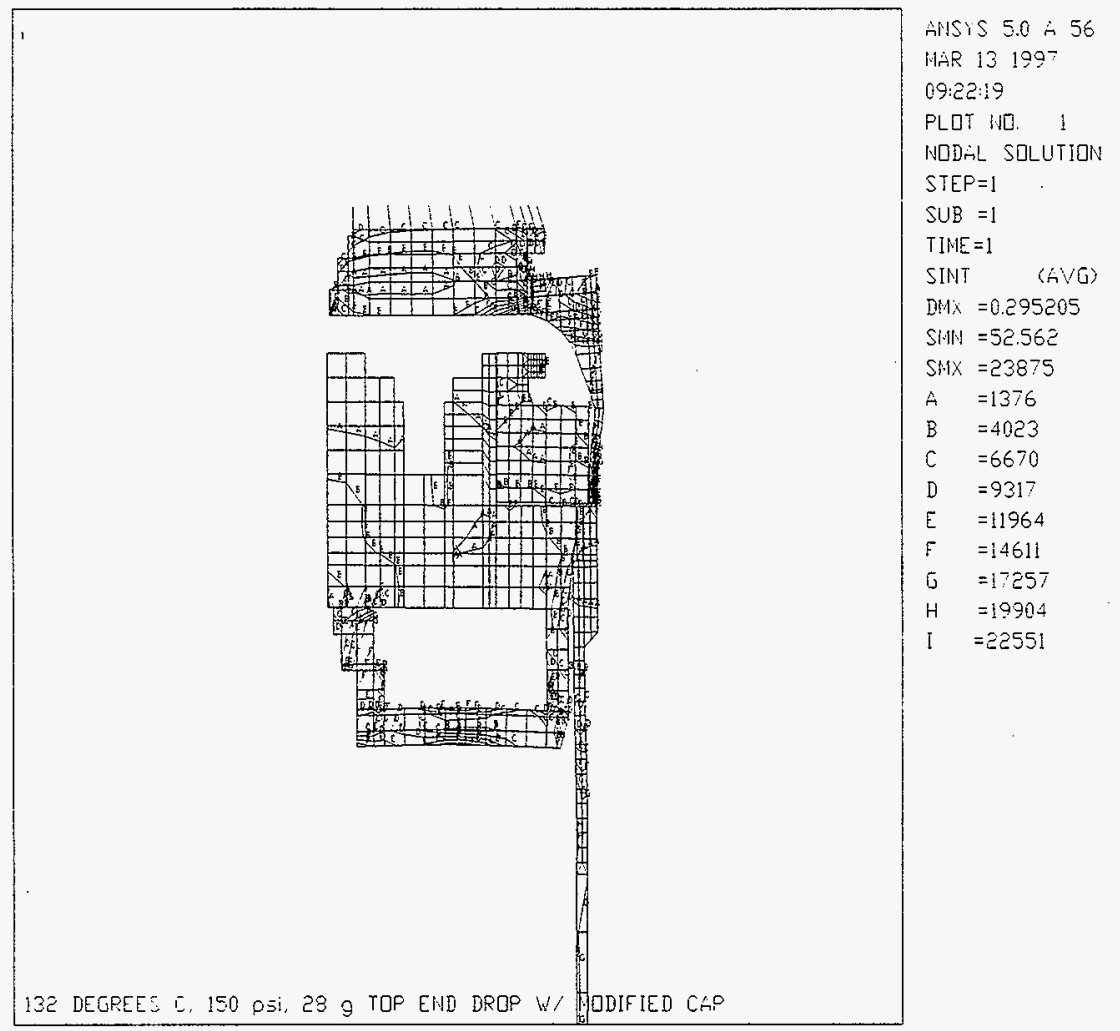

Figure 27: Top End Drop with Modified Cap and Upper Stress Intensities 
CLIENT: Duke Engineering \& Services Hanford

FILE NO: $\quad \mathrm{KH}-8009-8-03$ PROJECT: MCO Final Design DOC. NO.: HNF-SD-SNF-DR-003, Rev. 0, Appendix 5

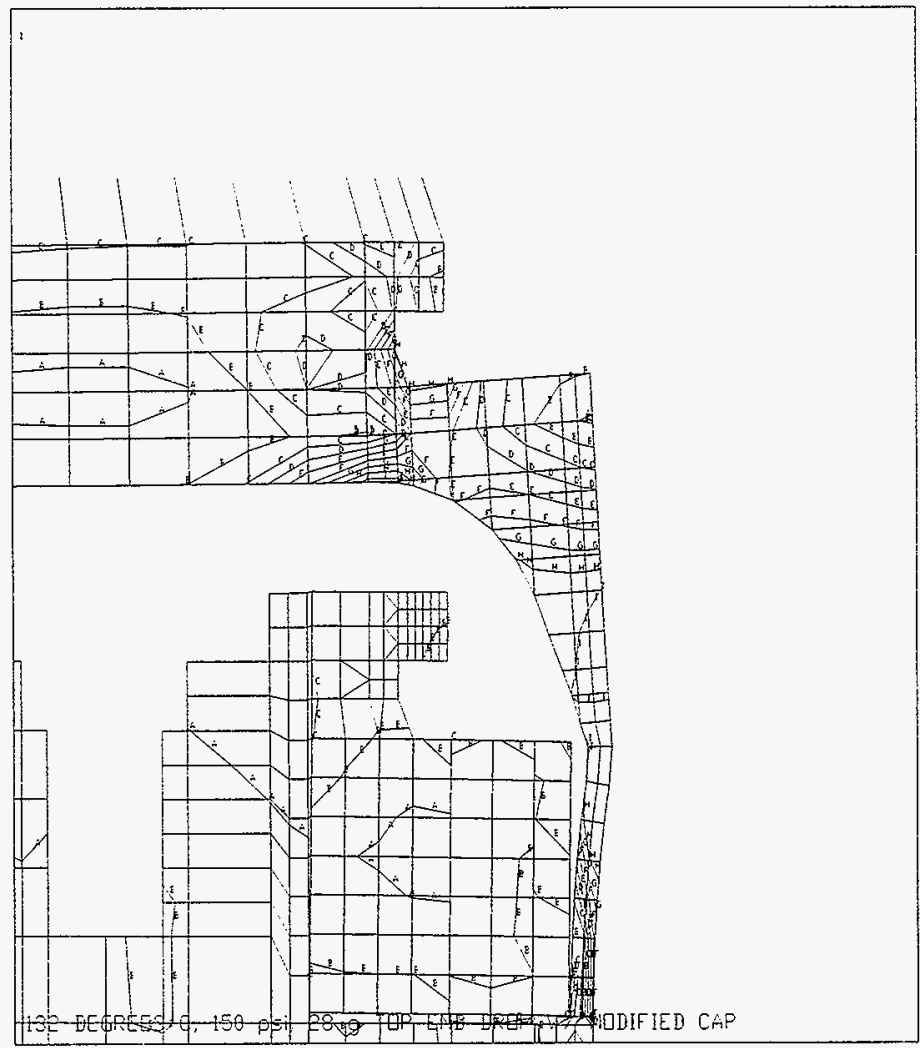

ANSIS 5.0 \& 56

WAR 131907

09:23:05

PLOT ND. 2

NODAL SOLUTION

STEP $=1$

SUB $=1$

TIME $=1$

SINT (AVG)

D.M $Y=0.295205$

SNN $=52.562$

$S M x=\hat{2} 3875$

A $\quad=1376$

E $=4023$

C $=6670$

D) $=9317$

$E \quad=11964$

$F \quad=14611$

o $=1725:$

$\mathrm{H}=19004$

$1=22551$

Figure 28: Top End Drop, Modified Cap @ Closure Weld, Stress Intensities 
CLIENT: Duke Engineering \& Services Hanford

FILE NO: KH-8009-8-03

PROJECT: MCO Final Design DOC. NO.: HNF-SD-SNF-DR-003, Rev. 0, Appendix 5

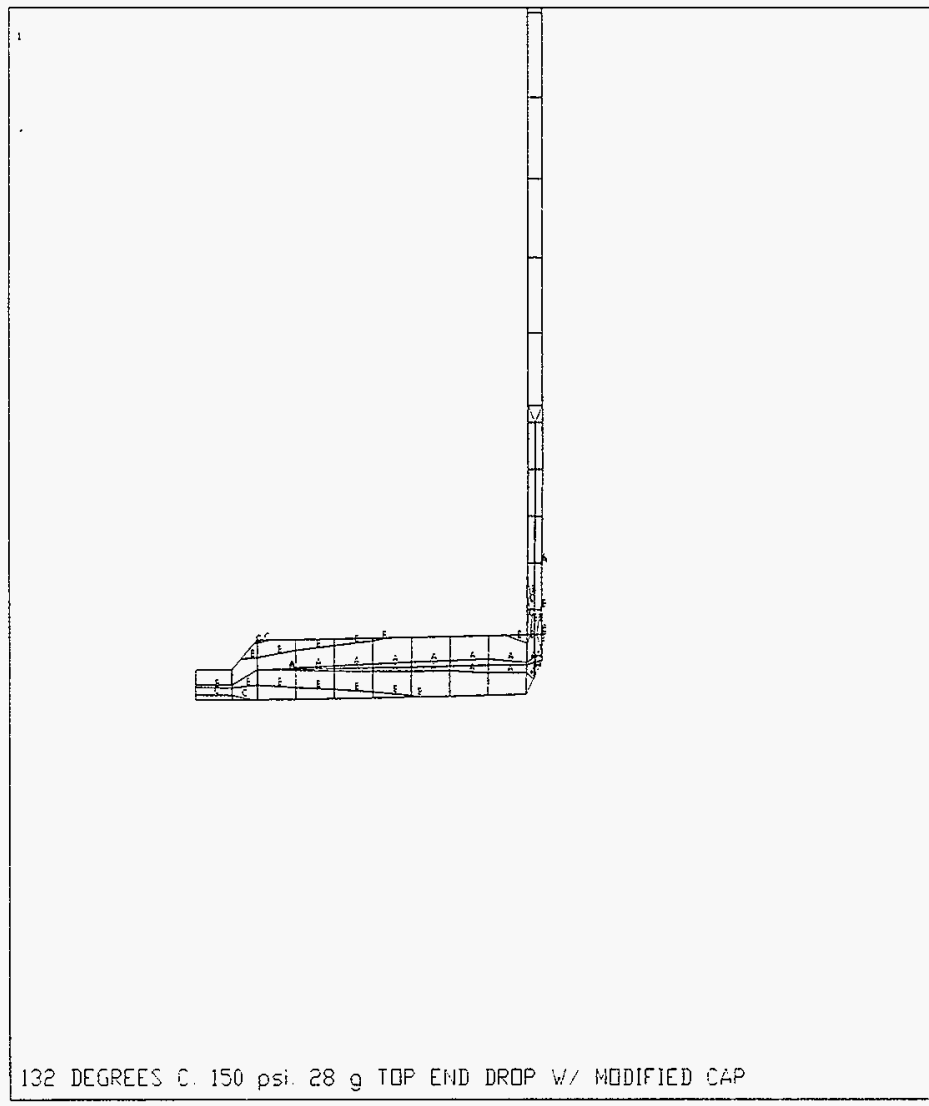

Alisis 5.0 a 56

HifR 13 lag:

09:23:53

PLOT 1.T. 3

IIODAL SDLUTIDN

STEF $=1$

SUE $=1$

TIME $=1$

SINT

(A) 10 )

DM: $=0.295205$

SMN $=52.562$

$S M x=23875$

$\therefore \quad=\$ 376$

$\mathrm{E}=4023$

C $=6670$

D) 931 ?

$E=11964$

$\mathrm{F}=14611$

I. $=1725$

$\mathrm{H}=19904$

$1=22551$

Figure 29: Top End Drop with Modified Cap Lower Stress Intensities 
CLIENT: Duke Engineering \& Services Hanford

FILE NO: $\quad \mathrm{KH}-8009-8-03$

PROJECT: MCO Final Design DOC. NO.: HNF-SD-SNF-DR-003, Rev. 0, Appendix 5

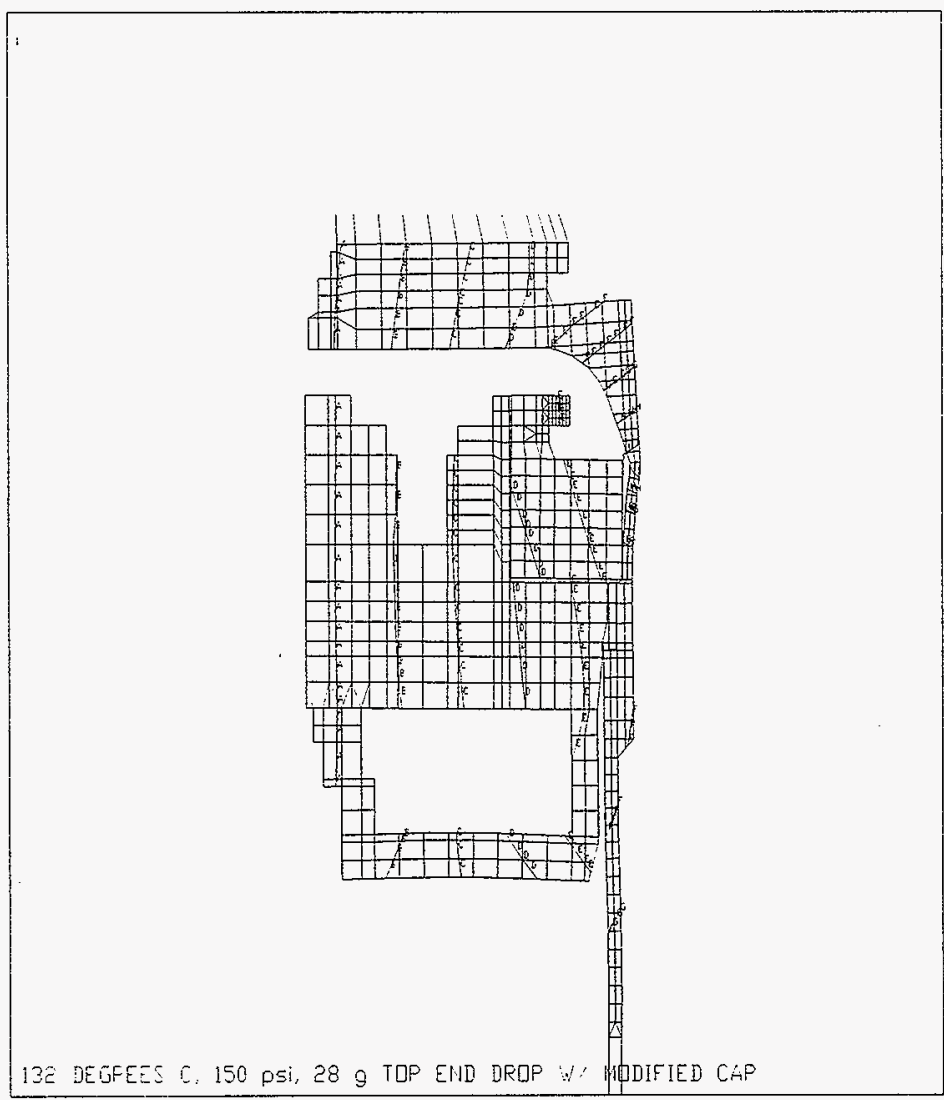

insis $5.0 \therefore 56$

VIAR 13 199-

$09: 24: 45$

PLOT ND. 4

WODAL SULUTION

$S T E P=1$

SUB $=1$

THHE $=1$

$\mathrm{U} \times$

RSIS $=0$

DH: $=0.295205$

$\sin x=0.03953$

$A \quad=0.002196$

B $=0.006588$

C $\quad=0.010981$

D $=0.015373$

E $\quad=0.019765$

$F=0.02415$ ?

ᄃ $=0.02855$

$H=0.032942$

I $=0.037334$

Figure 30: Top End Drop with Modified Cap and Radial Displacements

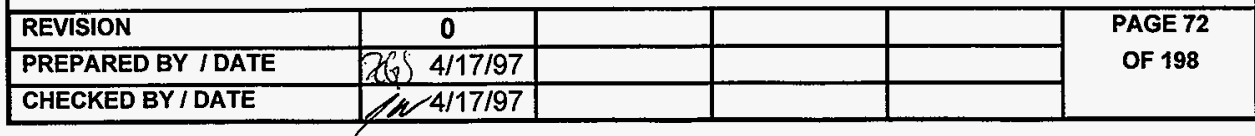


CLIENT: Duke Engineering \& Services Hanford FILE NO: KH-8009-8-03

PROJECT: MCO Final Design DOC. NO.: HNF-SD-SNF-DR-003, Rev. 0, Appendix 5

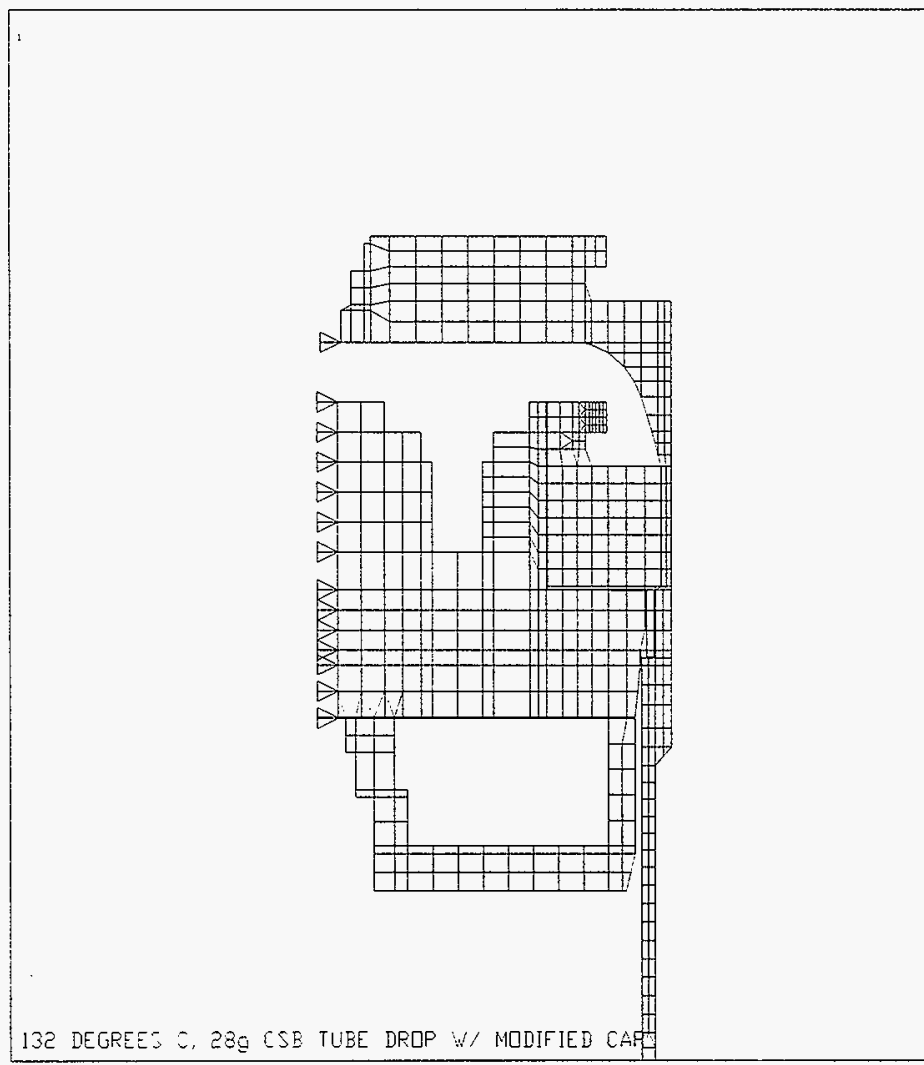

AlIS1S $500 \div 56$

HAR $13199^{-}$

$09: 57.52$

PLDT H. 6

ELEI-HENTS

TYPE NULA

U

$2 \mathrm{~V}=1$

*DIST $=1 \mathrm{~J} .386$

ㅅF $=4.938$

\%F $=\$ 53.63$

PRECISE HIDDEN

Figure 31: CSB Tube Drop with Modified Cap and Upper Boundary Conditions

0


PROJECT: MCO Final Design DOC. NO.: HNF-SD-SNF-DR-003, Rev. 0, Appendix 5

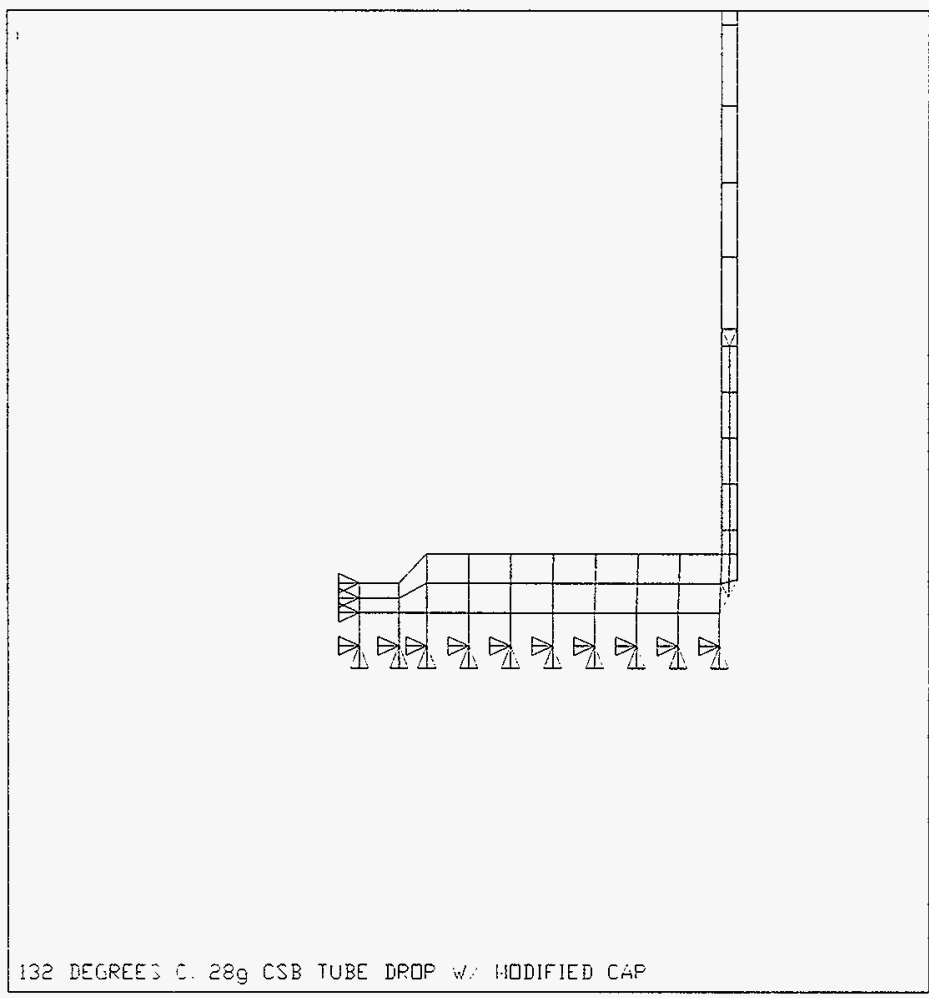

-ivs is 50 in 56

IIAR î 19:-

$09.5^{-} \cdot 28$

PLOT IV. 5

ELEMEITTS

TYPE IUUIF

U

$2: \quad=1$

*DIST $=14.604$

mXF $=3.466$

*YF $=1.952$

PRECISE HIDDEN

Figure 32: CSB Tube Drop with Modified Cap and Lower Boundary Conditions 
CLIENT: Duke Engineering \& Services Hanford

FILE NO: $\quad \mathrm{KH}-8009-8-03$

PROJECT: MCO Final Design

DOC. NO.: HNF-SD-SNF-DR-003, Rev. 0, Appendix 5

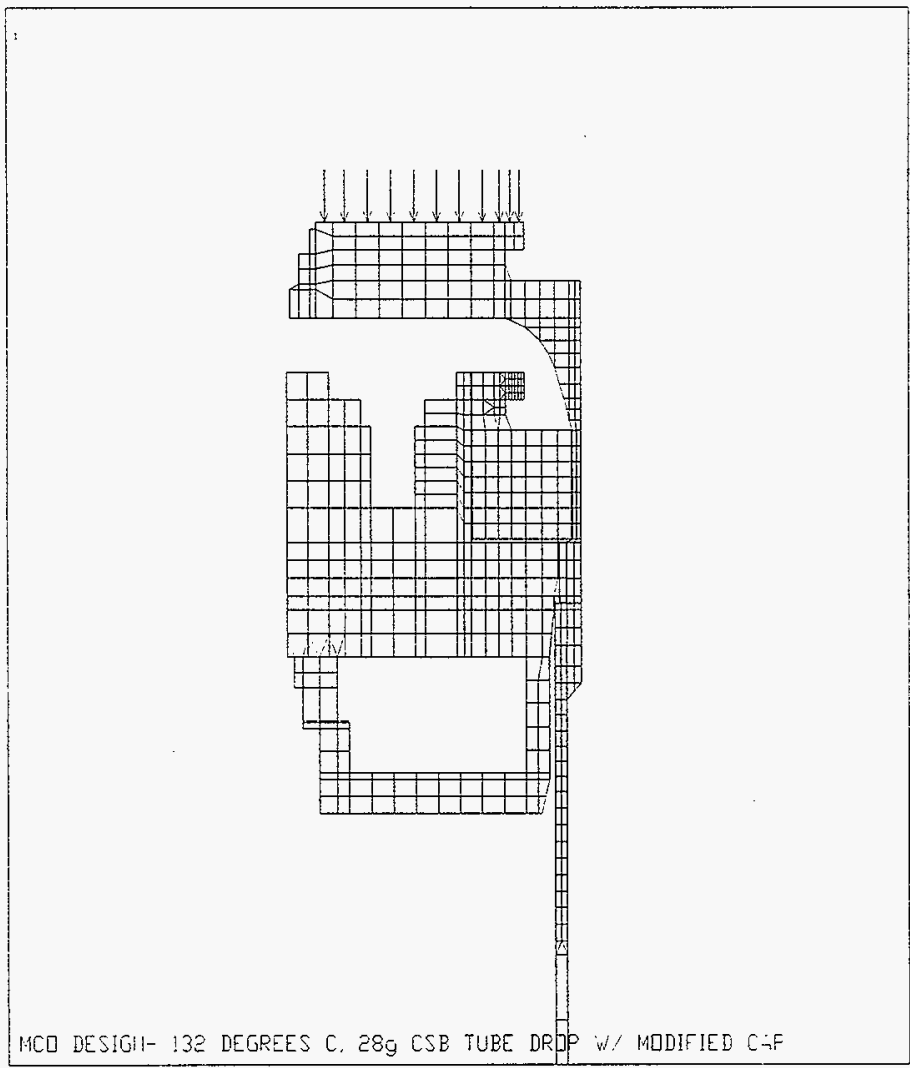

inlvis 5.0 in 56

Hifk le 190-

175636

PLOT HD. 4

ELEHENTS

TYPE NUM

PRES

$Z \mathrm{~V}=1$

*DIST $=19.375$

$* \times F=7.314$

*YF $=152.02$

PRECISE HIODEN

Figure 33: CSB Tube Drop with Modified Cap and Equivalent Pressure Distribution

0


CLIENT: Duke Engineering \& Services Hanford

FILE NO: KH-8009-8-03

PROJECT: MCO Final Design DOC. NO.: HNF-SD-SNF-DR-003, Rev. 0, Appendix 5

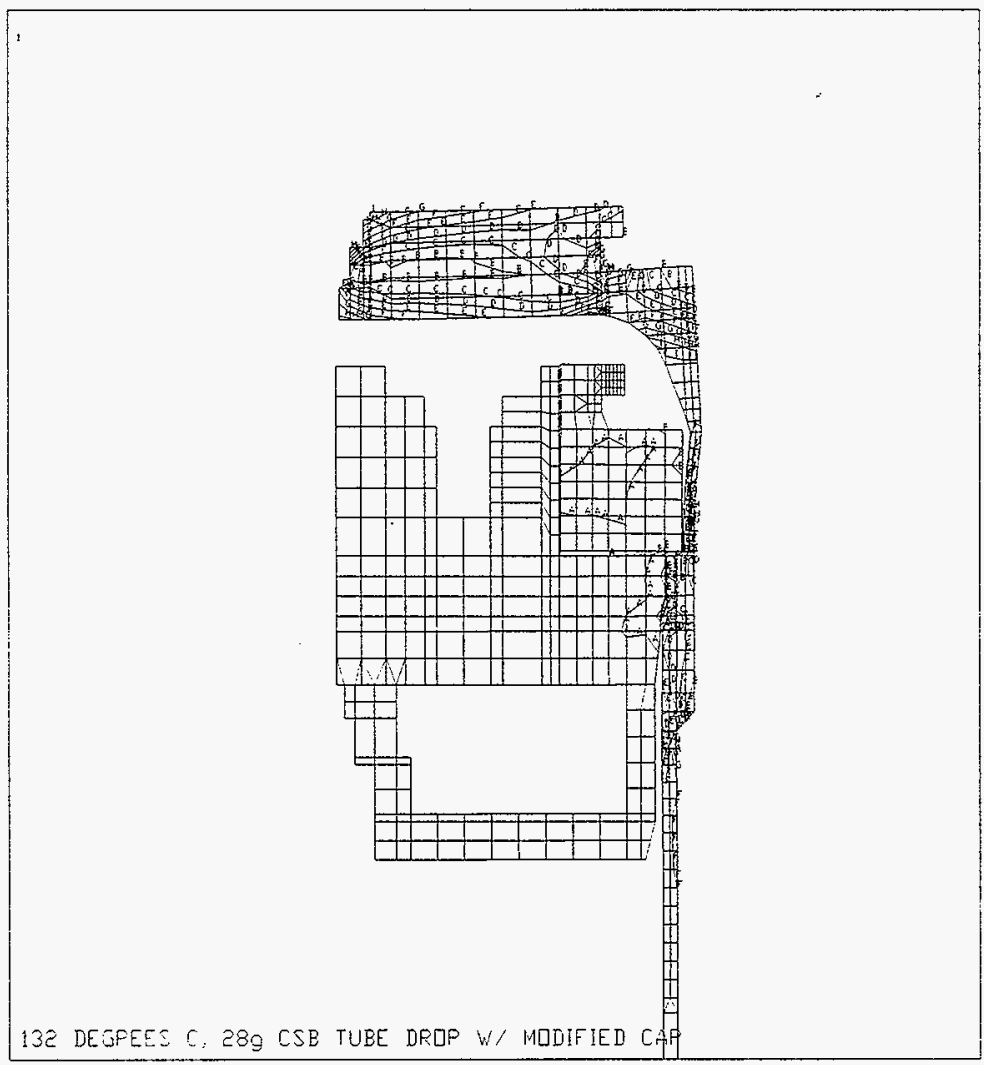

niNS Y 5.0 - 56

litif $1^{-} 199^{-}$

13:15:28

PLOT ND. 1

HODRL SOLUTIDN

STEP $=1$

SUB $=1$

TIN $\mathrm{HE}=1$

SINT

(ńv日)

DH $\lambda=0.215342$

SWIN $=2.965$

$\mathrm{SH}=2 \mathrm{X}=25895$

$A \quad=1275$

$\mathrm{B}=3818$

C $=6362$

$D=8905$

$E=11449$

$F \quad=13993$

C $=16536$

$\mathrm{H}=19080$

I $=21623$

Figure 34: CSB Tube Drop with Modified Cap and Upper Stress Intensities 
CLIENT: Duke Engineering \& Services Hanford

FILE NO: $\quad \mathrm{KH}-8009-8-03$ PROJECT: MCO Final Design $\quad$ DOC. NO.: HNF-SD-SNF-DR-003, Rev. 0, Appendix 5

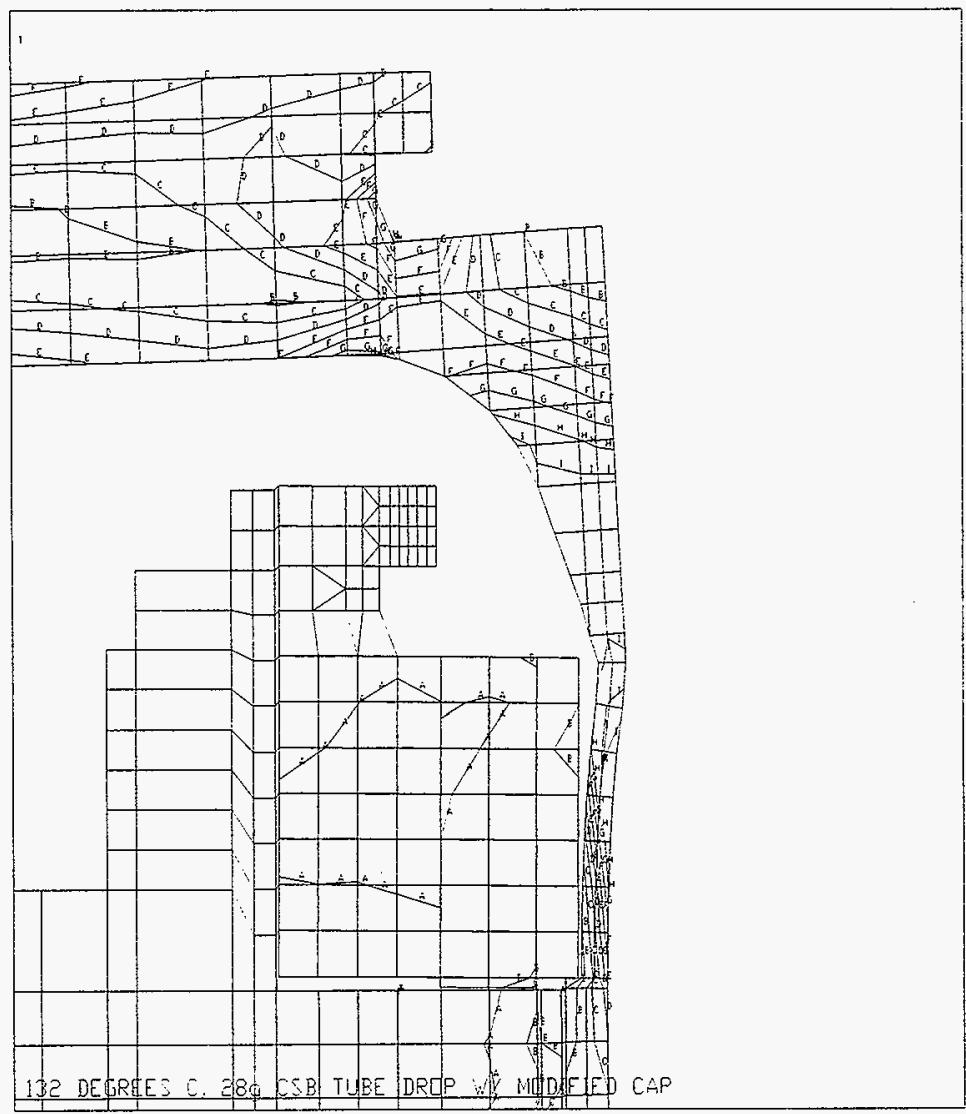

$$
\begin{aligned}
& \text { nilsis } 5.0 \text { - } 56 \\
& \text { MAR } 1^{-} 199^{-} \\
& \text {13:16:00 } \\
& \text { PLOT HO. } 2 \\
& \text { NOELL SLLUTIDU. } \\
& \text { STEP }=\text { : } \\
& \text { SUB }=1 \\
& \text { TIME }=1 \\
& \text { SINT } \\
& \text { (A) } \\
& \text { DHX }=0.215342 \\
& \text { SMH }=2.965 \\
& 54 \mathrm{X}=22895 \\
& \text { A }=1275 \\
& \text { B }=3818 \\
& \text { C }=6362 \\
& \text { D }=8905 \\
& \mathrm{E}=11449 \\
& F \quad=13993 \\
& \text { i. }=16536 \\
& \mathrm{H}=19080 \\
& \text { i }=21623
\end{aligned}
$$

Figure 35: CSB Tube Drop, Modified Cap @ Closure Weld Stress Intensities

\begin{tabular}{|l|c|l|l|l|}
\hline REVISION & 0 & & & \\
\hline PREPARED BY I DATE & $76 / 4 / 17 / 97$ & & & \\
\hline CHECKED BY I DATE & TM 4/17/97 & & & \\
\hline
\end{tabular}


CLIENT: Duke Engineering \& Services Hanford

PROJECT: MCO Final Design

FILE NO: KH-8009-8-03

DOC. NO.: HNF-SD-SNF-DR-003, Rev. 0, Appendix 5

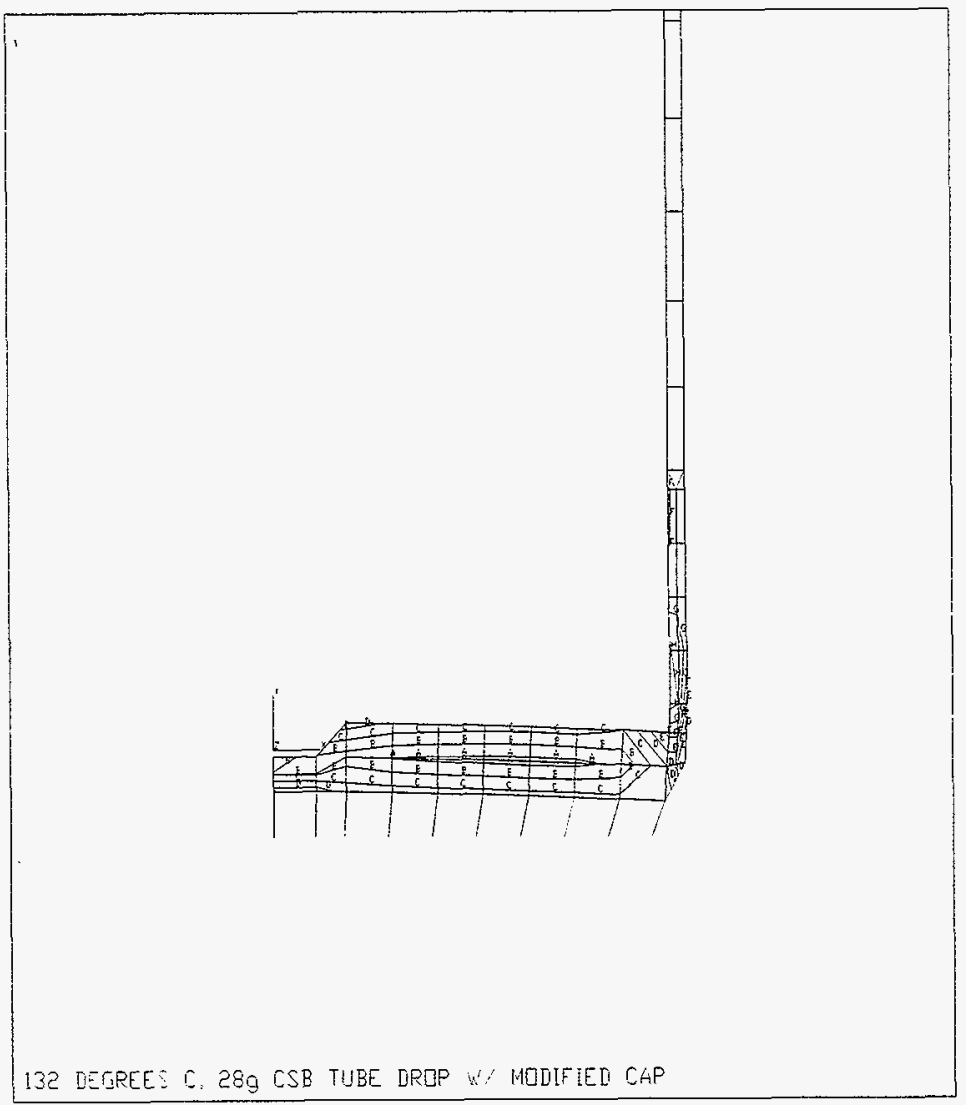

AiNS Ys 50 in 56

limR 1 : 199

13:16:41

PLOT HO. 3

MODÁ SOLUTIOH

$S T E F=1$

SUE $=1$

TIHE $=1$

SINT

(AVI)

DMX $=0.215342$

$S M N=2.965$

$\sin x=22895$

$\dot{A} \quad=1275$

$\mathrm{E}=381 \varepsilon$

C $=6360$

$\mathrm{D}=8905$

E $=11449$

$F \quad=13993$

(1) $=16536$

$\mathrm{H}=19080$

I $=21623$

132 DEFREE: C: 289 CSB TUBE DRDP W' MUDIFIED CAP

Figure 36: CSB Tube Drop with Modified Cap and Lower Stress Intensities 
CLIENT: Duke Engineering \& Services Hanford

\section{PROJECT: MCO Final Design DOC. NO.: HNF-SD-SNF-DR-003, Rev. 0, Appendix 5}

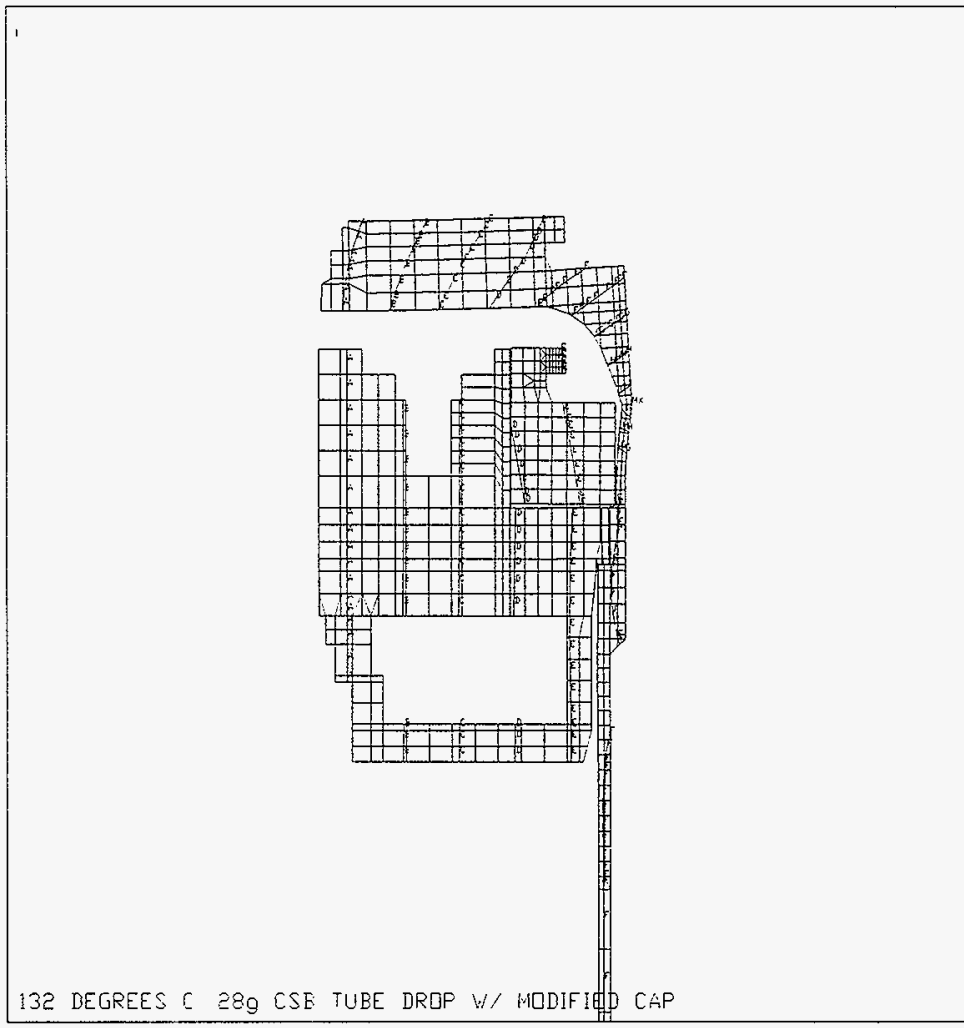

$$
\begin{aligned}
& \text { ANSIS } 5.0 \div 56 \\
& \text { I.IAR 1? 190? } \\
& \text { 13:1::31 } \\
& \text { PLOT NO. } 4 \\
& \text { NODÁL SGLUTIONN } \\
& \text { STEP }=1 \\
& \text { SUB }=1 \\
& \text { TIHE }=1 \\
& \text { UX } \\
& \text { RSYS }=0 \\
& \text { DHX }=0.215342 \\
& S H \%=0.038916 \\
& \therefore \quad=0.002162 \\
& B=0.006486 \\
& \text { C }=0.01081 \\
& \mathrm{D} \quad=0.015134 \\
& \text { E } \quad=0.019458 \\
& F \quad=0.023782 \\
& \text { J } \quad=0.028106 \\
& \mathrm{H}=0.03243 \\
& I \quad=0.036 \% 54
\end{aligned}
$$

132 BEGREES C 289 CSE TUBE DROP $W /$ MODIFIDD CAP

Figure 37: CSB Tube Drop with Modified Cap and Radial Displacements

|76s

\begin{tabular}{|c|c|}
\hline 0 & \\
\hline $4 / 17 / 97$ & \\
$-4 / 17 / 97$ & \\
\hline
\end{tabular}


CLIENT: Duke Engineering \& Services Hanford

PROJECT: MCO Final Design
FILE NO: $\quad \mathrm{KH}-8009-8-03$

DOC. NO:: HNF-SD-SNF-DR-003, Rev. 0, Appendix 5

\title{
Appendix A:
}

\section{Computer Run Output Sheets}

\&

\author{
Input File Listings
}


CLIENT: Duke Engineering \& Services Hanford $\quad$ FILE NO: KH-8009-8-03

PROJECT: MCO Final Design DOC. NO.: HNF-SD-SNF-DR-003, Rev. 0, Appendix 5

\section{COMPUTER RUN COVER SHEET}

Project Number:

Computer Code:

Software Version:

Computer System:

Computer Run File Number:

Unique Computer Run Filename:

Run Description:

Creation Date / Time:
KH-8009-8

ANSYS®-PC

$5.0 \mathrm{~A}$

MS-DOS, Pentium® Processor

$\mathrm{KH}-8009-8-03$

CSBMC.inp

MCO CSB Tube Drop with Lifting Cap

30 March 1997 10:55:18 AM
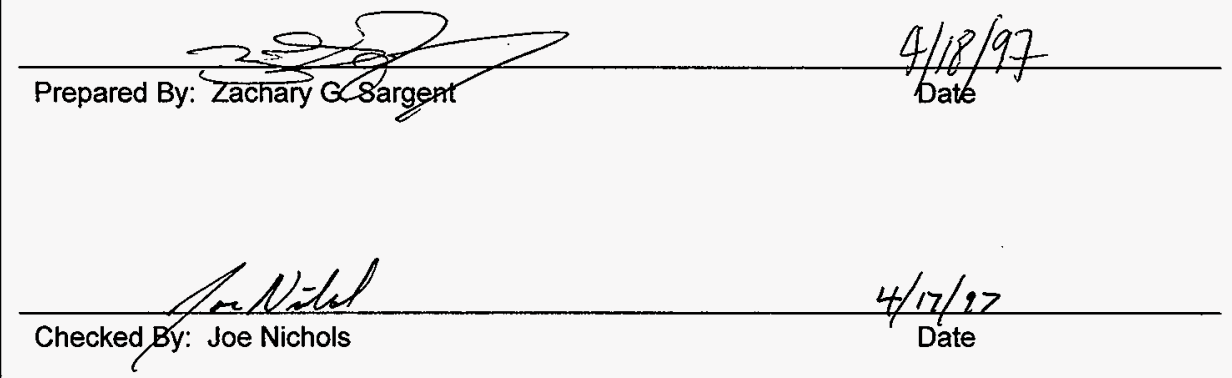

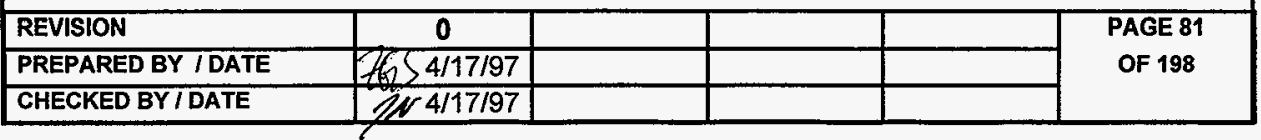


CLIENT: Duke Engineering \& Services Hanford

FILE NO: $\quad \mathrm{KH}-8009-8-03$

$\begin{array}{ll}\text { PROJECT: MCO Final Design } & \text { DOC. NO.: HNF-SD-SNF-DR-003, Rev. 0, Appendix } 5\end{array}$

\section{LISTING OF CSBMC.INP FILE}

IBATCH,LIST

IFILENAM,CSBMC

IPREP7

TITLE, 132 DEGREES C, $28 \mathrm{~g}$ CSB TUBE DROP W/ MODIFIED CAP

TREF,70

TUNIF,270

ETAN=0.006 I Tangent modulus

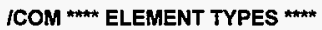

ET,1,42,,11 IShell

ET,2,42,,1 I Shield Plug

ET, 3,42,,1 I Lifting \& Locking Ring

ET,4,12 I Gap Elements Between Shield Plug \& Shell

$\mathrm{KEYOPT}, \mathbf{4 , 7 , 1} \quad$ I Bolt
$\mathrm{ET}, \mathbf{5 , 4 2 , 2}, \mathbf{1}$

ICOM REAL CONSTANTS FOR GAP ELEMENTS

R,4,-90,1.0E8,-0.06,3.0 IShell/Shield Plug, Initially Open .06"

R,5,0,1.0E8,2.95e-03 I L. Ring/Shield Plug, Under Bolt, Preloaded

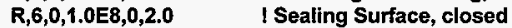

R,7,0,1.0E8,0,1.0 I Anchoring for drop, closed

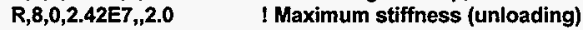

ICOM MATERIAL PROPERTIES

MP,DENS, 1,490/1728 I 304L SS

MP,NUXY, 1,0.3

MP,DENS,5,490/1728

MP,NUXY,5,0.3

TB,BKIN, 1,6

TBTEMP, 100

TBDATA, 1,25000, ETAN*28.1E+06

TBTEMP,200

TBDATA, 1,21300,ETAN*27.6E+06

TBTEMP, 300

TBDATA, 1,19100,ETAN ${ }^{27.0 E+06}$

TBTEMP, 400

TBDATA, 1,17500,ETAN*26.5E+06

TBTEMP, 500

TBDATA, 1,16300,ETAN*25.8E+06

TBTEMP, 600

TBDATA,1,15500,ETAN*25.3E+06

ICOM DEFINING TEMPERATURES FOR MPDATA *

MPTEMP, 1, 70,100,200,300,400,500

MPTEMP, $7,600,650,700,750$

ICOM \#\# DEFINING ELASTIC MODULI FOR 304L \& SA-193 ***

MPDATA,EX,1,1,28.3e+06,28.1e+06,27.6e+06,27.0e+06,26.5e+06,25.8e+06

\begin{tabular}{|l|c|l|l|l|}
\hline REVISION & 0 & & & \\
\hline PREPARED BY IDATE & I $S 4 / 17 / 97$ & & & \\
\hline CHECKED BY I DATE & $/ \sim-4 / 17 / 97$ & & & \\
\hline
\end{tabular}

PAGE 82

OF 198 


\section{르 PARSONS}

CLIENT: Duke Engineering \& Services Hanford

FILE NO: $\quad \mathrm{KH}-8009-8-03$

PROJECT: MCO Final Design DOC. NO.: HNF-SD-SNF-DR-003, Rev. 0, Appendix 5

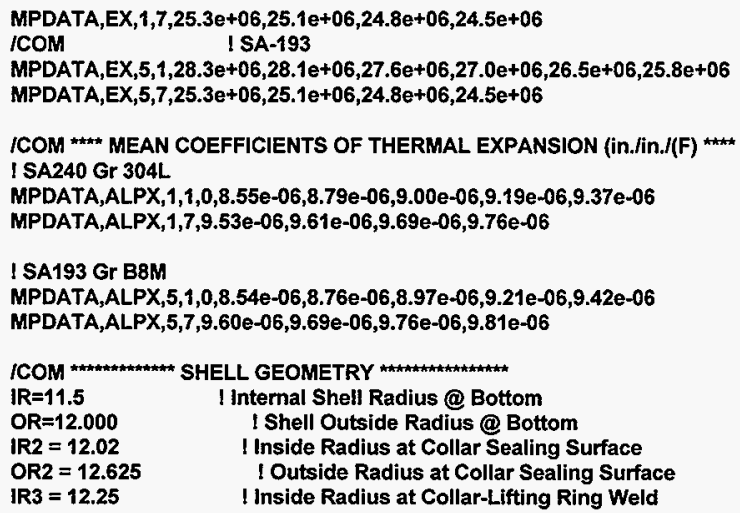

\begin{tabular}{|c|l|l|l|}
\hline 0 & & & \\
\hline $4 / 17 / 97$ & & & \\
\hline$/ 4 / 17 / 97$ & & & \\
\hline
\end{tabular}




\section{P. Parsons}

CLIENT: Duke Engineering \& Services Hanford

FILE NO: KH-8009-8-03

PROJECT: MCO Final Design

DOC. NO.: HNF-SD-SNF-DR-003, Rev. 0, Appendix 5

$\mathrm{N}, 101, \mathrm{OR}, 7.18$

I Outside

$N, 141$, OR,71.68

$\mathrm{N}, 181, \mathrm{OR}, 136.68$

FILL,100,140,20,2,2,1,2.0

FlLL $140,180,19,2,2,1, .5$

ICOM DOUBLE ROW SHELL

N,190,IR,137.18 ITransition to Double Row

$\mathrm{N}, 192, \mathrm{OR}, 137.18$

FILL

ICOM * BASE OF CASK THROAT--ELEVATION: 138 INCHES

$\mathrm{N}, 217,1 \mathrm{R}, 142.68$

! Transition to Double Row

$\mathrm{N}, \mathbf{2 1 9}, \mathrm{OR}, 142.68$

FILL

FILL,190,217,8,3,3,1 I Vertical Fill

ICOM BOTTOM OF COLLAR TRANSITION

N,235,IR,146.06 I Start of Transition to Large O.D \&

N,237,OR,146.06 I Assumed Location of Shield Plug Taper

FILL

$\mathrm{N}, 238, I R, 146.68$

$N, 240,0 R, 146.68$

FILL.

I Horizontal Fill

FILL,217,235,5,3,3,1 I Vertical Fill

ICOM ${ }^{\star * \star *}$ TOP OF COLLAR TRANSITION $* * \star *$

N,241,1R,147.31 I I Ind of Transition to Large O.D \&

$N, 243, O R, 147.31$

FILL

I Assumed Location of Shield Plug Taper

NGEN,2,3,241,243,1,0.75

ICOM *** COLLAR SEALING SURFACE **

$N, 247$, IR, 149.63 IInside Radius of Sealing Surface

$\mathrm{N}, 249, \mathrm{IR2}, 149.63$

1 Outside Radius at Sealing Surface

FILL

I Horizontal Fill

ICOM THICK WALL AT COLLAR TRANSITION

NGEN,2,10,240,249,3

I Nodes 250-259 Coincident w/240-249 (by 3)

$\mathrm{N}, 255, \mathrm{OR2}, 147.31$

$\mathrm{N}, 261, \mathrm{OR2}, 149.63$

IOutside Surface

$\mathrm{N}, 258, \mathrm{OR2}, 148.06$

lOutside Surface

$\mathrm{N}, 980, \mathrm{IR}, 149.38$

N,981,11.755,149.38

N,982,IR2,149.38

$\mathrm{N}, 983,12.317,149.38$

$\mathrm{N}, 984, \mathrm{OR2}, 149.38$

N,990,OR2, 146.68

FILL,240,990,1,251

NGEN, $2,5,980,984,1,-0.66$

FILL, 246,258, 1,257

FILL, 253,255,1,1,3,3

FILL, 237,990,1,991

ICOM COLLAR AT BOTTOM EDGE OF PLUG (.155" above Sealing Surface) **** NGEN,2,3,259,,,,0.245 I Nodes 262

\begin{tabular}{|l|c|l|l|l|}
\hline REVISION & 0 & & & \\
\hline PREPARED BY IDATE & ZG $\$ 4 / 17 / 97$ & & & \\
\hline CHECKED BY I DATE & IN $4 / 17 / 97$ & & & \\
\hline
\end{tabular}


CLIENT: Duke Engineering \& Services Hanford

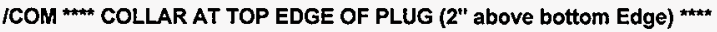

NGEN, $2,9,262, \ldots, 2.00$ ! Nodes 271

FILL,262,271,2

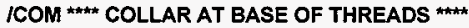

$\mathrm{N}, 274, \mathbf{I R 3}, 152.00$

$N, 1000, I R 2,152.00$

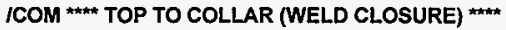

N,295,IR3,156.00

FILL, 274,295

NGEN,3,1,259,295,3,(OR2-IR2)/2

NGEN,3,1,274,295,3,(OR2-IR3)/2

ICOM

RING1 $=7.94$

RING2 $=9.375$

RING3 $=9.625$

RING4=10.19

RING5 $=12.23$

LOCAL, $11,0,152.00$ ! Local System $z=0$ at Base of Ring

CSYS, 11

ICOM TOP EDGE

N,401,RING1,6.13

CSYS,O

$\mathrm{N}, \mathbf{4 0 4 , 9 . 3 7 5 , 1 5 8 . 1 3}$

FILL, 401,404,,1

N,406,RING4, 158.13

FILL,404,406,,1 1 Top Edge

ICOM * LIFTING SURFACE \#**

CSYS,11

N,421, RING1,5.13

N,424, RING2,5.13

FILL,421,424

N,426,RING4,5.13

FILL,424,426

FiLL,401,421,1,10,6,1

N,431,RING1,6.13-1.56

N,434,RING2,6.13-1.56

FILL

ICOM ${ }^{\star * \star \star}$ BOLTING SURFACE ${ }^{\star \star \star \star}$

N,441,RING1,4

N,444,RING3,4

FILL

N,445,10.9375-.6875,4 ! Inside Edge of Bolt Hole

$\mathrm{N}, 447,10.9375+.6875,4 \quad$ ! Outside Edge of Bolt Hole

FILL

$\mathrm{N}, 910,10.9375-.6875,4$

$\mathrm{N}, 911,10.9375+.6875,4$

N,448,RING5,4

CSYS, 0

I O.D of Ring

N,924,10.25,152.00 ! Double Nodes @ Bolt for Gap elements

I Bolt Extension

\begin{tabular}{|l|c|l|l|l|}
\hline REVISION & 0 & & & \\
\hline PREPARED BY / DATE & $\mathscr{y} / 2 / 4 / 17 / 97$ & & & \\
\hline CHECKED BY I DATE & $\sim 4 / 17 / 97$ & & & \\
\hline
\end{tabular}


CLIENT: Duke Engineering \& Services Hanford

FILE NO: $\quad \mathrm{KH}-8009-8-03$

PROJECT: MCO Final Design DOC. NO.: HNF-SD-SNF-DR-003, Rev. 0, Appendix 5

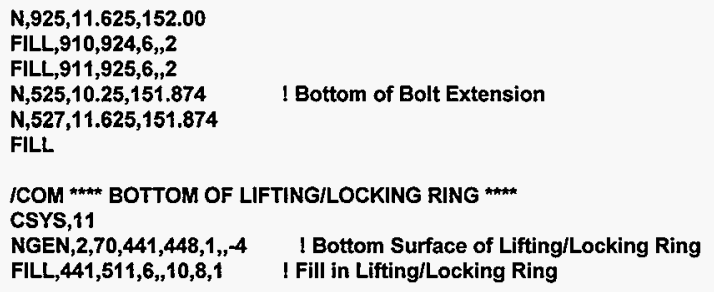

FILL, $910,924,6,2$

FILL,911,925,6,2

N,525,10.25,151.874 ! Bottom of Bolt Extension

$\mathrm{N}, 527,11.625,151.874$

FILL.

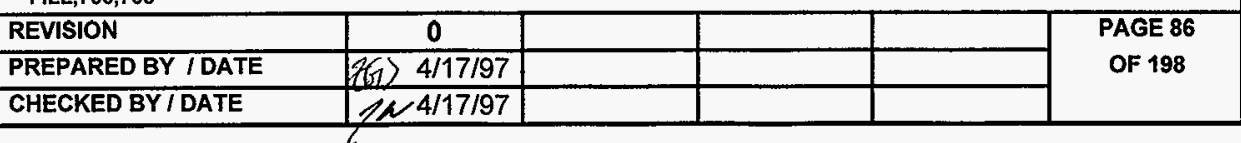


CLIENT: Duke Engineering \& Services Hanford

FILE NO: $\quad \mathrm{KH}-8009-8-03$

PROJECT: MCO Final Design DOC. NO.: HNF-SD-SNF-DR-003, Rev. 0, Appendix 5

NGEN $, 3,20,766,768,1,0.3125$

$\mathrm{N}, \mathbf{7 8 9 , 7 . 5 7 7 5 , - 1 . 5 6}$

N,796,7.5775,-5.56

FILL, 789,796,6

NGEN, 2,20,789,796,1,0.3125

NGEN, 3,20,777,783,1,0.3125

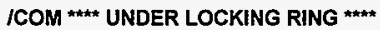

N, 824,8.5017,-6.25

$\mathrm{N}, 827,8.5017,-8.25$

FILL

$\mathrm{N}, \mathbf{8 2 8}, \mathbf{8 . 5 0 1 7 , - 8 . 7 5}$

$\mathrm{N}, 830,8.5017,-10.5$

FILL

NGEN,3,7,824,830,1,0.5616

NGEN, $2,7,838,844,1,0.625$

NGEN,2,7,845,851,1,0.6875 !Under Bolt

$\mathrm{N}, 859,11.625,-6.25$

$\mathrm{N}, 860,11.625,-6.917$

$\mathrm{N}, 861,11.625,-7.584$

N,862,PLUGR2,-8.25

N,863,PLUGR2,-8.75

N,865,PLUGR3,-10.5

FILL, $863,865,1$

N,866,PLUGR1-0.288,-6.25

$\mathrm{N}, 869, \mathrm{PLUGR} 1-0.288,-8.25$

FILL, 866,869,2

N,870,PLUGR1-0.288,-8.476

NGEN,2,5,866,870,1,0.288

ICOM ** REFINING LIFTING EAR **

CSYS, 0

$\mathrm{N}, 877,9.53,158.13$

$\mathrm{N}, 889,9.53,157.63$

$\mathrm{N}, 901,9.53,157.13$

FILL, $403,404,1,876$

FILL, $413,414,1,888$

FILL, $423,424,1,900$

FILL, $877,405,1,878$

FILL,405,406,2,879,1

FILL, 889,415, 1,890

FILL,415,416,2,891,1

FILL,404,414,1,881

FILL, $877,889,1,882$

FILL,878,890,1,883

FILL, $\mathbf{4 0 5 , 4 1 5 , 1 , 8 8 4}$

FILL, $879,891,1,885$

FILL,880,892,1,886

FILL,406,416,1,887

FILL,889,901,1,894

FILL,414,424,1,893

FILL,901,425,1,902

FILL,890,902,1,895

FILL,415,425,1,896

FILL,425,426,2,903,1

FILL,891,903,1,897

\begin{tabular}{|l|c|l|l|l|}
\hline REVISION & 0 & & & \\
\hline PREPARED BY IDATE & SF $4 / 17 / 97$ & & & \\
\hline CHECKED BYIDATE & IM $4 / 17 / 97$ & & & \\
\hline
\end{tabular}


CLIENT: Duke Engineering \& Services Hanford

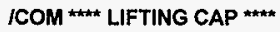

$N, 1001,0.125,161.167$

$\mathrm{N}, 1002,0.125,160.12$

NGEN,2,5,1001,1002,0.392

$\mathrm{N}, 1003,0.517,162.471$

$\mathrm{N}, 1004,0.517,161.9185$

$\mathbf{N}, \mathbf{1 0 0 5 , 0 . 5 1 7 , 1 6 1 . 3 6 6}$

$\mathbf{N}, \mathbf{1 0 0 8 , 1 . 0 0 , 1 6 3 . 3 6 6}$

NGEN, $2,6,1003,1007,0.483$

$\mathrm{N}, \mathbf{1 0 1 4 , 1 . 2 5 , 1 6 3 . 6 1 6}$

NGEN,2,7,1008,1013,0.25

$\mathrm{N}, 1021,1.9768,163.616$

$\mathrm{N}, 1023,1.9768,162.616$

FILL

$\mathrm{N}, 1024,1.9768,162.056$

$\mathrm{N}, 1025,1.9768,161.49$

$\mathrm{N}, 1027,1.9768,160.12$

FILL, 1025,1027

NGEN, $7,10,1021,1027,0.9884$

NGEN, $2,10,1081,1087,0.9888$

N,1101,RING2,163.616

N,1103,RING2,162.616

FILL.

N,1104,RING2,162.056

N,1105, RING2, 161.49

N,1107,RING2,160.12

FILL.

N,1108,RING2, 160.088

N,1112,RING4,163.616

N,1114,RING4,162.616

FILL

FILL,1101,1112,1,1109

FILL,1102,1113,1,1110

FILL,1103,1114,1,1111

N,1120,RING3,161.49

N,1122,RING3,160.12

FILL

NGEN, 2,3,1120,1122,0.629

NGEN,2,3,1123,1125, 0.614

NGEN, 2,3,1126,1128,,0.636

NGEN, 2,3,1129,1131, 0.621

N,1138,OR2,161.49

N,1140,OR2,160.12

FILL

FILL,1132,1138,1,1135

FILL,1133,1139,1,1136

FILL,1134,1140,1,1137

$\mathrm{N}, 1141,12.125,159.78$

N,1143,OR2,159.78

\begin{tabular}{|l|c|l|l|l|}
\hline REVISION & 0 & & & \\
\hline PREPARED BY IDATE & C & & & \\
\hline CHECKED BYI I DATE & $1 / 4 / 97$ & & & \\
\hline
\end{tabular}


CLIENT: Duke Engineering \& Services Hanford

FILE NO: KH-8009-8-03

PROJECT: MCO Final Design DOC. NO.: HNF-SD-SNF-DR-003, Rev. 0, Appendix 5

FILL.

NGEN, $3,3,1141,1143, \ldots-0.47$

NGEN, $2,3,1147,1150,,-0.53$

NGEN, $2,3,1150,1152,,,-0.60$

NGEN $, 2,3,1153,1155,,-0.54$

NGEN, $2,3,1156,1158,,-0.38$

NGEN, $2,3,1159,1161, \ldots, 0.41$

N,1168,RING3,160.032

$\mathrm{N}, \mathbf{1 1 6 9 , 1 0 . 2 5 4 , 1 5 9 . 7 8}$

$\mathrm{N}, \mathbf{1 1 7 0 , 1 0 . 8 6 8 , 1 5 9 . 7 8}$

$\mathrm{N}, 1174,11.504,159.78$

$\mathrm{N}, 1172,10.868,159.31$

$\mathrm{N}, 1173,11.504,159.31$

$\mathrm{N}, 1174,11.24,158.84$

$N, 1175,11.504,158.84$

$\mathrm{N}, 1176, \mathbf{1 1 . 5 0 4 , 1 5 8 . 3 1}$

$N, 1177,11.71,157.71$

N,1178,11.896,157.17

$\mathrm{N}, 1179, \mathbf{1 2 . 0 2 7 , 1 5 6 . 7 9}$

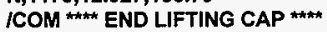

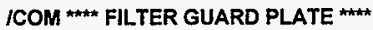

PLATE1 $=0.273$

PLATE2 $=0.6575$

PLATE3=1.357

PLATE4 $=10.25$

PLATE5=11.25

N,1200,PLATE4,146.78

N,1202,PLATE5, 146.78

FILL

NGEN $, 5,3,1200,1202,,,-0.85$

NGEN, $2,3,1212,1214,,-0.25$

N,1221,PLATE4, 141.88

$\mathrm{N}, \mathbf{1 2 2 2 , 1 0 . 7 5 , 1 4 1 . 8 8}$

$\mathrm{N}, 1223,10.915,141.88$

FILL,1215,1221,1,1218

FILL, 1223,1217,1,1220

FILL,1216,1222,1,1219

$\mathrm{N}, 1237,6.4375,143.38$

FiLL, 1212,1237,3,1225,4

$\mathrm{N}, 1249,3.578,143.38$

FILL,1237,1249,2,1241,4

NGEN, $2,1,1225,1249,4,-0.25$

NGEN,2,2,1226,1250,4,-1.25

FILLL, 1226,1228,1,1227, ,7,4

$\mathrm{N}, 1253,2.625,145.255$

$\mathrm{N}, 1254,2.625,145.005$

$\mathrm{N}, 1256,2.625,143.38$

FiLL, 1254, 1256

$\mathrm{N}, 1257,2.625,143.13$

$\mathrm{N}, 1259,2.625,141.88$

FILL,1257,1259

NGEN, $2,10,1253,1259,1,-0.5$

NGEN, $2,10,1263,1269,1,-0.768$

$\mathrm{N}, 1283,0.6575,145.255$

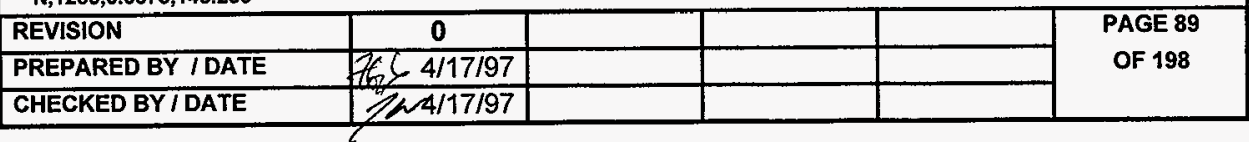




$\begin{array}{lll}\text { PROJECT: MCO Final Design DOC. NO.: HNF-SD-SNF-DR-003, Rev. 0, Appendix } 5 & \text { DO }\end{array}$

\author{
$\mathrm{N}, 1284,0.6575,145.005$ \\ $\mathrm{N}, 1260,2.125,147.63$ \\ $\mathrm{N}, 1270,1.357,147.63$ \\ $\mathrm{N}, 1280,0.6575,147.63$ \\ $N, 1290,0.273,147.63$ \\ NGEN, $3,1,1260,1290,10,-0.5625$
}

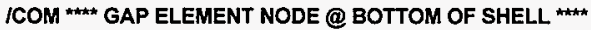
NGEN,2,2000,1,10,1, -1.00

ICOM **** COUPLING NODES ****

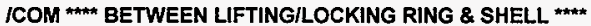

$\mathrm{CP}, 1, \mathrm{UY}, 508,277$

! Start Threads

$C P, 2, U Y, 498,280$

$C P, 3, U Y, 488,283$

$C P, 4, U Y, 478,286$

CP, $5, U Y, 468,289$

CP,6,UY,458,292

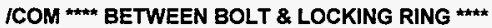

$\mathrm{CP}, \mathbf{7}, \mathrm{UY}, \mathbf{4 4 5 , 9 1 0}$

$\mathrm{CP}, 8, \mathrm{UX}, \mathbf{4 4 5 , 9 1 0}$

CP,9,UY, 447,911

CP, 10,UX,447,911

"DO,I,1,7

CP, 10+1,UY,445+10*1,910+2"1

*ENDDO

"DO, $1,1,7$

$C P, 17+1, U Y, 447+10^{*} 1,911+2 * \mid$

*ENDDO

*DO,1,1,7

$C P, 24+1, U X, 445+10 * 1,910+2 * 1$

*ENDDO

$\star D O, 1,1,7$

$C P, 31+1, U X, 447+10^{\star} 1,911+2 *$

*ENDDO

NALL

EALL

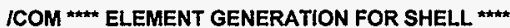

TYPE,1

MAT, 1

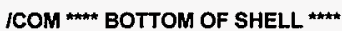

$E, 1,2,22,21$

$E, 2,3,23,22$

EGEN,8,1,-1

$E, 10,11,30$

$E, 21,22,42,41$

$E, 22,23,43,42$

EGEN,10,1,-1

$E, 11,31,30$

$E, 11,32,31$

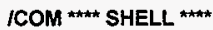

$E, 50,51,54,53$

\begin{tabular}{|l|c|l|l|l|}
\hline REVISION & 0 & & & \\
\hline PREPARED BY IDATE & t6S 4/17/97 & & & \\
\hline CHECKED BY I DATE & $1 / 4 / 17 / 97$ & & & \\
\hline
\end{tabular}


CLIENT: Duke Engineering \& Services Hanford

PROJECT: MCO Final Design DOC. NO.: HNF-SD-SNF-DR-003, Rev. 0, Appendix 5

FILE NO: $\quad \mathrm{KH}-8009-8-03$

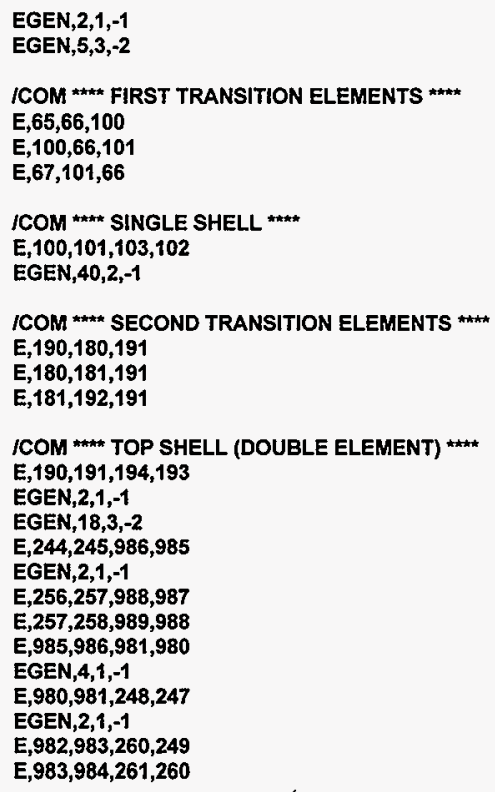

\begin{tabular}{|l|c|l|l|l|}
\hline REVISION & 0 & & & \\
\hline PREPARED BY IDATE & CGS 4/17/97 & & & \\
\hline CHECKED BY I DATE & D 4/17/97 & & & \\
\hline
\end{tabular}


PROJECT: MCO Final Design $\quad$ DOC. NO.: HNF-SD-SNF-DR-003, Rev. 0, Appendix 5

EGEN,2,1,-1

EGEN,2,10,-2

$E, 413,888,876,403$

$E, 881,404,876$

E,888,881,876

E,888,414,881

E,881,882,877,404

$E, 414,889,882,881$

E,882,883,878,877

E, $889,890,883,882$

$E, 883,884,405,878$

$E, 890,415,884,883$

$E, 884,885,879,405$

$E, 415,891,885,884$

$E, 885,886,880,879$

$E, 891,892,886,885$

E,886,887,406,880

$\mathrm{E}, 892,416,887,886$

$E, 423,900,888,413$

E,893,414,888

E,900,893,888

$E, 900,424,893$

$E, 893,894,889,414$

E,424,901,894,893

$E, 894,895,890,889$

E,901,902,895,894

$E, 895,896,415,890$

$E, 902,425,896,895$

$E, 896,897,891,415$

$E, 425,903,897,896$

$E, 897,898,892,891$

$E, 903,904,898,897$

$E, 898,899,416,892$

$E, 904,426,899,898$

E,431,432,422,421

$E, 905,423,422$

$E, 432,905,422$

$E, 432,433,905$

E,905,906,900,423

$E, 433,908,906,905$

E,906,907,424,900

E,908,434,907,906

$E, 441,442,432,431$

EGEN,2,1,-1

E,443,908,433

$E, 443,444,434,908$

$E, 451,452,442,441$

EGEN,3,1,-t

EGEN,7,10,-3

E,454,912,910,444

E,464,914,912,454

$E, 474,916,914,464$

$E, 484,918,916,474$

$E, 494,920,918,484$

$E, 504,922,920,494$

$E, 514,924,922,504$

\begin{tabular}{|l|c|c|c|c|c|}
\hline REVISION & 0 & & & & PAGE 92 \\
\hline PREPARED BY IDATE & OF 198 \\
\hline CHECKED BY IDATE & $4 / 17 / 97$ & & & & \\
\hline
\end{tabular}


Duke Engineering \& Services Hanford

FILE NO: $\quad \mathrm{KH}-8009-8-03$

$\begin{array}{ll}\text { PROJECT: MCO Final Design } & \text { DOC. NO.: HNF-SD-SNF-DR-003, Rev. 0, Appendix } 5\end{array}$

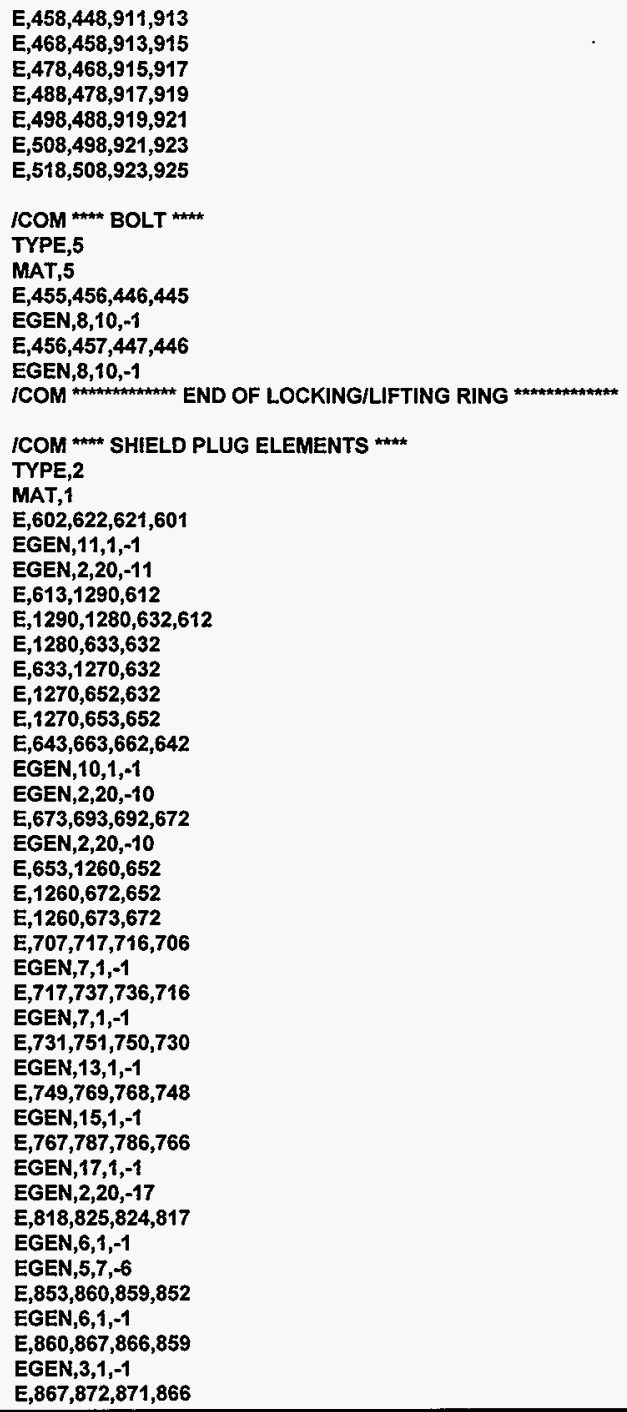


CLIENT: Duke Engineering \& Services Hanford

PROJECT: MCO Final Design

FILE NO: $\quad \mathrm{KH}-8009-8-03$

\section{MCO FinalDesign}

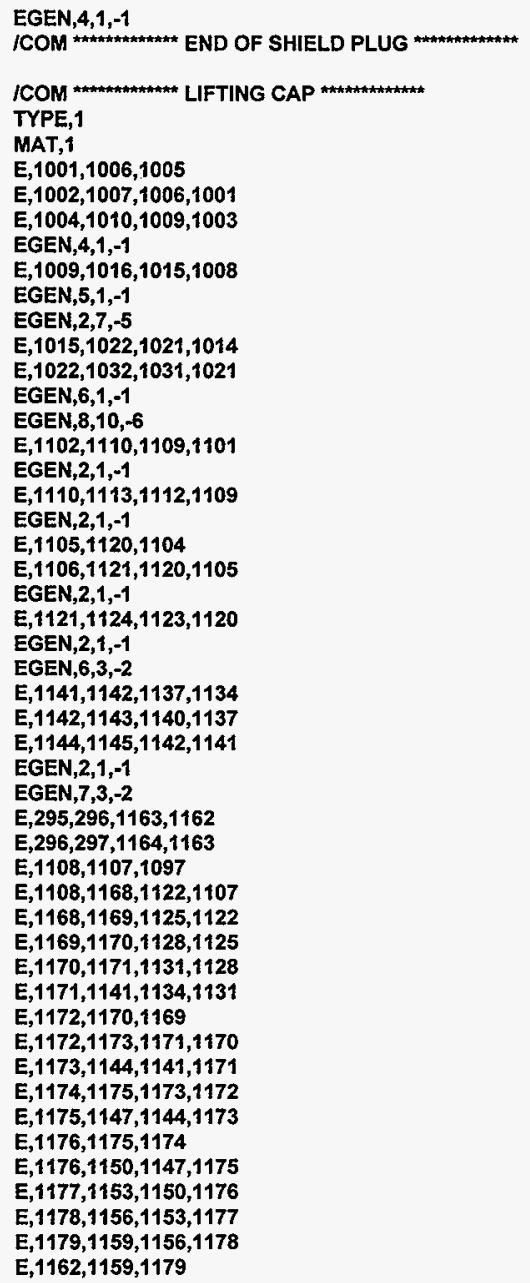


CLIENT: Duke Engineering \& Services Hanford

PROJECT: MCO Final Design

FILE NO: $\quad \mathrm{KH}-8009-8-03$

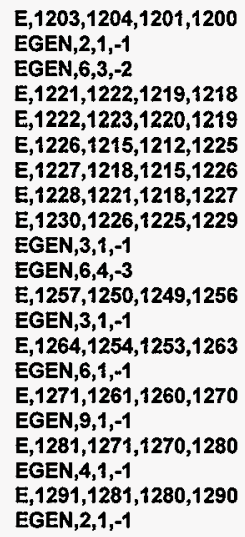

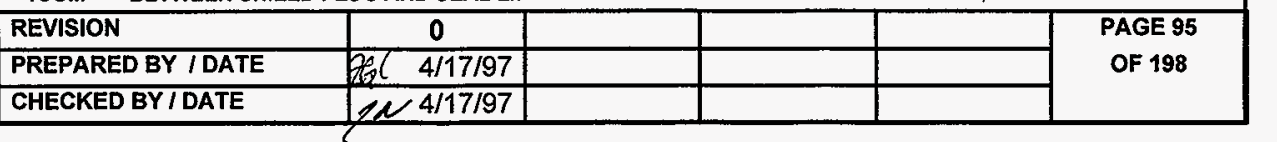


CLIENT: Duke Engineering \& Services Hanford

FILE NO: KH-8009-8-03

PROJECT: MCO Final Design DOC. NO.: HNF-SD-SNF-DR-003, Rev. 0, Appendix 5

TYPE,4

REAL, 6

$E, 248,870$

$E, 249,875$

TYPE, 4

REAL,8

$E, 248,869$

$E, 247,862$

ICOM *** BOTTOM GAP ELEMENTS **

TYPE, 4

REAL,7

$E, 2001,1$

EGEN,10,1,-1

ICOM END GAP ELEMENTS

ICOM BOUNARY CONDITIONS

CSYS, 0

NSEL,S,LOC, $X, 0$

NSEL, R, LOC, Y, $-1.33,163.616$

D,ALL,UX,O

NALL

EALL

NSEL,S,NODE,,1002

D,ALL,UX,0

NALL

EALL

NSEL,S,NODE,,2001,2010

D,ALL,ALL, 0

EALL

NALL

SAVE

ICOM *** LOAD 1: APPLYING 28g EQUIVALENT ACCELERATION ***

NSEL,S,LOC, $X, 0,10.2$

NSEL,R,LOC,Y,163.615,163.617

SF,ALL,PRES, 1662.94

NALL

EALL

SAVE

FINI

I

ICOM SOLUTION PHASE

ISOLUTION

SOLVE

SAVE

FINI

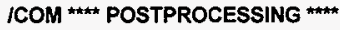

IPOST1

SET,LAST

TYPE,ALL,HIDC

IGLINE,ALL,0

RSYS, 0

REVISION

PREPARED BY I DATE

CHECKED BYI DATE

\begin{tabular}{|l|l|l|l|}
\hline 0 & & & \\
\hline $4 / 17 / 97$ & & & \\
\hline $4 / 17 / 97$ & & & \\
\hline
\end{tabular}


CLIENT: Duke Engineering \& Services Hanford

FILE NO: $\quad \mathrm{KH}-8009-8-03$

PROJECT: MCO Final Design

PLNSOL,S,INT

IDSCALE, 20

IREPLOT

NSEL,S,LOC,X,11.49,11.51

NSEL, R,LOC, $Y,=0.33,148$

PRNS,U,X

NALL

EALL

NSEL,S,LOC,X,1.356,11.26

NSEL,R,LOC,Y,141.87,143.39

PRNS,U,Y

NALL

EALL

PRRS,F

LPATH, 1,41

PRSECT

LPATH,6,46

PRSECT

LPATH, 10,50

PRSECT

LPATH,50,52

PRSECT

LPATH,62,64

PRSECT

LPATH, 65,67

PRSECT

LPATH, 100,101

PRSECT

LPATH,122, 123

PRSECT

LPATH,134, 135

PRSECT

LPATH,156, 157

PRSECT

LPATH,170,171

PRSECT

LPATH,180,181

PRSECT

LPATH,202,204

PRSECT

LPATH,235,237

PRSECT

LPATH,985,989

PRSECT

LPATH,262,264

PRSECT

LPATH,277,279

PRSECT

LPATH,292,294

PRSECT

LPATH,601,641

PRSECT

LPATH,601,613

PRSECT

L.PATH,603,703

PRSECT

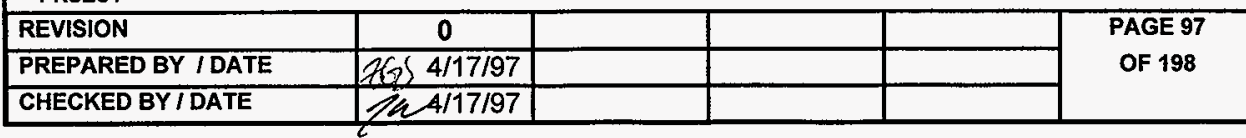


CLIENT: Duke Engineering \& Services Hanford

FILE NO: KH-8009-8-03

PROJECT: MCO Final Design DOC. NO.: HNF-SD-SNF-DR-003, Rev. 0, Appendix 5

LPATH,606,706
PRSECT
LPATH,766,806
PRSECT
LPATH,748,808
PRSECT
LPATH,736,815
PRSECT
LPATH,869,874
PRSECT
LPATH,870,875
PRSECT
LPATH,431,434
PRSECT
LPATH,406,426
PRSECT
LPATH,404,424
PRSECT
LPATH,1120,1097
PRSECT
LPATH,1120,1168
PRSECT
LPATH,1174,1149
PRSECT
LPATH,1176,1152
PRSECT
LPATH,1177,1155
PRSECT
LPATH,1178,1158
PRSECT
LPATH,1179,1161
PRSECT
LPATH,1162,1164
PRSECT
LPATH,295,297
PRSECT
LPATH,1021,1027
PRSECT
LPATH,1071,1077
PRSECT
LPATH,1138,1169
PRSECT
SAVE

LPATH,606,706

PRSECT

LPATH,766,806

PRSECT

PRSECT

LPATH,736,815

PRSECT

LPATH,869,874

PRSECT

LPATH, 431,434

PRSECT

LPATH,406,426

PRSECT

L.PATH,404,424

PRSECT

LPATH,1120,1097

PRSECT

LPATH,1174,1149

PRSECT

PRSECT

LPATH,1177,1155

LPATH,1178,1158

PRSECT

LPATH,1179,1161

PRSECT

LPATH,1162,1164

PRSECT

LPATH,295,297

PRSECT

LPATH,1021,1027

PRSECT

LPATH,1071,1077

PRSECT

PRSECT

SAVE 
CLIENT: Duke Engineering \& Services Hanford

PROJECT: MCO Final Design
FILE NO: KH-8009-8-03 DOC. NO.: HNF-SD-SNF-DR-003, Rev. 0, Appendix 5

\section{COMPUTER RUN COVER SHEET}

Project Number:

Computer Code:

Software Version:

Computer System:

Computer Run File Number:

Unique Computer Run Filename:

Run Description:

Run Date / Time:
$\mathrm{KH}-8009-8$

ANSYS\&-PC

$5.0 \mathrm{~A}$

MS-DOS, Pentium® Processor

KH-8009-8-03

CSBMC.out

MCO CSB Tube Drop with Lifting Cap

30 March $1997 \quad$ 11:48:18 AM
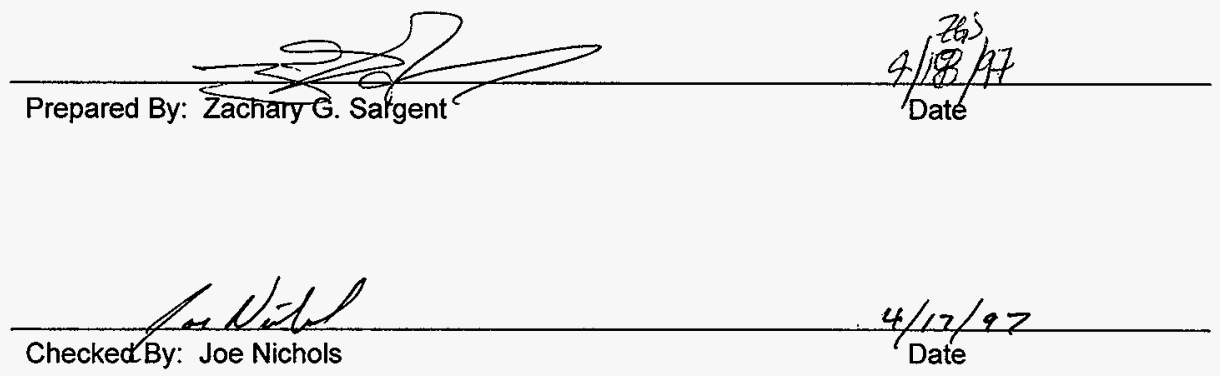

\begin{tabular}{|l|l}
\hline 0 & \\
\hline $4 / 17 / 97$ & \\
\hline $4 / 17 / 97$ & \\
\hline
\end{tabular}




\section{COMPUTER RUN COVER SHEET}

Project Number:

Computer Code:

Software Version:

Computer System:

Computer Run File Number:

Unique Computer Run Filename:

Run Description:

Creation Date / Time:
$\mathrm{KH}-8009-8$

ANSYS®-PC

$5.0 \mathrm{~A}$

MS-DOS, Pentium® Processor

$\mathrm{KH}-8009-8-03$

CSB.inp

MCO CSB Tube Drop without Lifting Cap

30 March $1997 \quad$ 10:53:20 AM
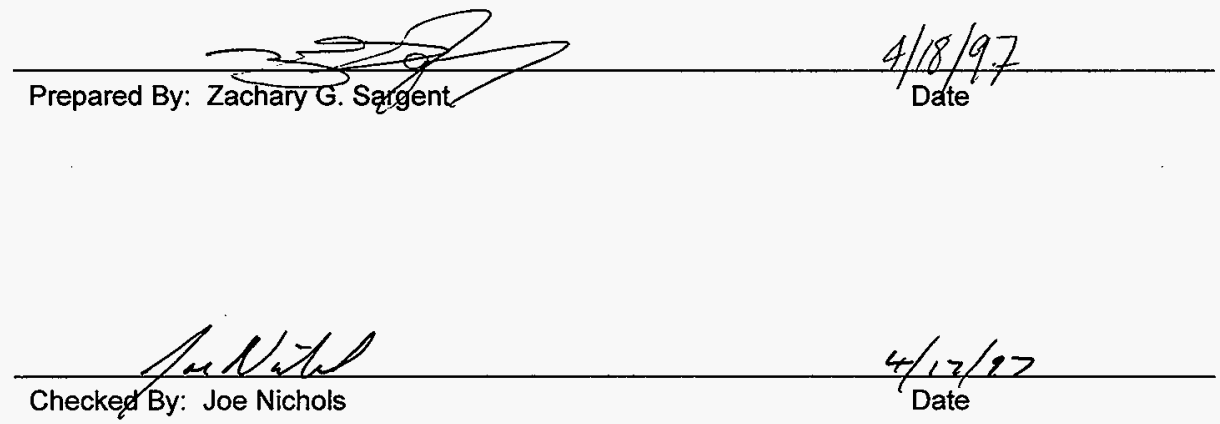

\begin{tabular}{|c|c|c|c|}
\hline 0 & & & \\
\hline $\operatorname{los} 4 / 17 / 97$ & & & \\
\hline $\operatorname{Re} 4 / 17 / 97$ & & & \\
\hline
\end{tabular}




\section{LISTING OF CSB.INP FILE}

/BATCH,LIST

IFILENAM,CSB

/PREP7

ITITLE,MCO DESIGN- 132 DEGREES C, $28 \mathrm{~g}$ CSB TUBE DROP

TREF,70

TUNIF, 270

ETAN $=0.006 \quad$ ! Tangent modulus

\begin{tabular}{|c|c|}
\hline ICOM ELEN & TYPES **** \\
\hline$E T, 1,42,,, 1$ & ! Shell \\
\hline $\mathrm{ET}, 2,42, \ldots, 1$ & ! Shield Plug \\
\hline $\mathrm{ET}, 3,42,, 1$ & ! Lifting \& Locking Ring \\
\hline $\begin{array}{l}\mathrm{ET}, 4,12 \\
\text { KEYOPT } 4,7,1\end{array}$ & ! Gap Elements Between Shield \\
\hline $\mathrm{ET}, 5,42_{n}, 1$ & ! Bolt \\
\hline
\end{tabular}

ICOM $\because$ REAL CONSTANTS FOR GAP ELEMENTS

R,4,-90,1.0e8,-0.06,3.0 !Shell/Shield Plug, Initially Open .06"

R,5,0,1.0e8,2.95e-03 ! L. Ring/Shield Plug, Under Bolt, Preloaded

R,6,0,1.0e8,0,2.0 ! Sealing Surface, closed

R,7,0,1.0e8,0,1.0 ! Bottom MCO Plate, closed

R,8,0,2.42e7,,2.0 ! Seal Spring, Max. Stiffness (Unloading Stif.)

ICOM

MP,DENS, $1,490 / 1728$ ! 304L SS

MP,NUXY, $1,0.3$

MP,DENS,5,490/1728 I SA193 Grade B8M

MP,NUXY,5,0.3

TB,BKIN, 1,6 ! Yield Stress \& Tangent Moduli v. Temp.

TBTEMP, 100

TBDATA, 1,25000,ETAN*28.1E+06

TBTEMP, 200

TBDATA, 1,21300,ETAN"27.6E+06

TBTEMP, 300

TBDATA,1,19100,ETAN*27.0E+06

TBTEMP, 400

TBDATA, $1,17500, E T A N * 26.5 E+06$

TBTEMP, 500

TBDATA, 1,16300, ETAN*25.8E+06

TBTEMP, 600

TBDATA 1,15500, ETAN*25.3E +06

ICOM DEFINING TEMPERATURES FOR MPDATA *

MPTEMP, $1,70,100,200,300,400,500$

MPTEMP $, 7,600,650,700,750$

/COM *** DEFINING ELASTIC MODULI FOR 304L \& SA-193 ***

MPDATA, EX, 1, 1,28.3e+06,28.1e+06,27.6e+06,27.0e+06,26.5e+06,25.8e+06

MPDATA,EX, 1,7,25.3e+06,25.1e+06,24.8e+06,24.5e+06

ICOM

! SA-193

\begin{tabular}{|l|c|l|l|l|}
\hline REVISION & 0 & & & \\
\hline PREPARED BY IDATE & G/S $4 / 17 / 97$ & & & \\
\hline CHECKED BY I DATE & $\mathbb{4} 4 / 17 / 97$ & & & \\
\hline
\end{tabular}


MPDATA,EX,5,1,28.3e+06,28.1e+06,27.6e+06,27.0e+06,26.5e+06,25.8e+06

MPDATA,EX,5,7,25.3e+06,25.1e+06,24.8e+06,24.5e+06

/COM MEAN COEFFICIENTS OF THERMAL EXPANSION (in./in./(F)

! SA240 Gr 304L.

MPDATA,ALPX, 1, 1,0,8.55e-06,8.79e-06,9.00e-06,9.19e-06,9.37e-06

MPDATA,ALPX, 1,7,9.53e-06,9.61e-06,9.69e-06,9.76e-06

! SA193 Gr B8M

MPDATA,ALPX,5, 1,0,8.54e-06,8.76e-06,8.97e-06,9.21e-06,9.42e-06

MPDATA,ALPX,5,7,9.60e-06,9.69e-06,9.76e-06,9.81e-06

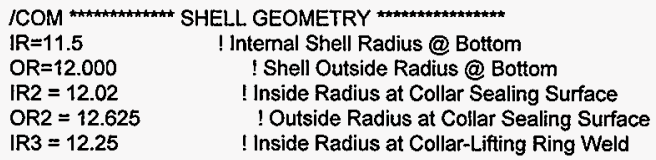

FILL

$\mathrm{N}, 41,0.00,-0.44$

! Row 3

$\mathrm{N}, 42,1.25,-0.44$ ! Row 1

$\mathrm{N}, 43,2.13,0.44$

$\mathrm{N}, 50, \mathrm{IR}, 0.44$

FILL,43,50

N,52,OR,0.44

FILL,50,52

FILL., 1,41,1,21,1,10 : Middle Row

FILL, 10,50,1,30

$\mathrm{N}, 32,12,-0.32$

FILL, 30,32

FILL, 10,32,1,11

$N, 53, I R, 1.17$

N,55,OR,1.17 ! Shell Stub/Weld

FILL, 53,55

/COM SHELL. [DWGS SK-2-300379 \& SK-2-300461] *

$\mathrm{N}, 65, \mathrm{IR}, 6.68$

N,67,OR,6.68

FILL

FILL, 53,65,3, 3,3,1

ICOM SINGLE ROW SHELL *

N, 100,IR,7.18

! Inside

N, 140,IR,71.68

$\mathrm{N}, \mathbf{1 8 0}, \mathrm{IR}, 136.68$

N,101,OR,7.18 I Outside

$N, 141, O R, 71.68$

REVISION

PREPARED BY I DATE CHECKED BY I DATE 
Duke Engineering \& Services Hanford

FILE NO: KH-8009-8-03

PROJECT: MCO Final Design DOC. NO.: HNF-SD-SNF-DR-003, Rev. 0, Appendix 5

$\mathrm{N}, 181, \mathrm{OR}, 136.68$

FILL, 100,140,20,2,2,1,2.0

FILL, $140,180,19,2,2,1, .5$

ICOM DOUBLE ROW SHELL

N, 190,IR,137.18 I Transition to Double Row

N, 192,OR, 137.18

FILL

ICOM BASE OF CASK THROAT-ELEVATION: 138 INCHES **

N,217,IR,142.68 I Transition to Double Row

N,219,OR, 142.68

FILL

FILL, 190,217,8, 3,3,1 IVertical Fil

ICOM BOTTOM OF COLLAR TRANSITION

N,235,IR, 146.06 1 Start of Transition to Large O.D \&

N,237,OR,146.06 ! Assumed Location of Shield Plug Taper

FILL

$\mathrm{N}, 238, \mathrm{IR}, 146.68$

N,240,OR, 146.68

FILL

! Horizontal Fill

FILL,217,235,5,3,3,1 ! Vertical Fil

ICOM TOP OF COLLAR TRANSITION ***

$N, 241, I R, 147.31$ ! End of Transition to Large O.D \&

$N, 243, O R, 147.31$

FILL

! Assumed Location of Shield Plug Taper

NGEN,2,3,241,243,1, 0.75

ICOM COLLAR SEALING SURFACE *

$N, 247, I R, 149.63$ ! Inside Radius of Sealing Surface

N,249,IR2,149.63 ! Outside Radius at Sealing Surface

FILL

! Horizontal Fill

ICOM THICK WALLAT COLLAR TRANSITION *

NGEN,2,10,240,249,3

! Nodes 250-259 Coincident w/240-249 (by 3)

N,255,OR2, 147.31

! Outside Surface

N,261,OR2, 149.63

! Outside Surface

$\mathrm{N}, 258$, OR2, 148.06

N,980,IR, 149.38

$N, 981,11.755,149.38$

N,982,IR2, 149.38

$N, 983,12.317,149.38$

N,984,OR2, 149.38

N,990,OR2, 146.68

FILL,240,990,1,251

NGEN, $2,5,980,984,1,,-0.66$

FILL,246,258,1,257

FILL,253,255, 1, 1,3,3

FILL,237,990,1,991

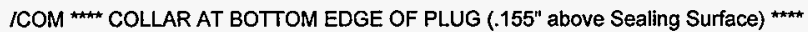

NGEN, $2,3,259,,, 0.245$ ! Nodes 262

/COM ${ }^{\star \star \star}$ COLLAR AT TOP EDGE OF PLUG (2" above bottom Edge) ***

\begin{tabular}{|l|c|l|l|l|}
\hline REVISION & 0 & & & \\
\hline PREPARED BY IDATE & $7 / 2) 4 / 17 / 97$ & & & \\
\hline CHECKED BY I DATE & $7 / 4 / 17 / 97$ & & & \\
\hline
\end{tabular}


NGEN,2,9,262,..,2.00 ! Nodes 271

FILL,262,271,2

ICOM COLLAR AT BASE OF THREADS

$N, 274, I R 3,152.00$

$\mathrm{N}, 1000$, IR2, 152.00

ICOM TOP TO COLLAR (WELD CLOSURE) $\cdots$

$\mathrm{N}, 295, \mathrm{IR} 3,156.00$

FILL,274,295

NGEN $, 3,1,259,295,3,($ OR2-IR2)/2

NGEN,3,1,274,295,3,(OR2-IR3)/2

ICOM LOCKING \& LIFTING RING GEOMETRY

RING1 $=7.94$

RING2 $=9.375$

RING3 $=9.625$

RING4 $=10.19$

RING5 $=12.23$

LOCAL, 11,0,152.00 ! Local System $z=0$ at Base of Ring

CSYS, 11

ICOM *** TOP EDGE ****

$N, 401, R I N G 1,6.13$

CSYS,O

$\mathrm{N}, 404,9.375,158.13$

FILL, $401,404,, 1$

N,406,RING4,158.13

FILL, $404,406, \ldots 1$ ! Top Edge

ICOM LIFTING SURFACE \#

CSYS, 11

N,421,RING1,5.13

N,424,RING2,5.13

FILL, 421,424

N,426, RING4,5.13

FILL,424,426

FILL,401,421,1,10,6,1

N,431,RING1,6.13-1.56

N,434,RING2,6.13-1.56

FILL

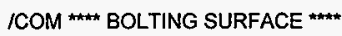

$\mathrm{N}, 441$, RING1,4

$\mathrm{N}, 444, \mathrm{RING} 3,4$

FILL

N,445,10.9375- 6875,4 ! Inside Edge of Bolt Hole

$\mathrm{N}, 447,10.9375+.6875,4$ ! Outside Edge of Bolt Hole

FILL

$\mathrm{N}, 910,10.9375-.6875,4$

$\mathrm{N}, 911,10.9375+.6875,4$

N,448, RING5,4

CSYS, 0

$\mathrm{N}, 924,10.25,152.00$

$\mathrm{N}, 925,11.625,152.00$

FILL, $910,924,6,2$

\begin{tabular}{|l|c|l|l|l|c|}
\hline REVISION & 0 & & & & PAGE 104 \\
PREPARED BY IDATE & OF 198 & \\
\hline CHECKED BY I DATE & $/ 1 / 17 / 97$ & & & & \\
\hline
\end{tabular}


CLIENT: $\quad$ Duke Engineering \& Services Hanford

FILE NO: $\quad \mathrm{KH}-8009-8-03$

PROJECT: MCO Final Design DOC. NO.: HNF-SD-SNF-DR-003, Rev. 0, Appendix 5

FILL,911,925,6,2

$N, 525,10.25,151.874$

! Bottom of Bolt Extension

$\mathrm{N}, 527,11.625,151.874$

FILL

ICOM BOTTOM OF LIFTING/LOCKING RING

CSYS, 11

NGEN, $2,70,441,448,1,-4$

FILL,441,511,6,10,8,1

! Bottom Surface of Lifting/Locking Ring

! Fill in Lifting/Locking Ring

ICOM SHIELD PLUG (offset $y$ by 158.25)

LOCAL, $20,0,158.13$

TYPE, 2

PLUGR1 $=11.975$

PLUGR2=11.45

PLUGR3=11.25

PLUGR4=7.89

1 COM NODES AT PLUG AXIS $(r=0)$

$\mathrm{N}, 601$

$\mathrm{N}, 602,0,-1$

$\mathrm{N}, 603,0,-1.994$

$\mathrm{N}, 606,0,-4.994$

FiLL, $603,606,2,604$

$\mathrm{N}, 607,0,-6.25$

$\mathrm{N}, 610,0,-8.25$

FILL, $607,610,2,608$

$\mathrm{N}, 611,0,-8.75$

$\mathrm{N}, 613,0,-10.5$

FILL, 611,613

ICOM NODAL GENERATION \#

NGEN, $2,20,601,613,1,0.8825$

NGEN, $2,20,621,633,1,0.8825$

NGEN,2,20,642,653, $1,0.6875$

NGEN,2,20,662,673, $1,0.6875$

NGEN,2,20,683,693,1,0.4235

NGEN,2,10,706,713,1,0.9515

! ld Large Opening

! ld Medium Opening

! Id Small Opening

I Center of Opening

! Od Small Opening

N,730,5.4665,-1.994
$N, 736,5.4665,-4.994$
FILL,730,736,5,731
N,737,5.4665,-6.25
$N, 740,5.4665,-8.25$
FILL, $737,740,2,738$
$N, 741,5.4665,-8.75$
$N, 743,5.4665,-10.5$
FILL,741,743
$N, 748,5.89,-1.0$

NGEN $, 2,20,730,743,1,0.4235$

FILL, 748,750

$N, 766,7.265,0$

NGEN $, 2,20,748,763,1,1.375$

FILL,766,768

NGEN $, 3,20,766,768,1,0.3125$

$\mathrm{N}, 789,7.5775,-1.56$

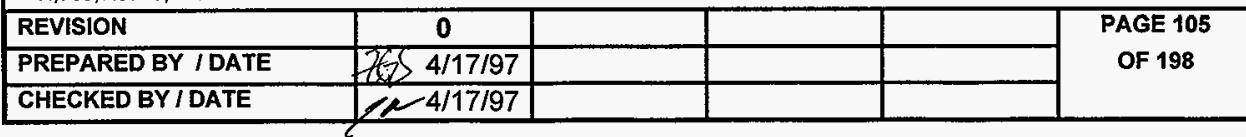


CLIENT: Duke Engineering \& Services Hanford

PROJECT: MCO Final Design
FILE NO: KH-8009-8-03

\author{
$\mathrm{N}, 796,7.5775,-5.56$ \\ FILL, 789,796,6 \\ NGEN, $2,20,789,796,1,0.3125$ \\ NGEN, $3,20,777,783,1,0.3125$
}

/COM UNDER LOCKING RING \#

$\mathrm{N}, 824,8.5017,-6.25$

$\mathrm{N}, 827,8.5017,-8.25$

FILL

$\mathrm{N}, 828,8.5017,-8.75$

$\mathrm{N}, 830,8.5017,-10.5$

FILL

NGEN, $3,7,824,830,1,0.5616$

NGEN, $2,7,838,844,1,0.625$

NGEN, $2,7,845,851,1,0.6875$ ! Under Bolt

$\mathrm{N}, 859,11.625,-6.25$

$\mathrm{N}, 860,11.625,-6.917$

N,861,11.625,-7.584

$\mathrm{N}, 862$, PLUGR2, -8.25

N,863,PLUGR2,-8.75

N,865,PLUGR3,-10.5

FILL,863,865,1

N,866,PLUGR1-0.255,-6.25

N,869,PLUGR1-0.255,-8.25

FILL,866,869,2

$\mathrm{N}, 870$, PLUGR 1-0.255,-8.476

NGEN $, 2,5,866,870,1,0.255$

ICOM **** REFINING LIFTING EAR ****

CSYS, 0

$N, 877,9.53,158.13$

$N, 889,9.53,157.63$

$\mathrm{N}, 901,9.53,157.13$

FILL, 403,404,1,876

FILL, $413,414,1,888$

FILL, $423,424,1,900$

FILL, $877,405,1,878$

FILLL,405,406,2,879,1

FILL, $889,415,1,890$

FILL,415,416,2,891,1

FILL, 404,414,1,881

FILL,877,889,1,882

FILL,878,890,1,883

FILL, 405,415, 1,884

FILL,879,891,1,885

FILL, $880,892,1,886$

FILL,406,416,1,887

FILL, $889,901,1,894$

FILL, $414,424,1,893$

FILL, $901,425,1,902$

FILL,890,902,1,895

FILL,415,425,1,896

FILL,425,426,2,903,1

FILL,891,903,1,897

FILL, $892,904,1,898$

FILL, 416,426, 1,899

\begin{tabular}{|l|c|l|l|l|l|}
\hline REVISION & 0 & & & & PAGE 106 \\
OF 198 \\
\cline { 1 - 5 } CHEPARED BY IDATE & ZGS 4/17/97 & & & & \\
\hline
\end{tabular}


CLIENT: Duke Engineering \& Services Hanford

PROJECT: MCO Final Design DOC. NO.: HNF-SD-SNF-DR-003, Rev. 0, Appendix 5

FILLL,424,434,1,907

FILL $, 433,434,1,908$

FILL $, 423,433,1,905$

FILL,905,907

ICOM *** FILTER GUARD PLATE ***

PLATE $1=0.273$

PLATE2 $=0.6575$

PLATE3=1.357

PLATE4 $=10.25$

PLATE5=11.25

N,1200,PLATE4,146.78

N,1202,PLATE5, 146.78

FILL.

NGEN,5,3,1200,1202, , -0.85

NGEN, $2,3,1212,1214, \ldots,-0.25$

$\mathrm{N}, 1221, \mathrm{PLATE} 4,141.88$

$N, 1222,10.75,141.88$

N,1223,10.915,141.88

FILL, 1215, 1221, 1,1218

FILL, 1223,1217, 1,1220

FILL, 1216,1222, 1,1219

$N, 1237,6.4375,143.38$

FILL, 1212,1237,3,1225,4

N, 1249,3.578,143.38

FILL, 1237,1249,2,1241,4

NGEN,2,1,1225,1249,4, -0.25

NGEN,2,2,1226,1250,4,,-1.25

FILL, 1226,1228,1,1227, ,7,4

$N, 1253,2.625,145.255$

N,1254,2.625,145.005

N, 1256,2.625,143.38

FILL, 1254, 1256

$N, 1257,2.625,143.13$

N,1259,2.625, 141.88

FILL, 1257,1259

NGEN $, 2,10,1253,1259,1,-0.5$

NGEN, $2,10,1263,1269,1,-0.768$

$\mathrm{N}, 1283,0.6575,145.255$

$\mathrm{N}, 1284,0.6575,145.005$

N, 1260,2.125,147.63

N, $1270,1.357,147.63$

$N, 1280,0.6575,147.63$

$N, 1290,0.273,147.63$

NGEN, $3,1,1260,1290,10,-0.5625$

/COM NODES AT BOTTOM GAP ELEMENTS \#

NGEN, 2,2000,1,10,1, -1.00

ICOM * COUPLING NODES ***

ICOM ** BETWEEN LIFTING/LOCKING RING \& SHELL \#

$\mathrm{CP}, 1, \mathrm{UY}, 508,277$

I Start Threads

CP, $2, U Y, 498,280$

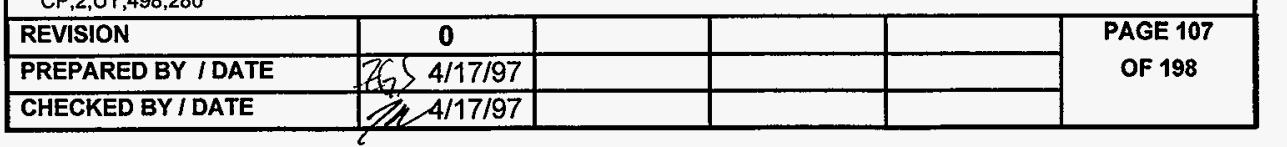


CLIENT: Duke Engineering \& Services Hanford

$C P, 3, U Y, 488,283$

CP, 4,UY,478,286

$C P, 5, \cup Y, 468,289$

CP, $6, U Y, 458,292$

/COM BETWEEN BOLT \& LOCKING RING *

CP, $7, U Y, 445,910$

$\mathrm{CP}, 8, \mathrm{UX}, 445,910$

$C P, 9, \cup Y, 447,911$

CP, 10,UX,447,911

*DO,I,1,7

CP, 10+1,UY,445+10*I,910+2*I

*ENDDO

*DO,I,1,7

CP, 17+1,UY,447+10*I,911+2*|

*ENDDO

${ }^{*} \mathrm{DO}, 1,1,7$

$C P, 24+1, U X, 445+10 * 1,910+2 * 1$

"ENDDO

${ }^{*} D O, 1,1,7$

$\mathrm{CP}, 31+1, \mathrm{UX}, 447+10^{\star} 1,911+2^{*} i$

*ENDDO

NALL

EALL

ICOM ELEMENT GENERATION FOR SHELL

TYPE, 1

MAT, 1

ICOM BOTTOM OF SHELL

$\mathrm{E}, 1,2,22,21$

$E, 2,3,23,22$

EGEN,8,1,-1

$E, 10,11,30$

$E, 21,22,42,41$

$E, 22,23,43,42$

EGEN, 10,1,-1

$E, 11,31,30$

$E, 11,32,31$

ICOM SHELL

$E, 50,51,54,53$

EGEN,2,1,-1

EGEN $, 5,3,-2$

ICOM FIRST TRANSITION ELEMENTS

$E, 65,66,100$

$E, 100,66,101$

$E, 67,101,66$

ICOM *** SINGLE SHELL *

$E, 100,101,103,102$

EGEN,40,2,-1

/COM SECOND TRANSITION ELEMENTS ****

$E, 190,180,191$

\begin{tabular}{|l|c|l|l|l|}
\hline REVISION & 0 & & & \\
\hline PREPARED BY IDATE & CES 4/17/97 & & & \\
\hline CHECKED BY IDATE & / $4 / 17 / 97$ & & & \\
\hline
\end{tabular}


CLIENT: Duke Engineering \& Services Hanford

PROJECT: MCO Final Design DOC. NO.: HNF-SD-SNF-DR-003, Rev. 0, Appendix 5

FILE NO: KH-8009-8-03

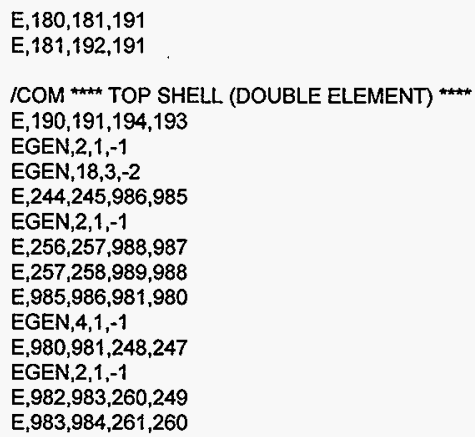


CLIENT: Duke Engineering \& Services Hanford

PROJECT: MCO Final Design DOC.NO.: HNF-SD-SNF-DR-003, Rev. 0, Appendix 5

$E, 885,886,880,879$

$E, 891,892,886,885$

$E, 886,887,406,880$

$E, 892,416,887,886$

$E, 423,900,888,413$

$E, 893,414,888$

$E, 900,893,888$

$E, 900,424,893$

$E, 893,894,889,414$

$E, 424,901,894,893$

$E, 894,895,890,889$

$E, 901,902,895,894$

$E, 895,896,415,890$

$\mathrm{E}, 902,425,896,895$

$E, 896,897,891,415$

$E, 425,903,897,896$

$E, 897,898,892,891$

$E, 903,904,898,897$

$E, 898,899,416,892$

E,904,426,899,898

$E, 431,432,422,421$

$E, 905,423,422$

$E, 432,905,422$

$E, 432,433,905$

$E, 905,906,900,423$

$E, 433,908,906,905$

$\mathrm{E}, 906,907,424,900$

$\mathrm{E}, 908,434,907,906$

$E, 441,442,432,431$

EGEN,2,1,-1

E, $443,908,433$

$E, 443,444,434,908$

$E, 451,452,442,441$

EGEN,3,1,-1

EGEN,7,10,-3

E,454,912,910,444

$E, 464,914,912,454$

E,474,916,914,464

$E, 484,918,916,474$

E,494,920,918,484

E,504,922,920,494

$E, 514,924,922,504$

$E, 458,448,911,913$

$E, 468,458,913,915$

E, $478,468,915,917$

$E, 488,478,917,919$

$E, 498,488,919,921$

E, $508,498,921,923$

E, $518,508,923,925$

ICOM ${ }^{\text {*a* }}$ BOLT *A**

TYPE, 5

MAT, 5

$E, 455,456,446,445$

EGEN,8,10,-1

$E, 456,457,447,446$

\begin{tabular}{|l|c|l|l|l|l|}
\hline REVISION & 0 & & & & $\begin{array}{c}\text { PAGE 110 } \\
\text { OF 198 }\end{array}$ \\
\hline PREPARED BY IDATE & J6S 4/17/97 & & & & \\
\hline CHECKED BYIDATE & / 4/17/97 & & & & \\
\hline
\end{tabular}


CLIENT: Duke Engineering \& Services Hanford

FILE NO: $\quad \mathrm{KH}-8009-8-03$

$\begin{array}{ll}\text { PROJECT: MCO Final Design } & \text { DOC. NO.: HNF-SD-SNF-DR-003, Rev. 0, Appendix } 5\end{array}$

EGEN, 8,10,-1

ICOM END OF LOCKINGILIFTING RING

ICOM SHIELD PLUG ELEMENTS

TYPE, 2

MAT, 1

E,602,622,621,601

EGEN,11,1,-1

EGEN,2,20,-11

$E, 613,1290,612$

$E, 1290,1280,632,612$

E, 1280,633,632

$E, 633,1270,632$

$E, 1270,652,632$

$E, 1270,653,652$

$E, 643,663,662,642$

EGEN,10,1,-1

EGEN, $2,20,-10$

$E, 673,693,692,672$

EGEN,2,20,-10

$E, 653,1260,652$

$E, 1260,672,652$

$\mathrm{E}, 1260,673,672$

$E, 707,717,716,706$

EGEN,7,1,-1

$\mathrm{E}, 717,737,736,716$

EGEN, $7,1,-1$

E, $731,751,750,730$

EGEN,13,1,-1

$E, 749,769,768,748$

EGEN,15,1,-1

E, $767,787,786,766$

EGEN,17,1,-1

EGEN,2,20,-17

$\mathrm{E}, 818,825,824,817$

EGEN, $6,1,-1$

EGEN, $5,7,-6$

$\mathrm{E}, 853,860,859,852$

EGEN,6,1,-1

$E, 860,867,866,859$

EGEN,3,1,-1

$E, 867,872,871,866$

EGEN,4,1,-1

/COM END OF SHIELD PLUG

ICOM FILTER GUARD PLATE

$E, 1200,1201,858,851$

E, 1201,1202,865,858

E, 1203,1204,1201,1200

EGEN, 2,1,-1

EGEN,6,3,-2

E, $1221,1222,1219,1218$

E, $1222,1223,1220,1219$

$E_{1} 1226,1215,1212,1225$

E, 1227,1218,1215,1226

$E, 1228,1221,1218,1227$

\begin{tabular}{|l|c|l|l|l|l|}
\hline REVISION & 0 & & & & PAGE 111 \\
PREPARED BY I DATE & PGS 4/17/97 & & & & \\
\cline { 1 - 4 } CHECKED BY I DATE & OF $4 / 17 / 97$ & & & & \\
\hline
\end{tabular}


CLIENT: Duke Engineering \& Services Hanford

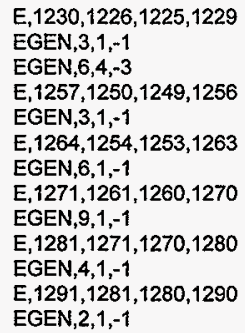


CLIENT: Duke Engineering \& Services Hanford

FILE NO: $\quad \mathrm{KH}-8009-8-03$

PROJECT: MCO Final Design DOC. NO.: HNF-SD-SNF-DR-003, Rev. 0, Appendix 5

$E, 247,862$

ICOM BOTTOM GAP ELEMENTS

TYPE, 4

REAL, 7

$E, 2001,1$

EGEN, 10,1,-1

NALL

EALL

ICOM END GAP ELEMENTS

ICOM BOUNDARY CONDITIONS B

CSYS, 0

NSEL,S,LOC,X,O

NSEL, R,LOC,Y,-1.33,158.14

$D, A L L, U X, 0$

NALL

EALL

NSEL,S,NODE, 2001,2010

$D, A L L, A L L, O$

EALL

NALL

SAVE

ICOM LOAD 1: APPLYING EQUIVALENT 28g CSB DROP

NSEL,S,NODE, ,601,641,20

NSEL,A,NODE,, $766,806,20$

SF,ALL,PRES, 13555

NALL

$128 \mathrm{~g} \times 19142=535976 \mathrm{LB}$

EALL

! Shield plug area $=39.54 \mathrm{in}^{\wedge} 2$

FINI

! $\mathrm{P} / \mathrm{A}=13555 \mathrm{psi}$

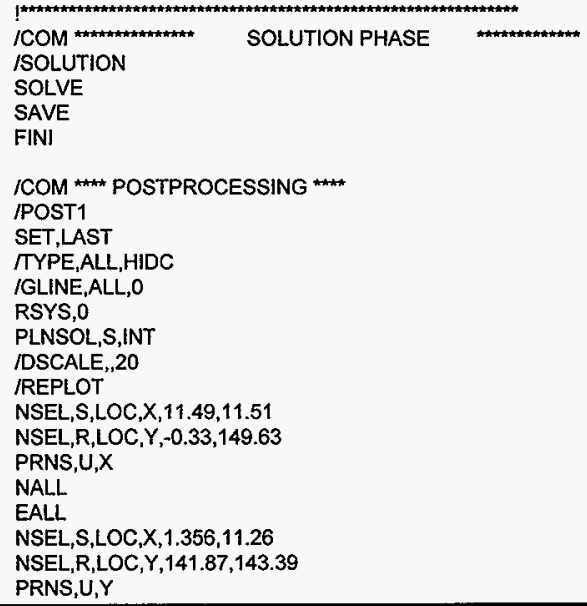

\begin{tabular}{|c|l|l|l|}
\hline 0 & & & \\
\hline $\log 4 / 17 / 97$ & & & \\
\hline $20-4 / 17 / 97$ & & & \\
\hline
\end{tabular}


CLIENT: Duke Engineering \& Services Hanford

PROJECT: MCO Final Design
FILE NO: $\quad \mathrm{KH}-8009-8-03$

NALL

EALL

LPATH, 1,41

PRSECT

LPATH, 6,46

PRSECT

LPATH, 10,50

PRSECT

LPATH,50,52

PRSECT

LPATH,62,64

PRSECT

LPATH,65,67

PRSECT

LPATH, 100,101

PRSECT

LPATH, 122, 123

PRSECT

LPATH, 134,135

PRSECT

LPATH, 156, 157

PRSECT

LPATH, 170,171

PRSECT

LPATH, 180,181

PRSECT

L.PATH,202,204

PRSECT

LPATH,235,237

PRSECT

LPATH,985,989

PRSECT

L.PATH,262,264

PRSECT

LPATH, 277,279

PRSECT

LPATH,292,294

PRSECT

LPATH,601,641

PRSECT

LPATH,601,613

PRSECT

LPATH,603,703

PRSECT

LPATH,606,706

PRSECT

LPATH,766,806

PRSECT

LPATH, 748,808

PRSECT

LPATH, 730,810

PRSECT

LPATH, 736,815

PRSECT

LPATH,869,874

PRSECT

\begin{tabular}{|l|c|l|l|l|}
\hline REVISION & 0 & & & \\
\hline PREPARED BY IDATE & $\operatorname{lE} \delta$ 4/17/97 & & & \\
\hline CHECKED BY IDATE & $\mathbb{2} 4 / 17 / 97$ & & & \\
\hline
\end{tabular}


CLIENT: Duke Engineering \& Services Hanford

PROJECT: MCO Final Design
FILE NO: KH-8009-8-03

DOC. NO.: HNF-SD-SNF-DR-003, Rev. 0, Appendix 5

LPATH, 870,875

PRSECT

LPATH, 431,434

PRSECT

LPATH,406,426

PRSECT

LPATH,404,424

PRSECT

SAVE

\begin{tabular}{|l|c|l|l|l|l|}
\hline REVISION & 0 & & & & PAGE 115 \\
OF 198 \\
\cline { 1 - 5 } PREPARED BY IDATE & CHECKED BY 4/17/97 & & & & \\
\hline
\end{tabular}


CLIENT: Duke Engineering \& Services Hanford

PARSDNS

PROJECT: MCO Final Design DOC. NO.: HNF-SD-SNF-DR-003, Rev. 0, Appendix 5

\section{COMPUTER RUN COVER SHEET}

Project Number:

Computer Code:

Software Version:

Computer System:

Computer Run File Number:

Unique Computer Run Filename:

Run Description:

Run Date / Time:
$\mathrm{KH}-8009-8$

ANSYS $B-P C$

$5.0 \mathrm{~A}$

MS-DOS, Pentium(8) Processor

$\mathrm{KH}-8009-8-03$

CSB.out

MCO CSB Tube Drop without Lifting Cap

30 March $1997 \quad$ 11:08:20 AM

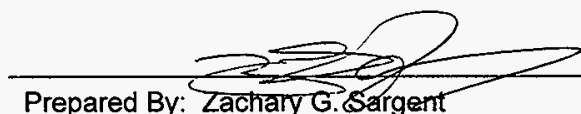

Prepared By: Zachaly G. Sargent

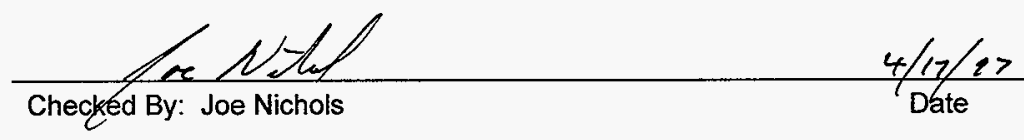

\begin{tabular}{|c|}
0 \\
\hline $\cos 54 / 17 / 97$ \\
\hline $4 / 17 / 97$ \\
\hline
\end{tabular}




\section{COMPUTER RUN COVER SHEET}

Project Number:

Computer Code:

Software Version:

Computer System:

Computer Run File Number:

Unique Computer Run Filename:

Run Description:

Creation Date / Time:
KH-8009-8

ANSYS $\circledast-P C$

$5.0 \mathrm{~A}$

MS-DOS, Pentium® Processor

$\mathrm{KH}-8009-8-03$

TEDMC.inp

MCO Top End Drop with Lifting Cap

30 March $1997 \quad$ 10:54:54 AM
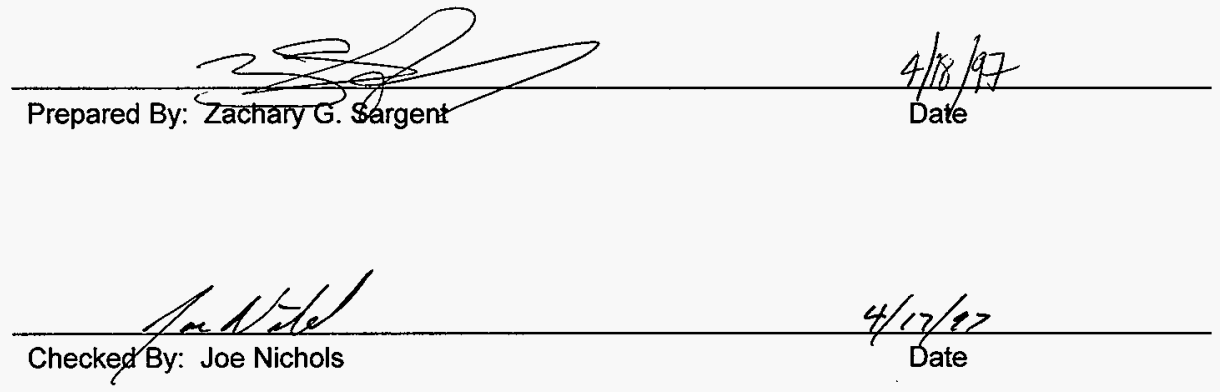

$\frac{125}{2}$

\begin{tabular}{|l|l|l|l|}
\hline 0 & & & \\
\hline $4 / 17 / 97$ & & & \\
\hline $4 / 17 / 97$ & & & \\
\hline
\end{tabular}


CLIENT: Duke Engineering \& Services Hanford

FILE NO: $\mathrm{KH}-8009-8-03$

$\begin{array}{ll}\text { PROJECT: MCO Final Design } & \text { DOC. NO.: HNF-SD-SNF-DR-003, Rev. 0, Appendix } 5\end{array}$

\section{LISTING OF TEDMC.INP FILE}

/BATCH,LIST

IFILENAM,TEDMC

IPREP7

TITLE, 132 DEGREES C, $150 \mathrm{psi}, 28 \mathrm{~g}$ TOP END DROP W/ MODIFIED CAP

TREF,70

TUNIF, 270

ETAN $=0.006 \quad$ ! Tangent modulus

ICOM *** ELEMENT TYPES ***

$\begin{array}{ll}E T, 1,42,, 1 & \text { ! Shell } \\ \text { ET,2,42,,,1 } & \text { ! Shield Plug } \\ \text { ET, 3,42,,1 } & \text { ! Lifting \& Locking Ring } \\ \text { ET, 4,12 } & \text { ! Gap Elements Between Shield Plug \& Shell }\end{array}$

KEY,4,12

ET, $5,42, \ldots 1$ ! Bolt

ICOM REAL CONSTANTS FOR GAP ELEMENTS

R,4,-90,1.0E8,-0.06,3.0 ! Shell/Shield Plug, Initially Open .06"

R,5,0,1.0E8,2.95e-03 ! L. Ring/Shield Plug, Under Boit, Preloaded

$R, 6,0,1.0 E 8,0,2.0$ ! Sealing Surface, closed

$R, 7,0,1.0 \mathrm{E} 8,0,1.0 \quad$ ! Anchoring for drop, closed

R,8,0,2.42e7,0,2.0 ISeal Spring, Max Stiffness

/COM MATERIAL PROPERTIES

MP,DENS, $1,490 / 1728$ ! 304L SS

MP, NUXY, $1,0.3$

MP,DENS,5,490/1728 ! SA193 Grade B8M

MP,NUXY,5,0.3

TB,BKIN, $1,6 \quad$ ! Yield Stress \& Tangent Moduli v. Temp.

TBTEMP, 100

TBDATA $1,25000, E T A N * 28.1 E+06$

TBTEMP, 200

TBDATA, 1,21300,ETAN*27.6E+06

TBTEMP, 300

TBDATA, 1, 19100,ETAN*27.0E+06

TBTEMP, 400

TBDATA, 1,17500, ETAN $* 26.5 E+06$

TBTEMP, 500

TBDATA, 1,16300, ETAN $\star 25.8 E+06$

TBTEMP,600

TBDATA, 1, 15500,ETAN*25.3E+06

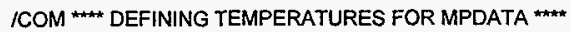

MPTEMP, $1,70,100,200,300,400,500$

MPTEMP, $7,600,650,700,750$

ICOM DEFINING ELASTIC MODULIFOR 304L \& SA-193 *\#*

MPDATA, EX, 1,1,28.3e+06,28.1e+06,27.6e+06,27.0e+06,26.5e+06,25.8e+06

\begin{tabular}{|l|c|l|l|l|}
\hline REVISION & 0 & & & \\
\hline PREPARED BY I DATE & fST, 4/17/97 & & & \\
\hline CHECKED BY I DATE & $7 / 4 / 17 / 97$ & & & \\
\hline
\end{tabular}


CLIENT: Duke Engineering \& Services Hanford

FILE NO: $\mathrm{KH}-8009-8-03$

PROJECT: MCO Final Design

DOC. NO.: HNF-SD-SNF-DR-003, Rev. 0, Appendix 5

MPDATA,EX,1,7,25.3e+06,25.1e+06,24.8e+06,24.5e+06

ICOM ISA-193

MPDATA, EX,5,1,28.3e+06,28.1e+06,27.6e+06,27.0e+06,26.5e+06,25.8e+06

MPDATA,EX,5,7,25.3e+06,25.1e+06,24.8e+06,24.5e+06

ICOM MEAN COEFFICIENTS OF THERMAL EXPANSION (in./in./(F)

I SA240 Gr 304L

MPDATA,ALPX,1,1,0,8.55e-06,8.79e-06,9.00e-06,9.19e-06,9.37e-06

MPDATA,ALPX,1,7,9.53e-06,9.61e-06,9.69e-06,9.76e-06

! SA193 Gr B8M

MPDATA,ALPX, 5,1,0,8.54e-06,8.76e-06,8.97e-06,9.21e-06,9.42e-06

MPDATA,ALPX, 5,7,9.60e-06,9.69e-06,9.76e-06,9.81e-06

ICOM SHELL GEOMETRY

IR=11.5 ! Internal Shell Radius @ Bottom

$\mathrm{OR}=12.000$

! Shell Outside Radius @ Bottom

IR2 $=12.02$

! Inside Radius at Collar Sealing Surface

$\mathrm{OR} 2=12.625$

! Outside Radius at Coliar Sealing Surface

IR3 $=12.25$

! Inside Radius at Collar-Lifting Ring Weld

ICOM BOTTOM COVER PLATE [DWG SK-2-300378]

$\mathrm{N}, 1,-1.32$

! Row 1

$N, 2,1,25,-1.32$

$\mathrm{N}, 3,2,13,-1.32$

$N, 10,11.423,-1.32$

FILL.

$\mathrm{N}, 41,0.00,-0.44$

! Row 3

$N, 42,1.25,-0.44$

$N, 43,2.13,0.44$

$\mathrm{N}, 50, \mathrm{IR}, 0.44$

FILL, 43,50

$\mathrm{N}, 52, \mathrm{OR}, 0.44$

FILL, 50,52

FILL, $1,41,1,21,1,10$

! Middle Row

FILL; $10,50,1,30$

$\mathrm{N}, 32,12,-0.32$

FILL, 30,32

FILL, 10,32,1,11

$\mathrm{N}, 53, \mathrm{IR}, 1.17$

$\mathrm{N}, 55, \mathrm{OR}, 1.17$

FILL, 53,55

! Shell StubMeld

ICOM SHELL [DWGS SK-2-300379 \& SK-2-300461]

$\mathrm{N}, 65, \mathrm{IR}, 6.68$

$\mathrm{N}, 67, \mathrm{OR}, 6.68$

FILL

FILL, $53,65,3,3,3,1$

ICOM SINGLE ROW SHELL

$N, 100, I R, 7.18$ ! Inside

N, 140,IR,71.68

$N, 180, I R, 136.68$

\begin{tabular}{|l|l|l|l|}
\hline & & & \\
\hline & & & \\
\hline
\end{tabular}


CLIENT: Duke Engineering \& Services Hanford

PROJECT: MCO Final Design

FILE NO: $\quad \mathrm{KH}-8009-8-03$

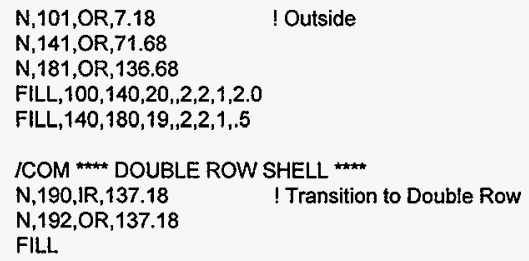

! Outside

$N, 141$, OR,71.68

N,181,OR, 136.68

FILL, 100,140,20, 2,2,1,2.0

FILL, $140,180,19,2,2,1, .5$

ICOM DOUBLE ROW SHELL

N,190.JR, 137.18 ! Transition to Double Row

N,192,OR, 137.18

FILL.

ICOM BASE OF CASK THROAT-ELEVATION: 138 INCHES

$N, 217, I R, 142.68$

! Transition to Double Row

N,219,OR, 142.68

FILL

FILL, 190,217,8,3,3,1 IVertical Fill

/COM ${ }^{\star \star \star \star}$ BOTTOM OF COLLAR TRANSITION

N,235,IR,146.06 IStart of Transition to Large O.D \&

N,237,OR,146.06 I Assumed Location of Shield Plug Taper

FILL

$\mathrm{N}, 238, \mathrm{IR}, 146.68$

N,240,OR, 146.68

FILL

! Horizontal Fil!

FILL,217,235,5,3,3,1 !Vertical Fill

ICOM TOP OF COLIAR TRANSITION

$N, 241, I R, 147.31 \quad$ ! End of Transition to Large O.D \&

$N, 243, O R, 147.31$

FILL ! Horizontal Fill

! Assumed Location of Shield Plug Taper

NGEN,2,3,241,243,1,0.75

ICOM COLLAR SEALING SURFACE

$N, 247, I R, 149.63$

N,249,IR2, 149.63

FILL.

! Inside Radius of Sealing Surface

! Outside Radius at Sealing Surface

! Horizontal Fill

ICOM *** THICK WALL AT COLLAR TRANSITION ***

NGEN,2,10,240,249,3

N,255,OR2,147.31

! Nodes 250-259 Coincident w/240-249 (by 3)

$\mathrm{N}, 261, \mathrm{OR} 2,149.63$

! Outside Surface

N,258,OR2,148.06

! Outside Surface

$\mathrm{N}, 980, \mathrm{IR}, 149.38$

N,981,11.755,149.38

N,982,IR2, 149.38

N,983,12.317,149.38

N,984,OR2, 149.38

N,990,OR2,146.68

FILL,240,990,1,251

NGEN,2,5,980,984, $1,-0.66$

FILL,246,258,1,257

FILL,253,255,1, 1,3,3

FILL, 237,990,1,991

/COM *** COLLAR AT BOTTOM EDGE OF PLUG (.155" above Sealing Surface) *** NGEN,2,3,259, ,.,0.245 1 Nodes 262

\begin{tabular}{|l|c|l|l|l|}
\hline REVISION & 0 & & & \\
\hline PREPARED BY I DATE & 2/ $/ 4 / 17 / 97$ & & & \\
\hline CHECKED BY I DATE & Z $4 / 17 / 97$ & & & \\
\hline
\end{tabular}


CLIENT: Duke Engineering \& Services Hanford

FILE NO: KH-8009-8-03

PROJECT: MCO Final Design

ICOM * COLLAR AT TOP EDGE OF PLUG (2" above bottom Edge) ****

NGEN, $2,9,262, \ldots, 2.00 \quad$ ! Nodes 271

FILL,262,271,2

/COM *** COLLAR AT BASE OF THREADS **

$N, 274, I R 3,152.00$

N.1000,IR2,152.00

ICOM TOP TO COLLAR (WELD CLOSURE)

$\mathrm{N}, 295, \mathrm{IR} 3,156.00$

FILL,274,295

NGEN,3,1,259,295,3,(OR2-IR2)/2

NGEN,3, 1,274,295,3,(OR2-IR3)/2

ICOM LOCKING \& LIFTING RING GEOMETRY

RING1 $=7.94$

RING2 $=9.376$

RING3 $=9.625$

RING4 $=10.19$

RING5 $=12.23$

LOCAL, 11,0,152.00 ! Local System $z=0$ at Base of Ring

CSYS, 11

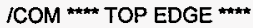

$N, 401, R I N G 1,6.13$

CSYS,O

$\mathrm{N}, 404,9.375,158.13$

FILL,401,404,,1

N,406,RING4,158.13

FILL,404,406,.,1 ! Top Edge

ICOM LIFTING SURFACE

CSYS, 11

$\mathrm{N}, 421$, RING1,5.13

$\mathrm{N}, 424, \mathrm{RING}, 5.13$

FILL, 421,424

$\mathrm{N}, 426, \mathrm{RING} 4,5.13$

FILL,424,426

FILL, 401,421,1, 10,6,1

N,431, RING1,6.13-1.56

N,434, RING2,6.13-1.56

FILL

ICOM BOLTING SURFACE *

N,441, RING1,4

N,444,RING3,4

FILL

$\mathrm{N}, 445,10.9375-.6875,4 \quad$ ! Inside Edge of Bolt Hole

$N, 447,10.9375+.6875,4$ ! Outside Edge of Bolt Hole

FILL

N,910,10.9375-.6875,4

$N, 911,10.9375+.6875,4$

N,448,RJNG5,4

CSYS, 0

$\mathrm{N}, 924,10.25,152.00$

! O.D of Ring

! Bolt Extension

REVISION

PREPARED BY IDATE I Double Nodes@ Bolt for Gap elements

CHECKED BY/DATE 
CLIENT: Duke Engineering \& Services Hanford

FILE NO: $\quad \mathrm{KH}-8009-8-03$

PROJECT: MCO Final Design DOC. NO.: HNF-SD-SNF-DR-003, Rev. 0, Appendix 5

$N, 925,11.625,152.00$

FILLL,910,924,6,2

FILL, $911,925,6,2$

$\mathrm{N}, 525,10.25,151.874 \quad$ ! Bottom of Bolt Extension

N, $527,11.625,151.874$

FILL

ICOM *** BOTTOM OF LIFTING/LOCKING RING ***

CSYS, 11

NGEN, $2,70,441,448,1,-4$ ! Bottom Surface of Lifting/Locking Ring

FILL,441,511,6,10,8,1 ! Fill in Lifting/Locking Ring

/COM

LOCAL, $20,0,158.13$

TYPE,2

PLUGR1 $=11.975$

PLUGR2 $=11.45$

PLUGR3 $=11.25$

PLUGR4=7.89

ICOM NODES AT PLUG AXIS (r=0)

$\mathrm{N}, 601$

$\mathrm{N}, 602,0,-1$

$\mathrm{N}, 603,0,-1.994$

$N, 606,0,-4.994$

FILL,603,606,2,604

$\mathrm{N}, 607,0,-6.25$

$\mathrm{N}, 610,0,-8.25$

FILL,607,610,2,608

$\mathrm{N}, 611,0,-8.75$

$\mathrm{N}, 613,0,-10.5$

FILL,611,613

ICOM NODAL GENERATION

NGEN, $2,20,601,613,1,0.8825$

NGEN, $2,20,621,633,1,0.8825$

NGEN $, 2,20,642,653,1,0.6875$

NGEN $, 2,20,662,673,1,0.6875$

NGEN, $2,20,683,693,1,0.4235$

NGEN $, 2,10,706,713,1,0.9515$

I Id Large Opening

! Id Medium Opening

! Id Small Opening

! Center of Opening

$N, 730,5.4665,-1.994$

$N, 736,5.4665,-4.994$

! Od Small Opening

FILL, $730,736,5,731$

$\mathrm{N}, 737,5.4665,-6.25$

$N, 740,5.4665,-8.25$

FILL, $737,740,2,738$

$N, 741,5.4665,-8.75$

$N, 743,5.4665,-10.5$

FILL, 741,743

$\mathrm{N}, 748,5.89,-1.0$

NGEN, $2,20,730,743,1,0.4235$

FILL, 748,750

$\mathrm{N}, 766,7.265,0$

NGEN, 2,20,748,763,1,1.375

FILL, 766,768

\begin{tabular}{|l|c|l|l|l|l|}
\hline REVISION & 0 & & & & PAGE 122 \\
PREPARED BY I DATE & OF 198 \\
\cline { 1 - 4 } CHECKED BY/ DATE & 4/17/97 & & & & \\
\hline
\end{tabular}


CLIENT: Duke Engineering \& Services Hanford

PROJECT: MCO Final Design
FILE NO: KH-8009-8-03

NGEN,3,20,766,768,1,0.3125

$N, 789,7.5775,-1.56$

$N, 796,7.5775,-5.56$

FILL, 789,796,6

NGEN, $2,20,789,796,1,0.3125$

NGEN, $3,20,777,783,1,0.3125$

ICOM UNDER LOCKING RING U

$\mathrm{N}, 824,8.5017,-6.25$

$\mathrm{N}, 827,8.5017,-8.25$

FILL

$\mathrm{N}, 828,8.5017,-8.75$

N,830,8.5017,-10.5

FIL.1.

NGEN, $3,7,824,830,1,0.5616$

NGEN, $2,7,838,844,1,0.625$

NGEN, $2,7,845,851,1,0.6875$ ! Under Bolt

$\mathrm{N}, 859,11.625,-6.25$

$\mathrm{N}, 860,11.625,-6.917$

$\mathrm{N}, 861,11.625,-7.584$

N,862,PLUGR2,-8.25

N,863,PLUGR2,-8.75

N,865,PLUGR3,-10.5

FILL,863,865,1

N,866,PLUGR1-0.288,-6.25

N,869,PLUGR1-0.288,-8.25

FILL, 866,869,2

N,870,PLUGR1-0.288,-8.476

NGEN, $2,5,866,870,1,0.288$

ICOM ${ }^{\star \star *}$ REFINING LIFTING EAR ***

CSYS, 0

$N, 877,9.53,158.13$

$\mathrm{N}, 889,9.53,157.63$

N,901,9.53,157.13

FILL,403,404,1,876

FILL,413,414,1,888

FILL,423,424,1,900

FILL,877,405,1,878

FILL,405,406,2,879,1

FILL,889,415,1,890

FILL,415,416,2,891,1

FILL, 404,414,1,881

FILL,877,889,1,882

FILL,878,890,1,883

FILL,405,415,1,884

FILL,879,891,1,885

FILL,880,892,1,886

FILL,406,416,1,887

FILL,889,901,1,894

FILL,414,424,1,893

FILL,901,425,1,902

FILL,890,902, 1,895

FILL,415,425, 1,895

FILL,425,426,2,903, 1

FILL,891,903,1,897

\begin{tabular}{|l|c|l|l|l|l|}
\hline REVISION & 0 & & & & PAGE 123 \\
PREPARED BY IDATE & OF 198 \\
\hline CHECKED BY I DATE & /N/17/97 & & & & \\
\hline
\end{tabular}


FILL, 892,904,1,898

FILL,416,426,1,899

FILL,424,434,1,907

FILL, $433,434,1,908$

FILL, 423,433,1,905

FILL, 905,907

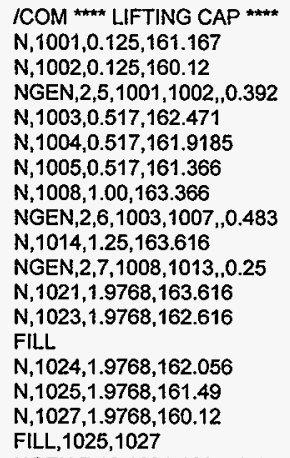

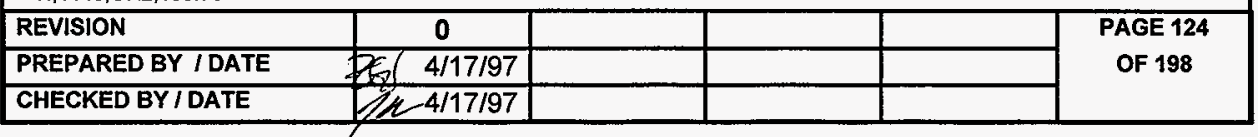


FILL

NGEN $, 3,3,1141,1143,,-0.47$

NGEN $, 2,3,1147,1150,,-0.53$

NGEN $, 2,3,1150,1152,,,-0.60$

NGEN $, 2,3,1153,1155,,-0.54$

NGEN, $2,3,1156,1158,,-0.38$

NGEN $, 2,3,1159,1161,,-0.41$

N,1168,RING3,160.032

$N, 1169,10.254,159.78$

$N, 1170,10.868,159.78$

$N, 1171,11.504,159.78$

$\mathrm{N}, 1172,10.868,159.31$

$\mathrm{N}, 1173,11.504,159.31$

$\mathrm{N}, 1174,11.24,158.84$

$\mathrm{N}, 1175,11.504,158.84$

N, $1176,11.504,158.31$

$N, 1177,11.71,157.71$

$N, 1178,11.896,157.17$

$N, 1179,12.027,156.79$

ICOM END LIFTING CAP

ICOM ** FILTER GUARD PLATE **

PLATE1 $=0.273$

PLATE2 $=0.6575$

PLATE3=1.357

PLATE4=10.25

PLATE5 $=11.25$

N,1200,PLATE4,146.78

N, 1202,PLATE5,146.78

FILL

NGEN,5,3,1200,1202,,,-0.85

NGEN,2,3,1212,1214,.,-0.25

N,1221,PLATE4,141.88

$N, 1222,10.75,141.88$

$\mathrm{N}, 1223,10.915,141.88$

FILL, 1215,1221,1,1218

FILL, 1223, 1217,1,1220

FILL,1216,1222,1,1219

$N, 1237,6.4375,143.38$

FILL, 1212, 1237,3,1225,4

$\mathrm{N}, 1249,3.578,143.38$

FILL, 1237, 1249,2, 1241,4

NGEN, $2,1,1225,1249,4,-0.25$

NGEN,2,2,1226,1250,4,,-1.25

FILL, 1226, 1228,1,1227, 7,4

$N, 1253,2.625,145.255$

$\mathrm{N}, 1254,2.625,145.005$

$N, 1256,2.625,143.38$

FILL, 1254,1256

$N, 1257,2.625,143.13$

N,1259,2.625,141.88

FILL, 1257,1259

NGEN,2, 10,1253, 1259,1,-0.5

NGEN, $2,10,1263,1269,1,-0.768$

$\mathrm{N}, 1283,0.6575,145.255$

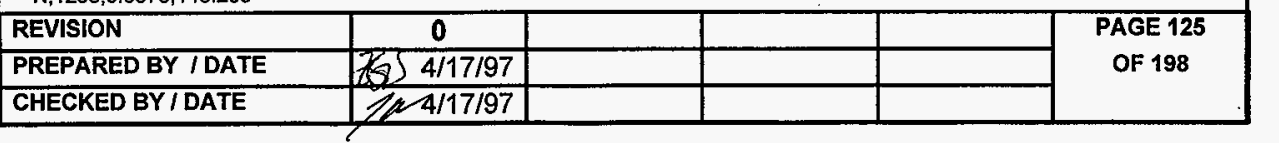


CLIENT: Duke Engineering \& Services Hanford

PROJECT: MCO Final Design DOC. NO.: HNF-SD-SNF-DR-003, Rev. 0, Appendix 5

\author{
$\mathrm{N}, 1284,0.6575,145.005$ \\ $\mathrm{N}, 1260,2.125,147.63$ \\ $\mathrm{N}, 1270,1.357,147.63$ \\ $\mathrm{N}, 1280,0.6575,147.63$ \\ $\mathrm{N}, 1290,0.273,147,63$ \\ NGEN,3,1,1260,1290,10, -0.5625
}

ICOM NODES FOR GAP ELEMENTS ATOP CAP

NGEN,2,1000,1014,.,,1.00

NGEN,2,1000,1021,1101,10,1.00

NGEN, $2,1000,1109,1112,3,1.00$

ICOM COUPLING NODES

ICOM BETWEEN LIFTING/LOCKING RING \& SHELL \#

$\mathrm{CP}, 1, \mathrm{UY}, 508,277$

! Start Threads

$C P, 2, U Y, 498,280$

$C P, 3, U Y, 488,283$

$C P, 4, U Y, 478,286$

$C P, 5, \cup Y, 468,289$

CP,6,UY,458,292

/COM BETWEEN BOLT \& LOCKING RING

$C P, 7, U Y, 445,910$

$C P, 8, U X, 445,910$

CP,, UY, 447,911

$C P, 10, \cup X, 447,911$

${ }^{*} \mathrm{DO}, \mathrm{I}, 1,7$

$C P, 10+1, U Y, 445+10 * 1,910+2 * 1$

"ENDDO

*DO,I,1,7

CP, $17+1, U Y, 447+10 * 1,911+2 * 1$

"ENDDO

${ }^{*} \mathrm{DO}, \mathrm{I}, 1,7$

$C P, 24+1, U X, 445+10^{*}|, 910+2 *|$

*ENDDO

*DO $, 1,1,7$

$\mathrm{CP}, 31+1, \mathrm{UX}, 447+10 * 1,911+2 * 1$

*ENDDO

NALL

EALL

ICOM ELEMENT GENERATION FOR SHELL *

TYPE, 1

MAT, 1

ICOM B* BOTTOM OF SHELL \#

$E, 1,2,22,21$

$E, 2,3,23,22$

EGEN,8,1,-1

$E, 10,11,30$

$E, 21,22,42,41$

$E, 22,23,43,42$

EGEN, 10,1,-1

$E, 11,31,30$

$E, 11,32,31$

\begin{tabular}{|l|c|l|l|l|l|}
\hline REVISION & 0 & & & & PAGE 126 \\
OF 198 \\
\hline CHEPARED BY IDATE & ChES $4 / 17 / 97$ & & & & \\
\hline
\end{tabular}


CLIENT: Duke Engineering \& Services Hanford

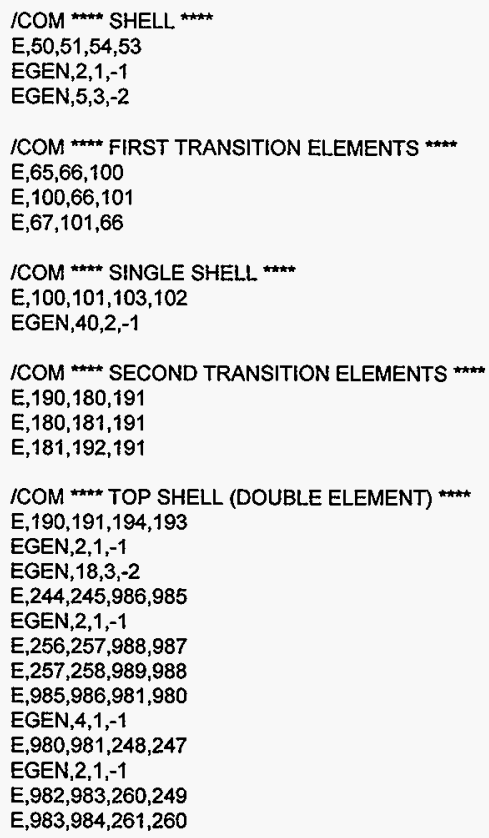

\begin{tabular}{|l|c|l|l|l|c|}
\hline REVISION & 0 & & & & PAGE 127 \\
PREPARED BY I DATE & OF 198 & \\
\hline CHECKED BY I DATE & OF $/ 17 / 97$ & & & & \\
\hline
\end{tabular}


PAASロNS

CLIENT: Duke Engineering \& Services Hanford

FILE NO: KH-8009-8-03

PROJECT: MCO Final Design

DOC. NO.: HNF-SD-SNF-DR-003, Rev. 0, Appendix 5

MAT, 1

$E, 411,412,402,401$

EGEN,2,1,-1

EGEN,2,10,-2

$E, 413,888,876,403$

E, $881,404,876$

E, 888,881,876

E,888,414,881

$E, 881,882,877,404$

$E, 414,889,882,881$

E,882,883,878,877

$E, 889,890,883,882$

$E, 883,884,405,878$

$E, 890,415,884,883$

E,884,885,879,405

$E, 415,891,885,884$

$E, 885,886,880,879$

$E, 891,892,886,885$

E,886,887,406,880

E, $892,416,887,886$

$E, 423,900,888,413$

E,893,414,888

$E, 900,893,888$

E, $900,424,893$

$E, 893,894,889,414$

$E, 424,901,894,893$

$E, 894,895,890,889$

$E, 901,902,895,894$

E,895,896,415,890

E,902,425,896,895

$E, 896,897,891,415$

$E, 425,903,897,896$

E,897,898,892,891

$E, 903,904,898,897$

$E, 898,899,416,892$

$E, 904,426,899,898$

$E, 431,432,422,421$

$E, 905,423,422$

E,432,905,422

$E, 432,433,905$

E, $905,906,900,423$

$E, 433,908,906,905$

$E, 906,907,424,900$

$E, 908,434,907,906$

$E, 441,442,432,431$

EGEN,2,1,-1

E,443,908,433

E,443,444,434,908

$E, 451,452,442,441$

EGEN, $3,1,-1$

EGEN, $7,10,-3$

$E, 454,912,910,444$

$E, 464,914,912,454$

$E, 474,916,914,464$

E,484,918,916,474

E,494,920,918,484

REVISION

PREPARED BY I DATE

CHECKED BY/DATE

\begin{tabular}{|l|l|l|l|}
\hline 0 & & & \\
\hline $4 / 17 / 97$ & & & \\
\hline $4 / 17 / 97$ & & & \\
\hline
\end{tabular}


CLIENT: Duke Engineering \& Services Hanford

$E, 504,922,920,494$

$E, 514,924,922,504$

$E, 458,448,911,913$

$E, 468,458,913,915$

$E, 478,468,915,917$

$E, 488,478,917,919$

$E, 498,488,919,921$

$E, 508,498,921,923$

$E, 518,508,923,925$

/COM BOLT

TYPE, 5

MAT, 5

$\mathrm{E}, 455,456,446,445$

EGEN,8,10,-1

$E, 456,457,447,446$

EGEN,8,10,-1

ICOM END OF LOCKING/LIFTING RING

ICOM SHIELD PLUG ELEMENTS

TYPE, 2

MAT, 1

$E, 602,622,621,601$

EGEN, 11, 1,-1

EGEN, $2,20,-11$

$E, 613,1290,612$

E, $1290,1280,632,612$

E, 1280,633,632

$E, 633,1270,632$

$E, 1270,652,632$

$E, 1270,653,652$

$E, 643,663,662,642$

EGEN,10,1,-1

EGEN, $2,20,-10$

$E, 673,693,692,672$

EGEN, 2,20,-10

$\mathrm{E}, 653,1260,652$

$E, 1260,672,652$

$E, 1260,673,672$

$E, 707,717,716,706$

EGEN,7,1,-1

$E, 717,737,736,716$

EGEN $7,1,-1$

$E, 731,751,750,730$

EGEN,13,1,-1

E, $749,769,768,748$

EGEN,15,1,-1

$E, 767,787,786,766$

EGEN,17,1,-1

EGEN, 2,20,-17

$E, 818,825,824,817$

EGEN,6,1,-1

EGEN,5,7,-6

$E, 853,860,859,852$

EGEN,6,1,-1

$E, 860,867,866,859$

\begin{tabular}{|l|c|l|l|l|}
\hline REVISION & 0 & & & \\
\hline PREPARED BY I DATE & $4 / 17 / 97$ & & & \\
\hline CHECKED BY I DATE & $/ 24 / 17 / 97$ & & & \\
\hline
\end{tabular}


CLIENT: Duke Engineering \& Services Hanford

EGEN, $3,1,-1$

$E, 867,872,871,866$

EGEN, 4,1,-1

ICOM END OF SHIELD PLUG

ICOM

TYPE, 1

MAT,1

E,1001,1006,1005

$E, 1002,1007,1006,1001$

$E, 1004,1010,1009,1003$

EGEN,4,1,-1

$E, 1009,1016,1015,1008$

EGEN,5,1,-1

EGEN, 2,7,-5

$E, 1015,1022,1021,1014$

$E, 1022,1032,1031,1021$

EGEN,6,1,-1

EGEN,8, 10,-6

$E, 1102,1110,1109,1101$

EGEN,2,1,-1

E, 1110,1113,1112,1109

EGEN,2,1,-1

E, 1105,1120,1104

$E, 1106,1121,1120,1105$

EGEN,2,1,-1

E,1121,1124,1123,1120

EGEN,2,1,-1

EGEN,6,3,-2

$E, 1141,1142,1137,1134$

E, 1142,1143,1140,1137

$E, 1144,1145,1142,1141$

EGEN, 2,1,-1

EGEN,7,3,-2

$E, 295,296,1163,1162$

$E, 296,297,1164,1163$

E,1108,1107,1097

$E, 1108,1168,1122,1107$

$E, 1168,1169,1125,1122$

$E, 1169,1170,1128,1125$

E,1170,1171,1131,1128

$E, 1171,1141,1134,1131$

E,1172,1170,1169

$E_{1} 1172,1173,1171,1170$

$E, 1173,1144,1141,1171$

$E, 1174,1175,1173,1172$

E,1175,1147,1144,1173

E, 1176,1175,1174

E,1176,1150,1147,1175

E, $1177,1153,1150,1176$

E,1178,1156,1153,1177

$E, 1179,1159,1156,1178$

$E, 1162,1159,1179$

/COM END OF LIFTING CAP

ICOM FILTER GUARD PLATE

\begin{tabular}{|l|c|l|l|l|}
\hline REVISION & 0 & & & \\
\hline PREPARED BY IDATE & ZKS 4/17/97 & & & \\
\hline CHECKED BY I DATE & / $/ 4 / 17 / 97$ & & & \\
\hline
\end{tabular}


CLIENT: Duke Engineering \& Services Hanford

FILE NO: KH-8009-8-03

PROJECT: MCO Final Design

DOC. NO.: HNF-SD-SNF-DR-003, Rev. 0, Appendix 5

\author{
$E, 1200,1201,858,851$ \\ $E, 1201,1202,865,858$ \\ $E, 1203,1204,1201,1200$ \\ EGEN,2,1,-1 \\ EGEN,6,3,-2 \\ E. $1221,1222,1219,1218$ \\ E, 1222,1223,1220,1219 \\ E, 1226,1215,1212,1225 \\ E,1227,1218,1215,1226 \\ $\mathrm{E}, 1228,1221,1218,1227$ \\ $E, 1230,1226,1225,1229$ \\ EGEN, 3,1,-1 \\ EGEN, $6,4,-3$ \\ E, $1257,1250,1249,1256$ \\ EGEN, $3,1,-1$ \\ $E, 1264,1254,1253,1263$ \\ EGEN,6, 1,-1 \\ $E, 1271,1261,1260,1270$ \\ EGEN, $9,1,-1$ \\ $E, 1281,1271,1270,1280$ \\ EGEN,4,1,-1 \\ $E, 1291,1281,1280,1290$ \\ EGEN,2,1,-1
}

/COM CONTACT ELEMENTS

/COM BETWEEN L.OCKING RING \& SHIELD PLUG *\#*

TYPE, 4

REAL, 4

$E, 806,401$

$\mathrm{E}, 807,411$

$E, 808,421$

$E, 809,431$

$E, 810,441$

$E, 811,451$

$E, 812,461$

$E, 813,471$

$E, 814,481$

$E, 815,491$

$\mathrm{E}, 816,501$

$E, 817,511$

/COM * ה" BETWEEN SHIELD PLUG \& BOTTOM OF BOLT

REAL,5

$E, 845,525$

$E, 852,526$

$E, 859,527$

ICOM ${ }^{\star \star \star \star ~ B E T W E E N ~ S H I E L D ~ P L U G ~ \& ~ S H E L L ~(A B O V E ~ S E A L) ~}$

REAL, 4

$E, 871,271$

$E, 872,268$

$E, 873,265$

$E, 874,262$

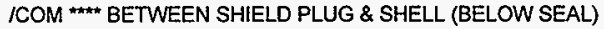

$E, 863,980$

REVISION

PREPARED BY I DATE CHECKED BYIDATE 
CLIENT: Duke Engineering \& Services Hanford

ICOM **** BETWEEN SHIELD PLUG AND SEAL. LIP

TYPE,4

REAL, 6

$E, 247,862$

$E, 248,870$

$E, 249,875$

TYPE, 4

REAL, 8

$E, 247,862$

$E, 248,869$

ICOM ABOVE LIFTING CAP \#*

TYPE, 4

REAL, 7

$E, 1014,2014$

$E, 1021,2021$

EGEN,9,10,-1

$E, 1109,2109$

EGEN,2,3,-1

/COM END GAP ELEMENTS

ICOM BOUNDARY CONDITIONS

CSYS, 0

NSEL, S, LOC, $X, O$

NSEL, R, LOC, Y, $-1.33,163.50$

$D, A L L, U X, 0$

NALL

EALL.

NSEL,S,NODE, , 1002

$D, A L L, U X, 0$

NALL

EALL

NSEL,S,NODE,,2021,2101,10

NSEL, A,NODE,,2109,2112,3

NSEL,A,NODE,,2014

D,ALL,ALL,O

EALL

NALL

SAVE

ICOM LOAD 1: 150 PSI INTERNAL. PRESSURE

NSEL,S,LOC,X,0,1.26

! Bottom Plate

NSEL,R,LOC,Y,-0.45,-0.43

SF,ALL,PRES, 150

NALL

EALL

NSEL,S,LOC,X,1.24,2.14

NSEL,R,LOC, Y,-0.45, 0.45

SF,ALL,PRES, 150

NALL

EALL

NSEL,S,LOC, $X, 2.12,11.51$

NSEL, R,LOC, Y, $0.43,0.45$

$S F, A L L, P R E S, 150$

REVISION

PREPARED BY / DATE

CHECKED BYIDATE 
CLIENT: Duke Engineering \& Services Hanford

PROJECT: MCO Final Design DOC. NO.: HNF-SD-SNF-DR-003, Rev. 0, Appendix 5

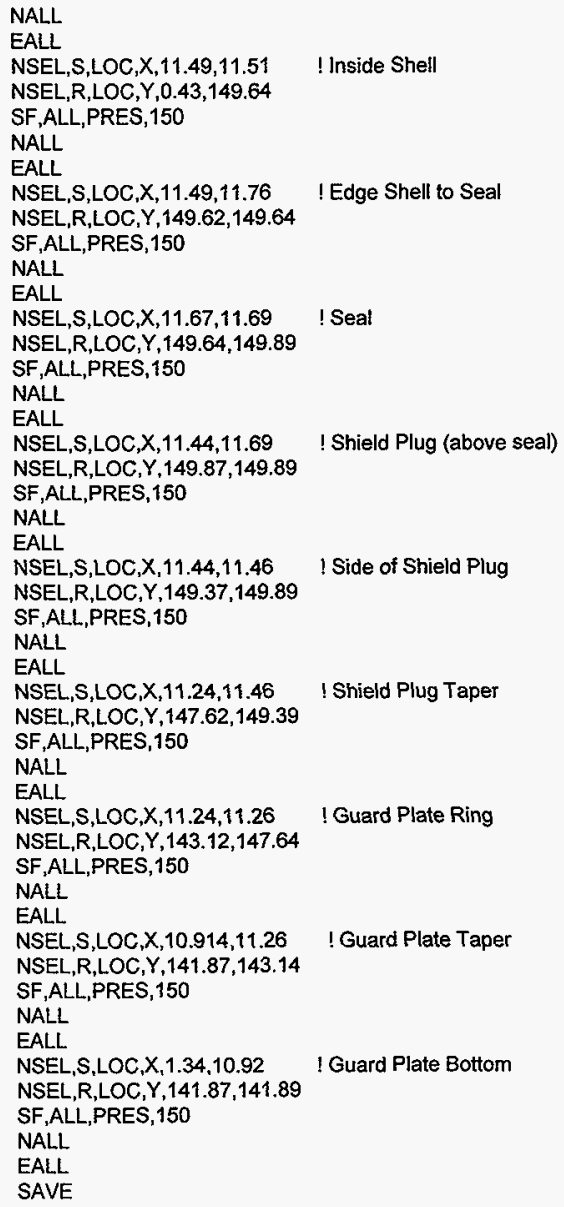


CLIENT: Duke Engineering \& Services Hanford

PROJECT: MCO Final Design

FILE NO: KH-8009-8-03

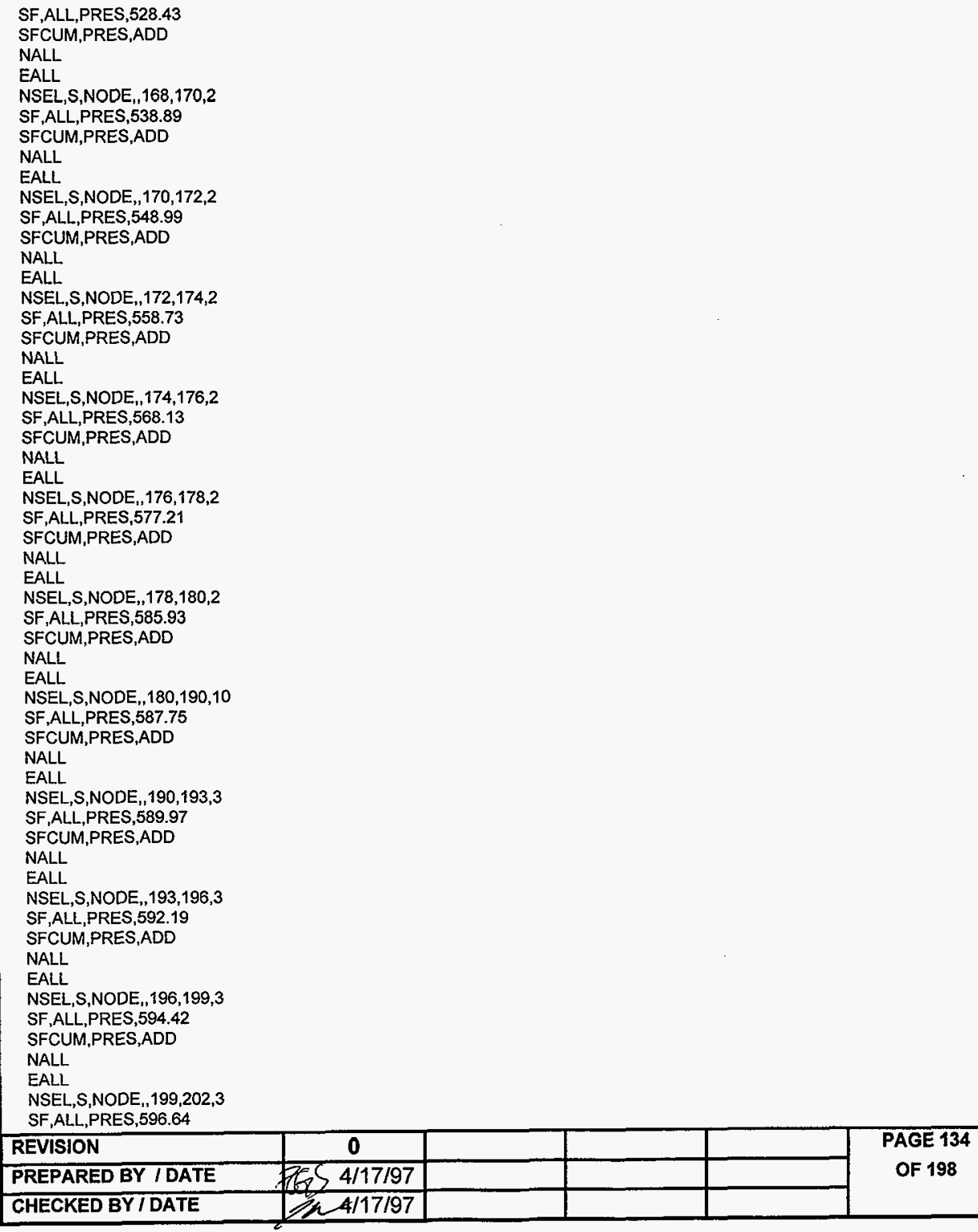

\begin{tabular}{|l|c|l|l|l|}
\hline REVISION & 0 & & & \\
\hline PREPARED BY I DATE & S 4/17/97 & & & \\
\hline CHECKED BY I DATE & \multicolumn{1}{|c|}{$4 / 17 / 97$} & & & \\
\hline
\end{tabular}


CLIENT: Duke Engineering \& Services Hanford FILE NO: $\mathrm{KH}-8009-8-03$

PROJECT: MCO Final Design DOC. NO.: HNF-SD-SNF-DR-003, Rev. 0, Appendix 5

SFCUM,PRES,ADD

NALL

EALL

NSEL,S,NODE,202,205,3

$\mathrm{SF}_{1} \mathrm{ALL}, \mathrm{PRES}, 598.90$

SFCUM,PRES,ADD

NALL

EALL

NSEL,S,NODE, ,205,208,3

SF,ALL,PRES,601.13

SFCUM,PRES,ADD

NALL

EALL

NSEL,S,NODE, ,208,211,3

SF,ALL,PRES, 603.35

SFCUM,PRES,ADD

NALL

EALL

NSEL,S,NODE, $, 211,214,3$

SF,ALL,PRES,605.60

SFCUM,PRES,ADD

NALL

EALL

NSEL,S,LOC,X,1.3565,10.9155

NSEL,R,LOC,Y,141.87,141.89

SF,ALL,PRES,1244.82 ! Vertical Pressure from 5 Baskets

SFCUM,PRES,ADD

! at 28 g's

NALL

EALL

SAVE

ICOM LOAD 3: APPLYING 28g ACCELERATION

ACEL, -28 ! Impose $28 \mathrm{~g}$ Acceleration Top Drop

SAVE

FINI

ICOM SOLUTION PHASE

ISOLUTION

SOLVE

SAVE

FINI

ICOM

IPOST1

SET,LAST

TYPE,ALL,HIDC

IGLINE,ALL,0

RSYS,O

PLNSOL,S,INT

IDSCALE, 20

IREPLOT

NSEL, $S, L O C, X, 11.49,11.51$

NSEL, $R, L O C, Y,-0.33,149.63$

PRNS, U,X

NALL

EALL

REVISION

PREPARED BY IDATE

CHECKED BYI DATE

\begin{tabular}{|c|c|c|c|}
\hline 0 & & & \\
\hline $7624 / 17 / 97$ & & & \\
\hline PN 4/17/97 & & & \\
\hline
\end{tabular}


NSEL,S,LOC,X, $1.356,11.26$

NSEL,R,LOC, $Y, 141.87,143.39$

PRNS,U,Y

NALL

EALL

LPATH, 1,41

PRSECT

LPATH,4,44

PRSECT

LPATH, 6,46

PRSECT

LPATH, 9,49

PRSECT

LPATH, 10,50

PRSECT

LPATH,50,52

PRSECT

LPATH,53,55

PRSECT

LPATH,62,64

PRSECT

LPATH,65,67

PRSECT

LPATH, 100,101

PRSECT

LPATH, 122,123

PRSECT

LPATH,134,135

PRSECT

LPATH,156,157

PRSECT

LPATH, 170,171

PRSECT

LPATH,180,181

PRSECT

LPATH,202,204

PRSECT

LPATH,235,237

PRSECT

LPATH,985,989

PRSECT

LPATH,262,264

PRSECT

LPATH,277,279

PRSECT

LPATH,292,294

PRSECT

LPATH,601,641

PRSECT

LPATH, 601,613

PRSECT

LPATH, 603,703

PRSECT

LPATH,606,706

PRSECT

LPATH,766,806

REVISION

PREPARED BY / DATE

CHECKED BY / DATE

\begin{tabular}{|c|l|l|l|}
\hline 0 & & & \\
\hline $\operatorname{lic} 4 / 17 / 97$ & & & \\
\hline $24 / 17 / 97$ & & & \\
\hline
\end{tabular}


CLIENT: Duke Engineering \& Services Hanford FILE NO: KH-8009-8-03

PROJECT: MCO Final Design

PRSECT

LPATH, 748,808

PRSECT

LPATH,736,815

PRSECT

LPATH, 862,873

PRSECT

LPATH, 869,874

PRSECT

LPATH $, 870,875$

PRSECT

LPATH, 1200,1202

PRSECT

LPATH,1209,1211

PRSECT

LPATH, 1229, 1232

PRSECT

LPATH,1237,1240

PRSECT

LPATH, 1245, 1248

PRSECT

LPATH, 1284, 1263

PRSECT

L.PATH, 1261,1291

PRSECT

LPATH, $\mathbf{4 3 1 , 4 3 4}$

PRSECT

LPATH,406,426

PRSECT

LPATH, 404,424

PRSECT

LPATH,1120,1097

PRSECT

LPATH,1120,1168

PRSECT

LPATH, 1174,1149

PRSECT

LPATH,1176,1152

PRSECT

LPATH,1177,1155

PRSECT

LPATH,1178,1158

PRSECT

LPATH,1179,1161

PRSECT

LPATH,1162,1164

PRSECT

LPATH,295,297

PRSECT

LPATH,1021,1027

PRSECT

LPATH, 1071,1077

PRSECT

LPATH,1138,1169

PRSECT

SAVE

REVISION

PREPARED BY I DATE

CHECKED BYIDATE 
CLIENT: $\quad$ Duke Engineering \& Services Hanford

FILE NO: KH-8009-8-03

PROJECT: MCO Final Design DOC. NO.: HNF-SD-SNF-DR-003, Rev. 0, Appendix 5

\section{COMPUTER RUN COVER SHEET}

Project Number:

$\mathrm{KH}-8009-8$

Computer Code:

ANSYS $B-P C$

Software Version:

$5.0 \mathrm{~A}$

Computer System:

MS-DOS, Pentium® Processor

Computer Run File Number:

KH-8009-8-03

Unique Computer Run Filename:

TEDMC.out

Run Description:

MCO Top End Drop with Lifting Cap

Run Date / Time:

30 March $1997 \quad$ 11:16:18 AM

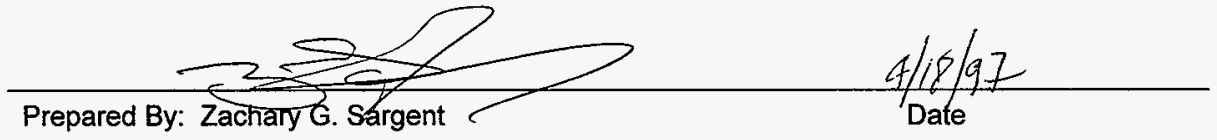

Checked By: Joe Nichols 
CLIENT: Duke Engineering \& Services Hanford FILE NO: KH-8009-8-03

PROJECT: MCO Final Design DOC. NO.: HNF-SD-SNF-DR-003, Rev. 0, Appendix 5

\section{COMPUTER RUN COVER SHEET}

Project Number:

Computer Code:

Software Version:

Computer System:

Computer Run File Number:

Unique Computer Run Filename:

Run Description:

Creation Date / Time:
$\mathrm{KH}-8009-8$

ANSYS $(8)-P C$

$5.0 \mathrm{~A}$

MS-DOS, Pentium ® Processor

$\mathrm{KH}-8009-8-03$

TED.inp

MCO Top End Drop without Lifting Cap

30 March $1997 \quad$ 10:54:30 AM

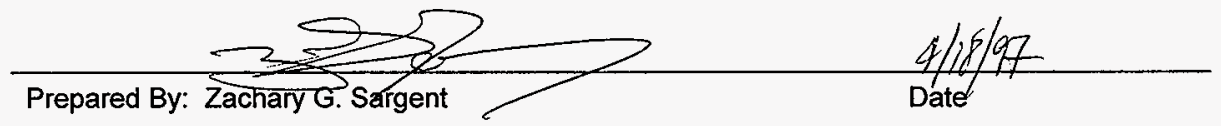

Checked By: Joe Nichols

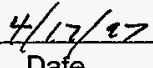

Date 


\section{EDASENS}

CLIENT: Duke Engineering \& Services Hanford

FILE NO: $\quad \mathrm{KH}-8009-8-03$

PROJECT: MCO Final Design DOC. NO.: HNF-SD-SNF-DR-003, Rev. 0, Appendix 5

\section{LISTING OF TED.INP FILE}

/BATCH,LIST

IFILENAM,TED

/PREP7

TITLE,MCO DESIGN- 132 DEGREES C, 150 psi, $28 \mathrm{~g}$ TOP END DROP W/O CAP

TREF,70

TUNIF, 270

ETAN $=0.006 \quad$ ! Tangent modulus

/COM ELEMENT TYPES

ET, $1,42, \ldots 1$ ! Shell

ET $, 2,42, \ldots 1$ ! Shield Plug

ET, $3,42,, 11$ ! Lifting \& Locking Ring

ET, 4,12 ! Gap Elements Between Shield Plug \& Shell

KEYOPT, 4,7,1

ET, $5,42, \ldots 1$ ! Boit

ICOM REAL CONSTANTS FOR GAP ELEMENTS

$\mathrm{R}, 4,-90,1.0 \mathrm{E} 8,-0.06,3.0$ ! Shell/Shield Plug, Initially Open .06"

R,5,0,1.0E8,2.95e-03 IL. Ring/Shield Plug, Under Bolt, Preloaded

$R, 6,0,1.0 \mathrm{E} 8,0,2.0$ ! Sealing Surface, closed

$\mathrm{R}, 7,0,1.0 \mathrm{E} 8,0,1.0$ : Anchoring for drop, closed

$\mathrm{R}, 8,0,2.42 \mathrm{e} 7,2.0$ ! Seal Spring, Max Stiffness

/COM

MP,DENS, $1,490 / 1728$ ! 304L SS

$M P, N U X Y, 1,0.3$

MP,DENS,5,490/1728 ! SA193 Grade B8M

MP,NUXY, $5,0.3$

TB,BKIN, 1,6 ! Yield Stress \& Tangent Moduli v. Temp.

TBTEMP, 100

TBDATA, 1,25000,ETAN*28.1E+06

TBTEMP, 200

TBDATA, 1,21300,ETAN*27.6E+06

TBTEMP, 300

TBDATA 1,19100, ETAN $27.0 E+06$

TBTEMP, 400

TBDATA 1,17500, ETAN $26.5 E+06$

TBTEMP, 500

TBDATA, 1,16300, ETAN*25.8E+06

TBTEMP,600

TBDATA, $1,15500, E T A N * 25.3 E+06$

ICOM ** DEFINING TEMPERATURES FOR MPDATA *\#*

MPTEMP, $1,70,100,200,300,400,500$

MPTEMP, $7,600,650,700,750$

/COM DEFINING ELASTIC MODULI FOR 304L \& SA-193

MPDATA, EX, 1, 1,28.3e+06,28.1e+06,27.6e+06,27.0e+06,26.5e+06,25.8e+06

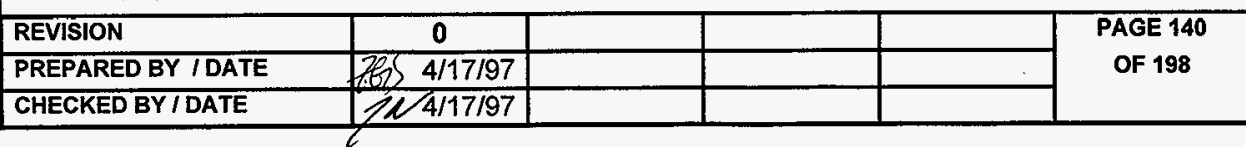


CLIENT: Duke Engineering \& Services Hanford

FILE NO: $\quad \mathrm{KH}-8009-8-03$

PROJECT: MCO Final Design

DOC. NO.: HNF-SD-SNF-DR-003, Rev. 0, Appendix 5

MPDATA,EX, 1,7,25.3e+06,25.1e+06,24.8e+06,24.5e+06

ICOM ! SA-193

MPDATA,EX,5,1,28.3e+06,28.1e+06,27.6e+06,27.0e+06,26.5e+06,25.8e+06

MPDATA,EX,5,7,25.3e+06,25.1e+06,24.8e+06,24.5e+06

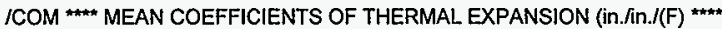

I SA240 Gr 304L

MPDATA,ALPX,1,1,0,8.55e-06,8.79e-06,9.00e-06,9.19e-06,9.37e-06

MPDATA,ALPX,1,7,9.53e-06,9.61e-06,9.69e-06,9.76e-06

! SA193 Gr B8M

MPDATA,ALPX,5, 1,0,8.54e-06,8.76e-06,8.97e-06,9.21e-06,9.42e-06

MPDATA,ALPX,5,7,9.60e-06,9.69e-06,9.76e-06,9.81e-06

ICOM SHELL GEOMETRY

IR=11.5 ! Internal Shell Radius @ Bottom

$\mathrm{OR}=12.000$

IR2 = 12.02

1 Shell Outside Radius @ Bottom

OR2 $=12.625$

! Inside Radius at Collar Sealing Surface

! Outside Radius at Collar Sealing Surface

IR3 $=12.25$

! Inside Radius at Collar-Lifting Ring Weld

/COM BOTTOM COVER PLATE [DWG SK-2-300378]

$\mathrm{N}, 1,-1.32$

! Row 1

$\mathrm{N}, 2,1.25,-1.32$

$\mathrm{N}, 3,2.13,-1.32$

$N, 10,11.423,-1.32$

FILL

$\mathrm{N}, 41,0.00,-0.44$

! Row 3

$\mathrm{N}, 42,1,25,-0.44$

$\mathrm{N}, 43,2.13,0.44$

$\mathrm{N}, 50, \mathrm{IR}, 0.44$

FILL, 43,50

$\mathrm{N}, 52, \mathrm{OR}, 0.44$

FILL, 50,52

FILL, 1,41,1,21,1,10 ! Middle Row

FILL, $10,50,1,30$

$\mathrm{N}, 32,12,-0.32$

FILL, 30,32

FILL, 10,32,1,11

$\mathrm{N}, 53, \mathrm{IR}, 1.17$

N.55,OR,1.17 IShell StubMeld

FILL, 53,55

/COM SHELL [DWGS SK-2-300379 \& SK-2-300461]

$\mathrm{N}, 65, \mathrm{IR}, 6.68$

$\mathrm{N}, 67, \mathrm{OR}, 6.68$

FILL

FILL, $53,65,3,3,3,1$

ICOM *** SINGLE ROW SHELL ***

$\mathrm{N}, 100, \mathbb{R}, 7.18 \quad$ ! Inside

$\mathrm{N}, 140, \mathrm{IR}, 71,68$

$\mathrm{N}, 180, \mathrm{IR}, 136.68$

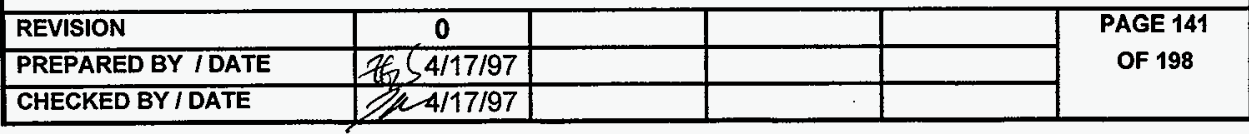


N, 101,OR,7.18

$\mathrm{N}, 141, \mathrm{OR}, 71.68$

$\mathrm{N}, 181, \mathrm{OR}, \mathbf{1 3 6 . 6 8}$

FILL,100,140,20,2,2,1,2.0

FILL, 140,180,19,2,2,1,.5

ICOM DOUBLE ROW SHELL

N,190,IR,137.18 I Transition to Double Row

$\mathrm{N}, 192, \mathrm{OR}, 137.18$

FILL

ICOM BASE OF CASK THROAT-ELEVATION: 138 INCHES

N,217,IR,142.68 ! Transition to Double Row

$N, 219, O R, 142.68$

FILL

FILL, 190,217,8, 3,3,1 IVertical Fill

ICOM BOTTOM OF COLLAR TRANSITION

$N, 235, I R, 146.06$ ! Start of Transition to Large O.D \&

$\mathrm{N}, 237, \mathrm{OR}, 146.06$ ! Assumed Location of Shield Plug Taper

FILL

$\mathrm{N}, 238, I R, 146.68$

$\mathrm{N}, 240, \mathrm{OR}, 146.68$

FILL

! Horizontal Fill

FILL,217,235,5,3,3,1 ! Vertical Fill

ICOM TOP OF COLLAR TRANSITION

$\mathrm{N}, 241, \mathrm{IR}, 147.31$ ! End of Transition to Large O.D \&

N,243,OR, 147.31 ! Assumed Location of Shield Plug Taper

FILL ! Horizontal Fill

NGEN, $2,3,241,243,1,0.75$

ICOM *** COLLAR SEALING SURFACE

$\mathrm{N}, 247, \mathrm{IR}, 149.63$

$N, 249, I R 2,149.63$

FILL

! Inside Radius of Sealing Surface

! Outside Radius at Sealing Surface

! Horizontal Fill

ICOM THICK WALL AT COLLAR TRANSITION

NGEN, 2, 10,240,249,3

! Nodes 250-259 Coincident w/240-249 (by 3)

N,255,OR2, 147.31

N,261,OR2,149.63

! Outside Surface

! Outside Surface

N,258,OR2,148.06

$\mathrm{N}, 980, \mathrm{IR}, 149.38$

N,981,11.755,149.38

N,982,IR2, 149.38

N,983,12.317,149.38

N,984, OR2, 149.38

N,990,OR2, 146.68

FILL,240,990,1,251

NGEN, $2,5,980,984,1,-0.66$

FILL,246,258,1,257

FILL,253,255,1, 1,3,3

FILL,237,990,1,991

ICOM COLLAR AT BOTTOM EDGE OF PLUG (.155" above Sealing Surface) NGEN $, 2,3,259, \ldots .245$ ! Nodes 262

\begin{tabular}{|l|c|l|l|l|}
\hline REVISION & 0 & & & \\
\hline PREPARED BY I DATE & $\operatorname{ZfS}$ 4/17/97 & & & \\
\hline CHECKED BY IDATE & N 4/17/97 & & & \\
\hline
\end{tabular}


CLIENT: Duke Engineering \& Services Hanford

\section{PROJECT: MCO Final Design

/COM COLLAR AT TOP EDGE OF PLUG (2" above bottom Edge)

NGEN,2,9,262,,,2.00 ! Nodes 271

FILL,262,271,2

ICOM COLLAR AT BASE OF THREADS $* \cdots$

$\mathrm{N}, 274, \mathrm{IR} 3,152.00$

$N, 1000$, IR2, 152.00

ICOM *** TOP TO COLLAR (WELD CLOSURE) ***

$\mathrm{N}, 295, \mathrm{IR} 3,156.00$

FILL,274,295

NGEN,3,1,259,295,3,(OR2-IR2)/2

NGEN,3,1,274,295,3,(OR2-IR3)/2

ICOM LOCKING \& LIFTING RING GEOMETRY

RING1 $=7.94$

RING2 $=9.375$

RING3 $=9.625$

RING4 $=10.19$

RING5 $=12.23$

LOCAL, 11,0,152.00 ! Local System $z=0$ at Base of Ring

CSYS, 11

/COM *** TOP EDGE ***

$\mathrm{N}, 401, \mathrm{RING} 1,6.13$

CSYS,O

$\mathrm{N}, 404,9.375,158.13$

FILL, $401,404,, 1$

$\mathrm{N}, 406, \mathrm{RING4}, 158.13$

Fil. $, 404,406,, 1$ ! Top Edge

ICOM "*IFTING SURFACE

CSYS, 11

N,421, RING1,5.13

$\mathrm{N}, 424, \mathrm{RING}, 5.13$

FILL, 421,424

N,426, RING4,5.13

FILL,424,426

FILL,401,421,1,10,6,1

N,431,RING1,6.13-1.56

N,434,RING2,6.13-1.56

FILL

ICOM *\# BOLTING SURFACE *

$\mathrm{N}, 441, \mathrm{RING} 1,4$

$\mathrm{N}, 444, \mathrm{RING} 3,4$

FILL

N,445, 10.9375- 6875,4 ! Inside Edge of Boit Hole

$\mathrm{N}, 447,10.9375+.6875,4 \quad$ ! Outside Edge of Bolt Hole

FILL

$N, 910,10.9375-.6875,4$

$N, 911,10.9375+.6875,4$

N,448,RING5,4

CSYS, 0

$\mathrm{N}, 924,10.25,152.00$

! O.D of Ring

! Bolt Extension

! Double Nodes @ Bolt for Gap elements

\begin{tabular}{|l|c|l|l|l|}
\hline REVISION & 0 & & & \\
\hline PREPARED BY I DATE & TS 4/17/97 & & & \\
\hline CHECKED BY I DATE & $2 \mu 4 / 17 / 97$ & & & \\
\hline
\end{tabular}


CLIENT: Duke Engineering \& Services Hanford

FILE NO: $\quad \mathrm{KH}-8009-8-03$

PROJECT: MCO Final Design

$N, 925,11.625,152.00$

FILL, $910,924,6,2$

FILL, $911,925,6,2$

$\mathrm{N}, 525,10.25,151.874 \quad$ ! Bottom of Bolt Extension

$N, 527,11.625,151.874$

FILL

ICOM BOTTOM OF LIFTINGLOCKING RING

CSYS, 11

NGEN $, 2,70,441,448,1,-4$ ! Bottom Surface of Lifting/Locking Ring

FILL,441,511,6,10,8,1 ! Fill in Lifting/Locking Ring

ICOM SHIELD PLUG (offset y by 158.25)

LOCAL, 20,0,158.13

TYPE, 2

PLUGR1=11.975

PLUGR2 $=11.45$

PLUGR3=11.25

PLUGR4=7.89

ICOM NODES AT PLUG AXIS $(r=0) \cdots$

$\mathrm{N}, 601$

$\mathrm{N}, 602,0,-1$

$\mathrm{N}, 603,0,-1.994$

$\mathrm{N}, 606,0,-4.994$

FILL,603,606,2,604

$\mathrm{N}, 607,0,-6.25$

$N, 610,0,-8.25$

FILL,607,610,2,608

$\mathrm{N}, 611,0,-8.75$

$\mathrm{N}, 613,0,-10.5$

FILL, 611,613

ICOM NODAL GENERATION

NGEN, 2,20,601,613,1,0.8825

NGEN $, 2,20,621,633,1,0.8825$

NGEN, $2,20,642,653,1,0.6875$

NGEN, $2,20,662,673,1,0.6875$

NGEN, $2,20,683,693,1,0.4235$

NGEN, $2,10,706,713,1,0.9515$

! Id Large Opening

! Id Medium Opening

! Id Small Opening

! Center of Opening

$\mathrm{N}, 730,5.4665,-1.994$

$\mathrm{N}, 736,5.4665,-4.994$

! Od Small Opening

FILL,730,736,5,731

$N, 737,5.4665,-6.25$

$N, 740,5.4665,-8.25$

FILL,737,740,2,738

$\mathrm{N}, 741,5.4665,-8.75$

$\mathrm{N}, 743,5.4665,-10.5$

FILL, 741,743

$\mathrm{N}, 748,5.89,-1.0$

NGEN, $2,20,730,743,1,0.4235$

FILL, 748,750

$\mathrm{N}, 766,7.265,0$

NGEN,2,20,748,763,1,1.375

FILL, 766,768 
CLIENT: Duke Engineering \& Services Hanford

PROJECT: MCO Final Design DOC. NO.: HNF-SD-SNF-DR-003, Rev. 0, Appendix 5

FILE NO: $\quad \mathrm{KH}-8009-8-03$

NGEN, $3,20,766,768,1,0.3125$

$N, 789,7.5775,-1.56$

$\mathrm{N}, 796,7.5775,-5.56$

FILL,789,796,6

NGEN, $2,20,789,796,1,0.3125$

NGEN,3,20,777,783,1,0.3125

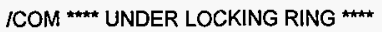

$\mathrm{N}, 824,8.5017,-6.25$

$\mathrm{N}, 827,8.5017,-8.25$

FILL

$\mathrm{N}, 828,8.5017,-8.75$

$\mathrm{N}, 830,8.5017,-10.5$

FILL

NGEN $, 3,7,824,830,1,0.5616$

NGEN $, 2,7,838,844,1,0.625$

NGEN, $2,7,845,851,1,0.6875$ ! Under Bolt

$\mathrm{N}, 859,11.625,-6.25$

$\mathrm{N}, 860,11.625,-6.917$

$\mathrm{N}, 861,11.625,-7.584$

N,862,PLUGR2,-8.25

N,863,PLUGR2,-8.75

N,865,PLUGR3,-10.5

FILL,863,865,1

N,866,PLUGR1-0.288,-6.25

N,869,PLUGR1-0.288,-8.25

FILL, $866,869,2$

N,870,PLUGR1-0.288,-8.476

NGEN, $2,5,866,870,1,0.288$

ICOM REFINING LIFTING EAR \#

CSYS, 0

$\mathrm{N}, 877,9.53,158.13$

$\mathrm{N}, 889,9.53,157.63$

$\mathrm{N}, 901,9.53,157.13$

FILL, $403,404,1,876$

FILL $, 413,414,1,888$

FILL, $423,424,1,900$

FILL, $877,405,1,878$

FILL, 405,406,2,879, 1

FILL, $889,415,1,890$

FILL, $415,416,2,891,1$

FiLL, 404,414,1,881

FILL, $877,889,1,882$

FILL, 878,890,1,883

FILL, $405, \mathbf{4 1 5}, 1,884$

FILL, $879,891,1,885$

FILL, $880,892,1,886$

FILL,406,416,1,887

FILL, 889,901, 1,894

FILL, 414,424,1,893

FILL, $901,425,1,902$

FILL,890,902, 1,895

FILL, $415,425,1,896$

FILL,425,426,2,903, 1

FILL, 891,903,1,897

\begin{tabular}{|l|c|l|l|l|l|}
\hline REVISION & 0 & & & \\
\cline { 1 - 4 } PREPARED BY IDATE & PAGE 4/17/97 & & & & OF 198 \\
\cline { 1 - 4 } CHECKED BYIDATE & /N 4/17/97 & & & & \\
\hline
\end{tabular}


CLIENT: Duke Engineering \& Services Hanford

PROJECT: MCO Final Design DOC. NO.: HNF-SD-SNF-DR-003, Rev. 0, Appendix 5

FILL, $892,904,1,898$

FILL,416,426,1,899

FILL,424,434,1,907

FILL,433,434, 1,908

FILL,423,433,1,905

FILL,905,907

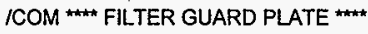

PLATE1 $=0.273$

PLATE2 $=0.6575$

PLATE3=1.357

PLATE4 $=10.25$

PLATE5 $=11.25$

N,1200,PLATE4, 146.78

$\mathrm{N}, 1202$, PLATE5, 146.78

FILL

NGEN $, 5,3,1200,1202, \ldots-0.85$

NGEN $2,3,1212,1214, \ldots,-0.25$

$\mathrm{N}, 1221, \mathrm{PLATE} 4,141.88$

$\mathrm{N}, 1222,10.75,141.88$

$\mathrm{N}, 1223,10.915,141.88$

FILL, 1215,1221,1,1218

FILL, 1223,1217,1,1220

FILL, 1216,1222,1,1219

$\mathrm{N}, 1237,6.4375,143.38$

FILL, 1212,1237,3,1225,4

$\mathrm{N}, 1249,3.578,143.38$

FILL, 1237, 1249,2,1241,4

NGEN, $2,1,1225,1249,4,-0.25$

NGEN,2,2, 1226, 1250,4,,-1.25

FILL, 1226,1228,1,1227,7,4

$\mathrm{N}, 1253,2.625,145.255$

$\mathrm{N}, 1254,2.625,145.005$

$\mathrm{N}, 1256,2.625,143.38$

FILL, 1254,1256

$N, 1257,2.625,143.13$

N, 1259,2.625,141.88

FILL, 1257,1259

NGEN, $2,10,1253,1259,1,-0.5$

NGEN,2,10,1263,1269,1,-0.768

$\mathrm{N}, 1283,0.6575,145.255$

$\mathrm{N}, 1284,0.6575,145.005$

$\mathrm{N}, 1260,2.125,147.63$

$\mathrm{N}, 1270,1.357,147.63$

$\mathrm{N}, 1280,0.6575,147.63$

$\mathrm{N}, 1290,0.273,147.63$

NGEN, $3,1,1260,1290,10,-0.5625$

ICOM NODES ABOVE PLUG \& RING

NSEL, $S, L O C, X, 0,10.20$

NSEL,R,LOC,Y,158.12,158.14

NGEN,2,2000,ALL,,,, 1.00

NALL.

ICOM COUPLING NODES

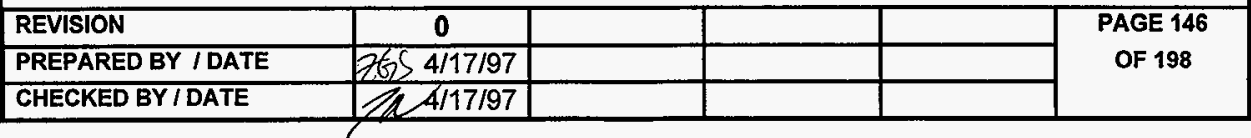


CLIENT: Duke Engineering \& Services Hanford

/COM BETWEEN LIFTING/LOCKING RING \& SHELL

$C P, 1, U Y, 508,277$

! Start Threads

$C P, 2, U Y, 498,280$

$C P, 3, U Y, 488,283$

$C P, 4, U Y, 478,286$

$\mathrm{CP}, 5, \mathrm{UY}, 468,289$

$\mathrm{CP}, 6, \mathrm{UY}, 458,292$

ICOM BETWEEN BOLT \& LOCKING RING

$\mathrm{CP}, 7, \mathrm{UY}, 445,910$

$\mathrm{CP}, 8, \mathrm{UX}, 445,910$

$\mathrm{CP}, 9, \mathrm{UY}, 447,911$

CP, 10,UX,447,911

${ }^{*} \mathrm{DO}, \mathrm{I}, 1,7$

CP, $10+1, \mathrm{UY}, 445+10 * 1,910+2 * 1$

*ENDDO

${ }^{*} \mathrm{DO}, \mathrm{I}, 1,7$

CP, $17+1, U Y, 447+10 * 1,911+2 * \mid$

*ENDDO

"DO, $1,1,7$

$\mathrm{CP}, 24+1, \mathrm{UX}, 445+10^{*}|, 910+2 *|$

*ENDDO

${ }^{*} D O, 1,1,7$

$C P, 31+1, U X, 447+10^{\star} \mid, 911+2 *$

*ENDDO

NALL

EALL

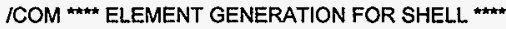

TYPE, 1

MAT, 1

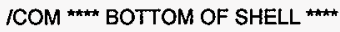

$E, 1,2,22,21$

$E, 2,3,23,22$

EGEN,8,1,-1

$E, 10,11,30$

$E, 21,22,42,41$

$E, 22,23,43,42$

EGEN, 10, $1,-1$

$E, 11,31,30$

$E, 11,32,31$

/COM SHELL

$E, 50,51,54,53$

EGEN,2,1,-1

EGEN,5,3,-2

ICOM \# FIRST TRANSITION ELEMENTS **

$\mathrm{E}, 65,66,100$

$E, 100,66,101$

$E, 67,101,66$

ICOM SINGLE SHELL

E.100,101,103,102

EGEN,40,2,-1

REVISION

PREPARED BY IDATE

CHECKED BYI DATE 


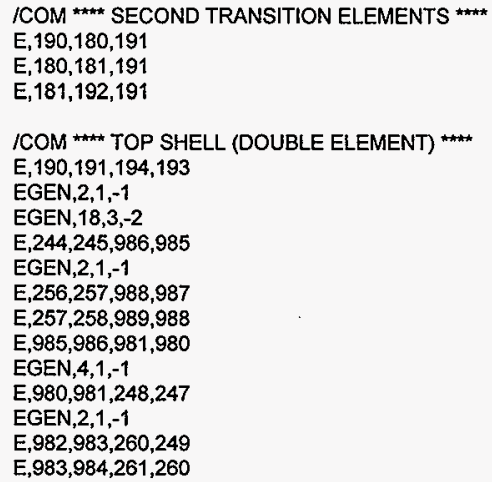

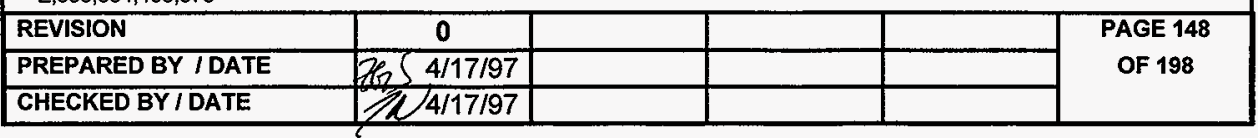


CLIENT: Duke Engineering \& Services Hanford

FILE NO: $\quad \mathrm{KH}-8009-8-03$

PROJECT: MCO Final Design $\quad$ DOC. NO.: HNF-SD-SNF-DR-003, Rev. 0, Appendix 5

$E, 890,415,884,883$

E,884,885,879,405

$\mathrm{E}, 415,891,885,884$

E,885,886,880,879

E,891,892,886,885

$E, 886,887,406,880$

$E, 892,416,887,886$

$E, 423,900,888,413$

$E, 893,414,888$

$E, 900,893,888$

E, $900,424,893$

E, 893,894,889,414

E,424,901,894,893

$E, 894,895,890,889$

$E, 901,902,895,894$

$E, 895,896,415,890$

E,902,425,896,895

$E, 896,897,891,415$

$E, 425,903,897,896$

$E, 897,898,892,891$

E, $903,904,898,897$

E,898,899,416,892

E, $904,426,899,898$

$E, 431,432,422,421$

$E, 905,423,422$

$E, 432,905,422$

$E, 432,433,905$

E, $905,906,900,423$

$E, 433,908,906,905$

E,906,907,424,900

E,908,434,907,906

$E, 441,442,432,431$

EGEN,2,1,-1

E, 443,908,433

$E, 443,444,434,908$

$E, 451,452,442,441$

EGEN,3,1,-1

EGEN, 7, 10,-3

$E, 454,912,910,444$

E,464,914,912,454

E,474,916,914,464

$E, 484,918,916,474$

E,494,920,918,484

E,504,922,920,494

E,514,924,922,504

$E, 458,448,911,913$

E,468,458,913,915

E,478,468,915,917

E,488,478,917,919

$E, 498,488,919,921$

$E, 508,498,921,923$

E,518,508,923,925

ICOM BOLT

TYPE, 5

MAT, 5

REVISION

PREPARED BY / DATE

CHECKED BY IDATE

PAGE 149

OF 198 
CLIENT: Duke Engineering \& Services Hanford

\section{PROJECT: MCO Final Design DOC. NO.: HNF-SD-SNF-DR-003, Rev. 0, Appendix 5}

$E, 455,456,446,445$

EGEN,8,10,-1

$E, 456,457,447,446$

EGEN $, 8,10,-1$

ICOM END OF LOCKING/LIFTING RING

ICOM SHIELD PLUG ELEMENTS

TYPE, 2

MAT, 1

$E, 602,622,621,601$

EGEN,11,1,-1

EGEN,2,20,-11

E,613,1290,612

$E, 1290,1280,632,612$

E, 1280,633,632

$E, 633,1270,632$

E, 1270,652,632

$E, 1270,653,652$

$E, 643,663,662,642$

EGEN,10,1,-1

EGEN,2,20,-10

$E, 673,693,692,672$

EGEN,2,20,-10

$E, 653,1260,652$

E,1260,672,652

$\mathrm{E}, 1260,673,672$

E,707,717,716,706

EGEN, $7,1,-1$

$E, 717,737,736,716$

EGEN, $7,1,-1$

E,731,751,750,730

EGEN,13,1,-1

$E, 749,769,768,748$

EGEN,15,1,-1

E, $767,787,786,766$

EGEN,17,1,-1

EGEN,2,20,-17

$E, 818,825,824,817$

EGEN,6,1,-1

EGEN, $5,7,-6$

$E, 853,860,859,852$

EGEN,6,1,-1

$E, 860,867,866,859$

EGEN, $3,1,-1$

$\mathrm{E}, 867,872,871,866$

EGEN,4,1,-1

/COM END OF SHIELD PLUG

ICOM FILTER GUARD PLATE

$E, 1200,1201,858,851$

$E, 1201,1202,865,858$

$E, 1203,1204,1201,1200$

EGEN, $2,1,-1$

EGEN,6,3,-2

$E, 1221,1222,1219,1218$

$E, 1222,1223,1220,1219$

REVISION

PREPARED BY IDATE

CHECKED BY I DATE 
CLIENT: Duke Engineering \& Services Hanford

PROJECT: MCO Final Design
FILE NO: $\quad \mathrm{KH}-8009-8-03$

\footnotetext{
$E, 1226,1215,1212,1225$

$E, 1227,1218,1215,1226$

$E, 1228,1221,1218,1227$

$E, 1230,1226,1225,1229$

EGEN, $3,1,-1$

EGEN, 6,4,-3

E, 1257,1250,1249,1256

EGEN, $3,1,-1$

$E, 1264,1254,1253,1263$

EGEN, 6,1,-1

$E, 1271,1261,1260,1270$

EGEN,9,1,-1

$E, 1281,1271,1270,1280$

EGEN,4,1,-1

$E, 1291,1281,1280,1290$

EGEN,2,1,-1
}

ICOM CONTACT ELEMENTS C

ICOM BETWEEN LOCKING RING \& SHIELD PLUG

TYPE, 4

REAL,4

E, 806,401

$E, 807,411$

E, 808,421

E, 809,431

E, 810,441

E,811,451

E,812,461

$E, 813,471$

E, 814,481

E.815,491

E,816,501

$E, 817,511$

ICOM * BETWEEN SHIELD PLUG \& BOTTOM OF BOLT

REAL,5

$E, 845,525$

$E, 852,526$

$E, 859,527$

/COM * BETWEEN SHELD PLUG \& SHELL (ABOVE SEAL)

REAL, 4

E,871,271

E, 872,268

E,873,265

$E, 874,262$

$/ C O M$ BETWEEN SHIELD PLUG \& SHELL (BELOW SEAL)

$E, 863,980$

ICOM * BETWEEN SHIELD PLUG AND SEAL LIP

TYPE,4

REAL,6

$E, 248,870$

E,249,875 
PROJECT: MCO Final Design DOC. NO.: HNF-SD-SNF-DR-003, Rev. 0, Appendix 5

TYPE, 4

REAL, 8

$E, 247,862$

$E, 248,869$

ICOM ABOVE PLUG \#

TYPE, 4

REAL., 7

$E, 601,2601$

EGEN,3,20,-1

$E, 766,2766$

EGEN,3,20,-1

$E, 401,2401$

EGEN,6,1,-1

$E, 876,2876$

EGEN,5,1,-1

ICOM END GAP ELEMENTS

ICOM BOUNDARY CONDITIONS

CSYS, 0

NSEL,S,LOC, $X, O$

NSEL, R,LOC,Y,-1.33,158.14

$D, A L L, U X, 0$

NALL

EALL

NSEL,S,NODE, ,2601,2641,20

NSEL,A,NODE, ,2766,2806,20

NSEL,A,NODE, 2401,2406,1

NSEL,A, NODE, $2876,2880,1$

$D, A L L, A L L, O$

EALL

NALL

SAVE

/COM LOAD 1: 150 PSI INTERNAL. PRESSURE

NSEL,S,LOC, X, $0,1.26$

! Bottom Plate

NSEL, R,LOC, Y, $-0.45,-0.43$

SF,ALL,PRES, 150

NALL

EALL.

NSEL, S,LOC, $X, 1.24,2.14$

NSEL, R,LOC,Y, $-0.45,0.45$

SF,ALL,PRES,150

NALL

EALL

NSEL,S,LOC, $X, 2.12,11.51$

NSEL,R,LOC,Y, $0.43,0.45$

SF,ALL,PRES, 150

NALL

EALL

NSEL,S,LOC,X,11.49,11.51 ! Inside Shell

NSEL,R,LOC, Y, $0.43,149.64$

SF,ALL,PRES, 150

NALL

EALL

NSEL,S,LOC, $X, 11.49,11.76$ ! Edge Shell to Seal

\begin{tabular}{|l|c|l|l|l|}
\hline REVISION & 0 & & & \\
\hline PREPARED BY IDATE & C 3 S $4 / 17 / 97$ & & & \\
\hline CHECKED BYIDATE & P $4 / 17 / 97$ & & & \\
\hline
\end{tabular}




\section{$\begin{array}{ll}\text { PROJECT: } & \text { MCO Final Design } \\ \end{array}$}

NSEL,R,LOC,Y,149.62,149.64

SF,ALL,PRES, 150

NALL

EALL

NSEL,S,LOC,X,11.67,11.69 ! Seal

NSEL,R,LOC,Y,149.64,149.89

SF,ALL,PRES, 150

NALL

EALL

NSEL,S,LOC,X,11.44,11.69 ! Shield Plug (above seal)

NSEL, R,LOC, Y, 149.87,149.89

SF,ALL,PRES, 150

NALL

EALL

NSEL,S,LOC,X,11.44,11.46 ! Side of Shield Plug

NSEL,R,LOC,Y,149.37,149.89

SF,ALL,PRES, 150

NALL

EALL

NSEL,S,LOC,X,11.24,11.46 ! Shield Plug Taper

NSEL,R,LOC,Y,147.62,149.39

SF,ALL,PRES, 150

NALL

EALL

NSEL,S,LOC,X,11.24,11.26 ! Guard Plate Ring

NSEL, R,LOC, Y, 143.12,147.64

SF,ALL,PRES, 150

NALL

EALL

NSEL, $S, L O C, X, 10.914,11.26$ ! Guard Plate Taper

NSEL,R,LOC,Y,141.87,143.14

SF,ALL,PRES, 150

NALL

EALL

NSEL, $S, L O C, X, 1.34,10.92$ ! Guard Piate Bottom

NSEL,R,LOC,Y,141,87, 141,89

SF,ALL,PRES, 150

NALL

EALL

SAVE

/COM LOAD 2: APPLYING PRESSURE GRADIENT (BASKETS)

NSEL,S,NODE, 164,166,2

SF,ALL,PRES, 258.79

! Bottom of Top Basket

SFCUM,PRES,ADD

NALL

EALL

NSEL,S,NODE,,166,168,2

SF,ALL,PRES,264.44

SFCUM,PRES,ADD

NALL

EALL

NSEL,S,NODE, $168,170,2$

SF,ALL,PRES,269.67

SFCUM,PRES,ADD

NALL

REVISION

PREPARED BY / DATE

CHECKED BY / DATE 
CLIENT: Duke Engineering \& Services Hanford

FILE NO: $\quad \mathrm{KH}-8009-8-03$

PROJECT: MCO Final Design DOC. NO.: HNF-SD-SNF-DR-003, Rev. 0, Appendix 5

EALL

NSEL,S,NODE,,170,172,2

SF,ALL,PRES, 274.72

SFCUM,PRES,ADD

NALL

EALL

NSEL,S,NODE, ,172,174,2

SF,ALL,PRES, 279.59

SFCUM,PRES,ADD

NALL

EALL

NSEL,S,NODE,,174,176,2

SF,ALL,PRES, 284.29

SFCUM,PRES,ADD

NALL

EALL

NSEL,S,NODE,,176,178,2

SF,ALL,PRES, 288.83

SFCUM,PRES,ADD

NALL

EALL

NSEL,S,NODE, ,178,180,2

SF,ALL,PRES, 293.19

SFCUM,PRES,ADD

NALL

EALL

NSEL,S,NODE, $180,190,10$

SF,ALL,PRES, 294.10

SFCUM,PRES,ADD

NALL

EALL

NSEL,S,NODE, 190,193,3

SF,ALL,PRES,295.21

SFCUM,PRES,ADD

NALL

EALL

NSEL,S,NODE, $193,196,3$

SF,ALL,PRES,296.32

SFCUM,PRES,ADD

NALL

EALL

NSEL,S,NODE, 196,199,3

SF,ALL,PRES, 297.43

SFCUM,PRES,ADD

NALL

EALL

NSEL,S,NODE,,199,202,3

SF,ALL,PRES,298.54

SFCUM,PRES,ADD

NALL

EALL.

NSEL,S,NODE,202,205,3

SF,ALL,PRES,299.67

SFCUM,PRES,ADD

NALL

EALL

\begin{tabular}{|l|c|c|c|c|c|}
\hline REVISION & 0 & & & & PAGE 154 \\
PREPARED BY IDATE & OF 198 \\
\hline CHECKED BY IDATE & P/17/97 & & & & \\
\hline
\end{tabular}


Duke Engineering \& Services Hanford

FILE NO: KH-8009-8-03

PROJECT: MCO Final Design DOC. NO.: HNF-SD-SNF-DR-003, Rev. 0, Appendix 5

NSEL,S,NODE, 205,208,3

SF,ALL,PRES, 300.79

SFCUM,PRES,ADD

NALL

EALL

NSEL,S,NODE, ,208,211,3

SF,ALL,PRES, 301.90

SFCUM,PRES,ADD

NALL

EALL

NSEL,S,NODE,,211,214,3

SF,ALL,PRES,303.01

SFCUM,PRES,ADD

NALL

EALL

NSEL, $S, L O C, X, 1.3565,10.9155$

NSEL, R,LOC, Y, 141.87, 141.89

SF,ALL,PRES, 1169.04 ! Vertical Pressure from 5 Baskets

SFCUM,PRES,ADD ! at $28 \mathrm{~g}$ 's

NALL

EALL

SAVE

ICOM LOAD 3: APPLYING 28g ACCELERATION

ACEL, ,-28 ! Impose $28 \mathrm{~g}$ Acceleration Top Drop

SAVE

FINI

ICOM SOLUTION PHASE

ISOLUTION

SOLVE

SAVE

FINI

/COM * POSTPROCESSING ***

IPOST1

SET,LAST

TYPE,ALL,HIDC

/GLINE,ALL, 0

RSYS, 0

PLNSOL,S,INT

/DSCALE, 20

IREPLOT

NSEL,S,LOC, X, 11.49, 11.51

NSEL,R,LOC,Y,-0.33,149.63

PRNS,U, $X$

NALL

EALL

NSEL,S,LOC,X,12.624,12.626

NSEL, R, LOC,Y,146.68,156.01

PRNS, U, $X$

NALL

EALL

NSEL,S,LOC,X,1.356,11.26

NSEL,R,LOC,Y,141.87,143.39

PRNS,U,Y

REVISION

PREPARED BY IDATE

CHECKED BY I DATE

\begin{tabular}{|c|c|}
\hline & \\
\hline $7635 / 17 / 97$ & \\
\hline$/ 24 / 17 / 97$ &
\end{tabular}


CLIENT: Duke Engineering \& Services Hanford

FILE NO: $\quad \mathrm{KH}-8009-8-03$

PROJECT: MCO Final Design DOC. NO.: HNF-SD-SNF-DR-003, Rev. 0, Appendix 5

NALL

EALL

LPATH,601,641

PRSECT

LPATH,766,806

PRSECT

LPATH, 1237, 1240

PRSECT

LPATH, 1245, 1248

PRSECT

LPATH,1229, 1232

PRSECT

LPATH, 1209,1211

PRSECT

LPATH, 1200,1202

PRSECT

LPATH, 1261,1291

PRSECT

LPATH, 100,101

PRSECT

LPATH,53,55

PRSECT

LPATH, 138, 140

PRSECT

L.PATH,150,152

PRSECT

LPATH,277,279

PRSECT

LPATH,193,195

PRSECT

LPATH, 1,41

PRSECT

L.PATH, 4,44

PRSECT

LPATH, 6,46

PRSECT

LPATH, 9,49

PRSECT

SAVE 
CLIENT: Duke Engineering \& Services Hanford

FILE NO: $\mathrm{KH}-8009-8-03$

PROJECT: MCO Final Design DOC. NO.: HNF-SD-SNF-DR-003, Rev. 0, Appendix 5

\section{COMPUTER RUN COVER SHEET}

Project Number:

Computer Code:

Software Version:

Computer System:

Computer Run File Number:

Unique Computer Run Filename:

Run Description:

Run Date / Time:

Prepared By: Zachary G.Sargent
$\mathrm{KH}-8009-8$

ANSYSß-PC

$5.0 \mathrm{~A}$

MS-DOS, Pentium® Processor

$\mathrm{KH}-8009-8-03$

TED.out

MCO Top End Drop without Lifting Cap

30 March 1997 11:00:16 AM

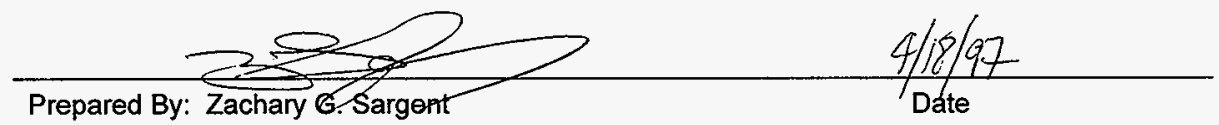
Hentol
Checked By: Joe Nichols

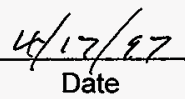

\begin{tabular}{|c|c}
\hline 1/17/97 & \\
\hline $4 / 17 / 97$ & \\
\hline
\end{tabular}


CLIENT: Duke Engineering \& Services Hanford

\section{COMPUTER RUN COVER SHEET}

Project Number:

Computer Code:

Software Version:

Computer System:

Computer Run File Number:

Unique Computer Run Filename:

Run Description:

Creation Date / Time:
KH-8009-8

ANSYS@-PC

$5.0 \mathrm{~A}$

MS-DOS, Pentium $\&$ Processor

$\mathrm{KH}-8009-8-03$

BED.inp

MCO Bottom End Drop

30 March 1997 11:32:14 AM

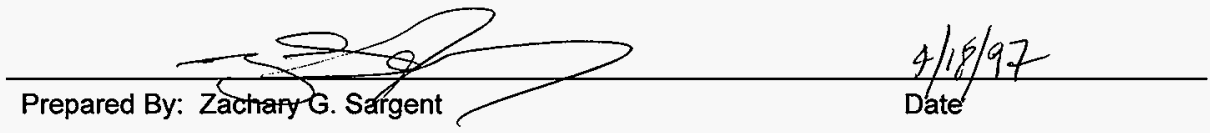

Checked By: Joe Nichols

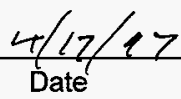

\begin{tabular}{|l|l|l|l|}
\hline 0 & & & \\
\hline $4 / 17 / 97$ & & & \\
\hline $4 / 17 / 97$ & & & \\
\hline
\end{tabular}


CLIENT: Duke Engineering \& Services Hanford

PROJECT: MCO Final Design

FILE NO: KH-8009-8-03

DOC. NO.: HNF-SD-SNF-DR-003, Rev. 0, Appendix 5

\section{LISTING OF BED.INP FILE}

/BATCH,LIST

IFILENAM,BED

IPREP7

TITLE,MCO DESIGN- 132 DEGREES C, 150 psi PRESSURE, $54 \mathrm{~g}$ ACCELERATION

TREF,70

TUNIF,270

ETAN $=0.006 \quad$ ! Tangent modulus

ICOM *** ELEMENT TYPES ****

ET, $1,42, \ldots 1$ ! Shell

ET,2,42,..1 ! Shield Plug

ET $, 3,42,,, 1$ ! Lifting \& Locking Ring

ET,4,12 ! Gap Elements Between Shield Plug \& Shell

KEYOPT,4,7,1

ET, $5,42,, 1$ ! Bolt

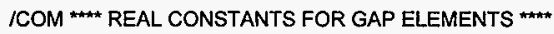

R,4,-90,1.0e8,-0.06,3.0 IShell/Shield Piug, Initially Open .06"

$\mathrm{R}, 5,0,1.0 \mathrm{e} 8,2.95 \mathrm{e}-03$ ! L. Ring/Shield Plug, Under Bolt, Preloaded

$\mathrm{R}, 6,0,1.0 \mathrm{e} 8,0,2.0 \quad$ ! Sealing Surface, closed

$\mathrm{R}, 7,0,1.0 \mathrm{e} 8,0,1.0 \quad$ ! Bottom MCO Plate, closed

R,8,0,2.42e7,2.0 I Seal Spring, Max. Stiffness

ICOM MATERIAL PROPERTIES

MP,DENS, $1,490 / 1728$ ! 304L SS

MP,NUXY,1,0.3

MP,DENS,5,490/1728 ! SA193 Grade B8M

MP,NUXY,5,0.3

TB,BKIN, $1,6 \quad$ ! Yield Stress \& Tangent Moduli v. Temp.

TBTEMP, 100

TBDATA, $1,25000, E T A N * 28.1 E+06$

TBTEMP, 200

TBDATA, 1,21300, ETAN*27.6E+06

TBTEMP, 300

TBDATA, $1,19100, E T A N * 27.0 \mathrm{E}+06$

TBTEMP, 400

TBDATA, 1,17500, ETAN"26.5E+06

TBTEMP, 500

TBDATA, 1, 16300,ETAN²5.8E+06

TBTEMP, 600

TBDATA, 1, 15500,ETAN\$25.3E+06

ICOM DEFINING TEMPERATURES FOR MPDATA **

MPTEMP, 1, 70,100,200,300,400,500

MPTEMP, $7,600,650,700,750$

/COM DEFINING ELASTIC MODULI FOR 304L \& SA-193

MPDATA,EX, 1,1,28.3e+06,28.1e+06,27.6e+06,27.0e+06,26.5e+06,25.8e+06

MPDATA,EX, 1,7,25.3e+06,25.1e+06,24.8e+06,24.5e+06

ICOM

! SA-193

\begin{tabular}{|l|c|c|c|c|}
\hline REVISION & 0 & & & \\
\hline PREPARED BY IDATE & ff/ $4 / 17 / 97$ & & & \\
\hline CHECKED BY I DATE & $/ \% 4 / 17 / 97$ & & & \\
\hline
\end{tabular}




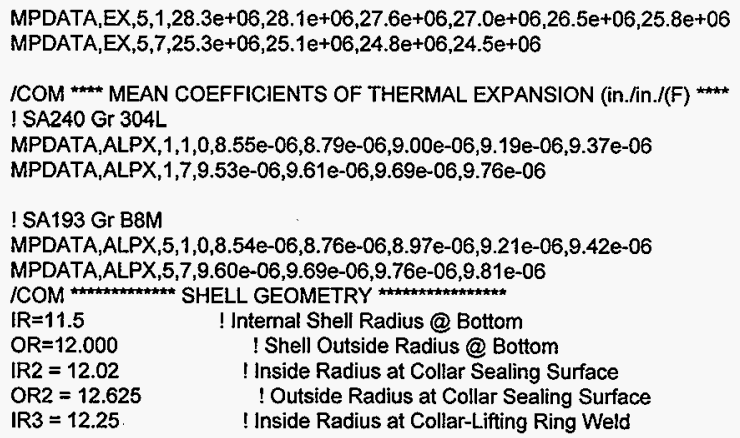




\section{P. PaAsons}

CLIIENT: Duke Engineering \& Services Hanford

FILE NO: $\quad \mathrm{KH}-8009-8-03$

PROJECT: MCO Final Design

FILL, 100,140,20, 2,2,1,2.0

FILL, $140,180,19,2,2,1, .5$

ICOM DOUBLE ROW SHELL

$N, 190, I R, 137.18 \quad$ ! Transition to Double Row

$\mathrm{N}, 192, \mathrm{OR}, 137.18$

FILL.

ICOM BASE OF CASK THROAT-ELEVATION: 138 INCHES *

$\mathrm{N}, 217, \mathrm{IR}, 142.68$ ! Transition to Double Row

$N, 219,0 R, 142.68$

FILL

FILL, 190,217,8,3,3,1 ! Vertical Fill

ICOM BOTTOM OF COLLAR TRANSITION

$N, 235, I R, 146.06 \quad$ ! Start of Transition to Large O.D \&

N,237,OR, 146.06 ! Assumed Location of Shield Plug Taper

FILL

$N, 238, I R, 146.68$

$N, 240, O R, 146.68$

FILL

! Horizontal Fill

FILL, $217,235,5,3,3,1 \quad$ IVertical Fill

ICOM TOP OF COLLAR TRANSITION **

$N, 241, I R, 147.31 \quad$ ! End of Transition to Large O.D \&

$\mathrm{N}, 243, \mathrm{OR}, 147.31$

FILL

! Assumed Location of Shield Plug Taper

NGEN, $2,3,241,243,1,0.75$

/COM COLLAR SEALING SURFACE \#

$N, 247, I R, 149.63$ ! Inside Radius of Sealing Surface

$\mathrm{N}, 249, \mathrm{IR} 2,149.63$

FILL

! Outside Radius at Sealing Surface

I Horizontal Fill

ICOM THICK WALL AT COLLAR TRANSITION

NGEN, $2,10,240,249,3$ ! Nodes $250-259$ Coincident w/240-249 (by 3 )

$\mathrm{N}, 255, \mathrm{OR} 2,147.31 \quad$ ! Outside Surface

N,261,OR2,149.63 I Outside Surface

$\mathrm{N}, 258$, OR2, 148.06

$\mathrm{N}, 980,1 \mathrm{R}, 149.38$

$N, 981,11.755,149.38$

N,982,IR2, 149.38

N, $983,12.317,149.38$

N,984, OR2, 149.38

N,990,OR2,146.68

FILL,240,990,1,251

NGEN $, 2,5,980,984,1,-0.66$

FILL,246,258,1,257

FILL,253,255,1,1,3,3

FILL,237,990,1,991

ICOM COLLAR AT BOTTOM EDGE OF PLUG (.155" above Sealing Surface) "

NGEN $, 2,3,259, \ldots, 0.245$ ! Nodes 262

ICOM COLLAR AT TOP EDGE OF PLUG (2" above bottom Edge)

NGEN $, 2,9,262, \ldots 2.00 \quad$ ! Nodes 271

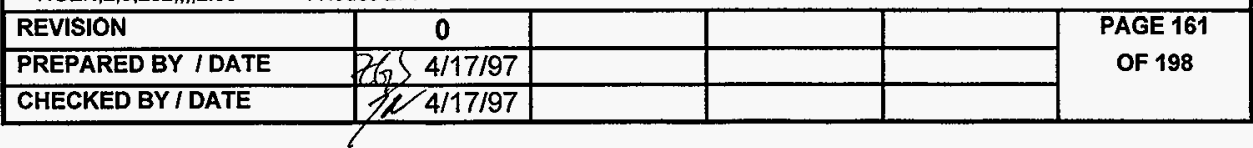


CLIENT: Duke Engineering \& Services Hanford

PROJECT: MCO Final Design

FILE NO: KH-8009-8-03

FILL,262,271,2

ICOM COLLAR AT BASE OF THREADS

N,274,IR3,152.00

N, 1000,IR2,152.00

ICOM TOP TO COLLAR (WELD CLOSURE)

N,295,IR3,156.00

FIL.L,274,295

NGEN, 3,1,259,295,3,(OR2-IR2)/2

NGEN, $3,1,274,295,3,($ OR2-IR3)/2

ICOM LOCKING \& LIFTING RING GEOMETRY

RING1 $=7.94$

RING2 $=9.375$

RING3 $=9.625$

RING4 $=10.19$

RING5 $=12.23$

LOCAL, 11,0,152.00 ! Local System $z=0$ at Base of Ring

CSYS, 11

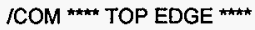

N,401,RING1,6.13

CSYS, $O$

$\mathrm{N}, 404,9.375,158.13$

FILL, 401,404,.,1

N,406,RING4,158.13

FILL,404,406,,,1 I Top Edge

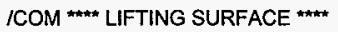

CSYS, 11

N,421,RING1,5.13

N,424,RING2,5.13

FILL, 421,424

N,426,RING4,5.13

FILL,424,426

FILL. 401,421,1,10,6,1

N,431,RING1,6.13-1.56

N,434,RING2,6.13-1.56

FILL

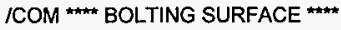

N,441,RING1,4

N,444,RING3,4

FILL

$\mathrm{N}, 445,10.9375-.6875,4 \quad$ ! Inside Edge of Bolt Hole

$\mathrm{N}, 447,10.9375 \div .6875,4 \quad$ ! Outside Edge of Bolt Hole

FILL

$N, 910,10.9375-.6875,4$

$N, 911,10.9375+.6875,4$

N,448,RING5,4

CSYS,0

$\mathrm{N}, 924,10.25,152.00$

N,925,11.625,152.00

DOC. NO.: HNF-SD-SNF-DR-003, Rev. 0, Appendix 5

FILL,910,924,6,22

FILL,911,925,6.,2

\begin{tabular}{|l|c|l|l|l|}
\hline REVISION & 0 & & & \\
\hline PREPARED BY IDATE & f(g) $4 / 17 / 97$ & & & \\
\hline CHECKED BYI DATE & / $4 / 17 / 97$ & & & \\
\hline
\end{tabular}


CLIENT: Duke Engineering \& Services Hanford

PROJECT: \begin{tabular}{ll} 
MCO Final Design DOC. NO.: HNF-SD-SNF-DR-003, Rev. 0, Appendix 5 \\
\hline
\end{tabular}

$\mathrm{N}, 525,10.25,151.874 \quad$ ! Bottom of Bolt Extension

$\mathrm{N}, 527,11.625,151.874$

FILL

ICOM ** BOTTOM OF LIFTING/LOCKING RING ***

CSYS, 11

NGEN, $2,70,441,448,1,-4$ ! Bottom Surface of Lifting/Locking Ring

FILL, $441,511,6,10,8,1 \quad$ ! Fill in Lifting/Locking Ring

ICOM SHIELD PLUG (offset y by 158.25)

LOCAL, 20,0,158.13

TYPE, 2

PLUGR $1=11.975$

PLUGR2=11.45

PLUGR3=11.25

PLUGR4 $=7.89$

ICOM NODES AT PLUG AXIS $(r=0) * \star * * *$

$\mathrm{N}, 601$

$\mathrm{N}, 602,0,-1$

$\mathrm{N}, 603,0,-1.994$

$N, 606,0,-4.994$

FILL,603,606,2,604

$\mathrm{N}, 607,0,-6.25$

$\mathrm{N}, 610,0,-8.25$

FILL, $607,610,2,608$

$N, 611,0,-8.75$

$N, 613,0,-10.5$

FILLL, 611,613

ICOM NODAL GENERATION

NGEN,2,20,601,613,1,0.8825

NGEN, $2,20,621,633,1,0.8825$

NGEN, $2,20,642,653,1,0.6875$

NGEN, $2,20,662,673,1,0.6875$

NGEN,2,20,683,693,1,0.4235

NGEN,2,10,706,713,1,0.9515

! ld Large Opening

! Id Medium Opening

!ld Small Opening

! Center of Opening

$\mathrm{N}, 730,5.4665,-1.994$

! Od Small Opening

$N, 736,5.4665,-4.994$

FILL,730,736,5,731

$\mathrm{N}, 737,5.4665,-6.25$

N, $740,5.4665,-8.25$

FILL, 737,740,2,738

$N, 741,5.4665,-8.75$

N, $743,5.4665,-10.5$

FILL,741,743

$\mathrm{N}, 748,5.89,-1.0$

NGEN, $2,20,730,743,1,0.4235$

FILL, 748,750

$N, 766,7.265,0$

NGEN,2,20,748,763,1,1.375

FILL,766,768

NGEN, $3,20,766,768,1,0.3125$

$\mathrm{N}, 789,7.5775,-1.56$

N, 796,7.5775, -5.56

\begin{tabular}{|l|c|c|c|c|c|}
\hline REVISION & 0 & & & & PAGE 163 \\
OF 198 \\
\hline PREPARED BY IDATE
\end{tabular}


CLIENT: Duke Engineering \& Services Hanford

FILE NO: $\quad \mathrm{KH}-8009-8-03$

PROJECT: MCO Final Design

FILL, 789,796,6

NGEN,2,20,789,796, 1, 0.3125

NGEN, $3,20,777,783,1,0.3125$

/COM UNDER LOCKING RING \#

$\mathrm{N}, 824,8.5017,-6.25$

$\mathrm{N}, 827,8.5017,-8.25$

FILLL

$\mathrm{N}, 828,8.5017,-8.75$

$\mathrm{N}, 830,8.5017,-10.5$

FILL

NGEN $, 3,7,824,830,1,0.5616$

NGEN, $2,7,838,844,1,0.625$

NGEN, $2,7,845,851,1,0.6875$ ! Under Bolt

$\mathrm{N}, 859,11.625,-6.25$

$\mathrm{N}, 860,11.625,-6.917$

$\mathrm{N}, 861,11.625,-7.584$

N,862,PLUGR2,-8.25

N,863,PLUGR2,-8.75

$\mathrm{N}, 865$, PLUGR3,-10.5

FILL,863,865,1

N,866,PLUGR1-0.288,-6.25

$\mathrm{N}, 869$, PLUGR1-0.288,-8.25

FILL, 866,869,2

N,870,PLUGR1-0.288,-8.476

NGEN,2,5,866,870,1,0.288

/COM REFINING LIFTING EAR

CSYS, 0

$\mathrm{N}, 877,9.53,158.13$

$\mathrm{N}, 889,9.53,157.63$

$\mathrm{N}, 901,9.53,157.13$

FILL, $403,404,1,876$

FILL, 413,414,1,888

FILL, $423,424,1,900$

FILL, $877,405,1,878$

FILL, $405,406,2,879,1$

FILL, $889,415,1,890$

FILL, $415,416,2,891,1$

FILL, $404,414,1,881$

FJLL $, 877,889,1,882$

FILL $, 878,890,1,883$

FILL, $405,415,1,884$

FILL, 879,891,1,885

FILL,880,892,1,886

FILL, 406,416,1,887

FILL, $889,901,1,894$

FILL, $414,424,1,893$

FILL, $901,425,1,902$

FILL, 890,902, 1,895

FILL, $415,425,1,896$

FILL, $425,426,2,903,1$

FILL, 891,903, 1,897

FILL, 892,904, 1,898

FILL,416,426,1,899

FILL, 424,434, 1,907

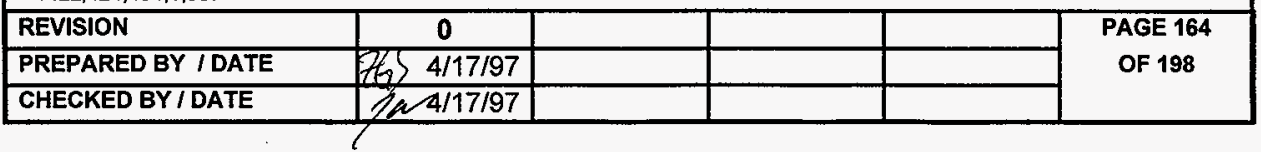


CLIENT: Duke Engineering \& Services Hanford

PROJECT: \begin{tabular}{ll} 
MCO Final Design DOC. NO.: HNF-SD-SNF-DR-003, Rev. 0, Appendix 5 \\
\hline
\end{tabular}

FILL,433,434,1,908

FILL,423,433,1,905

FILL, 905,907

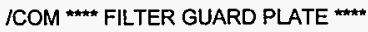

PLATE $1=0.273$

PLATE2 $=0.6575$

PLATE $3=1.357$

PLATE4 $=10.25$

PLATE5 $=11.25$

$N, 1200, P L A T E 4,146.78$

$\mathrm{N}, 1202, P L A T E 5,146.78$

FILL

NGEN, $5,3,1200,1202,,-0.85$

NGEN, $2,3,1212,1214, \ldots-0.25$

N, 1221,PLATE4,141.88

$\mathrm{N}, 1222,10.75,141.88$

$\mathrm{N}, 1223,10.915,141.88$

FiLL, 1215, 1221, 1,1218

FILL, 1223,1217,1,1220

FILL, 1216, 1222, 1,1219

$\mathrm{N}, 1237,6.4375,143.38$

FILL, 1212,1237,3,1225,4

N. 1249,3.578,143.38

FILL, 1237,1249,2,1241,4

NGEN, $2,1,1225,1249,4,-0.25$

NGEN,2,2,1226, 1250,4,,-1.25

FILL, 1226,1228,1,1227,,7,4

$N, 1253,2.625,145.255$

$N, 1254,2.625,145.005$

$\mathrm{N}, 1256,2.625,143.38$

FILL, 1254,1256

$\mathrm{N}, 1257,2.625,143.13$

$N,\{259,2.625,141.88$

FILL, 1257, 1259

NGEN,2,10,1253,1259,1,-0.5

NGEN $, 2,10,1263,1269,1,-0.768$

$\mathrm{N}, 1283,0.6575,145.255$

$\mathrm{N}, 1284,0.6575,145.005$

$\mathrm{N}, 1260,2.125,147.63$

$\mathrm{N}, 1270,1.357,147.63$

$\mathrm{N}, 1280,0.6575,147.63$

$\mathrm{N}, 1290,0.273,147.63$

NGEN, $3,1,1260,1290,10,-0.5625$

ICOM NODES AT BOTTOM GAP ELEMENTS *

NGEN,2,2000,1,10,1,,-1.00

$/$ COM **** COUPLING NODES ****

/COM BETWEEN LIFTING/LOCKING RING \& SHELL \#

CP, $1, U Y, 508,277$

CP,2,UY,498,280

CP, $3, \cup Y, 488,283$

\begin{tabular}{|l|c|l|l|l|c|}
\hline REVISION & 0 & & & & PAGE 165 \\
OF 198 \\
\hline PREPARED BY IDATE & CE) 4/17/97 & & & & \\
\hline CHECKED BY I DATE & /N 4/17/97 & & & & \\
\hline
\end{tabular}


CLIENT: Duke Engineering \& Services Hanford

FILE NO: KH-8009-8-03

PROJECT: MCO Final Design DOC. NO.: HNF-SD-SNF-DR-003, Rev. 0, Appendix 5

$C P, 4, U Y, 478,286$

CP, $5, U Y, 468,289$

$\mathrm{CP}, 6, \mathrm{UY}, 458,292$

ICOM B* BETWEEN BOLT \& LOCKING RING ***

CP, $7, U Y, 445,910$

$\mathrm{CP}, 8, \cup \times, 445,910$

CP,, UY $, 447,911$

CP, 10,UX,447,911

${ }^{*} D O, 1,1,7$

CP, $10+1, U Y, 445+10 * 1,910+2 * \mid$

*ENDDO

"DO, $1,1,7$

CP, 17+1,UY, 447+10*।,911+2*I

*ENDDO

${ }^{*} \mathrm{DO}, \mathrm{I}, 1,7$

CP, $24+1, U X, 445+10 * 1,910+2 * 1$

*ENDDO

*DO, $1,1,7$

$\mathrm{CP}, 31+1, \mathrm{UX}, 447+10^{\star} \mid, 911+2 * 1$

*ENDDO

NALL.

EALL

/COM ELEMENT GENERATION FOR SHELL

TYPE, 1

MAT,1

ICOM BOTTOM OF SHELL \#

$E, 1,2,22,21$

$E, 2,3,23,22$

EGEN,8,1,-1

E, 10,11,30

$E, 21,22,42,41$

$E, 22,23,43,42$

EGEN,10,1,-1

$E, 11,31,30$

$E, 11,32,31$

ICOM SHELL

$E, 50,51,54,53$

EGEN,2,1,-1

EGEN,5,3,-2

ICOM FIRST TRANSITION ELEMENTS

$\mathrm{E}, 65,66,100$

$E, 100,66,101$

$E, 67,101,66$

ICOM *\#* SINGLE SHELL **

$E, 100,101,103,102$

EGEN,40,2,-1

ICOM SECOND TRANSITION ELEMENTS

$E, 190,180,191$

E, $180,181,191$

REVISION

PREPARED BY IDATE

CHECKED BYIDATE 
CLIENT: Duke Engineering \& Services Hanford

FILE NO: KH-8009-8-03

PROJECT: MCO Final Design DOC. NO.: HNF-SD-SNF-DR-003, Rev. 0, Appendix 5

$E, 181,192,191$

ICOM TOP SHELL (DOUBLE ELEMENT)

$E, 190,191,194,193$

EGEN,2,1,1

EGEN, 18,3,-2

$E, 244,245,986,985$

EGEN,2,1,-1

$\mathrm{E}, 256,257,988,987$

$E, 257,258,989,988$

$E, 985,986,981,980$

EGEN, 4, 1,-1

E, $980,981,248,247$

EGEN,2,1,-1

$E, 982,983,260,249$

$\mathrm{E}, 983,984,261,260$

ICOM COLLAR TRANSITION \& THREADED REGIONS

$E, 237,991,251,240$

$E, 991,990,251$

$E, 240,251,254,253$

$\mathrm{E}, 251,990,255,254$

$E, 253,254,257,256$

EGEN,2,1,-1

$E, 259,260,263,262$

EGEN,2, 1,-1

EGEN, 12,3,-2

$E, 271,274,1000$

ICOM MERGE COINCIDENT NODES FOR SHELL *

ESEL,S,TYPE,,1

NSLE

NUMMRG,NODE,

EALL

NALL.

ICOM END OF SHELUCOLLAR ELEMENT GENERATION

ICOM *** LOCKING/LIFTING RING ELEMENTS ***

TYPE, 3

MAT, 1

$E, 411,412,402,401$

EGEN,2,1,-1

EGEN,2,10,-2

$E, 413,888,876,403$

$E, 881,404,876$

$E, 888,881,876$

$E, 888,414,881$

$E, 881,882,877,404$

$E, 414,889,882,881$

$E, 882,883,878,877$

$\mathrm{E}, 889,890,883,882$

$E, 883,884,405,878$

$E, 890,415,884,883$

$E, 884,885,879,405$

$E, 415,891,885,884$

$E, 885,886,880,879$

\begin{tabular}{|l|c|l|l|l|}
\hline REVISION & 0 & & & \\
\hline PREPARED BY IDATE & C/S 4/17/97 & & & \\
\hline CHECKED BY I DATE & $/ / 4 / 17 / 97$ & & & \\
\hline
\end{tabular}


$E, 891,892,886,885$

$E, 886,887,406,880$

$E, 892,416,887,886$

$E, 423,900,888,413$

$E, 893,414,888$

$E, 900,893,888$

$E, 900,424,893$

$E, 893,894,889,414$

$E, 424,901,894,893$

$E, 894,895,890,889$

$E, 901,902,895,894$

$E, 895,896,415,890$

$E, 902,425,896,895$

$E, 896,897,891,415$

$E, 425,903,897,896$

$E, 897,898,892,891$

E,903,904,898,897

$E, 898,899,416,892$

$E, 904,426,899,898$

$E, 431,432,422,421$

E,905,423,422

$E, 432,905,422$

$E, 432,433,905$

$E, 905,906,900,423$

E,433,908,906,905

$E, 906,907,424,900$

$E, 908,434,907,906$

$E, 441,442,432,431$

EGEN,2,1,-1

E, 443,908,433

$E, 443,444,434,908$

$E, 451,452,442,441$

EGEN,3,1,-1

EGEN,7,10,-3

$E, 454,912,910,444$

$E, 464,914,912,454$

$E, 474,916,914,464$

$E, 484,918,916,474$

$E, 494,920,918,484$

$E, 504,922,920,494$

$E, 514,924,922,504$

$E, 458,448,911,913$

$E, 468,458,913,915$

$E, 478,468,915,917$

$E, 488,478,917,919$

$E, 498,488,919,921$

$E, 508,498,921,923$

$\mathrm{E}, 518,508,923,925$

ICOM B* BOLT $* * * *$

TYPE, 5

MAT, 5

$E, 455,456,446,445$

EGEN,8, 10,-1

$E, 456,457,447,446$

EGEN, $8,10,-1$

\begin{tabular}{|l|c|l|l|l|l|}
\hline REVISION & 0 & & & & PAGE 168 \\
PREPARED BY I DATE & OF 198 \\
\cline { 1 - 4 } CHECKED BY I DATE & 4/17/97 & & & & \\
\hline
\end{tabular}


CLIENT: Duke Engineering \& Services Hanford

PROJECT: MCO Final Design

FILE NO: $\mathrm{KH}-8009-8-03$

ICOM

ICOM SHIELD PLUG ELEMENTS

TYPE, 2

MAT,1

$E, 602,622,621,601$

EGEN, $11,1,-1$

EGEN,2,20,-11

E,613,1290,612

E, $1290,1280,632,612$

E, 1280,633,632

E,633,1270,632

E, 1270,652,632

E, $1270,653,652$

E,643,663,662,642

EGEN, 10,1,-1

EGEN, 2,20,-10

E,673,693,692,672

EGEN,2,20,-10

$E, 653,1260,652$

E, $1260,672,652$

E, $1260,673,672$

E, $707,717,716,706$

EGEN, $7,1,-1$

E, $717,737,736,716$

EGEN,7,1,-1

$E, 731,751,750,730$

EGEN,13,1,-1

$E, 749,769,768,748$

EGEN,15,1,-1

E,767,787,786,766

EGEN, 17,1,-1

EGEN,2,20,-17

$E, 818,825,824,817$

EGEN,6,1,-1

EGEN, $5,7,-6$

$E, 853,860,859,852$

EGEN,6,1,-1

E,860,867,866,859

EGEN, $3,1,-1$

E,867,872,871,866

EGEN,4,1,-1

ICOM END OF SHIELD PLUG

ICOM FILTER GUARD PLATE

E, 1200,1201,858,851

$E, 1201,1202,865,858$

$E, 1203,1204,1201,1200$

EGEN, $2,1,-1$

EGEN,6,3,-2

E. 1221,1222,1219,1218

E, $1222,1223,1220,1219$

E, 1226,1215,1212,1225

E,1227,1218,1215,1226

E, 1228,1221,1218,1227

E, $1230,1226,1225,1229$

REVISION

PREPARED BY I DATE

CHECKED BY / DATE

\begin{tabular}{|c|c|l|l|l|} 
& 0 & & & \\
\hline & E $4 / 17 / 97$ & & & \\
\hline $2 / 1 / 17 / 97$ & & & \\
\hline
\end{tabular}


CLIENT: Duke Engineering \& Services Hanford

FILE NO: KH-8009-8-03

PROJECT: MCO Final Design

EGEN, 3,1,-1

EGEN, $6,4,-3$

$E, 1257,1250,1249,1256$

EGEN, $3,1,-1$

$E_{1} 1264,1254,1253,1263$

EGEN, 6,1,-1

$E, 1271,1261,1260,1270$

EGEN, $9,1,-1$

$E, 1281,1271,1270,1280$

EGEN, $4,1,-1$

$E, 1291,1281,1280,1290$

EGEN,2,1,-1

ICOM CONTACT ELEMENTS

ICOM BETWEEN LOCKING RING \& SHIELD PLUG

TYPE, 4

REAL, 4

E,806,401

E, 807,411

$E, 808,421$

$E, 809,431$

E,810,441

$E, 811,451$

$E, 812,461$

$E, 813,471$

E,814,481

$E, 815,491$

$E, 816,501$

$E, 817,511$

ICOM BETWEEN SHIELD PLUG \& BOTTOM OF BOLT

REAL, 5

E,845,525

$E, 852,526$

$E, 859,527$

ICOM BETWEEN SHIELD PLUG \& SHELL (ABOVE SEAL)

REAL, 4

$E, 271,871$

$E, 268,872$

$E, 265,873$

$E, 262,874$

$/$ COM *** BETWEEN SHIELD PLUG \& SHELL (BELOW SEAL)

$\mathrm{E}, 863,980$

ICOM B*tWEEN SHIELD PLUG AND SEAL LIP

TYPE, 4

REAL, 6

$E, 248,870$

$E, 249,875$

TYPE, 4

REAL, 8

$E, 247,862$

$E, 248,869$

REVISION

PREPARED BY / DATE

0

HELD PLUG 
CLIENT: Duke Engineering \& Services Hanford

FILE NO: $\quad \mathrm{KH}-8009-8-03$

PROJECT: MCO Final Design DOC. NO.: HNF-SD-SNF-DR-003, Rev. 0, Appendix 5 
PROJECT: MCO Final Design

FILE NO: $\quad \mathrm{KH}-8009-8-03$

DOC. NO.: HNF-SD-SNF-DR-003, Rev. 0, Appendix 5

SF,ALL,PRES, 150

NALL

EALL

NSEL,S,LOC,X,11.44,11.46 ! Side of Shield Plug

NSEL, R,LOC, Y, $149.37,149.89$

SF,ALL,PRES, 150

NALL

EALL

NSEL,S,LOC,X,11.24, 11.46 ! Shield Plug Taper

NSEL, R,LOC,Y,147.62,149.39

SF,ALL,PRES, 150

NALL

EALL

NSEL, S,LOC,X,11.24,11.26 ! Guard Plate Ring

NSEL, R,LOC, Y, 143.12,147.64

SF,ALL,PRES, 150

NALL

EALL

NSEL,S,LOC,X,10.914,11.26 ! Guard Plate Taper

NSEL, R,LOC,Y,141.87,143.14

SF,ALL,PRES, 150

NALL

EALL

NSEL,S,LOC,X,1.34,10.92 ! Guard Plate Bottom

NSEL, R,LOC,Y,141.87,141.89

SF,ALL,PRES, 150

NALL

EALL

SAVE

ICOM LOAD 2: APPLYING PRESSURE GRADIENT (BASKETS)

NSEL,S,NODE, 114,116,2 ! Top of bottom basket

SF,ALL,PRES, 623.86

SFCUM,PRES,ADD

NALL

EALL

NSEL,S,NODE $, 112,114,2$

SF,ALL,PRES, 633.05

SFCUM,PRES,ADD

NALL

EALL

NSEL,S,NODE, 110,112,2

SF,ALL,PRES, 641.94

SFCUM,PRES,ADD

NALL

EALL

NSEL,S,NODE, 108,110,2

$S F, A L L, P R E S, 650.52$

SFCUM,PRES,ADD

NALL

EALL

NSEL,S,NODE, ,106,108,2

SF,ALL,PRES, 658.80

SFCUM,PRES,ADD

NALL

EALL

REVISION

PREPARED BY / DATE

CHECKED BY I DATE

\begin{tabular}{|c|l|l|l|}
\hline 0 & & & \\
\hline $\cos 4 / 17 / 97$ & & & \\
\hline $1 / 4 / 17 / 97$ & & & \\
\hline
\end{tabular}


CLIENT: Duke Engineering \& Services Hanford

FILE NO: $\quad \mathrm{KH}-8009-8-03$

PROJECT: MCO Final Design DOC. NO.: HNF-SD-SNF-DR-003, Rev. 0, Appendix 5

\author{
NSEL,S,NODE, 104,106,2 \\ SF,ALL,PRES, 666.81 \\ SFCUM,PRES,ADD \\ NALL. \\ EALL \\ NSEL,S,NODE,,102,104,2 \\ SF,ALL,PRES, 674.54 \\ SFCUM,PRES,ADD \\ NALL \\ EALL \\ NSEL,S,NODE, 100,102,2 \\ SF,ALL,PRES, 682.01 \\ SFCUM,PRES,ADD \\ NALL \\ EALL \\ NSEL,S,NODE $, 65,100,35$ \\ SF,ALL,PRES, 683.77 \\ SFCUM,PRES,ADD \\ NALL \\ EALL \\ NSEL,S,NODE, 62,65,3 \\ SF,ALL,PRES,688.61 \\ SFCUM,PRES,ADD \\ NALL \\ EALL \\ NSEL,S,NODE, $59,62,3$ \\ SF,ALL,PRES, 693.45 \\ SFCUM,PRES,ADD \\ NALL \\ EALL \\ NSEL,S,NODE, $56,59,3$ \\ SF,ALL,PRES, 698.30 \\ SFCUM,PRES,ADD \\ NALL \\ EALL \\ NSEL,S,NODE, $53,56,3$ \\ SF,ALL,PRES, 703.14 \\ SFCUM,PRES,ADD \\ NALL \\ EALL. \\ NSEL,S,NODE, $, 50,53,3$
}

SF,ALL,PRES, 705.71

SFCUM,PRES,ADD

! Bottom of Bottom Basket

NALL

EALL

NSEL, S, LOC, X, 0, 1.26

NSEL,R,LOC,Y,-0.45,-0.43

SF,ALL,PRES, 2079.5

SFCUM,PRES,ADD

NALL

EALL

NSEL, S, LOC, $X, 1.24,2.14$

NSEL, R,LOC, $Y,-0.45,0.45$

SF,ALL,PRES, 2079.5

SFCUM,PRES,ADD

NALL

\begin{tabular}{|l|c|l|l|l|l|}
\hline REVISION & 0 & & & & PAGE 173 \\
PREPARED BY IDATE & 2198 \\
\hline CHECKED BY I DATE & $2 / 17 / 97$ & & & & \\
\hline
\end{tabular}




\section{EDAF5口NS}

CLIENT: Duke Engineering \& Services Hanford

FILE NO: $\quad \mathrm{KH}-8009-8-03$

PROJECT: MCO Final Design

DOC. NO.: HNF-SD-SNF-DR-003, Rev. 0, Appendix 5

EALL.

NSEL, S,LOC, $X, 2.12,11.51$

NSEL, R,LOC,Y, $0.43,0.45$

SF,ALL,PRES, 2079.5

SFCUM,PRES,ADD

NALL

EALL

SAVE

/COM LOAD 3: APPLYING 54g ACCELERATION

ACEL, 54 ! Impose $54 \mathrm{~g}$ Acceleration

SAVE

FINI

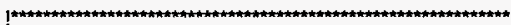

ICOM SOLUTION PHASE

ISOLUTION

SOLVE

SAVE

FINI

ICOM POSTPROCESSING

IPOST1

SET,LAST

ITYPE,ALL,HIDC

IGLINE,ALL,0

RSYS, 0

PLNSOL,S,INT

IDSCALE, 20

/REPLOT

NSEL,S,LOC, X, 11.49,11.51

NSEL,R,LOC,Y,-0.33, 149.63

PRNS, U, $X$

NALL

EALL

NSEL,S,LOC,X,12.624, 12.626

NSEL, R,LOC,Y,146.68,156.01

PRNS, U, $X$

NALL

EALL

NSEL,S,LOC,X,1.356,11.26 ! Guard Plate

NSEL,R,LOC,Y,141.87,143.39

PRNS, U, Y

NALL

EALL

LPATH,862,873

PRSECT

LPATH, 870,874

PRSECT

LPATH,870,875

PRSECT

LPATH, 1237, 1240

PRSECT

LPATH, 12.45, 1248

PRSECT

LPATH, 1229, 1232

\begin{tabular}{|l|c|l|l|l|c|}
\hline REVISION & 0 & & & & PAGE 174 \\
PREPARED BY IDATE & OF 198 \\
\hline CHECKED BYI DATE & $/ \mu / 17 / 97$ & & & & \\
\hline
\end{tabular}


CLIENT: Duke Engineering \& Services Hanford

PROJECT: MCO Final Design
FILE NO: $\quad \mathrm{KH}-8009-8-03$

PRSECT

LPATH, 1209,1211

PRSECT

LPATH, 1200,1202

PRSECT

LPATH, 1284,1263

PRSECT

LPATH, 1261,1291

PRSECT

LPATH, 100,101

PRSECT

LPATH, 116,117

PRSECT

LPATH, 130,132

PRSECT

LPATH,150,152

PRSECT

LPATH,277,279

PRSECT

LPATH, 50,52

PRSECT

L.PATH, 9,49

PRSECT

LPATH, 6,46

PRSECT

LPATH,4,44

PRSECT

LPATH, 1,41

PRSECT

SAVE 
CLIENT: Duke Engineering \& Services Hanford

PROJECT: MCO Final Design
FILE NO: $\quad \mathrm{KH}-8009-8-03$ DOC. NO.: HNF-SD-SNF-DR-003, Rev. 0, Appendix 5

\section{COMPUTER RUN COVER SHEET}

Project Number:

Computer Code:

Software Version:

Computer System:

Computer Run File Number:

Unique Computer Run Filename:

Run Description:

Run Date / Time:
$\mathrm{KH}-8009-8$

ANSYS®-PC

$5.0 \mathrm{~A}$

MS-DOS, Pentium® Processor

$\mathrm{KH}-8009-8-03$

BED.out

MCO Bottom End Drop

30 March $1997 \quad$ 11:39:38 AM

Prepared By: Zachary G. \&argent
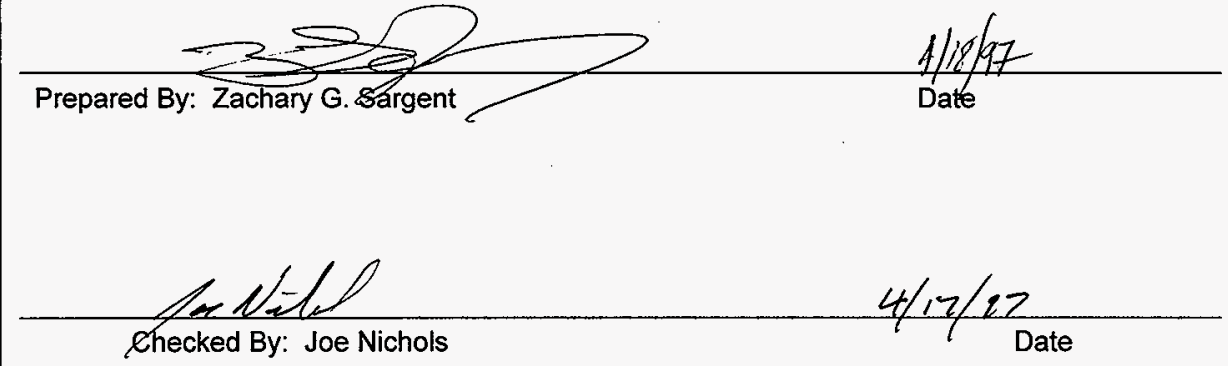
CLIENT: Duke Engineering \& Services Hanford

FILE NO: KH-8009-8-03

PROJECT: MCO Final Design DOC. NO.: HNF-SD-SNF-DR-003, Rev. 0, Appendix 5

\section{COMPUTER RUN COVER SHEET}

Project Number:

Computer Code:

Software Version:

Computer System:

Computer Run File Number:

Unique Computer Run Filename:

Run Description:

Creation Date / Time:
$\mathrm{KH}-8009-8$

ANSYS $B-P C$

$5.0 \mathrm{~A}$

MS-DOS, Pentium® Processor

$\mathrm{KH}-8009-8-03$

FINE.inp

MCO Bottom End Drop w/ Fine Mesh

3 April 1997 6:36:58 PM

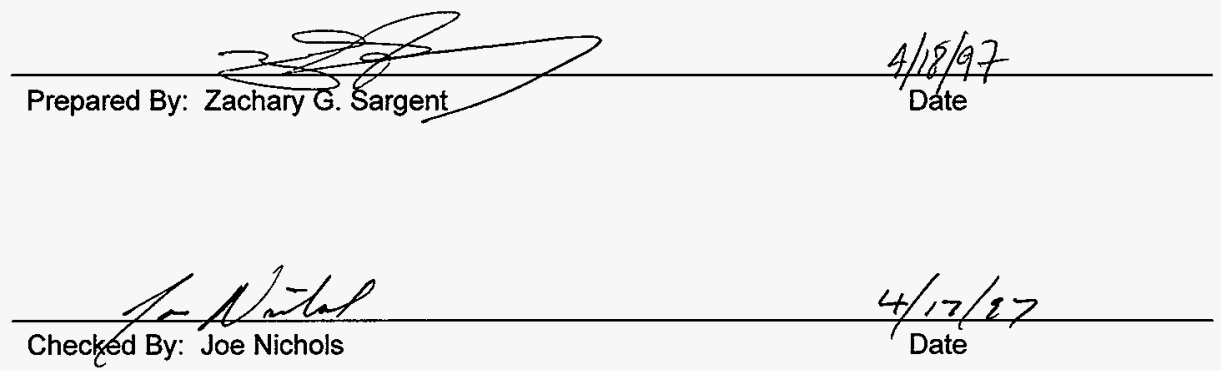




\section{OPARSDN5}

CLIENT: Duke Engineering \& Services Hanford

FILE NO: $\mathrm{KH}-8009-8-03$

PROJECT: MCO Final Design

DOC. NO.: HNF-SD-SNF-DR-003, Rev. 0, Appendix 5

\section{LISTING OF FINE.INP FILE}

/BATCH,LIST

/FILENAM,FINE

IPREP7

TITLE,MCO DESIGN- 132 DEGREES C, 150 psi PRESSURE, 54 g ACCELERATION

TREF,70

TUNIF, 270

ETAN $=0.006 \quad$ ! Tangent modulus

ICOM *** ELEMENT TYPES ***

ET, $1,42, \ldots 1$ ! Shell

ET, $2,42, \ldots 1$ ! Shield Plug

ET, 3,42,.., 1 I Lifting \& Locking Ring

ET,4,12 ! Gap Elements Between Shield Plug \& Shell

KEYOPT, 4,7,1

$\mathrm{ET}, 5,42,, 1$ ! Bolt

/COM REAL CONSTANTS FOR GAP ELEMENTS

R,4,-90,1.0e8,-0.06,3.0 ! Shell/Shield Plug, Initially Open .06"

R,5,0,1.0e8,2.95e-03 ! L. Ring/Shield Plug, Under Bolt, Preloaded

$R, 6,0,1.0 \mathrm{e} 8,0,2.0$ ! Sealing Surface, closed

$R, 7,0,1.0 \mathrm{e} 8,0,1.0 \quad$ ! Bottom MCO Plate, closed

$\mathrm{R}, 8,0,2.42 \mathrm{e}$, ,2.0 I Seal Spring, Max. Stiffness

ICOM MATERIAL PROPERTIES

MP,DENS, $1,490 / 1728$ !304L SS

MP,NUXY, $1,0.3$

MP,DENS, $5,490 / 1728 \quad$ ! SA193 Grade B8M

MP,NUXY,5,0.3

TB,BKIN,1,6 ! Yield Stress \& Tangent Moduli v. Temp.

TBTEMP, 100

TBDATA, 1,25000, ETAN $^{\star} 28.1 E+06$

TBTEMP, 200

TBDATA, 1,21300,ETAN*27.6E+06

TBTEMP, 300

TBDATA 1,19100, TAN $^{\star} 27.0 \mathrm{E}+06$

TBTEMP, 400

TBDATA, 1,17500, ETAN*26.5E+06

TBTEMP, 500

TBDATA, $1,16300, E T A N * 25.8 E+06$

TBTEMP, 600

TBDATA, $1,15500, E T A N * 25.3 E+06$

/COM *** DEFINING TEMPERATURES FOR MPDATA

MPTEMP, $1,70,100,200,300,400,500$

MPTEMP, $7,600,650,700,750$

/COM **** DEFINING ELASTIC MODULI FOR 304L \& SA-193****

MPDATA,EX, 1, 1,28.3e+06,28.1e+06,27.6e+06,27.0e+06,26.5e+06,25.8e+06

MPDATA,EX,1,7,25.3e+06,25.1e+06,24.8e+06,24.5e+06

ICOM !SA-193

\begin{tabular}{|l|c|c|c|c|c|}
\hline REVISION & 0 & & & & $\begin{array}{c}\text { PAGE 178 } \\
\text { OF 198 }\end{array}$ \\
\hline PREPARED BY IDATE & $2 B S$ 4/17/97 & & & & \\
\hline CHECKED BYIDATE & $2 \times 4 / 17 / 97$ & & & & \\
\hline
\end{tabular}


MPDATA,EX,5,1,28.3e+06,28.1e+06,27.6e+06,27.0e+06,26.5e+06,25.8e+06

MPDATA,EX,5,7,25.3e+06,25.1e+06,24.8e+06,24.5e+06

ICOM MEAN COEFFICIENTS OF THERMAL EXPANSION (in./in $/(F)$

! SA240 Gr 304L

MPDATA,ALPX, 1, 1,0,8.55e-06,8.79e-06,9.00e-06,9.19e-06,9.37e-06

MPDATA,ALPX, 1,7,9.53e-06,9.61e-06,9.69e-06,9.76e-06

! SA193 Gr B8M

MPDATA,ALPX,5,1,0,8.54e-06,8.76e-06,8.97e-06,9.21e-06,9.42e-06

MPDATA,ALPX $, 5,7,9.60 \mathrm{e}-06,9.69 \mathrm{e}-06,9.76 \mathrm{e}-06,9.81 \mathrm{e}-06$

ICOM SHELL GEOMETRY

$\mathrm{IR}=11.5$

! Internal Shell Radius @ Bottom

$\mathrm{OR}=12.000$

! Shell Outside Radius @ Bottom

IR2 $=12.02$

! Inside Radius at Collar Sealing Surface

OR2 $=12.625$

! Outside Radius at Collar Sealing Surface

IR3 $=12.25$

! Inside Radius at Collar-Lifting Ring Weld

ICOM BOTTOM COVER PLATE [DWG SK-2-300378]

$\mathrm{N}, 1,-1.32$

! Row 1

$\mathrm{N}, 2,1,25,-1.32$

$\mathrm{N}, 3,2.13,-1.32$

$\mathrm{N}, 10,11.423,-1.32$

FILL

$N, 41,0.00,-0.44$

! Row 3

$\mathrm{N}, 42,1.25,-0.44$

$N, 43,2.13,0.44$

$N, 50, I R, 0.44$

FiLL, 43,50

$\mathrm{N}, 52, \mathrm{OR}, 0.44$

FILL,50,52

FILL, $1,41,1,21,1,10$

FILL, $10,50,1,30$

$\mathrm{N}, 32,12,-0.32$

FILL, 30,32

FILL, 10,32,1,11

$\mathrm{N}, 53, \mathrm{IR}, 1.17$

$\mathrm{N}, 55, \mathrm{OR}, 1.17$

FILL, 53,55

FILL, $50,53,1,1101$

FILL, 51,54,1,1102

FILL,52,55,1,1103

ICOM SHELL [DWGS SK-2-300379 \& SK-2-300461]

$\mathrm{N}, 65, \mathrm{R}, 6.68$

$\mathrm{N}, 67, \mathrm{OR}, 6.68$

FILL

FILL, $53,65,3,3,3,1$

FILL,53,56,1,1104

FILL, 55,58,1,1106

FILL, 1104,1106

FJLL, 56,59,1,1107

FILLL, 58,61,1,1109

FILL, 1107,1109

\begin{tabular}{|l|c|l|l|l|c|}
\hline REVISION & 0 & & & & PAGE 179 \\
\hline PREPARED BY I DATE & OF 198
\end{tabular}


CLIENT: Duke Engineering \& Services Hanford

FILL,59,62,1,1110

FILL,61,64,1,1112

FILL, 1110,1112

FILL, 62,65,1,1113

FILL, $64,67,1,1115$

FILL, 1113,1115

ICOM SINGLE ROW SHELL \#*

$\begin{array}{ll}N, 100, I R, 7.18 & \text { ! Inside } \\ N, 140, I R, 71.68 & \\ N, 180, I R, 136.68 & \end{array}$

$\mathrm{N}, 101, \mathrm{OR}, 7.18 \quad$ ! Outside

$\mathrm{N}, 141, \mathrm{OR}, 71.68$

$\mathrm{N}, 181, \mathrm{OR}, 136.68$

FILL, $100,140,20,2,2,1,2.0$

FILL, 140,180,19,2,2,1,.5

FILL, 100,102,2,1116,2

FILL, 102,104,2,1120,2

FILL, 104, 106,2,1124,2

FILL, 106,108,2,1128,2

FILL, 108,110,2,1132,2

FILL, 110,112,2,1136,2

FILL, 112,114,2,1140,2

FILL, 114,116,2,1144,2

NGEN $2,1,1116,1146,2,0.50$

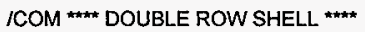

$\mathrm{N}, 190$, IR, 137.18 I Transition to Double Row

$\mathrm{N}, 192, \mathrm{OR}, 137.18$

FILL

/COM *** BASE OF CASK THROAT-ELEVATION: 138 INCHES ***

$\mathrm{N}, 217, \mathrm{IR}, 142.68$ ! Transition to Double Row

$N, 219,0 R, 142.68$

FILL

FILL, 190,217,8,3,3,1 ! Vertical Fill

ICOM BOTTOM OF COLLAR TRANSITION

$N, 235, I R, 146.06 \quad$ ! Start of Transition to Large O.D \&

$\mathrm{N}, 237, \mathrm{OR}, 146.06 \quad$ ! Assumed Location of Shield Plug Taper

FILL

$\mathrm{N}, 238, \mathrm{IR}, 146.68$

$N, 240, O R, 146.68$

FILL ! Horizontal Fill

FILL, 217,235,5,3,3,1 ! Vertical Fill

ICOM TOP OF COLLAR TRANSITION

$N, 241, I R, 147.31$ ! End of Transition to Large O.D \&

N,243,OR,147.31 ! Assumed Location of Shield Piug Taper

FILL ! Horizontal Fill

NGEN, $2,3,241,243,1,0.75$

$/$ COM $*$ COLLAR SEALING SURFACE ***

N,247,IR, 149.63 ! Inside Radius of Sealing Surface

$N, 249, I R 2,149.63 \quad$ ! Outside Radius at Sealing Surface

\begin{tabular}{|l|c|l|l|l|l|}
\hline REVISION & 0 & & & & PAGE 180 \\
OF 198 \\
PREPARED BY IDATE & CHE 4/17/97 & & & & \\
\hline
\end{tabular}


CLIENT: Duke Engineering \& Services Hanford

FILE NO: $\quad \mathrm{KH}-8009-8-03$

PROJECT: MCO Final Design DOC. NO.: HNF-SD-SNF-DR-003, Rev. 0, Appendix 5

FILL I Horizontal Fill

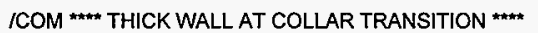

NGEN $, 2,10,240,249,3$

! Nodes 250-259 Coincident w/240-249 (by 3)

$N, 255, O R 2,147.31$

! Outside Surface

$\mathrm{N}, 261, \mathrm{OR} 2,149.63$

! Outside Surface

$\mathrm{N}, 258, \mathrm{OR} 2,148.06$

$\mathrm{N}, 980, \mathrm{IR}, 149.38$

$N, 981,11.755,149.38$

$\mathrm{N}, 982, \mathrm{IR2}, 149.38$

$\mathrm{N}, 983,12.317,149.38$

$\mathrm{N}, 984, \mathrm{OR} 2,149.38$

$\mathrm{N}, 990, \mathrm{OR} 2,146.68$

FILL,240,990, 1,251

NGEN $, 2,5,980,984,1,-0.66$

FILL,246,258, 1,257

FILL, $253,255,1,1,3,3$

FILL, 237,990, 1,991

/COM COLLAR AT BOTTOM EDGE OF PLUG (.155" above Sealing Surface)

NGEN, $2,3,259,, 0.245$ ! Nodes 262

ICOM COLLAR AT TOP EDGE OF PLUG (2" above bottom Edge)

NGEN $, 2,9,262, \ldots, 2.00 \quad$ ! Nodes 271

FILL,262,271,2

ICOM COLLAR AT BASE OF THREADS

$\mathrm{N}, 274, \mathrm{IR} 3,152.00$

N, 1000,IR2, 152.00

/COM **** TOP TO COLLAR (WELD CLOSURE) ****

N,295,IR3,156.00

FILL,274,295

NGEN,3,1,259,295,3,(OR2-IR2)/2

NGEN, $3,1,274,295,3,($ OR2-IR3)/2

ICOM LOCKING \& LIFTING RING GEOMETRY

RING1 $=7.94$

RING2 $=9.375$

RING3 $=9.625$

RING4 $=10.19$

RING5 $=12.23$

LOCAL, 11,0,152.00 ! Local System $z=0$ at Base of Ring

CSYS, 11

ICOM TOP EDGE

N,401,RING1,6.13

CSYS,O

$\mathrm{N}, 404,9.375,158.13$

FILL, 401,404,.,1

N,406,RING4,158.13

FILL, $404,406, . .1$ ! Top Edge

/COM LIFTING SURFACE

CSYS, 11

N,421,RING1,5.13

\begin{tabular}{|l|c|l|l|l|l|}
\hline REVISION & 0 & & & & $\begin{array}{c}\text { PAGE 181 } \\
\text { OF 198 }\end{array}$ \\
\hline PREPARED BY IDATE & CEC 4/17/97 & & & & \\
\hline CHECKED BY I DATE & $/ U / 4 / 17 / 97$ & & & & \\
\hline
\end{tabular}


CLIENT: Duke Engineering \& Services Hanford

FILE NO: $\quad \mathrm{KH}-8009-8-03$

PROJECT: MCO Final Design

DOC. NO.: HNF-SD-SNF-DR-003, Rev. 0, Appendix 5

$\mathrm{N}, 424, \mathrm{RING} 2,5.13$

FILL, 421,424

N,426,RING4,5.13

FILL,424,426

FILL, 401,421,1,10,6,1

$\mathrm{N}, 431, \mathrm{RING} 1,6.13-1.56$

N,434,RING2,6.13-1.56

FILL

ICOM BOLTING SURFACE

$\mathrm{N}, 441, \mathrm{RING} 1,4$

$\mathrm{N}, 444, \mathrm{RING}, 4$

FILL

N,445,10.9375-.6875,4 ! Inside Edge of Bolt Hole

$\mathrm{N}, 447,10.9375+.6875,4 \quad$ ! Outside Edge of Bolt Hole

FILL

$\mathrm{N}, 910,10.9375-.6875,4$

$\mathrm{N}, 911,10.9375+.6875,4$

N,448, RING5,4

CSYS, 0

! O.D of Ring

$\mathrm{N}, 924,10.25,152.00$

! Bolt Extension

$N, 925,11.625,152.00$

! Double Nodes@ Bolt for Gap elements

FILL, $910,924,6,2$

FILL, $911,925,6,2$

$\mathrm{N}, 525,10.25,151.874$

$N, 527,11.625,151.874$

! Bottom of Bolt Extension

FILL

ICOM BOTTOM OF LIFTING/LOCKING RING \#

CSYS, 11

NGEN, $2,70,441,448,1,-4$ ! Bottom Surface of Lifting/Locking Ring

FILL, 441,511,6, 10,8,1 . ! Fill in Lifting/Locking Ring

/COM SHIELD PLUG (offset y by 158.25)

LOCAL, 20,0,.158.13

TYPE, 2

PLUGR1 $=11.975$

PLUGR2=11.45

PLUGR3 $=11.25$

PLUGR4 $=7.89$

$/$ COM NODES AT PLUG AXIS $(r=0)$

$\mathrm{N}, 601$

$\mathrm{N}, 602,0,-1$

$\mathrm{N}, 603,0,-1.994$

$\mathrm{N}, 606,0,-4.994$

FILL, $603,606,2,604$

$\mathrm{N}, 607,0,-6.25$

$\mathrm{N}, 610,0,-8.25$

FILL, $607,610,2,608$

$\mathrm{N}, 611,0,-8.75$

$\mathrm{N}, 613,0,-10.5$

FILL, 611,613

/COM NODAL GENERATION *

NGEN, $2,20,601,613,1,0.8825$

\begin{tabular}{|c|c|c|c|c|}
\hline REVISION & 0 & & & \\
\hline PREPARED BY I DATE & $26 / 5 / 17 / 97$ & & & \\
\hline CHECKED BY/DATE & $1 / x-4 / 17 / 97$ & & & \\
\hline
\end{tabular}

PAGE 182

OF 198 
CLIENT: Duke Engineering \& Services Hanford

PROJECT: MCO Final Design

FILE NO: KH-8009-8-03

NGEN, $2,20,621,633,1,0.8825$

NGEN,2,20,642,653,1,0.6875

NGEN $2,20,662,673,1,0.6875$

NGEN, 2,20,683,693,1,0.4235

NGEN,2,10,706,713,1,0.9515

N,730,5.4665,-1.994

$N, 736,5.4665,-4.994$

FILL, 730,736,5,731

N,737,5.4665,-6.25

N,740,5.4665,-8.25

FILL,737,740,2,738

N,741,5.4665,-8.75

N,743,5.4665,-10.5

FILL, 741,743

$N, 748,5.89,-1.0$

NGEN, 2,20,730,743,1,0.4235

FILL, 748,750

N,766,7.265,0

NGEN $, 2,20,748,763,1,1.375$

FILL, 766,768

NGEN, $3,20,766,768,1,0.3125$

$N, 789,7.5775,-1.56$

N,796,7.5775,-5.56

FILL,789,796,6

NGEN, $2,20,789,796,1,0.3125$

NGEN, $3,20,777,783,1,0.3125$

ICOM \# UNDER LOCKING RING \#

$\mathrm{N}, 824,8.5017,-6.25$

$\mathrm{N}, 827,8.5017,-8.25$

FILL

N,828,8.5017,-8.75

$N, 830,8.5017,-10.5$

FILL

NGEN $, 3,7,824,830,1,0.5616$

NGEN,2,7,838,844,1,0.625

NGEN, $2,7,845,851,1,0.6875$ ! Under Bolt

$N, 859,11.625,-6.25$

$N, 860,11.625,-6.917$

N,861,11.625,-7.584

N,862,PLUGR2,-8.25

N,863,PLUGR2,-8.75

N,865,PLUGR3,-10.5

FILL,863,865,1

N,866,PLUGR1-0.288,-6.25

N,869,PLUGR1-0.288,-8.25

FILL,866,869,2

N,870,PLUGR1-0.288,-8.476

NGEN, $2,5,866,870,1,0.288$

ICOM REFINING LIFTING EAR \#

CSYS, 0

$\mathrm{N}, 877,9.53,158.13$

N, $889,9.53,157.63$

N,901,9.53,157.13
I Id Large Opening

! Id Medium Opening

! Id Small Opening

! Center of Opening

! Od Small Opening

\begin{tabular}{|l|c|c|c|c|c|}
\hline REVISION & 0 & & & & PAGE 183 \\
PREPARED BY IDATE & OF 198 & \\
\hline CHECKED BY I DATE & $4 / 17 / 97$ & & & & \\
\hline
\end{tabular}


CLIENT: Duke Engineering \& Services Hanford

FILE NO: $\quad \mathrm{KH}-8009-8-03$

PROJECT: MCO Final Design

DOC. NO.: HNF-SD-SNF-DR-003, Rev. 0, Appendix 5

FILL,403,404,1,876

FILL, $413,414,1,888$

FILL, $423,424,1,900$

FILL, 877,405,1,878

FILL, 405,406,2,879,1

FILL, 889,415,1,890

FILL, $415,416,2,891,1$

FILL, $404,414,1,881$

FILL,877,889,1,882

FILL, $878,890,1,883$

FILL, $405,415,1,884$

FILL, 879,891,1,885

FILL, $880,892,1,886$

FILL,406,416,1,887

FILL, 889,901,1,894

FILL, $414,424,1,893$

FILL, 901,425, 1,902

FILL,890,902,1,895

FILL $415,425,1,896$

FILL, 425,426,2,903,1

FILL, 891,903,1,897

FILL, 892,904,1,898

FILL, $416,426,1,899$

FILL, $424,434,1,907$

FILL, $433,434,1,908$

FILL, $423,433,1,905$

FILL, 905,907

ICOM FILTER GUARD PLATE

PLATE $1=0.273$

PLATE2 $=0.6575$

PLATE3 $=1.357$

PLATE4=10.25

PLATE5 $=11.25$

N, 1200,PLATE4, 146.78

N,1202,PLATE5, 146.78

FILL

NGEN, $5,3,1200,1202,,-0.85$

NGEN,2,3,1212,1214,,-0.25

$\mathrm{N}, 1221, \mathrm{PLATE} 4,141.88$

$N, 1222,10.75,141.88$

$N, 1223,10.915,141.88$

FILL, 1215,1221,1,1218

FILL, 1223,1217,1,1220

FILL, 1216,1222,1,1219

$\mathrm{N}, 1237,6.4375,143.38$

FILL, 1212,1237,3,1225,4

$\mathrm{N}, 1249,3.578,143.38$

FILL, 1237,1249,2,1241,4

NGEN $, 2,1,1225,1249,4, \cdots 0.25$

NGEN $, 2,2,1226,1250,4,-1.25$

FILL, 1226, 1228,1,1227, 7,4

$\mathrm{N}, 1253,2.625,145.255$

N,1254,2.625,145.005

\begin{tabular}{|l|c|c|c|c|c|}
\hline REVISION & 0 & & & & PAGE 184 \\
OF 198 \\
\cline { 1 - 5 }
\end{tabular}


Duke Engineering \& Services Hanford

FILE NO: KH-8009-8-03

PROJECT: MCO Final Design DOC. NO.: HNF-SD-SNF-DR-003, Rev. 0, Appendix 5

N.1256,2.625,143.38
FILL, 1254,1256
N,1257,2.625,143.13
N,1259,2.625,141.88
FlLL, 1257,1259
NGEN,2,10,1253,1259,1,-0.5
NGEN,2,10,1263,1269,1,-0.768
N,1283,0.6575,145.255
N,1284,0.6575,145.005
N, 1260,2.125,147.63
N,1270,1.357,147.63
N,1280,0.6575,147.63
N,1290,0.273,147.63
NGEN,3,1,1260,1290,10,-0.5625

ICOM NODES AT BOTTOM GAP ELEMENTS

NGEN,2,2000,1,10,1,-1.00

1 COM COUPLING NODES

ICOM BETWEEN LIFTING/LOCKING RING \& SHELL *

CP, $1, U Y, 508,277$ IStart Threads

CP, $2, U Y, 498,280$

$C P, 3, \cup Y, 488,283$

$\mathrm{CP}, 4, \mathrm{UY}, 478,286$

CP, $5, U Y, 468,289$

CP, $6, U Y, 458,292$

ICOM BETWEEN BOLT \& LOCKING RING \#

$\mathrm{CP}, 7, \mathrm{UY}, 445,910$

$C P, 8, U X, 445,910$

CP,, U, UY, 447,911

CP, $10, U X, 447,911$

"DO, $1,1,7$

CP, 10+1,UY,445+10*1,910+2*1

EENDDO

${ }^{*} \mathrm{DO}, \mathrm{I}, 1,7$

CP, 17+1,UY,447+10*1,911+2*1

*ENDDO

"DO, $1,1,7$

$C P, 24+1, U \times, 445+10^{\star} 1,910+2^{*} 1$

"ENDDO

*DO, $1,1,7$

$C P, 31+1, U X, 447+10^{*}|, 911+2 *|$

*ENDDO

NALL

EALL

ICOM ELEMENT GENERATION FOR SHELL **

TYPE, 1

MAT, 1

ICOM ** BOTTOM OF SHELL \#

$\mathrm{E}, 1,2,22,21$

$E, 2,3,23,22$

EGEN,8,1,-1

\begin{tabular}{|l|c|l|l|l|l|}
\hline REVISION & 0 & & & & PAGE 185 \\
PREPARED BY IDATE & OF 198 \\
\cline { 1 - 3 } CHECKED BYIDATE & 4/17/97 & & & & \\
\hline
\end{tabular}


$E, 10,11,30$

$E, 21,22,42,41$

$E, 22,23,43,42$

EGEN, 10,1,-1

$E, 11,31,30$

$E, 11,32,31$

1 COM $* * *$ SHELL $\cdots$

$E, 50,51,1102,1101$

EGEN, 2,1,-1

E, 1101,1102,54,53

EGEN, 2,1,-1

$E, 53,54,1105,1104$

EGEN,2,1,-1

$E, 1104,1105,57,56$

EGEN,2,1,-1

$E, 56,57,1108,1107$

EGEN,2,1,-1

$E, 1107,1108,60,59$

EGEN,2,1,-1

$E, 59,60,1111,1110$

EGEN,2,1,-1

E, 1110,1111,63,62

EGEN,2,1,-1

$E, 62,63,1114,1113$

EGEN,2,1,-1

$E, 1113,1114,66,65$

EGEN,2,1,-1

ICOM FIRST TRANSITION ELEMENTS

$E, 65,66,100$

$E, 100,66,101$

E, $67,101,66$

ICOM SINGLE SHELL ****

$\mathrm{E}, 100,101,1117,1116$

$\mathrm{E}, 1116,1117,1119,1118$

$E, 1118,1119,103,102$

$E, 102,103,1121,1120$

$E, 1120,1121,1123,1122$

$E, 1122,1123,105,104$

$E, 104,105,1125,1124$

E,1124,1125,1127,1126

$E, 1126,1127,107,106$

$\mathrm{E}, 106,107,1129,1128$

E, $1128,1129,1131,1130$

$\mathrm{E}, 1130,1131,109,108$

$E, 108,109,1133,1132$

$E, 1132,1133,1135,1134$

$E, 1134,1135,111,110$

$E, 110,111,1137,1136$

$E, 1136,1137,1139,1138$

$E, 1138,1139,113,112$

$\mathrm{E}, 112,113,1141,1140$

$E, 1140,1141,1143,1142$

$E, 1142,1143,115,114$

\begin{tabular}{|l|c|c|c|c|c|}
\hline REVISION & 0 & & & & PAGE 186 \\
PREPARED BY IDATE & OF 198
\end{tabular}


CLIENT: Duke Engineering \& Services Hanford

FILE NO: KH-8009-8-03

PROJECT: MCO Final Design

\author{
$E, 114,115,1145,1144$ \\ $E, 1144,1145,1147,1146$ \\ $E, 1146,1147,117,116$ \\ $E, 116,117,119,118$ \\ EGEN,32,2,-1
}

ICOM *** SECOND TRANSITION ELEMENTS ****

$E, 190,180,191$

$E, 180,181,191$

$E, 181,192,191$

/COM TOP SHELL (DOUBLE ELEMENT)

E,190,191,194,193

EGEN,2,1,-1

EGEN, 18,3,-2

$E, 244,245,986,985$

EGEN, 2,1,-1

$E, 256,257,988,987$

$E, 257,258,989,988$

$E, 985,986,981,980$

EGEN,4, $1,-1$

$E, 980,981,248,247$

EGEN,2, 1,-1

E, $982,983,260,249$

E, $983,984,261,260$

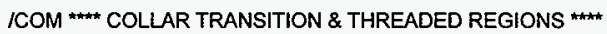

$E, 237,991,251,240$

E, $991,990,251$

E,240,251,254,253

$E, 251,990,255,254$

$E, 253,254,257,256$

EGEN, $2,1,-1$

$E, 259,260,263,262$

EGEN, 2,1,-1

EGEN, 12,3,-2

$E, 271,274,1000$

ICOM MERGE COINCIDENT NODES FOR SHELL *

ESEL,S,TYPE,, 1

NSLE

NUMMRG,NODE,

EALL

NALL

ICOM END OF SHELLCOLLAR ELEMENT GENERATION

ICOM LOCKINGILIFTING RING ELEMENTS

TYPE, 3

MAT, 1

$E, 411,412,402,401$

EGEN,2,1,-1

EGEN,2,10,-2

$E, 413,888,876,403$

$E, 881,404,876$

$E, 888,881,876$

$E, 888,414,881$

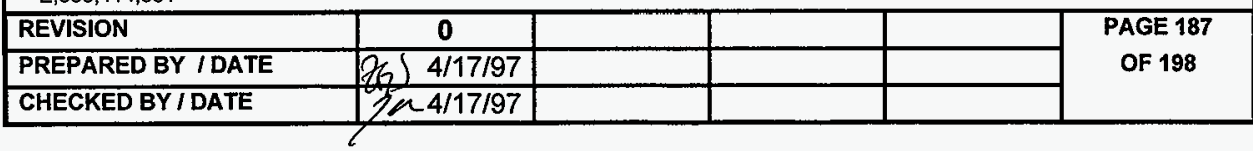


CLIENT: Duke Engineering \& Services Hanford

FILE NO: $\quad \mathrm{KH}-8009-8-03$

PROJECT: MCO Final Design

DOC. NO.: HNF-SD-SNF-DR-003, Rev. 0, Appendix 5

$E, 881,882,877,404$

E,414,889,882,881

$\mathrm{E}, 882,883,878,877$

$E, 889,890,883,882$

E, $883,884,405,878$

$E, 890,415,884,883$

$E, 884,885,879,405$

E, 415,891,885,884

$E, 885,886,880,879$

$E, 891,892,886,885$

E,886,887,406,880

$E, 892,416,887,886$

$E, 423,900,888,413$

E,893,414,888

$E, 900,893,888$

E, $900,424,893$

$E, 893,894,889,414$

$E, 424,901,894,893$

$E, 894,895,890,889$

$E, 901,902,895,894$

E,895,896,415,890

$E, 902,425,896,895$

E,896,897,891,415

$E, 425,903,897,896$

E,897,898,892,891

E,903,904,898,897

$E, 898,899,416,892$

E, $904,426,899,898$

$E, 431,432,422,421$

E, $905,423,422$

$E, 432,905,422$

$E, 432,433,905$

$E, 905,906,900,423$

$E, 433,908,906,905$

E,906,907,424,900

$E, 908,434,907,906$

$E, 441,442,432,431$

EGEN, $2,1,-1$

$E, 443,908,433$

E,443,444,434,908

$E, 451,452,442,441$

EGEN,3,1,-1

EGEN,7,10,-3

$E, 454,912,910,444$

$E, 464,914,912,454$

E,474,916,914,464

E,484,918,916,474

E,494,920,918,484

$E, 504,922,920,494$

E, 514,924,922,504

$E, 458,448,911,913$

$E, 468,458,913,915$

$E, 478,468,915,917$

$E, 488,478,917,919$

$E, 498,488,919,921$

$E, 508,498,921,923$

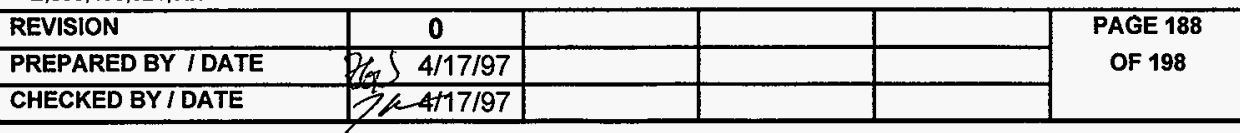


CLIENT: Duke Engineering \& Services Hanford

ICOM ${ }^{\star n \star *}$ BOLT

TYPE, 5

MAT,5

$E, 455,456,446,445$

EGEN,8,10,-1

$\mathrm{E}, 456,457,447,446$

EGEN,8,10,-1

ICOM END OF LOCKING/LIFTING RING

ICOM **** SHIELD PLUG ELEMENTS ***

TYPE, 2

MAT, 1

$E, 602,622,621,601$

EGEN, 11,1,-1

EGEN,2,20,-11

$E, 613,1290,612$

$E, 1290,1280,632,612$

$E, 1280,633,632$

$E, 633,1270,632$

E, $1270,652,632$

$E, 1270,653,652$

$E, 643,663,662,642$

EGEN, 10,1,-1

EGEN,2,20,-10

$E, 673,693,692,672$

EGEN,2,20,-10

$\mathrm{E}, 653,1260,652$

$E, 1260,672,652$

E, $1260,673,672$

$E, 707,717,716,706$

EGEN,7, $1,-1$

$E, 717,737,736,716$

EGEN, $7,1,-1$

E, $731,751,750,730$

EGEN, 13,1,-1

E, $749,769,768,748$

EGEN, 15,1,-1

E,767,787,786,766

EGEN,17,1,-1

EGEN,2,20,-17

$E, 818,825,824,817$

EGEN, $6,1,-1$

EGEN, $5,7,-6$

$E, 853,860,859,852$

EGEN,6,1,-1

$E, 860,867,866,859$

EGEN,3,1,-1

E,867,872,871,866

EGEN,4,1,-1

ICOM END OF SHIELD PLUG

ICOM FILTER GUARD PLATE

$\mathrm{E}, 1200,1201,858,851$

$E, 1201,1202,865,858$

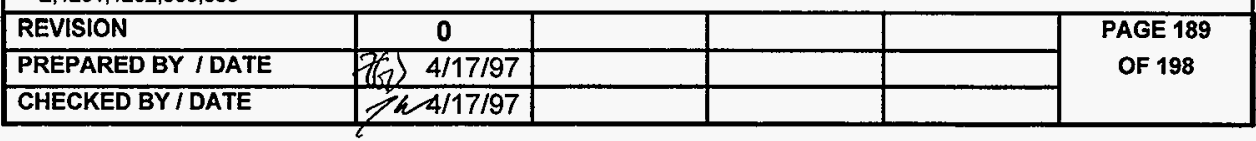


Duke Engineering \& Services Hanford

FILE NO: KH-8009-8-03

PROJECT: MCO Final Design DOC. NO.: HNF-SD-SNF-DR-003, Rev. 0, Appendix 5

$E, 1203,1204,1201,1200$

EGEN, 2,1,-1

EGEN, $6,3,-2$

$E, 1221,1222,1219,1218$

$E_{1} 1222,1223,1220,1219$

$E, 1226,1215,1212,1225$

$E, 1227,1218,1215,1226$

$E, 1228,1221,1218,1227$

E, 1230,1226,1225,1229

EGEN,3,1,-1

EGEN,6,4,-3

$E, 1257,1250,1249,1256$

EGEN,3,1,-1

$E, 1264,1254,1253,1263$

EGEN,6, 1,-1

$E, 1271,1261,1260,1270$

EGEN,9,1,-1

$E, 1281,1271,1270,1280$

EGEN,4,1,-1

$E, 1291,1281,1280,1290$

EGEN,2,1,-1

ICOM

/COM *** BETWEEN LOCKING RING \& SHIELD PLUG

TYPE, 4

REAL, 4

$E, 806,401$

$E, 807,411$

$E, 808,421$

$E, 809,431$

E, 810,441

$E, 811,451$

$E, 812,461$

$E, 813,471$

$E, 814,481$

$E, 815,491$

$E, 816,501$

$E, 817,511$

ICOM ${ }^{\star \star \star \star ~ B E T W E E N ~ S H I E L D ~ P L U G ~ \& ~ B O T T O M ~ O F ~ B O L T ~}$

REAL,5

$E, 845,525$

$E, 852,526$

$E, 859,527$

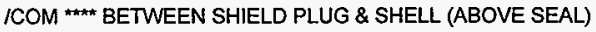

REAL, 4

$E, 271,871$

$E, 268,872$

$E, 265,873$

$E, 262,874$

/COM ${ }^{\star \star \star \star}$ BETWEEN SHIELD PLUG \& SHELL (BELOW SEAL)

$E, 863,980$

/COM BETWEEN SHIELD PLUG AND SEAL LIP

\begin{tabular}{|l|c|l|l|l|l|}
\hline REVISION & 0 & & & & PAGE 190 \\
OFEPARED BY I DATE & Of $4 / 17 / 97$ & & & & \\
\hline CHECKED BYIDATE & $/ 44 / 17 / 97$ & & & & \\
\hline
\end{tabular}


CLIENT: Duke Engineering \& Services Hanford

FILE NO: KH-8009-8-03

PROJECT: MCO Final Design DOC. NO.: HNF-SD-SNF-DR-003, Rev. 0, Appendix 5

TYPE, 4

REAL, 6

$E, 248,870$

$E, 249,875$

TYPE,4

REAL, 8

$E, 247,862$

$E, 248,869$

/COM ${ }^{\star \star A}$ BOTTOM GAP ELEMENTS

TYPE, 4

REAL, 7

$E, 2001,1$

EGEN, 10,1,-1

NALL

EALL

/COM END GAP ELEMENTS

ICOM

CSYS, 0

NSEL,S,LOC,X,O

NSEL,R,LOC, Y,-1.33,158.14

D,ALL,UX,O

NALL

EALL

NSEL,S,NODE,2001,2010

D,ALL,ALL, 0

EALL

NALL

SAVE

/COM *\# LOAD 1: 150 PSI INTERNAL PRESSURE *

NSEL,, LOC, $X, 0,1.26$

NSEL, R, LOC, Y, $-0.45,-0.43$

! Bottom Plate

SF,ALL,PRES, 150

NALLL

EALL

NSEL,S,LOC, $X, 1.24,2.14$

NSEL, R,LOC, Y, $-0.45,0.45$

SF,ALL,PRES, 150

NALL

EALL

NSEL,S,LOC, $X, 2.12,11.51$

NSEL, R,LOC, Y, $0.43,0.45$

SF,ALL,PRES, 150

NALL

EALL

NSEL,S,LOC, $X, 11.49,11.51$ ! Inside Shell

NSEL, R,LOC,Y,0.43,149.64

SF,ALL,PRES, 150

NALL

EALL

NSEL,S,LOC,X,11,49,11.76 ! Edge Shell to Seal

NSEL,R,LOC, Y, 149.62, 149.64

SF,ALL,PRES, 150

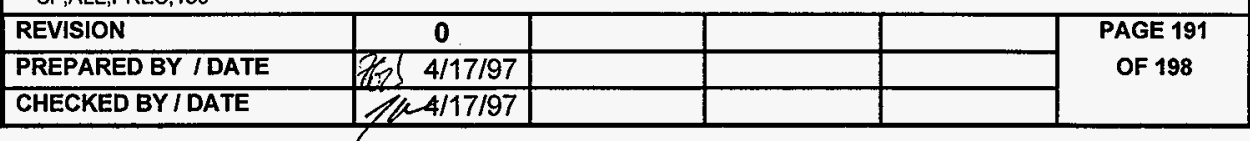


CLIENT: Duke Engineering \& Services Hanford

PROJECT: MCO Final Design DOC. NO.: HNF-SD-SNF-DR-003, Rev. 0, Appendix 5

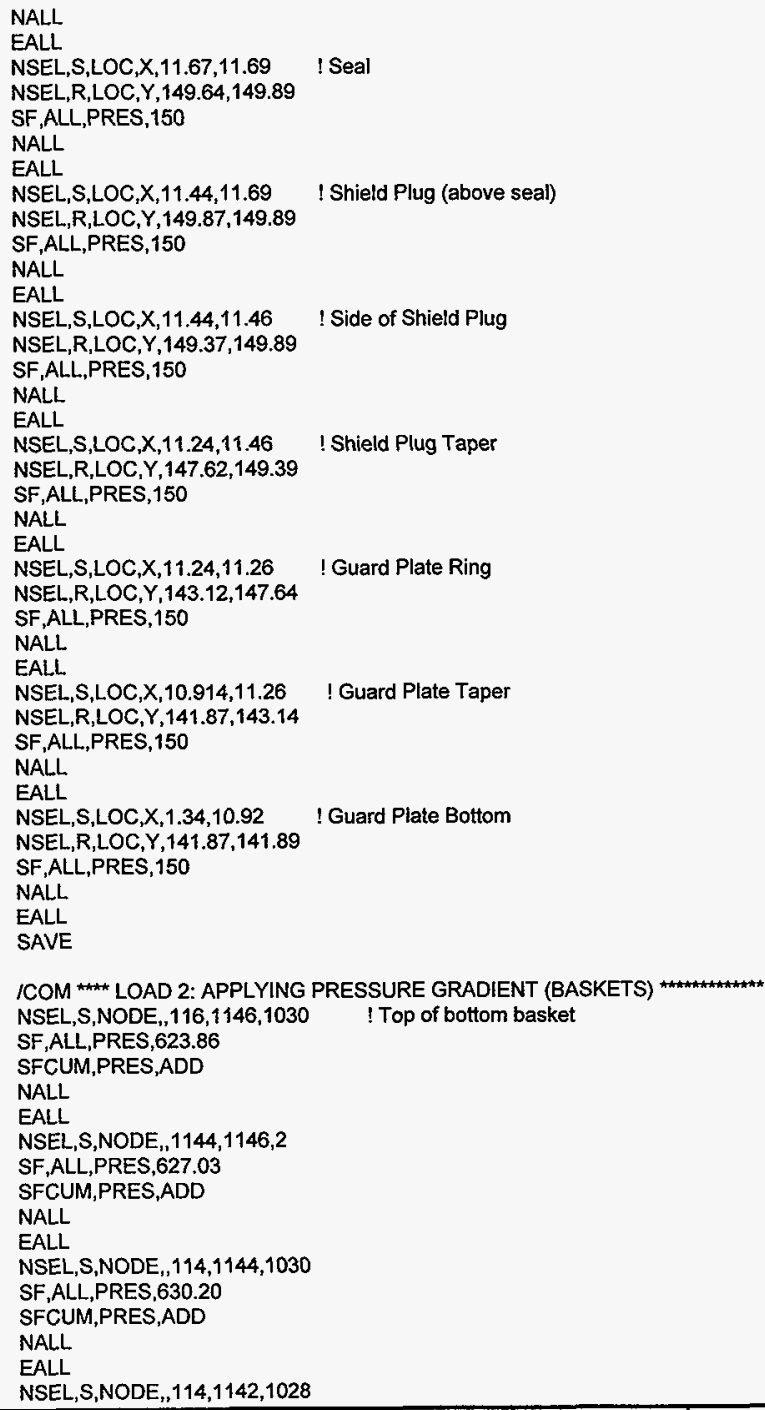

NAL.L

EALL.

NSEL,S,LOC, $X, 11.67,11.69 \quad$ ! Seal

NSEL,R,LOC,Y, 149.64,149.89

SF,ALL,PRES, 150

NALL

EALL

NSEL,S,LOC,X,11.44,11.69 ! Shield Plug (above seal)

NSEL,R,LOC,Y,149.87,149.89

SF,ALL,PRES, 150

NALL

EALL

NSEL, S,LOC,X,11.44,11.46 ! Side of Shield Plug

NSEL, R,LOC,Y,149.37,149.89

SF,ALL,PRES, 150

NALL

EALL

NSEL,S,LOC,X,11.24,11.46 ! Shield Plug Taper

NSEL,R,LOC,Y,147.62,149.39

SF,ALL,PRES, 150

NALL

EALL

NSEL,S,LOC,X,11.24,11.26 ! Guard Plate Ring

NSEL,R,LOC,Y,143.12,147.64

SF,ALL,PRES, 150

NALL

EALL

NSEL,S,LOC,X, 10.914,11.26 ! Guard Plate Taper

NSEL,R,LOC,Y,141.87,143.14

SF,ALL,PRES, 150

NALL.

EALL

NSEL,S,LOC,X, 1.34,10.92 ! Guard Plate Bottom

NSEL,R,LOC,Y,141.87,141.89

SF,ALL,PRES, 150

NALL

EALL

SAVE

ICOM LOAD 2: APPLYING PRESSURE GRADIENT (BASKETS)

NSEL, S,NODE, 116,1146,1030 ! Top of bottom basket

SF,ALL,PRES, 623.86

SFCUM,PRES,ADD

NALL

EALL

NSEL,S,NODE, $1144,1146,2$

SF,ALL,PRES, 627.03

SFCUM,PRES,ADD

NALL

EALL

NSEL,S,NODE,,114,1144,1030

SF,ALL,PRES, 630.20

SFCUM,PRES,ADD

NALLL.

EALL

NSEL,S,NODE, $114,1142,1028$

\begin{tabular}{|l|c|c|l|l|}
\hline REVISION & 0 & & & \\
\hline PREPARED BY IDATE & TK $4 / 17 / 97$ & & & \\
\hline CHECKED BY I DATE & $/ \mu / 17 / 97$ & & & \\
\hline
\end{tabular}


CLIENT: Duke Engineering \& Services Hanford

PROJECT: MCO Final Design DOC. NO.: HNF-SD-SNF-DR-003, Rev. 0, Appendix 5

SF,ALL,PRES, 633.27

SFCUM,PRES,ADD

NALL

EALL

NSEL,S,NODE, $1140,1142,2$

SF,ALL,PRES, 636.33

SFCUM,PRES,ADD

NALL

EALL

NSEL,S,NODE,,112,1140,1028

SF,ALL,PRES, 639.40

SFCUM,PRES,ADD

NALI.

EALL

NSEL,S,NODE, $112,1138,1026$

SF,ALL,PRES, 642.36

SFCUM,PRES,ADD

NALL

EALL

NSEL,S,NODE,1136,1138,2

SF,ALL,PRES, 645.32

SFCUM,PRES,ADD

NALL.

EALL

NSEL,S,NODE, $110,1136,1026$

SF,ALL,PRES,648.28

SFCUM,PRES,ADD

NALL.

EALL

NSEL,S,NODE, 110,1134,1024

SF,ALL,PRES,651.14

SFCUM,PRES,ADD

NALL

EALL

NSEL,S,NODE, 1132,1134,2

SF,ALL,PRES, 654.00

SFCUM,PRES,ADD

NALL

EALL

NSEL,S,NODE, 108,1132,1024

SF,ALL,PRES, 656.86

SFCUM,PRES,ADD

NALL

EALL

NSEL,S,NODE, $108,1130,1022$

SF,ALL,PRES, 659.62

SFCUM,PRES,ADD

NALL

EALL

NSEL,S,NODE, $, 1128,1130,2$

SF,ALL,PRES, 662.38

SFCUM,PRES,ADD

NALL

EALL

NSEL,S,NODE, 106, 1128,1022

SF,ALL,PRES,665.14

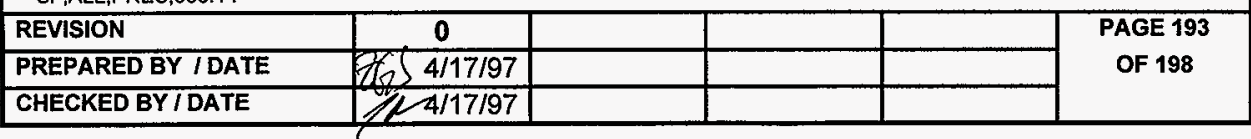


CLIENT: $\quad$ Duke Engineering \& Services Hanford

FILE NO: $\quad \mathrm{KH}-8009-8-03$

PROJECT: MCO Final Design DOC. NO.: HNF-SD-SNF-DR-003, Rev. 0, Appendix 5

SFCUM,PRES,ADD

NALL

EALL

NSEL,S,NODE, ,106,1126,1020

SF,ALL,PRES,667.81

SFCUM,PRES,ADD

NALL

EALL

NSEL,S,NODE „1124,1126,2

SF,ALL,PRES, 670.48

SFCUM,PRES,ADD

NALL

EALL

NSEL,S,NODE, 104,1124,1020

SF,ALL,PRES,673.15

SFCUM,PRES,ADD

NALL

EALL

NSEL, S,NODE, ,104,1122,1018

SF,ALL,PRES,675.73

SFCUM,PRES,ADD

NALL

EALL

NSEL,S,NODE,.1120,1122,2

SF,ALL,PRES,678.31

SFCUM,PRES,ADD

NALL

EALL

NSEL,S,NODE,,102,1120,1018

SF,ALL,PRES, 680.89

SFCUM,PRES,ADD

NALL.

EALL.

NSEL,S,NODE,,102,1118,1016

SF,ALL,PRES, 683.38

SFCUM,PRES,ADD

NALL

EALL

NSEL,S,NODE,,1116,1118,2

SF,ALL,PRES,685.87

SFCUM,PRES,ADD

NALL

EALL

NSEL,S,NODE, 100,1116,1016

SF,ALL,PRES, 688.36

SFCUM,PRES,ADD

NALL

EALL

NSEL,S,NODE,,65, 100,35

SF,ALL,PRES, 690,12

SFCUM,PRES,ADD

NALL

EALL

NSEL,S,NODE, $65,1113,1048$

SF,ALL,PRES,692.54

SFCUM,PRES,ADD

\begin{tabular}{|l|c|l|l|l|}
\hline REVISION & 0 & & & \\
\hline PREPARED BY IDATE & Te $S$ 4/17/97 & & & \\
\hline CHECKED BYIDATE & S $4 / 17 / 97$ & & & \\
\hline
\end{tabular}


CLIENT: Duke Engineering \& Services Hanford

FILE NO: KH-8009-8-03

$\begin{array}{lll}\text { PROJECT: } & \text { MCO Final Design } & \text { DOC. NO.: HNF-SD-SNF-DR-003, Rev. 0, Appendix } 5\end{array}$

NALL

EALL

NSEL, S,NODE, $62,1113,1051$

SF,ALL,PRES, 694.96

SFCUM,PRES,ADD

NALL

EALL

NSEL,S,NODE,,62,1110,1048

SF,ALL,PRES, 697.38

SFCUM,PRES,ADD

NALL

EALL

NSEL,S,NODE, $59,1110,1051$

SF,ALL,PRES, 699.80

SFCUM,PRES,ADD

NALL

EALL

NSEL,S,NODE, $, 59,1107,1048$

SF,ALL,PRES,702.22

SFCUM,PRES,ADD

NALL

EALL

NSEL,S,NODE, ,56,1107,1051

SF,ALL,PRES,704.64

SFCUM,PRES,ADD

NALL

EALL

NSEL,S,NODE, $56,1104,1048$

SF,ALL,PRES,707.06

SFCUM,PRES,ADD

NALL

EALL

NSEL,S,NODE, $53,1104,1051$

SF,ALL,PRES,709.48

SFCUM,PRES,ADD

NALL

EALL

NSEL, S,NODE, $53,1101,1048$

SF,ALL,PRES,710.76

SFCUM,PRES,ADD

NALL

EALL

NSEL,S,NODE,,50,1101,1051

SF,ALL,PRES,712.04 I Bottom of Bottom Basket

SFCUM,PRES,ADD

NALL

EALL

NSEL,S,LOC, $X, 0,1.26$

NSEL,R,LOC,Y, $-0.45,-0.43$

SF,ALL,PRES,2079.5

SFCUM,PRES,ADD

! Vertical Pressure from 5 Baskets

NALL

EALL

NSEL,S,LOC, X, 1.24,2.14

NSEL,R,LOC, $Y,-0.45,0.45$

SF,ALL,PRES,2079.5

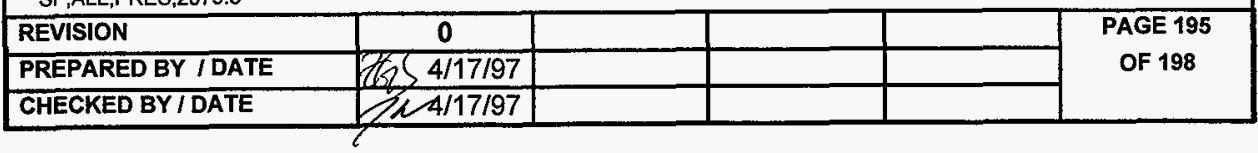


CLIENT: Duke Engineering \& Services Hanford

FILE NO: $\quad \mathrm{KH}-8009-8-03$

PROJECT: MCO Final Design DOC. NO.: HNF-SD-SNF-DR-003, Rev. 0, Appendix 5

\section{SFCUM,PRES,ADD}

NALL

EALL

NSEL,S,LOC,X,2.12,11.51

NSEL,R,LOC,Y, $0.43,0.45$

SF,ALL,PRES, 2079.5

SFCUM,PRES,ADD

NALL

EALL

SAVE

/COM **** LOAD 3: APPLYING 54g ACCELERATION ****

ACEL,,54 ! Impose $54 \mathrm{~g}$ Acceleration

SAVE

FINI

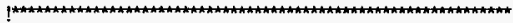

/COM ${ }^{\star \star \star \star \star \star \star \star \star \star \star \star \star \star \star \star \star \star \star \star ~ S O L U T I O N ~ P H A S E ~}$

ISOLUTION

NEQIT, 50

NSUBST,50

SOLVE

SAVE

FINI

/COM POSTPROCESSING *

IPOST1

SET,LAST

ITYPE,ALL,HIDC

/GLINE,ALL, 0

RSYS, 0

PLNSOL,S,INT

IDSCALE, 20

/REPLOT

NSEL,S,LOC,X,11.49,11.51

NSEL,R,LOC, Y, $-0.33,149.63$

PRNS, U, $X$

NALL

EALL

NSEL,S,LOC,X, $12.624,12.626$

NSEL, $R, L O C, Y, 146.68,156.01$

PRNS, U, $X$

NALL

EALL

NSEL,S,LOC, X, 1.356,11.26 ! Guard Plate

NSEL,R,LOC,Y,141.87,143.39

PRNS,U,Y

NALL

EALL

LPATH $, 862,873$

PRSECT

LPATH, 870,874

PRSECT

LPATH, 870,875

PRSECT

LPATH,1237,1240

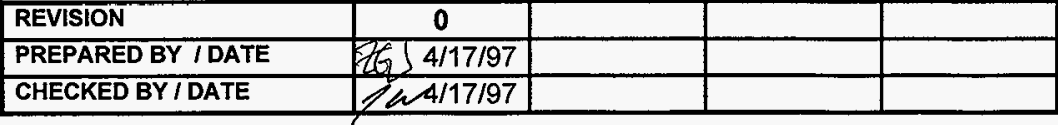


CLIENT: Duke Engineering \& Services Hanford

FILE NO: $\quad \mathrm{KH}-8009-8-03$

PROJECT: MCO Final Design DOC. NO.: HNF-SD-SNF-DR-003, Rev. 0, Appendix 5

PRSECT

LPATH, 1245, 1248

PRSECT

LPATH,1229,1232

PRSECT

LPATH,1209,1211

PRSECT

LPATH,1200,1202

PRSECT

LPATH, 1284,1263

PRSECT

LPATH,1261,1291

PRSECT

LPATH,100,101

PRSECT

LPATH,116,117

PRSECT

LPATH, 130,132

PRSECT

LPATH,150,152

PRSECT

LPATH,277,279

PRSECT

LPATH, 50,52

PRSECT

LPATH,9,49

PRSECT

LPATH, 6,46

PRSECT

LPATH,4,44

PRSECT

LPATH, 1,41

PRSECT

SAVE

\begin{tabular}{|c|c|c|c|}
\hline 0 & & & \\
\hline & & & \\
\hline $2 / 4 / 17 / 97$ & & & \\
\hline
\end{tabular}


CLIENT: Duke Engineering \& Services Hanford FILE NO: KH-8009-8-03

PROJECT: MCO Final Design DOC. NO.: HNF-SD-SNF-DR-003, Rev. 0, Appendix 5

\section{COMPUTER RUN COVER SHEET}

Project Number:

Computer Code:

Software Version:

Computer System:

Computer Run File Number:

Unique Computer Run Filename:

Run Description:

Run Date / Time:
KH-8009-8

ANSYS $\otimes-P C$

$5.0 \mathrm{~A}$

MS-DOS, Pentium® Processor

KH-8009-8-03

FINE.out

MCO Bottom End Drop w/ Fine Mesh

3 April 1997 8:12:20 PM

Prepared By: Zachary G. Sargent

(a)
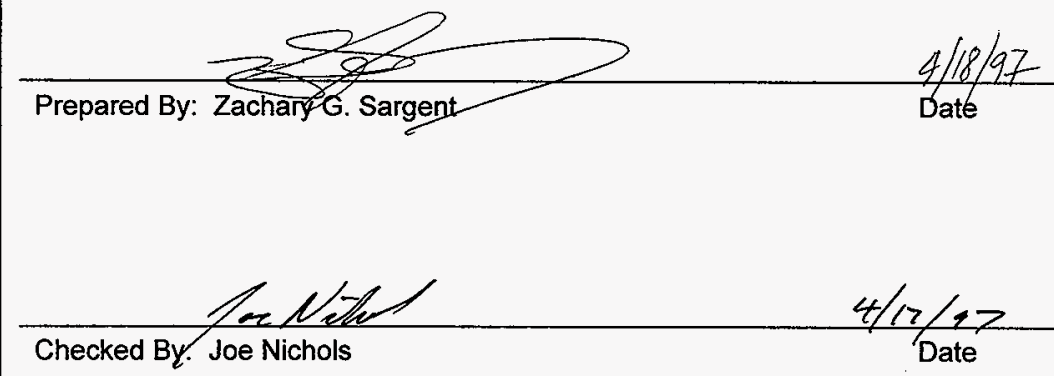

\begin{tabular}{|l|l|l|l|}
\hline 0 & & & \\
\hline $4 / 17 / 97$ & & & \\
\hline $4 / 17 / 97$ & & & \\
\hline
\end{tabular}


This document was too large to scan as a whole document, therefore it required breaking into smaller sections.

Document number:_SA-SNF-AR-003

Section 2 of 3

Title: Ohueti-Canister Qwergack Design Report

Date: 6/9.197 Revision: A000

Originator: Smith $\mathrm{KE}$

Co: hos st

Recipient:

Co:

References: EDT -620/06 


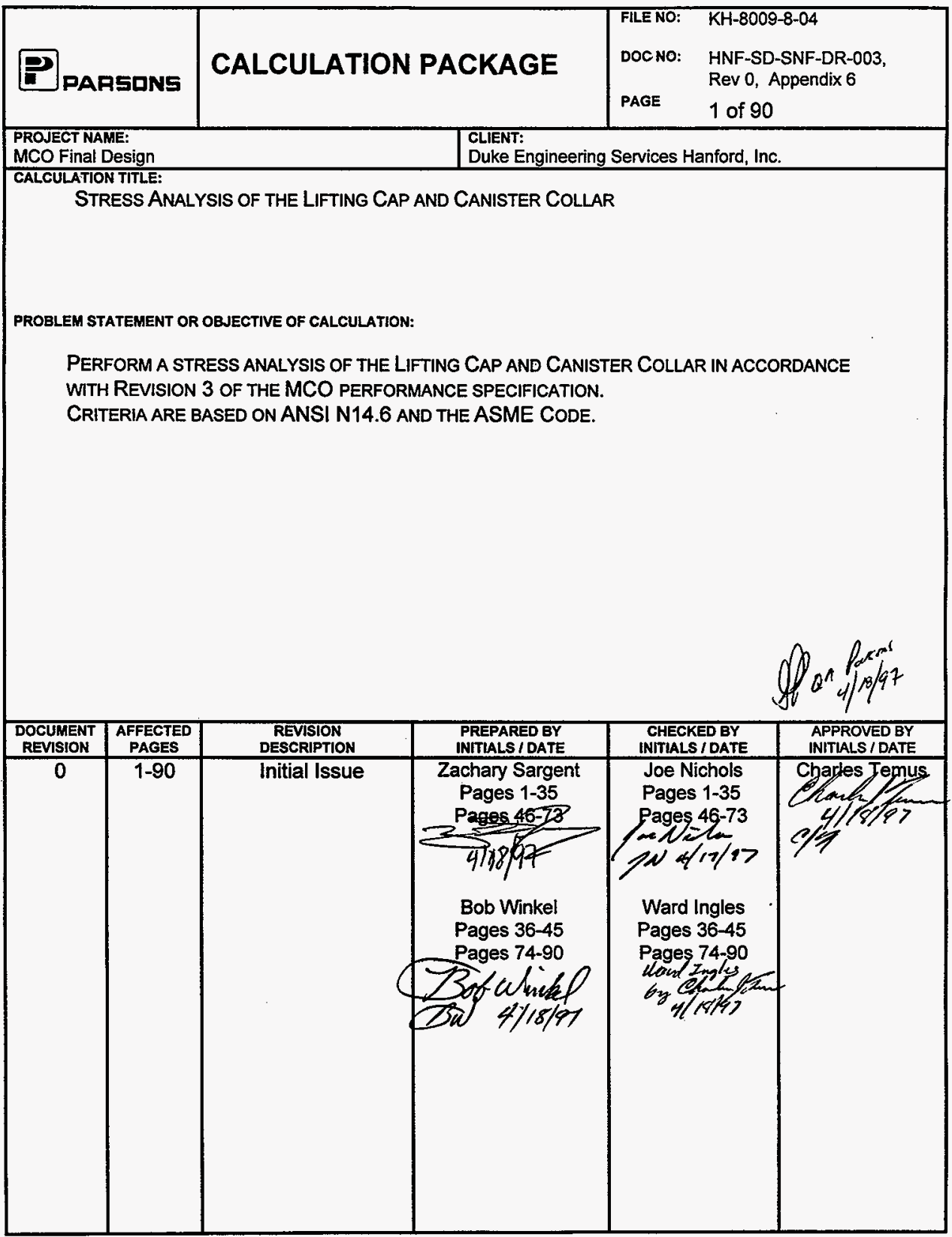


CLIENT: DUKE ENGINEERING SERVICES HANFORD, INC

FILE NO: $\quad \mathrm{KH}-8009-8-04$

PROJECT: MCO Final Design DOC. NO.: HNF-SD-SNF-DR-003, Rev. 0, Appendix 6

\section{CONTENTS}

Page

CONTENTS

LIST OF TABLES

LIST OF FIGURES

APPENDICES

1. INTRODUCTION 5

2. REFERENCES

3. ASSUMPTIONS 6

4. GEOMETRY 6

5. MATERIAL PROPERTIES 12

6. ACCEPTANCE CRITERIA

6.1 LIFTING LOADS

6.2 PREssure LoAds

6.3 WELD

7. LOAD CONDITIONS \& COMBINATIONS

14

8. STRESS ANALYSIS - HAND CALCULATIONS

14

8.1 LIFTING LOAD

8.2 PRESSURE LOAD

8.3 LIFTING AND PRESSURE LOADS

9. ANSYS@ ANALYSIS

9.1 STRESS ANALYSIS

10. DETAILED THREAD/CLOSURE EVALUATION

10.1 ANALYSIS DISCUSSION

10.2 ANALYSIS RESULTS

10.3 THREAD/CLOSURE ANALYSIS CONCLUSIONS 


\section{$\underline{\text { LIST OF TABLES }}$}

$\underline{\text { Page }}$

Table 1: ASME Code Material Properties for Type 304L Stainless Steel

Table 2: Level A Allowables

Table 3: Canister Collar Thread Relief Hand Calculation Results

Table 4: Hand vs. ANSYS Results

Table 5: ANSYS Results -- Pressure + Lifting

Table 6: ANSYS Results - Pressure + Lifting with Modifications

Table 7: ANSYS Models Stress Report Sections

\section{LIST OF FIGURES}

Page

Figure 1: Lifting Cap Top View

Figure 2: Lifting Cap With Gripping Shoe Configuration

Figure 3: Lifting Cap Cross Section

Figure 4: Canister Collar Cross Section

Figure 5: Modified Lifting Cap

Figure 6: Lifting Cap-Canister Collar Interface

Figure 7: Oblique and Isometric Views of ANSYSC model

Figure 8: Oblique and Isometric Views of ANSYS Model with Modified Cap 20

Figure 9: Detail of ANSYS Model Modified Cap 21

Figure 10: Top View of ANSYS@ model With Boundary Conditions 22

Figure 11: ANSYSC Model With Boundary Conditions 23

Figure 12: ANSYS Model with Modified Cap and Boundary Conditions 24

Figure 13: ANSYSC Model Lifting Area $\quad 26$

Figure 14: Stress Intensities - Front View 30

Figure 15: Stress Intensities -- Back View 31

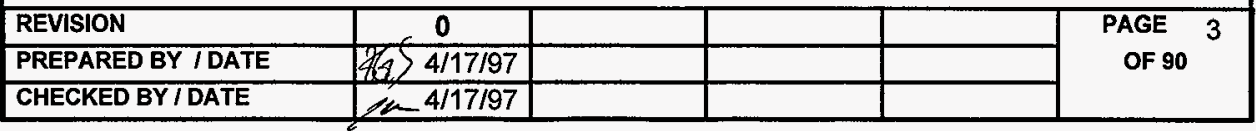


Figure 16: Stress Intensities --Top View

Figure 17: Stress Intensities in Lifting Ear

Figure 18: Front and Back ANSYS Model with Modified Cap and Stress Intensities

Figure 19: ANSYS Model Stress Intensity Details-Top View and Center

Figure 20: Thread Detail Geometry

Figure 21: Finite Element Model of MCO Thread/Closure Region

Figure 22. Stress Intensity Contours, Bottom Threads

Figure 23. Equivalent Plastic Strain Contours, Bottom Threads

Figure 24: Shell Load Distribution at the Closure Threads

Figure 25: Radial Displacement Contours, MCO Closure Thread Region

\section{APPENDICES}

Appendix A: Computer Run Output Sheets \& Input File Listings 
PROJECT: MCO Final Design DOC. NO.: HNF-SD-SNF-DR-003, Rev. 0, Appendix 6

\section{INTRODUCTION}

The canister collar is welded to the shell to provide a sealing surface for the shield plug seal and has a double lead buttress thread for positioning of the locking ring. After Hot Conditioning of the MCO is completed, a cap (referenced here as the lifting cap) is placed over the shield plug and welded to the canister collar. This cap is designed to accommodate a lifting grapple with six gripping shoes. The cap may be modified to include a rupture disc and cover flange.

This calculation documents the evaluation of the lifting cap with and without modifications, and canister collar under lifting and pressure loads. It also documents the evaluation of the weld at the lifting cap-canister collar interface. The evaluations are performed based on the criteria of the ASME Code and ANSI N14.6. A combination of hand calculations and ANSYSC analysis is used.

\section{REFERENCES}

1. "Performance Specification for the Spent Nuclear Fuel Multi-Canister Overpack," Specification HNF-S-0426, Revision 3, February 1997

2. ASME Boiler and Pressure Vessel Code, Section II - Materials, Part D - Properties, 1995 Edition with 1995 Addenda.

3. "American National Standard for Radioactive Materials - Special Lifting Devices for Shipping Containers Weighing 10000 Pounds (4500 kg) or More", ANSI N14.6-1993, American National Standards Institute, New York.

4. Roark, Raymond J., \& Young, Warren C., "Formulas for Stress and Strain", 5th Edition, McGraw-Hill Book Company, New York, 1975.

5. ASME Boiler and Pressure Vessel Code, Section III, Subsection NG - Material, 1995 Edition with Addenda

6. Duke Engineering \& Services Hanford, Inc, Specifications Drawings, Drawing H-2828042, Sheets 1,2 and 3, Revision C.

7. Swanson Analysis System, Inc., ANSYS@ Engineering Analysis System User's Manual, Volumes I, II and III, Version 5.0A, 23 December 1992. 
CLIENT: DUKE ENGINEERING SERVICES HANFORD, INC

PROJECT: MCO Final Design DOC. NO.: HNF-SD-SNF-DR-003, Rev. 0, Appendix 6

8. "Buttress Inch Screw Threads- $7^{\circ} / 45^{\circ}$ Form With 0.6 Pitch Basic Height of Thread Engagement," ANSI B1.9 - 1973, American Society of Mechanical Engineers, New York, New York.

9. Green, R. E. and McCauley, C. J., Machinery's Handbook, 25th Edition, Industrial Press, New York, New York, 1996.

\section{ASSUMPTIONS}

1. Pressure is applied uniformly

2. Others as noted

\section{GEOMETRY}

Figures 1 through 6 show the primary components analyzed and their interface. 
CLIENT: DUKE ENGINEERING SERVICES HANFORD, INC

FILE NO: KH-8009-8-04

PROJECT: MCO Final Design DOC. NO.: HNF-SD-SNF-DR-003, Rev. 0, Appendix 6

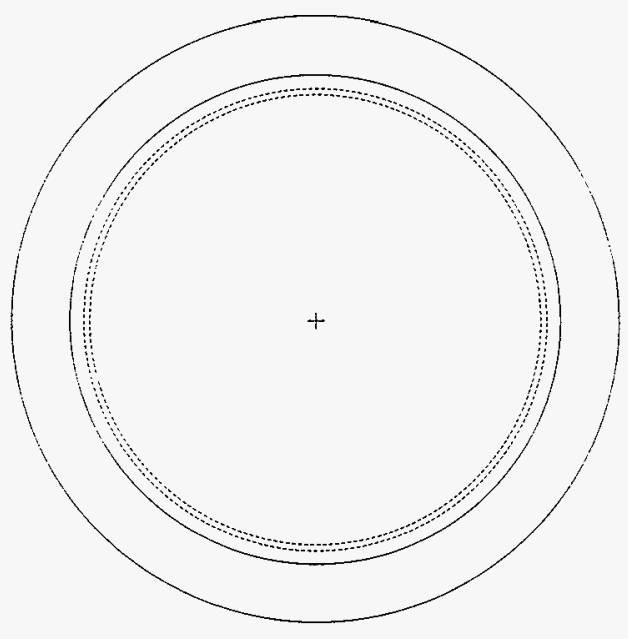

Figure 1: Lifting Cap Top View 


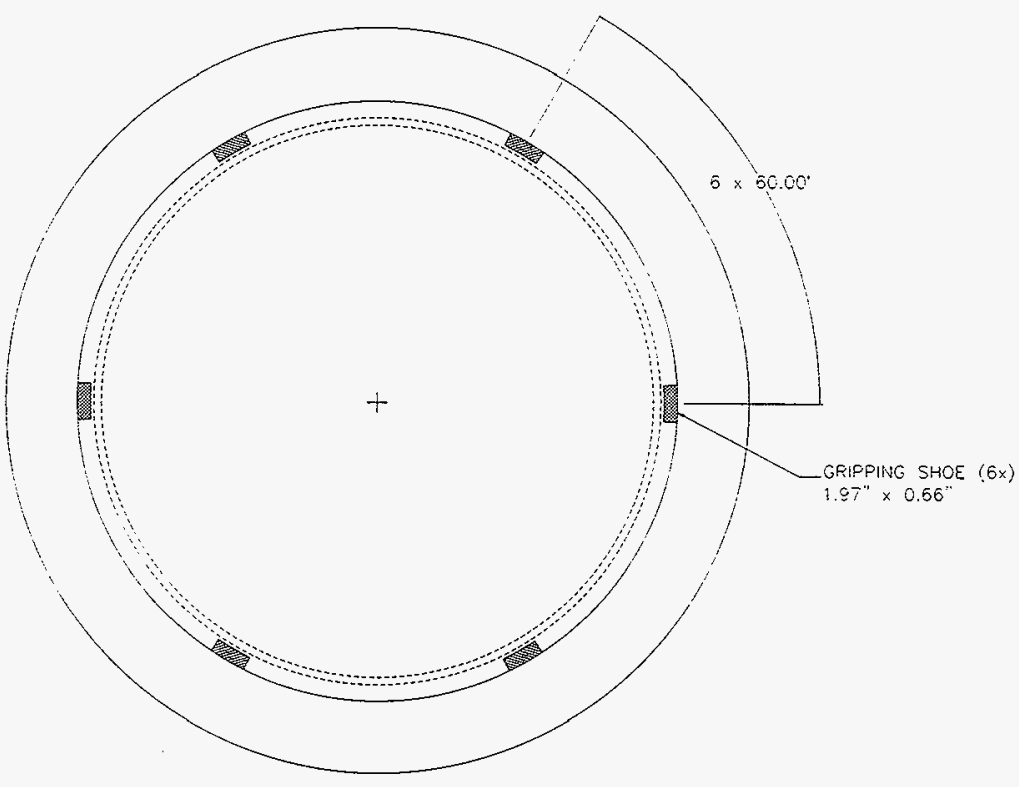

Figure 2: Lifting Cap With Gripping Shoe Configuration

\begin{tabular}{|c|l|l|l|}
\hline 0 & & & \\
\hline $2 \times 54 / 17 / 97$ & & & \\
\hline $2 \times 4 / 17 / 97$ & & & \\
\hline
\end{tabular}


CLIENT: DUKE ENGINEERING SERVICES HANFORD, INC

FILE NO: $\quad \mathrm{KH}-8009-8-04$

PROJECT: MCO Final Design DOC. NO.: HNF-SD-SNF-DR-003, Rev. 0, Appendix 6

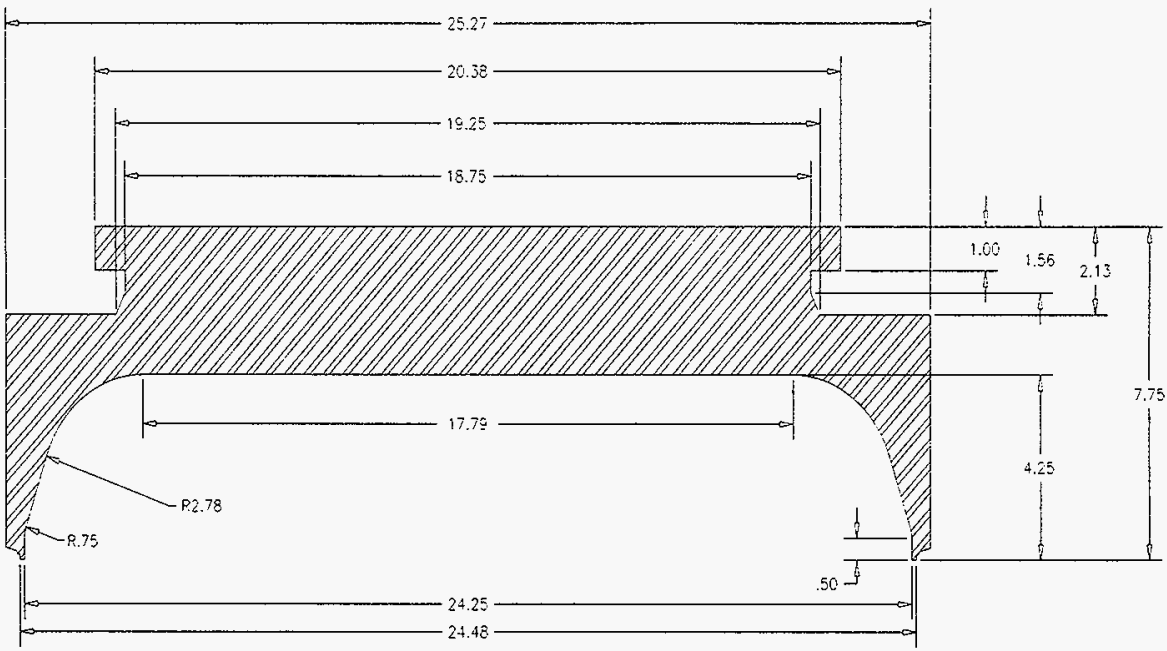

Figure 3: Lifting Cap Cross Section

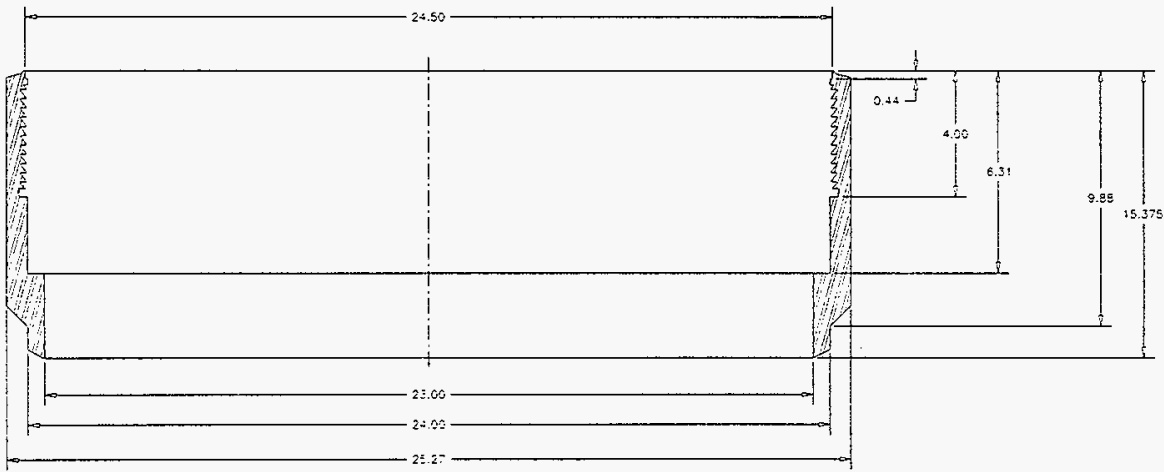

Figure 4: Canister Collar Cross Section

\begin{tabular}{|c|l|l|l|}
\hline 0 & & & \\
\hline $27 / 54 / 17 / 97$ & & & \\
\hline$/ 24 / 17 / 97$ & & & \\
\hline
\end{tabular}


CLIENT: DUKE ENGINEERING SERVICES HANFORD, INC

FILE NO: $\quad \mathrm{KH}-8009-8-04$

PROJECT: MCO Final Design DOC. NO.: HNF-SD-SNF-DR-003, Rev. 0, Appendix 6

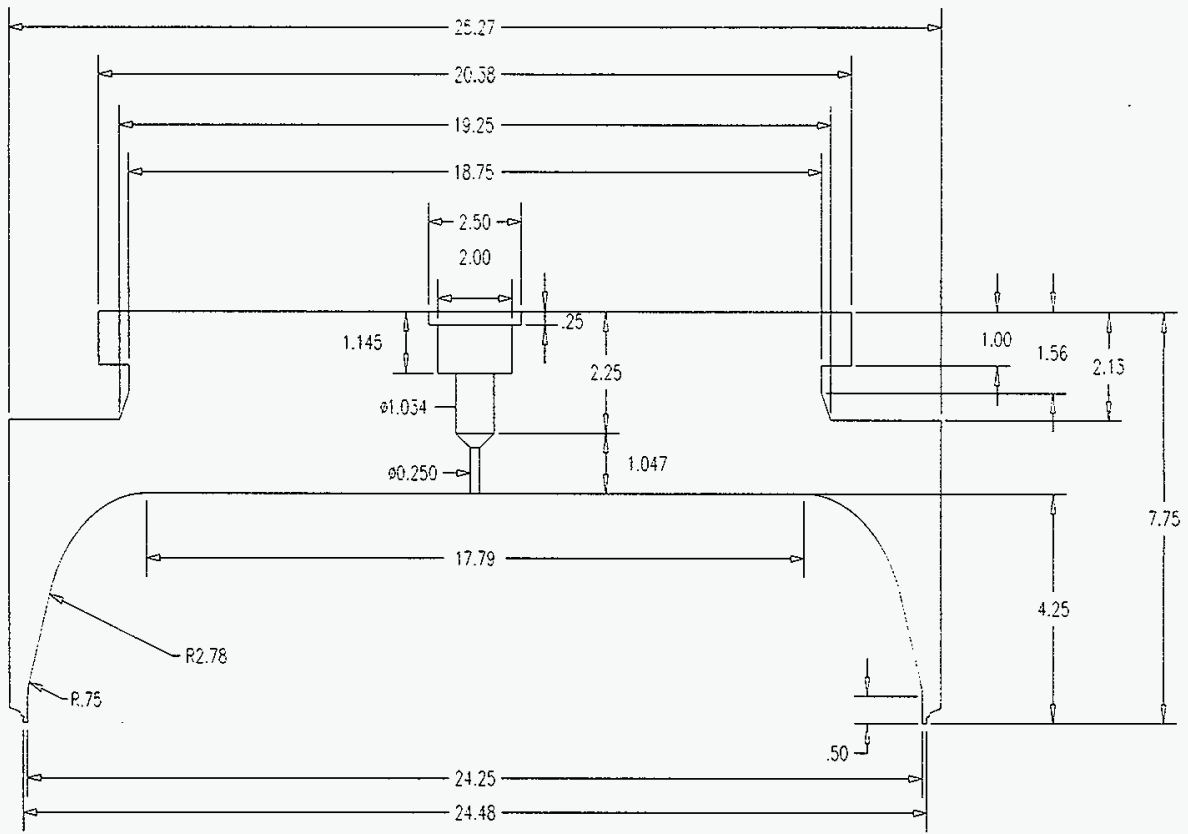

Figure 5: Modified Lifting Cap 
CLIENT: DUKE ENGINEERING SERVICES HANFORD INC

FILE NO: $\quad \mathrm{KH}-8009-8-04$

PROJECT: MCO Final Design DOC. NO.: HNF-SD-SNF-DR-003, Rev. 0, Appendix 6

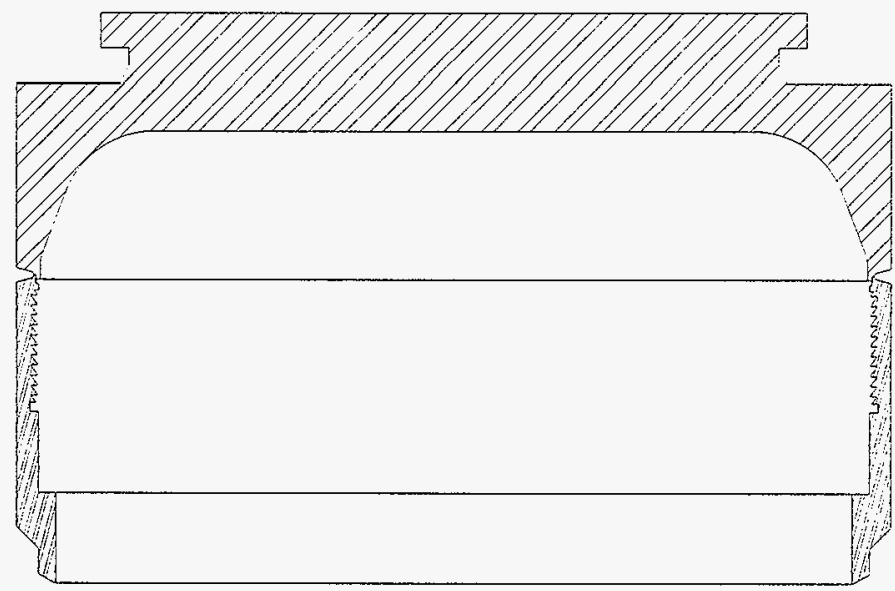

Figure 6: Lifting Cap-Canister Collar Interface

One must note that the interface with the modified lifting cap is the same as shown in Figure 6.

\begin{tabular}{|c|c|c|c|}
\hline 0 & & & \\
\hline (16) $4 / 17 / 97$ & & & \\
\hline / $-4 / 17 / 97$ & & & \\
\hline
\end{tabular}


CLIENT: DUKE ENGINEERING SERVICES HANFORD, INC

FILE NO: $\mathrm{KH}-8009-8-04$

PROJECT: MCO Final Design DOC. NO.: HNF-SD-SNF-DR-003, Rev. 0, Appendix 6

\section{MATERIAL PROPERTIES}

Both lifting cap and canister collar are fabricated from Type 304L stainless steel. For this analysis, only elastic modulus and allowable stress values are needed. Values are taken from Section II, Part D of the Code (See [2]) and are listed in Table 1.

\section{Table 1: ASME Code Material Properties for Type 304L Stainless Steel}

\begin{tabular}{|c|c|c|c|c|c||}
\hline \multicolumn{2}{|c|}{ Temperature } & \multicolumn{1}{|c|}{$\begin{array}{c}\mathrm{E}^{1} \\
\left(\times 10^{6} \mathrm{psi}\right)\end{array}$} & $\begin{array}{c}\mathrm{S}_{\mathrm{y}}{ }^{2} \\
(\mathrm{ksi})\end{array}$ & $\begin{array}{c}\mathrm{S}_{\mathrm{m}}{ }^{3} \\
(\mathrm{ksi})\end{array}$ & $\begin{array}{c}\mathrm{S}_{\mathrm{u}}{ }^{4} \\
(\mathrm{ksi})\end{array}$ \\
\hline${ }^{\circ} \mathrm{F}$ & ${ }^{\circ} \mathrm{C}$ & 25.0 & 16.7 & 70.0 \\
\hline-20 & -29 & 28.3 & 25.0 & 16.7 & 70.0 \\
\hline 70 & 21 & $28.1^{5}$ & 25.0 & 16.7 & 70.0 \\
\hline 100 & 38 & 27.6 & 21.3 & 16.7 & 66.2 \\
\hline 200 & 93 & 27.2 & 19.8 & 16.7 & 62.5 \\
\hline 270 & 132 & 27.0 & 19.1 & 16.7 & 60.9 \\
\hline 300 & 149 & 26.5 & 17.6 & 15.9 & 58.7 \\
\hline 392 & 200 & 26.5 & 17.5 & 15.8 & 58.5 \\
\hline 400 & 204 & 25.8 & 16.3 & 14.8 & 57.8 \\
\hline 500 & 260 & 25.3 & 15.5 & 14.0 & 57.0 \\
\hline 600 & 316 & 24.8 & 14.9 & 13.5 & 56.2 \\
\hline 700 & 371 & 24.8 & 14.9 & 13.5 & 56.2 \\
\hline 707 & 375 & 24.1 & 14.4 & 13.0 & 55.5 \\
\hline 800 & 427 & & & & \\
\hline
\end{tabular}

1 Table TM-1, Material Group G, P. 614

${ }^{2}$ Table Y-1, P. 524

${ }^{3}$ Table 2A, P. 322

${ }^{4}$ Table U, P. 441

${ }^{5}$ Underlined values determined by inear interpolation, all others taken from ASME Code, Section It, Part D.

\begin{tabular}{|l|l|l|l|}
\hline 0 & & & \\
\hline $4 / 17 / 97$ & & & \\
\hline $4 / 17 / 97$ & & & \\
\hline
\end{tabular}


For the lifting cap and canister collar, Type 304L stainless steel may be replaced with Type $304 \mathrm{~N}$ or $304 \mathrm{LN}$ at the discretion of the designer. The effects of thermal conductivity, thermal expansion, minimum yield strength and minimum tensile strength have been evaluated and it is the conclusion of the preparer that by using $304 \mathrm{~N}$ or $304 \mathrm{LN}$ the minimum tensile and yield strengths are increased to $70 \mathrm{ksi}$ and $30 \mathrm{ksi}$, respectively, for each material, thereby decreasing the appropriate stress ratios of this calculation.

\section{ACCEPTANCE CRITERIA}

This calculation considers (1) lifting loads and (2) pressure loads. Criteria for each are described below

\subsection{Lifting Loads}

Per Section 4.11 of the MCO Specification (See [1]), the lifting cap design "shall be capable/configured for lifting the MCO with the same equipment described in Section 4.13." Section 4.13 of [1] describe such equipment as a "lifting ring with a 12 ton lifting capacity." Therefore the lifting cap shall have a lifting capacity of 12 ton. Furthermore "the lifting ring design must exhibit the safety factors (for non-critical lifts) required by the American National Standards Institute (ANSI) N14.6-1986: Special Lifting Devices for Shipping Containers Weighing 10,000 Pounds $(4500 \mathrm{~kg}$ ) or more." For non-critical lifts, Section 4.2.1.1 of N14.6 (See [3]) establishes factors safety of 3 against yield stress and 5 against tensile stress. Thus criteria of N14.6 is equivalent to an allowable stress of the lesser of $S_{y} / 3$ or $S_{4} / 5$. These allowables are applied to the "membrane plus bending" component of stress. At the maximum lifting temperature of $132^{\circ} \mathrm{C}$, the allowables are:

$$
\begin{aligned}
& \frac{S_{y}}{3}=\frac{19.8 \mathrm{ksi}}{3}=6.6 \mathrm{ksi} \\
& \frac{S_{u}}{5}=\frac{62.5 \mathrm{ksi}}{5}=12.5 \mathrm{ksi} \\
& \Rightarrow \text { use: } P_{m}+P_{b} \leq 6.6 \mathrm{ksi}
\end{aligned}
$$

\subsection{Pressure Loads}

Per Section 4.11 of [1], "the cap shall be capable of withstanding the pressure rating of 150 psig at $132^{\circ} \mathrm{C}$." The MCO specification does not provide criteria for the lifting cap and canister collar under these loads, thus the normal (Level A) condition criteria of Subsection NG will be used. For membrane and membrane plus bending stresses the allowable stresses of Table 2 are applied.

\begin{tabular}{|l|l|}
\hline REVISION & \\
\hline PREPARED BY I DATE & $($ (A) \\
\hline CHECKED BYI DATE & /P \\
\hline
\end{tabular}

\begin{tabular}{|l|l|l|l|}
\hline 0 & & & \\
\hline $4 / 17 / 97$ & & & \\
\hline $4 / 17 / 97$ & & & \\
\hline
\end{tabular}


CLIENT: DUKE ENGINEERING SERVICES HANFORD, INC

FILE NO: $\quad \mathrm{KH}-8009-8-04$

PROJECT: MCO Final Design

DOC. NO.: HNF-SD-SNF-DR-003, Rev. 0, Appendix 6

\section{$6.3 \quad$ Weld}

Per Section 4.17 of [1], "All MCO pressure boundary welds and welds bearing the fully loaded MCO must be designed for and pass $100 \%$ volumetric examination (x-rays of ultrasonic) per ASME requirements" and "All MCO fabricator pressure boundary welds shall be made in accordance with ASME Section III, Division I, NB-3350". Therefore the stress limits for full penetration groove weld shall not exceed the stress values for the base metal being joined and the allowables of Table 2 apply.

Table 2: Level A Allowables

\begin{tabular}{|c|c|c|c|c|}
\hline \multicolumn{2}{|c|}{ Temperature } & $\mathrm{S}_{\mathrm{m}}$ & \multicolumn{2}{c|}{ Design / Level A Stress Limits } \\
\hline${ }^{\circ} \mathrm{F}$ & ${ }^{\circ} \mathrm{C}$ & (From Table 1) & $\begin{array}{c}\mathrm{P}_{\mathrm{m}} \\
\left(\mathrm{S}_{\mathrm{m}}\right)\end{array}$ & $\begin{array}{c}\left(\mathrm{P}_{\mathrm{m}} \text { or } \mathrm{P}_{\mathrm{L}}\right)+\mathrm{P}_{\mathrm{b}} \\
\left(1.5 \mathrm{~S}_{\mathrm{m}}\right)\end{array}$ \\
\hline 270 & 132 & $16.7 \mathrm{ksi}$ & $16.7 \mathrm{ksi}$ & $25.1 \mathrm{ksi}$ \\
\hline
\end{tabular}

Note: Design \& Level A stress limits for NG-3221 \& NG-3222, respectively.

\section{LOAD CONDITIONS \& COMBINATIONS}

The MCO lifting cap and canister collar are evaluated for the following case:

1. Lifting of the $\mathrm{MCO}$ and contents while at $132^{\circ} \mathrm{C}$ and $150 \mathrm{psig}$. This loading is evaluated using criteria based on the safety factors incorporated in ANSI N-14.6 for the lifting cap. The canister collar and the weld at the cap-collar interface are evaluated using Subsection NG.

\section{STRESS ANALYSIS - HAND CALCULATIONS}

The lifting cap and canister collar are evaluated using hand calculations. Since there are no practical hand calculations that may verify the stresses incurred in the lifting ear due to lifting, the following sections (8.1 thru 8.3) are merely a proof that the pressure and lifting loads were applied properly in the ANSYSC analysis. The analysis is described in the

\begin{tabular}{|l|c|l|l|l|l|}
\hline REVISION & 0 & & & & PAGE 14 \\
OF 90
\end{tabular}


CLIENT: DUKE ENGINEERING SERVICES HANFORD, INC

PROJECT: MCO Final Design DOC. NO.: HNF-SD-SNF-DR-003, Rev. 0, Appendix 6

following sections.

\subsection{Lifting Load}

The lifting cap must support the total weight of the $\mathrm{MCO}$ and contents for lifting. Per Section 4.13 of [1], the lifting cap must also have a total lifting capacity of 12 ton $(24,000 \mathrm{lb}$.). A lifting grapple with six (6) gripping shoes will be used to lift the MCO and its contents by the lifting cap. Figure 2 displays the gripping shoe configuration for the lifting grapple - lifting cap interface. The analysis presented here will cover only a $60^{\circ}$ sector $\left(360^{\circ} / 6\right.$ shoes).

Each gripping shoe will carry a weight $W$ of

$$
P=\frac{24000 \mathrm{lb}}{6}=4000 \mathrm{lbs}
$$

The outer diameter of the MCO shell is $24.00 \mathrm{in}$. and the inner diameter is $23.00 \mathrm{in}$. Therefore the cross sectional area of the shell is

$$
\begin{aligned}
& A_{\text {shell }}=\frac{\pi\left(D_{o}^{2}-D_{i}^{2}\right)}{4}=\frac{\pi\left(24.00^{2}-23.00^{2}\right)}{4} \\
& A_{\text {shell }}=36.92 \mathrm{in}^{2}
\end{aligned}
$$

and the area of the section is

$$
A_{\text {section }}=\frac{36.92 \mathrm{in}^{2}}{6}=6.16 \mathrm{in}^{2}
$$

Therefore the stress through the shell due to the lifting load is

$$
\sigma_{L}=\frac{P}{A_{\text {section }}}=\frac{4000}{6.16}=649 \mathrm{psi}
$$

The thinnest point in the shell is located at the thread relief in the canister collar. Since it will also see the lifting load through its section, it is analyzed.

The outer diameter of the MCO shell at the canister collar is 25.27 inches and the inner diameter at the base of the threads is 24.572 inches. The cross-sectional area of the shell through that section is

\begin{tabular}{|c|c|c|c|}
\hline 0 & & & \\
\hline X/1 4/17/97 & & & \\
\hline /N 4/17/97 & & & \\
\hline
\end{tabular}


CLIENT: DUKE ENGINEERING SERVICES HANFORD, INC

$$
A_{\text {collar }}=27.32 \mathrm{in}^{2}
$$

and the area of the section is

$$
A_{\text {collar section }}=4.55 \mathrm{in}^{2}
$$

Therefore the stress through the canister collar due to the lifting load is

$$
\sigma_{\text {collar }}=\frac{P}{A_{\text {collar sec cion }}}=\frac{4000}{4.55}=879 \mathrm{psi}
$$

\subsection{Pressure Load}

As stated in Section 6.2 above, the lifting cap must be able to withstand an internal pressure of 150 psig. The MCO shell has a thickness of 0.5 in. and its inside diameter is $23.00 \mathrm{in}$. The stress through the shell due to the pressure load is then

$$
\sigma_{P}=\frac{p R}{t}
$$

where $p=$ internal pressure $=150 \mathrm{psig}$

$$
\begin{aligned}
& R=\text { mean radius }=(24.00+23.00) / 4=11.75 \mathrm{in} . \\
& t=\text { thickness of } M C O \text { shell }=0.5 \mathrm{in} .
\end{aligned}
$$

Therefore

$$
\sigma_{P}=\frac{(150)(11.75)}{0.50}=3525 \text { psi or } 3.53 \mathrm{ksi}
$$

At its thinnest point, the shell has a thickness of 0.349 inches (with tolerancing) at the canister collar buttress thread relief. The stress through the collar due to the pressure is then:

$$
\begin{aligned}
& R=(25.27+24.572) / 4=12.46 \mathrm{in} . \\
& \sigma_{P}=\frac{(150)(12.46)}{0.349}=5355 \mathrm{psi} \text { of } 5.36 \mathrm{ksi}
\end{aligned}
$$

\begin{tabular}{|c|l|l|l|}
\hline 0 & & & \\
\hline $4 / 17 / 97$ & & & \\
\hline $4 / 17 / 97$ & & & \\
\hline
\end{tabular}


CLIENT: DUKE ENGINEERING SERVICES HANFORD, INC

FILE NO: $\quad \mathrm{KH}-8009-8-04$

PROJECT: MCO Final Design DOC. NO.: HNF-SD-SNF-DR-003, Rev. 0, Appendix 6

\subsection{Lifting and Pressure Loads}

When both lifting and pressure loads are applied together, the stress through the shell due to these loads then becomes

$$
\begin{aligned}
& \sigma_{L+P}=\frac{p R}{2 t}+\frac{P}{A_{\text {sec tion }}}+\frac{p R}{t} \\
& \sigma_{L+P}=1763+649+3525=5937 \mathrm{psi}
\end{aligned}
$$

The stress through the thinnest point, due to lifting and pressure, in the collar becomes

$$
\begin{aligned}
\sigma_{\text {THINEST }} & =\frac{p R}{2 t}+\frac{P}{A_{\text {COLLARSECTION }}}+\frac{p R}{t} \\
\sigma_{\text {THINNEST }} & =2678+879+5355=8912 \mathrm{psi}
\end{aligned}
$$

Since the allowed stress is $25.1 \mathrm{ksi}$, the stress ratio is, as shown in Table $3,0.36$. Since the weld at the collar-lifting cap interface can bo no deeper than 0.349 inches, and is subject to Subsection NG of the ASME, $t$

Table 3: Canister Collar Thread Relief Hand Calculation Results

\begin{tabular}{|c|c|c|c|c|}
\hline Stress & Stress Category & Allowed & $\begin{array}{c}\text { Section } 8 \\
\text { Results }\end{array}$ & Ratio \\
\hline$\sigma_{\mathrm{L}}$ & $\mathrm{P}_{\mathrm{M}}$ & $16.7 \mathrm{ksi}$ & $879 \mathrm{psi}$ & 0.05 \\
\hline$\sigma_{\mathrm{HOOP}}$ & $\mathrm{P}_{\mathrm{M}}$ & $16.7 \mathrm{ksi}$ & $5355 \mathrm{psi}$ & 0.32 \\
\hline$\sigma_{\mathrm{L}+\mathrm{P}}$ & $\mathrm{P}_{\mathrm{M}}$ & $16.7 \mathrm{ksi}$ & $8912 \mathrm{psi}$ & 0.53 \\
\hline
\end{tabular}

Table 3 is a compilation of hand calculation results of Section 8.1 through 8.3 for the thinnest section at the canister collar.

\begin{tabular}{|l|l|l|}
\hline & & \\
\hline & & \\
\hline & & \\
\hline
\end{tabular}




\section{ANSYSO ANALYSIS}

in addition to the hand calculations described in Section 8 , an evaluation of the lifting cap and canister is performed using the finite element model shown in Figure 7. The model is a 3-D, $60^{\circ}$ section and is developed with SOLID45 elements with 3 degrees of freedom at each node. Figure 7 offers 2 views of the section for better viewing.

Furthermore, a modified version of the lifting cap is also evaluated using ANSYS.

The model is a $3-D, 60^{\circ}$ section and is developed with SOLID45 elements with 3 degrees of freedom at each node. The modifications consists of a hole with chamfers to accommodate a rupture disc and cover flange, located at the center of the lifting cap. Figure 5 is a drawing with dimensions of such model. Figure 8 offers 2 views of the section for better viewing. Figure 9 offers a close-up of the lifting cap modifications. 
CLIENT: DUKE ENGINEERING SERVICES HANFORD, INC

FILE NO: $\quad \mathrm{KH}-8009-8-04$

PROJECT: MCO Final Design DOC. NO.: HNF-SD-SNF-DR-003, Rev. 0, Appendix 6

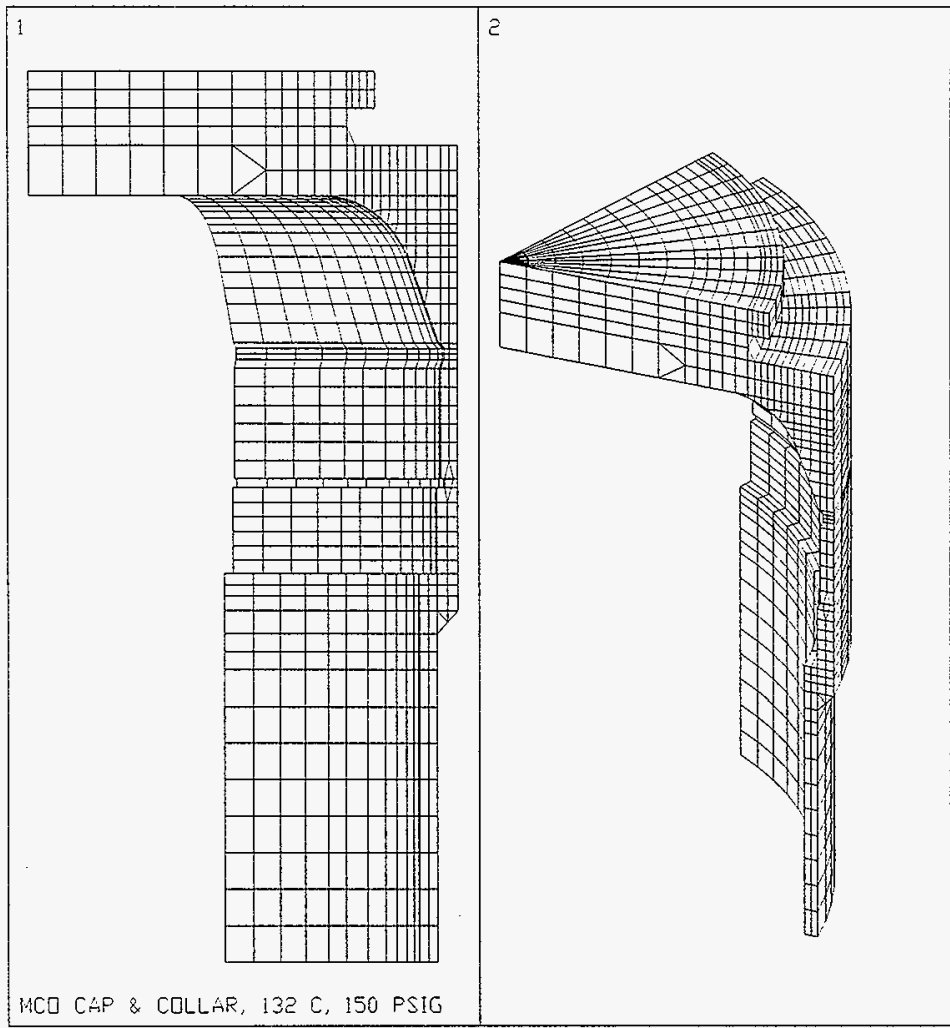

ANSIS 50 i 56

Felo 23 ig9.

$18: 32: 36$

PLOT NO. 1

ELEMENTS

TYPE NUM

$\underline{Z} \mathrm{~V}=1$

DIST $=13.888$

$X F=6.313$

$Y F=4.158$

$Z F=-5.467$

PRECISE HIDDEN

$W[N D=2$

$X_{V}^{\prime}=0.267261$

$Y Y=0.534522$

$Z Y=0.801784$

DIST $=16.978$

$Y F=6.313$

$Y F=4.158$

$Z F=-5.46^{-}$

PRECISE HIDDEI.

Figure 7: Oblique and Isometric Views of ANSYS $\odot$ model

\begin{tabular}{|l|c|c|c|c|c|}
\hline REVISION & 0 & & & & PAGE \\
\hline PREPARED BY IDATE & $\not{f} / 2 / 4 / 17 / 97$ & & & OF 90 \\
\hline CHECKED BY/DATE & $\not \mathscr{N} 4 / 17 / 97$ & & & & \\
\hline
\end{tabular}


CLIENT: DUKE ENGINEERING SERVICES HANFORD, INC

FILE NO: KH-8009-8-04

PROJECT: MCO Final Design DOC. NO.: HNF-SD-SNF-DR-003, Rev. 0, Appendix 6

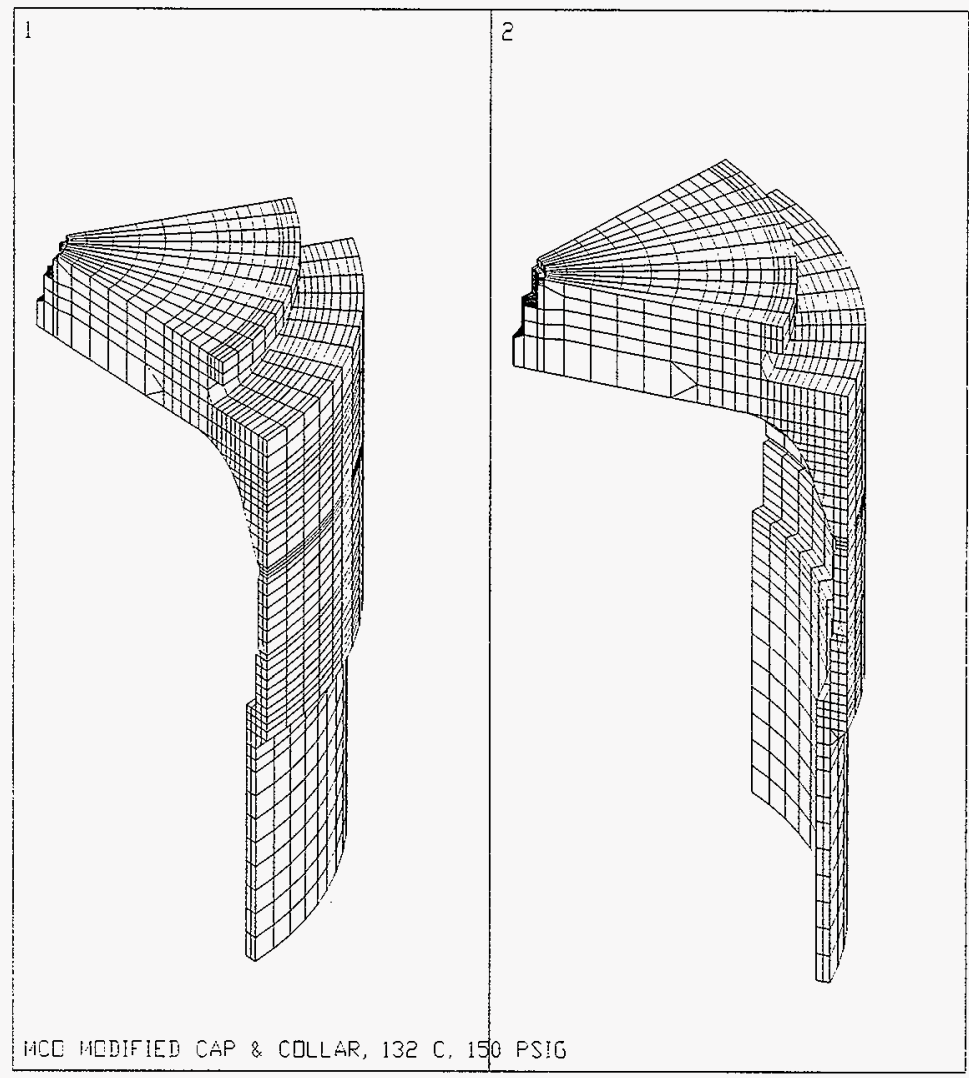

SiNSYS $5.0 \div 56$

HirR $18 \quad 199-$

$14: 02: 25$

PLOT MO. 1

ELEHENTS

TYPE NUM

i. $=1$

$\because=1$

$\exists \because=1$

DIST $=18.276$

$\therefore F=6.344$

if $=4.221$

$Z F=-5.467$

FRECISE HIDDEN

* $[M \bar{D}=5$

, $=1$

$\therefore \quad=2$

Z: $=3$

EIST $=16.913$

$X F=6.344$

iF $=4.221$

IF $=-5.467$

PRECISE HIDDEN

Figure 8: Oblique and Isometric Views of ANSYS Model with Modified Cap

\begin{tabular}{|l|c|l|l|l|l|}
\hline REVISION & 0 & & & & PAGE 20 \\
OF 90
\end{tabular}




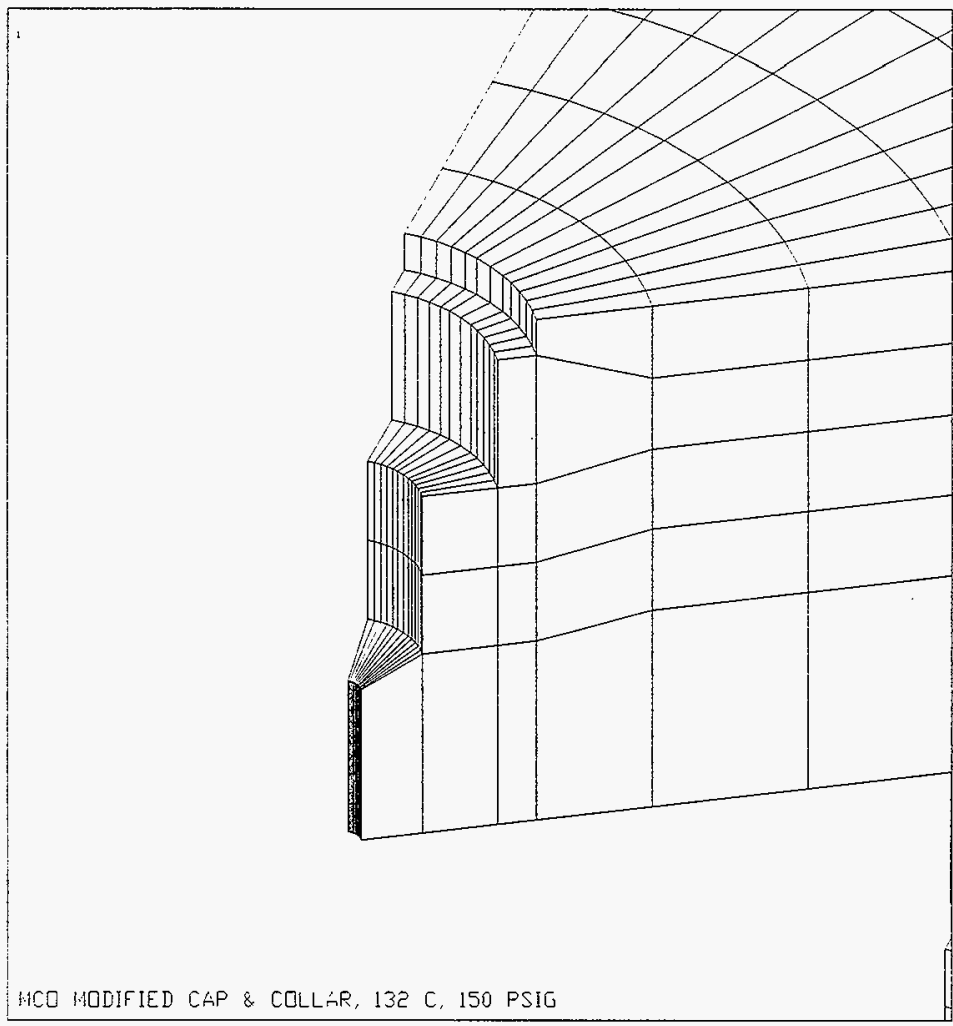

ANSYS 50 A 56 Nin 18 199:

13:10:10

FLOT NO. 2

ELEMENTS

TYPE NUM

$X_{V}=-0.633975$

$Y_{Y}=2$

$Z Y=3.098$

*DIST $=2.971$

$* X F \quad=2.796$

*YF $=9.084$

$* Z F \quad=-9.332$

PRECISE HIDDEN

Figure 9: Detail of ANSYS Model Modified Cap

\begin{tabular}{|c|c|c|c|}
\hline 0 & & & \\
\hline$z f_{2} 24 / 17 / 97$ & & & \\
\hline f/ $4 / 17 / 97$ & & & \\
\hline
\end{tabular}




\subsection{Stress Analysis}

\subsubsection{Boundary Conditions}

A $60^{\circ}$ section of the lifting cap and canister collar is modeled using ANSYS 5.0A Finite Element Analysis. Symmetry boundary conditions are applied at the edges of the models (See Figures $8 \& 9$ for lifting cap without modifications and Figures $10 \& 11$ for lifting with modifications). The bottom edge is fixed in the vertical direction to approximate the lifting configuration.
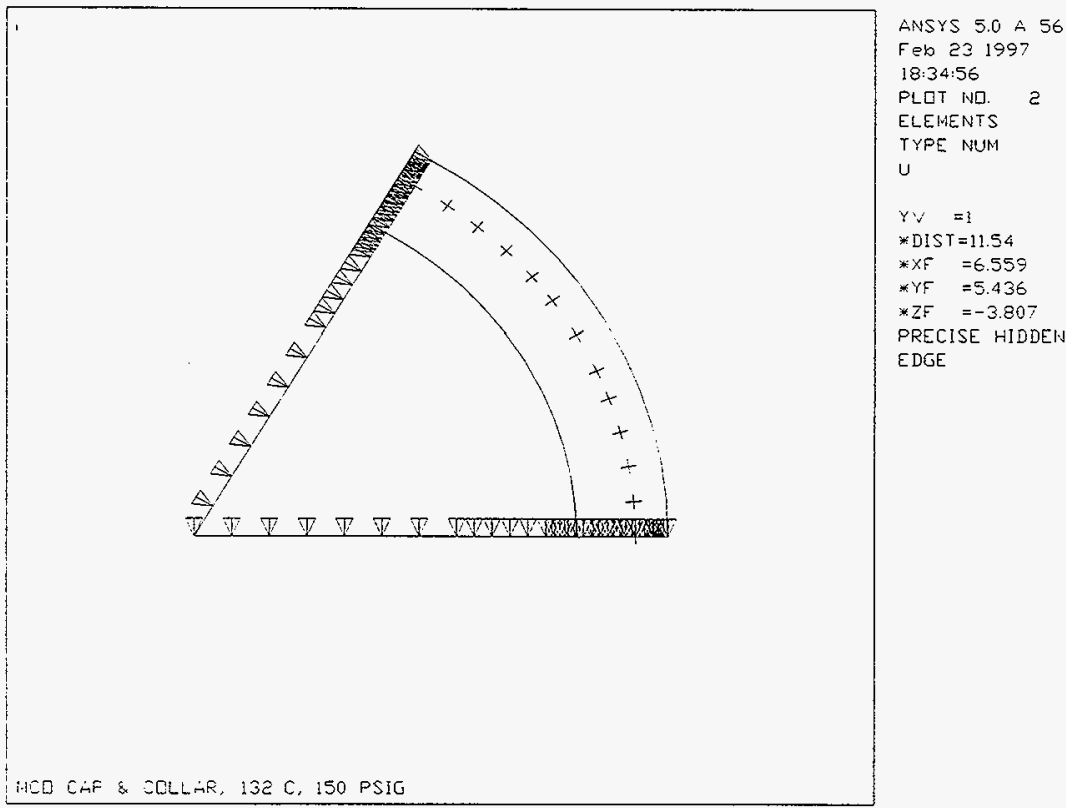

Figure 10: Top View of ANSYSC model With Boundary Conditions

\begin{tabular}{|l|l|l|l|}
\hline 0 & & & \\
\hline $4 / 17 / 97$ & & & \\
\hline $4 / 17 / 97$ & & & \\
\hline
\end{tabular}


P.

CLIENT: DUKE ENGINEERING SERVICES HANFORD, INC

FILE NO: KH-8009-8-04
DOC. NO.: HNF-SD-SNF-DR-003, Rev. 0, Appendix 6

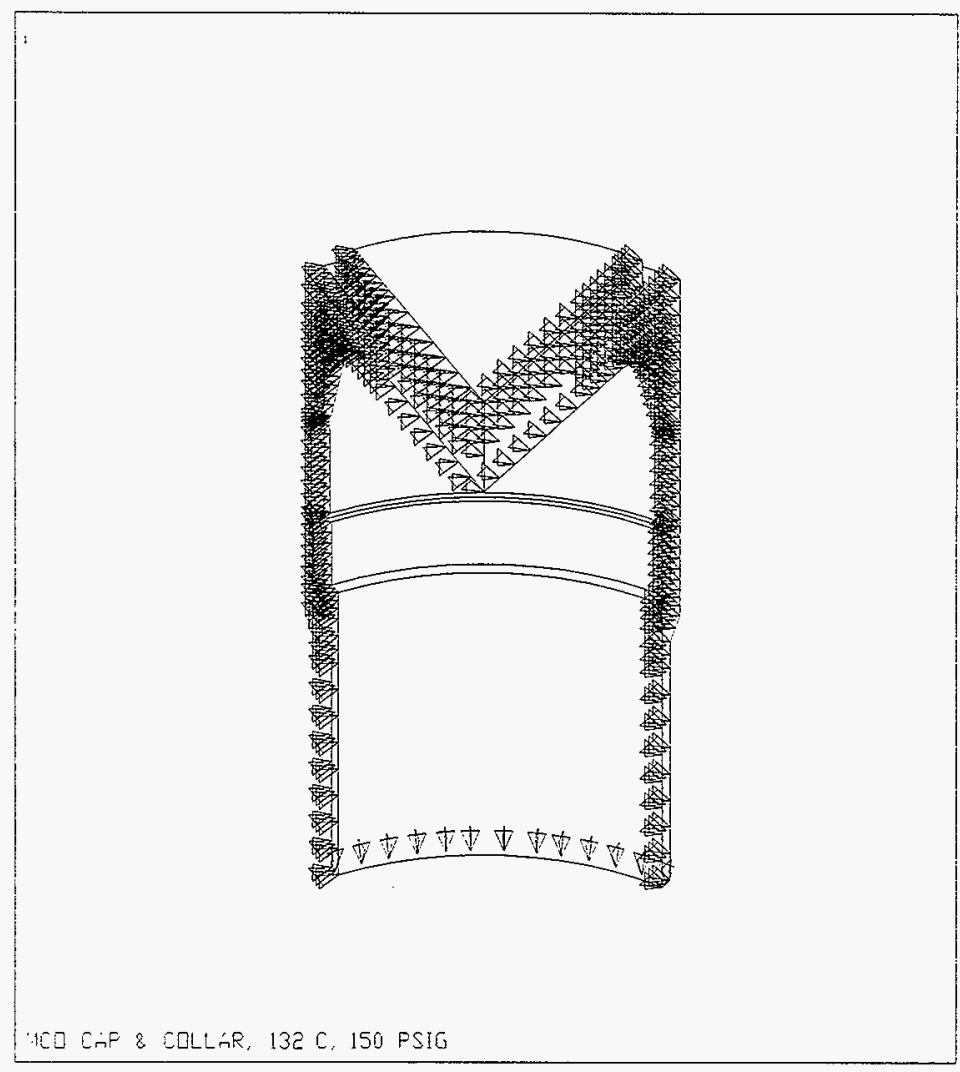

nilsis $5.0+56$

Feb 23199 ?

18:34:09

FLOT NO. 1

ELEWENTS

TYPE NUM

$U$

$x y=-2.639$

$\because y=5$

$I \because \quad=1.742$

\#DIST $=16.636$

$* \times F=7.619$

\#YF $=5.436$

$* Z F=-4.954$

PRECISE HIDDEN

EDGE

Figure 11: ANSYS@ Model With Boundary Conditions

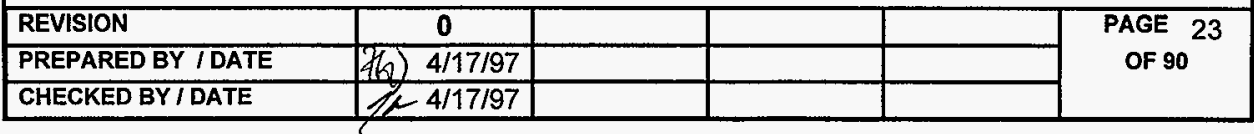


CLIENT: DUKE ENGINEERING SERVICES HANFORD, INC

FILE NO: KH-8009-8-04

PROJECT: MCO Final Design DOC. NO.: HNF-SD-SNF-DR-003, Rev. 0, Appendix 6

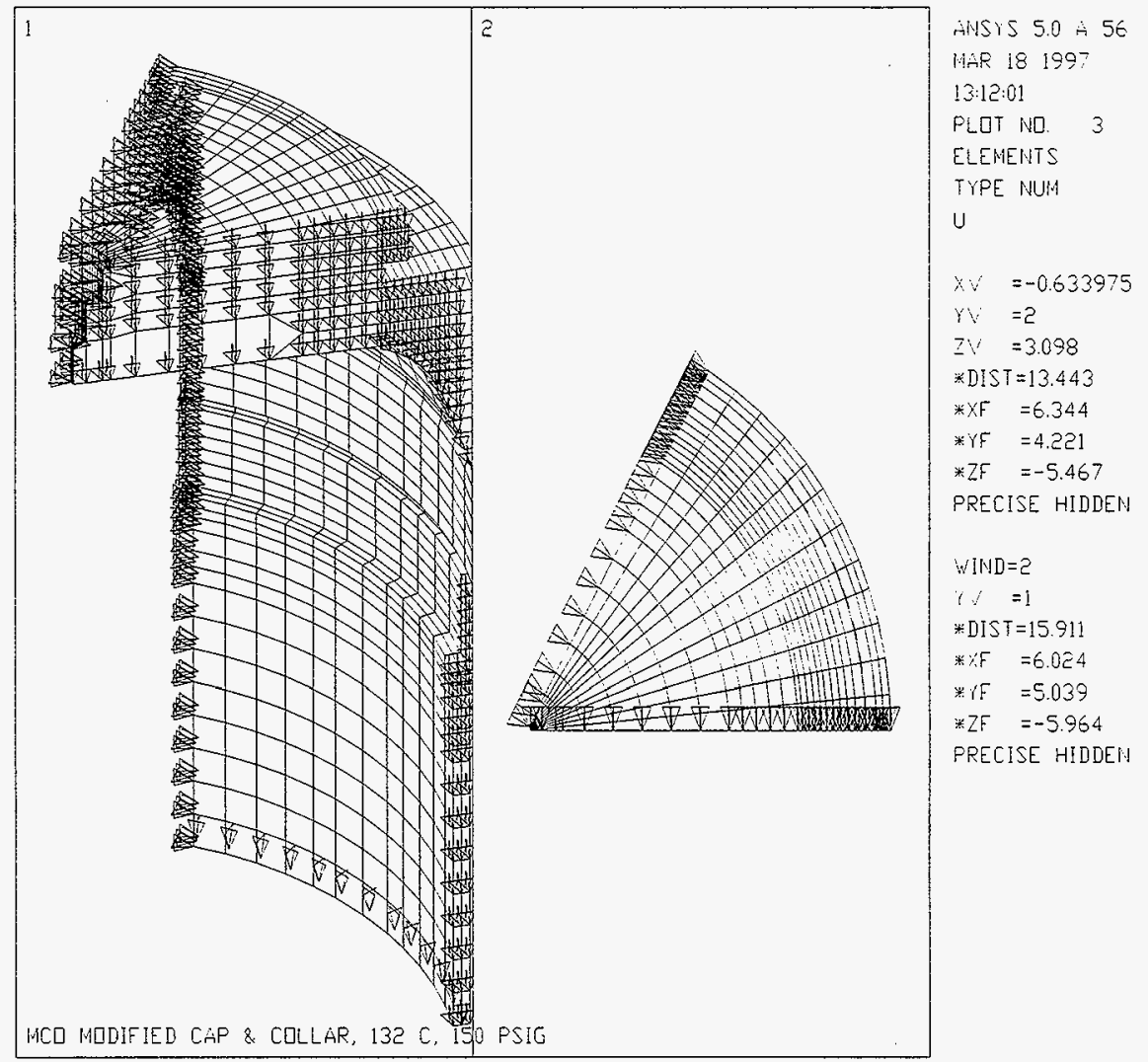

Figure 12: ANSYS Model with Modified Cap and Boundary Conditions

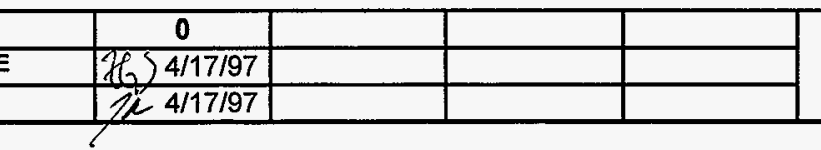




\subsubsection{Loading}

Two loads are applied to the models. A 150 psi pressure is applied uniformly to the inside of the model . In order to apply the lifting load properly, the model is built using a series of slices of different thicknesses and refining the mesh in the appropriate area. The intent of this is to apply the lifting load on few elements which represent the area of a gripping shoe. Per Section 4.13 of [1] "The shield plug shall feature an integrally machined axisymmetric lifting ring with a 12 ton lifting capacity when gripped with six equally spaced 1.97 " tangential length by 0.66 " radial contact length grippers". The area of the gripper is approximated to be $1.3 \mathrm{in}^{2}\left(1.97^{\prime \prime} \times 0.66^{\prime \prime}\right)$. Figure 13 shows the six elements representing that area. Since the griping shoe has an area of $1.3 \mathrm{in}^{2}$, the total load $\mathrm{F}$ applied for the $60^{\circ}$ section is

$$
\begin{aligned}
& A_{\text {shoe }}=1.3 \mathrm{in}^{2} \\
& P=4000 \mathrm{lbs} \text { (See Section 8.1) } \\
& F=\frac{P}{A_{\text {shoe }}}=\frac{4000}{1.3}=3077 \mathrm{psi}
\end{aligned}
$$

Since this pressure was applied on six elements of unequal areas, it is necessary to adjust the pressure accordingly. Two faces equal $0.4428 \mathrm{in}^{2}\left(0.2214 \mathrm{in}^{2}\right.$ each), two more equal $0.4332 \mathrm{in}^{2}\left(0.2166 \mathrm{in}^{2}\right.$ each), and finally two at $0.4234 \mathrm{in}^{2}\left(0.2117 \mathrm{in}^{2}\right)$. The two outermost faces will receive each:

$$
P_{\circ}=\frac{(.2166)}{(.2214)} \times(3077)=3010 p s i
$$

The two center faces will receive each:

$$
P_{c}=\frac{(.2166)}{(.2166)} \times(3077)=3077 p s i
$$

and the two innermost faces will receive each:

$$
P_{i}=\frac{(.2166)}{(.2117)} \times(3077)=3148 p s i
$$

Review of the stress analysis output file show that the reported reaction forces in the Zdirection for the lifting load only is $3997.10 \mathrm{lb}$ for cap without modifications and $3998.80 \mathrm{lb}$ 
CLIENT: DUKE ENGINEERING SERVICES HANFORD, INC

FILE NO: KH-8009-8-04

PROJECT: MCO Final Design

DOC. NO.: HNF-SD-SNF-DR-003, Rev. 0, Appendix 6

for cap with modifications. This is comparable to the value of $4000 \mathrm{lb}$. given above.

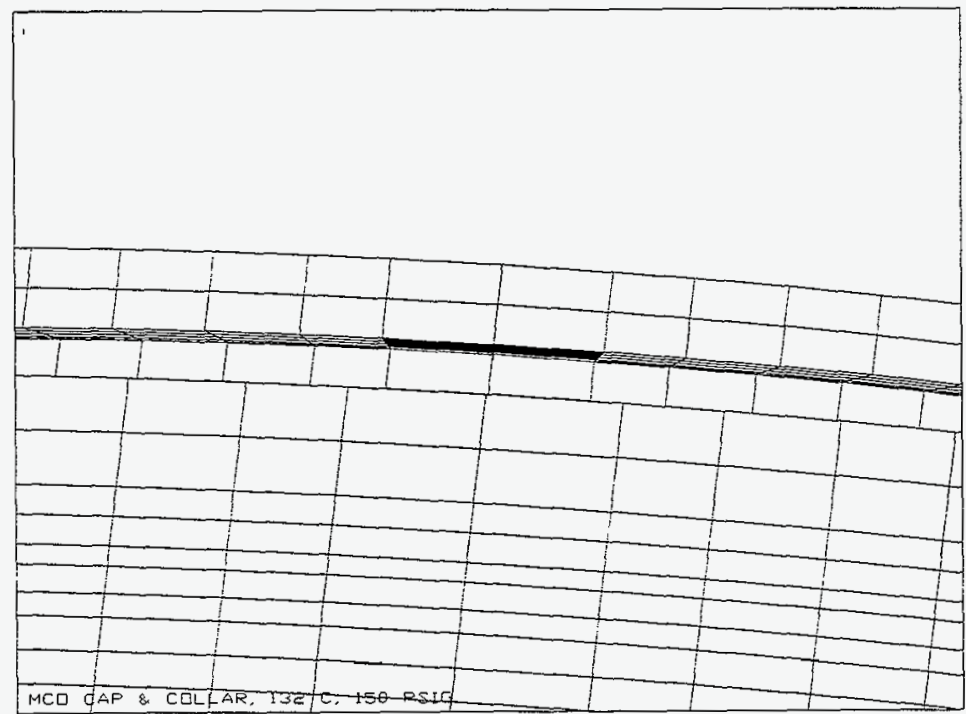

ANSYS 5.0 A 56

Feib 23199 ?

$18: 39: 49$

PLOT NO. I

ELEMENTS

TYPE NUIM

PRES.

$x V=0.852869$

$Y V=-0.150384$

$Z V=-0.5$

DIST $=4.327$

$* \times F \quad=8.558$

*YF $=15,125$

$\times Z F \quad=-4.919$

$A-Z S=-5.038$

PRECISE HIDDEN

Figure 13: ANSYSC Model Lifting Area

\subsubsection{Results}

The SOLID45 elements used for this analysis report stresses at the location of the gripping shoe, at the canister collar-lifting cap weld and at the rupture disc location. Stresses are classified as membrane plus bending stresses: $P_{m}+P_{B}$. Since ANSI standards limits these stresses to membrane plus bending, peak stresses are ignored.

Table 4 compares the calculated results of Sections 8.1 thru 8.3 to the ANSYS analysis results. ANSYS results were obtained using LPATH/PRSECT command. Stress results for several locations on the assembly are summarized and ratioed to the allowables in Tables 5 and 6. 
CLIENT: DUKE ENGINEERING SERVICES HANFORD, INC

FILE NO: $\mathrm{KH}-8009-8-04$

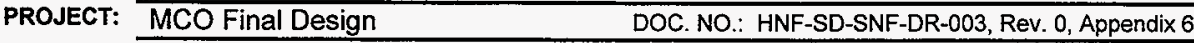

Table 4: Hand vs. ANSYS Results

\begin{tabular}{||c|c|c|c|c|c|c|c|}
\hline Stress & $\begin{array}{c}\text { Stress } \\
\text { Category }\end{array}$ & Allowed & $\begin{array}{c}\text { Section 8 } \\
\text { Results }\end{array}$ & $\begin{array}{c}\text { ANSYS } \\
\text { Results }\end{array}$ & Ratio & $\begin{array}{c}\text { ANSYS Results } \\
\text { (modified cap) }\end{array}$ & $\begin{array}{c}\text { Ratio (modified } \\
\text { cap) }\end{array}$ \\
\hline$\sigma_{\mathrm{L}}$ & $\mathbf{P}_{\mathrm{M}}$ & $16.7 \mathrm{ksi}$ & $649 \mathrm{psi}$ & $637.6 \mathrm{psi}$ & 0.04 & $637.9 \mathrm{psi}$ & 0.04 \\
\hline$\sigma_{\text {Hoop }}$ & $\mathbf{P}_{\mathrm{M}}$ & $16.7 \mathrm{ksi}$ & $3525 \mathrm{psi}$ & $3380 \mathrm{psi}$ & 0.20 & $3380 \mathrm{psi}$ & 0.20 \\
\hline$\sigma_{\mathrm{L}+\mathrm{P}}$ & $\mathbf{P}_{\mathrm{M}}$ & $16.7 \mathrm{ksi}$ & $2412 \mathrm{psi}$ & $2303 \mathrm{psi}$ & 0.14 & $2271 \mathrm{psi}$ & 0.14 \\
\hline
\end{tabular}

Ratio $=\frac{\text { ANSYS }}{\text { Allowed }}$

Table 5: ANSYS Results -- Pressure + Lifting

\begin{tabular}{|c|c|c|c|c|}
\hline \multirow{2}{*}{ Location } & \multirow{2}{*}{ Criteria } & \multicolumn{2}{|c|}{ Stress Intensities } & \multirow{2}{*}{ Ratio } \\
\cline { 3 - 4 } & & Maximum & $\begin{array}{c}\text { Allowed } \\
P_{M}+P_{B}\end{array}$ & \\
\hline Lifting Ear & ANSI N14.6 & $5.84 \mathrm{ksi}$ & $6.6 \mathrm{ksi}$ & 0.88 \\
\hline Lifting Cap Mid-Radius & ANSI N14.6 & $2.25 \mathrm{ksi}$ & $6.6 \mathrm{ksi}$ & 0.34 \\
\hline Weld & ASME Section NG & $7.32 \mathrm{ksi}$ & $25.1 \mathrm{ksi}$ & 0.29 \\
\hline Bottom of Collar & ASME Section NG & $5.24 \mathrm{ksi}$ & $25.1 \mathrm{ksi}$ & 0.21 \\
\hline
\end{tabular}

Although it is non-conservative to include pressure, the effect is small

\begin{tabular}{|c|c|c|c|c|}
\hline REVISION & $\mathbf{0}$ & & & \\
\hline PREPARED BY / DATE & 得) 4/17/97 & & & \\
\hline CHECKED BY / DATE & $4 / 17 / 97$ & & & \\
\hline
\end{tabular}




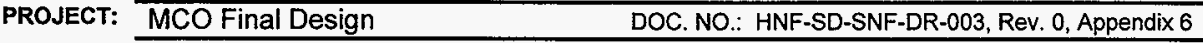

Table 6: ANSYS Results - Pressure + Lifting with Modifications

\begin{tabular}{|c|c|c|c|c|}
\hline \multirow{2}{*}{ Location } & \multirow{2}{*}{ Criteria } & \multicolumn{2}{|c|}{ Stress Intensities } & \multirow{2}{*}{ Ratio } \\
\cline { 3 - 4 } & & Maximum & $\begin{array}{c}\text { Allowed } \\
\mathrm{P}_{\mathrm{M}}+\mathrm{P}_{\mathrm{B}}\end{array}$ & \\
\hline Lifting Ear & ANSI N14.6 & $5.85 \mathrm{ksi}$ & $6.6 \mathrm{ksi}$ & 0.89 \\
\hline Lifting Cap Mid-Radius & ANSI N14.6 & $2.20 \mathrm{ksi}$ & $6.6 \mathrm{ksi}$ & 0.33 \\
\hline Weld & ASME Section NG & $7.09 \mathrm{ksi}$ & $25.1 \mathrm{ksi}$ & 0.28 \\
\hline Bottom of Collar & \\
\hline
\end{tabular}

On April 1,1997, the dimensions for the locking ring were revised. The most significant dimension change is increasing the reduction of the lifting ring thickness on the locking ring to $1.00+0 /-0.02$ inches. This reduction would increase the stress ratio to 0.924 . Although technically acceptable, increasing the stress ratio and reducing the margin on the MCO is not recommended. If other alternatives are available they should be investigated.

7 Although it is non-conservative to include pressure, the effect is small

\begin{tabular}{|l|c|l|l|l|}
\hline REVISION & 0 & & & \\
\hline PREPARED BY I DATE & fo/ $/ 4 / 17 / 97$ & & & \\
\hline CHECKED BYI DATE & $/ / 24 / 17 / 97$ & & & \\
\hline
\end{tabular}


Table 7: ANSYS Models Stress Report Sections

\begin{tabular}{|l|c|c|}
\hline \multicolumn{1}{|c|}{ Component } & Inside Node & Outside Node \\
\hline \multirow{4}{*}{ Top Plate } & 6002 & 6132 \\
\cline { 2 - 3 } & 6004 & 6134 \\
\cline { 2 - 3 } & 6004 & 6018 \\
\cline { 2 - 3 } & 6055 & 6015 \\
\cline { 2 - 3 } & 6056 & 6016 \\
\cline { 2 - 3 } & 6704 & 6700 \\
\cline { 2 - 3 } & 6705 & 6701 \\
\hline \multirow{3}{*}{ to Cylindrical } & 6057 & 6017 \\
\cline { 2 - 3 } & 6128 & 6096 \\
\hline Start Weld & 6170 & 6197 \\
\cline { 2 - 3 } & 6188 & 6197 \\
\hline Below Weld & 6245 & 6247 \\
\hline Bottom of Locking Ring Position & 6255 & 6257 \\
\hline Transition to Collar & 6285 & 6297 \\
\hline Lower collar & 6485 & 6487 \\
\hline
\end{tabular}

For the lifting cap without modifications, the corresponding ANSYS input and output files are NEWCAP.inp and NEWCAP.out, respectively.

For the lifting cap with modifications, the corresponding ANSYS input and output files are MODCAP.inp and MODCAP.out, respectively. 
CLIENT: DUKE ENGINEERING SERVICES HANFORD, INC

FILE NO: KH-8009-8-04

PROJECT: MCO Final Design DOC. NO.: HNF-SD-SNF-DR-003, Rev. 0, Appendix 6

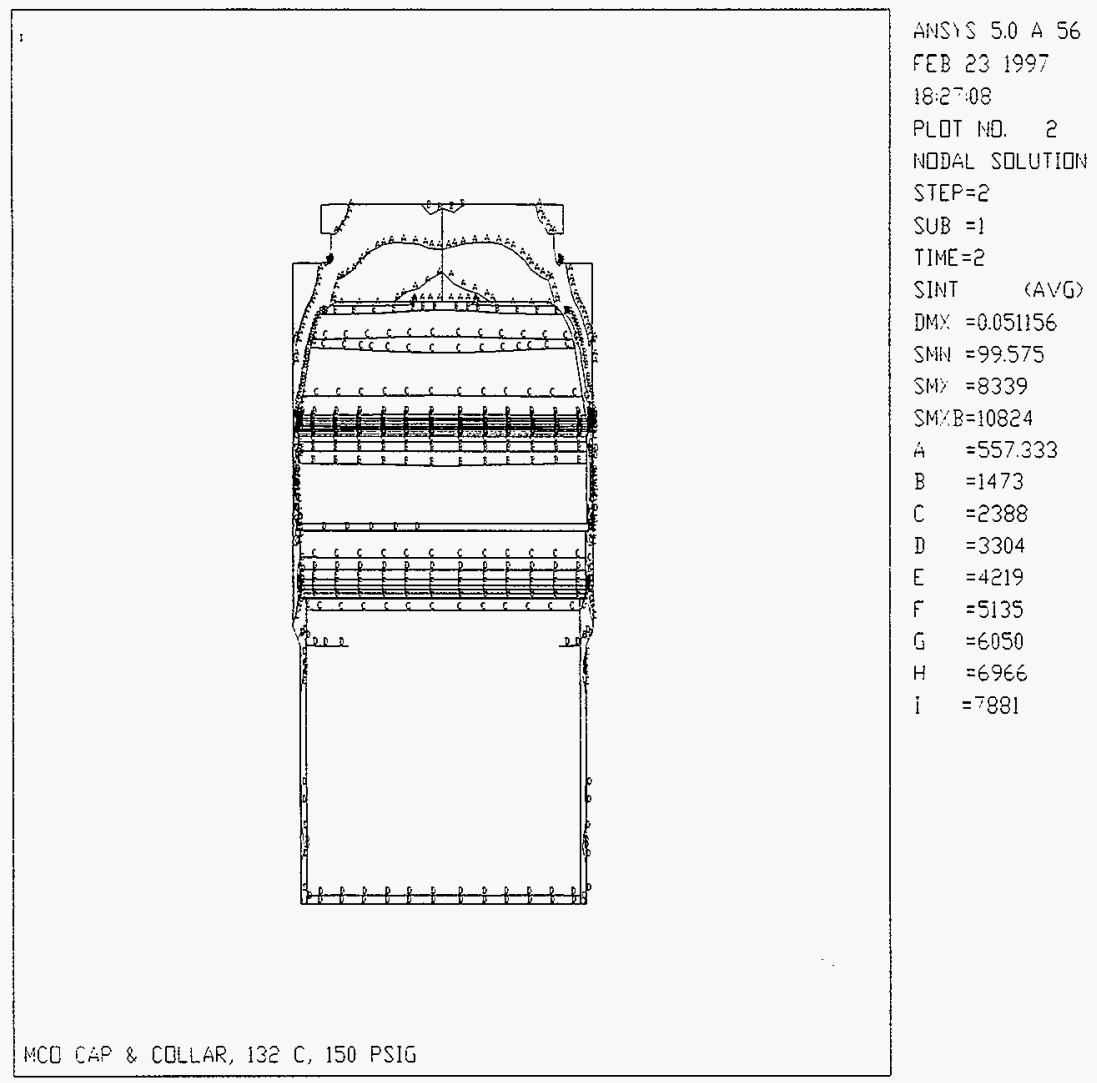

Figure 14: Stress Intensities -- Front View 
CLIENT: DUKE ENGINEERING SERVICES HANFORD INC

PROJECT: MCO Final Design
FILE NO: KH-8009-8-04

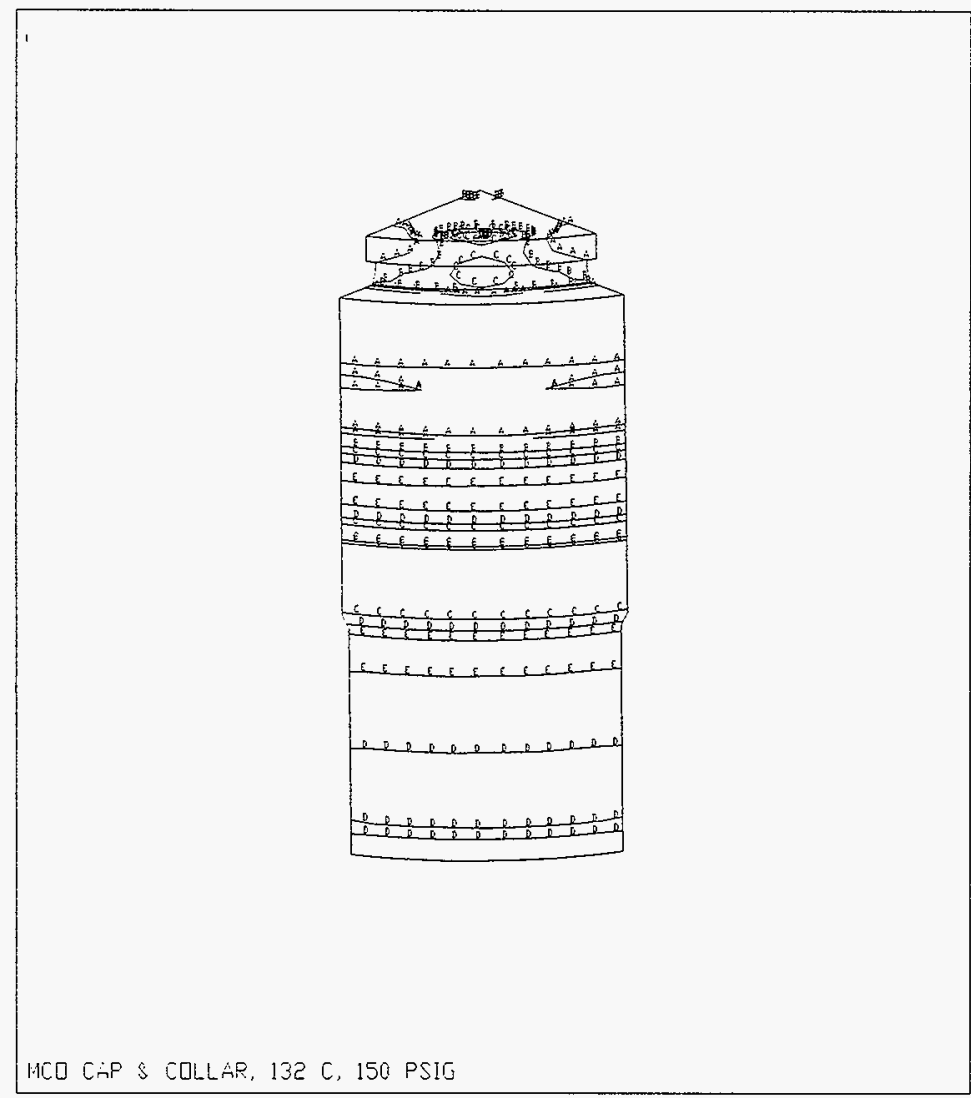

ANSYS $5.0+56$

FEB $23 \quad 1997$

18:31:54

PLOT ND. 4 NEDAL SDLUTION

$S T E P=2$

SUB $=1$

TIM $M=2$

SINT

DMX $=0.051156$

$\sin (N=99.575$

$\sin X=8339$

$\operatorname{Sin} \times B=10824$

$A \quad=557.333$

B $=1473$

C $=2388$

I) $=3304$

$E \quad=4219$

$F \quad=5135$

6 $=6050$

$\mathrm{H}=6966$

$\mathrm{I}=7881$

HCO CAP \& COLLAR, 132 C, 150 PSIT

Figure 15: Stress Intensities -- Back View

\begin{tabular}{|l|l|l|l|}
\hline 0 & & & \\
\hline $4 / 17 / 97$ & & & \\
\hline $4 / 17 / 97$ & & & \\
\hline
\end{tabular}


CLIENT: DUKE ENGINEERING SERVICES HANFORD, INC

FILE NO: KH-8009-8-04

PROJECT: MCO Final Design DOC. NO.: HNF-SD-SNF-DR-003, Rev. 0, Appendix 6

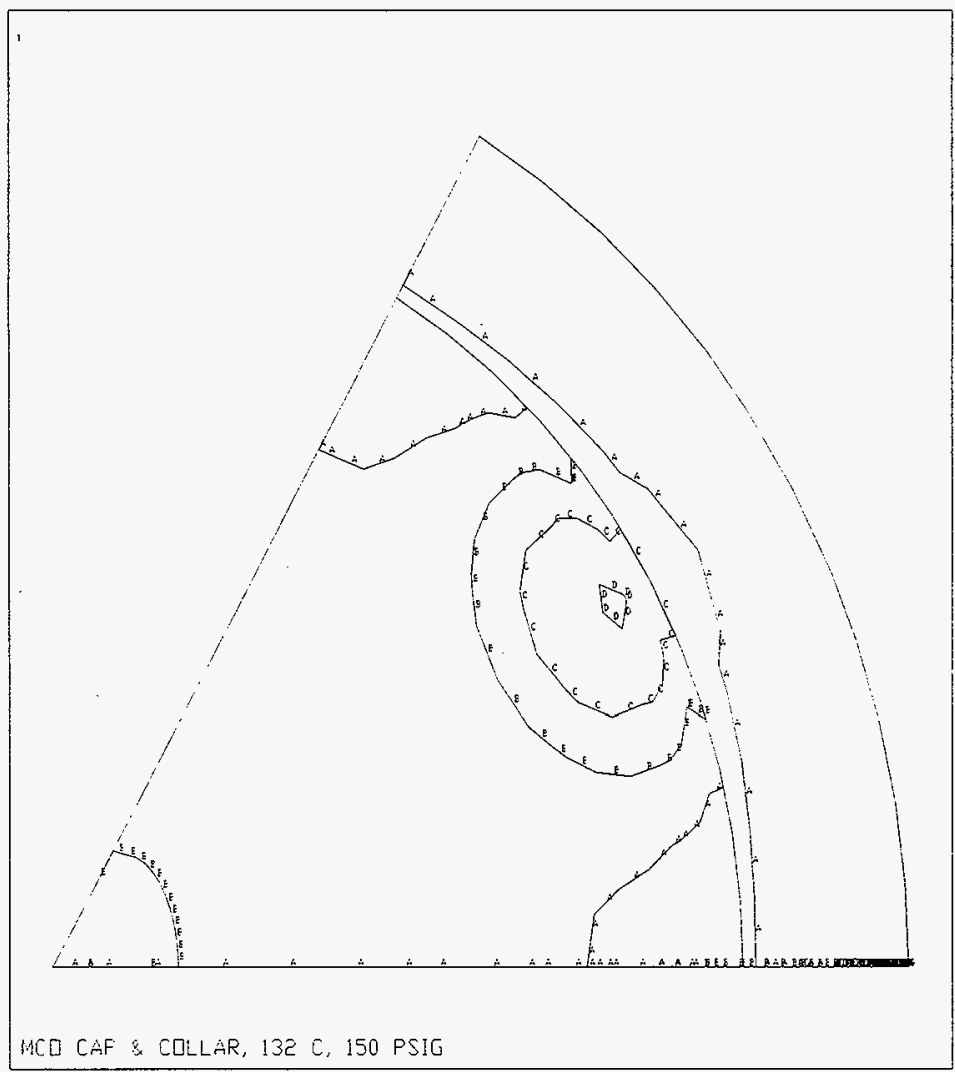

ANSYS 5.0 \& 56

FEB $23 \quad 1997$

$18: 25: 15$

PLGT NL. 1 NODAL SOLUTIDIN

STEP $=\hat{c}$

SUB $=1$

TIME =?

SINT (AVG)

DMY $=0.051156$

SiNN $=99.575$

$\operatorname{SMX}=8339$

$\operatorname{Sin} \times B=10824$

$A \quad=557.333$

B $\quad=1473$

C $=2388$

$\mathrm{D}=3304$

$E=4219$

$\mathrm{F}=5135$

[ $\quad=6050$

$H \quad=6966$

$\mathrm{I}=7881$

Figure 16: Stress Intensities --Top View 
CLIENT: DUKE ENGINEERING SERVICES HANFORD INC

PROJECT: MCO Final Design DOC. NO.: HNF-SD-SNF-DR-003, Rev. 0, Appendix 6

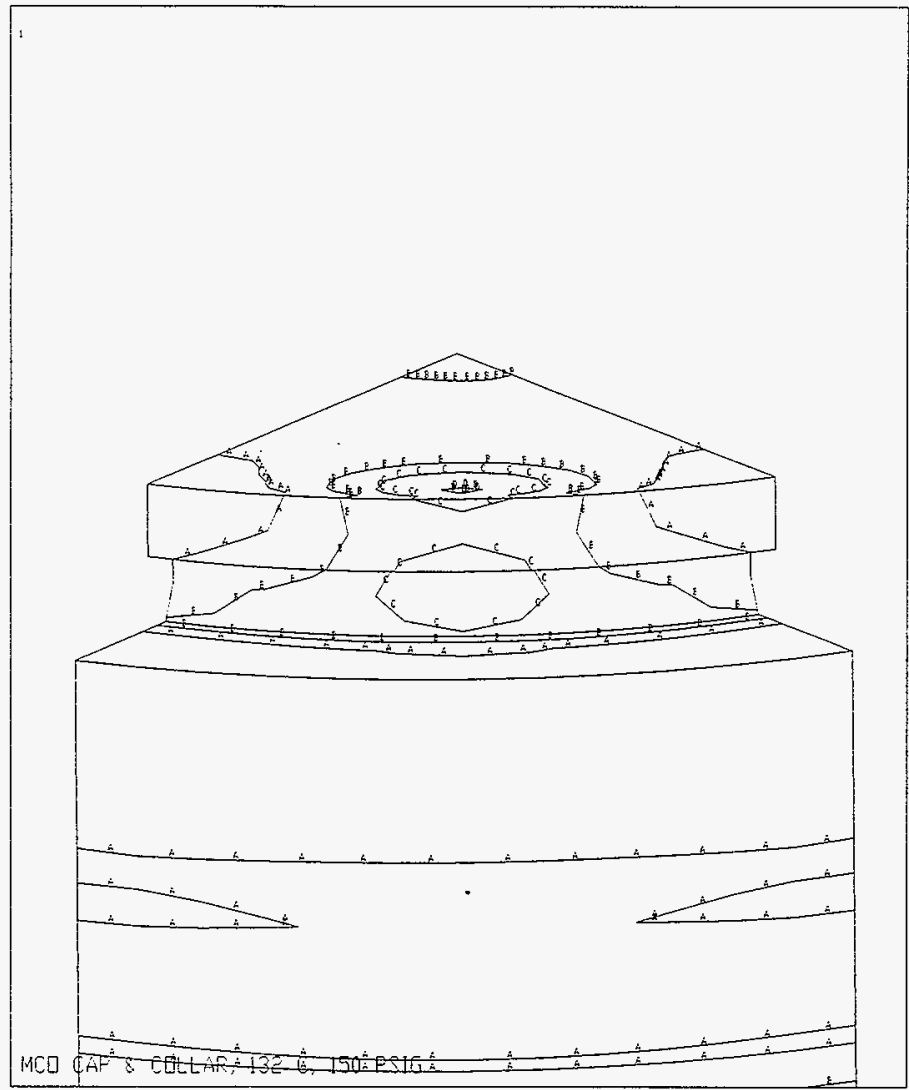

ANSIS 50 A 56

FEB 231997

18:30:19

PLOT NO. 3

NODAL SOLUTIOH

STEF $=2$

SUB $=1$

$T H E=?$

SINT

〈AVG)

DMX $=0.051156$

SMN $=99.575$

$S M Y=8339$

$S M X B=10824$

A $\quad=557.333$

$B=1473$

C $\quad=2388$

D $=3304$

$E=4219$

$F \quad=5135$

f) $=6050$

$H=6366$

I $=7881$

Figure 17: Stress Intensities in Lifting Ear 
CLIENT: DUKE ENGINEERING SERVICES HANFORD, INC

FILE NO: KH-8009-8-04

PROJECT: MCO Final Design DOC. NO.: HNF-SD-SNF-DR-003, Rev. 0, Appendix 6

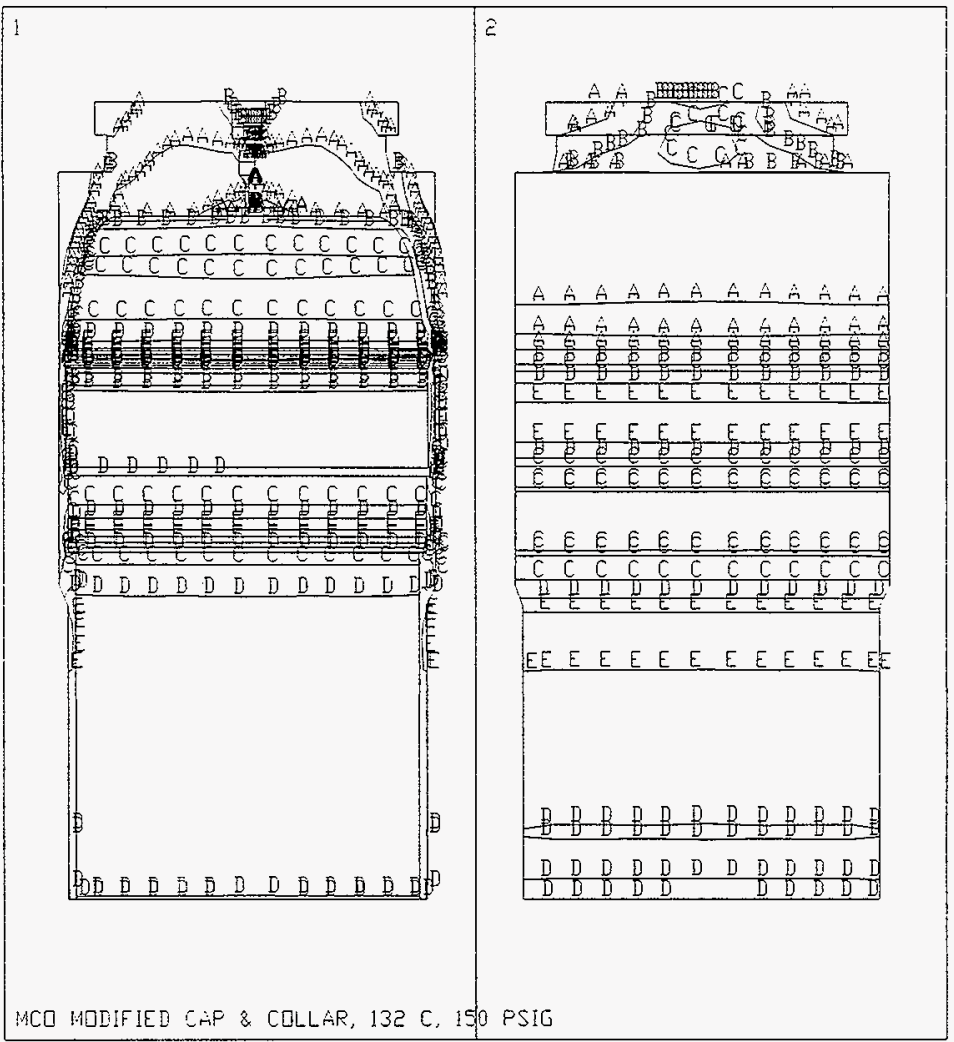

ANSYS $5.0 \rightarrow 56$

MIR 18 199:

15:08:25

PLOT HQ. 1

HODÁL SOLUTIOM

STEP $=2$

SUB $=1$

TIME $=2$

SINT

(AvG)

DMX $=0.051317$

SHN $=109.253$

SH. $1 \mathrm{X}=8092$

$S H \times B=10520$

$x y=-0.866025$

$Z Y=0.5$

*DIST $=16.805$

$\because F \quad=10.112$

$\because F \quad=4.22 \hat{C}$

$* 2 F \quad=-6.167$

$\therefore-Z S=0.854 \mathrm{E}-06$

PRECISE HIDDEIJ

EDGE

$\dot{A} \quad=552.718$

B $=\$ 440$

C $=2327$

D $=3214$

$E=4100$

$F \quad=4987$

G $=5874$

$\mathrm{H}=6761$

I $=7648$

Figure 18: Front and Back ANSYS Model with Modified Cap and Stress Intensities

\begin{tabular}{|c|}
\hline 0 \\
\hline $1 / 4 \sqrt{4 / 17 / 97}$ \\
\hline$/ 2-4 / 17 / 97$
\end{tabular}




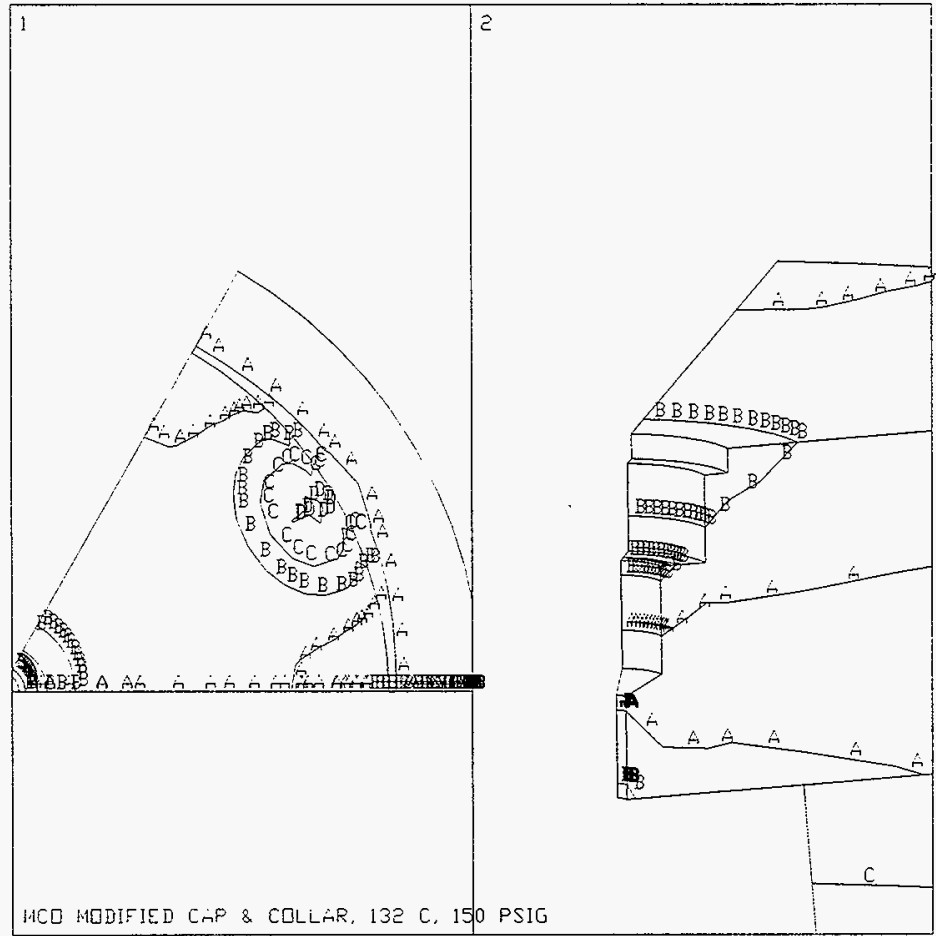

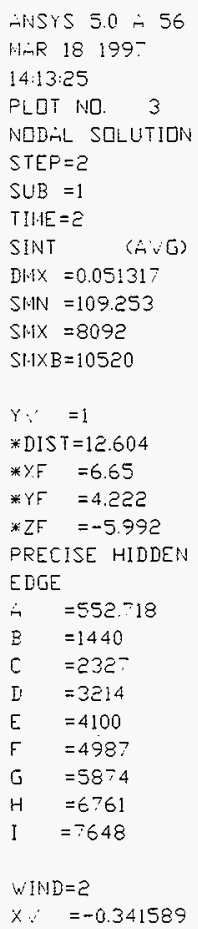

Figure 19: ANSYS Model Stress Intensity Details-Top View and Center 
CLIENT: DUKE ENGINEERING SERVICES HANFORD, INC

FILE NO: $\mathrm{KH}-8009-8-04$

PROJECT: MCO Final Design DOC. NO.: HNF-SD-SNF-DR-003, Rev. 0, Appendix 6

\section{DETAILED THREAD/CLOSURE EVALUATION}

Due to the importance of the buttress closure threads in providing and maintaining the MCO seal loading, a detailed analysis of the threads and other closure hardware is performed.

The detailed analysis approach, results, and conclusions follows below.

\subsection{Analysis Discussion}

The locking ring thread design involves buttress threads with a specified pitch of 0.25 in. and an engagement length of 2.875 in. The buttress thread design details are shown in Figure 20 and are in conformance with ANSI B1.9 [8]. As an initial check on the thread adequacy for the the bolt loading, thread stripping (thread shear) calculations are performed on the locking ring threads. Since ANSI B1.9 does not specify a formula for external thread stripping area, a conservative approximation from Machinery's Handbook [9] is used. On page 1718 of [9], a stripping/shear area, per inch of engagement, for Acme threads is given as

$$
A_{s}=3.1416 \cdot D_{1} \max \left[0.5+n \tan 14.5^{\circ}\left(D_{2} \min \cdot-D_{1} \max \right)\right]
$$

where, $D_{1}$ max is the maximum minor diameter of the internal thread and $D_{2}$ min is the minimum pitch diameter of the external thread.

Conservatively using the minor diameter of the locking ring external threads, and conservatively ignoring the 2nd term inside the brackets, results in the following thread stripping area:

$$
\begin{aligned}
& 1_{\text {engage }}:=2.875 \cdot \text { in } \\
& D_{\min }:=24.168 \cdot \text { in } \\
& A_{s}:=\pi \cdot D_{\min ^{*} \cdot 0.5 \cdot 1_{\text {engage }}} \\
& A_{s}=109.144 \cdot \text { in }^{2}
\end{aligned}
$$


From Appendix 4 (Calculation $\mathrm{KH}-8009-8-02$ ), the maximum bolt in-service loading is $216,000 \mathrm{lb}$, resulting in the following thread shear stress (allowable thread stress is $0.6 \mathrm{~S}_{\mathrm{m}}$ per ASME Section III, Subsection NB-3227.2):

$$
\begin{aligned}
& P_{\max }:=216000 \cdot 1 b \\
& \tau_{s}:=\frac{P_{\max }}{A_{s}} \\
& \tau_{s}=1.979 \cdot 10^{3} \cdot \frac{\mathrm{lb}}{\mathrm{in}^{2}} \\
& s_{m 707}:=13500 \cdot \frac{\mathrm{lb}}{\mathrm{in}^{2}} \\
& \text { Ratio : }=\frac{\tau_{\mathrm{s}}}{0.6 \cdot \mathrm{s}_{\mathrm{m} 707}}
\end{aligned}
$$

Ratio $=0.244$

Thus, the thread stripping area is adequate for the maximum jacking bolt loading.

Although the thread shear area is adequate, preliminary analyses of the shell, immediately below the threads, indicates that the membrane plus bending stresses exceed the material yield strength, for the upper bound preload. Subsection NB-3227.3 of the ASME Code addresses "nonintegral connections", including "screwed in plugs" and "shear ring closures", which are subject to failure "by bell mouthing or other types of progressive deformation". Such failures are addressed in NB-3227.3 by limiting the primary plus secondary stress intensities to the material yield strength. In order to accurately address the primary plus secondary stresses in the vicinity of the threads, a detailed finite element analysis of the thread/closure region is performed.

The failure modes addressed in NB-3227.3 are of a "ratchetting" nature, requiring cyclic loading. Although there will be some cyclic themal loading, the dominant closure load (bolt preload) is not cycled. However, any shell stresses in the closure load path which exceed the material yield strength, raises the concern for seal preload losses. This potential for seal load losses and potential seal leakage is the primary motivation for performing the

\begin{tabular}{|l|c|c|c|c|c|}
\hline REVISION & 0 & & & & $\begin{array}{c}\text { PAGE 37 } \\
\text { OF 90 }\end{array}$ \\
\hline PREPARED BY IDATE CHECKED BY/DATE & Su $/ 4 / 17 / 97$ & & & & \\
\hline
\end{tabular}


detailed thread/closure analysis.

The detailed thread/closure model developed is shown in Figure 21. Contact elements are used at the threads, boit interface, and seal stop interface. The model is subjected to the following load sequence: (1) The maximum room temperature preload of $190,000 \mathrm{lb}$ (conservative upper bound from Appendix 4). The preload is iteratively obtained using an interference fit at the bolt/shield plug interface. (2) Bolt preload plus $150 \mathrm{psi}$ at $200^{\circ} \mathrm{C}$. (3) Bolt preload plus $75 \mathrm{psi}$ at $375^{\circ} \mathrm{C}$. Note that the model details focus on the thread region. Other areas are modeled in much less detail, e.g. the shield plug and the locking ring, away from the threads. Also, some minor changes in dimensions occurred after the model was developed. Both the modeling approximations and the dimensional changes are judged to have a small effect on the results. The only boundary constraint is an axial displacement constraint at the bottom of the shell.

To account for nonlinear effects occurring during the load sequence, bilinear plasticity (BKIN) and large displacements (NLGEOM) are both enabled in the ANSYS analysis. Note, from Figure 21, that the full bolt width of $1.5 \mathrm{in}$. is modeled using axisymmetric ring elements. The full bolt width is used to bound the closure response in a cross section passing through a bolt. To provide the correct bolt stiffness, the elastic modulus of the bolt material is reduced by the ratio of the actual bolt area divided by the bolt ring area. The effective bolt length is assumed to project through the first few bolt threads $(0.25 \mathrm{in}$.).

\subsection{Analysis Results}

The ANSYS input and output files for the thread/closure analysis are THRD3.inp and THRD3.out, respectively. A summary of the results for the load sequence discussed in Section 10.1 follows below.

The stress/strain state of the thread region following the three step load sequence is summarized in Figure 22 and Figure 23. Note, from Figure 23, that the peak equivalent plastic strain of $0.16 \%$ occurs in the thread relief region below the bottom thread. Plastic straining, of lesser magnitude, also occurs in the root of the two bottom threads. This concentration of plastic straining in the bottom threads is anticipated since the thread load progressively diminishes above the bottom thread. The thread loading versus thread number is extracted from the ANSYS output and is provided in Figure 24.

The plastic straining results in an outward movement of the top of the shell which is illustrated in the radial displacement contours provided in Figure 25. Note that the maximum radial displacement (UX) at the top of the shell is nearly one-tenth of an inch (SMX $=0.0908$ in.). The shell radial displacement is of concern due to the potential for

\begin{tabular}{|l|c|l|l|l|l|}
\hline REVISION & 0 & & & & $\begin{array}{c}\text { PAGE 38 } \\
\text { OF 90 }\end{array}$ \\
\hline PREPARED BY I DATE CHECKED BY I DATE & GSU 4/17/97 & & & & \\
\hline
\end{tabular}


thread separation (thread engagement depth $\approx 0.14$ in). However, most of this displacement is due to thermal growth $\left(\alpha * \Delta T * R=9.69 \times 10^{-6} \times 637 \times 12.63=0.078\right.$ in. $)$. Thus, the relative growth is about $0.091-0.078=0.013$ in., which is about one-tenth of the depth of thread engagement.

The metallic closure seal is positioned next to the seal stop and is not included in the thread/closure model. Since the seal stop is much stiffer than the seal, as long as the seal stop remains in contact with the MCO shell, there will be a negligible change in the seal loading, as indicated by the seal load evaluation presented in Section 12 of Appendix 4. For the maximum preload case considered in this analysis, the seal stop load is reduced by about $30 \%$ when the pressure loading is added, but remains in compression (did not gap). This is consistent with Section 12 of Appendix 4 which also reports that the seal stop remains in compression for the "maxbolt" load condition.

\subsection{Thread/Closure Analysis Conclusions}

Although the upper bound primary plus secondary stress intensities exceed the material yield stress (NB-3227.3 stress limit), a detailed elastic/plastic analysis indicates that the plastic strain magnitudes are small $(<0.2 \%)$ and confined to the inside surface near the bottom threads. The corresponding deformations are also relatively small, indicating an adequate margin against thread separation. Concerning the seal loading, the plastic strains associated with the upper bound preload, do not result in gapping at the seal stop. Thus, it is concluded that the thread/closure design is adequate for maintaining the closure seal.

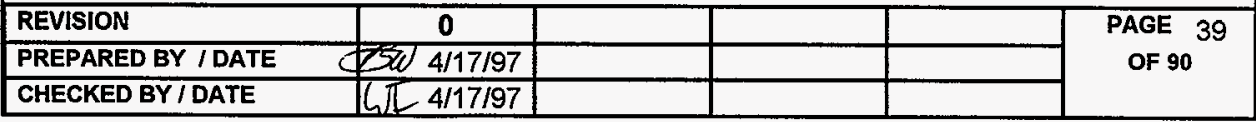


CLIENT: DUKE ENGINEERING SERVICES HANFORD, INC

FILE NO: KH-8009-8-04

PROJECT: MCO Final Design DOC. NO.: HNF-SD-SNF-DR-003, Rev. 0, Appendix 6

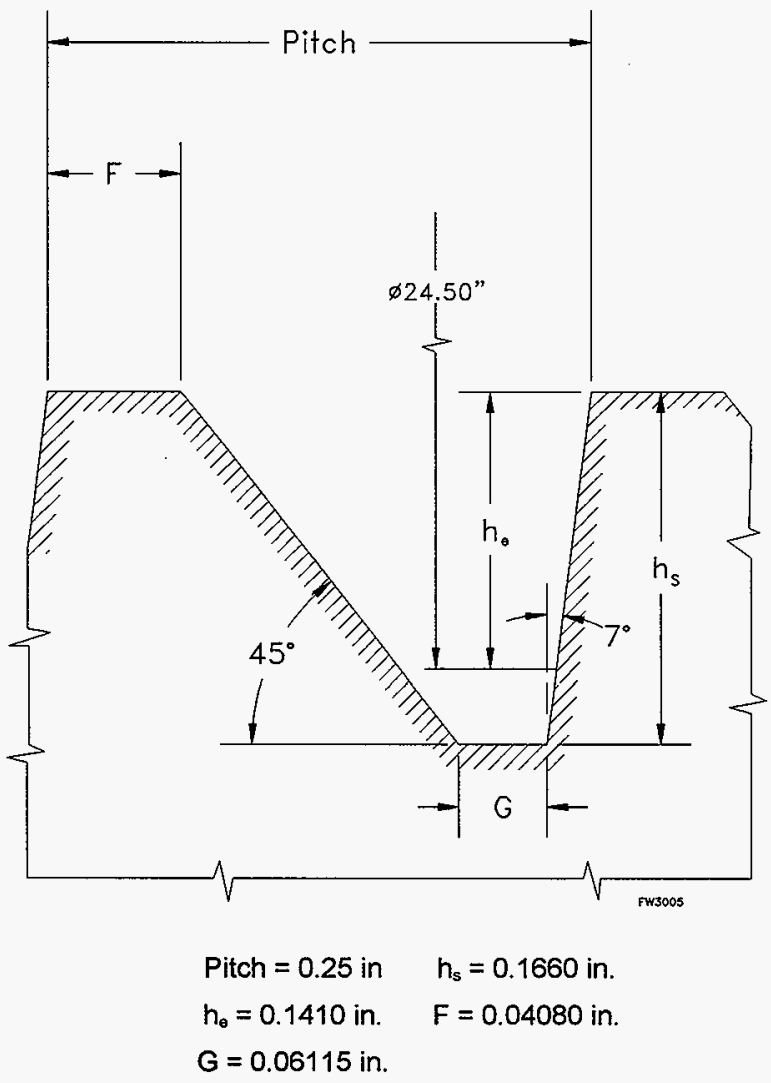

Figure 20: Thread Detail Geometry 
CLIENT: DUKE ENGINEERING SERVICES HANFORD, INC

PROJECT: MCO Final Design $\quad$ DOC. NO.: HNF-SD-SNF-DR-003, Rev. 0, Appendix 6

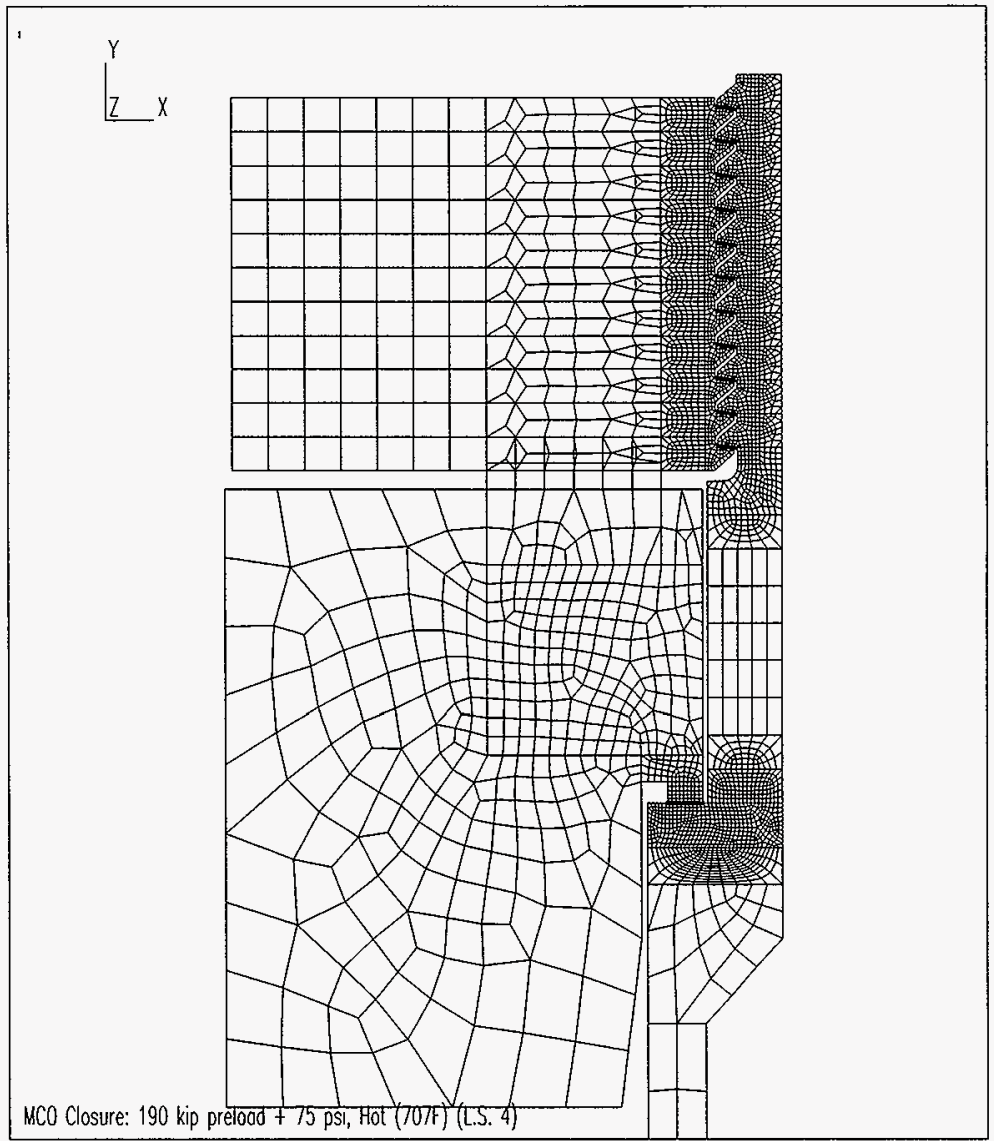

ANSYS 5.3

APR 161997

09:30:09

PLOT NO. 2

ELEMENTS

TYPE NUN

$2 N=1$

$*$ DIST $=4.182$

$* X F=10.227$

$¥ Y F=-.679697$

Figure 21: Finite Element Model of MCO Thread/Closure Region

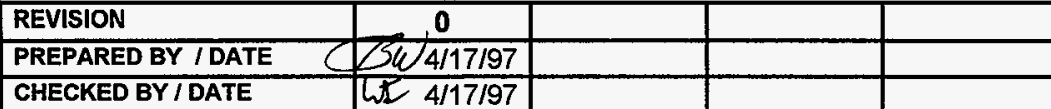


CLIENT: DUKE ENGINEERING SERVICES HANFORD, INC

PROJECT: MCO Final Design

FILE NO: KH-8009-8-04

DOC. NO.: HNF-SD-SNF-DR-003, Rev. 0, Appendix 6

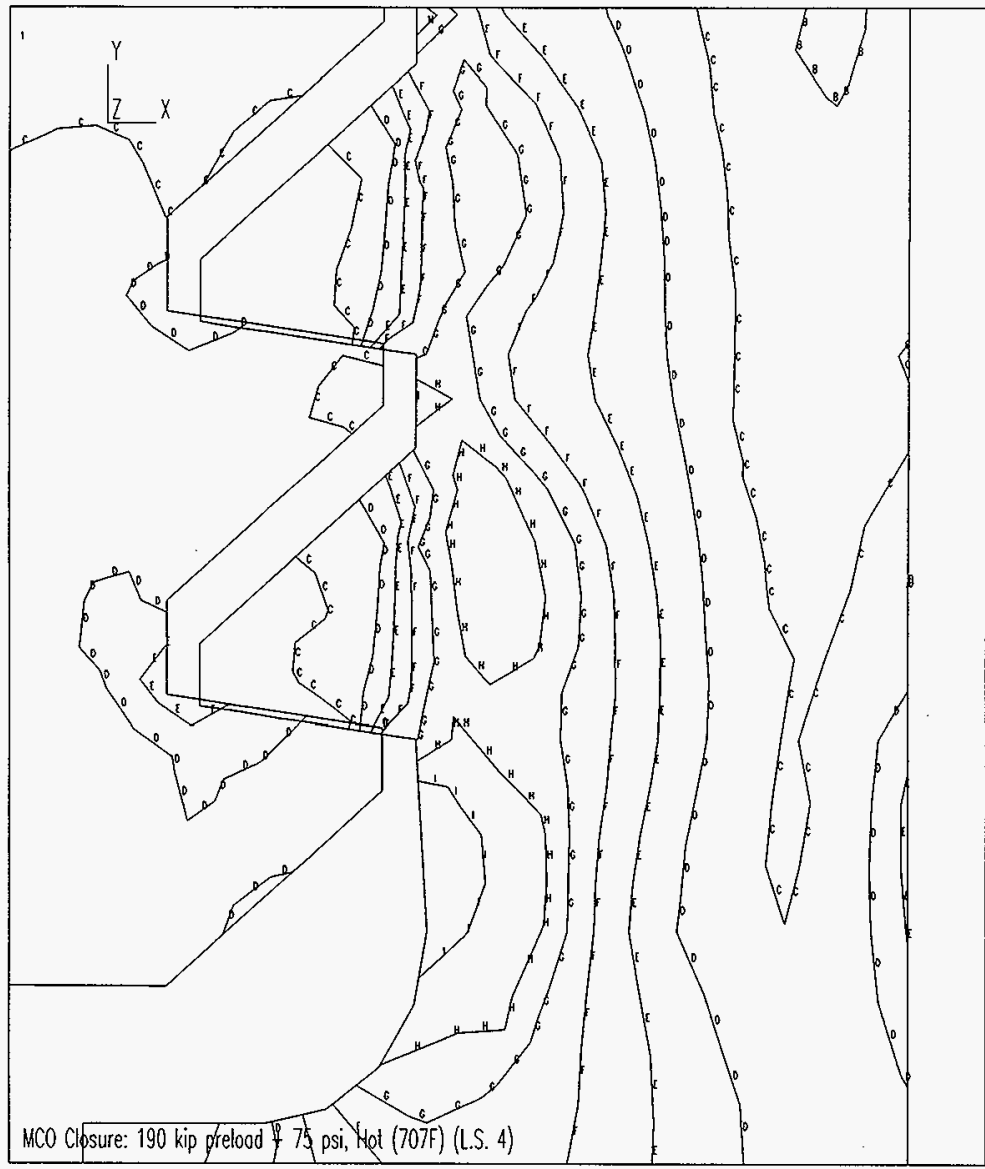

ANSYS 5.3

APR 161997

09:33:28

PLOT NO. 3

NODAL SOLUTION

STEP $=4$

SUB $=4$

TMEE $=4$

SINT (AVG)

OMX $=.139382$

SMN $=195.262$

SMX $=17264$

$A=1144$

$B=3040$

$C=4937$

$0=6833$

$E=8730$

$F=10626$

$G=12523$

$H=14419$

$1=16316$

Figure 22. Stress Intensity Contours, Bottom Threads

\begin{tabular}{|l|l|l|}
\hline & & \\
\hline & & \\
\hline & & \\
\hline
\end{tabular}


FILE NO: $\mathrm{KH}-8009-8-04$

PROJECT: MCO Final Design DOC. NO.: HNF-SD-SNF-DR-003, Rev. 0, Appendix 6

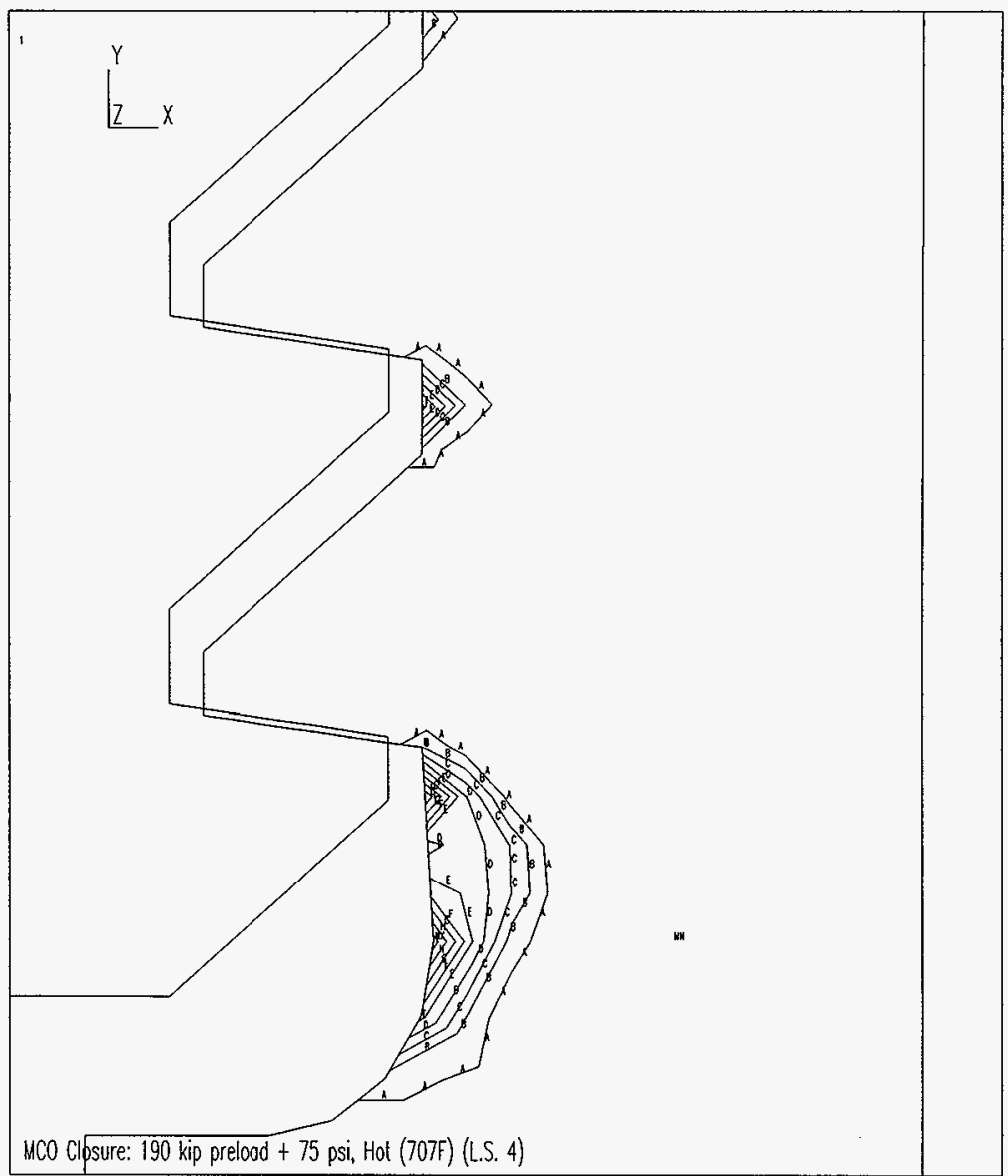

ANSYS 5.3

APR 161997

$09: 36: 25$

PLOT NO. 5

NODAL SOLUTION

STEP $=4$

SUB $=4$

TIME $=4$

EPPLEQ (AVG)

OMX $=.139382$

SMX $=.001676$

$A=.931 E-04$

$B=.279[-03$

$C=.466 \mathrm{E}-03$

$0=.652 \mathrm{E}-03$

$\mathrm{E}=.838 \mathrm{E}-03$

$F=.001024$

$G=.00121$

$H=.001397$

$1=.001583$

Figure 23. Equivalent Plastic Strain Contours, Bottom Threads

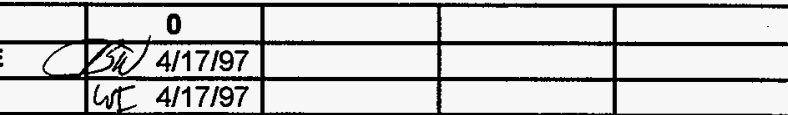


CLIENT: DUKE ENGINEERING SERVICES HANFORD, INC

PROJECT: MCO Final Design

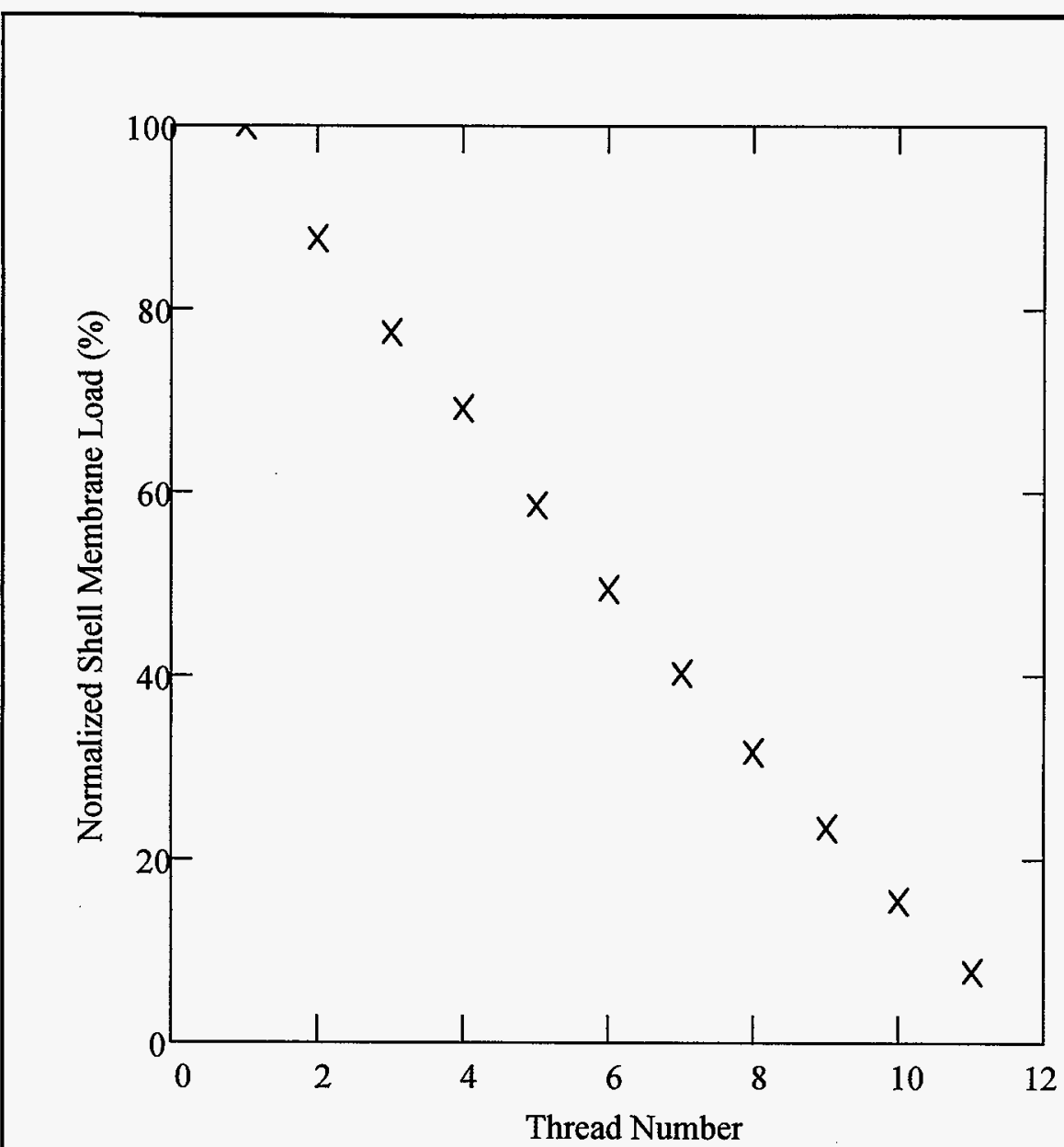

Figure 24: Shell Load Distribution at the Closure Threads

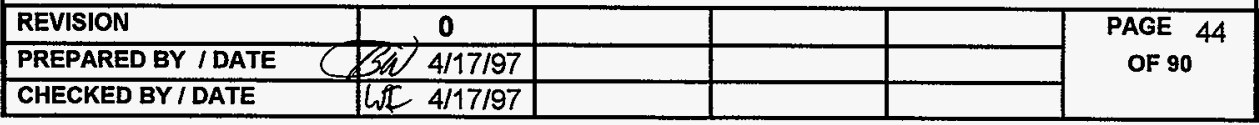


FILE NO: $\mathrm{KH}-8009-8-04$

PROJECT: MCO Final Design DOC. NO.: HNF-SD-SNF-DR-003, Rev. 0, Appendix 6

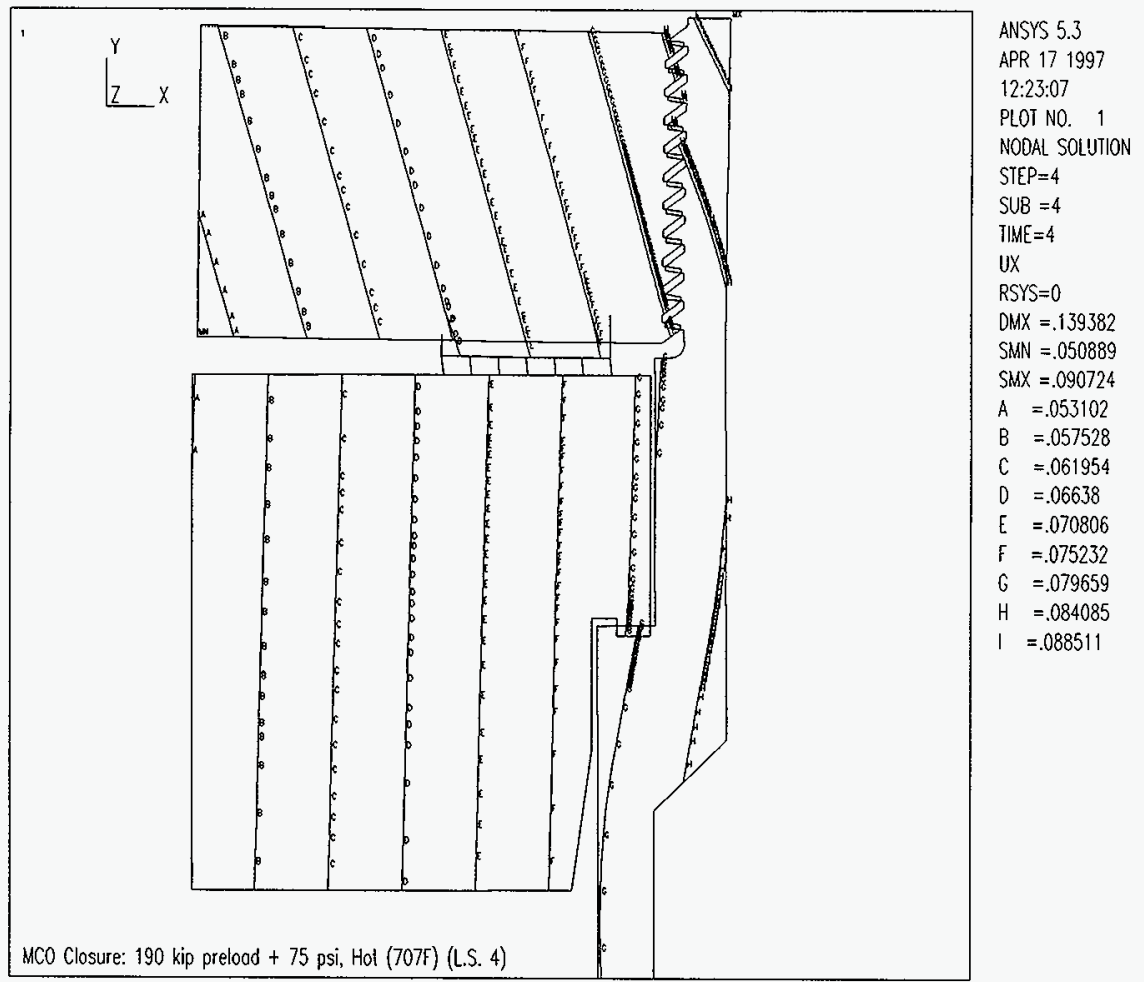

Figure 25: Radial Displacement Contours, MCO Closure Thread Region

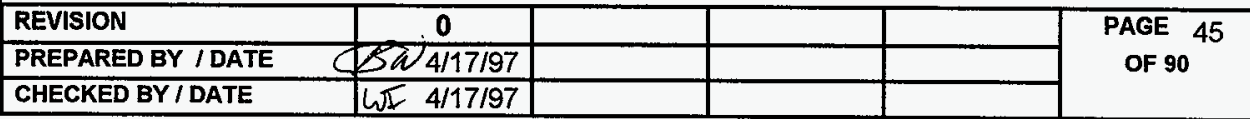




\section{Appendix A:}

\section{Computer Run Output Sheets}

\&

Input File Listings 
CLIENT: DUKE ENGINEERING SERVICES HANFORD, INC

FILE NO: KH-8009-8-04
PROJECT: MCO Final Design DOC. NO.: HNF-SD-SNF-DR-003, Rev. 0, Appendix 6

\section{COMPUTER RUN COVER SHEET}

Project Number:

Computer Code:

Software Version:

Computer System:

Computer Run File Number:

Unique Computer Run Filename:

Run Description:

Creation Date / Time:
$\mathrm{KH}-8009-8$

ANSYS®-PC

$5.0 \mathrm{~A}$

MS-DOS, Pentium \& Processor

KH-8009-8-04

NEWCAP.inp

Stress Analysis of Lifting Cap

28 March 1997
11:31:54 AM
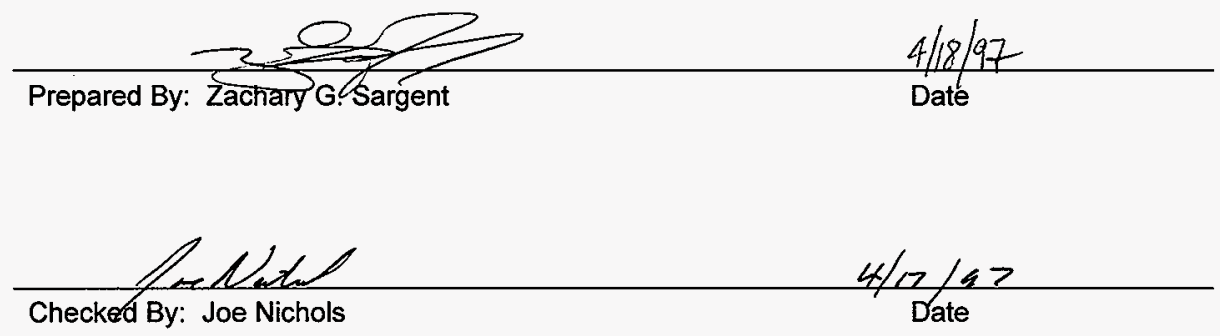

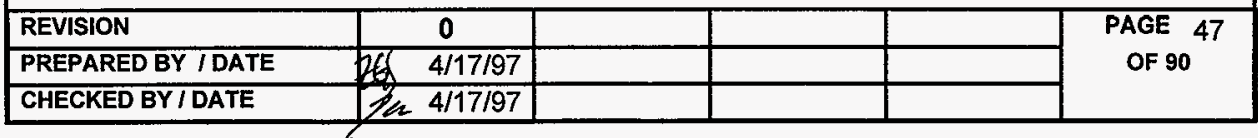


CLIENT: DUKE ENGINEERING SERVICES HANFORD, INC

$\begin{array}{ll}\text { PROJECT: MCO Final Design } & \text { DOC. NO.: HNF-SD-SNF-DR-003, Rev. 0, Appendix } 6\end{array}$

\section{LISTING OF NEWCAP.INP FILE}

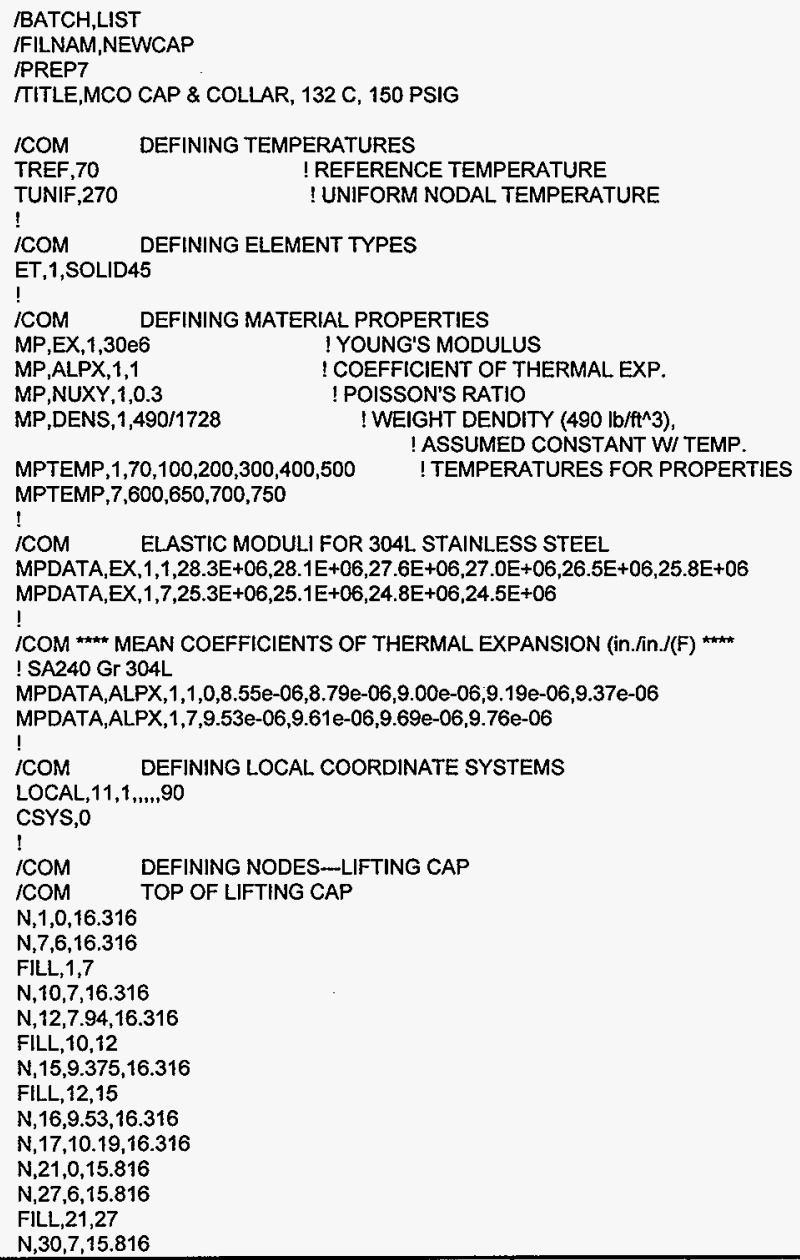




\section{PROJECT: MCO Final Design

CLIENT: DUKE ENGINEERING SERVICES HANFORD, INC

FILE NO: $\quad \mathrm{KH}-8009-8-04$

$N, 32,7.94,15.816$

FILL, 30,32

$N, 35,9.375,15.816$

FILL,32,35

$\mathrm{N}, 36,9.53,15.816$

$N, 37,10.19,15.816$

$\mathrm{N}, 41,0,15.316$

$N, 47,6,15.316$

FILL, 41,47

N, $50,7,15.316$

$N, 52,7.94,15.316$

FILL, 50,52

$\mathrm{N}, 55,9.375,15.316$

FILL,52,55

N, $56,9.53,15.316$

$\mathrm{N}, 57,10.19,15.316$

$\mathrm{N}, 61,0,14.816$

$\mathrm{N}, 67,6,14.816$

FILL, 61,67

$\mathrm{N}, 70,7,14.816$

N, $72,7.94,14.816$

FiLL, 70,72

$N, 75,9.375,14.816$

FILL, 72,75

$\mathrm{N}, 81,0,14.316$

$\mathrm{N}, 87,6,14.316$

FILL, 81,87

$\mathrm{N}, 90,7,14.316$

$\mathrm{N}, 92,7.94,14.316$

FILL, 90,92

$N, 95,9.375,14.316$

FILL, 92,95

$\mathrm{N}, 96,9.625,14.316$

N,102,11.125,14.316

FILL, 96,102

$\mathrm{N}, 103,11,4035,14.316$

N,104,11.75,14.316

$\mathrm{N}, 105,12.125,14.316$

$\mathrm{N}, 106,12.375,14.316$

$\mathrm{N}, 107,12.625,14.316$

$\mathrm{N}, 131,0,12.946$

$\mathrm{N}, 137,6,12.946$

FILL, 131,137

$\mathrm{N}, 140,7,12.946$

$\mathrm{N}, 142,7.94,12.946$

FILL, 140,142

$N, 145,9.375,12.946$

FILL, 142,145

$\mathrm{N}, 146,9,625,12.946$

$\mathrm{N}, 152,11.125,12.946$

FILL, 146,152

$N, 153,11.4035,12.946$

$\mathrm{N}, 154,11.75,12.946$

$\mathrm{N}, 155,12.125,12.946$ 
CLIENT: DUKE ENGINEERING
MCO Final Design

$\mathrm{N}, 156,12.375,12.946$

$N, 157,12.625,12.946$

SAVE

FILL,90,140,1,110

FILL,91,141,1,111

FILL,92,142,1,112

FILL,93,143,1,113

FILL,94, 144,1,114

FILL,95, 145,1,115

FILL,96,146,1,116

FILL,97,147,1,117

FILL, $98,148,1,118$

FILL,99,149,1,119

FILL, 100,150,1,120

FILL, 101,151,1,121

FILL, 102, 152,1,122

FIL.L, 103,153,1,123

FILL,104,154,1,124

FILL, 105,155,1,125

FILL, 106,156,1,126

FILL, 107,157,1,127

N,700,9.75,16.316

$\mathrm{N}, 701,9.97,16.316$

N,704,9.75,15.316

N,705,9.97,15.316

FILL,700,704,1,702

FILL, 701,705,1,703

!

ICOM SIDE OF LIFTING CAP

$\mathrm{N}, 128,9.375,12.8519$

$\mathrm{N}, 129,9.625,12.7692$

N, 130,9.875,12.6589

$\mathrm{N}, 158,10.125,12.5171$

$\mathrm{N}, 159,10.375,12.571$

N, 160,10.625,12.571

N, 161, 10.875,12.571

$\mathrm{N}, 162,11.125,12.571$

$N, 163,11.4035,12.571$

$\mathrm{N}, 164,11.75,12.571$

$\mathrm{N}, 165,12.125,12.571$

N, 166,12.375,12.571

$\mathrm{N}, 167,12.625,12.571$

$\mathrm{N}, 169,10.375,12.3375$

$\mathrm{N}, 170,10.5687,12.1661$

N,171,10.875,12.196

$\mathrm{N}, 172,11.125,12.196$

$\mathrm{N}, 173,11.4035,12.196$

$\mathrm{N}, 174,11.75,12.196$

N, $175,12.125,12.196$

$N, 176,12.375,12.196$

$N, 177,12.625,12.196$

$\mathrm{N}, 181,10.7726,11.946$

$\mathrm{N}, 182,11.125,11.946$

N, 183,11.4035,11.946

\begin{tabular}{|l|c|l|l|l|l|}
\hline REVISION & 0 & & & & PAGE 50 \\
OF 90
\end{tabular}


CLIENT: DUKE ENGINEERING

$N, 184,11.75,11.946$

$\mathrm{N}, 185,12.125,11.946$

$\mathrm{N}, 186,12.375,11.946$

$\mathrm{N}, 187,12.625,11.946$

$\mathrm{N}, 188,11.0198,11.596$

$\mathrm{N}, 192,11.125,11.596$

$\mathrm{N}, 193,11.4035,11.596$

$\mathrm{N}, 194,11.75,11.596$

$\mathrm{N}, 195,12.125,11.596$

$\mathrm{N}, 196,12.375,11.596$

$\mathrm{N}, 197,12.625,11.596$

$\mathrm{N}, 202,11.1753,11.296$

$\mathrm{N}, 203,11.4035,11.296$

$\mathrm{N}, 204,11.75,11.296$

$\mathrm{N}, 205,12.125,11.296$

$\mathrm{N}, 206,12.375,11.296$

$\mathrm{N}, 207,12.625,11.296$

$N, 208,11.3421,10.869$

$\mathrm{N}, 209,11.75,10.869$

$\mathrm{N}, 210,12.125,10.869$

$\mathrm{N}, 211,12.375,10.869$

$\mathrm{N}, 212,12.625,10.869$

$\mathrm{N}, 213,11.5046,10.4420$

$\mathrm{N}, 214,11.75,10.4420$

$N, 215,12.125,10.4420$

$\mathrm{N}, 216,12.375,10.4420$

$\mathrm{N}, 217,12.625,10.4420$

$\mathrm{N}, 219,11.6672,10.0146$

$\mathrm{N}, 220,12.125,10.0146$

$\mathrm{N}, 221,12.375,10.0146$

$\mathrm{N}, 222,12.625,10.0146$

$\mathrm{N}, 224,11.8682,9.4866$

$\mathrm{N}, 225,12.125,9.4866$

$\mathrm{N}, 226,12.375,9.4866$

$\mathrm{N}, 227,12.625,9.4866$

$\mathrm{N}, 234,12.0691,8.9586$

$!$

ICOM WELD AREA BETWEEN LIFTING CAP \& CANISTER COLLAR

$\mathrm{N}, 235,12.125,8.94$

$\mathrm{N}, 236,12.375,8.94$

$\mathrm{N}, 237,12.625,8.94$

$\mathrm{N}, 245,12.225,8.793$

$\mathrm{N}, 246,12.375,8.793$

$\mathrm{N}, 247,12.625,8.793$

$\mathrm{N}, 255,12.225,8.646$

$\mathrm{N}, 256,12.375,8.646$

$\mathrm{N}, 257,12.625,8.646$

!

ICOM CANISTER COLLAR THREADS

$\mathrm{N}, 265,12.225,8.5$

$\mathrm{N}, 266,12.375,8.5$

$\mathrm{N}, 267,12.625,8.5$

$\mathrm{N}, 285,12.125,8.25$

$\mathrm{N}, 297,12.625,8.25$

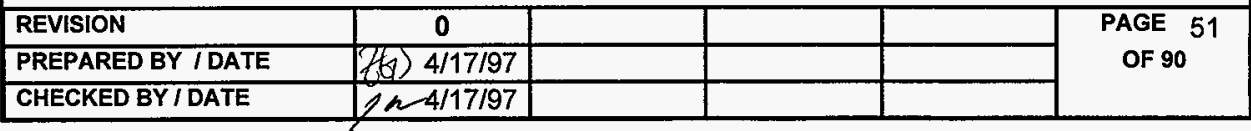




\section{PROJECT: MCO Final Design DOC. NO.: HNF-SD-SNF-DR-003, Rev. 0, Appendix 6}

CLIENT: DUKE ENGINEERING SERVICES HANFORD INC

FILL, 285,297,1,291

$\mathrm{N}, 305,12.125,7.75$

$N, 317,12.625,7.75$

FILL,305,317,1,311

$\mathrm{N}, 405,12.125,5.25$

FILL,305,405,4,325

$\mathrm{N}, 410,12.25,5.25$

$N, 391,12.375,5.75$

ILOWER OUTER THREADS

FILL,311,391,3,331

$N, 416,12.5,5.25$

$\mathrm{N}, 417,12.625,5.25$

FILL,317,417,4,337

$N, 430,12,25,5$

$\mathrm{N}, 435,12.01,5$

$\mathrm{N}, 437,12.625,5$

$\mathrm{N}, 445,12.01,4.6$

$N, 447,12.625,4.6$

FILL, 445,447

FILL, 430,437, 1,436

$N, 485,12.01,3$

FILL, 445,485,3,455

$\mathrm{N}, 487,12,625,3$

FILL,437,487,4,447

FILL,485,487, 1,486

FILL,446,486,3,456

$\mathrm{N}, 493,11.5,2.63$

$\mathrm{N}, 495,12.01,2.63$

FILL,493,495

$\mathrm{N}, 497,12.625,2.63$

FILL, 495,497

$\mathrm{N}, 503,11.5,2.33$

$\mathrm{N}, 505,12.01,2.33$

FILL,503,505

$\mathrm{N}, 507,12.625,2.33$

FILL,505,507

$\mathrm{N}, 513,11.5,2.03$

$\mathrm{N}, 515,12.01,2.03$

FILL, 513,515

$\mathrm{N}, 517,12.625,2.03$

FILL, 515,517

$\mathrm{N}, 523,11.5,1.63$

$\mathrm{N}, \mathbf{5 2 5}, 12.01,1.63$

FILL, 523,525

$\mathrm{N}, 527,12.625,1.63$

FILL, 525,527

$\mathrm{N}, 530,12.318,1.315$

N,533,11.5,1

$\mathrm{N}, 535,12.01,1$

FILL, 533,535

$\mathrm{N}, 553,11.5,0$

$\mathrm{N}, 555,12.01,0$

FILL, 533,553,1,543

FILL, 553,555

FILL, 534,554, 1,544

! LOWER LIFTING CAP

\begin{tabular}{|l|c|c|c|c|c|}
\hline REVISION & 0 & & & & PAGE 52 \\
OF 90
\end{tabular}


CLIENT: DUKE ENGINEERING SERVICES HANFORD, INC

FILE NO: KH-8009-8-04

PROJECT: MCO Final Design DOC. NO.: HNF-SD-SNF-DR-003, Rev. 0, Appendix 6

FILL,535,555,1,545

$\mathrm{N}, 633,11,5,-8$

FILL, $553,633,7,563$

$\mathrm{N}, 635,12,01,-8$

FILL, $555,635,7,565$

FILLL, 563,565

FILIL.573,575

FILL, 583,585

FILL.,593,595

FILL, 603,605

FILL,613,615

FILL, 623,625

FILL, 633,635

SAVE

!

ICOM CHANGING TO LOCAL COORDINATE SYSTEM

CSYS,11

ICOM NODAL GENERATION 60 DEGREE SWEEP

NGEN,5,1000,ALL,,.,-5

NGEN, $2,1000,4000,4999,1,-4.27$

NGEN, $3,1000,5000,5999,1,-5.73$

NGEN, $2,1000,7000,7999,1,-4.27$

NGEN $, 5,1000,8000,8999,1,,-5$

CSYS, 0

!

/COM ELEMENT GENERATION

ICOM

TYPE, 1

$E, 22,1022,1021,21,2,1002,1001,1$ ! TOP PLATE

EGEN,4,20,-1

$E, 132,1132,1131,131,82,1082,1081,81$

$E, 23,1023,1022,22,3,1003,1002,2$

EGEN $5,1,-1$

$E, 43,1043,1042,42,23,1023,1022,22$

EGEN,5,1,-1

$E, 63,1063,1062,62,43,1043,1042,42$

EGEN,5,1,-1

$E, 83,1083,1082,82,63,1063,1062,62$

EGEN,5,1,-1

$E, 133,1133,1132,132,83,1083,1082,82$

EGEN, $5,1,-1$

$E, 30,1030,1027,27,10,1010,1007,7$

EGEN,4,20,-1

$E, 87,90,110,110,1087,1090,1110,1110$

$E, 87,110,137,137,1087,1110,1137,1137$

$E, 110,140,137,137,1110,1140,1137,1137$

$E, 31,1031,1030,30,11,1011,1010,10$

EGEN,4,20,-1

$E, 32,1032,1031,31,12,1012,1011,11$

EGEN,4,20,-1

$E, 33,1033,1032,32,13,1013,1012,12$

EGEN,4,20,-1

$E, 34,1034,1033,33,14,1014,1013,13$

EGEN,4,20,-1

! GRAPPLE SHOE AREA (1.97"X0.66")

\begin{tabular}{|l|c|l|l|l|c|}
\hline REVISION & 0 & & & & PAGE 53 \\
OF 90 \\
\cline { 1 - 4 } CHECKED BY I DATE & (f) $4 / 17 / 97$ & & & & \\
\hline
\end{tabular}


FILE NO: $\quad \mathrm{KH}-8009-8-04$

PROJECT: MCO Final Design DOC. NO: HNF-SD-SNF-DR-003, Rev. 0, Appendix 6

$E, 35,1035,1034,34,15,1015,1014,14$

EGEN,4,20,-1

$E, 36,1036,1035,35,16,1016,1015,15$

EGEN,2,20,-1

$E, 702,1702,1036,36,700,1700,1016,16$

$E, 704,1704,1056,56,702,1702,1036,36$

$E, 703,1703,1702,702,701,1701,1700,700$

EGEN,2,2,-1

E,37,1037,1703,703,17,1017,1701,701

E, $57,1057,1705,705,37,1037,1703,703$

$E, 75,96,95,95,1075,1096,1095,1095$

$E, 111,1111,1110,110,91,1091,1090,90$

$E, 112,1112,1111,111,92,1092,1091,91$

$E, 113,1113,1112,112,93,1093,1092,92$

EGEN, $3,1,-1$

E, $116,1116,1115,115,96,1096,1095,95$

E,117,1117,1116,116,97,1097,1096,96

EGEN, 11,1,-1

E, $141,1141,1140,140,111,1111,1110,110$

EGEN, 17,1,-1

$E, 144,146,128,128,1144,1145,1128,1128$

$E, 129,1129,1128,128,146,1146,1145,145$

$E, 130,1130,1129,129,147,1147,1146,146$

E,158,1158,1130,130,148,1148,1147,147

E, $159,1159,1158,158,149,1149,1148,148$

EGEN, $9,1,-1$

$E, 158,159,169,169,1158,1159,1169,1169$

E, $170,1170,1169,169,160,1160,1159,159$

EGEN,8,1,-1

E, $170,171,181,181,1170,1171,1181,1181$

$E, 182,1182,1181,181,172,1172,1171,171$

EGEN, $6,1,-1$

E, 192,1192,1188,188,182,1182,1181,181

E, 193,1193,1192,192,183,1183,1182,182

EGEN, $5,1,-1$

$E, 188,192,202,202,1188,1192,1202,1202$

$E, 203,1203,1202,202,193,1193,1192,192$

EGEN,5,1,-1

$E, 202,203,208,208,1202,1203,1208,1208$

$E, 209,1209,1208,208,204,1204,1203,203$

EGEN, $4,1,-1$

$E, 214,1214,1213,213,209,1209,1208,208$

$E, 215,1215,1214,214,210,1210,1209,209$

EGEN, $3,1,-1$

$E, 213,214,219,219,1213,1214,1219,1219$

$\mathrm{E}, 220,1220,1219,219,215,1215,1214,214$

EGEN, $3,1,-1$

$E, 225,1225,1224,224,220,1220,1219,219$

EGEN,3,1,-1

$\mathrm{E}, 235,1235,1234,234,225,1225,1224,224$

EGEN,3,1,-1

$E, 234,235,245,245,1234,1235,1245,1245$

$E, 246,1246,1245,245,236,1236,1235,235$

$E, 247,1247,1246,246,237,1237,1236,236$

REVISION

PREPARED BY I DATE

CHECKED BYIDATE

0

. 
CLIENT: DUKE ENGINEERING SERVICES HANFORD, INC

FILE NO: $\quad \mathrm{KH}-8009-8-04$

PROJECT: MCO Final Design DOC. NO.: HNF-SD-SNF-DR-003, Rev. 0, Appendix 6

$E, 256,1256,1255,255,246,1246,1245,245$

$E, 257,1257,1256,256,247,1247,1246,246$

$E, 266,1266,1265,265,256,1256,1255,255$

$\mathrm{E}, 267,1267,1266,266,257,1257,1256,256$

$E, 291,1291,1285,285,266,1266,1265,265$

$E, 297,1297,1291,291,267,1267,1266,266$

E,311,1311,1305,305,291,1291,1285,285

EGEN,5,20,-1

$E, 317,1317,1311,311,297,1297,1291,291$

EGEN,5,20,-1

E.410,1410,1405,405,391,1391, 1385,385

$E, 391,416,410,410,1391,1416,1410,1410$

$E, 417,1417,1416,416,397,1397,1391,391$

E,436,1436,1430,430,416,1416,1410,410

$E, 437,1437,1436,436,417,1417,1416,416$

$E, 446,1446,1445,445,430,1430,1435,435$

$E, 430,436,446,446,1430,1436,1446,1446$

$E, 447,1447,1446,446,437,1437,1436,436$

$E, 456,1456,1455,455,446,1446,1445,445$

EGEN,4,10,-1

$E, 496,1496,1495,495,486,1486,1485,485$

$E, 457,1457,1456,456,447,1447,1446,446$

EGEN,4,10,-1

$E, 497,1497,1496,496,487,1487,1486,486$

$\mathrm{E}, 506,1506,1505,505,496,1496,1495,495$

EGEN,2,10,-1

$E, 526,1526,1525,525,516,1516,1515,515$

$E, 525,526,530,530,1525,1526,1530,1530$

E,507,1507,1506,506,497,1497,1496,496

EGEN,2,10,-1

E,527,1527,1526,526,517,1517,1516,516

$E, 526,527,530,530,1526,1527,1530,1530$

$E, 525,530,535,535,1525,1530,1535,1535$

E,504,1504,1503,503,494,1494,1493,493

EGEN,2,10,-1

E,505,1505,1504,504,495,1495, 1494,494

EGEN,2,10,-1

$E, 524,1524,1523,523,514,1514,1513,513$

EGEN, $2,1,-1$

E,534,1534,1533,533,524,1524,1523,523

EGEN, $2,1,-1$

$E, 544,1544,1543,543,534,1534,1533,533$

EGEN, 10,10,-1

E, $545,1545,1544,544,535,1535,1534,534$

EGEN, 10,10,-1

SAVE

ESEL,ALL

EGEN, $12,1000, \mathrm{ALL}$

$!$

/COM MERGING COINCIDENT LABELS

NUMMRG,NODE IMERGE ALL COINCIDENT NODES

ICOM BOUNDARY CONDITIONS

CSYS, 11 ! CYLINDRICAL

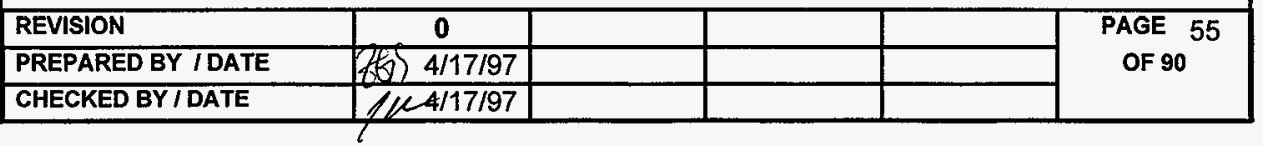


NROTAT,ALL

NSEL,S,NODE, 1,705

DSYM,SYMM,Y,11

NSEL,ALL

NSEL,S,NODE, ,12002,12705

DSYM,SYMM,Y,11

NSEL,ALL

NSEL,S,NODE, 634,12634,1000

$\mathrm{D}, \mathrm{ALL}, \mathrm{UZ}$

\section{! APPLY DOF CONSTRAINTS}

\section{ROTATE ALL NODES IN CURRENT SYS \\ ! SELECT ALL NODES ON FIRST SLICE! \\ I SYMMETRY CONSTRAINT}

! SELECT NODES ON LAST SLICE

SAVE

FINI

$!$

ICOM SOLUTION PHASE

ISOLU

NEQIT,25

OUTPR,LAST

OUTRES, LAST

!

ICOM LOAD STEP 1: LIFTING LOAD

NALL

NSEL,S,NODE, $5057,6057,1000$

NSEL,A,NODE, $5705,6705,1000$

SF,ALL,PRES, 3010

NALL

NSEL,S,NODE, $6057,7057,1000$

NSEL,A,NODE, ,6705,7705,1000

SF,ALL,PRES, 3010

NALL

NSEL,S,NODE, $5705,6705,1000$

NSEL, A,NODE, ,5704,6704,1000

SF,ALL,PRES,3077

NALL

NSEL,S,NODE, ,6705,7705,1000

NSEL,A,NODE, ,6704,7704,1000

SF,ALL,PRES,3077

NALL

NSEL,S,NODE, $5704,6704,1000$

NSEL,A,NODE, $5056,6056,1000$

SF,ALL,PRES, 3148

NALL

NSEL,S,NODE, $6704,7704,1000$

NSEL,A,NODE,,6056,7056,1000

SF,ALL,PRES,3148

NALL

EALL

LSWRITE, 1

!

ICOM LOAD STEP 2: APPLYING 150 PSI INTERNAL PRESSURE

!

NALL

EALL

NSEL, S,LOC,X,11.45,11.55

NSEL,R,LOC, $Z, 8.1,-2.64$

I LOWER HALF

REVISION

PREPARED BY / DATE

CHECKED BY I DATE

$4 / 17 / 97$

\begin{tabular}{|l|l|l|l|}
\hline & & & \\
\hline & & \\
\hline & & \\
\hline
\end{tabular}


FILE NO: $\quad \mathrm{KH}-8009-8-04$

PROJECT: MCO Final Design DOC. NO.: HNF-SD-SNF-DR-003, Rev. 0, Appendix 6

SF,ALL,PRES, 150

NALL

EALL

NSEL,S,LOC,X,11.48,12.03

NSEL,R,LOC, $Z,-2.61,-2.65$

SF,ALL,PRES, 150

NALL

EALL

NSEL,S,LOC,X,11.99,12.03

NSEL, R,LOC, Z,-2.61,-3.02

SF,ALL,PRES, 150

NALL

EALL

NSEL,S,LOC, $X, 12,12.128$

NSEL,R,LOC,Z,-2.64,-8.52

SF,ALL,PRES, 150

NALL.

EALL

NSEL,S,LOC, $X, 11.99,12.27$

NSEL,R,LOC, $Z,-4.98,-5.02$

SF,ALL,PRES, 150

NALL

EALL

NSEL,S,LOC, $X, 12.23,12.27$

NSEL,R,LOC, $Z,-4.95,-5.27$

SF,ALL,PRES, 150

NALL

EALL

NSEL,S,LOC, X,12.123,12.27

NSEL,R,LOC,Z,-5.23,-5.27

SF,ALL,PRES, 150

NALL

EALL

NSEL,S,NODE, ,265,12265,1000

NSEL,A,NODE, ,255, 12255,1000

NSEL,A,NODE, ,245, 12245,1000

NSEL,A,NODE,,234,12234,1000

NSEL,A,NODE, ,224,12224,1000

NSEL,A,NODE, ,219,12219,1000

NSEL,A,NODE, ,213,12213,1000

NSEL,A,NODE, ,208,12208,1000

NSEL,A,NODE, ,202,12202,1000

NSEL,A,NODE, , 188, 12188,1000

NSEL,A,NODE, $181,12181,1000$

NSEL,A,NODE, $170,12170,1000$

NSEL,A,NODE, $169,12169,1000$

NSEL,A,NODE, $158,12158,1000$

NSEL,A,NODE,, $129,12129,1000$

NSEL, $A, N O D E, 128,12128,1000$

NSEL,A,NODE, , $144,12144,1000$

SF,ALL,PRES,150

NALL

EALL

NSEL,S,LOC, X,0,8.9
! THREAD AREA

! START WELD AREA

! END WELD AREA

I STARTING INSIDE RADIUS

! END RADIUS

! INNER TOP PLATE 
CLIENT: DUKE ENGINEERING SERVICES HANFORD, INC

PROJECT: MCO Final Design

NSEL,R,LOC,Z,-12.943,-12.948

SF,ALL,PRES, 150

NALL

EALL

LSWRITE,2

LSSOLVE, 1,2

SAVE

FINI

!.

ICOM POSTPROCESSING

IPOST1

SET, 1

RSYS, 11

PLNS,S,INT

PRRS, F

LPATH 6613,6615

PRSECT

!

SET,LAST

TYPE,ALL,HIDC

IEDGE,ALL, 1,60

/GLINE,ALL, 0

RSYS, 11

PLNSOL, S,INT

PRRS, $F$

LPATH, 6055,6015

PRSECT

LPATH,6056,6016

PRSECT

LPATH 6704,6700

PRSECT

LPATH,6705,6701

PRSECT

LPATH,6057,6017

PRSECT

LPATH, 6128,6096

PRSECT

LPATH,6170,6197

PRSECT

LPATH, 6188,6197

PRSECT

LPATH, 6245,6247

PRSECT

LPATH, 6255,6257

PRSECT

LPATH, 6285,6297

PRSECT

LPATH,6485,6487

PRSECT

LPATH,6543,6545

PRSECT

LPATH, 6613,6615

PRSECT

SAVE

! READ LAST LOAD STEP

! DEFINE TYPE OF DISPLAY

! DIPLAY ONLY THE 'EDGES'

! DETERMINE ELEMENT OUTLINE STYLE

! DISPLAY STRESS INTENSITY

! PRINT REACTION FORCES

! LIFTING EAR (INSIDE)

! LIFTING EAR (INSIDE EDGE OF SHOE)

! LIFTING EAR (INSIDE SHOE)

! LIFTING EAR (INSIDE SHOE)

! OUTSIDE EDGE OF LIFTING SHOE

! START RADIUS (TOP TO CYLINDRICAL)

IMID-RADIUS

I END RADIUS, START TRANSITION

I END TRANSITION, WELD AREA

! THRU WELD

! BELOW WELD

! BOTTOM OF LOCKING RING POSITION

! LR TRANSITION TO SHELLL

! SHELL 


\section{COMPUTER RUN COVER SHEET}

Project Number:

Computer Code:

Software Version:

Computer System:

Computer Run File Number:

Unique Computer Run Filename:

Run Description:

Run Date / Time:
$\mathrm{KH}-8009-8$

ANSYS®-PC

$5.0 \mathrm{~A}$

MS-DOS, Pentium® Processor

KH-8009-8-04

NEWCAP.out

Stress Analysis of Lifting Cap

28 March 1997 12:36:42 PM
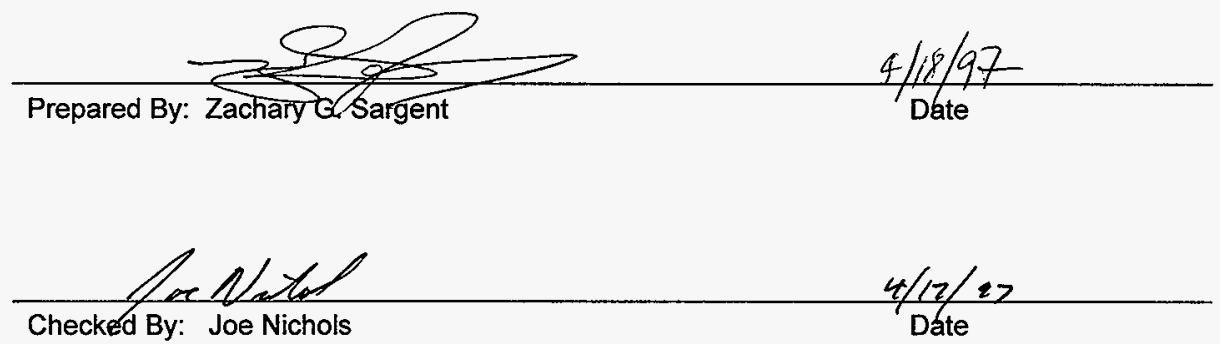

\begin{tabular}{|l|c|l|l|l|l|}
\hline REVISION & 0 & & & & PAGE 59 \\
OF 90
\end{tabular}




PROJECT: MCO Final Design DOC. NO.: HNF-SD-SNF-DR-003, Rev. 0, Appendix 6

\section{COMPUTER RUN COVER SHEET}

Project Number:

Computer Code:

Software Version:

Computer System:

Computer Run File Number:

Unique Computer Run Filename:

Run Description:

Creation Date / Time:
$\mathrm{KH}-8009-8$

ANSYS\&-PC

$5.0 \mathrm{~A}$

MS-DOS, Pentium ${ }^{\circledR}$ Processor

$\mathrm{KH}-8009-8-04$

MODCAP.inp

Stress Analysis of the Modified Lifting Cap 28 March $1997 \quad$ 11:33:14 AM
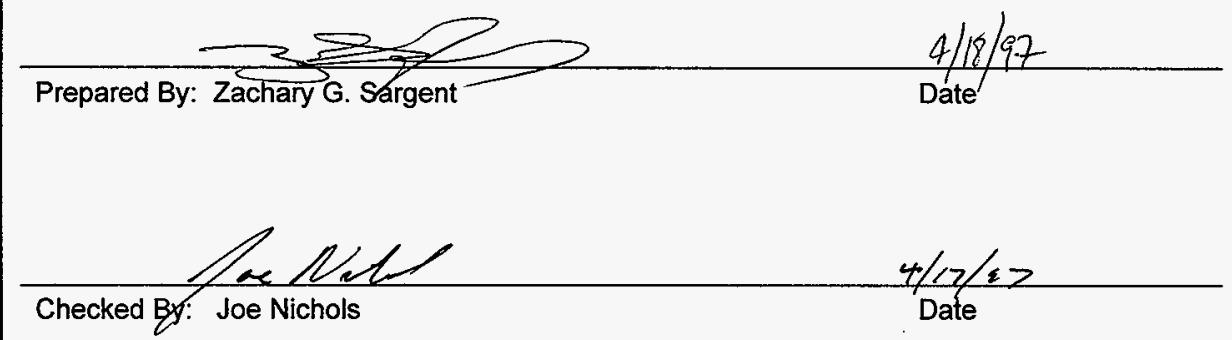

\begin{tabular}{|l|l|l|l|}
\hline 0 & & & \\
\hline $4 / 17 / 97$ & & & \\
\hline $4 / 17 / 97$ & & & \\
\hline
\end{tabular}


CLIENT: DUKE ENGINEERING SERVICES HANFORD, INC

FILE NO: $\quad \mathrm{KH}-8009-8-04$

PROJECT: MCO Final Design DOC. NO.: HNF-SD-SNF-DR-003, Rev. 0, Appendix 6

\title{
LISTING OF MODCAP.INP FILE
}

\author{
/BATCH,LIST \\ IFILNAM,MODCAP \\ IPREP7 \\ TIITLE,MCO MODIFIED CAP \& COLLAR, 132 C, 150 PSIG
ICOM DEFINING TEMPERATURES
TREF,70 ! REFERENCE TEMPERATURE \\ TUNIF,270 ! UNIFORM NODAL TEMPERATURE \\ /COM DEFINING ELEMENT TYPES \\ ET,1,SOLID45 \\ ICOM DEFINING MATERIAL PROPERTIES \\ MP,EX, 1,30e6 \\ MP,ALPX,1,1 \\ MP,NUXY,1,0.3 \\ MP,DENS, $1,490 / 1728$ \\ ! YOUNG'S MODULUS \\ ! COEFFICIENT OF THERMAL EXP. \\ ! POISSON'S RATIO \\ I WEIGHT DENDITY (490 lb/ $\left./ \mathrm{t}^{\wedge} 3\right)$, \\ ! ASSUMED CONSTANT WI TEMP. \\ MPTEMP $1,70,100,200,300,400,500$ I TEMPERATURES FOR PROPERTIES \\ MPTEMP, $7,600,650,700,750$
}

ICOM ELASTIC MODULI FOR 304L. STAINLESS STEEL

MPDATA, EX,1,1,28.3E+06,28.1E+06,27.6E+06,27.0E+06,26.5E+06,25.8E+06

MPDATA, EX,1,7,25.3E+06,25.1E+06,24.8E+06,24.5E+06

/COM MEAN COEFFICIENTS OF THERMAL EXPANSION (in.fin./(F)

MPDATA,ALPX, 1,1,0,8.55e-06,8.79e-06,9.00e-06,9.19e-06,9.37e-06

MPDATA,ALPX, 1,7,9.53e-06,9.61e-06,9.69e-06,9.76e-06

ICOM DEFINING LOCAL COORDINATE SYSTEMS

LOCAL, $11,1, \ldots, 90$

CSYS.0

ICOM DEFINING NODES--LIFTING CAP

/COM TOP OF LIFTING CAP

$\mathrm{N}, 2,1.25,16.442$

$\mathrm{N}, 3,2,16.442$

$N, 7,6,16.442$

FILL, 3,7

$\mathrm{N}, 10,7,16.442$

$\mathrm{N}, 12,7.94,16.442$

FILL, 10,12

$N, 15,9.375,16.442$

FILL, 12,15

$\mathrm{N}, 16,9.53,16.442$

N.17,10.19,16.442

$\mathrm{N}, 21,1,16.192$

$\mathrm{N}, 22,1.25,16.192$

$N, 23,2,15.942$

\begin{tabular}{|l|c|l|l|l|}
\hline REVISION & 0 & & & \\
\hline PREPARED BY I DATE & f/ $S$ S/17/97 & & & \\
\hline CHECKED BY I DATE & //K 4/17/97 & & & \\
\hline
\end{tabular}


CLIENT: DUKE ENGINEERING SERVICES HANFORD, INC

$\mathrm{N}, 27,6,15.942$

FILLL,23,27

$\mathrm{N}, 30,7,15.942$

$N, 32,7.94,15.942$

FILL,30,32

$\mathrm{N}, 35,9.375,15.942$

FILL,32,35

$\mathrm{N}, 36,9.53,15.942$

$\mathrm{N}, 37,10.19,15.942$

$\mathrm{N}, 40,0.517,15.297$

$\mathrm{N}, 41,1,15.297$

$\mathrm{N}, 42,1.25,15.297$

$\mathrm{N}, 43,2,15.442$

$N, 47,6,15.442$

FILL, 43,47

$\mathrm{N}, 50,7,15.442$

$\mathrm{N}, 52,7.94,15.442$

FILL, 50,52

$\mathrm{N}, 55,9.375,15.442$

FILL, 52,55

$\mathrm{N}, 56,9.53,15.442$

$\mathrm{N}, \mathbf{5 7}, \mathbf{1 0 . 1 9}, \mathbf{1 5 . 4 4 2}$

$\mathrm{N}, 60,0.517,14.7445$

$\mathrm{N}, 61,1,14.7445$

$\mathrm{N}, 62,1.25,14.7445$

$\mathrm{N}, 63,2,14.882$

$\mathrm{N}, 67,6,14.882$

FILL, 63,67

$\mathrm{N}, 70,7,14,882$

N,72,7.94,14.882

FILL, 70,72

$\mathrm{N}, 75,9.375,14.882$

FILL, 72,75

$\mathrm{N}, 77,0.125,13.993$

$\mathrm{N}, 80,0.517,14.192$

$\mathrm{N}, 81,1,14.192$

$N, 82,1.25,14.192$

$\mathrm{N}, 83,2,14.316$

$\mathrm{N}, 87,6,14.316$

FILL, 83,87

$N, 90,7,14.316$

$\mathrm{N}, 92,7.94,14.316$

FILL, 90,92

$\mathrm{N}, 95,9.375,14.316$

FILL, 92,95

$\mathrm{N}, 96,9.625,14.316$

$\mathrm{N}, 102,11.125,14.316$

FILL, 96,102

$\mathrm{N}, 103,11.4035,14.316$

$\mathrm{N}, 104,11.75,14.316$

$\mathrm{N}, 105,12.125,14.316$

$\mathrm{N}, 106,12.375,14.316$

$\mathrm{N}, 107,12.625,14.316$

$\mathrm{N}, 18,0.125,12.946$

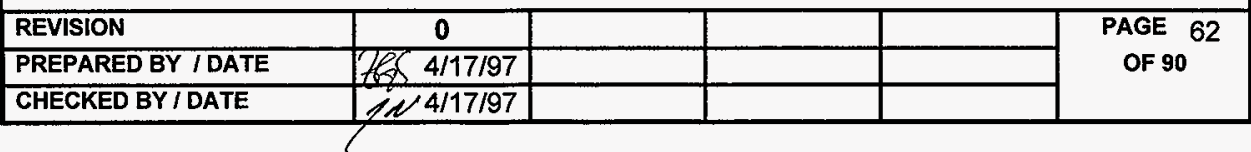


CLIENT: DUKE ENGINEERING SERVICES HANFORD, INC

PROJECT: MCO Final Design $\quad$ DOC. NO.: HNF-SD-SNF-DR-003, Rev. 0, Appendix 6

$N, 19,0.517,12.946$

$\mathrm{N}, 20,1,12.946$

$N, 132,1.25,12.946$

$\mathrm{N}, 133,2,12.946$

$\mathrm{N}, 137,6,12.946$

FILL, 133, 137

$N, 140,7,12.946$

$\mathrm{N}, 142,7.94,12.946$

FILL, 140,142

$N, 145,9.375,12.946$

FILL, 142,145

N, 146,9.625,12.946

N, 152, 11.125,12.946

FILL, 146,152

N, 153,11.4035,12.946

$N, 154,11.75,12.946$

N,155,12.125,12.946

N,156, 12.375,12.946

N,157,12.625,12.946

SAVE

FILL,90,140,1,110

FILL, 91,141,1,111

FILL,92,142,1,112

FILL, 93, 143,1,113

FILL, 94, 144,1,114

FILL,95,145,1,115

FILL, 96,146,1,116

FILL, 97,147,1,117

FILL,98,148,1,118

FILL,99,149,1,119

FILL, 100,150,1,120

FILL, 101,151,1,121

FILL, 102,152,1,122

FILL, 103,153,1,123

FILL, 104,154,1,124

FILL, 105,155,1,125

FILL, 106,156,1,126

FILL, 107,157,1,127

$\mathbf{N}, 700,9.75,16.442$

N,701,9.97,16.442

! LIFTING EAR

$\mathrm{N}, 704,9.75,15.442$

$\mathrm{N}, 705,9.97,15.442$

FILL, $700,704,1,702$

FILL, $701,705,1,703$

ICOM SIDE OF LIFTING CAP

N, $128,9.375,12.8519$

$N_{1} 129,9.625,12.7692$

$N, 130,9.875,12.6589$

$\mathrm{N}, 158,10.125,12.5171$

$\mathrm{N}, 159,10.375,12.571$

$\mathrm{N}, 160,10.625,12.571$

$N, 161,10.875,12.571$

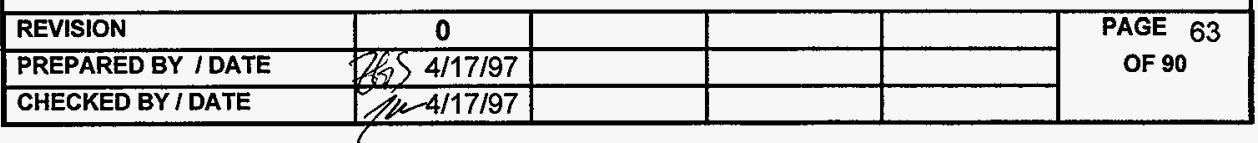


FILE NO: KH-8009-8-04

PROJECT: MCO Final Design DOC. NO.: HNF-SD-SNF-DR-003, Rev. 0, Appendix 6

$\mathrm{N}, 162,11.125,12.571$

$N, 163,11.4035,12.571$

$\mathrm{N}, 164,11.75,12.571$

$N, 165,12.125,12.571$

$\mathrm{N}, 166,12.375,12.571$

N,167,12.625, 12.571

$\mathrm{N}, 169,10.375,12.3375$

$\mathrm{N}, 170,10.5687,12.1661$

$N, 171,10.875,12.196$

$N, 172,11.125,12.196$

$N, 173,11.4035,12.196$

$\mathrm{N}, 174,11.75,12.196$

$\mathrm{N}, 175,12.125,12.196$

$\mathrm{N}, 176,12.375,12.196$

$\mathrm{N}, 177,12.625,12.196$

$\mathrm{N}, 181,10.7726,11.946$

$\mathrm{N}, 182,11.125,11.946$

$N, 183,11.4035,11.946$

$\mathrm{N}, 184,11.75,11.946$

$\mathrm{N}, 185,12.125,11.946$

$\mathrm{N}, 186,12.375,11.946$

$\mathrm{N}, 187,12.625,11.946$

$\mathrm{N}, 188,11.0198,11.596$

N, 192,11.125,11.596

$\mathrm{N}, 193,11.4035,11.596$

$\mathrm{N}, 194,11.75,11.596$

N,195,12.125,11.596

$\mathrm{N}, 196,12.375,11.596$

$\mathrm{N}, 197,12.625,11.596$

$N, 202,11.1753,11.296$

$\mathrm{N}, 203,11.4035,11.296$

N,204,11.75,11.296

N,205,12.125,11.296

N,206,12.375,11.296

N.207,12.625,11.296

$N, 208,11.3421,10.869$

$\mathrm{N}, 209,11.75,10.869$

$\mathrm{N}, 210,12.125,10.869$

$\mathrm{N}, 211,12.375,10.869$

$\mathrm{N}, 212,12.625,10.869$

$\mathrm{N}, 213,11.5046,10.4420$

$\mathrm{N}, 214,11.75,10.4420$

$\mathrm{N}, 215,12.125,10.4420$

$\mathrm{N}, 216,12.375,10.4420$

$N, 217,12.625,10.4420$

$N, 219,11.6672,10.0146$

N,220,12.125,10.0146

$\mathrm{N}, 221,12.375,10.0146$

$\mathrm{N}, 222,12.625,10.0146$

$\mathrm{N}, 224,11.8682,9.4866$

$N, 225,12.125,9.4866$

$N, 226,12.375,9.4866$

$\mathrm{N}, 227,12.625,9.4866$

$\mathrm{N}, 234,12.0691,8.9586$

\begin{tabular}{|l|c|l|l|l|l|}
\hline REVISION & 0 & & & & PAGE 64 \\
OF 90
\end{tabular}


FILE NO: $\quad \mathrm{KH}-8009-8-04$

$\begin{array}{ll}\text { PROJECT: MCO Final Design } & \text { DOC. NO.: HNF-SD-SNF-DR-003, Rev. 0, Appendix } 6\end{array}$

ICOM WELD AREA BETWEEN LIFTING CAP \& CANISTER COLLAR

$\mathrm{N}, 235,12.125,8.94$

N,236, 12.375,8.94

$\mathrm{N}, 237,12.625,8.94$

$\mathrm{N}, 245,12.225,8.793$

$\mathrm{N}, 246,12.375,8.793$

$\mathrm{N}, 247,12.625,8.793$

$\mathrm{N}, 255,12.225,8.646$

$\mathrm{N}, 256,12.375,8.646$

$\mathrm{N}, 257,12.625,8.646$

ICOM CANISTER COLLAR THREADS

$\mathrm{N}, 265,12.225,8.5$

$\mathrm{N}, 266,12.375,8.5$

! UPPER OUTER THREADS

$\mathrm{N}, 267,12.625,8.5$

$\mathrm{N}, 285,12.125,8.25$

$\mathrm{N}, 297,12.625,8.25$

FILL,285,297,1,291

$\mathrm{N}, 305,12.125,7.75$

$\mathrm{N}, 317,12.625,7.75$

FILL, 305,317,1,311

$\mathrm{N}, 405,12.125,5.25$

FILL, 305,405,4,325

$\mathrm{N}, 410,12.25,5.25$

$\mathrm{N}, 391,12.375,5.75$

! LOWER OUTER THREADS

FILL,311,391,3,331

$\mathrm{N}, 416,12.5,5.25$

$\mathrm{N}, 417,12.625,5.25$

FILL,317,417,4,337

$\mathrm{N}, 430,12.25,5$

$N, 435,12.01,5$

$\mathrm{N}, 437,12.625,5$

$\mathrm{N}, 445,12.01,4.6$

$N, 447,12.625,4.6$

FILL, 445,447

FILL, $430,437,1,436$

$\mathrm{N}, 485,12.01,3$

FILL, 445,485,3,455

$\mathrm{N}, 487,12.625,3$

FILL,437,487,4,447

FILL,485,487, 1,486

FILL, 446,486,3,456

$\mathrm{N}, 493,11.5,2.63$

$\mathrm{N}, 495,12.01,2.63$

FILL, 493,495

$\mathrm{N}, 497,12.625,2.63$

FILL, 495,497

$\mathrm{N}, 503,11,5,2.33$

$\mathrm{N}, 505,12.01,2.33$

FILL,503,505

$\mathrm{N}, 507,12.625,2.33$

FILL, 505,507

$\mathrm{N}, 513,11.5,2.03$

! LOWER LIFTING CAP

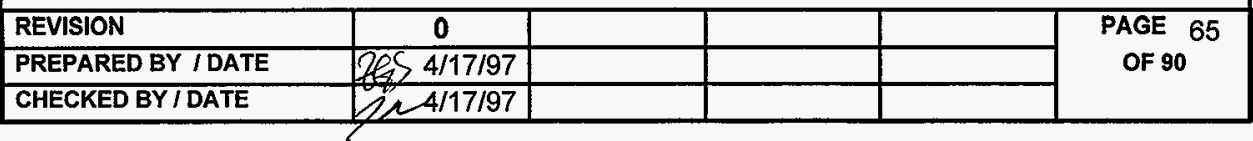


CLIENT: DUKE ENGINEERING SERVICES HANFORD, INC

FILE NO: $\quad \mathrm{KH}-8009-8-04$

$\begin{array}{ll}\text { PROJECT: MCO Final Design } & \text { DOC. NO.: HNF-SD-SNF-DR-003, Rev. 0, Appendix } 6\end{array}$

$\mathrm{N}, 515,12.01,2.03$

FIL.L, 513,515

$\mathrm{N}, 517,12.625,2.03$

FIL.L,515,517

$\mathrm{N}, 523,11.5,1.63$

$\mathrm{N}, 525,12.01,1.63$

FILL,523,525

$\mathrm{N}, 527,12.625,1.63$

FILL,525,527

$\mathrm{N}, 530,12.318,1.315$

$\mathrm{N}, 533,11.5,1$

N, $535,12.01,1$

FILL,533,535

$\mathrm{N}, 553,11.5,0$

$\mathrm{N}, 555,12.01,0$

FILL,533,553,1,543

FlLL, 553,555

FILL,534,554,1,544

FlLL, 535,555,1,545

N,633,11.5,-8

FILL,553,633,7,563

$N, 635,12.01,-8$

FILL,555,635,7,565

FILL,563,565

FILL,573,575

FILL,583,585

FILL,593,595

FILL,603,605

FILL,613,615

FILL, 623,625

FILL, 633,635

SAVE

1 BOTTOM

ICOM CHANGING TO LOCAL COORDINATE SYSTEM

CSYS, 11

/COM NODAL GENERATION 60 DEGREE SWEEP

NGEN, $5,1000, \mathrm{ALL}_{1, \ldots},-5$

I 5 DEGREE INCREMENTS

NGEN $, 2,1000,4000,4999,1,-4.27$

NGEN, $3,1000,5000,5999,1,-5.73$

NGEN, $2,1000,7000,7999,1,-4.27$

! GRAPPLE SHOE AREA (1.97"X0.66")

NGEN, $5,1000,8000,8999,1,-5$

CSYS, 0

/COM ELEMENT GENERATION

ICOM LIFTING CAP

TYPE, 1

$E, 42,1042,1041,41,22,1022,1021,21$

EGEN,3,20,-1

$E, 61,1061,1060,60,41,1041,1040,40$

EGEN,2,20,-1

E, $19,1019,1018,18,80,1080,1077,77$

$E, 20,1020,1019,19,81,1081,1080,80$

$E, 132,1132,1020,20,82,1082,1081,81$

$E, 23,1023,1022,22,3,1003,1002,2$

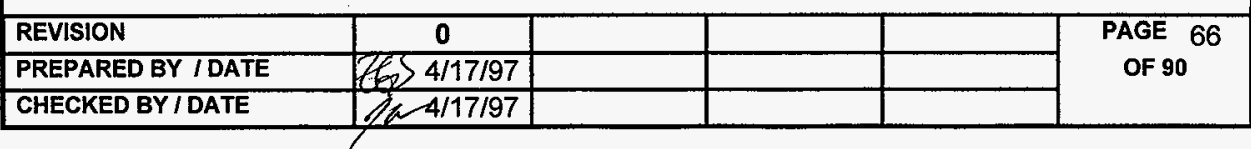


EGEN,5,1,-1

$E, 43,1043,1042,42,23,1023,1022,22$

EGEN, $5,1,-1$

$E, 63,1063,1062,62,43,1043,1042,42$

EGEN,5,1,-1

$E, 83,1083,1082,82,63,1063,1062,62$

EGEN,5,1,-1

E.133,1133,1132,132,83,1083,1082,82

EGEN,5,1,-1

$E, 30,1030,1027,27,10,1010,1007,7$

EGEN,4,20,-1

$E, 87,90,110,110,1087,1090,1110,1110$

E, $87,110,137,137,1087,1110,1137,1137$

$E, 110,140,137,137,1110,1140,1137,1137$

$E, 31,1031,1030,30,11,1011,1010,10$

EGEN,4,20,-1

$E, 32,1032,1031,31,12,1012,1011,11$

EGEN,4,20,-1

$E, 33,1033,1032,32,13,1013,1012,12$

EGEN,4,20,-1

$E, 34,1034,1033,33,14,1014,1013,13$

EGEN,4,20,-1

$E, 35,1035,1034,34,15,1015,1014,14$

EGEN, $4,20,-1$

$E, 36,1036,1035,35,16,1016,1015,15$

EGEN,2,20,-1

$E, 702,1702,1036,36,700,1700,1016,16$

E, $704,1704,1056,56,702,1702,1036,36$

$E, 703,1703,1702,702,701,1701,1700,700$

EGEN $2,2,-1$

$E, 37,1037,1703,703,17,1017,1701,701$

$E, 57,1057,1705,705,37,1037,1703,703$

$\mathrm{E}, 75,96,95,95,1075,1096,1095,1095$

$E, 111,1111,1110,110,91,1091,1090,90$

$E, 112,1112,1111,111,92,1092,1091,91$

$E, 113,1113,1112,112,93,1093,1092,92$

EGEN,3,1,-1

E, $116,1116,1115,115,96,1096,1095,95$

E,117,1117,1116,116,97,1097,1096,96

EGEN, $11,1,-1$

$E, 141,1141,1140,140,111,1111,1110,110$

EGEN, 17,1,-1

$E, 144,145,128,128,1144,1145,1128,1128$

$E, 129,1129,1128,128,146,1146,1145,145$

$E, 130,1130,1129,129,147,1147,1146,146$

E, $158,1158,1130,130,148,1148,1147,147$

$E, 159,1159,1158,158,149,1149,1148,148$

EGEN, $9,1,-1$

E, $158,159,169,169,1158,1159,1169,1169$

$E, 170,1170,1169,169,160,1160,1159,159$

EGEN,8,1,-1

E, 170,171,181,181,1170,1171,1181,1181

$E, 182,1182,1181,181,172,1172,1171,171$

EGEN,6,1,-1

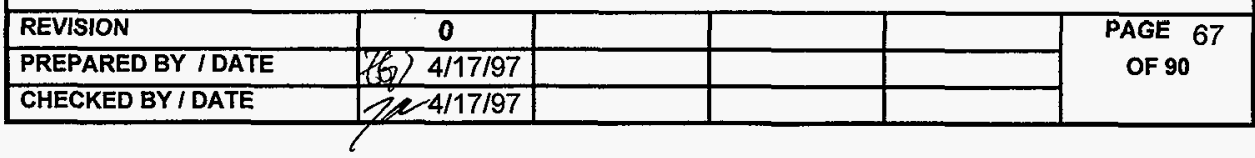


CLIENT:

PROJECT: MCO Final Design
FILE NO: KH-8009-8-04

$E, 192,1192,1188,188,182,1182,1181,181$

$E, 193,1193,1192,192,183,1183,1182,\{82$ EGEN, $5,1,-1$

$E, 188,192,202,202,1188,1192,1202,1202$ $E, 203,1203,1202,202,193,1193,1192,192$ EGEN $, 5,1,-1$

$\mathrm{E}, 202,203,208,208,1202,1203,1208,1208$ $E, 209,1209,1208,208,204,1204,1203,203$ EGEN,4,1,-1

$E_{,} 214,1214,1213,213,209,1209,1208,208$ $E, 215,1215,1214,214,210,1210,1209,209$ EGEN, $3,1,-1$

$E, 213,214,219,219,1213,1214,1219,1219$ $E, 220,1220,1219,219,215,1215,1214,214$ EGEN, $3,1,-1$

$E, 225,1225,1224,224,220,1220,1219,219$

EGEN,3,1,-1

$E, 235,1235,1234,234,225,1225,1224,224$

EGEN,3,1,-1

$E, 234,235,245,245,1234,1235,1245,1245$

$E, 246,1246,1245,245,236,1236,1235,235$

$E, 247,1247,1246,246,237,1237,1236,236$

$E, 256,1256,1255,255,246,1246,1245,245$

$E, 257,1257,1256,256,247,1247,1246,246$

E,266,1266,1265,265,256,1256,1255,255

E,267,1267,1266,266,257,1257,1256,256

$E, 291,1291,1285,285,266,1266,1265,265$

E,297,1297, 1291,291,267, 1267,1266,266

$E, 311,1311,1305,305,291,1291,1285,285$

EGEN, $5,20,-1$

E,317,1317,1311,311,297, 1297,1291,291

EGEN, $5,20,-1$

$E, 410,1410,1405,405,391,1391,1385,385$

$E, 391,416,410,410,1391,1416,1410,1410$

$E, 417,1417,1416,416,397,1397,1391,391$

$E, 436,1436,1430,430,416,1416,1410,410$

$E, 437,1437,1436,436,417,1417,1416,416$

$E, 446,1446,1445,445,430,1430,1435,435$

$E, 430,436,446,446,1430,1436,1446,1446$

$E, 447,1447,1446,446,437,1437,1436,436$

$E, 456,1456,1455,455,446,1446,1445,445$

EGEN, 4, 10,-1

$E, 496,1496,1495,495,486,1486,1485,485$

$E, 457,1457,1456,456,447,1447,1446,446$

EGEN,4,10,-1

$E, 497,1497,1496,496,487,1487,1486,486$

$E, 506,1506,1505,505,496,1496,1495,495$

EGEN,2,10,-1

$E, 526,1526,1525,525,516,1516,1515,515$

$E, 525,526,530,530,1525,1526,1530,1530$

$E, 507,1507,1506,506,497,1497,1496,496$

EGEN,2,10,-1

E,527,1527,1526,526,517,1517,1516,516

$\mathrm{E}, 526,527,530,530,1526,1527,1530,1530$

\begin{tabular}{|l|c|l|l|l|l|}
\hline REVISION & 0 & & & & $\begin{array}{c}\text { PAGE } 68 \\
\text { OF } 90\end{array}$ \\
\hline PREPARED BY I DATE & $2 / 8 / 17 / 97$ & & & & \\
\hline CHECKED BY I DATE & $1 / 4 / 17 / 97$ & & & & \\
\hline
\end{tabular}


CLIENT: DUKE ENGINEERING SERVICES HANFORD, INC

FILE NO: $\quad \mathrm{KH}-8009-8-04$

PROJECT: MCO Final Design DOC. NO.: HNF-SD-SNF-DR-003, Rev. 0, Appendix 6

$E, 525,530,535,535,1525,1530,1535,1535$

$E, 504,1504,1503,503,494,1494,1493,493$

EGEN,2,10,-1

$E, 505,1505,1504,504,495,1495,1494,494$

EGEN $, 2,10,-1$

$E, 524,1524,1523,523,514,1514,1513,513$

EGEN,2,1,-1

$E, 534,1534,1533,533,524,1524,1523,523$

EGEN,2,1,-1

$E, 544,1544,1543,543,534,1534,1533,533$

EGEN,10,10,-1

$E, 545,1545,1544,544,535,1535,1534,534$

EGEN,10,10,-1

SAVE

ESEL,ALL

EGEN, 12,1000,ALL

ICOM MERGING COINCIDENT LABELS

NUMMRG,NODE IMERGE ALL COINCIDENT NODES

ICOM BOUNDARY CONDITIONS

CSYS, 11

I CYLINDRICAL

NROTAT,ALL

I ROTATE ALL NODES IN CURRENT SYS

NSEL,S,NODE, $, 1,705$

DSYM, SYMM, Y, 11

! SELECT ALL NODES ON FIRST SLICE!

NSEL,ALL

NSEL,S,NODE $, 12002,12705$

I SYMMETRY CONSTRAINT

DSYM,SYMM, Y, 11

NSEL,ALL

NSEL,S,NODE, $634,12634,1000$ ! SELECT NODES AT BOTTOM OF C.COLLAR

D,ALL,UZ

! APPLY DOF CONSTRAINTS

SAVE

FINI

ICOM SOLUTION PHASE

ISOLU

NEQIT,25

OUTPR, LAST

OUTRES, LAST

/COM LOAD STEP 1: LIFTING LOAD

NALL

NSEL,S,NODE, $5057,6057,1000$

NSEL,A,NODE, $, 5705,6705,1000$

SF,ALL,PRES, 3010

NALL

NSEL,S,NODE, $6057,7057,1000$

NSEL,A,NODE, $6705,7705,1000$

SF,ALL,PRES, 3010

NALL

NSEL,S,NODE, $5705,6705,1000$

NSEL,A,NODE, $5704,6704,1000$

SF,ALL,PRES, 3077

NALL.

! SELECT NODES ON LAST SLICE 
CLIENT: DUKE ENGINEERING SERVICES HANFORD, INC

FILE NO: KH-8009-8-04

PROJECT: MCO Final Design

NSEL,S,NODE, $6705,7705,1000$

NSEL,A,NODE, $6704,7704,1000$

SF,ALL,PRES,3077

NALL

NSEL,S,NODE, $5704,6704,1000$

NSEL,A,NODE, $5056,6056,1000$

SF,ALL,PRES, 3148

NALL.

NSEL,S,NODE, $6704,7704,1000$

NSEL,A,NODE, $6056,7056,1000$

SF,ALL,PRES, 3148

NALL.

EALL

LSWRITE, 1

ICOM LOAD STEP 2: APPLYING 150 PSI INTERNAL PRESSURE

NALL

EALL

NSEL,S,LOC, $X, 11.45,11.55$

NSEL,R,LOC,Z,8.1,-2.64

! LOWER HALF

SF,ALL,PRES, 150

NALL.

EALL.

NSEL, S,LOC, $X, 11.48,12.03$

NSEL,R,LOC,Z,-2.61,-2.65

SF,ALL,PRES, 150

NALL

EALL

NSEL,S,LOC,X,11.99, 12.03

NSEL,R,LOC, $Z,-2.61,-3.02$

SF,ALL,PRES, 150

NALL

EALL

NSEL,S,LOC,X,12,12.128

NSEL,R,LOC, Z,-2.64,-8.52

! THREAD AREA

SF,ALL,PRES, 150

NALL

EALL

NSEL,S,LOC, X,11.99,12.27

NSEL,R,LOC, Z,-4.98, -5.02

SF,ALL,PRES, 150

NALL

EALL

NSEL,S,LOC, X, 12.23,12.27

NSEL,R,LOC,Z,-4.95,-5.27

SF,ALL,PRES, 150

NALL.

EALL

NSEL,S,LOC, X, 12.123,12.27

NSEL,R,LOC, Z,-5.23,-5.27

SF,ALL,PRES, 150

NALL

EALL.

NSEL,S,NODE,_285,12285,1000

REVISION

PREPARED BY I DATE

CHECKED BY/DATE 
CLIENT: DUKE ENGINEERING SERVICES HANFORD, INC

FILE NO: $\quad \mathrm{KH}-8009-8-04$

PROJECT: MCO Final Design DOC. NO.: HNF-SD-SNF-DR-003, Rev. 0, Appendix 6

NSEL,A,NODE, 265,12265,1000

NSEL,A,NODE, $255,12255,1000$

NSEL,A,NODE, 245,12245,1000

NSEL, A,NODE, $234,12234,1000$

NSEL,A,NODE, 224,12224,1000

NSEL, $A, N O D E, 219,12219,1000$

NSEL, A,NODE, $213,12213,1000$

NSEL,A,NODE, 208,12208,1000

NSEL,A,NODE, 202, 12202, 1000

NSEL,A,NODE, , $188,12188,1000$

NSEL,A,NODE, ,181,12181,1000

NSEL,A,NODE, $170,12170,1000$

NSEL,A,NODE, , 169,12169,1000

NSEL,A,NODE,, 158,12158,1000

NSEL,A,NODE, ,129,12129,1000

NSEL,A,NODE, , 128,12128,1000

NSEL,A,NODE, ,144,12144,1000

SF,ALL,PRES, 150

NALL

EALL

NSEL,S,LOC,X,0,8.9

NSEL,R,LOC,Z,-12.943,-12.948

SF,ALL,PRES, 150

NALL

EALL

LSWRITE,2

LSSOLVE, 1,2

SAVE

FINI

ICOM POSTPROCESSING

IPOST1

SET, 1

RSYS, 11

PLNS,S,INT

PRRS,F

LPATH, 6613,6615

PRSECT

SET,LAST

TYPE,ALL,HIDC

/EDGE,ALL, 1,60

/GLINE,ALL, 0

RSYS,11

PLNSOL,S,INT

PRRS,F

LPATH,6004,6134

PRSECT

LPATH,6002,6132

PRSECT

LPATH,6004,6018

PRSECT

LPATH,6055,6015

PRSECT

LPATH,6056,6016
I START WELD AREA

! END WELD AREA

! STARTING INSIDE RADIUS

! END RADIUS

! INNER TOP PLATE
! READ LAST LOAD STEP
! DEFINE TYPE OF DISPLAY
! DIPLAY ONLY THE 'EDGES'
! DETERMINE ELEMENT OUTLINE STYLE

! DISPLAY STRESS INTENSITY

! PRINT REACTION FORCES

! TOP PLATE

! TOP PLATE

! TOP PLATE

! LIFTING EAR (INSIDE)

! LIFTING EAR ( INSIDE EDGE OF SHOE) 
$\begin{array}{ll}\text { PROJECT: MCO Final Design } & \text { DOC. NO: HNF-SD-SNF-DR-003, Rev. 0, Appendix } 6\end{array}$

PRSECT

LPATH, 6704,6700

PRSECT

LPATH,6705,6701

PRSECT

LPATH, 6057,6017

PRSECT

LPATH,6128,6096

PRSECT

LPATH, 6170,6197

PRSECT

LPATH,6188,6197

PRSECT

LPATH, 6245,6247

PRSECT

LPATH, 6255,6257

PRSECT

LPATH,6285,6297

PRSECT

LPATH, 6485,6487

PRSECT

LPATH, 6543,6545

PRSECT

LPATH,6613,6615

PRSECT

SAVE
! LIFTING EAR (INSIDE SHOE)

! LIFTING EAR (INSIDE SHOE)

! OUTSIDE EDGE OF LIFTING SHOE

! START RADIUS (TOP TO CYLINDRICAL)

! MID-RADIUS

! END RADIUS, START TRANSITION

! END TRANSITION, WELD AREA

! THRU WELD

! BELOW WELD

! BOTTOM OF LOCKING RING POSITION

! LR TRANSITION TO SHELLL

! SHELL 


\section{COMPUTER RUN COVER SHEET}

Project Number:

Computer Code:

Software Version:

Computer System:

Computer Run File Number:

Unique Computer Run Filename:

Run Description:

Run Date / Time:
$\mathrm{KH}-8009-8$

ANSYS®-PC

$5.0 \mathrm{~A}$

MS-DOS, Pentium® Processor

$\mathrm{KH}-8009-8-04$

MODCAP.out

Stress Analysis of the Modified Lifting Cap

28 March $1997 \quad$ 11:47:42 AM
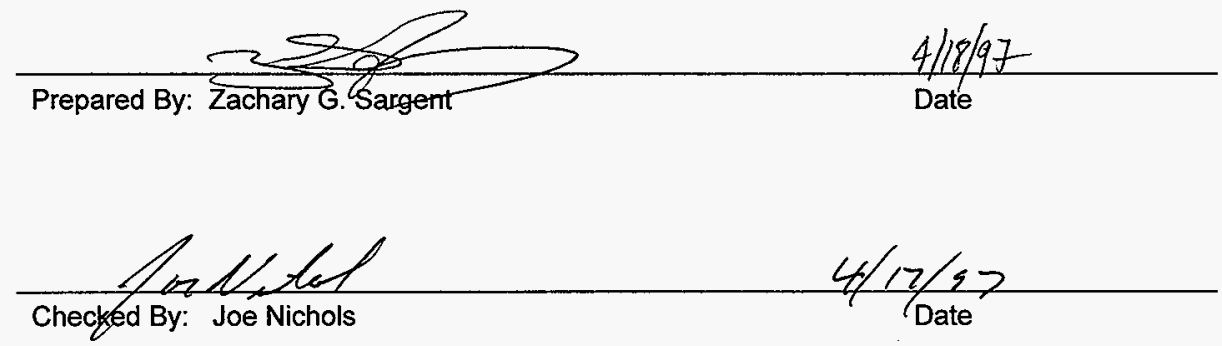


\section{Q}

CLIENT: DUKE ENGINEERING SERVICES HANFORD, INC
FILE NO: $\quad \mathrm{KH}-8009-8-04$
PROJECT: MCO Final Design DOC. NO.: HNF-SD-SNF-DR-003, Rev. 0, Appendix 6

\section{INPUT FILE COVER SHEET}

Project Number:

$\mathrm{KH}-8009-8$

Computer Code:

ANSYS $^{\circledR}$

Software Version:

n/a (See Note)

Computer System:

n/a (See Note)

Computer Run File Number:

KH-8009-8-04

Unique Input Filename:

THRD3.inp

Input File Description:

Stress Analysis of the MCO Thread/Closure

Creation Date / Time:

16 April 1997

10:28 AM

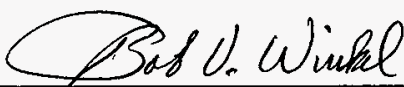

Prepared By: Bob V. Winkel

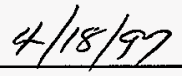

Date
See Note

Checked By: Ward Ingles

Note: This input file will run under multiple versions of ANSYS on different platforms. The input file was not checked, the output generated by the input file was checked.

\begin{tabular}{|l|c|c|c|c|c|}
\hline REVISION & 0 & & & & PAGE 74 \\
OF 90
\end{tabular}


CLIENT: DUKE ENGINEERING SERVICES HANFORD, INC

FILE NO: $\mathrm{KH}-8009-8-04$

PROJECT: MCO Final Design DOC. NO.: HNF-SD-SNF-DR-003, Rev. 0, Appendix 6

\section{LISTING OF THRD3.INP FILE}

/batch, list

title, Mco closure Threads, Preload only, No Friction

/filname, thrd3

/prep7

/triad, 1top

*afun, deg

/com element types for shell collar (internal threads)

et, 2, plane $42,, 1$ ! axisymmetxic quads

/com element types for jacking bolts

et,3,plane42,,1 ! axisymmetric quads

/com element types for lifting \& locking ring (external threads)

et, 4,plane $42, \ldots$ ! axisymmetric quads

/com element types for shield plug

et, 5, plane $42,, 1$ ! axisymmetric quads

et, 7, contac 48 ! contact surface (gap) elements at threads

keyopt, $7,7,1$

$r, 7,1.0 \mathrm{E}+06$

et, 8, contac 12

! contact elements under jacking bolts

keyopt, $8,7,1$

$r, 8,0,1.0 E+05, .304,1.0$ ! initial interference for preload

/com define variables

hs $=.1657$

! thread height

he $=.1406$

$F=.0408$

! height of thread engagment.

Dpitch $=24.5$

Rpitch=Dpitch $/ 2$

pitch $=.25$ ! pitch

/com shell dimensions

RSin $=24.04 / 2$

RSout $=25.31 / 2$

! collar inside radius (below threads)

! collar outside radius

IRshe111 $=11.50$

! shell inside radius (lower section)

ORshel11 $=12.00$

! shell outside radius (lower section)

REmin=Rpitch-hs

! external thread (locking \& lifting ring)

REmax=Rpitch

RImin=Rpitch-he ! internal thread (shell collar)

RImax=Rpitch-he+hs

RSavg $=($ RImax + RSOut $) / 2 \cdot$ ! average radius at threads

Rrelief $=24.567 / 2$

BoltCirc $=21.75$

! bolt circle diameter

REVISION

PREPARED BY / DATE 10

CHECKED BY / DATE 
CLIENT: $\quad$ DUKE ENGINEERING SERVICES HANFORD, INC

FILE NO: $\quad \mathrm{KH}-8009-8-04$

PROJECT: MCO Final Design

DOC. NO.: HNF-SD-SNF-DR-003, Rev. 0, Appendix 6

BoltSize=1.500 ! bolt nominal diameter

BoltIR $=($ BoltCirc-BoltSize $) / 2$ ! diameter at intside edge jacking bolts

BoltOR= (BoltCirc + BoltSize $) / 2$ ! diameter at outside edge jacking bolts

Bolt $A=3.1415926^{*}((B \circ 1 t O R * B \circ 1 t O R)-(B \circ I t I R * B O I t I R))$

/com scale factor for bolt $\mathrm{E} \& \mathrm{Sy}$ (modeled as axisymmetric)

RealArea $=18 * 1.41$ ! tensile area of jacking bolts

$\mathrm{SF}=$ RealArea/BoltA i $\mathrm{SF}=$ actual bolt area/modeled bolt area

elsize $=.7 * 0.05$ ! element size for thread mesh

/com thread dimensions

$\mathrm{Y} 2=0$

$\mathrm{Y} 3=($ hs $-\mathrm{he}) * \tan (7)$

$\mathrm{Y} 4=($ hs $* \tan (7))$

$\mathrm{Y} 5=(\mathrm{hs} * \tan (7))+\mathrm{F}$

Y6=pitch-Y5

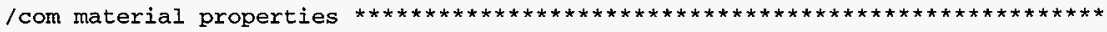
/com $304 \mathrm{~L}$

mp, nuxy, 1,.3 ! poisson constant with temperature

mp, dens,1,493/1728 ! weight density (493 lb/ft^3), assumed constant w/temp.

/com nonlinear properties - 304L

etan $=.006$ ! use $5 \%$ tangent modulus

tb,bkin,1,6 ! yield stress and tangent moduli $\mathrm{v}$. temperature

tbtemp, 100

tbdata, 1,25000, etan*28.1E+06

tbtemp, 300

tbdata 1,19100 , etan $* 27.0 \mathrm{E}+06$

tbtemp, 400

tbdata, 1, 17500, etan $* 26.5 \mathrm{E}+06$

tbtemp, 500

tbdata, 1,16300 , etan $\star 25.8 \mathrm{E}+06$

tbtemp, 700

tbdata, 1, 14900, etan*24.8E+06

tbtemp, 750

tbdata, 1, 14700, etan*24.5E+06

mptemp, $1,70,100,200,300,400,500$

mptemp, $7,600,650,700,750$

/com elastic moduli for $304 \mathrm{~L}$ stainless steel

mpdata, ex, $1,1,28.3 \mathrm{E}+06,28.1 \mathrm{E}+06,27.6 \mathrm{E}+06,27.0 \mathrm{E}+06,26.5 \mathrm{E}+06,25.8 \mathrm{E}+06$

mpdata, ex, $1,7,25.3 \mathrm{E}+06,25.1 \mathrm{E}+06,24.8 \mathrm{E}+06,24.5 \mathrm{E}+06$

/com instantaneous coefficients of thermal expansion, 304L stainlesss

mpdata, alpx, 1, 1, 8.46E-06,8.63E-06,9.08E-06,9.46E-06,9.80E-06, 10.10E-06

mpdata, alpx, 1,7,10.38E-06,10.50E-06,10.60E-06,10.70E-06

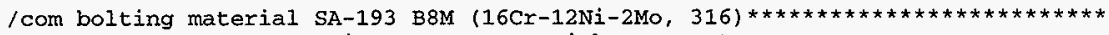

mp, nuxy, $2, .3 \quad$ ! poisson constant with temperature

mp, dens, 2,493/1728 ! weight density (493 1b/ft^3), assumed constant w/temp.

/com nonlineax properties - bolting material SA-193 B8M (16Cr-12Ni-2Mo, 316)

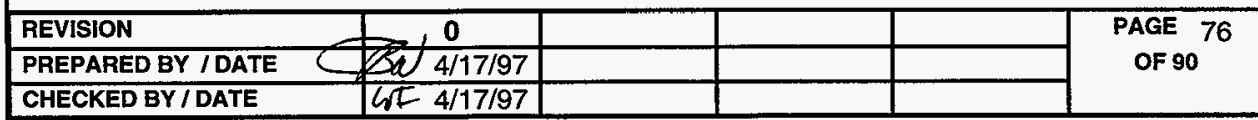


tb,bkin,2,6 ! yield stress and tangent moduli v. temperature

tbtemp, 100

tbdata, $1, \mathrm{SF} * 30000$, etan $* 28.1 \mathrm{E}+06$

tbtemp, 300

tbdata , 1, SF 23300 , etan $* 27.0 \mathrm{E}+06$

tbtemp, 400

tbdata , 1, SF $* 21400$, etan $* 26.5 E+06$

tbtemp, 500

tbdata , $1, S F \star 19900$, etan $* 25.8 E+06$

tbtemp, 700

tbdata, $1, \mathrm{SF} * 18100$, etan $* 24.8 \mathrm{E}+06$

tbtemp, 750

tbdata , $1, \mathrm{SF} * 17800$, etan $* 24.5 \mathrm{E}+06$

mptemp, 1, $70,100,200,300,400,500$

mptemp, $7,600,650,700,750$

/com elastic moduli for $16 \mathrm{Cr}-12 \mathrm{Ni}$ (same as $304,18-8$ )

mpdata, ex, $2,1, \mathrm{SF} \star 28,3 \mathrm{E}+06, \mathrm{SF} * 28.1 \mathrm{E}+06, \mathrm{SF} * 27.6 \mathrm{E}+06, \mathrm{SF} \star 27,0 \mathrm{E}+06, \mathrm{SF} * 26.5 \mathrm{E}+06$,

mpdata, ex $, 2,6, \mathrm{SF} * 25.8 \mathrm{E}+06, \mathrm{SF} * 25.3 \mathrm{E}+06, \mathrm{SF} * 25.1 \mathrm{E}+06, \mathrm{SF} * 24.8 \mathrm{E}+06, \mathrm{SF} * 24.5 \mathrm{E}+06$

/com instantaneous coefficients of thermal expansion, 16Cr-12Ni-2Mo High Alloy

mpdata, alpx, $2,1,8.42 \mathrm{E}-06,8.59 \mathrm{E}-06,9.09 \mathrm{E}-06,9.56 \mathrm{E}-06,9.95 \mathrm{E}-06,10.25 \mathrm{E}-06$

mpdata, alpx, 2,7,10.51E-06,10.64E-06,10.76E-06,10.87E-06

/com list material properties

mplist

tblist

/com Shell Collar (internal thread) $* * * * \star * * * * * * * \star * * * * * * * * * * * * * * * * * * * * * * * * * *$

$\mathrm{k}, 10$, IRshel11, -12.00

$k, 11$, ORshell1, -12.00

$k, 12$, IRshell1, -4.00

$k, 13$, ORshell1, -4.00

! bottom of transistion - outer shell

$k, 14$, RSout , -3.37

k,15, IRshell1, -2.97 ! top of transistion - outer shell

k, 16, RSout, -2.97

$k, 17$, IRshell1, -2.70

$k, 18$, RSout, -2.70

$k, 19$, IRshell1, -2.37 ! sealing surface

$k, 20,23.375 / 2,-2.37$ ! sealing surface

$k, 21$, RSin, -2.37 ! sealing surface

$k, 22$, RSout, -2.37 ! sealing surface

$k, 31$, RSin, -2.12

$k, 32$, RSout, -2.12

$k, 33$, RSin, -1.87

$k, 34$, RSout, -1.87

k, 35, RSin, -.5

\begin{tabular}{|l|c|l|l|l|l|}
\hline REVISION & 0 & & & & PAGE 77 \\
OF 90
\end{tabular}


$k, 36$, RSout, -.5

$k, 37, \operatorname{RSin},-.25$

$\mathrm{k}, 38$, RSout, -.25

$k, 39$, RSin, 0

$\mathrm{k}, 40$, Rrelief $-.125,0$

! bottom of thread relief

$\mathrm{k}, 41$, RSavg, . 125

! bottom of thread relief - tangent point

$\mathrm{k}, 42$, RSout, .125

! centerpoint of thread relief at vert. tangent

! outside of thread relief at vertical tangent

k, 43, Rrelief, . 125

$k, 44$, Rrelief-.125, .125

! thread relief vertical tangent point

! thread relief center of curvature

/com area ? - first thread

$k, 105$, RSout, 0.25

$k, 106$, RSavg, 0.25

$k, 107$, RImax, 0.25

$k, 108$, RIminthe, . $25+Y 3$

$\mathrm{k}, 109$, RImin, .25+Y4

k, 110, RImin, . .25+Y5

$\mathrm{k}, 111$, RImax, .25+Y6

k,112, RImax, .25+Pitch

$\mathrm{k}, 113$, RSavg, .25+Pitch

k, 114, RSout, .25+Pitch

/com lines for collar at thread relief

$1,37,38$

$1,38,42$

$1,42,41$

$1,41,43$

larc, $43,40,44, .125$

$1,40,39$

$1,39,37$
a, $10,11,13,12$
a, $12,13,14,16,15$
a, $15,16,18,17$
nominal shell
collar transition
a, $17,18,22,21,20,19$
a, $21,22,32,31$
a, $31,32,34,33$
a, $33,34,36,35$
a, $35,36,38,37$
area at sealing surface
collar above sealing surface
! collar - medium elements
1 collar - coarse element
! collar - medium elements

al, $1,2,3,4,5,6,7$

!a, 37, 38,42,41,40,39 ! collar below thread relief

/com generate thread areas
a, $43,41,106,107$
a, $41,42,105,106$
a, 107, 106, 113,112,111,110,109,108
! thread relief - inside
a, $106,105,114,113$
! thread relief - outside
t thread tooth
! thread outside

/com Lifting \& Locking Ring (external thread) local, 11,0, . .08

$k, 1000,15.9 / 2$

$k, 1001$, BoltIR

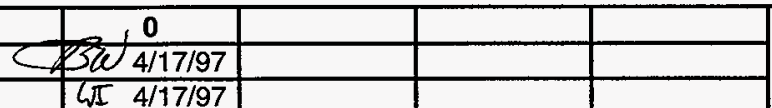


$\mathrm{k}, 1002$, Boltor

$k, 1003,12.0$

$k, 1004$, REmin

$k, 1005, R E \max , Y 6-Y 5$

$k, 1006, R E \max , Y 6-Y 4$

$k, 1007$, REmax-he, $Y 6-Y 3$

$\mathrm{k}, 1008$, REmin, Y6-Y2

k, 1009, REmin,pitch

k, 1010,12.0,pitch

$k, 1011$, Boltor, pitch

$k, 1012$, BoltIR, pitch

k, 1013,15.9/2,pitch

/com area 5

$a, 1003,1004,1005,1006,1007,1008,1009,1010$

! thread profile

/com area ?

a, $1002,1003,1010,1011$

! outer (fine) transition

/com area ?

a, 1001,1002,1011,1012

/com area?

$\mathrm{a}, 1000,1001,1012,1013$

! inner (coarse) transition

! ring

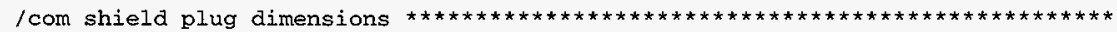

rplugl $=15.78 / 2$

rplug $2=22.90 / 2$

rplug $3=23.95 / 2$

$x$ seal $1=23.4 / 2$

rseal2 $=23.375 / 2$

! o-ring groove

/com shield plug

! o-ring groove

local, $12,0, .-2.37$

/com shield plug outline

$k, 2000, x p \operatorname{lug} 1,-2.25$

$\mathrm{k}, 2001$, rplug $2-.181,-2.25$

$k, 2002$,rplug2, -1

$k, 2003$, rplug2, . 155

$k, 2004$, rplug $3-.300, .155$

$k, 2005$, rplug $3-.300, .001$

$k, 2006$,rplug3, .001

$k, 2007$,rplug3, . 155

$k, 2008$,rplug3,2.31

k, 2009, rplug1,2.31

/com other locations on shield plug

k, 2100, BoltIR, 2.31

k, 2101, BoltoR, 2.31

k, 2102, BoltIR, 1.75

k, 2103, Boltor, 1.75

k, 2104,rplug3, 1.75

$k, 2105$, BoltIR, . 35

$k, 2106,11.9, .35$

$k, 2107$,plugr $3, .35$

$\mathrm{k}, 2107$, rplug3, . 35

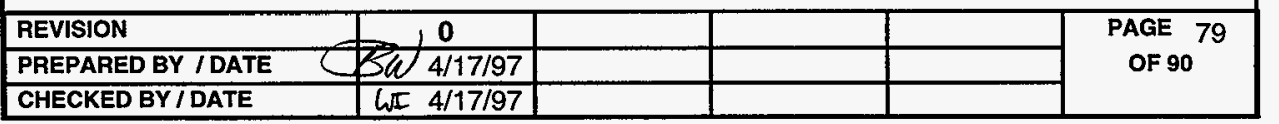


$k, 2108$, rplug $2, .35$

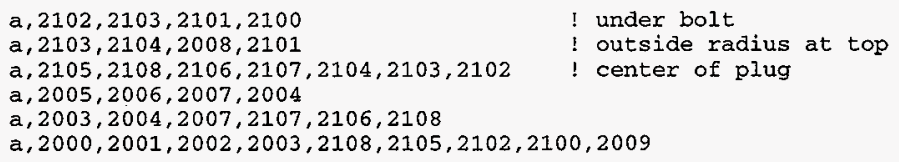


CLIENT: DUKE ENGINEERING SERVICES HANFORD, INC

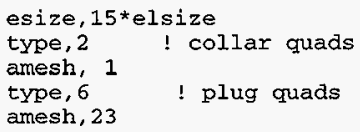


.

1sel,s,line, ,52 ! ring - 1st thread

nsll, , 1

cm, t1ring, node

lsel,s,line, 104 ! ring - thread 2

nsll, 1

cm, t2ring, node

Isel,s,line, 132 ! ring - thread 3

nsl1, 1

$\mathrm{cm}$, t3ring, node

lsel,s,1ine, 160 ! ring - thread 4

ns 11,1

$\mathrm{cm}, \mathrm{t} 4 \mathrm{ring}$, node

Isel,s,Iine, 188 ! ring - thread 5

nsl1, 1

$\mathrm{cm}, \mathrm{t}$ 5ring, node

Isel,s,1ine, 216 ! ring - thread 6

ns11, 1

$\mathrm{cm}, \mathrm{t} 6 \mathrm{ring}$, node

lsel,s,line, 244 ! ring - thread 7

ns 11,1

cm, t7ring, node

lsel,s,line, 272 ! ring - thread 8

ns11, 1

$\mathrm{cm}$, t8ring, node

lsel, s,line, 300 ! ring - thread 9

nsll, 1

cm, t9ring, node

lsel,s,line, 328 ! ring - thread 10

$\mathrm{ns} 11,1$

$\mathrm{cm}$, t10ring, node

lsel,s,1ine, 356 ! ring - thread 11

$\mathrm{ns} 11,1$

cm, t11ring, node

/com surfaces at o-ring

lsel,s,line, 21,22 ! collax

ns 11, 1

cm, cseal, node

lsel,s,line,, $78 \quad$ ! plug

nsl1, 1

cm, pseal, node

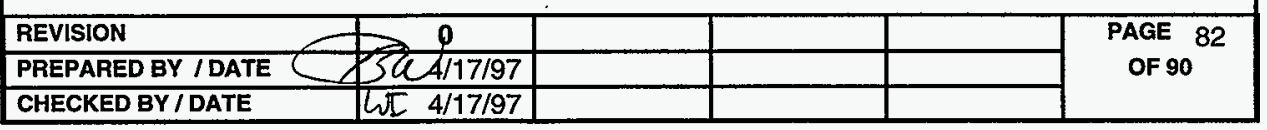


1 sel, all

nal1

/com generate gap elements

type, 7

real, 7

gogen, t1ring, t1shell

gcgen, t2ring, t2shell

gcgen, $t 3$ ring, $t 3$ shell

gcgen, t4ring, t4shell

gcgen, t5ring, t5shell

gcgen, t6ring, t6shell

gcgen, t7ring, t7shell

gcgen, t8ring, t8shell

gcgen, t9ring, t9shel1

gcgen, t10ring, t10 shell

gcgen, t11ring, t11shell

gcgen, pseal, cseal

/com bolts

csys, 12

$\mathrm{k}, 3000$, BoltIR, $2.310+.001$

$\mathrm{k}, 3001$, Boltor, $2.310+.001$
$\mathrm{a}, 3000,3001,1011,1012$

! same as shield plug local, $12,0,-2.37$

! k, 2100,BoltIR, 2.00

! k, 2101, BoltoR, 2.000

type, 3

real, 1

mat, 2

esize, $8^{*}$ elsize

amesh, 84

/com merge coincident nodes on area boundaries

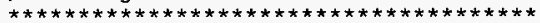

esel,s,type, 2 ! shell collar

nsle

! select nodes based on elements

$\mathrm{ksln}$

i select keypoints based on nodes

nummerg, node

nummrg, elem

nummerg, $\mathrm{kp}$

esel,s,type, 4 ! lifting \& locking ring

nsle

$\mathrm{ks} \ln$

! select keypoints based on nodes

nummerg, node

nurmrg, elem

nummerg, $\mathrm{kp}$

esel,s,type, $6 \quad$ ! shield plug

nsle

$\mathrm{ksln}$

nummerg, node

nummrg, elem

nummerg, $\mathrm{kp}$

a11s

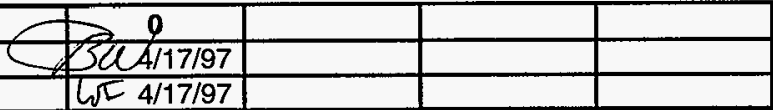


/com generate gap elements

lsel, s, 1ine, 370

! Iine on "bolt"

ns 11,1

! select nodes on line

$\mathrm{cm}$, boltgaps, node

! group nodes as "boltgaps"

1 sel, s, 1ine,, 68

! line on plug

ns 11,1

! select nodes on line

cm, pluggaps, node

! group nodes as "pluggaps"

*get, num bolt, node, count

! number of nodes = "num_bolts"

* dim, node_i, num bolt

! dimension arrays for nodes

*dim, node_j, , num_bolt

/com sort nodes on bolt

cmsel, , boltgaps

* get, yval, node, , mnloc, y

*do, i, 1, num bolt *get, min $r$, node, mnloc, $x$ node_i $(i)=$ node $\left(\min \_r, y v a l, 0\right)$

*enddo nsel, $u$, node, node_i (i)

! get $y$-value

! get value of minimum radius

! get node number at minimum radius

! remove selected node from group

/ com sort nodes on plug

cmse1, , pluggaps

* get, yval, node, , mnloc, y

^do, i, 1, num_bolt *get, min_r, node, , mnloc, $x$ node_j $(i)=$ node $($ min_r, rva 1,0$)$

* enddo nsel, $u$, node, node_j (i)

! get $y$-value

! get value of minimum radius

! get node number at minimum radius

! remove selected node from group

alls

/com generate elements

type, 8

real, 8

*do, $i, 1$, num_bolt e, node_j (i), node_i (i)

*enddo

nall

eal1.

/com relax convergence tolerances

cnvtol,f, .01 ! 18 on force (10*default)

! cnvtol,m, ..01 ! $1 \%$ on moment (10*default)

lcom boundary conditions csys, 0

nsel, s, loc, $x$, rplugl

id, al1, ux, 0.0

! inside of plug

! fix radially

nsel, s, loc,y,-12.0 ! base of shell

d,all, uy, 0.0 ! fix vertical (rollers)

\begin{tabular}{c|c|l|l|l|}
\hline & & & \\
\hline $\operatorname{san} 4 / 17 / 97$ & & & \\
\hline $\ln 4 / 17 / 97$ & & &
\end{tabular}


CLIENT: DUKE ENGINEERING SERVICES HANFORD, INC

FILE NO: $\mathrm{KH}-8009-8-04$

PROJECT: MCO Final Design DOC. NO.: HNF-SD-SNF-DR-003, Rev. 0, Appendix 6

nall

eall

save

fini

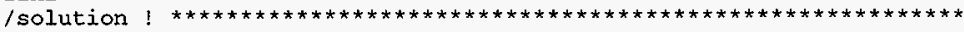

/com first step to close gaps

tunif, 70

tref, 70

/title, MCO Closure Threads - Load Step 1 - Thread Closure

$\mathrm{kbc}, 1$

! set change loads for load step 1

nsubst, 1

! number of substeps

neqit, 10

! number of equilibrium interations

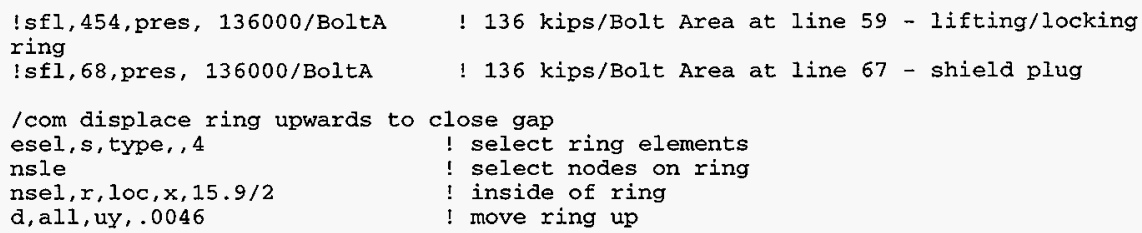

alls

lswrite

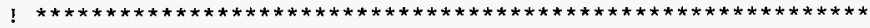

/title,MCO Closure: 190 kip preload, No Friction, 70F (L.S. 2)

$\mathrm{kbc}, 0$

nsubst, 10

neqit, 10

! ramp change loads for load step 2

autots, on

! number of substeps

! number of equilibrium interations

nropt, auto

nlgeom, on

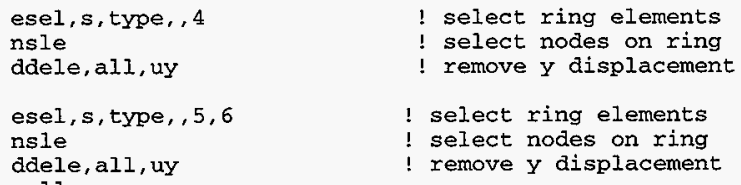


DOC. NO.: HNF-SD-SNF-DR-003, Rev. 0, Appendix 6

/title,MCO closure: $190 \mathrm{kip}$ preload $+150 \mathrm{psi}, 392 \mathrm{~F}$ (L.S. 3 )
kbc,
nsubst, 5

autots, on

nropt, auto

/com apply pressure load

PA_int $=150$

PA_model $=($ rplug $2 *$ rplug2 $)-($ pplug $1 *$ rplug1 $)$

P_int_p=P_int*PA_real/PA_model ! corrected pressure on plug bottom
lsel, s, line,, 82
lsel, a, line, , 86, 87
Isel, $a$, line, 11
lsel, a, line, 15
lsel, $a, 1$ ine,, 18
lsel, a, line, , 23
lsel, a, line, , 22
sfgrad
sfl,all, pres, P_int
! shield plug - seal groove
! shield plug side
! collar at base
! collar at taper
! collar at seal
! reset gradient (none used)
! apply pressure

/com shield plug bottom

lsels, s, line, , 85

sfl,all, pres, $P_{\text {_int }} p$

alls

sftran

tunif, 392

! shield plug bottom

! transfer line loads to elements

! max. temp. a 150 psi (200 degC)

\section{save}

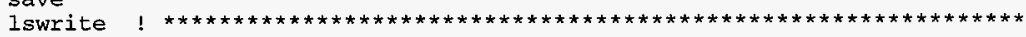

/title, MCO closure: 190 kip preload + 75 psi, Hot (707F) (L.S. 4)

$\mathrm{kbc}, 0$

nsubst, 5

! ramp change loads for load step 3

autots, on

! number of substeps

nropt, auto

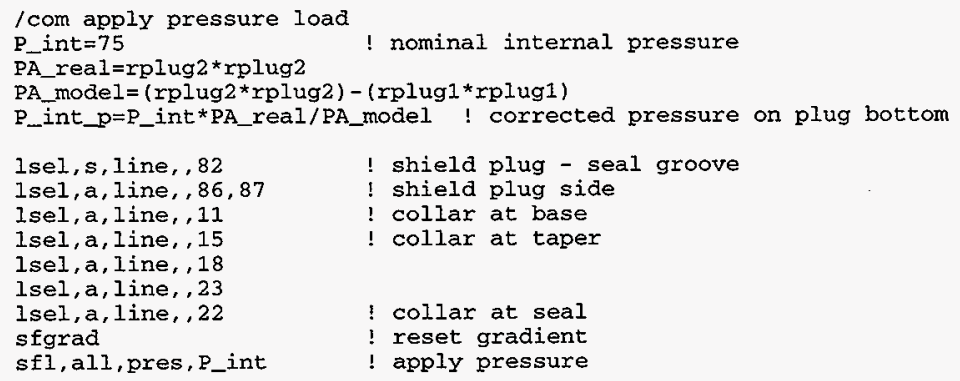

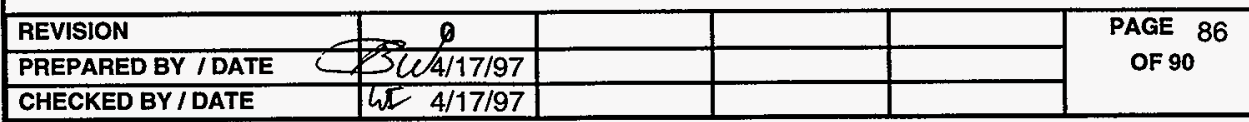


/com shield plug bottom

lsels, s, line,, 85

! shield plug bottom

sfl, all, pres, P_int_p

a11.s

tunif, 707

! max. temp. a 75 psi (375 degc)

save

Iswrite

Is solve, 1,4

save

fini

/post 1

set, 2

! PRELOAD ONLY

/com - sections through collar at base of threads (from bottom to top) esel, s, type, 2

! select collar elements only

1path, 12,675

! thread 1 (bottom)

prsect, -1

lpath, 44,687

! thread 2

prsect, -1

Ipath, 1849,1928 ! thread 3

prsect, -1

Ipath, 2129, 2208

! thread 4

prsect, -1

Ipath, 2409,2488

!hread 5

prsect, -1

1path, 2689,2768

! thread 6

prsect, -1

lpath, 2969,3048

! thread 7

prsect, -1

lpath, 3249,3328

1 thread 8

prsect, -1

1path, 3529,3608

prsect, -1

Ipath, 3809,3888

! thread 9

prsect, -1

lpath, 4089,4168

prsect, -1

! thread 10

! thread 11 (top)

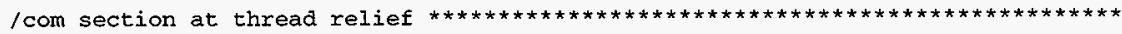

$\star d o, i, 2,4,1$ set, $i$

lpath, 2, 671

* enddo

prsect, -1

al1s

lcom - bolt loads

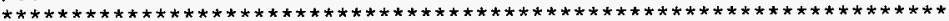

$\star$ do, $i, 2,4,1$

/gopr

set, $i$ 
/com bolt preload - membrane stress

esel, s, type, 3

! select bolt elements

lpath, 4642,990

! section at midthickness

prsect, -1

/com bolt preload - gap element loads

esel, $\mathrm{s}$, type, 8

! select contac12 gap elements

etable, boltload, smisc, 1

! store fn (normal force)

*enddo pretab

! list normal force

etable, eras

/com - seal stop loads $* \star$ (nforce)

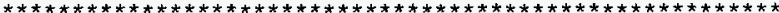

esel, s, type, , 6

nsle

1sel, s, line, 78

$n \leq 11, r, 1$
! shield plug elements

! elements on shield plug

! line at seal stop

! select nodes on line

$\star$ do $, i, 2,4,1$

gopr

set, $i$

nforce

* endao

/com - seal stop loads $\star *$ (gap element loads)

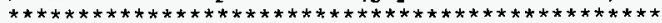

/page, , 10000

isel, s, 1ine, , 21, 22

! lines of output per page

Isel, s, line, 78

! lines on collar

ns 11

! line on plug

esln

! select nodes on 1 ines

esel, $x$, type,, 7

i select elements on nodes

! reselect contac48 gap elements only

$\star$ do, $i, 2,4,1$

/gopr

set, $\mathbf{i}$

etable, sealstop, smisc, 1 ! store normal force

*enddo

pretab

! print normal force

lcom - print reaction forces

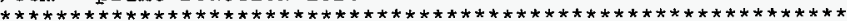

al1s

$\star$ do, $i, 2,4,1$

/gopr

set, $i$

prrs

! print reaction forces

*endao

al1s

\begin{tabular}{|l|c|l|l|l|}
\hline REVISION & 0 & & & \\
\hline PREPARED BY IDATE C & So/4/17/97 & & & \\
\hline CHECKED BY I DATE & $\cot 4 / 17 / 97$ & & & \\
\hline
\end{tabular}


CLIENT: DUKE ENGINEERING SERVICES HANFORD, INC

FILE NO: $\quad \mathrm{KH}-8009-8-04$

PROJECT: MCO Final Design DOC. NO.: HNF-SD-SNF-DR-003, Rev. 0, Appendix 6

/com, Generate contour plots

set, last

/dscale, , 1

ledge, , 1

eplot

plnsol, s, int

PLNSOL, $U, X, 0$

FINISH

exit, nosa 


\section{COMPUTER RUN COVER SHEET}

Project Number:

Computer Code:

Software Version:

Computer System:

Computer Run File Number:

Unique Computer Run Filename:

Run Description:

Run Date / Time:

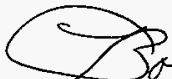

Prepared By: Bob V. Winkel

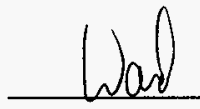

Checked By: Wakd Ingles

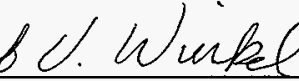

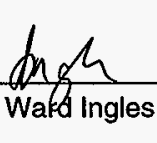

$\mathrm{KH}-8009-8$

ANSYS $^{\circledR} \cdot$ PC $386 / 486$

Version 5.3 Release 2

Windows NT 3.51 (Build 1057)

Pentium Pro 200 Processor

KH-8009-8-04

THRD3.out

Stress Analysis of the MCO Thread/Closure 16 April 1997 $12: 43: 44$ AM

$4-17.97$

\section{Date}

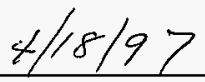

\section{Date}

\begin{tabular}{|l|c|l|l|l|}
\hline REVISION & 0 & & & \\
\hline PREPARED BY / DATE & $4 / 17 / 97$ & & & \\
\hline CHECKED BY / DATE & $\operatorname{lnE} 4 / 17 / 97$ & & & \\
\hline
\end{tabular}




\begin{tabular}{|l|l|l|l|}
\hline PILENO: & $\begin{array}{l}\text { KH-8009-8-05 } \\
\text { PARSDNS }\end{array}$ & CALCULATION PACKAGE & $\begin{array}{l}\text { DOC. NO. } \\
\text { HNF-SD-SNF-DR-003, Rev. 0, } \\
\text { Appendix } 7 \\
1 \text { of } 85\end{array}$ \\
\hline
\end{tabular}

PROJECT NAME:

MCO Final Design

CLIENT:

CALCULATION TITLE:

STRESS ANALYSIS OF THE MARK 1A STORAGE AND SCRAP BASKETS

PROBLEM STATEMENT OR OBJECTIVE OF CALCULATION:

PERFORM A STRESS ANALYSIS OF THE MARK 1A STORAGE AND SCRAP BASKETS IN ACCORDANCE WITH REVISION 3 OF THE MULTI-CANISTER OVERPACK (MCO) PERFORMANCE SPECIFICATION (REFERENCE 1). FOUR LOADING CONDITIONS ARE CONSIDERED:

1. LIFTING AT A MAXIMUM TEMPERATURE OF $100^{\circ} \mathrm{C}$.

2. DEADWEIGHT STACKING INSIDE THE MCO AT A DESIGN TEMPERATURE OF $375^{\circ} \mathrm{C}$.

3. VERTICAL DROP LOADING OF $35 \mathrm{G}^{\prime} S$ AT A MAXIMUM TEMPERATURE OF $132^{\circ} \mathrm{C}$.

4. HORIZONTAL DROP LOADING OF 101 G'S AT A MAXIMUM TEMPERATURE OF $132^{\circ} \mathrm{C}$.

CRITERIA ARE BASED ON ANSI N14.6 AND THE ASME CODE, SUBSECTION NG.

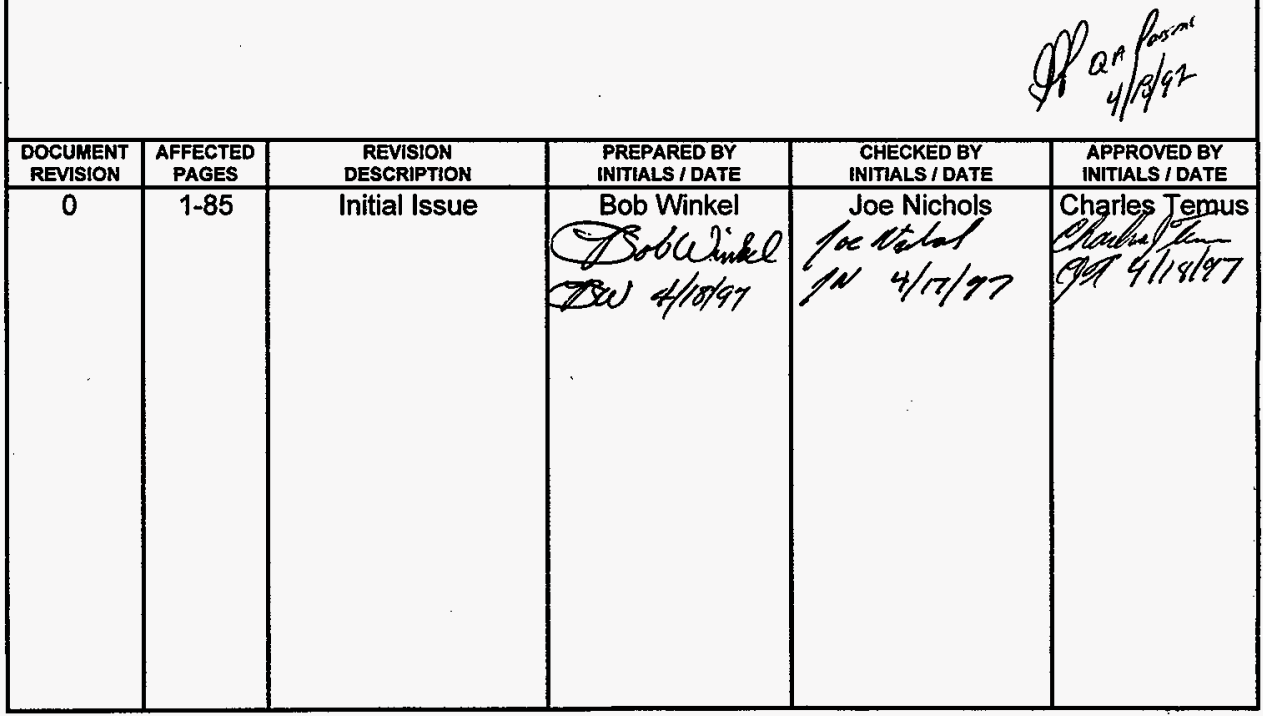


CLIENT: Duke Engineering \& Services Hanford

FILE NO: $\quad \mathrm{KH}-8009-8-05$

PROJECT: MCO Final Design DOC. NO.: HNF-SD-SNF-DR-003, Rev. 0, Appendix 7

\section{TABLE OF CONTENTS}

1. INTRODUCTION

2. REFERENCES

3. ASSUMPTIONS.

4. GEOMETRY.

5. MATERIAL PROPERTIES

6. ACCEPTANCE CRITERIA

6.1 LIFTING LOADS

6.2 DEADWEIGHT LOADS

6.3 DROP LOADING CONDITIONS.

6.4 WELD CRITERIA

7. LOAD CONDITIONS \& COMBINATIONS

8. STRESS ANALYSIS CALCULATIONS

8.1 CENTER PIPE

8.1.1 Vertical Drop Load Condition

8.1.2 Horizontal Drop Load Condition

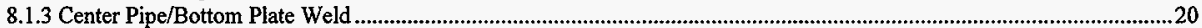

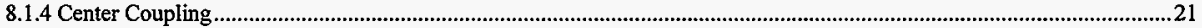

8.2 SUPPORT ROD

8.3 BOTTOM PLATE

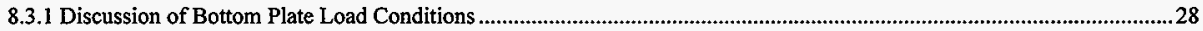

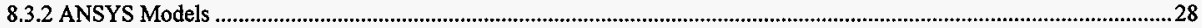

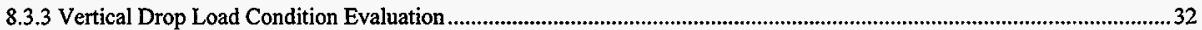

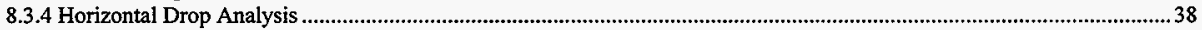

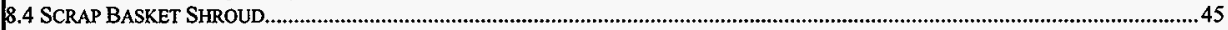

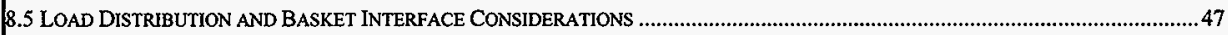

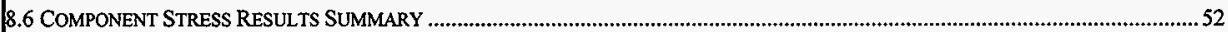

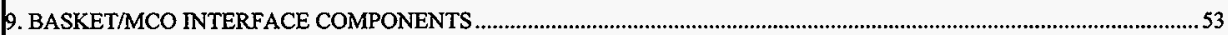

\begin{tabular}{|l|c|l|l|l|}
\hline REVISION & 0 & & & \\
\hline PREPARED BY IDATE & S & & \\
\hline CHECKED BY I DATE & $/ N 4 / 17 / 97$ & & & \\
\hline
\end{tabular}


PARSDNS

PROJECT: MCO Final Design $\quad$ DOC.NO.: HNF-SD-SNF-DR-003, Rev. 0, Appendix 7

\section{LIST OF FIGURES}

FIGURE $1.60^{\circ}$ SECTOR OF MARK 1A STORAGE BASKET STRUCTURAL COMPONENTS.

FIGURE 2. ILLUSTRATION OF SUPPORT ROD LOAD ECCENTRICITY. 27

FIGURE 3. 60 DEGREE SECTOR MODEL OF BASKET WITH HOLES.

FIGURE 4. 60 DEGREE SECTOR ANSYS MODEL OF BASKET WITHOUT HOLES. 31

FIGURE 5. 180 DEGREE ANSYS MODEL OF BASKET USED FOR HORIZONTAL DROP ANALYSES.

FIGURE 6. ELASTIC STRESS INTENSITY CONTOURS, 35G VERTICAL DROP, NO-HOLE MODEL.

FIGURE 7. ELASTIC STRESS INTENSITY CONTOURS, 35G DROP LOADING, DETAILED HOLE MODEL. 36

FIGURE 8. ELASTIC/PLASTIC STRESS INTENSITY CONTOURS, 35G VERTICAL DROP, NO-HOLE MODEL. 39

FIGURE 9. PLASTIC STRAIN CONTOURS, 35G VERTICAL DROP, NO-HOLE MODEL. 4

FIGURE 10. BOTTOM PLATE DISTORTION ILLUSTRATION FOR HORIZONTAL DROP MODELING. 4

FIGURE 11. 101G HORIZONTAL DROP STRESS INTENSITY CONTOURS, INELASTIC ANALYSIS.

FIGURE 12. HORIZONTAL DROP G LOAD VERSUS MAX. VERTICAL DISPLACEMENT, INELASTIC ANALYSIS. 44

FIGURE 13. SUPPORT ROD DEFORMED SHAPE PREDICTIONS, 0.030 " VERTICAL DEFLECTION.

FIGURE 14. DEFORMATION RESPONSE OF MARK IA BASKET SUPPORT RODS.

\begin{tabular}{|l|c|l|l|l|l|}
\hline REVISION & 0 & & & & PAGE 3
\end{tabular}

\begin{tabular}{|l|l|l|l|}
\hline PREPARED BY IDATE OF 85 & \\
\hline CHECKED
\end{tabular}

CHECKED BYIDATE 
CLIENT: Duke Engineering \& Services Hanford

\begin{tabular}{llll} 
PROJECT: & MCO Final Design & DOC. NO.: & HNF-SD-SNF-DR-003, Rev. 0, Appendix 7 \\
\cline { 2 - 3 }
\end{tabular}

\section{LIST OF TABLES}

TABLE 1. MARK 1A BASKET STRUCTURAL COMPONENTS

TABLE 2 ASME CODE MATERIAL PROPERTIES FOR TYPE 304L STAINLESS STEEL

TABLE 3 ALLOWABLE STRESSES - DEADWEIGHT

TABLE 4. ALLOWABLE STRESSES FOR DROP LOADING

TABLE 5. VERTICAL DROP STRESS INTENSITY RESULTS SUMMARY.

TABLE 6. SUMMARY OF MARK IA STORAGE BASKET STRESS RESULTS 
E paRSONS

CLIENT: Duke Engineering \& Services Hanford

FILE NO: KH-8009-8-05

\section{LIST OF APPENDICES}

APPENDIX A 


\section{P. PARSDNS}

CLIENT: Duke Engineering \& Services Hanford FILE NO: KH-8009-8-05

PROJECT: MCO Final Design $\quad$ DOC. NO.: HNF-SD-SNF-DR-003, Rev. 0, Appendix 7

\section{INTRODUCTION}

This calculation documents the evaluation of the Mark IA Storage and Scrap Baskets for lifting, deadweight, and drop accident loading. The structural adequacy evaluation is based upon ANSI N14.6 for the lifting loading and Section III, Subsection NG of the ASME Code for the deadweight stacking and drop load conditions. As discussed in Section 4, most of the Mark 1A Storage and Scrap Basket structural components are identical. This permitted the combining of the Mark $1 \mathrm{~A}$ basket evaluations into a single report.

\section{REFERENCES}

1. DE\&S, 1997, Performance Specification for the Spent Nuclear Fuel Multi-Canister Overpack, HNF-S-0426, Rev. 3, Duke Engineering and Services Hanford, Richland, Washington.

2. DE\&S, 1996, "K-Basin SNF Storage Basket Mock-Up Mark 1A" and "K-Basin SNF Scrap Basket Mock-Up Mark 1A", Drawing Nos. H-2-82860 \& H-2-82865, Rev. 0, Duke Engineering and Services Hanford, Richland, Washington.

3. ASME, 1995, ASME Boiler and Pressure Vessel Code, Section II, Materials, Part D-Properties, American Society of Mechanical Engineers, New York, New York.

4. ASME, 1995, ASME Boiler and Pressure Vessel Code, Section III, Subsection NG, American Society of Mechanical Engineers, New York, New York.

5. ASME, 1995, ASME Boiler and Pressure Vessel Code, Section III, Subsection NF, American Society of Mechanical Engineers, New York, New York.

6. ANSI, 1993, ANSI National Standard for Radioactive Materials- Special Lifting Devices for Containers Weighing 10,000 Pounds $(4500 \mathrm{~kg})$ or More, American National Standards Institute, New York, New York.

7. Roark, R. J. and Young, W. C., 1975, Formulas for Stress and Strain, 5th Edition, McGraw-Hill, New York, New York.

8. Baumeister, T., editor, 1967, Standard Handbook for Mechanical Engineers, 7th Edition, McGraw-Hill, New York, New York.

4

\begin{tabular}{|c|c|}
\hline 0 & \\
\hline $4 / 17 / 97$ & \\
\hline $4 / 17 / 97$ &
\end{tabular}




\section{DPARSRNS}

CLIENT: $\quad$ Duke Engineering \& Services Hanford FILE NO: KH-8009-8-05

PROJECT: MCO Final Design $\quad$ DOC. NO.: $\quad$ HNF-SD-SNF-DR-003, Rev. 0, Appendix 7

9. Myers, J. A., 1962, Handbook of Equations for Mass and Area Properties of Various Geometrical Shapes, US Naval Ordnance Test Station, China Lake, California.

10. Bowles, J. E., 1988, Foundation Analysis and Design, 4th Edition, McGraw-Hill, New York City, New York.

11. AISC, 1989, Manual of Steel Construction, Ninth Edition, American Institute of Steel Construction, Chicago, Illinois.

12. Pugh, C. E, et al, 1972, Currently Recommended Constitutive Equations for Inelastic Design Analysis of FFTF Components, ORNL-TM-3602, Oak Ridge National Laboratory, Oak Ridge, Tennesee.

\section{ASSUMPTIONS}

1. All welds made and inspected in accordance with ASME Code, Section III, Subsection NG (Reference 4). For the Mark 1A Storage and Scrap Basket welds, a "surface visual examination" is assumed.

2. For the vertical drop loading when the baskets are stacked within the MCO, it was conservatively assumed that the basket support rods are in alignment, with the exception of the bottom basket, which is rotated $30^{\circ}$ relative to the baskets above. This configuration produces the maximum bending in the basket bottom plate.

3. Since the Reference 1 Performance Specification does not specify the density of the scrap material in a loaded scrap basket, a density is calculated based upon the assumption that the total basket scrap weight is equal to the total fuel weight ( 48 fuel rods) in a loaded Mark 1A storage basket.

4. For the horizontal drop evaluation, it was assumed that the top end support for the center pipe, which interfaces with either the basket above or the bottom of the shield plug assembly, is maintained throughout the drop. See Section 9 for a discussion and justification of this assumption.

5. Other assumptions as noted within the calculation documentation. 


\section{Fipansons}

CLIENT: Duke Engineering \& Services Hanford FILE NO: KH-8009-8-05

PROJECT: MCO Final Design $\quad$ DOC. NO.: HNF-SD-SNF-DR-003, Rev. 0, Appendix 7

\section{GEOMETRY}

The Mark IA Storage and Scrap Basket geometry's are defined in Drawing Nos. H-2828060 and $\mathrm{H}-2-828065$, respectively. The primary structural components for both basket designs (bottom plate, support rods, and center pipe) are identical. The primary structural components and other significant components are identified in the Figure 1 sketch of the storage basket. The scrap basket has an additional component in the form of six radial stiffener plates which span between each support rod and the center pipe. The radial plates are welded to both the support rods and center pipe, and therefore enhance the buckling strength of these components. Thus, these components are evaluated for the more conservative application, i.e. the storage basket.

A summary of the function of each structural component is provided in Table 1. The bottom plate is constructed from a 1-1/8-in. plate with 1/4-inch flow holes on one-inch centers (equilateral triangle grid pattern). Final machining operations will bring the minimum thickness to nearly one inch. A uniform thickness of one inch is therefore conservatively assumed for the calculations within this report.

The center pipe is fabricated from six-inch, XXS pipe having a wall thickness of 0.864 inches. The trapezoidal geometry of the support rods is selected to provide the maximum cross section for the available space. A fourth structural component is the "center coupling", located at the top of the center pipe. The center coupling interfaces with a grappling device during lifting operations and slips into the adjacent basket, above, when the baskets are stacked inside the MCO. The center coupling is attached to the center pipe using four $1 / 2$-in. coil type spring pins. A single $1 / 2$-in. spring pin is used to attach the center bushing to the bottom of the center pipe as shown. These spring pins have a structural function during lifting operations, and are evaluated for structural adequacy. There is also a thick aluminum fuel-positioning plate (storage basket only) which sits above the bottom plate and a 50-mil sheet metal shroud enveloping the lower half of the basket. These components, plus the center bushing are primarily functional and are not subjected to a structural evaluation.

Both the storage and scrap basket designs include a 0.050 -in. sheet metal shroud located on the outside circumference of the baskets (half-height on the storage baskets and full height on the scrap basket). Since the fuel spacer plate in the storage basket prevents significant loading to the shroud, the storage basket shroud is considered to be nonstructural. The scrap basket shroud, however, is subjected to a relatively low pressure loading due to the scrap pieces bearing against the shroud, and is subjected to a structural evaluation.

\begin{tabular}{|l|l|l|l|}
\hline & & & \\
\hline & & & \\
\hline
\end{tabular}


CLIENT: Duke Engineering \& Services Hanford FILE NO: KH-8009-8-05 PROJECT: MCO Final Design $\quad$ DOC. NO.: HNF-SD-SNF-DR-003, Rev. 0, Appendix 7

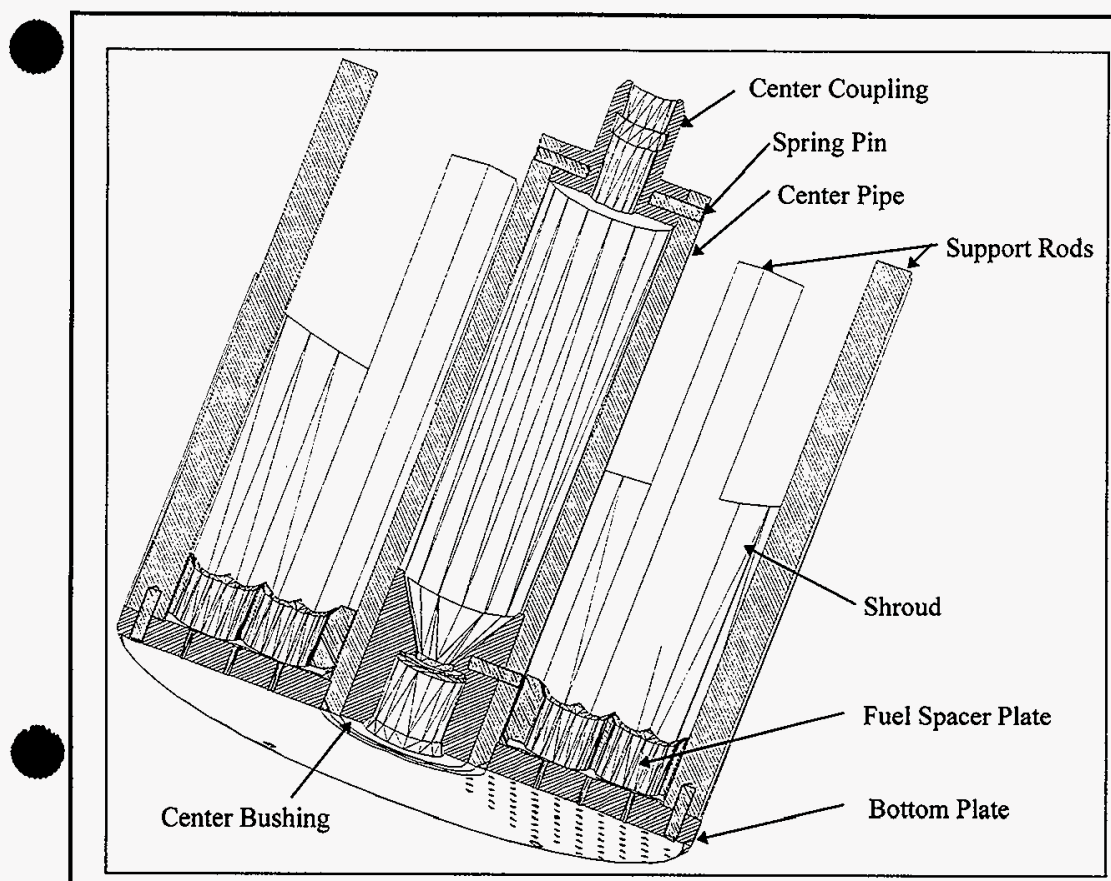

Figure 1. $60^{\circ}$ Sector of Mark 1A Storage Basket Structural Components.

\begin{tabular}{|l|c|l|l|l|l|}
\hline REVISION & 0 & & & & PAGE 9 \\
\hline PREPARED BY IDATE & OF 85 & \\
\hline CHECKED BY I DATE & PW 4/17/9/97 & & & & \\
\hline
\end{tabular}


CLIENT: Duke Engineering \& Services Hanford

FILE NO: $\quad \mathrm{KH}-8009-8-05$

PROJECT: MCO Final Design $\quad$ DOC. NO.: HNF-SD-SNF-DR-003, Rev. 0, Appendix 7

Table 1. Mark 1A Basket Structural Components

\begin{tabular}{|c|c|c|}
\hline $\begin{array}{l}\text { Component } \\
\text { Name }\end{array}$ & $\begin{array}{l}\text { Component Part } \\
\text { No. in Dwgs. } \\
\text { H-2-828060 } \\
\text { \& H-2-828065 }\end{array}$ & Structural Functions \\
\hline Center Pipe & 8 & $\begin{array}{l}\text { (1) Primary load carrying component during } \\
\text { lifting operations. (2) Provide support to } \\
\text { above baskets when stacked inside the } \\
\text { MCO. (3) Provides "void space" boundary } \\
\text { for criticality safety, which must be } \\
\text { maintained during normal operations and } \\
\text { following drop accidents (Reference } 1 \text {, } \\
\text { Section 4.18.3). }\end{array}$ \\
\hline $\begin{array}{l}\text { Center } \\
\text { Coupling }\end{array}$ & 6 & $\begin{array}{l}\text { Primary load bearing component during } \\
\text { lifting operations and a shear support for the } \\
\text { center pipe during a horizontal drop } \\
\text { accident. }\end{array}$ \\
\hline Spring Pins & 15 & $\begin{array}{l}\text { Shear pin support for lifting loads for the } \\
\text { center coupling and center bushing. }\end{array}$ \\
\hline $\begin{array}{l}\text { Support } \\
\text { Rod }\end{array}$ & 12 & $\begin{array}{l}\text { Provide support to above baskets when } \\
\text { stacked inside the MCO for normal } \\
\text { operations and during vertical drop } \\
\text { accidents. }\end{array}$ \\
\hline $\begin{array}{l}\text { Bottom } \\
\text { Plate }\end{array}$ & 9 & $\begin{array}{l}\text { Support the fuel during normal operations } \\
\text { and maintain the position of the center pipe } \\
\text { during drop accidents. }\end{array}$ \\
\hline $\begin{array}{l}\text { Scrap } \\
\text { Basket } \\
\text { Sheet Metal } \\
\text { Shroud }\end{array}$ & 13 & $\begin{array}{l}\text { Carries the radial pressure due to fuel scrap } \\
\text { pieces bearing against the shroud. }\end{array}$ \\
\hline
\end{tabular}


CLIENT: Duke Engineering \& Services Hanford

FILE NO: KH-8009-8-05

\begin{tabular}{llll} 
PROJECT: & MCO Final Design & DOC. NO.: & HNF-SD-SNF-DR-003, Rev. 0, Appendix 7 \\
\hline
\end{tabular}

\section{MATERIAL PROPERTIES}

Per the Reference 2 drawings, the Mark $1 \mathrm{~A}$ Basket components are to be fabricated from 304L stainless steel. The only exceptions are the spring pin design which specifies an SAE 30302 or SAE 30304 material and the non-structural aluminum fuel rack. For this analysis, the only mechanical properties of interest are the elastic modulus, yield strength, ultimate strength, and ASME stress allowable, $S_{m}$. The appropriate values are extracted from Reference 3, and are listed in Table 2.

\section{ACCEPTANCE CRITERIA}

For the lifting, dead weight stacking, and drop loadings considered, the appropriate acceptance criteria is discussed below.

\subsection{Lifting Loads}

Per Section 4.12.3 of the Reference 1 Performance Specification, the Mark1A basket designs "shall meet the safety factors required by the American National Standards Institute (ANSI) N14.6-1986. The ANSI safety factors apply from $5^{\circ} \mathrm{C}$ to $100^{\circ} \mathrm{C}$ ". Section 4.2.1.1 of N14.6 specifies that "the load bearing members of a special lifting device shall be capable of lifting three times the combined weight of the shipping container with which it will be used, plus the weight of intervening components of the special lifting device, without generating a combined shear stress or maximum tensile stress at any point in the device in excess of the corresponding minimum tensile yield strength of their materials of construction. They shall also be capable of lifting five times that weight without exceeding the ultimate tensile strength of the materials." ANSI N14.6 also states that the shear stress shall be taken as an average value over the cross section, and that the tensile stress may be due to direct or bending loads. The bending stress is defined as being linear over the cross section.

\begin{tabular}{|l|c|l|l|l|}
\hline REVISION & 0 & & & \\
\hline PREPARED BY I DATE & $5 a / 4 / 17 / 97$ & & & \\
\hline CHECKED BY/DATE & $\mathrm{s} w 4 / 17 / 97$ & & & \\
\hline
\end{tabular}




\section{P Pabsons}

CLIENT: Duke Engineering \& Services Hanford FILE NO: KH-8009-8-05 PROJECT: MCO Final Design $\quad$ DOC. NO.: HNF-SD-SNF-DR-003, Rev. 0, Appendix 7

Table 2

ASME Code Material Properties for Type 304L Stainless Steel

\begin{tabular}{|c|c|c|c|c|c|}
\hline \multicolumn{2}{|c|}{ Temperature } & \multirow{2}{*}{$\begin{array}{c}E \\
\text { Table TM-1, } \\
\text { Group G }\end{array}$} & \multirow{2}{*}{$\begin{array}{c}\mathrm{S}_{\mathrm{m}} \\
\text { Table 2A, p.322 }\end{array}$} & \multirow{2}{*}{$\begin{array}{c}\text { Sy } \\
\text { Table } Y-1, p .524\end{array}$} & \multirow{2}{*}{$\begin{array}{c}\mathrm{Su}_{\mathrm{u}} \\
\text { Table U, P. } 441\end{array}$} \\
\hline${ }^{\circ} \mathrm{F}$ & ${ }^{\circ} \mathrm{C}$ & & & & \\
\hline-20 & - & - & $16.7 \mathrm{ksi}$ & $25.0 \mathrm{ksi}$ & $70.0 \mathrm{ksi}$ \\
\hline 70 & -- & $28.3 E+06$ & - & - & - \\
\hline 100 & - & - & $16.7 \mathrm{ksi}$ & $25.0 \mathrm{ksi}$ & $70.0 \mathrm{ksi}$ \\
\hline 200 & -- & $27.6 \mathrm{E}+06$ & $16.7 \mathrm{ksi}$ & $21.3 \mathrm{ksi}$ & $66.2 \mathrm{ksi}$ \\
\hline 212 & 100 & $27.5 \mathrm{E}+06$ & $16.7 \mathrm{ksi}$ & $21.0 \mathrm{ksi}$ & $65.6 \mathrm{ksi}$ \\
\hline 300 & -- & $27.0 E+06$ & $16.7 \mathrm{ksi}$ & $19.1 \mathrm{ksi}$ & $60.9 \mathrm{ksi}$ \\
\hline 700 & $\overline{--}$ & $24.8 E+06$ & $13.5 \mathrm{ksi}$ & $14.9 \mathrm{ksi}$ & $56.2 \mathrm{ksi}$ \\
\hline 707 & 375 & $24.8 E+06$ & $13.5 \mathrm{ksi}$ & $14.9 \mathrm{ksi}$ & $56.2 \mathrm{ksi}$ \\
\hline 750 & -- & - & $13.3 \mathrm{ksi}$ & $14.7 \mathrm{ksi}$ & $55.9 \mathrm{ksi}$ \\
\hline 800 & & $24.1 E+06$ & - & - & - \\
\hline
\end{tabular}

Notes 1: Underlined values determined by linear interpolation,

s4allow.xls other values taken from Section II, Part D of the ASME

2: Value of $E$ taken from Table TM-1 for Material Group

The "load bearing members of a special lifting device" are interpreted to apply to all components of the storage baskets in the load path between the lifting grapple and fuel. At the maximum lifting temperature of $100^{\circ} \mathrm{C}$, the ANSI $N 14.6$ allowables are:

$$
\begin{aligned}
& \frac{S y}{3}=\frac{21.04 k s i}{3}=7.01 k s i \\
& \frac{S u}{5}=\frac{65.6 \mathrm{ksi}}{5}=13.12 \mathrm{ksi} \\
& \Rightarrow \text { Use: } P m+P b \leq 7.01 \mathrm{ksi}
\end{aligned}
$$

\begin{tabular}{|c|l|l|l|}
0 & & & \\
\hline $3 u / 4 / 17 / 97$ & & & \\
\hline $\operatorname{m-4/17/97}$ & & & \\
\hline
\end{tabular}


CLIENT: Duke Engineering \& Services Hanford FILE NO: KH-8009-8-05

PROJECT: MCO Final Design DOC.NO.: HNF-SD-SNF-DR-003, Rev. 0, Appendix 7

\subsection{Deadweight Loads}

Per Section 4.12.3 of Reference 1, the Mark IA baskets "shall meet the intent of ASME Boiler and Pressure Vessel Code, Section III, subsection NG". For primary membrane and primary membrane plus bending stresses, the allowable stresses of Table 3 apply. The dead weight stacking basket configuration is identical to the vertical drop accident configuration. Since the loading differences far exceed the allowable differences, the vertical drop accident condition obviously bounds the dead weight condition.

Table 3

Allowable Stresses - Deadweight

\begin{tabular}{|c|c|c|c|c|c|}
\hline \multirow{2}{*}{\multicolumn{2}{|c|}{ Temperature }} & \multirow{3}{*}{$\begin{array}{c}S_{m} \\
\text { [Table 2] }\end{array}$} & \multicolumn{3}{|c|}{ Design/Level A Stress Limits } \\
\hline & & & \multirow{2}{*}{$\begin{array}{c}P_{m} \\
\left(S_{m}\right)\end{array}$} & \multirow{2}{*}{$\begin{array}{c}P_{L} \\
\left(1.5 S_{m}\right) \\
\end{array}$} & \multirow{2}{*}{$\begin{array}{c}\left(P_{m} \text { or } P_{L}\right)+P_{b} \\
\left(1.5 S_{m}\right)\end{array}$} \\
\hline${ }^{\circ} \mathrm{F}$ & ${ }^{\circ} \mathrm{C}$ & & & & \\
\hline 212 & 100 & $16.7 \mathrm{ksi}$ & $16.7 \mathrm{ksi}$ & $25.1 \mathrm{ksi}$ & $25.1 \mathrm{ksi}$ \\
\hline 707 & 375 & $13.5 \mathrm{ksi}$ & $13.5 \mathrm{ksi}$ & $20.3 \mathrm{ksi}$ & $20.3 \mathrm{ksi}$ \\
\hline
\end{tabular}

Notes 1: Design \& Level A Stress Limits from NG-3221 \& NG-3222, respectively.

2: Axial compressive stresses must be limited to values established in accordance with one of the following:

- NB-3133.3 (external pressure)

- NB-3133.6 (axial compression on cylindrical shells)

- NF-3322.1(c) (column type members)

- Code Case N-284 (shell structures)

\subsection{Drop Loading Conditions}

From Table 3 of the Reference 1 Performance Specification, the bounding vertical drop loading is $35 \mathrm{~g}$ 's and the horizontal drop loading is $101 \mathrm{~g}$ 's. The only potential sequential drop (vertical followed by horizontal) in the Performance Specification is the "Dropped with Cask" case, which specifies a $33.5 \mathrm{~g}$ vertical(corner) $/ 101 \mathrm{~g}$ horizontal. In this report, the sequential drop is conservatively evaluated as a $35 \mathrm{~g} / 101 \mathrm{~g}$ combination. A maximum drop temperature of $132^{\circ} \mathrm{C}\left(270^{\circ} \mathrm{F}\right)$ is specified in Rev. 3 of Reference 1 , which was released following the completion of the Mark1A basket analyses. The previous revision of Reference 1 specified a maximum drop temperature of $200^{\circ} \mathrm{C}$. Since the existing analyses were conservative, not all of the analyses were repeated when Rev. 3 was released. 
CLIENT: $\quad$ Duke Engineering \& Services Hanford

FILE NO: KH-8009-8-05

PROJECT: MCO Final Design $\quad$ DOC.NO.: HNF-SD-SNF-DR-003, Rev. 0, Appendix 7

However, the summary table given in Section 8.6 was modified to reflect the Rev. 3 drop temperature reduction. For Level D events, the ASME Subsection NG acceptance criteria is specified in Appendix F, Para. F-1440, which refers to Para. F-1300, with some specified exceptions. The appropriate allowable stresses are listed in Table 4.

Note that allowables are listed for both the original and revised drop temperatures. As indicated, the decrease in drop temperature has a significant influence on the allowables, particularly for allowables which are a function of the ultimate strength.

\section{Table 4. Allowable Stresses for Drop Loading}

\begin{tabular}{|c|c|c|c|c|}
\hline Stress Category & $\begin{array}{l}\text { ASME Appendix } \vec{F} \\
\text { Paragraph No. }\end{array}$ & $\begin{array}{l}\text { Stress Limit } \\
\text { Criteria }\end{array}$ & $\begin{array}{c}\text { Allowable Stress } \\
\text { Value @ 200 } \\
\text { (ksi) }\end{array}$ & $\begin{array}{c}\text { Allowable Stress } \\
\text { Value @ } 132^{\circ} \mathrm{C} \\
\text { (ksi) }\end{array}$ \\
\hline \multirow{2}{*}{$\begin{array}{c}\text { Primary } \\
\text { Membrane }\end{array}$} & F-1331.1(a) & $\begin{array}{c}2.4 \mathrm{~S}_{\mathrm{m}}{ }^{1} \\
\text { (Elastic Analysis) }\end{array}$ & 38.2 & 40.1 \\
\hline & $F-1341.2(a)$ & $\begin{array}{c}0.7 S_{u} \\
\text { (Plastic Analysis) }\end{array}$ & 41.1 & 47.1 \\
\hline \multirow{2}{*}{$\begin{array}{c}\text { Primary } \\
\text { Membrane + } \\
\text { Bending }\end{array}$} & $F-1331.1(c)(1)$ & $\begin{array}{c}1.5\left(2.4 S_{m}\right)^{1} \\
\text { (Elastic Analysis) }\end{array}$ & 57.2 & 60.1 \\
\hline & F-1341.2(b) & $\begin{array}{c}0.9 S u \\
\text { (Plastic Anaiysis) }\end{array}$ & 52.8 & 60.6 \\
\hline $\begin{array}{c}\text { Ave. Primary } \\
\text { Shear }\end{array}$ & F-1331.1(d) & $0.42 S_{u}$ & 24.7 & 28.3 \\
\hline $\begin{array}{l}\text { Center Pipe } \\
\text { Compression }\end{array}$ & $F-1331.5(b)$ & $\begin{array}{l}150 \% \text { of NB-3133 } \\
\text { Limit }\end{array}$ & - & - \\
\hline $\begin{array}{l}\text { Support Rod } \\
\text { Buckling }\end{array}$ & $F-1334.3^{2}$ & $\begin{array}{c}F-1334.3(1) P / P_{y} \\
\text { Equations }\end{array}$ & - & -- \\
\hline
\end{tabular}

Notes: $\quad$ 1. Based upon the lesser of $2.4 S_{m}$ and $0.7 S_{u}$.

2. Linear type component support criteria used for support rod column buckling.

In addition to the Table 4 stress limits, Section 4.19 .3 of the Reference 1 Performance Specification stipulates a nuclear criticality safety requirement that a nominal void of 6.625 in. in diameter be maintained at the basket centerline. For all load conditions including the

\begin{tabular}{|c|c|c|c|}
0 & & & \\
\hline $304 / 17 / 97$ & & & \\
\hline $1 N 4 / 17 / 97$ & & & \\
\hline
\end{tabular}


CLIENT: $\quad$ Duke Engineering \& Services Hanford FILE NO: KH-8009-8-05

PROJECT: MCO Final Design DOC.NO.: HNF-SD-SNF-DR-003, Rev. 0, Appendix 7

drop accidents, this centerline void cannot deviate from the centerline by more than two inches. For the vertical drop, this requirement is met by demonstrating conformance to the ASME Code center pipe buckling requirements. For the horizontal drop, this requirement is addressed by predicting the maximum transverse deformation (elastic/plastic) in the center pipe for the horizontal drop loading.

\subsection{Weld Criteria}

There is only one structural weld on the Mark IA Storage Basket, the center pipe/bottom plate connection weld. Weld design criteria for Subsection NG is specified in Para. NG3350. Table NG-3352-1 specifies weld quality factors for various types of welds and examination methods. For the Mark IA Storage Baskets, a "surface visual examination" was assumed. Para. NG-3352 states that the quality factors are applied by "multiplying the allowable stress limit" by the appropriate quality factor specified in Table NG-3352-1. However, NG-3352 has no specific direction for defining the "allowable stress limit" for fillet and groove welds. Based upon a detailed fillet weld analysis described in Appendix 8, a reasonably conservative approach is to use the base metal stress allowables as the "allowable stress limit" or more specifically, the weld load divided by the throat area is compared to the base metal membrane stress allowable. As indicated above discussion, the weld design is controlled by the drop accident conditions. Thus, the appropriate base metal allowable is $2.4 \mathrm{Sm}$ or $38.2 \mathrm{ksi}$. For the basket weld, the appropriate quality factor from Table NG-3352 is 0.35 (single fillet/single groove weld, visual inspection), resulting in a weld allowable of $38.2(0.35)=13.4 \mathrm{ksi}$. For a double fillet weld, the weld quality factor is 0.4 , resulting in an allowable of $38.2(0.4)=15.3 \mathrm{ksi}$.

\section{LOAD CONDITIONS \& COMBINATIONS}

As discussed above, the Mark IA Storage Baskets are evaluated for four load cases: (1) lifting, (2) dead weight stacking inside the MCO, (3) a $35 \mathrm{~g}$ vertical drop, and (4) a $101 \mathrm{~g}$ horizontal drop. Each of these load conditions are independent, and are not combined. There is, however, the concern for a sequential drop, i.e. an end drop followed by a horizontal drop. This concern is addressed by considering the examining the maximum plastic distortion occurring in the vertical drop, which could potentially impact the buckling strength for a subsequent horizontal drop.

(

$\frac{254}{124}$

0
$4 / 17 / 97$ $4 / 17 / 97$ 


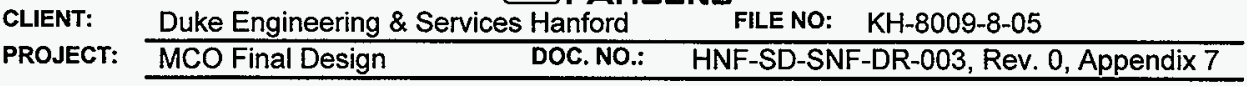

\section{STRESS ANALYSIS CALCULATIONS}

The Mark IV Storage Baskets are evaluated using both hand calculations (Mathcad) and finite element calculations (ANSYS). The finite element calculations are limited to stress predictions for the relatively complex bottom plate (Section 8.3).

\section{CALCULATION INPUT PARAMETERS:}

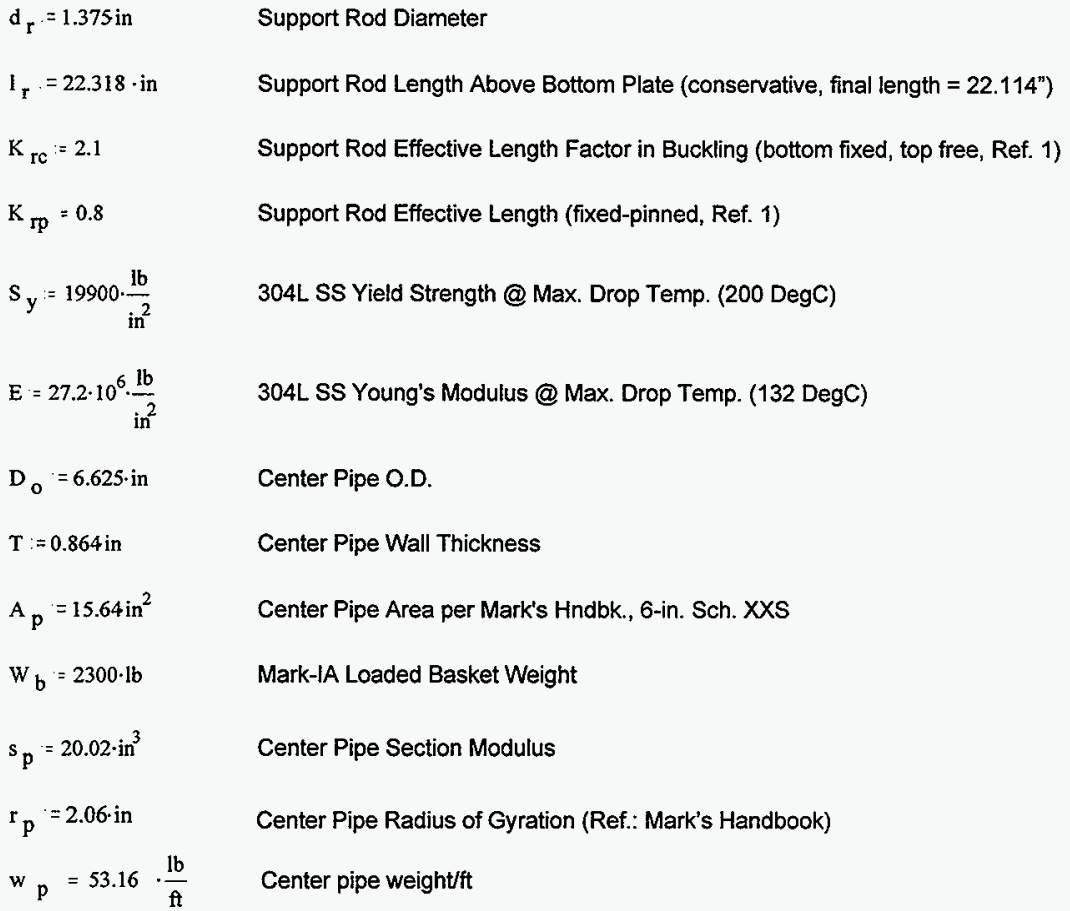

(1)


Cl.IENT: Duke Engineering \& Services Hanford FILE NO: KH-8009-8-05

PROJECT: MCO Final Design $\quad$ DOC. NO.: HNF-SD-SNF-DR-003, Rev. 0, Appendix 7

\subsection{Center Pipe}

The center pipe was evaluated for both a $35 \mathrm{~g}$ vertical drop and a $101 \mathrm{~g}$ horizontal drop event. For the vertical drop, the controlling ASME Code limit is axial compression (buckling). For the horizontal drop, beam bending (Pm) controls.

\subsubsection{Vertical Drop Load Condition}

Criteria: ASME Appendix F, Para. F-1331.5(b)

$$
\begin{aligned}
& \mathrm{D}_{0}=6.625 \mathrm{in} \\
& \mathrm{T}:=0.864 \mathrm{in} \\
& \mathrm{A}:=\frac{0.125}{\frac{\mathrm{D}_{\mathrm{o}}}{\frac{2}{T}}-\mathrm{T}} \mid \\
& \mathrm{A}=0.044 \\
& \mathrm{~B}=12200 \cdot \frac{\mathrm{lb}}{\mathrm{in}^{2}}
\end{aligned}
$$

From Fig. HA-3 \& Corresponding Table, $>S_{m}$ - Use B for Center Pipe Capacity

$$
\begin{aligned}
& \mathrm{A}_{\mathrm{p}}=15.64 \cdot \mathrm{in}^{2} \quad \text { Pipe Area per Mark's Hndbk., 6-in. Sch. XXS } \\
& \mathrm{P}_{\mathrm{pc}}=\mathrm{B} \cdot \mathrm{A}_{\mathrm{p}} \cdot 1.5 \\
& \mathrm{P}_{\mathrm{pc}}=2.862 \cdot 10^{5} \cdot \mathrm{lb} \quad \text { Center Pipe Capacity, Level D Event }
\end{aligned}
$$

\begin{tabular}{|l|l|l|}
\hline & & \\
\hline & & \\
\hline
\end{tabular}


CLIENT: PROJECT: $\begin{array}{lll}\text { MCO Final Design } & \text { DOC. NO.: } & \text { HNF-SD-SNF-DR-003, Rev. 0, Appendix } 7\end{array}$

\section{Check Center Pipe for Column Buckling:}

Using F-1334.3(b) (Max. Axial Compression Load for Linear Support):

$$
\begin{aligned}
& \mathrm{E}=27.2 \cdot 10^{6} \cdot \frac{\mathrm{lb}}{\mathrm{in}^{2}} \\
& \mathrm{~K}_{\mathrm{p}}:=0.8 \quad \text { Conservatively assumed pinned @ Top (Reference 1, Table C-C2.1) } \\
& l_{p}=l_{r} \\
& r_{p}:=2.06 \cdot \text { in } \\
& \lambda:=\frac{\mathrm{K}_{\mathrm{p}} \cdot \mathrm{l} \mathrm{p}}{\mathrm{r}_{\mathrm{p}}} \cdot \frac{1}{\pi} \cdot \sqrt{\frac{\mathrm{S} \mathrm{y}}{\mathrm{E}}} \\
& \lambda=0.073 \quad \text { 1st Equa. in F-1334.3 Applies } \\
& P_{y}:=S_{y} \cdot A_{p} \\
& P_{y}=3.112 \cdot 10^{5} \cdot l b \\
& P:=\frac{1-\frac{\lambda^{2}}{4}}{1.11+0.50 \lambda+\left(0.17 \lambda^{2}-0.28 \lambda^{3}\right)} \cdot \mathrm{P} y \\
& \mathrm{P}=2.709 \cdot 10^{5} \cdot 1 \mathrm{~b} \quad<\text { Axial Cylinder Capacity, Column Buckling Controls } \\
& \frac{P}{35 \cdot W_{b^{-5}}}=0.673
\end{aligned}
$$

Thus, the bottom basket center pipe Code capacity $=67.3 \%$ of the total $35 \mathrm{~g}$ Vertical Drop Loading. As discussed in Section 8.5, the center pipe/support rod vertical load sharing is a function of the component length-related tolerances. Section 8.5 demonstrates that for the specified tolerances, the center pipe and support rods will share the combined loading before reaching individual component capacities. Thus, the appropriate safety margin is for the center pipe/support rod combined capacity. As shown at the end of section 8.2 , the minimum combined center pipe/support rod capacity exceeds the total vertical drop loading by over $4 \%$. 
CLIENT: Duke Engineering \& Services Hanford

CLIENT: Duke Engineering \& Services Hanford FILE NO: KH-8009-8-05

PROJECT: MCO Final Design DOC. NO.: HNF-SD-SNF-DR-003, Rev. 0, Appendix 7

\subsubsection{Horizontal Drop Load Condition}

For the horizontal drop, the center pipe is loaded in beam bending. The bounding beam bending stress can be calculated by assuming a propped-cantilever beam. The loading consists of a $101 \mathrm{~g}$ loading of 8 fuel elements $\left(60^{\circ}\right.$ section), plus the pipe inertia.

$$
\begin{aligned}
& w_{t}=w_{p}+\frac{39.7 \cdot l b \cdot 8}{{ }_{1}} \quad \begin{array}{l}
\text { Single Fuel Pin Weight }=39.7 \\
\text { lb per Reference 1 }
\end{array} \\
& w_{t}=223.928 \cdot \frac{l b}{\mathrm{ft}}
\end{aligned}
$$

The maximum moment in the center pipe due to this fuel inertial loading can be obtained by assuming a simply-supported or a propped cantilever beam (same moment):

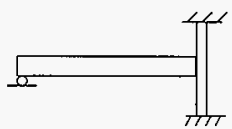

$$
\begin{aligned}
& M_{p}:=\frac{w_{t} \cdot p^{2}}{8} \cdot 101 \quad \text { Moment for 101g horizontal drop } \\
& M_{p}=9.779 \cdot 10^{3} \cdot l b \cdot f t \\
& f_{b}=\frac{M_{p}}{s_{p}} \\
& f_{b}=5.859 \cdot 10^{3} \cdot \frac{l b}{\mathrm{in}^{2}}
\end{aligned}
$$

From $F-1334.4$, the allowable bending stress is $f\left(S_{y}\right)$, where $f$ is the plastic shape factor.

Conservatively assuming $f=1$,

$$
F_{b}=s_{y}
$$


CLIENT: Duke Engineering \& Services HanSDNS \begin{tabular}{llll} 
PROJECT: & \multicolumn{1}{l}{ Duke Engineering \& Services Hanford } & FlLE NO: & KH-8009-8-05 \\
\cline { 2 - 3 } & MCO Final Design & DOC. NO.: & HNF-SD-SNF-DR-003, Rev. 0, Appendix 7 \\
\cline { 2 - 4 }
\end{tabular}

$$
\text { Ratio }=\frac{f_{b}}{F_{b}}
$$

Ratio $=\mathbf{0 . 3 3 3}$ Center Pipe OK for Hor. Drop

\subsubsection{Center Pipe/Bottom Plate Weld}

The critical loads for the center pipe/bottom plate weld are the drop loads. For the vertical drop load, it was assumed that $42 \%$ of the fuel inertia loading is carried by the weld, as discussed in Section 8.5. For the horizontal drop, it was conservatively assumed that the weld carries the maximum bending moment in the pipe (fixed-end moment, plate fixed against rotation).

Vertical Drop Load:

$$
\begin{aligned}
& \mathrm{w}:=\frac{48 \cdot 39.7 \cdot \mathrm{lb} \cdot 35 \cdot .42}{\mathrm{D}_{\mathrm{o}} \cdot \pi} \\
& \mathrm{w}=1.346 \cdot 10^{3} \cdot \frac{\mathrm{lb}}{\text { in }}
\end{aligned}
$$

Horizontal Drop Load:

$$
\begin{aligned}
& \mathrm{w}=\mathrm{f}_{\mathrm{b}} \cdot \mathrm{T} \\
& \mathrm{w}=5.062 \cdot 10^{3} \cdot \frac{\mathrm{lb}}{\mathrm{in}} \quad \text { Maximum Force/in., Horizontal Drop Load Critical } \\
& \sigma_{\mathrm{w}}:=\frac{\mathrm{w}}{\left.\frac{\sqrt{2}}{2}\right) \cdot \frac{3}{8} \cdot \mathrm{in} \cdot 2} \\
& \sigma_{\mathrm{w}}=9.545 \cdot 10^{3} \cdot \frac{\mathrm{lb}}{\mathrm{in}^{2}}
\end{aligned}
$$


CLIENT: $\quad$ Duke Engineering \& Services Hanford

FILE NO: KH-8009-8-05

PROJECT: MCO Final Design $\quad$ DOC.NO.: HNF-SD-SNF-DR-003, Rev. 0, Appendix 7

$$
\begin{aligned}
& \mathrm{n}:=0.35 \quad \text { Weld Quality Factor } \\
& \text { Ratio }:=\frac{\sigma_{\mathrm{w}}}{\mathrm{n} \cdot 2.4 \mathrm{~S}_{\mathrm{m}}} \\
& \text { Ratio }=0.715 \quad 3 / 8 \text { " Welds OK }
\end{aligned}
$$

\subsubsection{Center Coupling}

The center coupling is an extension of the center pipe which interfaces with adjacent baskets and the MCO shield plug. It is loaded during lifting and during a horizontal drop. During deadweight stacking, the center coupling interface with the center bushing is dimensioned to prevent loading of the center coupling. It attaches to the center pipe with four $1 / 2$ " spring pins. The minimum section of the coupling is the lifting grapple interface, which has an O.D.II.D. of 2.66"/2.00":

Check net section for tension (lifting load) and shear (hor. drop loading)

$$
\begin{aligned}
& A_{\min }:=\left[(2.66 \mathrm{in})^{2}-(2.00 \mathrm{in})^{2}\right] \cdot \frac{\pi}{4} \\
& A_{\min }=2.416 \cdot \mathrm{in}^{2} \\
& \sigma_{\text {lift }}:=\frac{\mathrm{W}_{\mathrm{b}}}{\mathrm{A}_{\text {min }}} \\
& \sigma_{\text {lift }}=972.855 \cdot \frac{\mathrm{lb}}{\mathrm{in}^{2}} \\
& \text { Ratio }:=\frac{\sigma_{\text {lift }}}{\frac{\mathrm{s}_{\mathrm{y}}}{3}}
\end{aligned}
$$

Ratio $=0.166$

9

\begin{tabular}{|l|l|l|l|}
\hline 0 & & & \\
\hline $4 / 17 / 97$ & & & \\
\hline $4 / 17 / 97$ & & & \\
\hline
\end{tabular}


CLIENT: Duke Engineering \& Services Hanford

PROJECT: MCO Final Design DOC NO
FILE NO: KH-8009-8-05

HNF-SD-SNF-DR-003, Rev. 0, Appendix 7

Thus, the center coupling is O.K. for the lifting load condition. For the horizontal drop, check the minimum section for average shear adequacy. Conservatively ignore bending resistance of bottom plate connection (top end reaction $=1 / 2$ of $101 \mathrm{~g}$ loading of eight 39.7 ib fuel pins).

$$
\begin{aligned}
& \tau_{\text {drop }}:=\frac{8 \cdot 39.7 \cdot \mathrm{lb} \cdot 101}{2 \cdot \mathrm{A} \min } \\
& \tau_{\text {drop }}=6.64 \cdot 10^{3} \cdot \frac{\mathrm{lb}}{\mathrm{in}^{2}} \\
& \mathrm{~S}_{\mathrm{u}}:=58800 \frac{\mathrm{lb}}{\mathrm{in}^{2}} \\
& \text { Ratio }:=\frac{\tau_{\text {drop }}}{0.42 \mathrm{~S}} \\
& \text { Ratio }=0.269
\end{aligned}
$$

Therefore, the center coupling is also adequate for the horizontal drop $101 \mathrm{~g}$ loading.

The center pipe/center coupling connector pins (pin every $90^{\circ}=4$ pins total) are specified as 1/2", heavy duty SAE 30302 or SAE 30304 coiled type spring pins, conforming to ASME Standard B18.8.2, Table11. The ASME standard specified shear strength of the pins is $25,000 \mathrm{lb}$ in double shear $(12,500 \mathrm{lb}$, single shear). The only significant pin loads occur during lifting operations, which requires a limit of $1 / 5$ of the ultimate strength.

$$
\begin{aligned}
& f_{s u}:=12500 \cdot l b \\
& W_{b}:=2350 \cdot 1 b \\
& \text { Ratio }:=\frac{W_{b}}{\frac{4 \cdot f_{s u}}{5}} \\
& \text { Ratio }=0.235
\end{aligned}
$$




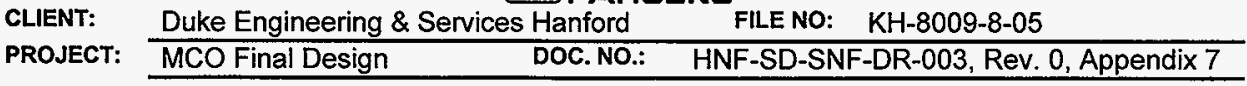

Thus, the center coupling pins are adequate for the lifting load condition.

This same 1/2" $302 / 304$ spring pin design is also used to connect the bottom bushing to the center pipe. The only load this pin carries is the dead weight of the bushing. A

conservative dead weight of the bushing can be obtained using the O.D. (5.00") and the minimum 1.D. $\left(2.65^{\prime \prime}\right)$ :

$$
\begin{aligned}
& W_{b}=(\pi / 4)\left[(5.00)^{2}-(2.65)^{2}\right](0.286)=4.04 \mathrm{lb} . \\
& \text { Ratio }=4.04 /(12,500 / 5)=0.002 \quad O . K .
\end{aligned}
$$

\subsection{Support Rod}

Support rod capacity calculations were performed for both a pinned and free top boundary condition. As indicated, the support rod capacity is controlled by buckling.

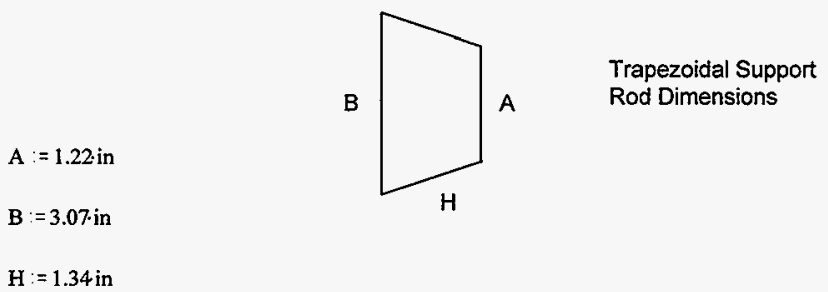

$$
\begin{array}{ll}
I_{x} \cdot \frac{H^{3} \cdot\left(A^{2}+4 \cdot A \cdot B+B^{2}\right)}{36 \cdot(A+B)} & \text { Reference } 9 \\
I_{x}=0.403 \cdot \text { in }^{4} & \text { Reference } 9
\end{array}
$$

\begin{tabular}{|c|l|l|l|}
\hline 0 & & & \\
\hline $3 a 4 / 17 / 97$ & & & \\
\hline $\ln 4 / 17 / 97$ & & & \\
\hline
\end{tabular}


CLIENT: \begin{tabular}{llll} 
PROJECT: & \multicolumn{1}{l}{ Duke Engineering \& Services Hanford } & FILE NO: & KH-8009-8-05 \\
\cline { 2 - 4 } & MCO Final Design & DOC. NO.: & HNF-SD-SNF-DR-003, Rev. 0, Appendix 7 \\
\hline
\end{tabular}

$$
\begin{aligned}
& \mathrm{I}_{\mathrm{y}}=1.307 \cdot \text { in }^{4} \\
& \mathrm{I}_{\mathrm{r}}:=22.318 \cdot \text { in } \\
& \mathrm{A}_{\mathrm{r}}:=\frac{\mathrm{A}+\mathrm{B}}{2} \cdot \mathrm{H} \\
& \mathrm{A}_{\mathrm{r}}=2.874 \quad \text { in } 2 \\
& \mathrm{~K}_{\mathrm{rc}}:=2.1 \quad \text { Cantilevered (No lateral restr. @ top, Reference 1, Table C-C2.1) } \\
& \mathrm{K}_{\mathrm{rp}}:=0.8 \quad \text { Pinned (lateral restr. @ top, Reference 1, Table C-C2.1) } \\
& \frac{\mathrm{r}_{\mathrm{x}}:=\sqrt{\mathrm{I}_{\mathrm{y}}}}{\mathrm{K}_{\mathrm{rp}} \cdot \mathrm{I}_{\mathrm{r}}}=26.477 \\
& \mathrm{r}_{\mathrm{x}} \\
& \mathrm{r}_{\mathrm{X}}=0.674 \cdot \text { in } \\
& \mathrm{r}_{\mathrm{r}} \cdot 1 \mathrm{r}
\end{aligned}
$$

For Level D Support Rod Capacity, Use F-1334.3(b) (Max. Axial Compression Load for Linear Support):

$$
\begin{aligned}
& S_{y}=19900 \cdot \frac{\mathrm{lb}}{\mathrm{in}^{2}} \\
& E=27.2 \cdot 10^{6} \cdot \frac{\mathrm{lb}}{\mathrm{in}^{2}} \\
& \lambda_{\mathrm{c}}:=\frac{\mathrm{K}_{\mathrm{rc}} \cdot \mathrm{l}_{\mathrm{r}}}{\mathrm{r}_{\mathrm{x}}} \cdot \frac{1}{\pi} \cdot \sqrt{\frac{\mathrm{S}_{\mathrm{y}}}{\mathrm{E}}} \\
& \lambda_{\mathrm{c}}=1.06 \quad \text { 2nd Yield Strength @ Max. Drop Temp. (200 degC) }
\end{aligned}
$$




\section{OPARSDNS}

CLIENT: Duke Engineering \& Services Hanford

FILE NO: $\mathrm{KH}-8009-8-05$

PROJECT: MCO Final Design DOC. NO.: HNF-SD-SNF-DR-003, Rev. 0, Appendix 7

$$
\lambda_{\mathrm{p}}=\frac{\mathrm{K}_{\mathrm{rp}} \cdot \mathrm{l}_{\mathrm{r}}}{\mathrm{r}_{\mathrm{x}}} \cdot \frac{1}{\pi} \cdot \sqrt{\frac{\mathrm{S}_{\mathrm{y}}}{\mathrm{E}}}
$$

$\lambda_{p}=0.404$

1st Equa. Applies

$P_{y}:=S_{y} \cdot A_{r}$

$P_{y}=5.72 \cdot 10^{4} \cdot 1 \mathrm{~b}$

$P_{c}=\frac{2}{3} \cdot\left(1-\frac{\lambda_{c}^{2}}{4}\right) \cdot P_{y}$

$P_{c}=2.741 \cdot 10^{4} \cdot 1 b$

Rod Capacity (Cantilevered)

$\mathrm{P}_{\mathrm{p}}:=\frac{1-\frac{\left(\lambda_{\mathrm{p}}\right)^{2}}{4}}{\left.1.11+0.50 \lambda_{\mathrm{p}}+\left(0.17 \lambda_{\mathrm{p}}{ }^{2}-0.28 \lambda_{\mathrm{p}}\right)^{3}\right)} \cdot \mathrm{P}_{\mathrm{y}}$

$P_{p}=4.152 \cdot 10^{4} \cdot \mathrm{lb} \quad$ Rod Capacity (Pinned)

For six Rods,

$$
6 \cdot P_{c}=1.645 \cdot 10^{5} \cdot 1 b
$$

Total Rod Capacity per ASME Appendix F, Linear Support (Cantilevered)

$$
6 \cdot \mathrm{P}_{\mathrm{p}}=2.491 \cdot 10^{5} \cdot \mathrm{lb} \quad \text { Pinned Rod Capacity }
$$

\begin{tabular}{|l|l|l|l|}
\hline 0 & & & \\
\hline $4 / 17 / 97$ & & & \\
\hline $4 / 17 / 97$ & & & \\
\hline
\end{tabular}




\section{国 PAasons,}

CLIENT: Duke Engineering \& Services Hanford FILE NO: KH-8009-8-05

PROJECT: MCO Final Design $\quad$ DOC. NO.: HNF-SD-SNF-DR-003, Rev. 0, Appendix 7

$\mathrm{W}_{\mathrm{b}}=2300 \cdot \mathrm{lb} \quad$ Mark-IA Loaded Basket Weight (Conservative), Bottom Basket Carries 5 Baskets

$\frac{6 \cdot P_{c}}{35 \cdot W_{b} \cdot 5}=0.409 \quad$ Support Rod Code Capacity (Cantilevered) carries $40.9 \%$ of $35 \mathrm{~g}$ Drop Load

$\frac{6 \cdot \mathrm{P}_{\mathrm{p}}}{35 \cdot \mathrm{W}_{\mathrm{b}} \cdot 5}=0.619 \quad$ Support Rod Code Capacity (Pinned) carries $61.9 \%$ of $35 \mathrm{~g}$ Drop Load

Thus, using the ASME Code Appendix F allowables, the support rods Code carry $40.9 \%$ (cantilevered) to $61.9 \%$ of the total $35 \mathrm{~g}$ drop loading. Based upon the Section 8.5 postbuckling calculations, there is adequate friction to provide lateral support at the top of the rods. However, conservatively using the cantilevered capacity, the support rod/center pipe combined capacity (see Section 8.5 ) is $40.9 \%+67.3 \%=108.2 \%$, which demonstrates that the support rod capacity, when combined with the center pipe capacity is sufficient to carry the $35 \mathrm{~g}$ drop loading. The resulting maximum stress ratio for the center pipe/support rod combined capacity is therefore:

$$
\begin{aligned}
& \text { Ratio }=\frac{35 \cdot \mathrm{W}_{\mathrm{b}} \cdot 5}{6 \cdot \mathrm{P}_{\mathrm{C}}+\mathrm{P}} \\
& \text { Ratio }=0.924
\end{aligned}
$$

Since this stress ratio is high, potential unconservatisms in the calculated support rod capacity is an issue. The most significant potential unconservatism is the effect of load eccentricities. Ignoring load eccentricities is judged to be acceptable for the following reasons:

(1) The basket interface geometry results in significant "self correcting" of load eccentricities, as shown in Figure 2. That is, the rotation of the top of the support rod, resulting from an eccentric load, will move the load center toward the high point of the support rod and reduce the eccentricity.

(2) A 0.25-inch load eccentricity was considered in the Section 8.5 non-linear instability analysis and the resulting support rod capacity exceeded the calculated code capacity.

(3) As mentioned in the last paragraph of Section 8.5, the 0.25 -inch chamfer on the bottom plate (see Figure 2) biases the eccentricity towards the center of the basket, resulting in

(1)




\section{Fiansows}

CLIENT: Duke Engineering \& Services Hanford FILE NO: KH-8009-8-05

PROJECT: MCO Final Design $\quad$ DOC.NO.: HNF-SD-SNF-DR-003, Rev. 0, Appendix 7

a preferential buckling direction toward the I.D. of the MCO shell. The MCO shell constraint results in a higher buckling failure mode and a higher support rod capacity.

(4) The non-linear/large deflection buckling analysis, discussed in Section 8.5, included a sensitivity study on the effect of friction on the buckling capacity of the support rods. A friction coefficient of 0.1 was found to be adequate to prevent lateral movement of the top of the rods. Accounting for lateral constraint at the top of the rods significantly increases the margin.

(5) Paragraph F-1322.3 (c) permits an adjustment of the stress-strain properties to account for strain rate effects. The increase in the yield strength for the drop accident load cases was conservatively ignored.

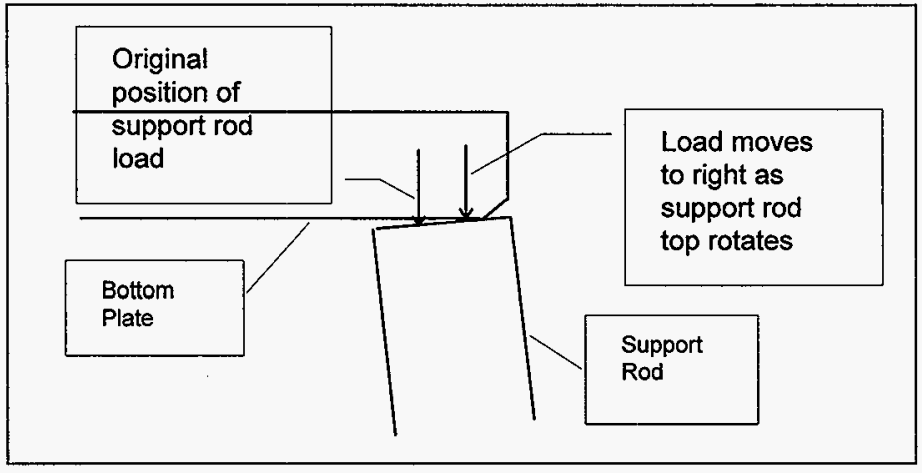

Figure 2. Illustration of Support Rod Load Eccentricity.

\subsection{Bottom Plate}

The Mark IA bottom plate was evaluated for normal operation and drop accident conditions. As indicated in the following subsection, the bottom plate design is controlled by the vertical drop load event. 


\section{P. PARSRNS}

CLIENT: Duke Engineering \& Services Hanford

FILE NO: $\quad \mathrm{KH}-8009-8-05$

PROJECT:

MCO Final Design

DOC. NO.:

HNF-SD-SNF-DR-003, Rev. 0, Appendix 7

\subsubsection{Discussion of Bottom Plate Load Conditions}

As indicated in Appendix A of the Performance Specification (Reference 1), a loaded MCO consists of six Mark IA baskets. The basket bottom plate stresses would be relatively low if the basket support rods are all alligned. However, since the baskets are not indexed to assure support rod allignment, rotational offsets are expected. The maximum bottom plate bending occurs when the support rods are midway between the above basket rods $\left(30^{\circ}\right.$ offset). The critical bottom plate is the next-to-the-bottom basket with the bottom basket rotated $30^{\circ}$ from the basket above. For this condition, the critical bottom plate rods react the loading from the four top baskets. This support rod offset produces significant bending stresses in the bottom plate, as demonstrated below.

The bounding drop loads for the bottom plate are a vertical drop of $\mathbf{3 5} \mathrm{g}$ 's and a horizontal drop of $101 \mathrm{~g}$ 's. As indicated below, the critical drop for the bottom plate is the $35 \mathrm{~g}$ vertical drop, except for the plate-to-pipe weld, where the horizontal drop is critical. Since the drop load stresses are much greater than the normal operation stresses within the MCO, the drop load condition controls the bottom plate design. However, since the boundary conditions and acceptance criteria of the single basket lift condition are much different than for the drop conditions within the MCO, the lifting condition is also addressed to confirm adequacy for this load condition.

\subsubsection{ANSYS Models}

Three ANSYS models were developed for evaluating the Mark IA Basket bottom plates. The first model generated was a sector model complete with holes, as shown in Figure 3. The mesh refinement necessary to properly define the holes resulted in a relatively large number of elements which ran relatively slow. Therefore, a second model without holes was developed, as shown in Figure 4. The majority of the bottom plate evaluations were run using the second model. The first model was used as a final confirmation using the final bottom plate design parameters (plate thickness, hole size/pattern, etc). Note that in both models, shell elements (SHELL43 and SHELL63) were used to model the center pipe and bottom plate, while solid elements (SOLID45) were used to model the support rod. Also note that both models were limited to $60^{\circ}$ sectors to take advantage of the $60^{\circ}$ symmetry (support rods every $60^{\circ}$ and $a 30^{\circ}$ repeating hole pattern).

Symmetry boundary conditions (no rotation about a radius line nor no displacement in the tangential direction) were used at the zero and 60 degree edges of the models. For the stacking load condition within the $\mathrm{MCO}$, it was assumed that the supporting basket below was rotated $30^{\circ}$, resulting in the support rods beneath being centered on the edges of the model. The precise bottom plate to support rod contact locations were not known. To

$\$ 2$

\begin{tabular}{|c|l|l|l|}
\hline & & & \\
\hline $\cos 4 / 17 / 97$ & & & \\
\hline $\ln 4 / 17 / 97$ & & & \\
\hline
\end{tabular}


CLIENT:

PROJECT:
Duke Engineering \& Services Hanford DOC. NO.:
FILE NO: KH-8009-8-05

address this contact issue, gap elements were placed at the bottom plate/support rod interface.

A $180^{\circ}$ degree symmetry model, shown in Figure 1, was developed for the horizontal drop evaluation. The primary purpose of this model is to demonstrate conformance to the twoinch distortion limit on the center pipe (Section 4.9.3 of the Reference 1 Performance Specification). The bottom plate and center pipe were modeled using ANSYS SHELL43 elements. The support rods are modeled with beam elements (BEAM4). Note that beam elements were also used at the support rod/bottom plate connection locations to spread the support rod moments over a reasonable area. Although the support rods structural adequacy is not an issue for the horizontal drop, the support rods were modeled to introduce the support rod inertia loads (moments) to the bottom plate. Gap elements (CONTAC52) were used at the drop-side interface with the MCO in order to achieve a reasonable interface load distribution. Since the horizontal drop can be preceded by a vertical drop, rigid links (BEAM4) were used to account for a potential axial reaction offset due to plastic distortion in the bottom plate occurring during the vertical drop. An offset equal to half of the plate thickness (1/2-inch) was used, as justified in Section 8.3 .

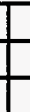


CLIENT: Duke Engineering \& Services Hanford

FILE NO: KH-8009-8-05

PROJECT: MCO Final Design $\quad$ DOC.NO.: HNF-SD-SNF-DR-003, Rev. 0, Appendix 7

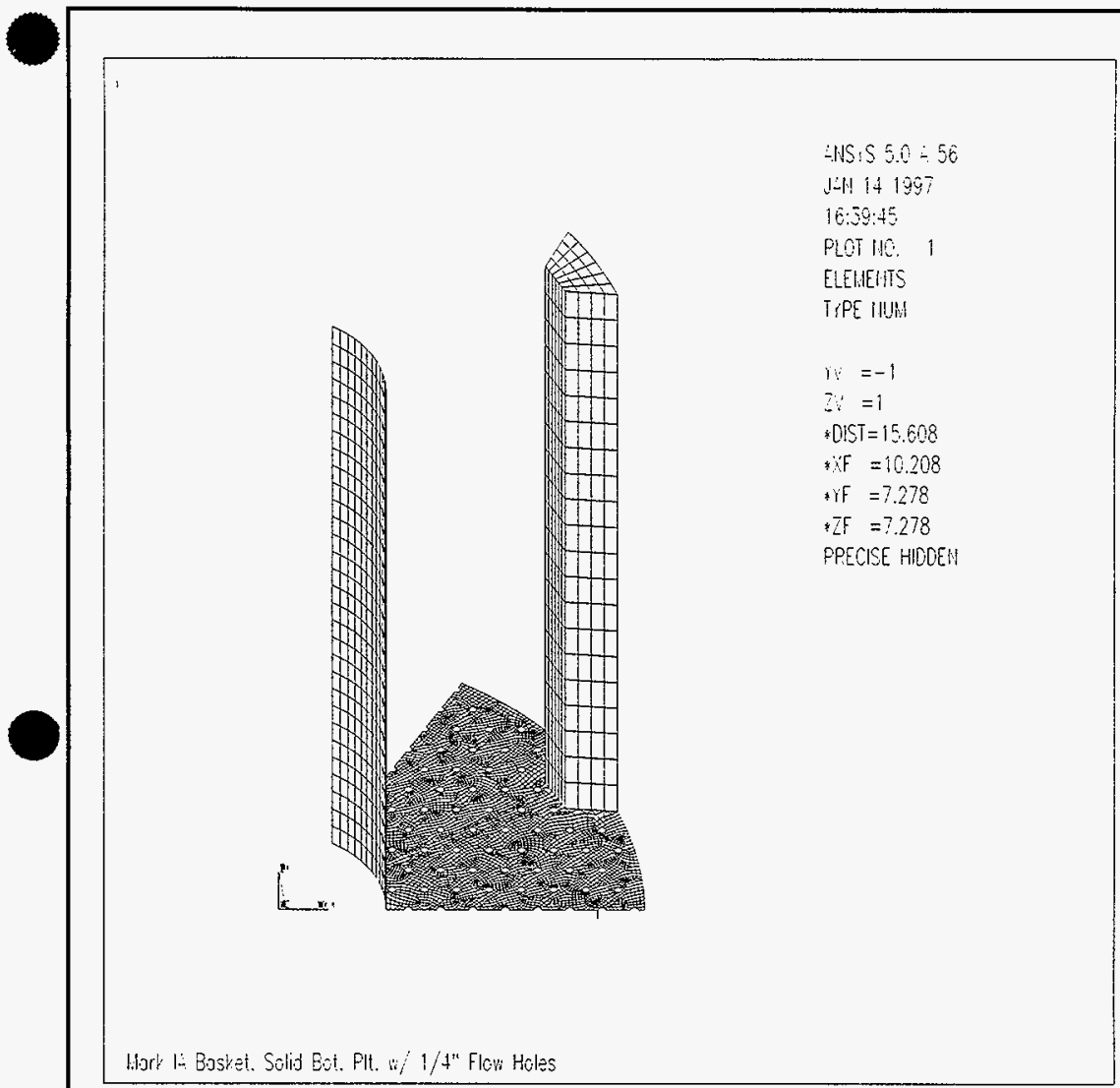

Figure 3. 60 Degree Sector Model of Basket with Holes.

\begin{tabular}{|l|c|c|c|c|c|}
\hline REVISION & 0 & & & & PAGE 30 \\
OF 85 \\
\cline { 1 - 5 }
\end{tabular}


CLIENT: $\quad$ Duke Engineering \& Services Hanford

FILE NO: $\mathrm{KH}-8009-8-05$

\begin{tabular}{lll} 
PROJECT: & MCO Final Design & DOC. NO.: HNF-SD-SNF-DR-003, Rev. 0, Appendix 7 \\
\cline { 2 - 4 }
\end{tabular}

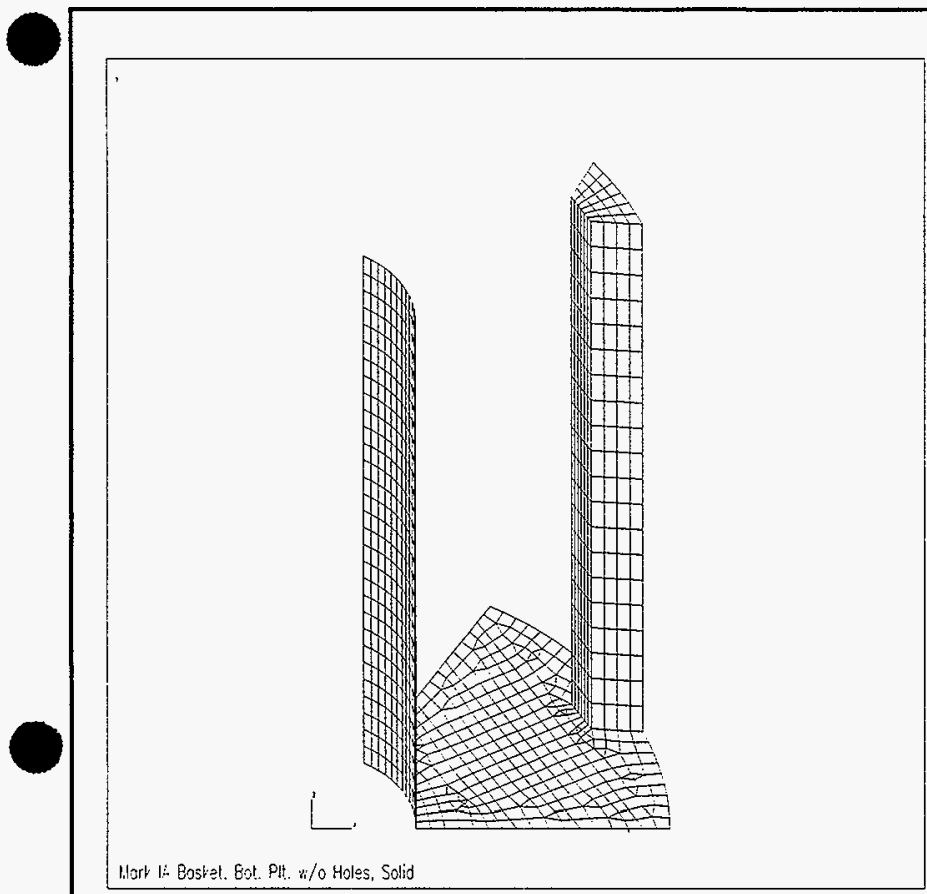

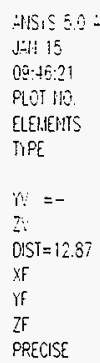

Figure 4. 60 Degree Sector ANSYS Model of Basket Without Holes. 
CLIENT: $\quad$ Duke Engineering \& Services Hanford

FILE NO: KH-8009-8-05

PROJECT: MCO Final Design $\quad$ DOC. NO.: HNF-SD-SNF-DR-003, Rev. 0, Appendix 7

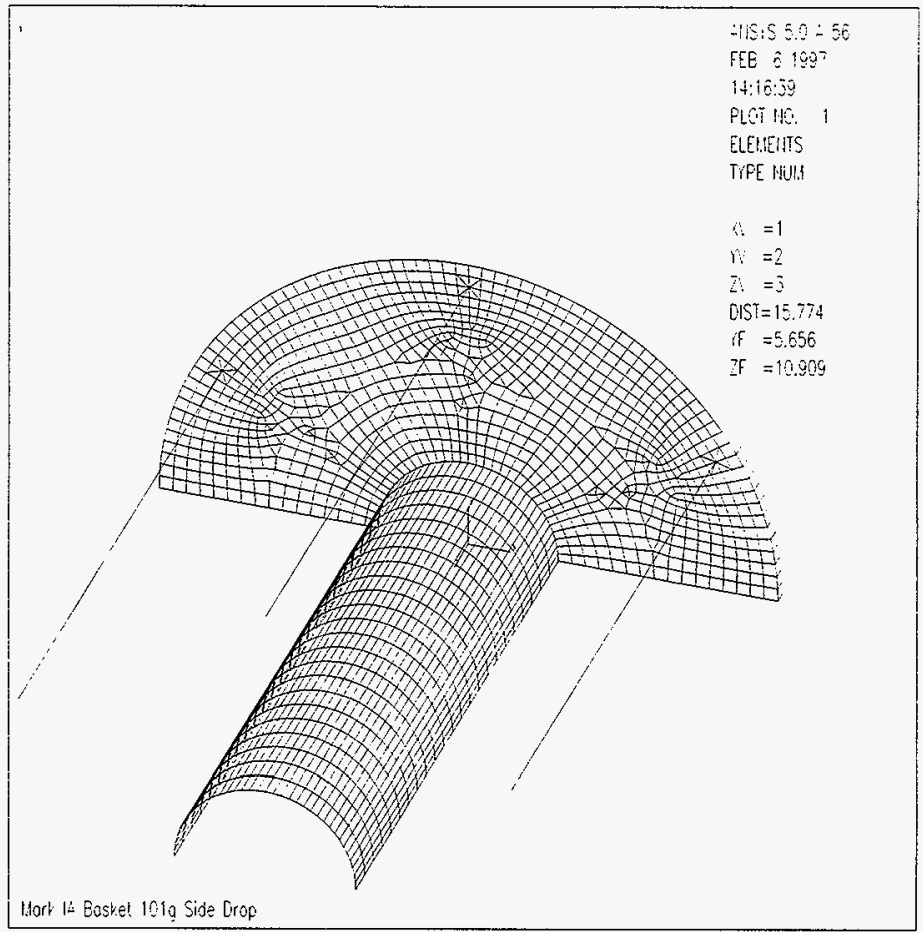

Figure 5. 180 Degree ANSYS Model of Basket Used for Horizontal Drop Analyses.

\subsubsection{Vertical Drop Load Condition Evaluation}

Using the Figure 4 no-hole model, an initial estimate of the $35 \mathrm{~g}$ vertical drop stresses are generated. The loading/boundary conditions corresponded to a next-to-the-bottom basket, with four Mark IA baskets above. Prior to the vertical drop analysis, a run is made to estimate the fuel load distribution between the center pipe and support rods by applying a uniform pressure to the bottom plate to simulate the fuel inertia loading which was reacted

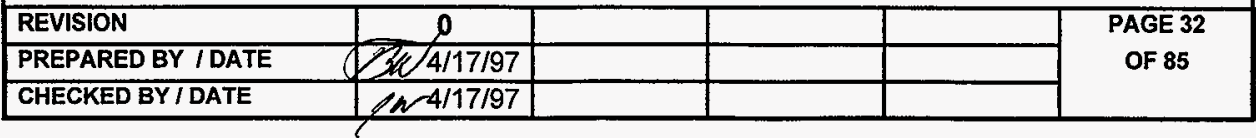


CLIENT: Duke Engineering \& Services Hanford

FILE NO: $\quad \mathrm{KH}-8009-8-05$

PROJECT:

MCO Final Design

DOC. NO.:

HNF-SD-SNF-DR-003, Rev. 0, Appendix 7

through the center pipe and support rod. As discussed in Section 8.5 , for an elastically loaded "perfect fit", $58 \%$ of the fuel loading is reacted through the support rods and $42 \%$ of the fuel loading is reacted through the center pipe. This support rod/center pipe load distribution provides a reasonable, and likely conservative, estimate of the support rod loading in the elastic/plastic vertical drop analysis described below. See Section 8.5 for a discussion of this load distribution.

From Appendix A of the Performance Specification (Reference 1), the loaded Mark IA basket weight is $2153 \mathrm{lb}$ (basket weight $=247 \mathrm{lb}$, fuel weight $=1906 \mathrm{lb}$ ). Subsequent basket design changes have increased the basket weight to approximately $400 \mathrm{lb}$. For analysis purposes, a total loaded basket weight of $2350 \mathrm{lb}$ was used. Using this conservative weight estimate, the $35 \mathrm{~g}$, four basket loading is

$$
35(2350)(4)=329,000 \mathrm{lbs} .
$$

For an elastic/plastic drop analysis, it is reasonable to assume that the support rods equally share the support rod portion (58\%) of the 329,000 drop load, resulting in a support rod loading for the next-to-the-bottom basket of

$$
F_{f}=0.58(329,000) / 6=31,803 \mathrm{lbs} .
$$

The center pipe load estimate for a $60^{\circ}$ sector is

$$
F_{c p}=0.42(329,000) / 6=23,030 \mathrm{lbs} .
$$

These loads were imposed on the Figure 4 model in the form of pressures on top of the support rod and center pipe (11,066 psi and 8835 psi, respectively). Vertical constraints were introduced at the bottom of the center pipe and at the interface with the bottom basket support rods.

Fuel inertia loading on the bottom plate was input as an equivalent pressure, using 39.7 Ib/fuel rod per Reference 1, Appendix A:

$$
\begin{aligned}
& F_{f}=48(39.7)(35)=66,696 \mathrm{lb} \\
& A_{p}=\left[(22.625)^{2}-(6.625)^{2}\right](\pi / 4)=367.57 \mathrm{in}^{2}
\end{aligned}
$$

\begin{tabular}{c|c|l|l}
0 & & \\
\hline $5 \pi / 4 / 17 / 97$ & & \\
\hline $\ln 4 / 17 / 97$ & &
\end{tabular}




\section{(1) parsons}

CLIENT: Duke Engineering \& Services Hanford \begin{tabular}{llll} 
PROJECT: & MCO Final Design & DOC. NO.: & HNF-SD-SNF-DR-003, Rev. 0, Appendix 7 \\
\cline { 2 - 4 }
\end{tabular}

Justification for using the Figure 4 "no-hole model" for the elastic/plastic analysis was obtained by performing elastic drop analyses on both the Figure 3 and Figure 4 models. The elastic analysis results are summarized in the Figure 6 and Figure 7 stress intensity contour plots. Note that with the exception of the very local stresses immediately adjacent to the flow holes, the results are very similar. Also note that the peak stress intensity for both models occurs near the support rod constraints and are very close to the same magnitude (65.2 ksi and $65.5 \mathrm{ksi})$. Using the results for the more detailed model (Figure 5), the maximum plate bending stress of $65.5 \mathrm{ksi}$ compares to an ASME III-NG Level D allowable stress intensity of $57.2 \mathrm{ksi}\left(1.5 \times 2.4 \mathrm{Sm} @ 200^{\circ} \mathrm{C}\right)$, indicating a small overstress for the elastic analysis limits. This elastic overstress was resolved by performing the elastic/plastic analysis described below.

0
$\sin / 4 / 17 / 97$
$\operatorname{s/n} 4 / 17 / 97$


CLIENT: Duke Engineering \& Services Hanford \begin{tabular}{llll} 
PROJECT: & MCO Final Design & DOC. NO.: & HNF-SD-SNF-DR-003, Rev. 0, Appendix 7 \\
\cline { 2 - 4 }
\end{tabular}

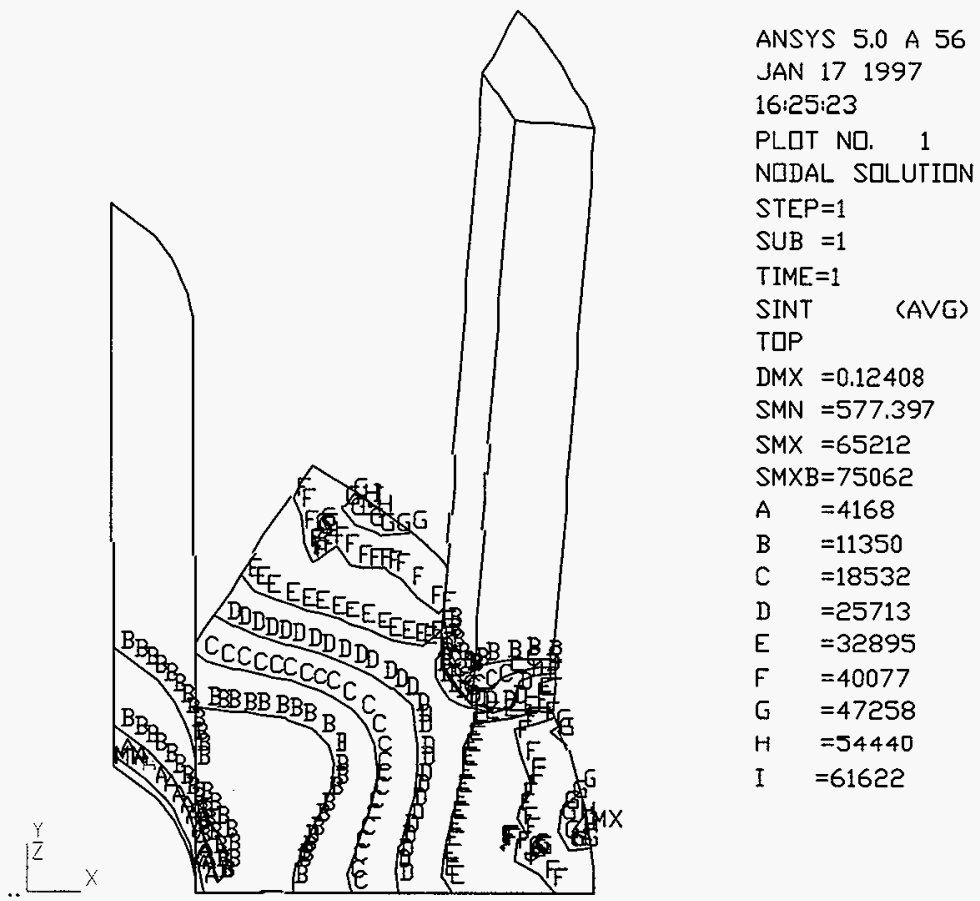

Figure 6. Elastic Stress Intensity Contours, 35g Vertical Drop, No-Hole Model.

\begin{tabular}{|c|c|c|c|}
\hline 0 & & & \\
\hline $151 / 4 / 17 / 97$ & & & \\
\hline $12-4 / 17 / 97$ & & & \\
\hline
\end{tabular}


CLIENT: Duke Engineering \& Services Hanford FILE NO: KH-8009-8-05 \begin{tabular}{llll} 
PROJECT: & MCO Final Design & DOC. NO.: & HNF-SD-SNF-DR-003, Rev. 0, Appendix 7 \\
\cline { 2 - 3 }
\end{tabular}

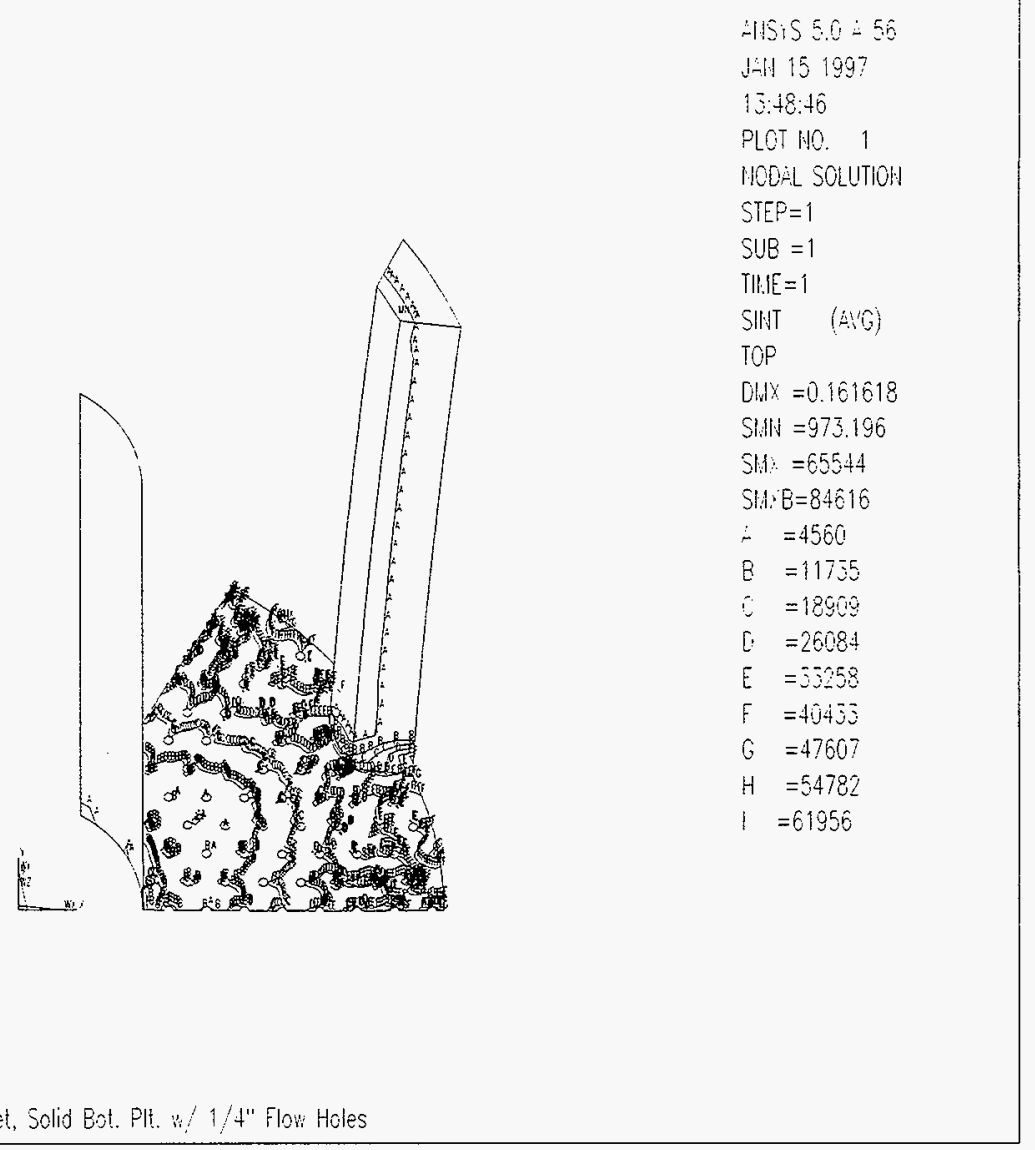

Figure 7. Elastic Stress Intensity Contours, $35 \mathrm{~g}$ Drop Loading, Detailed Hole Model. 
CLIENT: Duke Engineering \& Services Hanford

FILE NO: KH-8009-8-05

PROJECT: MCO Final Design $\quad$ DOC. NO.: $\quad$ HNF-SD-SNF-DR-003, Rev. 0, Appendix 7

Using the Figure 4 model, an elastic/plastic analysis of the $35 \mathrm{~g}$ drop loading was performed, assuming bilinear plasticity (ANSYS input/output files: pltnhp.inp and pltnhp.out). The method for developing the bilinear stress-strain curve was obtained from Reference 12, adapted from 304 SS data. Reference 12 indicates that the strain hardening coefficient is relatively independent of temperature. A value of $0.16 \times 10^{6} \mathrm{psi}$ was obtained from Table B.1 of Reference 12, for a conservative maximum strain of 5\%. Since 304 SS and 304L SS are nearly identical materials, it is reasonable to use the same strain hardening coefficient and the $17.8 \%$ yield strength increase. The ANSYS input and output files are contained in the attached disk (pltnhp.inp and pltnhp.out).

The vertical drop stress intensity contours are shown in Figure 8. A tabular summary of the membrane and membrane plus bending results are provided in Table 5. Note that all predicted stress intensities ratios are less than one, indicating that the results are within ASME Code allowables.

Table 5. Vertical Drop Stress Intensity Results Summary.

\begin{tabular}{|c|c|c|c|c|c|}
\hline \multicolumn{2}{|c|}{ Component } & \multirow{2}{*}{$\begin{array}{c}\text { Stress } \\
\text { Category }\end{array}$} & Stress Intensities, ksi & \multirow{2}{*}{} \\
\cline { 3 - 6 } & & Maximum & Allowable & \multirow{2}{*}{ Ratio } \\
\hline \multirow{3}{*}{ Bottom Plate } & Middle & $\mathrm{Pm}$ & 19.6 & 41.1 & 0.48 \\
\cline { 2 - 6 } & $\mathrm{Top}$ & $\mathrm{Pm}+\mathrm{Pb}$ & 24.5 & 52.8 & 0.46 \\
\cline { 2 - 6 } & Bottom & $\mathrm{Pm}+\mathrm{Pb}$ & 24.5 & 52.8 & 0.46 \\
\hline \multirow{3}{*}{ Center Pipe } & Middle & $\mathrm{Pm}$ & 14.7 & 41.1 & 0.36 \\
\cline { 2 - 6 } & Top & $\mathrm{Pm}+\mathrm{Pb}$ & 18.8 & 52.8 & 0.36 \\
\cline { 2 - 6 } & Bottom & $\mathrm{Pm}+\mathrm{Pb}$ & 22.5 & 52.8 & $0 . .43$ \\
\hline
\end{tabular}

The plastic strain contours are shown in Figure 9 . Note that a maximum plastic strain of about $1 \%$ was predicted, which is well below the $5 \%$ maximum assumed for the strain hardening coefficent selection above. Also note that a maximum displacement of 0.068 in.

\begin{tabular}{|l|c|l|l|l|l|}
\hline REVISION & 0 & & & & PAGE 37 \\
PREPARED BY IDATE 85 \\
\cline { 1 - 4 } CHECKED BYIDATE & OFaC 4/17/97 & & & & \\
\hline
\end{tabular}


CLIENT: Duke Engineering \& Services Hanford

PROJECT: MCO Final Design $\quad$ DOC. NO.: HNF-SD-SNF-DR-003, Rev. 0, Appendix 7

was predicted. This maximum displacement occurs in the vertical direction and occurs in the plate below the support rod. The bottom plate distortion is of interest because of the potential for a horizontal drop following a vertical drop. As indicated in Section 8.3.4, this maximum plate distortion was considered in the horizontal drop evaluation.

\subsubsection{Horizontal Drop Analysis}

Using the $180^{\circ}$ model shown in Figure 5, an elastic/plastic analysis was performed for the $101 \mathrm{~g}$ horizontal drop load condition. The plastic analysis option was selected for two reasons: (1) an initial elastic analysis predicted local stresses which exceeded the elastic allowables, (2) total transverse distortion (elastic plus plastic) predictions were needed to demonstrate conformance to the Reference 1 , Section 4.19.3, nuclear criticality safety requirement that the "void space centerline shall not deviate more than two inches from the MCO centerline".

As indicated by the small elements on the right side (impact side) of the Figure 5 model, line elements (BEAM4) were used to account for the potential offset associated with bottom plate plastic distortions occurring during a preceding vertical drop. Note from Figure 10, that offset from the bottom plate centerline can also be affected by the bottom plate edge contact. Although the vertical drop distortion was less than 0.1 inch, an offset of 0.5 " was conservatively assumed. Although the actual offset would be limited to a small area (near a support rod), a uniform offset was conservatively assumed.

\begin{tabular}{|c|c|c|c|}
\hline 0 & & & \\
\hline$\sum \omega^{4 / 17 / 97}$ & & & \\
\hline$\mu N^{4 / 17 / 97}$ & & & \\
\hline
\end{tabular}


CLIENT: Duke Engineering \& Services Hanford FILE NO: KH-8009-8-05 PROJECT: \begin{tabular}{lll} 
MCO Final Design & DOC.NO.: HNF-SD-SNF-DR-003, Rev. 0, Appendix 7 \\
\cline { 2 - 3 }
\end{tabular}

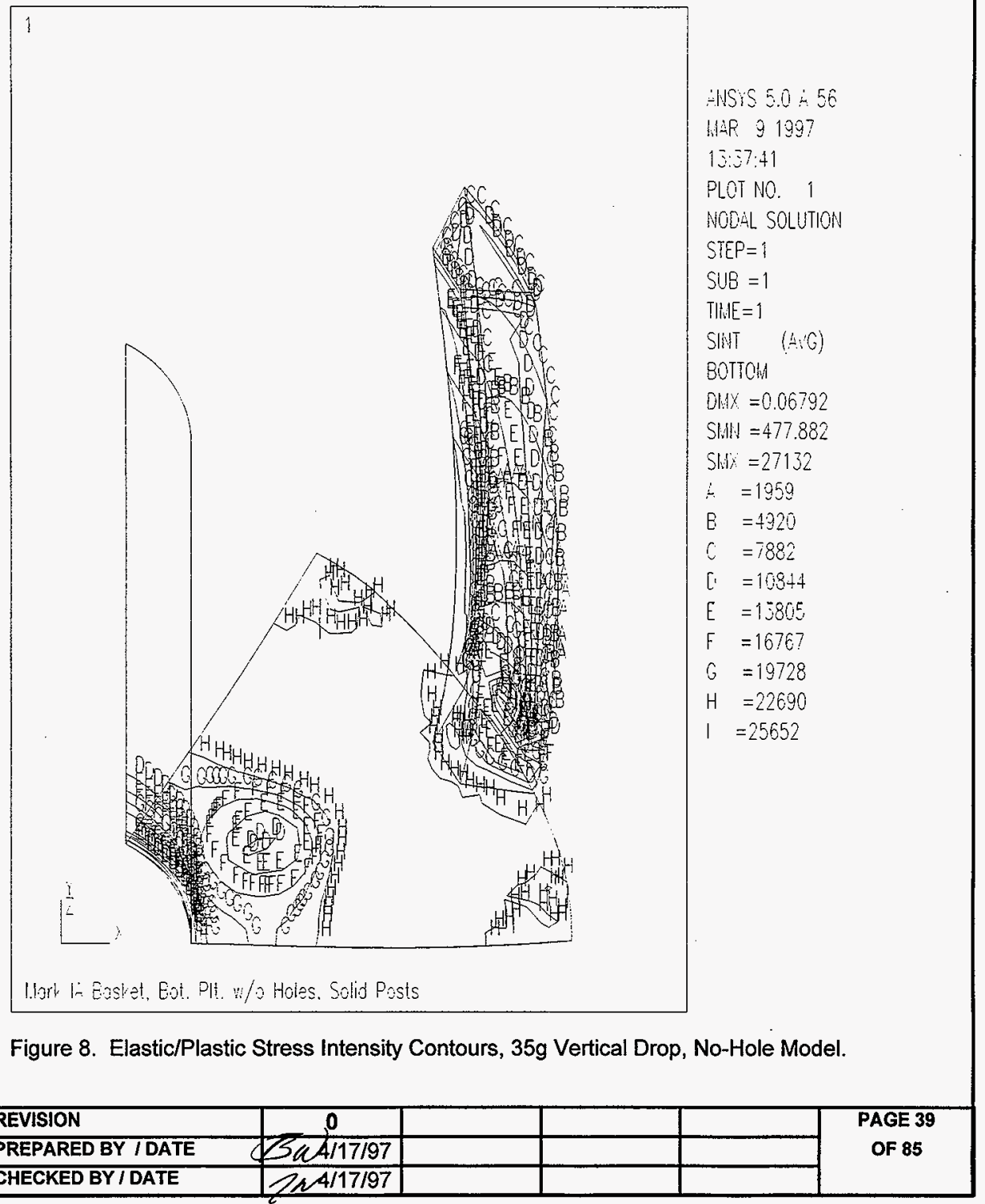


CLIENT: Duke Engineering \& Services Hanford

FILE NO: $\quad \mathrm{KH}-8009-8-05$

PROJECT: MCO Final Design DOC.NO.: HNF-SD-SNF-DR-003, Rev. 0, Appendix 7

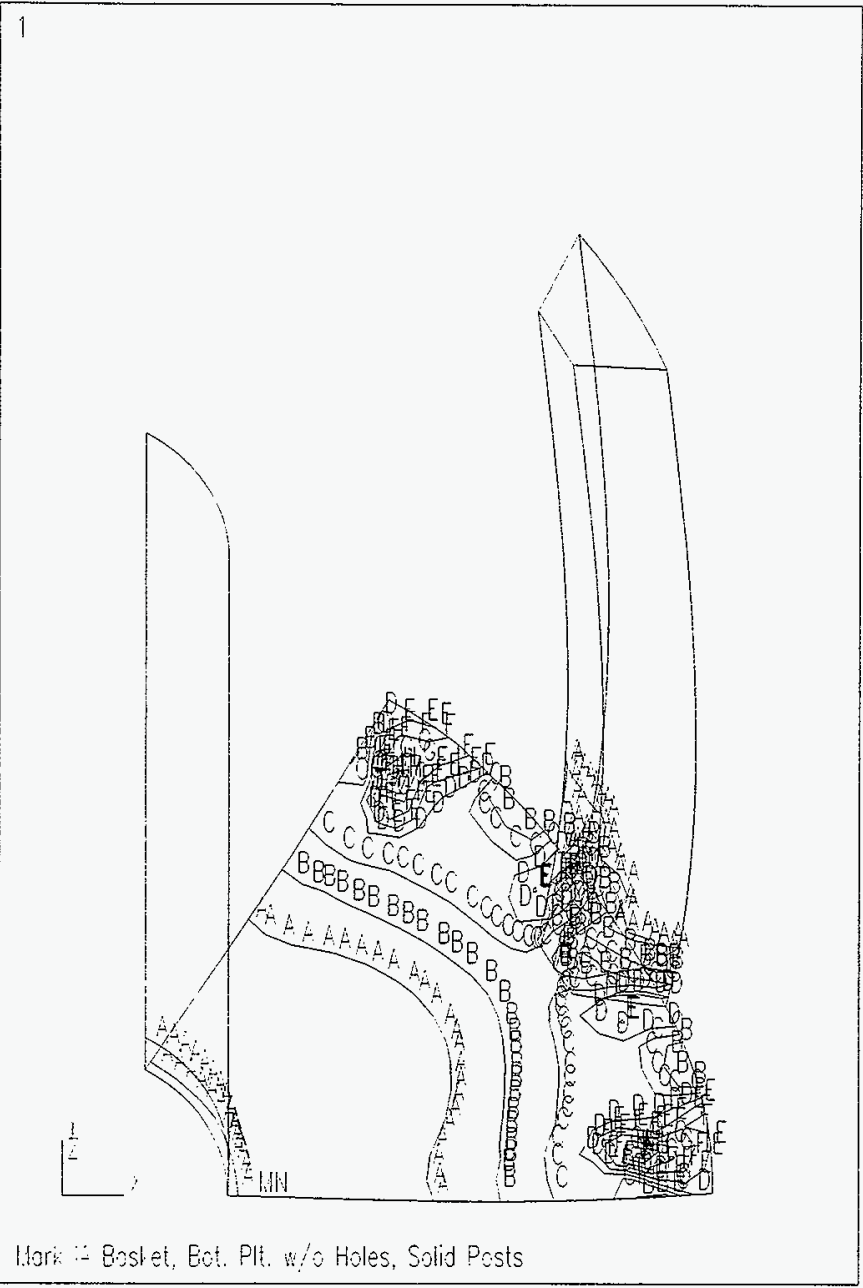

$\therefore 15,550-50$

II:R 9 1097

15.5804

PLOT NO. 2

HOCAL SOLUTIOH

STEP $=1$

SUB $=1$

TIULE $=1$

EPPLEQ (LOO)

EOTTOH:

$01.1 \%=0.0 .0792$

SHXX $=0.009790$

$\therefore \quad=0.544 E-03$

$B=0.001 \hat{6} 33$

C $=0.002721$

D $=0.00581$

$E=0.0048 \div 8$

$F=0,00508 \hat{r}$

$\hat{\sigma}=0.007075$

$H=0.008165$

I $=0.00925 ?$

Figure 9. Plastic Strain Contours, 35g Vertical Drop, No-Hole Model.

\begin{tabular}{|c|l|l|l|}
\hline 0 & & & \\
\hline$S^{4} / / 17 / 97$ & & & \\
\hline$/ N^{4 / 17 / 97}$ & & & \\
\hline
\end{tabular}




\section{F PaAsons}

CLIENT: Duke Engineering \& Services Hanford FILE NO: KH-8009-8-05

PROJECT: \begin{tabular}{lll} 
MCO Final Design & DOC. NO.: & HNF-SD-SNF-DR-003, Rev. 0, Appendix 7 \\
\hline
\end{tabular}

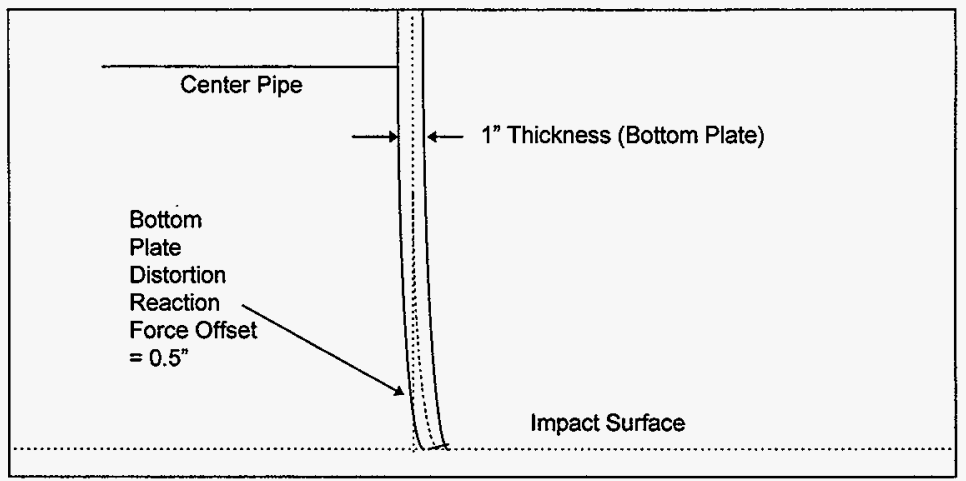

Figure 10. Bottom Plate Distortion Illustration for Horizontal Drop Modeling.

Gap elements (ANSYS CONTAC52) were used to account for the circumferentially varying gap between the outside of the bottom plate and the inside of the MCO (3/8" diameter difference). Assuming the same bilinear plasticity used for the vertical drop analysis above, the elastic/plastic response of the Figure 5 model was predicted. The potential for a basket instability (elastic/plastic buckling) was included in the ANSYS run by activating the large deflection/strain option (NLGEOM,ON command). The g loading was increased to 1.5 times the specified drop loading of $101 \mathrm{~g}$ 's to assure that the ASME Level D buckling requirements are met (Para. F-1331.5(a), loading < 2/3 buckling capacity). An earlier elastic buckling analysis, with no bottom plate offset, indicated that the elastic buckling strength was in excess of $1000 \mathrm{~g}$ 's (stress $>$ yield), which indicates that the actual buckling failure mode is inelastic.

The nonlinear results for the horizontal drop evaluation are summarized in Figure 11 and Figure 12. Note, in Figure 11, that a maxium stress intensity of $30.0 \mathrm{ksi}$ was predicted for the bottom side of the bottom plate. The corresponding maximums for the shell middle and top surfaces are 14.6 and $26.9 \mathrm{ksi}$, respectively. Since the maximum surface stress intensity is more than $50 \%$ higher than the mid-surface results, membrane plus bending stress intensity is the critical value for the horizontal drop. From Table 4, the allowable membrane plus bending stress intensity (plastic analysis) is $52.8 \mathrm{ksi}$, resulting in a ratio of 


\section{PDARSRS}

CLIENT: $\quad$ Duke Engineering \& Services Hanford FILE NO: KH-8009-8-05

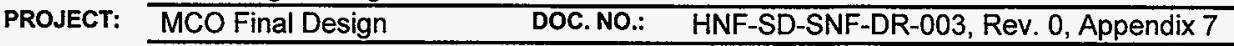

$$
\text { Ratio }=30.0 / 52.8=0.57
$$

Figure 12 provides an indication of the plastic instability response for a horizontal drop. As indicated, a g loading of 1.5 times the $101 \mathrm{~g}$ drop loading $(151.5 \mathrm{~g}$ 's) does not result in an unstable response. Thus, the ASME Level $D$ requirement (load $<2 / 3$ buckling strength) is met.

A criticality control limit in Section 4.19.3 of the Reference 1 Performance Specification, specifies that the center pipe cannot deviate from the MCO centerline by more than 2.00 in. From Figure 11, a maximum horizontal drop displacement of $0.59 \mathrm{in}$. is indicated in the figure legend (101g loading). This maximum displacement occurs at the top of a support rod, which has no criticality concern. From Figure 11, the maximum displacement in the bottom plate is only 0.02 in. for a $101 \mathrm{~g}$ loading. Combining this value with the $3 / 16$-in. radial displacement due to the basket O.D.MCO I.D. difference, results in a deviation of 0.21 in. The resulting allowable ratio is

$$
\text { Ratio }=0.21 / 2.00=0.11
$$


CLIENT: $\quad$ Duke Engineering \& Services Hanford

FILE NO: $\quad \mathrm{KH}-8009-8-05$ PROJECT: MCO Final Design DOC.NO.: HNF-SD-SNF-DR-003, Rev. 0, Appendix 7

1

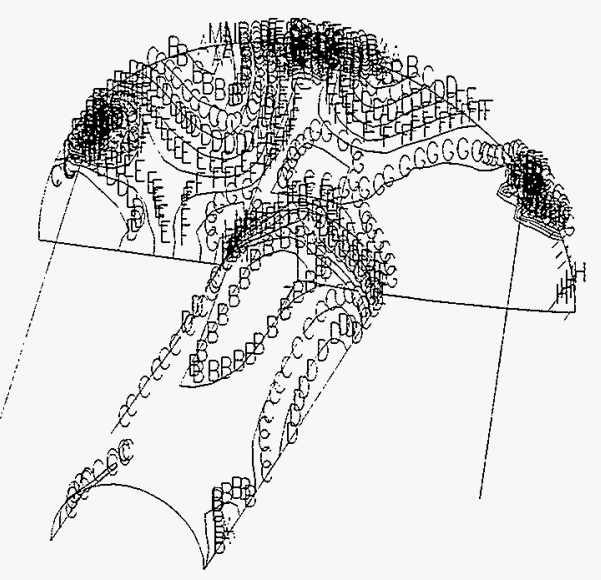

Wiark Boskel loig Side Crop

Figure 11. 101g Horizontal Drop Stress Intensity Contours, Inelastic Analysis.

$-115 i s 5.0 \div 5 \hat{0}$

L.HF 91997

160201

PLOT Ho. 6

NOOAL SOLUTION

STEP $=1$

SUE $=38$

TIH:SE $=1$

SIHT $(A, G)$

BOTTOL:

ONX $=0.59014 t$

St:11 $=1135$

Slid $X=30034$

$\therefore=2739$

$B=5950$

$\hat{\hat{C}}=\hat{y} \mid \hat{c} \hat{1}$

C $=12572$

$E=15584$

$F=18795$

$6=22006$

$H=25217$

I $=28428$

\begin{tabular}{|c|c|c|c|}
\hline 0 & & & \\
\hline S\&/4/17/97 & & & \\
\hline $\ln 4 / 17 / 97$ & & & \\
\hline
\end{tabular}


CLIENT: Duke Engineering \& Services Hanford

FILE NO: KH-8009-8-05

PROJECT: MCO Final Design $\quad$ DOC. NO.: $\quad$ HNF-SD-SNF-DR-003, Rev. 0, Appendix 7

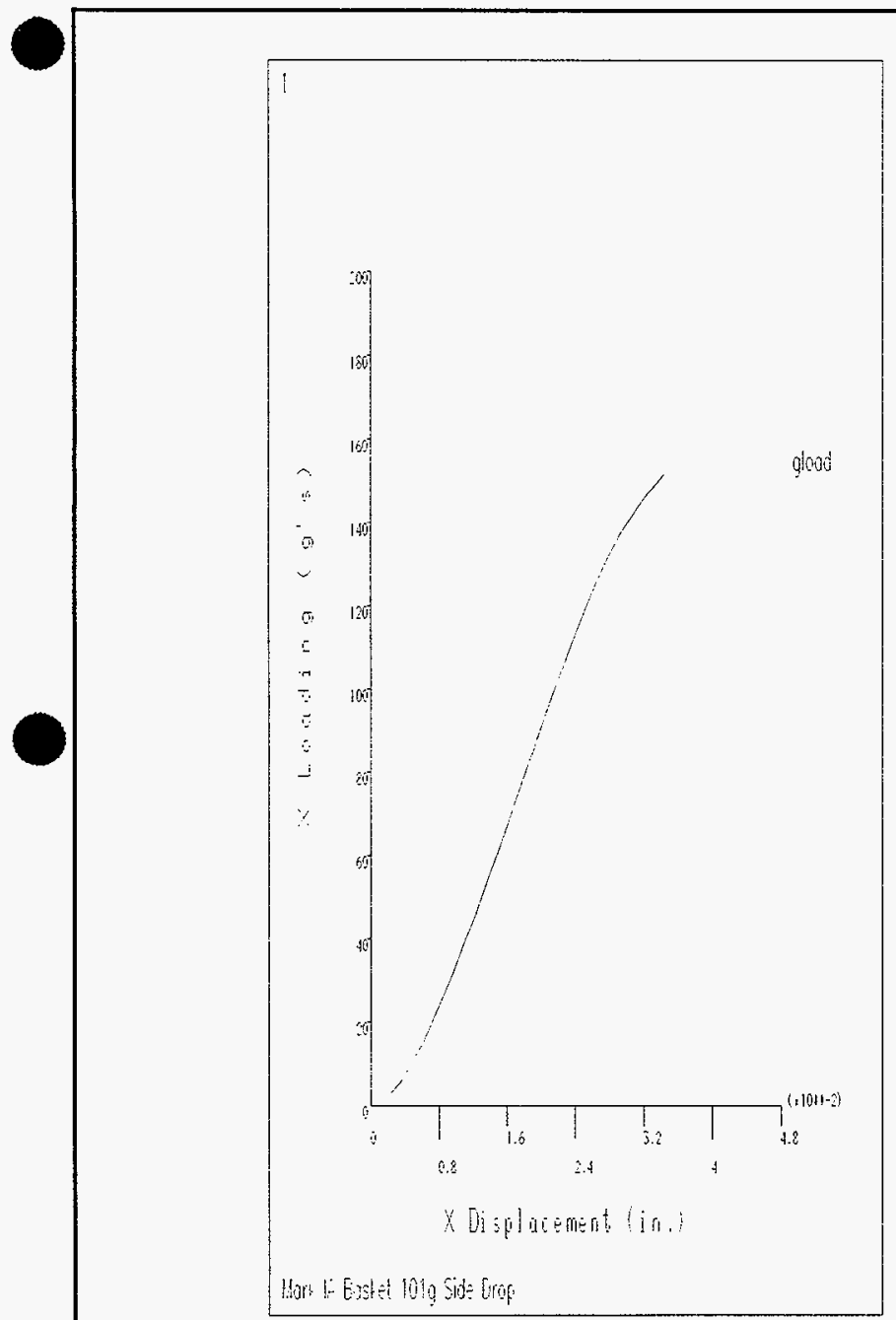

45650450

I. 4 . 9199 :

15.30:10.9.

FLit 4

Pojt?to

$N=i$

$015 \mathrm{~T}=0.75$

$\mathrm{XF}=0.5$

YF $=0.5$

If $=0.5$

YPTO $=0.681818$

PRECTEE HEOCEN

eqise

Figure 12. Horizontal Drop G Load Versus Max. Vertical Displacement, Inelastic Analysis.

$+\frac{1}{1+1}$

$+$


CLIENT: $\quad$ Duke Engineering \& Services Hanford

FILE NO: $\mathrm{KH}-8009-8-05$

PROJECT: $\begin{array}{lll}\text { MCO Final Design } & \text { DOC.NO.: HNF-SD-SNF-DR-003, Rev. 0, Appendix } 7\end{array}$

\subsection{Scrap Basket Shroud}

The scrap basket shroud extends the full height of the basket and is used to contain the scrap pieces. The scrap pieces vary in size and shape, and the resulting pressure will vary significantly. A reasonable estimate of the scrap pressure can be obtained by considering wall pressures associated with wall pressures associated with angular rock (limestone, iron ore, etc). The scrap pressure during drop loading was not considered, since it is assumed that the drop loading scrap pressure is carried by the MCO wall (see Appendix 5). The following evaluation of the wall pressure and corresponding structural evaluation of the shroud was performed on Mathcad and inserted into the report.

Assume that the scrap weight is equal to 48 fuel assemblies @ $39.7 \mathrm{lb}$.

$$
\begin{array}{ll}
\mathrm{W}_{\mathrm{s}}:=48 \cdot 39.7 \cdot \mathrm{lb} & \\
\mathrm{W}_{\mathrm{s}}=1.906 \cdot 10^{3} \cdot \mathrm{lb} & \\
\mathrm{h}_{\mathrm{s}}=22.00 \cdot \mathrm{in} & \text { Shroud Height } \\
\mathrm{d}_{\mathrm{s}}=22.525 \cdot \mathrm{in} & \text { Shroud Inside Radius } \\
\mathrm{t}_{\mathrm{s}}=0.048 \cdot \mathrm{in} & \text { Shroud Nominal Thickness } \\
\mathrm{V}_{\mathrm{s}}:=\frac{\pi}{4} \cdot\left(\mathrm{d}_{\mathrm{s}}\right)^{2} \cdot \mathrm{h}_{\mathrm{s}} & \\
\mathrm{v}_{\mathrm{s}}=8.767 \cdot 10^{3} \cdot \mathrm{in}^{3} & \text { Scrap Volume } \\
\delta_{\mathrm{s}}=\frac{\mathrm{W}_{\mathrm{s}}}{\mathrm{V}_{\mathrm{s}}} & \\
\delta_{\mathrm{s}}=0.217 \cdot \frac{\mathrm{lb}}{\mathrm{in}^{3}} & \text { Scrap Weight Density }
\end{array}
$$

\begin{tabular}{|l|l|l|}
\hline & & \\
\hline & & \\
\hline
\end{tabular}


CLIENT:

CLIENT: Duke Engineering \& Services Hanford FILE NO: KH-8009-8-05

PROJECT: MCO Final Design $\quad$ DOC. NO.: $\quad$ HNF-SD-SNF-DR-003, Rev. 0, Appendix 7

Assume a reasonable equivalent fluid pressure coefficient:

$$
\begin{aligned}
& \phi:=33 \cdot \operatorname{deg} \quad \begin{array}{l}
\text { Minimum of iron ore/coal/lime angle of } \\
\text { repose with 2 degree uncertainty, } \\
\text { Bowles (Ref. 11), Table 11-8 }
\end{array} \\
& \mathrm{K}_{\mathrm{a}}=\frac{1-\sqrt{1-\cos (\phi)^{2}}}{1+\sqrt{1-\cos (\phi)^{2}}} \quad \text { Bowles, Eq. 11-7a, Rankine pressure coeficient } \\
& \mathrm{K}_{\mathrm{a}}=0.295 \\
& \mathrm{~K}_{\mathrm{a}}=0.3 \\
& \mathrm{p}_{\text {base }}=\mathrm{K} \cdot \mathrm{a}^{\cdot \delta} \mathrm{s}^{\cdot \mathrm{h}_{\mathrm{s}}}
\end{aligned}
$$

Check maximum membrane pressure stress in the sheet metal shroud (lifting load condition):

$$
\begin{aligned}
& P_{m}=p_{\text {base }} \cdot \frac{d_{s}}{2 \cdot t_{s}} \\
& P_{m}=336.61 \cdot \frac{\mathrm{lb}}{\text { in }^{2}} \\
& \text { Ratio }=\frac{P_{m}}{\frac{S y}{3}}
\end{aligned}
$$

Ratio $=0.048$

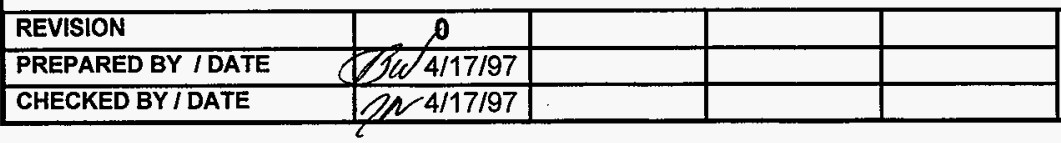


CLIENT: Duke Engineering \& Services Hanford FILE NO: KH-8009-8-05

PROJECT: MCO Final Design $\quad$ DOC. NO.: $\quad$ HNF-SD-SNF-DR-003, Rev. 0, Appendix 7

Check Shroud Welds (size corresponds to shroud thickness of $0.048^{\prime \prime}$ ):

1) Vertical Seam Weld (2" long welds @ 4" centers, intermittent)

$\mathrm{n}=0.3 \quad$ Weld quality factor, Table NG-3352-1, conservatively use "intermittent fillet or plug" weld with surface visual exam

$$
\text { Ratio }=\frac{2 \cdot \mathrm{P}_{\mathrm{m}}}{\mathrm{n} \cdot\left(\frac{\mathrm{s}_{\mathrm{y}}}{3}\right)}
$$

Ratio $=\mathbf{0 . 3 2} \quad$ Vertical Seam Weld O.K.

2) Shroud-to-Bottom-Plate Weld (2" @ 4" centers)

The shroud-to-bottom plate welds have no structural function because the pressure induced by the scrap is carried by the hoop strength of the shroud. Since there is no significant vertical uplift load on the shroud, the weld has no significant structural function and was not evaluated.

As indicated, the shroud and shroud welds are adequate. Although there are significant uncertainties in the load magnitude (pressure coefficient), the design margins are relatively large and no further refinement is warranted.

\subsection{Load Distribution and Basket Interface Considerations}

When the baskets are stacked inside the MCO, the center pipe and support rod load distribution is very sensitive to the interface geometry. Reasonable fabrication tolerances will result in an imperfect fit, which will likely result in a three-point contact at the basket interface (three rods or two rods and the center pipe). There is also the possibility that the center pipe will carry the entire load. 


\section{PaAsons}

CLIENT: Duke Engineering \& Services Hanford FILE NO: KH-8009-8-05

PROJECT: MCO Final Design DOC.NO.: HNF-SD-SNF-DR-003, Rev. 0, Appendix 7

Even for the case of a perfect fit, the stack loading will not be evenly shared between the center pipe and rods. Using the Figure 3 ANSYS model, the "perfect fit" load distribution was estimated by applying the $35 \mathrm{~g}$ fuel pressure to the bottom plate, and obtaining the reactions at the support rod and center pipe (ANSYS files: pltnhld.inp and pltnhld.out). From the ANSYS output, the support rod and center pipe reactions were $7226 \mathrm{lb}$ and 5166 $\mathrm{lb}$, respectively $(12,392 \mathrm{lb}$ total). The center pipe load ratio is $5166 / 12,392=0.42$.

However, for the reasonable fabrication tolerances discussed below, it is possible for the center pipe load ratio to range from zero to one, during normal stacking. As demonstrated below, for the drop condition, distortion/load redistribution will occur, and the 0.42 ratio can be used as a reasonable estimate of the center pipe load ratio.

In order to establish reasonable fabrication tolerances on the basket interface geometry, capacity force/deflection response predictions were made for the basket support rods. By knowing the force/deflection response, the effect of component length differences on the component load sharing can be evaluated. The capacity force/deflection response was obtained using the ANSYS plastic beam element (BEAM23), with large deflections/strain enabled. Buckling was initiated by assuming a 0.25 -in. offset of the vertical load. This load offset assumption is reasonable for the Mark $1 \mathrm{~A}$ support rods due to a 1/4-in. chamfer on the outside of the bottom of the basket, resulting in the basket load being biased to the inside of the rod.

The model consisted of two support rods, each having 20 BEAM23 elements. One of the rods was 0.030 -in. shorter than the other. By using gap/friction elements (CONTAC12) between the loaded node and the top of each support rod, the shorter rod would not begin to be loaded until the longer rod had experienced a 0.030 -in. vertical deformation. To enhance solution stability, the loading was applied as a vertical deformation, with a total deformation of 0.100 in. By multiplying the rod responses by a factor of 3.0 , the conservative case of three equal-length rods with three 0.030 -in shorter equal-length rods could be evaluated (no support from the center pipe). The purpose of the evaluation was to investigate the load capacity of the rods for relatively large vertical deformations.

The support rod deformation predictions for a vertical deformation load of 0.100 -in are shown in Figure 13 (ANSYS input/output files: Rodb2.inp/Rodb2.out) Note that a maximum horizontal deformation of 0.339 -in. was predicted for the longer rod. The support rod force/deflection prediction is shown in Figure 14 (3fy1 curve). Note that very little support rod capacity losses are predicted for a vertical deformation of 0.100 -in. Also note that the six-rod capacity is insufficient to carry the bottom basket $35 \mathrm{~g}$ loading. The top horizontal line shows the ASME Code Level D combined capacity of the center pipe and six support rods. Thus, this analysis indicates that relatively loose fabrication tolerances are possible. However, to account for analysis uncertainties, it is recommended that a maximum range of 0.030 -in. be imposed on support rod and support rod/center pipe fabrication length differences.

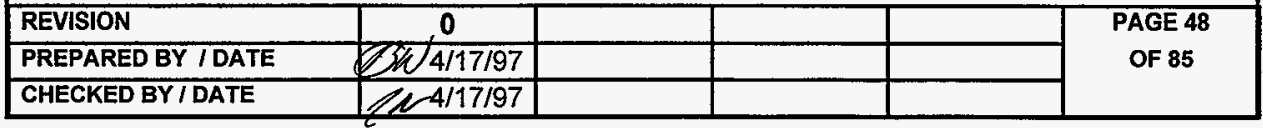


CLIENT: $\quad$ Duke Engineering \& Services Hanford FILE NO: KH-8009-8-05

PROJECT: MCO Final Design DOC. NO.: HNF-SD-SNF-DR-003, Rev. 0, Appendix 7

Another support rod design issue is the need for horizontal constraints at the top of the support rods. In the analysis described above, a friction coefficient of 0.10 was assumed, with no slippage predicted. From Table 1 of Section 3 of Mark's Handbook, the lowest steel-to-steel dynamic friction coefficient shown is 0.42 , indicating that friction is adequate to constrain the top of the support rods.

As mentioned above, the basket interface results in a support rod load offset which will bias the buckling to be radially outward in direction. This "preferential buckling" towards the inside wall of the MCO would enhance the support rod buckling strength. That is, as the support rods bows outward, the MCO will prevent collapse until a higher buckling mode is achieved. By using gap elements to represent the space between the support rod and the inside of the MCO, this buckling strength enhancement was evaluated with an ANSYS run. A buckling strength increase of about $50 \%$ was predicted. This buckling strength enhancement is supplemental in nature and is not needed for design verification. Therefore, the ANSYS input/output files are not included with this report.

+




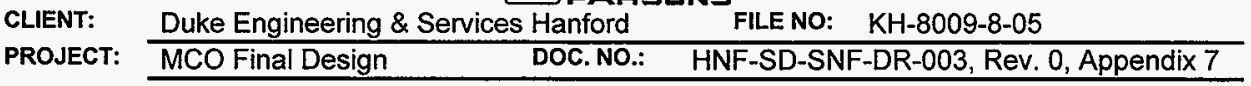
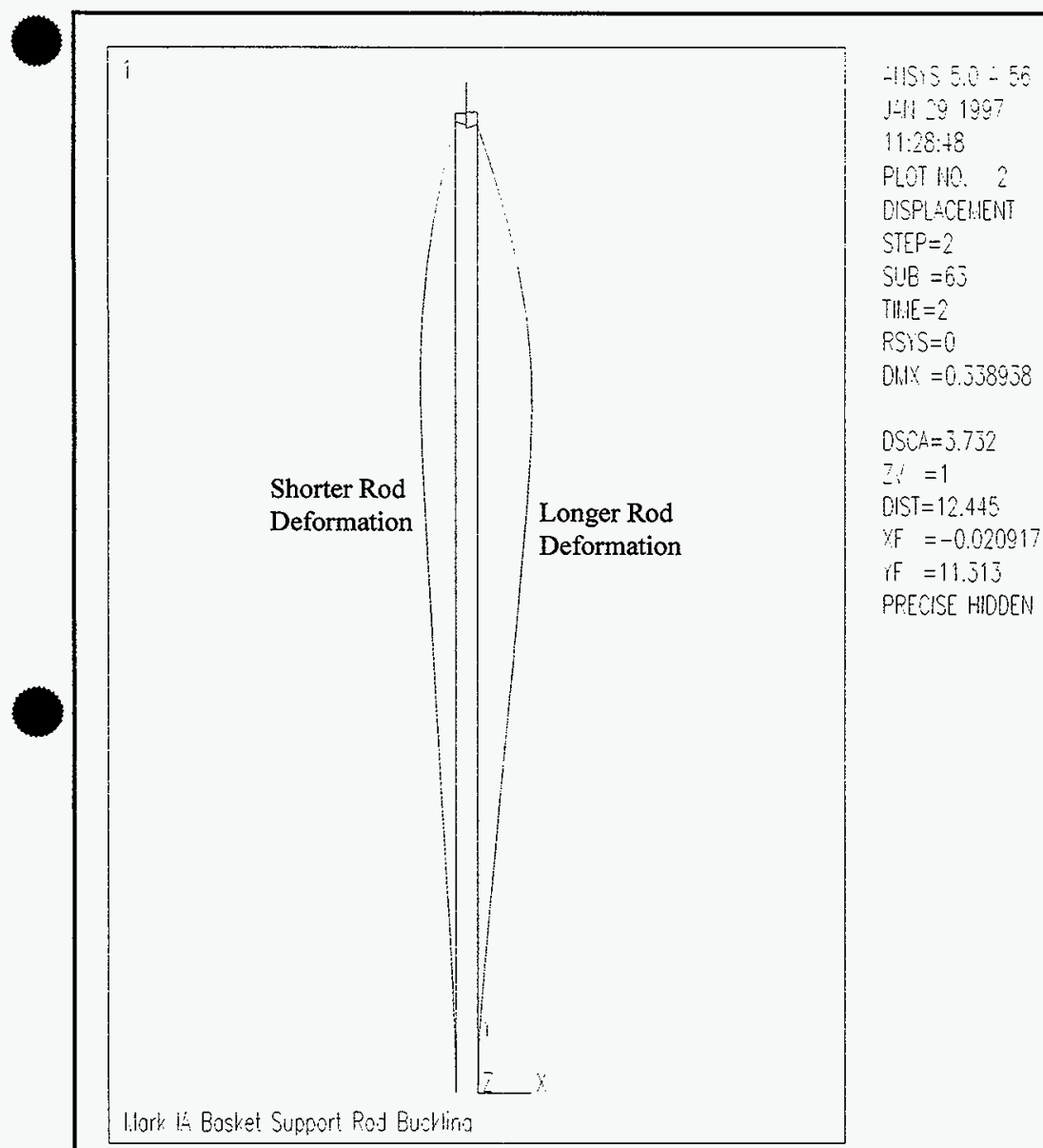

Figure 13. Support Rod Deformed Shape Predictions, 0.030" Vertical Deflection.

\begin{tabular}{l|l}
\hline 0 & \\
\hline $4 / 17 / 97$ & \\
\hline $4 / 17 / 97$ & \\
\hline
\end{tabular}


$\square$ PARSロNS

\begin{tabular}{llll} 
CLIENT: & \multicolumn{1}{l}{ Duke Engineering \& Services Hanford } & FILE NO: & KH-8009-8-05 \\
\cline { 2 - 3 } PROJECT: & MCO Final Design & DOC. NO.: & HNF-SD-SNF-DR-003, Rev. 0, Appendix 7 \\
\cline { 2 - 3 }
\end{tabular}

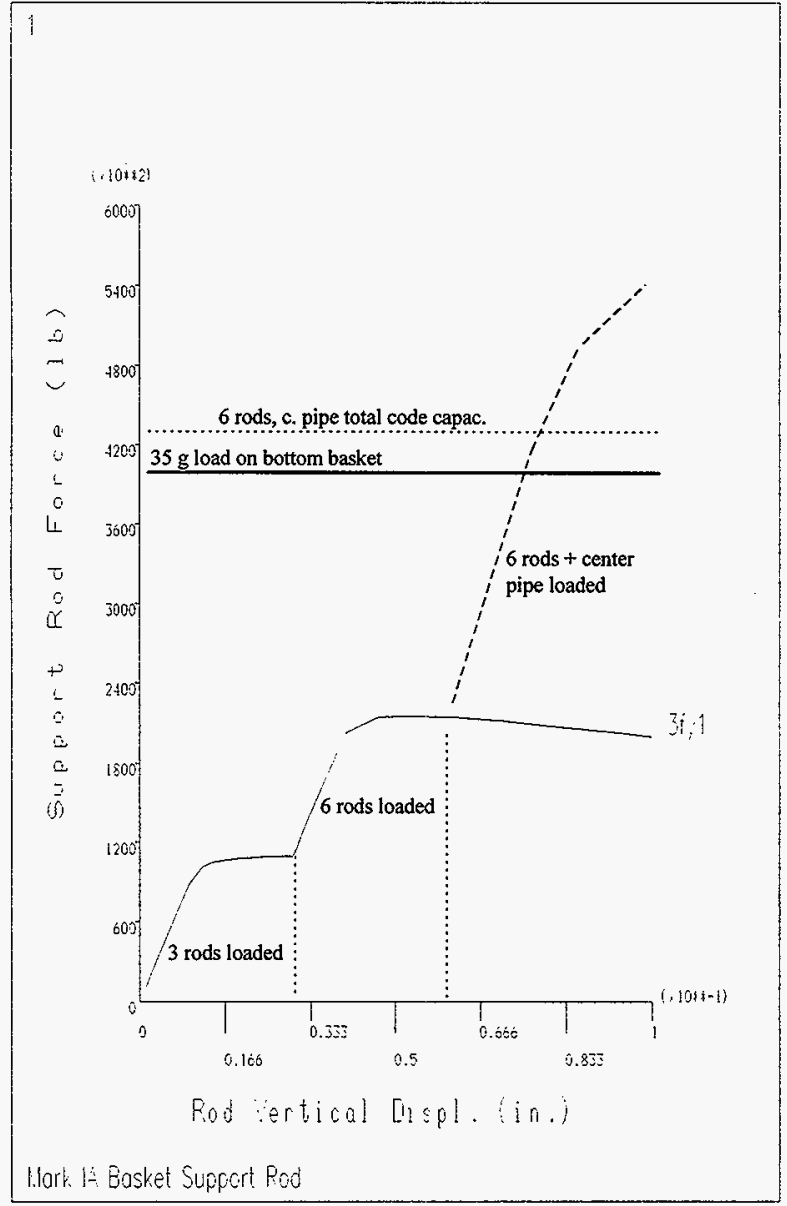

Figure 14. Deformation Response of Mark 1A Basket Support Rods.

$\because 15=500$

$1,1: 29$

11.260

Floitho.

POST?

$\because=1$

$\mathrm{OST}=0.7$

if

if

if

$X$ PTO $=0.68181$

PREQISE 


\section{(P)}

CLIENT: Duke Engineering \& Services Hanford FILE NO: KH-8009-8-05

PROJECT: MCO Final Design $\quad$ DOC. NO.: HNF-SD-SNF-DR-003, Rev. 0, Appendix 7

\subsection{Component Stress Results Summary}

From the calculations above, a summary of the component stress analysis results was compiled into Table 6 . Note that the predicted maximums are below allowables for all components and conditions. As indicated in Section 6.3, for the maximum drop temperatures from the current revision of the Reference 1 Performance Specification, the drop condition allowable stresses are conservative (approximately 5 to 14\%).

Table 6. Summary of Mark 1A Storage Basket Stress Results

\begin{tabular}{|c|c|c|c|c|c|}
\hline Component & $\begin{array}{l}\text { Critical Load } \\
\text { Condition }\end{array}$ & $\begin{array}{l}\text { Stress } \\
\text { Category }\end{array}$ & $\begin{array}{l}\text { Predicted } \\
\text { Maximum }\end{array}$ & Allowable & Ratio \\
\hline Center Pipe & $\begin{array}{c}35 g \text { Vertical } \\
\text { Drop }\end{array}$ & Buckling & $14,111 \mathrm{psi}^{\mathrm{t}}$ & $14,699 \mathrm{psi}$ & $0.92^{1}$ \\
\hline $\begin{array}{c}\text { Center Pipe } \\
\text { Criticality } \\
\text { Control }\end{array}$ & $\begin{array}{c}\text { Sequential } \\
35 \mathrm{~g} \text { Vertical } \& \\
101 \mathrm{~g} \text { Horiz. } \\
\text { Drops }\end{array}$ & $\begin{array}{c}\text { Plastic } \\
\text { Distortion \& } \\
\text { Potential } \\
\text { Instability }\end{array}$ & $\begin{array}{l}0.28 \text { in. radial } \\
\text { displacement }\end{array}$ & 2.00 in. & 0.14 \\
\hline $\begin{array}{l}\text { C. Pipe/B. } \\
\text { Plate Weld }\end{array}$ & $\begin{array}{l}101 g \text { Horiz. } \\
\text { Drop }\end{array}$ & $\mathrm{Pm}$ & 9545 psi & 13,400 psi & 0.71 \\
\hline $\begin{array}{l}\text { Center } \\
\text { Coupling }\end{array}$ & $\begin{array}{l}101 \mathrm{~g} \text { Horiz. } \\
\text { Drop }\end{array}$ & Shear & 6640 psi & 24,696 psi & 0.27 \\
\hline $\begin{array}{c}\text { Center } \\
\text { Coupling Pins }\end{array}$ & Lifting & Shear & $2350 \mathrm{lb}$ & $10,000 \mathrm{lb}$ & 0.24 \\
\hline $\begin{array}{c}\text { Center } \\
\text { Bushing Pin }\end{array}$ & Lifting & Shear & 4.04 & 2500 & 0.002 \\
\hline Support Rods & $\begin{array}{l}35 \mathrm{~g} \text { Vertical } \\
\text { Drop }\end{array}$ & Buckling & $11,110 \mathrm{psi}^{1}$ & 11,573 psi & $0.92^{t}$ \\
\hline Bottom Plate & $\begin{array}{l}35 g \text { Vertical } \\
\text { Drop }\end{array}$ & $\mathrm{Pm}$ & 19,600 & 41,100 & 0.48 \\
\hline
\end{tabular}

1 Based upon combined capacity of center pipe and support rods. See conclusion to Section 8.2. 
CLIENT: $\quad$ Duke Engineering \& Services Hanford

FILE NO: $\quad \mathrm{KH}-8009-8-05$

PROJECT: MCO Final Design DOC. NO.: HNF-SD-SNF-DR-003, Rev. 0, Appendix 7

\section{BASKET/MCO INTERFACE COMPONENTS}

An important issue in the Section 8.3.4 horizontal drop evaluation is the assumption that the top end of each Mark 1A basket (center post) remains inside, i.e. overlaps, the mating component during the drop. This assumption is vital to the horizontal drop calculations because the finite element analysis assumed a transverse support for the top end of the center pipe. Also, Section 4.19.3 of the Reference 1 Performance Specification (criticality control) requires that the center pipe cannot deviate by more than two inches from the MCO. centerline. This section provides justification for the assumption that the Mark 1A baskets will not come apart at the interfaces.

The basket/MCO interfaces were designed to assure that, during normal operations, the center coupling maintains at least a one-inch overlap with the shield plug assembly. During a vertical drop, if the "MCO Basket Support Plates" (Drawing No. H-2-828053) collapse, the center coupling overlap may be lost. Therefore, the basket support plates were evaluated to assure that the ASME Code Level D axial compression limits are met for a vertical drop. The Mathcad evaluation follows below:

\section{MATHCAD SUPPORT PLATES CALCULATIONS}

Support Plate Dimensions (six radial spokes @ 60 deg. intervals):

$$
\begin{array}{ll}
{ }^{t_{p}}=0.5 \cdot \text { in } & \text { Support Plate Width } \\
{ }^{l_{p}}=9.71 \cdot \mathrm{in} & \text { Support Plate Length } \\
\mathrm{h}_{\mathrm{p}}:=1.49 \cdot \mathrm{in} & \text { Support Plate Height }
\end{array}
$$

Per inch length of plate:

$$
\begin{aligned}
& I:=\frac{1 \cdot \operatorname{inr}\left(\mathrm{p}^{3}\right.}{12} \\
& \mathrm{I}=0.01 \cdot \mathrm{in}^{4} \\
& \mathrm{r}:=\sqrt{\frac{\mathrm{I}}{1 \cdot \mathrm{int} \mathrm{p}}} \\
& \mathrm{r}=0.144 \cdot \text { in }
\end{aligned}
$$

\begin{tabular}{|c|l|l|l|}
\hline 0 & & & \\
\hline per/17/97 & & & \\
\hline $\ln 4 / 17 / 97$ & & & \\
\hline
\end{tabular}


\begin{tabular}{llll} 
CLIENT: & \multicolumn{1}{l}{ Duke Engineering \& Services Hanford } & FILE NO: & KH-8009-8-05 \\
\cline { 2 - 3 } PROJECT: & MCO Final Design & DOC. NO.: & HNF-SD-SNF-DR-003, Rev. 0, Appendix 7 \\
\cline { 2 - 3 }
\end{tabular}

$\mathrm{K}=2.1 \quad$ Effective length, fixed/free (Ref.: AISC)

$\mathrm{S}_{\mathrm{y}}:=19760 \cdot \frac{\mathrm{lb}}{\mathrm{in}^{2}} \quad 304 \mathrm{~L}$ yield stress @ max. drop temp. of $132 \mathrm{deg} \mathrm{C}$

$\mathrm{E}:=27.2 \cdot 10^{6} \cdot \frac{\mathrm{lb}}{\mathrm{in}^{2}} \quad 304 \mathrm{~L}$ Young's Modulus @ max. drop temp.

ASME F-1334.3 Axial Compression Evaluation:

$$
\begin{aligned}
& \lambda=\left(\frac{\mathrm{K} \cdot \mathrm{h} p}{\mathrm{r}}\right) \cdot \frac{1}{\pi} \cdot \sqrt{\frac{\mathrm{S} y}{\mathrm{E}}} \\
& \begin{array}{l}
\lambda=0.186 \quad 0<\lambda<1,1 \mathrm{st} \mathrm{Eq.} \mathrm{Applies} \\
\sigma_{\text {allow }}=\mathrm{S}_{\mathrm{y}} \cdot \frac{\lambda^{2}}{1.11+0.50 \cdot \lambda+0.17 \cdot \lambda^{2}-0.28 \cdot \lambda^{3}}
\end{array} \\
& \sigma_{\text {allow }}=1.623 \cdot 10^{4} \cdot \frac{\mathrm{lb}}{\mathrm{in}^{2}}
\end{aligned}
$$

The maximum vertical $\mathrm{g}$ level for a sequential drop is $32.9 \mathrm{~g}$ 's:

$$
\begin{aligned}
& W_{b}:=2350 \cdot l b \quad \text { Upper Bound Basket Weight } \\
& \sigma:=\frac{6 \cdot W_{b} \cdot 27}{6 \cdot 1 p^{\cdot t} p} \\
& \sigma=1.592 \cdot 10^{4} \cdot \frac{\mathrm{lb}}{\text { in }^{2}}
\end{aligned}
$$

$\mid \begin{gathered}0 \\ \mid \operatorname{los} 4 / 17 / 97 \\ \ln 4 / 17 / 97\end{gathered}$


CLIENT: Duke Engineering \& Services Hanford

FILE NO: $\quad \mathrm{KH}-8009-8-05$

\begin{tabular}{llll} 
PROJECT: & MCO Final Design & DOC. NO.: & HNF-SD-SNF-DR-003, Rev. 0, Appendix 7 \\
\hline
\end{tabular}

$$
\text { Ratio }=\frac{\sigma}{\sigma_{\text {allow }}}
$$

Ratio $=0.981 \quad$ Basket Support Plate OK

Since the safety margin is small, it is noted that the results are conservative in that no credit is taken for either the vertical weld on the inside end of the plate or the lateral resistance for friction.

\section{Check Support Plate Welds:}

$$
\begin{aligned}
& f_{p}=\sigma \cdot t_{p} \\
& f_{p}=7.685 \cdot 10^{3} \cdot \frac{\mathrm{lb}}{\text { in }} \quad \text { Support plate drop loading/inch }
\end{aligned}
$$

Assume a maximum load offset $=1 / 2$ of plate thickness:

$$
\begin{aligned}
& M_{p}=f_{p} \cdot \frac{t_{p}}{2} \\
& M_{p}=1.921 \cdot 10^{3} \cdot l b \cdot \frac{\text { in }}{\text { in }}
\end{aligned}
$$

The $3 / 8^{\prime \prime}$ weld stress in the throat is

$$
\begin{aligned}
& \sigma_{\mathrm{w}}=\frac{\mathrm{M}_{\mathrm{p}}}{\mathrm{t} \mathrm{p} \cdot \frac{\sqrt{2}}{2} \cdot \frac{3}{8} \cdot \mathrm{in}} \\
& \sigma_{\mathrm{w}}=1.449 \cdot 10^{4} \cdot \frac{\mathrm{lb}}{\mathrm{in}^{2}}
\end{aligned}
$$

From Section 6.4, the allowable double fillet weld drop accident stress is $15.3 \mathrm{ksi}$, resulting in the following stress ratio: 
CLIENT: $\quad$ Duke Engineering \& Services Hanford FILE NO: KH-8009-8-05

PROJECT: MCO Final Design DOC. NO.: HNF-SD-SNF-DR-003, Rev. 0, Appendix 7

$$
\text { Ratio }=\frac{\sigma_{\mathrm{w}}}{15300 \cdot \frac{\mathrm{lb}}{\mathrm{in}^{2}}}
$$

Ratio $=0.947$

Thus, the basket support plates and attachment welds meet the ASME Level D compression load requirements, and the overlapping (telescoping) of the baskets is not jeopardized.

At the top end of the basket stack, the basket interfaces with the bottom of the shield plug assembly (specifically, the basket stabilizer, shield ring, and shield plate, part nos. 21, 15, and 14 of Drawing H-2-828041). For a top end drop, the basket loading would be reacted by the one-inch thick shield ring $\left(\right.$ area $\left.=67.5 \mathrm{in}^{2}\right)$ and the basket stabilizer. The shield ring area alone is more than double the bottom basket interface support plate area. Thus, the top axial support is adequate by comparison to the bottom basket interface.

For a horizontal drop, the top basket relies upon the shield plate for transverse support. For normal conditions, the shield plate position is maintained through a weld connection to the shield ring which in turn is welded to the shield plug. If either or both of these welds should fail during a horizontal drop, the shield plate would be captured between the top basket and shield plug and would continue to support the top end of the basket. Therefore, the maximum center pipe movement would be the 0.25 -inch difference between the inside radius of the $\mathrm{MCO}$ and the outside radius of the shield ring, which is well below the two-inch Performance Specification limit (Reference 1, Section 4.19.3).

\begin{tabular}{|l|c|l|l|l|l|}
\hline REVISION & 0 & & & & PAGE 56 \\
OF 85 \\
\cline { 1 - 4 } CHECKERED BY I DATE & $3 \mathrm{~W} / 1 / 17 / 97$ & & & & \\
\hline
\end{tabular}


CLIENT:

CLIENT: Duke Engineering \& Services Hanford FILE NO: KH-8009-8-05

PROJECT: MCO Final Design $\quad$ DOC.NO.: HNF-SD-SNF-DR-003, Rev. 0, Appendix 7

\author{
APPENDIX A
}

COMPUTER RUN OUTPUT SHEETS

AND

INPUT FILE LISTINGS 


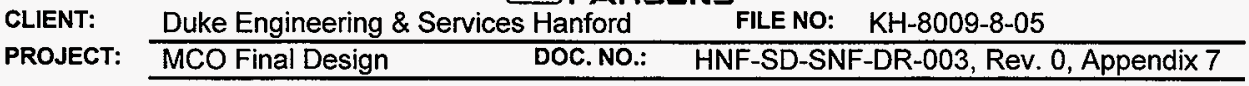

\section{COMPUTER RUN COVER SHEET}

Project Number:

$\mathrm{KH}-8009-8$

Computer Code:

ANSYS®-PC

Software Version:

$5.0 \mathrm{~A}$

Computer System:

MS-DOS, Pentium® Processor

Computer Run File Number:

$\mathrm{KH}-8009-8-05$

Unique Computer Run File Name:

Plth.inp

Run Description:

Elastic Drop Analysis of the Mark IA Storage Basket, Holes in Bottom Plate

Creation Date/Time:

15 January 1997/1:09:36pm

\section{Coob.c. Wiblal}

Prepared By: Bob V. Winkel

\section{Date}

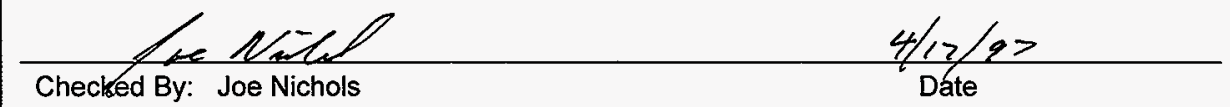

$\mid \begin{gathered}0 \\ 24 / 4 / 17 / 97 \\ 2 / 17 / 97\end{gathered}$




PROJECT: MCO Final Design DOC. NO.: HNF-SD-SNF-DR-003, Rev. 0, Appendix 7

\section{LISTING OF PLTH.INP FILE}

/batch, list

/filenam, plth

/prep7

/title, Mark IA Basket, Solid Bot. PIt. w/ 1/4" Flow Holes

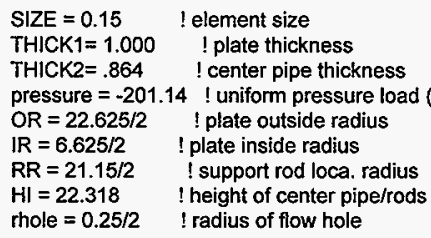

r, 1, THICK1

$r, 2$, THICK2

$r, 4,1 \mathrm{e} 6,0,2$

dens, $1, .2854$

ex, $1,26.5 E+06$

nuxy, 1,3

dens, 2,0 .

ex $2,30 . e 6$

nuxy, 2,3

/com define bottom plate

csys,1 ! global cylindrical
$k, 15,0,0$
$\mathrm{k}, 1, \mathrm{IR}, 0$
$k, 2,6.5,0$
k, 3,8.5,0
$k, 4,10.5,0$
$\mathrm{k}, 5, \mathrm{OR}, 0$
k,6,OR,30
k,7,9.868,30
$k, 8,9.093,30$
$k, 9,7.361,30$
$k, 10,5.629,30$
$k, 11,1 R, 30$
$k, 12$, OR,22.201
$k, 13,9.887,26.463$

$\begin{array}{ll}1,1,2 & \text { ! line 1 } \\ 1,2,3 & \text { ! line 2 } \\ 1,3,4 & \text { ! line 3 } \\ 1,4,5 & \text { ! line 4 }\end{array}$


larc,5,12,15, OR ! line 5

I,6,7

lesize,6,, 4

$1,7,8$

$1,8,9$

$1,9,10$

$1,10,11$

larc, $1,11,15, \mathrm{IR}$ ! line 11

lesize, $11,5 \quad$ I 5 degree segments for inside arc

csys, 0

$1,2,10 \quad$ ! line 12

$1,3,9$

$1,4,8$

! line 14

$1,7,13$

lesize, $15,, 3$

$\mathrm{I}, 13,12 \quad$ ! line 16

lesize, $16, . .4$

csys, 1

$1,12,6 \quad$ ! line 17

lesize, $17, \ldots 3$

/com bottom plate areas

al, 1,12,10,11 !A1

al, $2,13,9,12$

al, $3,14,8,13$

al, $4,5,16,15,7,14$ !A4

/com define center pipe

$\mathrm{k}, 20,0,0, \mathrm{HI}$

$k, 21, I R, 0, H I$

$\mathrm{k}, 25, \mid \mathrm{R}, 30, \mathrm{HI}$

larc, $25,21,20, \mathbb{R}$ ! line 18

lesize, 18,5 ! 5 degree segments for inside arc

$\mathbf{I , 1 , 2 1}$ ! vertical line 19 at 0 degrees

$1,11,25$ ! vertical line 20 at 30 degrees

/com area $5=$ center pipe

al, $18,19,20,11$

/com create sup. rod area \& ext. into solid eles.

al $6,15,16,17$ ! A6

/com define holes in bottom plate

boptn,yes ! to save holes

*afun,deg

${ }^{*}$ do, $, \mathrm{i}, 4,11$ ! Row 1 holes, areas 7 - 14

$\mathrm{xh}=\mathrm{i}$

$\mathrm{yh}=0$

wplane, $x h, y h$

pcirc,rhole

"enddo

*do,i,4,11 ! Row 2 holes, areas $15-22$

$\mathrm{xh}=\mathrm{i}-0.5$

$\mathrm{yh}=\cos (30)$

wplane, $x h, y h$

pcirc, rhole

*enddo

REVISION

PREPARED BY I DATE

\begin{tabular}{l}
0 \\
$4 / 17 / 97$ \\
\hline
\end{tabular}

PAGE 60 
CLIENT: DARSENS \begin{tabular}{llll} 
PROJECT: & \multicolumn{1}{l}{ Duke Engineering \& Services Hanford } & FILE NO: $\quad$ KH-8009-8-05 \\
\cline { 2 - 3 } & MCO Final Design & DOC. NO.: & HNF-SD-SNF-DR-003, Rev. 0, Appendix 7
\end{tabular}

*do,i,4,10 ! Row 3 holes, areas $23-29$

$\mathrm{xh}=\mathrm{i}$

yh $=2^{*} \cos (30)$

wplane, ,xh,yh

pcirc, ihole

"enddo

*do,i,5, 11 ! Row 4 holes, areas $30-36$

$\mathrm{xh}=\mathrm{i}-.5$

yh $=3^{*} \cos (30)$

wplane, $x h, y h$

pcirc, rhole

*endido

*do,i,6,10 ! Row 5 holes, areas $37-41$

$\mathrm{xh}=\mathrm{i}$

$y h=4^{*} \cos (30)$

wplane, $x h, y h$

pcirc, ihole

*enddo

*do,i,8,9 ! Row 6 holes, areas 42 - 43

$\mathrm{xh}=\mathrm{i}-0.5$

$y h=5^{*} \cos (30)$

wplane, $x h, y h$

pcirc,rhole

*enddo

aplot,all

/com subtract holes from bottom plate

Icom A1

asba, 1,7 ! A1 - A6 -> A44, etc

asba, 44,8

asba, 45,9

asba, 46,15

asba, 47,16

asba, 48,17

asba, 49,23

asba, 50,24

asba,51,30 ! A52

lcom A2

asba,2,10 ! A53

asba,53,11

asba, 54,18

asba, 55,19

asba,56,25

asba, 57,26

asba,58,31

asba, 59,32

asba,60,37! $\mathrm{A61}$

/com A3

asba,3,12 ! A62

asba, 62,13

asba, 63,20

asba,64,21

asba, 65,27

asba, 66,28

asba, 67,33

asba, 68,34

REVISION

PREPARED BY I DATE

CHECKED BY/DATE

\begin{tabular}{|l|l|l|}
\hline & & \\
\hline & & \\
\hline
\end{tabular}


CLIENT: Duke Engineering \& Services Hanford

FILE NO: KH-8009-8-05

PROJECT: MCO Final Design $\quad$ DOC. NO.: $\quad$ HNF-SD-SNF-DR-003, Rev. 0, Appendix 7

asba, 69,38

asba, 70,39

asba,71,42 ! A72

Icom A4

asba,4,14 !A73

asba, 73,22

asba, 74,29

asba, 75,35

asba, 76,36

asba, 77,40

asba, 78,41

asba,79,43 ! A80

/com delete unused areas

adele, 1,4

adele, 7,51

adele, 53,60

adele,62,71

adele,73,79

/com merge areas and mesh

nummerg,all

esize,SIZE

amesh,52,61,9 ! bottom plate

amesh,72,80,8

real, 2

esize, 75

amesh, 5

amesh,6 I support rod area

/com repeat mesh to obtain 60 degree model \& merge nodes

csys

clocal, $11,,,, 30$

arsym, y,all

lcom extrude sup. rod

type, 3

esize, 25

vext, $2,6,4, \ldots, \mathrm{HI}$

csys

nummrg, node

/com add gap elements at support rods beneath

$n, 11444,9.870,613,-1$

$\mathrm{n}, 11995,11.199,1.602,-1$

$\mathrm{n}, 14514,5.466,8.241,-1$

$\mathrm{n}, 15064,6.987,8.897,-1$

d,11444,all

d,11995, all

d, 14514 , all

d, 15064,all

type, 4

real, 4

e, 11444,1444

e,11995, 1995

e, 14514,4514

$e, 15064,5064$

REVISION

PREPARED BY / DATE

CHECKED BY I DATE 


\begin{tabular}{llll} 
CLIENT: & \multicolumn{1}{l}{ Duke Engineering \& Services Hanford } & FILE NO: & KH-8009-8-05 \\
\cline { 2 - 4 } PROJECT: & MCO Final Design & DOC. NO.: & HNF-SD-SNF-DR-003, Rev. 0, Appendix 7 \\
\cline { 2 - 4 }
\end{tabular}

/com constrain edges of plate ( 60 deg. symm.)

nsel,s,loc,y, 0

csys, 1

nrotat,all

d,all,uy, ,., rotx, rotz

nsel,all

nsel,s,loc,y,60

csys, 1

nrotat,all

d, all,uy ,..., rotx, rotz

nsel,all

/com axial constraint

nsel,s,loc,z,0

nsel, $r$,loc, $\mathrm{x}, \mathrm{IR}$

d,all,uz

nsel,all

Id, 1444,uz I support from rods beneath

Id, 1995,uz

Id, 4514,uz

Id ,5064,uz

/com fuel loading (pressure) \& accel.

asel,s,loc, $z, 0$

asel,u,area, ,2,6,4

nsla,s, 1

sf,all,pres,pressure

asel,all

nal!

nsel,s,loc,z, HI

nsel,r,loc,x,10,15

sf,all,pres, 8048

nall

nsel,s,loc,z,HI

nsel, $r$, loc, $x, 0,7$

sf,all,pres, 7600

nal:

fี่า

save

isolu

acel,,,-35

solve

fini 
CLIENT: Duke Engineering \& Services Hanford

PROJECT: MCO Final Design $\quad$ DOC. NO.: HNF-SD-SNF-DR-003, Rev. 0, Appendix 7

\section{COMPUTER RUN COVER SHEET}

Project Number:

Computer Code:

Software Version:

Computer System:

Computer Run File Number:

Unique Computer Run File Name:

Run Description:

Run Date/Time:

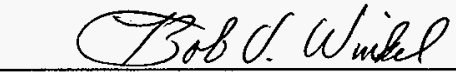

Prepared By: Bob V. Winkel
$\mathrm{KH}-8009-8$

ANSYS®-PC

$5.0 \mathrm{~A}$

MS-DOS, Pentium® Processor

$\mathrm{KH}-8009-8-05$

Plth.out

Elastic Drop Analysis of the Mark IA Storage Basket, Holes in Bottom Plate

15 January 1997/1:41:26pm
Chegked By: Joe Nichols

Date 
CLIENT: Duke Engineering \& Services Hanford

PROJECT: MCO Final Design $\quad$ DOC. NO.: HNF-SD-SNF-DR-003, Rev. 0, Appendix 7

\section{COMPUTER RUN COVER SHEET}

Project Number:

Computer Code:

Software Version:

Computer System:

Computer Run File Number:

Unique Computer Run File Name:

Run Description:

Creation Date/Time:

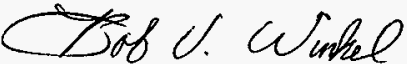

Prepared By: Bob V. Winkel
$\mathrm{KH}-8009-8$

ANSYS $B-P C$

$5.0 \mathrm{~A}$

MS-DOS, Pentium® Processor

KH-8009-8-05

Pltnhld.inp

Elastic Drop Analysis of the Mark IA Storage Basket, No Holes in Bottom Plate

3 February 1997/11:38:26am

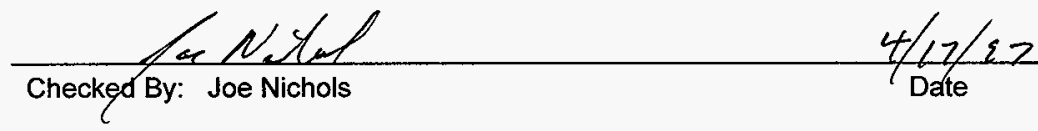




PROJECT: MCO Final Design $\quad$ DOC. NO.: HNF-SD-SNF-DR-003, Rev. 0, Appendix 7

\section{LISTING OF PLTNHLD.INP FILE}

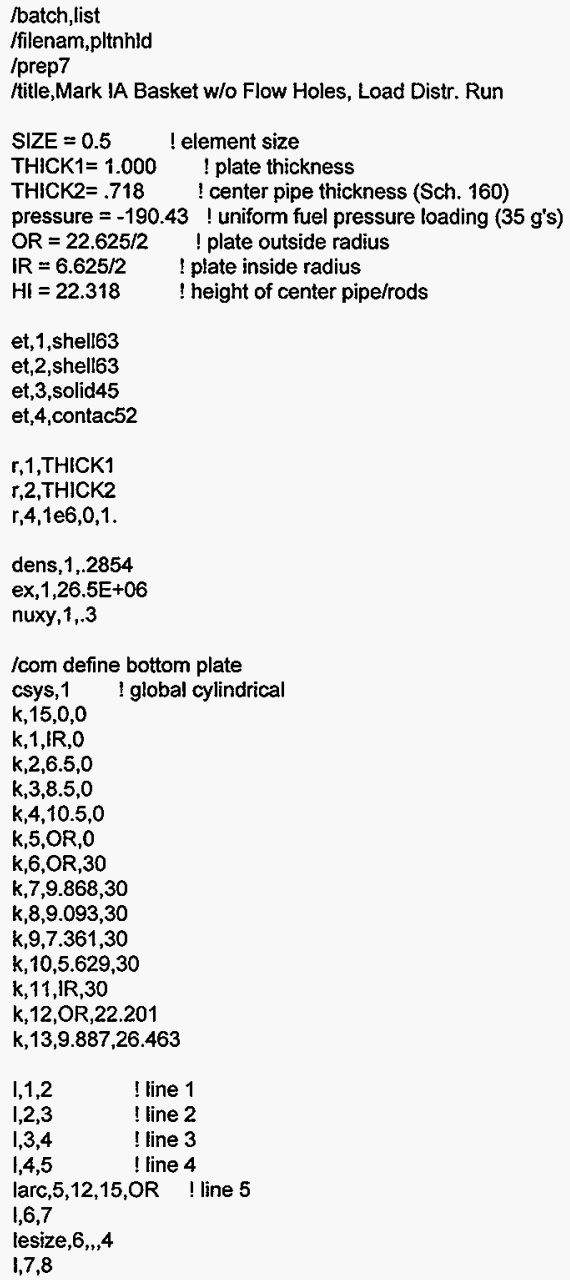


CLIENT: Duke Engineering \& Servics PARSINS

CLIENT: Duke Engineering \& Services Hanford

FILE NO: $\mathrm{KH}-8009-8-05$

PROJECT: MCO Final Design $\quad$ DOC. NO.: HNF-SD-SNF-DR-003, Rev. 0, Appendix 7

$1,8,9$

$1,9,10$

$\mathrm{I}, 10,11$

larc,1,11,15,IR ! line 11

lesize, $11,5 \quad 15$ degree segments for inside arc

csys, 0

I,2,10

$1,3,9$

$1,4,8$

$1,7,13$

! line 12

! line 14

lesize, $15,,, 3$

$1,13,12$ ! line 16

lesize, $16, \ldots 4$

csys, 1

I,12,6 ! line 17

lesize, $17, \ldots 3$

/com bottom plate areas

al, 1,12,10,11 !A1

al $, 2,13,9,12$

al, $3,14,8,13$

al, $4,5,16,15,7,14 \quad$ ! A4

/com define center pipe

$\mathrm{k}, 20,0,0, \mathrm{HI}$

$\mathrm{k}, 21, \mathrm{IR}, \mathrm{O}, \mathrm{H}$

$\mathrm{k}, 25, \mathrm{IR}, 30, \mathrm{HI}$

larc, $25,21,20,1 R$ ! line 18

lesize, 18,5 I 5 degree segments for inside arc

$1,1,21 \quad$ I vertical line 19 at 0 degrees

$1,11,25$ ! vertical line 20 at 30 degrees

/com area 5 = center pipe

al, $18,19,20,11$

/com create sup. rod area \& ext. into solid eles.

al, $6,15,16,17$ ! A6

icom merge areas and mesh

nummerg, all

esize,SIZE

amesh,1,4 ! bottom plate

amesh,6 ! support rod area

real, 2

esize, 75

amesh,5

/com repeat mesh to obtain 60 degree model \& merge nodes

csys

clocal, $11, \ldots, 30$

arsym,y,all

csys

/com support rod

type, 3

esize, 20

vext, $6,12,6, \ldots \mathrm{HI}$

REVISION

PREPARED BY IDATE

CHECKED BY I DATE

PAGE 67

OF 85 
CLIENT: Duke Engineering \& Services Hanford

FILE NO: KH-8009-8-05

PROJECT: MCO Final Design $\quad$ DOC. NO.: $\quad$ HNF-SD-SNF-DR-003, Rev. 0, Appendix 7

nummrg,node

/com constrain edges of plate $(60 \mathrm{deg}$. symm.)

nsel,s,loc,y,0

csys, 1

nrotat, all

d,all,uy,,,, rotx,rotz

nsel,all

nsel,s,loc,y,60

csys, 1

nrotat, all

d,all,uy ,.,.,rotx,rotz

nsel,all

/com axial constraint @ center pipe \& support rod

nsel,s,loc, z, Hl

d,alt,uz

nalt

Icom fuel loading (pressure) \& acel

asel, $s$, loc, $z, 0$

asel,u,area, $6,12,6$

sfa,all, pres,pressure

asel,all

acel,, 35

nall

fini

/solu

loutpr,basic, 1

solve

finj

/post1

set, last

prrs

nsel,s,loc, $x, 0,4$

prrs

nsel,s,loc, x,9,12

prrs

fini

lexit 


\begin{tabular}{llll} 
CLIENT: & \multicolumn{1}{l}{ Duke Engineering \& Services Hanford } & FILE NO: & KH-8009-8-05 \\
\cline { 2 - 3 } PROJECT: & MCO Final Design & DOC. NO.: & HNF-SD-SNF-DR-003, Rev. 0, Appendix 7 \\
\cline { 2 - 3 }
\end{tabular}

\section{COMPUTER RUN COVER SHEET}

Project Number:

Computer Code:

Software Version:

Computer System:

Computer Run File Number:

Unique Computer Run File Name:

Run Description:

Run Date/Time:
KH-8009-8

ANSYS $®-P C$

$5.0 \mathrm{~A}$

MS-DOS, Pentium® Processor

$\mathrm{KH}-8009-8-05$

Pltnhld.out

Elastic Drop Analysis of the Mark IA Storage Basket, No Holes in Bottom Plate

3 February 1997/11:40:50am

Prepared By: Bob V. Winkel

Date

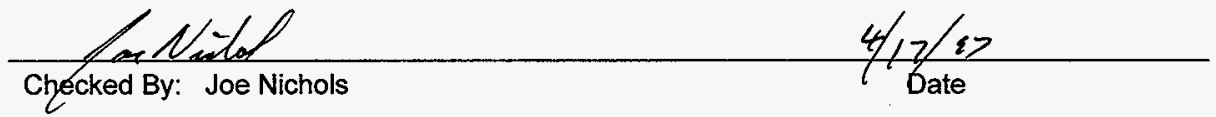


CLIENT: Duke Engineering \& Services Hanford

FILE NO: $\mathrm{KH}-8009-8-05$

PROJECT: MCO Final Design $\quad$ DOC. NO.: $\quad$ HNF-SD-SNF-DR-003, Rev. 0, Appendix 7

\section{COMPUTER RUN COVER SHEET}

Project Number:

Computer Code:

Software Version:

Computer System:

Computer Run File Number:

Unique Computer Run File Name:

Run Description:

Creation Date/Time:
$\mathrm{KH}-8009-8$

ANSYSQ-PC

$5.0 \mathrm{~A}$

MS-DOS, Pentium® Processor

KH-8009-8-05

Pltnhp.inp

Plastic Drop Analysis of the Mark IA Storage Basket Bottom Plate

5 March 1997/12:12:54pm

\section{YBob U. Wimbel}

Prepared By: Bob V. Winkel

\section{$4 / 18 / 97$}

Date

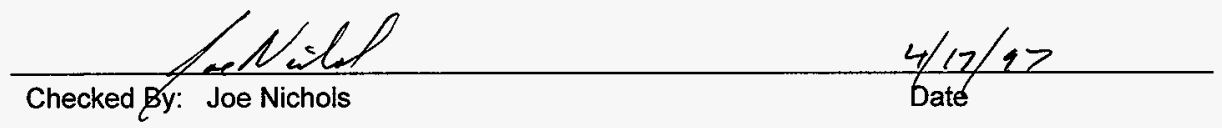

REVISION

PREPARED BY I DATE

CHECKED BY I DATE

12

\begin{tabular}{|c|l|l|l|}
\hline 0 & & & \\
\hline 2a 4/17/97 & & & \\
\hline
\end{tabular}




\begin{tabular}{llll} 
CLIENT: & \multicolumn{1}{l}{ Duke Engineering \& Services Hanford } & FLLE NO: & KH-8009-8-05 \\
\cline { 2 - 4 } PROJECT: & MCO Final Design & DOC.NO.: & HNF-SD-SNF-DR-003, Rev. 0, Appendix 7 \\
\cline { 2 - 4 } & &
\end{tabular}

/batch, list

\section{LISTING OF PLTNH.INP FILE}

filenam,pltnhp

/prep7

Aitle,Mark IA Basket, Bot. Plt. w/o Holes, Solid Posts

THICK $1=1.000 \quad$ ! plate thickness

THICK2 $=.864$ ! center pipe thickness (Sch. 160)

pressure $=-190.4 \quad$ ! uniform pressure load

$\mathrm{OR}=22.625 / 2 \quad$ i plate outside radius

$\mathrm{IR}=6.625 / 2-\mathrm{THiCK} 2 / 2$ ! center pipe mid-radius

$H I=22.1 \quad$ ! height of center pipe/rods

et, 1,shell43

et, 2, shell 43

et, 3,solid45

et, 4, contac52

$r, 1$, THICK 1

$r, 2$, THICK2

$r, 4,1 e 7,0,1$.

dens, 1,2854

ex, $1,26.5 E+06$

nuxy, $1, .3$

tb, bkin, 1,2

tbtemp,0

tbdata, 1,20.7e3,.16e6

tbtemp,200

tbdata,1,20.7e3,.16e6

/com define bottom plate

csys,1 ! global cylindrical

$k, 15,0,0$

$k, 1,1 R, 0$

$k, 2,6.5,0$

$k, 3,8.5,0$

$k, 4,10.5,0$

$\mathrm{k}, 5, \mathrm{OR}, 0$

$k, 6, O R, 30$

$k, 7,9.868,30$

$k, 8,9.093,30$

$k, 9,7.361,30$

$k, 10,5.629,30$

$k, 11, I R, 30$

$k, 12, O R, 22.201$

$k, 13,9.887,26.463$

1,1,2 I line 1

$1,2,3$ ! line 2

$1,3,4 \quad$ ! line 3

$1,4,5 \quad$ ! line 4

larc,5,12,15, OR ! line 5

$1,6,7$ 
CLIENT: Duke Engineering \& Services PARSDNS

CLIENT: Duke Engineering \& Services Hanford FILE NO: KH-8009-8-05

\begin{tabular}{llll} 
PROJECT: & MCO Final Design & DOC. NO.: & HNF-SD-SNF-DR-003, Rev. 0, Appendix 7 \\
\hline
\end{tabular}

lesize, $6,, 4$

$1,7,8$

$1,8,9$

$1,9,10$

$1,10,11$

larc, 1,11,15,JR ! line 11

lesize,11,5 ! 5 degree segments for inside arc

csys, 0

$1,2,10$

$1,3,9$

$1,4,8$

! line 12

! line 14

$1,7,13$

lesize, $15,, 3$

$\mathrm{I}, 13,12$

! line 16

lesize, $16,, 4$

csys, 1

$\mathrm{I}, 12,6$

I line 17

lesize, $17,, 3$

/com bottom plate areas
al, 1, 12,10,11 ! A1
al, $2,13,9,12$
al, $3, \uparrow 4,8,13$
al,4,5,16,15,7,14 I A4

/com define center pipe

$k, 20,0,0, \mathrm{HI}$

$\mathrm{K}, 21, \mathrm{IR}, \mathrm{O}, \mathrm{HI}$

$k, 25, I R, 30, H I$

larc,25,21,20,IR ! tine 18

lesize, 18,5 ! 5 degree segments for inside arc

$1,1,21 \quad$ ! vertical line 19 at 0 degrees

$1,11,25 \quad$ ! vertical line 20 at 30 degrees

/com area $5=$ center pipe

al, $18,19,20,11$

/com create sup. rod area \& ext. into solid eles.

a! $6,15,16,17$ ! $\mathrm{A} 6$

/com merge areas and mesh

nummerg,all

esize,SIZE

amesh, 1,4 ! bottom plate

amesh,6 ! support rod area

real,2

esize, 75

amesh, 5

/com repeat mesh to obtain 60 degree model \& merge nodes

csys

clocal, $11, \ldots, 30$

arsym,y,all

csys

/com support rod

type, 3

REVISION

PREPARED BY IDATE

\begin{tabular}{|c|c|c|c|}
\hline 0 & & & \\
\hline$/ 44 / 17 / 97$ & & & \\
\hline
\end{tabular}


CLIENT: Duke Engineering \& Services Hanford

FILE NO: KH-8009-8-05

PROJECT: MCO Final Design $\quad$ DOC. NO.: HNF-SD-SNF-DR-003, Rev. 0, Appendix 7

esize, 20

vext,6,12,6, , HI

nummrg,node

Icom gap elements at support rod

$\mathrm{n}, 2537,5.727,8.267,-1$

$\mathrm{n}, 2581,6.871,8.987,-1$

$\mathrm{n}, 2158,11.218,1.457,-1$

$\mathrm{n}, 2114,10.023, .827,-1$

type, 4

real, 4

e,2537,537

e,2581,581

$e, 2114,114$

e,2158,158

/com constrain edges of plate ( $60 \mathrm{deg}$. symm.)

nsel,s,loc,y,0

csys, 1

nrotat, all

d,all, uy, , , , rotx, rotz

nsel,all

nsel,s,loc,y, 60

csys, 1

nrotat,all

d,all,uy,.,,,rotx,rotz

nsel,all

Icom axial constraint

csys, 1

d,2537, all ! corners of sup. rods below

d,2114, alt

d,2158, all

$d, 2581$, all

nsel,s,loc,z,0 ! center pipe below

nsel, $r, l o c, x, I R$

d,all,uz

nall

/com bottom plate fuel loading (pressure)

asel, $s, l o c, z, 0$

asel,u,area, $6,12,6$

sfa,all, pres,pressure

asel,all

lcom $35 \mathrm{~g}$ loading from four baskets above

nsel,s,loc,z,HI

nsel, $r, l o c, x, 2,5$

sf,all,pres, 8835

nall

nsel,s,loc,z,HI

nsel, $r, 10 c, x, 7,12$

sf,all,pres,11066

d,all,ux,.,.,uy ! hor. constr. on top of sup. rod (friction)

nall

fini

REVISION

PREPARED BY IDATE

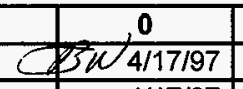

\begin{tabular}{|l|l|l|l|}
\hline & & & \\
\hline
\end{tabular}


CLIENT: Duke Engineering \& Services Hanford

FILE NO: $\quad \mathrm{KH}-8009-8-05$

PROJECT: MCO Final Design DOC. NO.: HNF-SD-SNF-DR-003, Rev. 0, Appendix 7

/solu

acel, , 35

!outpr, basic, 1

nsubst, 10

solve

fini

Iposts

set, last

/ANGLE, 1,-30,XS,1

MIN, 1, SQUARE

ledge,, 1

shell,bot

/show,graphics,pic, 1

plnsol,s, int

PLNSOL,EPPL,EQV

PLNSOL,U , Z

Ipath, 168,202

prsect

fini

lexit 


\section{PDARSDNS}

CLIENT: Duke Engineering \& Services Hanford

FILE NO: KH-8009-8-05

PROJECT: MCO Final Design $\quad$ DOC.NO.: HNF-SD-SNF-DR-003, Rev. 0, Appendix 7

\section{COMPUTER RUN COVER SHEET}

Project Number:

$\mathrm{KH}-8009-8$

Computer Code:

ANSYS®-PC

Software Version:

$5.0 \mathrm{~A}$

Computer System:

MS-DOS, Pentium $(B)$ Processor

Computer Run File Number:

$\mathrm{KH}-8009-8-05$

Unique Computer Run File Name:

Pltnhp.out

Run Description:

Plastic Drop Analysis of the Mark IA Storage

Basket Bottom Plate

Run Date/Time:

5 March 1997/12:12:54pm
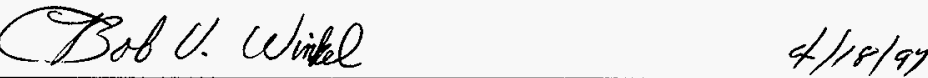

Prepared By: Bob V. Winkel

Date

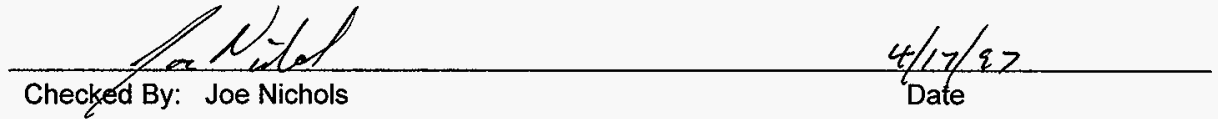

1




\begin{tabular}{llll} 
PROJENT: & \multicolumn{1}{l}{ Duke Engineering \& Services Hanford } & FILE NO: & KH-8009-8-05 \\
\cline { 2 - 3 } & MCO Final Design & DOC. NO.: & HNF-SD-SNF-DR-003, Rev. 0, Appendix 7 \\
\cline { 2 - 3 }
\end{tabular}

\section{COMPUTER RUN COVER SHEET}

Project Number:

Computer Code:

Software Version:

Computer System:

Computer Run File Number:

Unique Computer Run File Name:

Run Description:

Creation Date/Time:
$\mathrm{KH}-8009-8$

ANSYS@-PC

$5.0 \mathrm{~A}$

MS-DOS, Pentium $($ Processor

$\mathrm{KH}-8009-8-05$

H180p.inp

Horizontal Drop Analysis of the Mark IA Storage Basket

3 March 1997/2:49:04pm

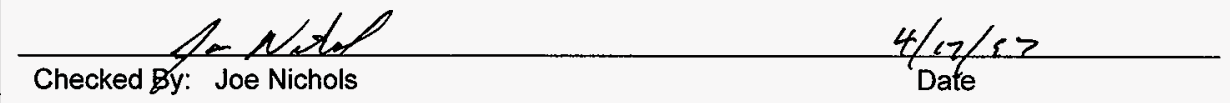

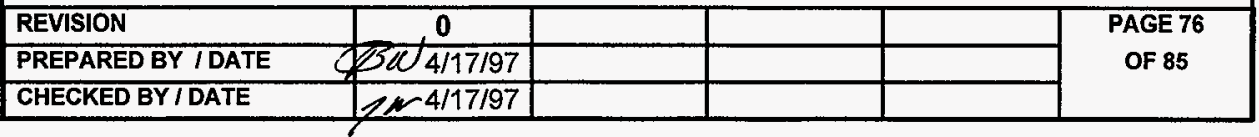




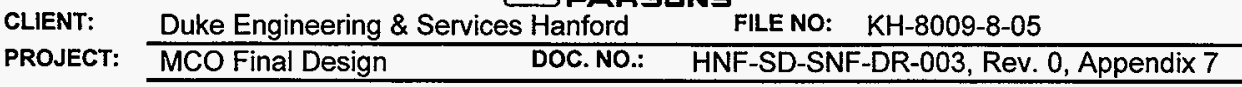

\section{LISTING OF H180P.INP FILE}

/batch,list

filenam, hi80p

/prep7

/titte, Mark IA Basket 101g Side Drop

SIZE $=1.00 \quad$ ! element size

THICK1 $=1.20$ ! plate thickness

THICK2 $=.864$ ! center pipe thickness

GLD $=101 \quad$ ! 101g Side Drop Load

$\mathrm{OR}=22.625 / 2 \quad$ ! plate outside radius

IR $=6.625 / 2-T H I C K / 2$ ! plate inside radius

$\mathrm{HI}=22.1 \quad$ ! height of center pipe/rods

*afun,deg ! use degrees for function input/output

et, 1 , shell 43

et, 2 , shell 43

et,3, beam4 ! 3-d elastic beam

et, 4, mass $21,,, 2$

et, 5, contac $52,, \ldots 1$

et,7,link11 ! centerline spring @ drop interface

r, 1, THICK1

$r, 2$, THICK2

$r, 3,10,100,100,10,10$ ! rigid link

r,4,18.5 ! weight/5 (lb) from adjacent pipe

$r, 5,1 \mathrm{e} 6, ., 1 \mathrm{e} 6 \quad$ ! Gap element properties

$r, 6,2.874, .403,1.307,1.34,3.07$ ! Support Rod prop.

$r, 7,1$ e4 ! Centerline Link Element

dens, 1,2854

ex, $1,27 \mathrm{E}+06$

nuxy, 1,3

ex, $2,27 \mathrm{e}+06$

dens,2,5.786 ! pseudo density of upper pipe elements for fuel load

ex, $3,27 \mathrm{e}+06$

dens,3,0.0 ! massless rigid links

tb,bkin,1,2 !Plas. Prop.

tbtemp, 0

tbdata, 1,21.e3,0.16e6

tbtemp, 200

tbdata, 1,21.e3,0.16e6

/com define bottom plate

csys,1 ! global cylindrical

$k, 10,0,0$

$k, 1, I R, 0$.

$k, 2, O R, 0$

$\mathrm{k}, 3, \mathrm{OR}, 30$

$k, 4, O R, 60$

$\mathrm{k}, 5, \mathrm{IR}, 60$

$1,1,2$

! line 1

\begin{tabular}{|c|l|l|l|}
\hline 0 & & & \\
\hline \multicolumn{6}{|c|}{$4 / 17 / 97$} & & & \\
\hline $1 / 4 / 17 / 97$ & & & \\
\hline
\end{tabular}


PROJECT: MCO Final Design DOC.NO.: HNF-SD-SNF-DR-003, Rev. 0, Appendix 7

larc,2,3,10,OR ! line 2

lesize, $2,2.5 \quad 12.5$ deg. segments, outside arc

larc, $3,4,10, \mathrm{OR}$ ! line 3

lesize,3,2.5 ! 2.5 deg. segments

$1,4,5 \quad$ ! line 4

larc, $5,1,10, \mathrm{IR}$ ! line 5

lesize, $5,10 \quad$ ! 5 degree segments for inside arc

Icom area 1 = bottom plate

al, $1,2,3,4,5$

Icom define center pipe

$k, 20,0,0, \mathrm{H}$

$k, 21, I R, 0, H I$

$k, 25, \mid R, 60, H I$

larc, $25,21,20, \mathrm{IR}$ ! line 6

lesize, $6,10 \quad$ ! 5 degree segments for inside arc

$1,1,21 \quad$ I vertical line (7) at 0 degrees

$1,5,25$ ! vertical line (8) at 60 degrees

lcom area 2 = center pipe

al, $, 5,6,7,8$

Icom mesh areas

/com bottom plate

esize,SIZE

mat, 1

real, 1

amesh,1

/com center pipe

esize, 1.5

type, 2

real, 2

amesh,2

/com repeat mesh for 180 deg. model

csys, 1

agen, 3, all, ,.,60

/com merge coincident nodes

nummerg,node

/com add masses to center pipe hole to account for adj. pipe load

type, 4

real, 4

e,1

e.43

egen, $4,1,-1$

Icom modify 30 arc of pipe elements to add fuel mass

csys, 1

nsel,s,loc, $x, \mid R$

nsel,, loc, $y, 150,180$

esin,s, 1

emodif,all,mat, 2

\begin{tabular}{|l|c|c|c|c|c|}
\hline REVISION & $\rho$ & & & & PAGE 78 \\
OF 85
\end{tabular}


PARSDNS PROJECT: MCO Final Design DOC.NO.: HNF-SD-SNF-DR-003, Rev. 0, Appendix 7

esel,all

/com add support rods

type, 3

real, 6

mat, 1

n,2114,10.586,30.2,22.318

$n, 2412,10.586,90.2,22.318$

n, 2710,10.586,150.2,22.318

e. 114,2114

$e, 412,2412$

e,710,2710

type, 3

real, 6

e,114,131 ISpread rod interface to adjacent nodes

e, 114,81

e, 114,80

e, 114,132

$e, 412,429$

$e, 412,379$

e,412,378

$e, 412,430$

e,710,727

e, 710,677

e, 710,676

e, 710,728

lcom define offset nodes on drop side of bottom plate n,5001, OR,0,-.5

$\mathrm{n}, 5013, \mathrm{OR}, 30,-.5$

fill, 5001,5013

real, 3

type,3

mat, 3

e,2,5001

$e, 11,5002$

egen, $11,1,-1$

e, 10,5013

/com Bottom Plate interface w/ MCO

lcom shift origin to MCO center to define MCO ID

$\mathrm{n}, 6001, \mathrm{OR}+.001,0,-.5$

csys, 1

$j=0.001$

*do,i,1,12

$\mathrm{j}=\mathrm{j}+.002 \quad$ ! gap increases 0.002 " for every $2.5 \mathrm{degr}$.

$\mathrm{n}, 6001+\mathrm{i}, \mathrm{OR}+\mathrm{j}, 2.5^{*} \mathrm{i},-.5$

*enddo

nrotat,6002,6013

d, 6001 , all.,.6013

type,7 I Soft Spring @ MCO I.D. (model symm. line)

real, 7

e, 5001,6001

type, 5

real, 5

e,5001,6001

REVISION PREPARED BY I DATE CHECKED BY I DATE

! Gap elements @ MCO I.D.

CHECKED BYIDATE

\begin{tabular}{|c|c|}
\hline & 0 \\
\hline$\overline{\mathrm{TE}}$ & $3 W_{4 / 17 / 97}$ \\
\hline & $/ 4-4 / 17 / 97$ \\
\hline
\end{tabular}


CLIENT: Duke Engineering \& Services Hanford

\begin{tabular}{llll} 
PROJECT: & MCO Final Design & DOC. NO.: & HNF-SD-SNF-DR-003, Rev. 0, Appendix 7 \\
\cline { 2 - 3 }
\end{tabular}

\section{$e, 5002,6002$}

egen, $12,1,-1$

/com constrain edges of center pipe at top

csys, 1

nsel,s,loc,z, $\mathrm{Hl}$

nsel, $r, 10 c, x$, IR

d,all,ux I $x$ constraint, pipe end

nsel, $r$, loc, $y, 0,5$

d,all,uz

nsel,all

lcom symm bound. cond.

csys, 0

nsel,s,loc,y,0 I select nodes at $y=0$

d,all, uy, $0.0,,$, rotx, rotz $\quad$ ! symmetry bc at $y=0$

nall

eall

/com apply $\mathrm{g}$ load

acel, $-1.5^{\star}$ GLD

fini

/solu

loutpr, basic,last

outres, 1

autots, on

nlgeom,on

nsubst, 50

neqit, 50

cnvtol, f,..005

cnvtol,m,..005

solve

save

fini

loost1

set,last

/show, graphics,pic,1

NIEW,ALL, 1, 2, 3

eplot

prrs

esel,u,type, 3,5

ledge,, 1

plnsol,s,int

plnsol,eppl,eqv

fini

lexit

\begin{tabular}{|c|c|c|c|}
\hline 0 & & & \\
\hline $\operatorname{la}_{5} / 4 / 17 / 97$ & & & \\
\hline
\end{tabular}


CLIENT: Duke Engineering \& Services Hanford

PROJECT: MCO Final Design DOC. NO.: HNF-SD-SNF-DR-003, Rev. 0, Appendix 7

\section{COMPUTER RUN COVER SHEET}

Project Number:

Computer Code:

Software Version:

Computer System:

Computer Run File Number:

Unique Computer Run File Name:

Run Description:

Run Date/Time:

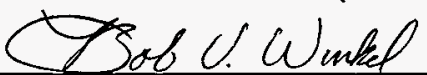

Prepared By: Bob V. Winkel

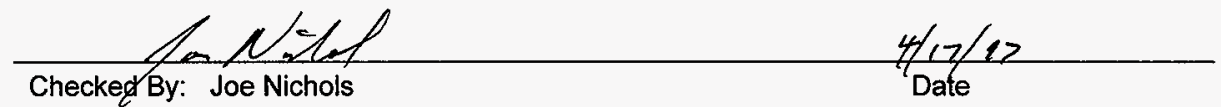

$\mathrm{KH}-8009-8$

ANSYS $\otimes-P C$

$5.0 \mathrm{~A}$

MS-DOS, Pentium $\circledast$ Processor

KH-8009-8-05

H180p.out

Horizontal Drop Analysis of the Mark IA Storage Basket

8 March 1997/2:49:04pm

\section{Date}


CLIENT: $\quad$ Duke Engineering \& Services Hanford

FILE NO: $\quad \mathrm{KH}-8009-8-05$

PROJECT: MCO Final Design $\quad$ DOC.NO.: HNF-SD-SNF-DR-003, Rev. 0, Appendix 7

\section{COMPUTER RUN COVER SHEET}

Project Number:

Computer Code:

Software Version:

Computer System:

Computer Run File Number:

Unique Computer Run File Name:

Run Description:

Creation Date/Time:
$\mathrm{KH}-8009-8$

ANSYS $B-P C$

$5.0 \mathrm{~A}$

MS-DOS, Pentium® Processor

$\mathrm{KH}-8009-8-05$

Rbuc2.inp

Mark IA Basket Support Rod Buckling

Two Rods

3 February 1997/1:33:18pm
Oob U. Wintal

Prepared By: Bob V. Winkel
Date

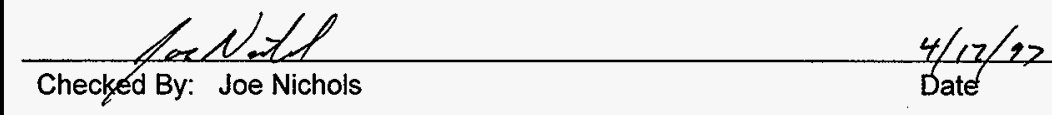


CLIENT: Duke Engineering \& Services Hanford

FILE NO: $\quad \mathrm{KH}-8009-8-05$

PROJECT: MCO Final Design $\quad$ DOC.NO.: HNF-SD-SNF-DR-003, Rev. 0, Appendix 7

\section{LISTING OF RBUC2.INP FILE}

/batch, list

/filenam,rodb2

tprep7

title,Mark IA Basket Support Rod Buckling, Two Rods

!et, 1 , beam $23, \ldots,,, 4$

et, 1,beam23 ! rectangle

et, 2 , beam 23

et, 3, contac12

Ir,1,2.874,1.34,6.384,3.072,2.916,1.897,2.816 ! general shape

$r, 1,2.874, .430,1.34$ ! equivalent rectangle

$r, 2,10,83.333,10$

$r, 3,0,1 e 8,1.0$ ! Longer Rod, no gap

$r, 4,0,1 e 8,-.03$ ! Shorter Rod, 30 mil gap

dens, 1,2854

ex, 1,27.6E+06

nuxy, 1,1

to,bkin, 1,2

tbtemp, 0

tbdata, 1, 17.6e3,0.3e6

tbtemp,200

tbdata, 1,17.6e3,0.3e6

dens, $2, .2854$

ex,2,27.6E+06

nuxy, 2,3

mu,2,0.1

tb,bkin, 2,2

tbtemp,0

tbdata, 1, 17.6e6,0.3e6

tbtemp,200

todata, 1,17.6e6,0.3e6

$\mathrm{n}, 1$,

$\mathrm{n}, 21,0,22.318$

fill, 1,21

$\mathrm{n}, 22,-.25,22.318$

$\mathrm{n}, 23,-.25,23$.

$n, 101,-.5$

$\mathrm{n}, 121,-.5,22.288$

fill, 101,121

n, 122,-.25,22.288

type, 1

real, 1

$e, 1,2$

egen, $20,1,1$

e, 101,102

egen,20,1,-1

type, 2

real,2

REVISION

PREPARED BY IDATE

CHECKED BY I DATE

$\frac{0}{4 / 17 / 97}$

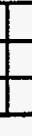




\begin{tabular}{llll} 
CLIENT: & \multicolumn{1}{l}{ Duke Engineering \& Services Hanford } & FILE NO: & KH-8009-8-05 \\
\cline { 2 - 3 } PROJECT: & MCO Final Design & DOC. NO.: & HNF-SD-SNF-DR-003, Rev. 0, Appendix 7 \\
\cline { 2 - 4 } & &
\end{tabular}

mat,2

e,21,22 I rigid link for load offset

e,121,122 ! 2nd rod rigid link

type, 3

real, 3

e, 22,23

real,4

e, 122,23

eplot

Icom Boundary Conditions

$d, 1$,all

d, 101,all

d,23,all

fini

/solu

d, 23,uy, -.012

autots, on

nigeom,on

cnvtol, $f_{1}, 01$

cnvtoi, $m,-1$

outres,all, 1

time, 1

nsubst, 10

loutpr,basic, 10

Iswrite, 1

time,2

d,23,uy, -10

nsubst, 1000

outres,all,2

loutpr,basic, 10

Iswrite,2

Issolve, 1,2

fini

/post26

NSOL,2,23,U,Y,uy23

RFORCE, 3,23,F,Y,fy23

prod $4,3, \ldots, 3 f y 1, \ldots-3$

ADD $, 5,2, \ldots, \ldots-1$

prvar, $2,3,4,5$

xvar, 5

WIN, 1,SQUARE

IAXLAB, X, Rod Vertical Displ. (in.)

IAXLAB, Y, Support Rod Force (Ib)

/show,graphics,pic, 1

plvar, 4

fini

/post1

set,last

pldisp

fini

lexit 


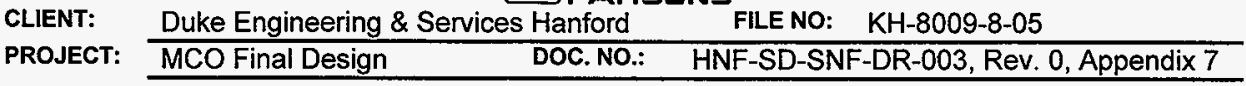

\section{COMPUTER RUN COVER SHEET}

Project Number:

Computer Code:

Software Version:

Computer System:

Computer Run File Number:

Unique Computer Run File Name:

Run Description:

Run Date/Time: $\longrightarrow \frac{18}{10}$

Prepared By: Bob V. Winkel
$\mathrm{KH}-8009-8$

ANSYS®-PC

$5.0 \mathrm{~A}$

MS-DOS, Pentium® Processor

KH-8009-8-05

Rbuc2.out

Mark IA Basket Support Rod Buckling

Two Rods

3 February 1997/1:36:56pm

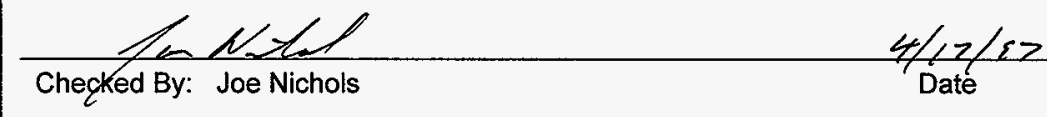




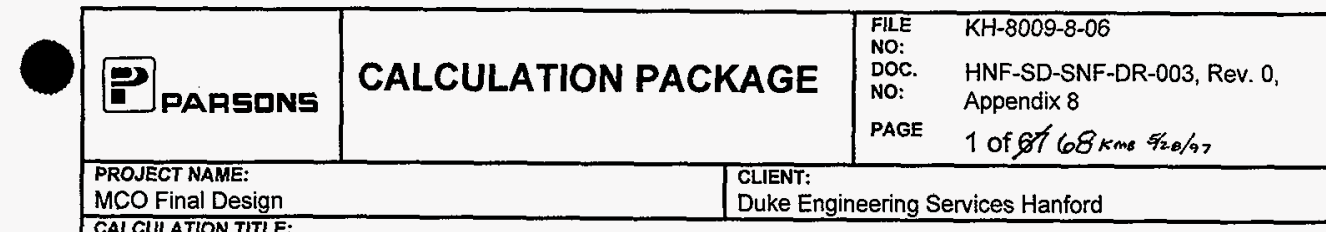

STRESS ANALYSIS OF THE MARK IV STORAGE BASKET

\section{PROBLEM STATEMENT OR OBJECTIVE OF CALCULATION:}

Perform a stress analysis of the Mark IV Storage Basket in accordance with Revision 3 of the MCO Performance Specification. Two loading conditions are considered:

1. Lifting at a max. temperature of $100^{\circ} \mathrm{C}$.

2. Deadweight stacking within the MCO at a design temperature of $375^{\circ} \mathrm{C}$.

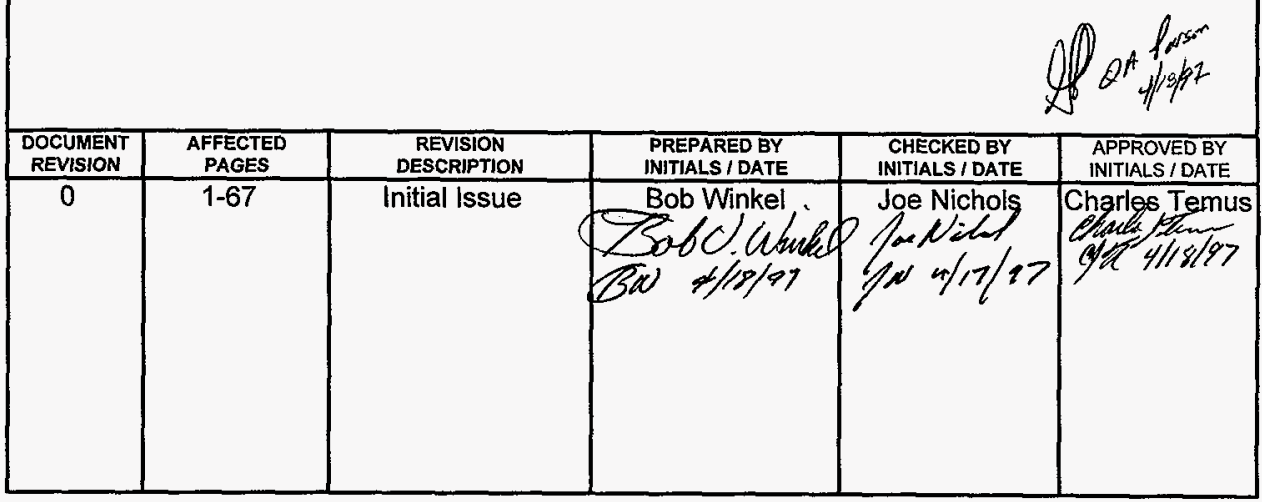


CLIENT: Duke Engineering Services Hanford

FILE NO: $\quad \mathrm{KH}-8009-8-06$

PROJECT: MCO Final Design DOC NO: HNF-SD-SNF-DR-003, Rev. 0, Appendix 8

TABLE OF CONTENTS

1. INTRODUCTION 6

2. REFERENCES 6

3. ASSUMPTIONS 7

4. GEOMETRY 7

5. MATERIAL PROPERTIES $\quad 9$

6. ACCEPTANCE CRITERIA 9

$\begin{array}{ll}\text { 7. LOAD CONDITIONS \& COMBINATIONS } & 15\end{array}$

8. STRESS ANALYSIS CALCULATIONS

$\begin{array}{ll}8.1 \text { Center Pipe } & 18\end{array}$

8.2 Support Rod $\quad 22$

8.3 Fuel Retaining Bars $\quad 23$

8.4 Support Ring 26

8.5 Bottom Plate $\quad 28$

8.5.1 Deadweight Stacking Load Condition 28

8.5.2 Lifting Load Condition 31

8.6 Load Distribution and Basket Interface Considerations 35

$\begin{array}{ll}8.7 \text { Fillet Weld Finite Element Analysis } & 39\end{array}$

8.8 Component Stress Results Summary 40 
CLIENT: Duke Engineering Services Hanford

PROJECT: MCO Final Design DOC NO

FILE NO: $\quad \mathrm{KH}-8009-8-06$

\section{LIST OF TABLES}

TABLE 1. MARKIV STORAGE BASKET STRUCTURAL COMPONENTS.

TABLE 2 ASME CODE MATERIAL PROPERTIES FOR TYPE 304L. STAINLESS STEEL

TABLE 3. ALLOWABLE STRESSES - DEADWEIGHT.

TABLE 4. MARK IV STORAGE BASKET WELD STRESS ALLOWABLES.

TABLE 5. SUMMARY OF MARK IV BASKET STRUCTUAL EVALUATION. 
CLIENT: Duke Engineering Services Hanford
FILE NO: $\mathrm{KH}-8009-8-06$

PROJECT: MCO Final Design DOC NO

HNF-SD-SNF-DR-003, Rev. 0, Appendix 8

\section{LIST OF FIGURES}

FIGURE 1. SIXTY-DEGREE SECTOR IDENTIFYING MARK IV STORAGE BASKET PRIMARY STRUCTURAL COMPONENTS.

FIGURE 2. SIXTY-DEGREE SECTOR FINITE ELEMENT MODEL OF THE MARK IV STORAGE BASKET. FIGURE 3. STRESS INTENSITY CONTOUR PLOT, DEADWEIGHT STACKING LOAD CONDITION.

FIGURE 4. STRESS INTENSITY CONTOUR PLOT FOR AN INSIDE LIGAMENT THICKNESS OF 0.085 IN.

FIGURE 5. STRESS INTENSITY CONTOUR PLOT FOR AN INSIDE LIGAMENT THICKNESS OF $0.210 \mathrm{IN}$. 
CLIENT: Duke Engineering Services Hanford

FILE NO: $\quad \mathrm{KH}-8009-8-06$

PROJECT: MCO Final Design DOC NO:

HNF-SD-SNF-DR-003, Rev. 0, Appendix 8

\section{LIST OF APPENDICES}

APPENDIX A. COMPUTER RUN COVER SHEETS AND INPUT FILE LISTINGS 
CLIENT: Duke Engineering Services Hanford

PROJECT: MCO Final Design

FILE NO: $\mathrm{KH}-8009-8-06$

DOC NO: HNF-SD-SNF-DR-003, Rev. 0, Appendix 8

\section{INTRODUCTION}

This calculation documents the evaluation of the Mark IV Storage Basket for lifting and deadweight loading. The structural adequacy evaluation is based upon ANSI N14.6 for the lifting loading and Section III of the ASME Code for the deadweight stacking loading within the MCO.

\section{REFERENCES}

1. Duke, 1996, Performance Specification for the Spent Nuclear Fuel Multi-Canister Overpack, HNF-S-0426, Rev. 3, Duke Engineering and Services Hanford, Richland, Washington.

2. Duke, 1996, "MCO Mark IV SNF Storage Basket", Drawing No. H-2-828070, Rev. C, Duke Engineering and Services Hanford, Richland, Washington.

3. ASME, 1995, ASME Boiler and Pressure Vessel Code, Section II, Materials, Part D-Properties, American Society of Mechanical Engineers, New York, New York.

4. ASME, 1995, ASME Boiler and Pressure Vessel Code, Section III, Subsection NG, American Society of Mechanical Engineers, New York, New York.

5. ASME, 1995, ASME Boiler and Pressure Vessel Code, Section III, Subsection NF, American Society of Mechanical Engineers, New York, New York.

6. ANSI, 1993, ANSI National Standard for Radioactive Materials- Special Lifting Devices for Containers Weighing 10,000 Pounds (4500 kg) or More, American National Standards Institute, New York, New York.

7. Roark, R. J. and Young, W. C., 1975, Formulas for Stress and Strain, 5th Edition, McGraw-Hill, New York, New York.

8. AISC, 1989, Manual of Steel Construction, Ninth Edition, American Institute of Steel Construction, Chicago, Illinois.

0
$2 x / 4 / 17 / 97$
$2 n / 4 / 17 / 97$




\section{ASSUMPTIONS}

1. All welds made and inspected in accordance with ASME Code, Section III, Subsection NG (Reference 4). For the Mark IV Storage Basket welds, a "surface visual examination" was assumed.

2. For the dead weight loading when the baskets are stacked within the MCO, it was conservatively assumed that the center tube carries the weight of the baskets above, for center tube structural adequacy evaluations, and for the support rod evaluations, it was conservatively assumed that the support rods carry the full weight of the baskets above.

3. Other assumptions as noted within the calculation documentation.

\section{GEOMETRY}

The Mark IV Storage Basket geometry is defined in Drawing No. H-2-828070. The storage basket primary structural components are identified in the $60^{\circ}$ sector shown in Figure 1. The geometry pattern shown in Figure 1 is repeated every $60^{\circ}$, including the Support Rod. Each hole in the bottom plate is designed to contain a single fuel rod, resulting in a capacity of 54 fuel rods per basket.

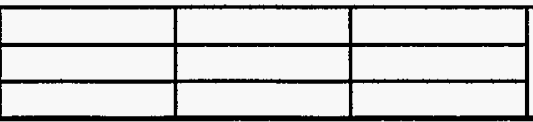


PROJECT: MCO Final Design DOC NO: HNF-SD-SNF-DR-003, Rev. 0, Appendix 8

FILE NO: KH-8009-8-06

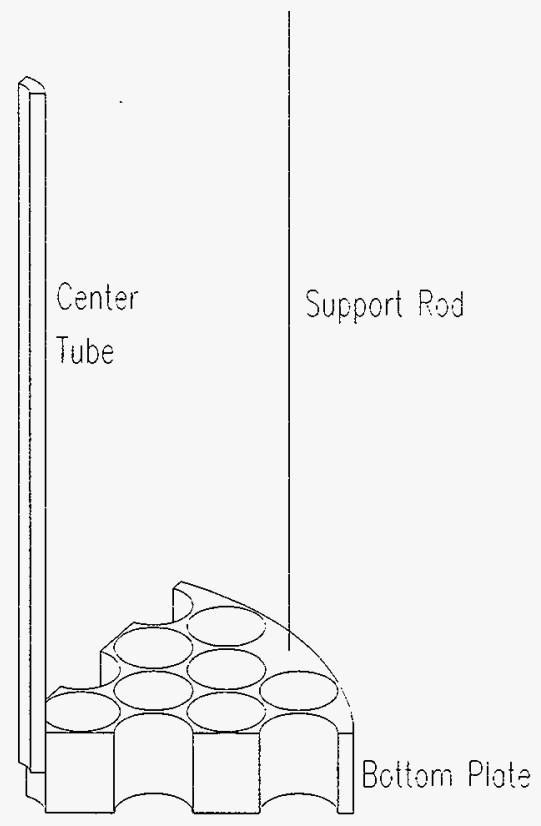

Figure 1. Sixty-Degree Sector Identifying Mark IV Storage Basket Primary Structural Components.

The Support Rods are constructed from 1-1/4 in. round bar which are shrunk fit into the bottom plate. The Center Tube is fabricated from 2.75 in. O.D., 1.75 I.D. tubing and is also connected to the bottom plate using a shrink fit. In addition to the shrink fit, the center tube is attached to the bottom plate with fillet welds. The baskets are designed to be stacked within the MCO such that the center tube and six support rods share the weight of the baskets stacked above. As noted in Section 3, establishing the center tube/support

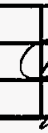

\begin{tabular}{|l|l|l|l|}
\hline & & & \\
\hline & & & \\
\hline
\end{tabular}


PROJECT: MCO Final Design DOC NO: HNF-SD-SNF-DR-003, Rev. 0, Appendix 8

rods load division was avoided by conservatively assuming that either the center tube or support rods carried the entire stack weight.

Not shown are a series of $1 / 4$-in. $\times 3 / 8$-in. fuel retaining bars which cross the bottom diameter of each hole to prevent the fuel from passing through the hole. Each hole contains a single retaining bar which extends inside a groove connecting a line of holes and is weided to each ligament between the holes. Also not shown is a 1-1/4-in. wide, 3/8-in. high support ring which is welded to the bottom of the bottom plate near the O.D. The support ring interfaces with the six Support Rods of a basket immediately below when the baskets are stacked.

A third component not shown is a 0.05 -in. thick, 11.0-in. high sheet metal band at the basket O.D. immediately above the bottom plate. During normal operations, this sheet metal shroud is not subjected to significant loading and is considered to be non-structural.

The Mark IV components which were subjected to a structural evaluation are listed in Table 1. Structural adequacy of each component is addressed in Section 8. A summary of the evaluation results is provided in Section 8.6.

\section{MATERIAL PROPERTIES}

Per the Reference 2 drawing, the specified material for the Mark IV Storage Basket components is $304 \mathrm{~L}$ stainless steel. For this analysis, the only mechanical properties of interest are the elastic modulus, yield strength, ultimate strength, and ASME stress allowable, $S_{m}$. The appropriate values were extracted from Reference 3, and are listed in Table 2.

\section{ACCEPTANCE CRITERIA}

For the lifting and dead weight stacking loadings considered, the appropriate acceptance criteria is discussed below.

\subsection{Lifting Loads}

Per Section 4.12.3 of the Reference 1 Performance Specification, the basket design "shall meet the safety factors required by the American National Standards Institute (ANSI) N14.6-1986. The ANSI safety factors apply from $5^{\circ} \mathrm{C}$ to $100^{\circ} \mathrm{C}$. Section 4.2.1.1 of N14.6 specifies that "the load bearing members of a special lifting device shall be

\begin{tabular}{|l|l|l|l|}
\hline & & \\
\hline & & & \\
\hline & & \\
\hline
\end{tabular}


CLIENT: Duke Engineering Services Hanford

PROJECT: MCO Final Design

FILE NO: $\quad \mathrm{KH}-8009-8-06$

DOC NO: HNF-SD-SNF-DR-003, Rev. 0, Appendix 8

Table 1. Mark IV Storage Basket Structural Components.

\begin{tabular}{|c|c|c|}
\hline $\begin{array}{l}\text { Component } \\
\text { Name }\end{array}$ & $\begin{array}{l}\text { Component Part No. } \\
\text { in Dwg. H-2-828070 }\end{array}$ & Component Function \\
\hline Center Tube & 5 & $\begin{array}{l}\text { (1) Provide support to above } \\
\text { baskets when stacked inside the } \\
\text { MCO (2) Provide dip tube } \\
\text { access to the bottom of the MCO } \\
\text { (3) Lifting grapple interface }\end{array}$ \\
\hline Support Rod & 6 & $\begin{array}{l}\text { Provide support to above } \\
\text { baskets when stacked inside the } \\
\text { MCO }\end{array}$ \\
\hline Bottom Plate & 2 & $\begin{array}{l}\text { (1) Mounting base for center } \\
\text { pipe and support rods. (2) } \\
\text { Maintain position of spent fuel } \\
\text { rods after the rods are inserted } \\
\text { into the basket }\end{array}$ \\
\hline $\begin{array}{l}\text { Retaining } \\
\text { Ring }\end{array}$ & 11 & $\begin{array}{l}\text { Bearing surface for adjacent } \\
\text { (below) basket support rods }\end{array}$ \\
\hline $\begin{array}{c}\text { Fuel } \\
\text { Retaining Bar }\end{array}$ & 12 & $\begin{array}{l}\text { Vertical support for spent fuel } \\
\text { rods }\end{array}$ \\
\hline $\begin{array}{l}\text { Center Tube } \\
\text { Welds }\end{array}$ & - & $\begin{array}{l}\text { Center tube structural } \\
\text { attachment to Bottom Plate }\end{array}$ \\
\hline $\begin{array}{l}\text { Support Ring } \\
\text { Welds }\end{array}$ & - & $\begin{array}{l}\text { Support Ring structural } \\
\text { attachment to Bottom Plate }\end{array}$ \\
\hline $\begin{array}{l}\text { Fuel } \\
\text { Retaining Bar } \\
\text { Welds }\end{array}$ & - & $\begin{array}{l}\text { Fuel Retaining Bar structural } \\
\text { attachment welds }\end{array}$ \\
\hline
\end{tabular}


CLIENT: Duke Engineering Services Hanford

PROJECT: MCO Final Design DOC NO: HNF-SD-SNF-DR-003, Rev. 0, Appendix 8

Table 2

ASME Code Material Properties for Type 304L Stainless Steel

\begin{tabular}{|c|c|c|c|c|c|}
\hline \multicolumn{2}{|c|}{ Temperature } & \multirow{2}{*}{$\begin{array}{c}E \\
\text { ble TM-1, Group }\end{array}$} & \multirow{2}{*}{$\begin{array}{c}S_{m} \\
\text { Table 2A, p.322 }\end{array}$} & \multirow{2}{*}{$\begin{array}{c}S_{y} \\
\text { Table } Y-9, p .524\end{array}$} & \multirow{2}{*}{$\begin{array}{c}S_{u} \\
\text { Table U. P. } 441\end{array}$} \\
\hline${ }^{\circ} \mathrm{F}$ & ${ }^{\circ} \mathrm{C}$ & & & & \\
\hline-20 & - & - & $16.7 \mathrm{ksi}$ & $25.0 \mathrm{ksi}$ & $70.0 \mathrm{ksi}$ \\
\hline 70 & - & $28.3 E+06$ & - & - & - \\
\hline 100 & - & - & $16.7 \mathrm{ksi}$ & $25.0 \mathrm{ksi}$ & $70.0 \mathrm{ksi}$ \\
\hline 200 & - & $27.6 \mathrm{E}+06$ & $16.7 \mathrm{ksi}$ & $21.3 \mathrm{ksi}$ & $66.2 \mathrm{ksi}$ \\
\hline 212 & 100 & $27.5 E+06$ & $16.7 \mathrm{ksi}$ & $21.0 \mathrm{ksi}$ & $65.6 \mathrm{ksi}$ \\
\hline 300 & - & $27.0 \mathrm{E}+06$ & $16.7 \mathrm{ksi}$ & $19.1 \mathrm{ksi}$ & $60.9 \mathrm{ksi}$ \\
\hline 700 & - & $24.8 E+06$ & $13.5 \mathrm{ksi}$ & $14.9 \mathrm{ksi}$ & $56.2 \mathrm{ksi}$ \\
\hline 707 & 375 & $24.8 \mathrm{E}+06$ & $13.5 \mathrm{ksi}$ & $14.9 \mathrm{ksi}$ & $56.2 \mathrm{ksi}$ \\
\hline 750 & - & - & $13.3 \mathrm{ksi}$ & $14.7 \mathrm{ksi}$ & $55.9 \mathrm{ksi}$ \\
\hline 800 & & $24.1 E+06$ & - & - & - \\
\hline
\end{tabular}

Notes 1: Underlined values determined by linear interpolation, all other values taken from Section II, Part D of the ASME Code.

2: Value of $E$ taken from Table TM-1 for Material Group G.

capable of lifting three times the combined weight of the shipping container with which it will be used, plus the weight of intervening components of the special lifting device, without generating a combined shear stress or maximum tensile stress at any point in the device in excess of the corresponding minimum tensile yield strength of their materials of construction. They shall also be capable of lifting five times that weight without exceeding the ultimate tensile strength of the materials." ANSI N14.6 also states that the shear stress shall be taken as an average value over the cross section, and that the tensile stress may be due to direct or bending loads. The bending stress is defined as being linear over the cross section.

The "load bearing members of a special lifting device" are interpreted to apply to all components of the storage baskets in the load path between the lifting grapple and fuel. At the maximum lifting temperature of $100^{\circ} \mathrm{C}$, the ANSI N14.6 allowables are:

\begin{tabular}{|l|l|l|l|}
\hline & & \\
\hline
\end{tabular}


CLIENT: Duke Engineering Services Hanford

FILE NO: KH-8009-8-06

PROJECT: MCO Final Design DOC NO: HNF-SD-SNF-DR-003, Rev. 0, Appendix 8

$$
\begin{aligned}
& \frac{S y}{3}=\frac{21.04 k s i}{3}=7.01 \mathrm{ksi} \\
& \frac{S u}{5}=\frac{65.6 \mathrm{ksi}}{5}=13.12 \mathrm{ksi} \\
& \Rightarrow U_{s e}: P m+P b \leq 7.01 \mathrm{ksi}
\end{aligned}
$$

\subsection{Deadweight Loads}

Per Section 4.12.3 of Reference 1, the "baskets will be able to support the fuel at $1.0 \mathrm{~g}$ while at $375^{\circ} \mathrm{C}$ ". Reference 1 does not specify the acceptance for this loading. For consistency with the Mark $1 \mathrm{~A}$ basket criteria, Reference 4 (Subsection NG) was assumed. For membrane and membrane plus bending stresses, the allowable stresses of Table 3 are

\begin{tabular}{|c|c|c|c|c|c|}
\hline \multirow{2}{*}{\multicolumn{2}{|c|}{ Temperature }} & \multirow{3}{*}{$\begin{array}{c}S_{m} \\
\text { Table 2] }\end{array}$} & \multicolumn{3}{|c|}{ Design/Level A Stress Limits } \\
\hline & & & \multirow{2}{*}{$\begin{array}{c}P_{m} \\
\left(S_{m}\right)\end{array}$} & \multirow{2}{*}{$\begin{array}{c}P L \\
\left(1.5 S_{m}\right) \\
\end{array}$} & \multirow{2}{*}{$\begin{array}{c}\left(P_{m} \circ P_{L}\right)+P_{b} \\
\left(1.5 S_{m}\right)\end{array}$} \\
\hline${ }^{\circ} \mathrm{F}$ & ${ }^{\circ} \mathrm{C}$ & & & & \\
\hline 212 & 100 & $16.7 \mathrm{ksi}$ & $16.7 \mathrm{ksi}$ & $25.1 \mathrm{ksi}$ & $25.1 \mathrm{ksi}$ \\
\hline 707 & 375 & $13.5 \mathrm{ksi}$ & $13.5 \mathrm{ksi}$ & $20.3 \mathrm{ksi}$ & $20.3 \mathrm{ksi}$ \\
\hline
\end{tabular}
applied.

Table 3. Allowable Stresses - Deadweight.

stallow.xds

Notes 1: Design \& Level A Stress Limits fromNG-3221 \&NG-3222, respectively.

2: Avial compressive stresses must be limited to values

established in accordance with one of the following:

- NG-3133.3 (external pressure)

- NG-3133.6 (axial compression on cylindrical shells)

- NF-3322.1(c) (column type members)

- Code Case N284 (shell structures)

\begin{tabular}{|l|l|l|l|}
\hline & & & \\
\hline & & & \\
\hline
\end{tabular}


CLIENT: Duke Engineering Services Hanford

PROJECT: MCO Final Design $\quad$ DOC NO: HNF-SD-SNF-DR-003, Rev. 0, Appendix 8

The bottom basket center pipe and support rods are subjected to compression loading, with the potential for column buckling. Since Subsection NG does not address column buckling, Subsection NG was supplemented by Subsection NF. For the center pipe, the more restrictive of NG-3133.6 (shell buckling) or NF-3322.1(c)(2) was used. For the support rods, the NF criteria was used.

\subsection{Weld Criteria}

As indicated in Table 1, the Mark IV Storage Basket design includes three structural welds. Two of the welds are fillet welds (retaining bars and support ring). The support ring weld is loaded only during lifting operations, supports only the deadweight of the support ring, and is not significantly stressed. The support ring weld is controlled by minimum weld size requirements, rather than stress limits. The third weld (center pipe/bottom plate) was originally a fillet weld, but was later changed to a bevel weld when the inside diameter of the bottom plate was reduced in order to thicken the innermost ligaments. The acceptance criteria for these two weld types are addressed separately below.

\subsubsection{Fillet Welds}

For deadweight stacking loading $\left(375^{\circ} \mathrm{C}\right)$, the ASME-NG allowable fillet weld stresses are controlled by Para. NG-3350. Table NG-3352-1 specifies weld quality factors for various types of welds and examination methods. For the Mark IV Storage Baskets, a "surface visual examination" was assumed. Para. NG-3352 states that the quality factors are applied by "multiplying the allowable stress limit" by the quality factor specified in Table NG3352-1. However, NG-3352 has no specific direction for defining the "allowable stress limit" for fillet welds. A conservative approach would be to apply the quality factors to the "pure shear" limit of $0.6 \mathrm{~S}_{\mathrm{m}}$, (NG-3227.2). Based upon a detailed finite element analysis of a typical fillet weld (Section 8.7), a more reasonable approach is to multiply the weld quality factor times the membrane stress limit of $S_{m}$, using the throat area of the fillet weld. From Table NG-3352-1, the appropriate weld quality factors range from 0.35 to 0.4 , resulting in fillet weld throat stress allowables ranging from $4.7 \mathrm{ksi}$ to $5.4 \mathrm{ksi}$.

For the lifting load condition, ANSI N14.6 applies. As indicated in Section 6.0, above, the limiting criteria for lifting for $304 \mathrm{~L}$ SS is one third of the yield strength. For a fillet weld loaded in shear, a reasonable estimate of the shear yield strength is 0.6 times the tensile yield (see, e.g., Para. NG-3227.2), the lifting loading weld stress limits become $0.6 \times 5 y / 3=$ $0.2 \mathrm{Sy}$. For the $100^{\circ} \mathrm{C}$ max. lifting temperature, this results in an allowable of $0.2 \times 21.04=$ $4.21 \mathrm{ksi}$, which is less than the deadweight stacking allowable stress. Since the retaining

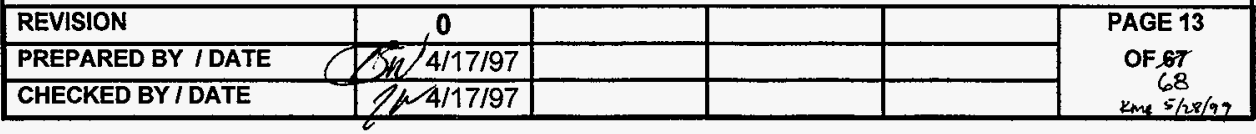


CLIENT: $\quad$ Duke Engineering Services Hanford

FILE NO: $\mathrm{KH}-8009-8-06$

PROJECT: MCO Final Design DOC NO: HNF-SD-SNF-DR-003, Rev. 0, Appendix 8

bar weld loading is the same for both the deadweight stacking and lifting load conditions, the allowable is controlled by the lifting load condition.

\subsubsection{Bevel Weld}

The center pipe/bottom plate bevel weld is loaded in compression bearing during deadweight stacking loading. The weld joint was sized to assure that there was sufficient bearing area for the deadweight stacking load condition. As indicated in Table 9.1 of Reference 8 , a partial penetration groove weld joint loaded in compression bearing, the allowable stresses are the same as the base metal. Therefore, it is not necessary to apply the weld quality factors for the deadweight stacking load condition. Base metal membrane and membrane plus bending load limits were applied to the deadweight stacking load condition.

For the lifting load condition, the center pipe/bottom plate weld is loaded in tension. The maximum principal (conservative relative to stress intensity or equivalent stress) membrane plus bending stresses in the weld were compared to the (yield stress) $/ 3$ limit as an acceptance criteria.

Table 4. Mark IV Storage Basket Weld Stress Allowables.

\begin{tabular}{|c|c|c|c|}
\hline $\begin{array}{c}\text { Weld } \\
\text { Location }\end{array}$ & Critical Load Condition & $\begin{array}{c}\text { Quality } \\
\text { Factor, } \mathbf{n}\end{array}$ & $\begin{array}{c}\text { Allowable } \\
\text { Stress, ksi }\end{array}$ \\
\hline $\begin{array}{c}\text { Center Pipe } \\
\text { to Bottom } \\
\text { Plate }\end{array}$ & Deadweight Stacking & 0.4 & $\begin{array}{c}0.24 \mathrm{Sm}= \\
3.24\end{array}$ \\
\hline $\begin{array}{c}\text { Fuel } \\
\text { Support } \\
\text { Bar to } \\
\text { Bottom } \\
\text { Plate }\end{array}$ & $\left(375^{\circ} \mathrm{C}\right)$ & 0.35 & $\begin{array}{c}0.21 \mathrm{Sm}= \\
2.84\end{array}$ \\
\hline $\begin{array}{c}\text { Support } \\
\text { Ring to } \\
\text { Bottom } \\
\text { Plate }\end{array}$ & $\left(375^{\circ} \mathrm{C}\right)$ & N/A & $0.2 S y=$ \\
\end{tabular}


CLIENT: Duke Engineering Services Hanford

FILE NO: KH-8009-8-06

PROJECT: MCO Final Design DOC NO: HNF-SD-SNF-DR-003, Rev. 0, Appendix 8

\section{LOAD CONDITIONS \& COMBINATIONS}

As previously mentioned, the Mark IV Storage Baskets were evaluated for two load cases:

1. Lifting of the basket and contents with a maximum temperature of $100^{\circ} \mathrm{C}$. This load condition was evaluated using ANSI N-14.6 safety factors.

2. Deadweight stacking of the baskets inside the $\mathrm{MCO}$ at the design temperature of $375^{\circ} \mathrm{C}$. A full stack consists of five baskets, resulting in the bottom basket supporting four baskets above.

No other loads were considered. Note that Section 4.11 of the performance specification exempts the Mark IV baskets from drop accident loading.

\section{STRESS ANALYSIS CALCULATIONS}

The Mark IV Storage Baskets were evaluated using both hand calculations (Mathcad) and finite element calculations (ANSYS). The finite element calculations were limited to stress predictions in the relatively complex bottom plate (Section 8.5).

The Mathcad calculations pages were copied from the Mathcad file. The first few pages provide the basic Mathcad input parameters and other preliminaries used in the calculations. The Mathcad pages for each component evaluated follow in the appropriate sections below.

\section{INPUT PARAMETERS:}

$$
\begin{array}{ll}
\mathrm{d}_{\mathrm{r}}=1.25 \cdot \mathrm{in} & \begin{array}{l}
\text { Support Rod Diameter } \\
\text { Support Rod Length Extending Above Bottom Plate }
\end{array} \\
\mathrm{I}_{\mathrm{r}}=24.817 \cdot \text { in } & 304 \mathrm{~L} \text { Yield Strength @ Design Temp. of } 375 \mathrm{degC} \\
\mathrm{S}_{\mathrm{y}}=14900 \frac{\mathrm{lb}}{\mathrm{in}^{2}} &
\end{array}
$$


CLIENT: Duke Engineering Services Hanford

PROJECT: MCO Final Design DOC NO: HNF-SD-SNF-DR-003, Rev. 0, Appendix 8

FILE NO: $\quad \mathrm{KH}-8009-8-06$

$\mathrm{E}=24.810^{6} \cdot \frac{\mathrm{lb}}{\mathrm{in}^{2}}$

304L Young's Modulus @ Design Temp.

$D_{0}:=2.75 \cdot$ in

Center Pipe O.D.

$\mathrm{T}:=0.5 \cdot$ in

Center Pipe Wall Thickness

$A_{p}:=\frac{\pi}{4} \cdot\left[D_{0}^{2}-\left(D_{0}-2 \cdot T\right)^{2}\right]$

$\mathrm{A}_{\mathrm{p}}=3.534 \cdot \mathrm{in}^{2}$

Center Pipe Area

$I_{p}:=\frac{\pi}{64} \cdot\left[D_{0}{ }^{4}-\left(D_{0}-2 \cdot T\right)^{4}\right]$

$I_{p}=2.347 \cdot$ in $^{4} \quad$ Center Pipe Mom. of Inertia

$r_{p}=\frac{1}{4} \cdot \sqrt{D_{o}^{2}-\left(D_{o}-2 \cdot T\right)^{2}}$

$r_{p}=0.446$ in Center Pipe Rad. of Gyration

$\mathrm{W}_{\mathrm{b}}:=\mathbf{3 2 0 0 \cdot \mathrm { lb }}$

Mark-IV Loaded Basket Upper Bound Weight, Appendix 3 of Design Report: $3190 \mathrm{lb}$

$D_{p i}:=2.50 \cdot$ in

Inside Dia. of Bottom Plate

\section{ALLOWABLE STRESSES}

\section{LIFTING LOAD CONDITION:}

Criteria: ANSI N14.6, Temp. $=100 \mathrm{deg}$, Establish Allowable Stresses:

Basic tension allowable (304L Material)-

0


CLIENT: Duke Engineering Services Hanford

PROJECT: MCO Final Design DOC NO: HNF-SD-SNF-DR-003, Rev. 0, Appendix 8

$$
\begin{aligned}
& \mathrm{S}_{\mathrm{ul} 00}:=65600 \frac{\mathrm{lb}}{\mathrm{in}^{2}} \\
& \frac{\mathrm{S}_{\mathrm{u} 100}}{5}=1.31210^{4} \cdot \frac{\mathrm{lb}}{\mathrm{in}^{2}}
\end{aligned}
$$

Base material lifting allowable controlled by yield strength

$$
\begin{aligned}
& \mathrm{S}_{\mathrm{y} 100}:=21040 \frac{\mathrm{lb}}{\mathrm{in}^{2}} \\
& \frac{\mathrm{S}_{\mathrm{y} 100}}{3}=7.013 \cdot 10^{3} \cdot \frac{\mathrm{lb}}{\mathrm{in}^{2}}
\end{aligned}
$$

Fillet Weld Allowable Stresses:

For a fillet weld, a direct application of ANSI N14.6 is to limit the shear stress in the throat of the weld to $1 / 3$ of the yield stress in pure shear. Since the shear yield stress is close to $60 \%$ of the tensile yield (see, e.g. Para. NG-3227.2: pure shear allow. $=0.6 \mathrm{Sm}$ ), this results in a weld shear stress allowable of $0.2 \mathrm{Sy}$.

\section{DESIGN CONDITION:}

Criteria: Assume ASME NG allowables, Design Temp. = $375 \operatorname{deg} C$ (707 degF),

$$
S_{\mathrm{m} 375}=13500 \cdot \frac{\mathrm{lb}}{\mathrm{in}^{2}}
$$


CLIENT: Duke Engineering Services Hanford

PROJECT: MCO Final Design DOC NO: HNF-SD-SNF-DR-003, Rev. 0, Appendix 8

$$
\mathrm{S}_{\mathrm{y} 375}=14900 \cdot \frac{\mathrm{b}}{\mathrm{in}^{2}}
$$

Fillet Weld Allowable Stress:

Section 6.3 of this calculation report addresses the Mark IV fillet weld allowable stresses, which are applied to the Mark IV basket fillet welds below.

\subsection{Center Pipe}

Membrane Stress (controlled by design condition)

$$
P_{m}:=\frac{4 \cdot W_{b}}{A_{p}}
$$

Conservatively assume all four baskets above bottom basket carried by center pipe

$$
\begin{aligned}
& \mathrm{P}_{\mathrm{m}}=3.622 \cdot 10^{3} \cdot \frac{\mathrm{lb}}{\mathrm{in}^{2}} \\
& \text { Ratio }=\frac{\mathrm{P}_{\mathrm{m}}}{\mathrm{S}_{\mathrm{m} 375}} \quad \text { Membrane stress OK }
\end{aligned}
$$

Check Buckling per ASME NG-3133.6 (also consider NF-3322.1(c)(2) for column buckling)

$$
\mathrm{T}=0.5 \cdot \text { in }
$$


CLIENT: Duke Engineering Services Hanford

PROJECT: MCO Final Design DOCNO: HNF-SD-SNF-DR-003, Rev. 0, Appendix 8

$$
\begin{aligned}
& R:=\frac{D_{0}}{2}-T \\
& R=0.875 \cdot \text { in } \\
& A:=\frac{0.125}{\left(\frac{R}{T}\right)}
\end{aligned}
$$

$\mathrm{A}=0.071$

Obtain B value from ASME Code, Section II, Figure HA-3

$$
\begin{aligned}
& \text { B }:=7560 \cdot \frac{\mathrm{lb}}{\mathrm{in}^{2}} \\
& \text { Ratio }:=\frac{\mathrm{P}_{\mathrm{m}}}{\mathrm{B}}
\end{aligned}
$$

Ratio $=0.476$ Axial compressive stress limit OK per ASME NG

Checking column buckling per ASME NF 3322.1:

$$
\begin{aligned}
& \mathrm{E}=2.48 \cdot 10^{7} \cdot \frac{\mathrm{lb}}{\mathrm{in}^{2}} \\
& \mathrm{I}_{\mathrm{p}}=2.347 \cdot \mathrm{in}^{4} \\
& \mathrm{~K}_{\mathrm{p}}:=0.8 \quad \text { Effective length factor, pinned @ top, Reference } 8 \\
& \mathrm{I}_{\mathrm{p}}=\mathrm{I}_{\mathrm{r}} \\
& \mathrm{r}_{\mathrm{p}}=0.53 \cdot \mathrm{in}
\end{aligned}
$$

\begin{tabular}{|c|l|l|l|}
\hline 0 & & & \\
\hline$/ \operatorname{su}_{4 / 17 / 97}$ & & & \\
\hline
\end{tabular}


CLIENT: Duke Engineering Services Hanford

FILE NO: $\mathrm{KH}-8009-8-06$

PROJECT: MCO Final Design DOC NO: HNF-SD-SNF-DR-003, Rev. 0, Appendix 8

$$
\begin{aligned}
& \frac{\mathrm{K}_{\mathrm{p}} \cdot \mathrm{p}}{\mathrm{r}_{\mathrm{p}}}=37.436<120, \text { use Eq. (6a) } \\
& \mathrm{F}_{\mathrm{a}}:=\mathrm{S}_{\mathrm{y} 375}\left[\begin{array}{r}
\frac{\mathrm{K}_{\mathrm{p}} \cdot \mathrm{l} p}{\mathrm{r}_{\mathrm{p}}} \\
444
\end{array}\right] \quad<\mathrm{B} \text {, above, column buckling controls } \\
& \begin{array}{l}
\mathrm{F}_{\mathrm{a}}=5.747 \cdot 10^{3} \cdot \frac{\mathrm{lb}}{\mathrm{in}^{2}} \\
\text { Ratio: }=\frac{\mathrm{P}_{\mathrm{m}}}{\mathrm{F}_{\mathrm{a}}} \quad<1.0, \text { ASME NF limits met }
\end{array} \\
& \text { Ratio }=0.63
\end{aligned}
$$

Evaluate Center Pipe/Bottom Plate Weld:

Weld geometry:

\section{Center Pipe}

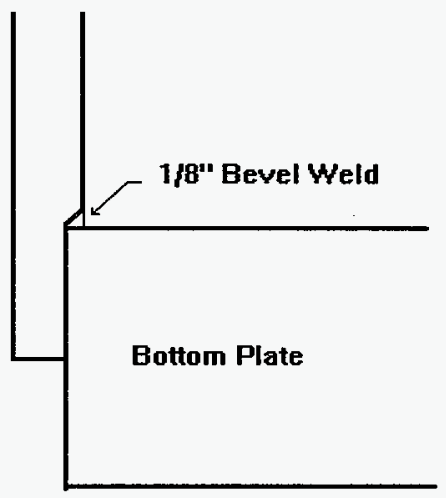


CLIENT: Duke Engineering Services Hanford

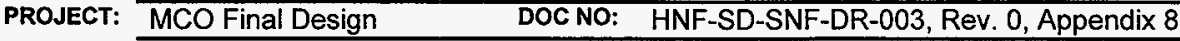

\section{BEVEL WELD SIZING:}

Check membrane stress in weld for deadweight stacking for a 1/8" bevel weld:

$$
\begin{aligned}
& \mathrm{A}_{\text {weld }}:=\frac{\pi}{4} \cdot\left[(2.75 \text { in })^{2}-(2.5 \cdot \mathrm{in})^{2}\right] \\
& P_{\mathrm{m}}: \frac{4 \cdot \mathrm{W}_{\mathrm{b}}}{\mathrm{A}_{\text {weld }}} \\
& P_{\mathrm{m}}=1.242 \cdot 10^{4} \cdot \frac{\mathrm{lb}}{\mathrm{in}^{2}} \\
& \text { Ratio }=\frac{\mathrm{P}_{\mathrm{m}}}{\mathrm{S}_{\mathrm{m} 375}}
\end{aligned}
$$

Ratio $=0.92 \quad 1 / 8^{\prime \prime}$ weld is sufficient for deadweight stacking loading

Check $1 / 8^{\prime \prime}$ Bevel Weld for lifting load condition (maximum lifting temperature $=100^{\circ} \mathrm{C}$ ):

$$
\begin{aligned}
& \mathrm{P}_{\mathrm{m}}:=\frac{\mathrm{W}_{\mathrm{b}}}{\mathrm{A}_{\text {weld }}} \\
& \mathrm{P}_{\mathrm{m}}=3.104 \cdot 10^{3} \cdot \frac{\mathrm{lb}}{\mathrm{in}^{2}} \\
& \frac{\mathrm{S}_{\mathrm{y} 100}}{3}=7.013 \cdot 10^{3} \cdot \frac{\mathrm{lb}}{\mathrm{in}^{2}}
\end{aligned}
$$

$\mid$\begin{tabular}{c|}
0 \\
$\mid 3 w 4 / 17 / 97$ \\
$1 / 4 / 17 / 97$
\end{tabular}

\begin{tabular}{|l|l|l|}
\hline & & \\
\hline & & \\
\hline & & \\
\hline
\end{tabular}


CLIENT: Duke Engineering Services Hanford

FILE NO: KH-8009-8-06

PROJECT: MCO Final Design DOCNO: HNF-SD-SNF-DR-003, Rev. 0, Appendix 8

$$
\text { Ratio }=\frac{\mathrm{P}_{\mathrm{m}}}{\left(\frac{\mathrm{S}_{\mathrm{y} 100}}{3}\right)}
$$

Ratio $=0.443$

Thus, the bevel weld is adequate per the Reference 6 allowable of Sy/3.

\subsection{Support Rod}

$$
\begin{aligned}
& \mathrm{d}_{\mathrm{r}}:=1.25 \mathrm{in} \\
& \mathrm{I}_{\mathrm{r}}:=\frac{\pi \cdot \mathrm{d}_{\mathrm{r}}^{4}}{64} \\
& \mathrm{I}_{\mathrm{r}}=0.12 \cdot \mathrm{in}^{4} \\
& \mathrm{~K}_{\mathrm{r}}=2.1 \quad \text { Effective length factor, fixed-free, Reference 8 } \\
& \mathrm{I}_{\mathrm{r}}=24.817 \cdot \mathrm{in} \\
& \mathrm{r}_{\mathrm{r}}:=\frac{\mathrm{d}_{\mathrm{r}}}{4} \\
& \mathrm{r}_{\mathrm{r}}=0.313 \cdot \mathrm{in} \\
& \mathrm{A}_{\mathrm{r}}=\frac{\pi \cdot \mathrm{d}_{\mathrm{r}}^{2}}{4} \\
& \mathrm{~A}_{\mathrm{r}}=1.227 \cdot \mathrm{in}^{2}
\end{aligned}
$$

\begin{tabular}{|c|}
\hline$\underline{0}$ \\
\hline $5 / 4 / 17 / 97$ \\
\hline$/ \mu / 4 / 17 / 97$ \\
\hline
\end{tabular}

Using ASME NF 3322.1(c)(2) 
CLIENT: Duke Engineering Services Hanford

PROJECT: MCO Final Design DOC NO: HNF-SD-SNF-DR-003, Rev. 0, Appendix 8

FILE NO: KH-8009-8-06

$$
\begin{aligned}
& \frac{\mathrm{K}_{r^{-1}}{ }_{r_{r}}}{{ }^{1}}=166.77 \quad>120 \text {, use Eq. }(6 b) \\
& F_{a}=S_{y 375} \cdot\left[\begin{array}{r}
\frac{K_{r} \cdot r_{r}}{r_{r}} \\
600
\end{array}\right] \\
& \mathrm{F}_{\mathrm{a}}=1.806 \cdot 10^{3} \cdot \frac{\mathrm{lb}}{\mathrm{in}^{2}}
\end{aligned}
$$

Conservatively assuming the bottom basket support rods carry the full weight of the four baskets above,

$$
\begin{aligned}
& F_{r}=\frac{4 \cdot W_{b}}{6 \cdot A_{r}} \\
& F_{r}=1.738 \cdot 10^{3} \cdot \frac{1 b}{i^{2}} \\
& \text { Ratio }=\frac{F_{r}}{F_{a}}
\end{aligned}
$$

Ratio $=0.962<1.0$, ASME NF requirements met for support rods

\subsection{Fuel Retaining Bars}

Critical load condition is lifting due to the ANSI N-14.6 lower allowables.

$$
b:=0.25 \text { in Retaining bar cross section per dwg.H-2-828070 }
$$

\section{$b:=0.25 \mathrm{in} \quad$ Retaining bar cross section per dwg. $\mathrm{H}-2-828070$}


PROJECT: MCO Final Design DOC NO: HNF-SD-SNF-DR-003, Rev. 0, Appendix 8

$$
\begin{aligned}
& h=0.375 \mathrm{in} \\
& I_{b}:=\frac{b \cdot h^{3}}{12} \\
& I_{b}=0.001 \cdot \text { in }^{4}
\end{aligned}
$$

Due to the deflection of the bar, the load would tend to concentrate near the O.D. of the hole. However, even if it is conservatively assuming a simply supported beam with the fuel rod load concentrated at the center, the support bar is adequate:

$$
\begin{aligned}
& W_{f}:=55.4 \mathrm{lb} \\
& I_{b}:=2.75 \cdot \mathrm{in} \\
& \sigma_{b}:=\frac{W_{f^{1}} \cdot \frac{h}{2}}{4 \cdot I_{b}} \\
& \sigma_{b}=6.5 \cdot 10^{3} \cdot \frac{\mathrm{lb}}{\mathrm{in}^{2}}
\end{aligned}
$$

Using ANSI N-14.6, the bending stress is limited to Sy/3 @ 100 degC-

$$
\begin{aligned}
& \frac{\mathrm{S}_{\mathrm{y} 100}}{3}=7.013 \cdot 10^{3} \cdot \frac{\mathrm{lb}}{\mathrm{in}^{2}} \\
& \text { Ratio }:=\frac{\sigma_{\mathrm{b}}}{\left(\frac{\mathrm{S}_{\mathrm{y} 100}}{3}\right)}
\end{aligned}
$$

Ratio $=0.927$

Fuel retaining bar OK in bending.

Checking shear in the retaining bar,

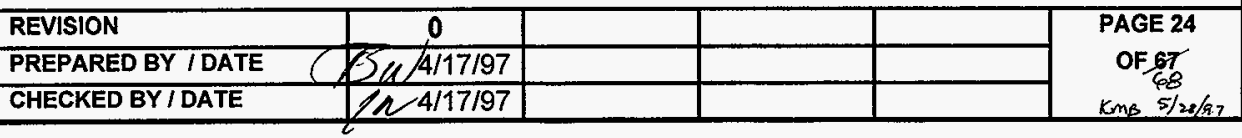


CLIENT: Duke Engineering Services Hanford

PROJECT: MCO Final Design DOC NO: HNF-SD-SNF-DR-003, Rev. 0, Appendix 8

$$
\begin{aligned}
& F_{s}=\frac{W_{f}}{2 \cdot b \cdot h} \\
& F_{S}=295.467 \cdot \frac{1 b}{\text { in }^{2}}
\end{aligned}
$$

The yield strength in shear is about $60 \%$ of the tensile yield, resulting in a lifting allowable of $0.6(\mathrm{~S} y / 3)=0.2 \mathrm{Sy}$.

$$
\begin{aligned}
& \text { Ratio }=\frac{F_{S}}{0.2 \cdot S_{y 100}} \\
& \text { Ratio }=0.07
\end{aligned}
$$

Retaining bar OK in shear

Check the length of the fillet welds attaching the fuel retaining bars to bottom plate.

Assume 1/8" welds (ASME NG \& NF minimum), on one side of the bar. Since the available weld length is less than $4 \times 1 / 8^{\prime \prime}=1 / 2^{\prime \prime}$, the maximum effective weld size is $0.375^{\prime \prime} / 4=3 / 32^{\prime \prime}$ (ASME NF-3324.5(d)(3)(b)):

$$
\begin{aligned}
& \mathrm{A}_{\mathrm{w}}:=\frac{\sqrt{2}}{2} \cdot \frac{3}{32} \cdot \mathrm{in} \cdot 0.375 \cdot \mathrm{in} \\
& \mathrm{A}_{\mathrm{w}}=0.025 \cdot \mathrm{in}^{2} \\
& \text { Ratio }=\frac{\frac{\mathrm{W}_{\mathrm{f}}}{0.35 \cdot 0.6 \cdot \mathrm{S}} \mathrm{m} 375}{\mathrm{~A}_{\mathrm{w}}} \\
& \text { Ratio }=0.786
\end{aligned}
$$

Thus, a single $1 / 8^{\prime \prime}$ fillet weld, the full height ( $\left.3 / 8^{\prime \prime}\right)$ of the bar, at each ligament is adequate.

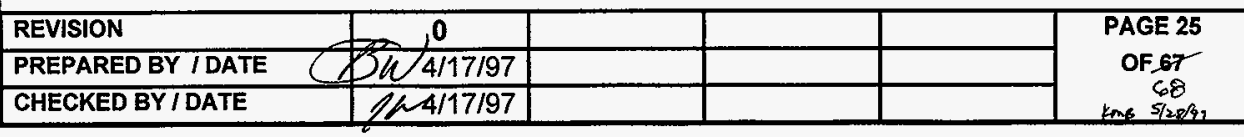


PROJECT: MCO Final Design DOC NO:

FILE NO: KH-8009-8-06

\subsection{Support Ring}

The critical loading for the support ring occurs in the next-to-the-bottom basket when the baskets are stacked inside the MCO at the design temperature. The critical support ring location corresponds to a support rod position from the bottom basket being centered on the fuel hole support ring span, as shown below. Conservatively assume bottom basket rod supports $1 / 6$ of weight of 4 baskets stacked above (no support from center tube):

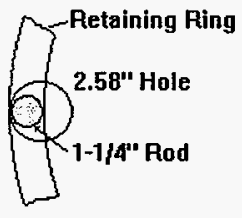

$$
\begin{aligned}
& F_{r}:=\frac{4 \cdot W_{b}}{6} \\
& F_{r}=2.118 \cdot 10^{3} \cdot 1 b
\end{aligned}
$$

Assume a $3 / 8^{\prime \prime}$ thick, 2.58 " $\times 1.25^{\prime \prime}$ rectangular plate simply supported on 3 sides. As the plate deflects under the support rod loading, the load would tend to concentrate furthest from the free edge. Thus, it is reasonable to assume that the loading linearly increases away from the free edge. Using Table 26, Case 2d. from Roark 5th Ed. (Reference 7) and the rectangular approximation below,

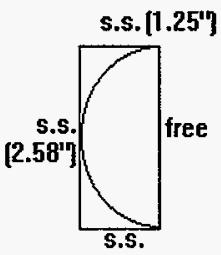

$$
q=\frac{2 \cdot F_{\mathrm{I}}}{(2.58 \cdot \operatorname{in} 1.25 \cdot \mathrm{in})}
$$

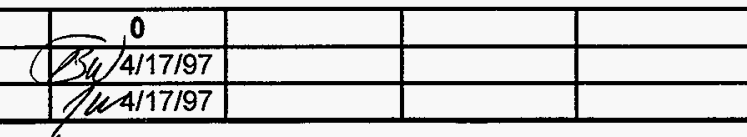


CLIENT: Duke Engineering Services Hanford

PROJECT: MCO Final Design DOC NO: HNF-SD-SNF-DR-003, Rev. 0, Appendix 8

$$
\begin{aligned}
& q=1.323 \cdot 10^{3} \cdot \frac{\mathrm{lb}}{\mathrm{in}^{2}} \\
& \beta=0.11 \quad \mathrm{a} / \mathrm{b}=0.50 \\
& \mathrm{~b}=2.58 \cdot \text { in } \\
& \mathrm{t}:=0.375 \cdot \text { in } \quad \text { Rectangular Plate Approximation } \\
& P_{b}:=\frac{\beta \cdot \mathrm{q} \cdot \mathrm{b}^{2}}{\mathrm{t}^{2}} \quad \mathrm{P}_{\mathrm{b}}=6.889 \cdot 10^{3} \cdot \frac{\mathrm{lb}}{\mathrm{in}^{2}}
\end{aligned}
$$

ASME III-NG Design Cond. Allowable (Para. NG-3221.2):

$$
\begin{aligned}
& 1.5 \cdot \mathrm{S}_{\mathrm{m} 375}=2.025 \cdot 10^{4} \cdot \frac{\mathrm{lb}}{\mathrm{in}^{2}} \\
& \text { Ratio }:=\frac{\mathrm{P}_{\mathrm{b}}}{1.5 \cdot \mathrm{S}_{\mathrm{m} 375}} \\
& \text { Ratio }=0.34 \quad<1.0 \text { O.K. }
\end{aligned}
$$

The fillet welds which attach the support ring to the bottom plate are loaded only during lifting operations. During lifting, the weld stresses are very small, since they carry only the dead weight of the support ring:

Assume 1" welds @ 30 deg. (5.92") spacing (12 welds total):

$$
\begin{aligned}
& \mathrm{W}_{\text {ring }}=\frac{\pi}{4} \cdot\left[(22.375 \cdot \mathrm{in})^{2}-(19.875 \cdot \mathrm{in})^{2}\right] \cdot 0.375 \cdot \mathrm{in} 0.286 \cdot \frac{\mathrm{lb}}{\mathrm{in}^{3}} \\
& \mathrm{~W}_{\text {ring }}=8.897 \cdot \mathrm{lb}
\end{aligned}
$$


CLIENT: Duke Engineering Services Hanford

PROJECT: MCO Final Design

FILE NO: KH-8009-8-06

For 1/8" fillet welds (Min. size per ASME III, Fig. NG 4427-1), the throat shear stress is

$$
\begin{aligned}
& \tau:=\frac{W_{\text {ring }}}{0.125 \cdot \operatorname{in} \frac{\sqrt{2}}{2} \cdot 12 \cdot 1 \cdot \text { in }} \\
& \tau=8.388 \cdot \frac{\mathrm{lb}}{\mathrm{in}^{2}} \\
& 0.2 \cdot \mathrm{S}_{\mathrm{y} 100}=4.208 \cdot 10^{3} \cdot \frac{\mathrm{lb}}{\mathrm{in}^{2}} \\
& \text { Ratio }=\frac{\tau}{0.2 \cdot \mathrm{S}_{\mathrm{y} 100}} \quad<<1.0, \text { weld stresses O.K. } \\
& \text { Ratio }=0.002
\end{aligned}
$$

The fillet welds which attach the support ring to the bottom plate are loaded only during lifting operations. During lifting operations, the weld stresses are negligible, since they carry only the dead weight of the support ring. However, Fig. NG-4427-1 of Subsection NG specifies a minimum fillet weld dimension of $1 / 8$ ". However, since the weld is essentially non-structural, a smaller weld size is permissible.

\subsection{Bottom Plate}

The bottom plate was evaluated for both the deadweight stacking condition inside the MCO and the lifting condition. These load conditions are evaluated in the subsections that follow.

\subsubsection{Deadweight Stacking Load Condition}

The critical bottom plate for this load condition is the second basket from the bottom with the bottom basket support rods rotated $30^{\circ}$ relative to the support rods for the basket immediately above. It was conservatively assumed that the weight of the top three baskets is carried entirely by the support rods of the fourth basket. This configuration develops the maximum bending stress in the bottom plate.

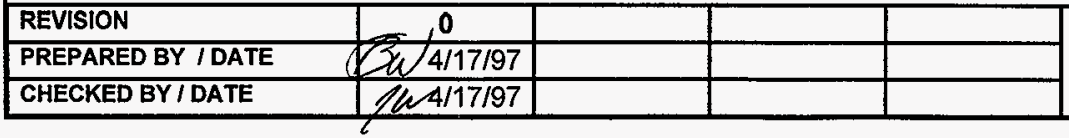


CLIENT: Duke Engineering Services Hanford

FILE NO: KH-8009-8-06

PROJECT: MCO Final Design DOC NO: HNF-SD-SNF-DR-003, Rev. 0, Appendix 8

For this load condition, the fourth basket support rods would each carry one-sixth of the weight of the top three baskets (single basket weight $=3177 \mathrm{lb}$ per Appendix A of the Performance Spec.):

$$
F_{r}=(3 \times 3177) / 6=1588 \mathrm{lbs}
$$

In addition to the loading on the support rods, the bottom plate also carries the weight of the fuel rods in the basket. The fuel rod weight was applied as an equivalent pressure (Single rod weight $=55.4 \mathrm{lb}$ per App. A of the Performance Spec.):

$$
\begin{aligned}
& \text { Area }=1 / 4\left[(22.625)^{2}-(2.75)^{2}\right] \pi-1 / 4(2.58)^{2}(54 \pi)=113.8 \mathrm{in}^{2} \\
& \text { Press }=54(55.4) / \text { Area }=26.3 \mathrm{psi} .
\end{aligned}
$$

The equivalent pressure approach was judged to be conservative because it moves the center of loading radially outward, relative to the actual fuel support locations, resulting in higher bending moments at the maximum stress locations (outer ligaments, as shown below).

Due to the $60^{\circ}$ symmetry, only one-sixth of the basket was modeled. The $60^{\circ}$ sector ANSYS finite element model of the Mark IV Storage Basket is shown in Figure 2. The bottom plate and center pipe were modelled using SOLID45 elements, and the support rod was modelled using BEAM4 elements. To account for a 1/8-inch reduction of wall thickness at the bottom of the center pipe, the thinner wall dimension was conservatively used for the full height of the pipe.

For the deadweight stacking load condition, the loading was comprised of a $1588 \mathrm{lb}$ downward vertical load at the top of the support rod, a 26.3 psi downward pressure on the bottom plate, and a $1.0 \mathrm{~g}$ deadweight acceleration. The model was constrained vertically at the bottom outer corners (adjacent basket, below, support rod locations, $30^{\circ}$ rotation). The ANSYS input data for the deadweight stacking load case is provided in the pltstk.inp and plstk.out files.

The deadweight stacking load case results are summarized in the form of a stress intensity contour plot shown in Figure 3. Note that the higher stress intensity values occur in the outermost ligaments. The maximum stress intensity location is the bottom (inside) of the ligaments adjacent to the support rod constraints. As indicated in the legend, the maximum stress intensity is just under $10 \mathrm{ksi}$. As directed by Table NG-3217-1 of the ASME Code, it is permissable to define the primary bending stress in a ligament as the average of the

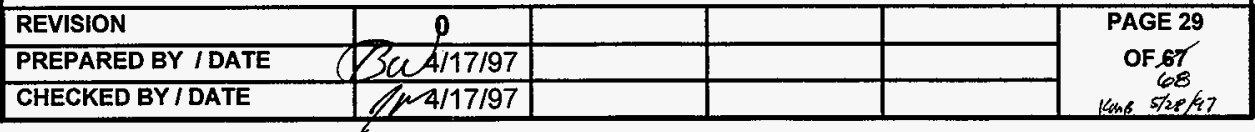


CLIENT: Duke Engineering Services Hanford

PROJECT: MCO Final Design DOC NO: HNF-SD-SNF-DR-003, Rev. 0, Appendix 8

surface stress through the width of a ligament. However, for simplicity, the maximum ligament stress of $7.3 \mathrm{ksi}$ was used. From Table 3 , the allowable primary membrane plus bending stress intensity is $20.3 \mathrm{ksi}$, resulting is a stress ratio of $7.3 / 20.3=0.36$ for the deadweight stacking load case. This relatively low stress ratio indicates that further refinement of the modeling and load application is not warranted.

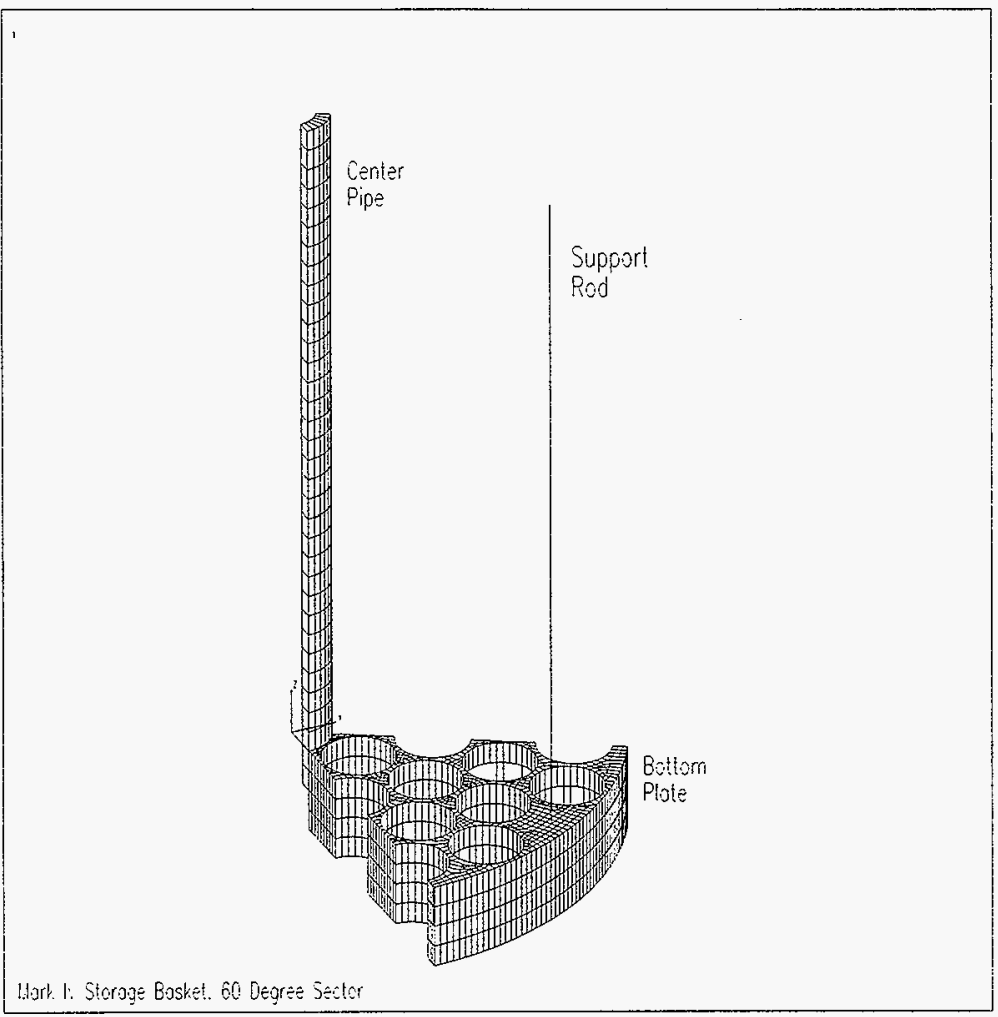

$41151550 \div 50$

OE: 311996

10:10:16

PLOT NO.

ELEIIENTS

TIPE WUW

$x=0.784886$

$n=-0.365998$

Z.: $=0.5$

DIST $=\$ 6.607$

if $=5.875$

if $=4.898$

if $=10.39$

$\therefore-7 S=-76.876$

PPECISE HIOCEH

Figure 2. Sixty-Degree Sector Finite Element Model of the Mark IV Storage Basket. 
CLIENT: Duke Engineering Services Hanford

PROJECT: MCO Final Design DOC NO: HNF-SD-SNF-DR-003, Rev. 0, Appendix 8

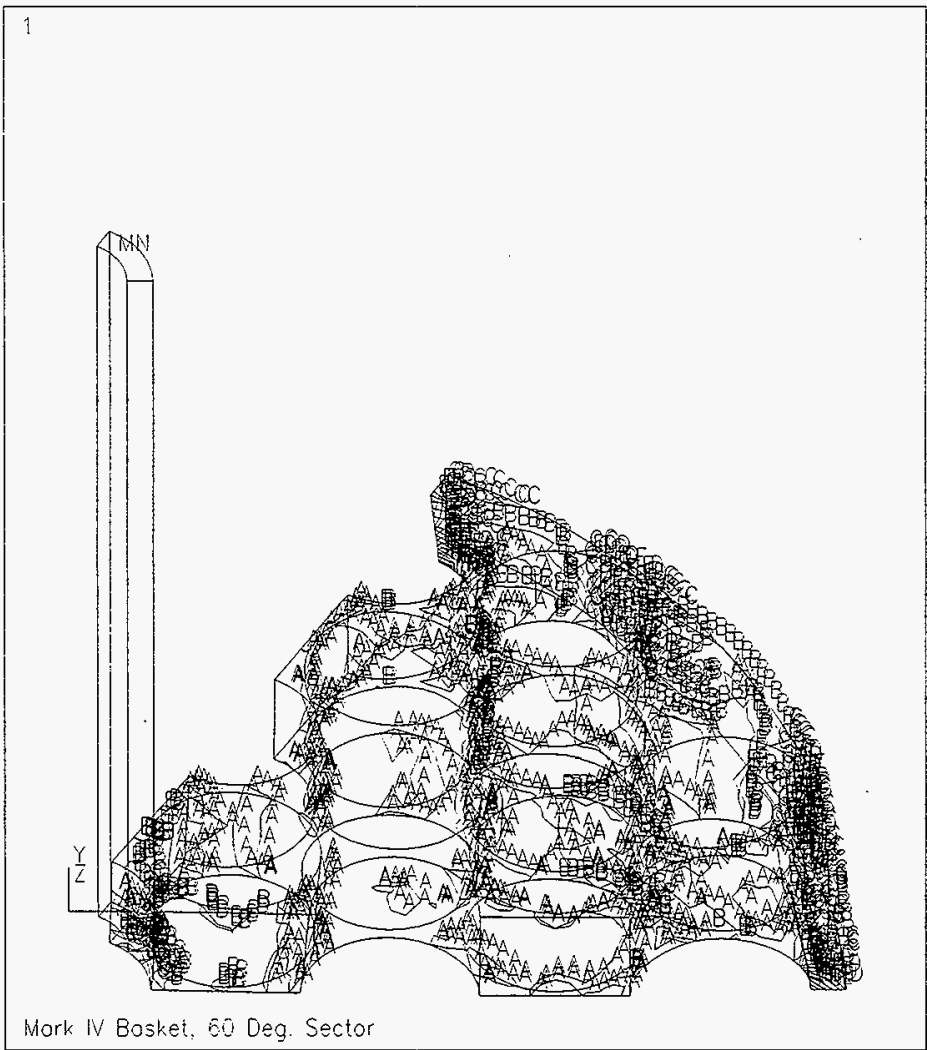

ANSYS 5.0 A 50

JâH 231907

15:27:42

PLOT NO

NOOAL SOLUTION

STEP $=1$

SUE $=1$

$\mathrm{T} \mid \mathrm{ME}=$ ?

SiNT

(AVQ)

TOP

$D M \lambda=0.002123$

SMN $=0.078918$

SMX $=7765$

SM: $B=1064^{7}$

$A=431.464$

$B=1294$

C $=2157$

$\mathrm{O}=3020$

$E=3883$

$F=474$

$\hat{G}=5008$

$H=54 ? 1$

$1=-534$

Figure 3. Stress Intensity Contour Plot, Deadweight Stacking Load Condition.

\subsubsection{Lifting Load Condition}

The Figure 3 ANSYS model was also used for the lifting load case, with boundary condition changes. The only constraint was a vertical constraint at the top of the center pipe (lifting location). The loading included $1.0 \mathrm{~g}$ gravity loading and the $26.3 \mathrm{psi}$ downward pressure

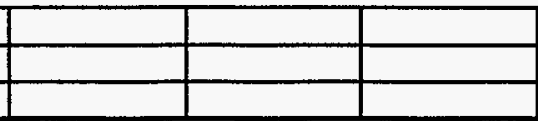


representing the gravity loading from the fuel. The lifting load results are summarized in the stress intensity plot provided in Figure 4. Note that a maximum stress intensity of about 9 ksi occurred in the bottom of the ligament at the inside radius of the plate. This maximum ligament stress is essentially all primary bending stress and exceeds the allowable lifting stress of $7.0 \mathrm{ksi}$ discussed in Section 6.1 .

The inner-most ligaments, per the $\mathrm{H}-2-827591$ drawing, are only 0.085 inches thick. By decreasing the O.D. of the center pipe, the thickness of the inner-most ligaments can be correspondingly increased. The currently specified O.D. of the center pipe is $2.75 \mathrm{in}$. If the center pipe O.D. is decreased to 2.50 in., the ligament thickness is increased by $1 / 8$-in., resulting in a ligament thickness of 0.210 in. This increased ligament thickness geometry modification was evaluated with the ANSYS model (ANSYS Files: plttft.inp and pltff.out). The results are summarized in the Figure 6 contour plot. Note that the maximum stress intensity $(5.5 \mathrm{ksi})$ occurs at a different location than for the thinner ligament model and is less than the $7.01 \mathrm{ksi}$ allowable:

$$
\text { Ratio : }=\frac{5463}{7010}
$$

Ratio $=0.779$

\begin{tabular}{l}
\hline REVISION \\
\hline PREPARED BY I DATE \\
\hline CHECKED BY I DATE
\end{tabular}

$\frac{1}{1 / 4} \frac{0}{4 / 17 / 97}$


CLIENT: Duke Engineering Services Hanford

FILE NO: KH-8009-8-06 PROJECT: MCO Final Design $\quad$ DOC NO: HNF-SD-SNF-DR-003, Rev. 0, Appendix 8

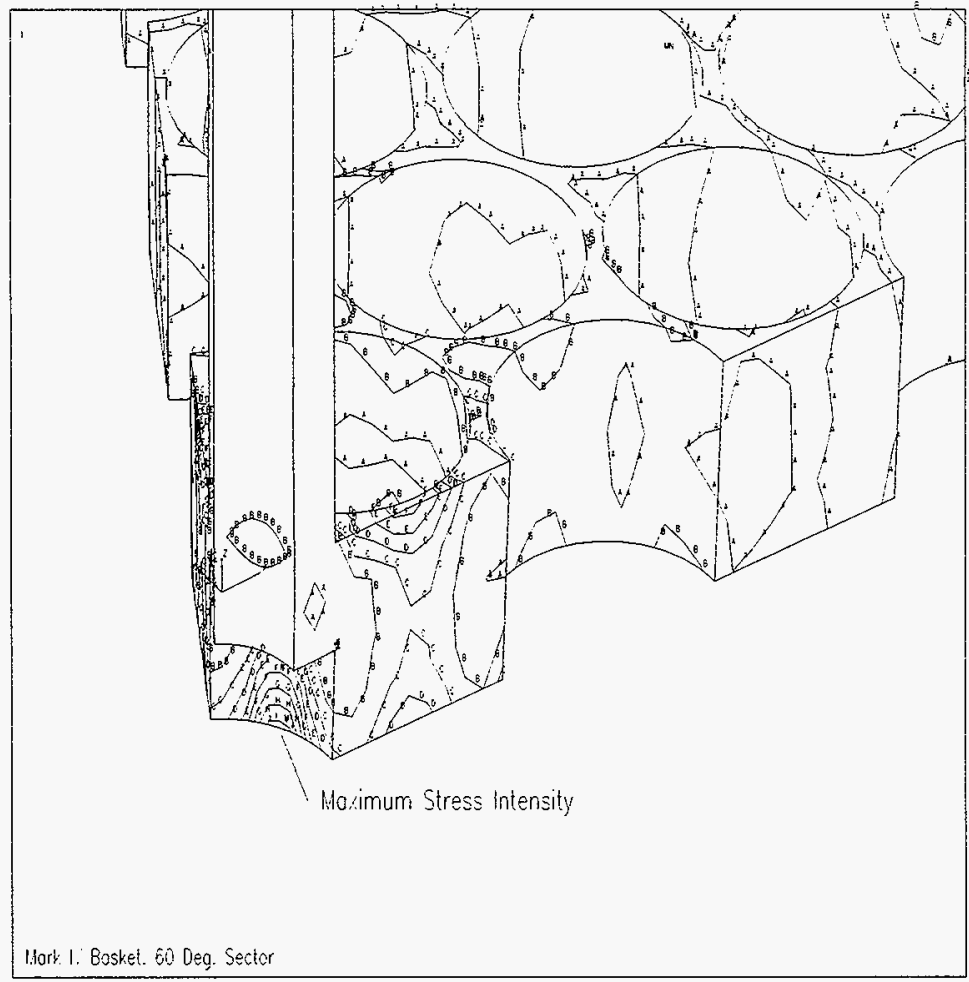

-15 is 50.56

DÊ if $130 \hat{0}$

15.58:?0

PLOT NQ. I

HOCAL SOLUTIOH

$S I E P=1$

SUE $=1$

TWE = ?

SINT $\quad A: G)$

TOP

$D k X=0.005706$

$S M N=14.535$

$5 \mathrm{~W} X=9185$

$S M \times B=10826$

$A=523.817$

$\mathrm{B}=1543$

C $=2562$

D $=3581$

$E=4600$

$F=5519$

$G=6058$

$\mathrm{H}=7657$

I $=867 \hat{0}$

Figure 4. Stress Intensity Contour Plot for an Inside Ligament Thickness of 0.085 in. 
CLIENT: Duke Engineering Services Hanford

PROJECT: MCO Final Design DOC NO: HNF-SD-SNF-DR-003, Rev. 0, Appendix 8

FILE NO: KH-8009-8-06

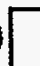

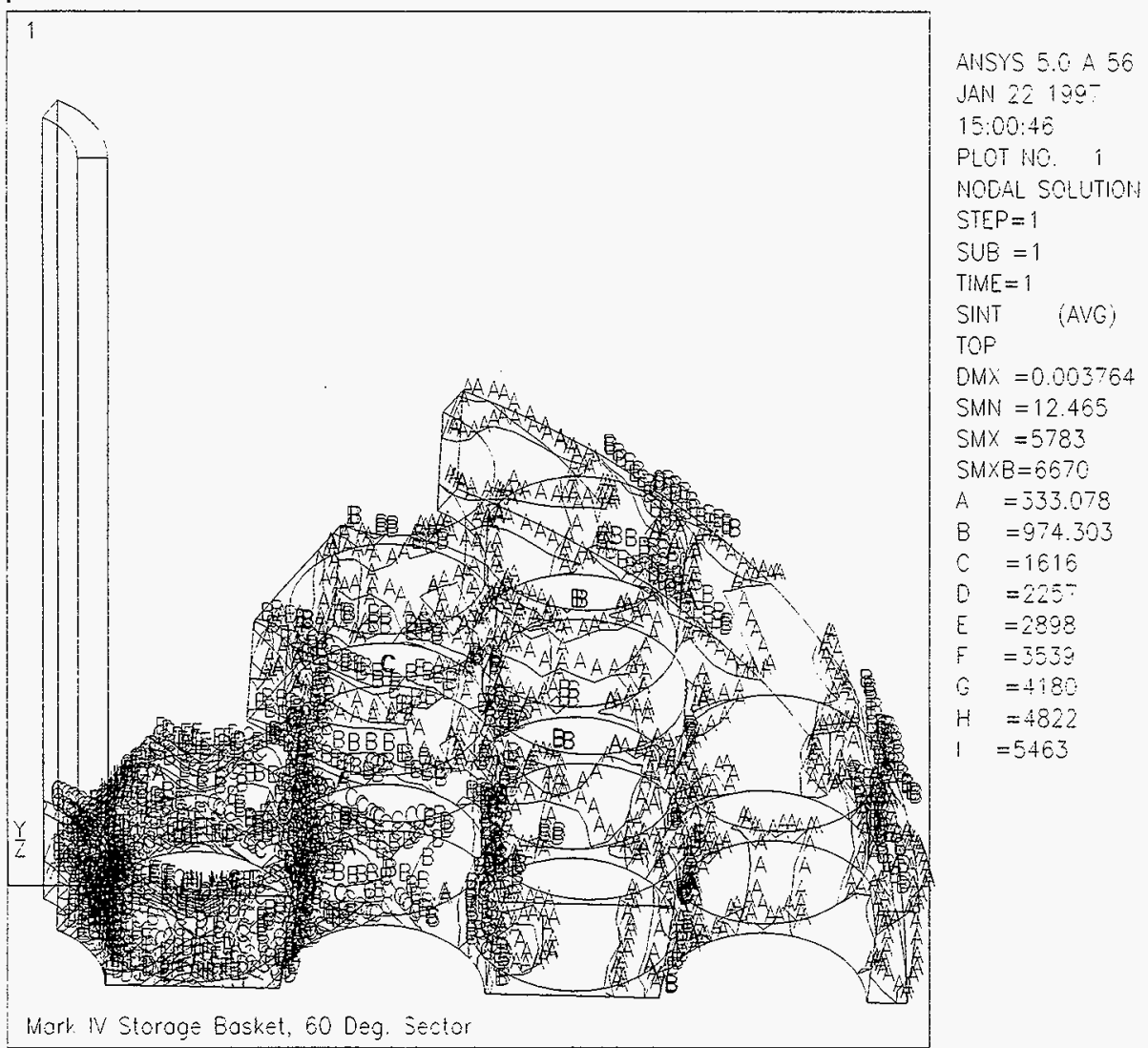

Figure 5. Stress Intensity Contour Plot for an Inside Ligament Thickness of 0.210 in.

\begin{tabular}{|c|c|c|}
\hline 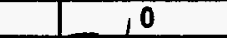 & & \\
\hline b/1/ 4/17/97 & & \\
\hline I/ 4/17/97 & & \\
\hline
\end{tabular}




\subsection{Load Distribution and Basket Interface Considerations}

When the baskets are stacked inside the MCO, the center pipe and support rod load distribution is very sensitive to the interface geometry. Reasonable fabrication tolerances will result in an imperfect fit, which will likely result in a three-point contact at the basket interface (three rods or two rods and the center pipe). There is also the possibility that the center pipe will carry the entire load.

Even for the case of a perfect fit, the stack loading will not be evenly shared between the center pipe and rods. Using the Figure 3 ANSYS model, the perfect fit load distribution was calculated by applying the fuel deadweight pressure to the bottom plate, and obtaining the reactions at the support rod and center pipe (ANSYS files: pitld.inp and pltld.out). From the ANSYS output, the support rod and center pipe reactions were $397 \mathrm{lb}$ and $137 \mathrm{lb}$, respectively ( $534 \mathrm{lb}$ total). The center pipe load ratio is $137 / 534=0.26$. However, for the imperfect fit, the center pipe load ratio could range from zero to one.

In order to establish reasonable tolerances on the basket interface dimensions, capacity force/deflection response predictions were made for both a support rod and the center pipe. By knowing the force/deflection response, the effect of component length differences on the component load sharing can be evaluated. The capacity force/deflection response was obtained using the ANSYS plastic beam element (BEAM23), with large deflections/strain enabled. Buckling was initiated by assuming a 0.25 -in. offset of the vertical load.

Assuming the top of the support rod is unrestrained laterally, the predicted support rod force/deflection response is shown in Figure 7 (ANSYS input/output files: rodb.inp/rodb.out). Assuming that three support rods are supporting four baskets above, the force per rod is $4(3177) / 3=4236 \mathrm{lb}$. Note, from Figure 7, that a deflection of about 0.070 inches is achieved in a support rod before the load capacity drops below $4236 \mathrm{lb}$. A less conservative force/deflection rod response was obtained by using a gap/friction element on top of the rod to account for lateral constraint due to friction. Using a conservative friction coefficient of 0.1 , the response shown in Figure 8 was obtained (ANSYS input/output files: rodbf.inp/rodbf.out). Note that a much higher capacity and deformation was obtained, when rod frictional constraint was considered.

The force/deflection response of the center pipe was also obtained as shown in Figure 9 (ANSYS input/output files: pipeb.inp/pipeb.out). Assuming that the bottom center pipe carries the full load from the four baskets above, the center pipe loading is $4(3177)=$ $12,710 \mathrm{lb}$, as shown in the figure. As indicated in the figure, the center pipe capacity is well in excess of the loading. Also note that with an 1/8-in. center pipe deflection, the plastic buckling mode has not been reached.

\begin{tabular}{l}
\hline REVISION \\
\hline PREPARED BY I DATE \\
\hline CHECKED BY / DATE
\end{tabular}

18

$\frac{34}{24}$

0

Trent

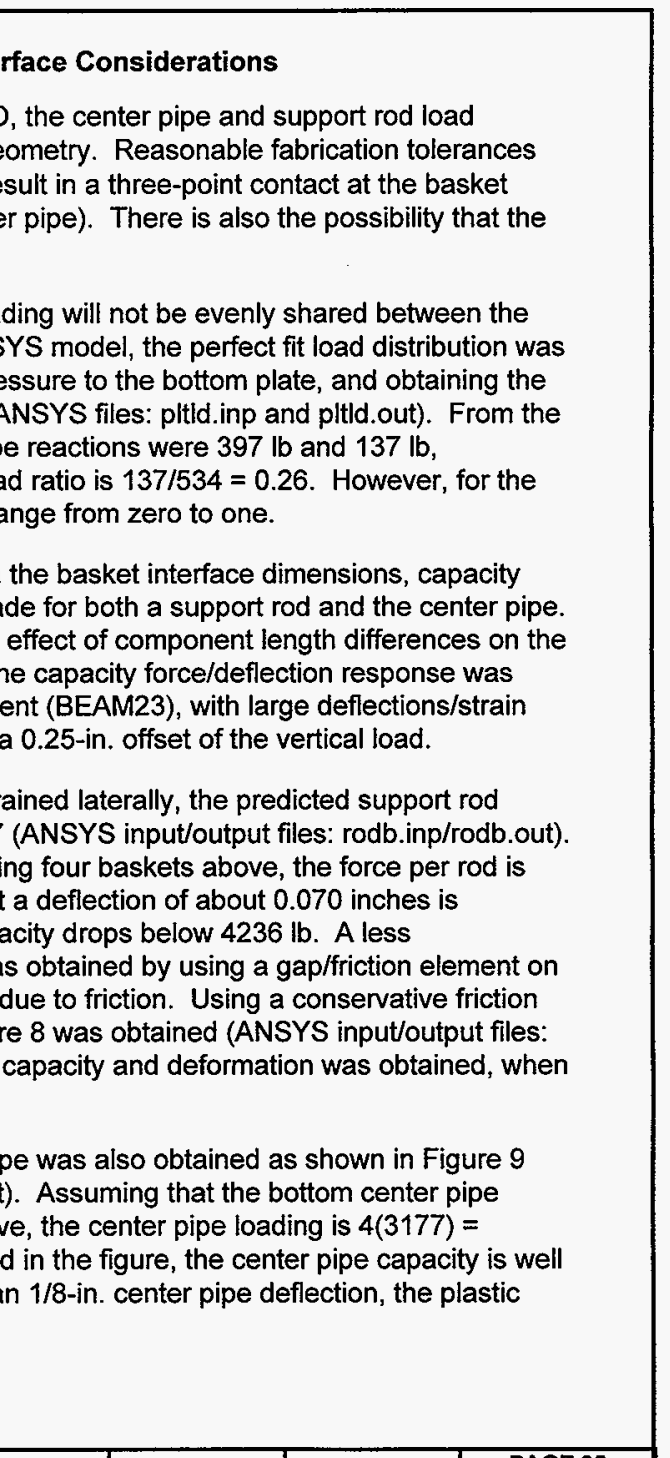


PROJECT: MCO Final Design $\quad$ DOC NO: HNF-SD-SNF-DR-003, Rev. 0, Appendix 8

The above force/deflection results indicate that tight basket interface tolerances are not necessary. Conservatively using half of the $4236 \mathrm{lb}$ deflection of $0.070 \mathrm{in}$. from Figure 7 , fabrication height differences of 0.035 in., or greater, are acceptable. It appears that the interface fabrication tolerance issue may be controlled by functional, rather than structural considerations. For example, a 0.035 in. height difference between the center pipe and support rods, results in a horizontal tipping distance of about $0.10 \mathrm{in}$.

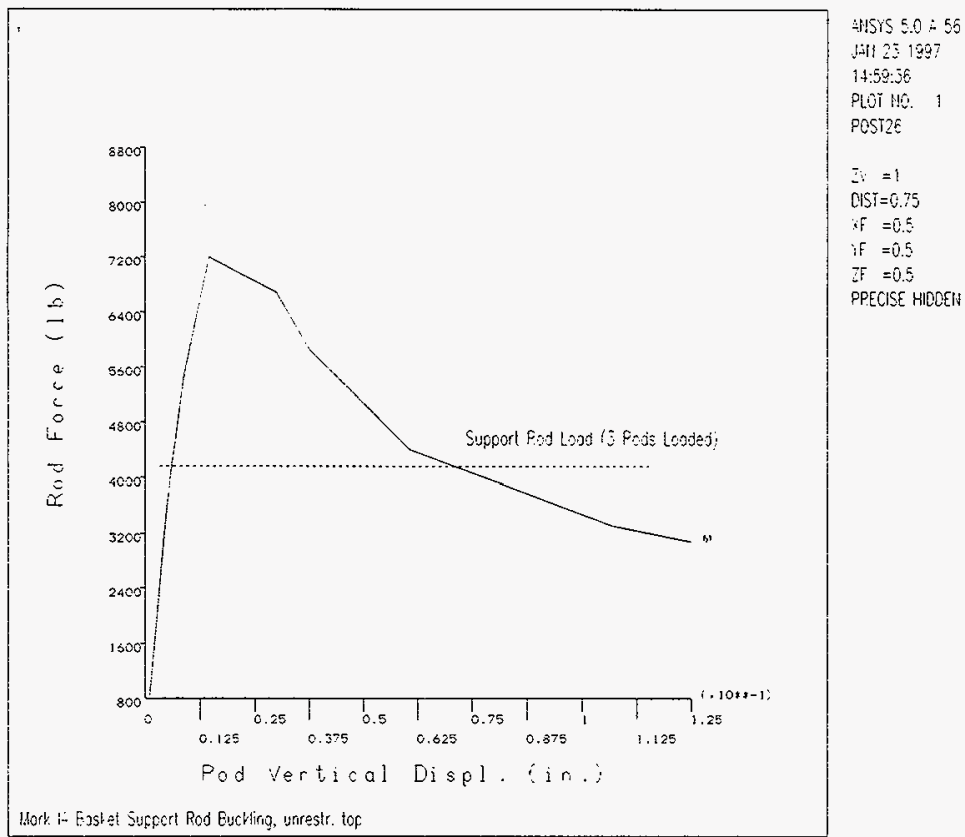

Figure 7. Force/Deflection Response of Support Rod, No Top Constraint.

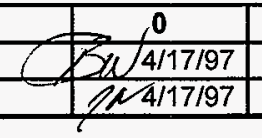


CLIENT:

Duke Engineering Services Hanford

PROJECT: MCO Final Design DOC NO:
FILE NO: $\mathrm{KH}-8009-8-06$

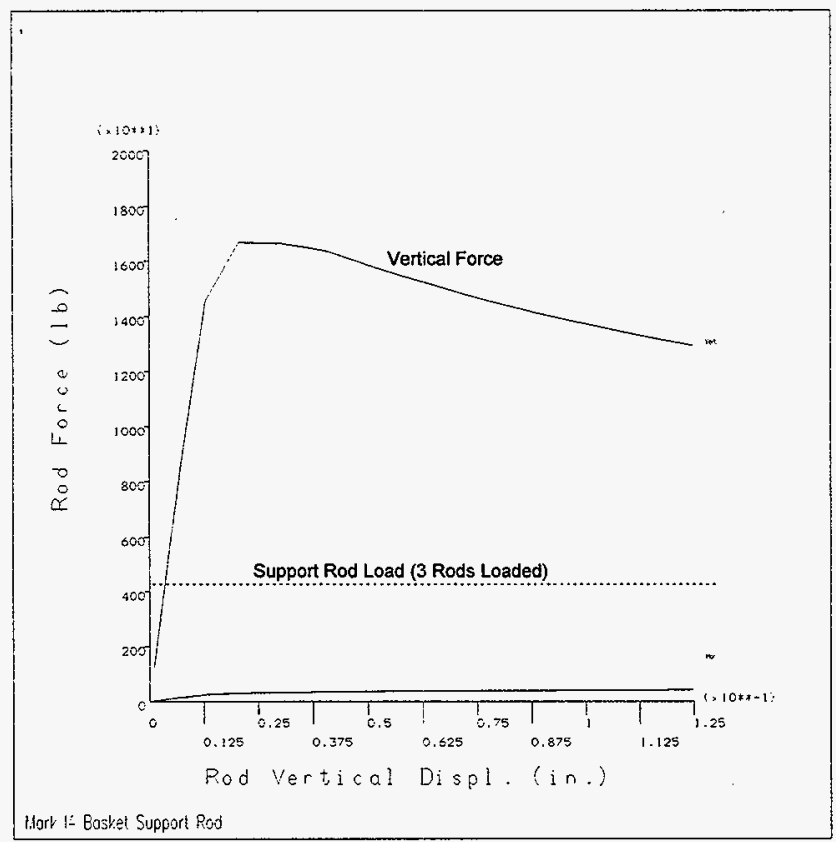

$415 i 550:$

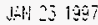

$14000 \hat{0}$

PLOT HOD

Pistre

$\therefore=1$

$015 T=0.75$

XF $=0.5$

it

IF $=0.5$

PPECISE

Figure 8. Support Rod Force/Deflection Response w/ Friction Constraint. 
CLIENT: Duke Engineering Services Hanford

\section{PROJECT: MCO Final Design DOC NO: HNF-SD-SNF-DR-003, Rev. 0, Appendix 8}

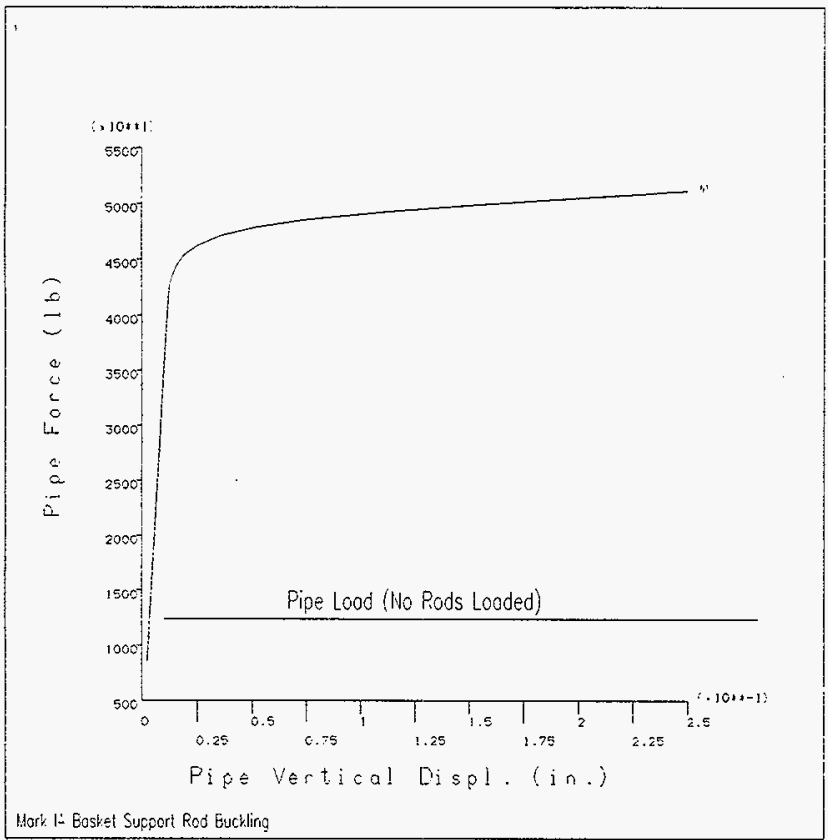

$-4515500: 56$

JiH $2+1957$

$0.51: 38$

PLOT HO. 1

PCSTES

$3:=1$

DSST $=0.75$

if $=0.5$.

if $=0.5$

IF $=0.5$

PRECISE HIOCEN

Mork Ii Bosket Support Rod Euckling

Figure 9. Center Pipe Force/Deflection Response. 
Whe Engineering Services HaRSONS

FILE NO: KH-8009-8-06

PROJECT: MCO Final Design

DOC NO: HNF-SD-SNF-DR-003, Rev. 0, Appendix 8

\subsection{Fillet Weld Finite Element Analysis}

As part of the development of the acceptance criteria for the basket fillet welds, a detailed ANSYS model of a 1/4" center pipe/bottom plate fillet weld was generated, as shown in Figure 10. This weld was later changed to a bevel weld, but the ANSYS results for the earlier fillet weld design were retained as part of the justification for the fillet weld acceptance criteria.

The weld was subjected to the dead weight of two baskets $(2 \times 3177 \mathrm{lb})$. Using the LPATH and PRSECT ANSYS commands, the membrane and membrane plus bending stress intensity values were obtained for the critical weld section. See fillet.inp and fillet.out for the ANSYS input and output file listings. The ANSYS results indicated a membrane stress intensity of $4005 \mathrm{psi}$ and a membrane plus bending stress intensity of $4938 \mathrm{psi}$. Since the membrane plus bending was less than 1.5 times the membrane stress, the membrane stress controls, which is limited to $n S_{m}$ ( $n=$ weld quality factor).

Using the throat area of the weld, the estimated membrane stress is

$$
\begin{aligned}
& \mathrm{P}_{\mathrm{m}}:=\frac{2 \cdot 3177 \cdot \mathrm{lb}}{2.75 \cdot \mathrm{in} \cdot \pi \cdot \frac{\sqrt{2}}{2} \cdot 0.25 \cdot \mathrm{in}} \\
& \mathrm{P}_{\mathrm{m}}=4.16 \cdot 10^{3} \cdot \frac{\mathrm{lb}}{\mathrm{in}^{2}}
\end{aligned}
$$

which is conservative relative to the ANSYS membrane stress prediction of 4005 psi. Thus, based upon the detailed ANSYS analysis, a reasonably conservative approach for the 1/4" fillet weld is to use a stress based upon the weld throat area and a stress limit of $n S_{m}$.

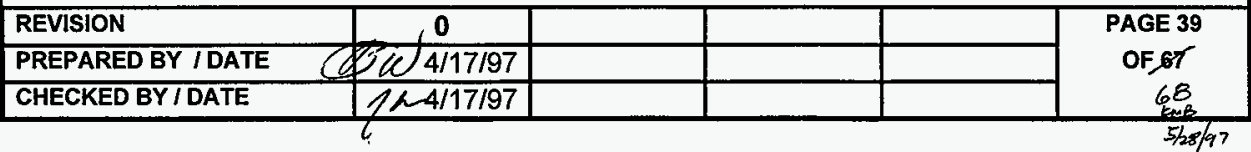


CLIENT: Duke Engineering Services Hanford

PROJECT: MCO Final Design
FILE NO: $\quad \mathrm{KH}-8009-8-06$

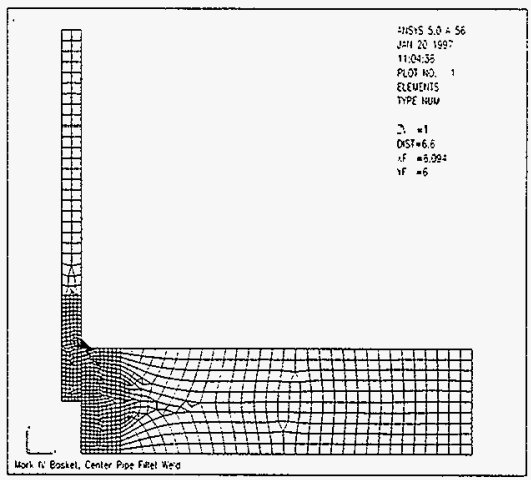

Figure 10. ANSYS Model of a 1/4" Center Pipe/Bottom Plate Fillet Weld.

\subsection{Component Stress Results Summary}

From the calculations above, a summary of the component stress analysis results was compiled into Table 5. Note that the predicted stresses are below allowables for all components. 


\section{EDARERNS}

CLIENT: $\quad$ Duke Engineering Services Hanford

FILE NO: KH-8009-8-06

PROJECT: MCO Final Design DOC NO: HNF-SD-SNF-DR-003, Rev. 0, Appendix 8

Table 5. Summary of Mark IV Basket Structual Evaluation.

\begin{tabular}{|c|c|c|c|c|c|}
\hline Item & $\begin{array}{l}\text { Critical } \\
\text { Load } \\
\text { Condition }\end{array}$ & Stress Category & $\begin{array}{c}\text { Maximum } \\
\text { Stress } \\
(p s i)\end{array}$ & Allowable & Ratio \\
\hline $\begin{array}{c}\text { Center } \\
\text { Pipe }\end{array}$ & $\begin{array}{l}\text { Dead } \\
\text { Weight } \\
\text { Stacking }\end{array}$ & Buckling & 3622 & 5747 & 0.63 \\
\hline $\begin{array}{l}\text { C. Pipe/B. } \\
\text { Plate } \\
\text { Weld }\end{array}$ & $\begin{array}{l}\text { Dead } \\
\text { Weight } \\
\text { Stacking }\end{array}$ & Pm & 12,420 & 13,500 & 0.92 \\
\hline $\begin{array}{l}\text { Support } \\
\text { Rod }\end{array}$ & $\begin{array}{l}\text { Dead } \\
\text { Weight } \\
\text { Stacking }\end{array}$ & Buckling & 1738 & 1806 & 0.96 \\
\hline $\begin{array}{c}\text { Retaining } \\
\text { Bar }\end{array}$ & Lifting & Maximum Bending Stress & 6500 & 7013 & 0.93 \\
\hline $\begin{array}{l}\text { R. Bar } \\
\text { Welds }\end{array}$ & $\begin{array}{l}\text { Dead } \\
\text { Weight } \\
\text { Stacking }\end{array}$ & Pure Shear & 922 & 4208 & 0.22 \\
\hline $\begin{array}{l}\text { Support } \\
\text { Ring }\end{array}$ & $\begin{array}{c}\text { Dead } \\
\text { Weight } \\
\text { Stacking }\end{array}$ & $\mathrm{Pb}$ & 6675 & 20,100 & 0.33 \\
\hline $\begin{array}{l}\text { Support } \\
\text { Ring } \\
\text { Welds }\end{array}$ & Lifting & Pure Shear & 8 & 4100 & 0.002 \\
\hline $\begin{array}{c}\text { Bottom } \\
\text { Plate }\end{array}$ & Lifting & $\mathrm{Pm}+\mathrm{Pb}$ & 5463 & 7013 & 0.78 \\
\hline
\end{tabular}

$E \quad \frac{1}{0} \frac{1}{4 / 17 / 97}$




\section{DPARSロN5}

CLIENT: Duke Engineering Services Hanford

FILE NO: KH-8009-8-06

\section{APPENDIX A}

COMPUTER RUN OUTPUT SHEETS

AND

INPUT FILE LISTINGS 
PROJECT: MCO Final Design
FILE NO: $\mathrm{KH}-8009-8-06$

\section{COMPUTER RUN COVER SHEET}

Project Number:

Computer Code:

Software Version:

Computer System:

Computer Run File Number:

Unique Computer Run File Name:

Run Description:

Creation Date/Time:
$\mathrm{KH}-8009-8$

ANSYS\&-PC

$5.0 \mathrm{~A}$

MS-DOS, Pentium® Processor

$\mathrm{KH}-8009-8-06$

Pltstk.inp

Stress Analysis of the Mark IV Storage Basket Stacking Load Condition

22 January 1997/10:31:40am

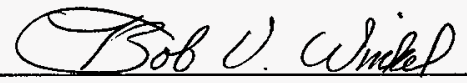

Prepared By: Bob V. Winkel

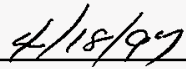

Date

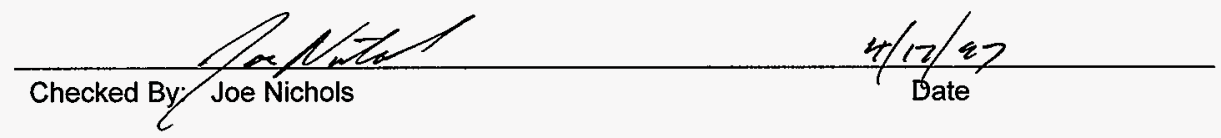

\begin{tabular}{|l|l|l|}
\hline & & \\
\hline & & \\
\hline & & \\
\hline
\end{tabular}


PROJECT: MCO Final Design DOC NO: HNF-SD-SNF-DR-003, Rev. 0, Appendix 8

\section{LISTING OF PLTSTK.INP FILE}

/batch,list

ffilenam,pltstk

/prep7

/title,Mark IV Basket, 60 Deg. Sector

$\begin{array}{ll}\text { SIZE }=0.24 & \text { ! element size }(.24 \text { best mesh) } \\ \text { THICK1 }=3.00 & \text { ! plate thickness } \\ \text { THICK2 }=.375 & \text { ! center pipe thickness } \\ \text { pressure }=26.1 & \text { ! uniform pressure load } \\ \text { OR }=22.625 / 2 & \text { ! plate outside radius } \\ \text { IR }=2.50 / 2 & \text { ! plate inside radius } \\ \mathrm{RR}=21.275 / 2 & \text { ! support rod loca. radius } \\ \mathrm{HI}=24.125 & \text { ! height of center pipe/rods } \\ \text { rhole }=2.58 / 2 & \text { ! radius of fuel support hole }\end{array}$

/com hole locations - global cartesian

$\mathrm{X} 1 \mathrm{=} \mathbf{2 . 3 8 2}$

$x 2=4.763$

$x 3=7.145$

$x 4=9.526$

$y 1=0$

$y 2=1.3750$

$y 3=2.750$

$y 4=4.125$

y5 $=5.500$

$y 6=6.875$

$y 7=8.250$

$y 8=9.625$

*afun, deg

! use degrees for function inputfoutput

et, 1 ,shell 63

et, 2 ,shell63

et, 3 , beam4

et, 4 , solid 45

et,5,beam4 ! Beam Element for Fuel Rod

et,6,mass21,.,2 ! Mass Element for Fuel Rod

$r, 1$, THICK1

$\mathrm{r}, 2$, THICK2

$r, 3,1.227, .120, .120,1.25$ ! Support Rod

$r, 5,100,1000,1000,2$ ! Rigid Fuel Element

$r, 6,55.4$ ! Mass of Fuel Element

$r, 7,27.7$ ! Fuel Ele. Mass on Symmetry Axis (Half Mass)

dens, 1,2854

ex, $1,26.5 E+06$

nuxy, 1,3

Icom define bottom plate

csys, 1 ! global cylindrical

$k, 10,0,0$

$k, 1, I R, 0$

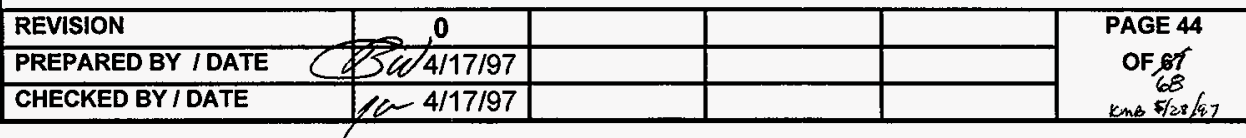


CLIENT: Duke

\section{$k, 2, \mathrm{OR}, 0$ \\ $k, 3, \mathrm{OR}, 30$ \\ $k, 4, R R, 30$ \\ $k, 5, I R, 30$}

\section{I,1,2 ! line 1}

larc,2,3,10, OR ! line 2

$1,3,4 \quad$ ! line 3

$\mathbf{1 , 4 , 5} \quad$ ! line 4

larc, $5,1,10$, IR ! line 5

lesize,5,5 $\quad 15$ degree segments for inside arc

/com area $1=$ bottom plate

al, $1,2,3,4,5$

/com define center pipe

$k, 20,0,0, \mathrm{HI}$

$k, 21,1 R, 0, \mathrm{HI}$

$k, 25, I R, 30, \mathrm{HI}$

larc, $25,21,20, \mathrm{IR}$ ! line 6

lesize,6,10 ! 5 degree segments for inside arc

$k, 26,0,0,-1.5$

k,27,IR, $0 .,-1.5$

$k, 28, I R, 30,-1.5$

larc, $28,27,26, I R \quad$ ! line $7 @$ bottom end of center pipe

lesize, 7,10

$1,27,21 \quad$ ! vertical line (8) at 0 degrees

$1,28,25 \quad$ I vertical line (9) at 30 degrees

/com area 2 = center pipe

al, $6,7,8,9$

/com define support rod

$\mathrm{k}, 30, \mathrm{RR}, 30, \mathrm{HI}$

$1,4,30 \quad$ ! vertical line $(10)$ at 30 degrees

lesize, $10, \ldots 4$

/com define holes in bottom plate

wplane, $, x 4, y 1$

pcirc, rhole

wplane, $x 4, y 3$

pcirc, rhole

wplane, $x 3, y 2$

pcirc, thole

wplane, $\times 3, y 4$

pcirc,rhole $\quad$ larea 6

wplane, $, x 2, y 1$

pcirc, rhole

wplane, $, x 2, y 3$

pcirc, rhole

wplane, ,x1,y2

peirc, rhole

wplane,,defa

wpstyle

! area 3

! area 4

larea 5

! area 7

! area 8

! area 9

Icom subtract holes from bottom plate

boptn,yes !To save areas

asba, 1,3 
CLIENT: Duke Engineering Services Hanford

PROJECT: MCO Final Design
FILE NO: KH-8009-8-06

DOC NO: HNF-SD-SNF-DR-003, Rev. 0, Appendix 8

asba, 10,4 :A10 created from previous subtraction

asba,11,5 IA11 created from prev. subtr.

asba, 12,6

asba, 13,7

asba, 14,8

asba, 15,9

! bottom plate is now area 16

adele, 1

adele, 3,15

/com mesh areas (30 degrees)

/com bottom plate

esize,SIZE

mat, 1

real, 1

amesh, 16

/com center pipe

esize, THICK1/4

type, 2

real,2

amesh,2

/com repeat mesh to obtain 60 degree model

csys

clocal, $11, \ldots, 30$

arsym, $y, 2,16,14$

/com nodes in cylindrical coordinates

csys, 1

nrotat,all

/com support rod

type, 3

real, 3

Imesh,10

/com generate solid elements

type. 4

esize, 4 lesize $=\mathbf{4}$ for final model

/com bottom plate extrusion

vext, $3, \ldots, \ldots-3$

vext, $16, \ldots,-3$

lcom center pipe extrusion

esize,.,75

vext, 1,2,-,THICK2

/com remove shell elements

asel,all

asel,s,type,,2

aclear,all

asel,all

asel,s,type, 1

aclear,all

REVISION

PREPARED BY / DATE

CHECKED BY/DATE 
csys, 0

lcom rotate all nodes into cyl. coord. system and merge

csys, 1

nrotate, all

nummerg,a!l

/com constrain edges of plate (60 deg. symm.)

nsel, $s$, loc $, y, 0$

csys, 1

nrotat, all

d,all,uy,

nsel,all

nsel,s,loc, $y, 60$

csys, 1

nrotat,all

d,all,uy

nsel,all

Icom axial constrain @ center pipe support

nsel,s,loc, $x, I R$

nsel, $r$, loc, $z,-3$

d,all,uz

nall

/com axial constraint @ support rods underneath

csys, 1

nsel, s,loc, $z,-3$

nsel, $r$, loc, $y, 0,2$

nsel, $r$, loc, $x$, OR-1, OR

d,all,uz

nall

nsel,s,loc,,,-3

nsel,, , loc, $, y, 58,60$

nsel, r, loc, $x$, OR-1,OR

d,all,uz

nall

Icom couple nodes adjacent to rod support

nsel,, loc, $\mathbf{z}, 0$

nsel, $r$, loc,, , 28, 32

nsel, r, loc, $x$, RR $-.3, \mathrm{RR}+.3$

$\mathrm{cp}, 1, \mathrm{uz}, \mathrm{all}$

nall

Icom constrain rota. dof @ rod connection

csys

$\mathrm{dk}_{,}, 4$, rotx, , ,roty, rotz

sbctran

fini

Isolu

/com fuel loading (pressure), sup. rod ( 3 baskets above) \& acel

asel, $,, l o c, z, 0$

sfa,all,pres,pressure ! fuel pressure

asel,all

REVISION

PREPARED BY I DATE

CHECKED BY / DATE

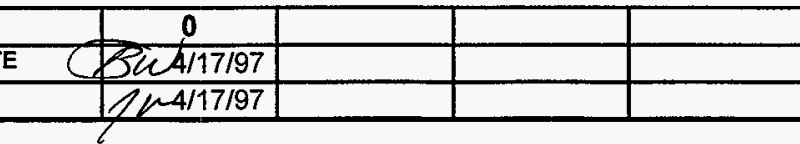


CLIENT: Duke Engineering Services Hanford
FILE NO: KH-8009-8-06

\section{PROJECT: MCO Final Design DOC NO: HNF-SD-SNF-DR-003, Rev. 0, Appendix 8}

\section{$\mathrm{f}, 1005, \mathrm{fz},-1588$}

acel $_{\text {,., } 1.0}$ ! $1 \mathrm{~g}$ vertical

solve

save

fini

\section{/post1}

MIN, 1,SQUARE

/ANGLE, 1,-30,XS,1

/show,graphics,pic, 1

eplot

ledge,, 1

set, last

pinsol,s,int

/show,vga

fini

lexit

\begin{tabular}{|l|l|l|}
\hline & & \\
\hline & & \\
\hline
\end{tabular}


CLIENT: Duke Engineering Services Hanford
PARSDNS

PROJECT: MCO Final Design DOC NO: HNF-SD-SNF-DR-003, Rev. 0, Appendix 8

\section{COMPUTER RUN COVER SHEET}

Project Number:

Computer Code:

Software Version:

Computer System:

Computer Run File Number:

Unique Computer Run File Name:

Run Description:

Creation Date/Time:
$\mathrm{KH}-8009-8$

ANSYS@-PC

$5.0 \mathrm{~A}$

MS-DOS, Pentium® Processor

KH-8009-8-06

Pltlft.inp

Stress Analysis of the Mark IV Storage Basket Stacking Load Condition

22 January 1997/2:43:56pm

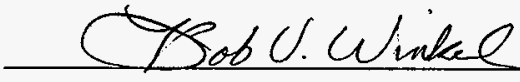

Prepared By: Bob V. Winkel
$4 / 18 / 97$

Date

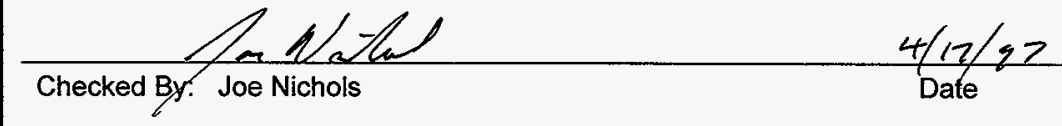


foatch, list

/filenam,pltift

/prep7

/title,Mark IV Storage Basket, $60 \mathrm{Deg}$. Sector

$\begin{array}{lc}\text { SIZE }=0.24 & \text { ! element size (.24 best mesh) } \\ \text { THICK1 }=3.00 & \text { ! plate thickness } \\ \text { THICK2 }=.375 & \text { ! center pipe thickness } \\ \text { pressure }=26.1 & \text { ! uniform pressure load } \\ \text { OR }=22.625 / 2 & \text { ! plate outside radius } \\ \text { IR }=2.50 / 2 & \text { ! plate inside radius } \\ \text { RR }=21.275 / 2 & \text { ! support rod loca. radius } \\ \text { HI }=23.785 & \text { ! height of center pipe/rods } \\ \text { rhole }=2.58 / 2 & \text { ! radius of fuel support hole }\end{array}$

/com hole locations - global cartesian

$\mathbf{x 1}=2.382$

$\times 2=4.763$

$\times 3=7.145$

$\times 4=9.526$

$y 1=0$

y2 $=1.3750$

y3 $=2.750$

$y 4=4.125$

y5 $=5.500$

y $6=6.875$

$y 7=8.250$

$y 8=9.625$

*afun,deg

I use degrees for function input/output

et, 1, shell63

et, 2, shell63

et, 3 , beam 4

et, 4, solid 45

et, 5 , beam 4

et, 6 ,solid45

! 3-d elastic beam

! Bottom Plate

! Beam Element for Fuel Rod

! Center Pipe

$r, 1$, THICK1

$r, 2$, THICK2

$r, 3,1.227, .120,120,1.25$ ! Support Rod

dens, 1,2854

ex, $1,26.5 \mathrm{E}+06$

nuxy, 1,3

/com define bottom plate

csys, 1 ! global cylindrical

$k, 10,0,0$

$k, 1, \mathbb{R}, 0$

$k, 2,0 R, 0$

$k, 3, \mathrm{OR}, 30$

$k, 4, R R, 30$ 
$k, 5, I R, 30$

I,1,2 ! line 1

larc,2,3,10, OR ! line 2

$1,3,4 \quad$ ! line 3

$1,4,5 \quad$ ! line 4

larc, $5,1,10$, IR ! line 5

lesize,5,5 5 degree segments for inside arc

/com area $1=$ bottom plate

al, $1,2,3,4,5$

/com define center pipe

$k, 20,0,0, \mathrm{HI}$

$k, 21, \mid R, 0, H I$

$k, 25, \mid R, 30, H I$

larc, $25,21,20, \mathrm{IR}$ ! line 6

lesize, 6,5 ! 5 degree segments for inside arc

$k, 26,0,0,-1.5$

$k, 27, I R, 0 .,-1.5$

$\mathrm{k}, 28, \mathrm{IR}, 30,-1.5$

larc,28,27,26,IR ! line 7 @ bottom end of center pipe

lesize, $7, .5$

$1,27,21 \quad$ I vertical line (8) at 0 degrees

$1,28,25 \quad$ I vertical line $(9)$ at 30 degrees

lesize, 8,750

lesize, 9,750

/com area 2 = center pipe

al, $6,7,8,9$

Jcom define support rod

$\mathrm{k}, 30, \mathrm{RR}, 30, \mathrm{HI}$

$1,4,30 \quad$ I vertical line $(10)$ at 30 degrees

lesize, $10_{n}, 4$

/com define holes in bottom plate

wplane, $x 4, y 1$

pcirc,inole

wplane, $x 4, y 3$

pcirc,inole

wplane, $x 3, y 2$

pcirc, rhole

wplane, $\times 3, y 4$

pcirc, rhole

wplane, $x 2, y 1$

pcirc, rhole

wplane, $x 2, y 3$

pcirc,rhole

wplane , $x 1, y 2$

pcirc, rhole

wplane,,defa

wpstyle

! area 3

! area 4

! area 5

! area 6

! area 7

! area 8

! area 9

/com subtract holes from bottom plate

boptn,yes !To save areas

asba, 1,3

asba, 10,4 !A10 created from previous subtraction

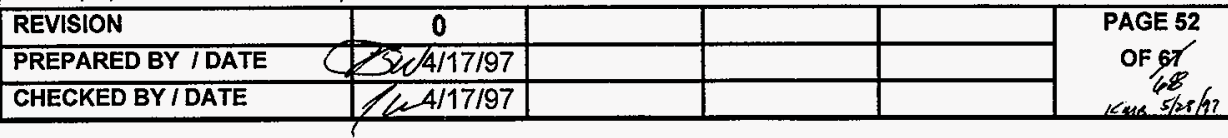




\section{CLIENT:}

Duke Engineering Services Hanford

PROJECT: MCO Final Design

asba,11,5 !A11 created from prev. subtr.

asba, 12,6

asba, 13,7

asba, 14,8

asba, 15,9 ! bottom plate is now area 16

adele, 1

adele,3,15

Icom mesh areas (30 degrees)

/com bottom plate

esize,SIZE

mat, 1

real, 1

amesh, 16

/com center pipe

type, 2

real,2

amesh,2

Icom repeat mesh to obtain 60 degree model

csys

clocal, $11_{, \ldots, 30}$

arsym,y,2,16,14

Icom nodes in cylindrical coordinates

csys, 1

nrotat,all

/com support rod

type, 3

real, 3

Imesh, 10

/com generate solid elements

type, 4

esize, 4 lesize $=4$ for final model

Icom bottom plate extrusion

vext, $3, \ldots,,-3$

vext, $16, \ldots, .3$

/com center pipe extrusion

type, 6

esize, 2

vext, 1,2,--THICK2

lcom remove shell elements

asel,ali

asel,s,type,,2

aclear,all

asel,all

asel,s,type,,1

aclear,all

csys, 0

REVISION

PREPARED BY IDATE 
lcom merge nodes

csys, 1

esel,s,type.,4

nsle,s

nummerg,node, 02 ! merge bottom plate nodes

esel,s,type, 6

nsle,s

nummerg,node,.02 ! merge center pipe nodes

nall

eall

nsel,s,loc, $x, 1.24,1.26$

nsel,, loc, $, z,-.1,0.1$

nummerg,node,.02 ! connect pipe/plate @ top of plate only (conservative)

nall

/com constrain edges of plate ( 60 deg. symm.)

nsel,s, loc, $y, 0$

csys, 1

nrotat,all

d,all,uy,

nsel,all

nsel,s,loc, $y, 60$

csys, 1

nrotat,all

d,all,uy

nsel,all

/com axial constraint

nsel, s, loc, $\mathrm{z}, \mathrm{HI}$

nsel,, , Ioc, $x, 0,1 R+.1$

d,all,uz

nsel,all

/com constrain rota. dof @ rod connection

csys

$\mathrm{dk}, 4$, rotx, , , roty, rotz

sbctran

fini

Isolu

/com fuel loading (pressure), sup. rod ( 3 baskets above) \& acel

asel,s,loc, $z, 0$

sfa,all,pres,pressure ! fuel pressure

asel,all

acel,.., 1.0 ! 1 g vertical

solve

save

fini

/post1

MIN, 1,SQUARE

IZOOM, OFF

IANGLE, $1,-30, X S, 1$

/show,graphics,pic,1

eplot

REVISION

PREPARED BY IDATE

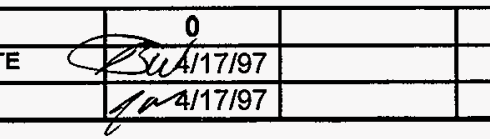


CLIENT: Duke Engineering Services Hanford

FILE NO: $\quad \mathrm{KH}-8009-8-06$

PROJECT: MCO Final Design DOC NO: HNF-SD-SNF-DR-003, Rev. 0, Appendix 8

ledge, 1

set,last

plnsol,s,int

/show,vga

fini

lexit 


\section{COMPUTER RUN COVER SHEET}

Project Number:

Computer Code:

Software Version:

Computer System:

Computer Run File Number:

Unique Computer Run File Name:

Run Description:

Run Date/Time:

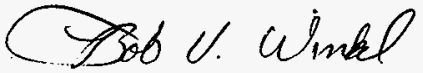

Prepared By: Bob V. Winkel

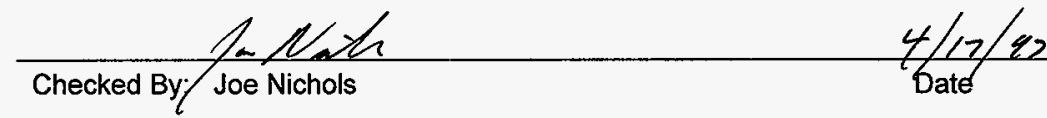

$\mathrm{KH}-8009-8$

ANSYS $\circledast-P C$

$5.0 \mathrm{~A}$

MS-DOS, Pentium® Processor

$\mathrm{KH}-8009-8-06$

Pitlft.out

Stress Analysis of the Mark IV Storage Basket Stacking Load Condition

22 January 1997/3:15:30pm 
CLIENT: Duke Engineering Services Hanford

PROJECT: MCO Final Design DOC NO: HNF-SD-SNF-DR-003, Rev. 0, Appendix 8

\section{COMPUTER RUN COVER SHEET}

Project Number:

Computer Code:

Software Version:

Computer System:

Computer Run File Number:

Unique Computer Run File Name:

Run Description:

Creation Date/Time:

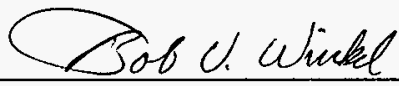

Prepared By: Bob V. Winkel

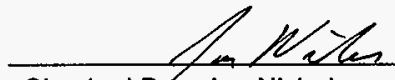

Checked By: Joe Nichols
Bob O. Wirelel

Prepared By. Bob V. Whkel
$\mathrm{KH}-8009-8$

ANSYS $\otimes-P C$

$5.0 \mathrm{~A}$

MS-DOS, Pentium® Processor

$\mathrm{KH}-8009-8-06$

Rodb.inp

Stress Analysis of the Mark IV Storage Basket Stacking Load Condition

23 January 1997/1:38:20pm

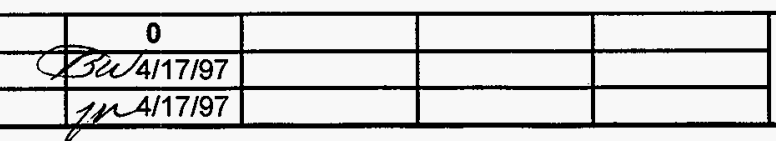


CLIENT: Duke Engineering Services Hanford

\section{LISTING OF RODB.INP FILE}

Ibatch,list

Ifilenam, rodb

fprep7

Atitle,Mark IA Basket Support Rod Buckling

et, 1, beam $23, \ldots, \ldots 4$

let,1,beam23

et, 2 , beam 23

et, 3, contac 12

$r, 1,2.874,1.34,6.384,3.072,2.916,1.897,2.816$

I $r, 1,2.874, .430,1.34$

$r, 2,10,83.333,10$

$r, 3,0,1 e 6,1.0$

dens, 1,2854

ex, 1,27.6E+06

nuxy, 1,3

tb, bkin, 1,2

totemp,0

thdata, $1,17.6 \mathrm{e} 3,0.9 \mathrm{e} 6$

tbtemp,200

tbdata, 1,17.6e3,0.9e6

dens, 2, 2854

ex,2,27.6E+06

nuxy, 2,3

tb,bkin,2,2

tbtemp, 0

tbdata, 1,17.6e6,0.9e6

tbtemp,200

tbdata, 1,17.6e6,0.9e6

mu,2,0.1 ! Rod/basket friction coefficient

n, 1 ,

$n, 21,0,22.318$

fill, 1,21

$\mathrm{n}, 22,-.25,22.318$

$n, 23,-.25,23$

type, 1

real, 1

e,1,2

egen,20,1,1

type,2

mat,2

real,2

e,21,22

type, 3

mat,2

real, 3

e, 22,23

eplot

REVISION

PREPARED BY I DATE

0

DOC NO:

FILE NO: $\quad \mathrm{KH}-8009-8-06$

HNF-SD-SNF-DR-003, Rev. 0, Appendix 8




PROJECT: MCO Final Design DOC NO: HNF-SD-SNF-DR-003, Rev. 0, Appendix 8

Icom Boundary Conditions

d,1,all

d,23,all

fini

Isolu

autots, on

nigeom,on

d,23,uy, -0.012 ! Initial Displacement

cnvtol, , , , , 005

cnvtol, $m,-1$

time, 1

nsubst, 1

outpr,basic, 1

Iswrite, 1

time, 2

d, 23, uy, -.100

nsubst, 2000

outres,all,2

outpr,basic, 10

Iswrite,2

time, 3

nsubst,2000

d,23,uy, -.2

Issolve, 1,2

fini

/post26

NSOL,2,22,U,Y, uY22

RFORCE, $3,1, F, Y$, fy1

prvar, 2,3

$A D D, 4,2, \ldots, \ldots-1$

xvar, 4

IAXLAB, X,Rod Vertical Displ. (in.)

IAXLAB, Y, Rod Force (Ib)

!/show,graphics,pic, 1

plvar,3 
CLIENT:

PROJECT: MCO Final Design
FILE NO: KH-8009-8-06 DOCNO: HNF-SD-SNF-DR-003, Rev. 0, Appendix 8

\section{COMPUTER RUN COVER SHEET}

Project Number:

Computer Code:

Software Version:

Computer System:

Computer Run File Number:

Unique Computer Run File Name:

Run Description:

Run Date/Time:

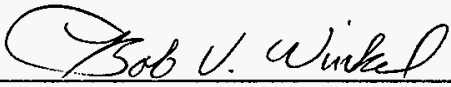

Prepared By: Bob V. Winkel

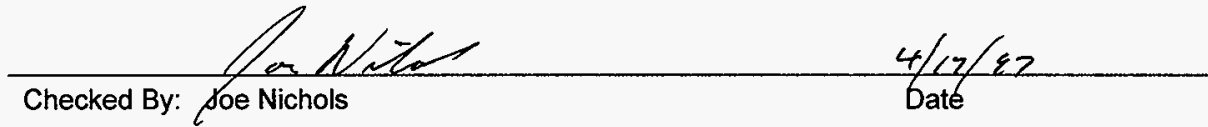

$\mathrm{KH}-8009-8$

ANSYS@-PC

$5.0 \mathrm{~A}$

MS-DOS, Pentium® Processor

KH-8009-8-06

Rodb.out

Stress Analysis of the Mark IV Storage Basket Stacking Load Condition

23 January 1997/1:40:26pm 
CLIENT: Duke Engineering Services Hanford

PROJECT: MCO Final Design DOC NO: HNF-SD-SNF-DR-003, Rev. 0, Appendix 8

\section{COMPUTER RUN COVER SHEET}

Project Number:

Computer Code:

Software Version:

Computer System:

Computer Run File Number:

Unique Computer Run File Name:

Run Description:

Creation Date/Time:

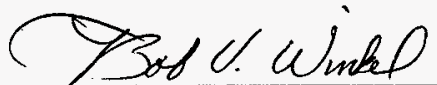

Prepared By: Bob V. Winkel

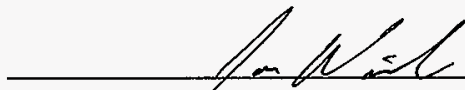

Checked By: Joe Nichols
$\mathrm{KH}-8009-8$

ANSYS $\otimes-P C$

$5.0 \mathrm{~A}$

MS-DOS, Pentium® Processor

$\mathrm{KH}-8009-8-06$

Rodbf.inp

Stress Analysis of the Mark IV Storage Basket Stacking Load Condition

23 January 1997/4:15:56pm
$4 / 8 / 97$

Date 
CLIENT: Duke Engineering Services Hanford

PROJECT: MCO Final Design DOC NO: HNF-SD-SNF-DR-003, Rev. 0, Appendix 8

\section{LISTING OF RODBF.INP FILE}

/batch, list

/filenam,roobf

/prep7

/title,Mark IA Basket Support Rod Buckling, unrestr. top

et, 1 , beam $23, \ldots, \ldots, 2$

et,2,contac12

\section{$r, 1,1.25$}

$r, 2,10$.

$r, 3,0,1 e 8,1.0$

dens, 1,2854

ex, $1,27.6 \mathrm{E}+06$

nuxy, 1,3

tb, bkin, 1,2

tbtemp, 0

tbdata, $1,21.3 \mathrm{e} 3,0.3 \mathrm{e} 6$

tbtemp,200

tbdata, 1,21.3e3,0.3e6

mu,2,0.1 ! Rod/basket friction coefficient

$\mathrm{n}, 1$,

$n, 21,0,24.125$

fill, 1,21

$n, 22,-.25,24.125 \quad$ ! 1/4" offset

!n,23, . 25,25.

type,1

real, 1

e, 1,2

egen,20,1,1

real, 2

$e, 21,22$ ! rigid link for load offset

type, 2

mat, 2

real, 3

le,22,23

eplot

/com Boundary Conditions

d, 1 , all

fini

isolu

/com Apply displ. in y direction

d, 22,uy,-.005

neqit, 50

autots, on

nlgeom,on 
PROJECT: MCO Final Design DOC NO: HNF-SD-SNF-DR-003, Rev. 0, Appendix 8

cnvtol, $f_{,}, 01$

cnvtol, m,-1

time, 1

nsubst, 10

outpr,basic, 10

Iswrite, 1

time, 2

d,22,uy, -.125

nsubst, 1000

outres, all, 2

outpr,basic, 10

Iswrite, 2

Issolve, 1,2

fini

\section{Ipost26}

NSOL,2,22,U,Y,uy22

RFORCE, 3, 1,F,Y,fy1

prvar,2,3

ADD $, 4,2, \ldots,,-1$

xvar, 4

IAXLAB,X,Rod Vertical Displ. (in.)

IAXLAB,Y, Rod Force (lb)

Ishow,graphics,pic, I

plvar, 3

fini

lexit 
CLIENT: Duke Engineering Services Hanford

PROJECT: MCO Final Design
FILE NO: $\mathrm{KH}-8009-8-06$

\section{COMPUTER RUN COVER SHEET}

Project Number:

Computer Code:

Software Version:

Computer System:

Computer Run File Number:

Unique Computer Run File Name:

Run Description:

Run Date/Time:

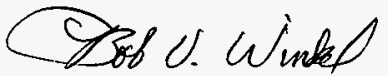

Prepared By: Bob V. Winkel

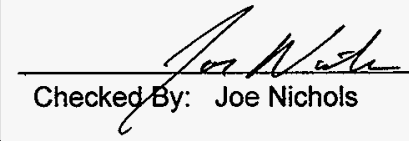

Prepared By: Bob V. Whinel
KH-8009-8

ANSYS®-PC

$5.0 \mathrm{~A}$

MS-DOS, Pentium® Processor

$\mathrm{KH}-8009-8-06$

Rodbf.out

Stress Analysis of the Mark IV Storage Basket Stacking Load Condition

23 January 1997/4:17:06pm

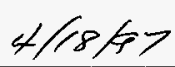

Date

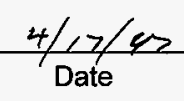


CLIENT: Duke Engineering Services Hanford

PROJECT: MCO Final Design
FILE NO: $\mathrm{KH}-8009-8-06$

\section{COMPUTER RUN COVER SHEET}

Project Number:

Computer Code:

Software Version:

Computer System:

Computer Run File Number:

Unique Computer Run File Name:

Run Description:

Creation Date/Time:

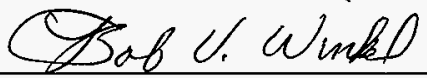

Prepared By: Bob V. Winkel

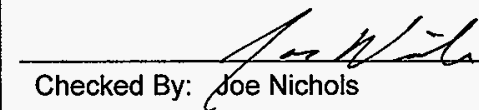

$\mathrm{KH}-8009-8$

ANSYSB-PC

$5.0 \mathrm{~A}$

MS-DOS, Pentium® Processor

KH-8009-8-06

Weld.inp

Stress Analysis of the Mark IV Storage Basket Stacking Load Condition

27 January 1997/8:36:28am
4/18/97

Date

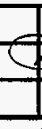


CLIENT: Duke Engineering Services Hanford

FILE NO: KH-8009-8-06

PROJECT: MCO Final Design DOC NO: HNF-SD-SNF-DR-003, Rev. 0, Appendix 8

\section{LISTING OF WELD.INP FILE}

/ batch,list

/filenam, fillet

/prep7

title,Mark IV Basket, Center Pipe Fillet Weld

THICK $1=3.00 \quad$ ! plate thickness

THICK2 $=.500$ ! center pipe thickness

$\mathrm{OR}=22.625 / 2$ ! plate outside radius

$\mathrm{IR}=2.50 / 2 \quad$ ! plate inside radius

fsize $=0.125$ ! fillet weld size

et, 1, plane $42, ., 1$

dens, 1,2854

ex, $1,26.5 E+06$

nuxy, 1,3

Icom Define Area Keypoints

$k, 1, I R, 0$

$k, 2, \mathrm{OR}, 0$

$k, 3, \mathrm{OR}, 3$

$k, 4, I R, 3$

$k, 5, I R, 1.75$

$k, 6, I R, 1.75$ - fsize

$k, 7,1.375,3$

$k, 8,1.375,3+$ fsize

$k, 9, \mathrm{JR}-.5,1.75$

$k, 10, l R-.5,12$

k, 11,1.375,12

$k, 12, I R-f s i z e, 1.75$

$k, 13, I R+1,0$

$k, 14, I R+1,3$

$k, 15, I R-.5,4.5$

$k, 16,1.375,4.5$

$k, 17, I R, 2$

$\mathrm{k}, 18, \mathrm{IR}, 1,75$

/com Define Areas

A, 13,2,3,14

A, $1,13,14,7,4,17,5,6$

A, $9,12,18,4,8,16,15$

$A, 4,7,8$

A, $15,16,11,10$

/com Develop Mesh

esize,.02

amesh, 4

esize, .08

amesh,2,3

esize, 3

amesh, 1

amesh, 5

save 
CLIENT: Duke Engineering Services Hanford

FILE NO: KH-8009-8-06

PROJECT: MCO Final Design DOCNO: HNF-SD-SNF-DR-003, Rev. 0, Appendix 8

fini

Isolu

/com Vertical Constraint at Top of Center Pipe

nsel,s,loc,y, 12

d,all,uy

nall

/com Pressure Load on Bottom Plate

nsel,s,loc, $y, 3$

nsel, $r, l o c, x, 1.375,15$

sf,all,pres, 8.017

nall

solve

save

fini

/post1

set, last

WIN, 1,SQUARE

/show,graphics,pic, 1

EPLOT

IZOOM, 1, $1.8250,2.9745,0.00000 E+00,1.4232$

IEDGE, 1

PLNSOL,S ,INT

Ipath, 2,10

prsect

Ishow,vga

fini

lexit

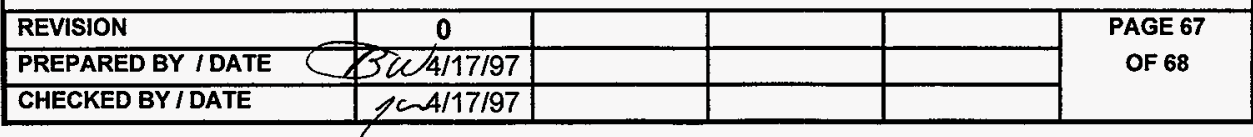


CLIENT: Duke Engineering Services Hanford

FILE NO: $\mathrm{KH}-8009-8-06$

PROJECT: MCO Final Design DOC NO: HNF-SD-SNF-DR-003, Rev. 0, Appendix 8

\section{COMPUTER RUN COVER SHEET}

Project Number:

$\mathrm{KH}-8009-8$

Computer Code:

ANSYS $\otimes-P C$

Software Version:

$5.0 \mathrm{~A}$

Computer System:

MS-DOS, Pentium (8) Processor

Computer Run File Number:

$\mathrm{KH}-8009-8-06$

Unique Computer Run File Name:

Weld.out

Run Description:

Stress Analysis of the Mark IV Storage Basket Stacking Load Condition

Run Date/Time:

27 January 1997/8:18:24am

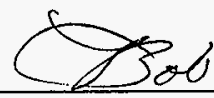

Prepared By: Bob V. Winkel

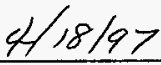

Date

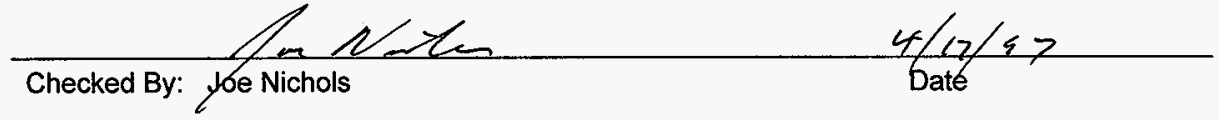


CALCULATION TITLE: Duke Engineering \& Services Hanford, Inc.

STRESS ANALYSIS OF THE MARK IV SCRAP BASKET

PROBLEM STATEMENT OR OBJECTIVE OF CALCULATION:

PERFORM A STRESS ANALYSIS OF THE MARK IV SCRAP BASKET IN ACCORDANCE WITH

REVISION 2 OF THE MCO PERFORMANCE SPECIFICATION. TWO LOADS ARE CONSIDERED:

1. LIFTING

2. DEADWEIGHT

CRITERIA ARE BASED ON ANSI N14.6 AND THE ASME CODE.

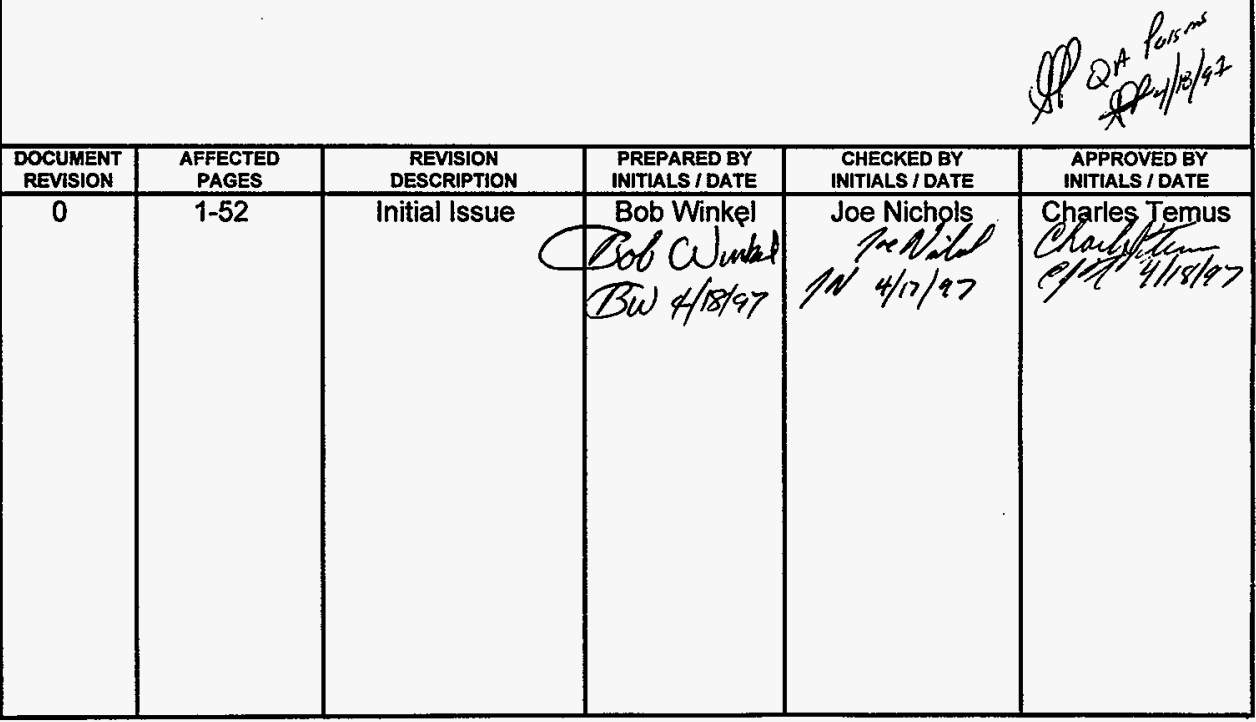




\section{CONTENTS}

CONTENTS

LIST OF TABLES

LIST OF FIGURES

1. INTRODUCTION

2. REFERENCES

3. ASSUMPTIONS

4. GEOMETRY

5. MATERIAL PROPERTIES

6. ACCEPTANCE CRITERIA

6.1 Lifting Loads

6.2 Deadweight Loads

7. LOAD CONDITIONS \& COMBINATIONS

8. STRESS ANALYSIS - HAND CALCULATIONS

8.1 Center Pipe

8.2 Bottom Plate

8.3 Center Pipe to Coupling Pipe and Coupling Pipe to Bottom Plate Welds

8.4 Stiffener Plate to Bottom Plate Welds

8.5 Stiffener Plate to Center Pipe Weld

8.6 Stiffener Plate to Outer (Shell) Plate Weld

8.7 Outer (Shell) Plate to Bottom Plate Weld

\begin{tabular}{|l|l|l|l|}
\hline & & & \\
\hline & & & \\
\hline
\end{tabular}


CLIENT: Duke Engineering Services Hanford

PROJECT: MCO Final Design
FILE NO: KH-8009-8-07

DOC NO: HNF-SD-SNF-DR-003, Rev. 0, Appendix 9

9. ANSYS ANALYSIS

9.1 Stress Analysis 
CLIENT: Duke Engineering Services Hanford

PROJECT: MCO Final Design
FILE NO: $\quad \mathrm{KH}-8009-8-07$

DOC NO: HNF-SD-SNF-DR-003, Rev. 0, Appendix 9

\section{LIST OF TABLES}

Page

TABLE 1 MARK IV SCRAP BASKET GEOMETRY

TABLE 2 ASME CODE MATERIAL PROPERTIES FOR TYPE 304L STAINLESS STEEL

TABLE 3 ALLOWABLE STRESSES - DEADWEIGHT

TABLE 4 WELD CRITERIA - DEADWEIGHT

TABLE 5 ANSYS MODEL WEIGHT

TABLE 6 EVALUATION OF STIFFENER TO BOTTOM PLATE WELD USING ANSYS RESULTS

TABLE 7 EVALUATION OF STIFFENER TO CENTER PIPE WELD USING ANSYS RESULTS

TABLE 8 EVALUATION OF STIFFENER TO OUTER (SHELL) PLATE WELD USING ANSYS RESULTS

TABLE 9 EVALUATION OF CENTER PIPE TO BOTTOM PLATE WELD USING ANSYS RESULTS

TABLE 10 EVALUATION OF OUTER SHELL TO BOTTOM PLATE WELD USING ANSYS RESULTS

TABLE 11 ANSYS RESULTS - DEADWEIGHT ALLOWABLES

TABLE 12 ANSYS RESULTS - LIFTING ALLOWABLES 


\section{LIST OF FIGURES}

FIGURE 1 ANSYS MODEL

FIGURE 2 BOUNDARY CONDITIONS

FIGURE 3 BOUNDARY CONDITIONS- TOP VIEW

FIGURE 4 STRESS INTENSITY

FIGURE 5 STRESS INTENSITY IN BOTTOM PLATE

FIGURE 6 STRESS INTENSITY IN CENTER PIPE

FIGURE 7 STRESS INTENSITY IN CENTER PIPE AT STIFFENER PLATE CONNECTION

FIGURE 8 STRESS INTENSITY IN OUTER (SHELL) PLATE

FIGURE 9 STRESS INTENSITY IN STIFFENER PLATE 
CLIENT: Duke Engineering Services Hanford

FILE NO: KH-8009-8-07

PROJECT: MCO Final Design

DOC NO: HNF-SD-SNF-DR-003, Rev. 0, Appendix 9

\section{LIST OF APPENDICES}

APPENDIX A 


\section{INTRODUCTION}

This calculation documents the evaluation of the Mark IV scrap basket for lifting and deadweight loads. The evaluations are performed based on the criteria of the ASME Code and ANSI N14.6. A combination of hand calculations and ANSYS analysis is used.

\section{REFERENCES}

1. "Performance Specification for the Spent Nuclear Fuel Multi-Canister Overpack," Specification HNF-S-0426, Revision 3, February 1996.

2. "K-Basin Spent Nuclear Fuel Scrap Basket", Prepared for U.S.D.O.E. (Richland Operations) by DE\&S Hanford, Drawing Number H-2-828075, Revsion C.

3. ASME Boiler and Pressure Vessel Code, Section II - Materials, Part D - Properties, 1995 Edition with 1995 Addenda.

4. ASME Boiler and Pressure Vessel Code, Section III, Subsection NG, "Core Support Structures", 1995 Edition with 1995 Addenda.

5. ASME Boiler and Pressure Vessel Code, Section III, Subsection NF, "Component Supports", 1995 Edition with 1995 Addenda.

6. "American National Standard for Radioactive Materials - Special Lifting Devices for Shipping Containers Weighing 10000 Pounds (4500 kg) or More", ANSI N14.6-1993, American National Standards Institute, New York.

7. Roark, Raymond J., \& Young, Warren C., "Formulas for Stress and Strain", 5th Edition, McGraw-Hill Book Company, New York, 1975.

8. AISC, 1989, Manual of Steel Construction, Ninth Edition, American Institute of Steel Construction, Chicago, Illinois

\section{ASSUMPTIONS}

1. All welds are made in accordance with NG-4000 and are inspected in accordance with NG 5260.

3. Others as noted

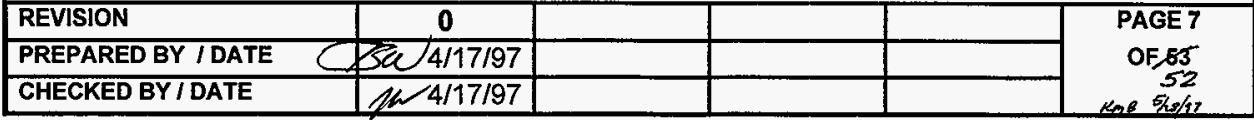


CLIENT: Duke Engineering Services Hanford

PROJECT: MCO Final Design DOCNO: HNF-SD-SNF-DR-003, Rev. 0, Appendix 9

\section{GEOMETRY}

The primary structural components of the Mark IV scrap basket, as defined in the Reference 2 drawing, are as listed in Table 1:

Table 1

Mark IV Scrap Basket Geometry

\begin{tabular}{|c|c|}
\hline Component & Description \\
\hline Center Pipe & $\begin{array}{l}\text { Tubular Section with Outside diameter of } 2.75 \text { inches and a nominal } \\
\text { wall thickness of } 0.50^{\prime \prime} \text {. Section Properties are: } \\
\qquad \begin{aligned} A & =\frac{\pi}{4}\left(O D^{2}-I D^{2}\right) \\
& =\frac{\pi}{4}\left((2.75 \mathrm{in})^{2}-(1.75 \mathrm{in})^{2}\right) \\
& =3.53 \mathrm{in}^{2} \\
I & =\frac{\pi}{64}\left(O D^{4}-I D^{4}\right) \\
& =\frac{\pi}{64}\left((2.75 \mathrm{in})^{4}-(1.75 \mathrm{in})^{4}\right) \\
& =2.35 \mathrm{in}^{4} \\
r & =\sqrt{1 / \mathrm{A}} \\
& =\sqrt{2.35 \mathrm{in}^{4} / 3.53 \mathrm{in}^{2}} \\
& =.815 \mathrm{in}^{2}\end{aligned}\end{array}$ \\
\hline Bottom Plate & $\begin{array}{l}\text { The bottom plate is a } 1 / 4 \text { " thick plate with } 1 / 4 \text { " diameter holes on a } \\
1-1 / 2 \text { triangular pitch. }\end{array}$ \\
\hline
\end{tabular}

$\log _{4}$


CLIENT: Duke Engineering Services Hanford
FILE NO: $\quad \mathrm{KH}-8009-8-07$

DOC NO: HNF-SD-SNF-DR-003, Rev. 0, Appendix 9
Outer (Shell) Plate
The outer shell plate is 12 gage sheet metal (.109" thick). The OD of the shell is 22.50 inches. Other section properties are:

$$
\begin{aligned}
I D & =O D-2 t \\
& =22.50 \mathrm{in}-2(.109 \mathrm{in}) \\
& =22.28 \mathrm{in} \\
I R & =I D / 2 \\
& =11.14 \mathrm{in} \\
A & =\frac{\pi}{4}\left(O D^{2}-I D^{2}\right) \\
& =\frac{\pi}{4}\left((22.50 \mathrm{in})^{2}-(22.28 \mathrm{in})^{2}\right) \\
& =7.74 \mathrm{in}^{2}
\end{aligned}
$$

There are 6 radial stiffener plates connecting the center pipe to the bottom plate and the shell plate. The stiffener plates are 10 gage $\left(.109^{\prime \prime}\right)$ thick stainless steel.

Center Pipe to Coupling Ring Weld

The center pipe to coupling ring weld is specified as a continuous $1 / 4$ " fillet weld.

Coupling Ring to Bottom Plate Weld

The center pipe to coupling ring weld is specified as a continuous $1 / 4^{\prime \prime}$ fillet weld.

Outer (Shell) Plate to Bottom Plate Weld

The shell plate to bottom plate weld is specified as a continuous butt weld.

Stiffener Plate to Center Pipe Weld

Stiffener Plate to Outer (Shell) Plate Weld

Stiffener Plate to Bottom Plate Weld
The stiffener plate to center pipe weld(s) is specified as a double sided intermittent 0.109 " fillet weld, 2 " welds on 4.43 " centers.

The stiffener plate to outer plate weld(s) is specified as a double sided intermittent 0.109 " fillet weld, 2 " welds on 4.43 " centers.

The stiffener plate to bottom plate weld(s) is specified as a double sided $1 / 16$ " fillet weld (2" on 4 " centers).

0

(

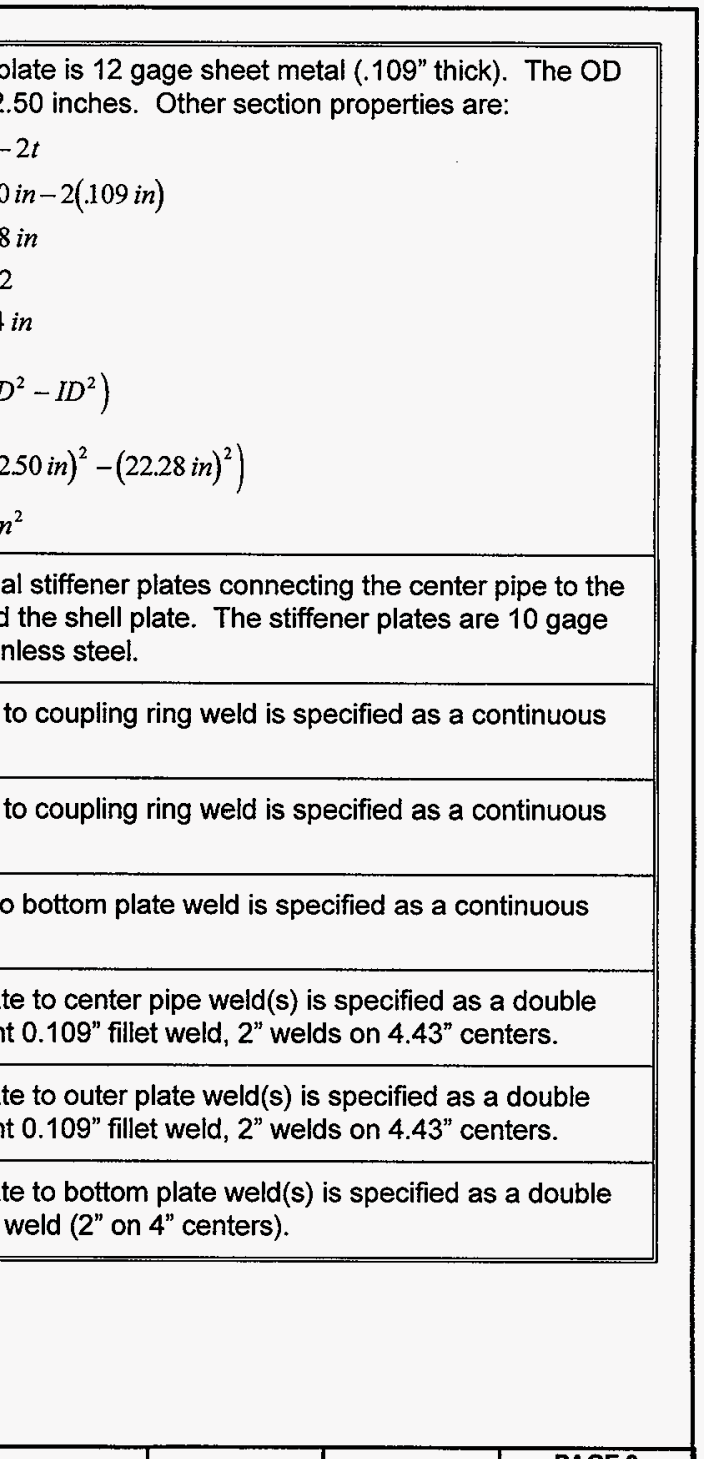


CLIENT: Duke Engineering Services Hanford

\section{MATERIAL PROPERTIES}

The Mark IV scrap basket may fabricated from Type 304L or Type 304 stainless steel. Only Type $304 \mathrm{~L}$ material properties are used in this analysis to preserve conservatism.

For this analysis, only elastic modulus and allowable stress values are needed. Values are taken from Section II, Part D of the Code (See [3]) and are listed in Table 2.

Table 2

ASME Code Material Properties for Type 304L Stainless Steel

\begin{tabular}{|c|c|c|c|c|c|}
\hline \multicolumn{2}{|c|}{ Temperature } & \multirow{2}{*}{$\begin{array}{c}E \\
\text { ble } T M-1 \text {, Group }\end{array}$} & \multirow{2}{*}{$\underset{\text { Table 2A, p.322 }}{S_{m}}$} & \multirow{2}{*}{$\begin{array}{c}S_{y} \\
\text { Table Y-1, p.524 }\end{array}$} & \multirow{2}{*}{$\begin{array}{c}S_{u} \\
\text { Table U, P. 441 }\end{array}$} \\
\hline${ }^{\circ} \mathrm{F}$ & ${ }^{\circ} \mathrm{C}$ & & & & \\
\hline-20 & -- & - & $16.7 \mathrm{ksi}$ & $25.0 \mathrm{ksi}$ & $70.0 \mathrm{ksi}$ \\
\hline 70 & - & $28.3 E+06$ & - & - & - \\
\hline 100 & - & - & $16.7 \mathrm{ksi}$ & $25.0 \mathrm{ksi}$ & $70.0 \mathrm{ksi}$ \\
\hline 200 & - & $27.6 E+06$ & $16.7 \mathrm{ksi}$ & $21.3 \mathrm{ksi}$ & $66.2 \mathrm{ksi}$ \\
\hline 212 & 100 & $27.5 \mathrm{E}+06$ & $16.7 \mathrm{ksi}$ & $21.0 \mathrm{ksi}$ & $65.6 \mathrm{ksi}$ \\
\hline 300 & - & $27.0 \mathrm{E}+06$ & $16.7 \mathrm{ksi}$ & $19.1 \mathrm{ksi}$ & $60.9 \mathrm{ksi}$ \\
\hline 700 & - & $24.8 \mathrm{E}+06$ & $13.5 \mathrm{ksi}$ & $14.9 \mathrm{ksi}$ & $56.2 \mathrm{ksi}$ \\
\hline 707 & 375 & $24.8 \mathrm{E}+06$ & $13.5 \mathrm{ksi}$ & $14.9 \mathrm{ksi}$ & $56.2 \mathrm{ksi}$ \\
\hline 750 & - & - & $13.3 \mathrm{ksi}$ & $14.7 \mathrm{ksi}$ & $55.9 \mathrm{ksi}$ \\
\hline 800 & & $24.1 E+06$ & - & - & - \\
\hline
\end{tabular}

s4allow:xis

Notes 1: Underlined values determined by linear interpolation, all other values taken from Section II, Part D of the ASME Code.

2: Value of $E$ taken from Table TM-1 for Material Group $G$. 


\section{ACCEPTANCE CRITERIA}

This calculation considers (1) lifting loads and (2) deadweight loads. Criteria for each are described below

\subsection{Lifting Loads}

Per Section 4.12.3 of the MCO Specification (See [1]), the basket design is "shall meet the safety factors required by the American National Standards Institute (ANSI) N14.6-1986. THE ANSI safety factors apply from $5^{\circ} \mathrm{C}$ to $100^{\circ} \mathrm{C}$." For nonessential lifts, Section 4.2.1.1 of N14.6 (See [6]) establishes factors safety of 3 against yield stress and 5 against tensile stress. Thus criteria of N14.6 is equivalent to an allowable stress of the lesser of $S_{y} / 3$ or $\mathrm{S}_{\mathrm{u}} / 5$. At the maximum lifting temperature of $100^{\circ} \mathrm{C}$, the allowables are:

$$
\begin{aligned}
& \frac{S_{y}}{3}=\frac{25.0 \mathrm{ksi}}{3}=8.33 \mathrm{ksi} \\
& \frac{S_{u}}{5}=\frac{70.0 \mathrm{ksi}}{5}=14.00 \mathrm{ksi} \\
& \Rightarrow \text { use: } P_{m}+P_{b} \leq 8.33 \mathrm{ksi}
\end{aligned}
$$

\subsection{Deadweight Loads}

Per Section 4.12.3 of [1], all baskets must be able to support the fuel at $1.0 \mathrm{~g}$ while at $375^{\circ} \mathrm{C}$. The specification does not provide criteria for the Mark IV baskets under these loads, thus the normal (Level A) condition criteria of Subsection NG will be used. As described in the following paragraphs, the criteria of NG is supplemented by the criteria of Subsection NF for the center pipe. For membrane and membrane plus bending stresses the allowable stresses of Table 3 are applied.

The scrap baskets may be located at the top or the bottom of the MCO. Thus, in addition to self weight, the Mark IV scrap basket must be able to support the weight of 4 additional baskets. Under these compressive loads, stability of the basket must be evaluated. 
Table 3

Allowable Stresses - Deadweight

\begin{tabular}{|c|c|c|c|c|c|}
\hline \multirow{2}{*}{ Temperature } & \multirow{2}{*}{$\mathrm{S}_{\mathrm{m}}$} & \multicolumn{3}{|c|}{ Design/Level A Stress Limits } \\
\cline { 5 - 6 } & $\mathrm{P}_{\mathrm{m}}$ & $\mathrm{P}_{\mathrm{L}}$ & $\left(\mathrm{P}_{\mathrm{m}}\right.$ or $\left.\mathrm{P}_{\mathrm{L}}\right)+\mathrm{P}_{\mathrm{b}}$ \\
$\left({ }^{\circ} \mathrm{F}\right.$ & ${ }^{\circ} \mathrm{C}$ & Table 2] & $\left(\mathrm{S}_{\mathrm{m}}\right)$ & $\left(1.5 \mathrm{~S}_{\mathrm{m}}\right)$ & $\left(1.5 \mathrm{~S}_{\mathrm{m}}\right)$ \\
\hline 212 & 100 & $16.7 \mathrm{ksi}$ & $16.7 \mathrm{ksi}$ & $25.1 \mathrm{ksi}$ & $25.1 \mathrm{ksi}$ \\
\hline 707 & 375 & $13.5 \mathrm{ksi}$ & $13.5 \mathrm{ksi}$ & $20.3 \mathrm{ksi}$ & $20.3 \mathrm{ksi}$ \\
\hline
\end{tabular}

s4allow.xls

Notes 1: Design \& Level A Stress Limits from NG-3221 \& NG-3222, respectively.

2: Axial compressive stresses must be limited to values established in accordance with one of the following:

- NG-3133.3 (external pressure)

- NG-3133.6 (axial compression on cylindrical shells)

- NF-3322.1(c) (column type members)

- Code Case N-284 (shell structures)

\subsubsection{Outer Shell}

\subsubsection{Shell Buckling}

The shell buckling allowable for the outer shell is determined in the following steps using NG-3133.6. Note that NG-3133.6 assumes that both the top and bottom edges of the shell are constrained by stiffening rings and/or vessel heads. This constraint is not provided at the top of the basket (although the radial stiffeners do provide intermittent support).

1. Determine the factor $A$ using a value of $\mathbf{1 1 . 1 4}$ inches for the shell inside radius (See Table 1 for dimensions):

$$
\begin{aligned}
& A=\frac{.125}{R / t} \\
& =\frac{.125}{11.14 \mathrm{in} / 0.109 \mathrm{in}}=0.0012
\end{aligned}
$$


2. From the ASME Code, Section II, Part D, Figure HA-3 for Type 304L stainless steel at $375^{\circ} \mathrm{C}$, the allowable compressive stress is equal to Factor $\mathrm{B}$ :

$$
B=4100 \text { psi }
$$

\subsubsection{Panel Buckling}

Critical load will be investigated using Table 35, Case 13 of [7]. Each $60^{\circ}$ segment of the basket is modeled as a curved panel with a uniform compression load on the curved edges. This formulation does not include the safety factors built into the Code equations.

$$
\sigma^{\prime}=\frac{1}{6} \cdot \frac{E}{1-v^{2}} \cdot\left[\sqrt{12\left(1-v^{2}\right)\left(\frac{t}{r}\right)^{2}+\left(\frac{\pi t}{b}\right)^{4}}+\left(\frac{\pi t}{b}\right)^{2}\right]
$$

Terms in this equation are:

$$
\begin{aligned}
r & =\text { radius, use OR of } 11.25 \mathrm{in} \\
a & =\text { panel height }=26.85 \mathrm{in} \\
b & =\text { arc length } \\
& =\alpha \cdot O R=60^{\circ}\left(\frac{\pi}{180^{\circ}}\right) 11.25 \mathrm{in}, \\
& =11.78 \mathrm{in} \\
t & =\text { panel thickness }=.109 \mathrm{in}
\end{aligned}
$$

Substituting the dimensions into the buckling equation to find the critical buckling stress and using $\mathrm{E}$ at $375^{\circ} \mathrm{C}$ from Table 2:

$$
\begin{aligned}
\sigma^{\prime} & =\frac{1}{6} \cdot \frac{24.8 \cdot 10^{6} p s i}{1-.3^{2}} \cdot\left[\sqrt{12\left(1-3^{2}\right)\left(\frac{.109 \mathrm{in}}{11.25 \mathrm{in}}\right)^{2}+\left(\frac{\pi \cdot .109 \mathrm{in}}{11.25 \mathrm{in}}\right)^{4}}+\left(\frac{\pi \cdot .109 \mathrm{in}}{11.25 \mathrm{in}}\right)^{2}\right. \\
& =150 \mathrm{ksi}
\end{aligned}
$$

This stress is much larger than the value determined for shell buckling in Section 6.2.1.1, therefore the shell buckling allowable will be used (Note also that this value is greater than the yield stress, thus elastic buckling will not control).

\subsubsection{Center Pipe}

For the center pipe, criteria will be developed based on the more restrictive of NG-3133.6 (shell buckling) or NF-3322.1(c)(2) (buckling of stainless steel columns).

\begin{tabular}{|l|c|l|l|l|}
\hline REVISION & 0 & & & \\
\hline PREPARED BY I DATE & C 4/17/97 & & & \\
\hline CHECKED BY I DATE & /S 4/17/97 & & & \\
\hline
\end{tabular}


CLIENT: Duke Engineering Services Hanford
FILE NO: $\quad \mathrm{KH}-8009-8-07$

PROJECT: MCO Final Design

DOC NO: HNF-SD-SNF-DR-003, Rev. 0, Appendix 9

6.2.2.1 Shell Buckling - NG-3133.6

Determine factor $A$ where $R$ is the center pipe inside radius of $1.375^{\prime \prime}$

$$
\begin{aligned}
A & =\frac{.125}{R / t} \\
& =\frac{.125}{.875 \mathrm{in} / 0.50 \mathrm{in}}=.071
\end{aligned}
$$

From Figure $\mathrm{HA}-3$ for Type $304 \mathrm{~L}$ stainless steel at $375^{\circ} \mathrm{C}$, the allowable compressive stress is equal to the Factor $B$ :

$$
B=8500 p s i
$$

\subsubsection{Column Buckling - NF-3322(c)(2):}

The center pipe is approximately 28.0 " tall. At the bottom of the basket, the pipe is restrained by the bottom plate. At the top of the basket, the center pipes interlock such that lateral restraint is provided. Thus the pipe is modeled as pinned at both the top and bottom ( $k=1.0$, Reference 8 ). Using the radius of gyration calculated in Table 1, the equivalent buckling length is:

$$
\frac{k l}{r}=\frac{1.0 \cdot 28.6 \mathrm{in}}{.815 \mathrm{in}}=35.1
$$

For this value of $\mathrm{k} / \mathrm{r}$, the allowable axial compressive stress is:

$$
F_{a}=S_{y}\left(0.47-\frac{(k 1 / r)}{444}\right)
$$

At the deadweight temperature of $375^{\circ} \mathrm{C}$, this becomes

$$
\begin{aligned}
F_{a} & =14.9 \mathrm{ksi}\left(0.47-\frac{35.1}{444}\right) \\
& =5.83 \mathrm{ksi}
\end{aligned}
$$

This value is less than the value for shell buckling, thus the allowable compressive stress on the center pipe will be conservatively taken as value for column buckling.

\begin{tabular}{|c|l|l|l|}
0 & & & \\
\hline $5 / 4 / 17 / 97$ & & & \\
\hline$/ 24 / 17 / 97$ & & & \\
\hline
\end{tabular}




\subsubsection{Weld Criteria}

Criteria for welds will be developed based on the quality factors provided in Table NG-33521. As stated in NG-3352, "The quality factor is used by multiplying the allowable stress limit for primary and secondary stress categories times the quality factor in evaluating the design." For deadweight loads, this is interpreted as factoring the allowable membrane plus bending stress (listed in

Table 3) by the appropriate quality factor from Table NG-3352-1. These values are listed and allowables developed in Table 4.

Table 4

Weld Criteria - Deadweight

\begin{tabular}{||l|l|l|c|c||}
\hline \multicolumn{1}{|c|}{ Weld Type } & Location(s) & $\begin{array}{c}\text { Quality } \\
\text { Factor }\end{array}$ & $\begin{array}{c}\text { Allowable } \\
\text { Primary } \\
\text { Stress }\end{array}$ & $\begin{array}{c}\text { Allowable } \\
\text { Weld } \\
\text { Stress }\end{array}$ \\
\hline $\begin{array}{l}\text { Continuous Fillet Weld } \\
\text { (Type VII) }\end{array}$ & $\begin{array}{l}\text { 1. Center Pipe to Coupling } \\
\text { Ring Weld } \\
\text { 2. Coupling Ring to Bottom } \\
\text { Plate Weld }\end{array}$ & $\mathrm{n}=0.35$ & $20.3 \mathrm{ksi}$ & $7.11 \mathrm{ksi}$ \\
\hline $\begin{array}{l}\text { Intermittent butt weld; 2" } \\
\text { welds on 4" center }\end{array}$ & $\begin{array}{l}\text { 3. Shell plate to bottom plate } \\
\text { weld }\end{array}$ & $\begin{array}{l}n=0.30 \\
\text { (Note 1) }\end{array}$ & $20.3 \mathrm{ksi}$ & $6.03 \mathrm{ksi}$ \\
\hline $\begin{array}{l}\text { Double sided intermittent } \\
\text { fillet weld (1/8"); } \\
2^{n} \text { welds on 4" centers. }\end{array}$ & $\begin{array}{l}\text { 4. Stiffener Plate to Center } \\
\text { Pipe Weld } \\
\text { 5. Stiffener Plate to Outer } \\
\text { (Shell) Plate Weld } \\
\text { 6. Stiffener Plate to Bottom } \\
\text { Plate Weld }\end{array}$ & $\mathrm{n}=0.30$ & $20.3 \mathrm{ksi}$ & $6.03 \mathrm{ksi}$ \\
\hline
\end{tabular}

Notes:

1. No criteria are provided for intermittent butt welds, therefore the quality factor for intermittent fillet welds is conservatively applied.

2. All welds are assumed to be inspected using surface visual examination in accordance with NG-5260.

\section{LOAD CONDITIONS \& COMBINATIONS}

The Mark IV scrap baskets are evaluated for two load cases:

\begin{tabular}{|l|l|l|l|}
\hline 0 & & & \\
\hline $4 / 17 / 97$ & & & \\
\hline $4 / 17 / 97$ & & & \\
\hline
\end{tabular}


CLIENT: Duke Engineering Services Hanford
FILE NO: $\quad \mathrm{KH}-8009-8-07$

PROJECT: MCO Final Design

DOCNO:

HNF-SD-SNF-DR-003, Rev. 0, Appendix 9

1. Lifting of the basket and contents while at $100^{\circ} \mathrm{C}$. This loading is evaluated using criteria based on the safety factors incorporated in ANSI N-14.6.

2. Deadweight of the basket and contents while at $375^{\circ} \mathrm{C}$. The basket inside the $M C O$ is considered the limiting case. The basket may be at the top of the MCO (and thus be required to support only it's own weight) or at the bottom of the MCO where it is required to support the dead load of 4 added baskets.

No other loads are considered. Section 4.12 .2 of the performance specification (See [1]) exempts the Mark IV baskets from consideration of drop, or other, accident loads.

An analysis weight of 3200 pounds (see Table 1 of Appendix 3 ) is used for each basket.

\section{STRESS ANALYSIS - HAND CALCULATIONS}

The Mark IV scrap basket will be evaluated using hand calculations. The analysis model used for each component is described in the following sections: As appropriate, separate evaluations are performed for lifting and deadweight and/or, tensile and compressive loadings.

\subsection{Center Pipe}

\subsubsection{Lifting}

The center pipe must support the total weight of the basket and contents for lifting.

Stresses will be calculated over the gross cross section and at the reduced cross section at the grapple attachment. Note that local effects at the grapple attachment are not included in the scope of this calculation. The nominal cross sectional area is calculated in Table 1. At the grapple, the OD of the pipe remains 2.75 in, however the inside diameter increases from 1.75 inch to:

$$
I D=1.75 \text { in }+2(.125 \text { in })=2.00 \text { in }
$$

The cross sectional area is:

$$
\begin{aligned}
A & =\frac{\pi}{4}\left(O D^{2}-I D^{2}\right) \\
& =\frac{\pi}{4}\left[(2.75 \mathrm{in})^{2}-(2.00 \mathrm{in})^{2}\right] \\
& =2.80 \mathrm{in}^{2}
\end{aligned}
$$

Stress at the grapple ring

$\mathrm{Cac}^{4}$




$$
f_{a}=\frac{3200 / b}{2.80 \mathrm{in}^{2}}=1.14 \mathrm{ksi}
$$

Stress over the nominal cross section:

$$
f_{a}=\frac{3200 \mathrm{lb}}{3.53 \mathrm{in}^{2}}=.91 \mathrm{ksi}
$$

Ratios of calculated to allowable stress are:

$$
\text { Ratio: } \begin{aligned}
& \frac{1.14 \mathrm{ksi}}{8.33 \mathrm{ksi}}=.14 \leq 1.0 \Rightarrow O K \quad \text { (at the grapple ring) } \\
& \frac{0.91 \mathrm{ksi}}{8.33 \mathrm{ksi}}=.11 \leq 1.0 \Rightarrow \text { OK (at the nominal cross section) }
\end{aligned}
$$

\subsubsection{Deadweight}

Under deadweight loading, the critical load in the center pipe is axial compression resulting from the weight of the 4 upper baskets (for the bottom basket in the $\mathrm{MCO}$ ). Assuming the center pipe carries the total weight of the upper baskets and neglecting the self weight of the center pipe in the bottom basket, the axial compression stress is:

$$
f_{a}=\frac{(4 \text { baskets }) 3200 \mathrm{lb} / \text { basket }}{3.53 \mathrm{in}^{2}}=3.63 \mathrm{ksi}
$$

Compared to the allowable column buckling stress determined in Section 6.2.2:

$$
\text { Ratio: } \frac{3.63 \mathrm{ksi}}{5.83 \mathrm{ksi}}=.62 \leq 1.0 \Rightarrow \text { OK }
$$

\subsection{Bottom Plate}

To evaluate the bottom plate using hand calculations, the plate is modeled as a uniformly loaded circular sector. Equations are provided in [6] for two type of boundary conditions (1) all edges simply supported and (2) the sides simply supported and the outside edge fixed. Although this analysis model does not correspond directly with the loaded condition, it will provide an indication of the stress level in the bottom plate. Using this model, stresses in the bottom plate are the same for the lifting and deadweight conditions.

\begin{tabular}{|l|l}
\hline 0 & \\
\hline $4 / 17 / 97$ & \\
\hline $4 / 17 / 97$ &
\end{tabular}


For both sets of boundary conditions, the applied pressure is taken as the plate self weight plus the fuel weight distributed over the annular plate area. Plate area is (using OD of the coupling ring and ID of the shell plate):

$$
\begin{aligned}
A_{\text {plate }} & =\frac{\pi\left(O D^{2}-I D^{2}\right)}{4} \\
& =\frac{\pi\left((3.25 \mathrm{in})^{2}-(22.28 \mathrm{in})^{2}\right)}{4}=381.6 \mathrm{in}^{2}
\end{aligned}
$$

The applied pressure load is:

$$
\begin{aligned}
q & =\frac{W_{\text {FUEL }}}{A_{\text {PLATE }}}+\gamma_{\text {STEEL }} t_{\text {PLATE }} \\
& =\frac{3010 \mathrm{lb}}{381.6 \mathrm{in}^{2}}+\left(.286 \mathrm{lb} / \mathrm{in}^{3}\right)(.25 \mathrm{in}) \\
& =7.96 \mathrm{lb} / \mathrm{in}^{2} \Rightarrow \text { use } 8.0 \mathrm{lb} / \mathrm{in}^{2}
\end{aligned}
$$

Reference: fuel weight from Appendix 3, Table 1, assuming scrap weight = total fuel weight, 304 SS density from Mil Hndbk 5.

Case 1 - All edges Simply supported ([7], Table 24, Case 27 (Page 370):

The maximum bending stress is given as:

$$
\sigma_{\max }=\frac{\beta_{1} \cdot q \cdot a^{2}}{t^{2}}
$$

For $\theta$ equal to $60^{\circ}$ ( 6 stiffeners), the factor $\beta_{1}$ is .155 . Using the shell plate OD to calculate the stress:

$$
\begin{aligned}
\sigma_{\max } & =\frac{.155 \cdot 8.0 \mathrm{lb} / \mathrm{in}^{2} \cdot(11.25 \mathrm{in})^{2}}{(.25 \mathrm{in})^{2}} \\
& =2.51 \mathrm{ksi}
\end{aligned}
$$

Case 2 - Outside edge fixed ([7], Table 24, Case 28 (Page 371):

The maximum bending stress is calculated using the same equation listed in the preceding paragraph. For the outside edge fixed and $\theta$ equal to $60^{\circ}$ (6 stiffeners), the factor $\beta$ is .204 . The stress is: 


$$
\begin{aligned}
\sigma_{\max } & =\frac{.204 \cdot 8.0 \mathrm{lb} / \mathrm{in}^{2} \cdot(11.25 \mathrm{in})^{2}}{(25 \mathrm{in})^{2}} \\
& =3.30 \mathrm{ksi}
\end{aligned}
$$

Case 2 controls. The ratio of calculated to allowable stress is determined for both lifting and deadweight allowables. The calculated stress in bending in the bottom plate. Thus the allowable for membrane plus bending stress is applied:

$$
\text { Ratio: } \begin{aligned}
& \frac{3.30 \mathrm{ksi}}{8.33 \mathrm{ksi}}=.40 \leq 1.0 \Rightarrow \text { OK } \quad(\text { lifting }) \\
& \frac{3.30 \mathrm{ksi}}{20.3 \mathrm{ksi}}=.16 \leq 1.0 \Rightarrow \text { OK } \quad(\text { deadweight })
\end{aligned}
$$

\subsection{Center Pipe to Coupling Pipe and Coupling Pipe to Bottom Plate Welds}

Both welds will be evaluated using a weld area based on the outside diameter of the center pipe:

$$
\begin{aligned}
A_{w} & =\pi \cdot O D \cdot t_{w} \\
& =\pi(2.75 \mathrm{in})(.25 \mathrm{in}) \\
& =2.16 \mathrm{in}^{2}
\end{aligned}
$$

\section{Lifting}

Under lifting loads, the welds are assumed to carry the total weight of the basket and contents. Weld stress is:

$$
\begin{aligned}
f_{w} & =\frac{\text { Weight }}{A_{w}} \\
& =\frac{3200 \mathrm{lb}}{2.16 \mathrm{in}^{2}} \sqrt{2} \\
& =2.10 \mathrm{ksi} \quad \text { (at the weld throat) }
\end{aligned}
$$

\section{$\underline{\text { Deadweight }}$}

Under deadweight loads, the weld in the bottom basket is assumed to carry half of the total load from the upper 4 baskets. This assumption is reasonable since the center pipe is welded to the stiffeners, which transfer much of load directly to the bottom plate.

\begin{tabular}{|c|c|c|c|}
\hline 0 & & & \\
\hline $\operatorname{lac} 4 / 17 / 97$ & & & \\
\hline
\end{tabular}




$$
\begin{aligned}
f_{w} & =\frac{4 \cdot \text { Weight }}{A_{w}} \\
& =\frac{4 \cdot 3200 \mathrm{lb}}{2.16 \mathrm{in}^{2}} \sqrt{2} \\
& =8.38 \mathrm{ksi} \text { (at the weld throat) }
\end{aligned}
$$

Using the allowable weld stress from Table 4, the ratio of calculated to allowable stress is:

$$
\text { Ratio: } \frac{8.38 \mathrm{ksi} / 2}{7.11 \mathrm{ksi}}=0.59 \leq 1.0 \Rightarrow O K
$$

\subsection{Stiffener Plate to Bottom Plate Welds}

The loading in the stiffener plate to bottom plate welds is the same for deadweight and lifting. Stresses in the 2"-4" skip welds are calculated based on double fillets with $1 / 16$ " leg. The effective weld throat is compared to the stiffener plate thickness to determine the controlling element:

$$
t_{w}=\frac{(2 \text { sides })(.0625 \mathrm{in})}{\sqrt{2}}=.088 \mathrm{in}
$$

The combined thickness of the double sided weld is slightly less than the plate thickness of .109 inches. In the initial analysis of this weld, the weld area was larger than the plate thickness and the stress was calculated based on the plate thickness. As noted in Table 11 , below, the calculated margin is sufficient to cover this weld size change.

There are 6 stiffener plates (spaced at $60^{\circ}$ ) around the basket. Based on a coupling ring OD of 3.25 in and a shell plate ID of 11.14 inches, and using the plate thickness, the weld area is:

$$
\begin{aligned}
A_{w} & =L_{w} \cdot t_{\text {platc }}=6 \text { stiffeners }(22.28 \mathrm{in}-3.25 \mathrm{in})(.109 \mathrm{in}) / 2 \\
& =(57.1 \mathrm{in})(.109 \mathrm{in}) \\
& =6.22 \mathrm{in}^{2}
\end{aligned}
$$

Assuming that the weld carries the total load of $3200 \mathrm{lb}$ :

$$
\begin{aligned}
f_{w} & =\frac{3200 \mathrm{lb}}{6.22 \mathrm{in}^{2}} \\
& =0.51 \mathrm{ksi}
\end{aligned}
$$


CLIENT: Duke Engineering Services Hanford
FILE NO: KH-8009-8-07

PROJECT: MCO Final Design DOC NO: HNF-SD-SNF-DR-003, Rev. 0, Appendix 9

Using the allowable weld stress from Table 4, the ratio of calculated to allowable stress is:

$$
\text { Ratio: } \frac{0.51 \mathrm{ksi}}{6.03 \mathrm{ksi}}=0.08 \leq 1.0 \Rightarrow O K
$$

\subsection{Stiffener Plate to Center Pipe Weld}

The loading in the stiffener plate to center pipe welds is considered to be the same for deadweight and lifting. Stresses in the welds are calculated based on five (5) 2" long segments of double fillet welds with $1 / 8$ " leg. The effective weld throat is compared to the stiffener plate thickness to determine the controlling element:

$$
t_{\mathrm{w}}=\frac{(2 \text { sides })(.125 \mathrm{in})}{\sqrt{2}}=.177 \mathrm{in}
$$

The combined thickness of the double sided weld is greater than the plate thickness of .109 inches. Therefore, stress will be calculated based on the plate thickness.

There are 6 stiffener plates (spaced at $60^{\circ}$ ) around the basket. The total weld area is:

$$
\begin{aligned}
A_{w} & =L_{w} \cdot t_{\text {plate }} \\
& =(6 \text { stiffeners })(5 \text { welds } / \text { stiffener })(2 \text { in } / \text { weld })(109 \mathrm{in}) \\
& =6.54 \mathrm{in}^{2}
\end{aligned}
$$

Assuming that the total load of 3200 pounds is carried in the welds, the stress is:

$$
\begin{aligned}
f_{w} & =\frac{3200 \mathrm{lb}}{6.54 \mathrm{in}^{2}} \\
& =0.49 \mathrm{ksi}
\end{aligned}
$$

Using the allowable stress for intermittent fillet welds from Table 4, the ratio of calculated to allowable stress is:

$$
\text { Ratio: } \frac{0.49 \mathrm{ksi}}{6.03 \mathrm{ksi}}=0.08 \leq 1.0 \Rightarrow O K
$$




\subsection{Stiffener Plate to Outer (Shell) Plate Weld}

The configuration of the stiffener plate to outer (shell) plate welds is identical to the configuration of the stiffener plate to center pipe welds evaluated in Section 8.5 (2" double fillet welds on 4" centers). Therefore, the qualification of the stiffener plate to center pipe welds in Section 8.5 also demonstrates qualfication of the stiffener to outer plate weld. No additional analysis is required.

\subsection{Outer (Shell) Plate to Bottom Plate Weld}

The outer plate to bottom plate weld is specified as a continuous butt weld. For a shell diameter of approximately $22.5^{\prime \prime}$, there will be welding for $1 / 2$ of the circumference and the weld thickness will be equal to the outer plate thickness of .109 in. Weld area is:

$$
\begin{aligned}
A_{w} & =L_{w} t_{w} \\
& =(\pi O D) t_{w} \\
& =(\pi 22.5 \mathrm{in}) .109 \mathrm{in} \\
& =7.7 \mathrm{in}^{2}
\end{aligned}
$$

Lifting

Assuming the weld carries the total load of 3200 pounds, the stress is:

$$
\begin{aligned}
f_{w} & =\frac{3200 \mathrm{lb}}{7.7 \mathrm{in}^{2}} \\
& =0.42 \mathrm{ksi}
\end{aligned}
$$

Using the allowable stress for intermittent fillet welds from Table 4, the ratio of calculated to allowable stress is:

$$
\text { Ratio: } \frac{0.42 \mathrm{ksi}}{6.03 \mathrm{ksi}}=0.07 \leq 1.0 \Rightarrow O K
$$

\section{Deadweight}

Under deadweight loads, it is assumed that the total weight of the upper baskets is transmitted through the outer shell(s). The load on the weld will be the weight of the 4 upper baskets plus the weight of the outer plate of the bottom basket. The weight of the shell plate will be neglected and the load is:

$$
\begin{aligned}
f & =(4 \text { baskets })(3200 \mathrm{lb} / \text { basket }) \\
& =12,800 \mathrm{lb}
\end{aligned}
$$


CLIENT:

PROJECT: MCO Final Design

The stress is:

$$
\begin{aligned}
f_{w} & =\frac{12,800 \mathrm{lb}}{3.85 \mathrm{in}^{2}} \\
& =3.32 \mathrm{ksi}
\end{aligned}
$$

And the ratio of calculated to allowable stress is:

$$
\text { Ratio: } \frac{3.32 \mathrm{ksi}}{6.03 \mathrm{ksi}}=0.55 \leq 1.0 \Rightarrow O K
$$

\begin{tabular}{|l|l|l|}
\hline & & \\
\hline & & \\
\hline & & \\
\hline
\end{tabular}




\section{ANSYS ANALYSIS}

In addition to the hand calculations described in Section 8, an evaluation of the Mark IV scrap basket was performed using the finite element model shown in Figure 1. The model was developed with SHELL63 elements with 6 degrees of freedom at each node.

Thicknesses were input for each component of the model as listed below:

$\begin{array}{cc}\text { Component } & \text { Analysis Thickness } \\ \text { Bottom Plate } & .25 \text { in } \\ \text { Center Pipe } & .50 \text { in } \\ \text { Outer (Shell) Plate } & .109 \text { in } \\ \text { Stiffener Plate } & .109 \text { in }\end{array}$

The mid-thicknesses of the center pipe and outer plates were modeled at radii of 1.375" and 11.25 ", respectively. (Note: The correct mid-thickness of the center pipe is 1.125". Since the effect of this change would be small, and since the stress ratios are relatively low, a reanlysis was not necessary.) The basket height was modeled as $26.85^{\prime \prime}$. To simplify the modeling, the stiffener plate was modeled as rectangular with a uniform height of 20.6".

The basket was modeled as 4 separate parts. At the welded connections, coincident nodes were coupled in translation. The forces at these coupled nodes are used to evaluate the connecting welds. All welds were modeled as being continuous. Since the welds are intermittent (2" welds at 4 " spacing), the calculated weld stresses will be doubled.

\subsection{Stress Analysis}

\subsubsection{Boundary Conditions}

A $60^{\circ}$ section of the basket was modeled. Symmetry boundary conditions were applied at the edges of the model (See Figure 2 \& Figure 3). The top edge of the center pipe was fixed in the vertical direction to approximate the lifting configuration. These boundary conditions are also used for deadweight stresses. This is conservative since, under deadweight, additional support will be provided at the outside edge of the basket (by the bottom of the MCO for the bottom basket or by support rods for a top basket).

\subsubsection{Loading}

A uniform pressure load was applied to the bottom plate. The load was calculated (within ANSYS) using the fuel weight of $3010 \mathrm{lb}$ (see Table 1 of Appendix 3, assuming the scrap

$C_{\ln 4}$


weight equal to the total fuel weight) over the annular area of the bottom plate. In addition, a $1.0 \mathrm{~g}$ load was applied in the vertical direction. Thus the vertical reaction load should be equal to the basket self weight plus $3010 \mathrm{lb}$. The weight of the model ( $1 / 6$ of a basket) is calulated in Table 5 using the input dimensions:

\begin{tabular}{|c|c|}
\hline Component & Weight (As Modeled): \\
\hline Bottom Plate & $\begin{aligned} W_{\text {BOTTOM }} & =\left(\frac{60^{\circ}}{360^{\circ}}\right) \pi\left(O R^{2}-I R^{2}\right) \cdot t_{\text {BOTJOM }} \cdot \gamma_{\text {STEEL }} \\
& =\left(\frac{60^{\circ}}{360^{\circ}}\right) \pi\left[(1125 \mathrm{in})^{2}-(1375 \mathrm{in})^{2}\right] \cdot 25 \mathrm{in} \cdot 2854 \mathrm{lb} / \mathrm{in}^{3} \\
& =4.658 \mathrm{lb}\end{aligned}$ \\
\hline Center Pipe & $\begin{aligned} W_{C . P I P E} & =\alpha \cdot I R \cdot t_{C P P P E} \cdot h \cdot \gamma_{\text {STEEL }} \\
& =60^{\circ}\left(\frac{\pi}{180^{\circ}}\right) \cdot 1.375 \mathrm{in} \cdot .50 \mathrm{in} \cdot 26.85 \mathrm{in} \cdot .2854 \mathrm{lb} / \mathrm{in}^{3} \\
& =5.517 \mathrm{lb}\end{aligned}$ \\
\hline Outer Plate & $\begin{aligned} W_{\text {SHELL }} & =\alpha \cdot O R \cdot t_{\text {SHELL }} \cdot h \cdot \gamma_{\text {STEEL }} \\
& =60^{\circ}\left(\frac{\pi}{180^{\circ}}\right) \cdot 11.25 \mathrm{in} \cdot . .109 \mathrm{in} \cdot 26.85 \mathrm{in} \cdot .2854 \mathrm{lb} / \mathrm{in}^{3} \\
& =9.840 \mathrm{lb}\end{aligned}$ \\
\hline Stiffener Plate & $\begin{aligned} W_{\text {SIIFEENER }} & =(O R-I R) \cdot t_{\text {SIIFFENER }} \cdot h_{\text {SIIFFENER }} \cdot \gamma_{\text {STEEL }} \\
& =(11.25 \mathrm{in}-1.375 \mathrm{in}) \cdot .109 \mathrm{in} \cdot 20.6 \mathrm{in} \cdot .2854 \mathrm{lb} / \mathrm{in}^{3} \\
& =6.328 \mathrm{lb}\end{aligned}$ \\
\hline TOTAL WEIGHT & 26.3 pounds ( $1 / 6$ Basket) \\
\hline
\end{tabular}

Under the $1 \mathrm{~g}$ lifting load, the total reaction should be approximately:

$$
\begin{aligned}
F_{Z} & =W_{\text {FUEL }}+W_{\text {BASKET }} \\
& =\frac{3010 \mathrm{lb}}{6}+26.3 \mathrm{lb} \\
& =528.0 \mathrm{lb}
\end{aligned}
$$

\begin{tabular}{|c|c|c|c|}
\hline 0 & & & \\
\hline (13/17/97 & & & \\
\hline $1 / \mathrm{h} / 1 / 17 / 97$ & & & \\
\hline
\end{tabular}


Review of the stress analysis output file shows that the reported reaction force in the $Z$ direction is $527.36 \mathrm{lb}$. This is acceptable compared to the value calculated above.

\subsubsection{Results}

The SHELL63 elements used for this analysis report stresses at the top and bottom surfaces, and at the midthickness of the element. in this analysis, the midthickness stress is classified as membrane stress. Since stresses are low, stresses at all locations are conservatively classifed as general membrane stresses, $P_{m}$. Stresses at the top and bottom surface stresses are classified as membrane plus bending stresses: $P_{m}+P_{b}$.

Stress results for each part of the scrap basket were listed in the ANSYS analysis. Results are summarized in Table 11 for lifting and in Table 12 for deadweight (conservatively, the analyses are identical, only the allowables are different) In addition, stress contour plots are included (for the top surface stresses only) for each component (See Figure 4 through Figure 9).

\subsubsection{Weld Evaluation}

Welds are evaluated in the following tables:

$\begin{array}{lc}\text { Stiffener to Bottom Plate Weld } & \text { Table } 6 \\ \text { Stiffener to Center Pipe Weld } & \text { Table 7 } \\ \text { Stiffener to Outer (Shell) Plate Weld } & \text { Table 8 } \\ \text { Center Pipe to Bottom Plate Weld } & \text { Table } 9 \\ \text { Outer (Shell) Plate to Bottom Plate Weld } & \text { Table } 10\end{array}$

Evaluation of all welds follows the same methodology.

1. Length of each segment of weld, $L_{w}$, is determined based on node point coordinates

2. Forces at each node are taken from the ANSYS output and a resultant force calculated as:

$$
F_{R E S U L T A N T}=\sqrt{F_{X}^{2}+F_{Y}^{2}+F_{Z}^{2}}
$$

3. Weld load (pounds per inch) is calculated as: $F_{\text {RESULTANI }} / L_{w}$.

\begin{tabular}{|c|c|c|}
\hline 0 & & \\
\hline (4/17/97 & & \\
\hline $1 / 4 / 17 / 97$ & & \\
\hline
\end{tabular}


4. Weld stress is calculated based on the weld load and the weld area. Area used for each weld is listed in the notes at the bottom of the tables.

5. The stress ratio (calculated stress / allowable stress) is calculated. The allowable stress is listed at the bottom of each table. The intermittent fillet welds (2" welds on 4" centers) were modeled as continuous, therefore the calculated stresses are doubled (ratio of $4 " / 2 ")$.

As shown in the weld tables, all stresses are below the allowable values.

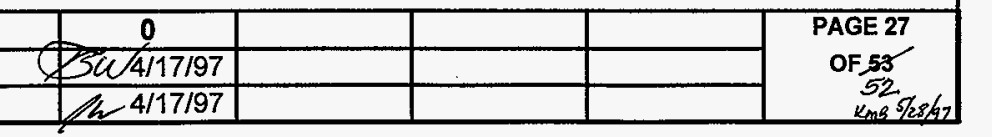


CLIENT: Duke Engineering Services Hanford

PROJECT: MCO Final Design DOC NO: HNF-SD-SNF-DR-003, Rev. 0, Appendix 9

FILE NO: $\quad \mathrm{KH}-8009-8-07$

Table 6

Evaluation of Stiffener to Bottom Plate Weld Using ANSYS Results

Stiffener to Bottom Plate Weld

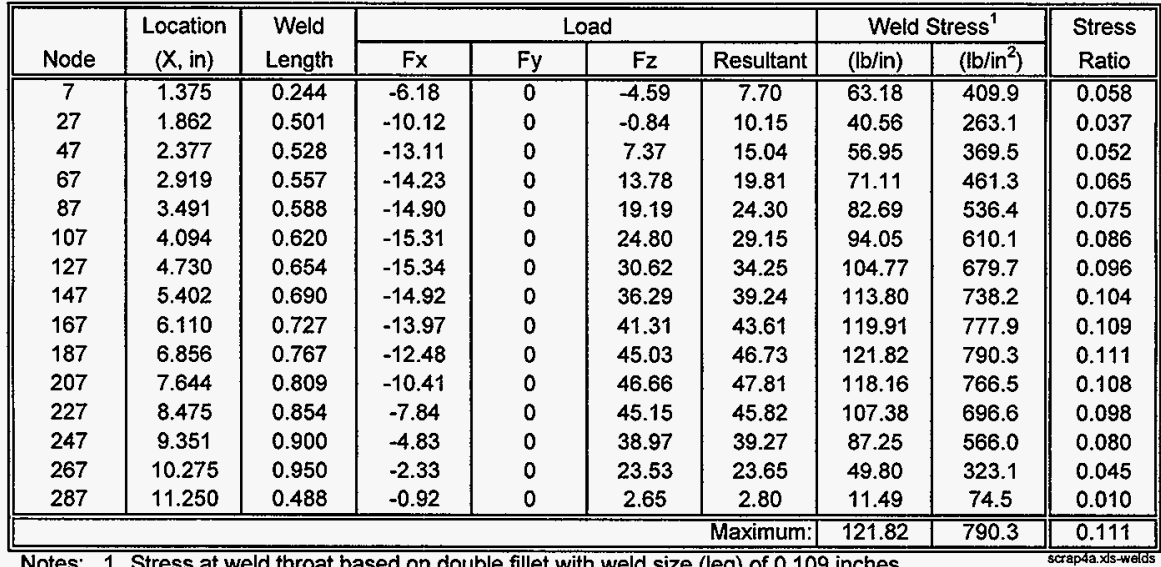

Notes: 1. Stress at weld throat based on double fillet with weld size (leg) of 0.109 inches.

2. Stress ratio based on an allowable, Fw, of $7.11 \mathrm{ksi}$ for continuous fillet welds

3. The stiffener to Center Pipe weld is an intermittent weld (2" on 4" centers), since only half the length is welded, stresses are factored by 2 .

Note: Current design is for $1 / 16$ " welds, but welds are obviously adequate due to large margins.

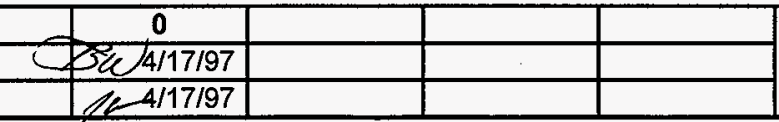


PROJECT: MCO Final Design DOC NO: HNF-SD-SNF-DR-003, Rev. 0, Appendix 9

Table 7

Evaluation of Stiffener to Center Pipe Weld Using ANSYS Results

Stiffener to Center Pipe Weld

\begin{tabular}{|c|c|c|c|c|c|c|c|c|c|}
\hline \multirow[b]{2}{*}{ Node } & \multirow{2}{*}{$\begin{array}{c}\text { Location } \\
(x, \text { in })\end{array}$} & \multirow{2}{*}{$\begin{array}{l}\text { Weld } \\
\text { Length }\end{array}$} & \multicolumn{4}{|c|}{ Load } & \multicolumn{2}{|c|}{ Weld Stress $^{(1,3)}$} & \multirow{2}{*}{$\begin{array}{l}\text { Stress } \\
\text { Ratio }\end{array}$} \\
\hline & & & Fx & Fy & $\mathrm{Fz}$ & Resultant & $(\mid \mathrm{b} / \mathrm{in})$ & $\left(\mathrm{lb} / \mathrm{in}^{2}\right)$ & \\
\hline 7 & 0.000 & 0.251 & -6.18 & 0 & -4.59 & 7.70 & 61.29 & 397.6 & 0.066 \\
\hline 1027 & 0.502 & 0.502 & -4.78 & 0 & -9.98 & 11.06 & 44.02 & 285.6 & 0.047 \\
\hline 1047 & 1.005 & 0.502 & -1.89 & 0 & -12.57 & 12.71 & 50.60 & 328.2 & 0.054 \\
\hline 1067 & 1.507 & 0.502 & 0.78 & 0 & -13.18 & 13.20 & 52.55 & 340.9 & 0.057 \\
\hline 1087 & 2.010 & 0.502 & 2.23 & 0 & -13.05 & $\$ 3.24$ & 52.70 & 341.9 & 0.057 \\
\hline 1107 & 2.512 & 0.502 & 2.65 & 0 & -12.61 & 12.89 & 51.29 & 332.8 & 0.055 \\
\hline 1127 & 3.015 & 0.502 & 2.49 & 0 & -12.11 & 12.36 & 49.21 & 319.2 & 0.053 \\
\hline 1147 & 3.517 & 0.502 & 2.08 & 0 & -11.60 & 11.79 & 46.91 & 304.3 & 0.050 \\
\hline 1167 & 4.020 & 0.502 & 1.60 & 0 & -11.11 & 11.22 & 44.68 & 289.8 & 0.048 \\
\hline 1187 & 4.522 & 0.502 & 1.09 & 0 & -10.64 & 10.70 & 42.57 & 276.2 & 0.046 \\
\hline 1207 & 5.024 & 0.502 & 0.59 & 0 & -10.19 & 10.21 & 40.63 & 263.6 & 0.044 \\
\hline 1227 & 5.527 & 0.502 & 0.11 & 0 & -9.79 & 9.79 & 38.96 & 252.8 & 0.042 \\
\hline 1247 & 6.029 & 0.502 & -0.35 & 0 & -9.43 & 9.43 & 37.55 & 243.6 & 0.040 \\
\hline 1267 & 6.532 & 0.502 & -0.76 & 0 & -9.11 & 9.14 & 36.40 & 236.1 & 0.039 \\
\hline 1287 & 7.034 & 0.502 & -1.13 & 0 & -8.84 & 8.92 & 35.49 & 230.2 & 0.038 \\
\hline 1307 & 7.537 & 0.502 & -1.45 & 0 & -8.62 & 8.74 & 34.81 & 225.8 & 0.037 \\
\hline 1327 & 8.039 & 0.502 & -1.72 & 0 & -8.45 & 8.62 & 34.32 & 222.7 & 0.037 \\
\hline 1347 & 8.542 & 0.502 & -1.94 & 0 & -8.32 & 8.54 & 34.01 & 220.6 & 0.037 \\
\hline 1367 & 9.044 & 0.502 & -2.12 & 0 & -8.24 & 8.50 & 33.85 & 219.6 & 0.036 \\
\hline 1387 & 9.546 & 0.503 & -2.25 & 0 & -8.19 & 8.50 & 33.81 & 219.4 & 0.036 \\
\hline 1407 & 10.049 & 0.502 & -2.33 & 0 & -8.20 & 8.52 & 33.91 & 220.0 & 0.036 \\
\hline 1427 & 10.551 & 0.503 & -2.35 & 0 & -8.24 & 8.57 & 34.10 & 221.2 & 0.037 \\
\hline 1447 & 11.054 & 0.503 & -2.33 & 0 & -8.32 & 8.64 & 34.40 & 223.1 & 0.037 \\
\hline 1467 & 11.556 & 0.503 & -2.25 & 0 & -8.45 & 8.74 & 34.80 & 225.8 & 0.037 \\
\hline 1487 & 12.059 & 0.503 & -2.12 & 0 & -8.62 & 8.88 & 35.33 & 229.2 & 0.038 \\
\hline 1507 & 12.561 & 0.502 & -1.92 & 0 & -8.84 & 9.04 & 36.03 & 233.7 & 0.039 \\
\hline 1527 & 13.063 & 0.503 & -1.65 & 0 & -9.11 & 9.25 & 36.83 & 238.9 & 0.040 \\
\hline 1547 & 13.566 & 0.503 & -1.31 & 0 & -9.43 & 9.52 & 37.87 & 245.7 & 0.041 \\
\hline 1567 & 14.068 & 0.503 & -0.88 & 0 & -9.81 & 9.85 & 39.20 & 254.3 & 0.042 \\
\hline 1587 & 14.571 & 0.503 & -0.35 & 0 & -10.26 & 10.27 & 40.86 & 265.1 & 0.044 \\
\hline 1607 & 15.073 & 0.503 & 0.30 & 0 & -10.79 & 10.79 & 42.96 & 278.7 & 0.046 \\
\hline 1627 & 15.576 & 0.503 & 1.08 & 0 & -11.40 & 11.45 & 45.58 & 295.7 & 0.049 \\
\hline 1647 & 16.078 & 0.502 & 2.04 & 0 & -12.14 & 12.31 & 49.04 & 318.1 & 0.053 \\
\hline 1667 & 16.580 & 0.502 & 3.22 & 0 & -12.99 & 13.38 & 53.27 & 345.5 & 0.057 \\
\hline 1687 & 17.083 & 0.503 & 4.72 & 0 & -14.05 & 14.82 & 59.00 & 382.7 & 0.063 \\
\hline 1707 & 17.585 & 0.503 & 6.68 & 0 & -15.23 & 16.63 & 66.19 & 429.4 & 0.071 \\
\hline 1727 & 18.088 & 0.502 & 9.28 & 0 & -16.77 & 19.16 & 76.28 & 494.8 & 0.082 \\
\hline 1747 & 18.590 & 0.503 & 12.58 & 0 & -18.39 & 22.28 & 88.68 & 575.3 & 0.095 \\
\hline 1767 & 19.093 & 0.503 & 16.96 & 0 & -20.81 & 26.85 & 106.85 & 693.2 & 0.115 \\
\hline 1787 & 19.595 & 0.502 & 22.68 & 0 & -24.07 & 33.07 & 131.63 & 853.9 & 0.142 \\
\hline 1807 & 20.098 & 0.503 & 42.78 & 0 & -28.93 & 51.64 & 205.55 & 1333.4 & 0.221 \\
\hline 1827 & 20.600 & 0.251 & 56.94 & 0 & -34.82 & 66.74 & 531.81 & 3450.0 & 0.572 \\
\hline & & & & & & Maximum: & 531.81 & 3450.0 & 0.572 \\
\hline
\end{tabular}

Notes: 1 . Stress on weld throat based on double fillet with weld size (leg) of 0.109 inches.

2. Stress ratio based on an allowable, Fw, of $6.03 \mathrm{ksi}$ for intermittent fillet welds.

3. The stiffener to Center Pipe weld is an intermittent weld ( $2^{\prime \prime}$ on $4^{\prime \prime}$ centers), since only half the length is welded, stresses are factored by 2 .

Note: Weld spacing increased to 4.43 ". Margins are adequate to cover this minor change.

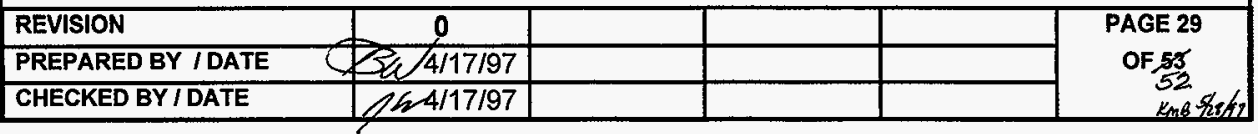


Table 8

Evaluation of Stiffener to Outer (Shell) Plate Weld Using ANSYS Results

Stiffener to Outer Shell Weld

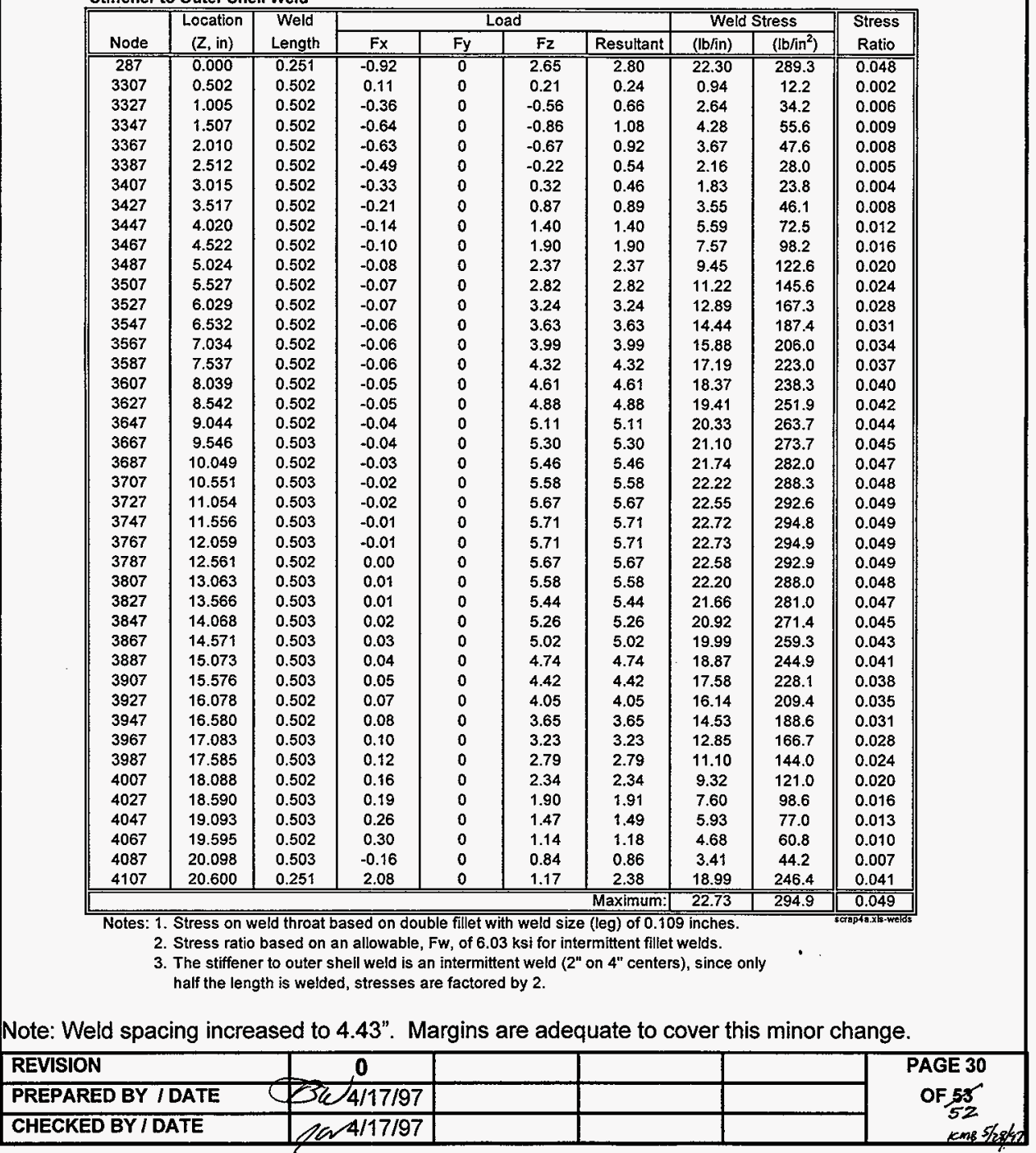

Note: Weld spacing increased to 4.43". Margins are adequate to cover this minor change. 
CLIENT: Duke Engineering Services Hanford

PROJECT: MCO Final Design DOC NO: HNF-SD-SNF-DR-003, Rev. 0, Appendix 9

Table 9

Evaluation of Center Pipe to Bottom Plate Weld Using ANSYS Results Stiffener to Bottom Plate Weld

\begin{tabular}{|c|c|c|c|c|c|c|c|c|c|}
\hline \multirow[b]{2}{*}{ Node } & \multirow{2}{*}{$\begin{array}{l}\text { Location } \\
(\mathrm{X}, \text { in })\end{array}$} & \multirow{2}{*}{$\begin{array}{l}\text { Weld } \\
\text { Length }\end{array}$} & \multicolumn{4}{|c|}{ Load } & \multicolumn{2}{|c|}{ Weld Stress ${ }^{1}$} & \multirow{2}{*}{$\begin{array}{c}\text { Stress } \\
\text { Ratio } \\
\end{array}$} \\
\hline & & & $F x$ & Fy & $F_{z}$ & Resultant & $(\mathrm{lb} / \mathrm{in})$ & $\left(\mathrm{lb} / \mathrm{in}^{2}\right)$ & \\
\hline 7 & 1.375 & 0.244 & -6.18 & 0 & -4.59 & 7.70 & 63.18 & 409.9 & $0 \overline{068}$ \\
\hline 27 & 1.862 & 0.501 & -10.12 & 0 & -0.84 & 10.15 & 40.56 & 263.1 & 0.044 \\
\hline 47 & 2.377 & 0.528 & -13.11 & 0 & 7.37 & 15.04 & 56.95 & 369.5 & 0.061 \\
\hline 67 & 2.919 & 0.557 & -14.23 & 0 & 13.78 & 19.81 & 71.11 & 461.3 & 0.076 \\
\hline 87 & 3.491 & 0.588 & -14.90 & 0 & 19.19 & 24.30 & 82.69 & 536.4 & 0.089 \\
\hline 107 & 4.094 & 0.620 & -15.31 & 0 & 24.80 & 29.15 & 94.05 & 610.1 & 0.101 \\
\hline 127 & 4.730 & 0.654 & -15.34 & 0 & 30.62 & 34.25 & 104.77 & 679.7 & 0.113 \\
\hline 147 & 5.402 & 0.690 & -14.92 & 0 & 36.29 & 39.24 & 113.80 & 738.2 & 0.122 \\
\hline 167 & 6.110 & 0.727 & -13.97 & 0 & 41.31 & 43.61 & 119.91 & 777.9 & 0.129 \\
\hline 187 & 6.856 & 0.767 & -12.48 & 0 & 45.03 & 46.73 & 121.82 & 790.3 & 0.131 \\
\hline 207 & 7.644 & 0.809 & -10.41 & 0 & 46.66 & 47.81 & 118.16 & 766.5 & 0.127 \\
\hline 227 & 8.475 & 0.854 & -7.84 & 0 & 45.15 & 45.82 & 107.38 & 696.6 & 0.116 \\
\hline 247 & 9.351 & 0.900 & -4.83 & 0 & 38.97 & 39.27 & 87.25 & 566.0 & 0.094 \\
\hline 267 & 10.275 & 0.950 & -2.33 & 0 & 23.53 & 23.65 & 49.80 & 323.1 & 0.054 \\
\hline 287 & 11.250 & 0.488 & -0.92 & 0 & 2.65 & 2.80 & 11.49 & 74.5 & 0.012 \\
\hline & & & & & & Maximum: & 121.82 & 790.3 & 0.131 \\
\hline
\end{tabular}

Notes: 1. Stress at weld throat based on double fillet with weld size (leg) of 0.109 inches.

2. Stress ratio based on an allowable, $\mathrm{Fw}$, of $6.03 \mathrm{ksi}$ for intermittent fillet welds.

3. The stiffener to Center Pipe weld is an intermittent weld (2" on 4" centers), since only half the length is welded, stresses are factored by 2 .

Note: Current weld design is continuous. Above evaluation is conservative.

\begin{tabular}{|c|l|l|l|}
\hline 0 & & & \\
\hline $\sec 4 / 17 / 97$ & & & \\
\hline
\end{tabular}


CLIENT: Duke Engineering Services Hanford

PROJECT: MCO Final Design

DOC NO: HNF-SD-SNF-DR-003, Rev. 0, Appendix 9

Table 10

Evaluation of Outer Shell to Bottom Plate Weld Using ANSYS Results

Outer Shell to Bottom Plate Weld

\begin{tabular}{|c|c|c|c|c|c|c|c|c|c|}
\hline \multirow[b]{2}{*}{ Node } & \multirow{2}{*}{$\begin{array}{c}\text { Location } \\
(\theta)\end{array}$} & \multirow{2}{*}{$\begin{array}{l}\text { Weld } \\
\text { Length }\end{array}$} & \multicolumn{4}{|c|}{ Load } & \multicolumn{2}{|c|}{ Weld Stress } & \multirow{2}{*}{$\begin{array}{l}\text { Stress } \\
\text { Ratio }\end{array}$} \\
\hline & & & $\mathbf{F}_{\mathrm{R}}$ & $F_{\theta}$ & $F z$ & Resultant & (lb/in) & $\left(\mathrm{lb} / \mathrm{in}^{2}\right)$ & \\
\hline 281 & -30.0 & 0.491 & -1.85 & 5.38 & -8.76 & 10.44 & 42.54 & 390.3 & 0.055 \\
\hline 282 & -25.0 & 0.982 & -3.59 & -3.06 & -17.08 & 17.72 & 36.10 & 331.2 & 0.046 \\
\hline 283 & -20.0 & 0.982 & -3.29 & -5.62 & -15.69 & 16.99 & 34.61 & 317.5 & 0.044 \\
\hline 284 & -15.0 & 0.982 & -2.83 & -7.16 & -13.13 & 15.22 & 31.00 & 284.4 & 0.040 \\
\hline 285 & -10.0 & 0.982 & -2.19 & -7.33 & -8.89 & 11.73 & 23.89 & 219.1 & 0.031 \\
\hline 286 & -5.0 & 0.982 & -1.31 & -5.48 & -0.35 & 5.65 & 11.50 & 105.5 & 0.015 \\
\hline 287 & 0.0 & 0.982 & 0.14 & 0.00 & 5.53 & 5.53 & 11.27 & 103.4 & 0.014 \\
\hline 288 & 5.0 & 0.982 & -1.31 & 5.48 & -0.35 & 5.65 & 11.50 & 105.5 & 0.015 \\
\hline 289 & 10.0 & 0.982 & -2.19 & 7.33 & -8.89 & 11.73 & 23.89 & 219.1 & 0.031 \\
\hline 290 & 15.0 & 0.982 & -2.83 & 7.16 & -13.13 & 15.22 & 31.00 & 284.4 & 0.040 \\
\hline 291 & 20.0 & 0.982 & -3.29 & 5.62 & -15.69 & 16.99 & 34.61 & 317.5 & 0.044 \\
\hline 292 & 25.0 & 0.982 & -3.59 & 3.06 & -17.08 & 17.72 & 36.10 & 331.2 & 0.046 \\
\hline 293 & 30.0 & 0.491 & -1.85 & -5.38 & -8.76 & 10.44 & 42.54 & 390.3 & 0.055 \\
\hline & & & & & & Maximum: & 42.54 & 390.3 & 0.055 \\
\hline
\end{tabular}

Notes: 1. Length is $\alpha R$, where $\alpha$ is in radians and $R$ is the IR of the outer shell of $\approx 11.25$ in.

2. Weld stress based on single groove weld aliowable for a weld thickness of 0.109 inches.

3. Stress ratio based on an allowable, Fw, of $6.03 \mathrm{ksi}$ for single groove welds.

4. The outer shell to bottom plate weld is an intermittent weld (2" on 4" centers), since only half the length is welded, stresses are factored by 2 .

Note: The above analysis is conservative for the current design, which specifies a continuous weld. 
Table 11

ANSYS Results - Deadweight Allowables

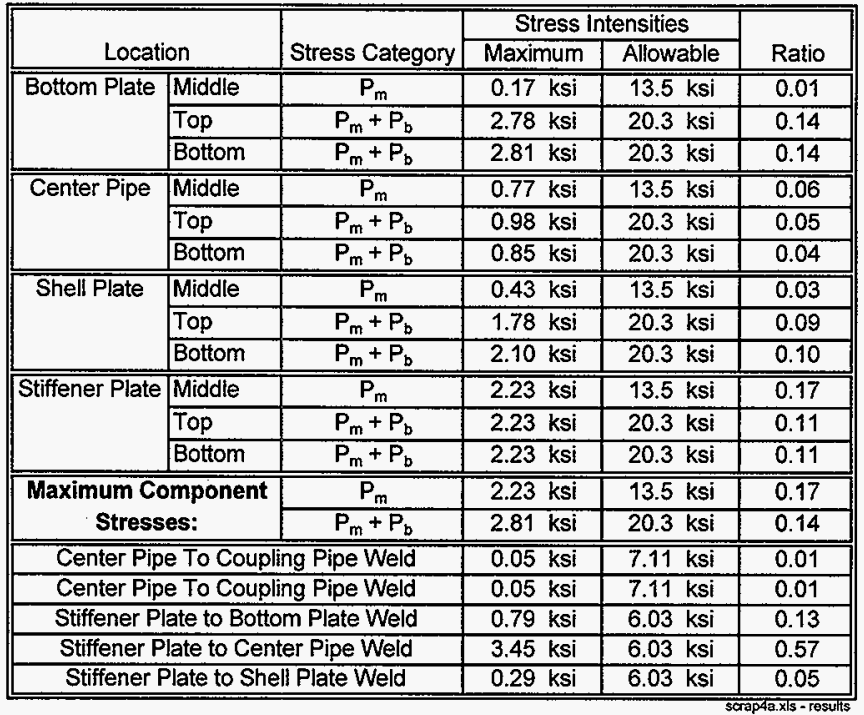

\begin{tabular}{c|c|l|l|l|}
0 & & & \\
\hline fow 4/17/97 & & & \\
\hline $2 / 4 / 17 / 97$ & & & \\
\hline
\end{tabular}


PROJECT: MCO Final Design DOC NO: HNF-SD-SNF-DR-003, Rev. 0, Appendix 9

FILE NO: $\quad \mathrm{KH}-8009-8-07$

\section{Table 12}

\section{ANSYS Results - Lifting Allowables}

\begin{tabular}{|c|c|c|c|c|c|}
\hline \multirow{2}{*}{\multicolumn{2}{|c|}{ Location }} & \multirow[b]{2}{*}{ Stress Category } & \multicolumn{2}{|c|}{ Stress Intensities } & \multirow[b]{2}{*}{ Ratio } \\
\hline & & & Maximum & Allowable & \\
\hline \multirow[t]{3}{*}{ Bottom Plate } & Middle & $\overline{P_{m}}$ & $0.17 \mathrm{ksi}$ & $8.33 \mathrm{ksi}$ & 0.02 \\
\hline & Top & $P_{m}+P_{b}$ & $2.78 \mathrm{ksi}$ & $8.33 \mathrm{ksi}$ & 0.33 \\
\hline & Bottom & $P_{m}+P_{b}$ & $2.81 \mathrm{ksi}$ & $8.33 \mathrm{ksi}$ & 0.34 \\
\hline \multirow[t]{3}{*}{ Center Pipe } & Middle & $\overline{\bar{P}}$ & $0.77 \mathrm{ksi}$ & $8.33 \mathrm{ksi}$ & 0.09 \\
\hline & Top & $P_{m}+P_{b}$ & $0.98 \mathrm{ksi}$ & $8.33 \mathrm{ksi}$ & 0.12 \\
\hline & Bottom & $P_{m}+P_{b}$ & $0.85 \mathrm{ksi}$ & $8.33 \mathrm{ksi}$ & 0.10 \\
\hline \multirow[t]{3}{*}{ Shell Plate } & Middle & $\overline{\overline{P_{m}}}$ & $0.43 \mathrm{ksi}$ & $8.33 \mathrm{ksi}$ & 0.05 \\
\hline & Top & $P_{m}+P_{b}$ & $1.78 \mathrm{ksi}$ & $8.33 \mathrm{ksi}$ & 0.21 \\
\hline & Bottom & $P_{m}+P_{b}$ & $2.10 \mathrm{ksi}$ & $8.33 \mathrm{ksi}$ & 0.25 \\
\hline \multirow[t]{3}{*}{ Stiffener Plate } & Middle & $P_{m}$ & $2.23 \mathrm{ksi}$ & $8.33 \mathrm{ksi}$ & 0.27 \\
\hline & Top & $P_{m}+P_{b}$ & $2.23 \mathrm{ksi}$ & $8.33 \mathrm{ksi}$ & 0.27 \\
\hline & Bottom & $P_{m}+P_{b}$ & $2.23 \mathrm{ksi}$ & $8.33 \mathrm{ksi}$ & 0.27 \\
\hline \multicolumn{3}{|c|}{ Maximums } & $2.81 \mathrm{ksi}$ & $8.33 \mathrm{ksi}$ & 0.34 \\
\hline
\end{tabular}


CLIENT: Duke Engineering Services Hanford

PROJECT: MCO Final Design
FILE NO: $\quad \mathrm{KH}-8009-8-07$

DOC NO: HNF-SD-SNF-DR-003, Rev. 0, Appendix 9
Figure 1

ANSYS Model

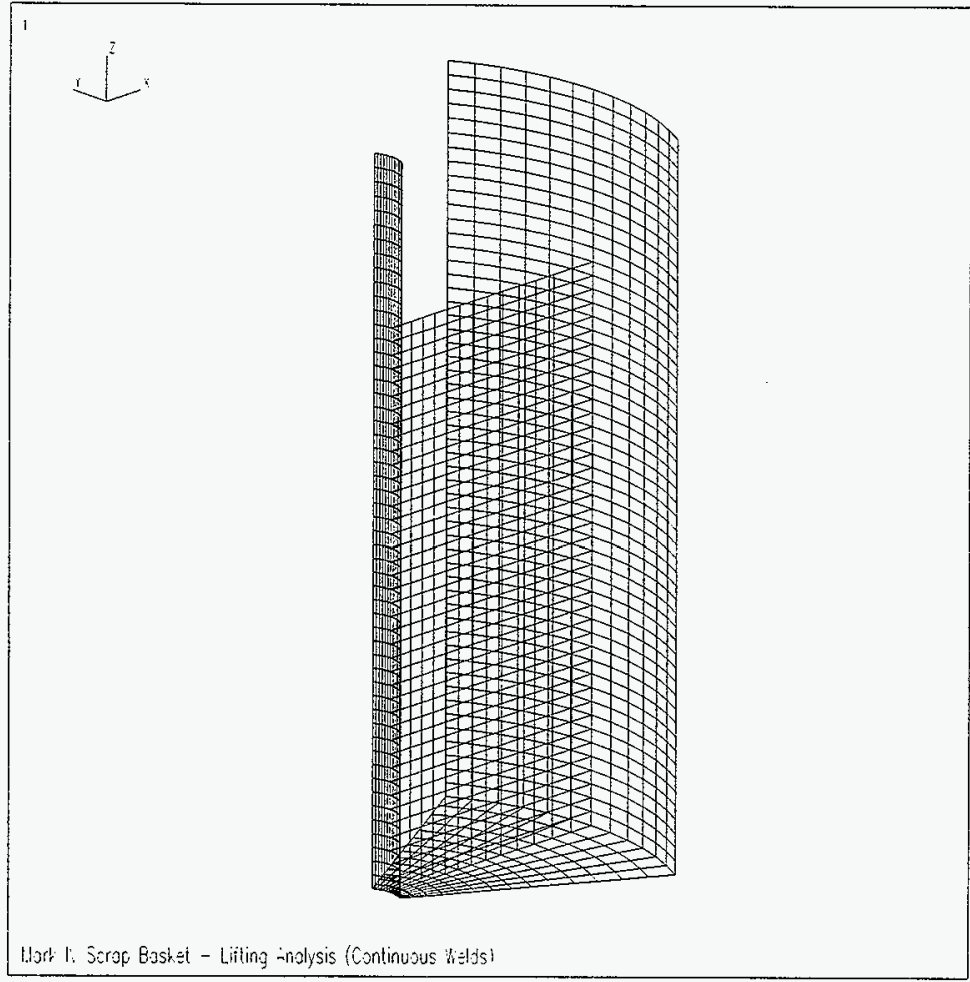

ANSBS $50 \div 50$

JAtl 16 19g9

$11: 09: 25$

PLOT HON. 1

ELEHENTS

TYPE NUNM

$$
\begin{aligned}
& X:=-i \\
& Y=-1 \\
& Z Y=0.5 \\
& \text { OIST }=16.685 \\
& X F=6.22 \\
& \text { 2F }=15.425 \\
& Y U F=?
\end{aligned}
$$

\begin{tabular}{|c|c|c|c|c|}
\hline & 0 & & & \\
\hline S6/4/17/97 & & & \\
\hline $2 / 4 / 17 / 97$ & & & \\
\hline
\end{tabular}


CLIENT:

PROJECT: MCO Final Design
FILE NO: $\quad \mathrm{KH}-8009-8-07$

DOC NO: HNF-SD-SNF-DR-003, Rev. 0, Appendix 9

Figure 2

Boundary Conditions

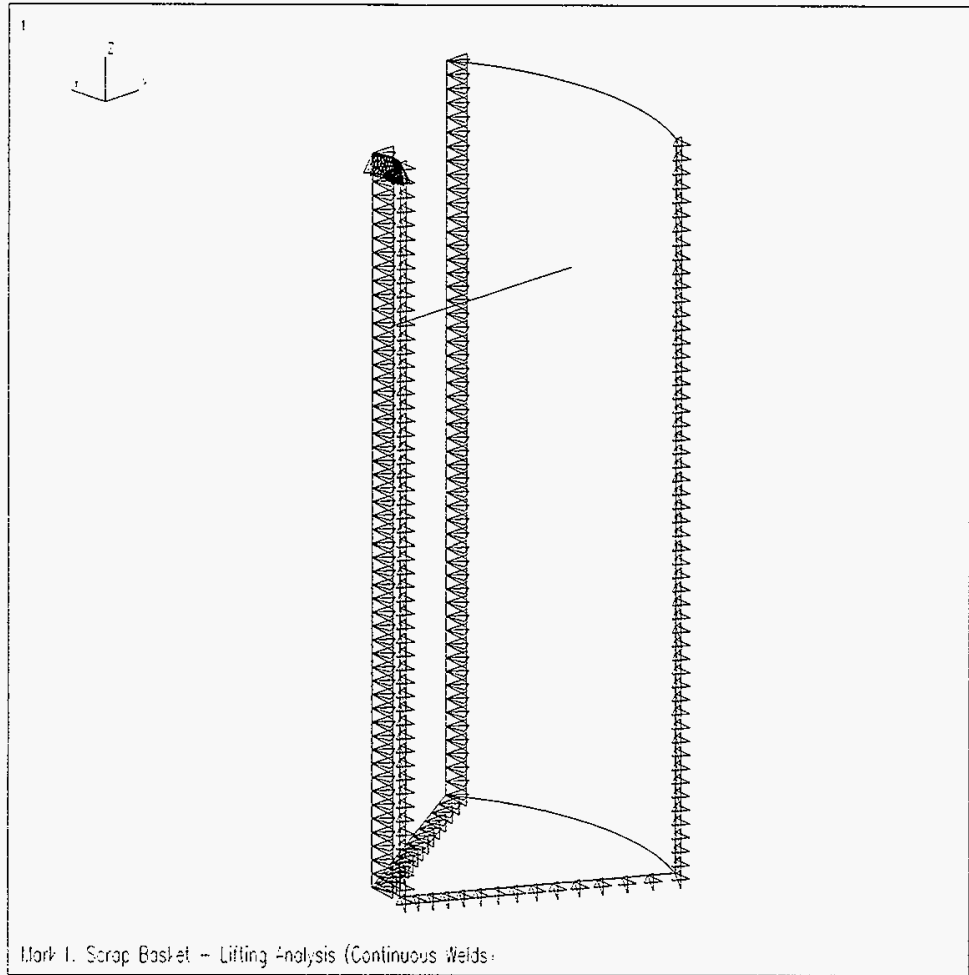

ANSIS 500450

JAM 18 1997

11.09 .39

PLOT NO. 4

ELEIIENTS

TYPE NUI,

u

$X y=-1$

$Y Y=-1$

$Z=0.5$

DIST $=16.685$

$X F=\hat{F} .22$

If $=15.425$

:IJP $=2$

EOGE

\begin{tabular}{|c|c|l|l|l|}
\hline 0 & & & \\
\hline & $=1 / 17 / 97$ & & & \\
\hline $2 / 4 / 17 / 97$ & & & \\
\hline
\end{tabular}


CLIENT: Duke Engineering Services Hanford

PROJECT: MCO Final Design
FILE NO: KH-8009-8-07

DOCNO: HNF-SD-SNF-DR-003, Rev. 0, Appendix 9

Figure 3

Boundary Conditions- Top View

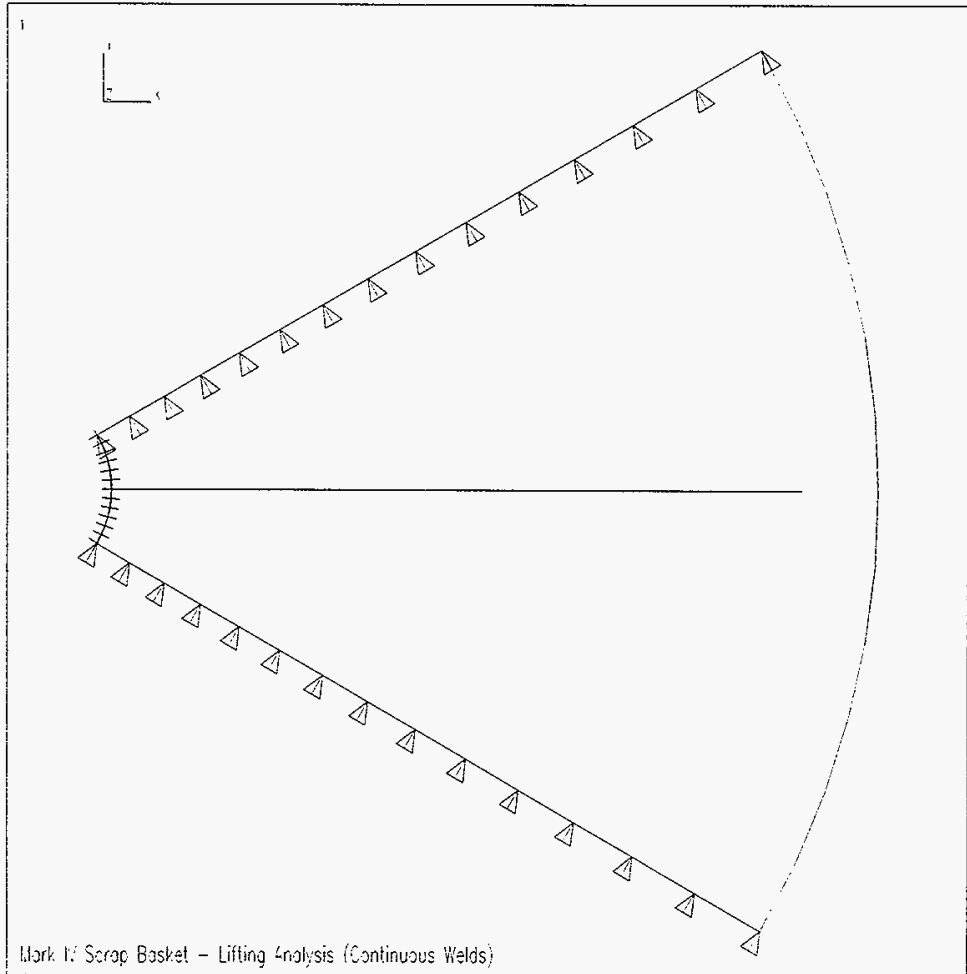

AHSis $5.0+56$

JAN $1 E: 997$

$11: 09: 38$

PLOT HO. 3

ELEI:HENTS

TIPE FINA

y

$2:=1$

OIST $=6.188$

$\mathrm{XF}=6.22$

ZF $=13.425$

EDGE

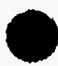

li: Snrac 8astat - Liting -righese (intinuous walds) 
Figure 5

Stress Intensity in Bottom Plate

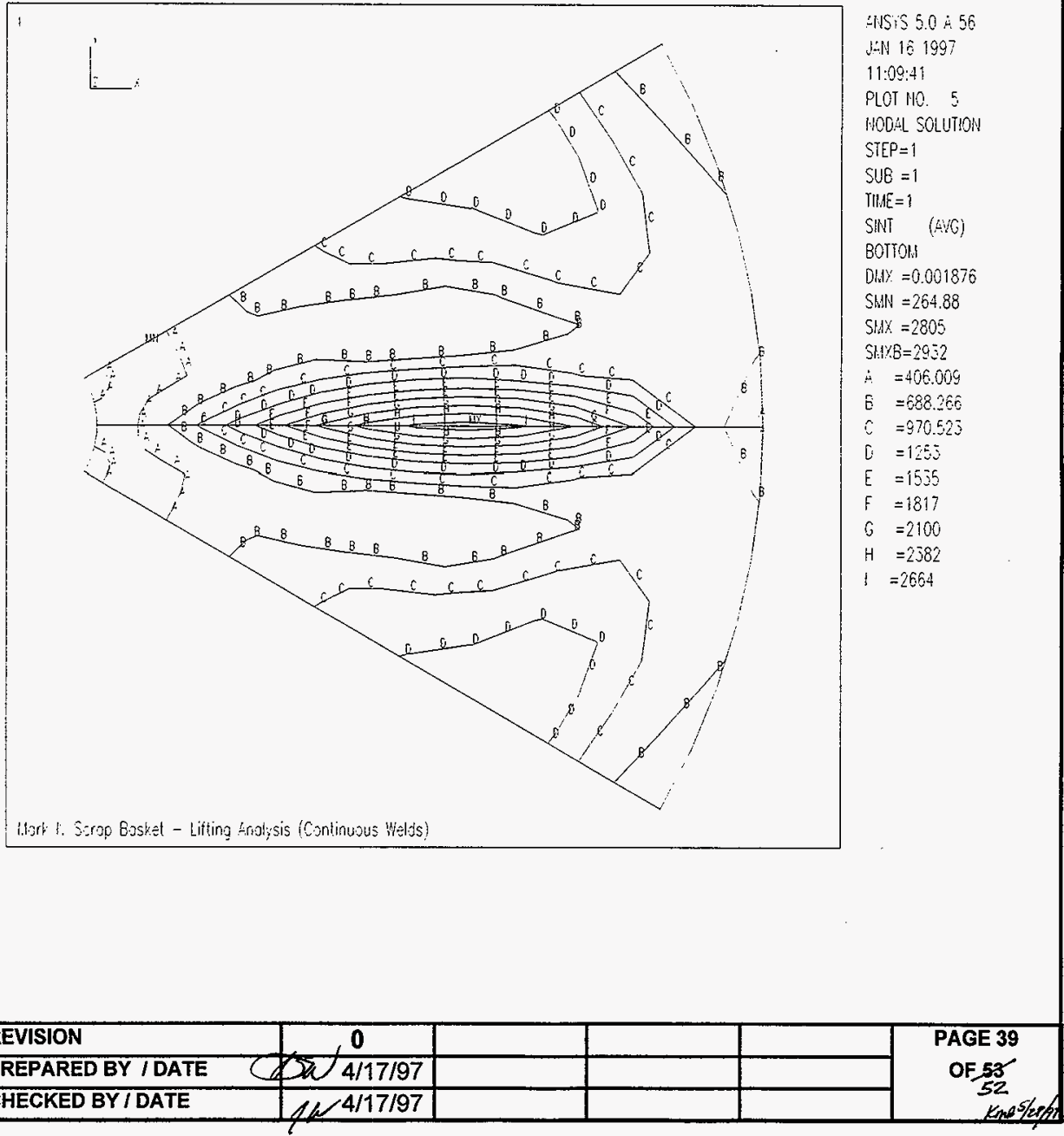


CLIENT: Duke Engineering Services Hanford

PROJECT: MCO Final Design DOC NO: HNF-SD-SNF-DR-003, Rev. 0, Appendix 9

FILE NO: $\quad \mathrm{KH}-8009-8-07$

Figure 6

Stress Intensity in Center Pipe

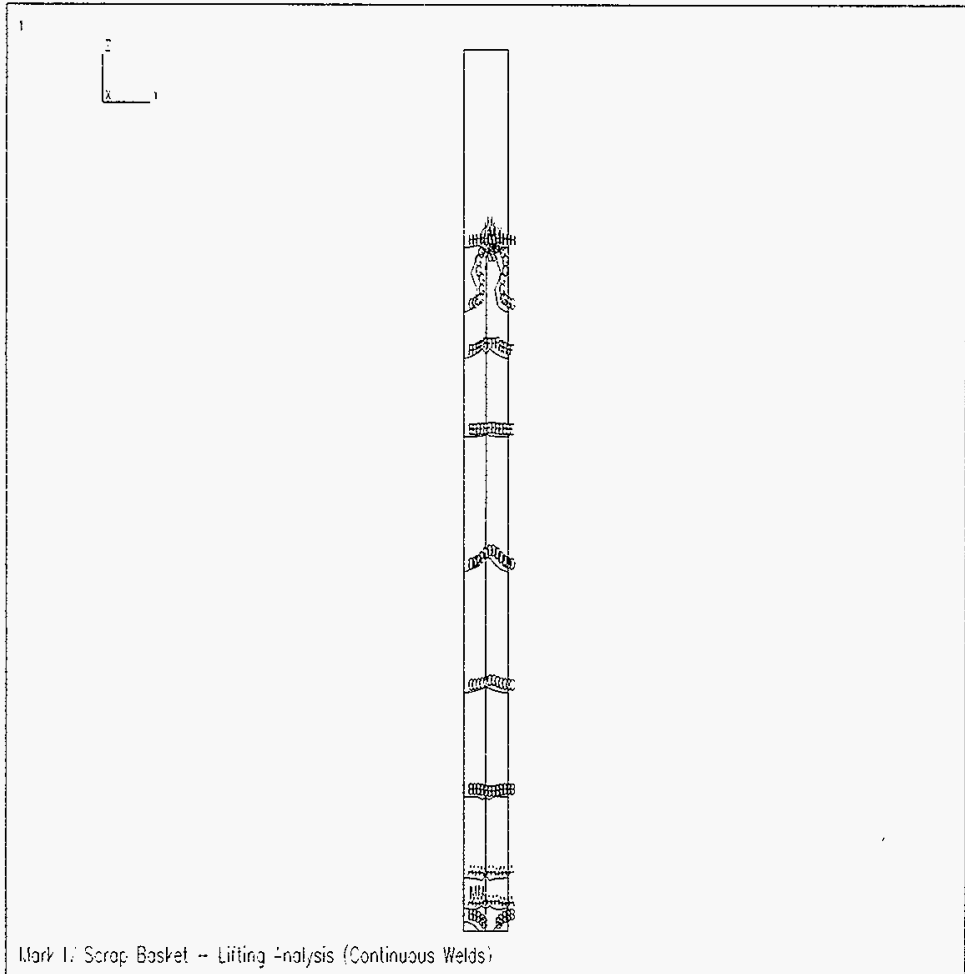

HSYS $5.0+5 \hat{6}$

JiA 106997

$11: 09: 44$

PLOT HO. 6

IODAL SOLUTHON

STEP $=1$

SUB $=1$

THWE $=1$

SINT $(A, C)$

BOTTOLS

$01 \mathrm{X}=0.00187 \overline{\mathrm{C}}$

$\operatorname{S!NN}=13,342$

$5 \mathrm{~N} X=850.306$

S1:1\%: $\%=958.508$

$\therefore \quad=59.84$

$8=152.85$

$\hat{6}=245.352$

$0=538.828$

$E=431.824$

$F=524.82$

$G=617.816$

H $=710.812$

$1=803.508$ 
Figure 7

Stress Intensity in Center Pipe at Stiffener Plate Connection

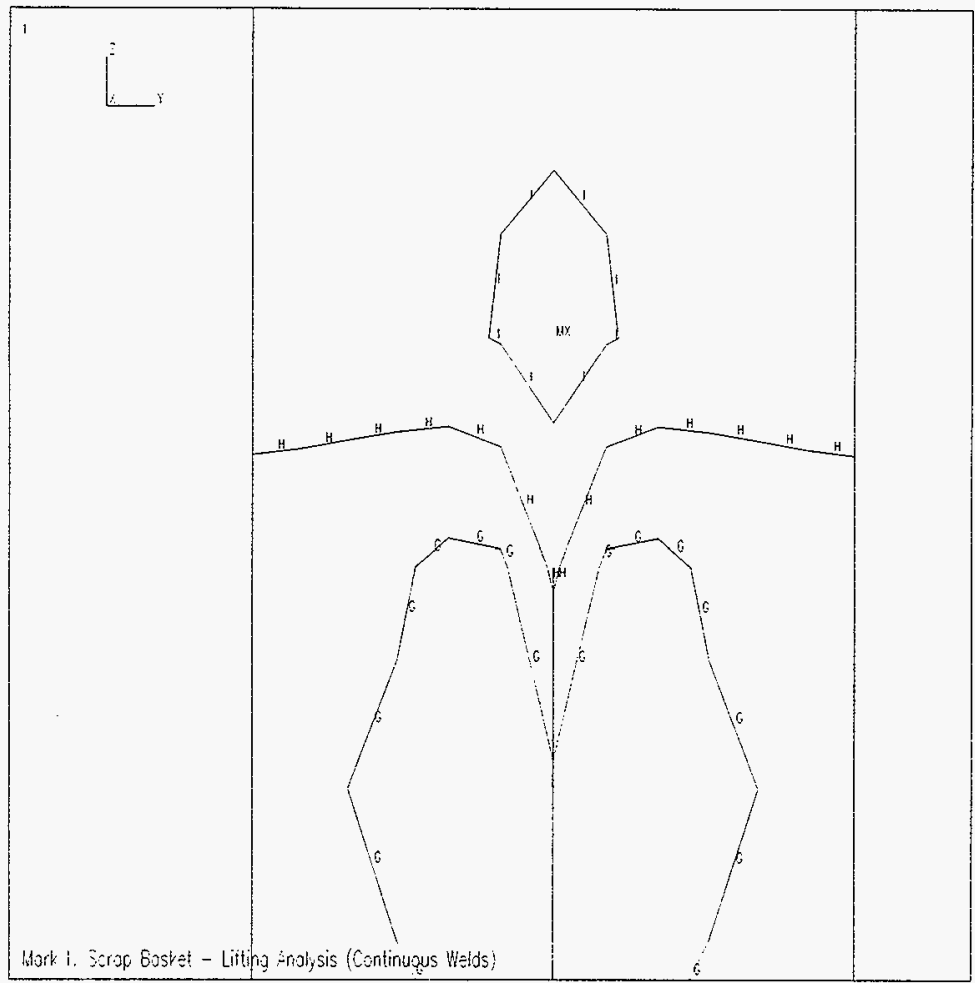

AHSTS $50.0 \% 5 \hat{0}$

J1910 1697

$11.09: 40$

PLOT NO. 7

NOOLL SOLUTION

STEP $=1$

SUB $=1$

$T H M E=1$

SINT $\quad(A \cup C)$

BOTIOL:1

OW $=0.001876$

SHAH $=15.342$

$S H X=850.306$

SH:X $=958.508$

$\therefore \quad=59.84$

$B=152.236$

$C=245.832$

$D=338.828$

$E=431.824$

$F=524.82$

$G=617.816$

$\mathrm{H}=710.812$

I $=803.808$

\begin{tabular}{|c|c|l|l|}
\hline 0 & & & \\
\hline Se/4/17/97 & & & \\
\hline$/ 1 / 4 / 17 / 97$ & & & \\
\hline
\end{tabular}


Figure 8

\section{Stress Intensity in Outer (Shell) Plate}

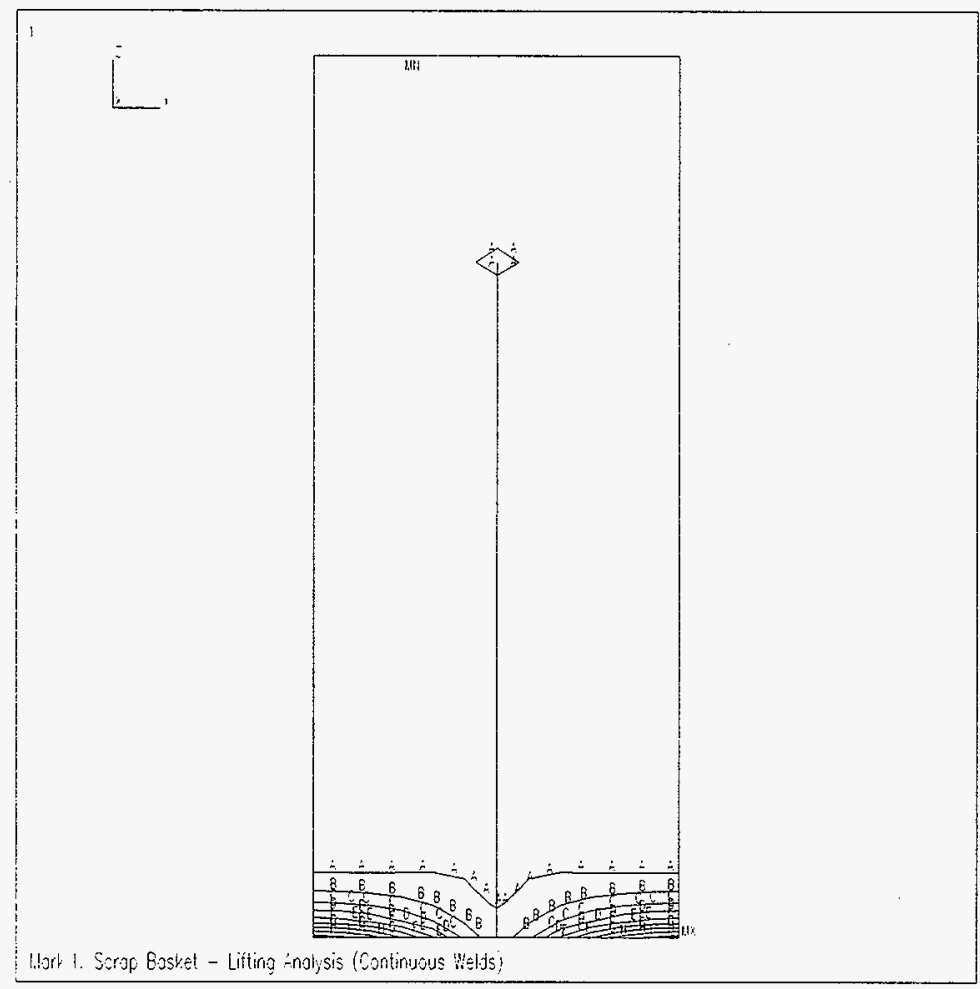

Ansis $5.0 \div 56$

J-4it 16.997

$11: 09: 51$

FLOT NO. a

HOOLL SOLUTIOH

STEP $=1$

SU $8=1$

THME $=1$

SHIJT $\{\mathrm{AU}$

BOTTOL:

OAd $=0.00187 \hat{\sigma}$

SI.HA $=1.417$

SWI: $=2095$

$51 .: 8=210^{2}$

$\therefore \quad=117.740$

$\theta=350.412$

$\hat{\hat{C}}=583.075$

[ $\quad=815.738$

$E=1048$

$F=1281$

$G=1514$

$H=1748$

I $=1979$

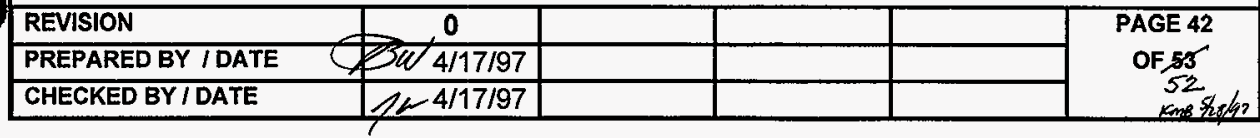


CLIENT: Duke Engineering Services Hanford

Figure 9

Stress Intensity in Stiffener Plate

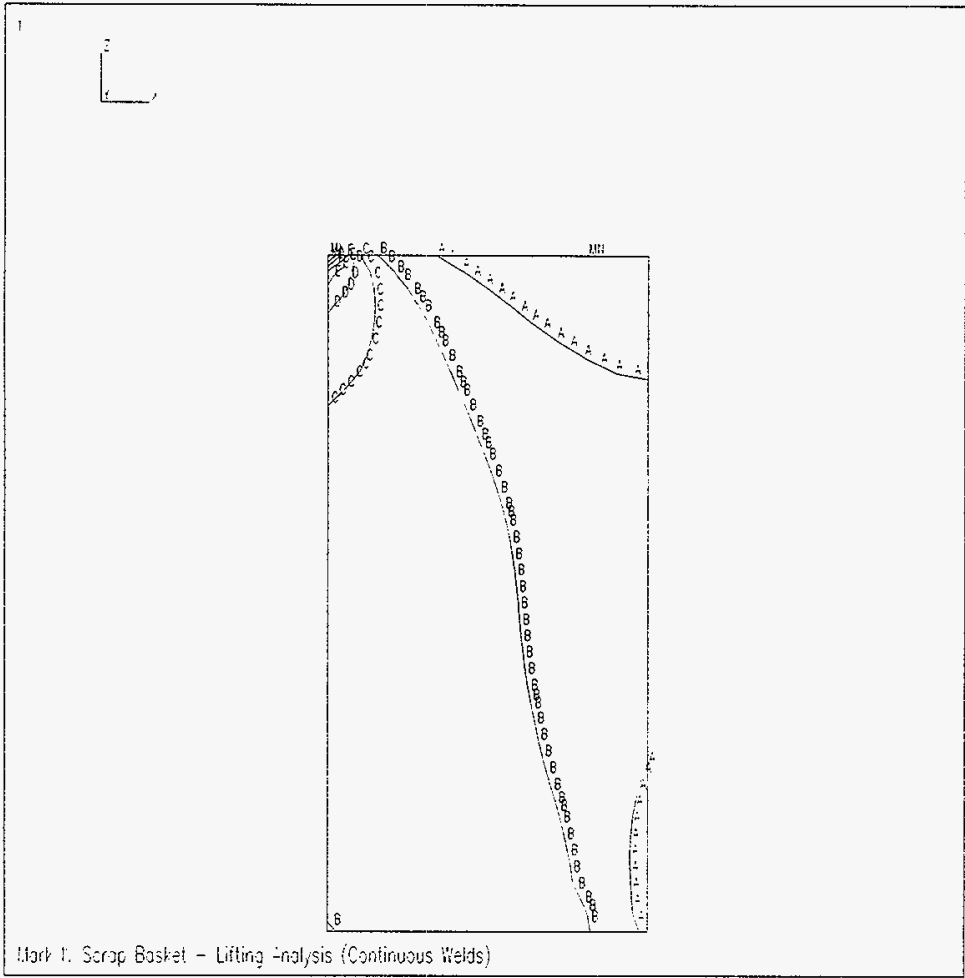

$45155.0-56$

ILit 10 1903

$11: 09: 54$

PLOT 10. 10

Hof́L SOLUTOH

STEP $=1$

SUB $=1$

TII:SE $=1$

SHT $\$ \mathrm{AOO}$

EOTTO

OH: $=0.001876$

SIIIII $=10.761$

$51.1 \cdot=2227$

S1:1: $B=2828$

$\therefore=135.871$

$\mathrm{E}=383.89$

$\therefore \quad=3.30 .307$

$0 \quad=872.595$

$E=1119$

$F=13 \hat{0} 5$

$\hat{\hat{u}}=\hat{\imath} \hat{\mathrm{s}} 11$

$H=185.7$

$1=2104$

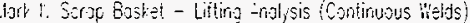


CLIENT: Duke Engineering Services Hanford

PROJECT: MCO Final Design DOCNO: HNF-SD-SNF-DR-003, Rev. 0, Appendix 9

FILE NO: $\quad \mathrm{KH}-8009-8-07$
APPENDIX A

COMPUTER RUN OUTPUT SHEETS

AND

INPUT FILE LISTINGS 
PROJECT: MCO Final Design DOC NO: HNF-SD-SNF-DR-003, Rev. 0, Appendix 9

\section{COMPUTER RUN COVER SHEET}

Project Number:

Computer Code:

Software Version:

Computer System:

Computer Run File Number:

Unique Computer Run File Name:

Run Description:

Creation Date/Time:

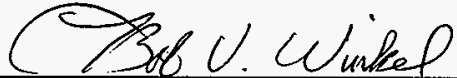

Prepared By: Bob V. Winkel
$\mathrm{KH}-8009-8$

ANSYS $\otimes-P C$

$5.0 \mathrm{~A}$

MS-DOS, Pentium® Processor

KH-8009-8-07

Scrap4a.inp

Stress Analysis of the Mark IV Scrap Basket

16 January 1997/11:02:38am

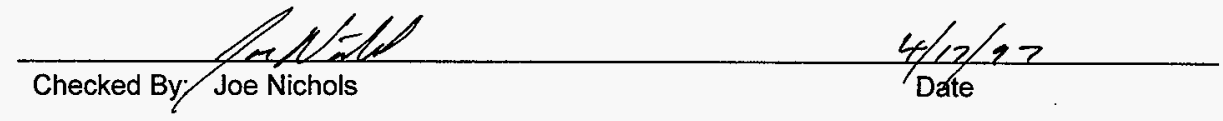




\section{LISTING OF SCRAP4A.INP}

Ibatch, list

Ifilenam, scrap4a

/title,Mark IV Scrap Basket - Lifting Analysis (Continuous Welds)

Icom 60 Degree section of Mark IV scrap basket.

lcom Lifting load is evaluated by restraining the center pipe in the axial

/com direction and applying a uniform pressure load to the bottom plate

lcom plus $1 \mathrm{~g}$ in the axial (z) direction. Pressure load is taken as the

lcom weight of fuel in a Mark IV fuel basket over the area of the annular

fcom bottom plate. The basket is modeled with shell elements with

Icom thicknesses input.

Icom

Icom Each part (e.g., bottom plate, center pipe) is modeled separately.

Icom At weld locations, the parts are joined by merging coincident nodes.

lcom
lcom This analysis assumes continuous welds between all parts.
lcom

/prep7

tref,400 levaluate at $662 \mathrm{~F}(350 \mathrm{C})$

et, 1 , shell63 ! bottom plate

et,2,shell63 ! center pipe

et,3,shell63 ! outer shell

et,4,shell63 ! stiffener

r, 1,0.25 ! bottom plate thickness

$r, 2,0.50$ ! center pipe thickness

$r, 3,0.109$ ! outer shell thickness

$r, 4,0.109$ ! stiffener thickness

$\mathrm{pi}=3.1415926$

ir $=1.375$

or $=11.25$

hi=26.85

fuelwt $=3010$

! weight of fuel in Mark IV basket

area $=p^{\star}{ }^{*}\left((\right.$ or*or $\left.)-\left(i i^{*} i r\right)\right)$

! total area of bottom plate

deadload $=$ fuelwt/area

! fuel pressure on bottom plate

Itriad, Itop

/com material properties

dens, 1,2854

nuxy, 1,3

mptemp, $1,70,100,200,300,400,500$

mptemp, $7,600,650,700,750$

/com elastic moduli for $304 \mathrm{~L}$ stainless steel

mpdata,ex, 1, 1,28.3E+06,28.1E+06,27.6E+06,27.0E+06,26.5E+06,25.8E+06

mpdata,ex, 1,7,25.3E+06,25.1E+06,24.8E+06,24.5E+06

/com list material properties

mplist 
CLIENT: Duke Engineering Services Hanford

FILE NO: $\quad \mathrm{KH}-8009-8-07$

PROJECT: MCO Final Design DOCNO: HNF-SD-SNF-DR-003, Rev. 0, Appendix 9

csys, 1

/com bottom plate

$\mathrm{n}, 1, \mathrm{ir},-30$ ! inside arc

$\mathrm{n}, 13, \mathrm{ir}, 30$

fill, 1,13

$n, 281$, or $_{2}-30 \quad$ ! outside arc

$n, 293$, or, 30

fill, 281,293

fill, $1,281,13,20,13,1,2.0$

type, 1

real, 1

e, $1,21,22,2$

egen, $12,1,-1$

egen, $14,20,-12$

save

lcom center pipe

ngen, $2,1000,1,13,1$

ngen,42,20,1001,1013,1, , 20.6/41

ngen, $13,20,1821,1833,1_{1},(26.85-20.6) / 12$

type, 2

real, 2

$e, 1001,1002,1022,1021$

egen, $12,1,-1$

egen, $53,20,-12$

lcom outside shell

ngen, $2,3000,281,293,1$

ngen, $42,20,3281,3293,1,, 20,6 / 41$

ngen, $13,20,4101,4113,1,,(26.85-20.6) / 12$

type, 3

real, 3

e,3281,3282,3302,3301

egen, $12,1,-1$

egen, $53,20,-12$

/com stiffener plate

$\mathrm{n}, 5001$, ir

$n, 5015$, or

fill, $5001,5015,13,1,1,2.0$

ngen, $42,20,5001,5015,1,, 20.6 / 41$

Ingen, $13,20,5821,5835,1, \ldots(26.85-20.6) / 12$

type, 4

real, 4

e,5001,5002,5022,5021

egen, $14,1,-1$

egen, $41,20,-14$

save 
/com nodes in cylindrical system

nall

nrotat,all

/com boundary conditions

lcom symmetry

csys, 1

nsel,s,loc,y, 30

nsel, a, loc, y, -30

nplot

d, all,uy, $0.0, \ldots$, rotx, rotz

/com top of center pipe for lifting

nsel,s,loc, $x$, ir

nsel,, loc, $z$, hi

d, all, uz, 0

nall

save

lcom merge coincident nodes at welded connections

/com center pipe to bottom plate weld

esel,s,type, 1 ! bottom plate

esel,a,type,,2 ! center pipe

nsle ! select nodes associated w/elements

nummrg,node

Icom bottom plate to outer shell (assume - verify rotation)

esel,s,type, 1 ! bottom plate

esel,a,type, 3 ! outer shell

nsle I select nodes associated w/elements

nummrg,node

/com outer shell to stiffener (assume continuous)

esel,s,type, 4 ! stiffener

esel,a,type,,3 ! outer shell

nsle ! select nodes associated w/elements

nummrg,node

Icom bottom plate to stiffener (assume continuous)

esel,s,type, 4 ! stiffener

esel,a,type, 1 ! bottom plate

nsle ! select nodes associated w/elements

nummrg, node

/com center pipe to stiffener (assume continuous)

esel,s,type, 4 ! stiffener

esel,a,type,2 ! center pipe

nsle ! select nodes associated w/elements

nummrg, node

nall

eall

/com applied loads

esel,s,type, , 1

sfe,all,2,pres, deadload ! pressure load

eall

REVISION

PREPARED BY / DATE

DOC NO:

9


CLIENT: Duke Engineering Services Hanford

PROJECT: MCO Final Design

FILE NO: KH-8009-8-07

nall

acel,.,1.0 ! gravity load

save

fini

Isolu

solve

fini

/post1

set,last

rsys,1 ! results in global cylindrical

Icom stiffener to bottom plate weld

esel,s,type, 4 ! select stiffener elements

nsle ! select stiffener element nodes

nsel,r,loc,, 0 ! nodes at base

nlist

nforce

/com stiffener to center pipe weld

nsle ! select stiffener element nodes

nsel,r,loc,x,ir ! nodes at center pipe

nlist

nforce

Icom stiffener to outer shell weld

nsle I select stiffener element nodes

nsel,r,loc,x,or ! nodes at outer shell

nlist

nforce

Icom center pipe to bottom plate weld

esel,s,type,1 I select bottom plate

nsle I select bottom plate element nodes

nsel,r,loc,x,ir I nodes at center pipe

nlist

nforce

Icom outer shell to bottom plate weld

nsle ! select bottom plate element nodes

nsel,r,loc,x,or ! nodes at outer shell

nlist

nforce

nail

eall

lcom print stress results

/page...800 1800 lines per page (elimate headers)

/com stress results in bottom plate

esel,s,type ,1 ! bottom plate

shell,mid

prns,s,prin ! membrane

shell,top

prns,s,prin ! membrane + bending

shell, bot

prns,s,prin ! membrane + bending

\begin{tabular}{|l|c|l|l|l|}
\hline REVISION & 0 & & & \\
\hline PREPARED BY IDATE & $\mathrm{S} / 4 / 17 / 97$ & & & \\
\hline CHECKED BYIDATE & $/ \Omega 4 / 17 / 97$ & & & \\
\hline
\end{tabular}




\section{MCO Final Design}

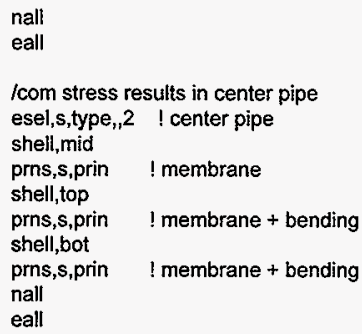


CLIENT: Duke Engineering Services Hanford

PROJECT: MCO Final Design
FIL.E NO: $\quad \mathrm{KH}-8009-8-07$

Iview, $,-1,-1, .5$

eplot

Ipbc,u,0 ! hide transfational restraints

esel,s,type, 1 ! bottom plate

iview, $1,, 1$

Nup, y

plns,s,int

esel,s,type,,2 1 center pipe

Niew, 1,1

Ivup,, 2

plns, s,int

ZOOM, 1,6.2204,-0.14295,20.764,1.0978

/zoom, off

esel,s,type, $3 \quad$ ! outer shell

plns,s,int

esel,s,type,1 4 ! stiffener plate

/view,..1

p!ns,s,int

nall

eall

fini

exit

\begin{tabular}{|c|c|c|c|}
\hline 0 & & & \\
\hline$\not \mathbb{N} 4 / 17 / 97$ & & & \\
\hline
\end{tabular}




\section{COMPUTER RUN COVER SHEET}

Project Number:

Computer Code:

Software Version:

Computer System:

Computer Run File Number:

Unique Computer Run File Name:

Run Description:

Run Date/Time:
$\mathrm{KH}-8009-8$

ANSYS®-PC

$5.0 \mathrm{~A}$

MS-DOS, Pentium@ Processor

KH-8009-8-07

Scrap4a.out

Stress Analysis of the Mark IV Scrap Basket 16 January 1997/11:09:56am

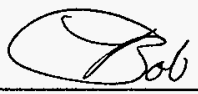

Prepared By: Bob V. Winkel

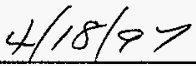

Date

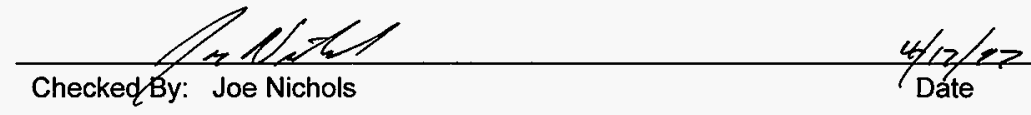




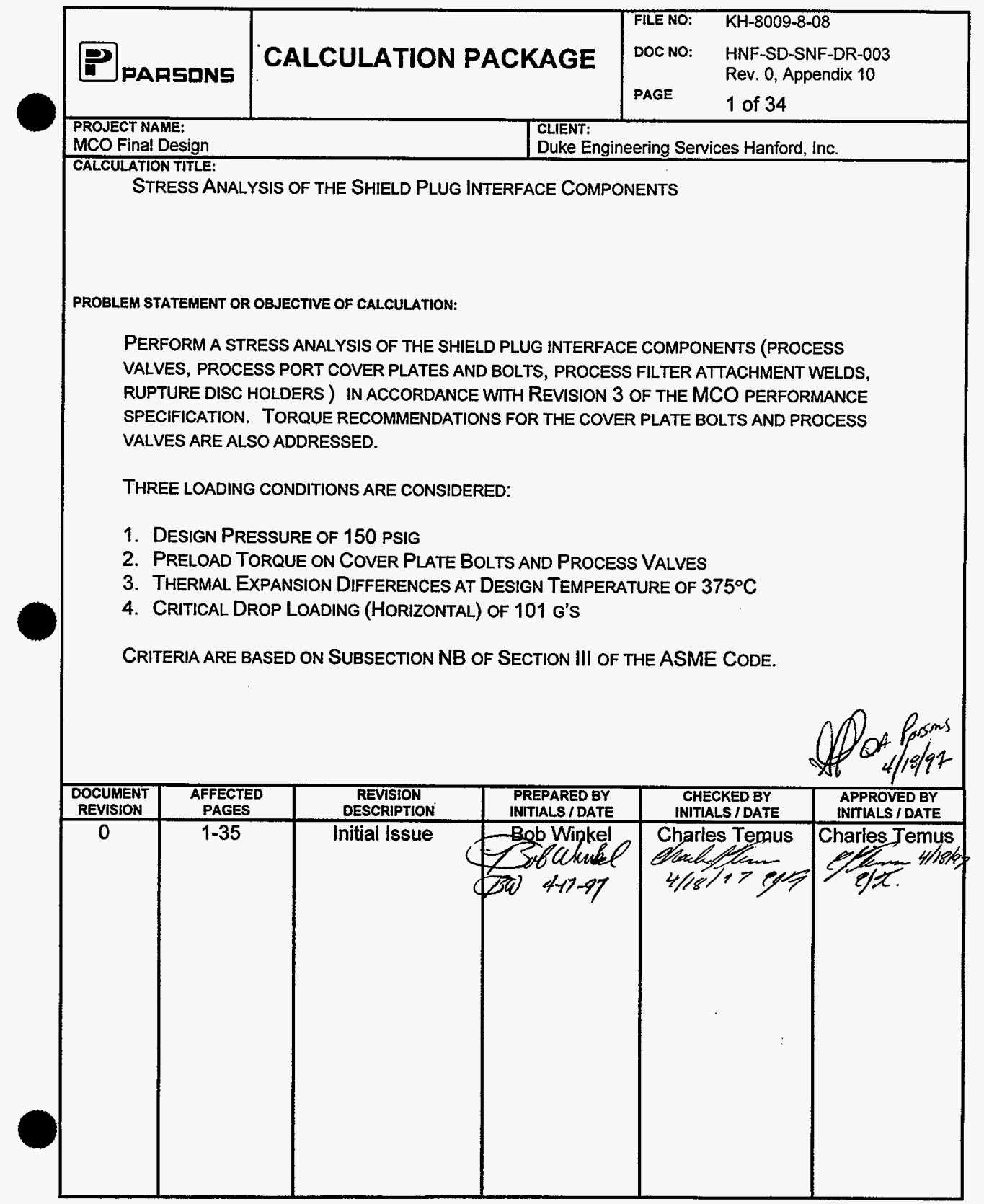


CLIENT: Duke Engineering Services Hanford, INC

FILE NO: $\mathrm{KH}-8009-8-08$

PROJECT: MCO Final Design DOC. NO.: HNF-SD-SNF-DR-003, Rev. 0, Appendix 10

TABLE OF CONTENTS

1. INTRODUCTION

2. REFERENCES 5

3. ASSUMPTIONS 6

4. GEOMETRY

5. MATERIAL PROPERTIES

6. ACCEPTANCE CRITERIA

7. LOAD CONDITIONS AND COMBINATIONS

8. STRESS ANALYSIS CALCULATIONS

$\begin{array}{ll}\text { 8.1 Process Port Cover Plate and Bolts } & 11\end{array}$

$\begin{array}{lr}\text { 8.1.1 Cover Plate Bolts } & 13\end{array}$

$\begin{array}{lr}\text { 8.1.2 Cover Plate Evaluation } & 16\end{array}$

$\begin{array}{lr}\text { 8.2 Process Valve Plug Evaluation } & 17\end{array}$

$\begin{array}{lr}\text { 8.3 Rupture Disc Holder } & 25\end{array}$

$\begin{array}{ll}\text { 8.4 Torque Recommendations } & 31\end{array}$

$\begin{array}{ll}\text { 8.5 Process Filter Attachment Welds } & 33\end{array}$

\begin{tabular}{|c|l|l|l|}
\hline $\operatorname{sat}_{4 / 18 / 97}$ & & & \\
\hline $\cos 4 / 18 / 97$ & & & \\
\hline
\end{tabular}


CLIENT: Duke Engineering Services Hanford, INC

FILE NO: KH-8009-8-08

PROJECT: MCO Final Design DOC. NO.: HNF-SD-SNF-DR-003, Rev. 0, Appendix 10

\section{LIST OF FIGURES}

FIGURE 1. SHIELD PLUG INTERFACE COMPONENT GEOMETRY. 
CLIENT: $\quad$ Duke Engineering Services Hanford, INC

FILE NO: KH-8009-8-08

PROJECT: MCO Final Design DOC. NO.: HNF-SD-SNF-DR-003, Rev. 0, Appendix 10

\section{LIST OF TABLES}

TABLE 1. MATERIAL LISTING FOR SHIELD PLUG INTERFACE COMPONENTS

TABLE 2. ASME CODE MATERIAL PROPERTIES FOR TYPE 304L STAINLESS STEEL.

TABLE 3. ASME DESIGN TEMPERATURE MATERIAL PROPERTIES FOR COVER PLATE BOLTS AND PROCESS VALVES. 
CLIENT: Duke Engineering Services Hanford, INC

FILE NO: $\quad \mathrm{KH}-8009-8-08$

PROJECT: MCO Final Design

DOC. NO.: HNF-SD-SNF-DR-003, Rev. 0, Appendix 10

\section{INTRODUCTION}

This calculation documents the evaluation of the MCO process port cover plates, cover plate bolts, process valves, and the process filter attachment welds. The special process valves containing rutpure discs are included in the evaluation, as well as the rupture disc unit which will be inserted into the canister cover. The structural adequacy evaluation is based upon Subsection NB of Section III of the ASME Code (Reference 3). Component loading includes preload from torquing of bolts and process valve bodies, design pressure, and drop loading.

\section{REFERENCES}

1. DE\&S, 1997, Performance Specification for the Spent Nuclear Fuel Multi-Canister Overpack, WHC-S-0426, Rev. 3, Duke Engineering and Services Hanford, Richland, Washington.

2. ASME, 1995, ASME Boiler and Pressure Vessel Code, Section II, Materials, Part D-Properties, American Society of Mechanical Engineers, New York, New York.

3. ASME, 1995, ASME Boiler and Pressure Vessel Code, Section III, Subsection NB, American Society of Mechanical Engineers, New York, New York.

4. Roark, R. J. and Young, W. C., 1975, Formulas for Stress and Strain, 5th Edition, McGraw-Hill, New York, New York.

5. Baumeister, T., editor, 1967, Standard Handbook for Mechanical Engineers, 7th Edition, McGraw-Hill, New York, New York.

6. Parsons, 1997, "Stress Analysis of the Mark 1A Storage and Scrap Baskets," Calculation No. KH-8009-8-05, Parsons Infrastructure and Technology Group, Inc., Richland, Washington.

7. Bickford, J. H., 1990, An Introduction to the Design and Behavior of Bolted Joints, 2nd Edition, Marcel Dekker, Inc., New York City, New York.

8. Shigley, J. E. and Mischke, C. R., Mechanical Engineering Design, 5th Edition, McGrawHill, New York, New York.

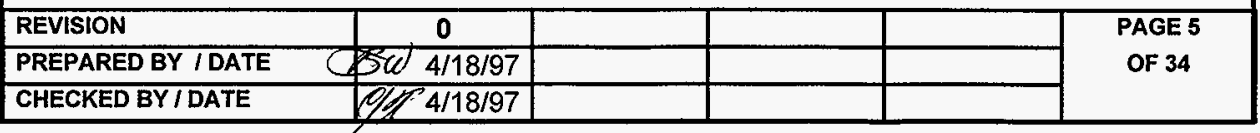


CLIENT: Duke Engineering Services Hanford, INC

PROJECT: MCO Final Design DOC. NO.: HNF-SD-SNF-DR-003, Rev. 0, Appendix 10

9. IFI, 1988, Fastener Standards, 6th Edition, Industrial Fasteners Institute, Cleveland, Ohio.

10. Horton, H. L., 1974, Machinery's Handbook, 19th Edition, Industrial Press, Inc., New York, New York.

\section{ASSUMPTIONS}

1. Preloads from torquing the cover plate bolts and process valves are assumed to be accurate to within $+30 \%$ and $-35 \%$. This range is assumed to include the standard preload/torque uncertainties, including operator and tool inaccuracies. A "Never-Sieze" lubricant is assumed for the preload/torque calculations, using "Nut-Factor" values from Reference 7. It is conservatively assumed that the maximum temperature during torque application (insertion and removal) is the design temperature of $375^{\circ} \mathrm{C}$. It is also assumed that the Reference 7 nut factors are not affected by temperature. As discussed in Section 8.1.4, the adequacy of the uncertainty range and the mean nut factor, for the cover plate bolts and process valves must be verified by test.

2. It is assumed that the threaded process valve bodies can be appropriately evaluated as bolts, relative to the ASME Code design stress limits.

3. It is assumed that the torquing tool, used for inserting and preloading the rupture disc type process plug, will extend beyond the holes in the hex head. That is, the hex head minimum cross section, at the vent holes, will not experience the full torque during torquing operations.

4. Some of the dimensions of the rupture disc valve (e.g. rupture disc outside diameter) are based upon a specific rupture disc manufacturer. Since the dimensions are vendor dependent, some of the calculations may need to be modified, depending on the final rupture disc manufacturer selected.

5. Other assumptions as noted within the calculation documentation.

\section{GEOMETRY}

The geometry of the shield plug interface components are defined on the assembly drawing ( $\mathrm{H}-2-828041)$, the shield plug drawing $(\mathrm{H}-2-828045)$, the process plug valve drawing $(\mathrm{H}-2-$ 828047), the cover plate/bolt drawing ( $\mathrm{H}-2-828048)$, and the process filter drawing $(\mathrm{H}-2-$ 828049). The structural components are identified in Figure 1. There are four process ports in the shield plug. The structural components of the port closures are identical, except some of the cover plates have four bolts and some have five. Only the weaker four-bolt

\begin{tabular}{|c|c|c|c|}
\hline \multirow{2}{*}{$\begin{array}{l}\text { REVISION } \\
\text { PREPARED BY / DAT }\end{array}$} & \multicolumn{3}{|l|}{0} \\
\hline & 5y) $4 / 18 / 97$ & & \\
\hline CHECKED BY IDATE & c/ $/ 4 / 18 / 97$ & & \\
\hline
\end{tabular}




\section{Parsans}

CLIENT: Duke Engineering Services Hanford, INC

FILE NO: KH-8009-8-08

PROJECT: MCO Final Design

DOC. NO.: HNF-SD-SNF-DR-003, Rev. 0, Appendix 10

configurations are analyzed in this report. The purpose of the cover plates is to provide secondary containment for the process valve seals, and to protect the valves during handling operations.

The top of the process filter valve bodies consists of a hex head used for torquing the threaded valve bodies into the four shield plug port holes. Sealing for both the process valves and the cover plates is achieved using a C-seal requiring a minimum seating load of $300 \mathrm{lb} / \mathrm{in}$.

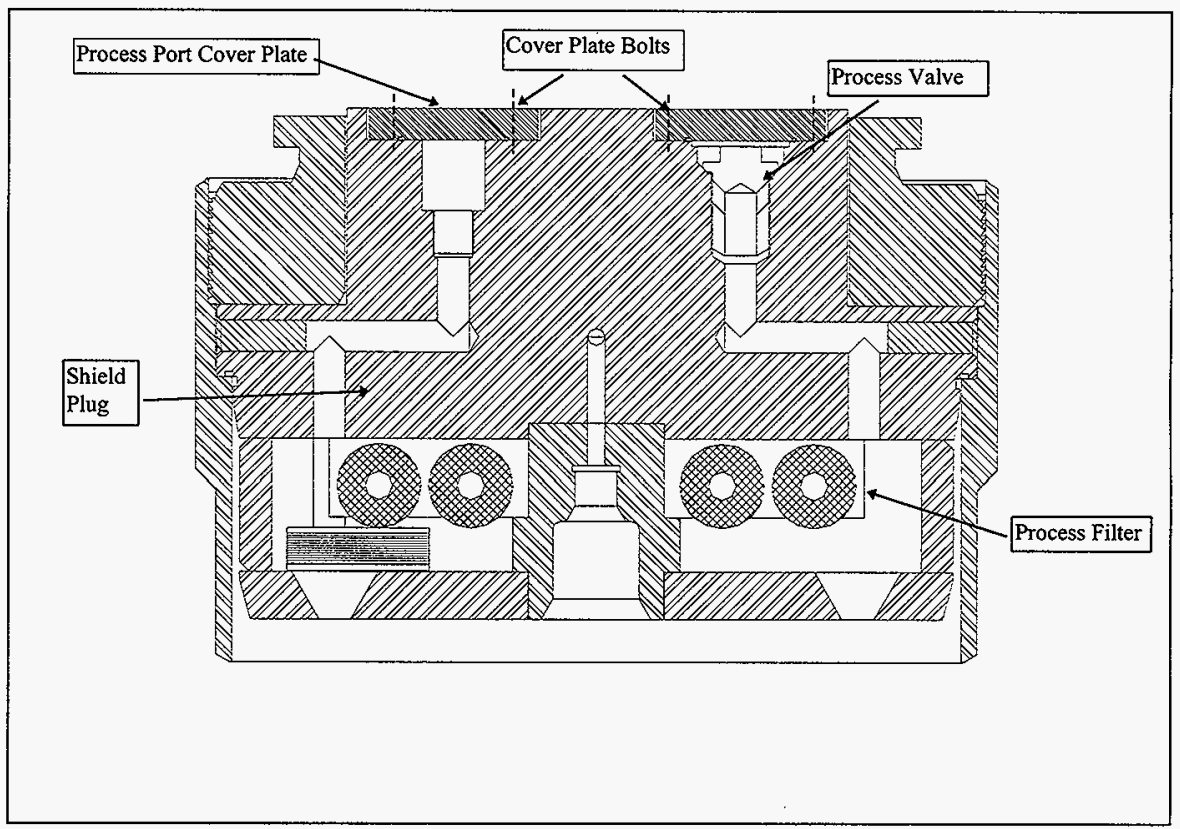

Figure 1. Shield Plug Interface Component Geometry.

The process filter is welded to the bottom of the shield plug as shown. The filter details are not specified by the drawing because it is being supplied by others. Only the $1 / 8$-in. filter

$\mid$\begin{tabular}{c|c}
0 \\
$4 / 18 / 97$ \\
$0 / 84 / 18 / 97$
\end{tabular}


CLIENT: $\quad$ Duke Engineering Services Hanford, INC

FILE NO: $\mathrm{KH}-8009-8-08$

PROJECT: MCO Final Design DOC. NO.: HNF-SD-SNF-DR-003, Rev. 0, Appendix 10

attachment welds are evaluated by this calculation. A bounding filter assembly weight of 50 lb is specified by the Reference 1 Performance Specification.

The process valve bodies are identical except for the type that contain rupture discs. The rupture disc type valve bodies are in two pieces which are welded together by a fullcircumference structural groove weld. The critical loading for this weld is the torque applied during insertion and removal.

In addition to the process valve design which contains a rupture disc, there is a rupture disc pressure relief device (hereafter referred to as "rupture disc holder") which can be inserted either into Port 1 or the canister cover. The threaded portion of this pressure relief component is smaller ( $3 / 4$-in. NPSM thread) than the $1-7 / 8$-in process valve threads and is also addressed in the calculations below.

\section{MATERIAL PROPERTIES}

The materials included in the shield plug interface components are listed in Table 1. The structural properties of interest for 304L stainless steel are provided in Table 2. The cover plate bolt and process valve material properties at the MCO design temperature of $375^{\circ} \mathrm{C}$ are listed in Table 3. It is noted that the process valves and rupture disc holder are constructed from 304LN stainless steel, which is a standard ASME pressure boundary material, but $304 \mathrm{LN}$ is not listed under the ASME bolting material section (Section II, Part D, Table 4). Since the valve bodies function much like a bolt (external threads, hex head, provide preload to seal), they were evaluated using ASME rules for bolts. The 304LN $S_{m}$ value listed in the table is the pressure vessel material value which is essentially twice the bolt material allowable. This difference is accounted for in the stress calculations.

Since the process filters are being designed by others, process filter material properties are not addressed. The process filters will be welded to the $304 \mathrm{~L}$ shield plugs. Therefore, the $304 \mathrm{~L}$ base material allowables, Table 2, were applied to the attachment welds.

Table 1. Material Listing for Shield Plug Interface Components

\begin{tabular}{|c|c|c|}
\hline Component & Material & ASME Spec No. \\
\hline Shield Plug, Process Port Cover Plate & 304 L SS & SA-182 \\
\hline Cover Plate Bolts & 22Cr-13Ni-5Mn & SA-193-B8R \\
\hline Process Valves \& Rupture Disc Holder & 304 LN SS & SA-479 (Bar Stock) \\
\hline
\end{tabular}

\begin{tabular}{|l|c|c|c|c|c|}
\hline REVISION & 0 & & & \\
\cline { 1 - 2 } \\
OF 34
\end{tabular}


CLIENT: Duke Engineering Services Hanford, INC

FILE NO: KH-8009-8-08

PROJECT: MCO Final Design

DOC. NO.: HNF-SD-SNF-DR-003, Rev. 0, Appendix 10

Table 2. ASME Code Material Properties for Type 304L Stainless Steel.

\begin{tabular}{|c|c|c|c|c|c|}
\hline \multicolumn{2}{|c|}{ Temperature } & $E$ & $S_{m}$ & $S_{y}$ & \\
\hline${ }^{\circ} \mathrm{F}$ & ${ }^{\circ} \mathrm{C}$ & ble TM-1, Group & Table $2 A, p .322$ & Table $Y=1, p .524$ & Table U.P. 441 \\
\hline-20 & $\cdots$ & $-\ldots$ & $16.7 \mathrm{ksi}$ & $25.0 \mathrm{ksi}$ & $70.0 \mathrm{ksi}$ \\
\hline 70 & -- & $28.3 E+06$ & -- & -- & -- \\
\hline 100 & -- & - & $16.7 \mathrm{ksi}$ & $25.0 \mathrm{ksi}$ & $70.0 \mathrm{ksi}$ \\
\hline 200 & -- & $27.6 E+06$ & $16.7 \mathrm{ksi}$ & $21.3 \mathrm{ksi}$ & $66.2 \mathrm{ksi}$ \\
\hline 212 & 100 & $27.5 E+06$ & $16.7 \mathrm{ksi}$ & $21.0 \mathrm{ksi}$ & $65.6 \mathrm{ks} \mathrm{i}$ \\
\hline 300 & -- & $27.0 E+06$ & $16.7 \mathrm{ks} \mathrm{i}$ & $19.1 \mathrm{ksi}$ & $60.9 \mathrm{ksi}$ \\
\hline 700 & -- & $24.8 E+06$ & $13.5 \mathrm{ksi}$ & $14.9 \mathrm{ksi}$ & $56.2 \mathrm{ksi}$ \\
\hline 707 & 375 & $24.8 E+06$ & $13.5 \mathrm{ksi}$ & $14.9 \mathrm{ksi}$ & $56.2 \mathrm{ksi}$ \\
\hline 750 & -- & -- & $13.3 \mathrm{ksi}$ & $14.7 \mathrm{ksi}$ & $55.9 \mathrm{ksi}$ \\
\hline 800 & & $24.1 E+06$ & -- & -- & -- \\
\hline
\end{tabular}

Notes 1: Underlined values determ ined by linear interpolation, all other values taken from Section II, Part D of the ASME Code.

2: Value of $E$ taken from $T$ able $T M-1$ for $M$ aterial Group $G$.

Table 3. ASME Design Temperature Material Properties for Cover Plate Bolts and Process Valves.

\begin{tabular}{|c|c|c|c|}
\hline Material & Elastic Modulus, psi & $\begin{array}{c}\text { Mean Thermal } \\
\text { Expansion Coefficient } \\
\left(70-707^{\circ} \mathrm{F}, \text { in } / \text { in } /{ }^{\circ} \mathrm{F}\right)\end{array}$ & $\mathrm{S}_{\mathrm{m}}, \mathrm{psi}$ \\
\hline $\begin{array}{c}\text { SA 193-B8 Cover } \\
\text { Plate Bolts }\end{array}$ & $26.2 \times 10^{6}$ & $9.16 \times 10^{-6}$ & 12,100 \\
\hline $\begin{array}{c}\text { 304LN Process Valves } \\
\text { \& Rupture Disc Holder }\end{array}$ & $24.8 \times 10^{6}$ & $9.77 \times 10^{-6}$ & 15,900 \\
\hline
\end{tabular}


CLIENT: Duke Engineering Services Hanford, INC

FILE NO: KH-8009-8-08

PROJECT: MCO Final Design $\quad$ DOC. NO.: HNF-SD-SNF-DR-003, Rev. 0, Appendix 10

\section{ACCEPTANCE CRITERIA}

For the process port valves, cover plates, and cover plate bolts, the critical load condition is the design condition loading: 150 psi design pressure @ $375^{\circ} \mathrm{C}$ combined with the preload torque loading. (Note: Near the end of the MCO design process, the design condition was changed to two temperature/pressure combinations-- $150 \mathrm{psi} @ 200^{\circ} \mathrm{C}$ and $75 \mathrm{psi} @$ $375^{\circ} \mathrm{C}$. In this appendix, the conservative envelope of $150 \mathrm{psi} @ 375^{\circ} \mathrm{C}$ is conservatively used.) Torsional stresses in the process valve bodies are also addressed. It is conservatively assumed that the preload is applied at the full design temperature, since the valves will be tightened and loosened during processing operations. For the process filter attachment welds, the critical loading is the $101 \mathrm{~g}$ horizontal drop.

Since the Reference 1 Performance Specification specifies that the MCO is to be designed to the intent of Subsection NB of the ASME Code, the ASME Code requirements are used as the acceptance criteria for the shield plug interface components. All of the interface components are part of the MCO pressure boundary with the exception of the process filter attachment welds. For the process filter attachment welds, it is assumed that the inspection will be limited to a visual examination and appropriate weld quality factors from Subsection NG are applied. The full design temperature allowables are conservatively applied to the attachment weld evaluation.

As mentioned in Sections 3 and 5, since the process valves are constructed in the form of a bolt, and are required to maintain the preload on the pressure boundary seal, ASME bolt stress limits are applied to the process valve bodies. Since the $304 \mathrm{LN}$ valve plug material is not listed in the bolt section of the Code material properties, Table 4 of Reference 2, the appropriate bolt-type Sm was derived using Article III-2120 from Section III of the ASME Code $(1 / 3$ yield).

A rupture disc is included in one of the process filter designs and in the canister cover, which results in a two-piece design assembled with a seal weld at the edge of the disc and a structural connection weld. The structural weld is subjected to both torque loading and the design pressure. The weld is a groove weld which is questionable relative to the ASME Subsection NB weld configuration requirements. The Subsection NG weld quality factors are applied to the rupture disc valve structural weld, assuming a surface only dye penetrant examination. As indicated in the calculations below, the resulting design margin is large, which minimizes potential concerns relative to the structural adequacy of the rupture disc valve structural weld.

\begin{tabular}{|l|c|l|l|l|l|}
\hline REVISION & 0 & & & & PAGE 10 \\
PREPARED BY IDATE OF 34 \\
CHECKED BYIDATE & SW 4/18/97 & & & & \\
\hline
\end{tabular}


CLIENT: Duke Engineering Services Hanford, INC

FILE NO: KH-8009-8-08

PROJECT: MCO Final Design

DOC. NO.: HNF-SD-SNF-DR-003, Rev. 0, Appendix 10

\section{LOAD CONDITIONS AND COMBINATIONS}

It is expected that the initial attachment of the process valves and cover plates to the shield plug will be conducted at room temperature. For later processing operations, torquing of both the cover plate bolts and process valves may occur at higher temperatures. To cover the possibility of torquing operations at higher temperatures, it is conservatively assumed that torquing could occur at the full design temperature of $375^{\circ} \mathrm{C}$. Another conservative assumption is that the maximum torque to the process valves could occur simultaneously with the full design pressure, e.g. when the break away torque is applied during valve opening.

As stated in Section 6, the critical loading for the process valves and cover plates is the torquing preload combined with the design pressure and temperature. Due to the relative size of these components, the vertical drop inertia loading is less than the pressure loading. For the more severe horizontal drop, the parts bear against the sides of shield plug holes (cover plate sits in a recess). Appendix F of the ASME Code does not limit bearing stresses. Thus, only the filter attachment welds are evaluated for the drop loading.

\section{STRESS ANALYSIS CALCULATIONS}

The shield plug interface components structural evaluations were limited to hand calculations. Calculation details for each component follows below.

\subsection{Process Port Cover Plate and Bolts}

\section{Calculation Parameters:}

$n_{b}=4 \quad$ Minimum number of bolts

$A_{b}:=\frac{\pi}{4} \cdot(.490 \cdot i n)^{2} \quad$ Bolt area above threads

$$
A_{b}=0.189 \cdot \text { in }^{2}
$$

\begin{tabular}{c|c|c|c} 
& 0 & & \\
\hline $3 W^{4 / 18 / 97}$ & & \\
\hline / $/ / 18 / 97$ & &
\end{tabular}


CLIENT: $\quad$ Duke Engineering Services Hanford, INC

FILE NO: $\quad \mathrm{KH}-8009-8-08$

PROJECT: MCO Final Design

DOC. NO.: HNF-SD-SNF-DR-003, Rev. 0, Appendix 10

$\mathrm{A}_{\text {et }}=0.998 \cdot \frac{\mathrm{in}^{2}}{\text { in }} \quad 5 / 8-11$ UNC thread stripping area, Reference 9

$\mathrm{A}_{\text {it }}=1.42 \cdot \frac{\mathrm{in}^{2}}{\text { in }} \quad$ Internal thread stripping area, Reference 9

$1_{\text {thread }}=0.75 \cdot$ in $\quad$ Bolt thread length

$d_{s}:=3.555 \cdot$ in $\quad$ Outside diameter of seal

${ }^{t} p=1.00 \cdot$ in $\quad$ Plate thickness

$d_{b}:=0.625 \cdot$ in $\quad$ Nominal bolt diameter

$d_{b c}=4.375 \cdot$ in $\quad$ Diameter of bolt circle

$f_{s p}:=300 \cdot \frac{b}{\text { in }} \quad$ Minimum seal preload, see Appendix 14

pres $=150 \cdot \frac{\mathrm{lb}}{\mathrm{in}^{2}} \quad$ Design pressure

$\mathrm{S}_{\mathrm{mb}}=12100 \cdot \frac{\mathrm{lb}}{\mathrm{in}^{2}} \quad \mathrm{SA} 193 \mathrm{~B} 8 \mathrm{R}$ bolt material $\mathrm{Sm}$, design temp. $=707 \mathrm{degF}$

$\mathrm{S}_{\mathrm{mp}}=13500 \cdot \frac{\mathrm{lb}}{\mathrm{in}^{2}} \quad 304 \mathrm{~L}$ plate, design temperature

$\alpha_{p}=9.70 \cdot 10^{-6} \cdot \frac{\text { in }}{\text { in } \cdot R} \quad 304 \mathrm{~L}$ mean expansion coefficient, 70 to $707 \mathrm{degF}$

$\alpha_{b}:=9.16 \cdot 10^{-6} \cdot \frac{\text { in }}{\mathrm{in} \cdot \mathrm{R}} \quad$ SA $193 \mathrm{Gr}$ B8R bolt expansion coefficient, $70-707 \mathrm{deg} F$

$E_{b}:=26.2 \cdot 10^{6} \cdot \frac{\mathrm{lb}}{\mathrm{in}^{2}} \quad \mathrm{SA} 193 \mathrm{Gr}$ B8R bolt, Elastic Modulus, $707 \mathrm{deg} \mathrm{F}$

$\mathrm{K}_{\min }=0.11 \quad$ Minimum nut factor, "Never-Seize"

lubricant, Table 5.1 of Reference 7

$\mathrm{K}_{\max }:=0.21 \quad$ Maximum nut factor, Reference 7 
CLIENT: Duke Engineering Services Hanford, INC

FILE NO: $\mathrm{KH}-8009-8-08$

PROJECT: MCO Final Design

DOC. NO.: HNF-SD-SNF-DR-003, Rev. 0, Appendix 10

$\mathrm{K}_{\text {mean }}=0.17 \quad$ Mean nut factor, Reference 7.

Note that $K_{\max }=K_{\text {mean }}+24 \% \& K_{\min }=K_{\text {mean }}-35 \%$, which is close to the $30 \%$ variance frequently recommended for bolt/preload predictions (Reference 7 ). For establishing the minimum torque required for seal seating, a $\mathrm{K}_{\text {mean }}+30 \%$ was conservatively used.

\subsubsection{Cover Plate Bolts}

Bolt Area Requirement (See Appendix E, ASME Code):

Appendix $E$ requires that a bolt have sufficient area to carry the required seal load plus the pressure loading.

$$
\begin{aligned}
& \mathrm{H}:=\frac{\pi}{4} \cdot \mathrm{d}_{\mathrm{s}}^{2} \cdot \mathrm{pres} \quad \text { Pressure load } \\
& \mathrm{H}=1.489 \cdot 10^{3} \cdot \mathrm{lb} \\
& \mathrm{H}_{\mathrm{p}}=\pi \cdot \mathrm{d}_{\mathrm{s}} \cdot \mathrm{f}_{\mathrm{sp}} \quad \text { Minimum preload } \\
& \mathrm{H}_{\mathrm{p}}=3.351 \cdot 10^{3} \cdot \mathrm{lb} \\
& \mathrm{A}_{\text {req }}:=\frac{\mathrm{H}+\mathrm{H}_{\mathrm{p}}}{\mathrm{S}_{\mathrm{mb}}} \\
& \mathrm{A}_{\text {req }}=0.4 \cdot \mathrm{in}_{\text {Ratio }}=\frac{\mathrm{A}_{\text {req }}}{\mathrm{n}_{\mathrm{b}} \cdot \mathrm{A}_{\mathrm{b}}} \quad \text { Adequate Bolt Area } \\
& \text { Ratio }=0.53
\end{aligned}
$$

Pressure load

Minimum preload

Adequate Bolt Area for preload + pressure

$C$

\begin{tabular}{|c|}
0 \\
\hline $4 / 18 / 97$ \\
\hline
\end{tabular}


CLIENT: Duke Engineering Services Hanford, INC

FILE NO: $\mathrm{KH}-8009-8-08$

PROJECT: MCO Final Design $\quad$ DOC. NO.: HNF-SD-SNF-DR-003, Rev. 0, Appendix 10

Check Bolt Adequacy for In-Service Loads:

The ASME Code requires that the maximum in-service bolt stress not exceed $2 \mathrm{Sm}$.

1) Thermal Loading

$$
\begin{aligned}
& \sigma_{b t h}:=E_{b} \cdot\left(\alpha_{p}-\alpha_{b}\right) \cdot(707 \cdot R-70 \cdot R) \\
& \sigma_{b t h}=9.012 \cdot 10^{3} \cdot \frac{\mathrm{lb}}{\text { in }^{2}} \quad \text { Preload increase due to relative thermal growth }
\end{aligned}
$$

2) Maximum Preload

Torque, per bolt, required to assure required minimum preload $\left(f_{\mathrm{Sp}}\right)$ on seal:

$$
\begin{aligned}
& T_{\text {in }}:=\frac{f_{s p} \cdot \pi \cdot d_{s}}{n_{b}} \cdot K_{\text {mean }^{1}} \cdot 3 \cdot d_{b} \\
& T_{\text {in }}=9.641 \cdot l b \cdot f t
\end{aligned}
$$

Maximum preload per bolt for a torque of $\mathrm{T}_{\text {in }}$ :

$$
\begin{aligned}
& F_{\text {pmax }}:=\frac{T_{\text {in }}}{K_{\min ^{2} \mathrm{~b}}} \\
& F_{\text {pmax }}=1.683 \cdot 10^{3} \cdot 1 \mathrm{~b} \\
& \sigma_{\mathrm{bp}}:=\frac{F_{\text {pma }}}{\mathrm{A}_{\mathrm{b}}} \\
& \sigma_{\mathrm{bp}}=8.924 \cdot 10^{3} \cdot \frac{\mathrm{lb}}{\mathrm{in}^{2}}
\end{aligned}
$$


CLIENT: Duke Engineering Services Hanford, INC

FILE NO: KH-8009-8-08

PROJECT: MCO Final Design DOC. NO.: HNF-SD-SNF-DR-003, Rev. 0, Appendix 10

Bolt stress from pressure loading (conservatively assume bolts carry entire pressure load):

$$
\begin{aligned}
& \sigma_{\text {bpres }}:=\frac{H}{\mathrm{n}_{\mathrm{b}} \cdot \mathrm{A}_{\mathrm{b}}} \\
& \sigma_{\text {bpres }}=1.974 \cdot 10^{3} \cdot \frac{\mathrm{lb}}{\mathrm{in}^{2}}
\end{aligned}
$$

Maximum combined in-service stress in bolts:

$$
\begin{aligned}
& \sigma_{\text {btot }}:=\sigma_{\text {bp }}+\sigma_{\text {bpres }}+\sigma_{\text {bth }} \\
& \sigma_{\text {btot }}=1.991 \cdot 10^{4} \cdot \frac{\mathrm{lb}}{\text { in }^{2}}
\end{aligned}
$$

Comparing the total in-service stress to the allowable of $2 S_{m}$ :

$$
\text { Ratio }:=\frac{\sigma_{\text {btot }}}{2 \cdot \mathrm{S}_{\mathrm{m}}}
$$

Ratio $=0.823 \quad$ Cover bolts $O K$ for in-service loading

\section{CHECK BOLT THREAD STRIPPING:}

Since the $X M-19$ bolt strength is much higher than the $304 \mathrm{~L}$ shield plug strength, the shield plug internal threads control.

$$
\begin{aligned}
& \mathrm{S}_{\mathrm{m}}:=13500 \cdot \frac{\mathrm{lb}}{\mathrm{in}^{2}} \quad \text { Shield plug membrane stress allowable } \\
& \sigma_{\text {thread }}:=\frac{2 \cdot \mathrm{S} \mathrm{mb}^{\mathrm{A}} \cdot \mathrm{A}}{\mathrm{A}^{\mathrm{it}}{ }^{-1} \text { thread }} \quad \text { Thread stress, bolt @ allow. stress }
\end{aligned}
$$


CLIENT: Duke Engineering Services Hanford, INC

FILE NO: $\quad \mathrm{KH}-8009-8-08$

PROJECT: MCO Final Design DOC. NO.: HNF-SD-SNF-DR-003, Rev. 0, Appendix 10

$$
\begin{aligned}
& \sigma_{\text {thread }}=4.285 \cdot 10^{3} \cdot \frac{\mathrm{lb}}{\mathrm{in}^{2}} \\
& \text { Ratio }=\frac{\sigma \text { thread }}{0.6 \cdot \mathrm{S}_{\mathrm{m}}} \\
& \text { Ratio }=0.529
\end{aligned}
$$

\subsubsection{Cover Plate Evaluation}

Consider a simply-supported circular plate having a diameter equal to the bolt-circle diameter (Reference 4, Table 24):

$$
v:=0.3
$$

$$
\begin{aligned}
& M_{\text {max }}:=\frac{\text { pres } \cdot\left(\frac{d_{b c}}{2}\right)^{2} \cdot(3+v)}{16} \\
& M_{\text {max }}=148.041 \cdot 1 b \cdot \frac{\text { in }}{\text { in }} \\
& \sigma_{p}:=6 \cdot \frac{M_{\text {max }}}{t_{p}{ }^{2}} \\
& \sigma_{p}=888.245 \cdot \frac{1 b}{\text { in }^{2}} \\
& \text { Ratio }:=\frac{\sigma_{p}}{1.5 \cdot \mathrm{s}_{\mathrm{m}}}
\end{aligned}
$$

Ratio $=0.044$

Thus, the cover plate has a large margin, relative to Code allowables. 


\section{E]paAsRNS}

CLIENT: Duke Engineering Services Hanford, INC FILE NO: KH-8009-8-08

PROJECT: MCO Final Design DOC. NO.: HNF-SD-SNF-DR-003, Rev. 0, Appendix 10

\subsection{Process Valve Plug Evaluation}

The process valve plug is threaded and was evaluated using bolt requirements (NB-3230).

\section{Parameter Definitions:}

$$
\begin{aligned}
& \mathrm{d}_{\mathrm{p}}:=1.875 \text {-in } \quad \text { Valve nominal diameter (1 7/8-12 UN-2A Thread) } \\
& 1_{\text {thread }}=2.266 \cdot \text { in } \quad \text { Valve thread engagement length } \\
& A_{\text {vsol }}=2.53 \cdot \text { in }^{2} \quad \text { Tensile area of valve/bolt (solid), Reference } 10 \\
& d_{\text {eff }}=\sqrt{\frac{A}{\pi} \text { vsol }^{4}} \\
& \mathrm{~d}_{\text {eff }}=1.795 \text { in } \quad \text { Valve plug effective outside diameter } \\
& \mathrm{d}_{\mathrm{h}}=0.688 \cdot \text { in } \quad \text { Valve plug radial hole diam. ( } 3 \text { holes @ } 120 \text { degrees) } \\
& \mathrm{d}_{\mathrm{i}}:=1.00 \cdot \text { in } \quad \text { Valve plug inside diameter } \\
& A_{\text {vnet }}=A_{\text {vsol }}-\frac{\pi \cdot d_{i}^{2}}{4}-3 \cdot d_{h} \cdot\left(\frac{d_{\text {eff }}-d_{i}}{2}\right) \\
& A_{\text {vnet }}=0.924 \cdot \text { in }^{2} \quad \text { Valve plug net area @ radial holes } \\
& \mathrm{S}_{\mathrm{mv}}:=5856 \cdot \frac{\mathrm{lb}}{\mathrm{in}^{2}}
\end{aligned}
$$

$S_{m v}=$ valve plug design temperature material allowable, SA-479, 304LN divided by 3 to correspond to bolt $S_{m}$ value.

$$
\mathrm{E}_{\mathrm{v}}:=24.8 \cdot 10^{6} \cdot \frac{\mathrm{lb}}{\mathrm{in}^{2}} \quad \text { Valve plug modulus of elasticity }
$$

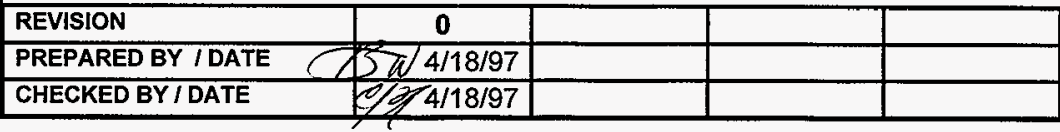


CLIENT: Duke Engineering Services Hanford, INC

FILE NO: KH-8009-8-08

PROJECT: \begin{tabular}{lll} 
MCO Final Design $\quad$ DOC. NO.: HNF-SD-SNF-DR-003, Rev. 0, Appendix 10 \\
\hline
\end{tabular}
$\mathrm{d}_{\mathrm{s}}=2.00 \cdot$ in $\quad$ Conservative seal diameter

Required Valve Plug Area:

Per ASME Code requirements, bolt (valve plug) area must be sufficient to carry the required seal load plus the full pressure load.

$$
\begin{aligned}
& \mathrm{H}:=\frac{\pi}{4} \cdot \mathrm{d}_{\mathrm{s}}{ }^{2} \cdot \text { pres } \\
& \mathrm{H}=471.239 \cdot \mathrm{lb} \\
& \mathrm{H}_{\mathrm{p}}=\pi \cdot \mathrm{d}_{\mathrm{s}} \cdot \mathrm{f}_{\mathrm{sp}} \\
& \mathrm{H}_{\mathrm{p}}=1.885 \cdot 10^{3} \cdot \mathrm{bb} \\
& \mathrm{A}_{\text {req }}=\frac{\mathrm{H}+\mathrm{H}_{\mathrm{p}}}{\mathrm{S}_{\mathrm{mv}}} \\
& \mathrm{A}_{\text {req }}=0.402 \cdot \text { in }^{2} \\
& \text { Ratio }:=\frac{\mathrm{A}_{\text {req }}}{\mathrm{A}_{\text {vnet }}}
\end{aligned}
$$$$
\text { Ratio }=0.435
$$

Net area of plug is adequate for axial loading

\section{Check Valve Plug Adequacy for In-Service Loads}

1) Thermal Loading

There is no thermal loading in the valve plug since the expansion coefficients for the valve and shield plug are the same. 
CLIENT: Duke Engineering Services Hanford, INC

FILE NO: KH-8009-8-08

PROJECT: MCO Final Design DOC. NO.: HNF-SD-SNF-DR-003, Rev. 0, Appendix 10

2) Maximum Preload

Torque required to maintain required minimum preload $\left(f_{s p}\right)$ on seal:

$$
\begin{aligned}
& T_{\text {in }}=\left(f_{\text {sp }} \cdot \pi \cdot d_{s}\right) \cdot K_{\text {mean }} \cdot 1.3 \cdot d_{p} \\
& T_{\text {in }}=65.09 \cdot 1 \mathrm{~b} \cdot \mathrm{ft}
\end{aligned}
$$

Maximum preload:

$$
\begin{aligned}
& F_{\text {pmax }}=\frac{T_{\text {in }}}{K_{\min ^{\cdot} \cdot d_{p}}} \\
& F_{\text {pmax }}=3.787 \cdot 10^{3} \cdot \mathrm{lb} \\
& \sigma_{\text {bp }}:=\frac{F_{\text {pmax }}}{A_{\text {vnet }}} \\
& \sigma_{\text {bp }}=4.097 \cdot 10^{3} \cdot \frac{\mathrm{lb}}{\text { in }^{2}}
\end{aligned}
$$

Plug stress from pressure loading (conservatively assume plug carries entire pressure load):

$$
\begin{aligned}
& \sigma_{\text {bpres }}=\frac{H}{\mathrm{~A}_{\text {vnet }}} \\
& \sigma_{\text {bpres }}=509.794 \cdot \frac{\mathrm{lb}}{\mathrm{in}^{2}}
\end{aligned}
$$


CLIENT: Duke Engineering Services Hanford, INC

FILE NO: KH-8009-8-08

PROJECT: MCO Final Design $\quad$ DOC. NO.: HNF-SD-SNF-DR-003, Rev. 0, Appendix 10

Maximum combined in-service stress in plug:

$$
\begin{aligned}
& \sigma_{\text {btot }}=\sigma_{\text {bp }}+\sigma_{\text {bpres }} \\
& \sigma_{\text {btot }}=4.607 \cdot 10^{3} \cdot \frac{\mathrm{lb}}{\mathrm{in}^{2}}
\end{aligned}
$$

Comparing the total in-service stress to the allowable of $2 S_{m}$ :

$$
\begin{aligned}
& \text { Ratio }=\frac{\sigma_{\text {btot }}}{2 \cdot \mathrm{S}_{\mathrm{mv}}} \\
& \text { Ratio }=0.393 \quad \text { Process valve plug meets Code bolt stress requirements }
\end{aligned}
$$

Because the valve plug net area is small relative to the thread engagement length, the axial stress obviously controls relative to thread stripping. Therefore, thread stripping calculations were not performed.

\section{Check Valve Plug Adequacy for Torque Loading:}

Conservatively assume that the minimum cross-section of the valve plug experiences the full torque. The polar moment of inertia can be approximated from $3\left(A_{n e t} / 3\right) d_{c}{ }^{2}$, where $d_{c}$ is the midwall radius:

$$
\begin{aligned}
& d_{\text {eff }}=1.795 \cdot \text { in } \\
& J_{\text {net }}:=3 \cdot\left(\frac{\mathrm{A}_{\text {vnet }}}{3}\right) \cdot\left(\frac{\frac{\mathrm{d}_{\text {eff }}}{2}+\frac{\mathrm{d}_{\mathbf{i}}}{2}}{2}\right)^{2} \\
& \mathrm{~J}_{\text {net }}=0.451 \cdot \text { in }^{4}
\end{aligned}
$$

\begin{tabular}{|c|l|l|l|}
\hline 0 & & & \\
\hline $4 / 18 / 97$ & & & \\
\hline $74 / 18 / 97$ & & & \\
\hline
\end{tabular}


PROJECT: \begin{tabular}{ll} 
MCO Final Design $\quad$ DOC. NO.: HNF-SD-SNF-DR-003, Rev. 0, Appendix 10 \\
\cline { 2 - 2 }
\end{tabular}

For comparison, check $J$ for section w/o holes:

$$
\begin{aligned}
& J=\frac{\pi}{32}\left(\mathrm{~d}_{\text {eff }}^{4}-\mathrm{d}_{\mathrm{i}}^{4}\right) \\
& \mathrm{J}=0.921 \cdot \mathrm{in}^{4}
\end{aligned}
$$

$J_{\text {net }}$ appears to be overly conservative (ignores centroidal inertia). A more reasonable value can be obtained from Reference 8, p. 752, for a hollow shaft with two holes:

$$
\begin{array}{ll}
d_{h}=0.688 \cdot \text { in } & \text { Hole diameter } \\
d_{\text {heff }}=d_{h} \cdot \sqrt{1.5} & \\
d_{\text {heff }}=0.843 \cdot \text { in } & \text { Two-hole equivalent diameter } \\
\frac{d_{\text {heff }}}{d_{\text {eff }}}=0.469 & \\
\frac{d_{i}}{d_{\text {eff }}}=0.557 &
\end{array}
$$

From Table A-16 of Reference 8, an effective $J$ coefficient, A, was obtained:

$$
\begin{array}{ll}
\mathrm{A}=0.58 & \text { Approximate, use } 0.5 \\
\tau:=\frac{\mathrm{T}_{\text {in }} \cdot \frac{\mathrm{d} \text { eff }}{2}}{0.5 \cdot J} & \\
\tau=1.523 \cdot 10^{3} \cdot \frac{\mathrm{lb}}{\mathrm{in}^{2}} & \quad \text { Pure shear allowable, Sm doubled } \\
\tau \text { allow }=0.6 \cdot 2 \cdot \mathrm{S}_{\mathrm{mv}} & \text { for bolt in-service stress }
\end{array}
$$

Ratio $=0.217 \quad$ Torsional stress relatively small

\begin{tabular}{|c|l|l|l|}
\hline Sud & & & \\
\hline $\cos 4 / 18 / 97$ & & & \\
\hline
\end{tabular}


CLIENT: Duke Engineering Services Hanford, INC

FILE NO: $\quad \mathrm{KH}-8009-8-08$

PROJECT: MCO Final Design

DOC. NO.: HNF-SD-SNF-DR-003, Rev. 0, Appendix 10

Although the torsional stress is small, it is additive to the preload stress and potentially the pressure stress. Combining the valve plug axial and torsional stresses follows below.

$$
\begin{aligned}
& \sigma_{1}:=\frac{\sigma_{\text {btot }}}{2}+\sqrt{\frac{\sigma_{\text {btot }}^{2}}{4}+\tau^{2}} \\
& \sigma_{1}=5.065 \cdot 10^{3} \cdot \frac{\mathrm{lb}}{\mathrm{in}^{2}} \\
& \sigma_{2}=\frac{\sigma_{\text {btot }}}{2}-\sqrt{\frac{\sigma_{\text {btot }}^{2}}{4}+\tau^{2}} \\
& \sigma_{2}=-457.983 \cdot \frac{\mathrm{lb}}{\mathrm{in}^{2}} \\
& P_{\mathrm{m}}=\sigma_{1}-\sigma_{2} \\
& P_{\mathrm{m}}=5.523 \cdot 10^{3} \cdot \frac{1 \mathrm{~b}}{\mathrm{in}^{2}} \\
& \text { Ratio }=\frac{\mathrm{P} \mathrm{m}}{2 \cdot \mathrm{S} \mathrm{mv}} \\
& \text { Ratio }=0.472
\end{aligned}
$$

Thus, the valve plug is adequate for a combined axial stress (preload/pressure) and torsional stress (65 $\mathrm{ft}-1 \mathrm{~b}$ torque). Note from Section 8.4 , a maximum torque of $105 \mathrm{ft}-\mathrm{lbs}$ is recommended, which increases the above stress ratio to 0.74 , indicating the valve plug is adequate for the maximum torque as well.

\section{Rupture Disc Process Plug Torque Stress:}

The hex head of the process plug, which contains the rupture disc, is hollow which introduces the possibility of a torque overstress, which does not exist for the solid hex heads of the standard process valves. The hollow hex head torsional stress evaluation follows below. For analysis simplification, the outside surface was assumed to be round, conservatively using the minimum diameter (flat-to-flat).

\begin{tabular}{|l|c|l|l|l|}
\hline REVISION & 0 & & & \\
\hline PREPARED BY IDATE $\backslash$ SU 4/18/97 & & & \\
\hline CHECKED BY I DATE & OPY 4/18/97 & & & \\
\hline
\end{tabular}


Duke Engineering Services Hanford, INC

FILE NO: $\quad \mathrm{KH}-8009-8-08$

PROJECT: MCO Final Design DOC. NO.: HNF-SD-SNF-DR-003, Rev. 0, Appendix 10

$$
\begin{aligned}
& d_{0}=1.312 \cdot \text { in } \\
& \mathrm{d}_{\mathrm{i}}=1.1 \cdot \mathrm{in} \\
& J:=\frac{\pi}{32} \cdot\left(d_{0}{ }^{4}-d_{i}^{4}\right) \\
& J=0.147 \cdot \text { in }^{4} \\
& \mathrm{~T}_{\max }:=105 \cdot \mathrm{ft} \cdot \mathrm{lb} \quad \text { Maximum torque (Section 8.4) } \\
& \tau:=\frac{T_{\max } \frac{d_{0}}{2}}{J} \\
& \tau=5.617 \cdot 10^{3} \cdot \frac{1 \mathrm{~b}}{\text { in }^{2}} \\
& \tau_{\text {allow }}=2 \cdot 0.6 \cdot \mathrm{S}_{\mathrm{mv}} \\
& \tau_{\text {allow }}=7.027 \cdot 10^{3} \cdot \frac{\mathrm{lb}}{\text { in }^{2}} \\
& \text { Ratio }=\frac{\tau}{\tau \text { allow }} \\
& \text { Ratio }=0.799 \quad \text { Hex head torsional stress OK }
\end{aligned}
$$

\section{Weld Sizing for Rupture Disc Process Valve}

The process valve which contains a rupture disc, requires a two-piece construction connected with a structural weld. This weld potentially carries both the pressure loading and the torsional stress during torquing (removal).

\begin{tabular}{|l|l|l|l|}
\hline 0 & & & \\
\hline $4 / 18 / 97$ & & & \\
\hline $4 / 18 / 97$ & & & \\
\hline
\end{tabular}




\section{P. PARSDNS}

CLIENT: Duke Engineering Services Hanford, INC

FILE NO: $\mathrm{KH}-8009-8-08$

PROJECT: MCO Final Design DOC. NO.: HNF-SD-SNF-DR-003, Rev. 0, Appendix 10

$\begin{array}{ll}d_{w}=2.00 \cdot \text { in } & \text { Structural weld diameter } \\ T_{\text {max }}=105 \cdot f t-l b & \text { Maximum preload torque (above) }\end{array}$

Try a $1 / 8$-in groove weld:

$$
\begin{aligned}
& t_{w}:=0.125 \cdot \text { in } \\
& f_{w}:=\frac{T_{\text {max }}}{\pi \cdot \frac{d_{w}^{2}}{2}} \quad \text { Circumferential force/in @ weld } \\
& f_{w}=200.535 \quad \cdot \frac{\text { in }}{\text { in }} \quad \text { Circumferential shear stress in weld } \\
& \tau_{w}:=\frac{f_{w}}{t_{w}} \quad \text { Weld shear stress } \\
& \tau_{w}=1.604 \cdot 10^{3} \cdot \frac{l b}{\text { in }^{2}} \quad \\
& n:=0.4 \quad \text {. }
\end{aligned}
$$

$\mathrm{n}$ = groove weld quality factor per ASME NG-3352-1 (Surface PT inspection)

$$
\begin{gathered}
\text { ratio : } \frac{{ }^{\tau}{ }_{w}}{0.6 \cdot 2 \cdot S_{m v^{n}}} \quad \text { Stress ratio using } 0.6 \times 2 \times S m v \text { allow. per ASME NB-3227.2 } \\
\text { ratio }=0.571
\end{gathered}
$$

The groove weld also carries the axial pressure loading.

$$
\text { pres }=150 \cdot \frac{\mathrm{lb}}{\mathrm{in}^{2}}
$$

\begin{tabular}{|l|}
\hline REVISION \\
\hline PREPARED BY I DATE \\
\hline CHECKED BY I DATE \\
\hline
\end{tabular}


CLIENT: Duke Engineering Services Hanford, INC

FILE NO: KH-8009-8-08

PROJECT: MCO Final Design DOC. NO.: HNF-SD-SNF-DR-003, Rev. 0, Appendix 10

$$
\begin{aligned}
& \mathrm{d}_{\text {seal }}:=1.37 \cdot \text { in } \\
& \tau_{\mathrm{wp}}=\frac{\text { pres } \cdot \pi \cdot \frac{\mathrm{d}_{\text {seal }}{ }^{2}}{4}}{\pi \cdot \mathrm{d}_{\mathrm{w}} \cdot \mathrm{t}_{\mathrm{w}}} \\
& \tau_{\mathrm{wp}}=281.535 \cdot \frac{\mathrm{bb}}{\mathrm{in}^{2}}
\end{aligned}
$$

Conservatively adding the torsional and axial shear stresses:

$$
\begin{aligned}
& \tau_{\text {tot }}=\tau_{\mathrm{w}}+\tau_{\mathrm{wp}} \\
& \text { ratio }=\frac{\tau_{\text {tot }}}{0.6 \cdot 2 \cdot \mathrm{S}_{\mathrm{mv}} \mathrm{n}} \quad \text { Weld OK for torsion + pressure }
\end{aligned}
$$

\subsection{Rupture Disc Holder}

The rupture disc pressure relief device which may be inserted into both Port 1 and the canister cover, has a specified thread of 3/4" NPSM. The rupture disc holder body is

\begin{tabular}{|c|c|c|c|c|}
\hline REVISION & 0 & & & \\
\hline PREPARED BY / DATE & 730 4/18/97 & & & \\
\hline CHECKED BY / DATE & O//2 4/18/97 & & & \\
\hline
\end{tabular}
evaluated below, following a similar procedure as for the process valve plug. One significant difference is that the rupture disc holder will be torqued at room temperature only and torsion will not be combined with pressure.

\section{Parameter Definitions:}

$$
\begin{array}{ll}
d_{b}:=1.0 \cdot \text { in } & \text { Thread area nominal diameter }(3 / 4-\text { in NPSM) } \\
1_{\text {thread }}:=0.79 \cdot \text { in } & \text { Valve thread engagement length } \\
d_{0}=0.982 \cdot \text { in } \quad \text { Min. pitch diameter, } p .1777 \text { of Reference } 10 \\
d_{i}:=0.75 \cdot \text { in } \quad \text { Inside diameter, threaded region }
\end{array}
$$


CLIENT: Duke Engineering Services Hanford, INC

FILE NO: $\quad \mathrm{KH}-8009-8-08$

PROJECT: \begin{tabular}{ll} 
MCO Final Design DOC. NO.: HNF-SD-SNF-DR-003, Rev. 0, Appendix 10 \\
\hline
\end{tabular}
$A=\frac{\pi}{4} \cdot\left(d_{0}^{2}-d_{i}^{2}\right)$
$\mathrm{A}=0.316 \cdot \mathrm{in}^{2} \quad$ Cross sectional area, threaded region

Body design temperature material allowable, SA-479, 304LN yield strength divided by 3 to correspond to bolt $S_{m}$ value:

$$
\begin{aligned}
& \mathrm{S}_{\mathrm{mv}}=5856 \cdot \frac{\mathrm{lb}}{\mathrm{in}^{2}} \\
& \mathrm{~d}_{\mathrm{s}}=1.158 \cdot \text { in } \quad \text { Seal diameter }
\end{aligned}
$$

\section{Required Holder Body Area:}

$$
\begin{aligned}
& H:=\frac{\pi}{4} \cdot d_{s}^{2} \cdot \text { pres } \quad \text { Pressure load } \\
& H=157.979 \cdot 1 b \\
& H_{p}:=\pi \cdot d_{s} \cdot f_{s p} \quad \text { Minimum preload } \\
& H_{p}=1.091 \cdot 10^{3} \cdot \mathrm{lb} \\
& A_{\text {req }}:=\frac{H+H_{p}}{S_{m v}} \\
& A_{\text {req }}=0.213 \cdot \text { in }^{2} \\
& \text { Ratio }=\frac{A_{\text {req }}}{A^{-}}
\end{aligned}
$$$$
\text { Ratio }=0.676 \quad \text { Body area is adequate for preload }+ \text { pressure }
$$

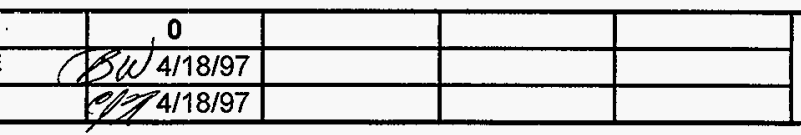




\section{EaASENS}

CLIENT: Duke Engineering Services Hanford, INC

FILE NO: $\mathrm{KH}-8009-8-08$

PROJECT: MCO Final Design DOC. NO.: HNF-SD-SNF-DR-003, Rev. 0, Appendix 10

\section{Check Holder Body Adequacy for In-Service Loads}

1) Thermal Loading

No thermal loading (expansion coefficients are same)

2) Maximum Preload

Torque required to achieve required minimum preload $\left(f_{s p}\right)$ on seal:

$$
\begin{aligned}
& \mathrm{T}_{\text {in }}:=\left(\mathrm{f}_{\mathrm{sp}} \cdot \pi \cdot \mathrm{d}_{\mathrm{s}}\right) \cdot \mathrm{K}_{\text {mean }} \cdot 1.3 \cdot \mathrm{d}_{\mathrm{b}} \\
& \mathrm{T}_{\text {in }}=20.1 \cdot \mathrm{lb} \cdot \mathrm{ft}
\end{aligned}
$$

Maximum preload for $T_{\text {in }}$ torque:

$$
\begin{aligned}
& F_{\text {pmax }}=\frac{\mathrm{T}_{\text {in }}}{\mathrm{K}_{\min ^{\cdot \mathrm{d}_{\mathrm{b}}}}} \\
& \mathrm{F}_{\mathrm{pmax}}=2.193 \cdot 10^{3} \cdot \mathrm{lb} \\
& \sigma_{\mathrm{bp}}=\frac{\mathrm{F} \text { pmax }}{\mathrm{A}} \\
& \sigma_{\mathrm{bp}}=6.948 \cdot 10^{3} \cdot \frac{\mathrm{lb}}{\mathrm{in}^{2}}
\end{aligned}
$$

Body axial stress from pressure loading (conservatively assume body carries entire pressure load):

$$
\begin{aligned}
& \sigma_{\text {bpres }}:=\frac{H}{A} \\
& \sigma_{\text {bpres }}=500.579 \cdot \frac{\mathrm{lb}}{\mathrm{in}^{2}}
\end{aligned}
$$

\begin{tabular}{|c|c|c|c|}
0 & & & \\
\hline
\end{tabular}




\section{PARSDNS}

CLIENT: Duke Engineering Services Hanford, INC

FILE NO: KH-8009-8-08

PROJECT: MCO Final Design

DOC. NO.: HNF-SD-SNF-DR-003, Rev. 0, Appendix 10

Maximum combined in-service stress in holder body:

$$
\begin{aligned}
& \sigma_{\text {btot }}=\sigma_{\text {bp }}-\sigma_{\text {bpre }} \\
& \sigma_{\text {btot }}=7.448 \cdot 10^{3} \cdot \frac{\mathrm{lb}}{\mathrm{in}^{2}}
\end{aligned}
$$

Comparing the total in-service stress to the allowable of $2 \mathrm{~S}_{\mathrm{m}}$ :

$$
\text { Ratio }=\frac{\sigma_{\text {btot }}}{2 \cdot S_{\mathrm{mv}}}
$$

Ratio $=0.636$

Body meets Code bolt stress requirements for the seal seating load + pressure

Check Thread Stripping in Holder Body:

$$
\begin{aligned}
& \mathrm{T}_{\text {max }}=35 \cdot \mathrm{ft} \cdot 1 \quad \text { Maximum recommended torque, Section } 8.4 \\
& F_{\text {pmax }}=\frac{T_{\text {max }}}{K_{\text {min }} \cdot d_{b}} \\
& F_{\text {max }}=3.818 \cdot 10^{3} \cdot \mathrm{lb} \\
& \mathrm{A}_{\text {et }}=1.66 \cdot \frac{\mathrm{in}^{2}}{\text { in }} \quad \text { Approximate external thread stripping area ( } 1 \text { " bolt) }
\end{aligned}
$$

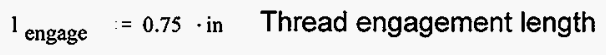

$$
\begin{aligned}
& \tau_{\mathrm{s}}:=\frac{\mathrm{F}_{\text {pmax }}}{\mathrm{A}_{\text {et }}{ }^{-1} \text { engage }} \\
& \tau_{s}=3.067 \cdot 10^{3} \cdot \frac{\mathrm{lb}}{\mathrm{in}^{2}}
\end{aligned}
$$

\begin{tabular}{|l|l|l|l|}
\hline 0 & & & \\
\hline $4 / 18 / 97$ & & & \\
\hline $4 / 18 / 97$ & & & \\
\hline
\end{tabular}




\section{DPARSDNS}

CLIENT: Duke Engineering Services Hanford, INC

FILE NO: $\quad \mathrm{KH}-8009-8-08$

PROJECT: MCO Final Design

DOC. NO.: HNF-SD-SNF-DR-003, Rev. 0, Appendix 10

$$
\text { Ratio }=\frac{\tau \mathrm{s}}{0.6 \cdot 2 \cdot \mathrm{s}_{\mathrm{mv}}}
$$

Ratio $=0.256$

Thread stripping OK

\section{Check Disc Holder Adequacy for Torque Loading:}

Conservatively assuming that the minimum cross-section of the body experiences the full torque, find the allowable torque which will bring the holder body to the allowable stress. Assume that the maximum torque will be applied only at room temperature.

$$
\begin{aligned}
& \mathrm{J}:=\frac{\pi}{32} \cdot\left(\mathrm{d}_{\mathrm{o}}^{4}-\mathrm{d}_{\mathrm{i}}^{4}\right) \\
& \mathrm{J}=0.06 \cdot \mathrm{in}^{4} \\
& \mathrm{~T}_{\max }=50 \cdot \mathrm{ft} \cdot \mathrm{lb} \quad \text { Maximum torque (iteratively obtained) } \\
& \tau=\frac{\mathrm{T} \max \cdot \frac{\mathrm{d}}{2}}{\mathrm{~J}} \\
& \tau=4.891 \cdot 10^{3} \cdot \frac{\mathrm{lb}}{\mathrm{in}^{2}}
\end{aligned}
$$

Combining torsional stress with preload stress:

$$
\begin{aligned}
& F_{\text {pmax }}:=\frac{T_{\text {max }}}{K_{\text {min }}{ }^{d_{b}}} \\
& F_{\text {pmax }}=5.455 \cdot 10^{3} \cdot \mathrm{bb} \\
& \sigma_{b p}:=\frac{F_{p m a}}{A} \\
& \sigma_{b p}=1.728 \cdot 10^{4} \cdot \frac{1 b}{\mathrm{in}^{2}} \\
& \sigma_{b \text { tot }}:=\sigma_{b p}
\end{aligned}
$$


CLIENT: Duke Engineering Services Hanford, INC

FILE NO: $\mathrm{KH}-8009-8-08$

PROJECT: MCO Final Design DOC. NO.: HNF-SD-SNF-DR-003, Rev. 0, Appendix 10

$$
\begin{aligned}
& \sigma_{1}:=\frac{\sigma_{\text {btot }}}{2}+\sqrt{\frac{\sigma_{\text {btot }}^{2}}{4}+\tau^{2}} \\
& \sigma_{1}=1.857 \cdot 10^{4} \cdot \frac{\mathrm{lb}}{\text { in }^{2}} \\
& \sigma_{2}=\frac{\sigma_{\text {btot }}}{2}-\sqrt{\frac{\sigma_{\text {btot }}^{2}}{4}+\tau^{2}} \\
& \sigma_{2}=-1.288 \cdot 10^{3} \cdot \frac{\mathrm{lb}}{\text { in }^{2}} \\
& P_{m}:=\sigma_{1}-\sigma_{2} \\
& P_{m}=1.986 \cdot 10^{4} \cdot \frac{1 b}{\text { in }^{2}} \\
& S_{m v}=10000 \cdot \frac{1 b}{\text { in }^{2}} \quad \text { One third of room temperature yield strength } \\
& \text { Ratio }:=\frac{P_{m}}{2 \cdot S_{m}} \quad
\end{aligned}
$$

Ratio $=0.993 \quad$ Thus, a torque of $50 \mathrm{ft}$-lbs is the maximum allowed.

\section{Check rupture disc holder weld:}

The specified rupture disc holder weld is a circumferential 1/8-in. groove weld at a diameter of $1.75 \mathrm{in}$. The weld size is equal to the rupture disc process valve. Since the maximum allowable torque ( $50 \mathrm{ft}-\mathrm{lbs}$ ) is approximately half of the $105 \mathrm{ft}-\mathrm{lb}$ maximum for the process valve, and since the rupture disc holder will only be torqued at room temperature, the weld is structurally adequate by comparison.

\begin{tabular}{|l|c|l|l|l|}
\hline REVISION & 0 & & & \\
\hline PREPARED BY IDATE & SU/4/18/97 & & & \\
\hline CHECKED BY IDATE & S// $4 / 18 / 97$ & & & \\
\hline
\end{tabular}


CLIENT: Duke Engineering Services Hanford, INC

FILE NO: KH-8009-8-08

PROJECT: MCO Final Design

DOC. NO.: HNF-SD-SNF-DR-003, Rev. 0, Appendix 10

\subsection{Torque Recommendations}

The minimum torque values, $T_{\text {in }}$, required to seat the seals is established above for the cover plate bolts, process valves, and rupture disc holder. It is emphasized that preload torque relationship is conditional on the adequacy of the "nut factors" extracted from Reference 7, assuming a "Never Sieze" lubricant. Reference 7, in turn, emphasizes that nut factors "can only be determined experimentally, and experience shows that we really have to redetermine it for each new application. Even then it is not a single number. Experience shows that for accurate prediction we have to make a number of experiments to determine the mean $\mathrm{K}$, standard deviation, etc. Having done this, however, we can indeed predict the minimum and maximum preload we're going to achieve for a given input torque, at a predictable confidence level."

With the above qualifier in mind, a maximum torque can be estimated as the torque which would cause a component to reach it's Code allowable.

\section{Cover Plate Bolts:}

$$
\text { Minimum torque }=9.6 \mathrm{ft}-\mathrm{lb}\left(\mathrm{T}_{\text {in }} \text { for cover bolts }\right)
$$

From the in-service stress evaluation above, the maximum stress ratio for the cover plate bolts is 0.823 , which correponds to the $T_{\text {in }}$ torque value. The maximum torque permitted, $T_{\max }$, is the torque which results in a Code stress ratio of 1.0. The stresses and allowables associated with this stress margin are as follows:

$$
\begin{array}{ll}
\sigma_{\mathrm{bp}}=8924 \cdot \frac{\mathrm{lb}}{\mathrm{in}^{2}} & \text { Preload torque stress, } 9.6 \mathrm{ft}-\mathrm{lb} \text { torque (above) } \\
\sigma_{\text {bpress }}=1974 \cdot \frac{\mathrm{lb}}{\mathrm{in}^{2}} & \text { Pressure stress (above) } \\
\sigma_{\text {ballow }}:=2 \cdot \mathrm{S}_{\mathrm{mb}} & \text { Allowable in-service stress, 2Sm } \\
\mathrm{T}_{\text {incr }}=\frac{\sigma_{\text {ballow }}-\sigma_{\text {bpress }}}{\sigma_{\mathrm{bp}}} & \text { Allowable torque increase ratio } \\
\mathrm{T}_{\text {incr }}=2.491 & \\
\mathrm{~T}_{\text {max }}=\mathrm{T}_{\text {incr }} \cdot 9.6 \cdot \mathrm{ft} \cdot \mathrm{bb} &
\end{array}
$$




\section{PaAsRns}

CLIENT: Duke Engineering Services Hanford, INC

FILE NO: KH-8009-8-08

PROJECT: MCO Final Design

DOC. NO.: HNF-SD-SNF-DR-003, Rev. 0, Appendix 10

$$
\mathrm{T}_{\max }=23.91 \cdot \mathrm{lb} \cdot \mathrm{ft}
$$

Thus, the maximum torque range for the cover plate bolts is 10 to $24 \mathrm{ft}-\mathrm{lb}$. To allow some margin on both seal load and bolt overstress, a torque of $17 \pm 5 \mathrm{ft}-\mathrm{lb}$ is recommended. As indicated above, a testing/calibration program is recommended to finalize the bolt torque range.

\section{Process Valves:}

Following the same procedure as above for the process valve torquing, yields the following:

$$
\sigma_{b p}=4097 \cdot \frac{\mathrm{lb}}{\mathrm{in}^{2}} \quad \text { Preload stress for } \mathrm{T}_{\text {in }}=65 \mathrm{ft}-\mathrm{lb} \text { torque }
$$

Ignoring small contribution from torsional stress as indicated above):

$$
\begin{array}{ll}
\sigma_{\text {bpress }}=509.8 \cdot \frac{\mathrm{b}}{\mathrm{in}^{2}} & \text { Pressure stress } \\
\sigma_{\text {ballow }}:=2 \cdot \mathrm{S} \mathrm{mv} & \text { Allowable in-service stress } \\
\mathrm{T}_{\text {incr }}=\frac{\sigma_{\text {ballow }}-\sigma_{\text {bpress }}}{\sigma_{\mathrm{bp}}} & \\
\mathrm{T}_{\text {incr }}=2.734 & \\
\mathrm{~T}_{\text {in }}=65.09 \cdot \mathrm{lb} \cdot \mathrm{ft} & \mathrm{T}_{\max }=\mathrm{T}_{\text {incr }} \cdot \mathrm{T}_{\text {in }} \\
\mathrm{T}_{\max }=177.972 \cdot \mathrm{lb} \cdot \mathrm{ft}
\end{array}
$$

Thus, a torque of $178 \mathrm{ft}$-lb will cause the axial stress to reach the allowable stress of $2 \mathrm{~S}_{\mathrm{m}}$. However, as shown above, the rupture disc valve hex head will be overstressed by a torque exceeding $130 \mathrm{ft}-\mathrm{lbs}$. An appropriate torque value is one which assures an adequate seal preload, but low enough to prevent damage to the hollow hex head and not damage the seal. A reasonable recommended torque is $90 \pm 15 \mathrm{ft}-\mathrm{lb}$ ). The need for a

\begin{tabular}{|c|c|c|c|}
\hline 0 & & & \\
\hline $5 \% / 4 / 18 / 97$ & & & \\
\hline $1 / 24 / 18 / 97$ & & & \\
\hline
\end{tabular}




\section{PARSロNS}

CLIENT: Duke Engineering Services Hanford, INC

FILE NO: $\mathrm{KH}-8009-8-08$

PROJECT: MCO Final Design

DOC. NO.: HNF-SD-SNF-DR-003, Rev. 0, Appendix 10

testing/calibration program, discussed above, is especially important for the process valves because the geometry is significantly different from a solid cylinder.

\section{Rupture Disc Holder:}

As shown in Section 8.3, a minimum torque of $20 \mathrm{ft}-\mathrm{lb}$ is required to preload the seal and a torque in excess of $50 \mathrm{ft}$-lbs will overstress the disc holder minimum cross section. To assure an adequate preload and to avoid damaging the seal and/or the holder, a torque magnitude of $30 \pm 5 \mathrm{ft}-\mathrm{lbs}$ ) is recommended.

\subsection{Process Filter Attachment Welds}

Drawings $\mathrm{H}-2-828041$ and $\mathrm{H}-2-828049$ specifies 1/8-in. fillet welds on both sides of the filter (11.5 in. on one side and $16.38 \mathrm{in.}$ on the other side).

$$
\begin{aligned}
& \mathrm{L}_{\text {sw }}=11.5 \cdot \text { in } \\
& \mathrm{L}_{\text {lw }}=16.38 \cdot \text { in }
\end{aligned}
$$

The filter is relatively flat, allowing the simplifying assumption that the filter inertia loading is in the plane of the welds. Assuming the center of gravity is midway between the welded edges, the worst drop direction is parallel to the weld axes. Moment equilibrium requires that the force on each weld be equal.

$$
\mathrm{W}_{\mathrm{f}}=50 \cdot \mathrm{lb}
$$

Maximum filter weight, Reference 1

$$
\begin{aligned}
& F_{1}=\frac{W_{f^{101}}}{2} \\
& F_{1}=2.525 \cdot 10^{3} \cdot \mathrm{lb} \\
& \sigma_{w}:=\frac{F_{1}}{L_{\mathrm{sw}} \cdot 0.125 \cdot \text { in } \cdot 0.707} \\
& \sigma_{w}=2.484 \cdot 10^{3} \cdot \frac{\mathrm{lb}}{\mathrm{in}^{2}}
\end{aligned}
$$

\begin{tabular}{|c|l|l|l|}
\hline 0 & & & \\
\hline (3) $4 / 18 / 97$ & & & \\
\hline
\end{tabular}


CLIENT: Duke Engineering Services Hanford, INC

FILE NO: KH-8009-8-08

PROJECT: MCO Final Design DOC.NO.: HNF-SD-SNF-DR-003, Rev. 0, Appendix 10

The inertia force, being in the same plane as the welds, results in a shear stress with a throat stress allowable of $0.6 \mathrm{~S}_{\mathrm{m}}$.

$$
\begin{array}{ll}
n=0.35 & \text { Weld efficiency factor, surface visual inspection } \\
\mathrm{S}_{\mathrm{m}}=13500 \cdot \frac{\mathrm{b}}{\mathrm{in}^{2}} & 304 \mathrm{~L} @ \text { design temperature } \\
\text { Ratio }=\frac{\sigma_{\mathrm{w}}}{0.6 \cdot \mathrm{S}_{\mathrm{m}} \cdot \mathrm{n}} &
\end{array}
$$$$
\text { Ratio }=0.876 \quad \text { Filter attachment weids OK }
$$

Filter attachment weids OK 
This document was too large to scan as a whole document, therefore it required breaking into smaller sections.

Document number: $S A-S N F-D R-003$

Section $\underline{3}$ of 3

Title:Ohueti-Canister Qvergack Design) Report

Date: $6 / 9.97$ Revision: A000

Originator: Smith $K \varepsilon$

co: hos st

Recipient:

Co:

References: EDT -620/06 


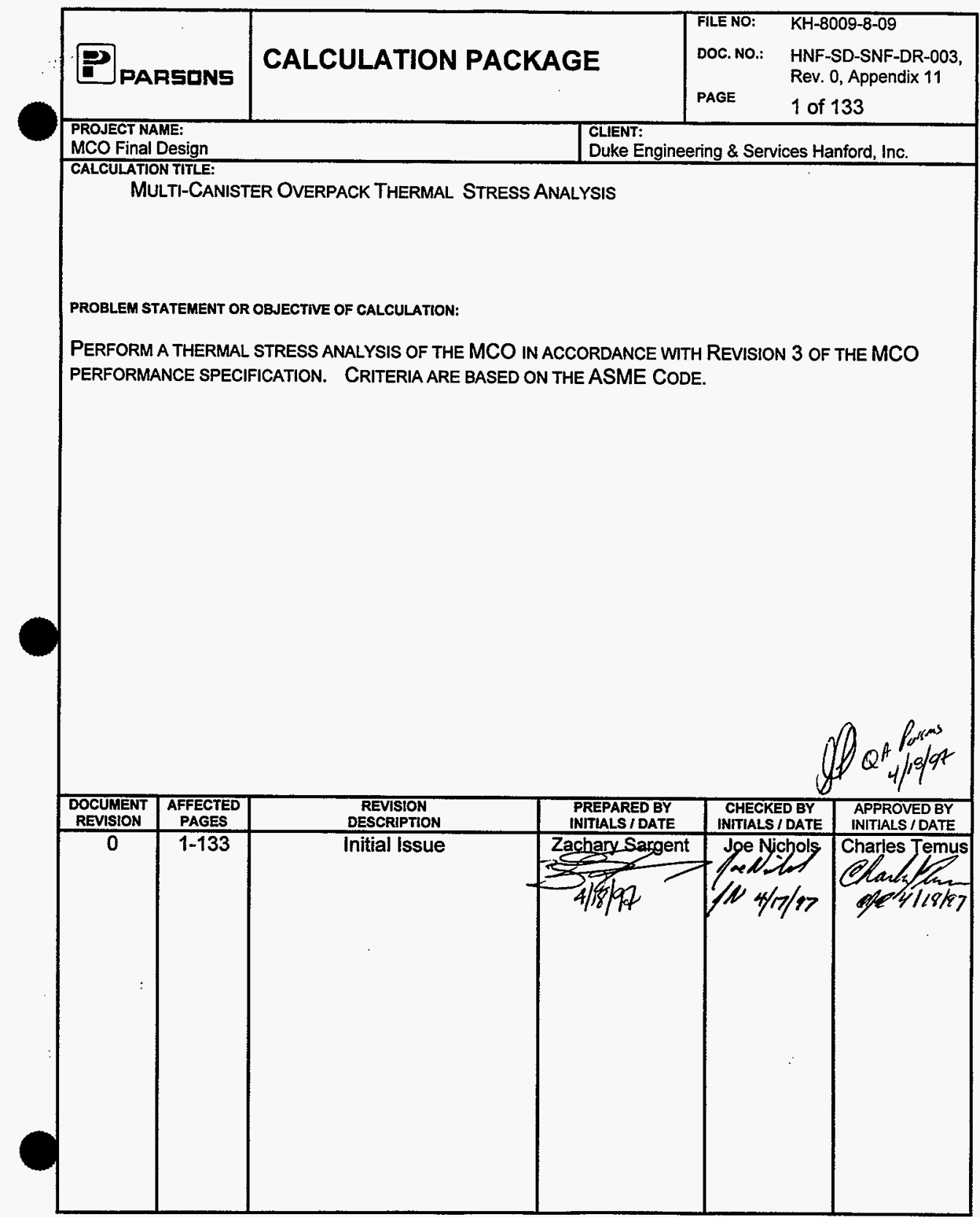


CLIENT: DUKE ENGINEERING \& SERVICES HANFORD, INC.

FILE NO: $\quad \mathrm{KH}-8009-8-09$

PROJECT: MCO Final Design

CONTENTS

LIST OF TABLES

LIST OF FIGURES

1. INTRODUCTION

2. REFERENCES

3. ASSUMPTIONS

4. MATERIAL PROPERTIES

5. ACCEPTANCE CRITERIA

6. SHELL DESIGN

6.1 Internal Pressure

6.2 External Pressure

7. STRESS ANALYSIS

7.1 Computer Model

7.2 Load Cases

7.3 Results 
CLIENT: DUKE ENGINEERING \& SERVICES HANFORD, INC.

FILE NO: $\quad \mathrm{KH}-8009-8-09$

PROJECT: MCO Final Design DOC. NO.: HNF-SD-SNF-DR-003, Rev. 0, Appendix 11

\section{LIST OF TABLES}

Page

TABLE 1: ASME CODE MATERIAL PROPERTIES FOR TYPE 304L STAINLESS STEEL

TABLE 2: ASME CODE MATERIAL PROPERTIES FOR TYPE 304 STAINLESS STEEL

TABLE 3: ALLOWABLE LEVEL A STRESS INTENSITY LIMITS FOR TYPE 304L

TABLE 4: ALLOWABLE LEVEL A STRESS INTENSITY LIMITS FOR TYPE 304

TABLE 5: ANSYS MODEL STRESS REPORT SECTIONS

TABLE 6: SUMMARY OF MAXIMUM STRESS INTENSITIES FOR LOAD CASE 1

TABLE 7: SUMMARY OF MAXIMUM STRESS INTENSITIES FOR LOAD CASE 2

TABLE 8: SUMMARY OF MAXIMUM STRESS INTENSITIES FOR LOAD CASE 3

TABLE 9: SUMMARY OF MAXIMUM STRESS INTENSITIES FOR LOAD CASE 4

TABLE 10: SUMMARY OF MAXIMUM STRESS INTENSITIES FOR LOAD CASE 5

TABLE 11: SUMMARY OF MAXIMUM STRESS INTENSITIES FOR LOAD CASE 6

\section{LIST OF FIGURES}

Page

FIGURE 1: AXISYMMETRIC MODEL WITH BOUNDARY CONDITIONS, UPPER SECTION 12

FIGURE 2: AXISYMMETRIC MODEL WITH COUPLED NODES

FIGURE 3: AXISYMMETRIC MODEL WITH BOUNDARY CONDITIONS, LOWER SECTION 14

FIGURE 4: LOAD CASE 1 - UPPER SECTION STRESS INTENSITIES 22

FIGURE 5: LOAD CASE 1 -- LOWER SECTION STRESS INTENSITIES

FIGURE 6: LOAD CASE 2 - UPPER SECTION STRESS INTENSITIES . 24

FIGURE 7: LOAD CASE 3 - UPPER SECTION STRESS INTENSITIES

\begin{tabular}{|l|c|l|l|l|}
\hline REVISION & O & & & \\
\hline PREPARED BY I DATE $\quad$ G (T) 4/17/97 & & & \\
\hline CHECKED BY I DATE & IN 4/17/97 & & & \\
\hline
\end{tabular}


CLIENT: DUKE ENGINEERING \& SERVICES HANFORD, INC.

FILE NO: $\quad \mathrm{KH}-8009-8-09$

PROJECT: MCO Final Design DOC. NO.: HNF-SD-SNF-DR-003, Rev. 0, Appendix 11

FIGURE 8: LOAD CASE 3 - LOWER SECTION STRESS INTENSITIES

FIGURE 9: LOAD CASE 4 - UPPER SECTION STRESS INTENSITIES

FIGURE 10: LOAD CASE 4 - LOWER SECTION STRESS INTENSITIES

FIGURE 11: LOAD CASE 5 - UPPER AND LOWER MCO TEMPERATURE DISTRIBUTION

FIGURE 12: LOAD CASE 5 - UPPER SECTION STRESS INTENSITIES

FIGURE 13: LOAD CASE 5 - LOWER SECTION STRESS INTENSITIES

FIGURE 14: LOAD CASE 6 - UPPER SECTION STRESS INTENSITIES @ HEAT-UP

FIGURE 15: LOAD CASE 6 - LOWER SECTION STRESS INTENSITIES @ HEAT-UP

FIGURE 16: LOAD CASE 6 - UPPER SECTION STRESS INTENSITIES @ COOL-DOWN

FIGURE 17: LOAD CASE 6 - LOWER SECTION STRESS INTENSITIES @ COOL-DOWN

\section{APPENDICES}

APPENDIX A: 
CLIENT: DUKE ENGINEERING \& SERVICES HANFORD, INC. FILE NO: KH-8009-8-09

PROJECT: MCO Final Design DOC. NO.: HNF-SD-SNF-DR-003, Rev. 0, Appendix 11

\section{INTRODUCTION}

The Multi-Canister Overpack (MCO) assembly is a single purpose Spent Nuclear Fuel (SNF) that is capable of maintaining subcriticality at all times and maintain SNF containment and confinement after being closed and sealed. The MCO assembly consists of a shell, a shield plug, a locking ring and jacking screws.

This calculation documents the evaluation of the MCO shell under different Process Operating Conditions. These evaluations are as follows:

1. $\mathrm{MCO}$ at $75^{\circ} \mathrm{C}\left(167^{\circ} \mathrm{F}\right)$ with full internal vacuum and $25 \mathrm{psig}$ external pressure.

2. $\mathrm{MCO}$ at $375^{\circ} \mathrm{C}\left(707^{\circ} \mathrm{F}\right)$ with full internal vacuum and 0 psig external pressure.

3. $\mathrm{MCO}$ at $375^{\circ} \mathrm{C}\left(707^{\circ} \mathrm{F}\right)$ with $150 \mathrm{psig}$ internal pressure and $\mathrm{O}$ psig external pressure.

4. Lifting of the $\mathrm{MCO}$ at $132^{\circ} \mathrm{C}\left(270^{\circ} \mathrm{F}\right)$ and $150 \mathrm{psig}$.

5. Thermal gradient of a maximum of $100^{\circ} \mathrm{C}\left(180^{\circ} \mathrm{F}\right)$ between the outside of the $\mathrm{MCO}$ shell and the center of the MCO shield plug.

6. Heat-up / Cool Down thermal transient.

The evaluations are performed based on the criteria of the ASME Code. A combination of hand calculations and ANSYSO analysis is used.

\section{REFERENCES}

1. "Performance Specification for the Spent Nuclear Fuel Multi-Canister Overpack," Specification HNF-S-0426, Revision 3, February 1997.

2. ASME Boiler and Pressure Vessel Code, Section II - Materials, Part D - Properties, 1995 Edition with 1995 Addenda.

3. ASME Boiler and Pressure Vessel Code, Section III - Division I, Subsection NB, 1995 Edition with 1995 Addenda.

4. Swanson Analysis System, Inc., ANSYS@ Engineering Analysis System User's Manual, Volumes I, II and III, Version 5.0A, 23 December 1992. 
CLIENT: DUKE ENGINEERING \& SERVICES HANFORD, INC.

FILE NO: $\quad \mathrm{KH}-8009-8-09$

PROJECT: MCO Final Design

DOC. NO.: HNF-SD-SNF-DR-003, Rev. 0, Appendix 11

5. Duke Éngineering Services Hanford, Specifications Drawings, Drawing H-2-828041, Revision C.

\section{ASSUMPTIONS}

1. Pressure is applied uniformly

2. Others as noted

\section{MATERIAL PROPERTIES}

The MCO assembly is fabricated from Type 304L stainless steel, except for the jacking screws which are fabricated ASTM A193 Grade B8M. The MCO shell is fabricated out of Type 304L stainless steel with minimum tensile and yield strengths of Type 304 stainless steel. For this analysis, values for material properties are taken from Section II, Part D of the Code (See [2]) and are listed in Table 1.

For the shield plug, locking ring, lifting cap and canister collar, Type $304 \mathrm{~L}$ stainless steel may be replaced with Type $304 \mathrm{~N}$ or Type $304 \mathrm{LN}$, at the discretion of the designer. The effects of thermal conductivity, thermal expansion, minimum yield strength and minimum tensile strength have been evaluated and it is the conclusion of the preparer that the use of these stainless steels does not, in any way, alter the contents of this calculation.

\section{ACCEPTANCE CRITERIA}

This calculation considers thermal and pressure loads. The allowable stress intensities are specified by NB-3220 of the ASME Code [3]. For normal condition loading, the MCO is analyzed according to Level $A$ stress intensity limits, as listed in Table 2 below. 
CLIENT: DUKE ENGINEERING \& SERVICES HANFORD, INC.

FILE NO: $\quad \mathrm{KH}-8009-8-09$

PROJECT: MCO Final Design DOC. NO.: HNF-SD-SNF-DR-003, Rev. 0, Appendix 11

Table 1: ASME Code Material Properties for Type 304L Stainless Steel

\begin{tabular}{|c|c|c|c|c|c|c|c|}
\hline \multicolumn{2}{|c|}{ Temperature } & \multirow{2}{*}{$\begin{array}{c}E^{\prime} \\
\left(\times 10^{6} \mathrm{psi}\right)\end{array}$} & \multirow{2}{*}{$\begin{array}{c}\alpha^{2} \\
\text { (in. } / \mathrm{in} . f^{\circ} \mathrm{F} \text { ) }\end{array}$} & \multirow{2}{*}{$\begin{array}{c}\beta^{3} \\
\left(B t u / H r-i n .-^{\circ} F\right) \\
\end{array}$} & \multirow{2}{*}{$\begin{array}{c}S_{y}{ }^{4} \\
(\mathrm{ksi}) \\
\end{array}$} & \multirow{2}{*}{$\begin{array}{l}S_{m}{ }^{5} \\
(k s i)\end{array}$} & \multirow{2}{*}{$\begin{array}{l}\mathrm{S}_{\mathrm{u}}{ }^{6} \\
(\mathrm{ksi})\end{array}$} \\
\hline${ }^{\circ} \mathrm{F}$ & ${ }^{\circ} \mathrm{C}$ & & & & & & \\
\hline-20 & -29 & & - & - & 25.0 & 16.7 & 70.0 \\
\hline 70 & 21 & 28.3 & - & 0.716 & 25.0 & 16.7 & 70.0 \\
\hline 100 & 38 & $28.1^{7}$ & 8.55 & 0.725 & 25.0 & 16.7 & 70.0 \\
\hline 200 & 93 & 27.6 & 8.79 & 0.775 & 21.3 & 16.7 & 66.2 \\
\hline 270 & 132 & 27.2 & 8.94 & 0.807 & 19.8 & 16.7 & 62.5 \\
\hline 300 & 149 & 27.0 & 9.00 & 0.817 & 19.1 & 16.7 & 60.9 \\
\hline 392 & 200 & 26.5 & 9.18 & 0.863 & 17.6 & 15.9 & 58.7 \\
\hline 400 & 204 & 26.5 & 9.19 & 0.867 & 17.5 & 15.8 & 58.5 \\
\hline 500 & 260 & 25.8 & 9.37 & 0.908 & 16.3 & 14.8 & 57.8 \\
\hline 600 & 316 & 25.3 & 9.53 & 0.942 & 15.5 & 14.0 & 57.0 \\
\hline 700 & 371 & 24.8 & 9.69 & 0.983 & 14.9 & 13.5 & 56.2 \\
\hline 707 & 375 & 24.8 & 9.70 & 0.986 & 14.9 & 13.5 & 56.2 \\
\hline 800 & 427 & 24.1 & 9.82 & 1.02 & 14.4 & 13.0 & 55.5 \\
\hline
\end{tabular}

Table TM-1, Material Group G, P. 614

${ }^{2}$ Table TE-1, P.590-591

${ }^{3}$ Table TCD, P. 606

${ }^{4}$ Tabie Y-1, P. 524

${ }^{5}$ Table 2A, P. 322

${ }^{6}$ Table U, P. 441

${ }^{7}$ Underlined values determined by linear interpolation, all others taken from ASME Code, Section II, Part D. 
CLIENT: DUKE ENGINEERING \& SERVICES HANFORD, INC. FILE NO: KH-8009-8-09

PROJECT: MCO Final Design DOC. NO.: HNF-SD-SNF-DR-003, Rev. 0, Appendix 11

Table 2: ASME Code Material Properties for Type 304 Stainless Steel

\begin{tabular}{|c|c|c|c|c|c|c|c|}
\hline \multicolumn{2}{|c|}{ Temperature } & \multirow{2}{*}{$\begin{array}{c}E^{8} \\
\left(\times 10^{6} \text { psi }\right) \\
\end{array}$} & \multirow{2}{*}{$\begin{array}{c}\alpha^{9} \\
\text { (in./in./解) }\end{array}$} & \multirow{2}{*}{$\begin{array}{c}\beta^{10} \\
\left(B t u / H r-j n--^{\circ} F\right)\end{array}$} & \multirow{2}{*}{$\begin{array}{l}\mathrm{S}_{\mathrm{y}}^{11} \\
\text { (ksi) }\end{array}$} & \multirow{2}{*}{$\begin{array}{l}\mathrm{S}_{\mathrm{m}}{ }^{12} \\
\text { (ksi) }\end{array}$} & \multirow{2}{*}{$\begin{array}{l}\mathrm{S}_{\mathrm{u}}{ }^{13} \\
(\mathrm{ksi})\end{array}$} \\
\hline${ }^{\circ} \mathrm{F}$ & ${ }^{\circ} \mathrm{C}$ & & & & & & \\
\hline-20 & -29 & & - & - & 30.0 & 20.0 & 75.0 \\
\hline 70 & 21 & 28.3 & - & 0.716 & 30.0 & 20.0 & 75.0 \\
\hline 100 & 38 & $28.1^{14}$ & 8.55 & 0.725 & 30.0 & 20.0 & 75.0 \\
\hline 200 & 93 & 27.6 & 8.79 & 0.775 & 25.1 & 20.0 & 71.0 \\
\hline 270 & 132 & 27.2 & 8.94 & 0.807 & 23.3 & 20.0 & 67.5 \\
\hline 300 & 149 & 27.0 & 9.00 & 0.817 & 22.5 & 20.0 & 66.0 \\
\hline 392 & 200 & 26.5 & 9.18 & 0.863 & 20.9 & 18.8 & 64.5 \\
\hline 400 & 204 & 26.5 & 9.19 & 0.867 & 20.8 & 18.7 & 64.4 \\
\hline 500 & 260 & 25.8 & 9.37 & 0.908 & 19.4 & 17.5 & 63.5 \\
\hline 600 & 316 & 25.3 & 9.53 & 0.942 & 18.3 & 16.4 & 63.5 \\
\hline 700 & 371 & 24.8 & 9.69 & 0.983 & 17.7 & 16.0 & 63.5 \\
\hline 707 & 375 & 24.8 & 9.70 & 0.986 & 17.6 & 16.0 & 63.5 \\
\hline 800 & 427 & 24.1 & 9.82 & 1.02 & 16.9 & 15.2 & 62.7 \\
\hline
\end{tabular}

Table 3: Allowable Level A Stress Intensity Limits for Type 304L

\begin{tabular}{|c|c|c|c|c|}
\cline { 2 - 5 } \multicolumn{1}{c|}{} & \multicolumn{4}{c|}{ Allowable Stress Intensity Limits (ksi) } \\
\hline Stress Intensity & Formula & $132^{\circ} \mathrm{C}\left(270^{\circ} \mathrm{F}\right)$ & $75^{\circ} \mathrm{C}\left(167^{\circ} \mathrm{F}\right)$ & $375^{\circ} \mathrm{C}\left(707^{\circ} \mathrm{F}\right)$ \\
\hline $\mathrm{P}_{M}$ & $1.0 \mathrm{~S}_{\mathrm{M}}$ & 16.7 & 16.7 & 13.5 \\
\hline $\mathrm{P}_{\mathrm{L}}$ & $1.5 \mathrm{~S}_{\mathrm{M}}$ & 25.1 & 25.1 & 20.3 \\
\hline $\mathrm{P}_{\mathrm{L}}+\mathrm{P}_{\mathrm{B}}$ & $1.5 \mathrm{~S}_{\mathrm{M}}$ & 25.1 & 25.1 & 20.3 \\
\hline $\mathrm{P}_{\mathrm{L}}+\mathrm{P}_{\mathrm{B}}+\mathrm{Q}$ & $3.0 \mathrm{~S}_{\mathrm{M}}$ & 50.1 & 50.1 & 40.5 \\
\hline $\mathrm{P}_{\mathrm{M}}+\mathrm{P}_{\mathrm{B}}+\mathrm{Q}+\mathrm{F}$ & \multicolumn{3}{c|}{$\mathrm{N} / \mathrm{A}^{15}$} \\
\hline
\end{tabular}

${ }^{8}$ Table TM-1, Material Group G, P. 614

9 Table TE-1, P. 590-591

${ }^{10}$ Table TCD, P. 606

11 Table Y-1, P. 530-531

${ }^{12}$ Table 2A, P. 326

${ }^{13}$ Table U, P. 441

${ }^{14}$ Underlined values determined by linear interpolation, all others taken from ASME Code, Section II, Part D.

${ }^{15}$ Not applicable because fatigue is not being considered.

REVISION

PREPARED BY IDATE

CHECKED BYIDATE

PAGE 8

OF 133 
CLIENT: DUKE ENGINEERING \& SERVICES HANFORD, INC.

FILE NO: KH-8009-8-09

PROJECT: MCO Final Design

DOC. NO.: HNF-SD-SNF-DR-003, Rev. 0, Appendix 11

Table 4: Allowable Level A Stress Intensity Limits for Type 304

\begin{tabular}{|c|c|c|c|c||}
\cline { 2 - 5 } \multicolumn{1}{c|}{} & \multicolumn{4}{c||}{ Allowable Stress Intensity Limits (ksi) } \\
\hline Stress Intensity & Formula & $132^{\circ} \mathrm{C}\left(270^{\circ} \mathrm{F}\right)$ & $75^{\circ} \mathrm{C}\left(167^{\circ} \mathrm{F}\right)$ & $375^{\circ} \mathrm{C}\left(707^{\circ} \mathrm{F}\right)$ \\
\hline $\mathrm{P}_{\mathrm{M}}$ & $1.0 \mathrm{~S}_{\mathrm{M}}$ & 20.0 & 20.0 & 16.0 \\
\hline $\mathrm{P}_{\mathrm{L}}$ & $1.5 \mathrm{~S}_{\mathrm{M}}$ & 30.0 & 30.0 & 24.0 \\
\hline $\mathrm{P}_{\mathrm{L}}+\mathrm{P}_{\mathrm{B}}$ & $1.5 \mathrm{~S}_{\mathrm{M}}$ & 30.0 & 30.0 & 24.0 \\
\hline $\mathrm{P}_{\mathrm{L}}+\mathrm{P}_{\mathrm{B}}+\mathrm{Q}$ & $3.0 \mathrm{~S}_{\mathrm{M}}$ & 60.0 & 60.0 & 48.0 \\
\hline $\mathrm{P}_{\mathrm{M}}+\mathrm{P}_{\mathrm{B}}+\mathrm{Q}+\mathrm{F}$ & \multicolumn{3}{c}{$\mathrm{N} / \mathrm{A}^{16}$} \\
\hline
\end{tabular}

\section{SHELL DESIGN}

The MCO shell and bottom plate are analyzed for internal pressure using classical methods. The allowable external pressure for the shell is calculated per the rules of Paragraph NB-3133.2 Reference 3. The design internal pressure of the MCO is 150 psi. There is also a pressure on the bottom of the MCO due to the weight of the fuel. From Appendix $A$ of [1], the weight of the contents, $W_{F}$, is approximately $16,000 \mathrm{lbs}$.

Given the inside radius of the MCO shell $R=11.50$ inches, the area of the bottom plate is:

$$
\mathrm{A}_{\mathrm{BP}}=\pi\left(R^{2}\right)=415.48 \mathrm{in}^{2}
$$

Therefore, the pressure from the fuel on the bottom plate, $P_{F}$, is

$$
\mathrm{P}_{\mathrm{F}}=\frac{W_{F}}{A_{B P}}=38.51 \mathrm{psi} \text { or } 39.00 \mathrm{psi}
$$

The fuel is conservatively assumed to act as a fluid, resulting in lateral pressure against the shell walls. Therefore, the total internal pressure is $150+39=189 \mathrm{psi}$.

${ }^{16}$ Not applicable because fatigue is not being considered. 
CLIENT: DUKE ENGINEERING \& SERVICES HANFORD, INC.

FILE NO: $\quad \mathrm{KH}-8009-8-09$

PROJECT: MCO Final Design

DOC. NO.: HNF-SD-SNF-DR-003, Rev. 0, Appendix 11

\subsection{Internal Pressure}

The inside diameter of the MCO shell is 23.00 inches and its outer diameter is 24.00 inches.

The wall thickness is therefore $0.5 \mathrm{inch}$. The stress through the shell due to the pressure load is then

$$
\sigma_{P}=\frac{p R}{t}
$$

where

$$
\begin{aligned}
& p=\text { internal pressure }=189 \mathrm{psig} \\
& R=\text { Mean Radius }=(24.00+23.00) / 4=11.75 \mathrm{in} . \\
& T=\text { thickness of } M C O \text { shell }=0.5 \mathrm{in} .
\end{aligned}
$$

Therefore

$$
\sigma_{P}=\frac{(189)(11.75)}{0.50}=4442 \mathrm{psi}
$$

\subsection{External Pressure}

In Process Operating Condition 1, the MCO is subjected to a full internal vacuum with a 25 psig external pressure; equivalent to external pressures of $14.7 \mathrm{psi}+25 \mathrm{psi}$ or $40 \mathrm{psi}$ at $75^{\circ} \mathrm{C}\left(167^{\circ} \mathrm{F}\right)$.

Given the following parameters:

$$
\begin{aligned}
& T=\text { Shell thickness }=0.50 \text { inches } \\
& D_{0}=\text { Shell outside diameter }=24.00 \text { inches } \\
& L=\text { Shell unsupported length }=143.55 \text { inches }(139.76+1 / 3(0.88)+1 / 3(10.5))[6] \\
& D_{0} / T=48.0 \\
& L / D_{0}=5.98
\end{aligned}
$$

\begin{tabular}{|c|l|l|l|}
\hline 0 & & & \\
\hline $2654 / 17 / 97$ & & & \\
\hline $2 \mu 4 / 17 / 97$ & & & \\
\hline
\end{tabular}


CLIENT: DUKE ENGINEERING \& SERVICES HANFORD, INC.

FILE NO: $\quad \mathrm{KH}-8009-8-09$

PROJECT: MCO Final Design

DOC. NO.: HNF-SD-SNF-DR-003, Rev. 0, Appendix 11

$$
\begin{aligned}
& A=\text { Geometric factor, from Figure } G \text { of }[2]=0.0006 \\
& B=\text { Stress factor, from Figure HA-1 of }[2]=6,500 \text { psi } \\
& P_{a}=\text { Allowed external pressure } \\
& P_{a}=\frac{4 B}{3\left(\frac{D_{o}}{T}\right)}=181 \mathrm{psi}
\end{aligned}
$$

This value is greater than the $40 \mathrm{psi}$ maximum external pressure, therefore the cylindrical portion of the shell is adequate for external pressure.

\section{STRESS ANALYSIS}

A stress analysis of the MCO assembly is performed using the computer analysis program ANSYS, Reference 5. For normal conditions six load cases are evaluated as described in Section 7.2.

\subsection{Computer Model}

The ANSYS model is built using two-dimensional axisymmetric elements. To model the threads between the shell and locking ring, coincident nodes are coupled. Coupled nodes are also used to model the threads between the locking ring and the jacking screw. Symmetry boundary conditions are applied to all nodes along the centerline.

The axisymmetric model used in this analysis is shown in Figures 1,2 and 3.

\begin{tabular}{|l|c|l|l|l|c|}
\hline REVISION & 0 & & & & PAGE 11 \\
OF 133 \\
\cline { 1 - 4 } CHECKED BY I DATE & z6/ 4/17/97 & & & & \\
\hline
\end{tabular}


CLIENT: DUKE ENGINEERING \& SERVICES HANFORD, INC.

FILE NO: $\quad \mathrm{KH}-8009-8-09$

PROJECT: MCO Final Design DOC. NO.: HNF-SD-SNF-DR-003, Rev. 0, Appendix 11

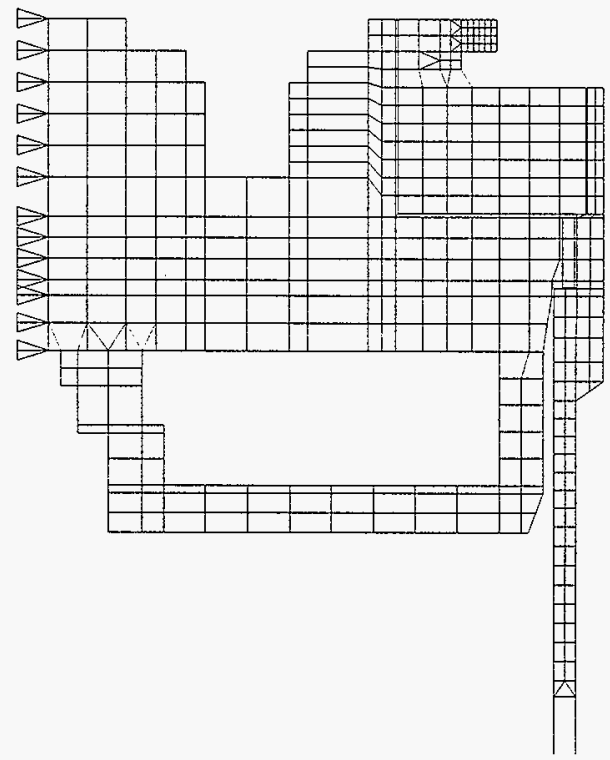

Figure 1: Axisymmetric Model with Boundary Conditions, Upper Section

\begin{tabular}{|c|c|c|c|}
\hline 0 & & & \\
\hline 7/55 $4 / 17 / 97$ & & & \\
\hline $1 / 4 / 17 / 97$ & & & \\
\hline
\end{tabular}


CLIENT: $\quad$ DUKE ENGINEERING \& SERVICES HANFORD, INC. FILE NO: KH-8009-8-09

PROJECT: MCO Final Design $\quad$ DOC. NO.: HNF-SD-SNF-DR-003, Rev. 0, Appendix 11

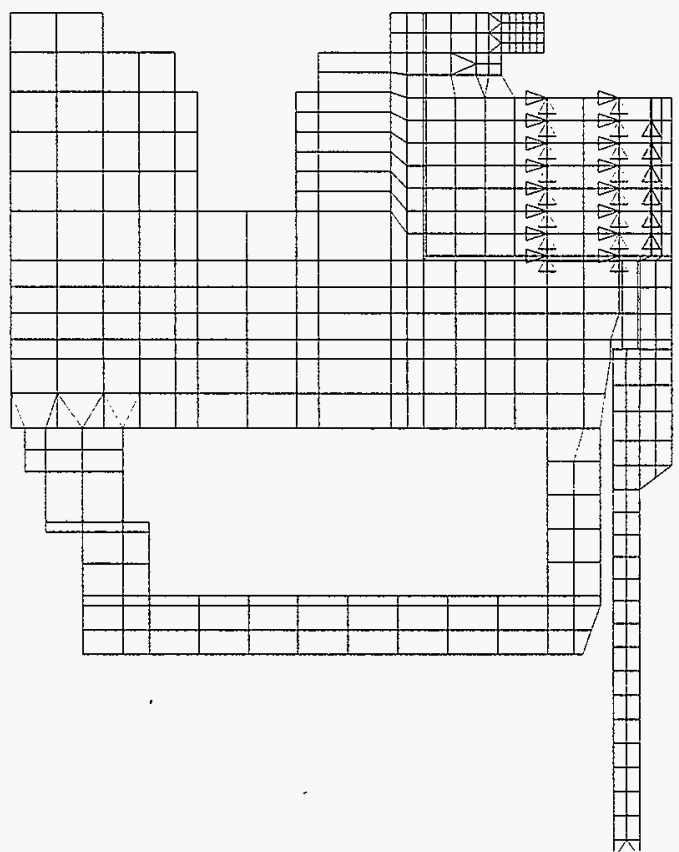

Figure 2: Axisymmetric Model with Coupled Nodes

\begin{tabular}{|c|l|l|l|}
\hline 0 & & & \\
\hline $7654 / 17 / 97$ & & & \\
\hline $2 / 4 / 17 / 97$ & & & \\
\hline
\end{tabular}


CLIENT: $\quad$ DUKE ENGINEERING \& SERVICES HANFORD, INC.

FILE NO: KH-8009-8-09

PROJECT: MCO Final Design DOC. NO.: HNF-SD-SNF-DR-003, Rev. 0, Appendix 11

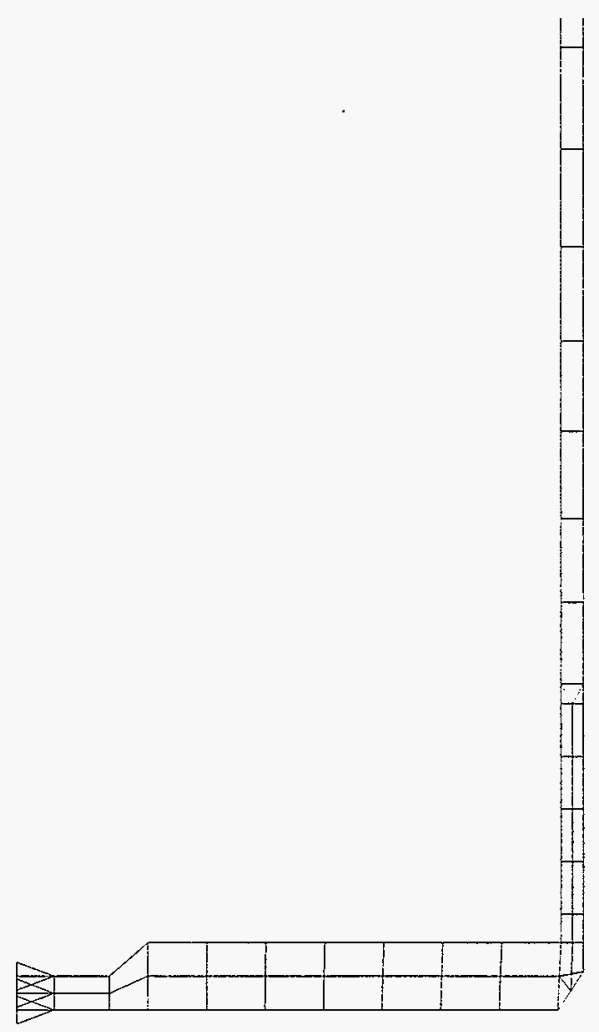

Figure 3: Axisymmetric Model with Boundary Conditions, Lower Section 
CLIENT: $\quad$ DUKE ENGINEERING \& SERVICES HANFORD, INC. FILE NO: KH-8009-8-09 PROJECT: MCO Final Design DOC. NO.: HNF-SD-SNF-DR-003, Rev. 0, Appendix 11

\subsection{Load Cases}

Six Process Operating Condition load cases are analyzed in this calculation.

1. Full internal vacuum with $25 \mathrm{psig}$ external pressure; equivalent to external pressures of $14.7 \mathrm{psi}+25 \mathrm{psi}$ or $40 \mathrm{psi}$, at $75^{\circ} \mathrm{C}\left(167^{\circ} \mathrm{F}\right)$ uniform temperature. All stresses for this load case are classified as primary stresses $\left(P_{M}\right.$ or $\left.P_{L}+P_{B}\right)$.

2. Full internal vacuum with 0 psig external pressure; equivalent to external pressure of $14.7 \mathrm{psi}$ or $15 \mathrm{psi}$, at $375^{\circ} \mathrm{C}\left(707^{\circ} \mathrm{F}\right)$ uniform temperature. All stresses for this load case are classified as primary stresses.

3. $189 \mathrm{psi}$ internal pressure at $375^{\circ} \mathrm{C}\left(707^{\circ} \mathrm{F}\right)$ uniform temperature. This value represents $150 \mathrm{psi}$ for the design pressure and $39 \mathrm{psi}$ for the fuel weight. All stresses for this load case are classified as primary stresses.

4. Lifting of the $\mathrm{MCO}$ with $189 \mathrm{psi}$ internal pressure at $132^{\circ} \mathrm{C}\left(270^{\circ} \mathrm{F}\right)$ uniform temperature. All stresses for this load case are classified as primary stresses.

5. Differential temperature: shell at $375^{\circ} \mathrm{C}\left(707^{\circ} \mathrm{F}\right)$ and shield plug at $275^{\circ} \mathrm{C}\left(527^{\circ} \mathrm{F}\right)$, at 189 psi internal pressure. All stresses for this load case are classified as primary plus secondary $\left(P_{L}+P_{B}+Q\right)$, since thermal stresses are secondary stresses $(Q)$. The primary stresses for this load case are the same as load case 1.

6. Heat up / cool down transient: shell temperature is different at various locations, timed at 24 hours (heat up) and at 73 hours (cool down), with 189 psi internal pressure. Shell temperatures are obtained from Section 4.9.2 and Figure 1 of [1]. All stresses for this load case are classified as primary plus secondary $\left(P_{L}+P_{B}+Q\right)$, since thermal stresses are secondary stresses $(Q)$. The primary stresses for this load case are the same as load case 1.

For Load Case 1, the corresponding ANSYS input and output files are POC1.inp and POC1.out, respectively.

For Load Case 2, the corresponding ANSYS input and output files are POC2.inp and POC2.out, respectively.

For Load Case 3, the corresponding ANSYS input and output files are MCO375.inp and MCO375.out, respectively.

For Load Case 4, the corresponding ANSYS input and output files are POC4.inp and POC4.out, respectively. 
CLIENT: DUKE ENGINEERING \& SERVICES HANFORD, INC.

FILE NO: KH-8009-8-09

PROJECT: MCO Final Design

DOC. NO.: HNF-SD-SNF-DR-003, Rev. 0, Appendix 11

For Load Case 5, the corresponding ANSYS input and output files are TG275.inp and

TG275.out, respectively.

For Load Case 6 at Heat-up, the corresponding ANSYS input and output files are TT24.inp and TT24.out, respectively.

For Load Case 6 at Cool-down, the corresponding ANSYS input and output files are TT73.inp and TT73.out, respectively.

\subsection{Results}

Stresses are reported along the sections is listed in Table 4. A summary of the maximum stress intensities is presented in Tables 5 through 9.

For load cases 1,2,3 and 4 (internal and external pressures), the primary membrane stress $P_{M}$, is compared to the allowable membrane stress, $S_{M}$; the membrane plus bending stress, $P_{L}+P_{B}$, is compared to $1.5 S_{M}$. For load cases 5 and 6 , the total stress $P_{L}+P_{B}+Q$, is compared to $3 S_{M}$.

The results show that for all load cases, the computed stress intensities are lower than the allowable stress intensities.

Figure 11 is a temperature distribution plot for Load Case 6. Per the MCO Performance Specification, one can notice that the shell radial temperature difference does not exceed $5^{\circ} \mathrm{C}\left(9^{\circ} \mathrm{F}\right)$.

2e

\begin{tabular}{|c|l|l|l|}
\hline 0 & & & \\
\hline $4 / 17 / 97$ & & & \\
\hline$/ 4 / 17 / 97$ & & & \\
\hline
\end{tabular}




\section{(P) PaAsons}

CLIENT: DUKE ENGINEERING \& SERVICES HANFORD, INC.

FILE NO: $\quad \mathrm{KH}-8009-8-09$

$\begin{array}{lll}\text { PROJECT: } & \text { MCO Final Design } & \text { DOC. NO.: HNF-SD-SNF-DR-003, Rev. 0, Appendix } 11\end{array}$

Table 5: ANSYS Model Stress Report Sections

\begin{tabular}{|c|c|c|}
\hline Component & Inside Node & OUtside Node \\
\hline \multirow[t]{3}{*}{ Bottom Plate } & 1 & 41 \\
\hline & 6 & 46 \\
\hline & 10 & 50 \\
\hline \multirow[t]{5}{*}{ Lower Shell } & 50 & 52 \\
\hline & 50 & 55 \\
\hline & 53 & 55 \\
\hline & 62 & 64 \\
\hline & 65 & 67 \\
\hline \multirow[t]{6}{*}{ Mid-Shell } & 100 & 101 \\
\hline & 122 & 123 \\
\hline & 134 & 135 \\
\hline & 156 & 157 \\
\hline & 170 & 171 \\
\hline & 180 & 181 \\
\hline \multirow[t]{6}{*}{ Upper Shell } & 202 & 204 \\
\hline & 235 & 237 \\
\hline & 985 & 989 \\
\hline & 262 & 264 \\
\hline & 277 & 279 \\
\hline & 292 & 294 \\
\hline \multirow[t]{11}{*}{ Shield Plug } & 601 & 641 \\
\hline & 601 & 613 \\
\hline & 603 & 703 \\
\hline & 606 & 706 \\
\hline & 706 & 736 \\
\hline & 766 & 806 \\
\hline & 748 & 808 \\
\hline & 730 & 810 \\
\hline & 736 & 815 \\
\hline & 869 & 874 \\
\hline & 870 & 875 \\
\hline \multirow{3}{*}{ Locking Ring } & 431 & 434 \\
\hline & 404 & 424 \\
\hline & 406 & 426 \\
\hline
\end{tabular}

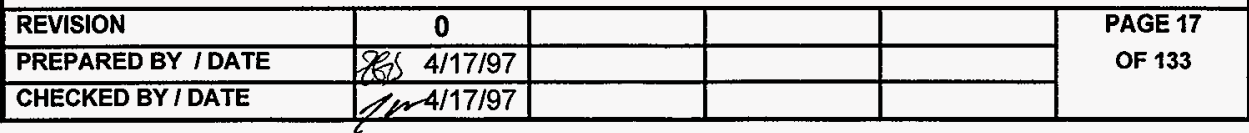


CLIENT: DUKE ENGINEERING \& SERVICES HANFORD, INC.

FILE NO: $\quad \mathrm{KH}-8009-8-09$

PROJECT: MCO Final Design

DOC. NO.: HNF-SD-SNF-DR-003, Rev. 0, Appendix 11

For the following tables the lower shell, middle shell and upper shell have the properties of Type 304 stainless steel. The bottom plate, shield plug and locking ring have the properties of Type 304L stainless steel.

Therefore, in calculating the stress ratios, the following apply:

Type 304:

$$
\begin{aligned}
& S_{M}=20.0 \mathrm{ksi} @ 75^{\circ} \mathrm{C}\left(167^{\circ} \mathrm{F}\right) \\
& S_{M}=16.0 \mathrm{ksi} @ 375^{\circ} \mathrm{C}\left(707^{\circ} \mathrm{F}\right) \\
& S_{M}=20.0 \mathrm{ksi} @ 132^{\circ} \mathrm{C}\left(270^{\circ} \mathrm{F}\right)
\end{aligned}
$$

Type 304L:

$$
\begin{aligned}
& S_{M}=16.7 \mathrm{ksi} @ 75^{\circ} \mathrm{C}\left(167^{\circ} \mathrm{F}\right) \\
& S_{M}=13.5 \mathrm{ksi} @ 375^{\circ} \mathrm{C}\left(707^{\circ} \mathrm{F}\right) \\
& S_{M}=16.7 \mathrm{ksi} @ 132^{\circ} \mathrm{C}\left(270^{\circ} \mathrm{F}\right)
\end{aligned}
$$

Table 6: Summary of Maximum Stress Intensities for Load Case 1

\begin{tabular}{|l|c|c|c|c|}
\hline Component & $P_{M}(\mathrm{ksi})$ & Stress Ratio & $P_{\mathrm{L}}+\mathrm{P}_{\mathrm{B}}(\mathrm{ksi})$ & Stress Ratio \\
\hline Bottom Plate (304L) & 0.94 & 0.06 & 2.56 & 0.11 \\
\hline Lower Shell (304) & 1.02 & 0.05 & 1.88 & 0.08 \\
\hline Middle Shell (304) & 1.01 & 0.05 & 1.03 & 0.04 \\
\hline Upper Shell (304) & 6.69 & 0.33 & 10.28 & 0.43 \\
\hline Shield Plug (304L) & 6.58 & 0.39 & 7.57 & 0.30 \\
\hline Locking Ring (304L) & 1.18 & 0.07 & 1.69 & 0.07 \\
\hline
\end{tabular}

Note: Stress Ratio $=\frac{P_{M}}{S_{M}}$ or $\frac{P_{L}+P_{B}}{15 S_{M}} \quad \mathrm{~S}_{\mathrm{M}}$ at $75^{\circ} \mathrm{C}\left(167^{\circ} \mathrm{F}\right)$ 
CLIENT: $\quad$ DUKE ENGINEERING \& SERVICES HANFORD, INC. FILE NO: KH-8009-8-09

PROJECT: MCO Final Design DOC. NO.: HNF-SD-SNF-DR-003, Rev. 0, Appendix 11

Table 7: Summary of Maximum Stress Intensities for Load Case 2

\begin{tabular}{|l|c|c|c|c||}
\hline Component & $P_{M}(\mathrm{ksi})$ & Stress Ratio & $P_{\mathrm{L}}+\mathrm{P}_{\mathrm{B}}(\mathrm{ksi})$ & Stress Ratio \\
\hline Bottom Plate (304L) & 0.35 & 0.03 & 0.96 & 0.05 \\
\hline Lower Shell (304) & 0.38 & 0.02 & 0.71 & 0.03 \\
\hline Middle Shell (304) & 0.38 & 0.02 & 0.39 & 0.02 \\
\hline Upper Shell (304) & 5.48 & 0.34 & 8.62 & 0.36 \\
\hline Shield Plug (304L) & 7.20 & 0.53 & 7.64 & 0.38 \\
\hline Locking Ring (304L) & 1.26 & 0.09 & 1.74 & 0.08 \\
\hline
\end{tabular}

Note: Stress Ratio $=\frac{P_{M}}{S_{M}}$ or $\frac{P_{L}+P_{B}}{15 S_{M}} \quad \mathrm{~S}_{\mathrm{M}}$ at $375^{\circ} \mathrm{C}\left(707^{\circ} \mathrm{F}\right)$

Table 8: Summary of Maximum Stress Intensities for Load Case 3

\begin{tabular}{||c|c|c|c|c|}
\hline Component & $\mathrm{P}_{\mathrm{M}}(\mathrm{ksi})$ & Stress Ratio & $\mathrm{P}_{\mathrm{L}}+\mathrm{P}_{\mathrm{B}}(\mathrm{ksi})$ & Stress Ratio \\
\hline Bottom Plate (304L) & 4.41 & 0.33 & 12.05 & 0.59 \\
\hline Lower Shell (304) & 4.84 & 0.30 & 11.46 & 0.48 \\
\hline Middle Shell (304) & 4.76 & 0.30 & 4.86 & 0.20 \\
\hline Upper Shell (304) & 6.25 & 0.39 & 10.35 & 0.43 \\
\hline Shield Plug (304L) & 4.47 & 0.33 & 6.46 & 0.32 \\
\hline Locking Ring (304L) & 1.65 & 0.12 & 2.27 & 0.11 \\
\hline
\end{tabular}

Note: Stress Ratio $=\frac{P_{M}}{S_{M}}$ or $\frac{P_{L}+P_{B}}{1.5 S_{M}} \quad \mathrm{~S}_{\mathrm{M}}$ at $375^{\circ} \mathrm{C}\left(707^{\circ} \mathrm{F}\right)$ 
CLIENT: DUKE ENGINEERING \& SERVICES HANFORD, INC.

FILE NO: KH-8009-8-09

$\begin{array}{ll}\text { PROJECT: MCO Final Design } & \text { DOC. NO.: HNF-SD-SNF-DR-003, Rev. 0, Appendix } 11\end{array}$

Table 9: Summary of Maximum Stress Intensities for Load Case 4

\begin{tabular}{|l|c|c|c|c|}
\hline Component & $\mathrm{P}_{\mathrm{M}}(\mathrm{ksi})$ & Stress Ratio & $\mathrm{P}_{\mathrm{L}}+\mathrm{P}_{\mathrm{B}}(\mathrm{ksi})$ & Stress Ratio \\
\hline Bottom Plate (304L) & 4.48 & 0.27 & 12.29 & 0.49 \\
\hline Lower Shell (304) & 4.81 & 0.24 & 8.52 & 0.28 \\
\hline Middle Shell (304) & 4.78 & 0.24 & 4.86 & 0.16 \\
\hline Upper Shell (304) & 6.89 & 0.35 & 11.53 & 0.38 \\
\hline Shield Plug (304L) & 4.36 & 0.26 & 6.39 & 0.26 \\
\hline Locking Ring (304L) & 1.85 & 0.11 & 3.24 & 0.13 \\
\hline \hline
\end{tabular}

Note: Stress Ratio $=\frac{P_{M}}{S_{M}}$ or $\frac{P_{L}+P_{B}}{1.5 S_{M}} \quad \mathrm{~S}_{\mathrm{M}}$ at $132^{\circ} \mathrm{C}\left(270^{\circ} \mathrm{F}\right)$

Table 10: Summary of Maximum Stress Intensities for Load Case 5

\begin{tabular}{|l|c|c|}
\cline { 2 - 3 } \multicolumn{1}{c|}{} & \multicolumn{2}{c|}{ Differential Temperature } \\
\hline Component & $\mathrm{P}_{\mathrm{L}}+\mathrm{P}_{\mathrm{B}}+\mathrm{Q}$ (ksi) & Stress Ratio \\
\hline Bottom Plate (304L) & 11.79 & 0.29 \\
\hline Lower Shell (304) & 9.45 & 0.20 \\
\hline Middle Shell (304) & 4.86 & 0.10 \\
\hline Upper Shell (304) & 10.85 & 0.23 \\
\hline Shield Plug (304L) & 21.71 & 0.54 \\
\hline Locking Ring (304L) & 3.09 & 0.08 \\
\hline
\end{tabular}

Note: Stress Ratio $=\frac{P_{L}+P_{B}+Q}{3 S_{M}} \quad \mathrm{~S}_{M}$ at $375^{\circ} \mathrm{C}$ for Shell, rest at $275^{\circ} \mathrm{C}$ 
CLIENT: DUKE ENGINEERING \& SERVICES HANFORD, INC.

FILE NO: KH-8009-8-09

PROJECT: MCO Final Design DOC.NO.: HNF-SD-SNF-DR-003, Rev. 0, Appendix 11

Table 11: Summary of Maximum Stress Intensities for Load Case 6

\begin{tabular}{|l|c|c|c|c||}
\cline { 2 - 5 } \multicolumn{1}{c|}{} & \multicolumn{2}{c|}{ Heat Up (24 hr.) } & \multicolumn{2}{c|}{ Cool-Down (73 hr.) } \\
\hline \hline Component & $P_{\mathrm{L}}+P_{\mathrm{B}}+Q$ (ksi) & Stress Ratio & $P_{\mathrm{L}}+P_{\mathrm{B}}+Q$ (ksi) & Stress Ratio \\
\hline Bottom Plate (304L) & 11.83 & 0.29 & 11.66 & 0.29 \\
\hline Lower Shell (304) & 9.33 & 0.19 & 9.87 & 0.21 \\
\hline Middle Shell (304) & 4.85 & 0.10 & 4.90 & 0.10 \\
\hline Upper Shell (304) & 10.62 & 0.22 & 11.07 & 0.23 \\
\hline Shield Plug (304L) & 8.13 & 0.20 & 6.24 & 0.15 \\
\hline Locking Ring (304L) & 1.76 & 0.04 & 2.43 & 0.06 \\
\hline
\end{tabular}

Note: Stress Ratio $=\frac{P_{L}+P_{B}+Q}{3 S_{M}}$

Temperatures are extracted from Figure 1 of [1] for the Heat-up and Cool-down. 
CLIENT: DUKE ENGINEERING \& SERVICES HANFORD, INC.

FILE NO: $\quad \mathrm{KH}-8009-8-09$

PROJECT: MCO Final Design

DOC. NO.: HNF-SD-SNF-DR-003, Rev. 0, Appendix 11

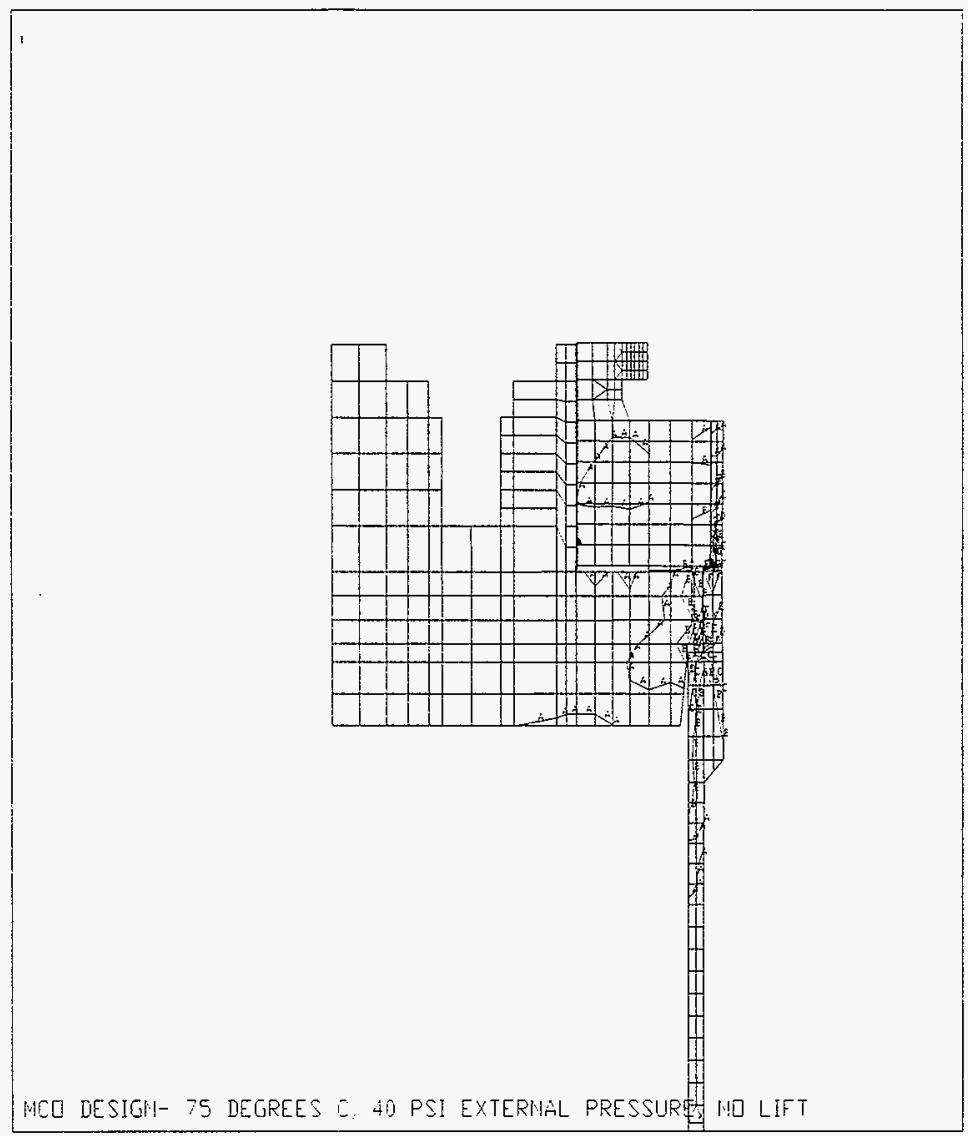

$-115: 550 \div 56$

Hif $z^{-}, 199^{-}$

$14: 1305$

PLOT NUD. I

HODAL SOLUTIOH

STEP $=1$

SUB $=1$

TIME =!

SINT

(Á)

$D H \times=0.135353$

SIM $=1.481$

SMY $=10451$

$S M_{Y} B=19015$

$Z Y=1$

*DIST $=15.631$

$* X F=5.08$

* IF $=154.37$

PRECISE HILUE!:

$A \quad=582.02^{7}$

$\mathrm{E}=1743$

ᄃ $=2704$

D) $=4065$

E $=5226$

$F=6387$

G $=7549$

$\mathrm{H}=8^{-10}$

$I=9871$

Figure 4: Load Case 1 - Upper Section Stress Intensities 
CLIENT: $\quad$ DUKE ENGINEERING \& SERVICES HANFORD, INC.

FILE NO: $\quad \mathrm{KH}-8009-8-09$

PROJECT: MCO Final Design DOC. NO.: HNF-SD-SNF-DR-003, ReV. 0, Appendix 11

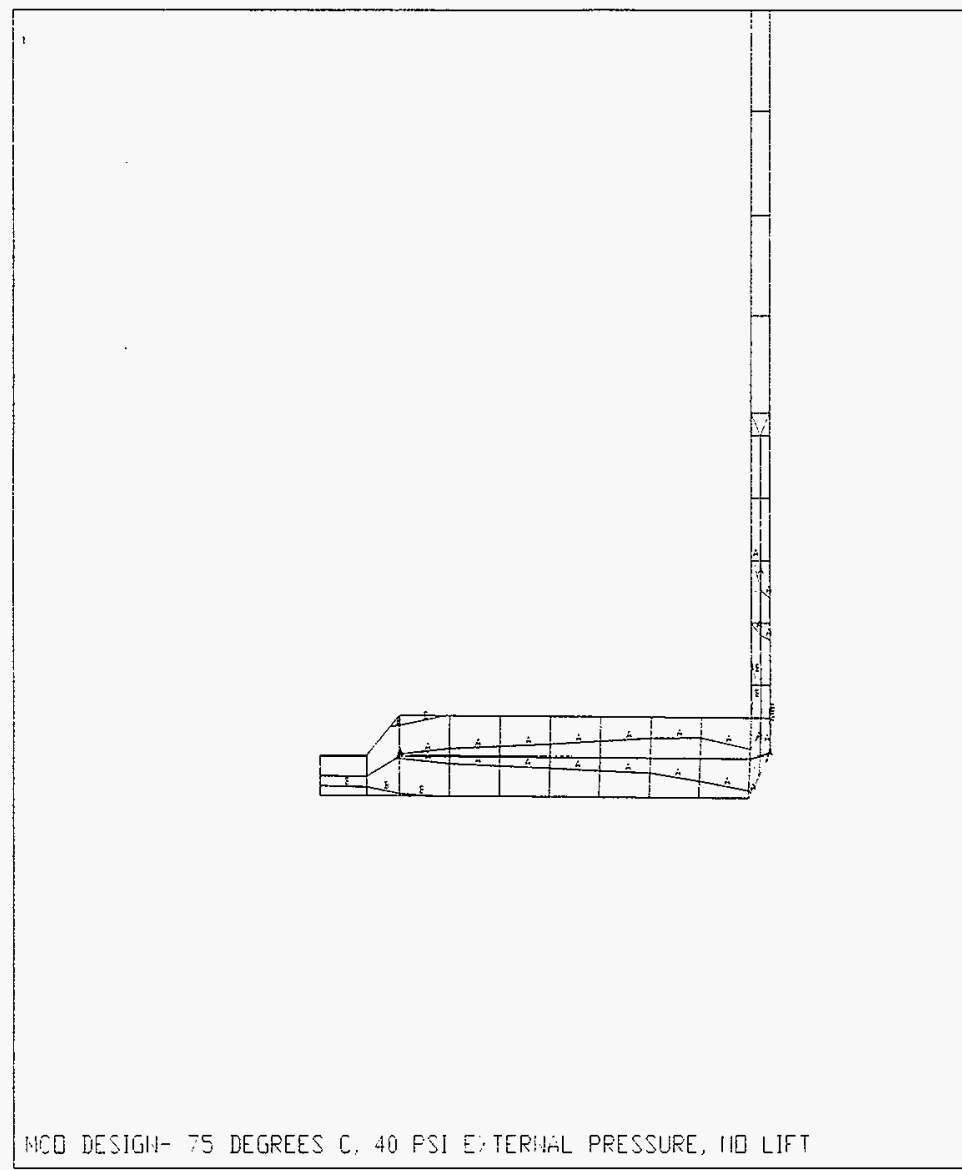

माइis 50 i 50

Hitis $z^{-}$

I $4: 32=$

FLoT IIO E

HUÉL SOLUTIOH

STEP $=1$

$S \cup B=1$

TIME $=1$

SIIT

\{AंU)

Div: $=0.135353$

SHI $=1.481$

$34 y=10451$

$S M: B=19015$

$\overline{-}=1$

$\because \mathrm{DIS} T=12.912$

$\because F \quad=4.6$

*if $=3.328$

FFECISE HIDIEL

$\therefore \quad=58202^{7}$

E $=17+3$

i $=2994$

I! $=4065$

$E=5226$

$F \quad=6397$

E $=7549$

$r \quad=870$

$\vdots \quad=927$

Figure 5: Load Case 1 -- Lower Section Stress Intensities 
CLIENT: $\quad$ DUKE ENGINEERING \& SERVICES HANFORD, INC. $\quad$ FILE NO: KH-8009-8-09

PROJECT: MCO Final Design DOC. NO.: HNF-SD-SNF-DR-003, Rev. 0, Appendix 11

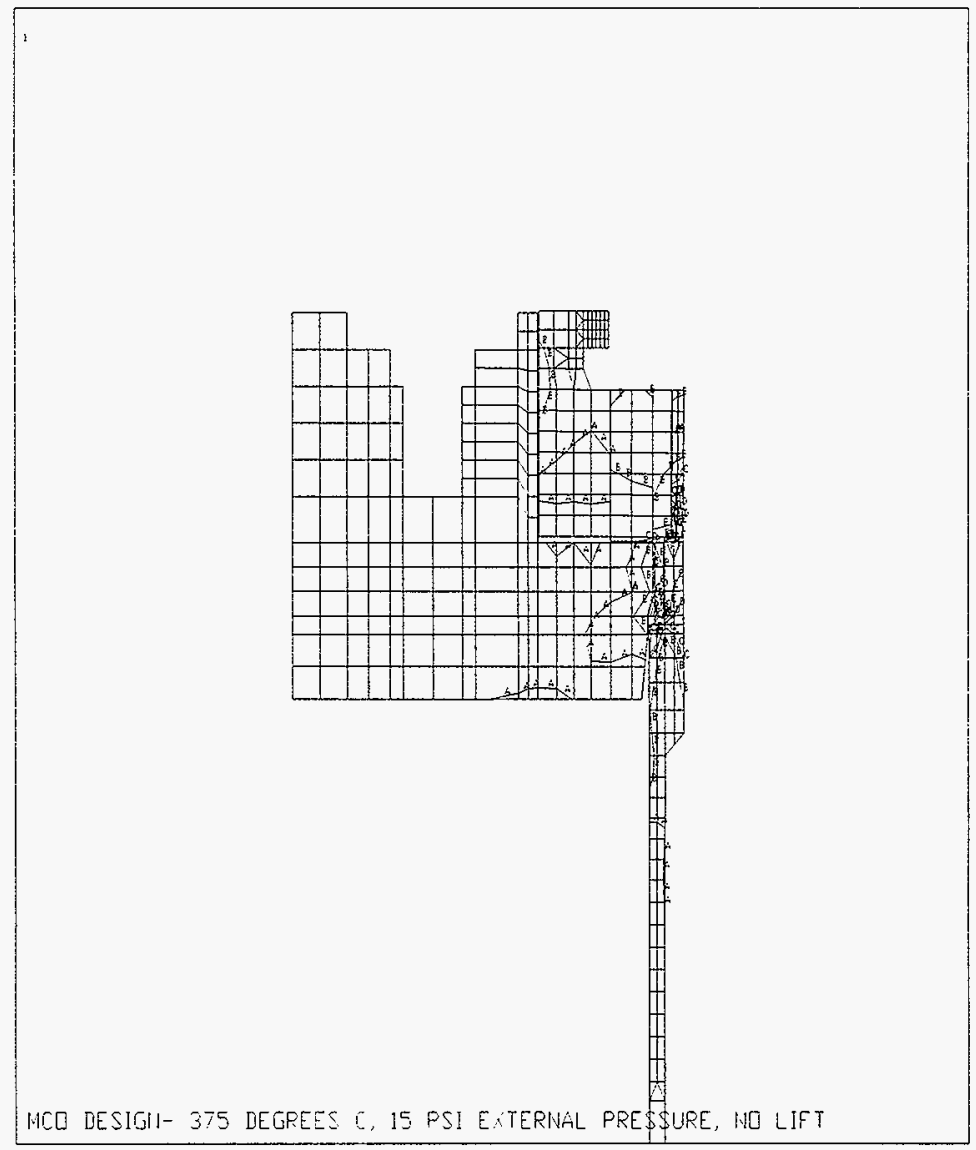

AISIS $50 \therefore 56$

MiR $2^{-199^{-}}$

14:18:44

PLOT IIG. 1

MODÁL SOLUTIOH

STEF $=1$

SUB $=1$

$T$ IME = 1

SINT (iivG)

DIA $=0.988178$

SWM $=2.766$

$S M \lambda=8767$

$\operatorname{SW} \times B=16230$

$Z !=1$

$* D I S T=17.271$

$* \% F=7.20 ?$

$*_{i} F=169.688$

PRECISE HIDDEII

$\therefore \quad=489.648$

E $=1463$

$5=2437$

D) $\quad=341 !$

$E \quad=4385$

$F \quad=5358$

(c) $=6332$

$H \quad=7306$

I $=8280$

Figure 6: Load Case 2 - Upper Section Stress Intensities 
CLIENT: $\quad$ DUKE ENGINEERING \& SERVICES HANFORD, INC. FILE NO: KH-8009-8-09

$\begin{array}{lll}\text { PROJECT: } & \text { MCO Final Design } & \text { DOC. NO.: HNF-SD-SNF-DR-003, Rev. 0, Appendix } 11\end{array}$

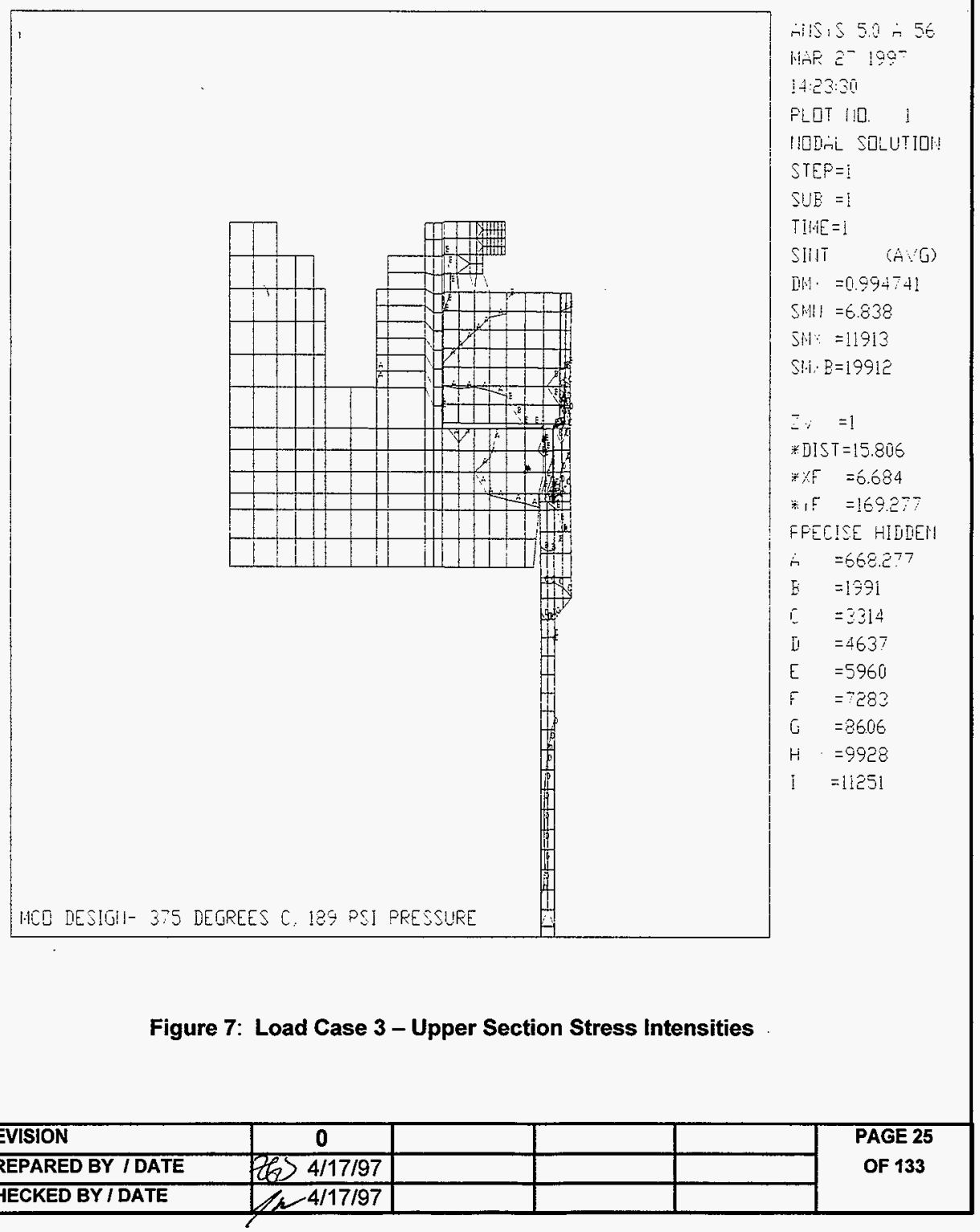


CLIENT: DUKE ENGINEERING \& SERVICES HANFORD, INC.

FILE NO: $\quad \mathrm{KH}-8009-8-09$

PROJECT: MCO Final Design DOC. NO.: HNF-SD-SNF-DR-003, Rev. 0, Appendix 11

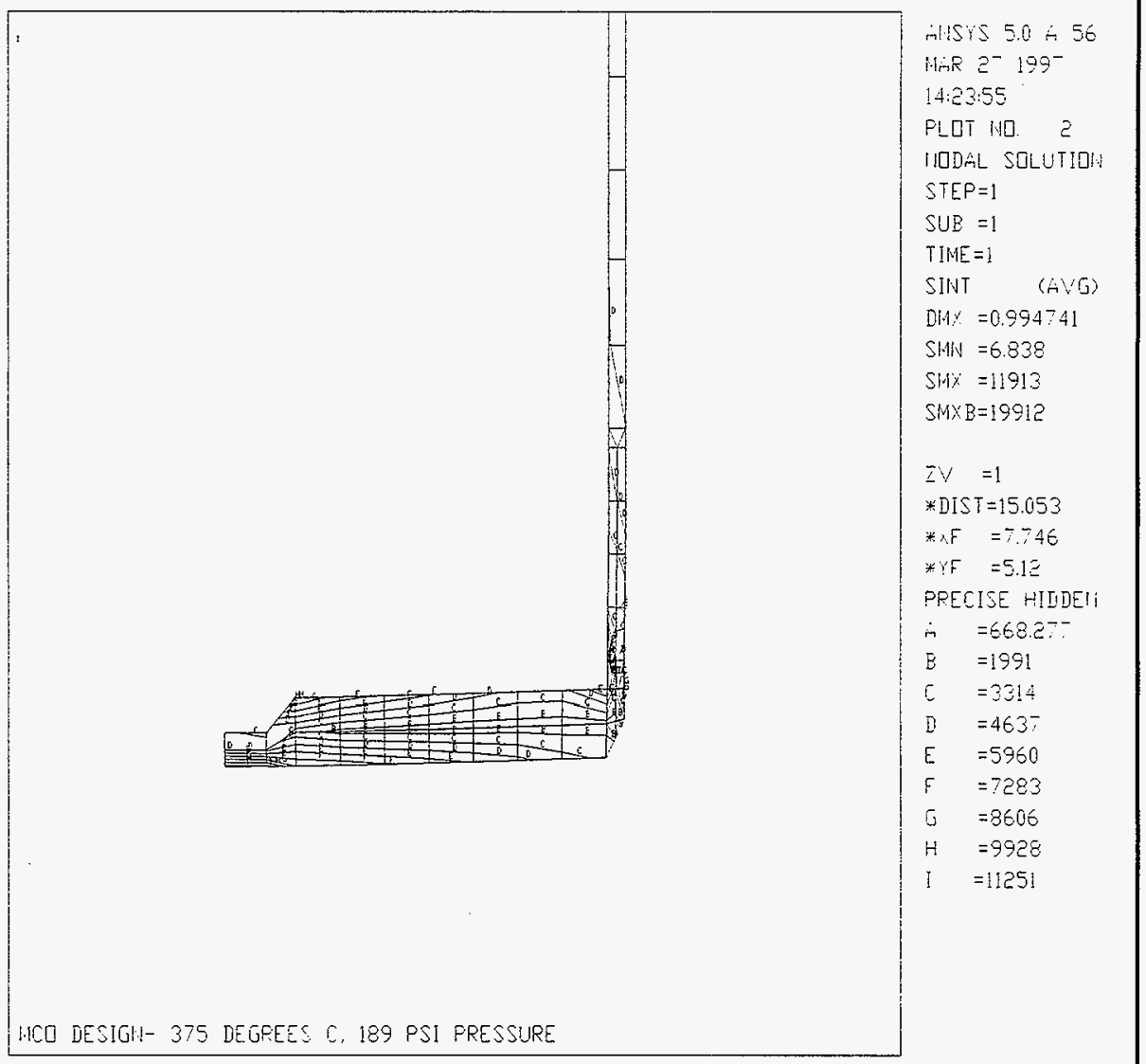

Figure 8: Load Case 3 - Lower Section Stress Intensities 


\section{P PaAsons}

CLIENT: $\quad$ DUKE ENGINEERING \& SERVICES HANFORD, INC. FILE NO: KH-8009-8-09

PROJECT: MCO Final Design DOC. NO.: HNF-SD-SNF-DR-003, Rev. 0, Appendix 11

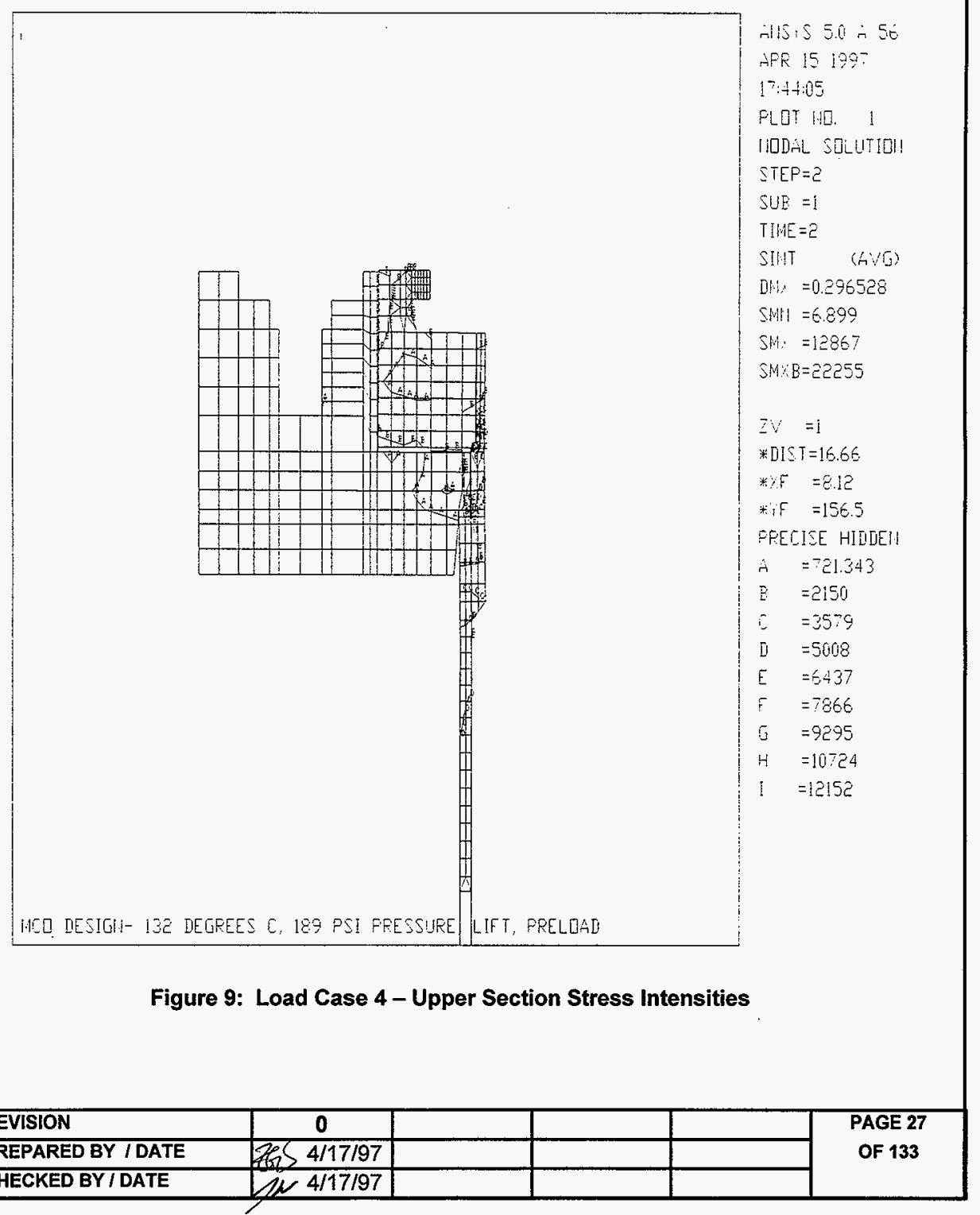


CLIENT: DUKE ENGINEERING \& SERVICES HANFORD, INC.

FILE NO: $\quad \mathrm{KH}-8009-8-09$

PROJECT:

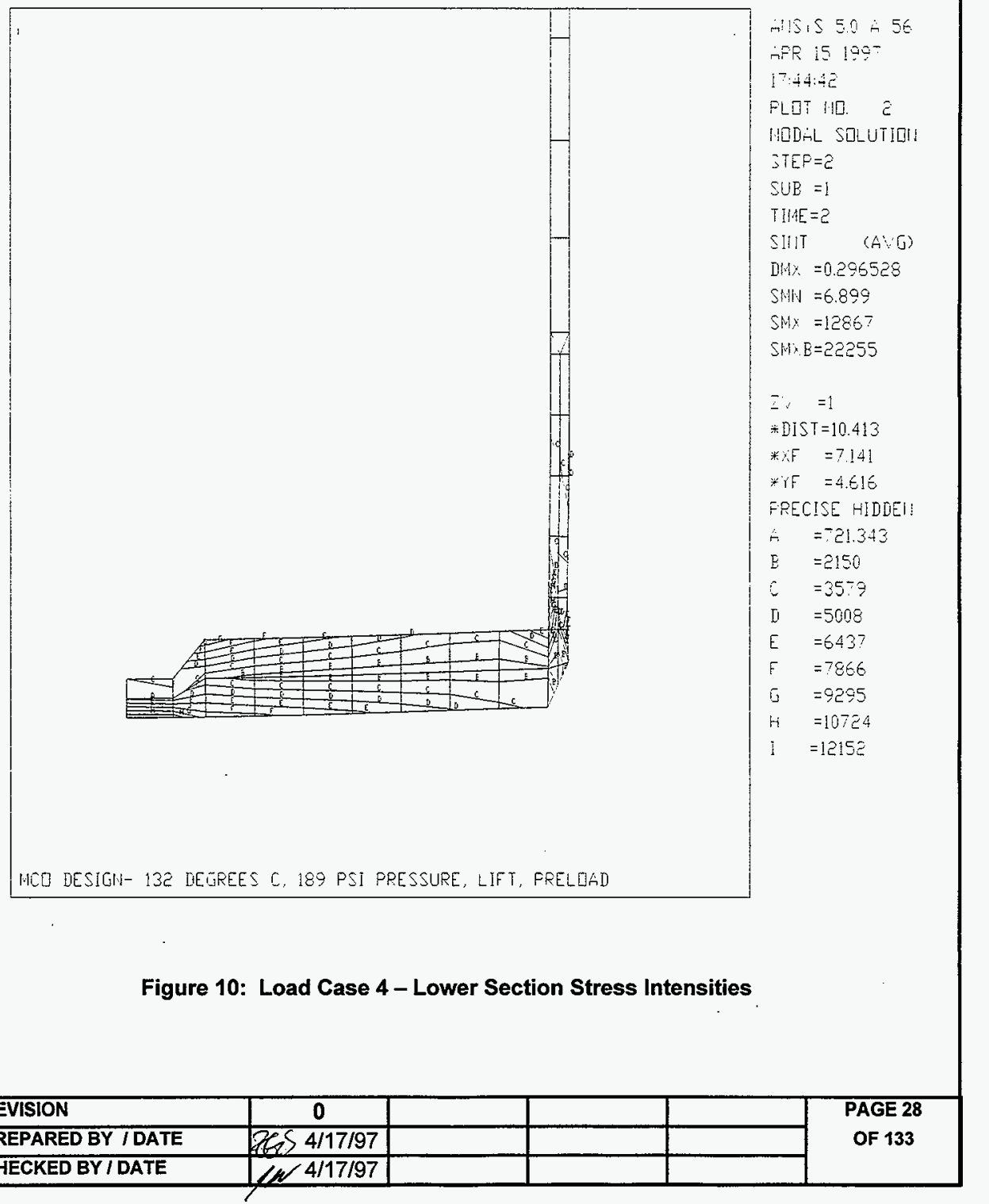


CLIENT: DUKE ENGINEERING \& SERVICES HANFORD, INC.

FILE NO: KH-8009-8-09

PROJECT: MCO Final Design DOC. NO.: HNF-SD-SNF-DR-003, Rev. 0, Appendix 11

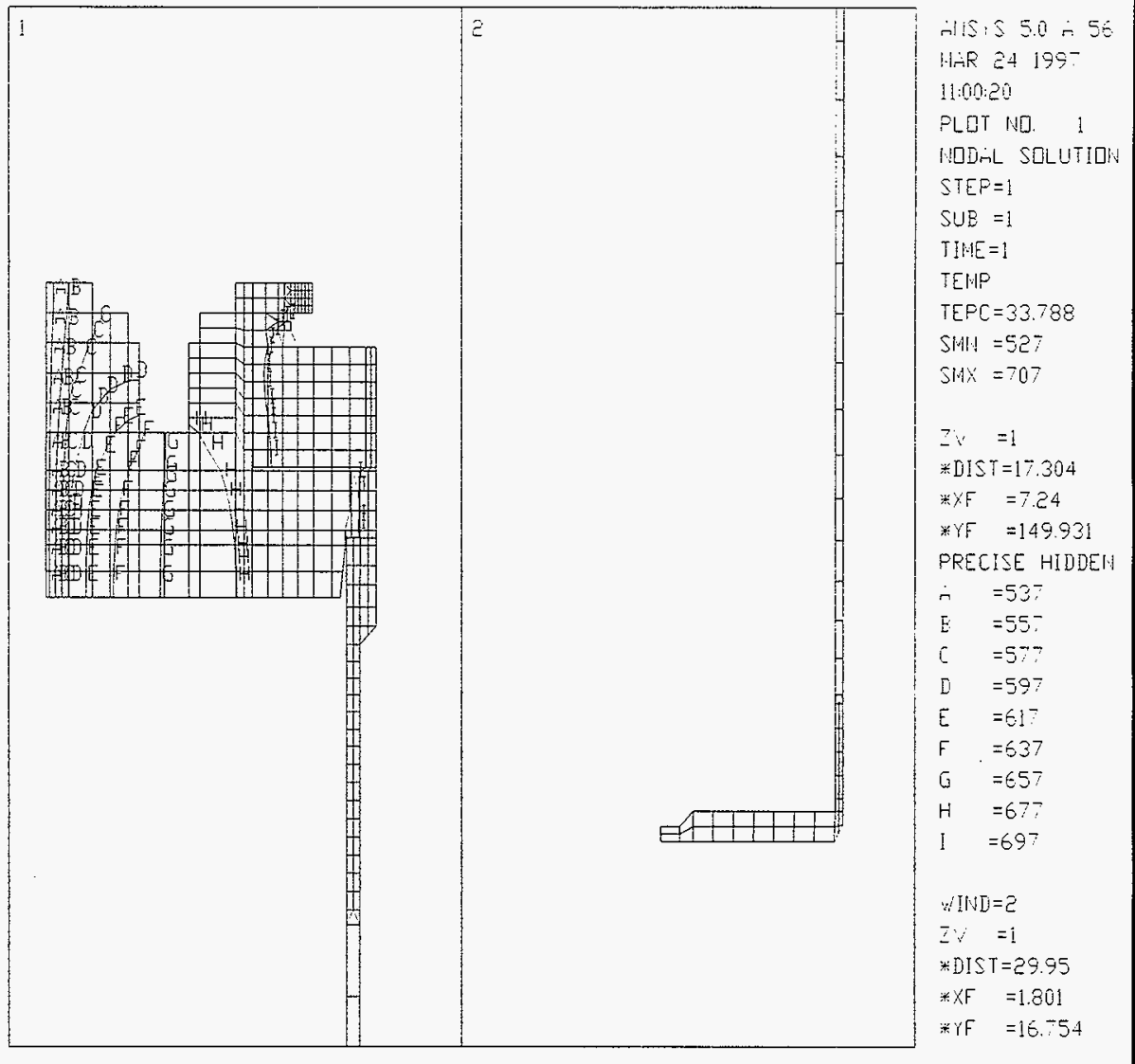

Figure 11: Load Case 5 - Upper and Lower MCO Temperature Distribution

Note: The temperature distribution for the lower MCO Assembly (pictured at right) shows no letters because it is a continuation of the upper shell, at $707^{\circ} \mathrm{F}$.

\begin{tabular}{|c|c|l|l|}
\hline 0 & & & \\
\hline Res $4 / 17 / 97$ & & & \\
\hline
\end{tabular}


CLIENT: DUKE ENGINEERING \& SERVICES HANFORD, INC.

FILE NO: $\quad \mathrm{KH}-8009-8-09$

PROJECT: MCO Final Design DOC. NO.: HNF-SD-SNF-DR-003, Rev. 0, Appendix 11

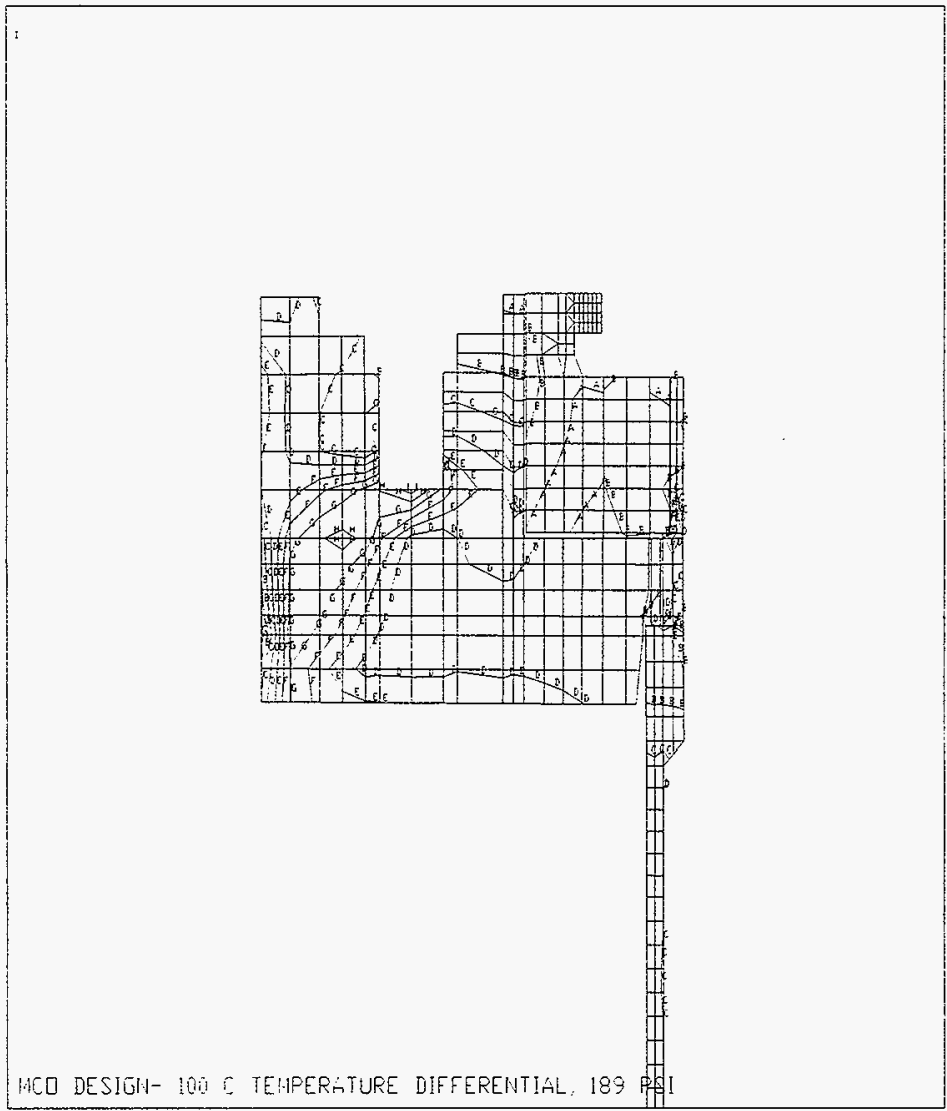

$\therefore 1 S$ : $5.0 \div 56$

MAR $\hat{c}^{-}$109-

: $2: \overline{2}+3 \mathrm{i}$

Pidi III. :

HDDRL SDLUTIOI!

STEP =1

SUB $=1$

TIME $=1$

SIHT

(A) 6 )

$\mathrm{DH} X=0.99423$

SWM $=3.8 .5$

$S H x=1548$ ?

SN, $\mathrm{B}=20451$

$2 \%=1$

*DIST $=14.919$

$\because F \quad=6811$

*if $=160.82 \varepsilon$

PFECISE HIIDEI!

$\therefore \quad=864.046$

$B=2584$

C $=4505$

j) $=6025$

$E \quad=7: 45$

$F=9466$

$\overline{1}=11186$

$\mathrm{H}=12900$

I $=1462$ ?

Figure 12: Load Case 5 - Upper Section Stress Intensities

\begin{tabular}{|l|l|l|l|}
\hline 0 & & & \\
\hline $4 / 17 / 97$ & & & \\
\hline $4 / 17 / 97$ & & & \\
\hline
\end{tabular}


CLIENT: DUKE ENGINEERING \& SERVICES HANFORD, INC.

FILE NO: KH-8009-8-09

PROJECT: MCO Final Design DOC. NO.: HNF-SD-SNF-DR-003, Rev. 0, Appendix 11

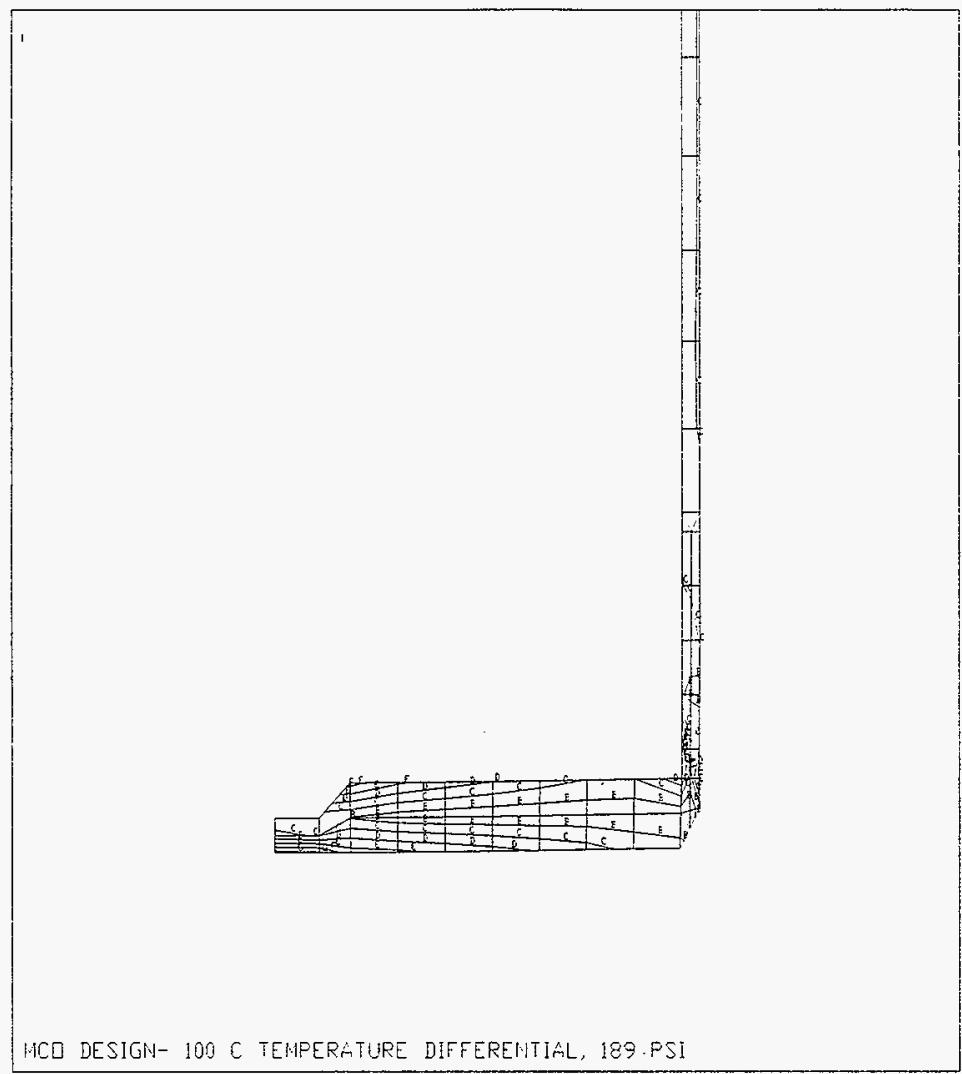

AIS is 5.0 in 56

HITR $\varepsilon^{-} \quad 199^{-}$

$12: 25: 12$

FLOT IIS. द

IIODAL SOLUTIOH

$S T E P=1$

SUE $=1$

TIME $=1$

SINT

(i) (j)

Dily $=0.99423$

SI: $=3.8: 5$

SH $=1548$ ?

$S|| B=$,2045 !

Z. $=1$

*DIST $=14.209$

*AF $=6.309$

* FF $=6.863$

PRECISE HIDDEI!

$\therefore \quad=864.046$

$E \quad=2584$

$C=4305$

D $=5025$

$E=-75$

$\mathrm{F}=9466$

$\mathrm{G}=111 \mathrm{e6}$

$\mathrm{H}=12906$

] $=14627$

Figure 13: Load Case 5 - Lower Section Stress Intensities

\begin{tabular}{|l|l|l|l|}
\hline 0 & & & \\
\hline $4 / 17 / 97$ & & & \\
\hline $4 / 17 / 97$ & & & \\
\hline
\end{tabular}


CLIENT: DUKE ENGINEERING \& SERVICES HANFORD, INC.

FILE NO: $\quad \mathrm{KH}-8009-8-09$

PROJECT: MCO Final Design DOC. NO.: HNF-SD-SNF-DR-003, Rev. 0, Appendix 11

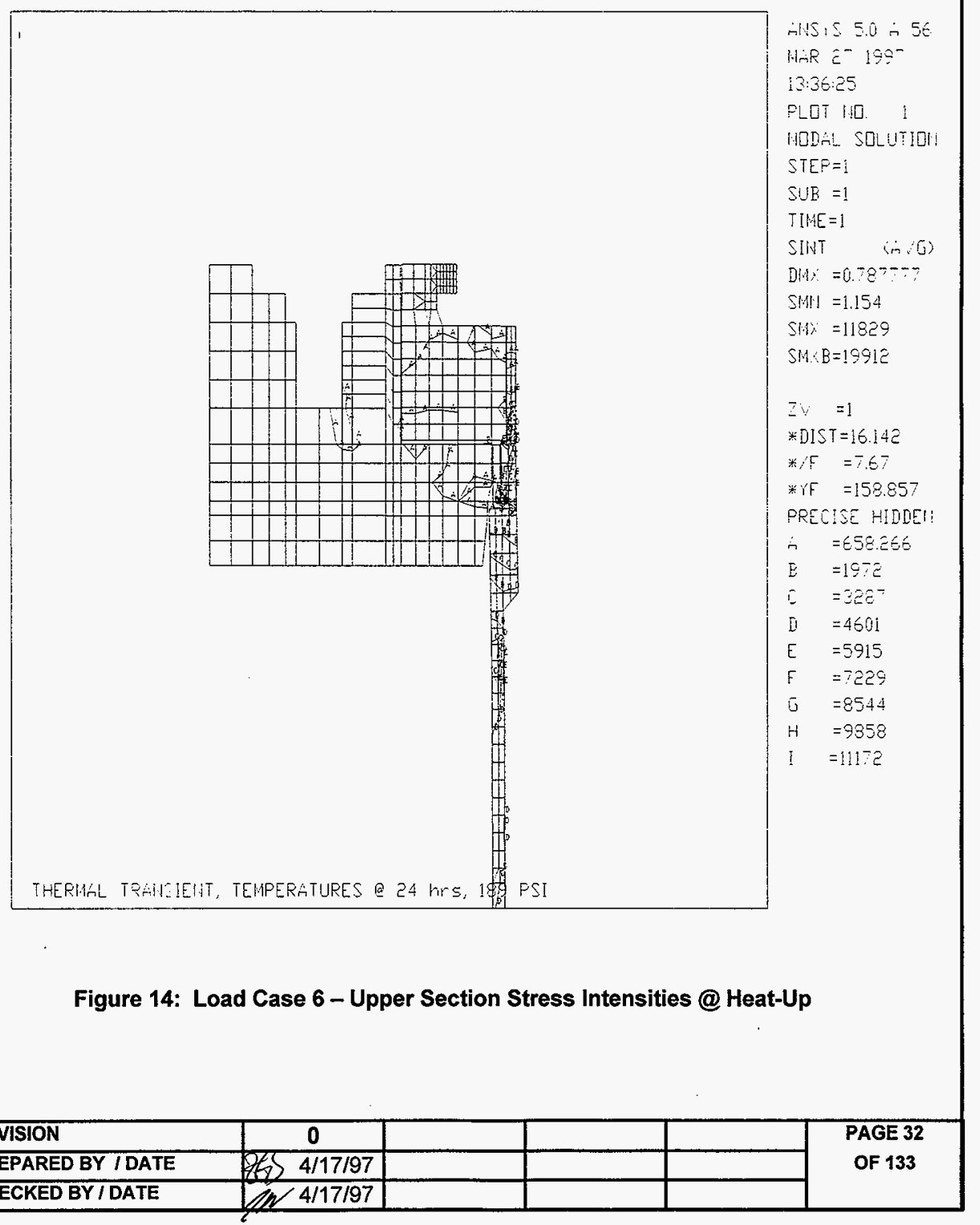


CLIENT: DUKE ENGINEERING \& SERVICES HANFORD, INC. FILE NO: KH-8009-8-09

PROJECT: MCO Final Design DOC. NO.: HNF-SD-SNF-DR-003, Rev. 0, Appendix 11

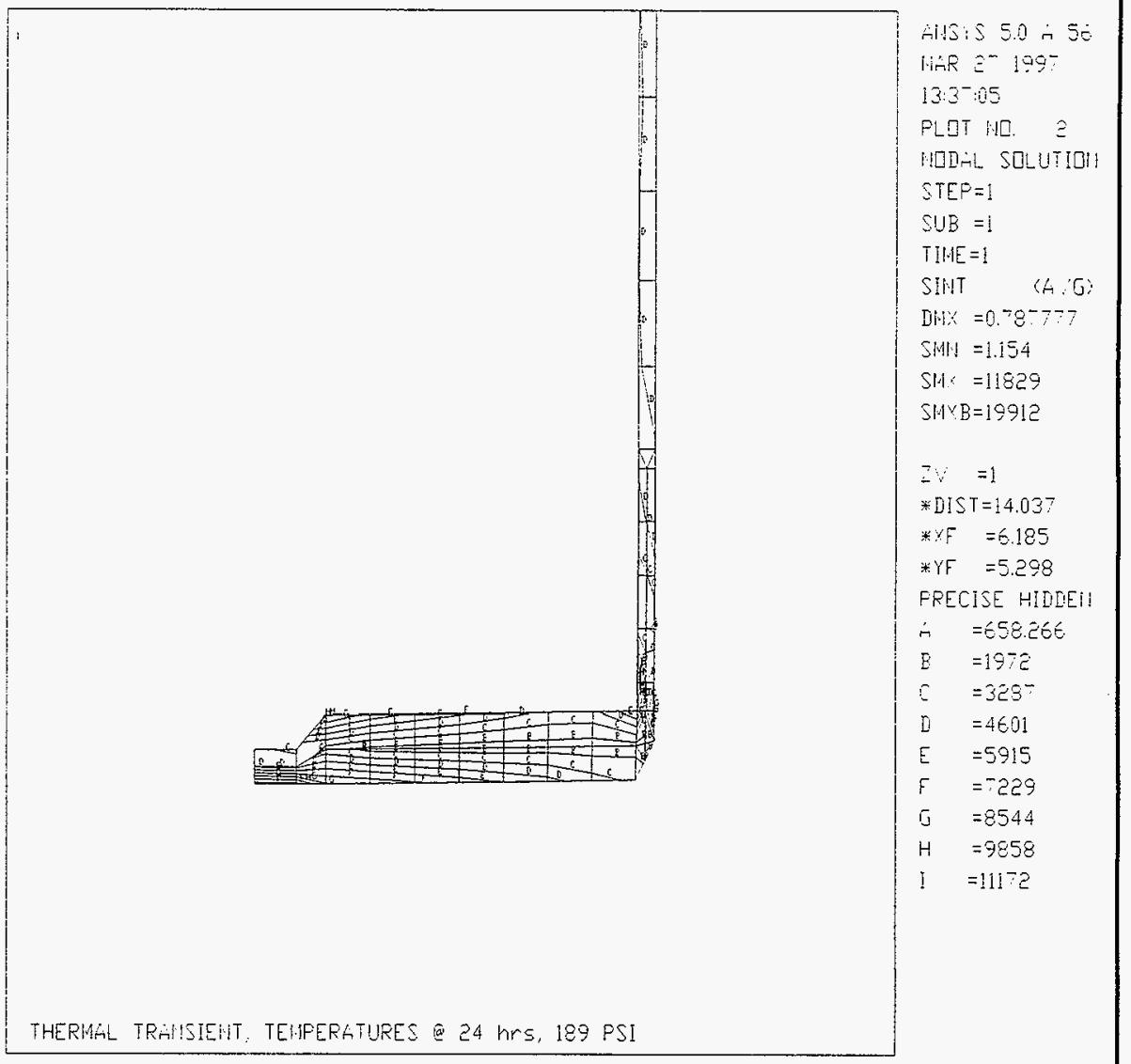

Figure 15: Load Case 6 - Lower Section Stress Intensities @ Heat-Up 
CLIENT: $\quad$ DUKE ENGINEERING \& SERVICES HANFORD, INC. FILE NO: KH-8009-8-09

PROJECT: MCO Final Design DOC. NO.: HNF-SD-SNF-DR-003, Rev. 0, Appendix 11

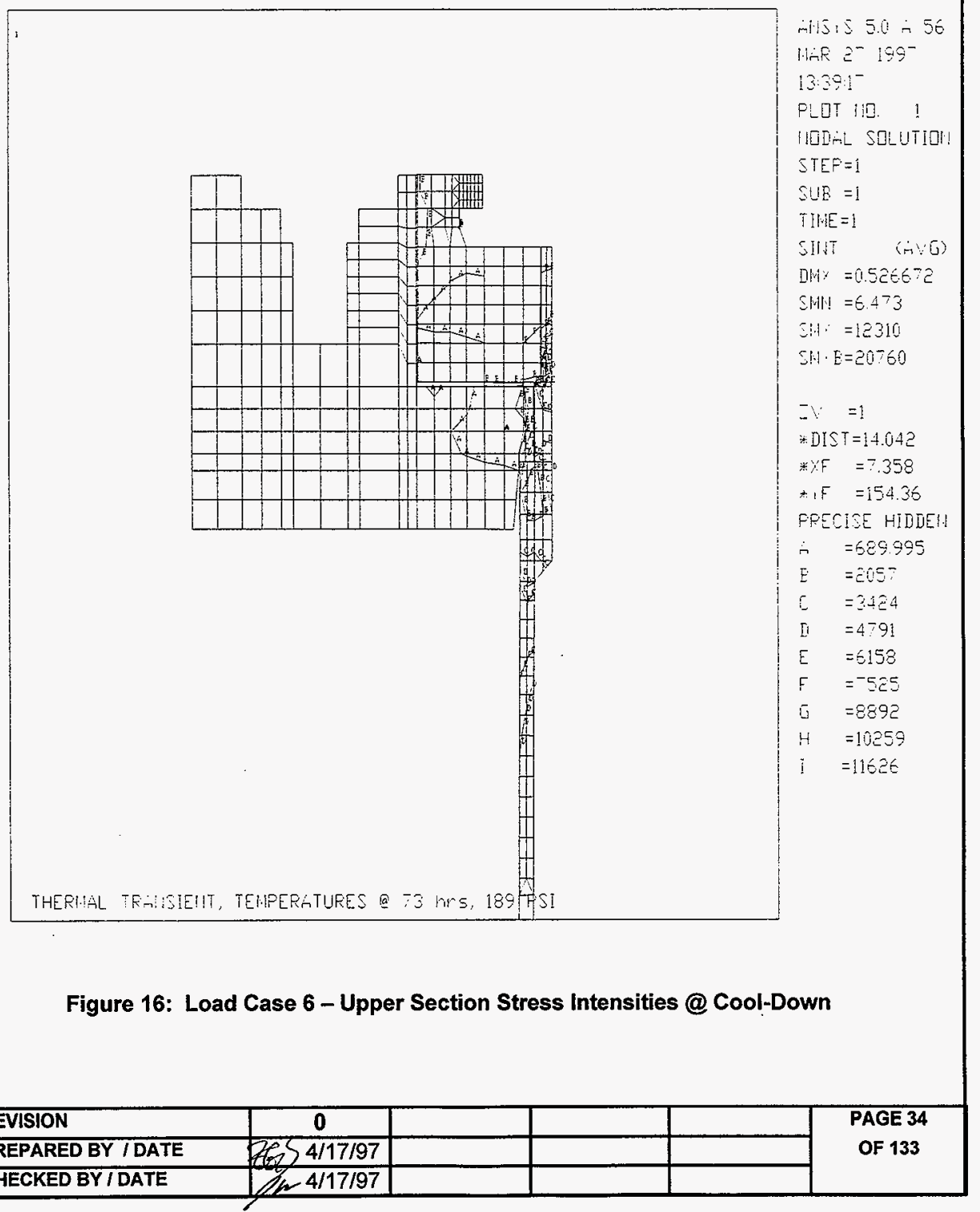


CLIENT: DUKE ENGINEERING \& SERVICES HANFORD, INC.

FILE NO: KH-8009-8-09

PROJECT: MCO Final Design DOC. NO.: HNF-SD-SNF-DR-003, Rev. 0, Appendix 11

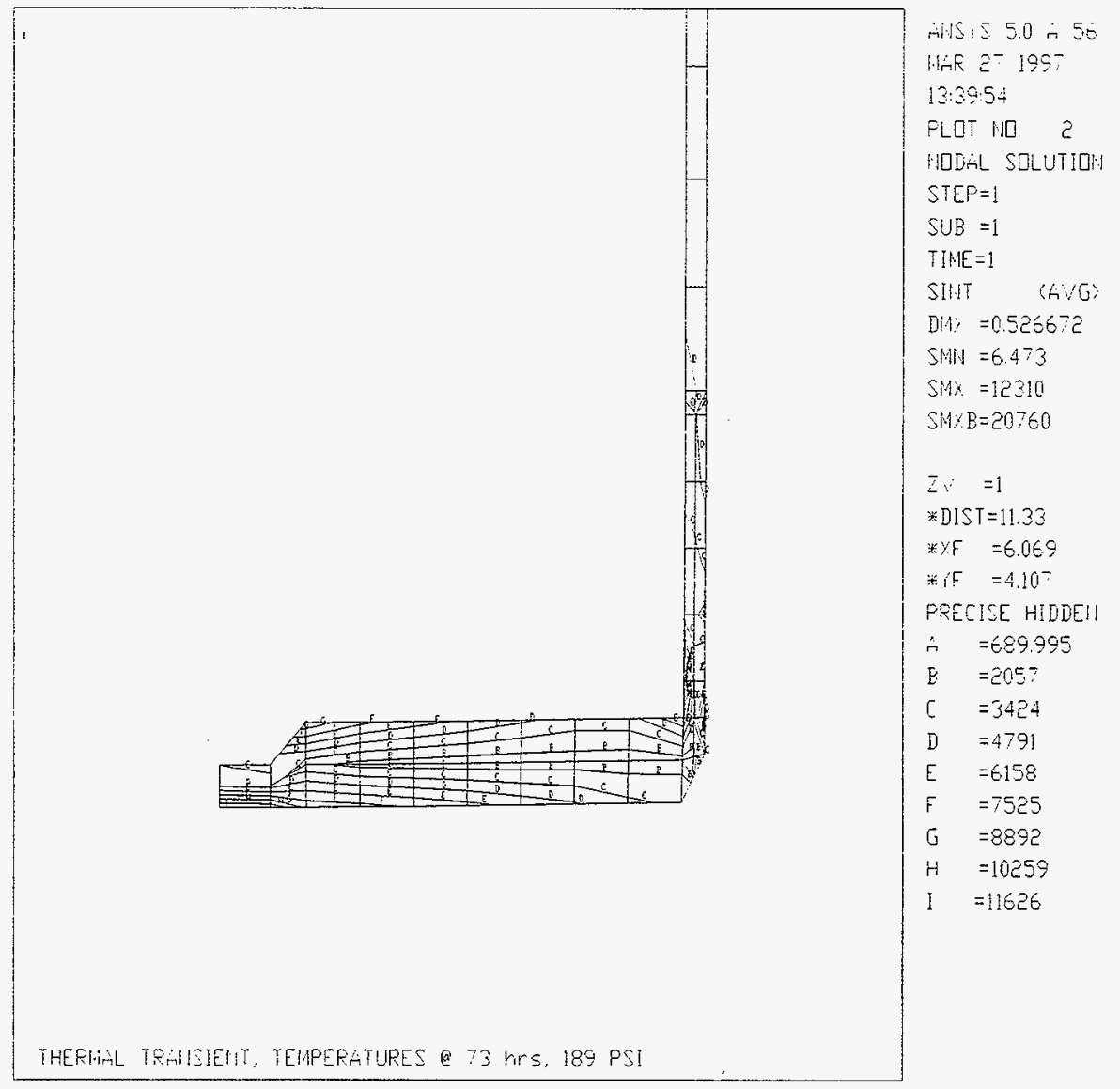

Figure 17: Load Case 6 - Lower Section Stress intensities @ Cool-Down 
CLIENT: DUKE ENGINEERING \& SERVICES HANFORD, INC.

FILE NO: $\quad \mathrm{KH}-8009-8-09$

PROJECT: $\begin{array}{ll}\text { MCO Final Design } & \text { DOC. NO.: HNF-SD-SNF-DR-003, Rev. 0, Appendix } 11\end{array}$

APPENDIX A:

Computer Run Output Sheets

\&

Input File Listings 
CLIENT: DUKE ENGINEERING \& SERVICES HANFORD, INC. FILE NO: KH-8009-8-09

PROJECT: MCO Final Design DOC. NO.: HNF-SD-SNF-DR-003, Rev. 0, Appendix 11

\section{COMPUTER RUN COVER SHEET}

Project Number:

Computer Code:

Software Version:

Computer System:

Computer Run File Number:

Unique Computer Run Filename:

Run Description:

Creation Date / Time:
$\mathrm{KH}-8009-8$

ANSYS®-PC

$5.0 \mathrm{~A}$

MS-DOS, Pentium® Processor

KH-8009-8-09

POC1.inp

Load Case 1: $40 \mathrm{psi}, 75^{\circ} \mathrm{C}$

27 March $1997 \quad 2: 08: 44$ PM
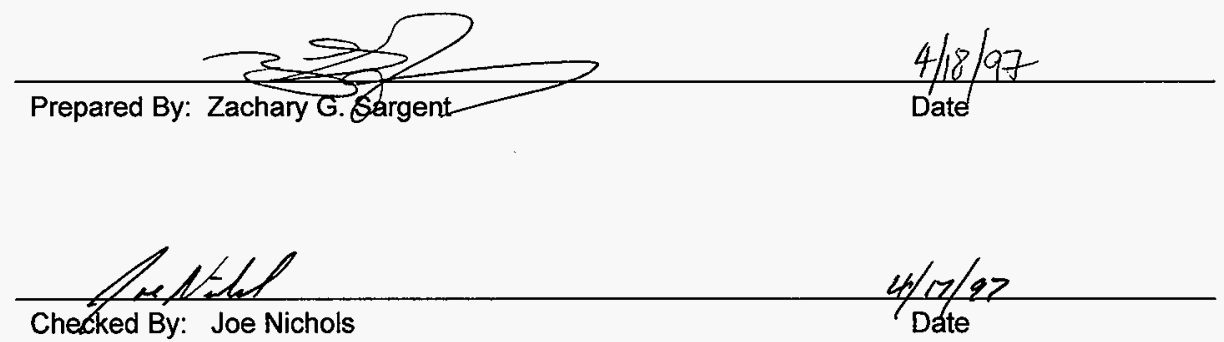
CLIENT: DUKE ENGINEERING \& SERVICES HANFORD, INC.

FILE NO: KH-8009-8-09

PROJECT: MCO Final Design DOC. NO.: HNF-SD-SNF-DR-003, Rev. 0, Appendix 11

\section{LISTING OF POC1.INP FILE}

IBATCH,LIST

IFILENAM,POC1

IPREP7

ITITLE,MCO DESIGN- 75 DEGREES C, 40 PSI EXTERNAL PRESSURE, NO LIFT

TREF,70

TUNIF,167

ICOM ELEMENT TYPES ***

ET,1,42,,1 I Shell

ET,2,42,,1 I Shield Plug

ET, 3,42,,1 ! Lifting \& Locking Ring

$E T, 4,12$

I Gap Elements Between Shield Plug \& Shel]

KEYOPT,4,7,1

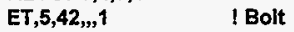

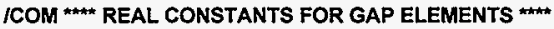

R,4,-90,1.0e8,-0.06,3.0 IShellishield Plug, Initially Open .06"

R,5,0,1.0e8,2.95e-03 I L. Ring/Shield Plug, Under Bolt, Preloaded

R,6,0,1.0E8,0,2.0 I Sealing Surface, closed

ICOM

MP,DENS, 1,490/1728 I 304L SS

MP,NUXY,1,0.3

MP,DENS,5,490/1728 ! SA193 Grade B8M

$M P, N U X Y, 5,0.3$

ICOM DEFINING TEMPERATURES FOR MPDATA *

MPTEMP,1, 70,100,200,300,400,500

MPTEMP, $7,600,650,700,750$

ICOM *\# DEFINING ELASTIC MODULI FOR 304L \& SA-193 *\#*

MPDATA,EX,1,1,28.3e+06,28.1e+06,27.6e+06,27.0e+06,26.5e+06,25.8e+06

MPDATA,EX,1,7,25.3e+06,25.1e+06,24.8e+06,24.5e+06

ICOM ISA-193

MPDATA,EX,5,1,28.3e+06,28.1e+06,27.6e+06,27.0e+06,26.5e+06,25.8e+06

MPDATA,EX,5,7,25.3e+06,25.1e+06,24.8e+06,24.5e+06

ICOM MEAN COEFFICIENTS OF THERMAL EXPANSION (in./in./(F)

1 SA240 Gr 304L

MPDATA,ALPX,1,1,0,8.55e-06,8.79e-06,9.00e-06,9.19e-06,9.37e-06

MPDATA,ALPX, 1,7,9.53e-06,9.61e-06,9.69e-06,9.76e-06

I SA193 Gr B8M

MPDATA,ALPX,5,1,0,8.54e-06,8.76e-06,8.97e-06,9.21e-06,9.42e-06

MPDATA,ALPX,5,7,9.60e-06,9.69e-06,9.76e-06,9.81e-06

ICOM SHELL GEOMETRY

IR=11.5 I Internal Shell Radius @ Bottom

OR=12.000 I Shell Outside Radius @ Bottom

IR2 $=\mathbf{1 2 . 0 2}$ ! Inside Radius at Collar Sealing Surface

OR2 $=12.625$ ! Outside Radius at Collar Sealing Surface

\begin{tabular}{|l|c|l|l|l|}
\hline REVISION & 0 & & & \\
\hline PREPARED BY I DATE & $Z(6)$ 4/17/97 & & & \\
\hline CHECKED BY I DATE & $2 \times 4 / 17 / 97$ & & & \\
\hline
\end{tabular}

PAGE 38

OF 133 
CLIENT: DUKE ENGINEERING \& SERVICES HANFORD, INC.

FILE NO: KH-8009-8-09

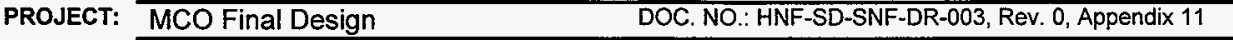

$$
\text { IR3 = } 12.25 \quad \text { I Inside Radius at Collar-Lifting Ring Weld }
$$

$I^{1 C O M}{ }^{\star \star \star \star}$ BOTTOM COVER PLATE [DWG SK-2-300378]

$N, 1,-1.32$ I ROW 1

$N, 2,1.25,-1.32$

$\mathrm{N}, 3,2.13,-1.32$

$N, 10,11.423,-1.32$

FILL

$$
\begin{aligned}
& \mathrm{N}, 41,0.00,-0.44 \\
& N, 42,1.25,-0.44 \\
& N, 43,2.13,0.44 \\
& N, 50,1 R, 0.44 \\
& \text { FILL,43,50 } \\
& \text { N,52,OR,0.44 } \\
& \text { FILL, } 50,52
\end{aligned}
$$

FILL, 1,41,1,21,1,10 I Middle Row

FILL, $10,50,1,30$

IRow 3

$N, 32,12,-0.32$

FILL, 30,32

FILL, 10,32,1,11

N,53,IR,1.17

$\mathrm{N}, 55, \mathrm{OR}, 1.17$

FILL, 53, 55

I Shell StubNeld

ICOM SHELL [DWGS SK-2-300379 \& SK-2-300461]

$\mathrm{N}, 65, \mathbf{I R , 6 . 6 8}$

$\mathrm{N}, 67,0 R, 6.68$

FILLL

FILL, $53,65,3,3,3,1$

ICOM ** SINGLE ROW SHELL *

$N, 100,1 R, 7.18 \quad$ I Inside

$N, 140, I R, 71.68$

$N, 180, I R, 136.68$

N,101,OR,7.18 I Outside

$\mathrm{N}, 141, \mathrm{OR}, 71.68$

$\mathrm{N}, 181, \mathrm{OR}, 136.68$

FILL, 100,140,20,2,2,1,2.0

FILL, 140,180,19,2,2,1,.5

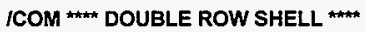

N,190,iR,137.18 I Transition to Double Row

$\mathrm{N}, 192, \mathrm{OR}, 137.18$

FILL

ICOM *\#* BASE OF CASK THROAT-ELEVATION: 138 INCHES

N,217,IR,142.68 ITransition to Double Row

$N, 219,0 R, 142.68$

FILL.

FILL, 190,217,8,,3,3,1 IVertical Fill

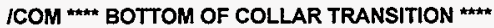

N,235,IR,146.06 IStart of Transition to Large O.D \&

\begin{tabular}{|l|c|l|l|l|}
\hline REVISION & 0 & & & \\
\hline PREPARED BY IDATE & ReS 4/17/97 & & & \\
\hline CHECKED BY I DATE & $\mathbb{2} 4 / 17 / 97$ & & & \\
\hline
\end{tabular}


CLIENT: DUKE ENGINEERING \& SERVICES HANFORD, INC.

FILE NO: $\quad \mathrm{KH}-8009-8-09$

PROJECT: MCO Final Design

DOC. NO.: HNF-SD-SNF-DR-003, Rev. 0, Appendix 11

$\mathrm{N}, 237, \mathrm{OR}, 146.06$

FILL

$\mathrm{N}, 238, \mathrm{IR}, 146.68$

$N, 240,0 R, 146.68$

FILL

FILL, 217,235,5,3,3,1

! Horizontal Fill

ICOM TOP OF COLLAR TRANSITION

$N, 241, I R, 147.31$

$\mathrm{N}, 243, \mathrm{OR}, \mathbf{1 4 7 . 3 1}$

FILL.

NGEN $, 2,3,241,243,1,0.75$

I End of Transition to Large O.D \&

I Assumed Location of Shield Plug Taper

I Horizontal Fill

ICOM *** COLLAR SEALING SURFACE ****

$\mathrm{N}, 247, \mathrm{IR}, 149.63$

$N, 249, I R 2,149.63$

FILL

I Inside Radius of Sealing Surface

1 Outside Radius at Sealing Surface

1 Horizontal Fill

\section{ICOM THICK WALL AT COLLAR TRANSITION}

NGEN, 2,10,240,249,3

$\mathrm{N}, \mathbf{2 5 5}, \mathrm{OR2}, 147.31$

$\mathrm{N}, \mathbf{2 6 1}, \mathrm{OR2}, 149.63$

I Nodes 250-259 Coincident w/240-249 (by 3)

1 Outside Surface

I Outside Surface

$\mathrm{N}, \mathbf{2 5 8 , O R 2 , 1 4 8 . 0 6}$

N,980,IR, 149.38

N,981,11.755,149.38

N,982,IR2,149.38

$\mathbf{N}, \mathbf{9 8 3}, 12.317,149.38$

$\mathrm{N}, 984, \mathrm{OR2}, 149.38$

N,990,OR2, 146.68

FILL,240,990,1,251

NGEN, $2,5,980,984,1,-0.66$

FILL, 246,258,1,257

FILL, 253,255,1,1,3,3

FILL,237,990,1,991

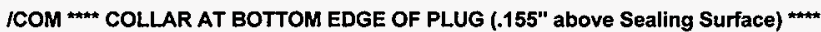

NGEN, $2,3,259, \ldots, 0.245$ ! Nodes 262

ICOM *** COLLAR AT TOP EDGE OF PLUG (2" above bottom Edge) **

NGEN $, 2,9,262,, 2.00$ I Nodes 271

FILL,262,271,2

ICOM COLLAR AT BASE OF THREADS \#*

$\mathrm{N}, 274, \mathrm{IR3}, 152.00$

$N, 1000$, IR2,152.00

ICOM *\#* TOP TO COLLAR (WELD CLOSURE) ***

$\mathrm{N}, 295,1 \mathrm{R} 3,156.00$

FILL,274,295

NGEN,3,1,259,295,3,(OR2-IR2)/2

NGEN,3,1,274,295,3,(OR2-1R3)/2

ICOM LOCKING \& LIFTING RING GEOMETRY

RING1 $=7.94$

RING2 $=9.375$

RING3 $=9.625$

\begin{tabular}{|l|c|l|l|l|}
\hline REVISION & 0 & & & \\
\hline PREPARED BY IDATE & R/ 4/17/97 & & & \\
\hline CHECKED BY I DATE & $/ / 2 / 17 / 97$ & & & \\
\hline
\end{tabular}


CLIENT: DUKE ENGINEERING \& SERVICES HANFORD, INC.

FILE NO: KH-8009-8-09

PROJECT: MCO Final Design DOC. NO.: HNF-SD-SNF-DR-003, Rev. 0, Appendix 11

RING4 $=10.19$

RING5 $=12.23$

LOCAL, $11,0,152.00 \quad$ ! Local System $z=0$ at Base of Ring

CSYS, 11

ICOM *** TOP EDGE ***

$N, \mathbf{4 0 1 , R I N G 1 , 6 . 1 3}$

CSYS,O

$\mathrm{N}, 404,9.375,158.13$

FILL,401,404,,1

N,406,RING4,158.13

FILL,404,406,,1 ITop Edge

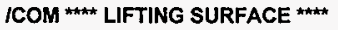

CSYS,11

N,421,RING1,5.13

N,424,RING2,5.13

FILL, 421,424

N,426,RING4,5.13

FILL,424,426

FILL,401,421,1,10,6,1

N,431,RING1,6.13-1.56

N,434,RING2,6.13-1.56

FILL

ICOM BOLTING SURFACE

N,441, RING1,4

N,444,RING3,4

FILL.

N,445,10.9375-.6875,4 IInside Edge of Bolt Hole

N,447,10.9375+.6875,4 I Outside Edge of Bolt Hole

FILL

$\mathrm{N}, 910,10.9375-.6875,4$

$\mathrm{N}, 911,10.9375+.6875,4$

N,448,RING5,4

CSYS, 0

10.D of Ring

$\mathrm{N}, 924,10.25,152.00$

I Bolt Extension

I Double Nodes @ Bolt for Gap elements

$\mathrm{N}, 925,11.625,152.00$

FILL,910,924,6,2

FILL,911,925,6,2

$\mathrm{N}, 525,10.25,151.874$

$\mathrm{N}, 527,11.625,151.874$

FILL

ICOM **** BOTTOM OF LIFTINGILOCKING RING ***

CSYS, 11

NGEN,2,70,441,448,1,-4 I Bottom Surface of Lifting/Locking Ring

FILL,441,511,6,10,8,1 I Fill in Lifting/Locking Ring

ICOM

LOCAL,20,0,158.13

TYPE,2

PLUGR1 $=11.975$

PLUGR2 $=11.45$

PLUGR3=11.25

PLUGR4=7.89

\begin{tabular}{|l|c|l|l|l|}
\hline REVISION & 0 & & & \\
\hline PREPARED BY I DATE & PfS 4/17/97 & & & \\
\hline CHECKED BY I DATE & P $4 / 17 / 97$ & & & \\
\hline
\end{tabular}


CLIENT: DUKE ENGINEERING \& SERVICES HANFORD, INC.

FILE NO: KH-8009-8-09

PROJECT: MCO Final Design DOC. NO.: HNF-SD-SNF-DR-003, Rev. 0, Appendix 11

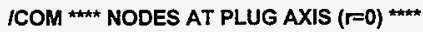

N,601

$N, 602,0,-1$

$\mathrm{N}, 603,0,-1.994$

N,606,0,-4.994

FILL,603,606,2,604

$N, 607,0,-6.25$

$N, 610,0,-8.25$

FILL,607,610,2,608

$\mathbf{N}, 611,0,-8.75$

$\mathrm{N}, 613,0,-10.5$

FILL,611,613
ICOM NODAL GENERATION *
NGEN, 2,20,601,613,1,0.8825
NGEN,2,20,621,633,1,0.8825
NGEN,2,20,642,653,1,0.6875
NGEN,2,20,662,673,1,0.6875
NGEN,2,20,683,693,1,0.4235
NGEN,2,10,706,713,1,0.9515

\section{Id Large Opening \\ I Id Medium Opening \\ I Id Small Opening \\ I Center of Opening}

I Od Small Opening

$N, 730,5.4665,-1.994$

N,736,5.4665,-4.994

FILL, 730,736,5,731

$N, 737,5.4665,-6.25$

$N, 740,5.4665,-8.25$

FILL, 737,740,2,738

$\mathbf{N}, 741,5.4665,-8.75$

$\mathbf{N}, 743,5.4665,-10.5$

FILL, 741,743

N,748,5.89,-1.0

NGEN,2, 20,730,743,1,0.4235

FILL,748,750

$\mathbf{N}, \mathbf{7 6 6 , 7 . 2 6 5 , 0}$

NGEN,2,20,748,763,1,1.375

FILL,766,768

NGEN, $3,20,766,768,1,0.3125$

N,789,7.5775,-1.56

N,796,7.5775,-5.56

FILL,789,796,6

NGEN, 2,20,789,796,1,0.3125

NGEN, $3,20,777,783,1,0.3125$

ICOM ** UNDER LOCKING RING ****

$N, 824,8.5017,-6.25$

$\mathrm{N}, 827,8.5017,-8.25$

FILL

$\mathrm{N}, 828,8.5017,-8.75$

N,830,8.5017,-10.5

FILL.

NGEN, 3,7,824,830,1,0.5616

NGEN,2,7,838,844,1,0.625

NGEN,2,7,845,851,1,0.6875 I Under Bolt

$N, 859,11.625,-6.25$

$\mathrm{N}, 860,11.625,-6.917$

\begin{tabular}{|l|c|c|c|c|c|}
\hline REVISION & 0 & & & & PAGE 42 \\
\hline PREPARED BY IDATE & OGS 4/17/97 & & & & OF 133 \\
\hline CHECKED BY I DATE & S $4 / 17 / 97$ & & & & \\
\hline
\end{tabular}


CLIENT: DUKE ENGINEERING \& SERVICES HANFORD, INC.

FILE NO: $\quad \mathrm{KH}-8009-8-09$

PROJECT: MCO Final Design DOC. NO.: HNF-SD-SNF-DR-003, Rev. 0, Appendix 11

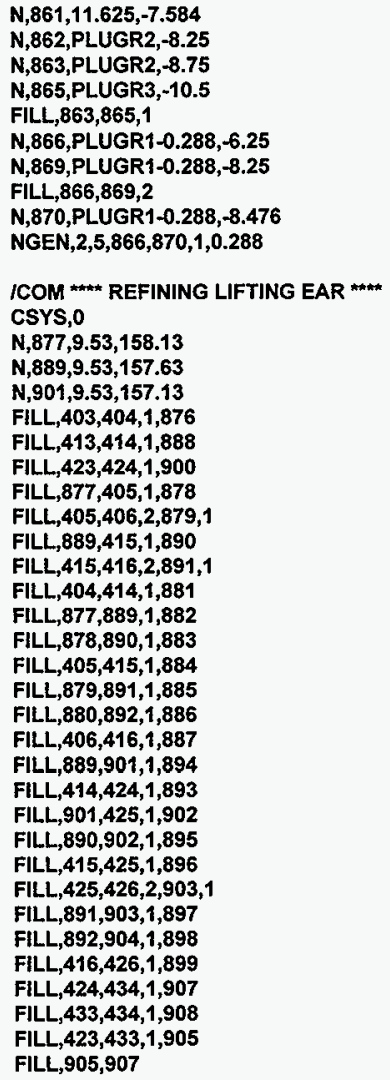

\begin{tabular}{|l|c|l|l|l|c|}
\hline REVISION & 0 & & & & PAGE 43 \\
PREPARED BY I DATE & OF 133 \\
\cline { 1 - 4 } CHECKED BY I DATE & $\mathbb{2} / 17 / 97 / 17 / 97$ & & & & \\
\hline
\end{tabular}


CLIENT: DUKE ENGINEERING \& SERVICES HANFORD, INC.

FILE NO: $\quad \mathrm{KH}-8009-8-09$

PROJECT: MCO Final Design

DOC. NO.: HNF-SD-SNF-DR-003, Rev. 0, Appendix 11

CP,9,UY,447,911

CP, 10,UX,447,911

*DO,l,1,7

$C P, 10+1, U Y, 445+10 * \mid, 910+2 * 1$

*ENDDO

*DO,I,1,7

$\mathrm{CP}, 17+1, \mathrm{UY}, 447+10^{*} 1,911+2 * \mid$

^ENDDO

"DO, I, 1,7

$C P, 24+1, U X, 445+10^{\star} \mid, 910+2 * 1$

*ENDDO

"DO, $, 1,7$

$C P, 31+1, U X, 447+10^{\star} 1,911+2 * \mid$

*ENDDO

NALL.

EALL

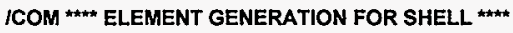

TYPE, 1

MAT,1

ICOM *** BOTTOM OF SHELL *

$E, 1,2,22,21$

$E, 2,3,23,22$

EGEN,8,1,-1

$E, 10,11,30$

$E, 21,22,42,41$

$E, 22,23,43,42$

EGEN,10,1,-1

$E, 11,31,30$

$E, 11,32,31$

ICOM *n* SHELL ***

$\mathrm{E}, \mathbf{5 0 , 5 1 , 5 4 , 5 3}$

EGEN,2,1,-1

EGEN,5,3,-2

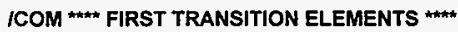

$E, 65,66,100$

$E, 100,66,101$

E,67,101,66

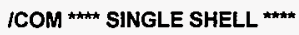

$E, 100,101,103,102$

EGEN,40,2,-1

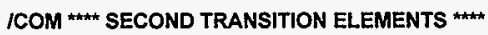

E,190,180,191

$E, 180,181,191$

$E, 181,192,191$

ICOM *** TOP SHELLL (DOUBLE ELEMENT)

$E, 190,191,194,193$

EGEN, $2,1,-1$

EGEN,18,3,-2

$E, 244,245,986,985$

\begin{tabular}{|l|c|l|l|l|}
\hline REVISION & 0 & & & \\
\hline PREPARED BY I DATE & S & & & \\
\hline CHECKED BY I DATE & / $/ 17 / 97$ & & & \\
\hline
\end{tabular}


CLIENT: DUKE ENGINEERING \& SERVICES HANFORD, INC.

FILE NO: KH-8009-8-09

PROJECT: MCO Final Design DOC. NO.: HNF-SD-SNF-DR-003, Rev. 0, Appendix 11

EGEN,2,1,-1

$E, 256,257,988,987$

$E, 257,258,989,988$

$E, 985,986,981,980$

EGEN,4,1,-1

$E, 980,981,248,247$

EGEN,2,1,-1

$E, 982,983,260,249$

$E, 983,984,261,260$

ICOM * COLLAR TRANSITION \& THREADED REGIONS ***

$E, 237,991,251,240$

$E, 991,990,251$

$E, 240,251,254,253$

$E, 251,990,255,254$

$E, 253,254,257,256$

EGEN,2,1,-1

$E, 259,260,263,262$

EGEN,2,1,-1

EGEN,12,3,-2

$E, 271,274,1000$

ICOM ** MERGE COINCIDENT NODES FOR SHELL

ESEL,S,TYPE,,1

NSLE

NUMMRG,NODE,

EALL

NALL

ICOM *ND OF SHELLCOLLAR ELEMENT GENERATION ***

ICOM * LOCKINGRIFTING RING ELEMENTS

TYPE, 3

MAT,1

$E, 411,412,402,401$

EGEN, 2,1,-1

EGEN,2,10,-2

$E, 413,888,876,403$

$E, 881,404,876$

$E, 888,881,876$

$E, 888,414,881$

$E, 881,882,877,404$

$E, 414,889,882,881$

$E, 882,883,878,877$

$E, 889,890,883,882$

$E, 883,884,405,878$

$E, 890,415,884,883$

$E, 884,885,879,405$

$E, 415,891,885,884$

$E, 885,886,880,879$

$E, 891,892,886,885$

$E, 886,887,406,880$

E,892,416,887,886

$E, 423,900,888,413$

$E, 893,414,888$

$E, 900,893,888$

$E, 900,424,893$

REVISION

PREPARED BY I DATE

CHECKED BYI DATE 
CLIENT: DUKE ENGINEERING \& SERVICES HANFORD, INC. FILE NO: KH-8009-8-09

PROJECT: MCO Final Design DOC. NO.: HNF-SD-SNF-DR-003, Rev. 0, Appendix 11

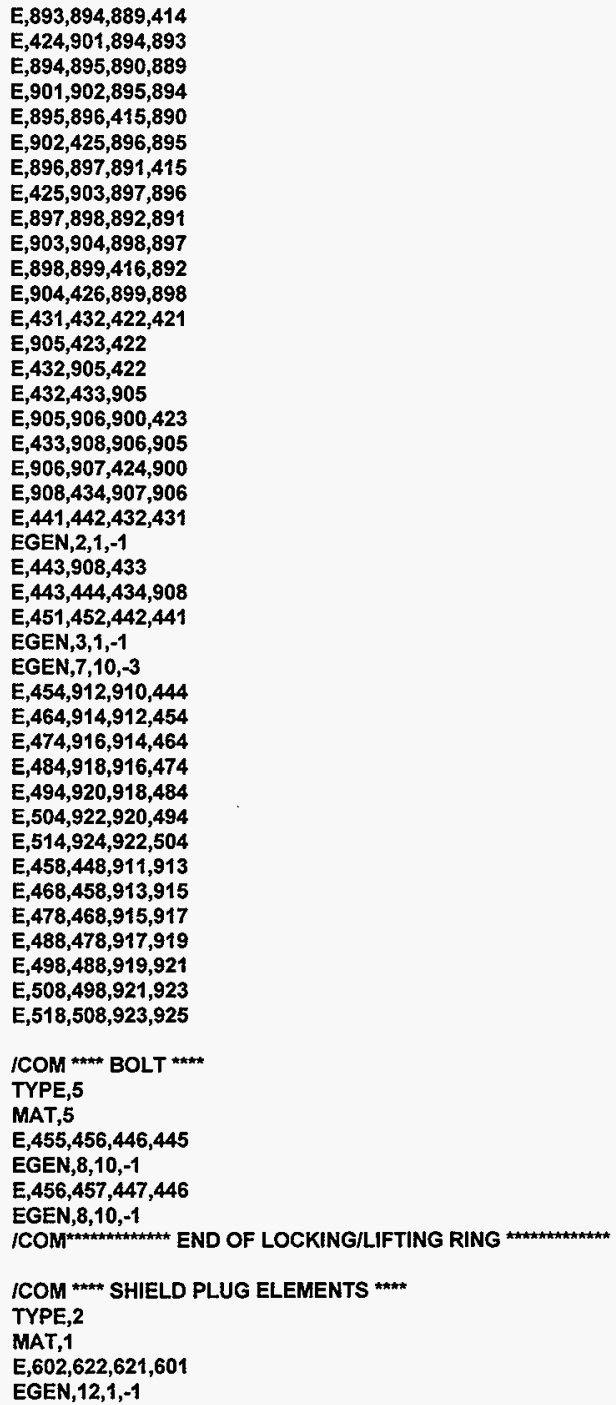


CLIENT: DUKE ENGINEERING \& SERVICES HANFORD, INC.

FILE NO: $\quad \mathrm{KH}-8009-8-09$

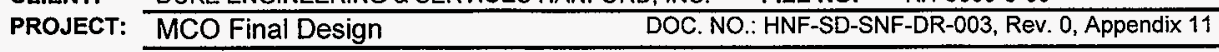

EGEN,2,20,-12

EGEN, $3,20,-11$

EGEN, 2,20,-10

$\mathrm{E}, 707,717,716,706$

EGEN, $7,1,-1$

E, $717,737,736,716$

EGEN,7,1,-1

E,731,751,750,730

EGEN,13,1,-1

$E, 749,769,768,748$

EGEN,15,1,-1

$\mathrm{E}, 767,787,786,766$

EGEN,17,1,-1

EGEN,2,20,-17

$E, 818,825,824,817$

EGEN,6,1,-1

EGEN, $5,7,-6$

$E, 853,860,859,852$

EGEN, 6,1,-1

$E, 860,867,866,859$

EGEN,3,1,-1

$E, 867,872,871,866$

EGEN,4,1,-1

ICOM END OF SHIELD PLUG

ICOM CONTACT ELEMENTS

ICOM * BETWEEN LOCKING RING \& SHIELD PLUG

TYPE, 4

REAL,4

$E, 806,401$

$E, 807,411$

$E, 808,421$

$E, 809,431$

$E, 810,441$

$E, 811,451$

$E, 812,461$

$E, 813,471$

$E, 814,481$

$E, 815,491$

$E, 816,501$

$E, 817,511$

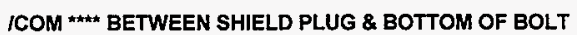

REAL, 5

$E, 845,525$

$E, 852,526$

$E, 859,527$

ICOM BATWEEN SHIELD PLUG \& SHELL (ABOVE SEAL)

REAL,4

E,871,271

$\mathrm{E}, 872,268$

E,873,265

$\mathrm{E}, 874,262$

ICOM ** BETWEEN SHIELD PLUG \& SHELL (BELOW SEAL)

REVISION

PREPARED BY IDATE

CHECKED BYIDATE

\begin{tabular}{|c|c|c|c|}
\hline 0 & & & \\
\hline $\mathcal{Z} S 4 / 17 / 97$ & & & \\
\hline $\operatorname{los} 4 / 17 / 97$ & & & \\
\hline
\end{tabular}


CLIENT: DUKE ENGINEERING \& SERVICES HANFORD, INC. FILE NO: KH-8009-8-09

PROJECT: MCO Final Design DOC. NO.: HNF-SD-SNF-DR-003, Rev. 0, Appendix 11

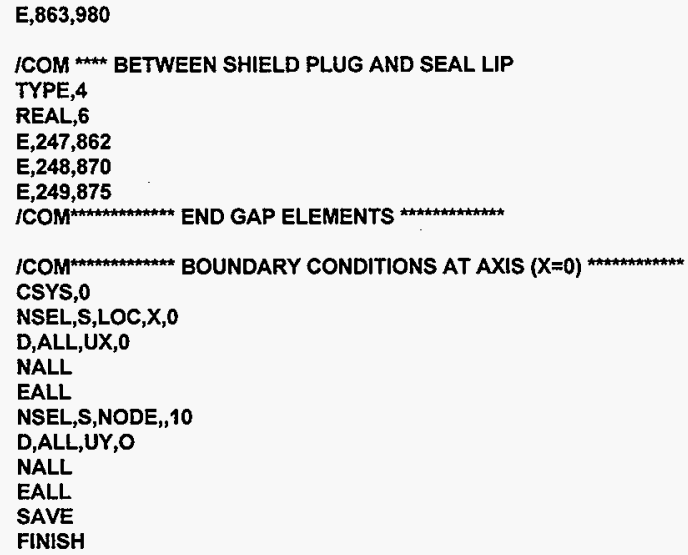

\begin{tabular}{|l|c|l|l|l|l|}
\hline REVISION & 0 & & & & PAGE 48 \\
\hline PREPARED BY I DATE & OF 133 \\
\hline CHECKED BY I DATE & T/L/4/17/9/97 & & & & \\
\hline
\end{tabular}


CLIENT: DUKE ENGINEERING \& SERVICES HANFORD, INC. FILE NO: KH-8009-8-09

PROJECT: MCO Final Design DOC. NO.: HNF-SD-SNF-DR-003, Rev. 0, Appendix 11

NSEL,R,LOC,Y,155.99,156.01

SF,ALL,PRES,40

NALL

EALL

NSEL,S,LOC, X,9.624,12.24 I Top of Locking Ring

NSEL,R,LOC, Y,155.99,156.01

SF,ALL,PRES,40

NALL

EALL

NSEL,S,LOC, X,9.374,9.626 I Transition to Lifting Ear

NSEL,R,LOC,Y,155.99,157.13

SF,ALL,PRES,40

NALL

EALL

NSEL,S,LOC,X,9.374,10.20 ! Underside of Lifting Ear

NSEL,R,LOC,Y,157.12,157.14

SF,ALL,PRES,40

NALL

EALL

NSEL,S,LOC,X,10.18,10.20 I Side of Lifting Ear

NSEL, R, LOC, $Y, 157.12,158.14$

SF,ALL,PRES, 40

NALL

EALL

NSEL,S,LOC,X,7.93,10.20 I Top of Lifting Ear

NSEL,R,LOC,Y,158.12,158.14

SF,ALL,PRES, 40

NALL

EALL

NSEL,S,LOC,X,7.25,7.90 ! Top of Shield plug

NSEL, R,LOC, $Y, 158.12,158.14$

SF,ALL,PRES, 40

NALL

EALL

NSEL,S,LOC,X,7,26,7,27 I Side of Siphon Port (Top)

NSEL,R,LOC, Y,157.12,158.14

SF,ALL,PRES, 40

NALL

EALL

NSEL,S,LOC,X,5.88,7.27 I Siphon Port Step (Top)

NSEL,R,LOC,Y,157.12,157.14

SF,ALL,PRES, 40

NALL

EALL

NSEL,S,LOC,X,5.88,5.90 ! Side of Siphon Port (Mid)

NSEL,R,LOC,Y,156.13,157.14

SF,ALL,PRES,40

NALL

EAL.L

NSEL,S,LOC,X,5.4,5.9 I Siphon Port Step (Mid)

NSEL,R,LOC, $Y, 156.13,156.15$

SF,ALL,PRES, 40

NALL

EALL.

NSEL,S,LOC, X, 5.46,5.47

NSEL,R,LOC,Y,153.0,156.37

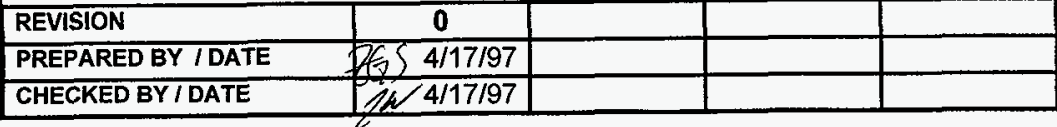


CLIENT: DUKE ENGINEERING \& SERVICES HANFORD, INC.

FILE NO: KH-8009-8-09

PROJECT: MCO Final Design

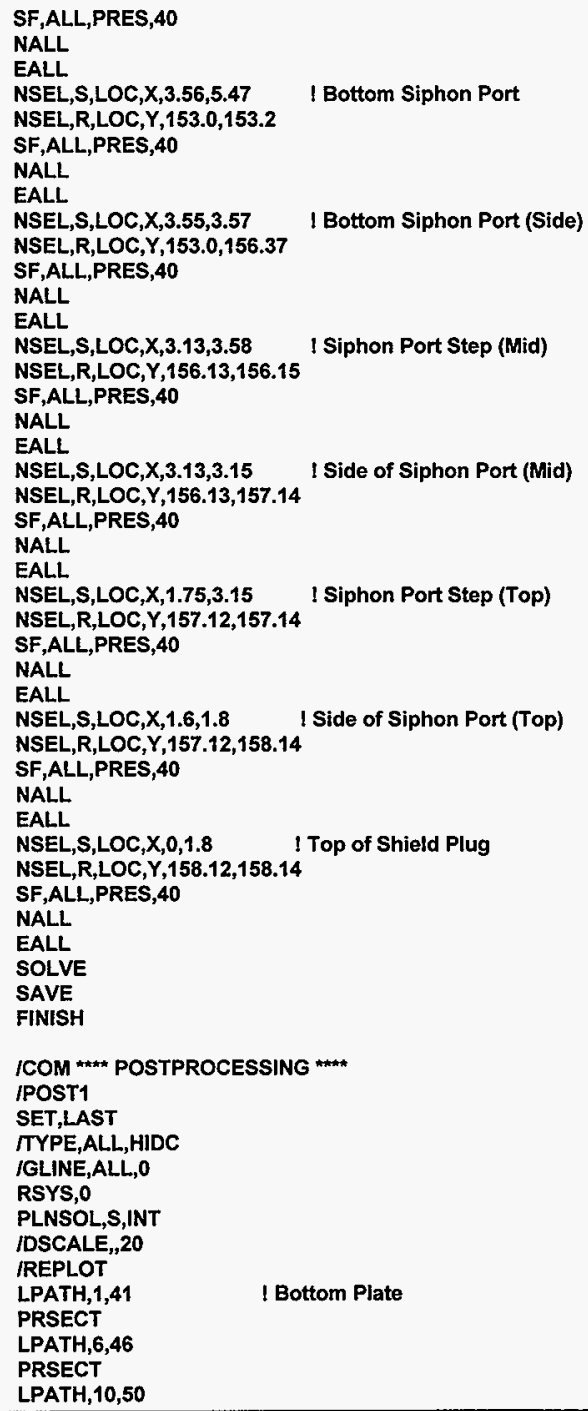

\begin{tabular}{|c|c|}
\hline REVISION & 0 \\
\hline PREPARED BY / DATE & the $4 / 17 / 97$ \\
\hline CHECKED BY/DATE & Se / 4/17/97 \\
\hline
\end{tabular}


CLIENT: $\quad$ DUKE ENGINEERING \& SERVICES HANFORD, INC. FILE NO: KH-8009-8-09

PROJECT: MCO Final Design DOC. NO.: HNF-SD-SNF-DR-003, Rev. 0, Appendix 11

PRSECT

LPATH, 50,52

PRSECT

LPATH, 62,64

PRSECT

LPATH, 65,67

PRSECT

LPATH,100,101

PRSECT

LPATH, 122,123

PRSECT

LPATH, 134,135

PRSECT

LPATH,156,157

PRSECT

LPATH, 170,171

PRSECT

LPATH, 180,181

PRSECT

LPATH,202,204

PRSECT

LPATH,235,237

PRSECT

LPATH, 985,989

PRSECT

LPATH,262,264

PRSECT

LPATH,277,279

PRSECT

LPATH,292,294

PRSECT

LPATH,601,641

PRSECT

LPATH, 601,613

PRSECT

LPATH, 603,703

PRSECT

LPATH,606,706

PRSECT

LPATH,766,806

PRSECT

LPATH,748,808

PRSECT

LPATH, 730,810

PRSECT

LPATH,736,815

PRSECT

LPATH,869,874

PRSECT

LPATH,870,875

PRSECT

LPATH,431,434

PRSECT

LPATH,406,426

PRSECT

LPATH,404,424

REVISION

PREPARED BY / DATE

CHECKED BY / DATE
! Lower Shell

! Mid Shell

I Upper Shell

I Shield Plug

! Locking Ring 
CLIENT: $\quad$ DUKE ENGINEERING \& SERVICES HANFORD, INC. FILE NO: KH-8009-8-09

PROJECT: MCO Final Design DOC. NO.: HNF-SD-SNF-DR-003, Rev. 0, Appendix 11

PRSECT

SAVE 
CLIENT: DUKE ENGINEERING \& SERVICES HANFORD, INC. FILE NO: KH-8009-8-09

$\begin{array}{ll}\text { PROJECT: MCO Final Design } & \text { DOC. NO.: HNF-SD-SNF-DR-003, Rev. 0, Appendix } 11\end{array}$

\section{COMPUTER RUN COVER SHEET}

Project Number:

$\mathrm{KH}-8009-8$

Computer Code:

ANSYS 8 -PC

Software Version:

$5.0 \mathrm{~A}$

Computer System:

MS-DOS, Pentium® Processor

Computer Run File Number:

KH-8009-8-09

Unique Computer Run Filename:

POC1.out

Run Description:

Load Case 1 Output

Run Date / Time:

27 March 1997

2:11:24 PM

Prepared By: Zachary G. Sargent
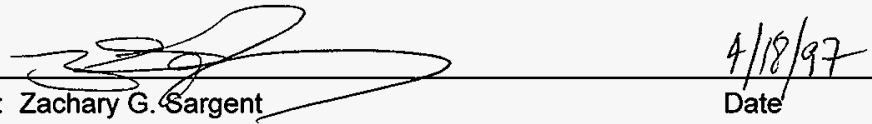

Checked By: Joe Nichols

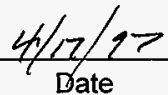


CLIENT: DUKE ENGINEERING \& SERVICES HANFORD, INC.

FILE NO: KH-8009-8-09

PROJECT: MCO Final Design DOC. NO.: HNF-SD-SNF-DR-003, Rev. 0, Appendix 11

\section{COMPUTER RUN COVER SHEET}

Project Number:

$\mathrm{KH}-8009-8$

Computer Code:

ANSYS\&-PC

Software Version:

$5.0 \mathrm{~A}$

Computer System:

MS-DOS, Pentium® Processor

Computer Run File Number:

KH-8009-8-09

Unique Computer Run Filename:

POC2.inp

Run Description:

Load Case 2: $15 \mathrm{psi}, 375^{\circ} \mathrm{C}$

Creation Date / Time:

27 March $1997 \quad$ 2:09:06 PM
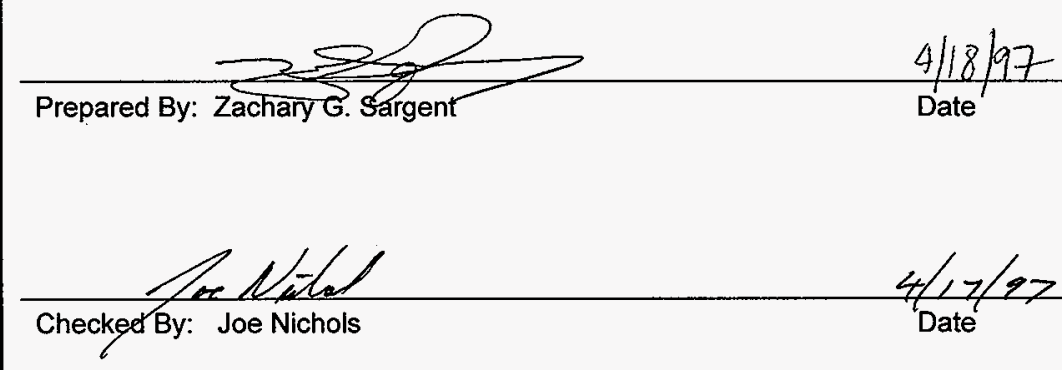

\begin{tabular}{|c|l|l|l|}
\hline 0 & & & \\
\hline $4 / 17 / 97$ & & & \\
\hline $4 / 17 / 97$ & & & \\
\hline
\end{tabular}


CLIENT: DUKE ENGINEERING \& SERVICES HANFORD, INC.

FILE NO: $\quad \mathrm{KH}-8009-8-09$

PROJECT: MCO Final Design DOC. NO.: HNF-SD-SNF-DR-003, Rev. 0, Appendix 11

\section{LISTING OF POC2.INP FILE}

IBATCH,LIST

IFILENAM,POC2

IPREP7

TITLE,MCO DESIGN- 375 DEGREES C, 15 PSI EXTERNAL PRESSURE, NO LIFT

TREF,70

TUNIF,707

\begin{tabular}{|c|c|}
\hline \multicolumn{2}{|c|}{$I^{\prime C O M} M^{\star \star \star \star}$ ELEMENT TYPES ${ }^{\star \star \star \star}$} \\
\hline $\mathrm{ET}, 1,42,, \ldots 1$ & I Shell \\
\hline ET $, 2,42, \ldots 1$ & I Shield Plug \\
\hline$E T, 3,42, \ldots 1$ & I Lifting \& Locking Ring \\
\hline$E T, 4,12$ & I Gap Elements Between Shield Plug \& Shell \\
\hline $\begin{array}{l}\text { KEYOPT,4,7,1 } \\
\text { ET,5,42,,1 }\end{array}$ & I Bolt \\
\hline
\end{tabular}

ICOM REAL CONSTANTS FOR GAP ELEMENTS ****

R,4,-90,1.0e8,-0.06,3.0 ! Shell/Shield Plug, Initially Open .06"

R,5,0,1.0e8,2.95e-03 I L. Ring/Shield Plug, Under Boit, Preloaded

$R, 6,0,1.0 E 8,0,2.0 \quad$ I Sealing Surface, closed

ICOM MATERIAL PROPERTIES

MP,DENS,1,490/1728 I 304L SS

MP,NUXY,1,0.3

MP,DENS,5,490/1728 I SA193 Grade B8M

MP,NUXY,5,0.3

ICOM DEFINING TEMPERATURES FOR MPDATA

MPTEMP, 1, 70,100,200,300,400,500

MPTEMP, $7,600,650,700,750$

ICOM *** DEFINING ELASTIC MODULI FOR 304L \& SA-193

MPDATA,EX,1,1,28.3e+06,28.1e+06,27.6e+06,27.0e+06,26.5e+06,25.8e+06

MPDATA,EX, 1,7,25.3e+06,25.1e+06,24.8e+06,24.5e+06

ICOM ISA-193

MPDATA,EX,5, $1,28.3 e+06,28.1 e+06,27.6 e+06,27.0 e+06,26.5 e+06,25.8 e+06$

MPDATA,EX,5,7,25.3e+06,25.1e+06,24.8e+06,24.5e+06

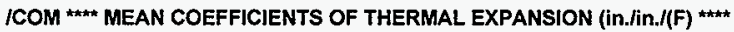

I SA240 Gr 304L

MPDATA,ALPX,1, 1,0,8.55e-06,8.79e-06,9.00e-06,9.19e-06,9.37e-06

MPDATA,ALPX,1,7,9.53e-06,9.61e-06,9.69e-06,9.76e-06

1 SA193 Gr B8M

MPDATA,ALPX,5,1,0,8.54e-06,8.76e-06,8.97e-06,9.21e-06,9.42e-06

MPDATA,ALPX,5,7,9.60e-06,9.69e-06,9.76e-06,9.81e-06

ICOM SHELL GEOMETRY

IR=11.5 I Internal Shell Radius @ Bottom

$O R=12.000$

IR2 $=\mathbf{1 2 . 0 2}$

$O R 2=12.625$

1 Shell Outside Radius @ Bottom

I Inside Radius at Coltar Sealing Surface

1 Outside Radius at Collar Sealing Surface

\begin{tabular}{|l|c|l|l|l|}
\hline REVISION & 0 & & & \\
\hline PREPARED BY I DATE & f & & & \\
\hline CHECKED B/17/97 & & & \\
\hline
\end{tabular}


CLIENT: $\quad$ DUKE ENGINEERING \& SERVICES HANFORD, INC. FILE NO: KH-8009-8-09 PROJECT: MCO Final Design DOC. NO.: HNF-SD-SNF-DR-003, Rev. 0, Appendix 11

IR3 = $12.25 \quad$ I Inside Radius at Collar-Lifting Ring Weld

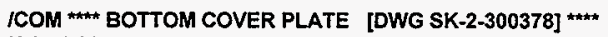

$N, 1,-1,32$

I Row 1

$\mathbf{N}, 2,1.25,-1.32$

$\mathbf{N}, 3,2.13,-1.32$

N, 10,11.423,-1.32

FILL.

$$
\begin{aligned}
& \mathrm{N}, \mathbf{4 1}, \mathbf{0 . 0 0},-0.44 \\
& \mathrm{~N}, \mathbf{4 2}, 1.25,-0.44 \\
& \mathrm{~N}, \mathbf{4 3}, \mathbf{2 . 1 3 , 0 . 4 4} \\
& \mathrm{N}, \mathbf{5 0}, \mathrm{IR}, \mathbf{0 . 4 4} \\
& \text { FILL, } 43,50 \\
& \text { N, 52, OR, } 0.44 \\
& \text { FILL,50,52 }
\end{aligned}
$$

FILL, $1,41,1,21,1,10$

FILL, 10,50,1,30

$\mathrm{N}, \mathbf{3 2 , 1 2 , - 0 . 3 2}$

FILL,30,32

FILL, 10,32,1,11

$\mathrm{N}, \mathbf{5 3}, \mathrm{IR}, \mathbf{1 . 1 7}$

$\mathrm{N}, 55, \mathrm{OR}, 1.17$

FILL, 53,55
1 Row 3

! Middle Row

\section{Shell Stub/Weld}

ICOM SHELL [DWGS SK-2-300379 \& SK-2-300461]

$\mathrm{N}, 65, \mathrm{IR}, 6.68$

$N, 67,0 R, 6.68$

FILLL

FILLL, $53,65,3,3,3,1$

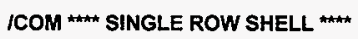

$N, 100, I R, 7.18$

$\mathrm{N}, 140, \mathrm{IR}, 71.68$

$\mathrm{N}, \mathbf{1 8 0}, \mathrm{IR}, \mathbf{1 3 6 . 6 8}$

N,101, OR,7.18

$\mathrm{N}, 141, \mathrm{OR}, 71.68$

$\mathrm{N}, \mathbf{1 8 1}, \mathrm{OR}, 136.68$

FILL, 100,140,20,2,2,1,2.0

FILL, $140,180,19,2,2,1, .5$

ICOM DOUBLE ROW SHELL

$\mathrm{N}, \mathbf{1 9 0 , I R , 1 3 7 . 1 8}$

I Transition to Double Row

$\mathrm{N}, \mathbf{1 9 2 , O R , 1 3 7 . 1 8}$

FILL

ICOM BASE OF CASK THROAT--ELEVATION: 138 INCHES

$N, 217, I R, 142.68$

$\mathrm{N}, \mathbf{2 1 9}, \mathrm{OR}, 142.68$

FILL

FILL, 190,217,8,3,3,1 I Vertical Fill

ICOM BOTTOM OF COLLAR TRANSITION *

$\mathrm{N}, \mathbf{2 3 5}, \mathrm{IR}, \mathbf{1 4 6 . 0 6}$

I Start of Transition to Large O.D \&

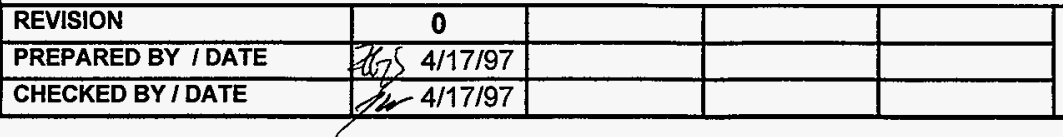


CLIENT: DUKE ENGINEERING \& SERVICES HANFORD, INC.

FILE NO: KH-8009-8-09

PROJECT: MCO Final Design DOC. NO.: HNF-SD-SNF-DR-003, Rev. 0, Appendix 11

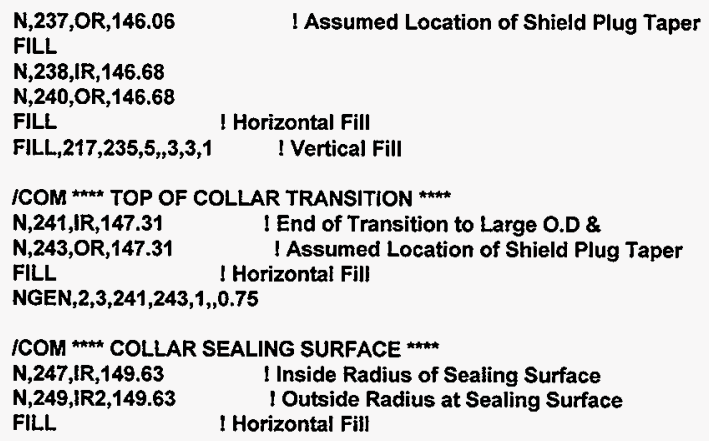

\begin{tabular}{|l|c|l|l|l|}
\hline REVISION & 0 & & & \\
\hline PREPARED BY IDATE & S 4/17/97 & & & \\
\hline CHECKED BY I DATE & L 4/17/97 & & & \\
\hline
\end{tabular}


CLIENT: DUKE ENGINEERING \& SERVICES HANFORD, INC. FILE NO: KH-8009-8-09

PROJECT: MCO Final Design DOC. NO.: HNF-SD-SNF-DR-003, Rev. 0, Appendix 11

RING4=10.19

RING5 $=12.23$

LOCAL, $11,0,152.00 \quad$ ! Local System $z=0$ at Base of Ring

CSYS, 11

ICOM *** TOP EDGE ***

$N, 401$, RING1,6.13

CSYS,O

$\mathrm{N}, 404,9.375,158.13$

FILL, 401,404,,1

N,406,RING4,158.13

FILL,404,406,,,1 I Top Edge

ICOM LIFTING SURFACE

CSYS, 11

N,421, RING1,5.13

N,424,RING2,5.13

FILL, 421, 424

N,426,RING4,5.13

FILL, 424,426

FILL,401,421,1, 10,6,1

N,431,RING1,6.13-1.56

N,434,RING2,6.13-1.56

FILL

ICOM BOLTING SURFACE *\#*

N,441,RING1,4

N,444,RING3,4

FILL

N,445,10.9375-.6875,4 ! Inside Edge of Bolt Hole

$\mathbf{N}, 447,10.9375+.6875,4 \quad$ ! Outside Edge of Bolt Hole

FILL

$N, 910,10.9375-6875,4$

$\mathrm{N}, 911,10.9375+.6875,4$

N,448,RING5,4

CSYS, 0

$\mathrm{N}, 924,10.25,152.00$

1 O.D of Ring

I Bolt Extension

$\mathrm{N}, 925,11.625,152.00$

1 Double Nodes@Bolt for Gap elements

FILL, $910,924,6,2$

FILL, $911,925,6,2$

$\mathrm{N}, 525,10.25,151.874$

$N, 527,11.625,151.874$

! Bottom of Boit Extension

FILL

ICOM BOTTOM OF LIFTINGILOCKING RING ***

CSYS, 11

NGEN,2,70,441,448,1,-4 I Bottom Surface of Lifting/Locking Ring

FILL,441,511,6,10,8,1 I Fill in Lifting/Locking Ring

ICOM SHIELD PLUG (offset y by 158.25)

LOCAL,20,0,,158.13

TYPE, 2

PLUGR1=11.975

PLUGR2 $=11.45$

PLUGR3=11.25

PLUGR4=7.89

\begin{tabular}{|l|c|c|c|c|c|}
\hline REVISION & 0 & & & PAGE 58 \\
PREPARED BY IDATE & OF 133 & \\
\cline { 1 - 4 } CHECKED BY I DATE & 4/17/97 & & & & \\
\hline
\end{tabular}


CLIENT: DUKE ENGINEERING \& SERVICES HANFORD, INC. FILE NO: KH-8009-8-09

PROJECT: MCO Final Design DOC. NO.: HNF-SD-SNF-DR-003, Rev. 0, Appendix 11

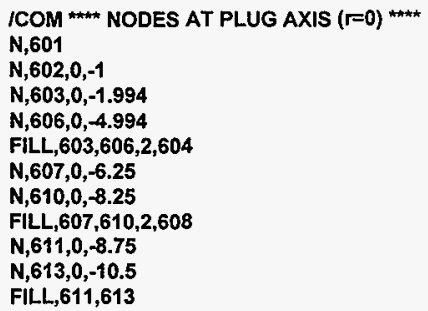

\section{Id Large Opening}

IId Medium Opening

IId Small Opening

! Center of Opening

IOd Small Opening 
CLIENT: $\quad$ DUKE ENGINEERING \& SERVICES HANFORD, INC. FILE NO: KH-8009-8-09

PROJECT: MCO Final Design DOC. NO.: HNF-SD-SNF-DR-003, Rev. 0, Appendix.11

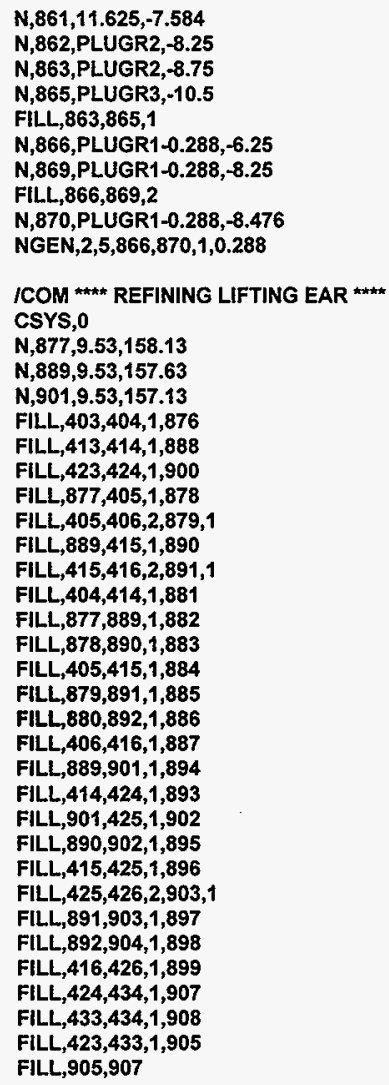

\begin{tabular}{|l|c|l|l|l|l|}
\hline REVISION & 0 & & & & PAGE 60 \\
PREPARED BY I DATE & OF 133 \\
\hline CHECKED BYI DATE & $4 / 17 / 97$ & & & & \\
\hline
\end{tabular}


CLIENT: $\quad$ DUKE ENGINEERING \& SERVICES HANFORD, INC. FILE NO: KH-8009-8-09

PROJECT: MCO Final Design DOC. NO.: HNF-SD-SNF-DR-003, Rev. 0, Appendix 11

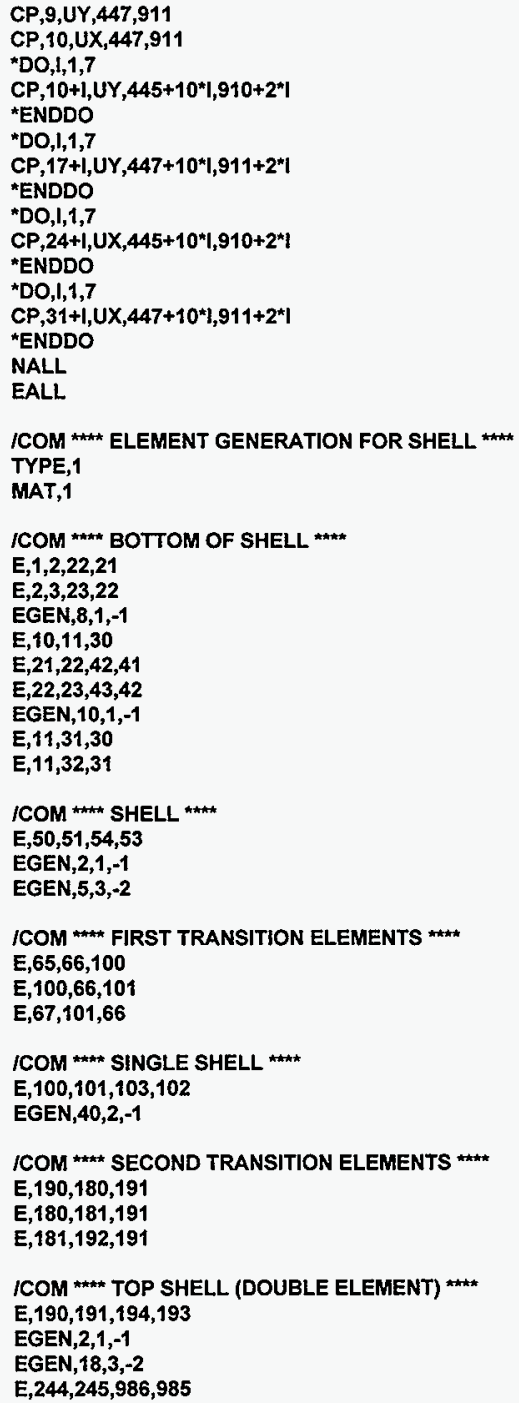


CLIENT: DUKE ENGINEERING \& SERVICES HANFASDNS

FILE NO: $\quad \mathrm{KH}-8009-8-09$

PROJECT: MCO Final Design

DOC. NO.: HNF-SD-SNF-DR-003, Rev, 0, Appendix 11

\author{
EGEN,2,1,-1 \\ $E, 256,257,988,987$ \\ $E, 257,258,989,988$ \\ $E, 985,986,981,980$ \\ EGEN,4,1,-1 \\ $E, 980,981,248,247$ \\ EGEN,2,1,-1 \\ $E, 982,983,260,249$ \\ $E, 983,984,261,260$
}

ICOM COLLAR TRANSITION \& THREADED REGIONS \#

$E, 237,991,251,240$

$E, 991,990,251$

$E, 240,251,254,253$

$E, 251,990,255,254$

E,253,254,257,256

EGEN, 2,1,-1

$E, 259,260,263,262$

EGEN, 2,1,-1

EGEN, 12,3,-2

$E, 271,274,1000$

ICOM MERGE COINCIDENT NODES FOR SHELL

ESEL,S,TYPE,,1

NSLE

NUMMRG,NODE,

EALL

NALL

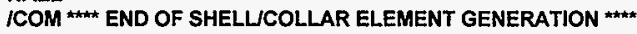

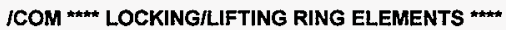

TYPE, 3

MAT,1

$E, 411,412,402,401$

EGEN,2,1,-1

EGEN, 2,10,-2

$E, 413,888,876,403$

$E, 881,404,876$

$E, 888,881,876$

$E, 888,414,881$

$E, 881,882,877,404$

$E, \mathbf{4 1 4 , 8 8 9 , 8 8 2 , 8 8 1}$

$E, 882,883,878,877$

$E, 889,890,883,882$

$E, 883,884,405,878$

$E, 890,415,884,883$

$E, 884,885,879,405$

$E, 415,891,885,884$

E,885,886,880,879

$E, 891,892,886,885$

$\mathrm{E}, \mathbf{8 8 6 , 8 8 7 , 4 0 6 , 8 8 0}$

$E, 892,416,887,886$

$E, 423,900,888,413$

$E, 893,414,888$

E, $900,893,888$

$E, 900,424,893$

REVISION

PREPARED BY / DATE

CHECKED BYIDATE 
CLIENT: $\quad$ DUKE ENGINEERING \& SERVICES HANFORD, INC. FILE NO: KH-8009-8-09 PROJECT: MCO Final Design DOC. NO.: HNF-SD-SNF-DR-003, Rev. 0, Appendix 11

$E, 893,894,889,414$

$E, 424,901,894,893$

$E, 894,895,890,889$

$E, 901,902,895,894$

$E, 895,896,415,890$

$E, 902,425,896,895$

$E, 896,897,891,415$

$E, 425,903,897,896$

$E, 897,898,892,891$

E,903,904,898,897

$E, 898,899,416,892$

E,904,426,899,898

$E, 431,432,422,421$

E, $905,423,422$

$E, 432,905,422$

$E, 432,433,905$

$E, 905,906,900,423$

$E, 433,908,906,905$

E,906,907,424,900

E,908,434,907,906

$E, 441,442,432,431$

EGEN, 2,1,-1

$E, 443,908,433$

$E, 443,444,434,908$

$E, 451,452,442,441$

EGEN,3,1,-1

EGEN, 7,10,-3

E,454,912,910,444

E,464,914,912,454

$E, 474,916,914,464$

$E, 484,918,916,474$

$E, 494,920,918,484$

$E, 504,922,920,494$

$E, 514,924,922,504$

$E, 458,448,911,913$

$E, 468,458,913,915$

$E, 478,468,915,917$

$E, 488,478,917,919$

$E, 498,488,919,921$

E,508,498,921,923

$E, 518,508,923,925$

ICOM *** BOLT ***

TYPE, 5

MAT, 5

E,455,456,446,445

EGEN,8,10,-1

$E, 456,457,447,446$

EGEN,8,10,-1

ICOM END OF LOCKING/LIFTING RING

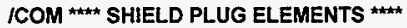

TYPE, 2

MAT, 1

$E, 602,622,621,601$

EGEN,12,1,-1

\begin{tabular}{|l|c|l|l|l|}
\hline REVISION & 0 & & & \\
\hline PREPARED BY I DATE & K//S 4/17/97 & & & \\
\hline CHECKED BY I DATE & /h/ $/ 4 / 17 / 97$ & & & \\
\hline
\end{tabular}

PAGE 63

OF 133 
CLIENT: $\quad$ DUKE ENGINEERING \& SERVICES HANFORD, INC. FILE NO: KH-8009-8-09

PROJECT: MCO Final Design DOC. NO.: HNF-SD-SNF-DR-003, Rev. 0, Appendix 11

EGEN,2,20,-12

EGEN,3,20,-11

EGEN,2,20,-10

$E, 707,717,716,706$

EGEN, $7,1,-1$

E, 717,737,736,716

EGEN, $7,1,-1$

$E, 731,751,750,730$

EGEN,13,1,-1

E,749,769,768,748

EGEN,15,1,-1

$E, 767,787,786,766$

EGEN,17,1,-1

EGEN,2,20,-17

E,818,825,824,817

EGEN, $6,1,-1$

EGEN, $5,7,-6$

$E, 853,860,859,852$

EGEN, 6,1,-1

E, 860,867,866,859

EGEN, 3,1,-1

$E, 867,872,871,866$

EGEN,4,1,-1

ICOM END OF SHIELD PLUG

ICOM CONTACT ELEMENTS

ICOM *\#" BETWEEN LOCKING RING \& SHIELD PLUG

TYPE, 4

REAL, 4

$E, 806,401$

$E, 807,411$

$E, 808,421$

$E, 809,431$

$E, 810,441$

$E, 811,451$

$E, 8+2,461$

$E, 813,471$

$E, 814,481$

$E, 815,491$

$E, 816,501$

$E, 817,511$

ICOM ** BETWEEN SHIELD PLUG \& BOTTOM OF BOLT

REAL,5

E,845,525

$E, 852,526$

$E, 859,527$

ICOM BETWEEN SHIELD PLUG \& SHELL (ABOVE SEAL)

REAL,4

$E, 871,271$

$E, 872,268$

$E, 873,265$

$E, 874,262$

ICOM ** BETWEEN SHIELD PLUG \& SHELL (BELOW SEAL)

\begin{tabular}{|l|c|l|l|l|}
\hline REVISION & 0 & & & \\
\hline PREPARED BY IDATE & S 4/17/97 & & & \\
\hline CHECKED BY I DATE & $\operatorname{se~4/17/97}$ & & & \\
\hline
\end{tabular}


CLIENT: $\quad$ DUKE ENGINEERING \& SERVICES HANFORD, INC.

FILE NO: KH-8009-8-09

PROJECT: MCO Final Design DOC. NO.: HNF-SD-SNF-DR-003, Rev. 0, Appendix 11

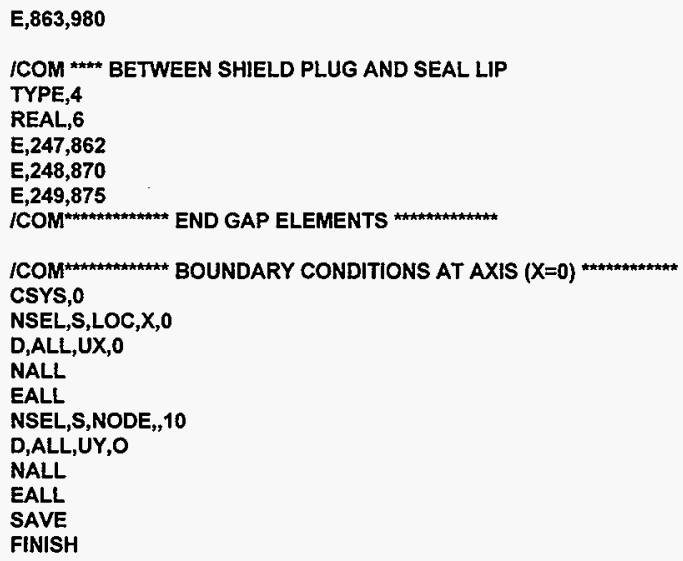


CLIENT: DUKE ENGINEERING \& SERVICES HANFORD, INC.

FILE NO: $\quad \mathrm{KH}-8009-8-09$

PROJECT: MCO Final Design DOC. NO.: HNF-SD-SNF-DR-003, Rev. 0, Appendix 11

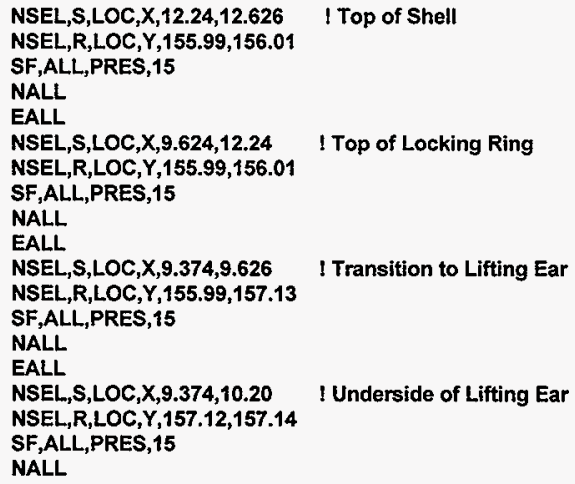

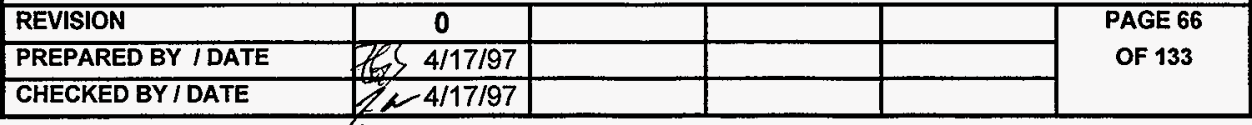


CLIENT: DUKE ENGINEERING \& SERVICES HANFORD, INC.

NSEL,R,LOC,Y,153.0,156.37

SF,ALL,PRES, 15

NALL

EALL

NSEL,S,LOC,X,3.56,5.47 ! Bottom Siphon Port

NSEL,R,LOC,Y,153.0,153.2

SF,ALL,PRES, 15

NALL

EALL

NSEL,S,LOC,X,3.55,3.57 ! Bottom Siphon Port (Side)

NSEL,R,LOC,Y,153.0,156.37

SF,ALL,PRES, 15

NALL

EALL

NSEL,S,LOC,X,3.13,3.58 I Siphon Port Step (Mid)

NSEL,R,LOC,Y,156.13,156.15

SF,ALL,PRES, 15

NALL

EALL

NSEL, S,LOC,X,3.13,3.15 I Side of Siphon Port (Mid)

NSEL,R,LOC,Y,156.13,157.14

SF,ALL,PRES, 15

NALL.

EALL

NSEL,S,LOC,X,1.75,3.15 I Siphon Port Step (Top)

NSEL,R,LOC,Y,157.12,157.14

SF,ALL,PRES, 15

NALL

EALL

NSEL,S,LOC,X,1.6,1.8 ISide of Siphon Port (Top)

NSEL,R,LOC,Y,157.12,158.14

SF,ALL,PRES, 15

NALL

EALL

NSEL, $S, L O C, X, 0,1.8 \quad$ I Top of Shield Plug

NSEL, R,LOC, Y,158.12,158.14

SF,ALL,PRES, 15

NALL.

EALL

SOLVE

SAVE

FINISH

ICOM POSTPROCESSING

IPOST1

SET,LAST

ITYPE,ALL,HIDC

IGLINE,ALL,O

RSYS,0

PLNSOL,S,INT

IDSCALE,,20

IREPLOT

LPATH, 1,41

PRSECT

LPATH, 6,46

REVISION

PREPARED BY / DATE

I Bottom Plate

CHECKED BY I DATE 
CLIENT: DUKE ENGINEERING \& SERVICES HANFORD, INC. FILE NO: KH-8009-8-09 PROJECT: MCO Final Design DOC. NO.: HNF-SD-SNF-DR-003, Rev. 0, Appendix 11

\section{PRSECT}

LPATH, 10,50

PRSECT

LPATH, 50,52

I Lower Shell

PRSECT

LPATH,62,64

PRSECT

LPATH, 65,67

PRSECT

LPATH,100,101

PRSECT

LPATH, 122,123

PRSECT

LPATH, 134,135

PRSECT

LPATH,156,157

PRSECT

LPATH,170,171

PRSECT

LPATH,180,181

PRSECT

LPATH,202,204

PRSECT

LPATH, 235,237

PRSECT

LPATH,985,989

PRSECT

LPATH,262,264

PRSECT

LPATH,277,279

PRSECT

LPATH,292,294

PRSECT

LPATH,601,641

PRSECT

L.PATH,601,613

PRSECT

LPATH, 603,703

PRSECT

LPATH,606,706

PRSECT

LPATH, 766,806

PRSECT

LPATH, 748,808

PRSECT

LPATH,730,810

PRSECT

LPATH;736,815

PRSECT

LPATH, 869,874

PRSECT

LPATH, 870,875

PRSECT

LPATH,431,434

PRSECT

! Mid Shell

I Upper Shell

I Shield Plug

LPATH, 406,426

\begin{tabular}{|l|c|l|l|l|}
\hline REVISION & 0 & & & \\
\hline PREPARED BY I DATE & I/ $/$ S 4/17/97 & & & \\
\hline CHECKED BY I DATE & / $/ 1 / 17 / 97$ & & & \\
\hline
\end{tabular}


CLIENT: $\quad$ DUKE ENGINEERING \& SERVICES HANFORD, INC.

FILE NO: KH-8009-8-09

\begin{tabular}{lll} 
PROJECT: MCO Final Design & DOC. NO.: HNF-SD-SNF-DR-003, Rev. 0, Appendix 11 \\
\cline { 2 - 3 }
\end{tabular}

PRSECT

LPATH,404,424

PRSECT

PRSECT

SAVE

प

\begin{tabular}{|l|l|l|}
\hline & & \\
\hline & & \\
\hline
\end{tabular}


CLIENT: DUKE ENGINEERING \& SERVICES HANFORD, INC.

FILE NO: $\quad \mathrm{KH}-8009-8-09$

PROJECT: MCO Final Design DOC. NO.: HNF-SD-SNF-DR-003, Rev. 0, Appendix 11

\section{COMPUTER RUN COVER SHEET}

Project Number:

Computer Code:

Software Version:

Computer System:

Computer Run File Number:

Unique Computer Run Filename:

Run Description:

Run Date / Time:
KH-8009-8

ANSYS®-PC

$5.0 \mathrm{~A}$

MS-DOS, Pentium® Processor

KH-8009-8-09

POC2.out

Load Case 2 Output

27 March 1997

2:16:10 PM
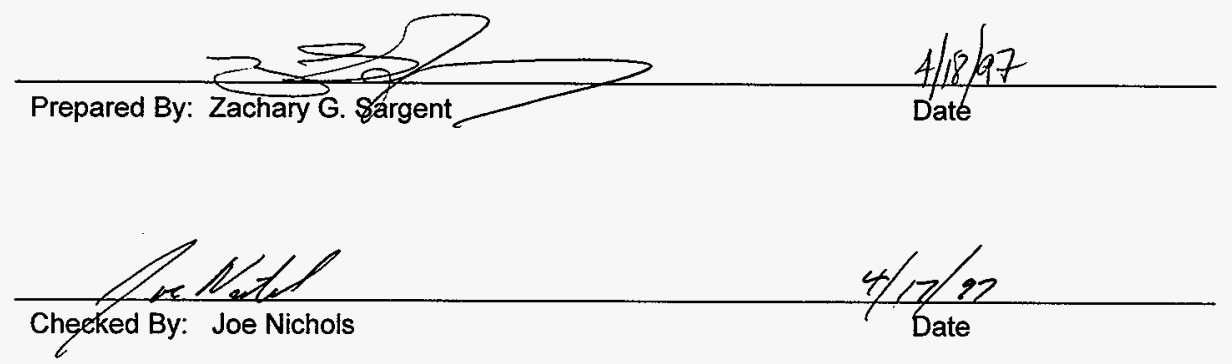
CLIENT: $\quad$ DUKE ENGINEERING \& SERVICES HANFORD, INC. FILE NO: KH-8009-8-09

PROJECT: MCO Final Design $\quad$ DOC. NO.: HNF-SD-SNF-DR-003, Rev. 0, Appendix 11

\section{COMPUTER RUN COVER SHEET}

Project Number:

$\mathrm{KH}-8009-8$

Computer Code:

ANSYSB-PC

Software Version:

$5.0 \mathrm{~A}$

Computer System:

MS-DOS, Pentium(B Processor

Computer Run File Number:

KH-8009-8-09

Unique Computer Run Filename:

MCO375.inp

Run Description:

Load Case 3: $189 \mathrm{psi}, 375^{\circ} \mathrm{C}$

Creation Date / Time:

27 March $1997 \quad$ 2:08:16 PM
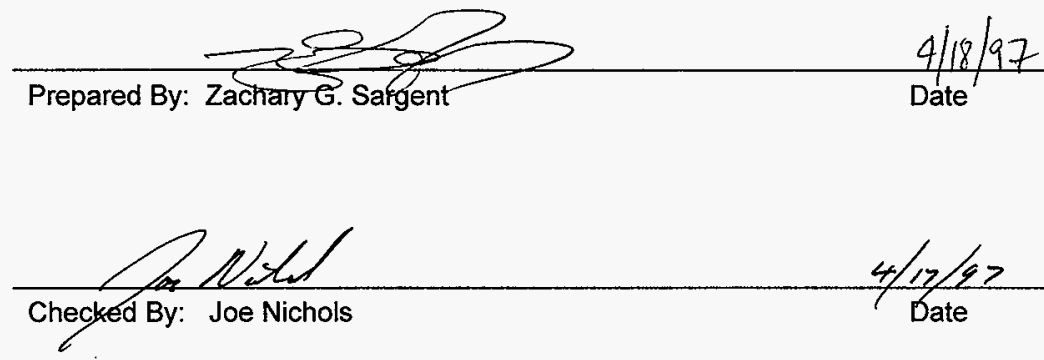
CLIENT: DUKE ENGINEERING \& SERVICES HANFORD, INC.

FILE NO: KH-8009-8-09

PROJECT: MCO Final Design DOC. NO.: HNF-SD-SNF-DR-003, Rev. 0, Appendix 11

\section{LISTING OF MCO375.INP FILE}

/BATCH,LIST

IFILENAM, MCO375

IPREP7

ITITLE,MCO DESIGN- 375 DEGREES C, 189 PSI PRESSURE

TREF,70

TUNIF,707

\begin{tabular}{|c|c|}
\hline ICOM *\# ELE & YPES \\
\hline$E T, 1,42,, 1$ & IShell \\
\hline $\mathrm{ET}, 2,42,, 1$ & I Shield Plug \\
\hline$E T, 3,42,, 1$ & I Lifting \& Locking Ring \\
\hline ET, 4,12 & IGap Elements Between Shield Plug \& Shell \\
\hline $\begin{array}{l}\text { KEYOPT,4,7,1 } \\
\text { ET,5,42,,1, }\end{array}$ & 1 Bolt \\
\hline
\end{tabular}

ICOM REAL CONSTANTS FOR GAP ELEMENTS **

R,4,-90,1.0e8,-0.06,3.0 IShell/Shield Plug, Initially Open .06"

R,5,0,1.0e8,2.95e-03 ! L. Ring/Shield Plug, Under Bolt, Preloaded

$\mathbf{R}, 6,0,1.0 e 8,0,2.0 \quad$ I Sealing Surface, initially closed

ICOM

MP,DENS, $1,490 / 1728$ ! $304 L$ SS

MP,NUXY, $1,0.3$

MP,DENS,5,490/1728 ISA193 Grade B8M

MP,NUXY,5,0.3

ICOM *** DEFINING TEMPERATURES FOR MPDATA ****

MPTEMP, 1, 70, 100,200,300,400,500

MPTEMP, $7,600,650,700,750$

ICOM ** DEFINING ELASTIC MODULI FOR 304L \& SA-193 ***

MPDATA,EX,1,1,28.3e+06,28.1e+06,27.6e+06,27.0e+06,26.5e+06,25.8e+06

MPDATA,EX,1,7,25.3e+06,25.1e+06,24.8e+06,24.5e+06

ICOM ISA-193

MPDATA,EX,5,1,28.3e+06,28.1e+06,27.6e+06,27.0e+06,26.5e+06,25.8e+06

MPDATA,EX,5,7,25.3e+06,25.1e+06,24.8e+06,24.5e+06

ICOM *** MEAN COEFFICIENTS OF THERMAL EXPANSION (in.fin./(F)

I SA240 Gr 304L

MPDATA,ALPX,1,1,0,8.55e-06,8.79e-06,9.00e-06,9.19e-06,9.37e-06

MPDATA,ALPX,1,7,9.53e-06,9.61e-06,9.69e-06,9.76e-06

I SA193 Gr B8M

MPDATA,ALPX,5,1,0,8.54e-06,8.76e-06,8.97e-06,9.21e-06,9.42e-06

MPDATA,ALPX,5,7,9.60e-06,9.69e-06,9.76e-06,9.81e-06

/COM SHELL GEOMETRY

IR=11.5 I Internal Shell Radius @ Bottom

OR=12.000 I Shell Outside Radius @ Bottom

IR2 = 12.02 I Inside Radius at Collar Sealing Surface

$O R 2=12.625 \quad$ I Outside Radius at Collar Sealing Surface

\begin{tabular}{|l|c|l|l|l|}
\hline REVISION & 0 & & & \\
\hline PREPARED BY I DATE & f $/ 5$ 4/17/97 & & & \\
\hline CHECKED BY I DATE & 4 4/17/97 & & & \\
\hline
\end{tabular}

PAGE 72

OF 133 
CLIENT: DUKE ENGINEERING \& SERVICES HANFORD, INC.

FILE NO: $\quad \mathrm{KH}-8009-8-09$

PROJECT: MCO Final Design DOC. NO.: HNF-SD-SNF-DR-003, Rev. 0, Appendix 11

IR3 $=\mathbf{1 2 . 2 5} \quad$ I Inside Radius at Collar-Lifting Ring Weld

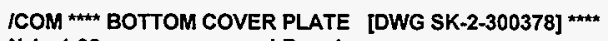

N,1, -1.32 I Row 1

$\mathrm{N}, \mathbf{2 , 1 . 2 5 , - 1 . 3 2}$

$N, 3,2.13,-1.32$

$N, 10,11.423,-1.32$

FILL.
$N, 41,0.00,-0.44$
$\mathrm{N}, 42,1.25,-0.44$
$\mathrm{N}, 43,2.13,0.44$
$\mathrm{N}, \mathbf{5 0}, \mathrm{IR}, 0.44$
FILL, 43,50
$\mathrm{N}, 52, \mathrm{OR}, 0.44$
FILL,50,52

I Row 3

FILL, 1,41,1,21,1,10 I Middle Row

FILL, 10,50,1,30

$\mathrm{N}, 32,12,-0.32$

FILL, 30,32

FILL, 10,32,1,11

$\mathrm{N}, 53, \mathrm{IR}, 1.17$

$\mathrm{N}, 55, \mathrm{OR}, 1.17$

FILL, 53,55

I Shell Stub/Weld

ICOM *** SHELL. [DWGS SK-2-300379 \& SK-2-300461] ***

$\mathbf{N}, 65, I R, 6.68$

$\mathrm{N}, 67, \mathrm{OR}, 6.68$

FILL

FILL, $53,65,3,3,3,1$

ICOM SINGLE ROW SHELL **

$\mathrm{N}, 100, \mathrm{IR}, 7.18 \quad$ I Inside

$\mathrm{N}, 140, \mathrm{IR}, 71.68$

$N, 180, I R, 136.68$

N,101,OR,7.18 IOutside

$\mathrm{N}, 141, \mathrm{OR}, 71.68$

$\mathrm{N}, 181, \mathrm{OR}, 136.68$

FILL, 100,140,20,2,2,1,2.0

FILL, $140,180,19,2,2,1, .5$

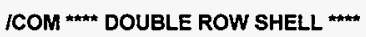

N,190,IR,137.18 I Transition to Double Row

N,192,OR, 137.18

FILL

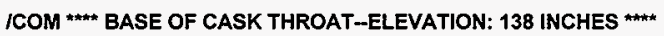

$\mathrm{N}, 217$, IR, 142.68 I Transition to Double Row

$\mathrm{N}, 219, \mathrm{OR}, 142.68$

FILL.

FILL, 190,217,8,3,3,1 IVertical Fill

ICOM ${ }^{\star * \star *}$ BOTTOM OF COLLAR TRANSITION ****

$\mathrm{N}, 235, I R, 146.06$ I Start of Transition to Large O.D \&

\begin{tabular}{|l|c|l|l|l|c|}
\hline REVISION & 0 & & & & PAGE 73 \\
\hline PREPARED BY IDATE & Of $4 / 17 / 97$ & & & & OF 133 \\
\hline CHECKED BY I DATE & /f $-4 / 17 / 97$ & & & & \\
\hline
\end{tabular}


CLIENT: $\quad$ DUKE ENGINEERING \& SERVICES HANFORD, INC.

FILE NO: KH-8009-8-09

PROJECT: MCO Final Design DOC. NO.: HNF-SD-SNF-DR-003, Rev. 0, Appendix 11

$\mathrm{N}, \mathbf{2 3 7}, \mathrm{OR}, \mathbf{1 4 6 . 0 6}$

FILL

$\mathrm{N}, \mathbf{2 3 8}, \mathrm{IR}, \mathbf{1 4 6 . 6 8}$

$N, 240,0 R, 146.68$

FILL

FILL,217,235,5,3,3,1

I Horizontal Fill

ICOM **** TOP OF COLLAR TRANSITION

$N, 241, I R, 147.31 \quad$ I End of Transition to Large O.D \&

$\mathrm{N}, \mathbf{2 4 3}, \mathrm{OR}, \mathbf{1 4 7 . 3 1}$

FILL.

I Assumed Location of Shield Plug Taper

1 Horizontal Fill

NGEN,2,3,241,243,1,0.75

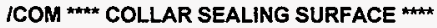

$\mathrm{N}, 247, \mathrm{IR}, 149.63$

$N, 249$, IR2,149.63

FILL

I Inside Radius of Sealing Surface

1 Outside Radius at Sealing Surface

I Horizontal Fill

ICOM ${ }^{\star * * *}$ THICK WALL AT COLLAR TRANSITION ***

NGEN,2,10,240,249,3

$N, 255, O R 2,147.31$

I Nodes 250-259 Coincident w/240-249 (by 3)

$N, 261, O R 2,149.63$

1 Outside Surface

I Outside Surface

$N, 258, O R 2,148.06$

$\mathrm{N}, \mathbf{9 8 0}, \mathrm{IR}, \mathbf{1 4 9 . 3 8}$

$\mathrm{N}, 981,11.755,149.38$

$\mathrm{N}, 982, \operatorname{IR2}, 149.38$

N,983,12.317, 149.38

$\mathrm{N}, 984, \mathrm{OR2}, 149.38$

$\mathrm{N}, 990, \mathrm{OR2}, 146.68$

FILL, 240,990,1,251

NGEN $, 2,5,980,984,1,-0.66$

FILL, 246,258,1,257

FILL, 253,255,1,1,3,3

FILL, 237,990,1,991

ICOM COLLAR AT BOTTOM EDGE OF PLUG (.155" above Sealing Surface)

NGEN, 2,3,259,,,0.245 I Nodes 262

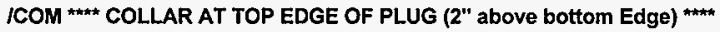

NGEN, 2,9,262,,,2.00 I Nodes 271

FILL,262,271,2

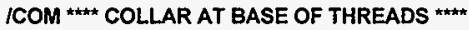

$\mathrm{N}, 274, \mathrm{IR} 3,152.00$

$N, 1000, I R 2,152.00$

ICOM I $^{\star \star \star}$ TOP TO COLLAR (WELD CLOSURE) ${ }^{\star \star \star \star ~}$

$\mathrm{N}, 295, \mathrm{IR3}, 156.00$

FILL, 274,295

NGEN,3,1,259,295,3,(OR2-IR2)/2

NGEN,3,1,274,295,3,(OR2-IR3)/2

ICOM"

RING1 $=7.94$

RING2 $=9.375$

RING3 $=9.625$

\begin{tabular}{|l|c|c|c|c|c|}
\hline REVISION & 0 & & & & PAGE 74 \\
\hline PREPARED BY IDATE & HE 4/17/97 & & & & OF 133 \\
\hline CHECKED BY I DATE & L/e -4/17/97 & & & & \\
\hline
\end{tabular}


CLIENT: DUKE ENGINEERING \& SERVICES HANFORD, INC.

FILE NO: KH-8009-8-09

PROJECT: MCO Final Design DOC. NO.: HNF-SD-SNF-DR-003, Rev. 0, Appendix 11

RING4 $=10.19$
RING5 $=12.23$

LOCAL, $11,0,152.00 \quad$ I Local System $z=0$ at Base of Ring
CSYS 11

ICOM *** TOP EDGE ***

N,401,RING1,6.13

CSYS,O

$N, 404,9.375,158.13$

FILL,401,404,,1

N,406,RING4,158.13

FILL,404,406,,,1

! Top Edge

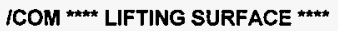

CSYS,11

N,421,RING1,5.13

N,424,RING2,5.13

FILL, 421,424

N,426,RING4,5.13

FILL, 424,426

FILL, 401,421,1,10,6,1

N,431, RING1,6.13-1.56

N,434,RING2,6.13-1.56

FILL

ICOM **** BOLTING SURFACE ****

N,441,RING1,4

$\mathrm{N}, \mathbf{4 4 4 , R I N G 3 , 4}$

FILL

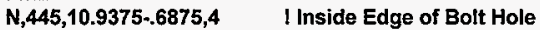

$\mathrm{N}, 447,10.9375+.6875,4 \quad$ ! Outside Edge of Bolt Hole

FILL

$\mathrm{N}, 910,10.9375-.6875,4$

$\mathrm{N}, 911,10.9375+.6875,4$

$\mathrm{N}, 448, \mathrm{RING5,4}$

CSYS, 0

$\mathrm{N}, 924,10.25,152.00$

$\mathrm{N}, 925,11.625,152.00$

FILL,910,924,6,,2

FILL,911,925,6,2

$\mathrm{N}, 525,10.25,151.874$

$\mathrm{N}, 527,11.625,151.874$

FILL

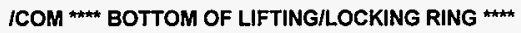

CSYS, 11

NGEN,2,70,441,448,1,-4 ! Bottom Surface of Lifting/Locking Ring

FILL,441,511,6,10,8,1

\author{
1 O.D of Ring \\ I Bolt Extension \\ ! Double Nodes @ Bolt for Gap elements
}

I Bottom of Bolt Extension

JCOM SHELD PLUG (offset y by 158.25)

LOCAL,20,0,158.13

TYPE, 2

PLUGR1 $=11.975$

PLUGR2=11.45

PLUGR3=11.25

PLUGR4=7.89

\begin{tabular}{|l|c|c|c|c|c|}
\hline REVISION & 0 & & & & PAGE 75 \\
\hline PREPARED BY I DATE & OF 133 \\
\hline CHECKED BY I DATE & 4/17/97 & & & & \\
\end{tabular}


CLIENT: DUKE ENGINEERING \& SERVICES HANFORD, INC. FILE NO: KH-8009-8-09 PROJECT: MCO Final Design DOC. NO.: HNF-SD-SNF-DR-003, Rev. 0, Appendix 11

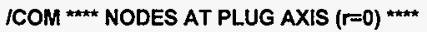

$\mathrm{N}, 601$

$\mathrm{N}, 602,0,-1$

$\mathrm{N}, 603,0,-1.994$

$\mathrm{N}, 606,0,-4.994$

FILL, 603,606,2,604

$\mathrm{N}, 607,0,-6.25$

$\mathrm{N}, 610,0,-8.25$

FILL,607,610,2,608

$\mathrm{N}, 611,0,-8.75$

$\mathrm{N}, 613,0,-10.5$

FILL, 611,613

ICOM NODAL GENERATION

NGEN, 2, 20,601, 613,1,0.8825

NGEN,2,20,621,633,1,0.8825

NGEN, 2,20,642,653,1,0.6875

NGEN, 2,20,662,673,1,0.6875

NGEN, $2,20,683,693,1,0.4235$

NGEN,2,10,706,713,1,0.9515

I Id Large Opening

I Id Medium Opening

I Id Small Opening

I Center of Opening

$N, 730,5.4665,-1.994$

I Od Small Opening

$\mathrm{N}, 736,5.4665,-4.994$

FILL, 730,736,5,731

$\mathrm{N}, 737,5.4665,-6.25$

$\mathrm{N}, 740,5.4665,-8.25$

FILL, $737,740,2,738$

$\mathrm{N}, 741,5.4665,-8.75$

$\mathrm{N}, 743,5.4665,-10.5$

FILL, 741,743

N,748,5.89,-1.0

NGEN, $2,20,730,743,1,0.4235$

FILL,748,750

N,766,7.265, 0

NGEN, 2,20,748,763,1,1.375

FILL,766,768

NGEN,3,20,766,768,1,0.3125

$\mathrm{N}, 789,7.5775,-1.56$

$\mathrm{N}, 796,7.5775,-5.56$

FILL, 789,796,6

NGEN,2,20,789,796,1,0.3125

NGEN,3,20,777,783,1,0.3125

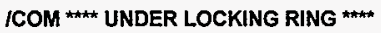

$\mathrm{N}, 824,8.5017,-6.25$

$\mathrm{N}, 827,8.5017,-8.25$

FILL.

$\mathrm{N}, 828,8.5017,-8.75$

$\mathrm{N}, 830,8.5017,-10.5$

FILL

NGEN, $3,7,824,830,1,0.5616$

NGEN, $2,7,838,844,1,0.625$

NGEN,2,7,845,851,1,0.6875 I Under Bolt

$N, 859,11.625,-6.25$

$\mathrm{N}, 860,11.625,-6.917$

\begin{tabular}{|l|c|l|l|l|c|}
\hline REVISION & 0 & & & & PAGE 76 \\
PREPARED BY IDATE & OF 133 \\
\cline { 1 - 4 } CHECKED BY I DATE & 4/17/97 & & & & \\
\hline
\end{tabular}


CLIENT: DUKE ENGINEERING \& SERVICES HANFORD, INC.

FILE NO: KH-8009-8-09

PROJECT: MCO Final Design DOC. NO.: HNF-SD-SNF-DR-003, Rev. 0, Appendix 11

\author{
$\mathrm{N}, 861,11.625,-7.584$ \\ N,862,PLUGR2,-8.25 \\ N,863,PLUGR2,-8.75 \\ N,865,PLUGR3,-10.5 \\ FILL,863,865,1 \\ N,866,PLUGR1-0.288,-6.25 \\ $\mathrm{N}, 869$, PLUGR $1-0.288,-8.25$ \\ FILL, 866,869,2 \\ N,870,PLUGR1-0.288, -8.476 \\ NGEN, 2,5,866,870,1,0.288
}

ICOM **** REFINING LIFTING EAR ****

CSYS, 0

$\mathrm{N}, 877,9.53,158.13$

$\mathrm{N}, 889,9.53,157.63$

$\mathrm{N}, 901,9.53,157.13$

FILL, $\mathbf{4 0 3 , 4 0 4 , 1 , 8 7 6}$

FILL,413,414, 1,888

FILL,423,424,1,900

FILL, $877,405,1,878$

FILL,405,406,2,879,1

FILL,889,415,1,890

FILL,415,416,2,891,1

FILL,404,414,1,881

FILL, 877,889,1,882

FILL, $878,890,1,883$

FILL,405,415,1,884

FILL,879,891,1,885

FILL,880,892,1,886

FILL,406,416,1,887

FILL, 889,901,1,894

FiLL,414,424,1,893

FILL, $901,425,1,902$

FILL,890,902,1,895

FILL, $415,425,1,896$

FILL, $425,426,2,903,1$

FILL,891,903,1,897

FILL, $892,904,1,898$

FILL, $416,426,1,899$

FILL, $424,434,1,907$

FILL, $433,434,1,908$

FILL, $423,433,1,905$

FILL, 905,907

ICOM *** COUPLING NODES *

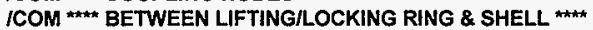

CP, $1, U Y, 508,277$

CP, $2, U Y, 498,280$

CP, $3, \cup Y, 488,283$

$C P, 4, U Y, 478,286$

CP, $5, U Y, 468,289$

$\mathrm{CP}, 6, \mathrm{UY}, 458,292$

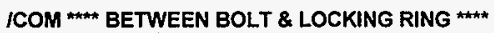

CP, $7, U Y, 445,910$

CP, $8, \mathrm{UX}, 445,910$

\begin{tabular}{|l|c|c|c|c|c|}
\hline REVISION & 0 & & & & PAGE 77 \\
\hline PREPARED BY I DATE & OF 133 \\
\hline CHECKED BY / DATE & 4/17/97 & & & & \\
\hline
\end{tabular}


CLIENT: $\quad$ DUKE ENGINEERING \& SERVICES HANFORD, INC.

FILE NO: $\quad \mathrm{KH}-8009-8-09$

PROJECT: MCO Final Design DOC. NO.: HNF-SD-SNF-DR-003, Rev. 0, Appendix 11

CP,9,UY,447,911

$C P, 10, \cup X, 447,911$

*DO,I,1,7

$C P, 10+1, U Y, 445+10 * 1,910+2 * \mid$

"ENDDO

*DO,I,1,7

$C P, 17+1, U Y, 447+10^{*} 1,911+2 * I$

*ENDDO

*DO,l,1,7

$C P, 24+1, U X, 445+10 * 1,910+2 * 1$

ENDDDO

*DO,l,1,7

$C P, 31+1, U X, 447+10 * 1,911+2$ *1

"ENDDO

NALL

EALL

ICOM *** ELEMENT GENERATION FOR SHELL ***

TYPE, 1

MAT,1

ICOM **** BOTTOM OF SHELL ****

$E, 1,2,22,21$

$E, 2,3,23,22$

EGEN, $8,1,-1$

$E, 10,11,30$

$E, 21,22,42,41$

$E, 22,23,43,42$

EGEN,10,1,-1

$E, 11,31,30$

$E, 11,32,31$

ICOM $\because$ SHELL $\cdots$

$E, 50,51,54,53$

EGEN, 2,1,-1

EGEN, $5,3,-2$

ICOM FIRST TRANSITION ELEMENTS *

$E, 65,66,100$

$E, 100,66,101$

$E, 67,101,66$

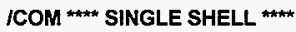

$E, 100,101,103,102$

EGEN,40,2,-1

ICOM *** SECOND TRANSITION ELEMENTS ***

$E, 190,180,191$

$E, \mathbf{1 8 0 , 1 8 1 , 1 9 1}$

$E, 181,192,191$

/COM *A* TOP SHELL (DOUBLE ELEMENT) ****

$E, 190,191,194,193$

EGEN, 2,1,-1

EGEN, 18,3,-2

$E, 244,245,986,985$

REVISION

PREPARED BY I DATE

CHECKED BY I DATE 
CLIENT: $\quad$ DUKE ENGINEERING \& SERVICES HANFORD, INC.

FILE NO: $\quad \mathrm{KH}-8009-8-09$

PROJECT: MCO Final Design DOC. NO.: HNF-SD-SNF-DR-003, Rev. 0, Appendix 11

EGEN,2,1,-1

$E, 256,257,988,987$

$E, 257,258,989,988$

$E, 985,986,981,980$

EGEN,4,1,-1

$E, 980,981,248,247$

EGEN,2,1,-1

$E, 982,983,260,249$

$E, 983,984,261,260$

ICOM *** COLLAR TRANSITION \& THREADED REGIONS ***

E,237,991,251,240

E,991,990,251

$E, 240,251,254,253$

$E, 251,990,255,254$

$E, 253,254,257,256$

EGEN,2,1,-1

$E, 259,260,263,262$

EGEN,2,1,-1

EGEN,12,3,-2

$E, 271,274,1000$

ICOM MERGE COINCIDENT NODES FOR SHELL ***

ESEL,S,TYPE,"1

NSLE

NUMMRG,NODE,

EALL

NALL

ICOM *ND OF SHELUCOLLAR ELEMENT GENERATION

ICOM LOCKING/LIFTING RING ELEMENTS **

TYPE,3

MAT, 1

$E, 411,412,402,401$

EGEN, 2,1,-1

EGEN,2,10,-2

$E, 413,888,876,403$

$E, 881,404,876$

$E, 888,881,876$

$E, 888,414,881$

$E, 881,882,877,404$

$E, 414,889,882,881$

$E, 882,883,878,877$

$E, 889,890,883,882$

$E, 883,884,405,878$

$E, 890,415,884,883$

$E, 884,885,879,405$

$E, 415,891,885,884$

$E, 885,886,880,879$

$E, 891,892,886,885$

$E, 886,887,406,880$

$E, 892,416,887,886$

$E, 423,900,888,413$

$E, 893,414,888$

$E, 900,893,888$

E, 900,424,893

\begin{tabular}{|l|c|l|l|l|}
\hline REVISION & 0 & & & \\
\hline PREPARED BY IDATE & Z $S$ 4/17/97 & & & \\
\hline CHECKED BY I DATE & /6 $-4 / 17 / 97$ & & & \\
\hline
\end{tabular}


CLIENT: DUKE ENGINEERING \& SERVICES HANFORD, INC.

PROJECT: MCO Final Design DOC. NO.: HNF-SD-SNF-DR-003, Rev. 0, Appendix 11

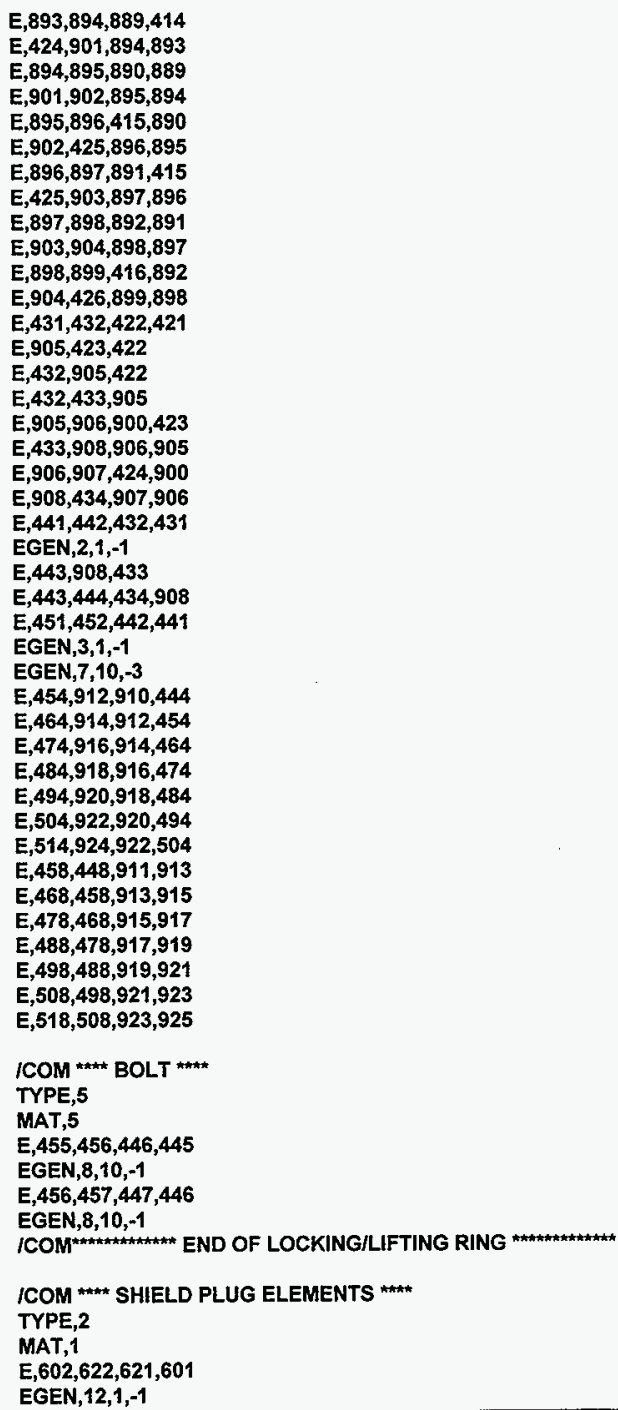

\begin{tabular}{|l|c|c|c|c|c|}
\hline REVISION & 0 & & & & PAGE 80 \\
\hline PREPARED BY I DATE & OF 133 & \\
\hline CHECKED BY I DATE & $4 / 17 / 97$ & & & & \\
\hline
\end{tabular}


CLIENT: $\quad$ DUKE ENGINEERING \& SERVICES HANFORD, INC. FILE NO: KH-8009-8-09

PROJECT: MCO Final Design DOC. NO.: HNF-SD-SNF-DR-003, Rev. 0, Appendix 11

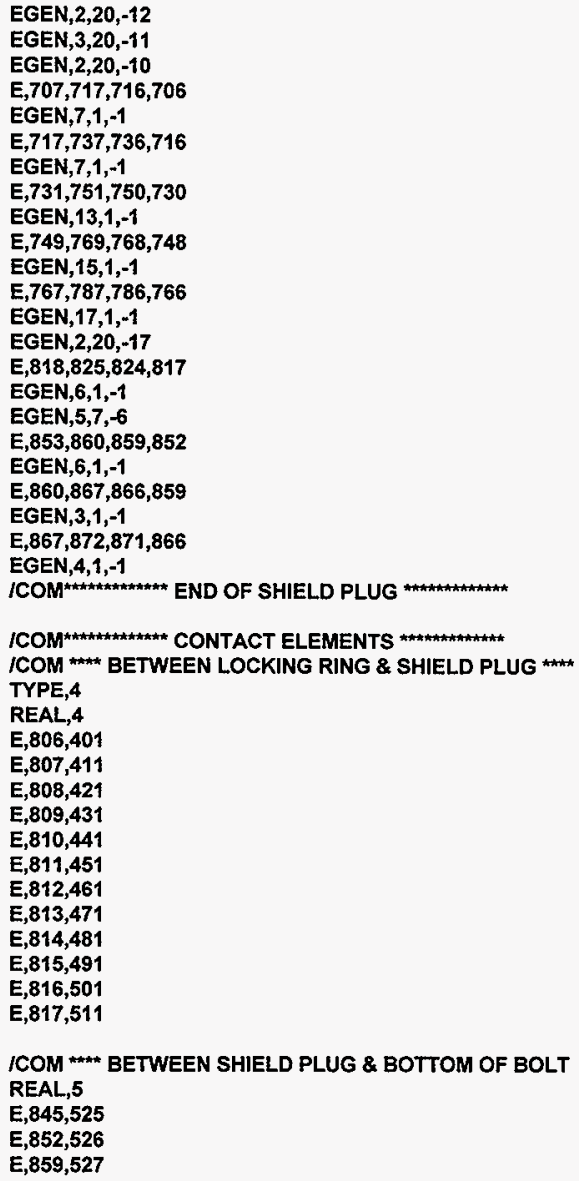


CLIENT: DUKE ENGINEERING \& SERVICES HANFORD, INC.

FILE NO: $\quad \mathrm{KH}-8009-8-09$

$\begin{array}{ll}\text { PROJECT: MCO Final Design } & \text { DOC. NO.: HNF-SD-SNF-DR-003, Rev. 0, Appendix } 11\end{array}$

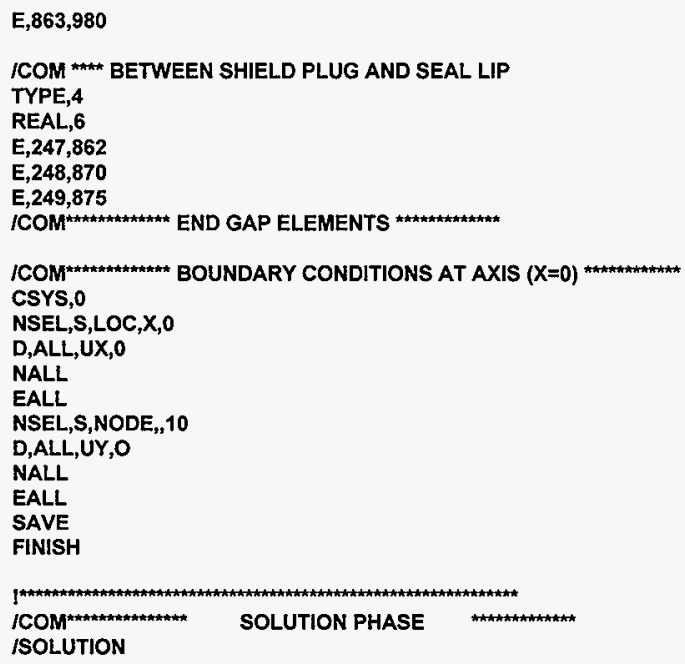

\begin{tabular}{|l|c|l|l|l|}
\hline REVISION & 0 & & & \\
\hline PREPARED BY IDATE & S $4 / 17 / 97$ & & & \\
\hline CHECKED BY IDATE & T/2 4/17/97 & & & \\
\hline
\end{tabular}


CLIENT: $\quad$ DUKE ENGINEERING \& SERVICES HANFORD, INC. FILE NO: KH-8009-8-09

PROJECT: MCO Final Design DOC. NO.: HNF-SD-SNF-DR-003, Rev. 0, Appendix 11

NSEL,R,LOC,Y,149.64,149.89

SF,ALL,PRES, 189

NALL

EALL

NSEL,S,LOC,X,11.44,11.69 I Shield Plug (above seal)

NSEL,R,LOC,Y,149.87,149.89

SF,ALL,PRES,189

NALL

EALL

NSEL,S,LOC, $X, 11.44,11.46$ 1 Side of Shield Plug

NSEL,R,LOC,Y,149.37,149.89

SF,ALL,PRES, 189

NALL

EALL

NSEL,S,LOC,X,11.24,11.46 I Shield Plug Taper

NSEL,R,LOC,Y,147.62,149.39

SF,ALL,PRES, 189

NALL

EALL

NSEL,S,LOC,X,0,11.26 I Bottom of Shield Plug

NSEL,R,LOC,Y,147.62,147.64

SF,ALL,PRES,189

NALL

EALL.

SOLVE

SAVE

FINISH

ICOM *** POSTPROCESSING ****

IPOST1

SET,LAST

ITYPE,ALL,HIDC

IGLINE,ALL, 0

RSYS, 0

PLNSOL,S,INT

IDSCALE,,20

IREPLOT

LPATH,1,41

I Bottom Plate

PRSECT

LPATH, 6,46

PRSECT

LPATH, 10,50

PRSECT

LPATH, 50,52

PRSECT

LPATH, 53,55

PRSECT

LPATH;62,64

PRSECT

LPATH, 65,67

PRSECT

LPATH,100,101

PRSECT

LPATH,122,123

PRSECT

LPATH,134,135

REVISION

PREPARED BY I DATE

! Lower Shell

CHECKED BY / DATE

I Mid Shell 
CLIENT: DUKE ENGINEERING \& SERVICES HANFORD, INC.

FILE NO: $\quad \mathrm{KH}-8009-8-09$

$\begin{array}{lll}\text { PROJECT: } & \text { MCO Final Design } & \text { DOC. NO.: HNF-SD-SNF-DR-003, Rev. 0, Appendix } 11\end{array}$

PRSECT

LPATH,156,157

PRSECT

LPATH, 170,171

PRSECT

LPATH, $\mathbf{1 8 0 , 1 8 1}$

PRSECT

LPATH,202,204

PRSECT

LPATH,235,237

PRSECT

LPATH,985,989

PRSECT

LPATH,262,264

PRSECT

LPATH,277,279

PRSECT

LPATH,292,294

PRSECT

LPATH,601,641

PRSECT

LPATH, 601,613

PRSECT

LPATH, 603,703

PRSECT

LPATH, 606,706

PRSECT

LPATH,766,806

PRSECT

LPATH, 748,808

PRSECT

LPATH,730,810

PRSECT

LPATH,736,815

PRSECT

LPATH,869,874

PRSECT

LPATH, 870,875

PRSECT

LPATH,431,434

PRSECT

LPATH,406,426

PRSECT

LPATH,404,424

PRSECT

SAVE
I Upper Shell

I Shield Plug

I Locking Ring

\begin{tabular}{|l|l|l|l|}
\hline & & & \\
\hline & & & \\
\hline
\end{tabular}


DKE ENGINEERING \& SERVICES HA

PROJECT: MCO Final Design DOC. NO.: HNF-SD-SNF-DR-003, Rev. 0, Appendix 11

\section{COMPUTER RUN COVER SHEET}

Project Number:

Computer Code:

Software Version:

Computer System:

Computer Run File Number:

Unique Computer Run Filename:

Run Description:

Run Date / Time:
$\mathrm{KH}-8009-8$

ANSYS ${ }^{\circledR}-P C$

5.0A

MS-DOS, Pentium $®$ Processor

$\mathrm{KH}-8009-8-09$

MCO375.out

Load Case 3 Output

27 March $1997 \quad$ 2:22:12 PM
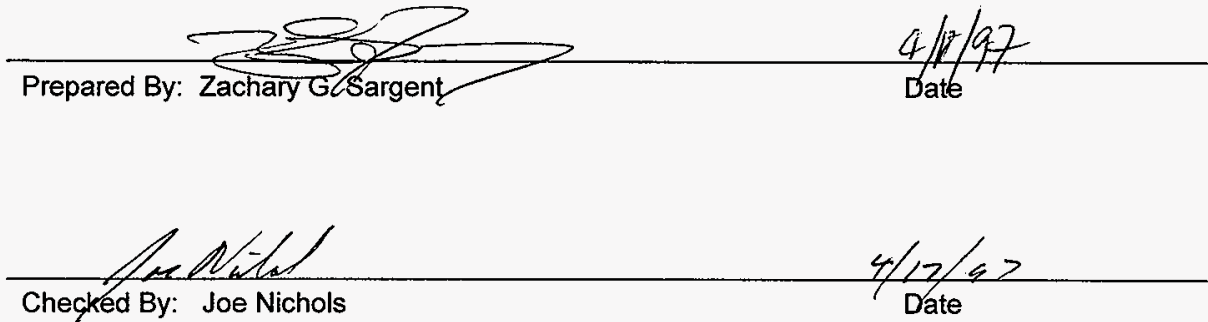

\begin{tabular}{|l|c|l|l|l|l|}
\hline REVISION & 0 & & & & PAGE 85 \\
PREPARED BY IDATE & OF 133 \\
\cline { 1 - 4 } CHECKED BY IDATE & $4 / 17 / 97$ & & & & \\
\hline
\end{tabular}


CLIENT: DUKE ENGINEERING \& SERVICES HANFORD INC.

FILE NO: $\quad \mathrm{KH}-8009-8-09$

$\begin{array}{ll}\text { PROJECT: MCO Final Design } & \text { DOC. NO.: HNF-SD-SNF-DR-003, Rev. 0, Appendix } 11\end{array}$

\section{COMPUTER RUN COVER SHEET}

Project Number:

Computer Code:

Software Version:

Computer System:

Computer Run File Number:

Unique Computer Run Filename:

Run Description:

Creation Date / Time:
$\mathrm{KH}-8009-8$

ANSYS®-PC

$5.0 \mathrm{~A}$

MS-DOS, Pentium® Processor

KH-8009-8-09

POC4.inp

Load Case 4: $189 \mathrm{psi}, 132^{\circ} \mathrm{C}$

15 April $1997 \quad 5: 17: 54$ PM
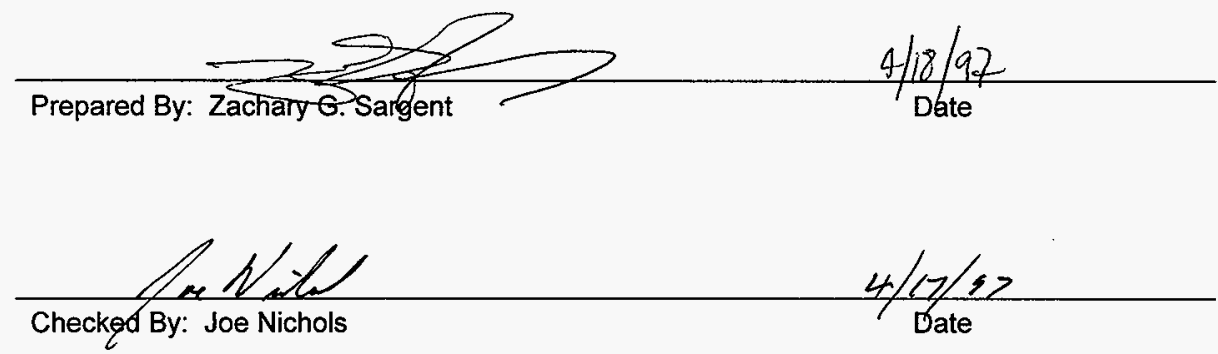

\begin{tabular}{|l|c|l|l|l|l|}
\hline REVISION & 0 & & & & PAGE 86 \\
PREPARED BY IDATE & $4 / 17 / 97$ & & & & OF 133 \\
\cline { 1 - 4 } CHECKED BY/DATE & $/ 1 / 4 / 17 / 97$ & & & & \\
\hline
\end{tabular}


CLIENT: DUKE ENGINEERING \& SERVICES HANFORD, INC.

FILE NO: $\quad \mathrm{KH}-8009-8-09$

PROJECT: MCO Final Design DOC. NO.: HNF-SD-SNF-DR-003, Rev. 0, Appendix 11

\section{LISTING OF POC4.INP FILE}

IBATCH,LIST

IFILENAM,POC4

IPREP7

ITITLE,MCO DESIGN- 132 DEGREES C, 189 PSI PRESSURE, LIFT, PRELOAD

TREF,70

TUNIF,270

1 COM $\cdots \cdots$

ET, $1,42, \ldots 1$ ! Shell

ET, $2,42, \ldots 1$ ! Shield Plug

ET, $3,42, \ldots 1$ ! Lifting \& Locking Ring

ET $, 4,12$ ! Gap Elements Between Shield Plug \& She!l

KEYOPT,4,7,1

ET $, 5,42, \ldots 1$ ! Bolt

/COM REAL CONSTANTS FOR GAP ELEMENTS

R,4,-90,1.0e8,-0.06,3.0 ! Shell/Shield Plug, Initially Open .06"

R,5,0,1.0e8,2.95e-03 ! L. Ring/Shield Plug, Under Bolt, Preloaded

$R, 6,0,1,0 E 8,0,2.0$ ! Sealing Surface, closed

/COM

MP,DENS, $1,490 / 1728 \quad$ ! 304L SS

MP,NUXY, 1,0.3

MP,DENS,5,490/1728 ! SA193 Grade B8M

MP,NUXY,5,0.3

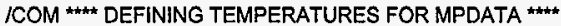

MPTEMP, $1,70,100,200,300,400,500$

MPTEMP, $7,600,650,700,750$

/COM ** DEFINING ELASTIC MODULI FOR 304L \& SA-193 ***

MPDATA,EX, 1, 1,28.3e+06,28.1e+06,27.6e+06,27.0e+06,26.5e+06,25.8e+06

MPDATA,EX, 1,7,25.3e $\div 06,25.1 \mathrm{e}+06,24,8 \mathrm{e}+06,24.5 \mathrm{e}+06$

ICOM ISA-193

MPDATA,EX,5, 1,28.3e+06,28.1e+06,27.6e+06,27.0e+06,26.5e+06,25.8e+06

MPDATA, EX,5,7,25.3e+06,25.1e+06,24.8e+06,24.5e+06

/COM MEAN COEFFICIENTS OF THERMAL EXPANSION (in./in./(F)

! SA240 Gr 304L

MPDATA,ALPX, 1,1,0,8.55e-06,8.79e-06,9.00e-06,9.19e-06,9.37e-06

MPDATA,ALPX, 1,7,9.53e-06,9.61e-06,9.69e-06,9.76e-06

! SA193 Gr B8M

MPDATA,ALPX,5,1,0,8.54e-06,8.76e-06,8.97e-06,9.21e-06,9.42e-06

MPDATA,ALPX,5,7,9.60e-06,9.69e-06,9.76e-06,9.81e-06

ICOM SHELL GEOMETRY

$\mathrm{IR}=11.5$

! Intemal Shell Radius @ Bottom

$\mathrm{OR}=12.000$

! Shell Outsicie Radius @ Bottom

IR2 $=12.02$

! Inside Radius at Collar Sealing Surface

\begin{tabular}{|l|c|l|l|l|}
\hline REVISION & 0 & & & \\
\hline PREPARED BY I DATE & f/G $4 / 17 / 97$ & & & \\
\hline CHECKED BY I DATE & / $/ 2-4 / 17 / 97$ & & & \\
\hline
\end{tabular}


CLIENT: DUKE ENGINEERING \& SERVICES HANFORD, INC.

FILE NO: KH-8009-8-09

PROJECT: MCO Final Design DOC. NO.: HNF-SD-SNF-DR-003, Rev. 0, Appendix 11

$\mathrm{OR} 2=12.625$

! Outside Radius at Collar Sealing Surface

IR3 $=12.25$

! Inside Radius at Collar-Lifting Ring Weld

ICOM BOTTOM COVER PLATE [DWG SK-2-300378] ***

$\mathrm{N}, 1,,-1.32$

! Row 1

$N, 2,1.25,-1.32$

$N, 3,2.13,-1.32$

$N, 10,11.423,-1.32$

FILL

$\mathrm{N}, 41,0.00,-0.44$

! Row 3

$N, 42,1.25,-0.44$

$\mathrm{N}, 43,2.13,0.44$

$\mathrm{N}, 50, \mathrm{IR}, 0.44$

FILL, 43,50

$\mathrm{N}, 52, \mathrm{OR}, 0.44$

FILL, 50,52

FILL, $1,41,1,21,1,10$

! Middle Row

FILL, $10,50,1,30$

$\mathrm{N}, 32,12,-0.32$

FILL, 30,32

FILL, 10,32,1,11

$\mathrm{N}, 53, \mathrm{IR}, 1,17$

$\mathrm{N}, 55, \mathrm{OR}, 1.17$

FILL,53,55

! She!l StubMeld

/COM SHELLL [DWGS SK-2-300379 \& SK-2-300461] ****

$\mathrm{N}, 65, \mathrm{IR}, 6.68$

$\mathrm{N}, 67, \mathrm{OR}, 6.68$

FILL

FILL, $53,65,3,, 3,3,1$

ICOM *** SINGLE ROW SHELL ***

$\mathrm{N}, 100, I R, 7.18 \quad$ ! Inside

$\mathrm{N}, 140, \mathrm{IR}, 71.68$

$\mathrm{N}, 180, \mathrm{IR}, 136.68$

$\mathrm{N}, 101, \mathrm{OR}, 7.18 \quad$ ! Outside

$N, 141,0 R, 71.68$

N $181,0 R, 136.68$

FILL, $100,140,20,2,2,1,2.0$

FILLL, 140,180,19,2,2,1,.5

ICOM DOUBLE ROW SHELL *

$\mathrm{N}, 190, \mathrm{IR}, 137.18$ ! Transition to Double Row

$\mathrm{N}, 192, \mathrm{OR}, 137.18$

FILL.

ICOM BASE OF CASK THROAT--ELEVATION: 138 INCHES

$\mathrm{N}, 217, \mathrm{IR}, 142.68$

! Transition to Double Row

$N, 219, \mathrm{OR}, 142.68$

FILL.

FILL, 190,217,8,3,3,1 ! Vertical Fill

/COM ** BOTTOM OF COLLAR TRANSITION **

REVISION

PREPARED BY IDATE

0

CHECKED BYIDATE 
CLIENT: DUKE ENGINEERING \& SERVICES HANFORD, INC.

FILE NO: $\quad \mathrm{KH}-8009-8-09$

PROJECT: MCO Final Design DOC. NO.: HNF-SD-SNF-DR-003, Rev. 0, Appendix 11

$\begin{array}{ll}\text { N,235,IR, 146.06 } & \text { ! Start of Transition to Large O.D \& } \\ \text { N,237,OR, 146.06 } & \text { ! Assumed Location of Shield Plug Taper }\end{array}$

F!LL

$\mathrm{N}, 238, I R, 146.68$

$\mathrm{N}, 240, \mathrm{OR}, 146.68$

FILL

! Horizontal Fill

FILL, 217,235,5,3,3,1 ! Vertical Fi!l

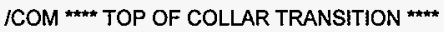

N,241,IR, $147.31 \quad$ ! End of Transition to Large O.D \&
N,243,OR,147.31
FILL

NGEN, $2,3,241,243,1,0.75$

/COM COLLAR SEALING SURFACE

$N, 247, I R, 149.63$ ! Inside Radius of Sealing Surface

$\mathrm{N}, 249, \mathrm{IR} 2,149.63$

FILL

! Horizontal Fill

/COM **** THICK WALL AT COLLAR TRANSITION ****

NGEN, $2,10,240,249,3$

! Nodes 250-259 Coincident w/240-249 (by 3)

$\mathrm{N}, 255, \mathrm{OR} 2,147.31$

! Outside Surface

$\mathrm{N}, 261, \mathrm{OR} 2,149.63$

! Outside Surface

$N, 258, O R 2,148.06$

$\mathrm{N}, 980,[R, 149.38$

N, $981,11.755,149.38$

$\mathrm{N}, 982, \mathrm{IR} 2,149.38$

$\mathrm{N}, 983,12.317,149.38$

$\mathrm{N}, 984, \mathrm{OR} 2,149.38$

N,990,OR2, 146.68

FILL, 240,990,1,251

NGEN, $2,5,980,984,1,-0.66$

FILL, 246,258, 1,257

FILL, 253,255,1,1,3,3

FILL,237,990,1,991

/COM ${ }^{\star \star \star \star}$ COLLAR AT BOTTOM EDGE OF PLUG (.155" above Sealing Surface) ${ }^{\star \star \star \star}$

NGEN, $2,3,259, \ldots, 0.245 \quad$ ! Nodes 262

ICOM COLLAR AT TOP EDGE OF PLUG (2" above bottom Edge) ***

NGEN $, 2,9,262, \ldots, 2.00 \quad$ ! Nodes 271

FILL,262,271,2

ICOM *** COLLAR AT BASE OF THREADS ***

$\mathrm{N}, 274$, IR $3,152.00$

$N, 1000, I R 2,152.00$

ICOM IN** TOP TO COLLAR (WELD CLOSURE) $^{\star \star \star *}$

$\mathrm{N}, 295, \mathrm{IR} 3,156.00$

FILL,274,295

NGEN $, 3,1,259,295,3,($ OR2-IR2)/2

NGEN,3,1,274,295,3,(OR2-IR3)/2

ICOM LOCKING \& LIFTING RING GEOMETRY

RING $1=7.94$

RING2 $=9.375$

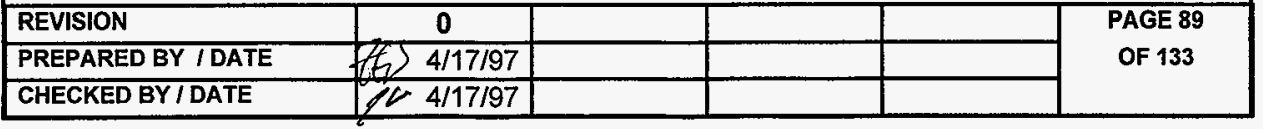


CLIENT: DUKE ENGINEERING \& SERVICES HANFORD, INC.

FILE NO: KH-8009-8-09

PROJECT: MCO Final Design

DOC. NO.: HNF-SD-SNF-DR-003, Rev. 0, Appendix 11

RING3 $=9.625$

RING4=10.19

RING5 $=12.23$

LOCAL, $11,0,152.00 \quad$ ! Local System $z=0$ at Base of Ring

CSYS, 11

ICOM $* * *$ TOP EDGE $* * * *$

$N, 401$, RING1,6.13

CSYS,O

$\mathrm{N}, 404,9.375,158.13$

FILL, $401,404,, 1$

N,406,RING4, 158.13

FILL,404,406,.,1 ! Top Edge

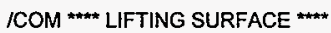

CSYS, 11

N,421,RING1,5.13

$N, 424$, RING2,5.13

FILL 421,424

N,426, RING4,5.13

FILL, 424,426

FILL, 401,421,1, 10,6,1

N,431,RING1,6.13-1.56

N,434,RING2,6.13-1.56

FILL

ICOM BOLTING SURFACE

$\mathrm{N}, 441, \mathrm{RING1,4}$

$\mathrm{N}, 444, \mathrm{RING} 3,4$

FILL.

N,445,10.9375- 6875,4 ! Inside Edge of Bolt Hole

$\mathrm{N}, 447,10.9375+.6875,4 \quad$ ! Outside Edge of Bolt Hole

FILL

N.910,10.9375- 6875,4

$\mathrm{N}, 911,10.9375+, 6875,4$

N,448,RING5,4

CSYS, 0

$\mathrm{N}, 924,10.25,152.00$

! O.D of Ring

! Bolt Extension

$\mathrm{N}, 925,11.625,152.00$

! Double Nodes @ Bolt for Gap elements

FILL,910,924,6,2

FILL, $911,925,6,2$

$\mathrm{N}, 525,10.25,151.874$

$\mathrm{N}, 527,11.625,151.874$

! Bottom of Bolt Extension

FILL

/COM **+* BOTTOM OF LIFTING/LOCKING RING ****

CSYS, 11

NGEN, $2,70,441,448,1,-4$
FILL,441,511,6,10,8,1 $\quad$ ! Bottom Surface of Lifting/Locking Ring

/COM SHIELD PLUG (offset y by 158.25)

LOCAL, $20,0,158.13$

TYPE, 2

PLUGR1 $=11.975$

PLUGR2 $=11.45$

PLUGR3=11.25

\begin{tabular}{|l|c|l|l|l|}
\hline REVISION & 0 & & & \\
\hline PREPARED BY IDATE & th $4 / 17 / 97$ & & & \\
\hline CHECKED BY I DATE & / $4 / 17 / 97$ & & & \\
\hline
\end{tabular}


CLIENT: $\quad$ DUKE ENGINEERING \& SERVICES HANFORD, INC. FILE NO: KH-8009-8-09

\section{PROJECT: MCO Final Design $\quad$ DOC. NO.: HNF-SD-SNF-DR-003, Rev. 0, Appendix 11}

PLUGR4 $=7.89$

$/ C O M \cdots$ NODES AT PLUG AXIS $(r=0)$

$\mathrm{N}, 601$

$N, 602,0,-1$

$\mathrm{N}, 603,0,-1.994$

$\mathrm{N}, 606,0,-4.994$

FILL, $603,606,2,604$

$N, 607,0,-6.25$

$\mathrm{N}, 610,0,-8.25$

FILL,607,610,2,608

$N, 611,0,-8.75$

$\mathrm{N}, 613,0,-10.5$

FILL, 611,613

/COM NODAL GENERATION ***

NGEN $, 2,20,601,613,1,0.8825$

NGEN, $2,20,621,633,1,0.8825$

NGEN, $2,20,642,653,1,0.6875$

NGEN, $2,20,662,673,1,0.6875$

NGEN $, 2,20,683,693,1,0.4235$

NGEN $, 2,10,706,713,1,0.9515$

I Id Large Opening

! Id Medium Opening

! ld Small Opening

! Center of Opening

I Od Small Opening

$\mathrm{N}, 730,5.4665,-1.994$

$\mathrm{N}, 736,5.4665,-4.994$

FILL,730,736,5,731

$\mathrm{N}, 737,5.4665,-6.25$

$\mathrm{N}, 740,5.4665,-8.25$

FILL, $737,740,2,738$

$\mathrm{N}, 741,5.4665,-8.75$

$\mathrm{N}, 743,5.4665,-10.5$

FILL, 741,743

$\mathrm{N}, 748,5.89,-1.0$

NGEN, $2,20,730,743,1,0.4235$

FILL, 748,750

$\mathrm{N}, 766,7.265,0$

NGEN $, 2,20,748,763,1,1.375$

FILL, 766,768

NGEN,3,20,766,768,1,0.3125

$\mathrm{N}, 789,7.5775,-1.56$

$\mathrm{N}, 796,7.5775,-5.56$

FILL $, 789,796,6$

NGEN $2,20,789,796,1,0.3125$

NGEN, $3,20,777,783,1,0.3125$

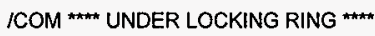

$\mathrm{N}, 824,8.5017,-6.25$

$N, 827,8.5017,-8.25$

FILL

$\mathrm{N}, 828,8.5017,-8.75$

$N, 830,8.5017,-10.5$

FILL

NGEN, $3,7,824,830,1,0.5616$

NGEN, $2,7,838,844,1,0.625$

NGEN, $2,7,845,851,1,0.6875$ ! Under Bolt

$\mathrm{N}, 859,11.625,-6.25$

\begin{tabular}{|l|c|l|l|l|}
\hline REVISION & 0 & & & \\
\hline PREPARED BY I DATE & C $4 / 17 / 97$ & & & \\
\hline CHECKED BY I DATE & J $4 / 17 / 97$ & & & \\
\hline
\end{tabular}


CLIENT: DUKE ENGINEERING \& SERVICES HANFORD, INC.

FILE NO: KH-8009-8-09

PROJECT: MCO Final Design DOC. NO.: HNF-SD-SNF-DR-003, Rev. 0, Appendix 11

N,860,11.625,-6.917
$N, 861,11.625,-7.584$
N,862,PLUGR2,-8.25
N,863,PLUGR2,-8.75
N,865,PLUGR3,-10.5
FILL,863,865,1
N,866,PLUGR1-0.288,--6.25
N,869,PLUGR1-0.288,-8.25
FILL,866,869,2
N,870,PLUGR1-0.288,-8.476
NGEN,2,5,866,870,1,0.288

ICOM *** REFINING LIFTING EAR ***

CSYS, 0

$\mathrm{N}, 877,9.53,158.13$

$\mathrm{N}, 889,9.53,157.63$

$\mathrm{N}, 901,9.53,157.13$

FILL, $403,404,1,876$

FILL, 413,414,1,888

FILL, 423,424,1,900

FILL, $877,405,1,878$

FILL, $405,406,2,879,1$

FILL, $889,415,1,890$

FILL, $415,416,2,891,1$

FILL, $404,414,1,881$

FILL, $877,889,1,882$

FILL, $878,890,1,883$

FILL, 405,415,1,884

FILL, $879,891,1,885$

FILL, $880,892,1,886$

FILL,406,416,1,887

FILL,889,901,1,894

FILL, $414,424,1,893$

FILL, $901,425,1,902$

FILL,890,902,1,895

FILL, $415,425,1,896$

FILL,425,426,2,903,1

FILL, $891,903,1,897$

FILL, $892,904,1,898$

FILL, 416,426, 1,899

FILL, 424,434,1,907

FILL, $433,434,1,908$

FILL, $423,433,1,905$

FILL,905,907

/COM $\cdots$ COUPLING NODES ***

/COM BETWEEN LIFTING/LOCKING RING \& SHELL

CP, 1, UY, 508,277 I Start Threads

$C P, 2, U Y, 498,280$

CP, $3, \mathrm{UY}, \mathbf{4 8 8 , 2 8 3}$

$C P, 4, U Y, 478,286$

$\mathrm{CP}, 5, \mathrm{UY}, 468,289$

$C P, 6, U Y, 458,292$

ICOM *** BETWEEN BOLT \& LOCKING RING *\#*

$\mathrm{CP}, 7, \mathrm{UY}, 445,910$

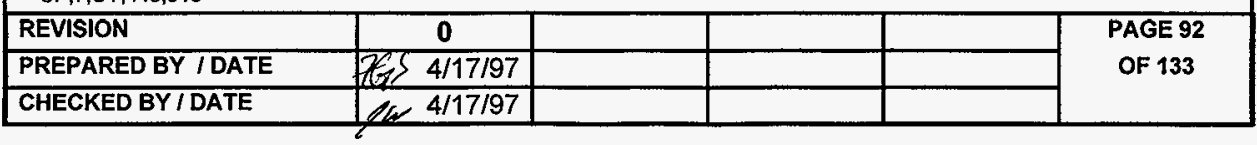


CLIENT: DUKE ENGINEERING \& SERVICES HANFORD, INC.

FILE NO: KH-8009-8-09

PROJECT: MCO Final Design DOC. NO.: HNF-SD-SNF-DR-003, Rev. 0, Appendix 11

$C P, 8, U X, 445,910$

CP,9,UY,447,911

$C P, 10, U X, 447,911$

${ }^{\star} D O, 1,1,7$

$C P, 10+1, U Y, 445+10 * 1,910+2 * 1$

"ENDDO

"DO, I, 1,7

CP, $17+1, U Y, 447+10^{*}, 911+2 * !$

*ENDDO

*DO,I,1,7

$C P, 24+1, U X, 445+10^{*} 1,910+2 * 1$

*ENDDO

${ }^{\star} D O, I, 1,7$

CP, $31+1, U X, 447+10^{*}, 911+2 * 1$

"ENDDO

NALL

EALL.

/COM **** ELEMENT GENERATION FOR SHELL ****

TYPE, 1

MAT, 1

/COM ** BOTTOM OF SHELL

$E_{1}, 1,2,22,21$

$E, 2,3,23,22$

EGEN,8,1,-1

E, $10,11,30$

$E, 21,22,42,41$

$E, 22,23,43,42$

EGEN, 10,1,-1

$E, 11,31,30$

$E, 11,32,31$

ICOM *** SHELL ****

$E, 50,51,54,53$

EGEN,2,1,-1

EGEN,5,3,-2

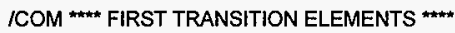

$E, 65,66,100$

$E, 100,66,101$

E, $67,101,66$

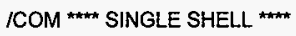

$E, 100,101,103,102$

EGEN,40,2,-1

ICOM SECOND TRANSITION ELEMENTS

$E, 190,180,191$

$E, 180,181,191$

$E, 181,192,191$

/COM TOP SHELL (DOUBLE ELEMENT)

E, 190,191,194,193

EGEN, $2,1,-1$

EGEN, 18,3,-2

REVISION

PREPARED BY I DATE

CHECKED BY/DATE 


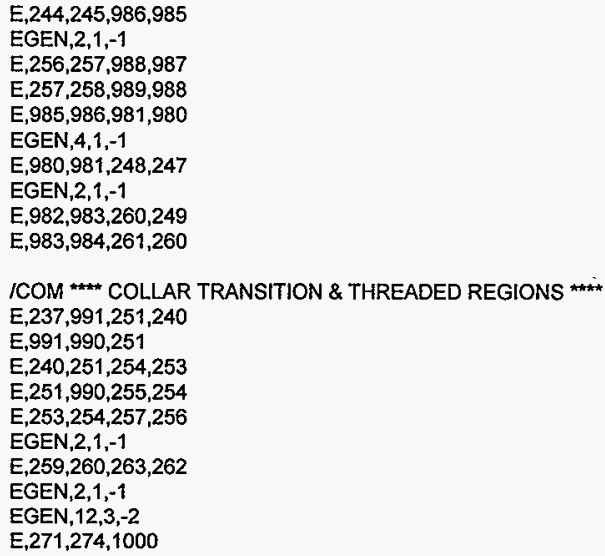


CLIENT: DUKE ENGINEERING \& SERVICES HANFORD, INC. FILE NO: KH-8009-8-09

PROJECT: MCO Final Design DOC. NO.: HNF-SD-SNF-DR-003, Rev. 0, Appendix 11

$E, 900,424,893$

$\mathrm{E}, 893,894,889,414$

$E, 424,901,894,893$

$E, 894,895,890,889$

$E, 901,902,895,894$

E,895,896,415,890

E,902,425,896,895

$E, 896,897,891,415$

E,425,903,897,896

E,897,898,892,891

$E, 903,904,898,897$

E,898,899,416,892

$E, 904,426,899,898$

$E, 431,432,422,421$

E,905,423,422

E,432,905,422

$E, 432,433,905$

E,905,906,900,423

$E, 433,908,906,905$

E,906,907,424,900

E,908,434,907,906

$E, 441,442,432,431$

EGEN, 2, 1,-1

E, $443,908,433$

$E, 443,444,434,908$

$E, 451,452,442,441$

EGEN, 3,1,-1

EGEN $7,10,-3$

E,454,912,910,444

E,464,914,912,454

$E, 474,916,914,464$

E,484,918,916,474

E,494,920,918,484

E, 504,922,920,494

E,514,924,922,504

E,458,448,911,913

$E, 468,458,913,915$

$E, 478,468,915,917$

$E, 488,478,917,919$

$E, 498,488,919,921$

E, $508,498,921,923$

E,518,508,923,925

ICOM **** BOLT ****

TYPE, 5

MAT, 5

$E, 455,456,446,445$

EGEN,8,10,-1

$E, 456,457,447,446$

EGEN $, 8,10,-1$

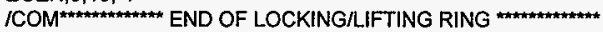

ICOM ${ }^{* \star *}$ SHIELD PLUG ELEMENTS ****

TYPE, 2

MAT, 1

$E, 602,622,621,601$

\begin{tabular}{|l|c|l|l|l|l|}
\hline REVISION & 0 & & & & $\begin{array}{c}\text { PAGE 95 } \\
\text { OF 133 }\end{array}$ \\
\hline PREPARED BY IDATE & RS 4/17/97 & & & & \\
\hline CHECKED BY I DATE & /W 4/17/97 & & & & \\
\hline
\end{tabular}


CLIENT: DUKE ENGINEERING \& SERVICES HANFORD, INC.

FILE NO: $\quad \mathrm{KH}-8009-8-09$

PROJECT: MCO Final Design

EGEN,12,1,-1

EGEN,2,20,-12

EGEN,3,20,-11

EGEN,2,20,-10

$E, 707,717,716,706$

EGEN, $7,1,-1$

$\mathrm{E}, 717,737,736,716$

EGEN, $7,1,-1$

$E, 731,751,750,730$

EGEN, 13,1,-1

$\mathrm{E}, 749,769,768,748$

EGEN,15,1,-1

$E, 767,787,786,766$

EGEN,17,1,-1

EGEN,2,20,-17

$E, 818,825,824,817$

EGEN, $6,1,-1$

EGEN, $5,7,-6$

$E, 853,860,859,852$

EGEN,6,1,-1

$E, 860,867,866,859$

EGEN,3,1,-1

$E, 867,872,871,866$

EGEN,4,1,-1

ICOM END OF SHIELD PLUG

ICOM CONTACT ELEMENTS

ICOM BETWEEN LOCKING RING \& SHIELD PLUG ***

TYPE, 4

REAL, 4

$E, 806,401$

$E, 807,411$

$E, 808,421$

$E, 809,431$

$E, 810,441$

$E, 811,451$

$E, 812,461$

$E, 813,471$

$\mathrm{E}, 814,481$

$E, 815,491$

$E, 816,501$

$E, 817,511$

ICOM * BETWEEN SHIELD PLUG \& BOTTOM OF BOLT

REAL, 5

$E, 845,525$

$E, 852,526$

$E, 859,527$

ICOM BETWEEN SHIELD PLUG \& SHELL (ABOVE SEAL)

REAL, 4

E,871,271

$E, 872,268$

$E, 873,265$

$E, 874,262$

\begin{tabular}{|l|l|l|l|}
\hline $\mathbf{0}$ & & & \\
\hline $4 / 17 / 97$ & & & \\
\hline $4 / 17 / 97$ & & & \\
\hline
\end{tabular}


CLIENT: DUKE ENGINEERING \& SERVICES HANFORD, INC.

FILE NO: $\quad \mathrm{KH}-8009-8-09$

PROJECT: MCO Final Design DOC. NO.: HNF-SD-SNF-DR-003, Rev. 0, Appendix 11

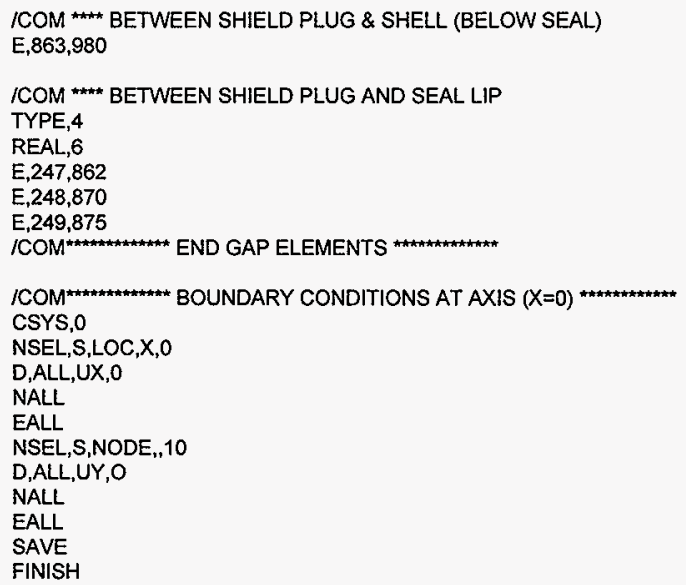

\begin{tabular}{|l|c|l|l|l|}
\hline REVISION & 0 & & & \\
\hline PREPARED BY I DATE & 765 4/17/97 & & & \\
\hline CHECKED BY I DATE & $/ 4 / 17 / 97$ & & & \\
\hline
\end{tabular}


CLIENT: DUKE ENGINEERING \& SERVICES HANFORD, INC.

FILE NO: $\quad \mathrm{KH}-8009-8-09$

PROJECT: MCO Final Design DOC. NO.: HNF-SD-SNF-DR-003, Rev. 0, Appendix 11

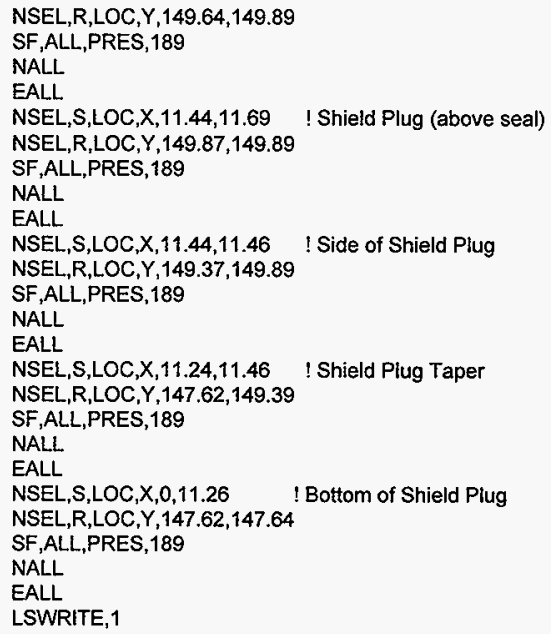


CLIENT: $\quad$ DUKE ENGINEERING \& SERVICES HANFORD, INC. FILE NO: KH-8009-8-09

PROJECT: MCO Final Design DOC. NO.: HNF-SD-SNF-DR-003, Rev. 0, Appendix 11

PRSECT

LPATH, 62,64

PRSECT

LPATH, 65,67

PRSECT

LPATH, 100,101

PRSECT

LPATH, 122,123

PRSECT

LPATH, 134, 135

PRSECT

LPATH, 156,157

PRSECT

LPATH, 170,171

PRSECT

LPATH, 180,181

PRSECT

LPATH,202,204

PRSECT

LPATH,235,237

PRSECT

LPATH, 985,989

PRSECT

LPATH,262,264

PRSECT

LPATH,277,279

PRSECT

LPATH,292,294

PRSECT

LPATH, 601,641

PRSECT

LPATH $, 601,613$

PRSECT

LPATH, 603,703

PRSECT

LPATH, 606,706

PRSECT

LPATH,766,806

PRSECT

LPATH, 748,808

PRSECT

LPATH, 730,810

PRSECT

LPATH, 736,815

PRSECT

LPATH, 869,874

PRSECT

LPATH $_{;} 870,875$

PRSECT

LPATH,431,434

PRSECT

LPATH,406,426

PRSECT

LPATH, 404,424

PRSECT

SAVE
! Mid Shell

! Upper Shell

! Shield Plug

! Locking Ring 
CLIENT: DUKE ENGINEERING \& SERVICES HANFORD, INC.

FILE NO: KH-8009-8-09

PROJECT: MCO Final Design DOC. NO.: HNF-SD-SNF-DR-003, Rev. 0, Appendix 11

\section{COMPUTER RUN COVER SHEET}

Project Number:

Computer Code:

Software Version:

Computer System:

Computer Run File Number:

Unique Computer Run Filename:

Run Description:

Run Date / Time:
$\mathrm{KH}-8009-8$

ANSYS@-PC

$5.0 \mathrm{~A}$

MS-DOS, Pentium® Processor

KH-8009-8-09

POC4.out

Load Case 4 Output

15 April $1997 \quad 5: 19: 56$ PM
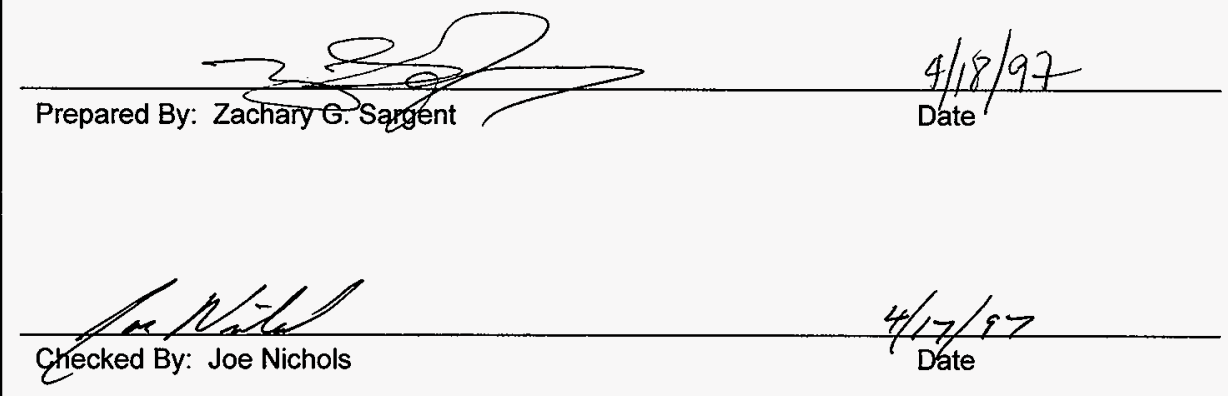

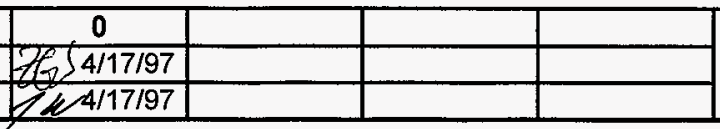


CLIENT: $\quad$ DUKE ENGINEERING \& SERVICES HANFORD, INC. FILE NO: KH-8009-8-09

PROJECT: MCO Final Design DOC. NO.: HNF-SD-SNF-DR-003, Rev. 0, Appendix 11

\section{COMPUTER RUN COVER SHEET}

Project Number:

$\mathrm{KH}-8009-8$

Computer Code:

ANSYS $\otimes-P C$

Software Version:

$5.0 \mathrm{~A}$

Computer System:

MS-DOS, Pentium® Processor

Computer Run File Number:

KH-8009-8-09

Unique Computer Run Filename:

TG275.inp

Run Description:

Load Case 5: Differential Temperature

Creation Date / Time:

27 March $1997 \quad$ 1:00:32 PM

Prepared By: Zachary G. Sargent

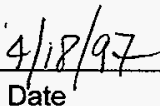

Checked By: Joe Nichols

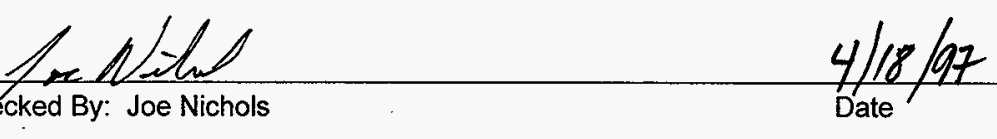

\begin{tabular}{|r|l|l|l|}
\hline 0 & & & \\
\hline $4 / 17 / 97$ & & & \\
\hline $1 / 4 / 17 / 97$ & & & \\
\hline
\end{tabular}


CLIENT: DUKE ENGINEERING \& SERVICES HANFORD, INC.

FILE NO: $\quad \mathrm{KH}-8009-8-09$

PROJECT: MCO Final Design DOC. NO.: HNF-SD-SNF-DR-003, Rev. 0, Appendix 11

\section{LISTING OF TG275.INP FILE}

IBATCH,LIST

IFILENAM,TG275

IPREP7

ITITLE,MCO DESIGN- 100 C TEMPERATURE DIFFERENTIAL, 189 PSI

TREF,70 I Reference Temperature of $70 \mathrm{~F}$.

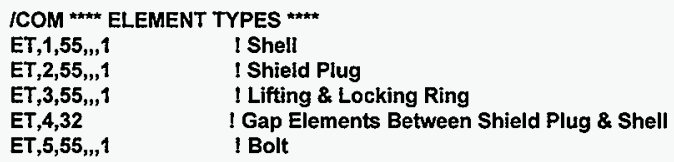

\begin{tabular}{|l|c|l|l|l|}
\hline REVISION & 0 & & & \\
\hline PREPARED BY IDATE & TE $4 / 17 / 97$ & & & \\
\hline CHECKED BY I DATE & P 4/17/97 & & & \\
\hline
\end{tabular}


CLIENT: DUKE ENGINEERING \& SERVICES HANFORD, INC.

FILE NO: $\quad \mathrm{KH}-8009-8-09$

PROJECT: MCO Final Design DOC. NO.: HNF-SD-SNF-DR-003, Rev. 0, Appendix 11

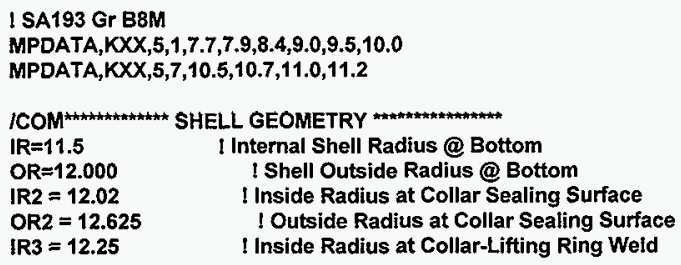

$N, 3,2.13,-1.32$

$N, 10,11.423,-1.32$

FILL

$$
\begin{aligned}
& \mathrm{N}, 41,0.00,-0.44 \\
& N, 42,1.25,-0.44 \\
& N, 43,2.13,0.44 \\
& N, 50, I R, 0.44 \\
& \text { FILL,43,50 } \\
& N, 52,0 R, 0.44 \\
& \text { FILL, } 50,52
\end{aligned}
$$$$
\text { ! Row } 3
$$

FILL, 1,41,1,21,1,10 I Middle Row

FILL, 10,50,1,30

$\mathrm{N}, 32,12,-0.32$

FILL, 30,32

FILL, $10,32,1,11$

$\mathrm{N}, \mathbf{5 3}, \mathrm{IR}, \mathbf{1 . 1 7}$

$\mathrm{N}, 55, \mathrm{OR}, 1.17$

FILL, 53,55

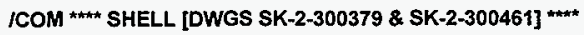

$\mathrm{N}, 65, \mathrm{IR}, 6.68$

$\mathrm{N}, 67, \mathrm{OR}, 6.68$

FILL.

FILL, $53,65,3,3,3,1$

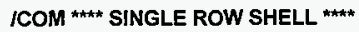

$\mathrm{N}, 100, \mathrm{IR}, 7.18 \quad$ ! Inside

$N, 140,1 R, 71.68$

$N, 180, I R, 136.68$

N,101,OR,7.18 I Outside

$\mathrm{N}, 141, \mathrm{OR}, 71.68$

$\mathrm{N}, 181, \mathrm{OR}, 136.68$

FILL,100,140,20,2,2,1,2.0

FILL,140,180,19,2,2,1,.5

ICOM *** DOUBLE ROW SHELL ***

N,190,IR,137.18 I Transition to Double Row

$\mathrm{N}, 192, \mathrm{OR}, 137.18$

\begin{tabular}{|l|c|l|l|l|}
\hline REVISION & 0 & & & \\
\hline PREPARED BY IDATE & 20, 4/17/97 & & & \\
\hline CHECKED BY I DATE & $/ / 4 / 17 / 97$ & & & \\
\hline
\end{tabular}


CLIENT: $\quad$ DUKE ENGINEERING \& SERVICES HANFORD, INC.

FILE NO: $\quad \mathrm{KH}-8009-8-09$

PROJECT: MCO Final Design DOC. NO.: HNF-SD-SNF-DR-003, Rev. 0, Appendix 11

FILL

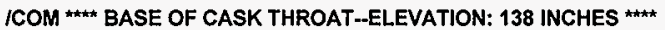

$N, 217, I R, 142.68$ I Transition to Double Row

N,219,OR,142.68

FILL

FILL,190,217,8,,3,3,1 ! Vertical Fill

ICOM *** BOTTOM OF COLLAR TRANSITION ****

N,235,IR,146.06 I Start of Transition to Large O.D \&

N,237,OR,146.06 I Assumed Location of Shield Plug Taper

FILL

$N, 238, I R, 146.68$

$\mathrm{N}, 240, \mathrm{OR}, 146.68$

FILL I Horizontal Fill

FILL, 217,235,5,3,3,1 IVertical Fill

ICOM TOP OF COLLAR TRANSITION

N,241,IR,147.31 ! End of Transition to Large O.D \&

N,243,OR,147.31 I Assumed Location of Shield Plug Taper

FILL I Horizontal Fill

NGEN,2,3,241,243,1,0.75

ICOM ** COLLAR SEALING SURFACE

$N, 247, I R, 149.63 \quad$ IInside Radius of Sealing Surface

N,249,IR2,149.63 ! Outside Radius at Sealing Surface

FILL.

! Horizontal Fill

ICOM THICK WALL AT COLLAR TRANSITION

NGEN, $2,10,240,249,3$

I Nodes 250-259 Coincident w/240-249 (by 3)

N,255,OR2,147.31

! Outside Surface

$N, 261$, OR2,149.63 I Outside Surface

$\mathrm{N}, \mathbf{2 5 8 , O R 2 , 1 4 8 . 0 6}$

N,980,IR, 149.38

N,981,11.755,149.38

N,982,IR2,149.38

$\mathrm{N}, 983,12.317,149.38$

$N, 984$, OR2, 149.38

N,990,OR2,146.68

FILL, 240,990,1,251

NGEN $, 2,5,980,984,1,-0.66$

FILL, 246,258,1,257

FILL, 253,255,1,1,3,3

FILL, 237,990,1,991

ICOM COLLAR AT BOTTOM EDGE OF PLUG (.155" above Sealing Surface)

NGEN $, 2,3,259,{ }_{m}, 0.245$ I Nodes 262

ICOM *** COLLAR AT TOP EDGE OF PLUG (2" above bottom Edge) ***

NGEN, 2,9,262,,,2.00 [ Nodes 271

FILL,262,271,2

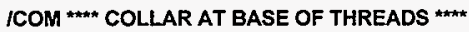

N,274,IR3, 152.00

$\mathrm{N}, 1000$, IR2, 152.00

REVISION

PREPARED BY IDATE

CHECKED BY IDATE 
CLIENT: DUKE ENGINEERING \& SERVICES HANFORD, INC.

FILE NO: $\quad \mathrm{KH}-8009-8-09$

PROJECT: MCO Final Design DOC. NO.: HNF-SD-SNF-DR-003, Rev. 0, Appendix 11

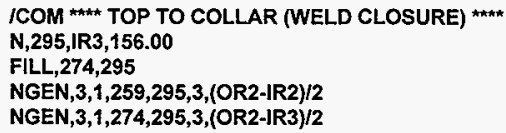


CLIENT: DUKE ENGINEERING \& SERVICES HANFORD, INC.

FILE NO: KH-8009-8-09

PROJECT: MCO Final Design DOC. NO.: HNF-SD-SNF-DR-003, Rev. 0, Appendix 11

NGEN, $2,70,441,448,1,-4$

FILL,441,511,6,10,8,1
I Bottom Surface of Lifting/Locking Ring

I Fill in Lifting/Locking Ring

ICOM SHIELD PLUG (offset y by 158.25)

LOCAL,20,0,158.13

TYPE, 2

PLUGR1=11.975

PLUGR2 $=11.45$

PLUGR3=11.25

PLUGR4 $=7.89$

ICOM *** NODES AT PLUG AXIS $(\mathrm{r}=0)^{\star \star \star \star}$

$\mathrm{N}, 601$

$\mathbf{N}, 602,0,-1$

$\mathrm{N}, 603,0,-1.994$

$\mathrm{N}, 606,0,-4.994$

FILL, $603,606,2,604$

$\mathbf{N}, 607,0,-6.25$

$\mathrm{N}, 610,0,-8.25$

FILL, 607,610,2,608

$\mathrm{N}, 611,0,-8.75$

N,613,0,-10.5

FILL,611,613

ICOM N* NODAL GENERATION

NGEN, 2,20,601,613,1,0.8825

NGEN, 2, 20,621, 633,1,0.8825

NGEN, $2,20,642,653,1,0.6875$

NGEN, 2, 20,662, 673,1,0.6875

NGEN, 2,20,683,693,1,0.4235

NGEN,2,10,706,713,1,0.9515

I Id Large Opening

I Id Medium Opening

I ld Small Opening

1 Center of Opening

$\mathrm{N}, 730,5.4665,-1.994$

N,736,5.4665,-4.994

I Od Small Opening

FILL, 730,736,5,731

$\mathbf{N}, 737,5.4665,-6.25$

$\mathbf{N}, 740,5.4665,-8.25$

FILL,737,740,2,738

$\mathbf{N}, 741,5.4665,-8.75$

$\mathrm{N}, 743,5.4665,-10.5$

FILL, 741,743

N,748,5.89,-1.0

NGEN,2,20,730,743,1,0.4235

FILL, 748,750

$\mathrm{N}, 766,7,265,0$

NGEN,2,20,748,763,1,1.375

FILL,766,768

NGEN,3,20,766,768,1,0.3125

$\mathrm{N}, 789,7.5775,-1.56$

N,796,7.5775,-5.56

FILL, 789,796,6

NGEN,2,20,789,796, $1,0.3125$

NGEN,3,20,777,783,1,0.3125

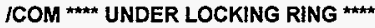

$\mathrm{N}, 824,8.5017,-6.25$

\begin{tabular}{|l|c|l|l|l|}
\hline REVISION & 0 & & & \\
\hline PREPARED BY IDATE & $6 / 4 / 17 / 97$ & & & \\
\hline CHECKED BY I DATE & $/ \mathrm{h} / 4 / 17 / 97$ & & & \\
\hline
\end{tabular}


CLIENT: DUKE ENGINEERING \& SERVICES HANFORD, INC.

FILE NO: $\quad \mathrm{KH}-8009-8-09$

PROJECT: MCO Final Design

$N, 827,8.5017,-8.25$

FILL

$N, 828,8.5017,-8.75$

$\mathrm{N}, 830,8.5017,-10.5$

FILL

NGEN, $3,7,824,830,1,0.5616$

NGEN, 2,7,838,844,1,0.625

NGEN, 2,7,845,851, 1,0.6875 IUnder Bolt

$\mathbf{N}, 859,11.625,-6.25$

$\mathbf{N}, 860,11.625,-6.917$

$\mathrm{N}, 861,11.625,-7.584$

$\mathrm{N}, 862$, PLUGR2, -8.25

N,863,PLUGR2,-8.75

N,865,PLUGR3,-10.5

FILL,863,865, 1

$\mathrm{N}, 866$, PLUGR1 $-0.288,-6.25$

N,869,PLUGR1-0.288,-8.25

FILL, 866,869,2

N;870,PLUGR1-0.288,-8.476

NGEN,2,5,866,870,1,0.288

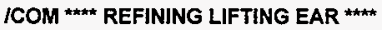

CSYS, 0

$\mathrm{N}, 877,9.53,158.13$

$\mathrm{N}, 889,9.53,157.63$

$\mathrm{N}, 901,9.53,157.13$

FILL, $403,404,1,876$

FILL, $413,414,1,888$

FILL, $\mathbf{4 2 3 , 4 2 4 , 1 , 9 0 0}$

FILL, 877,405,1,878

FILL,405,406,2,879,1

FILL, $889,415,1,890$

FILL,415,416,2,891,1

FILL, 404,414,1,881

FILL,877,889,1,882

FiLL, $878,890,1,883$

FILL,405,415,1,884

FILL,879,891,1,885

FILL,880,892,1,886

FILL,406,416,1,887

FILL,889,901,1,894

FILL, $414,424,1,893$

FILL, $901,425,1,902$

FILL,890,902,1,895

FILL,415,425,1,896

FILL,425,426,2,903,1

FILL,891,903,1,897

FILL,892,904,1,898

FILL,416,426;1,899

FILL,424,434,1,907

FILL,433,434,1,908

FILL,423,433,1,905

FILL,905,907

ICOM * COUPLING NODES ***

ICOM *** BETWEEN LIFTINGILOCKING RING \& SHELL ***

\begin{tabular}{|l|c|l|l|l|}
\hline REVISION & 0 & & & \\
\hline PREPARED BY I DATE & ZfS 4/17/97 & & & \\
\hline CHECKED BY I DATE & /h/ 4/17/97 & & & \\
\hline
\end{tabular}


CLIENT: DUKE ENGINEERING \& SERVICES HANFORD, INC. FILE NO: KH-8009-8-09

PROJECT: MCO Final Design DOC. NO.: HNF-SD-SNF-DR-003, Rev. 0, Appendix 11

CP, 1, TEMP, 508,277

! Start Threads

CP,2,TEMP,498,280

CP,3,TEMP,488,283

CP, 4, TEMP, 478,286

$C P, 5$, TEMP,468,289

CP,6,TEMP,458,292

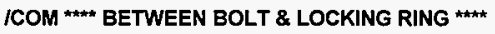

*DO,1,1,7

CP,8+1,TEMP, 445+10*1,910+2*I

¿ENDDO

"DO, I, 1,7

CP, 15+1,TEMP, $447+10 * 1,911+2 * \mid$

*ENDDO

NALL.

EALL

ICOM ${ }^{\star \star \star}$ ELEMENT GENERATION FOR SHELL

TYPE,1

MAT,1

ICOM BOTTOM OF SHELL

$E, 1,2,22,21$

$E, 2,3,23,22$

EGEN,8,1,-1

$E, 10,11,30$

$E, 21,22,42,41$

$E, 22,23,43,42$

EGEN,10,1,-1

$E, 11,31,30$

$E, 11,32,31$

ICOM *** SHELL ***

E,50,51,54,53

EGEN,2,1,-1

EGEN,5,3,-2

ICOM FIRST TRANSITION ELEMENTS

$E, 65,66,100$

$\mathrm{E}, \mathbf{1 0 0 , 6 6 , 1 0 1}$

$E, 67,101,66$

ICOM SINGLE SHELL *

$E, 100,101,103,102$

EGEN,40,2,-1

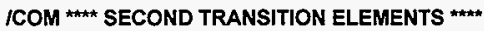

E, 190,180,191

$E, 180,181,191$

$E, 181,192,191$

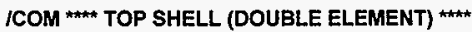

E,190,191,194,193

EGEN,2,1,-1

EGEN,18,3,-2

$E, 244,245,986,985$

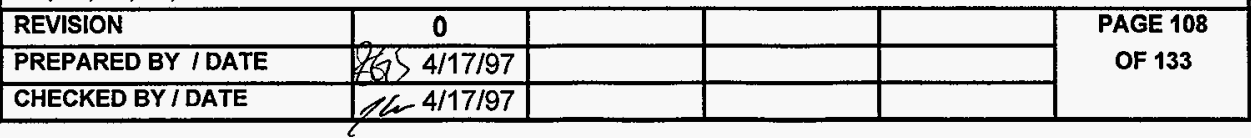


CLIENT: DUKE ENGINEERING \& SERVICES HANFORD, INC.

FILE NO: $\quad \mathrm{KH}-8009-8-09$

PROJECT: MCO Final Design DOC. NO.: HNF-SD-SNF-DR-003, Rev. 0, Appendix 11

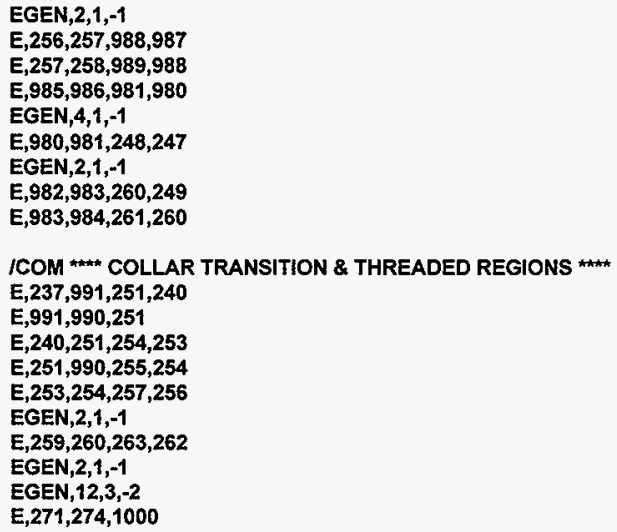

REVISION PREPARED BY I DATE CHECKED BY / DATE 
CLIENT: $\quad$ DUKE ENGINEERING \& SERVICES HANFORD, INC.

FILE NO: $\quad \mathrm{KH}-8009-8-09$

PROJECT: MCO Final Design DOC. NO.: HNF-SD-SNF-DR-003, Rev. 0, Appendix 11

$E, 893,894,889,414$

$E, 424,901,894,893$

$E, 894,895,890,889$

E,901,902,895,894

E,895,896,415,890

E,902,425,896,895

$E, 896,897,891,415$

$E, 425,903,897,896$

$E, 897,898,892,891$

E,903,904,898,897

$E, 898,899,416,892$

E,904,426,899,898

$E, 431,432,422,421$

$E, 905,423,422$

E,432,905,422

$E, 432,433,905$

E,905,906,900,423

$E, 433,908,906,905$

E,906,907,424,900

$E, 908,434,907,906$

$E, 441,442,432,431$

EGEN,2,1,-1

$E, 443,908,433$

$E, 443,444,434,908$

E,451,452,442,441

EGEN,3,1,-1

EGEN,7,10,-3

$E, 454,912,910,444$

E,464,914,912,454

$E, 474,916,914,464$

$E, 484,918,916,474$

$E, 494,920,918,484$

$E, 504,922,920,494$

E,514,924,922,504

$E, 458,448,911,913$

$E, 468,458,913,915$

$E, 478,468,915,917$

$E, 488,478,917,919$

$E, 498,488,919,921$

$E, 508,498,921,923$

E,518,508,923,925

/COM ${ }^{\star \star \star \star ~ B O L T ~}{ }^{\star \star \star \star *}$

TYPE,5

MAT,5

$\mathrm{E}, \mathbf{4 5 5}, \mathbf{4 5 6 , 4 4 6 , 4 4 5}$

EGEN,8,10,-1

$E, 456,457,447,446$

EGEN,8,10,-1

/COM

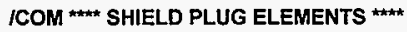

TYPE,2

MAT, 1

$E, 602,622,621,601$

EGEN, 12,1,-1

REVISION

PREPARED BY I DATE

CHECKED BY/DATE

PAGE 110

OF 133 
CLIENT: DUKE ENGINEERING \& SERVICES HANFORD, INC. FILE NO: KH-8009-8-09

PROJECT: MCO Final Design DOC. NO.: HNF-SD-SNF-DR-003, Rev. 0, Appendix 11

EGEN,2,20,-12

EGEN,3,20,-11

EGEN,2,20,-10

$E, 707,717,716,706$

EGEN, $7,1,-1$

E,717,737,736,716

EGEN, $7,1,-1$

E,731,751,750,730

EGEN,13,1,-1

E,749,769,768,748

EGEN,15,1,-1

$E, 767,787,786,766$

EGEN,17,1,-1

EGEN,2,20,-17

$E, 818,825,824,817$

EGEN, 6,1,-1

EGEN,5,7,-6

$E, 853,860,859,852$

EGEN,6,1,-1

$E, 860,867,866,859$

EGEN;3,1,-1

$E, 867,872,871,866$

EGEN,4,1,-1

ICOM END OF SHIELD PLUG

ICOM CONTACT ELEMENTS

ICOM * BETWEEN LOCKING RING \& SHIELD PLUG

TYPE, 4

REAL, 4

$E, 806,401$

E,807,411

$E, 808,421$

E,809,431

E,810,441

$E, 811,451$

$E, 812,461$

$E, 813,471$

$E, 814,481$

$E, 815,491$

$E, 816,501$

$E, 817,511$

ICOM *** BETWEEN SHIELD PLUG \& BOTTOM OF BOLT

REAL, 5

E,845,525

$E, 852,526$

$E, 859,527$

ICOM BETWEEN SHIELD PLUG \& SHELL (ABOVE SEAL)

REAL, 4

$E, 871,271$

$E, 872,268$

$E, 873,265$

$E, 874,262$

ICOM BETWEEN SHIELD PLUG \& SHELL (BELOW SEAL)

\begin{tabular}{|l|c|l|l|l|}
\hline REVISION & 0 & & & \\
\hline PREPARED BY I DATE & S $4 / 17 / 97$ & & & \\
\hline CHECKED BY I DATE & S $4 / 17 / 97$ & & & \\
\hline
\end{tabular}


CLIENT: DUKE ENGINEERING \& SERVICES HANFORD, INC. FILE NO: KH-8009-8-09

PROJECT: MCO Final Design DOC. NO.: HNF-SD-SNF-DR-003, Rev. 0, Appendix 11

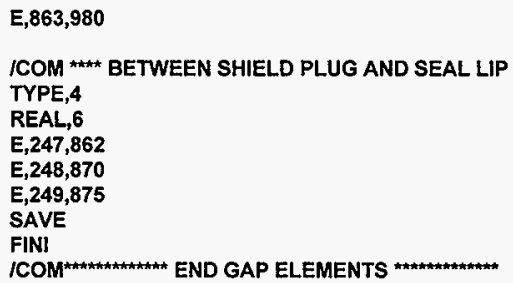

\begin{tabular}{|l|c|l|l|l|c|}
\hline REVISION & 0 & & & & PAGE 112 \\
PREPARED BY IDATE & OF 133 \\
\hline CHECKED BY I DATE & 4/17/97 & & & & \\
\end{tabular}


CLIENT: $\quad$ DUKE ENGINEERING \& SERVICES HANFORD, INC. FILE NO: KH-8009-8-09

PROJECT: MCO Final Design DOC. NO.: HNF-SD-SNF-DR-003, Rev. 0, Appendix 11

IPREP7

ICOM *** ELEMENT TYPES ***

$E T, 1,42,, 1$ ! Switches

ET, 2,42,,1 ! Thermal Elements PLANE55

ET, 3,42,,1 ! to

ET, 5,42,,1 ! Structural Elements PLANE42

ET,4,12 I Switches LINK32 to CONTACT12

KEYOPT,4,7,1

ICOM ** REAL CONSTANTS FOR GAP ELEMENTS ***

R,4,-90, 1.0e8,-0.06,3.0 IShell/Shield Plug, Initially Open .06"

R,5,0,1.0e8,2.95e-03 I L. Ring/Shield Plug, Under Bolt, Preloaded

$R, 6,0,1.0 e 8,0,2.0 \quad$ I Sealing Surface, initially closed

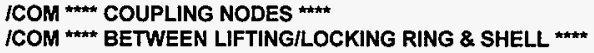

CP,1,UY,508,277 I Start Threads

$C P, 2, U Y, 498,280$

CP,3,UY,488,283

$C P, 4, U Y, 478,286$

$C P, 5, U Y, 468,289$

CP, $6, U Y, 458,292$

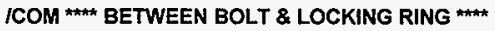

$\mathrm{CP}, 7, \mathrm{UY}, 445,910$

CP, $8, U X, 445,910$

CP,9,UY,447,911

CP, 10,UX,447,911

${ }^{*} \mathrm{DO}, \mathrm{l}, \mathbf{1 , 7}$

$C P, 10+1, U Y, 445+10^{\star} 1,910+2^{\star}$

*ENDDO

${ }^{*} \mathrm{DO}, \mathbf{1 , 1 , 7}$

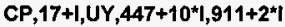

*ENDDO

${ }^{*} \mathrm{DO}, 1,1,7$

$C P, 24+1, U X, 445+10 * 1,910+2 \star \mid$

"ENDDO

*DO,I,1,7

CP,31+1,UX,447+10*1,911+2*1

"ENDDO

NALL

EALL

ICOM BOUNDARY CONDITIONS AT AXIS $(X=0)$

CSYS, 0

NSEL,S,LOC, $X, 0$

D,ALL,UX,0

ALLS

NSEL,S,NODE, ,10

D,ALL,UY,O

ALLS

SAVE

FINI

ICOM SOLUTION $\quad$ I Transfer Temperatures to Structural Model

\begin{tabular}{|l|c|l|l|l|}
\hline REVISION & 0 & & & \\
\hline PREPARED BY I DATE & / $/ 4 / 17 / 97$ & & & \\
\hline CHECKED BY I DATE & $/ 4 / 17 / 97$ & & & \\
\hline
\end{tabular}


CLIENT: DUKE ENGINEERING \& SERVICES HANFORD, INC. FILE NO: KH-8009-8-09

PROJECT: MCO Final Design DOC. NO.: HNF-SD-SNF-DR-003, Rev. 0, Appendix 11

ISOLU

LDREAD,TEMP,LAST,,,'TG275,RTH I Reads in Temperatures from TG275.RTH file.

ICOM APPLYING 189 PSI INTERNAL PRESSURE

NALL.

EALL

NSEL,S,LOC,X,0,1.26 ! Bottom Plate

NSEL,R,LOC,Y, $-0.45,-0.43$

SF,ALL,PRES, 189

NALL

EALL

NSEL,S,LOC, $X, 1.24,2.14$

NSEL, R,LOC,Y,-0.45, 0.45

SF,ALL,PRES, 189

NALL

EALL

NSEL,S,LOC,X,2.12,11.51

NSEL,R,LOC,Y,0.43,0.45

SF,ALL,PRES, 189

NALL

EALL

NSEL,S,LOC,X,11.49,11.51 IInside Shell

NSEL,R,LOC,Y, $0.43,149.64$

SF,ALL,PRES, 189

NALL

EALL

NSEL,S,LOC,X,11.49,11.76 I Edge Shell to Seal

NSEL,R,LOC,Y,149.62,149.64

SF,ALL,PRES, 189

NALL

EALL

NSEL,S,LOC,X,11.67,11.69 iseal

NSEL,R,LOC,Y,149.64,149.89

SF,ALL,PRES, 189

NALL

EALL

NSEL,S,LOC,X,11.44,11.69 I Shield Plug (above seal)

NSEL,R,LOC,Y,149.87,149.89

SF,ALL,PRES, 189

NALL

EALL

NSEL,S,LOC,X,11.44,11.46 I Side of Shield Plug

NSEL,R,LOC, Y, $149.37,149.89$

SF,ALL,PRES, 189

NALL

EALL

NSEL,S,LOC,X,11.24,11.46 I Shield Plug Taper

NSEL,R,LOC,Y,147.62, 149.39

SF,ALL,PRES, 189

NALL

EALL

NSEL, S,LOC, $X, 0,11.26$ I Bottom of Shield Plug

NSEL,R,LOC, Y,147.62, 147.64

SF,ALL,PRES, 189

NALL

EALL

\begin{tabular}{|l|c|l|l|l|}
\hline REVISION & 0 & & & \\
\hline PREPARED BY IDATE & Z4/ 4/17/97 & & & \\
\hline CHECKED BY I DATE & $/ 4 / 4 / 17 / 97$ & & & \\
\hline
\end{tabular}


CLIENT: DUKE ENGINEERING \& SERVICES HANFORD, INC.

FILE NO: $\quad \mathrm{KH}-8009-8-09$

PROJECT: MCO Final Design $\quad$ DOC. NO.: HNF-SD-SNF-DR-003, Rev. 0, Appendix 11

SOLVE

SAVE

FINI

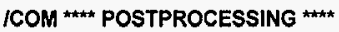

IPOST1

PLNSOL,S,INT

ITYPE,ALL,HIDC

IGLINE,ALL, 0

RSYS, 0

IDSCALE,,10

IREPLOT

LPATH,1,41

PRSECT

1 Bottom Plate

LPATH, 6,46

PRSECT

LPATH, 10,50

PRSECT

LPATH, 50,52

PRSECT

I Lower Shell

LPATH,62,64

PRSECT

LPATH, 65,67

PRSECT

LPATH,100,101

PRSECT

LPATH,122,123

PRSECT

LPATH,134,135

PRSECT

LPATH,156,157

PRSECT

LPATH,170,171

PRSECT

LPATH,180,181

PRSECT

LPATH,202,204

PRSECT

LPATH, 235, 237

PRSECT

LPATH,985,989

PRSECT

LPATH,262,264

PRSECT

LPATH,277,279

PRSECT

LPATH,292,294

PRSECT

LPATH,601,641

PRSECT

LPATH,601,613

PRSECT

LPATH, 603,703

PRSECT

LPATH,606,706

PRSECT

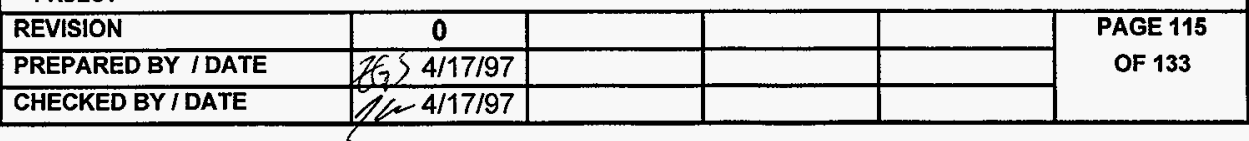


CLIENT: DUKE ENGINEERING \& SERVICES HANFORD, INC.

FILE NO: $\quad \mathrm{KH}-8009-8-09$

PROJECT: MCO Final Design DOC. NO.: HNF-SD-SNF-DR-003, Rev. 0, Appendix 11

LPATH,706,736

PRSECT

LPATH,766,806

PRSECT

LPATH,748,808

PRSECT

LPATH, 730,810

PRSECT

LPATH,736,815

PRSECT

LPATH,869,874

PRSECT

LPATH,870,875

PRSECT

LPATH, $\mathbf{4 3 1 , 4 3 4}$

PRSECT

I Locking Ring

LPATH,406,426

PRSECT

LPATH,404,424

PRSECT

SAVE

\begin{tabular}{|c|l|l|l|}
\hline 0 & & & \\
\hline $2654 / 17 / 97$ & & & \\
\hline $4 \times 4 / 17 / 97$ & & & \\
\hline
\end{tabular}


CLIENT: DUKE ENGINEERING \& SERVICES HANFORD, INC.

FILE NO: KH-8009-8-09

PROJECT: MCO Final Design DOC. NO.: HNF-SD-SNF-DR-003, Rev. 0, Appendix 11

\section{COMPUTER RUN COVER SHEET}

Project Number:

Computer Code:

Software Version:

Computer System:

Computer Run File Number:

Unique Computer Run Filename:

Run Description:

Run Date / Time:
$\mathrm{KH}-8009-8$

ANSYS $B-P C$

$5.0 \mathrm{~A}$

MS-DOS, Pentium \& Processor

KH-8009-8-09

TG275.out

Load Case 5 Output

27 March $1997 \quad$ 1:03:02 PM

Prepared By: Zachary G. Sargent
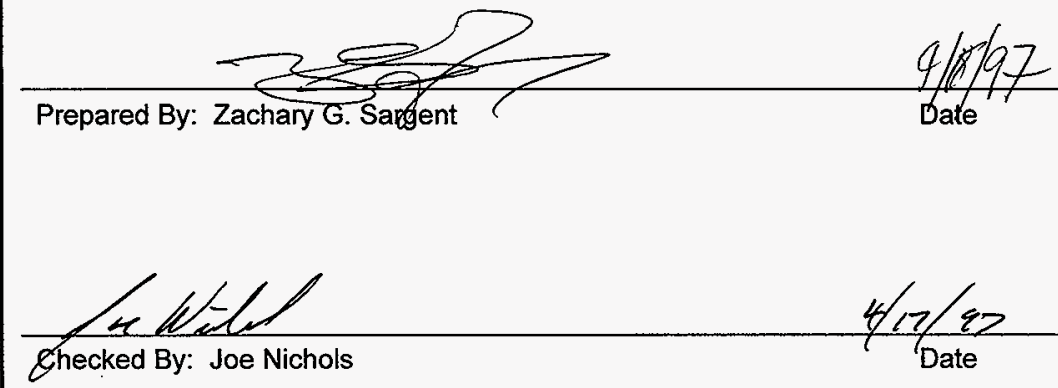

0

$4 / 17 / 97$


CLIENT: $\quad$ DUKE ENGINEERING \& SERVICES HANFORD, INC. FILE NO: KH-8009-8-09

PROJECT: MCO Final Design DOC. NO.: HNF-SD-SNF-DR-003, Rev. 0, Appendix 11

\section{COMPUTER RUN COVER SHEET}

Project Number:

Computer Code:

Software Version:

Computer System:

Computer Run File Number:

Unique Computer Run Filename:

Run Description:

Creation Date / Time:
$\mathrm{KH}-8009-8$

ANSYS\&-PC

$5.0 \mathrm{~A}$

MS-DOS, Pentium® Processor

$\mathrm{KH}-8009-8-09$

TT24.inp

Load Case 6: Thermal Transient

27 March $1997 \quad$ 1:28:56 PM
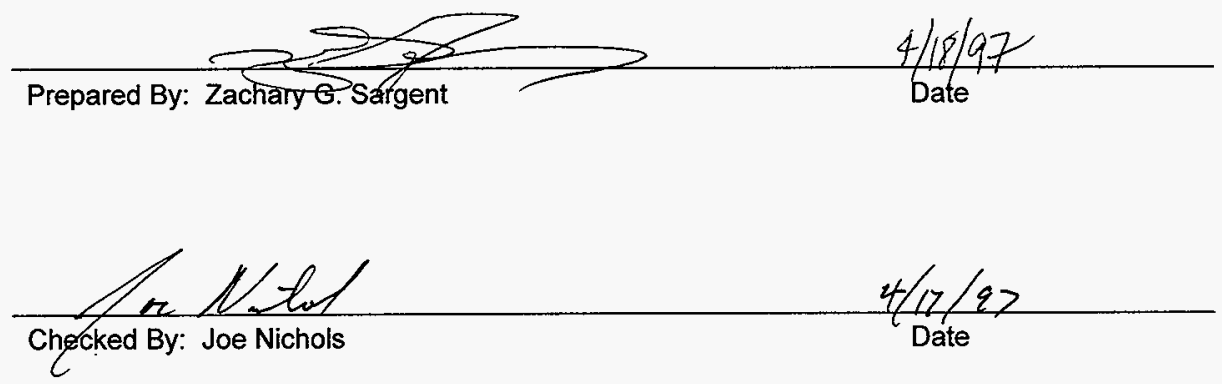

\begin{tabular}{|l|l|l|l|}
\hline 0 & & & \\
\hline $4 / 17 / 97$ & & & \\
\hline $4 / 17 / 97$ & & & \\
\hline
\end{tabular}


CLIENT: DUKE ENGINEERING \& SERVICES HANFORD, INC.

FILE NO: $\quad \mathrm{KH}-8009-8-09$

PROJECT: MCO Final Design DOC. NO.: HNF-SD-SNF-DR-003, Rev. 0, Appendix 11

\title{
LISTING OF TT24.INP FILE
}

\author{
/BATCH,LIST \\ IFILENAM,TT24 \\ IPREP7 \\ ITITLE, THERMAL TRANSIENT, TEMPERATURES @ $24 \mathrm{hrs,} 189$ PSI \\ TREF,70 I Reference Temperature of $70 \mathrm{~F}$.

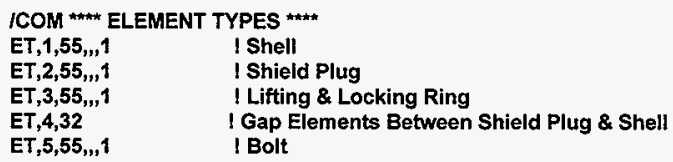

\begin{tabular}{|l|c|l|l|l|}
\hline REVISION & 0 & & & \\
\hline PREPARED BY I DATE & f6/ 4/17/97 & & & \\
\hline CHECKED BY I DATE & I/A 4/17/97 & & & \\
\hline
\end{tabular}


CLIENT: DUKE ENGINEERING \& SERVICES HANFORD, INC.

FILE NO: KH-8009-8-09

$\begin{array}{ll}\text { PROJECT: MCO Final Design } & \text { DOC. NO.: HNF-SD-SNF-DR-003, Rev. 0, Appendix } 11\end{array}$

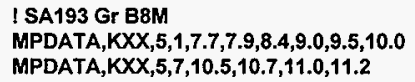

I SA193 Gr B8M

MPDATA,KXX,5,1,7.7,7.9,8.4,9.0,9.5,10.0

MPDATA,KXX,5,7,10.5,10.7,11.0,11.2

ICOM $M^{\star \star \star \star \star \star \star \star \star ~}$

IR=11.5 ! Internal Shell Radius @ Bottom

OR=12.000 I Shell Outside Radius @ Bottom

IR2 = 12.02 I Inside Radius at Collar Sealing Surface

OR2 $=12.625 \quad$ I Outside Radius at Collar Sealing Surface

IR3 = 12.25 I Inside Radius at Collar-Lifting Ring Weld

ICOM *** BOTTOM COVER PLATE [DWG SK-2-300378]

$\mathrm{N}, 1,-1.32$

! Row 1

$\mathbf{N}, 2,1.25,-1.32$

$N, 3,2.13,-1.32$

$\mathbf{N}, \mathbf{1 0}, \mathbf{1 1}, \mathbf{4 2 3},-\mathbf{- 1 . 3 2}$

FILL
$\mathbf{N}, 41,0.00,-0.44$
$\mathrm{N}, 42,1.25,-0.44$
$\mathbf{N}, 43,2.13,0.44$
$\mathrm{N}, 50,1 R, 0.44$
FILL, 43,50
N,52,OR,0.44
FILL,50,52

FILL,1,41,1,21,1,10

! Middle Row

FILL, 10,50,1,30

$\mathrm{N}, 32,12,-0.32$

FILL, 30,32

FILL,10,32,1,11

N,53,IR,1.17

N,55,OR, 1.17

FILL,53,55

! Row 3

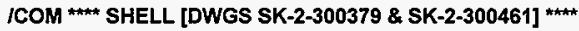

$\mathrm{N}, 65, \mathrm{IR}, 6.68$

$\mathrm{N}, 67, \mathrm{OR}, 6.68$

FILL

FILL, $53,65,3,3,3,1$

ICOM SINGLE ROW SHELL *

$\mathrm{N}, \mathbf{1 0 0 , I R , 7 . 1 8}$

! Inside

$\mathrm{N}, 140, \mathrm{IR}, 71.68$

$\mathrm{N}, \mathbf{1 8 0 , I R , 1 3 6 . 6 8}$

$\begin{array}{ll}N, 101,0 R, 7.18 & \text { I Outside }\end{array}$

$\mathrm{N}, 141, \mathrm{OR}, 71.68$

$\mathrm{N}, 181, \mathrm{OR}, 136.68$

FILL,100,140,20,2,2,1,2.0

FILL, 140,180,19,2,2,1,.5

ICOM *** DOUBLE ROW SHELL ****

N,190,IR,137.18 I Transition to Double Row

$\mathrm{N}, \mathbf{1 9 2 , O R , 1 3 7 . 1 8}$

REVISION

PREPARED BY / DATE

CHECKED BY / DATE

PAGE 120

OF 133 
CLIENT: DUKE ENGINEERING \& SERVICES HANFORD, INC.

FILE NO: KH-8009-8-09

$\begin{array}{ll}\text { PROJECT: MCO Final Design } & \text { DOC. NO.: HNF-SD-SNF-DR-003, Rev. 0, Appendix } 11\end{array}$

FILL

ICOM BASE OF CASK THROAT--ELEVATION: 138 INCHES ***

$N, 217, I R, 142.68$ ! Transition to Double Row

$N, 219,0 R, 142.68$

FILL.

FILL, 190,217,8,3,3,1 IVertical Fill

ICOM B*tTOM OF COLLAR TRANSITION

$N, 235, I R, 146.06 \quad$ I Start of Transition to Large O.D \&

N,237,OR,146.06 I Assumed Location of Shield Plug Taper

FILL

$\mathrm{N}, 238, I R, 146.68$

$N, 240, O R, 146.68$

FILL

! Horizontal Fill

FILL,217,235,5,3,3,1 IVertical Fill

ICOM *** TOP OF COLLAR TRANSITION ****

N,241,IR,147.31 ! End of Transition to Large O.D \&

N,243,OR,147.31 I Assumed Location of Shield Plug Taper

FILL ! Horizontal Fill

NGEN,2,3,241,243,1,0.75

ICOM $* *$ COLLAR SEALING SURFACE ****

N,247,IR,149.63 ! Inside Radius of Sealing Surface

N,249,IR2,149.63 I Outside Radius at Sealing Surface

FILL

I Horizontal Fill

ICOM THICK WALL AT COLLAR TRANSITION

NGEN,2,10,240,249,3

I Nodes 250-259 Coincident w/240-249 (by 3)

N,255,OR2,147.31

1 Outside Surface

$\mathrm{N}, 261, \mathrm{OR2}, 149.63$

I Outside Surface

$\mathrm{N}, 258, \mathrm{OR2}, 148.06$

N,980,IR,149.38

$\mathrm{N}, 981,11.755,149.38$

N,982,IR2, 149.38

$\mathrm{N}, 983,12.317,149.38$

$\mathrm{N}, 984, \mathrm{OR2}, 149.38$

N,990,OR2,146.68

FILL, 240,990,1,251

NGEN, $2,5,980,984,1,-0.66$

FILL, 246,258, 1,257

FILL, 253,255,1,1,3,3

FILL, 237,990,1,991

ICOM *** COLLAR AT BOTTOM EDGE OF PLUG (.155" above Sealing Surface) ****

NGEN,2,3,259,,,0.245 I Nodes 262

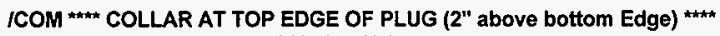

NGEN,2,9,262,,2, 2.00 ! Nodes 271

FILL, 262,271,2

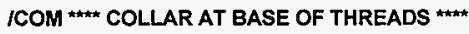

$\mathrm{N}, 274, \mathrm{IR} 3,152.00$

$\mathrm{N}, 1000, \mathrm{IR2}, 152.00$

\begin{tabular}{|l|c|l|l|l|l|}
\hline REVISION & 0 & & & & PAGE 121 \\
\cline { 1 - 4 } PREPARED BY IDATE & OF 133 \\
\hline CHECKED BY I DATE & 4/17/97/17/97 & & & & \\
\hline
\end{tabular}


CLIENT: DUKE ENGINEERING \& SERVICES HANFORD, INC.

FILE NO: $\quad \mathrm{KH}-8009-8-09$

PROJECT: MCO Final Design DOC. NO.: HNF-SD-SNF-DR-003, Rev. 0, Appendix 11

ICOM

N,295, IR3,156.00

FILL,274,295

NGEN,3,1,259,295,3,(OR2-IR2)/2

NGEN,3,1,274,295,3,(OR2-IR3)/2

ICOM

RING1=7.94

RING2 $=9.375$

RING3 $=9.625$

RING4=10.19

RING5=12.23

LOCAL,11,0,152.00 I Local System $z=0$ at Base of Ring

CSYS, 11

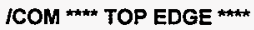

N,401,RING1,6.13

CSYS, $O$

$\mathrm{N}, 404,9.375,158.13$

FILL,401,404, ,1

N,406,RING4,158.13

FILL,404,406,,1 I Top Edge

ICOM LIFTING SURFACE

CSYS,11

N,421,RING1,5.13

N,424,RING2,5.13

FILL,421,424

N,426,RING4,5.13

FILL,424,426

FILL,401,421,1,10,6,1

N,431,RING1,6.13-1.56

N,434,RING2,6.13-1.56

FILL

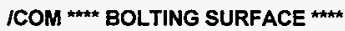

N,441,RING1,4

N,444,RING3,4

FILL

N,445, 10.9375-.6875,4 IInside Edge of Bolt Hole

$\mathrm{N}, 447,10.9375+.6875,4 \quad$ I Outside Edge of Bolt Hole

FILL

N,910,10.9375-.6875,4

$\mathrm{N}, 911,10.9375+.6875,4$

N,448,RING5,4

CSYS,0

I O.D of Ring

$N, 924,10.25,152.00$

! Bolt Extension

N,925,11.625,152.00

I Double Nodes @ Bolt for Gap elements

FILL, $910,924,6,2$

FILL,911,925,6,2

N,525,10.25,151.874

$\mathrm{N}, \mathbf{5 2 7}, 11.625,151.874$

FILL

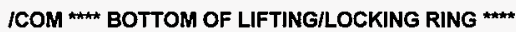

CSYS, 11

REVISION

PREPARED BY / DATE

I Bottom of Bolt Extension

CHECKED BY I DATE 
CLIENT: $\quad$ DUKE ENGINEERING \& SERVICES HANFORD, INC. FILE NO: KH-8009-8-09

PROJECT: MCO Final Design DOC. NO.: HNF-SD-SNF-DR-003, Rev. 0, Appendix 11

NGEN $, 2,70,441,448,1,-4$ I Bottom Surface of Lifting/Locking Ring

FILL,441,511,6,10,8,1 ! Fill in Lifting/Locking Ring

ICOM SHIELD PLUG (offset y by 158.25)

LOCAL,20,0,158.13

TYPE, 2

PLUGR1 $=11.975$

PLUGR2=11.45

PLUGR3=11.25

PLUGR4=7.89

ICOM NODES AT PLUG AXIS $(r=0)$ *

$\mathrm{N}, 601$

$\mathrm{N}, 602,0,-1$

$\mathrm{N}, 603,0,-1.994$

$\mathrm{N}, 606,0,-4.994$

FILL, 603,606,2,604

$\mathrm{N}, 607,0,-6.25$

$\mathrm{N}, 610,0,-8.25$

FILL, $607,610,2,608$

$\mathrm{N}, 611,0,-8.75$

$\mathrm{N}, 613,0,-10.5$

FILL,611,613

ICOM ** NODAL GENERATION *

NGEN,2,20,601,613,1,0.8825

NGEN, 2,20,621,633,1,0.8825

NGEN,2,20,642,653,1,0.6875

NGEN,2,20,662,673,1,0.6875

NGEN, 2,20,683,693,1,0.4235

NGEN,2,10,706,713,1,0.9515

I Id Large Opening

I Id Medium Opening

IId Small Opening

! Center of Opening

\section{$\mathrm{N}, 730,5.4665,-1.994$ \\ I Od Small Opening}

N,736,5.4665,-4.994

FILL, 730,736,5,731

$\mathrm{N}, 737,5.4665,-6.25$

$\mathrm{N}, 740,5.4665,-8.25$

FILL, 737,740,2,738

$N, 741,5.4665,-8.75$

$N, 743,5.4665,-10.5$

FILL, 741,743

$\mathrm{N}, 748,5.89,-1.0$

NGEN, $2,20,730,743,1,0.4235$

FILL, 748,750

$\mathrm{N}, 766,7.265,0$

NGEN, 2,20,748,763,1,1.375

FILL,766,768

NGEN, $3,20,766,768,1,0.3125$

$\mathrm{N}, 789,7.5775,-1.56$

$\mathbf{N}, 796,7.5775,-5.56$

FILL,789,796,6

NGEN,2,20,789,796,1,0.3125

NGEN,3,20,777,783, 1,0.3125

ICOM UNDER LOCKING RING

$\mathrm{N}, 824,8.5017,-6.25$

\begin{tabular}{|l|c|c|c|c|c|}
\hline REVISION & 0 & & & & PAGE 123 \\
PREPARED BY IDATE & OF 133 \\
\hline CHECKED BY I DATE & $4 / 17 / 97$ & & & & \\
\hline
\end{tabular}


CLIENT: DUKE ENGINEERING \& SERVICES HANFORD, INC. FILE NO: KH-8009-8-09

PROJECT: MCO Final Design DOC. NO.: HNF-SD-SNF-DR-003, Rev. 0, Appendix 11

$\mathbf{N}, \mathbf{8 2 7 , 8 . 5 0 1 7 , - 8 . 2 5}$

FILL.

$\mathrm{N}, 828,8.5017,-8.75$

$\mathbf{N}, 830,8.5017,-10.5$

FILL.

NGEN,3,7,824,830,1,0.5616

NGEN,2,7,838,844, 1,0.625

NGEN, $2,7,845,851,1,0.6875$ ! Under Bolt

N,859,11.625,-6.25

$\mathrm{N}, 860,11.625,-6.917$

$\mathrm{N}, 861,11.625,-7.584$

N,862,PLUGR2,-8.25

N,863,PLUGR2, -8.75

N,865,PLUGR3, -10.5

FILL,863,865, 1

N,866,PLUGR1-0.288,-6.25

N,869,PLUGRT-0.288,-8.25

FILL,866,869,2

N,870,PLUGRt $-0.288,-8.476$

NGEN,2,5,866,870,1,0.288

ICOM REFINING LIFTING EAR

CSYS, 0

N,877,9.53,158.13

N,889,9.53,157.63

N,901,9.53,157.13

FILL,403,404,1,876

FILL, 413,414,1,888

FILL, 423,424,1,900

FILL, 877,405,1,878

FilLL,405,406,2,879,1

FILL,889,415,1,890

FIL.L, 415,416,2,891,1

FILL,404,414,1,881

FILL, 877,889, 1,882

FILL, 878,890,1,883

FILL, 405,415,1,884

FILLL,879,891,1,885

FI느,880,892,1,886

FILL,406,416,1,887

FILL,889,901,1,894

FILL,414,424,1,893

FILL,901,425,1,902

FILL,890,902,1,895

FILL,415,425,1,896

FILLL,425,426,2,903,1

FILL,891,903,1,897

FILL,892,904, 1,898

FILL,416,426,1,899

FILL,424,434,1,907

FILL,433,434,1,908

FILL,423,433,1,905

FILL,905,907

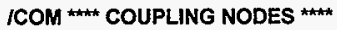

ICOM *** BETWEEN LIFTINGILOCKING RING \& SHELL \#*

\begin{tabular}{|l|c|l|l|l|}
\hline REVISION & 0 & & & \\
\hline PREPARED BY I DATE & $/ 4 / 17 / 97$ & & & \\
\hline CHECKED BY I DATE & $/ / 4 / 4 / 17 / 97$ & & & \\
\hline
\end{tabular}


FILE NO: $\quad \mathrm{KH}-8009-8-09$

PROJECT: MCO Final Design DOC. NO.: HNF-SD-SNF-DR-003, Rev. 0, Appendix 11

CP, 1, TEMP, 508,277
CP, 2, TEMP, 498,280
CP,3,TEMP,488,283
CP,4,TEMP,478,286
CP,5,TEMP,468,289
CP,6,TEMP,458,292

I Start Threads

ICOM BETWEEN BOLT \& LOCKING RING B*

${ }^{*} \mathrm{DO}, 1,1,7$

CP,8+1,TEMP,445+10*1,910+2*|

*ENDDO

*DO, $1,1,7$

CP,15+1,TEMP,447+10*1,911+2*|

*ENDDO

NALL

EALL

ICOM ELEMENT GENERATION FOR SHELL \#\#

TYPE,1

MAT,1

ICOM B\#* BOTTOM OF SHELL ****

$E, 1,2,22,21$

$E, 2,3,23,22$

EGEN,8,1,-1

$E, 10,11,30$

$E, 21,22,42,41$

$E, 22,23,43,42$

EGEN,10,1,-1

$E, 11,31,30$

$E, 11,32,31$

ICOM *** SHELL ***

$E, 50,51,54,53$

EGEN, 2,1,-1

EGEN, $5,3,-2$

ICOM FIRST TRANSITION ELEMENTS

$E, 65,66,100$

$E, 100,66,101$

$E, 67,101,66$

ICOM *** SINGLE SHELL ***

$E, 100,101,103,102$

EGEN,40,2,-1

ICOM * SECOND TRANSITION ELEMENTS

$E, 190,180,191$

$E, 180,181,191$

$E, 181,192,191$

ICOM *** TOP SHELL (DOUBLE ELEMENT)

$E, 190,191,194,193$

EGEN,2,1,-1

EGEN,18,3,-2

$E, 244,245,986,985$

\begin{tabular}{|l|c|l|l|l|}
\hline REVISION & 0 & & & \\
\hline PREPARED BY IDATE & TS $4 / 17 / 97$ & & & \\
\hline CHECKED BYIDATE & / $/ 17 / 97$ & & & \\
\hline
\end{tabular}


CLIENT: DUKE ENGINEERING \& SERVICES HANFORD, INC. FILE NO: KH-8009-8-09

PROJECT: MCO Final Design DOC. NO.: HNF-SD-SNF-DR-003, Rev. 0, Appendix 11

EGEN,2,1,-1

E,256,257,988,987

E,257,258,989,988

E,985,986,981,980

EGEN,4,1,-1

$E, 980,981,248,247$

EGEN,2,1,-1

$E, 982,983,260,249$

$E, 983,984,261,260$

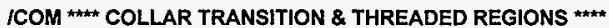

$E, 237,991,251,240$

E,991,990,251

E,240,251,254,253

E,251,990,255,254

$E, 253,254,257,256$

EGEN,2,1,-1

$E, 259,260,263,262$

EGEN, 2,1,-1

EGEN,12,3,-2

$E, 271,274,1000$

ICOM \#ERGE COINCIDENT NODES FOR SHELL \#*

ESEL,S,TYPE,,1

NSLE

NUMMRG,NODE,

EALL

NALL

ICOM END OF SHELLICOLLAR ELEMENT GENERATION **

ICOM LOCKINGLIFTING RING ELEMENTS

TYPE,3

MAT,1

$E, 411,412,402,401$

EGEN,2,1,-1

EGEN,2,10,-2

$E, 413,888,876,403$

$E, 881,404,876$

$E, 888,881,876$

$E, 888,414,881$

$\mathrm{E}, \mathbf{8 8 1 , 8 8 2 , 8 7 7 , 4 0 4}$

$E, 414,889,882,881$

$E, 882,883,878,877$

$E, 889,890,883,882$

E,883,884,405,878

E,890,415,884,883

$E, 884,885,879,405$

$E, 415,891,885,884$

$E, 885,886,880,879$

$E, 891,892,886,885$

$E, \mathbf{8 8 6}, \mathbf{8 8 7}, \mathbf{4 0 6 , 8 8 0}$

$E, 892,416,887,886$

$E, 423,900,888,413$

$E, 893,414,888$

$E, 900,893,888$

$E, 900,424,893$

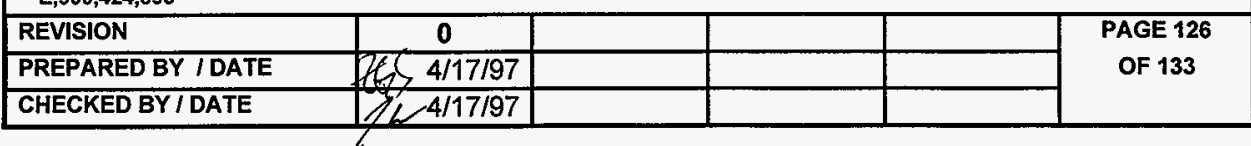


CLIENT: DUKE ENGINEERING \& SERVICES HANFORD, INC.

FILE NO: $\quad \mathrm{KH}-8009-8-09$

$\begin{array}{lll}\text { PROJECT: } & \text { MCO Final Design } & \text { DOC. NO.: HNF-SD-SNF-DR-003, Rev. 0, Appendix } 11\end{array}$

$E, 893,894,889,414$

$E, 424,901,894,893$

E,894,895,890,889

E,901,902,895,894

E,895,896,415,890

$E, 902,425,896,895$

E,896,897,891,415

$E, 425,903,897,896$

$E, 897,898,892,891$

E,903,904,898,897

E,898,899,416,892

E,904,426,899,898

$E, 431,432,422,421$

E,905,423,422

$E, 432,905,422$

E,432,433,905

$E, 905,906,900,423$

$E, 433,908,906,905$

E,906,907,424,900

E,908,434,907,906

$E, 441,442,432,431$

EGEN,2,1,-1

$E, 443,908,433$

$E, 443,444,434,908$

$E, 451,452,442,441$

EGEN,3,1,-1

EGEN,7,10,-3

E,454,912,910,444

$E, 464,914,912,454$

E,474,916,914,464

E,484,918,916,474

E,494,920,918,484

E,504,922,920,494

E,514,924,922,504

E,458,448,911,913

E,468,458,913,915

E,478,468,915,917

$E, 488,478,917,919$

E,498,488,919,921

E, $508,498,921,923$

$E, 518,508,923,925$

/COM **** BOLT ****

TYPE, 5

MAT, 5

$E, 455,456,446,445$

EGEN,8,10,-1

$E, 456,457,447,446$

EGEN,8,10,-1

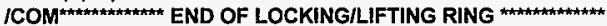

ICOM **** SHIELD PLUG ELEMENTS *kt*

TYPE,2

MAT, 1

$E, 602,622,621,601$

EGEN,12,1,-1

\begin{tabular}{|l|c|l|l|l|}
\hline REVISION & 0 & & & \\
\hline PREPARED BY IDATE & We $4 / 17 / 97$ & & & \\
\hline CHECKED BY IDATE & / $/ 4 / 17 / 97$ & & & \\
\hline
\end{tabular}


CLIENT: DUKE ENGINEERING \& SERVICES HANFORD, INC.

FILE NO: $\quad \mathrm{KH}-8009-8-09$

PROJECT: MCO Final Design DOC. NO.: HNF-SD-SNF-DR-003, Rev. 0, Appendix 11

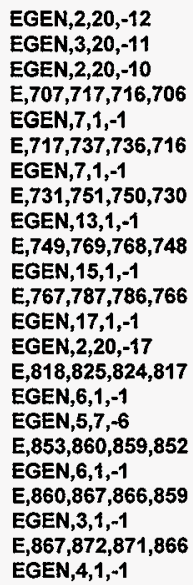

ICOM BETWEEN SHIELD PLUG \& SHELL (BELOW SEAL)

\begin{tabular}{|l|c|l|l|l|c|}
\hline REVISION & 0 & & & & PAGE 128 \\
PREPARED BY I DATE & OF 133 \\
\cline { 1 - 4 } CHECKED BY/DATE & $/ 1 / 17 / 97$ & & & & \\
\hline
\end{tabular}


CLIENT: $\quad$ DUKE ENGINEERING \& SERVICES HANFORD, INC.

FILE NO: $\quad \mathrm{KH}-8009-8-09$

PROJECT: MCO Final Design $\quad$ DOC. NO.: HNF-SD-SNF-DR-003, Rev. 0, Appendix 11

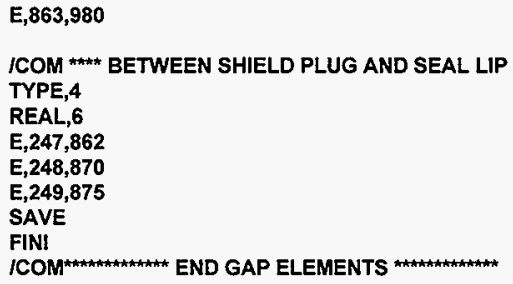

\begin{tabular}{|l|c|c|c|c|c|}
\hline REVISION & 0 & & & & PAGE 129 \\
OF 133 \\
PREPARED BY IDATE & CHECKED BY I DATE & $4 / 17 / 97$ & & & \\
\hline
\end{tabular}


CLIENT: $\quad$ DUKE ENGINEERING \& SERVICES HANFORD, INC. FILE NO: KH-8009-8-09

PROJECT: MCO Final Design DOC. NO.: HNF-SD-SNF-DR-003, Rev. 0, Appendix 11

$C P, 4, U Y, 478,286$

CP, $5, U Y, 468,289$

$\mathrm{CP}, 6, \mathrm{UY}, \mathbf{4 5 8 , 2 9 2}$

ICOM ** BETWEEN BOLT \& LOCKING RING **

$C P, 7, U Y, 445,910$

$\mathrm{CP}, 8, \mathrm{UX}, \mathbf{4 4 5 , 9 1 0}$

CP,9,UY,447,911

CP,10,UX,447,911

*DO, $1,1,7$

$\left.C P, 10+1, U Y, 445+10^{*} 1,910+2^{*}\right]$

*ENDDO

*DO,I, 1,7

CP, $17+1, U Y, 447+10^{*}\left|, 911+2^{*}\right|$

*ENDDO

${ }^{*} \mathrm{DO}, \mathrm{I}, 1,7$

$C P, 24+1, U X, 445+10 * 1,910+2 * 1$

*ENDDO

*DO,1,1,7

$C P, 31+1, U \times, 447+10 * 1,911+2 * \mid$

¿ENDDO

NALL

EALL

ICOM BOUNDARY CONDITIONS AT AXIS $(X=0)$

CSYS, 0

NSEL, $S, L O C, X, 0$

$D, A L L, U X, 0$

ALLS

NSEL,S,NODE, 10

D,ALL,UY,O

ALLS

SAVE

FINI

ICOM SOLUTION ITransfer Temperatures to Structural Model

ISOLU

LDREAD,TEMP,LAST,,",TT24,RTH I Reads in Temperatures from TT24.RTH file.

ICOM APPLYING 89 PSI INTERNAL PRESSURE

NALL.

EALL

NSEL,S,LOC, $X, 0,1.26$ ! Bottom Plate

NSEL, R, LOC, Y, $-0.45,-0.43$

SF,ALL,PRES, 189

NALL

EALL

NSEL,S,LOC, $X, 1.24,2.14$

NSEL,R,LOC,Y,-0.45,0.45

SF,ALL,PRES, 189

NALL

EALL

NSEL,S,LOC,X,2.12,11.51

NSEL, R,LOC,Y, $0.43,0.45$

SF,ALL,PRES, 189

NALL

\begin{tabular}{|l|c|l|l|l|c|}
\hline REVISION & 0 & & & & PAGE 130 \\
OF 133 \\
\cline { 1 - 4 } CHECKED BY I DATE & OF $4 / 17 / 97$ & & & & \\
\hline
\end{tabular}


CLIENT: DUKE ENGINEERING \& SERVICES HANFORD, INC.

FILE NO: $\quad \mathrm{KH}-8009-8-09$

PROJECT: MCO Final Design DOC. NO.: HNF-SD-SNF-DR-003, Rev. 0, Appendix 11

EALL

NSEL,S,LOC,X,11.49,11.51 IInside Shell

NSEL,R,LOC, Y, 0.43,149.64

SF,ALL,PRES, 189

NALL

EALL

NSEL,S,LOC,X,11.49,11.76 I Edge Shell to Seal

NSEL,R,LOC,Y,149.62,149.64

SF,ALL,PRES, 189

NALL

EALL

NSEL,S,LOC, $X, 11.67,11.69$ ! Seal

NSEL,R,LOC,Y,149.64,149.89

SF,ALL,PRES, 189

NALL

EALL

NSEL,S,LOC,X,11.44,11.69 I Shield Plug (above seal)

NSEL,R,LOC,Y,149.87,149.89

SF,ALL,PRES, 189

NALL

EALL

NSEL,S,LOC,X,11.44,11.46 ISide of Shield Plug

NSEL,R,LOC,Y,149.37,149.89

SF,ALL,PRES, 189

NALL

EALL

NSEL,S,LOC,X,11.24,11.46 ! Shield Plug Taper

NSEL, R,LOC, Y,147.62,149.39

SF,ALL,PRES, 189

NALL

EALL

NSEL, $S, L O C, X, 0,11.26$ I Bottom of Shield Plug

NSEL,R,LOC,Y,147.62,147.64

SF,ALL,PRES,189

NALL

EALL

SOLVE

SAVE

FINI

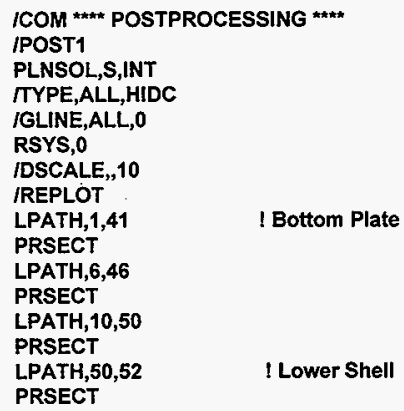

\begin{tabular}{|l|c|c|c|c|}
\hline REVISION & 0 & & & \\
\hline PREPARED BY IDATE & C $/ 4 / 17 / 97$ & & & \\
\hline CHECKED BY I DATE & $/ \mathrm{V} / \mathrm{4} / 17 / 97$ & & & \\
\hline
\end{tabular}


CLIENT: $\quad$ DUKE ENGINEERING \& SERVICES HANFORD, INC. FILE NO: KH-8009-8-09

$\begin{array}{ll}\text { PROJECT: MCO Final Design } & \text { DOC. NO.: HNF-SD-SNF-DR-003, Rev. 0, Appendix } 11\end{array}$

LPATH,62,64

PRSECT

LPATH,65,67

PRSECT

LPATH,100,101

PRSECT

LPATH,122,123

PRSECT

LPATH,134,135

PRSECT

LPATH,156,157

PRSECT

LPATH,170,171

PRSECT

LPATH,180,181

PRSECT

LPATH,202,204

PRSECT

LPATH,235,237

PRSECT

LPATH,985,989

PRSECT

LPATH,262,264

PRSECT

LPATH,277,279

PRSECT

LPATH,292,294

PRSECT

LPATH,601,641

PRSECT

LPATH, 601,613

PRSECT

LPATH, 603,703

PRSECT

LPATH,606,706

PRSECT

LPATH,766,806

PRSECT

LPATH, 748,808

PRSECT

LPATH,730,810

PRSECT

LPATH, 736,815

PRSECT

LPATH,869,874

PRSECT

LPATH, 870,875

PRSECT

LPATH,431,434

PRSECT

LPATH,406,426

PRSECT

LPATH, 404,424

PRSECT

SAVE
! Mid Shell

I Upper Shell

I Shield Plug

! Locking Ring

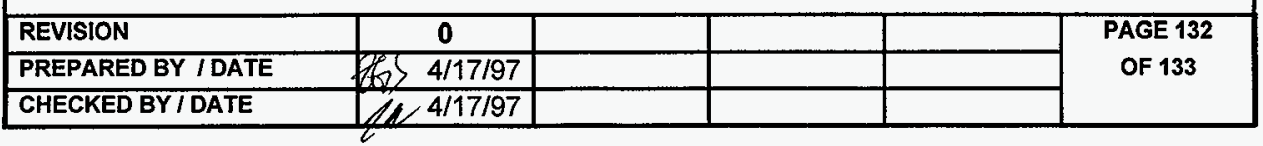




\section{QPARSDNE}

CLIENT: DUKE ENGINEERING \& SERVICES HANFORD, INC. FILE NO: KH-8009-8-09

PROJECT: MCO Final Design DOC. NO.: HNF-SD-SNF-DR-003, Rev. 0, Appendix 11

\section{COMPUTER RUN COVER SHEET}

Project Number:

Computer Code:

Software Version:

Computer System:

Computer Run File Number:

Unique Computer Run Filename:

Run Description:

Run Date / Time:
$\mathrm{KH}-8009-8$

ANSYS $\otimes-P C$

$5.0 \mathrm{~A}$

MS-DOS, Pentium® Processor

$\mathrm{KH}-8009-8-09$

TT24.out

Load Case 6 Output

27 March $1997 \quad$ 1:31:22 PM

Prepared By: Zachary G. Sargent

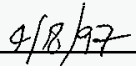


Document No. HNF-SD-SNF-DR-003,

Rev. 0, Appendix 12

\title{
MULTI-CANISTER OVERPACK DESIGN REPORT
}

\author{
RUPTURE DISK DATA
}

POTENTIAL RUPTURE DISK SUPPLIERS

Address: $\quad$ Fike Metal Products

704 South 10th Street

Blue Springs, MO 64015

Telephone: (816) 229-3405

Fax: $\quad$ (816) $228-9277$

Contact: Jason Patterson

Arthur Forsyth Co.

(206) 283-5716 phone

(206) 284-7269 fax

Address: Continental Disc Corporation

3160 West Heartland Drive

Liberty, MO 64068

Telephone: (816) 792-1500

Fax: $\quad$ (816) $792-5447$

Contact Michael Pruitt 


\title{
MULTI-CANISTER OVERPACK DESIGN REPORT
}

\author{
MAIN SEAL DATA
}

\section{CARBONE LORRAINE}

Product: $\quad$ Helicoflex Seals

Model/Part No.: $\quad$ H-305236 REV NC

Address: Helicoflex

2770 The Boulevard

P. O. Box 9889

Columbia, SC 28209

Telephone: $\quad$ (803) $783-1880$

Fax: $\quad$ (803) $783-4279$

Contact: $\quad$ Michel LeFrançois 
From: Michel LEFRANGOIS

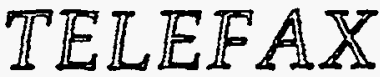

Cory :

To: Chuck TEMUS

Company: VECTRA TECHNOLCGIES

Addreas:

countryistata: WA

Tolotax of 2083742401

Columbla, Maren 3, 1997

SUbjoet: MCO CASK SEAL / HELICOFLEX H-305236 REV NC

กอ .:

Dear Sir,

Ro. your rocent conace with Gesard Anshoine, please find sketch atrached including seal description.

Please do not hesitate to call mo at the number above if you have any question.

Yours Sincerely, Micted Lefançois 


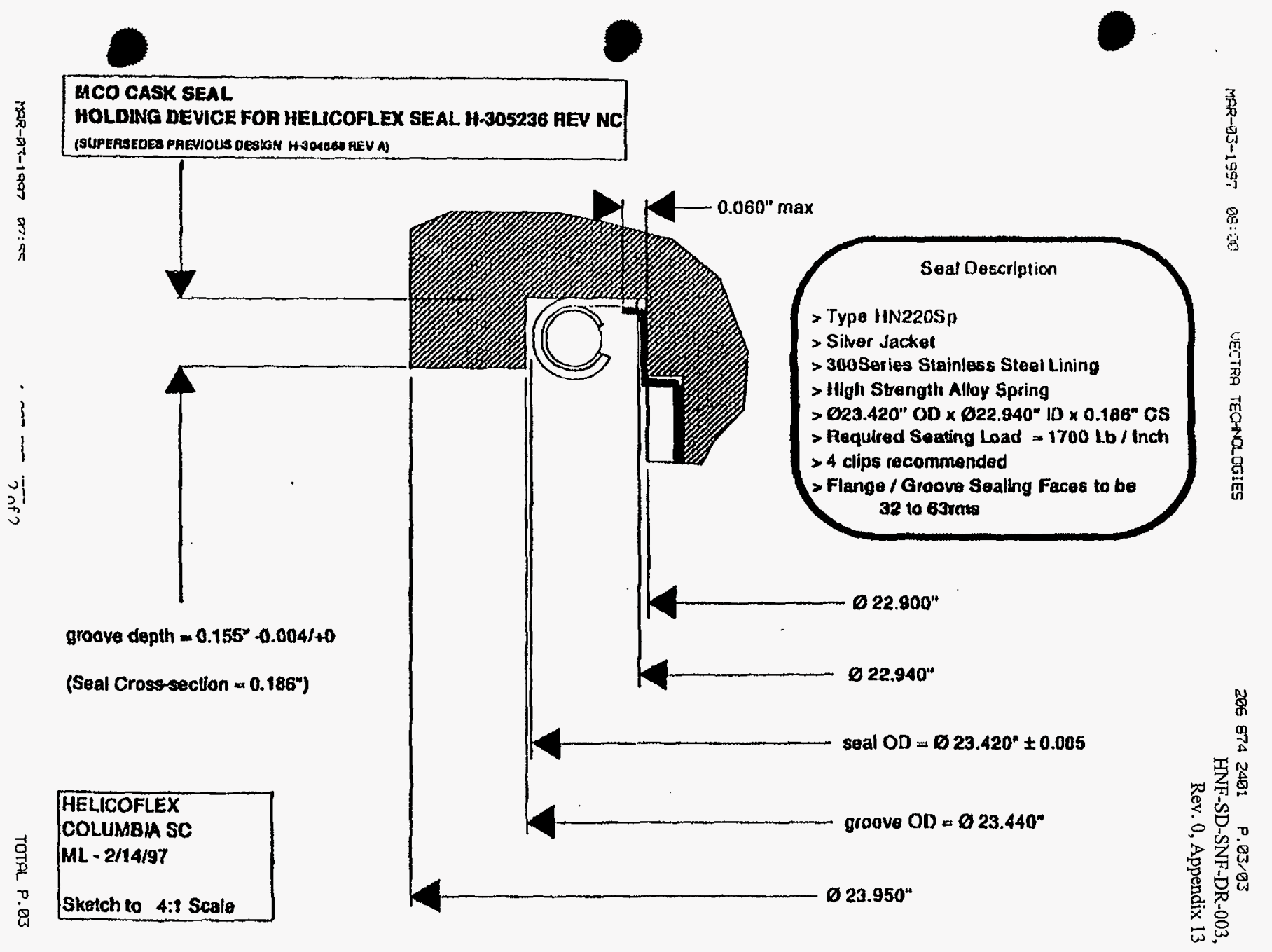


Document No. HNF-SD-SNF-DR-003,

Rev. 0, Appendix 14

\title{
MULTI-CANISTER OVERPACK DESIGN REPORT
}

\author{
SEAL DATA FOR PROCESS VALVE, \\ COVERS, AND FILTERS
}

EG\&G PRESSURE SCIENCE

Address: $\quad$ EG\&G Pressure Science

11642 Old Baltimore Pike

Beltsville, MA 20705-1294

Telephone: (301) 937-9654

Fax: $\quad$ (301) $937-7027$

Contact: $\quad$ Jeff Layer 
HNF-SD-SNF-DR-003, Rev. 0, Appendix 14

Contact Person : Jeff Layer

Phone / fax : (301) 937-9654 / (301) 937-7027

Address : EG\&G Pressure Science 11642 Old Baltimore Pike Beltsville Md. 20705

\section{Part Number Information}

- Modified Series 80 C-Seal (Boss Size $\$ 24) \quad$ PSI part number $\underline{13632}$

- inch O.D. silver plated C-Seal PSI part number $\underline{13503}$

- Series 80 C-Seal (Boss Size $\# 12$ )

- Series 80 C-Seal (Boss Size \#4)
PSI part number 801A91-0012 (INCO 718)

PSI part number 801A91-0004 (INCO 718) 
APR-10-97 THU 16:05

EG\&G

FAX NO. 3019377027

P. 04

HNF-SD-SNF-DR-003,

Rev. 0, Appendix 14

PERMISSION TO USE PAGE 23

EG\&G Pressure Science grants Duke Engineering Services Hanford and Parsons permission to use page 23 of our product catalog in any reports or publications necessary.

Jeff Layer

Engineering Manager

EG\&G Pressure Science

11642 Old Baltimore Pike

Beltsville Md. 21797

Signed : Jeff Layer

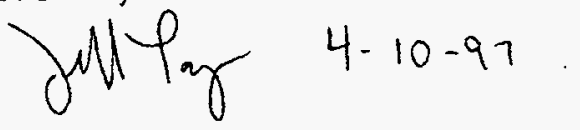

CC:

Transmitted by :

Dato

2 of 7 


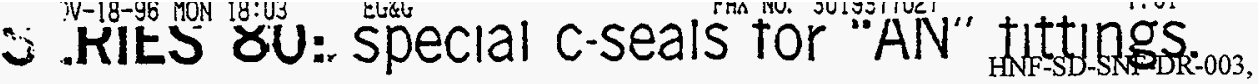
Designed specifleally for ANO 10049 (MS33650)

Rev. 0, Appendix 14 Type Fittings a AND 10050 (MS33649) Type Bosses.

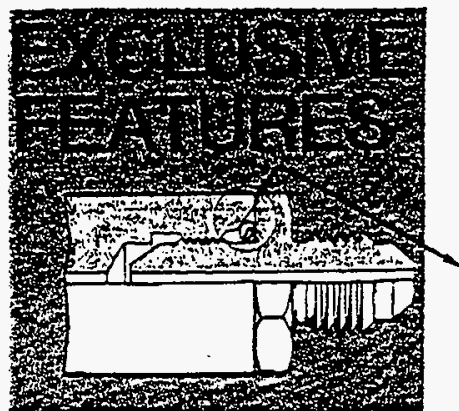

FOOLPROOF, its symmetrical and slips right on in either direction at installation.

IT'S SELF ALIGNING and installs easily regardless of attitude of the boss.

FOR EASY RE-USE, it stays with the fitzing after the first compression.

READILY "UNSCRENS" from the fitting when you want to discard it.

(Supersedes Series 70)

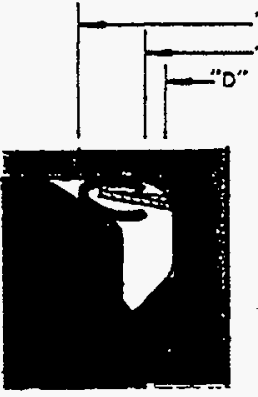

After tightening

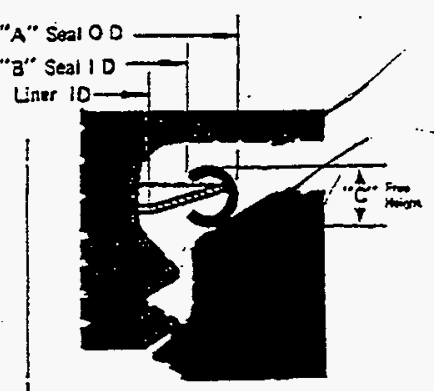

Before tightening $\sim$

HOW TO SPECIFY:

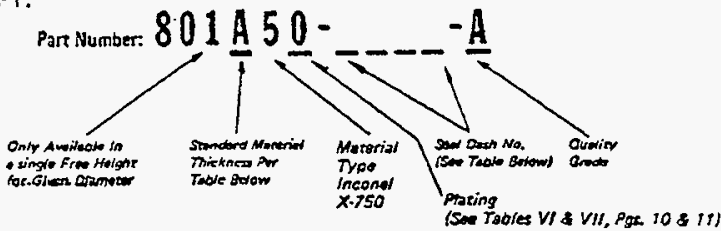

\begin{tabular}{|c|c|c|c|c|c|c|}
\hline $\begin{array}{c}\text { BOSS } \\
\text { SIZE No }\end{array}$ & $\begin{array}{c}\text { SEAL } \\
\text { OASHNO }\end{array}$ & $\operatorname{sEAL}^{-1{ }^{*}}$ & $\begin{array}{c}\text { "g" } \\
\text { SEAC 1.0 }\end{array}$ & $\begin{array}{c}\text { SEAL } \\
\text { FAEE HEIGHT } \\
\text { "ᄂ }\end{array}$ & $\begin{array}{l}\text { Lor } \\
\text { Liner }\end{array}$ & $\begin{array}{c}\text { STO WATERIAL } \\
\text { THICXNESS } \\
\text { ("A" in Ath digit } \\
\text { of PIW }\end{array}$ \\
\hline $\begin{array}{l}2 \\
3 \\
4 \\
5\end{array}$ & $\begin{array}{l}\infty 002 \\
0003 \\
\infty 004 \\
0,05\end{array}$ & $\begin{array}{l}.381 \\
.444 \\
.506 \\
.589\end{array}$ & $\begin{array}{l}.302 \\
.365 \\
.427 \\
.490\end{array}$ & $\begin{array}{l}.046 \\
.046 \\
.046 \\
.048\end{array}$ & $\begin{array}{l}.278 \\
.341 \\
.397 \\
.459\end{array}$ & $\begin{array}{l}.006 \\
.006 \\
006 \\
.006\end{array}$ \\
\hline $\begin{array}{l}8 \\
7 \\
8 \\
9\end{array}$ & $\begin{array}{l}0,008 \\
0007 \\
0008 \\
0009\end{array}$ & $\begin{array}{l}.831 \\
.694 \\
.819 \\
.882\end{array}$ & $\begin{array}{l}.552 \\
.815 \\
.740 \\
.803\end{array}$ & $\begin{array}{l}.046 \\
.048 \\
.048 \\
.046\end{array}$ & $\begin{array}{l}.677 \\
.579 \\
.699 \\
.761\end{array}$ & $\begin{array}{l}2008 \\
.008 \\
008 \\
.008\end{array}$ \\
\hline $\begin{array}{l}70 \\
11 \\
12 \\
14\end{array}$ & $\begin{array}{l}\infty 010 \\
\infty 11 \\
\infty 12 \\
\infty\end{array}$ & $\begin{array}{l}. .948 \\
1.100 \\
1.158 \\
1.281\end{array}$ & $\begin{array}{l}.865 \\
1.029 \\
1.051 \\
1.178\end{array}$ & $\begin{array}{l}.048 \\
.046 \\
.002 \\
.062\end{array}$ & $\begin{array}{r}.8 T 7 \\
.932 \\
.995 \\
.120\end{array}$ & $\begin{array}{l}006 \\
005 \\
010 \\
010\end{array}$ \\
\hline $\begin{array}{l}15 \\
18 \\
20 \\
24\end{array}$ & $\begin{array}{l}\infty 015 \\
0018 \\
\infty 020 \\
0024\end{array}$ & $\begin{array}{l}1.408 \\
1.593 \\
1.718 \\
1.968\end{array}$ & $\begin{array}{l}1.301 \\
1.488 \\
.1 .613 \\
1.863\end{array}$ & $\begin{array}{r}0.022 \\
.062 \\
.062 \\
.002\end{array}$ & $\begin{array}{l}7.245 \\
1.432 \\
1.557 \\
1.807\end{array}$ & $\begin{array}{l}\text { mo } \\
010 \\
010 \\
010\end{array}$ \\
\hline 32 & $\begin{array}{l}0028 \\
0032\end{array}$ & $\begin{array}{l}2.543 \\
2.594\end{array}$ & $\begin{array}{l}2.258 \\
2.498\end{array}$ & .002 & $\begin{array}{l}2182 \\
2.432\end{array}$ & $\begin{array}{l}.310 \\
.010\end{array}$ \\
\hline
\end{tabular}

Serits $\$ 0$ Seals are produead in only TWO Ouality Grades:

A - Best Seal Lino Finien

8- Loss Then Best Seol Line Finish.

Tolerences are the sarme for both Trode.

Both work at High and Low preners.

Seleez dies a for critical leakas. Cliows 8 for low cost. 
APR-IJ-91 IHU 16.05
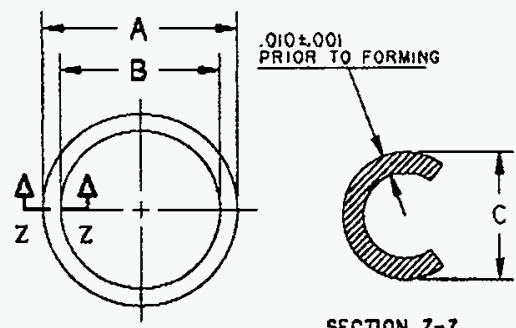

\begin{tabular}{|c|c|c|c|}
\hline \multicolumn{3}{|c|}{ REVISIONS } \\
\hline LTR & DATE & ECO NO & APPROVAL \\
\hline & & & \\
\hline & & & \\
\hline & & & \\
\hline
\end{tabular}

SECTION Z-Z

NOTES

I. TIG WELOING PRIOR-TO-FORHING PERMITTED.

(2) MAXIMUN OUT-OF-ROUNDNESS OF DIAMETERS: .016

3 INDIYIOUALLY PACKAGE PER STANDARO PRESSURESCIENCE NETHOOS.

4. MARK EACH PACKAGE AS SPECIFIEO ON SALES ORDER.

5. SEALING gURFACES TO CONFORM TO QCS 85345 .

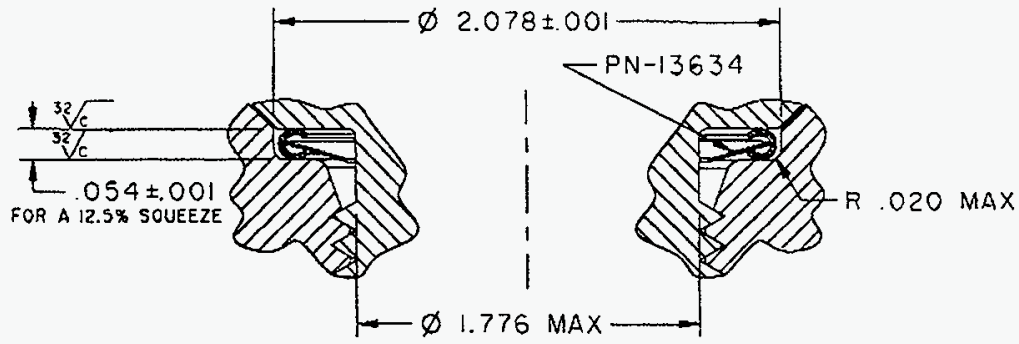

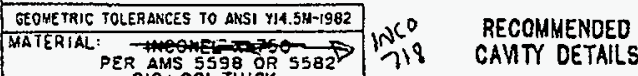

\begin{tabular}{|c|c|c|c|}
\hline \multicolumn{4}{|c|}{$\begin{array}{l}\text { HEAT TREATMENT: } \\
\text { PER PS OB8O SEC. } 11.0\end{array}$} \\
\hline \multicolumn{4}{|c|}{$\begin{array}{l}\text { UNLESS OTHERWISE SPECIFIED } \\
\text { DIMENSIONS ARE IN INCHES }\end{array}$} \\
\hline \multicolumn{4}{|c|}{ TOLERANCES ON } \\
\hline \multicolumn{3}{|c|}{ LINEAR DIMENSIONS } & ANGLES \\
\hline$x$ & $\begin{array}{r}. x \times \\
+.01\end{array}$ & $\begin{array}{r}. \times \times x \\
+.005\end{array}$ & \\
\hline
\end{tabular}

$$
\text { QA. }
$$$$
\pm .01 \pm .005
$$

PLATING

\begin{tabular}{|c|c|c|}
\hline \multicolumn{3}{|c|}{ SEAL DIMENSIONS } \\
\hline $\begin{array}{l}\text { AYERAGE O.O. } \\
-A- \\
\text { SEE NOTE } Q\end{array}$ & $\begin{array}{l}\text { AYG. MIN. I.0. } \\
\text { - B- } \\
\text { SEE NOTE 2) }\end{array}$ & $\begin{array}{l}\text { FREE } \\
\text { HEIGHT } \\
-\mathrm{C}-\end{array}$ \\
\hline $2.062+.000$ & 1.969 & $.062 \pm .002$ \\
\hline
\end{tabular}

\begin{tabular}{|l|l|l|}
\hline MFG & & \\
\hline ENG & & \\
\hline CHECKED & & \\
\hline
\end{tabular}

\begin{tabular}{|l|l|l|l|}
\hline DRAWN & IS ROWLANO & O5 MAR 97 \\
\hline
\end{tabular} APPROYED

\section{N EGEE PRESSURE SCIENCE}

11642 OLD BALTIMORE PIKE, BELTSVILLE. MARYLAND 20705-1294 PHONE (301) 937-4010 FAX (301) 937-0134 Compuser Generoted

C-SEAL 02.062 0.D. FACE TYPE INTERNAL PRESSURE 


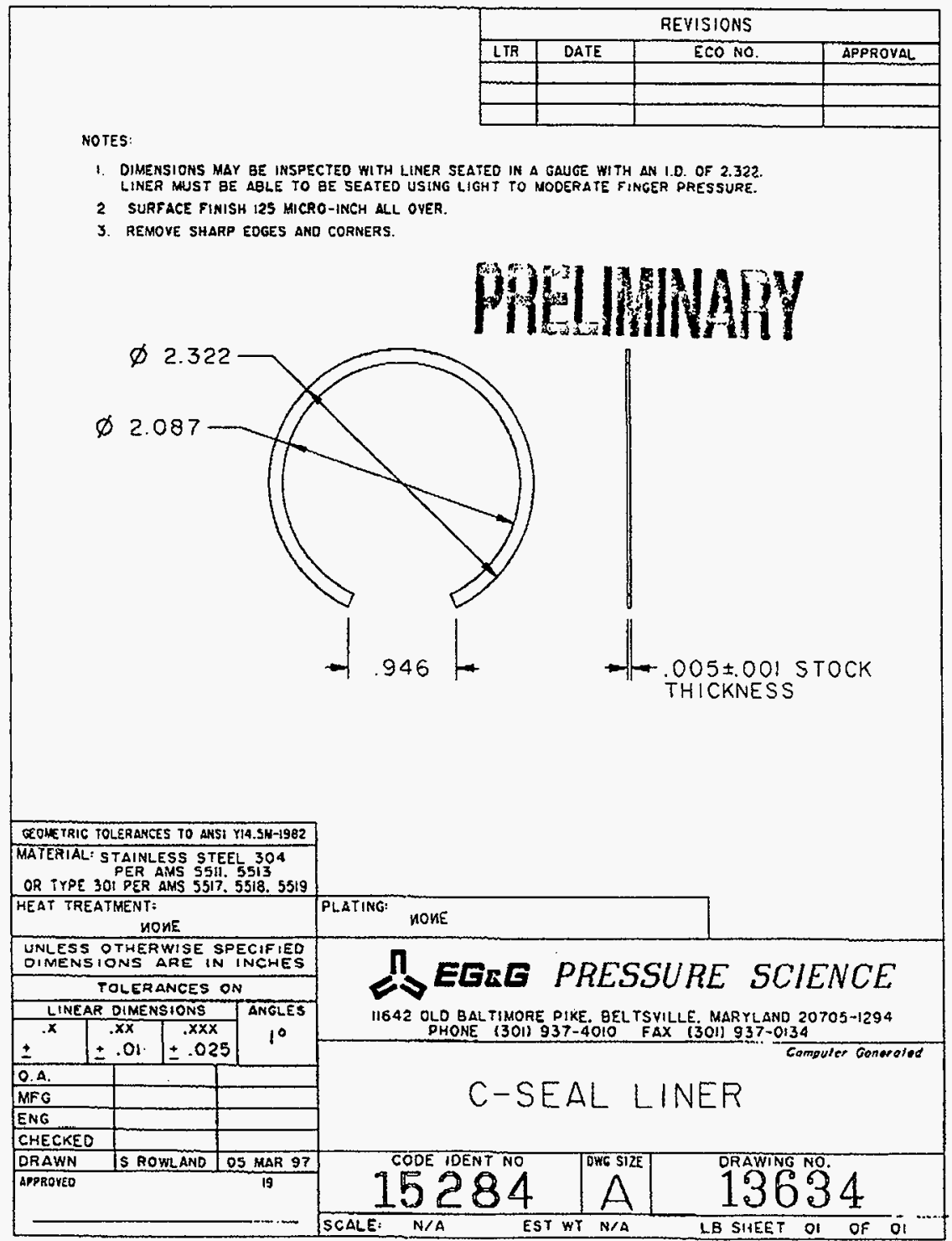


SEP-!6-96 MON 13:00 EC\&G

FAX NO. 3019377027

Y. 02

HNF-SD-SNF-DR-003,

Rev. 0, Appendix 14

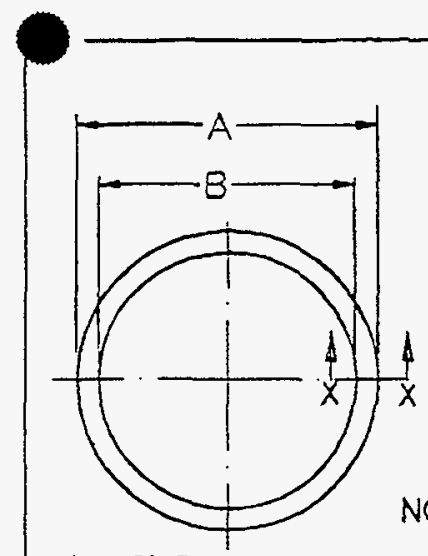

\begin{tabular}{|l|l|l|l|}
\hline \multicolumn{3}{|c|}{ REVISIONS } \\
\hline LTR & OATE & ECO NO. & APPR'L \\
\hline & & & \\
\hline & & & \\
\hline & & & \\
\hline
\end{tabular}

\begin{tabular}{|c|c|c|c|}
\hline $\begin{array}{l}\text { SEAL } \\
\text { DIMENSIONS }\end{array}$ & $\begin{array}{r}\text { AVG } 0.0 . \\
-A-A \\
\text { SEE NOTE } \Lambda \\
\end{array}$ & $\begin{array}{r}\text { AVG.NIN I.D. } \\
-B-B-1 \\
\text { SEE NOTE } 1 \\
\end{array}$ & $\begin{array}{c}\text { FREE HEIGHT } \\
-\mathrm{C}-\end{array}$ \\
\hline FLATING & $3.520 / 3.510$ & 3.310 & $.128 / .122$ \\
\hline $\begin{array}{l}\text { AFTER } \\
\text { PLATING }\end{array}$ & $(3.519)$ & $(3.305)$ & $(.129)$ \\
\hline
\end{tabular}

(.015) PRIOR

TO FORMING 7 द

1. REOUIRED OUT OF ROUNDNESS OF DIAMETERS : . .100-.130 2. PLATING RECUIRED OVER THIS AREA AS SPECIFIED.

3. PLATING OPTIONAL ANO MAY BE INCOMPLETE IN THIS AREA.

4. INOIVIOUALLY PACKAGE PER PSI SPECIFICATION.
5. MARK EACH PACKAGE AS SPECIFIED ON SALES ORDER.

6. VARIATION IN BASE MATERIAL MEIGHT SHALL 8E LESS THAN $.0005^{\circ} 10.5^{\circ}$ AROUNO ENTIRE PERMETER OF THE SEAL.

7. TIG WELDING PRIOR TO FORMING PERMITTED.

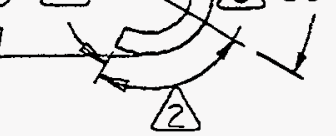

TIG WELDING PRIOR TO FORN

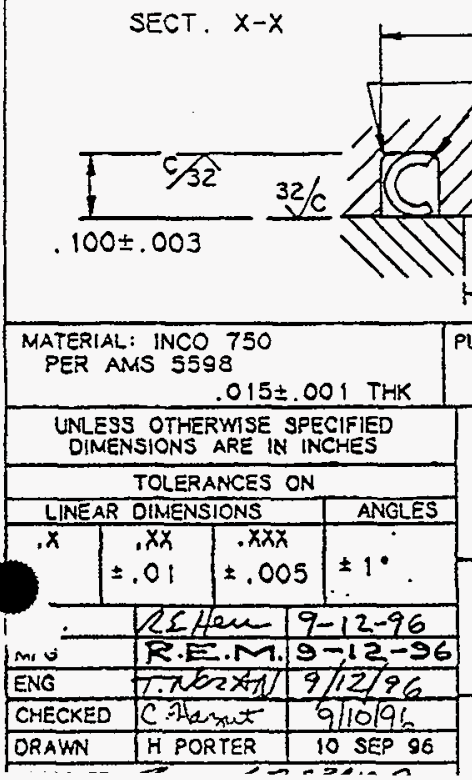

$\varnothing 3.555 \pm .005$

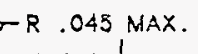


EG\&G Pressure Science has been a supplier of high performance metallic seals since 1959. Typical applications for these seals have been in aerospace, industrial, main frame computer and semiconductor processing equipment. Over the past 37 years, a large body of empirical, application-specific data has been gathered on the performance of EG\&G seals; this combined with economical mass-customization of core sealing technologies has led to the positive reputation enjoyed by EG\&G Pressure Science and to competitive advantages for our customers.

A Pressure Science C-Seal was chosen for this application because near zero leakage may be obtained in a cavity with a rough surface finish (up to 64 RMS). This is accomplished by plating the seal with a soft metalic plating that is smeared during compression of the seal. Gold plating is used on this seal because of the maximum temperature requirement (gold plating is used up to $1400^{\circ} \mathrm{F}$. C C-Seals undergo some plastic deformation when installed at the 10 to $20 \%$ squeeze recommended, however when re-used in their original cavities, they return to their original load.

In an effort to meet the re-sealability requirements (at least 5 re-seals while maintaining $1 \times 10^{-4} \mathrm{cc}$ per second Helium) on the Pressure Science SERIES 80 AN fitting seal (boss size 12 \&24), three different seal face angles were considered. A standard MS33649 boss $\left(30^{\circ}\right.$ angled face) and a modified MS33649 boss (with a $45^{\circ}$ angled face) were tested, and compared with data from a cavity with parallel sealing faces $\left(0^{\circ}\right.$ angle). These tests were periormed using a Varian Vacuum Products Mass Spectrometer Leak Detector, using Helium at $100 \mathrm{psi}$. All three configurations were capable of containing the maximum leakage to below $1 \times 10^{-9} \mathrm{cc}$ per second Helium on the initial seal. The fixture with parallel sealing faces $\left(0^{\circ}\right.$ angle) was capable of 10 reseals, staying in the $1 \times 10^{-8}$ range. The other two configurations were capable of up to eight reseals in the $1 \times 10^{-5}$ range, if everything was aligned and re-seated very carefully. The problem with the "conical" shaped cavity $\left(30^{\circ}-45^{\circ}\right.$ angled faces) is that the seal can seat un-parallel to the top (flat surface) sealing face. The degree of offset possible is not enough to affect the initial instaliation / compression of the seal. However once the seal has been deformed, a new seating position with a different amount of offset may create a gap that in some locations around the circumference of the seal is either larger or smaller than the previous gap. This could create large leak paths or locally over compress the seal. Evidence of this happening is the seal free height variation after multiple reseals (as much as $.005^{\circ}$ ). Normal free height variation of a compressed C-Seal is about .001 inches total. 


\title{
MULTI-CANISTER OVERPACK DESIGN REPORT
}

\author{
HEPA FILTER DATA
}




\section{MCO INTERNAL HEPA FILTER SPECIFICATION}

\subsection{INTRODUCTION}

\subsection{BACKGROUND}

The MCO internal filter is required for radioactive contaminant filtration inside the $\mathrm{MCO}$ to protect process equipment from contamination. The filter(s) will be filtering a saturated steam atmosphere at steam flows of 35 cubic feet per minute (cfm) at temperatures not to exceed $375^{\circ} \mathrm{C}$ inside a vacuum chamber. The filter(s) shall provide HEPA removal efficiency of 0.3 micron $(\mu \mathrm{m})$ particles and have a $40 \mathrm{gram}$ loading capacity with 10 inches water column differential pressure at $35 \mathrm{cfm}$ air flow. The filter(s) shall be sized such that 3.5 inches water column differential pressure at $35 \mathrm{cfm}$ air flow clean. The filter(s) shall be capable of regeneration by back flow of gas or liquid, and be moisture repellant to saturated steam. The filter/manifold(s) shall not be larger than the envelop described in drawing H-2-828049 and be no larger than 2.6 inches in diameter (see drawing for conceptual filter arrangement scheme). The filter/manifold(s) shall be constructed of all 316 stainless steel. The filter structure shall also withstand a $100 \mathrm{~g}$ drop and maintain a minimum of $50 \%$ of flow and filter loading capacity but HEPA efficiency is not required to be maintained after the drop.

\subsection{REQUIREMENTS}

\subsection{FILTER DOCUMENTATION REQUIREMENTS}

The bidders shall provide documentation and/or test results for the following requirements equivalent to or superseding the documents described below with their proposals:

\subsubsection{Filter Airflow Resistance}

Test reports or flow versus differential pressure curves for a clean airflow (Helium, steam) to meet 3.5 inches water column at $35 \mathrm{ACFM}$ air.

\subsubsection{Filter Removal Efficiency}

Test reports documenting DOP, or other approved test material, $0.3 \mu \mathrm{m}$ particulate removal efficiency of $99.97 \%$.

\subsubsection{Filter Loading Capacity}

Test reports documenting filter loading capacity in accordance with standard SAE J726 or ASHRAE 52-76 using ASHRAE fine test dust.

\subsubsection{Filter Moisture Repellency}

Test report shall document filter efficiency and differential pressure performance while subjected to water spray during ASHRAE particulate loading per Section 2.2.3. 


\subsubsection{Filter Regenerability}

Test reports documenting filter regeneration (minimally $70 \%$ clean differential pressure, loading capacity recovery, and $99.97 \%$ particulate removal efficiency after regeneration) per ASHRAE 5276.

\subsubsection{Filter Rough Handling}

Certificate of Conformance, test reports, or engineering calculations shall be provided confirming the filter will withstand a $100 \mathrm{~g}$ drop or 100 times the filter(s) weight in bending and maintain $50 \%$ of original rated flow and $50 \%$ of filter loading capacity after the drop. The filter shall sustain no visible integrity loss (cracks or punctures in the filter media, endcaps, or manifold hardware.)

\subsubsection{Filter Heating Resistance}

Test reports documenting resistance to heated air and maintain particulate removal efficiency during 240 hours of $375^{\circ} \mathrm{C}$ per standards UL-586 and MIL-F51068.

\subsection{FILTER DESIGN REQUIREMENTS}

\subsubsection{Filter Airflow Resistance}

The filter(s) shall be sized for a clean airflow (Helium, steam) of 3.5 inches water column at 35 ACFM air.

\subsubsection{Filter Removal Efficiency}

The filter(s) shall be designed to withstand a DOP challenge, or other approved test material, of 0.3 $\mu \mathrm{m}$ particulate and remove $99.97 \%$ of the particulate.

\subsubsection{Filter Loading Capacity and Surface Area Maximization}

The filter(s) shall provide maximum resistance of 10 inches water column when loaded with 40 grams of ASHRAE fine test dust at 35 ACFM air in accordance with standard SAE J726 or ASHRAE 52-76. The filter(s) shall also be designed to provide a maximum surface area square footage.

\subsubsection{Filter Moisture Repellency}

The filter(s) shall provide maximum repellency of 10 inches water column when the filter is challenged with supersaturated steam containing a minimum 1 gram of entrained water per 1 cubic foot of air at 35 ACFM.

\subsubsection{Filter Regenerability}

The filter(s) shall be capable of reverse back pulsing with gas to regain minimally $70 \%$ loading 
capacity as specified in Section 2.2.2 and differential pressure and $99.97 \%$ particulate filtration per ASHRAE 52-76. The gas reverse back pulse shall consist of rapid application of reverse flow of helium or argon to the filter manifold (all filters) from a reservoir with a maximum size of 5 cubic feet, at a maximum initial pressure of $100 \mathrm{psi}$ through a 20 foot length of 1 inch ID maximum discharge line.

\subsubsection{Filter Heating Resistance}

The filter shall be designed to with stand resistance to heated air and maintain particulate removal efficiency during 240 hours of $375^{\circ} \mathrm{C}$ per standards UL-586 and MIL-F51068.

\subsubsection{Filter Design Basis Accident Functionality}

The filter/manifold(s) shall be designed to withstand a $100 \mathrm{~g}$ drop or 100 times the filter(s) weight in bending and maintain $50 \%$ of original rated flow and $50 \%$ of filter loading capacity after the drop. The drop or force loading to the filter shall be applied midway on the filter in a radial direction normal to the longitudinal filter axis. The filter shall also be designed to physically withstand forces related to a $1035 \mathrm{kPa}(150 \mathrm{psig})$ pressure spike transient and maintain $50 \%$ flow and $50 \%$ loading capacity after the pressurized pulse. The intent of this requirement is to maintain a high removal efficiency (non-HEPA rated) by assuring no visible holes, punctures, cracks, or extensive deformations that circumvent or block the filter flow path.

\subsection{CONSTRUCTION REQUIREMENTS}

\subsubsection{Filter Media}

The filter(s) shall be all stainless steel construction--316L, or other suitable corrosion resistant materials. Filter end caps (if required) shall be constructed from 316L stainless steel and NOT attached to filter media by epoxies or sealants. Rolled or metallic end cap bonding such as welding or brazing may be appropriate. If weld bonding is utilized, it is suggested that low carbon base materials such as $316 \mathrm{~L}$ or other similar corrosion resistant materials be used.

\subsubsection{Filter Mounting Hardware}

The filter(s) mounting hardware (i.e. supports, manifolds, etc.) shall be constructed from 316L stainless steel, other low carbon base, or corrosion resistant material (painted surfaces shall not be acceptable). The filter(s) shall be mounted to manifold in such a manner to prevent build-up of excess free water inside the manifold. The filter(s) shall be mounted near ( $+/-0.0625$ inches) the bottom of the manifold and the manifold angled such that any free water in the manifold will drain back into the filter media. The total weight of the filter and mounting hardware shall not exceed $22.7 \mathrm{~kg}$ (50 lbs). Refer to drawing H-2-828049 for filter envelop space, mounting suggestions, and additional construction suggestions.

\subsubsection{Filter Environmental Conditions}


The filters shall be designed and constructed to withstand the following environmental conditions:

- Design Basis Accident equal to a $100 \mathrm{~g}$ drop or 100 times the filter(s) weight in bending per design stated above;

- Temperature ranges from -20 to $375^{\circ} \mathrm{C}\left(375^{\circ} \mathrm{C}\right.$ for a time period of 240 hours);

- Internal to external accidental pressure spike transient of $1035 \mathrm{kPa}$ (150 psig) as described above; and

- All gas atmospheres such as Nitrogen, Hydrogen, Helium, Argon, Oxygen, air, and steam.

\subsection{OPTIONS}

3.1 Optional bids may be submitted for filters meeting the following design conditions:

Option \#1: $35 \mathrm{acfm}$ flow at $6.0^{\prime \prime}$ water column with $0.3 \mu \mathrm{m}$ efficiency

Option \#2: 35 acfm flow at $3.5^{\prime \prime}$ water column with $0.5 . \mu \mathrm{m}$ efficiency

All other specification requirements remain in force for the optional bids. 


\title{
MULTI-CANISTER OVERPACK DESIGN REPORT
}

\author{
K BASIN MCO SHIELD PLUG THICKNESS \\ TECHNICAL EVALUATION
}




\section{TABLE OF CONTENTS}

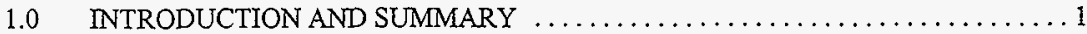

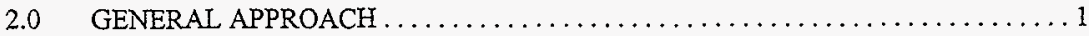

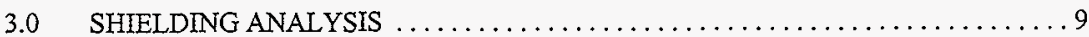

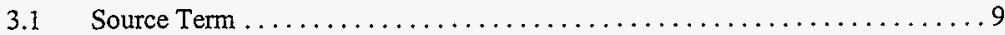

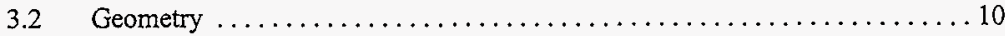

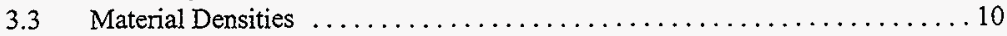

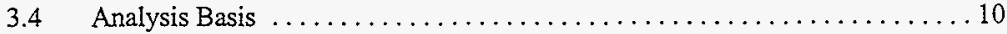

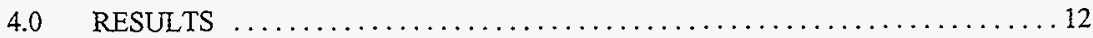

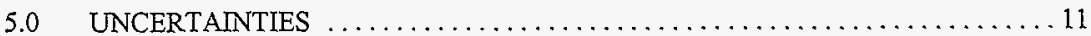

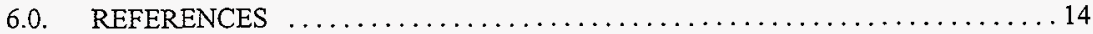

Appendices

Appendix A

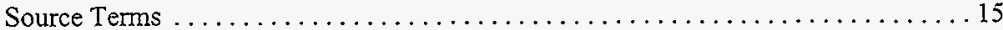

Appendix B

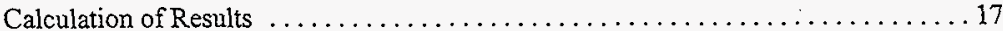

Appendix C

Ratio Based Internal Central Cylinder Plug $\ldots \ldots \ldots \ldots \ldots \ldots \ldots \ldots \ldots \ldots \ldots \ldots$ 


\subsection{INTRODUCTION AND SUMMARY}

This evaluation provides an assessment of dose as a function of plug thickness for the $\mathrm{K}$ Basin Multiple Canister Overpack (MCO), including an initial assessment of areal and centerline doses. Based on input from Westinghouse Hanford Company, areal averages were calculated for the more complex plug designs. The emphasis has been on obtaining the thickness that will result in an average dose rate of $100 \mathrm{mrem} / \mathrm{hr}$ or $(30 \mathrm{mrem} / \mathrm{hr})$ at contact on the plug for peak loads and nominal loads of fuel, respectively. The calculations are based on the design planned by Parsons Infrastructure \& Technology Group and summarized in Table 1-1 below. This report provides a summary of the technical assumptions, basis, and results of the calculations.

Table 1-1.

\begin{tabular}{|l|l|}
\hline Source Term & $\begin{array}{l}\text { Cleaned and reloaded Mark IV fuel elements; } \\
5.43 \mathrm{MTU} ; 11 \text { energy bins; } 8.29 \times 10+15 \\
\text { photons/s }\end{array}$ \\
\hline Model Geometry & $\begin{array}{l}27 \mathrm{~cm} \text { SS lid; } 4 \text { instrument penetrations; } 1 " \\
\text { central and lateral holes thru lid; } 4 \mathrm{~cm} \text { SS } \\
\text { plate under filters; collar in place }\end{array}$ \\
\hline Detector Geometry & $\begin{array}{l}\text { Tissue equivalent plastic in 3 thicknesses and } \\
8 \text { rings }\end{array}$ \\
\hline $\begin{array}{l}\text { Requirement --< } 100 \mathrm{mrem} / \mathrm{h} \text { contact, areal } \\
\text { average (Peak Load0 }\end{array}$ & $<7 \mathrm{mrem} / \mathrm{h}$ photons and neutrons \\
\hline $\begin{array}{l}\text { Requirement -- <30 mrem/h contact, areal } \\
\text { average (Nominal Load) }\end{array}$ & $<2 \mathrm{mrem} / \mathrm{h}$ photons and neutrons \\
\hline \hline
\end{tabular}

\subsection{GENERAL APPROACH}

For modeling purposes, each fuel element in the MCO is assumed to be 2 concentric shells of uranium, and each of these shells are treated as separate sources for analyses. This calculation uses MCNP 4A to allow a more realistic model of the $\mathrm{MCO}$ and to directly determine the impacts of scattering. The MCNP software is a Monte Carlo shielding analysis program, which assesses the shielding based on nuclear interactions. Figure 1-1 summarizes the presumed geometry of the fuel. The density of the uranium, steel, and air matrix is listed in Section III. Fifty-four fuel elements are assumed per layer, four layers, and no gap between layers. The center space (number 28 in Figure 2-1B) is occupied by an air filled stand pipe.

The basic assumptions are summarized below:

- The shielding calculation used the extremely conservative activity concentrations in

Table 3.8 of WHC-SD-SNF-TI-009, Rev. 0. These values were used to ensure a corresponding level of conservatism in the results. The photon emissions of these 
HNF-SD-SNF-DR-003, Rev. 0, Appendix 16

radionuclides were placed in 12 energy bins from $100 \mathrm{keV}$ to $3 \mathrm{MeV}$. If the total yield fraction is less than $1 \times 10^{-6}$ the contribution is treated as zero.

- Nominal fuei calculations are based on the an average 4-tier Concept B MCO of Mark IV fuel, as specified in Table 3.8 of WHC-SD-SNF-TI-009, Rev. 0.

- The dose is calculated by determining the energy absorbed per gram in the plastic material used in the ICRU sphere.

- The dose assumes a layer of this material directly above the top of the MCO.

- Three different thickness of material were assumed (i.e., $1 \mathrm{~cm}, 15 \mathrm{~cm}$, and $30 \mathrm{~cm}$ ) to provide a result comparable to the $15 \mathrm{~cm}$ radius ICRU sphere. Note that the detectors are cylindrical rings except for the inner most detector, which is a disk. The diameters of these rings were $1,5,10,15,20,25,30$, and $32.5 \mathrm{~cm}$ in radius.

- For the neutron dose assessment, the dose is assumed to be $10 \%$ of the gamma dose based on the information in the procurement documentation.

The geometry and shield data used in calculating the shielding are summarized in Figures 2-1A through 2-1D. The general model of the MCO, lid, and fuel used in the calculations is shown in Figure 2-1E. The current design is summarized in the drawings included in the reference listing. 
HNF-SD-SNF-DR-003, Rev. 0, Appendix 16

Figure 1-1

Fuel Geometry Information

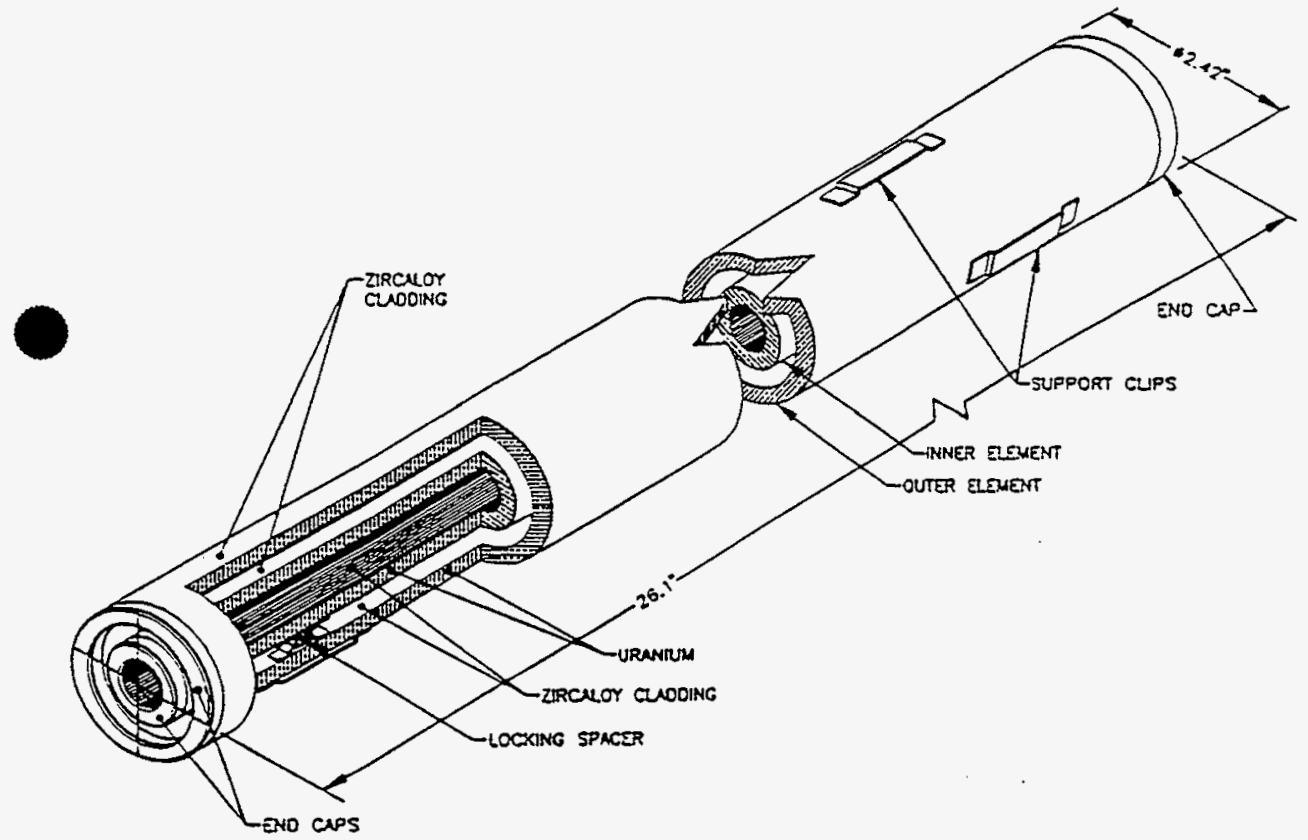

$\frac{105-N \text { REACTOR MARK IV FUEL ELEMENT ASSEMBLY }}{(0500015 \mathrm{~A})}$ 
Figure 2-1A

MCO Geometry

(Full length vertical cross section, dimensions in $\mathrm{cm}$ )
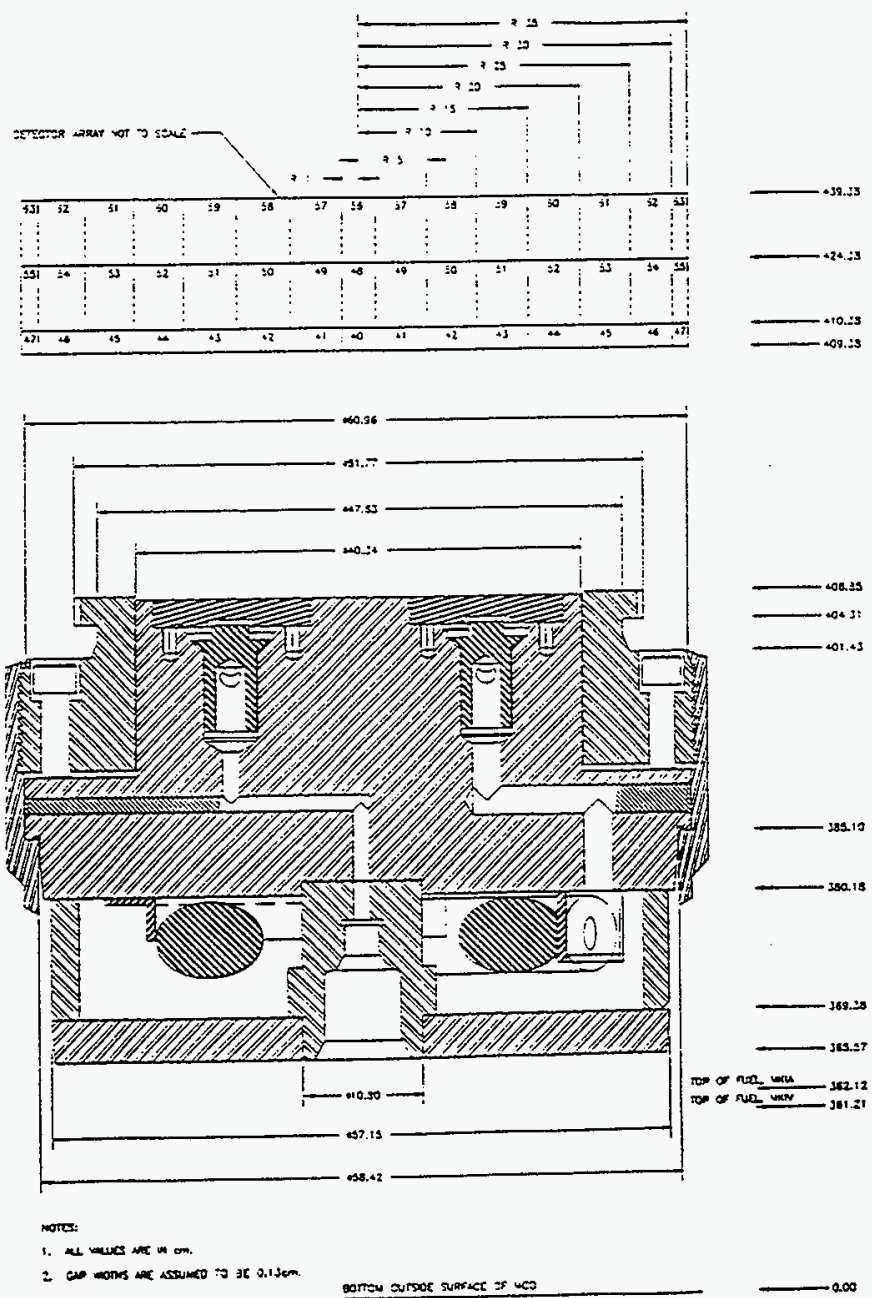
HNF-SD-SNF-DR-003, Rev. 0, Appendix 16

Figure 2-1B

MCO Geometry

(Fuel element horizontal cross section, dimensions in inches. Number 28 is an open pipe.)

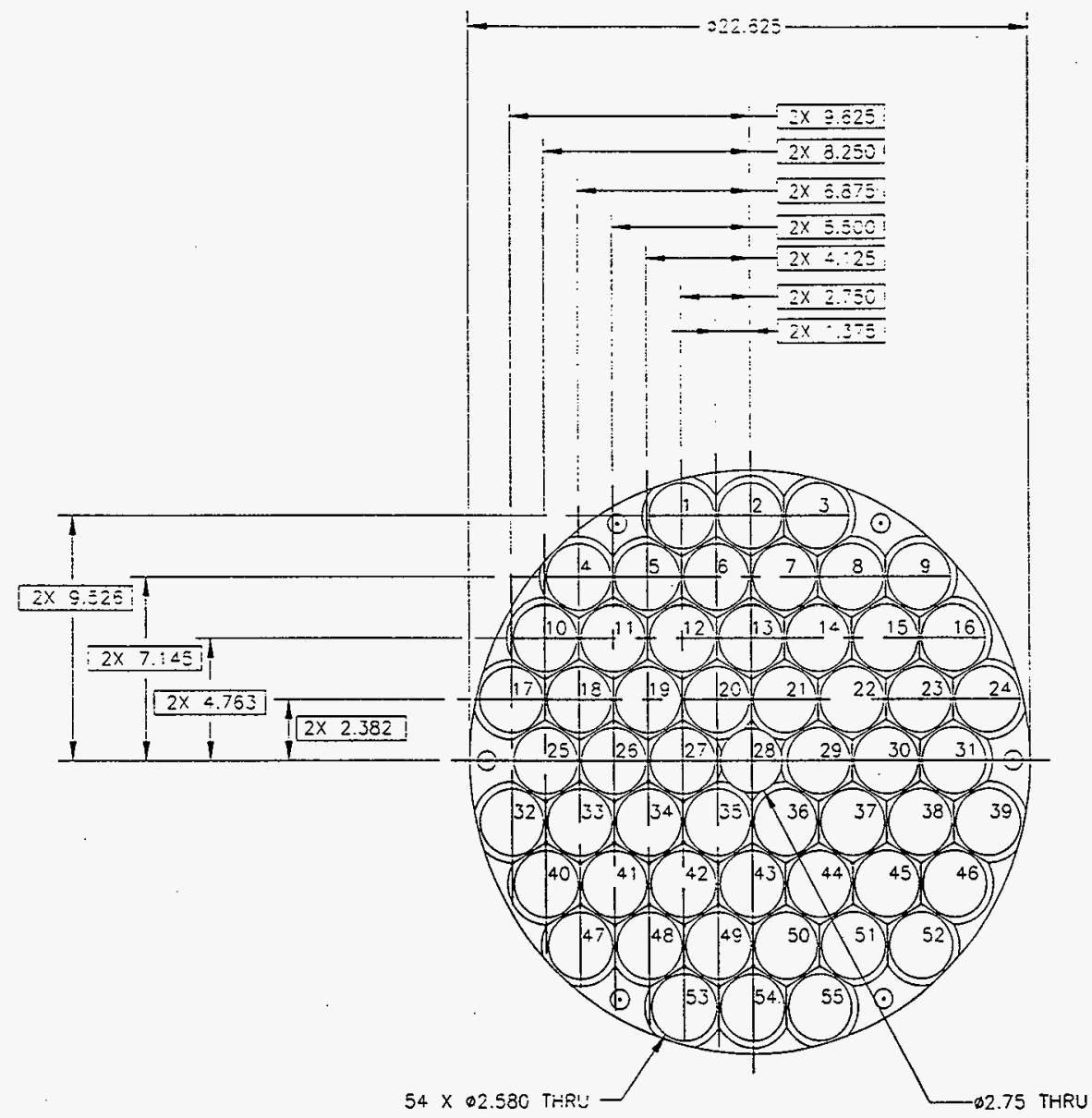


HNF-SD-SNF-DR-003, Rev. 0, Appendix 16

Figure 2-1C

MCO Geometry

(Lid vertical cross section, dimensions in inches)

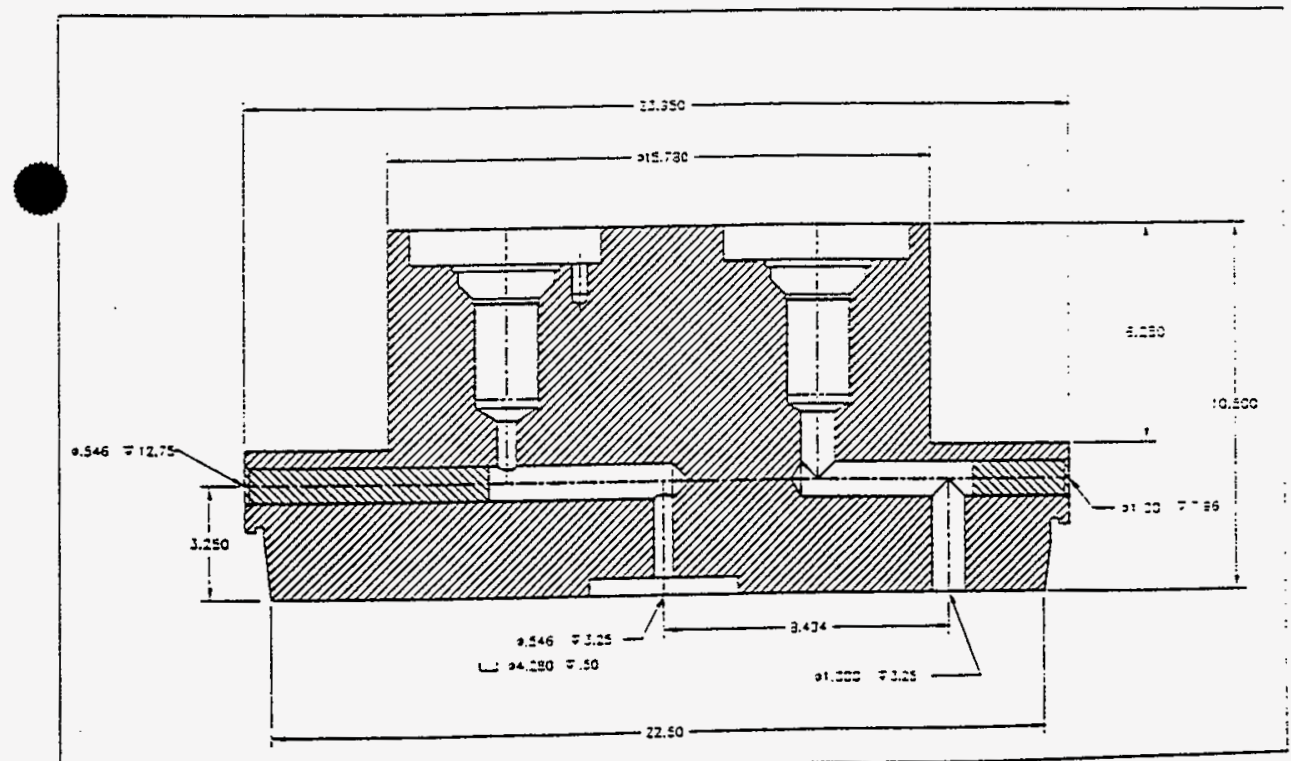


HNF-SD-SNF-DR-003, Rev. 0, Appendix 16

Figure 2-1D

MCO Geometry

(Lid Horizontal cross section, dimensions in inches)

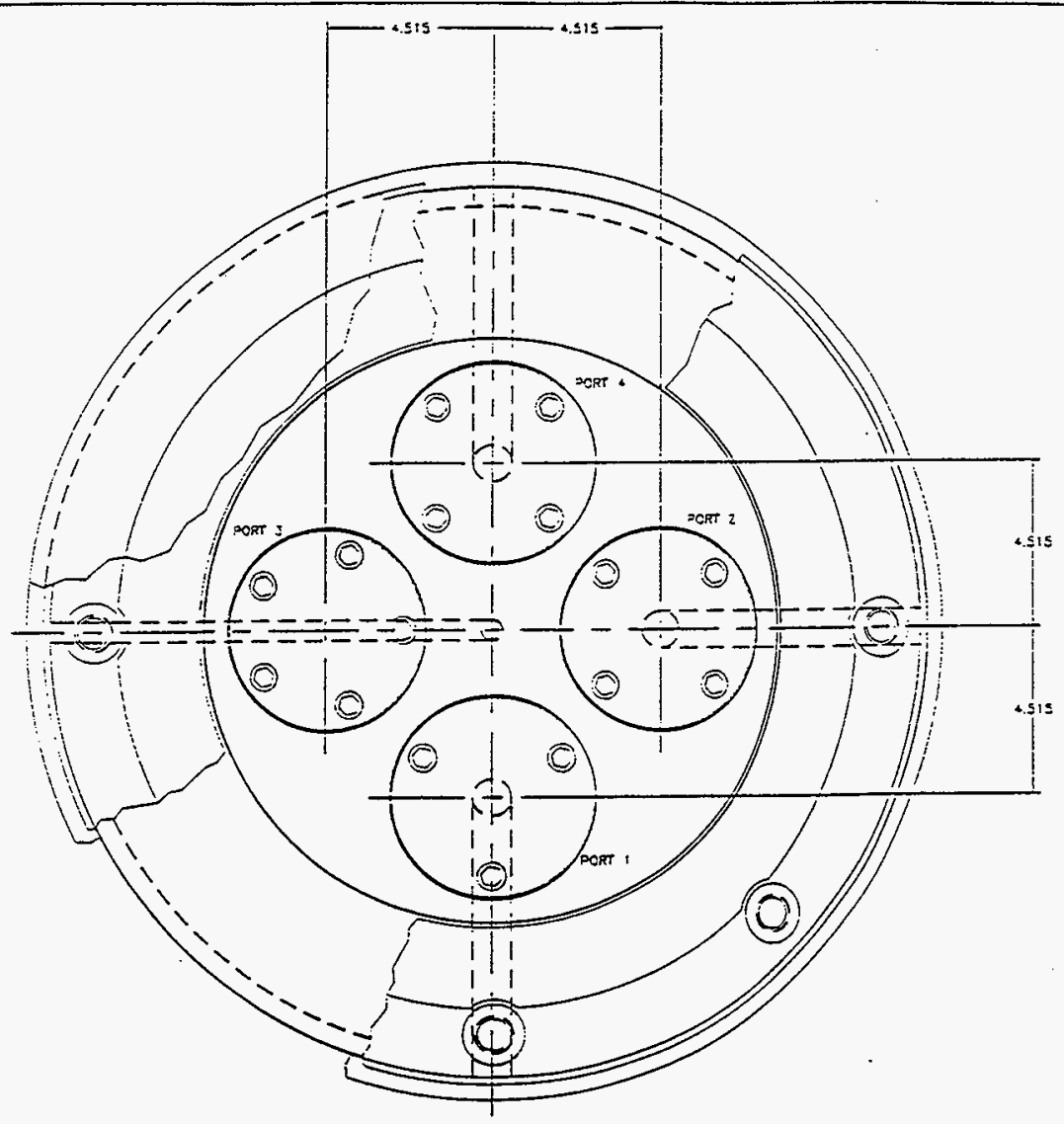


HNF-SD-SNF-DR-003, Rev. 0, Appendix 16

Figure 2-1E

Model of MCO and Fuel Used for Calculations
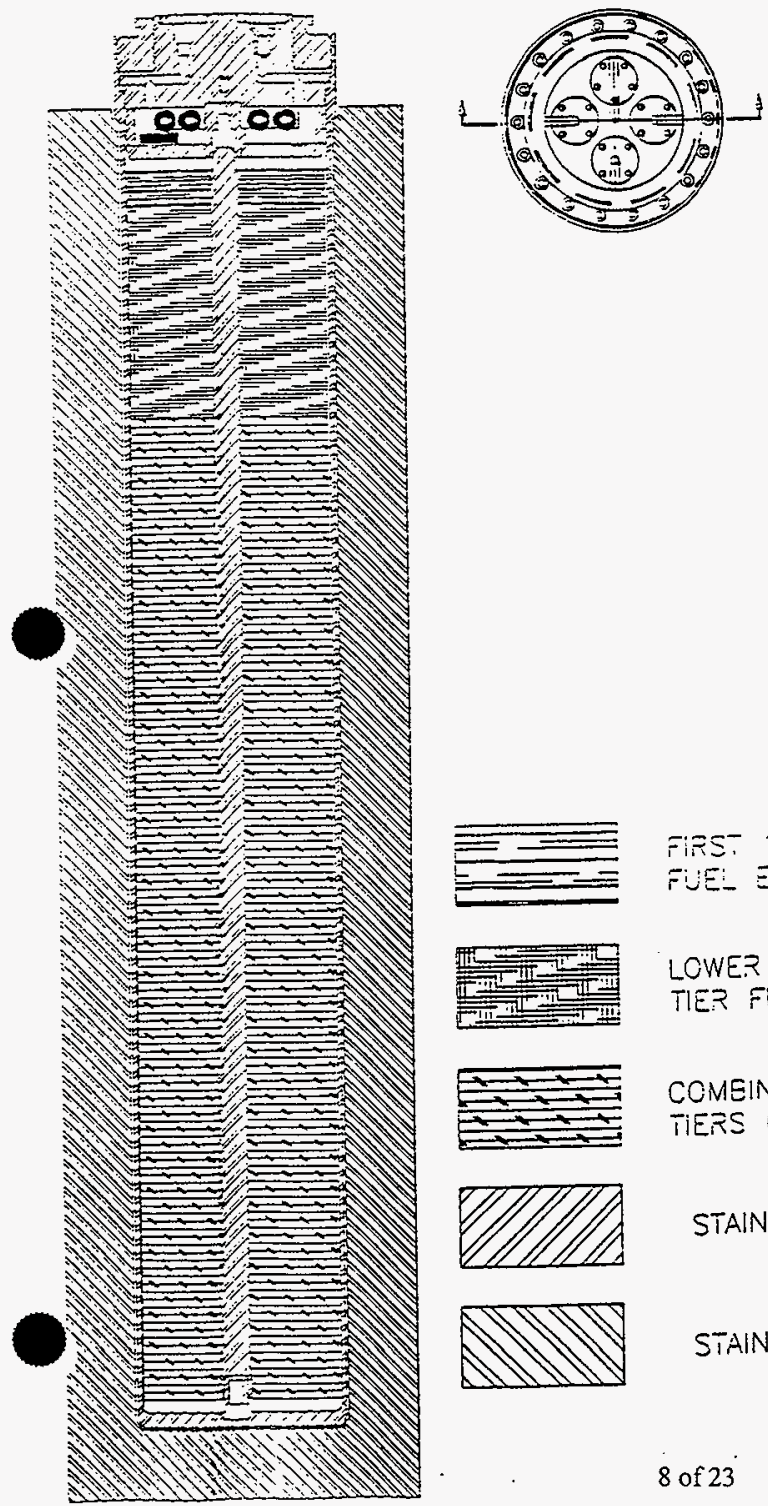

FEST IOCW OF TOF TER

FUE: E:EUENTS (SEE Fi. $i-:$ ).

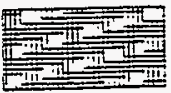

LOWER FORTION OF TOP TIER FUE' ELEMENTS.

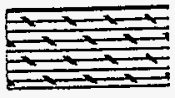

COMEINED LOWER 3

TIERS OF FUEL ELEVENTS.

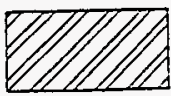

STAINLESS STEEL.

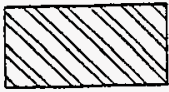

STAINLESS STEEL REFLECTOR. 


\subsection{SHIELDING ANALYSIS}

The shielding analysis used MCNP 4A, a computer application developed by Los Alamos National Laboratory. This computer program is documented in "MCNP 4A Monte Carlo N-Particle Transport Code System," Radiation Shielding Information Center (RSIC) document CCC-200 (see references). This software was validated in the Parsons Infrastructure \& Technology Group Computer Software Validation Record, consistent with the applicable procedures. The verification of performance (completed under another DOE contract and part of a prior task) was documented in the Parsons Infrastructure \& Technology Group Computer Software Verification Record number PD-A-VV-003.

The MCNP 4A output files, which include a reflection of the input deck, are maintained in the Parsons Infrastructure \& Technology Office in Richland, Washington as part of the quality records associated with this calculation.

\subsection{Source Term}

The fission and activation product inventory from Table 3.8 of WHC-SD-SNE-TI-009, Rev 0, was used as the source term for this calculation. This source has been corrected for decay to January 1, 1995, in this table. Loaded MCO handling will not begin until 1997; however, no adjustment from January 1, 1995 to the 1997 time frame was made, as an additional means to ensure conservatism. The uranium content in the fuel was based on the data in Table 3.8 of WHC-SD-SNF-TI-009, Rev. 0. The emission spectra are based on the information in the Brookhaven database (see Appendix A). The relative energy is calculated based on this data and is summarized in Table 3-1 of this document. As indicated previously, the energy distribution is binned as discussed in Section II. Each bin value represents the upper bound of the energy bin. Therefore, the Cs- $137662 \mathrm{keV}$ photon was treated as an $800 \mathrm{keV}$ photon. This is conservative by about a factor of 3-4. In the $600 \mathrm{keV}$ bin, it would have been non-conservative by about a factor of 2. The total fuel weight per MCO was 5.43 MTU. The total source strength was 8.294 $\times 10^{15}$ photons per second.

Table 3-1.

Energy Distribution

\begin{tabular}{|c|c|}
\hline Upper Energy Bound (keV) & Fraction \\
\hline 100 & 0.089 \\
\hline 200 & .206 \\
\hline 300 & .0032 \\
\hline 400 & .00037 \\
\hline 500 & .19 \\
\hline 600 & .0047 \\
\hline 800 & .496 \\
\hline
\end{tabular}


HNF-SD-SNF-DR-003, Rev. 0, Appendix 16

\begin{tabular}{|c|c|}
\hline 1000 & .0031 \\
\hline 1500 & .0059 \\
\hline 2000 & .00076 \\
\hline 2500 & .0000083 \\
\hline
\end{tabular}

\subsection{Geometry}

The geometry is shown in Figures 2-1A and 2-1B. It is assumed that the tolerance is $0.13 \mathrm{~cm}$; this is assumed to be the width of the gaps for assessing scattering. The ports at the top of the MCO shield plug are assumed to be filled with inserts which reduce the holes passing through the ports to one inch, except for the rupture disk port which has no insert. The port cover plates are not in place in any of the calculations.

\subsection{Material Densities}

The MCNP input includes the density and the makeup of the materials. This information is summarized in Table 3-2.

Table 3-2.

Material Properties

\begin{tabular}{|l|l|l|}
\hline Material & Density $\left(\mathrm{g} / \mathrm{cm}^{2}\right)$ & $\begin{array}{l}\text { Material Constituents (Cross-section file number [i.e., } \\
\text { MCNP cross-section file] }]^{*} \text { and weight fraction) }\end{array}$ \\
\hline Steel & 8.03 & $26000(1)$ \\
\hline Air (gas) & 0.001293 & $7000(0.755), 8000(0.232), 18000(0.013)$ \\
\hline Uranium & 17.86 & $92000(1)$ \\
\hline $\begin{array}{l}\text { Tissue } \\
\text { (ICRU) }\end{array}$ & 1.0 & $\begin{array}{l}1000(0.102), 6000(0.123), 7000(0.035), 8000 \\
(0.72893), 11000(0.0008), 12000(0.0002), 15000 \\
(0.002), 16000(0.005), 19000(0.003), 20000 \\
(0.00007)\end{array}$ \\
\hline $\begin{array}{l}\text { "The MCNP is the atomic number multiplied by 1000. For example, the atomic number of } \\
\text { carbon is 6, so the cross-section file number is 6000. }\end{array}$ \\
\hline
\end{tabular}

\subsection{Analysis Basis}

The shielding analysis was completed for each of the two uranium portions (i.e., concentric shells) of each fuel element for the top layer or tier of fuel elements (see Appendix B). However, to improve convergence of the results, the first $10 \mathrm{~cm}$ of each top tier fuel element is treated as a separate source. Representative fuel elements were selected for running after the top $10 \mathrm{~cm}$ of the first tier rather than running all 108 cases, as a means to reduce analysis time. The bottom 3 
tiers fuel element tiers are treated as a single unit and have negligible impact on the results, due to self-shielding effects. Calculations relative to the bottom 3 tiers fuel elements are not included in the spreadsheets referenced in the appendices of this document; however, they are included in the computer output files maintained with the quality assurance records.

\subsection{RESULTS}

MCNP was used to calculate the energy deposition per photon in a $1 \mathrm{~cm}$, then $14 \mathrm{~cm}$, and an additional $15 \mathrm{~cm}$ thick disk of ICRU tissue equivalent plastic. With the known photon flux the energy deposition per unit time was then calculated. This was then converted to dose using the definition of a rad which is $100 \mathrm{ergs} / \mathrm{g}$ of energy deposition in tissue. The choice of the detector thickness was chosen to provide worst case and an range which covers the ICRU $15 \mathrm{~cm}$ radius sphere (i.e., the basis for deep dose). What we calculated was the average energy per gram of tissue deposited in the first $\mathrm{cm}$ of tissue, a $15 \mathrm{~cm}$ thick layer of tissue, and a $30 \mathrm{~cm}$ thick layer of tissue. This provides a accurate direct method of assessing dose; although using a ICRU sphere center at each location would have been mor accurate, it was not technically feasible. This approach is consistent with the current ICRP approach for assessing the dose used by EPA, DOE, and NRC. The calculation of flux though a detector is an indirect rather than direct method Of assessing the dose and may be less accurate.

The results of the calculations are summarized in Table 4-1. These results were calculated using the spreadsheets referenced in Appendix B. As indicated in Section 2, data is given for a disk/ring source $1 \mathrm{~cm}, 15 \mathrm{~cm}$, and $30 \mathrm{~cm}$ thick; areal averages are given in the last two rows. The bottom row in this table includes the neutron dose consistent with the assumptions identified in the proposal (i.e., the neutron dose is $10 \%$ of the photon dose). The dose measured by an ionization chamber at about 1 to 2 inches above ihe MCO top would probably be similar to the doses specified for $1 \mathrm{~cm}$ thick disk. The doses for the 15 and $30 \mathrm{~cm}$ disks would provide the probable range of actual dose to an individual consistent with the ICRU model for deep dose.

The doses in Table 4-1 are for peak loading. The doses for the average fuel load would be about $26 \%$ of these values.

\subsection{UNCERTAINTIES}

The presence of a steel plug over the central cylindrical tube was not included in the original model. The reduction in doses from this plug were estimated using MCNP as described in Appendix C. This dose will range from $100 \%$ to $45 \%$ of the original values depending on the location. In the central area, where most of the areal dose is generated, the dose will range from about $100 \%$ to $65 \%$. Overall, the plug introduces less than $10 \%$ measure of conservatism for the doses. Convergence with this data is very high.

The MCNP software provides an assessment of convergence. When using a Monte Carlo program it is necessary to use enough particles (i.e., case studies) to adequately represent the behavior of the system (i.e., obtain convergence of the predicted results with the true results). This must always be a compromise between the time required to run the calculation and the amount of convergence obtained. Approximately 10,000,000 particles (i.e., cases studies) were 
run for each source term analyzed. This means since each fuel element or section of fuel element (i.e., the upper $10 \mathrm{~cm}$ of the first fuel element) has an inner and outer portion each of these portions were run for $10,000,000$ particles. Because of the limitation of time the goal was to achieve a convergence of about 0.3 or better for the most affected detector and less than 0.2 for areas that are significant dose contributors. Other detectors would have lower convergences for a specific source. Since the higher values of convergences have a larger uncertainty, the doses were recalculated using values with convergence results of less than 0.3 for one of the analyses. The results of this recalculation were comparable so it is reasonable to assume that the estimate is reasonable and conservative. Since the basic source geometry is simple it is a reasonable assumption that the area where there would be significant dose contributions would be the area where the convergence is low, since the photon flux in these area is the highest. Note, the top layer of source (i.e., the upper $10 \mathrm{~cm}$ of the first fuel element) requires 108 separate runs and each run requires about 7 hours to complete so the $10,000,000$ particles analysis reflects a reasonable limit for this analysis.

The results for the MCO without the cap (Table 4-1) indicate a higher degree of accuracy. In this scenario, convergence values are all lower than 0.3 except for the center detector (see Appendix B). Since there is not fuel directly below the center detector, the detector is not a significant contributor to the dose. This means that limited convergence for this case and any accompanying inaccuracies will have no significant impact on the overall dose estimate. Also, better results and convergence were possible in the calculations for this case from knowledge of the ratio of inner cylindrical section contribution relative to the outer cylindrical section contribution that had been ascertained from the first set of calculations (where the $\mathrm{MCO}$ cap was attached). With this ratio pre-determined (the value is constant for all scenarios) the data could be consolidated for the fuel sections, which had the impact of minimizing statistical uncertainties. Consequently, the calculations for the scenario without the MCO cap in place have much lower uncertainties and better accuracy, notwithstanding the same 10,000,000 particle case study Monte Carlo analysis was used. 
HNF-SD-SNF-DR-003, Rev. 0, Appendix 16

Table 4-1

Dose Results Without Cover Cap

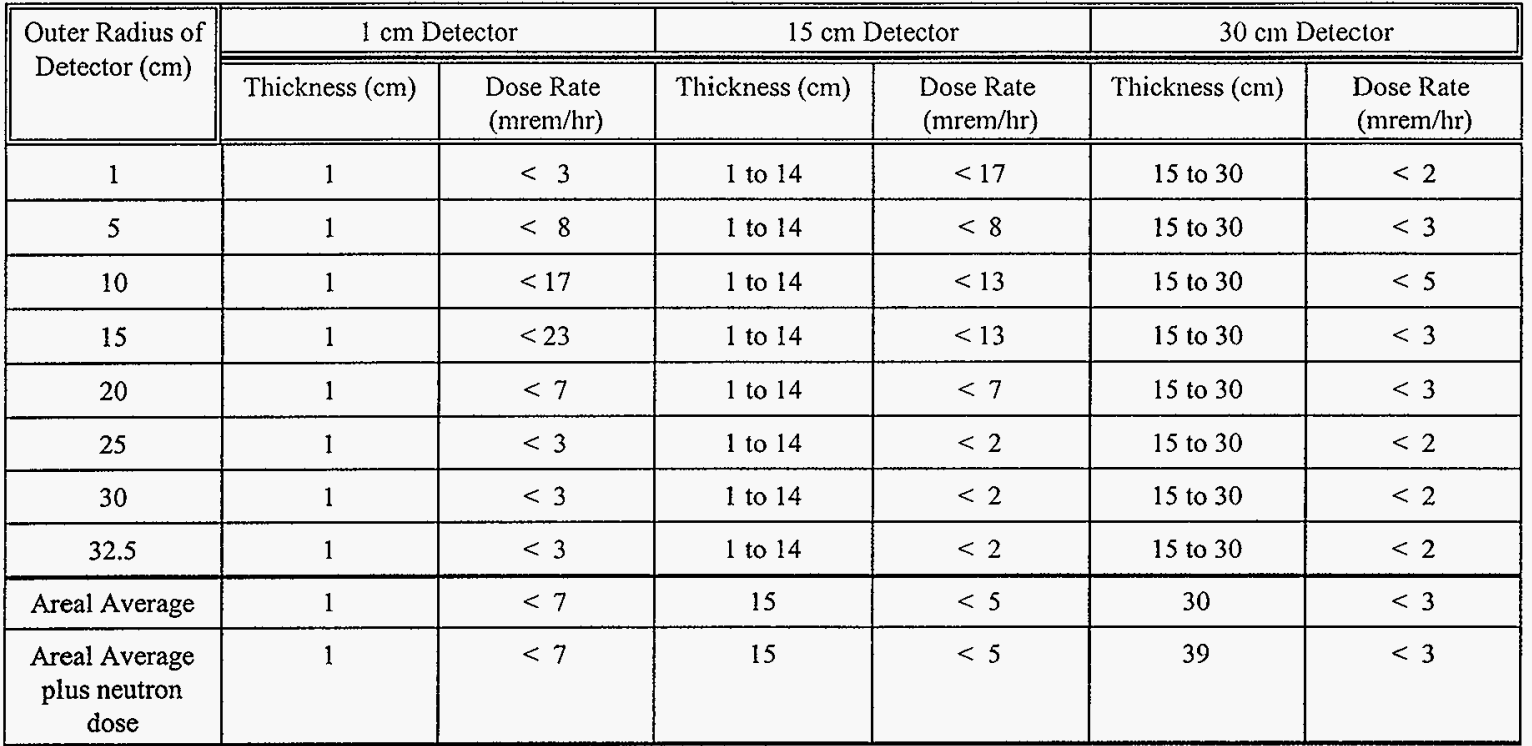


HNF-SD-SNF-DR-003, Rev. 0, Appendix 16

\subsection{REFERENCES}

Courtney, J. C., 1976. "A Handbook of Radiation Shielding Data," American Nuclear Society, ANS/SD-76/14.

Radiation Shielding Information Center (RSIC), 1989. "QAD-CGGP," CCC-493.

Rockwell, Theodore II, 1956. Reactor Shielding Design Manual, McGraw-Hill Book Co., Inc.

Los Alamos National Laboratory, 1995. "MCNP 4A Monte Carlo N-Particle Transport Code System," Radiation Shielding Information Center, (CCC-200).

Willis, W. L. and A. N. Praga, 1995. "105-K Basin Material Design Basis Feed Description for Spent Nuclear Project Facilities," WHC-SD-SNF-TI-009, Rev. 0.

Parsons Infrastructure \& Technology Group Internal Documentation:

Computer Software Verification Plan, Project No. PD-A-VV-003, MCNP 4A: A Monte Carlo N-Particle Transport Code, December 18, 1996.

Computer Software Validation Record, "MCNP 4A: A Monte Carlo N-Particle Transport Code, December 18, 1996.

Computer Software Verification Plan, Project No. 7282651-03000, Computer Software:

SUPERSHIELD, Discipline: Health Physics, Version: 1.0, draft November, 1995. 
HNF-SD-SNF-DR-003, Rev. 0, Appendix 16

Appendix A

Source Terms 


\section{Appendix A, Source Term}

The determination of the source term was based on the Brookhaven National Laboratory data from the Internet (i.e., www.nndc.bnl.com). This is the most current emission spectra data available. The calculations were performed using an EXCEL 7.0 spreadsheet, containing 3 sheets. The calculation sheets are described below, and the spreadsheet output is available in the Parsons Infrastructure \& Technology Group QA and/or project files for Project KH-8009-8, project number 730531.

Sheet 1 uses the photon data from sheet 2 to find the sum the photon emissions from each radionuclide for each of the energy bins in Table 3-1.

Sheet 2 is the data downloaded from the Brookhaven database.

Sheet 3, Cells B4 to X20 summarizes the photon emissions for each of the energy bins by radionuclides from Sheet 1 . Cells $\mathrm{C} 6$ to $\mathrm{Y} 6$ provide the activity of each radionuclide per MTU based on the data specified in WHC-SD-SNF-TI-009, Rev 0. Cell Z6 is the total activity. Cells $\mathrm{AC} 4$ to BA20 calculate the total photon emissions in each bin (i.e., BA4 to BA20 contain the totals from cells $\mathrm{AC} 4$ to $\mathrm{AZ20}$ ). Celis $\mathrm{AC4}$ to $\mathrm{AZ20}$ are the activities of the radionuclides times the photon emissions in the bin divided by the total activity. Cells E36 to E48 normalize the photon emissions to photon emission fractions based on cells BA4 to BA20. 
HNF-SD-SNF-DR-003, Rev. 0, Appendix 16

Appendix B

Calculation of Results 


\section{Appendix B, Calculation of Results}

The calculations were performed using an EXCEL 7.0 spreadsheet, containing 4 sheets. The calculation sheets are described below, and the spreadsheet output is available in the Parsons Infrastructure \& Technology Group QA and/or project files for Project KH-8009-8, project number 730531. Sheets 1 and 2 address the dose with the cover cap in place and sheets 5 and 6 address the dose without the cap in place.

\section{Dose With Cap}

In Sheet 1 the energy flux absorbed (i.e., $\mathrm{MeV} /$ photon/g) and the value of the convergence from the MCNP are included in the value column for each detector (i.e., see Table B-1 and Row 1 in the spreadsheet). The energy flux absorbed is in the odd number rows and the convergence value is in the even numbered columns for the various detector cell numbers. The first two entries for any MCNP cell (i.e., Column A) are the outer concentric ring of fuel material and the second two are the inner ring of fuel material for a fuel element. Matrix Total adds the inner and outer ring energy flux absorbed data. Odd number rows are set to one in the Convergence columns and the first three rows in the Matrix Total columns are set to zero so they have no impact on the results. The even numbered rows in the Convergence column are set to one if the convergence value is zero (i.e., no data detected); otherwise they are set to the value of the convergences. Row 225 has the total energy flux absorbed and the lowest value of the convergence for MCNP cell for the first layer of fuel (i.e., the top $10 \mathrm{~cm}$ of a first fuel element). Row number 450 has the same data for the second layer of fuel (i.e., the balance of the top fuel element). Row number 457 has this same data for both layers. The areas in light gray in the second layer are MNCP numbers; the other values are based on these numbers as shown in Table B-2. Note that MCNP cell 28 has no fuel in it so its values are zero - it is marked with dark gray.

Sheet 2 Rows 3 to 10 and columns E, L, and S contains the grand total energy flux absorbed. The dose is then calculated in columns F, M, and T using the values shown in rows 14 to 38 . The lowest applicable convergence values from Sheet 1 are summarized in columns $G, N$, and $U$. The total for each detector is then calculated in row 11. Row 12 is the cumulative dose from a $1 \mathrm{~cm}$ thick, $15 \mathrm{~cm}$ thick, and $30 \mathrm{~cm}$ thick detector. Similar values were calculated below this with the cover cap off using a Microshield correction factor for the reduced shielding (see Appendix $\mathrm{C}$ for the Microshield runs) and then for the same calculation using this Microshield data for cover cap off but with an additional $4 \mathrm{~cm}$ of shielding over the first $25 \mathrm{~cm}$ of the $\mathrm{MCO}$ top.

\section{Dose WithoutCap}

The layout and calculations in sheets 5 and 6 are the same as sheets 1 and 2 discussed above except as described below. The dose from the inner cylinder is based on the dose from the outer cylinder, corrected based on the relative ratio between the inner and outer cylinders from sheet 1 and averaged over all values with a convergence value of less than 0.3 . 
Table B-1.

Detectors

\begin{tabular}{|c|c|c|c|}
\hline Inner Radius (cm) & Outer Radius (cm) & Detector Thickness (cm) & $\begin{array}{l}\text { MCNP Cell } \\
\text { Number for } \\
\text { Detector }\end{array}$ \\
\hline 0 & 1 & 1 & 40 \\
\hline 1 & 5 & 1 & 41 \\
\hline 5 & 10 & 1 & 42 \\
\hline 10 & 15 & 1 & 43 \\
\hline 15 & 20 & 1 & 44 \\
\hline 20 & 25 & 1 & 45 \\
\hline 25 & 30 & 1 & 46 \\
\hline 30 & 32.5 & 1 & 47 \\
\hline 0 & 1 & 14 & 48 \\
\hline 1 & 5 & 14 & 49 \\
\hline 5 & 10 & 14 & 50 \\
\hline 10 & 15 & 14 & 51 \\
\hline 15 & 20 & 14 & 52 \\
\hline 20 & 25 & 14 & 53 \\
\hline 25 & 30 & 14 & 54 \\
\hline 30 & 32.5 & 14 & 55 \\
\hline 0 & 1 & 14 & 56 \\
\hline 1 & 5 & 15 & 57 \\
\hline 5 & 10 & 15 & 58 \\
\hline 10 & 15 & 15 & 59 \\
\hline 15 & 20 & 15 & 60 \\
\hline 20 & 25 & 15 & 61 \\
\hline 25 & 30 & 15 & 62 \\
\hline 30 & 32.5 & $\because 15$ & 63 \\
\hline
\end{tabular}


HNF-SD-SNF-DR-003, Rev. 0, Appendix 16

Table B-2.

MCNP Cell Equivalence for Second Layer

\begin{tabular}{|c|c|}
\hline $\begin{array}{c}\text { MCNP Cell Basis for } \\
\text { Unanalyzed Cells }\end{array}$ & Cell To Which This Applies \\
\hline$(2+17+24) / 3$ & $1,3,4,10,16,32,39,40,46,47,52,51,53,54,55$, \\
\hline 25 & $8,31,48,58$ \\
\hline 33 & $6,7,11,15,18,23,38,41,45,49,50$ \\
\hline$(26+30+44) / 3$ & $12,13,14,19,22,26,30,34,37,42,43,44$, \\
\hline 31 & $20,21,27,28,29,35,36$ \\
\hline
\end{tabular}


HNE-SD-SNF-DR-003, Rev. 0, Appendix 16

Appendix C

Ratio Based Internal Central Cylinder Plug 


\section{Appendix C, Ratio Based Internal Central Cylinder Plug}

Sheet 7 contains the MCNP run results with no cap on the MCO and no lid. The fuel is bare except for the small plug over the center cylinder (the "AP\#" files) and the same case with this small plug removed (the "AP\#\#" files). The ratio is calculated in the indicate column. The next two columns are the ratio minus the layer specific mean and minus the general mean, respectively. The calculations of the mean and standard deviation are shown, as well as the projected error from averaging, which is assumed to be twice the standard deviation.

Sheet 7 used data with no top or cap but with the plug in place. These values are calculated based on the steel plug compared to the ratio with a plug made only of air. This was calculated for cells $2,7,13$, and 20. The results for the first layer are summarized in Table C-1 and indicates the cells to which they would apply. Sheet 7 of the EXCEL spreadsheet can be found in the project files. 
Table C-1.

Plug Correction for the First Layer

\begin{tabular}{|c|c|c|c|c|c|c|c|c|c|}
\hline \multirow[t]{2}{*}{$\begin{array}{l}\text { Calculated } \\
\text { Cell }\end{array}$} & \multirow[t]{2}{*}{ Equivalent Cells } & \multicolumn{8}{|c|}{$\begin{array}{l}\text { Plug Shield Correction Factors for Each of the Detector Disks/Rings } \\
\qquad \pm 0.05 \text { (Radius of Disk/Ring in } \mathrm{cm} \text { ) }\end{array}$} \\
\hline & & 1 & 5 & 10 & 15 & 20 & 25 & 30 & 32.5 \\
\hline 2 & $\begin{array}{c}1,3,4,910,17,24,32,39,40,46,47,52 \\
53,54,55\end{array}$ & 1.00 & 1.00 & 1.00 & 1.00 & 1.00 & 1.00 & 1.00 & 1.00 \\
\hline 6 & $\begin{array}{c}5,7,8,11,15,18,22,25,30,31,33,37,41 \\
45,48,49,50,51\end{array}$ & 1.00 & 1.00 & 1.00 & 1.00 & 1.00 & .95 & .95 & .95 \\
\hline 13 & $12,14,19,23,26,34,38,42,43,44$ & .90 & .95 & .90 & .90 & .90 & .90 & .90 & .90 \\
\hline 20 & $16,21,27,29,35,36$ & .45 & .60 & .65 & .70 & .75 & .80 & .90 & .90 \\
\hline
\end{tabular}



HNF-SD-SNF-DR-003, Rev. 0, Appendix 17

\section{WAREHOUSE PLAN}

\section{FOR}

\section{MULTI-CANISTER OVERPACK}

Contract No. KH-8009

April 1997

Revision 0 


\section{-INF-SD-SNE-PLA- 021, Rev $\theta-6 / 9 / 97$ \\ HNF-SD-SNF-DR-003, Rev. 0, Appendix 17}

\section{TABLE OF CONTENTS}

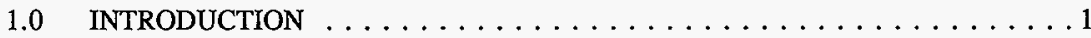

1.1 General Considerations . . . . . . . . . . . . . . . . 1

1.2 Container/Storage Configurations . . . . . . . . . . . . . 1

1.3 Warehouses/Storage Allocation of Components and Storage Areas . . . . 2

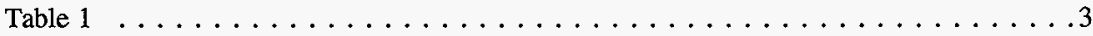

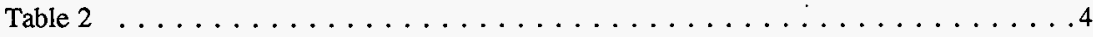

2.0 HANDLING AT WAREHOUSE $\ldots \ldots \ldots \ldots \ldots \ldots \ldots \ldots$

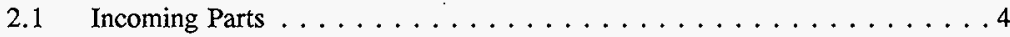

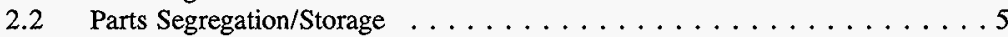

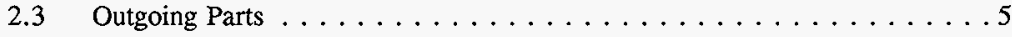

3.0 HANDLING AND USAGE OF MCO PARTS AT CSB \& $\mathrm{K}$ BASINS $\ldots \ldots \ldots 5$

3.1 Reception and Staging $\ldots \ldots \ldots \ldots \ldots \ldots \ldots \ldots \ldots \ldots \ldots \ldots \ldots \ldots$

Figure 1. Flowchart of MCO Component Warehousing, Handling, and Usage . . . . . . 7

3.2 Packaging Removal/Component Inspection . . . . . . . . . . 7

3.3 Component Assembly/Integration into $\mathrm{MCO} \ldots \ldots \ldots \ldots \ldots$

4.0 QUALITY ASSURANCE REQUIREMENTS $\ldots \ldots \ldots \ldots \ldots \ldots \ldots$

4.1 Incoming Parts Inspection $\ldots \ldots \ldots \ldots \ldots \ldots \ldots \ldots \ldots \ldots \ldots$

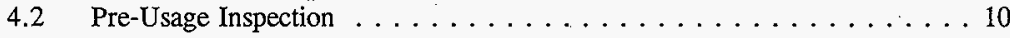

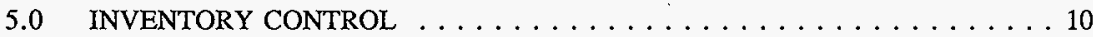

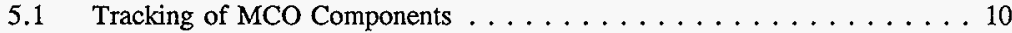

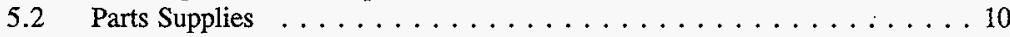

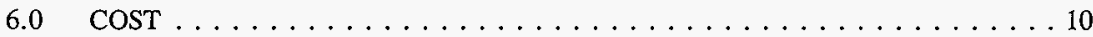

6.1 Warehouse Space Rental . . . . . . . . . . . . . . . . . 10

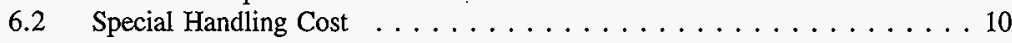




\subsection{INTRODUCTION}

The Multi-Canister Overpacks (MCO) will contain spent fuel removed from the $\mathrm{K}$ East and West Basins. Approximately four hundred MCOs will be fabricated for this purpose. The MCO consists of different components/sub-assemblies manufactured by one or more vendors. All components/sub-assemblies will be shipped to central warehouses which will inspect and store these components until they are required at the $\mathrm{K}$ Basins. The components will generally be stored as received from the manufacturer, with specified protective coatings, wrappings, and packaging intact, to maintain mechanical integrity of the components and to prevent corrosion. Components will be shipped as needed from the warehouses to the basins. At the basins, the components will be unwrapped, assembled as necessary and used in the MCO loading process.

\subsection{General Considerations}

Enough warehouse space is needed to house all MCO components ordered and to carry out the necessary inspections and staging activities. Warehouse facilities must maintain suitable environmental conditions to prevent corrosion of the components, and must have suitable storage shelving as required for smaller parts and ample floor space for larger components, such as the MCO shell and dip tubes. Detailed requirements on receiving, storage and handling of all MCO components are found in ASME NQA-1-1994 Edition, Subpart 2.2, "Quality Assurance Requirements for Packaging, Shipping, Receiving, Storage, and Handling of Items for Nuclear Power Plants." All warehousing, receiving, and handling of MCO components shall conform to the Level C Item requirements. Conformance to these requirements will satisfy the Quality Control and Assurance requirements for the warehousing activities. The preferred locations have easy access to the $\mathrm{K}$ Basins. The warehouse at Building $2101 \mathrm{M}$ in the 200 East area will be used for MCO components that are first sent to the CSB (shells, locking rings, and MCO caps), and a warehouse in the $100 \mathrm{~K}$ area in Building 190 Kanister Storage Building East (KE) will be used for all the other MCO components, or those that are used first at the K Basins.

A project records control center (Record Control) needs to be established to receive inspection, storage, handling, and usage records generated during project execution. Records Control will maintain a database reflecting status on all components. The database will list components by serial numbers and/or container numbers for unnumbered parts, as well as inventory numbers used in tracking (see Section 5.0). Records referencing usage of components by number will be forwarded to Records Control, which will track all activity in the database.

\subsection{Container/Storage Configurations}

Table 1 lists MCO components, container/packaging configurations, and storage configurations to be used for the given component. Standard packaging identification is performed consistent with ASME NQA-1-1994 Edition, Subpart 2.2, Section 3. (Also see the Fabrication 


\section{Specification.)}

\subsection{Warehouses/Storage Allocation of Components and Storage Areas}

There must be adequate warehouse space for staging components, and receiving and transferring components and MCO component sets as required. It is planned that two main warehousing locations will be used for MCO components:

Building 2101M, 200 East Area - Building $2101 \mathrm{M}$ is a large warehouse in current use for storage of equipment, waste containers, etc. It has in place the $\mathrm{QC}$ required item tracking system which must be used for MCO components stored here. The MCO components that are first used at the CSB (MCO shells \& locking ring assembly, and the MCO cap) will be stored in Building $2101 \mathrm{M}$, which is geographically close to the CSB. This warehouse has the capability to store up to $120 \mathrm{MCO}$ shells in packages of two as described in Table 1. The MCO caps would be stored here on a long-term basis until needed for welding onto the MCOs at the Hot Conditioning System (HCS) annex to the CSB.

Warehouse in 100K Area - A warehouse located in Building $190 \mathrm{KE}$ in the 100 area will be established for storage of all the other MCO components. Transfers of parts from this warehouse to poolside at the $\mathrm{K}$ Basins will be done so as to keep a steady supply of the parts available for ongoing fuel loading campaigns at the basins, without exceeding the limited staging space available for these parts within the basin facilities. Quality Control (QC) item tracking capability equivalent to that used at $2101 \mathrm{M}$ must be established and used at this warehouse.

Table 2 provides estimates of warehouse space needed for the components or subassemblies. 
Table 1

Packaging \& Storage Configurations

\begin{tabular}{|c|c|c|}
\hline MCO Component & Factory Packaging & Storage Configuration \\
\hline 1a. MCO Shell & $\begin{array}{l}\text {-Two shells per package, side by side, horizontally at } \\
\text { the same level in the package. Packages stackable up } \\
\text { three to four tiers deep. Packages not exceeding } \\
\text { dimensions of } 36^{\prime \prime} \times 53^{\prime \prime} \times 168^{\prime \prime} \text {. }\end{array}$ & $\begin{array}{l}\text { Storage will be as is in } \\
\text { manufacturer's packaging } \\
\text { and crating at Building } \\
2101 \mathrm{M} \text { warehouse. } \\
\text { (Stacked) }\end{array}$ \\
\hline 1b. Locking Ring & -Installed in the MCO shell at the factory. & $\begin{array}{l}\text { Storage will be as is in the } \\
\text { MCO shell and its } \\
\text { packaging. }\end{array}$ \\
\hline 2. Shield Plug & $\begin{array}{l}\text {-Each shield plug assembly separate on individual } \\
\text { holder/heavy-duty pallet. }\end{array}$ & $\begin{array}{l}\text { Storage will be as is in } \\
\text { manufacturer's packaging } \\
\text { and crating at Building } 190 \\
\text { KE warehouse. (No } \\
\text { Stacking). }\end{array}$ \\
\hline 3. Dip Tube & $\begin{array}{l}\text { - Manufacturer may package multiple dip tubes per } \\
\text { package, but the total package dimensions will not } \\
\text { exceed that of the MCO shell package described above. }\end{array}$ & $\begin{array}{l}\text { Storage will be as is in } \\
\text { manufacturer's packaging } \\
\text { and crating at Building } 190 \\
\text { KE warehouse. (No } \\
\text { Stacking) }\end{array}$ \\
\hline 4. MCO Cap & -Packaged/shipped on pallets (Two caps per pallet) & $\begin{array}{l}\text { Long-term (up to eight } \\
\text { years) storage required. } \\
\text { Store in original packaging } \\
\text { at Building } 2101 \mathrm{M} \\
\text { warehouse. (No Stacking) }\end{array}$ \\
\hline $\begin{array}{l}\text { 5. Mark IA } \\
\text { Basket; Mark IA } \\
\text { Scrap Basket; } \\
\text { Mark IV Basket; } \\
\text { Mark IV Scrap } \\
\text { Basket }\end{array}$ & $\begin{array}{l}\text { - Loaded on pallets, with a maximum of two baskets } \\
\text { per pallet }\end{array}$ & $\begin{array}{l}\text { Storage will be as is in } \\
\text { manufacturer's packaging } \\
\text { and crating at Building } 190 \\
\text { KE warehouse. }\end{array}$ \\
\hline $\begin{array}{l}\text { 6. 24" diameter } \\
\text { shield plug seal - } \\
\text { "helicoflex" seal }\end{array}$ & $\begin{array}{l}\text { - Individually wrapped and packed in crates; } \\
\text { approximately } 20 \text { seals/crate, } 24^{\prime \prime} \times 30^{\prime \prime} \times 30^{\prime \prime} \text { crate size. }\end{array}$ & $\begin{array}{l}\text { Storage will be as is in } \\
\text { manufacturer's packaging } \\
\text { and crating at Building } 190 \\
\text { KE warehouse. }\end{array}$ \\
\hline 7. Small parts & Packed in crates in conveniently sized lots. & $\begin{array}{l}\text { Storage will be as is in } \\
\text { manufacturer's packaging } \\
\text { and crating at Building } 190 \\
\text { KE warehouse. }\end{array}$ \\
\hline
\end{tabular}


HAF-SD-SNF-PLN-021, Rev 0

Table 2

Warehouse Space Estimates

\begin{tabular}{|c|c|}
\hline Component or Subassembly & Warehouse space needed for storage \\
\hline 1a. MCO Shell & $\begin{array}{l}\text { Assuming } 36^{\prime \prime} \times 53^{\prime \prime} \times 168^{\prime \prime} \text { packages, } 15 \text { to } 20 \text { warehouse spaces (for three } \\
\text { tier to four tier stacking) sized about } 4 \text { l } / 2 \text { by } 14 \text { feet (individual area of } 62 \\
\mathrm{ft}^{2} \text { ) are needed, for a total required storage area of at least } 930 \text { to } 1240 \mathrm{ft}^{2} \text {, } \\
\text { plus aisle access area as required. This assumes that the maximum } \\
\text { inventory of MCO shells requiring storage remains at or below } 120 \text { units. }\end{array}$ \\
\hline 1b. Locking Ring & Not applicable (locking rings are stored within MCO shells). \\
\hline 2. Shield Plug Assembly & $\begin{array}{l}\text { Assuming one shield plug per pallet, where pallet footprint is } 16 \mathrm{ft}^{2} \text {, and a } \\
\text { maximum inventory of } 120 \text { units, up to } 1920 \mathrm{ft}^{2} \text { will be needed. }\end{array}$ \\
\hline 3. Dip Tube & $\begin{array}{l}\text { Assuming a maximum inventory of } 120 \text { units, } 1 \text { " diameter dip tubes and } \\
\text { crating of } 20 \text { tubes per } 12^{n} \times 12^{\prime \prime} \times 156^{n} \text { package, a } 6 \mathrm{ft} \text { by } 13 \mathrm{ft} \text { foot storage } \\
\text { area will be needed, no stacking required. }\end{array}$ \\
\hline 4. MCO Cap & $\begin{array}{l}\text { Assuming maximum inventory will be all } 400 \text { caps, and that two caps can } \\
\text { be placed per pallet, } 20016 \mathrm{ft}^{2} \text { pallets covering a cumulative area of } 3200 \\
\mathrm{ft}^{2} \text { will need to be stored for up to five years. }\end{array}$ \\
\hline 5. Baskets (all types) & $\begin{array}{l}\text { Baskets placed two per pallet can be stored at the } 100 \mathrm{~K} \text { Area warehouse. } \\
\text { Storage area required for the baskets for two MCOs would be } 80 \mathrm{ft}^{2} \text {. The } \\
\text { total storage space needed for the baskets for the maximum inventory of } \\
120 \text { units would be } 4800 \mathrm{ft}^{2} \text {. }\end{array}$ \\
\hline $\begin{array}{l}\text { 6. } 24 \text { " diameter shield plug } \\
\text { seal - "helicoflex" seal }\end{array}$ & $\begin{array}{l}\text { Minor storage space requirement. If crated in lots of } 20 \text {, no more than } 6 \\
24^{\prime \prime} \times 30^{n} \times 30^{n} \text { crates would need to be stored. }\end{array}$ \\
\hline 7. Small parts & $\begin{array}{l}\text { Minor storage space requirement. Estimate that between } 20 \text { and } 40 \text { small } \\
\text { crates will need to be stored at any given time. These could be stacked. }\end{array}$ \\
\hline
\end{tabular}

\subsection{HANDLING AT WAREHOUSE}

\section{$2.1 \quad$ Incoming Parts}

All incoming parts are shipped by the manufacturer to the Hanford Site. They are received at the 1100 Area Central Warehouse, at which time a receipt inspection will be performed according to QA requirements (see NQA-1). Upon passing inspection, the parts are redirected to the proper warehouse destination on the basis of component type (Section 1.3 lists the warehouse destinations of the different $\mathrm{MCO}$ components). Arriving at the warehouse, they are unloaded, by forklift for palleted items, and are placed in the storage location designated for the particular component. The forklift used at the $2101 \mathrm{M}$ warehouse must have sufficient capacity to handle the MCO shell package. Items, except those small enough to be stored on shelving, will be on pallets and movable to their storage location on the warehouse floor by forklift. Items will be stacked as allowable, to minimize the storage cost billed to the SNF Project by the warehouses and/or maintain more efficient storage configurations. Written records prepared during the receiving process per NQA-1 will be forwarded to Records Control, and copies will follow the parts up to usage. 


\subsection{Parts Segregation/Storage}

Components will be separated based on the determined packaging and storage conditions for the given component, or other warehouse constraints. Warehouse managers should ensure that components are arranged and tracked so withdrawal of specified sets of components for given shipments to the CSB and $\mathrm{K}$ Basins can be rapidly performed. Also, to minimize risk of corrosion during extended storage, arrangements should be made in segregation and storage of the components to create turnover of existing warehouse inventories as new shipments of components are received from the manufacturers.

Storage of the parts will be performed according to NQA-1 requirements. Storage records are to be maintained and will be forwarded to Records Control; copies follow the parts up to usage.

Cleanliness control of all $\mathrm{MCO}$ components in all warehouses shall be performed in accordance with ASME NQA-1-1994 Edition, Subpart 2.2 requirements for Level C items.

\subsection{Outgoing Parts}

On demand from the designated SNF Project parts coordinator, the warehoused parts are to be collected and placed on a flatbed trailer or in trucks for transport to the CSB and K Basins as required. MCO shells (with pre-installed locking rings) are shipped to the CSB no less than one package at a time with two shells per package (more than one package can be shipped per shipment if requested).

Other parts are taken from the $190 \mathrm{KE}$ warehouse, loaded on a truck or carts as appropriate, and transferred per given directions to the $\mathrm{K}$ East or $\mathrm{K}$ West Basin.

Storage records will be updated to reflect the transfer of the parts out of the warehouse; the records will be forwarded to Records Control.

\subsection{HANDLING AND USAGE OF MCO PARTS AT CSB \& K BASINS}

Section 3 concerns handling and usage of $\mathrm{MCO}$ components received at the CSB and $\mathrm{K}$ Basins. CSB Operations and $\mathrm{K}$ Basin Operations will be responsible for these handling operations, and for providing designated areas or stations and equipment within the basins to accommodate staging, packaging removal and component assembly activities as required.

Figure 1 depicts the handling and usage of $\mathrm{MCO}$ components at the key facilities. Components and assemblies are shown passing from warehouse to facility or from facility to facility as labeled arrows. Staging, packaging removal, handling, and other processing activities associated with the components are listed as bulleted phrases within the facilities and facility areas where they are executed. 
HNF-\$P-SNE P4N-021, REFO

\subsection{Reception and Staging}

Each basin is to have a forklift on hand to remove all palleted $\mathrm{MCO}$ components brought from the warehouses on transport trucks. The palleted items need to be moved into a designated staging/assembly area within the facility where the items can be temporarily staged pending use in the MCO loading process. This area must be a non-radiological zone, so that packaging materials and pallets can be disposed and/or recycled without generating any radiological waste or requiring radiological survey of materials leaving this zone.

Upon notice from the coordinator, loading, transfer and unloading of the MCO shells from Building $2101 \mathrm{M}$ to the CSB will take approximately two working days. Shield plugs and crates of other parts from the $190 \mathrm{KE}$ warehouse can be delivered within one working day. Basin Operations is responsible for notifying warehouses in advance of needs for MCO components so as to assure timely availability of the components. $\mathrm{K}$ Basins Operations must coordinate with the CSB for timely arrival of cask/MCO shells from that facility. 
Figure 1. Flowchart of MCO Component Warehousing, Handling, and Usage

\subsection{Packaging Removal/Component Inspection}

MCO components should remain in the staging/assembly area, and in the original packaging materials until just prior to use, to minimize contamination and potential mechanical damage. At the same time, all protective coatings, plastic covers and caps, and other packaging materials should be removed prior to introduction of the components into radiological zones, for reasons stated above. Operators are to remove all packaging material, and otherwise ready the components for use according to directions listed in Table 3. Note that all activities described are performed prior to introduction of the completed assemblies into radiological zones.

Written records shall be made documenting the unpackaging, pre-use inspection, and use of MCO components. These records will be added to the other records accompanying the components, and the complete set of records will be forwarded to Records Control. Records Control will update the component database accordingly.

\subsection{Component Assembly/Integration into $\mathrm{MCO}$}

The steps that occur in the course of component assembly and integration into a loaded MCO are depicted in Figure 1. Some of the steps are sequential, but many activities will need to be conducted simultaneously to maintain production schedules and assure availability of parts. It is beyond the scope of this plan to develop a detailed time and motion study for these activities; however, this should be done to confirm that adequate personnel support, facility processing area, and other resources are available to meet processing needs.

\subsection{QUALITY ASSURANCE REQUIREMENTS}

\subsection{Incoming Parts Inspection}

Inspection of components will be completed at the fabrication site per NQA-1 requirements. Documentation of QA performed at the manufacturer will accompany each shipment. Incoming parts will be inspected for number and damage during shipping and handling prior to receipt at the warehouse only; inspections will be documented. If a shipment is received damaged and found not to meet the necessary criteria, the damaged units will be returned, at the manufacturer's expense, for replacement/repair. All deficiencies will be noted in a nonconformance report for each shipment. Specifics on receiving/inspection can be found in NQA-1-1994 Edition, Subpart 2.2, Section 5. 
IFF-SA-SNF-PLN- 021, Rev0 HNF-SD-SNF-DR-003, Rev. 0, Appendix 17

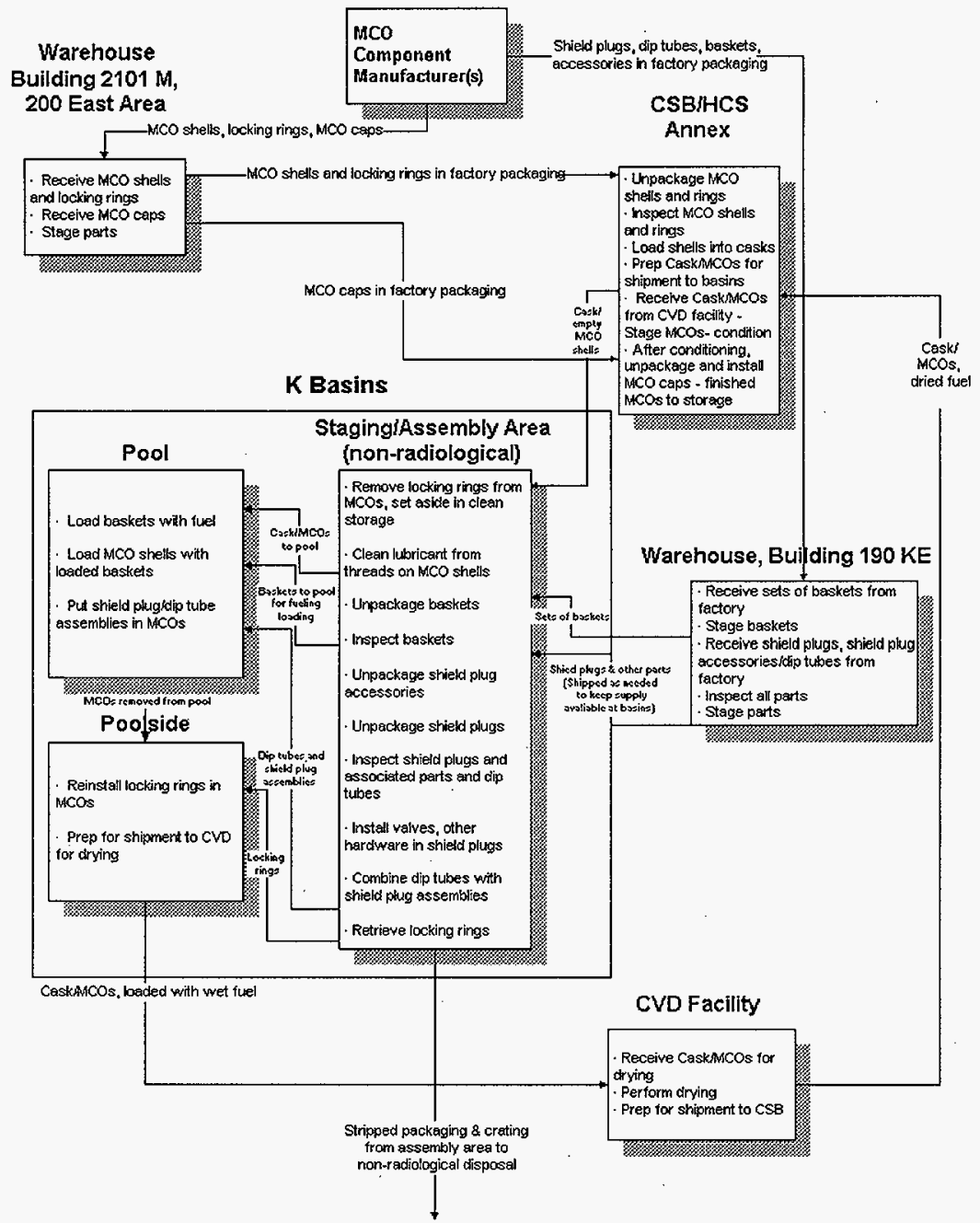


Table 3

Packaging Removal/Inspection Requirements

\begin{tabular}{|c|c|}
\hline Component or Subassembly & Packaging Removal/Inspection Requirements \\
\hline 1a. MCO Shell & $\begin{array}{l}\text { MCO shells are removed from packaging at the CSB. Protective } \\
\text { coatings are to be removed from any exposed sealing or threaded } \\
\text { surfaces on the shell and locking ring. Verify locking ring is } \\
\text { snugly screwed into place. A final inspection of integrity of the } \\
\text { shell may be performed here prior to loading the shell into an } \\
\text { empty cask. Following this, an inspection of the } \mathrm{MCO} / \text { cask } \\
\text { assembly may be required. }\end{array}$ \\
\hline 1b. Locking Ring & $\begin{array}{l}\text { The locking ring is removed from the } \mathrm{MCO} \text { shell upon receipt of } \\
\text { the cask/MCO from the CSB. Excess lubricant is to be wiped } \\
\text { from the ring before storage of the ring pending reinstallation of } \\
\text { the ring in the MCO after loading of fuel in the MCO is } \\
\text { complete. }\end{array}$ \\
\hline 2. Shield Plug & $\begin{array}{l}\text { Shield plugs are removed from packaging at the staging/assembly } \\
\text { areas in the basins. Port plugs and any protective coatings are } \\
\text { carefully removed, and surfaces cleaned if necessary. The safety } \\
\text { class rupture disk with orifice plate, the two process valves, and } \\
\text { the process relief valve, all installed at the manufacturer's shop, } \\
\text { will be inspected for correct installation to proper torque values. } \\
\text { This process should be done in conjunction with unpacking of } \\
\text { other shield plug hardware just previous to assembly of the } \\
\text { complete shield plug assembly. }\end{array}$ \\
\hline 3. Dip Tube & $\begin{array}{l}\text { Dip tubes are removed from factory crating at the } \\
\text { staging/assembly areas at the basins. Shrink-wrap, plastic caps, } \\
\text { and protective coatings on threads are to be removed from a given } \\
\text { dip tube as shield plug assemblies are completed. Inspection of } \\
\text { the tube will be performed, including thread integrity and length } \\
\text { measurements to ensure that the shield plug/dip tube assembly to } \\
\text { be made will fit in the MCO. }\end{array}$ \\
\hline 4. MCO Cap & $\begin{array}{l}\text { MCO caps will be unpackaged and inspected at the CSB. } \\
\text { Integrity of weld surfaces is to be verified. }\end{array}$ \\
\hline 5. Baskets (all types) & $\begin{array}{l}\text { Baskets are unpackaged and inspected at the basins } \\
\text { staging/assembly areas. }\end{array}$ \\
\hline $\begin{array}{l}\text { 6. } 24^{\prime \prime} \text { diameter shield plug seal - } \\
\text { "helicoflex" seal }\end{array}$ & Shield plug seals are unpackaged and inspected just prior to use. \\
\hline 7. Small parts & $\begin{array}{l}\text { Each part is to be removed from crating, removed from any of its } \\
\text { shrink-wrap or packaging, and visually inspected prior to use. }\end{array}$ \\
\hline
\end{tabular}

Physical inspection of all components is required before use of the components in the MCO assembly process. These inspections will occur at the basins staging/assembly areas, as per the specific requirements given in Section 3.2. The inspection will be documented for each part and subassembly. 


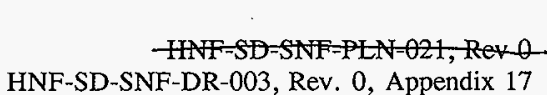

\subsection{INVENTORY CONTROL}

\subsection{Tracking of MCO Components}

MCO components will be tracked by an inventory number assigned. Travelers will be used to track pieces of the $\mathrm{MCO}$ as they are brought together.

For all received and outgoing MCO components (relative to the warehouses), entries in the Hanford Inventory Control System database are to be made, or alternately any functionally equivalent database may be used by Records Control for this function. Careful referencing of the serial numbers on parts (where applicable) is to be used in the database entries.

\subsection{Parts Supplies}

The facilities consuming $\mathrm{MCO}$ parts will coordinate with the warehouses to maintain a constant supply of these parts at the assembly areas. To accomplish this, operations at CSB and the $\mathrm{K}$ Basins will maintain an inventory of parts on hand, and replenish the supply by arranging for parts shipments from the warehouses when inventory drops below predetermined levels.

\section{$6.0 \operatorname{CosT}$}

\subsection{Warehouse Space Rental}

Cost will be dependent upon the space required, duration of storage, and location of the warehouse.

Building $2101 \mathrm{M}$ currently charges $\$ 8.50 / \mathrm{ft}^{2} /$ year for storage space. There is no ongoing cost to the SNF Project for storage space in the $190 \mathrm{KE}$ warehouse, once initial capital costs for readying the warehouse for use are made.

\subsection{Special Handling Cost}

Special handling cost will be determined based upon the packaging for each MCO part. 
HNF-SD-SNF-DR-003,

Rev. 0, Appendix 18

\section{MULTI-CANISTER OVERPACK DESIGN REPORT ASME CODE EXCEPTION REPORT}


HNF-SD-SNF-DR-003,

Rev. 0, Appendix 18

\subsection{Purpose}

This Code Exception Report is prepared to document and provide justifications for all deviations from the ASME Code Section III requirements. The MCO is a non-stamped Code vessel. The design of the $\mathrm{MCO}$ is required to be constructed to the intent of the ASME Code as specified in the Performance Specification. The MCO design criteria relative to Code requirements is specified in the following excerpt from the Performance Specification.

"The MCO shall be designed in accordance with DOE Order 6430.1A, General Design Criteria (DOE 1989). Safety Class (SC) and Safety Significant Components (SSCs), providing fuel containment, confinement, and criticality control, shall be constructed to meet the intent of ASME Boiler and Pressure Vessel Code, Section III, to the extent specified in this Performance Specification, Rules for Construction of Nuclear Power Plant Components, Subsection NB (ASME 1995) under the component safety group as guided by the NUREG/CR 3854, UCRL-53544, Fabrication Criteria for Shipping Containers, 1984. The Nuclear Regulatory Commission (NRC) positions in Regulatory Guides 1.84 and 1.85 on ASME Section III code cases shall be reviewed prior to using such code cases for safety class applications for the MCO. All deviations from Subsection NB shall be documented and justified."

In addition to NB, this Code Exception Report will also include a review of Sections NF and $N G$ for those elements of the design that are required to meet the intent of these sections. 
This section summarizes the description of the $\mathrm{MCO}$ components, the $\mathrm{MCO}$ function, and the application of the Code requirements to the MCO components.

\subsection{Component Description}

The MCO assembly is a single purpose SNF (Spent Nuclear Fuel) container that is capable of maintaining SNF subcriticality at all times and maintaining SNF containment after having been closed and sealed. The $\mathrm{MCO}$ assembly consists of a shell assembly, a shield plug assembly, and five to six SNF fuel baskets. It contains features that will allow processing of its SNF contents. The MCO package is an $\mathrm{MCO}$ assembly loaded with SNF.

The containment boundary is made up of the shell assembly and shield plug with process ports and fittings, main seal and the cover cap. The containment boundary items are to be constructed to the intent of the ASME Code Section III, Subsection NB. Since the locking ring provides structural support for the shield plug, it is also constructed to the intent of Subsection NB.

All MCO fabricator pressure boundary welds shall be made in accordance with ASME Section III, Division 1, NB-3350. All welds shall be sufficiently smooth to enable easy decontamination. Butt welds shall be ground flush to within .03 inches of base metal. Weld joint designs shall avoid potential contamination traps to the greatest extent practical. All MCO pressure boundary welds and welds bearing the weight of the fully loaded MCO must be designed for and pass $100 \%$ volumetric examination (x-rays or ultrasonic) per ASME requirements. Exceptions for field welds and the structural welds for the rupture disks shall be documented.

The Mark 1A baskets must comply with Subsection NG because geometry control is required for criticality control. The design shall meet Service Level A requirements for normal operating loads and Service Level $D$ for accident conditions under ASME Boiler and Pressure Vessel Code, Section III, Division I Subsection NG. The Mark IV baskets do not have any Code requirements.

\subsubsection{MCO Shell}

The MCO shell is a cylindrical vessel that provides access to its cavity through its top end and receives a shield plug at its top end for closing. The $\mathrm{MCO}$ shell has a bottom assembly that provides a permanent sealed closure on the shell bottom end. The MCO bottom assembly is nominally flat and must include an internal liquid collection sump at the $\mathrm{MCO}$ centerline. The MCO must be designed with a 1.49 inch nominal distance between the inside of the MCO bottom assembly and the bottom of the lowest basket. 
HNF-SD-SNF-DR-003,

Rev. 0, Appendix 18

The MCO must permit or allow loading and stacking of the fuel baskets within its cavity. The empty shell must be designed to load into and out of the transport cask.

\subsubsection{MCO Shield Plug}

The MCO shall be designed with a mechanical closure configuration. The closure shall rely on a mechanical crushable seal to maintain the containment and confinement requirement at the final closure interface. The closure system shall utilize the shield plug/shell interface as the closure boundary where the crushable seal shall be located. The shield plug shall be held in place via a locking ring threaded into the $\mathrm{MCO}$ shell. The locking ring shail contain screws that will be tightened to force the shield plug down against the crushable seal while pushing up on the locking ring.

The MCO shall be designed to incorporate a final welded closure cap over the shield plug. The cap shall be welded to the MCO shell. The cap shall be capable/configured for lifting the MCO. The closure cap shall be capable of being fitted with a replaceable rupture disk.

\subsubsection{MCO Fuel Baskets}

The MCO fuel baskets are categorized into two major configurations: 1) intact fuel element baskets, and, 2) scrap fuel (fragment) baskets. Fuel baskets must also maintain criticality control for the higher enriched (Mark IA) fuel. These basic requirements lead to four different basket types as follows:

- Type (1) must have the ability to hold 48 Mark IA (higher enriched) intact-fuel elements.

- Type (2) must have the ability to hold 54 Mark IV intact-fuel.

- Type (3) will hold Mark IA (higher enriched) scrap fuel (fragments).

- Type (4) will hold Mark IV scrap fuel (fragments).

\subsection{Functional Requirements}

The mission of the MCO is to confine, contain, and maintain the SNF in a critically safe array to insure the safe operations and support processing of removal from K Basin, Cold Vacuum Drying (CVD) Facility, transport to the Canister Storage Building(CSB), staging (short-term storage) in the CSB, hot conditioning in the Hot Conditioning System (HCS), and interim storage in the CSB. The MCO serves as the SNF processing vessel during both CVD and hot conditioning. 
The functions the $\mathrm{MCO}$ performs to accomplish this mission are defined below:

- Enable the reracking of fuel elements and fuel fragments/scrap;

- Maintain fuel elements and fuel fragments/scrap in a critically safe condition;

- At various times during its life provide confinement and/or containment for fuel elements and fuel fragments/scrap.

- Relieve excess internal pressure (provide pressure relief);

- Enable the processes to dewater (in preparation for sealing), CVD, and hot conditioning;

- Enable the functions to inert the MCO interior and to purge and vent gasses;

- Shield workers against gamma and neutron radiation traveling through the shield plug from the MCO interior; and

- Enable the handling of all MCO components individually, as well as a fully assembled and loaded MCO.

\subsection{Code Applicability}

The following sections of the ASME Code apply to the intent of construction of the MCO:

- Section II applies for materials

- Section III for materials, design, fabrication, testing, inspection, and overpressure protection

- Section V for inspection (as prescribed by the Performance Specification)

- Section IX for welder and procedure qualifications.

There are no exceptions taken for Sections II, V, and IX as prescribed by the Performance Specification. All Code Exceptions presented will refer to Section III.

\subsection{Code Exceptions}

This section contains specific information of where Code exceptions are taken and the reason for the exception. The Code exceptions are provided in Table 3.1 
HNF-SD-SNF-DR-003,

Rev. 0, Appendix 18

TABLE 3.1

Code Exceptions

\begin{tabular}{|c|c|c|c|c|c|c|}
\hline $\begin{array}{l}\text { Section / } \\
\text { Subsection }\end{array}$ & AIticle & Paragraph & $\begin{array}{l}\text { Section } \\
\text { used as } \\
\text { gufdance } \\
\text { (yeg / no) }\end{array}$ & $\begin{array}{c}\text { Bxception } \\
\text { Taken } \\
\text { (yes / no) }\end{array}$ & Exception & Justification \\
\hline Secrion III, NCA & & & no & & & \\
\hline Section III, NB & NB-1000 & & no & & & \\
\hline Section III, NB & NB-2000 & & yes & no & & \\
\hline \multirow[t]{14}{*}{ Section III, NB } & $\mathrm{NB}-3000$ & 3100 & yes & no & & \\
\hline & & 3110 & yes & no & & \\
\hline & & 3220 & yes & no & & \\
\hline & & 3130 & yes & no & & \\
\hline & & 3200 & yes & no & & \\
\hline & & 3210 & yes & no & & \\
\hline & & 3220 & yes & no & & \\
\hline & & 3230 & yes & no & & \\
\hline & & 3300 & yes & no & & \\
\hline & & 3310 & yes & no & & \\
\hline & & 3320 & yes & no & & \\
\hline & & 3330 & yes & no & & \\
\hline & & 3340 & yes & no & & \\
\hline & & 3350 & yes & Yes & $\begin{array}{l}\text { NB-335l defines weld } \\
\text { category according to } \\
\text { geometry. These weld } \\
\text { cypes are illustrated } \\
\text { in Figure NB-3351.1 of } \\
\text { NB-30oo. For designs } \\
\text { that are restricted to } \\
\text { cylindrical vessels } \\
\text { with nozzles, weld } \\
\text { categories are: } \\
\text { NB-3351.1, category A: } \\
\text { All longitudinal welds } \\
\text { and welds to spherical } \\
\text { sections. } \\
\text { Category A joints shall } \\
\text { meet the fabrication } \\
\text { requirements of NB-4241 } \\
\text { and shall be capable of } \\
\text { being examined in } \\
\text { accordance with NB- } \\
\text { s2lo. } \\
\text { NB-33sl.2, Category B: } \\
\text { All circumferential } \\
\text { welds to plates of } \\
\text { equal thickness except } \\
\text { to spherical sections } \\
\text { as defined in category } \\
\text { A. The weld angle } \\
\text { between plates can not } \\
\text { be less than } 150 \\
\text { degrees. } \\
\text { Category B joints shall } \\
\text { meet the fabrication } \\
\text { requirementsof NB-4242 } \\
\text { and shall be capable of } \\
\text { beingexamined in } \\
\text { accordancewith NB-5220. } \\
\text { Somejoints require } \\
\text { cyclic service analysis } \\
\text { pex NB-3222.4. }\end{array}$ & 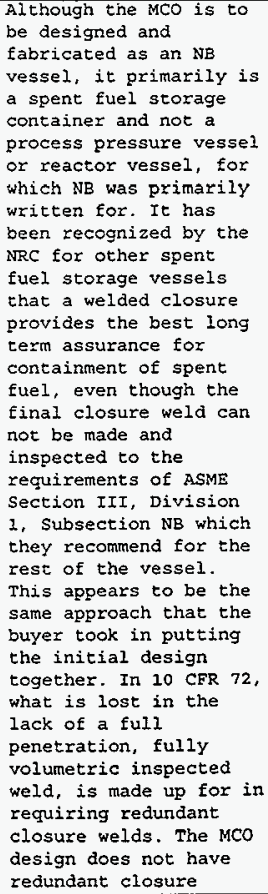 \\
\hline
\end{tabular}


HNF-SD-SNF-DR-003,

Rev. 0, Appendix 18

\begin{tabular}{|c|c|c|c|c|c|c|}
\hline $\begin{array}{l}\text { Section } / \\
\text { subsection }\end{array}$ & article & Paragraph & $\begin{array}{c}\text { Section } \\
\text { used as } \\
\text { guidance } \\
\text { (yes / no) }\end{array}$ & $\begin{array}{c}\text { Bxception } \\
\text { Taken } \\
\text { (yes / no) }\end{array}$ & Bxception & Justification \\
\hline & & & & & $\begin{array}{l}\text { NB-3351.3, Category C: } \\
\text { All circumferential } \\
\text { welds between cylinders } \\
\text { and flat plates or } \\
\text { flanges. } \\
\text { Category } \mathrm{C} \text { joints shall } \\
\text { meet the fabrication } \\
\text { requirements of NB-4243 } \\
\text { and shall be capable of } \\
\text { being exanined in } \\
\text { accordance with NB- } \\
\text { 5230. } \\
\text { NB-3351.4, category D: } \\
\text { All welds between } \\
\text { pressure boundaries and } \\
\text { nozzles. Due to the } \\
\text { geometry of a } \\
\text { circumferential butt } \\
\text { weld, the } \\
\text { classification of the } \\
\text { field closure weld is } \\
\text { considered to be a } \\
\text { category } \text { seld, even } \\
\text { though all the } \\
\text { requirements for a } \\
\text { Category B weld cannot } \\
\text { be met. The weld is } \\
\text { not a true full } \\
\text { penetration weld. The } \\
\text { welds used to assemble } \\
\text { the rupture disk in the } \\
\text { process plug do not } \\
\text { meet the requirements } \\
\text { of subsection NB. The } \\
\text { weld that seals the } \\
\text { rupture disk in place } \\
\text { and acts as a pressure } \\
\text { boundary is a seal weld } \\
\text { and has no structural } \\
\text { capabilities or } \\
\text { requirements. This is } \\
\text { permitted by the Code. } \\
\text { The weld that holds the } \\
\text { top of the process } \\
\text { plug to the bottom is a } \\
\text { non pressure boundary } \\
\text { weld. It provides } \\
\text { structural support to } \\
\text { the rupture disk and } \\
\text { also transmits the } \\
\text { operating torque from } \\
\text { the operator to the } \\
\text { closure threads. It, } \\
\text { being a partial } \\
\text { penetration weld, is an } \\
\text { exception to the code. }\end{array}$ & $\begin{array}{l}\text { weids. However, it does } \\
\text { have redundant seals in } \\
\text { the final form. Even } \\
\text { though not welded, the } \\
\text { mechanical seal plus } \\
\text { the welded closure cap } \\
\text { make up a redundant } \\
\text { seal system. In NRC } \\
\text { approved systems, it } \\
\text { should be noted that } \\
\text { neither one of the } \\
\text { redundant seal welds } \\
\text { are volumerrically } \\
\text { inspected nor } \\
\text { hydrostatically tested. } \\
\text { The welds used in the } \\
\text { installation of the } \\
\text { rupture disk are } \\
\text { acceptable, since the } \\
\text { seal weld is permitted } \\
\text { and is required to meet } \\
\text { the leaktightness } \\
\text { requirements. } \\
\text { The weld holding the } \\
\text { two parts of the } \\
\text { process plug together } \\
\text { is a structural weld } \\
\text { but not a pressure } \\
\text { boundary weld. it is a } \\
\text { partial penetration } \\
\text { weld, and can not be } \\
\text { volumetrically } \\
\text { inspected. However, its } \\
\text { function is to transmit } \\
\text { torque during operation } \\
\text { and function as support } \\
\text { to the edges of the } \\
\text { rupture disk. As shown } \\
\text { in Appendix lo, it has } \\
\text { very low stresses. It } \\
\text { is also inspected with } \\
\text { liquid penetrant } \\
\text { similar to partial } \\
\text { penetrant welds } \\
\text { permitted by subsection } \\
\text { NB in other } \\
\text { applications. }\end{array}$ \\
\hline & & 3360 & yes & Yes & $\begin{array}{l}\text { NB-3362, Bolted Flange } \\
\text { connections, states : } \\
\text { "It is recommended that } \\
\text { the dimensional } \\
\text { requirements of bolted } \\
\text { flange connections to } \\
\text { external piping conform }\end{array}$ & $\begin{array}{l}\text { The limited space } \\
\text { available at the } \\
\text { process piping } \\
\text { interface does not } \\
\text { permit the use of ANSI } \\
\text { fittings. Since the } \\
\text { DESH Performance }\end{array}$ \\
\hline
\end{tabular}


HNF-SD-SNF-DR-003,

Rev. 0, Appendix 18

\begin{tabular}{|c|c|c|c|c|c|c|}
\hline $\begin{array}{l}\text { Section / } \\
\text { subsection }\end{array}$ & Article & Paragraph & $\begin{array}{l}\text { Section } \\
\text { used as } \\
\text { guidance } \\
\text { (yes / no) }\end{array}$ & $\begin{array}{l}\text { Bxception } \\
\text { Taken } \\
\text { (yes / no) }\end{array}$ & Bxception & Justification \\
\hline & & & & & $\begin{array}{l}\text { to ANSI B16.5, Steel } \\
\text { pipe Flanges and } \\
\text { Flanged Fittings." The } \\
\text { MCO process hardware } \\
\text { interfaces do not use } \\
\text { standard ANSI flanges } \\
\text { and fittings. } \\
\text { NB-3363 states that } \\
\text { access openings may be } \\
\text { attached by studs or } \\
\text { bolts. The MCo opening } \\
\text { is not attached in this } \\
\text { way. }\end{array}$ & 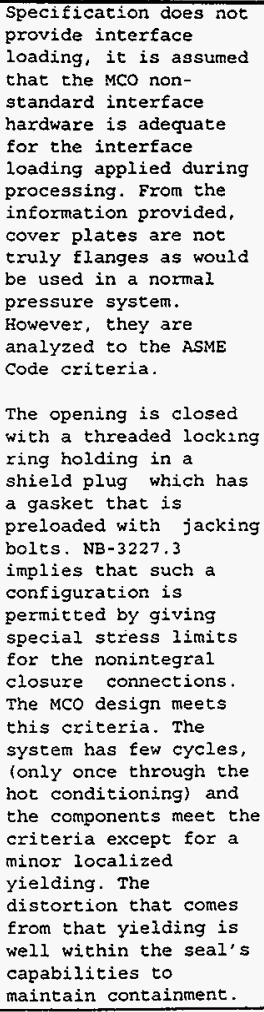 \\
\hline & & 3400 & yes & no & & \\
\hline & & 3410 & yes & no & & \\
\hline & & 3420 & yes & no & & \\
\hline & & 3430 & yes & no & & \\
\hline & & 3440 & yes & no & & \\
\hline & & 3500 & yes & no & & \\
\hline & & 3510 & Yes & no & & \\
\hline & & 3520 & yes & no & & \\
\hline & & 3530 & yes & no & & \\
\hline & & 3540 & yes & no & & \\
\hline & & 3550 & yes & no & & \\
\hline & & 3560 & yes & no & & \\
\hline & & 3570 & yes & no & & \\
\hline & & 3580 & yes & no & & \\
\hline & & 3590 & yes & yes & $\begin{array}{l}\text { NB-3594.1: Pressure } \\
\text { relief valve flanges } \\
\text { shall meet interface }\end{array}$ & $\begin{array}{l}\text { The non-standard } \\
\text { pressure relief } \\
\text { interface dimensions }\end{array}$ \\
\hline
\end{tabular}


HNF-SD-SNF-DR-003,

Rev. 0, Appendix 18

\begin{tabular}{|c|c|c|c|c|c|c|}
\hline $\begin{array}{l}\text { Section } / \\
\text { subsection }\end{array}$ & Article & Paragraph & $\begin{array}{c}\text { Section } \\
\text { used as } \\
\text { guidance } \\
\text { (yes / no) }\end{array}$ & $\begin{array}{c}\text { Exception } \\
\text { Taken } \\
\text { (yes / no) }\end{array}$ & Bxception & Justification \\
\hline & & & . & & $\begin{array}{l}\text { dimensions of ANSI } \\
\text { B16.5. The non-Code } \\
\text { relief devices use } \\
\text { threaded connections } \\
\text { with a metal seal. }\end{array}$ & $\begin{array}{l}\text { conform to the DESH } \\
\text { requirements. Since } \\
\text { there is no pressure } \\
\text { relief piping, the } \\
\text { interface has no } \\
\text { structural requirements } \\
\text { other than the loading } \\
\text { imposed by the pressure } \\
\text { relief device which has } \\
\text { been evaluated. The } \\
\text { relief valve selected } \\
\text { by the buyer is a non- } \\
\text { flanged connection, } \\
\text { therefore there is no } \\
\text { flange interface } \\
\text { requirement. The } \\
\text { interface is a threaded } \\
\text { connection. Threaded } \\
\text { connections are } \\
\text { permitted by subsection } \\
\text { NB for piping in the } \\
\text { sizes indicated and it } \\
\text { is not clear that they } \\
\text { are permitted for } \\
\text { attachments to the } \\
\text { vessel as in this case. } \\
\text { straight threads are } \\
\text { used in lieu of tapered } \\
\text { threads to allow the } \\
\text { used of a seal for easy } \\
\text { replacement and high } \\
\text { assurance of resealing. }\end{array}$ \\
\hline & & 3600 & yes & no & & \\
\hline & & 3610 & yes & no & & \\
\hline & & 3620 & yes & no & & \\
\hline & & 3630 & yes & no & & \\
\hline & & 3640 & yes & no & & \\
\hline & & 3650 & yes & no & & \\
\hline & & 3660 & $y e s$ & no & & \\
\hline & & 3670 & yes & yes & $\begin{array}{l}\text { NB-3671.1 permits } \\
\text { flanged joints. } \\
\text { NB-3671.7 permits } \\
\text { mechanical joints where } \\
\text { no standard exists if } \\
\text { either tested or } \\
\text { designed in accordance } \\
\text { with NB-3200. }\end{array}$ & $\begin{array}{l}\text { Although this section } \\
\text { of the code pertains to } \\
\text { piping components and } \\
\text { is not totaliy } \\
\text { applicable to the MCO, } \\
\text { it is noted that } \\
\text { mechanical joints such } \\
\text { as the MCO closure are } \\
\text { permitted when designed } \\
\text { in accordance with NB- } \\
3200 \text {. }\end{array}$ \\
\hline & & 3680 & no & no & & \\
\hline & & 3690 & no & no & & \\
\hline & $\mathrm{NB}-4000$ & 4100 & yes & no & & \\
\hline & & 4120 & Yes & yes & $\begin{array}{l}\text { Material and } \\
\text { fabrication } \\
\text { certification will not } \\
\text { be performed by a } \\
\text { certificate holder. }\end{array}$ & $\begin{array}{l}\text { Since, as directed by } \\
\text { the DESH performance } \\
\text { Specification, the MCo } \\
\text { will not be Code } \\
\text { stamped, fabricators } \\
\text { will not be limited to } \\
\text { certificate holder. }\end{array}$ \\
\hline & & 4130 & yes & no & & \\
\hline & & 4200 & yes & no & & \\
\hline
\end{tabular}


HNF-SD-SNF-DR-003,

Rev. 0, Appendix 18

\begin{tabular}{|c|c|c|c|c|c|c|}
\hline $\begin{array}{l}\text { Saction / } \\
\text { subaection }\end{array}$ & Article & Paragraph & $\begin{array}{c}\text { Section } \\
\text { used as } \\
\text { guidance } \\
\text { (yes / no) }\end{array}$ & $\begin{array}{c}\text { Exception } \\
\text { Taken } \\
\text { (yes / no) }\end{array}$ & Bxception & Justification \\
\hline & & 4210 & yes & no & & \\
\hline & & 4220 & yes & no & & \\
\hline & & 4230 & yes & no & & \\
\hline & & 4240 & yes & Yes & $\begin{array}{l}\text { N8-4242 "Category B } \\
\text { weld joints in vessels } \\
\text { and circumferential } \\
\text { weld joints in other } \\
\text { components shall be } \\
\text { full penetration butt } \\
\text { joints, except that } \\
\text { piping } 2 \text { in. noninal } \\
\text { pipe size and smaller } \\
\text { may be socket welded. } \\
\text { When used, backing } \\
\text { strips shall be } \\
\text { continuous in cross } \\
\text { section. Joints } \\
\text { prepared with opposing } \\
\text { lips to form an } \\
\text { integral backing strip } \\
\text { and joints with backing } \\
\text { strips which are not } \\
\text { later removed are } \\
\text { acceptable provided } \\
\text { that the requirements } \\
\text { of NB-3352.2 are met." } \\
\text { The field closure weld } \\
\text { for the MCo meets this } \\
\text { definition and is } \\
\text { fabricated to these } \\
\text { requirements. NB- } \\
3352 \text { evaluation is } \\
\text { not performed since } \\
\text { there are no cyclic } \\
\text { loads applied to the } \\
\text { joint. }\end{array}$ & $\begin{array}{l}\text { Since the weld meets } \\
\text { the fabrication } \\
\text { requirements, this is } \\
\text { not a true exception. } \\
\text { However, even though } \\
\text { the cyclic requirements } \\
\text { of w- } 3352.2 \text { are met, } \\
\text { the inspection } \\
\text { requirements for this } \\
\text { weld can not be met, } \\
\text { and therefore it is an } \\
\text { exception. } \\
\text { The grocess plug } \\
\text { rupture disk welds do } \\
\text { not meet any of the } \\
\text { requirements for the } \\
\text { weld configurations of } \\
\text { this section. The welds } \\
\text { are designed with high } \\
\text { margins of safety and } \\
\text { are inspected in } \\
\text { accordance with the } \\
\text { requirements of partial } \\
\text { penetration welds } \\
\text { permitted by this } \\
\text { section. }\end{array}$ \\
\hline & & 4250 & yes & no & & \\
\hline & & 4300 & yes & no & & \\
\hline & & 4310 & yes & no & & \\
\hline & & 4320 & yes & no & & \\
\hline & & 4330 & yes & no & & \\
\hline & & 4340 & yes & no & & \\
\hline & & 4350 & yes & no & & \\
\hline & & 4360 & yes & no & & \\
\hline & & 4400 & yes & no & & \\
\hline & & 4410 & yes & no & & \\
\hline & & 4420 & yes & yes & $\begin{array}{l}\text { NB-4424 (d) : NB- } \\
4424 \text { (d) states: } \\
\text { "Concavity on the root } \\
\text { side of a single-welded } \\
\text { circumferential butt } \\
\text { weld is permitted when } \\
\text { the resulting thickness } \\
\text { meets the requirements } \\
\text { of NB-3000." The field } \\
\text { closure weld on the MCo } \\
\text { can not be inspected to } \\
\text { verify this requirement } \\
\text { therefore an exception } \\
\text { is taken. }\end{array}$ & $\begin{array}{l}\text { Although, this } \\
\text { requirement cannot be } \\
\text { verified by inspection, } \\
\text { the weld design } \\
\text { configuration minimizes } \\
\text { the concavity } \\
\text { potential. }\end{array}$ \\
\hline & & 4430 & yes & no & & \\
\hline & & 4440 & yes & no & & \\
\hline
\end{tabular}


HNF-SD-SNF-DR-003,

Rev. 0, Appendix 18

\begin{tabular}{|c|c|c|c|c|c|c|}
\hline $\begin{array}{l}\text { Section / } \\
\text { Subsection }\end{array}$ & Axticle & Paragraph & $\begin{array}{c}\text { Section } \\
\text { uned as } \\
\text { gutdance } \\
\text { (Yos / no) }\end{array}$ & $\begin{array}{l}\text { Bxception } \\
\text { Taken } \\
\text { (yes / no) }\end{array}$ & Bxception & . Justification \\
\hline & & 4450 & yes & no & & \\
\hline & & 4500 & yes & no & & \\
\hline & & 4510 & yes & no & & \\
\hline & & 4520 & yes & no & & \\
\hline & & 4530 & yes & no & & \\
\hline & & 4540 & yes & no & & \\
\hline & & 4600 & yes & no & & \\
\hline & & 4620 & yes & no & & \\
\hline & & 4620 & yes & no & & \\
\hline & & 4630 & yes & no & & \\
\hline & & 4640 & yes & no & & \\
\hline & & 4650 & yes & no & & \\
\hline & & 4660 & yes & no & & \\
\hline & & 4700 & yes & no & & \\
\hline & & 4710 & yes & no & & \\
\hline & & 4720 & yes & no & & \\
\hline & & 4730 & yes & no & & \\
\hline & $\mathrm{NB}-5000$ & 5100 & yes & no & & \\
\hline & & 5110 & yes & no & & \\
\hline & & 5120 & yes & no & & \\
\hline & & 5130 & yes & no & & \\
\hline & & 5140 & yes & no & & \\
\hline & & 5200 & yes & no & & \\
\hline & & 5210 & yes & no & & \\
\hline & & 5220 & yes & yes & $\begin{array}{l}\text { NB-5221 For Vessel } \\
\text { Welded Joints states: } \\
\text { "Category B welded } \\
\text { joints in vessels shall } \\
\text { be examined by } \\
\text { radiography and either } \\
\text { the liquid penetrant or } \\
\text { magnetic particle } \\
\text { method." An exception } \\
\text { is taken to the Code } \\
\text { since the field closure } \\
\text { weld on the Mco can not } \\
\text { be radiographed due to } \\
\text { the lack of } \\
\text { accessibility and the } \\
\text { radiation field it is } \\
\text { in. } \\
\text { NB-5222 (b) Permits } \\
\text { circumferential partial } \\
\text { penetration welds in } \\
\text { piping, pump, and } \\
\text { valves to be examined } \\
\text { by liquid penetrant. } \\
\text { The weld on the process } \\
\text { plug/rupture disk } \\
\text { holders is considered } \\
\text { like the stem/bonnet of } \\
\text { a valve. Therefore } \\
\text { liquid penetrant } \\
\text { inspection for a } \\
\text { partial penetration } \\
\text { weld is permitted and } \\
\text { no other examination is } \\
\text { required. }\end{array}$ & $\begin{array}{l}\text { The weld configuration } \\
\text { is in accordance with } \\
\text { DESH direction. The } \\
\text { magnitude of the stress } \\
\text { levels indicate that a } \\
\text { fully radiographed weld } \\
\text { efficiency of } 100 \text { is } \\
\text { not necessary. } \\
\text { dltrasonic inspection } \\
\text { may be substituted in } \\
\text { accordance with NB- } \\
5279 \text {. } \\
\text { The Mco process plug to } \\
\text { rupture disk welds are } \\
\text { inspected to the } \\
\text { conditions of category } \\
\text { B partial penetration } \\
\text { welds for valves. }\end{array}$ \\
\hline & & 5230 & yes & no & & \\
\hline & & 5240 & yes & no & & \\
\hline
\end{tabular}


HNF-SD-SNF-DR-003,

Rev. 0, Appendix 18

\begin{tabular}{|c|c|c|c|c|c|c|}
\hline $\begin{array}{c}\text { Section / } \\
\text { subsection }\end{array}$ & Article & Paragraph & $\begin{array}{c}\text { Section } \\
\text { used as } \\
\text { guldance } \\
\text { (yes / no) }\end{array}$ & $\begin{array}{c}\text { Exception } \\
\text { Taken } \\
\text { (yes / no) }\end{array}$ & Exception & Justification \\
\hline & & 5260 & yes & no & & \\
\hline & & 5270 & yes & yes & $\begin{array}{l}\text { NB-5279 gives special } \\
\text { exception to joints } \\
\text { that cannot be } \\
\text { radiographed. Example } \\
\text { is given as a closure } \\
\text { weld. It permits the } \\
\text { use of ultrasonic } \\
\text { examination performed } \\
\text { in accordance with } \\
\text { Section v, along with } \\
\text { liquid penetrant } \\
\text { inspection. Detailed } \\
\text { written procedure must } \\
\text { be used which has been } \\
\text { proven by actual } \\
\text { demonstration to the } \\
\text { satisfaction of the } \\
\text { Inspector, as capable } \\
\text { of detecting and } \\
\text { locating defects } \\
\text { described in subsection } \\
\text { NB-533o. Since this is } \\
\text { a non-stamped vessel. } \\
\text { the Inspector would not } \\
\text { normally be part of the } \\
\text { acceptance process, } \\
\text { therefore an exception } \\
\text { is taken and the buyer } \\
\text { would be required to } \\
\text { find a suitable } \\
\text { substitute. }\end{array}$ & $\begin{array}{l}\text { The buyer will be } \\
\text { providing QA coverage } \\
\text { of the procurement of } \\
\text { these vessels and will } \\
\text { have inspection } \\
\text { personnel equivalent to } \\
\text { the "Inspector" as used } \\
\text { in the Code to perform } \\
\text { his function. }\end{array}$ \\
\hline & & 5300 & yes & no & & \\
\hline & & 5310 & yes & no & & \\
\hline & & 5320 & yes & no & & \\
\hline & & 5330 & yes & no & & \\
\hline & & 5340 & yes & no & & \\
\hline & & 5350 & yes & no & & \\
\hline & & 5360 & yes & no & & \\
\hline & & 5370 & yes & no & & \\
\hline & & 5380 & yes & no & & \\
\hline & & 5400 & yes & no & & \\
\hline & & 5410 & yes & no & & \\
\hline & & 5500 & Yes & no & & \\
\hline & & 5510 & yes & no & & \\
\hline & & 5520 & yes & no & & \\
\hline & & 5530 & yes & no & & \\
\hline & NB- 6000 & 6100 & yes & no & & \\
\hline & & 6110 & yes & yes & $\begin{array}{l}\text { NB-61Il requires all } \\
\text { components to be } \\
\text { pressure tested. The } \\
\text { cover cap and final } \\
\text { closure weld are not } \\
\text { pressure tested. } \\
\text { "Bolts, studs, nuts, } \\
\text { washers, and gaskets } \\
\text { are exempt from the } \\
\text { pressure test". This } \\
\text { allows the use of } \\
\text { elastomeric seals for } \\
\text { the performance of the } \\
\text { pressure test. Since }\end{array}$ & $\begin{array}{l}\text { Final assembly is in } \\
\text { the field with spent } \\
\text { fuel inside the } \\
\text { package. pressure } \\
\text { testing is difficult to } \\
\text { perform due to the } \\
\text { radiation field. It is } \\
\text { not in the best } \\
\text { interest of the health } \\
\text { and welfare of the } \\
\text { workers, or the public, } \\
\text { to pressure test } \\
\text { loaded canisters. }\end{array}$ \\
\hline
\end{tabular}


HNF-SD-SNF-DR-003,

Rev. 0, Appendix 18

\begin{tabular}{|c|c|c|c|c|c|c|}
\hline $\begin{array}{l}\text { Section } \\
\text { Subsection }\end{array}$ & Article & Paragraph & $\begin{array}{l}\text { Section } \\
\text { uged as } \\
\text { guidance } \\
\text { (yes / no) }\end{array}$ & $\begin{array}{c}\text { Bxception } \\
\text { Taken } \\
\text { (yos / no) }\end{array}$ & Exception & Justification \\
\hline & & & & & $\begin{array}{l}\text { Ehis is not a stamped } \\
\text { vessel, the pressure } \\
\text { test need not be } \\
\text { witnessed by a } \\
\text { certified Inspector. } \\
\text { The test need not be } \\
\text { done by a Certificate } \\
\text { holder since there is } \\
\text { no requirement for a } \\
\text { Certificate. }\end{array}$ & \\
\hline & & 6120 & yes & no & & \\
\hline & & 6200 & yes & no & & \\
\hline & & 6210 & yes & no & & \\
\hline & • & 6220 & yes & yes & $\begin{array}{l}\text { NB-6221 (a) states: } \\
\text { "The installed system } \\
\text { shall be } \\
\text { hydrostatically tested } \\
\text { at not less than } 1.25 \\
\text { times the lowest Design } \\
\text { Pressure of any } \\
\text { component within the } \\
\text { boundary protected by } \\
\text { the overpressure } \\
\text { protection devices } \\
\text { which satisfy the } \\
\text { requirements of NB- } \\
\text { 70oo." The Mco } \\
\text { assembly will be } \\
\text { hydrostatically tested } \\
\text { in the shop prior to } \\
\text { fuel loading, but not } \\
\text { after loading. The } \\
\text { field closure weld and } \\
\text { the cover cap will not } \\
\text { be hydrostatically } \\
\text { tested at any time. No } \\
\text { pneumatic testing will } \\
\text { be performed either, } \\
\text { since the vessel will } \\
\text { be loaded with fuel } \\
\text { whenever it is fully } \\
\text { assembled. }\end{array}$ & $\begin{array}{l}\text { The project position } \\
\text { resulting in a final } \\
\text { closure weld which } \\
\text { cannot be } \\
\text { hydrostatically tested } \\
\text { was established by } \\
\text { DESH. The inability to } \\
\text { perform a hydrostatic } \\
\text { test on the field } \\
\text { closure weld result in } \\
\text { a minimal risk, because } \\
\text { the calculated pressure } \\
\text { stresses in the weld } \\
\text { are relatively small. }\end{array}$ \\
\hline & & 6300 & Yes & Yes & $\begin{array}{l}\text { Pneumatic testing is } \\
\text { not permitted since } \\
\text { N8-6112.1 permits } \\
\text { pneumatic testing only } \\
\text { when the vessel cannot } \\
\text { be filled with water. } \\
\text { For the shop test, the } \\
\text { vessel can be filled } \\
\text { with water. For safety } \\
\text { reasons the field } \\
\text { closure can not be } \\
\text { tested either } \\
\text { gneumatically or } \\
\text { hydrostatically. }\end{array}$ & See above. \\
\hline & & 6310 & yes & no & & \\
\hline & & 6320 & yes & no & & \\
\hline & & 6400 & yes & no & & \\
\hline & & 6500 & yes & no & & \\
\hline & & 6610 & yes & no & & \\
\hline & & 6620 & yes & no & & \\
\hline
\end{tabular}


HNF-SD-SNF-DR-003,

Rev. 0, Appendix 18

\begin{tabular}{|c|c|c|c|c|c|c|}
\hline $\begin{array}{l}\text { Section / } \\
\text { Subsection }\end{array}$ & Articlo & Paragraph & $\begin{array}{l}\text { Section } \\
\text { used as } \\
\text { guidance } \\
\text { (yes / no) }\end{array}$ & $\begin{array}{c}\text { Exception } \\
\text { Taken } \\
\text { (yes / no) }\end{array}$ & Exception & Justification \\
\hline & NB- 7000 & 7100 & Yes & no & & \\
\hline & & 7110 & yes & yes & $\begin{array}{l}\text { Exact compliance with } \\
\text { the general } \\
\text { requirements can not be } \\
\text { made since no } \\
\text { overpressure Protection } \\
\text { Report is provided by } \\
\text { the buyer and this is } \\
\text { not a ASME code stamped } \\
\text { vessel. }\end{array}$ & $\begin{array}{l}\text { The general incend is } \\
\text { met by providing } \\
\text { overpressure protection } \\
\text { capabilities to the } \\
\text { extent specified in the } \\
\text { Performance } \\
\text { Specification. }\end{array}$ \\
\hline & & 7120 & yes & no & $\begin{array}{l}\text { overpressure protection } \\
\text { is required by the use } \\
\text { of pressure relief } \\
\text { devices and associated } \\
\text { pressure sensing } \\
\text { devices or a design } \\
\text { that does not exceed } \\
\text { the service Limits } \\
\text { specified in the Design } \\
\text { specifications for non } \\
\text { reactor components. In } \\
\text { accordance with the } \\
\text { gerformance } \\
\text { Specification, there } \\
\text { is a possibility that } \\
\text { the pressure may } \\
\text { exceed the service } \\
\text { Limits, thus requiring } \\
\text { overpressure } \\
\text { protection. The } \\
\text { overpressure protection } \\
\text { is not combined with } \\
\text { any associated pressure } \\
\text { sensing device. }\end{array}$ & $\begin{array}{l}\text { The storage system is } \\
\text { passive and there is a } \\
\text { low probability that } \\
\text { any overpressure } \\
\text { situation could occur. } \\
\text { The release from the } \\
\text { overpressure situation } \\
\text { would be inside } \\
\text { additional confinement } \\
\text { such as the cSB tube or } \\
\text { process area, therefore } \\
\text { there is little need of } \\
\text { sensing the pressure } \\
\text { within the MCo. }\end{array}$ \\
\hline & & 7130 & yes & yes & $\begin{array}{l}\text { Although the only } \\
\text { relief valve to be } \\
\text { provided in the system } \\
\text { is to be specified by } \\
\text { the Buyer and is a non- } \\
\text { Code component, an } \\
\text { exception is taken to } \\
\text { NB-7131, which requires } \\
\text { verification of the } \\
\text { operation of reclosing } \\
\text { pressure relief } \\
\text { devices. } \\
\text { No verification system } \\
\text { or indicator is build } \\
\text { into the system for } \\
\text { monitoring. }\end{array}$ & $\begin{array}{l}\text { The relief valve is a } \\
\text { non-Code item, per the } \\
\text { performance } \\
\text { Specification, anc } \\
\text { therefore does not have } \\
\text { to meet the code } \\
\text { requirements. The type } \\
\text { of system, passive } \\
\text { storage for the most } \\
\text { part, should not } \\
\text { generate any } \\
\text { significant events that } \\
\text { will cause the rupture } \\
\text { disks to burst. }\end{array}$ \\
\hline & & 7140 & yes & yes & $\begin{array}{l}\text { N8-7141 (b) states: } \\
\text { "The connection between } \\
\text { a system and its } \\
\text { pressure relief device } \\
\text { shall have a minimum } \\
\text { inside diameter equal } \\
\text { to or greater than the } \\
\text { nominal inside diameter } \\
\text { of the pressure relief } \\
\text { device inlet. The } \\
\text { opening in the } \\
\text { connection shall be } \\
\text { designed to provide }\end{array}$ & $\begin{array}{l}\text { Sizing of the } \\
\text { penetrations and relief } \\
\text { device connections, as } \\
\text { well as the discharge } \\
\text { restrictions, are } \\
\text { dictated by the } \\
\text { performance } \\
\text { Specification provided } \\
\text { by the Buyer. } \\
\text { Accumulation } \\
\text { information was not } \\
\text { provided, therefore } \\
\text { sizing to meet }\end{array}$ \\
\hline
\end{tabular}


HNF-SD-SNF-DR-003,

Rev. 0, Appendix 18

\begin{tabular}{|c|c|c|c|c|c|c|}
\hline $\begin{array}{l}\text { Section / } \\
\text { subsection }\end{array}$ & Article & Paragraph & $\begin{array}{l}\text { Section } \\
\text { used as } \\
\text { guidance } \\
\text { (yes / no) }\end{array}$ & $\begin{array}{c}\text { Exception } \\
\text { Taken } \\
(y \in s / \text { no) }\end{array}$ & Exception & Justification \\
\hline & & & & & $\begin{array}{l}\text { direct and unobstructed } \\
\text { flow between the system } \\
\text { and the pressure relief } \\
\text { device." } \\
\text { NB-7241 (c) states: } \\
\text { "The connection between } \\
\text { a system and its safety } \\
\text { valve shall be not } \\
\text { longer than the face- } \\
\text { to-face dimension of } \\
\text { the corresponding tee } \\
\text { fitting of the same } \\
\text { dimension and pressure } \\
\text { rating listed in ANsI } \\
\text { Bl6.11. Alternatively, } \\
\text { the connection shall } \\
\text { not result in } \\
\text { accumulative line } \\
\text { losses greater than } 28 \\
\text { of the relieving } \\
\text { pressure." } \\
\text { NB-714l (d) limits } \\
\text { total pressure loss to } \\
36 \text { of the relieving } \\
\text { pressure, regardless of } \\
\text { geometry. }\end{array}$ & $\begin{array}{l}\text { accumulation pxessure } \\
\text { requirements is not } \\
\text { part of this design. }\end{array}$ \\
\hline & & 7150 & yes & no & & \\
\hline & & 7160 & yes & no & & \\
\hline & & 7170 & Yes & no & & \\
\hline & & 7200 & yes & yes & $\begin{array}{l}\text { No Overpressure } \\
\text { Protection Report was } \\
\text { provided by } \\
\text { owner/buyer. Exception } \\
\text { to all references and } \\
\text { use of information } \\
\text { normally provided in } \\
\text { the report is taken. }\end{array}$ & $\begin{array}{l}\text { No Overpressure } \\
\text { Protection Report was } \\
\text { provided by DESH. }\end{array}$ \\
\hline & & 7210 & yes & no & & \\
\hline & & 7220 & yes & no & & \\
\hline & & 7230 & yes & no & & \\
\hline & & 7240 & yeg & no & & \\
\hline & & 7250 & yes & no & & \\
\hline & & 7300 & yes & no & & \\
\hline & & 7310 & yes & no & & \\
\hline & & 7320 & yes & no & & \\
\hline & & 7400 & yes & no & & \\
\hline & & 7410 & yes & no & & \\
\hline & & 7420 & yes & no & & \\
\hline & & 7500 & yes & no & $\begin{array}{l}\text { Relief valve to be used } \\
\text { in the system is non- } \\
\text { Code and is being } \\
\text { specified by the Buyer, } \\
\text { therefore this section } \\
\text { is not evaluated. }\end{array}$ & $\begin{array}{l}\text { NB-7500 is not applied } \\
\text { to the Buyer supplied } \\
\text { relief valve, since the } \\
\text { Performance } \\
\text { Specification makes the } \\
\text { relief valve a non-Code } \\
\text { item. }\end{array}$ \\
\hline & & 7510 & yes & no & & \\
\hline & & 7520 & yes & no & & \\
\hline & & 7530 & yes & no & & \\
\hline & & 7540 & yes & no & & \\
\hline & & 7600 & yes & no & & \\
\hline & & 7610 & yes & yes & NB-7610 (a) states: & The use of rupture \\
\hline
\end{tabular}


HNF-SD-SNF-DR-003,

Rev. 0, Appendix 18

\begin{tabular}{|c|c|c|c|c|c|c|}
\hline $\begin{array}{l}\text { Section / } \\
\text { Subsection }\end{array}$ & Article & Paragraph & $\begin{array}{l}\text { Section } \\
\text { used as } \\
\text { gutdance } \\
\text { (yes / 2o) }\end{array}$ & $\begin{array}{c}\text { Exception } \\
\text { Taken } \\
\text { (yes / no) }\end{array}$ & Bxception & Justification \\
\hline & & & & & $\begin{array}{l}\text { "rupture disk devices } \\
\text { shall not be used as } \\
\text { the sole pressure } \\
\text { relief devices: t An } \\
\text { exception is taken } \\
\text { since the current } \\
\text { specification states } \\
\text { that the other relief } \\
\text { devices are non-ASME } \\
\text { Code devices and hence } \\
\text { credit for their } \\
\text { performance can nor be } \\
\text { evaluated for in the } \\
\text { Code. }\end{array}$ & $\begin{array}{l}\text { disks as the sole } \\
\text { pressure relief devices } \\
\text { was specified by DESH. }\end{array}$ \\
\hline & & 7620 & yes & no & $\dot{5}$ & \\
\hline & & 7700 & yes & no & & \\
\hline & & 7710 & yes & no & & \\
\hline & & 7720 & yes & no & & \\
\hline & & 7730 & yes & no & & \\
\hline & & 7740 & yes & no & & \\
\hline & & 7800 & yes & no & & \\
\hline & & 7810 & yes & no & & \\
\hline & & 7820 & yes & yes & $\begin{array}{l}\text { NB-7822 requires the } \\
\text { rupture disk holders to } \\
\text { be marked in a certain } \\
\text { way. Due to the nature } \\
\text { of special holders } \\
\text { required for the MCo, } \\
\text { the full extent of the } \\
\text { marking cannot be made. }\end{array}$ & $\begin{array}{l}\text { The fabrication } \\
\text { specification requires } \\
\text { that components be } \\
\text { uniquely marked which } \\
\text { will allow for the } \\
\text { required tractability. }\end{array}$ \\
\hline & & 7830 & yes & no & & \\
\hline Section III, NC & & & no & & & \\
\hline Section III, ND & & & no & & & \\
\hline Section III, NE & & & no & & & \\
\hline Section III, NF & $\mathrm{NF}-1000$ & & no & & & \\
\hline & $\mathrm{NF}-2000$ & & no & & & \\
\hline & NF -3000 & $\begin{array}{c}\text { all } \\
\text { except } \\
\mathrm{NE}-3320\end{array}$ & no & & & \\
\hline & & 3320 & yes & no & $\begin{array}{l}\text { Note: applies for } \\
\text { buckling of support } \\
\text { rods and baskets only. }\end{array}$ & \\
\hline & $\mathrm{NE}-4000$ & & no & & & \\
\hline & $\mathrm{NF}-5000$ & & no & & & \\
\hline Section III, NG & NG-1000 & & no & & & \\
\hline & NG-2000 & & yes & yes & $\begin{array}{l}\text { Spring pins are to ASME } \\
\text { specifications, but not } \\
\text { to section III, } \\
\text { Subsection NG-2130. }\end{array}$ & $\begin{array}{l}\text { The spring pin } \\
\text { specification } \\
\text { (ASME B18.8.2-1994) was } \\
\text { "developed under } \\
\text { procedures accredited } \\
\text { as meeting the criteria } \\
\text { for American National } \\
\text { Standards", and is } \\
\text { "widely used in general } \\
\text { industrial } \\
\text { applications." } \\
\text { For the fuel basket } \\
\text { applications, the pin } \\
\text { loads are small } \\
\text { compared to the ASME } \\
\text { allowables, which } \\
\text { provides additional }\end{array}$ \\
\hline
\end{tabular}


HNF-SD-SNF-DR-003,

Rev. 0, Appendix 18

\begin{tabular}{|c|c|c|c|c|c|c|}
\hline $\begin{array}{l}\text { Section } / \\
\text { Subsection }\end{array}$ & Axticle & Paragraph & $\begin{array}{l}\text { Section } \\
\text { used as } \\
\text { guldance } \\
\text { (yeg / po) }\end{array}$ & $\begin{array}{c}\text { Bxception } \\
\text { Taken } \\
\text { (yes / no) }\end{array}$ & Exception & Justification \\
\hline & & & & & & $\begin{array}{l}\text { assurance that gpring } \\
\text { pin failures wili not } \\
\text { occur. } \\
\text { The specification for } \\
\text { the pin requires } \\
\text { material type and shear } \\
\text { strength to be } \\
\text { certified which are } \\
\text { the important } \\
\text { parameters. There is no } \\
\text { welding of the pins } \\
\text { which would require a } \\
\text { certified material test } \\
\text { report. }\end{array}$ \\
\hline & $\mathrm{NG}-3000$ & & yes & yes & $\begin{array}{l}\text { NG-3133.6 requirements } \\
\text { are taken exception to, } \\
\text { since both the center } \\
\text { tube and the basket } \\
\text { support rods behave as } \\
\text { colums and are } \\
\text { evaluated using the } \\
\text { requirements of } \\
\text { subsection NF. }\end{array}$ & $\begin{array}{l}\text { For the center pipe, } \\
\text { both Subsections NG and } \\
\text { NF were considered and } \\
\text { met. For the support } \\
\text { rods, subsection NF was } \\
\text { required since } \\
\text { subsection NG does not } \\
\text { address column } \\
\text { buckling. }\end{array}$ \\
\hline & NG -4000 & & yes & yes & $\begin{array}{l}\text { Non-stamped component. } \\
\text { Therefore, exception to } \\
\text { the requirement that } \\
\text { fabrication be by a } \\
\text { certificate holder is } \\
\text { taken (NG }-4120 \text { ). }\end{array}$ & $\begin{array}{l}\text { DESh specified that the } \\
\text { baskets were not to be } \\
\text { Code stamped. }\end{array}$ \\
\hline & NG-5000 & & yes & no & & \\
\hline $\begin{array}{l}\text { Mandatory } \\
\text { Appendices }\end{array}$ & $\begin{array}{l}\text { Appendix } \\
\text { I }\end{array}$ & & yes & & & \\
\hline & $\begin{array}{l}\text { Appendix } \\
\text { II }\end{array}$ & & no & no & & \\
\hline & $\begin{array}{l}\text { Appendix } \\
\text { III }\end{array}$ & & yes & no & & \\
\hline & $\begin{array}{l}\text { Appendix } \\
\text { IV }\end{array}$ & & no & no & & \\
\hline & $\begin{array}{l}\text { Appendix } \\
\mathrm{V}\end{array}$ & & no & no & $\begin{array}{l}\text { Reports not completed } \\
\text { and certified. }\end{array}$ & $\begin{array}{l}\text { DESH specified that the } \\
\text { components are non- } \\
\text { stamped, therefore } \\
\text { official documentation } \\
\text { sheets are not } \\
\text { generated. } \\
\text { Documentation of design } \\
\text { and fabrication data is } \\
\text { generated by other } \\
\text { means. }\end{array}$ \\
\hline & $\begin{array}{l}\text { Appendix } \\
\text { VI }\end{array}$ & & yes & no & & \\
\hline & $\begin{array}{l}\text { Appendix } \\
\text { VII }\end{array}$ & & no & no & & \\
\hline & $\begin{array}{l}\text { Appendix } \\
\text { XI }\end{array}$ & & no & no & & \\
\hline & $\begin{array}{l}\text { Appendix } \\
\text { XII }\end{array}$ & & yes & no & & \\
\hline & $\begin{array}{l}\text { Appendix } \\
\text { XIII }\end{array}$ & & no & no & & \\
\hline & $\begin{array}{l}\text { Appendix } \\
\text { XIV }\end{array}$ & & no & no & & \\
\hline & $\begin{array}{l}\text { Appendix } \\
\text { XVIII }\end{array}$ & & yes & 0 & & \\
\hline
\end{tabular}


HNF-SD-SNF-DR-003,

Rev. 0, Appendix 18

\begin{tabular}{|c|c|c|c|c|c|c|}
\hline $\begin{array}{l}\text { Section } / \\
\text { Subsection }\end{array}$ & Article & Paragraph & $\begin{array}{l}\text { Section } \\
\text { uged as } \\
\text { guldance } \\
\text { (yes / no) }\end{array}$ & $\begin{array}{c}\text { Exception } \\
\text { Taken } \\
\text { (yea / no }\end{array}$ & Exception & Justiflcation \\
\hline & $\begin{array}{l}\text { Appendix } \\
\text { XIX }\end{array}$ & & no & no & & \\
\hline & $\begin{array}{c}\text { Appendix } \\
\mathrm{XX}\end{array}$ & & no & no & & \\
\hline & $\begin{array}{l}\text { Appendix } \\
\text { XXI }\end{array}$ & & no & no & & \\
\hline & $\begin{array}{c}\text { Appendix } \\
\text { XXII }\end{array}$ & & no & no & & \\
\hline & $\begin{array}{c}\text { Appendix } \\
\text { XXIII }\end{array}$ & & yes & yes & $\begin{array}{l}\text { Since this is a non- } \\
\text { stamped vessel produced } \\
\text { by non-certificate } \\
\text { holders, the formal } \\
\text { qualification of } \\
\text { personnel and assigned } \\
\text { duties does not exist. } \\
\text { This includes all } \\
\text { aspects from owner's } \\
\text { design specification to } \\
\text { the certified data } \\
\text { reports. }\end{array}$ & $\begin{array}{l}\text { Alternate QA } \\
\text { organization and } \\
\text { personnel qualification } \\
\text { system is in place that } \\
\text { is acceptable to the } \\
\text { buyer. }\end{array}$ \\
\hline
\end{tabular}


HNF-SD-SNF-DR-003,

Rev. 0, Appendix 18

\subsection{Conclusion}

The ASME Code is used as a guideline for the construction of the MCO. The intent of the Code is to provide the design and construction requirements. The MCO does not require a Code stamp. 


\title{
MULTI-CANISTER OVERPACK
}

FABRICATION SPECIFICATION

\author{
Contract No. KH-8009
}

May 1997

Revision 1

\section{ORIGINAL}

Parsons Approved:

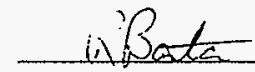

Date: $5 / 13 / 47$

\begin{tabular}{|c|c|c|c|c|c|}
\hline $\begin{array}{c}\text { DOCUMENT } \\
\text { REVISION }\end{array}$ & $\begin{array}{c}\text { AFFECTED } \\
\text { PAGES }\end{array}$ & $\begin{array}{c}\text { REVISION } \\
\text { DESCRIPTION }\end{array}$ & $\begin{array}{c}\text { DATE OF } \\
\text { REVISION }\end{array}$ & $\begin{array}{c}\text { REVISION } \\
\text { CHECKED BY }\end{array}$ & $\begin{array}{c}\text { REVISION } \\
\text { APPROVED BY }\end{array}$ \\
\hline & & & & & \\
\hline
\end{tabular}

Prepared for: Duke Engineering \& Services Hanford, Inc. Prepared by: Parsons Infrastructure and Technology Group, Inc. 


\section{TABLE OF CONTENTS}

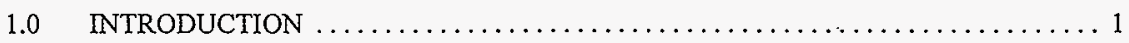

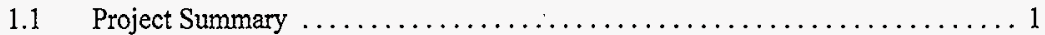

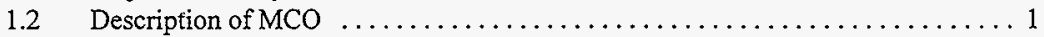

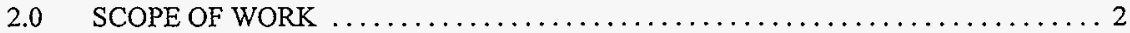

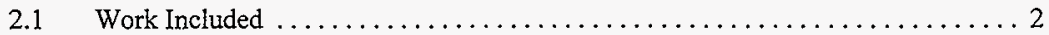

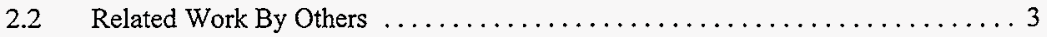

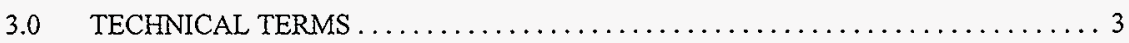

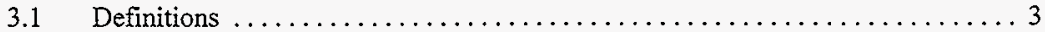

3.2 Abbreviations ..................................... 4

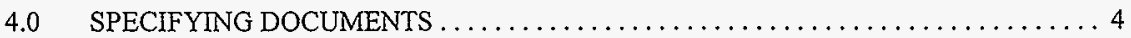

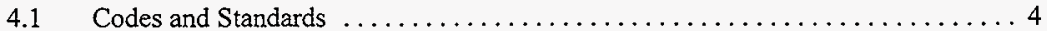

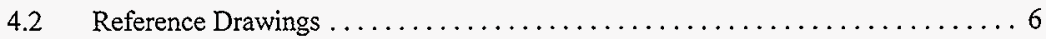

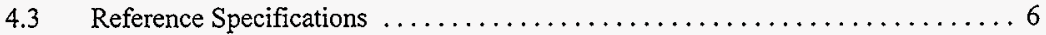

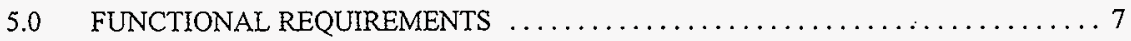

5.1 Dimensional Interfaces and Limitations $\ldots \ldots \ldots \ldots \ldots \ldots \ldots \ldots \ldots \ldots \ldots \ldots \ldots \ldots \ldots$

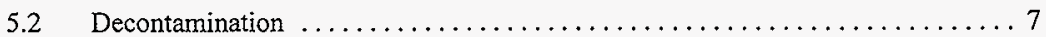

6.0 MANUFACTURING REQUIREMENTS $\ldots \ldots \ldots \ldots \ldots \ldots \ldots \ldots \ldots \ldots \ldots \ldots$

6.1 General Requirements $\ldots \ldots \ldots \ldots \ldots \ldots \ldots \ldots \ldots \ldots \ldots \ldots \ldots \ldots$

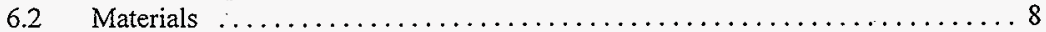

6.2.1 ASME Code Materials . . . . . . . . . . . . . . . . . . . . . 9

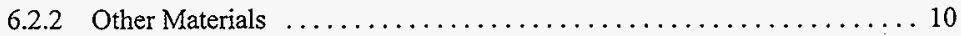

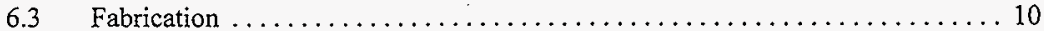

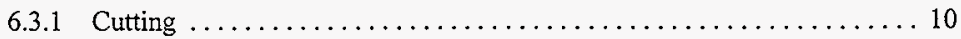

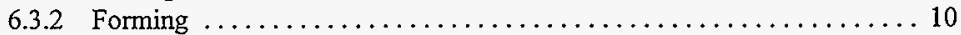

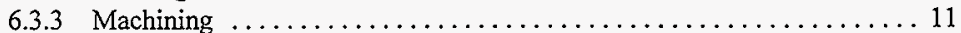

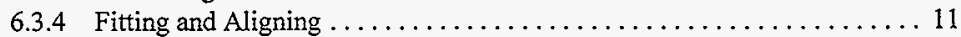

6.3 .5 Piece Part Tolerance ................................ 11

6.3.6 Dimensional Verification $\ldots \ldots \ldots \ldots \ldots \ldots \ldots \ldots \ldots \ldots \ldots \ldots \ldots$

Table 6.3.6-1

MCO SHELL CRITICAL DIMENSIONS $\ldots \ldots \ldots \ldots \ldots \ldots \ldots \ldots \ldots \ldots \ldots \ldots$

Table 6.3.6-2

PROCESS TUBE CRITICAL DIMENSIONS $\ldots \ldots \ldots \ldots \ldots \ldots \ldots \ldots \ldots \ldots \ldots \ldots \ldots$

Table 6.3.6-3

SHIELD PLUG CRITICAL DIMENSIONS $\ldots \ldots \ldots \ldots \ldots \ldots \ldots \ldots \ldots \ldots \ldots \ldots \ldots$ 
Table 6.3.6-4

LOCKING \& LIFTING RING CRITICAL DIMENSIONS

Table 6.3.6-5

CANISTER COVER CRITICAL DIMENSIONS $\ldots \ldots \ldots \ldots \ldots \ldots \ldots \ldots \ldots \ldots$

Table 6.3.6-6

MK IA STORAGE BASKET CRITICAL DIMENSIONS $\ldots \ldots \ldots \ldots \ldots \ldots \ldots \ldots$

Table 6.3.6-7

MK IA SCRAP BASKET CRITICAL DIMENSIONS $\ldots \ldots \ldots \ldots \ldots \ldots \ldots \ldots \ldots$

Table 6.3.6-8

MK IV STORAGE BASKET CRITICAL DIMENSIONS $\ldots \ldots \ldots \ldots \ldots \ldots \ldots \ldots$

Table 6.3.6-9

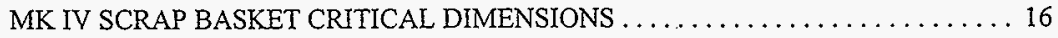

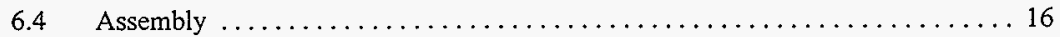

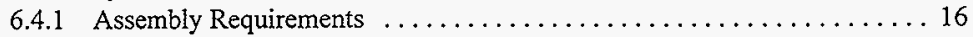

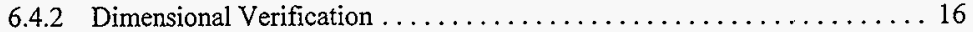

6.4.3 Marking (Labeling) of Completed Assemblies .............. 16

6.4.3.1 Shield Plug and Canister Cover ................... 17

6.4.3.2 Canister Shell and Locking Ring .................. 17

6.4 .3 .3 Storage and Scrap Baskets .................... 17

Table 6.4.3-1

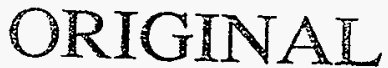

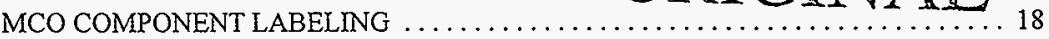

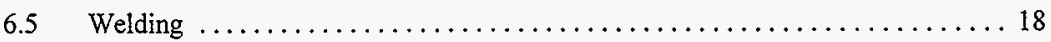

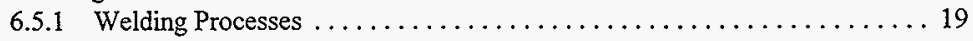

6.5.2 Welding Procedures and Qualifications . . . . . . . . . . . . . . 19

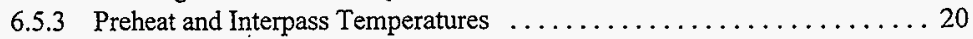

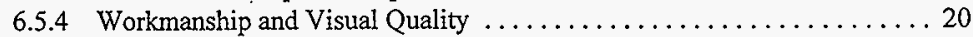

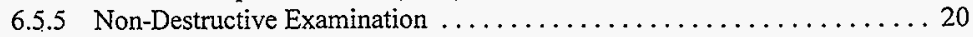

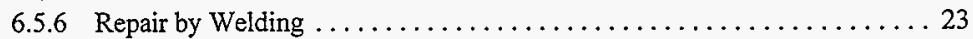

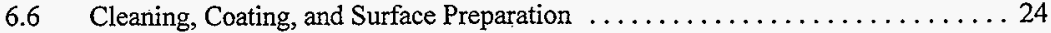

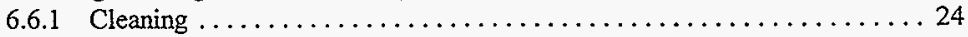

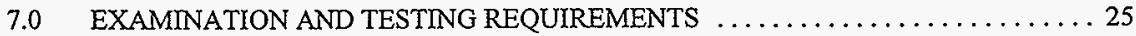

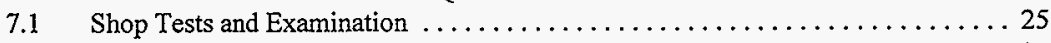

7.1.1 Ultrasonic Inspection of Plate Used For MCO Shell $\ldots \ldots \ldots \ldots \ldots \ldots 25$

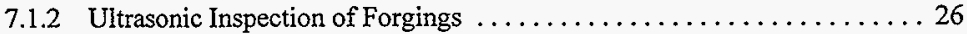

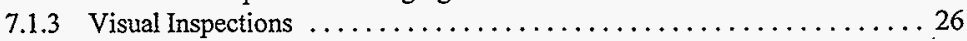




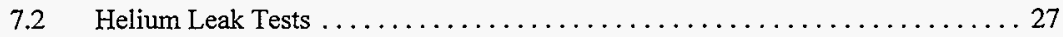

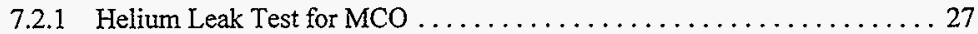

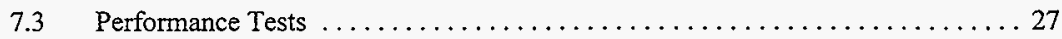

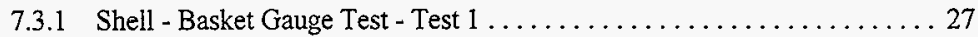

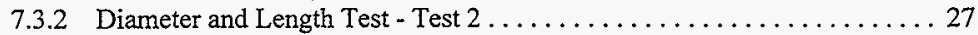

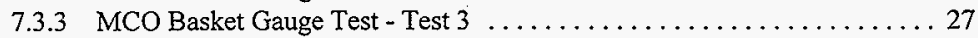

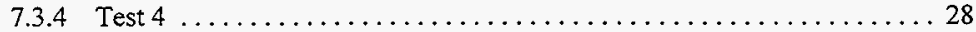

7.3.4.1 Shield Plug and Process Tube Insertion Test ............ 28

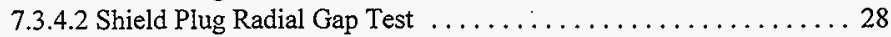

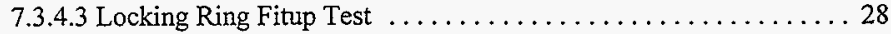

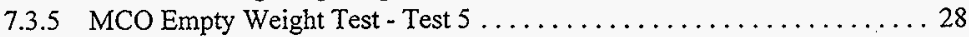

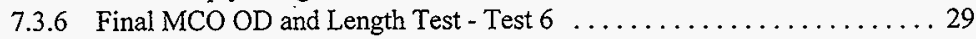

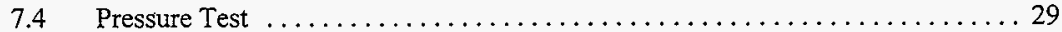

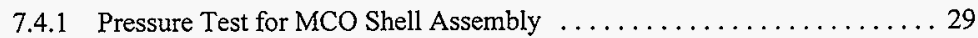

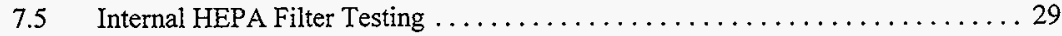

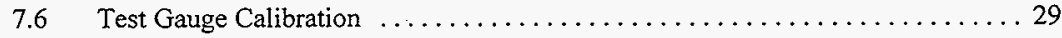

Table 7-1

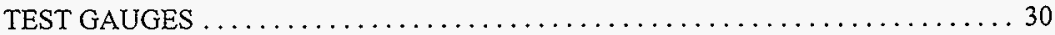

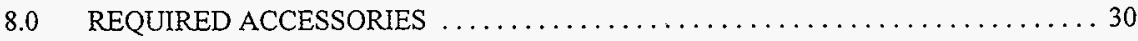

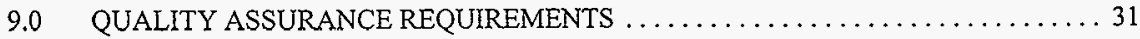

9.1 Procurement of Materials . . . . . . . . . . . . . . . . . . . . . . . 31

9.1.1 Procurement of ASME Code Category "SC" Materials $\ldots \ldots \ldots \ldots \ldots 31$

9.1.2 Procurement of ASME Code Category "SS" Materials . . . . . . . . . . 32

9.1.3 Procurement of Category "GS" Materials . . . . . . . . . . . . . 32

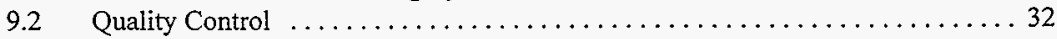

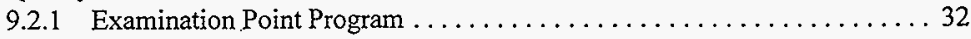

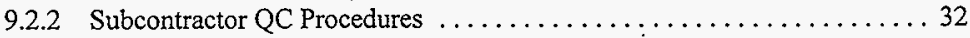

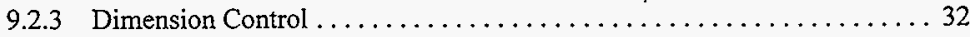

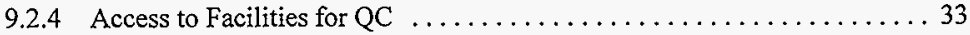

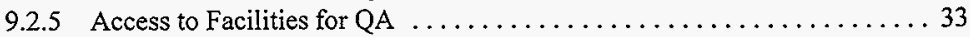

9.2.6 Nonconformance .................................. 33

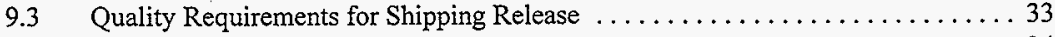

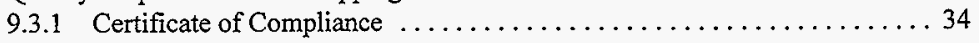

Table 9-1

MCO MATERIAL QUALITY CATEGORY DESIGNATION ............... 34

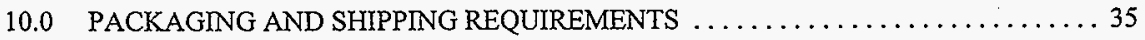

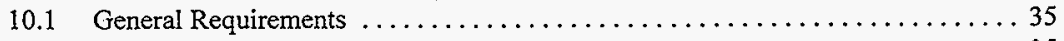

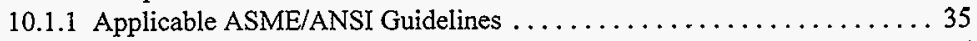

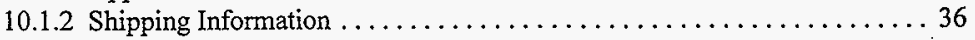




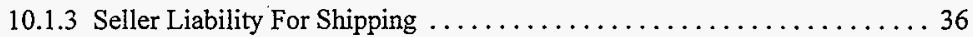

10.2 Detailed Requirements . . . . . . . . . . . . . . . . . . . . . . . 36

10.2.1 MCO, Locking Ring, and Shield Plug Preparation for Shipping $\ldots \ldots 36$

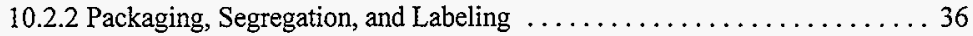

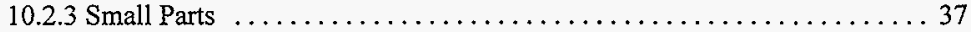

10.2.4 Packaging and Shipping Procedures $\ldots \ldots \ldots \ldots \ldots \ldots \ldots \ldots \ldots \ldots \ldots \ldots \ldots \ldots \ldots$

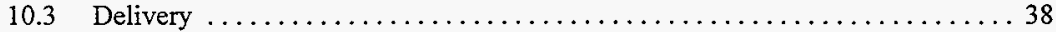

11.0 DOCUMENT AND RECORD SUBMITTAL REQUIREMENTS $\ldots \ldots \ldots \ldots \ldots \ldots 38$

11.1 Documents .......................................... 38

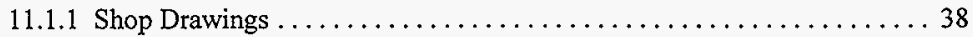

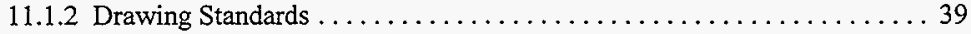

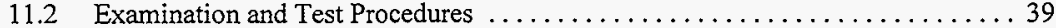

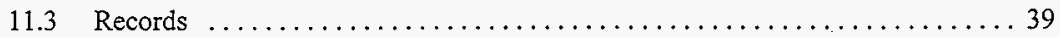

11.3.1 General Requirements for Document Packages ............... 39

Table 11-1

AS-BUILT DIMENSIONS REPORTS $\ldots \ldots \ldots \ldots \ldots \ldots \ldots \ldots \ldots \ldots \ldots \ldots$

Table $11-2$

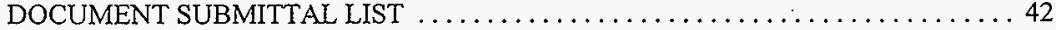

Table 11-3

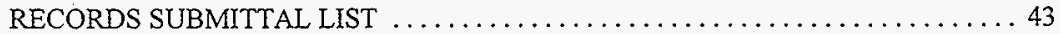

\section{APPENDICES}

Appendix 1: Intentionally left Blank. .........................

Appendix 2: MCO Internal HEPA Filter Specification (DESH) $\ldots \ldots \ldots \ldots \ldots \ldots$ A-2

Appendix 3: Potential Rupture Disk Suppliers ...................... A-3

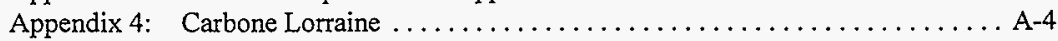

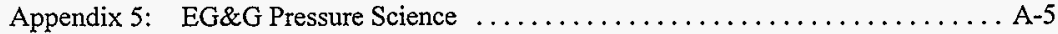

Appendix 6: Johnson Well Wire (Wheelabrator Engineered Systems, Inc.) . . . . . A A-6

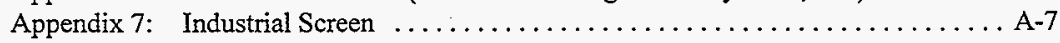

Appendix 8: MCO Critical Dimension Sketches $\ldots \ldots \ldots \ldots \ldots \ldots \ldots \ldots \ldots$ A-8 


\subsection{INTRODUCTION}

This Specification provides requirements for materials, marking fabrication, welding, examination, shop testing, quality assurance, documentation, packaging, and shipping for the Multi-Canister Overpack (MCO). The MCO consists of the shell assembly, shield plug, locking ring, canister cover and fuel baskets consisting of Mark IA storage, Mark 1A scrap, Mark IV storage and Mark IV scrap. The MCO shell shall be fabricated in accordance with the requirements of the ASME Boiler and Pressure Vessel Code Section III, Division 1, Subsection NB (Class 1 Components) unless noted otherwise by this Specification. The baskets shall be fabricated in accordance with the requirements of the ASME Boiler and Pressure Vessel Code Section III, Subsection NG, unless otherwise noted by this Specification. An ASME certification or N-stamp is not required.

\subsection{Project Summary}

The MCO provides a containment system for the vertical storage of Single Pass Reactor and NReactor Spent Nuclear Fuel (SNF) currently stored in the K Basins located at the Hanford Site. The MCOs will be placed in vertical storage tubes in the Canister Storage Building (CSB) located in the Hanford 200 Area. Individual MCOs and their associated transport equipment are designed for a range of capacities, fuel types, and fuel conditions associated with the subject fuel currently stored in the $\mathrm{K}$ East and $\mathrm{K}$ West Basins. The $\mathrm{MCO}$ is designed to store spent fuel assemblies or lesser quantities of damaged fuel, and fuel fragments.

\subsection{Description of MCO}

The major components of the MCO are as follows:
A. MCO Shell
B. Bottom Assembly
C. Canister Collar
D. Shield Plug Assembly
E. Locking Ring
F. Canister Cover
a. Mark IA regular basket
b. Mark IA scrap basket
c. Mark IV regular basket
d. Mark IV scrap basket

G. There are four types of fuel baskets used within the MCO:

The cylindrical shell, the bottom assembly, the canister collar, the shield plug, and the locking and lifting ring form the primary pressure retaining containment boundary for the spent nuclear fuel.

The MCO is equipped with a top end shield plug so that the radiation dose is minimized for drying, conditioning, sealing, and handling operations. The shield plug will be a single stainless steel forging. Major welded joints will attach the bottom assembly and canister collar to the MCO shell. 
The shield plug will be sealed with a mechanical seal preloaded with the locking ring, and Jacking boits/screws. The bottom assembly containment boundary welds are made during fabrication of the $\mathrm{MCO}$.

The following items are critical to the $\mathrm{MCO}$ and must be carefully controlled and verified during fabrication, shop testing, packaging and shipment:

A. The tolerances on the outer dimensions of the $\mathrm{MCO}$ must be maintained to ensure that the $\mathrm{MCO}$ can be inserted in the transport cask.

B. The fit up of the MCO top shield plug and locking ring require dimensional controls to allow these components to be placed remotely.

C. This Specification requires a full length gauge verification test for the as-fabricated basket assemblies with respect to the $\mathrm{MCO}$ shell. This requires that the dimensions of the MCO shell relative to the basket assemblies envelope be closely controlled during fabrication of the MCO basket assemblies and shell.

D. Basket tolerances must be such that alignment when assembled is easily repeated.

E. Basket tolerances must be such that criticality control is also met.

These requirements are delineated by this Specification and the associated Design Drawings.

\subsection{SCOPE OF WORK}

This Specification sets forth the requirements for the materials, fabrication, welding, examination, shop testing, quality assurance, documentation, packaging, and shipping of one or more MCOs specified in the purchase order. The MCOs shall be shipped to the location specified in the purchase order.

\section{$2.1 \quad$ Work Included}

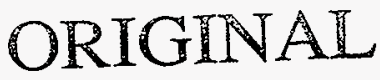

The Work set forth by this Specification shall include the following:

A. The preparation and submittal of a MCO manufacturing plan, schedule, and shop detail and assembly drawings to the Buyer for approval in accordance with the requirements of Section 11 of this Specification.

B. The preparation and submittal of MCO fabrication travelers, material certificates, fabrication procedures, welding procedures, examination procedures and reports, dimensional certificates, and other documentation as defined in Section 11 of this Specification, to verify that the material and completed Work conforms to the requirements of this Specification. 
C. The furnishing of materials, fabrication, welding, shop testing, examination, documentation, quality assurance, packaging, and shipping of a complete $\mathrm{MCO}$ are as described by the Design Drawings, this Specification, and the Purchase Order. Included in this Work is the gauge to be used for the basket assembly acceptance tests, the mechanical closure thread gauges, the fixture for the pressure test of the shell to bottom forge assembly weld joint, and the accessories listed on the Design Drawings and in Section 8 of this Specification. The fabricator shall be responsible for fabrication design, construction, storage prior to use, and maintenance of any test equipment required by this specification.

\section{$2.2 \quad$ Related Work By Others}

The following items related to the MCO system are not included and will be furnished by Others:

A. Design of the MCO.

B. Design, fabrication, and delivery of the on-site transport cask and related equipment, including the support skid, trailer, positioning equipment, lifting yoke, remote loading and assembly equipment, and vacuum drying processing system.

\subsection{TECHNICAL TERMS}

The following definitions and abbreviations shall apply as used within this Specification.

\subsection{Definitions}

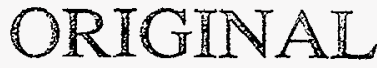

The following definitions of terms shall apply throughout this document.

A. Buyer is either DE\&S (Duke Engineering \& Services) Hanford, Inc., and/or Fluor Daniel Hanford, Inc., Richland, Washington.

B. Seller is the entity awarded the contract for furnishing the equipment specified herein.

C. Owner is the United States Department of Energy (DOE) Richland (Hanford Operations) to which the MCO is shipped.

D. The Work is any and all equipment, material, apparatus, item, process, and parts or portions thereof to be supplied by the Seller under the Contract.

E. Hold Point is a step in the fabrication/inspection process where the Seller must wait for signed approval of the Buyer to proceed. Hold Points are designated in the specification.

F. Witness Point is a step in the fabrication/inspection process where the Seller must contact the Buyer for authorization to proceed. Witness Points are designated in the specification. 
G. First Article of Production is the first component/subcomponent fabricated, i.e., the first MCO produced. The Seller shall allow the Buyer to inspect each First Article of Production. The Seller must wait for the Buyer's written approval of the First Article of Production before fabricating more components.

\subsection{Abbreviations}

The abbreviations applicable to this Specification are defined as follows:

$\begin{array}{ll}\text { AISC } & \text { American Institute of Steel Construction, Inc. } \\ \text { ANS } & \text { American Nuclear Society } \\ \text { ANSI } & \text { American National Standards Institute } \\ \text { ASME } & \text { American Society of Mechanical Engineers } \\ \text { ASME Code } & \text { ASME Boiler and Pressure Vessel Code } \\ \text { ASNT } & \text { American Society of Non-Destructive Testing } \\ \text { ASTM } & \text { American Society of Testing and Materials } \\ \text { AWS } & \text { American Welding Society } \\ \text { Cask } & \text { Hanford On-Site Transport Cask } \\ \text { CFR } & \text { Code of Federal Regulations } \\ \text { CMTR } & \text { Certified Material Test Report } \\ \text { CSB } & \text { Canister Storage Building } \\ \text { DESH } & \text { Duke Engineering \& Services Hanford } \\ \text { DOE } & \text { US Department of Energy } \\ \text { ISO } & \text { International Organization for Standardization } \\ \text { NRC } & \text { US Nuclear Regulatory Commission } \\ \text { SSPC } & \text { Steel Structures Painting Council }\end{array}$

\subsection{SPECIFYING DOCUMENTS}

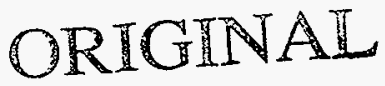

Equipment and/or services for the Work furnished in accordance with this Specification shall comply with all federal laws and with the following codes and standards to the extent referenced herein. Unless otherwise noted, the document with addenda, amendments, and revisions in effect on the date of the purchase order will apply. Later editions may be used if mutually consented to in writing between the Seller and the Buyer. Any conflicting requirements must be submitted to the Buyer in writing for resolution before proceeding with any phase of the Work.

\subsection{Codes and Standards}

The materials, fabrication, testing, examination, and shipping of the $\mathrm{MCO}$ shall be in accordance with the requirements of the following codes and standards. The Codes and Standards are applicable to the extent referenced in this fabrication specification and the associated Design Drawings.

A. ASME Code, Section II, 1995 Edition, with 1995 Addenda. 
HNF-SD-SNF-DR-003, Rev. 0

Appendix 19

B. ASME Code, Section III, Division 1, Subsection NB, 1995 Edition, with 1995 Addenda.

C. ASME Code, Section III, Division 1, Subsection NF, 19995 Edition, with 1995 Addenda.

D. ASME Code, Section III, Division 1, Subsection NG, 1995 Edition, with 1995 Addenda.

E. ASME Code, Section V, Nondestructive Examination, 1995 Edition, with 1995 Addenda.

Radiography: Subsection A, Article 2

Subsection B, Article 22

Ultrasonic: Subsection A, Article 5

Subsection B, Article 23

Liquid Penetrant: $\quad$ Subsection A, Article 6

Subsection B, Article 24

Visual: $\quad$ Subsection A, Article 9

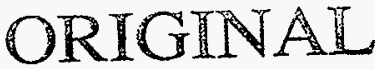

Subsection B, Article 28

F. ASME Code, Section IX, Welding and Brazing Qualifications, 1995 Edition, with 1995 Addenda, Part QW, Welding Articles 1,2,3, and 4.

G. CFR, Title 10, Part 21, "Reporting of Defects and Noncompliance."

H. $\quad$ CFR, Title 10, Part 71, Subpart H, "Quality Assurance."

I. ANSI N45.2.1, "Cleaning of Fluid Systems and Associated Components During Fabrication Phase of Nuclear Power Plants."

J. ANSI N45.2.2, "Packaging, Shipping, Receiving, Storage and Handling of Items for Nuclear Plants."

K. ANSI Y14.5M, "Dimensioning and Tolerancing:", 1994 Edition.

L. American Society of Non-Destructive Testing, Inc., SNT-TC-1A-92, December 1992 Edition, "Recommended Practice for Non-Destructive Testing Personnel Qualification and Certification."

M. "American National Standard for Radioactive Materials - Leakage Test on Packages for Shipments."

N. AWS D1.1, "Structural Welding Code: Steel." 
O. ASTM A380, "Standard Recommended Practice for Cleaning and Descaling Stainless Steel Parts, Equipment and Systems."

P. ASME SA-240, "Heat-Resisting Chromium and Chromium-Nickel Stainless Steel Plate, Sheet, and Strip for Pressure Vessels."

Q. ANSI N45.2.6, "Qualifications of Inspection, Examination, and Testing Personnel for Nuclear Power Plants." (Withdrawn Prior to 12/83. Incorporated with NQA-1-1983.)

R. AWS A2.4, "Weld Symbols."

S. USNRC Regulatory Guide 1.31, "Control of Ferrite Content in Stainless Steel Weld Metal."

T. Fastener/Thread standards as identified on drawings.

U. ASME SA-312/SA-312M, "Seamless and Welded Austenitic Stainless Steel Pipe."

V. ASME SA-182/SA-182M, "Forged or Rolled Alloy Steel Pipe Flanges, Forged Fittings, and Valves and Parts for High-Temperature Service."

W. ASME Y1.1, "Abbreviations - For Use on Drawings and in Text."

X. ANSI/ASME Y14.36, "Surface Texture Symbols."

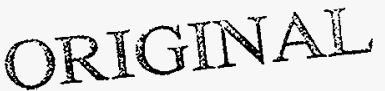

Y. ANSI/ASME B46.1, "Surface Texture (Surface Roughness, Waviness, and Lay):", 1985 Edition.

Z. Good Painting Practices, Steel Structures Painting Council, Vol. 1, 1993, SSPC-SP1, "Surface Preparation Specification No. 1 Solvent Cleaning."

AA. ANSI N14.6, "Special Lifting Devices for Shipping Containers Weighing 10,000 pounds or more", 1993 edition.

\subsection{Reference Drawings}

The Design Drawings specified in the Purchase Order are hereinafter referred to as Drawings or Design Drawings, and set forth the extent of the Work to be performed under this contract.

\subsection{Reference Specifications}

The following reference specifications shall apply to the Work to the extent referenced in this Fabrication Specification and the Design Drawings: 
A. Johnson Weld Wire, Johnsons Screens Catalog, Wheelabrator Clean Air Inc., (A Division of Wheelabrator-Frye), Minneapolis, Minnesota.

B. Continental Disc Corporation, Rupture Discs Catalog, Continental Disc Corporation, Liberty, Missouri.

C. Helicoflex seal, Helicoflex Components Division, Columbia, South Carolina.

D. EG\&G seals, EG\&G Pressure Science, Belesville, Maryland.

E. Relief valves to be specified by buyer.

F. HEPA filters per DESH specification.

G. Industrial Screen Products, Inc.

\subsection{FUNCTIONAL REQUIREMENTS}

It is critical to the safe, smooth, and proper use of the MCO that the specified dimensional tolerances for the $\mathrm{MCO}$ be maintained.

\subsection{Dimensional Interfaces and Limitations}

The critical dimensions Tables and sketches shown in Appendix 8, define the tolerances for the MCO (NOTE: If sketches, appendix 8 differ from design drawings, buyer will be notified immediately for resolution). It is important that these tolerances be met since the MCO must interface with the transport cask, the spent nuclear fuel assemblies, the $\mathrm{K}$ Basin fuel handling equipment, and the CSB. In addition, material thicknesses were established to provide adequate radiation shielding protection and structural margin for maximum design basis conditions. The material thicknesses are critical to final acceptance of the MCO. Where potential thinning of materials may occur during fabrication, the fabricator shall measure and verify thickness to assure that it is within that specified on the Design Drawings. The dimensions and tolerances on the final assembled components after welding and machining are the controlling dimensions. Refer to section 6.3.5 Piece Part Tolerance.

\subsection{Decontamination}

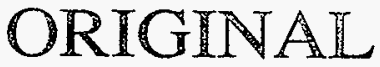

Exterior and the specific weld exterior surfaces of the MCO shall be readily decontaminable. This requires smooth surfaces on all sheet, plate, and welds, with no re-entrant corners. All exposed surfaces shall have a finish which meets or exceeds that specified on the Design Drawings. The MCO shell shall not be bead or sandblasted. 


\subsection{MANUFACTURING REQUIREMENTS}

\subsection{General Requirements}

The MCO materials, fabrication, welding, and examination shall comply with the requirements of the ASME Boiler and Pressure Vessel Code Section III, Division 1, Subsection NB for the MCO shell, bottom plate, shield plug, locking ring, and rupture disk. The materials and welds for the MKIA steel baskets are required to meet Subsection NG. The materials, fabrication, and examination for items with non-code requirements shall meet the applicable ASTM and AWS requirements specified on the Design Drawings and herein. Refer to table 9-1 for Safety Classification of MCO components.

All Work specified in this Specification, except as specifically noted, is nuclear safety-related and requires the application of the provisions of the Code of Federal Regulations, Title 10, Part 21 (10CFR21), "Reporting of Defects and Noncompliance." It is the responsibility of the Seller to implement the provisions of CFR, Title 10, Part 21, and to pass these requirements on to their subcontractors. All defects shall be reported to Buyer.

The Seller shall accept complete responsibility for all Work performed in compliance with this Specification. Review or approval of data or procedures by the Buyer with regard to work performed to accomplish the requirements of this Specification does not constitute acceptance of any materials or components which will not fulfill the requirements established by this Specification. The requirements of this Specification shall be met.

Alternative fabrication details proposed by the Seller shall be submitted to the Buyer in writing. These shall not be incorporated in Seller's fabrication drawings without written approval from the Buyer.

\subsection{Materials}

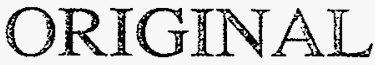

Procured materials shall meet the quality assurance requirements detailed in section 9.1 of this specification. All materials used for the fabrication of the MCO shall be in accordance with the Design Drawings. Material purchase specifications shall be prepared and submitted to the Buyer for approval prior to placing the material order. Certified Material Test Reports for all materials of fabrication, including welding materials, shall be provided to the Buyer. The Seller may propose to the buyer for approval to supply alternative materials, components, or parts other than those specified if, in the opinion of the Seller, the substitution is more economical, better qualified to operate under the conditions and performance requirements, and is equivalent to, and in compliance with, the applicable ASME Code requirements. All proposed substitutions shall be clearly defined by the Seller, with a complete description including supporting data establishing equivalence to the specified item. No materials may be substituted or changed without written approval of the Buyer. 


\subsubsection{ASME Code Materials}

All sheet, plate, pipe, forging, and rod materiais, as well as all weld consumables shall meet the applicable requirements of Section III, Division 1, Subsection NB, Article NB-2000 or NG-2000, of the ASME Code. The lowest service temperature is $32^{\circ} \mathrm{F}$. Certified material test reports shall accompany each lot or heat of the material supplied.

\section{A. Material Identification}

All materials shall be marked with the information required by the applicable ASME Section II, Material Specifications and Section III Sub-Articles NB-2150 or NG-2150, as applicable. Marking shall be done by any permanent method that will not result in harmful contamination or sharp discontinuities. Examples of such acceptable methods are etching or photo engraving or other method approved by the Buyer in writing. Marking of all components shall be maintained to provide traceability in the final assembly.

B. Examination and Repair of Material Defects

Examination and repair of the MCO shell materials shall be in accordance with the ASME Code, Section III, Division 1, Sub-Articles NB-2500 and NB-4130. Basket materials shall be examined and repaired in accordance with the ASME Code Section III, Sub-Articles NG-2500 and NG-4130. The requirements of the ASME Code Section II, Material Specification shall apply where they include provisions that are more restrictive than the ASME Code Section III requirements. Where repair of defects which exceed the lesser of $3 / 8$-inch or 10 percent of the section thickness are required, the Buyer shall be notified and no work shall be done until the Seller's written repair procedure has been approved by the Buyer.

\section{Welding Materials}

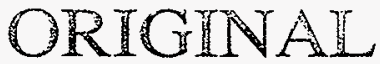

All welding filler metals and fluxes used in the fabrication and repair of the MCO components shall conform to the requirements of Article NB-2400 of Section III of the ASME Code. Welding filler metals and fluxes used in the fabrication and repair of the steel basket shall conform to the requirement of NG-2400. (Note: Welding materials may be purchased in accordance with the year and addenda of the ASME Code effective at the time the material purchase order is placed.) These filler metals and fluxes shall be tested as specified below. Certified material test reports shall accompany each lot or heat of the material covered.

\section{Stainless Steel to Stainless Steel Welds}

a. Stainless steel filler metals shall meet the ferrite requirements of USNRC Regulatory Guide 1.31 and NB-2433. All stainless steel filler metals shall be tested in accordance with NB-2432 "Chemical Analysis Test." 
b. Covered stainless steel electrodes for shielded metal arc welding shall conform with the requirements of ASME SFA-5.4, classification E308L-15, E308L-16, E309L-15, or E309L-16.

c. Stainless steel filler metal for gas tungsten arc welding or for gas metal arc welding shall conform with ASME SFA-5.9, classifications ER308L or ER309L.

\section{Shielding Gases}

All shielding gases shall be welding grade with dew point $-60^{\circ} \mathrm{F}$ or lower.

\subsubsection{Other Materials}

Piece parts and standard products incorporated into the $\mathrm{MCO}$ have been designated on the Design Drawings as ASTM materials or by manufacturer's call-out where applicable. These materials shall be purchased in accordance with the requirements of the applicable ASTM specifications or manufacturers standard specifications. The Seller shall supply Certificates of Conformance attesting that the item meets the applicable specifications.

\subsection{Fabrication}

All cutting, forming, machining and fitting operations required in the fabrication of the MCO shall be performed in accordance with approved shop drawings, the provisions of this Specification, the requirements of the ASME Code Section III, Article NB-4000 or NG-4000, as applicable, and manufacturer's recommended practices as applicable.

\subsubsection{Cutting}

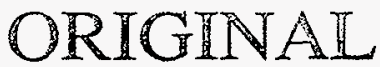

Preparation for welds shall be by mechanical means where practical. Plasma-arc cut surfaces shall be ground to provide slag-free metal and fitup equivalent to machining. Thermal cutting of austenitic stainless steel shall not be permitted without the Buyer's approval of the process to be used. See Section 6.5 .5 for enhanced non-destructive examination requirements for weld end preparation surfaces.

\subsubsection{Forming}

All plates if used for the MCO shell (in lieu of pipe sections) shall be rolled to final form and shall be accurately cut to size before welding, in accordance with of the ASME Code. Forming and bending shall be in accordance with the requirements of NB- 4200 of the ASME Code. Plates shall be formed to true shape with curvature continuous from the edges of the plate; flat spots shall meet the cylindricity tolerances specified on the Design Drawings and in the Codes and Standards. Corrections, if required, shall be made by the pressure process and not by hammer blows. 


\subsubsection{Machining}

Machining of the $\mathrm{MCO}$ and components shall be performed using tooling suitable for the task, capable of producing the required dimensional control and surface finishes specified on the Design Drawings.

\subsubsection{Fitting and Aligning}

Fitting and aligning of all parts is to be performed in accordance with the requirements of NB-4230 or NG-4230, as applicable, of the ASME Code. The accuracy of fitup shall be in compliance with the component tolerances specified on the Design Drawings.

\subsubsection{Piece Part Tolerance}

Where piece part tolerances are not shown on the Design Drawings, the Seller shall establish piece part tolerances which provide a finished product meeting the overall tolerances specified in section 6.3.6 of this specification. All piece part tolerances shall be stated on the shop drawings.

The Seller may recommend variations in the piece part tolerances established by the Design Drawings, provided a system is established which ensures that the critical dimensions and tolerances listed in section 6.3.6 are maintained.

\subsubsection{Dimensional Verification}

The Seller shall submit Measurement and Test Reports in accordance with section 11 of this specification which certify that each assembly/component, identified by a unique serial number, is in compliance with its applicable Table 6.3.6 and sketches Appendix 8 (NOTE: If sketches, appendix 8 differ from design drawings, buyer will be notified immediately for resolution).

Table 6.3.6-1

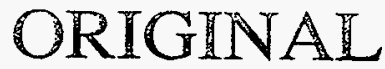

MCO SHELL CRITICAL DIMENSIONS

\begin{tabular}{|c|c|c|c|}
\hline Measurement & Minimum & Maximum & Method \\
\hline $\begin{array}{l}\text { OD and Straightness, canister } \\
\text { collar }\end{array}$ & $\mathrm{n} / \mathrm{a}$ & $25.310^{\prime \prime}$ & Insert into a Gage: ID $25.310^{\prime \prime}$, Length $10^{\prime \prime}$ \\
\hline $\begin{array}{l}\text { External Ovality, to Collar's } 45^{\circ} \\
\text { Shoulder }\end{array}$ & $\mathrm{n} / \mathrm{a}$ & $24.120^{\prime \prime}$ & $\begin{array}{l}\text { Pass through a ring gage: ID } 24.120^{\prime \prime} \text {, } \\
\text { Minimum Length } 4.030^{\prime \prime}\end{array}$ \\
\hline $\begin{array}{l}\text { Straightness, to Collar's } 45^{\circ} \\
\text { Shoulder }\end{array}$ & $\mathrm{n} / \mathrm{a}$ & $24.940 "$ & Insert into a Gage: ID $24.940 "$, Length 149" \\
\hline ID and Internal Straightness & $22.750^{\prime \prime}$ & $\mathrm{n} / \mathrm{a}$ & Pass Through a Gage: OD $22.750^{\prime \prime}$, Length $28^{\prime \prime}$ \\
\hline Minimum Wall Thickness & $0.425^{\prime \prime}$ & $\mathrm{n} / \mathrm{a}$ & $\begin{array}{l}25 \% \text { Surface Area UT, } \\
100 \% \text { Weld UT }\end{array}$ \\
\hline
\end{tabular}


HNF-SD-SNF-DR-003, Rev. 0

Appendix 19

\begin{tabular}{|c|c|c|c|}
\hline Measurement & Minimum & Maximum & Method \\
\hline $\begin{array}{l}\text { Process Tube Guide Cone OD } \\
\text { above shoulder }\end{array}$ & $2.645^{\prime \prime}$ & $2.655^{\prime \prime}$ & $\begin{array}{l}\text { Direct Measurement, after assembly of } \\
\text { baseplate, guide and basket support plates }\end{array}$ \\
\hline $\begin{array}{l}\text { Process Tube Guide Cone Top } \\
\text { Surface to the Top Surfaces of the } \\
6 \text { Support Plates }\end{array}$ & $2.525^{\prime \prime}$ & $2.565^{\prime \prime}$ & $\begin{array}{l}\text { Direct Measurement, after assembly of } \\
\text { baseplate, guide and basket support plates }\end{array}$ \\
\hline $\begin{array}{l}\text { Process Tube Guide Cone OD } \\
\text { concentric with MCO ID }\end{array}$ & $\mathrm{n} / \mathrm{a}$ & .015 & Direct Measurement \\
\hline $\begin{array}{l}\text { Support Plates Top Surfaces } \\
\text { Flatness }\end{array}$ & $\mathrm{n} / \mathrm{a}$ & $0.020^{\prime \prime}$ & $\begin{array}{l}\text { Direct Measurement, after assembly of } \\
\text { baseplate, guide and basket support plates }\end{array}$ \\
\hline Outside Length & $157.325^{\prime \prime}$ & $157.515^{\prime \prime}$ & Direct Measurement \\
\hline $\begin{array}{l}\text { Inside Length, Top Surface of } \\
\text { Support Plates to the Collar's } \\
\text { finished sealing surface }\end{array}$ & $147.930^{\prime \prime}$ & $148.200^{\prime \prime}$ & Direct Measurement \\
\hline Sealing Surface Flatness & $n / a$ & $0.002^{\prime \prime}$ & Direct Measurement \\
\hline Sealing Surface Lay & $\mathrm{n} / \mathrm{a}$ & Circular & Visual, Relative to MCO Centerline \\
\hline Sealing Surface Texture & $\mathrm{n} / \mathrm{a}$ & $0.000063^{\prime \prime}$ & $\begin{array}{l}\text { Projection Method: Four } 1.5^{\prime \prime} \text { Long segments a } \\
90^{\circ} \text { Spacing }\end{array}$ \\
\hline $\begin{array}{l}\text { ID between Sealing Surface and } \\
\text { Buttress Threads }\end{array}$ & $24.035^{\prime \prime}$ & $24.045^{\prime \prime}$ & Direct Measurement \\
\hline ID above Buttress Threads & $24.567^{\prime \prime}$ & $24.572^{\prime \prime}$ & Direct Measurement \\
\hline Collar Major OD & $25.280^{\prime \prime}$ & $25.310^{\prime \prime}$ & Direct Measurement \\
\hline \multicolumn{4}{|c|}{$\begin{array}{l}\text { Table } 6.3 .6-2 \\
\text { E CRITICAL DIMENSIONS }\end{array}$} \\
\hline Measurement & Minimum & Maximum & Method \\
\hline Length & $146.930^{\prime \prime}$ & $146.960^{\prime \prime}$ & Direct Measurement \\
\hline Threaded Length & $1.47^{\prime \prime}$ & $\mathrm{n} / \mathrm{a}$ & Direct Measurement \\
\hline Straightness Over Entire Length & $\mathrm{n} / \mathrm{a}$ & $0.125^{\prime \prime}$ & Direct Measurement \\
\hline
\end{tabular}

Table 6.3.6-3

SHIELD PLUG CRITICAL DIMENSIONS

\begin{tabular}{|l|r|r|l|}
\hline Measurement & \multicolumn{1}{|c|}{ Minimum } & \multicolumn{1}{c|}{ Maximum } & Method \\
\hline $\begin{array}{l}\text { Shield Plug Thickness prior to } \\
\text { assembly with Collar and Filter } \\
\text { Guard Plate }\end{array}$ & $10.485^{\prime \prime}$ & $10.515^{\prime \prime}$ & Direct Measurement \\
\hline Plug Major OD & $23.977^{\prime \prime}$ & $23.983^{\prime \prime}$ & Direct Measurement \\
\hline
\end{tabular}


HNF-SD-SNF-DR-003, Rev. 0

Appendix 19

\begin{tabular}{|c|c|c|c|}
\hline Measurement & Minimum & Maximum & Method \\
\hline Plug Minor OD (Top) & $15.775^{\prime \prime}$ & $15.785^{\prime \prime}$ & Direct Measurement \\
\hline $\begin{array}{l}\text { Plug Minor OD Total Run out with } \\
\text { respect to Rotation about the Major } \\
\text { Diameter's Axis }\end{array}$ & $\mathrm{n} / \mathrm{a}$ & $0.005^{\prime \prime}$ & Direct Measurement \\
\hline Plug Minor O.D. Bottom & $22.885^{\prime \prime}$ & $22.915^{\prime \prime}$ & Direct Measurement \\
\hline $\begin{array}{l}\text { Vertical Distance Between Top } \\
\text { Surface of the Plug and its Locating } \\
\text { Diameter }\end{array}$ & $8.545^{\prime \prime}$ & $8.575^{\prime \prime}$ & Direct Measurement \\
\hline Locating Diameter Flatness & $\mathrm{n} / \mathrm{a}$ & $0.003^{\prime \prime}$ & Direct Measurement \\
\hline $\begin{array}{l}\text { Vertical Distance Between Locating } \\
\text { Diameter and the Sealing Surface }\end{array}$ & $0.151^{\prime \prime}$ & $0.155^{\prime \prime}$ & Direct Measurement \\
\hline Sealing Surface Flatness & $n / a$ & $0.002^{\prime \prime}$ & Direct Measurement \\
\hline Sealing Surface Texture & $\mathrm{n} / \mathrm{a}$ & $0.000063^{\prime \prime}$ & $\begin{array}{l}\text { Projection Method: Four } 1.5^{\prime \prime} \text { Long } \\
\text { segments at } 90^{\circ} \text { Spacing }\end{array}$ \\
\hline Sealing Surface Lay & $\mathrm{n} / \mathrm{a}$ & Circular & Visual, Relative to MCO Centerline \\
\hline $\begin{array}{l}\text { Vertical Distance Between Locating } \\
\text { Diameter and the Guard Plate } \\
\text { Bottom Surface }\end{array}$ & $7.615^{\prime \prime}$ & $7.765^{\prime \prime}$ & Direct Measurement \\
\hline Basket Stabilizer ID & $2.710^{\prime \prime}$ & $2.720^{\prime \prime}$ & Direct Measurement \\
\hline $\begin{array}{l}\text { Basket Stabilizer Major Cutout } \\
\text { Height }\end{array}$ & $3.100^{\prime \prime}$ & $\mathrm{n} / \mathrm{a}$ & Direct Measurement \\
\hline $\begin{array}{l}\text { Perpendicularity between Basket } \\
\text { Stabilizer Thread ID and Locating } \\
\text { Diameter }\end{array}$ & $\mathrm{n} / \mathbf{a}$ & $0.010^{\prime \prime}$ & Direct Measurement \\
\hline $\begin{array}{l}\text { Vertical Distance Between Locating } \\
\text { Diameter and the Basket Stabilizer } \\
\text { Thread Relief Top Surface }\end{array}$ & $2.760^{\prime \prime}$ & $2.785^{\prime \prime}$ & Direct Measurement \\
\hline $\begin{array}{l}\text { Port Flange Cutout Flatness } \\
\text { (4 Places) }\end{array}$ & $\mathrm{n} / \mathrm{a}$ & $0.002^{\prime \prime}$ & Direct Measurement \\
\hline Port Flange Cutout Surface Texture & $\mathbf{n} / \mathbf{a}$ & $0.000032^{\prime \prime}$ & Projection Method \\
\hline $\begin{array}{l}\text { Port Flange C-seal Recess Depth } \\
\text { (4 Places) }\end{array}$ & $0.097^{\prime \prime}$ & $0.103^{\prime \prime}$ & Direct Measurement \\
\hline $\begin{array}{l}\text { Port Flange C-seal Recess Flatness } \\
\text { (4 Places) }\end{array}$ & $\mathrm{n} / \mathrm{a}$ & $0.002^{\prime \prime}$ & Direct Measurement \\
\hline $\begin{array}{l}\text { Port Flange C-seal Recess Sealing } \\
\text { Surface Texture }\end{array}$ & $\mathrm{n} / \mathrm{a}$ & $0.000032^{\prime \prime}$ & Projection Method \\
\hline
\end{tabular}


Table 6.3.6-4

LOCKING \& LIFTING RING CRITICAL DIMENSIONS

\begin{tabular}{|l|r|r|l|}
\hline Measurement & \multicolumn{1}{|c|}{ Minimum } & \multicolumn{1}{c|}{ Maximum } & Method \\
\hline Thickness & $5.970^{\prime \prime}$ & $6.030^{\prime \prime}$ & Direct Measurement \\
\hline Center ID & $15.900^{\prime \prime}$ & $15.910^{\prime \prime}$ & Direct Measurement \\
\hline $\begin{array}{l}\text { Center Cutout Concentricity with } \\
\text { Buttress Thread Axis of Rotation }\end{array}$ & $\mathrm{n} / \mathrm{a}$ & $0.005^{\prime \prime}$ & Direct Measurement \\
\hline Lifting Ring OD & $20.350^{\prime \prime}$ & $20.410^{\prime \prime}$ & Direct Measurement \\
\hline Lifting Ring ID & $18.720^{\prime \prime}$ & $18.780^{\prime \prime}$ & Direct Measurement \\
\hline
\end{tabular}

Table 6.3.6-5

CANISTER COVER CRITICAL DIMENSIONS

\begin{tabular}{|l|r|r|l|}
\hline Measurement & \multicolumn{1}{|c|}{ Minimum } & \multicolumn{1}{c|}{ Maximum } & Method \\
\hline Major Diameter & $25.280^{\prime \prime}$ & $25.310^{\prime \prime}$ & Direct Measurement \\
\hline Thickness & $7.970^{\prime \prime}$ & $8.030^{\prime \prime}$ & Direct Measurement (Prior to marking) \\
\hline Lifting Ring OD & $20.350^{\prime \prime}$ & $20.410^{\prime \prime}$ & Direct Measurement \\
\hline Lifting Ring ID & $18.720^{\prime \prime}$ & $18.780^{\prime \prime}$ & Direct Measurement \\
\hline Bottom Lip Recess Depth & $.110^{\prime \prime}$ & $.150^{\prime \prime}$ & Direct Measurement \\
\hline Bottom Lip Recess OD & $24.550^{\prime \prime}$ & $24.560^{\prime \prime}$ & Direct Measurement \\
\hline
\end{tabular}

Table 6.3.6-6

MK IA STORAGE BASKET CRITICAL DIMENSIONS

\begin{tabular}{|l|r|r|l|}
\hline Measurement & \multicolumn{1}{|c|}{ Minimum } & \multicolumn{1}{c|}{ Maximum } & Method \\
\hline Stack Height & $23.162^{\prime \prime}$ & $23.182^{\prime \prime}$ & $*$ \\
\hline Baseplate Thickness & $1.200^{\prime \prime}$ & $\mathrm{n} / \mathrm{a}$ & Direct Measurement \\
\hline Baseplate Flatness & $\mathrm{n} / \mathrm{a}$ & $0.010^{\prime \prime}$ & $* *$ \\
\hline Envelope Diameter & $\mathrm{n} / \mathrm{a}$ & $22.700^{\prime \prime}$ & Pass Through a Gage: ID 22.700", \\
\hline Fuel Vertical Room & $21.5^{\prime \prime}$ & $\mathrm{n} / \mathrm{a}$ & Direct Measurement \\
\hline Fuel Rack Hole ID x 48 & $2.570^{\prime \prime}$ & $\mathrm{n} / \mathrm{a}$ & Plug Gage: OD 2.570", Length 2.5" \\
\hline Coupling OD & $2.645^{\prime \prime}$ & $2.655^{\prime \prime}$ & Direct Measurement \\
\hline Coupling Stab Height & $\mathrm{n} / \mathrm{a}$ & $3.020^{\prime \prime}$ & Direct Measurement \\
\hline Bushing Receiver ID & $2.710^{\prime \prime}$ & $2.720^{\prime \prime}$ & Direct Measurement \\
\hline Bushing Reciever ID Height & 3.025 & $\mathrm{n} / \mathrm{a}$ & Direct Measurement \\
\hline
\end{tabular}

* Direct measurement between a reference plane which the basket is resting on and the top surfaces of the six outside support rods and the top surface of the center post.

** Direct measurements between reference plane which the basket is resting on and the bottom surface of the basket baseplate. 
Table 6.3.6-7

MK IA SCRAP BASKET CRITICAL DIMENSIONS

\begin{tabular}{|l|r|r|l|}
\hline Measurement & Minimum & \multicolumn{1}{|c|}{ Maximum } & Method \\
\hline Stack Height & $23.162^{\prime \prime}$ & $23.182^{\prime \prime}$ & $*$ \\
\hline Baseplate Thickness & $1.200^{\prime \prime}$ & $n / a$ & Direct Measurement \\
\hline Baseplate Bottom Flatness & $\mathrm{n} / \mathrm{a}$ & $0.010^{\prime \prime}$ & $* *$ \\
\hline Envelope Diameter & $\mathrm{n} / \mathrm{a}$ & $22.700^{\prime \prime}$ & $\begin{array}{l}\text { Pass Through a Gage: ID 22.700", } \\
\text { Minimum Length 24" }\end{array}$ \\
\hline Coupling OD & $2.645^{\prime \prime}$ & $2.655^{\prime \prime}$ & Direct Measurement \\
\hline Coupling Stab Height & $\mathrm{n} / \mathrm{a}$ & 3.020 & Direct Measurement \\
\hline Bushing, Receiver ID & $2.710^{\prime \prime}$ & $2.720^{\prime \prime}$ & Direct Measurement \\
\hline Bushing, Receiver ID Height & 3.025 & $\mathrm{n} / \mathrm{a}$ & Direct Measurement \\
\hline
\end{tabular}

*Direct measurement between a reference plane which the basket is resting on and the top surfaces of the six outside support rods and the top surface of the center post.

**Direct measurements between a reference plane which the basket is resting on and the bottom surface of the basket baseplate.

Table 6.3.6-8

MK IV STORAGE BASKET CRITICAL DIMENSIONS

\begin{tabular}{|l|r|r|l|}
\hline Measurement & \multicolumn{1}{|c|}{ Minimum } & \multicolumn{1}{|c|}{ Maximum } & Method \\
\hline Side Post Stack Height & $27.812^{\prime \prime}$ & $27.822^{\prime \prime}$ & $*$ \\
\hline Center Post Height & $30.387^{\prime \prime}$ & $30.407^{\prime \prime}$ & $* *$ \\
\hline Envelope Diameter & $\mathrm{n} / \mathrm{a}$ & $22.700^{\prime \prime}$ & $\begin{array}{l}\text { Pass Through a Gage: ID 22.700", } \\
\text { Minimum Length 28" }\end{array}$ \\
\hline Fuel Vertical Room & $26.7^{\prime \prime}$ & $\mathrm{n} / \mathrm{a}$ & Direct Measurement \\
\hline Fuel Rack Hole ID x 54 & $2.570^{\prime \prime}$ & $\mathrm{n} / \mathrm{a}$ & Plug Gage: OD 2.570", Length 2.5" \\
\hline Center Post Coupling OD & $2.645^{\prime \prime}$ & $2.655^{\prime \prime}$ & Direct Measurement \\
\hline Bottom Plate Receiver ID & $2.710^{\prime \prime}$ & $2.720^{\prime \prime}$ & Direct Measurement \\
\hline Bottom Plate Receiver Depth & $2.570^{\prime \prime}$ & $2.590^{\prime \prime}$ & Direct Measurement \\
\hline
\end{tabular}

*Direct measurement between a reference plane which the basket is resting on and the top surfaces of the six outside support rods.

**Direct measurement between a reference plane which the basket is resting on and the top surface of the center post. 
Table 6.3.6-9

MK IV SCRAP BASKET CRITICAL DIMENSIONS

\begin{tabular}{|l|r|r|l|}
\hline Measurement & \multicolumn{1}{|c|}{ Minimum } & \multicolumn{1}{c|}{ Maximum } & Method \\
\hline Stack Height & $27.812^{\prime \prime}$ & $27.822^{\prime \prime}$ & $*$ \\
\hline Center Post Height & $30.387^{\prime \prime}$ & $30.407^{\prime \prime}$ & $* *$ \\
\hline Envelope Diameter & n/a & $22.700^{\prime \prime}$ & $\begin{array}{l}\text { Pass Through a Gage: ID 22.700", } \\
\text { Minimum Length 28" }\end{array}$ \\
\hline Center Post Coupling OD & $2.645^{\prime \prime}$ & $2.655^{\prime \prime}$ & Direct Measurement \\
\hline Bottom Plate Receiver ID & $2.710^{\prime \prime}$ & $2.720^{\prime \prime}$ & Direct Measurement \\
\hline Bottom Plate Receiver Depth & $2.570^{\prime \prime}$ & $2.590^{\prime \prime}$ & Direct Measurement \\
\hline
\end{tabular}

*Direct measurement between a reference plane which the basket is resting on and the top surface of the outside shell.

**Direct measurement between a reference plane which the basket is resting on and the top surface of the center post.

\subsection{Assembly}

The Seller shall develop a manufacturing plan which provides sufficient detail to clearly define the proposed assembly sequence including the required cutting, fitting, aligning, and welding steps to ensure that the overall critical tolerances are maintained. The manufacturing plan shall be submitted to the Buyer for approval prior to start of fabrication.

\subsubsection{Assembly Requirements}

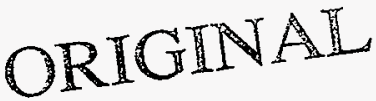

The Seller shall establish acceptable tolerance criteria for each sub-assembly, which shall be included on the shop drawings. Tolerances shall be consistent with the overall finished MCO tolerances for size and weight specified on the Design Drawings and this Specification.

Prior to inserting the $\mathrm{MCO}$ basket assemblies (for fit-up test or size verification) into the MCO shell assembly, the interior of the MCO shell assembly and the basket assemblies shall be cleaned in accordance with Section 6.6 of this Specification.

\subsubsection{Dimensional Verification}

The Seller shall submit measurement and test reports in accordance with Section 11 of this specification which certify that each assembly/component, identified by a unique serial number, is in compliance with the dimensions shown in Table 11-1.

\subsubsection{Marking (Labeling) of Completed Assemblies}

This section lists the requirements for permanent marking of completed $\mathrm{MCO}$ assemblies. It does NOT concern temporary markings used to maintain CMTR/heat/lot traceability during shop fabrication of piece-parts. 
Completed MCO components shall be sequentially identified with serial numbers per table 6.4.3-1 in order to provide traceability and nuclear material accountability.

\subsubsection{Shield Plug and Canister Cover}

A. Method: Alphanumeric labels shall be formed by bead welding directly to the top of the shield plug and canister cover using 308L weld rod. A method must be developed and used to provide uniform characters. Free hand welding without any guide or visual aid is not acceptable. Weld bead height is not to exceed $1 / 16^{\prime \prime}$. Weld profile shall be smooth with no undercutting or areas that could trap foreign materials. Two samples of welded aiphanumeric labels are to be submitted to the Buyer for approval prior to method use. Welds shall be by procedures and personnel qualified in accordance with this specification. Additionally, Rupture disk holder and Process plug shall be mark with a unique serial number traceable to manufacturing documentation.

B. Marking Text Font: The text font shall be an upright, sans-serif, modern type font such as Gothic, Arial, or equivalent. Height-to-width ratio of the letters and numbers in the font may be from $1 / 1$ to $2.2 / 1$. All letters shall be upper case.

C. Marking Text Height: Text height shall be a minimum of $5 \%$ ".

\subsubsection{Canister Shell and Locking Ring}

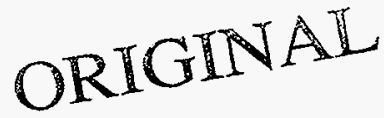

A. Method: Components shall be impression stamped using blunt-nosed continuous dies, or blunt-nosed interrupted dot die stamps.

B. Marking Text Font: The text font shall be an upright, sans-serif, modern type font such as Gothic, Aerial, or equivalent. Height-to-width ratio of the letters and numbers in the font may be from $1 / 1$ to $2.2 / 1$. All letters shall be upper case.

C. Marking Text Height: Text height shall be a minimum of $5 \%$ ".

D. Marking Die Stamp: die stamps shall be low-stress type stamps. The tip radius of the dies for $1 / 4^{\prime \prime}$ character size letters shall be $0.010^{\prime \prime}$ minimum. Die stamp marking shall be applied to a visible, low stress location. The material thickness of the item to be marked shall not be reduced by die stamping to less than the minimum specified on the component drawing. The impression depth shall not exceed 0.010".

\subsubsection{Storage and Scrap Baskets}

A. Method: Baskets shall be electrochemically etched. The depth of etching shall not exceed $0.003 "$. The electrolyte and neutralizer used for electrochemical etching shall be compatible with the material to be marked. 
B. Marking Text Font: The text font shall be an upright, sans-serif, modern type font such as Gothic, Arial, or equivalent. Height-to-width ratio of the letters and numbers in the font may be from $1 / 1$ to $2.2 / 1$. All letters shall be upper case.

C. Marking Text Height: Text height shall be 2".

Table 6.4.3-1

MCO COMPONENT LABELING

\begin{tabular}{|l|l|l|}
\hline \multicolumn{1}{|c|}{ COMPONENTS } & \multicolumn{1}{|c|}{\begin{tabular}{c}
\multicolumn{1}{|c|}{$\begin{array}{c}\text { SERIAL NUMBER } \\
\text { (First Item) }\end{array}$} \\
\hline Shield Plug
\end{tabular}} & \multicolumn{1}{|c|}{$\begin{array}{c}\text { LOCATION AND } \\
\text { ORIENTATION ON THE } \\
\text { COMPONENT }\end{array}$} \\
\hline Canister Shell & $\begin{array}{l}\text { H-001 } \\
\text { tracking) }\end{array}$ & Top surface, between process ports \\
\hline Locking \& Lifting Ring & R-001 MCO \# used for & $\begin{array}{l}\text { OD surface of collar, approx. 1" } \\
\text { from top edge, and near one of the } \\
\text { buttress thread starts. }\end{array}$ \\
\hline Canister Cover & C-001 & $\begin{array}{l}\text { Approx. centered on the top surface } \\
\text { of the lifting lip. }\end{array}$ \\
\hline Mark IA Storage Basket & IA-0001 & Approx. centered on the top surface. \\
\hline Mark IA Scrap Basket & LAS-0001 & $\begin{array}{l}\text { OD surface of the shroud, approx. 3" } \\
\text { from top edge. }\end{array}$ \\
\hline Mark IV Storage Basket & IV-0001 & $\begin{array}{l}\text { OD surface of the shroud, approx. 3" } \\
\text { from top edge. }\end{array}$ \\
\hline Mark IV Scrap Basket & IVS-0001 & $\begin{array}{l}\text { OD surface of the shroud, approx. 3" } \\
\text { below seal. }\end{array}$ \\
\hline
\end{tabular}

\subsection{Welding}

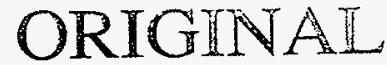

All welding shall conform to the requirements of Articles NB-4000 or NG-4000 as applicable for Class 1 plate and shell type supports, as applicable, of the ASME Code, Section III and this Specification, unless noted otherwise on the Design Drawings.

All welding shall be performed by welders and welding operators qualified in accordance with NB-4300 or NG-4300, as applicable. Copies of the welder's qualifications shall be submitted at the same time that each welding procedure is submitted to the Buyer for approval.

The use of temporary welded attachments during fabrication shall be avoided as much as possible. After fabrication is complete, temporary welded attachments and arc strikes shall be removed flush with the base material without encroaching on the specified minimum thickness. All areas from which temporary attachments have been removed shall be examined by the Liquid Penetrant method after the surface has been restored. 
Each piece (shell, collar, base plate, etc) is to be uniquely identified. The Seller shall establish a weld map, material map, and radiographic map for each MCO. The maps shall provide traceability of all materials to heat (lot) numbers throughout the fabrication sequence and final assembly. Each weld shall identify the welder, weld material by heat (lot) number and be traceable to applicable NDE reports. These maps shall be included in the final document package. Identification for traceability of MCO components is required. Vibra-etching, laser marking, or photo engraving is recommended for identification. High stress die stamping and pin stamping are not permitted (see section 6.4.3.2).

\subsubsection{Welding Processes}

Only the following welding processes with the restrictions listed are permitted:

A. Manual Shielded Metal-Arc

Electrodes conforming to the requirements of Section 6.2.1 Paragraph C.1 shall be used for joining stainless steel to stainless steel. Covered electrodes shall be stored and handled as recommended by the electrode manufacturer and in accordance with the applicable code requirements.

B. Gas Tungsten Arc

An inert gas backing purge must be used for the first 3/16 inch of deposited weld metal thickness for full penetration butt welds. Filler metal conforming to the requirements of Section 6.2.1 Paragraph C. 1 shall be used for joints in stainless steel.

C. Gas Metal Arc

This process, except for the short circuiting arc mode, may be used for any stainless steel weld joint. The short circuiting arc mode is prohibited for all construction covered by this Specification. Filler metals for welding stainless steel shall conform to the requirements of Section 6.2.1 Paragraph C.1.

\subsubsection{Welding Procedures and Qualifications}

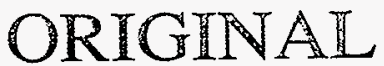

The Seller shall prepare written Welding Procedure Specifications (WPS). Each WPS shall be prepared and qualified in accordance with the requirements of ASME Section IX, ASME Section III, Subsections NB and NG, and this Specification. WPS and Procedure Qualification Records $(\mathrm{PQR})$ shall be in the form of clear, sharp, reproducible prints and shall be submitted on forms $Q W$ 482 and QW-483 as shown in the ASME Code, Section IX or similar forms or formats.

Approved WPS identifications and designations shall be shown on the Seller's shop drawings for each weld. Thirty days prior to the start of fabrication, the Seller shall submit to the Buyer, one copy of WPS and corresponding PQRs. The welding and quality control procedures shall include the limitation that no welder shall have in his possession more than one type of filler metal at any one time; except that a welder may have both bare wire and covered electrodes that deposit weld metal of the same A-number class. A technique shall be used to avoid weld splatter. 


\subsubsection{Preheat and Interpass Temperatures}

A. The maximum preheat and interpass temperature for welding austenitic stainless steel materials shail be $350^{\circ} \mathrm{F}$. The minimum preheat and Interpass temperature is $50^{\circ} \mathrm{F}$.

B. Preheat and Interpass temperature shall be determined by temperature indicating crayons, contact pyrometers, or other suitable means accepted by the Buyer. Temperature indicating crayons shall not be used on austenitic stainless steel, except for weld interpass temperature measurements, in which case all crayon markings shall be removed by using acetone or isopropyl alcohol prior to any heat treatment.

C. Interpass temperature requirements listed above shall also apply to tack welding, fillet welds, attachment welds, and thermal gouging and cutting.

\subsubsection{Workmanship and Visual Quality}

A. Each weld shall be essentially uniform in width and size throughout its full length. Each layer of welding shall be visually free of slag, inclusions, cracks, porosity, and lack of fusion.

B. Fillet welds shall be of the specified size with full throat thickness as required. Excessive convexity or concavity shall be avoided. Fillet welds shall always develop the minimum size required by the Design Drawing, but may vary in size above the minimum as long as a reasonably uniform appearance is maintained.

C. Elimination of defects and surface preparation of welds by chipping, grinding or gouging shall be done in such a manner as not to gouge, groove, or reduce the adjacent base-material thickness below the specified thickness. The Buyer approved repair procedures and verification reports shall be used for the Work.

D. Precautions should be taken to avoid weld splatter and arc strikes. If these occur, they shall be removed by procedures approved by the Buyer.

E. Peening shall not be used without prior written acceptance of the method and controls to be used. The use of pneumatic tools for slag removal is not considered peening, and is acceptable. Peening of root and cover passes is not acceptable.

F. Welds shall be ground flush within $+1 / 32^{\prime \prime},-0^{\prime \prime}$ from the high side of the weld. Weld caps on all seams shall be a maximum of $.03^{\prime \prime}$ high. If grinding is required to maintain the maximum weld height, the finish shall be equivalent to 80 grit.

\subsubsection{Non-Destructive Examination}

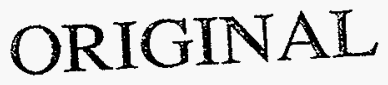

A. General

All machined surfaces shall be examined at completion of machining. Except as specified herein, all welds shall be non-destructively examined in accordance with the requirements 
of NB-5000 or NG-5000, as applicable, of the ASME Code, Section III. Personnel performing non-destructive examination shall be qualified in accordance with NB-5520 or NG-5520, as applicable, SNT-TC-1A, and their qualification records shall be available at the point of work. Only individuals qualified for NDT Level I, NDT Level II, or NDT Level III may perform non-destructive testing. Level I personnel shall not interpret the results of an examination or make determination of the acceptability of an examined part. Copies of the NDE personnel qualifications shall be submitted at the same time that each NDE procedure is submitted to the Buyer for approval.

The Seller shall prepare written NDE procedures which shall be submitted to the Buyer for approval. The Seller shall also submit a sample radiograph to the Buyer for approval.

The acceptance standards shall conform to the requirements of NB-5300 or NG-5300, as applicable, of the ASME Code, Section III, Division 1. Unacceptable welds shall be repaired in accordance with NB-4400 or NG-4400, as applicable, of the ASME Code, Section III, Division 1 and re-examined by the same method that detected the flaw.

\section{B. Radiography (RT)}

All welds designated on the Design Drawings to be radiographically examined shall be examined in accordance with the requirements of Section V, Article 2 of the ASME Code. The ASME Section III penetrameter chart shall be used for radiographic examination. Radiographic acceptance standards shall be as stated in NB-5320 of the ASME Code, Section III with the following additional comments:

1. Surface pinholes shall be removed by grinding and need not be repaired by welding, providing the minimum specified thickness is maintained and the depression is faired into the surrounding weld.

2. The radiographic film shall be fine grain, high contrast, at least equivalent to Eastman Kodak AA. Lead intensifying screens shall be used. The minimum film density shail be 2.0 for single viewing, and 2.6 for double composite film viewing. For the latter, the minimum density on a single film shall not be less than 1.3 . The maximum film density for both shall be 4.0 . The film shall be double loaded cassettes with both films submitted to the Buyer for review.

3. A written record shall be prepared for each weld radiographically examined. As a minimum the written record shall include: identification of part, joint thickness, material, examined area, the radiographic technique covering all essential variables, a shooting sketch showing the identification and location of the film and placement of the radioactive source, lead markers, pentameter used and which datum was visible, name and certification level of radiographer interpreting the film and the findings or disposition if any. The film negative plus any film negative from repaired areas made both before and after repair work shall be included. 
4. The Seller must use a calibrated densitometer when verifying the density of a radiograph. Comparator strips are not acceptable. Individual radiographs must be uniquely identified in order to correlate the radiographs with specific welds, weld locations and reader sheets for each canister.

5. The results of the radiographic inspections shall be made part of the document package. Radiographic films shall become the property of the Buyer after the contract is closed.

C. Liquid Penetrant (PT)

All welds designated on the Drawings to be examined by liquid penetrant examination shall be examined in accordance with the requirements of Section V, Article 6 of the ASME Code. For buttwelds requiring radiography, the buttweld end preparation surfaces for plate and forgings shall be examined by the liquid penetrant method. This requirement applies to all thicknesses of these product forms. The minimum acceptance standards for liquid penetrant examination shall be as specified in Section III, Division 1, Sub-Article NB-5350 or NG-5350, as applicable, of the ASME Code.

1. Liquid penetrant testing shall be by the solvent removal method. The penetration time shall be at least 5 minutes, but at no time shall the penetrant be allowed to dry or harden on the examined surfaces. The developer drying time shall be made no sooner than seven minutes nor later than 30 minutes after the developer has dried. Prior to the application of developer, the surface shall be wiped clean using solvent-damp lint-free cloth or paper towels. Flooding with liquid solvent is prohibited.

2. Penetrant testing materials (i.e., penetrant, cleaners, developers, etc.) used for examination of austenitic stainless steels and nickel-base alloys shall not cause corrosive or other harmful effects and shall not contain more than the amount of contaminant specified in Section V of the ASME Code. The penetrant materials shall be thoroughly removed immediately after the examination has been completed, followed by a swabbing or flushing of the area with demineralized water, acetone, or isopropyl alcohol.

3. A written report of each weld examined by liquid penetration shall be prepared. At a minimum, the written record shall include: Identification of part, material and/or area, name and level of examiner, and the findings or dispositions, if any.

D. Ultrasonic (UT)

All welds designated on the Design Drawings to be ultrasonically examined shall be examined in accordance with the requirements of Section V, Article 5 of the ASME Code. The minimum acceptance standards for UT examined welds shall be as specified in Section III, Division 1, Sub-Article NB-5330 or NG-5330, as applicable, of the ASME Code. 
1. Calibration holes or notches shall not be machined in the article to be examined. Calibration blocks of ultrasonically identical material with thickness no less than $90 \%$ and no more than $110 \%$ of the part to be examined shall be used.

2. Couplants used for ultrasonic examination of austenitic stainless steels and nickel-base alloys shall not cause corrosive or other harmful effects.

3. Couplants shall be removed after completion of the examination using materials and procedures approved by the Buyer.

4. A written report of each weld examined by ultrasonic methods shall be prepared. At a minimum, the written report shall include: identification of part, material and/or area, name and level of examiner, calibration block used, $\mathrm{dB}$ settings for searching and reporting frequency, couplant, transducer size, shape, type, and model, and the findings or dispositions, if any.

5. Calibration shall consist of $80 \%$ full-screen height signal from the $1 / 4 \mathrm{t}$ hole or notch. The Distance amplitude correction (DAC) shall plot 1/4t, 1/2t, and 3/4t reflectors. Electronic DAC evaluators are acceptable. All signals over 50\% DAC are reportable, all signals over $100 \% \mathrm{DAC}$ are rejectable, unless judged by a Level III examiner to be caused by geometry or other non-harmful phenomena.

\section{E. Visual Examination (VT)}

All welds shall be visually examined in accordance with the requirements of paragraph NB4424 or NG-4424, as applicable of Section III, and Article 9, Section V of the ASME Code. Only visual examiners qualified and certified in accordance with Seller's approved Quality Assurance Program may perform visual examination under this specification.

\subsubsection{Repair by Welding}

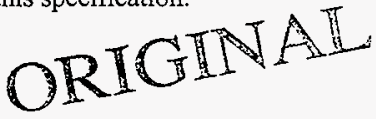

A. Weld Repair of Defects in Base Material:

Defects in plates, shapes, and bars shall be as specified in Section 6.2.1.B of this Specification.

B. Repair of Defects in Completed Welds:

Unacceptable weld defects shall be eliminated and repaired in accordance with Section III, Sub-Article NB-4450 or NG-4450, as applicable, of the ASME Code and visually re-examined prior to proceeding to any additional examinations required by this Specification. Repair welding shall be performed employing welding procedures and welders qualified in accordance with Section IX of the ASME Code. All repairs to completed welds are to be documented on an NDE/Weld History record which shall include the type and location of defect repaired, subsequent heat treatment, if performed, and the results of re-examination(s) performed after repair. In process repairs requiring removal of weld pass(es) must be documented even if they are considered "in progress". 


\subsection{Cleaning, Coating, and Surface Preparation}

Cleaning and surface preparation for the MCO and its sub-assemblies shall be as specified on the Design Drawings and the following:

\subsubsection{Cleaning}

A. All metal surfaces shall have a surface cleanliness classification $\mathrm{C}$ or better as defined in ASME NQA-1-1994 Edition, Subpart 2.1.

B. All welding by-products such as slag, splatter, or smoke stains shall be removed.

C. The use of any mechanical method which produces excessive roughness, or cleaning agents which may have corrosive effects or affect the performance of the material is prohibited.

D. No materials or methods used during fabrication, testing, examination, or cleaning shall contain appreciable amounts of halogens or other deleterious materials.

E. Written procedures prepared in accordance with ANSI N45.2.1, Section 2.2 for cleaning, examination, and testing of cleanness shall be submitted to the Buyer for approval. The Seller shall use ASTM A-380 as a guide for preparing the procedures.

F. Prior to welding, all components shall be degreased using a cleaning agent that does not contain more than $250 \mathrm{ppm}$ halogens. No markings made with markers shall remain. The cleaning technique shall be included in the cleaning procedure.

G. The inside and outside surfaces of the finished $\mathrm{MCO}$ shall be free from all mill scale, machining chips, grease, oil, weld splatter, arc strikes, or other foreign matter. It is essential that the MCO vessel, shield plug flow passages, process tube, strainer, and HEPA filter not contain foreign materials.

H. During fabrication, contact with CLEAN carbon steel bed plates, cutting tables, lathes, boring mills, tooling, handling equipment, testing equipment etc. is permitted. Any of the above carbon steel surfaces shall be clean of any loose scale, rust, or steel particles that could become embedded in stainless steel.

I. All grinding wheels shall be made of aluminum oxide, all wire brushes shall be Type 300 stainless steel, and both shall be used only on stainless steel material. MCO material shall be isolated from grinding or welding of carbon steels to avoid contamination.

J. Cutting oils, lubricants, inks, labels or other means of marking fabrication progress, and all other materials/chemicals used during the fabrication and inspection (such as liquid penetrant dye and developer, ultrasonic testing couplants, etc) shall have a maximum halogen content of $250 \mathrm{ppm}$. Certifications of halogen content shall be available for Buyer review. 


\subsection{EXAMINATION AND TESTING REQUIREMENTS}

The MCO shall undergo the shop tests, examinations, performance tests, leak tests and pressure test described herein. The purpose of these tests and examinations is to ensure the quality of workmanship and to guarantee that the MCO meets the functional requirements specified herein. Detailed test procedures, acceptance criteria, and a test schedule shall be provided by the Seller for the Buyer's approval. The Seller shall furnish all shop examination and test facilities, materials, and labor necessary for performance of tests and examinations, or for any modifications resulting from the tests or examinations. The Seller shall repair or replace all or any parts of the Work not in compliance with this Specification as determined by such examinations and tests.

The Buyer shall be notified in writing at least two weeks in advance of designated hold points or witness points. In addition, three days prior to these hold or witness points, the seller shall notify the Buyer by telephone. Hold or witness points may not be waived without specific written consent of the Buyer.

\subsection{Shop Tests and Examination}

The Seller shall submit to the Buyer documentation identifying the Seller's QA examination and test, witness and hold points which will occur during the manufacturing, assembly, and testing activities for the MCO. This list will be used to designate the Buyer surveillance hold and witness points.

The Buyer reserves the right to make arrangements with a qualified examination firm, or provide his own representative, to be present during the required hold or witness point activities. The presence of a representative from such a firm or the Buyer's representative does not relieve the Seller from performing his required in-house quality control functions. The Seller is responsible for first line examination and verification of items and services within the contractual scope of work. The Seller shall furnish the representative all necessary documents and records to perform the required surveillance during hold and witness point activities.

The Buyer will review the work to ensure that all items have been fabricated and tested in accordance with the Specification. All material used in the MCO shell assembly, shield plug, locking ring, and canister cover shall meet the requirements of Section III, Subsection NB-2000. All material used in the fabrication of Mark IA baskets shall meet the requirements of Section III, Subsection NB.

\subsubsection{Ultrasonic Inspection of Plate Used For MCO Shell}

The Seller shall develop and perform a procedure for ultrasonic testing of the plate material that is used to fabricate the pressure boundary shell of the $\mathrm{MCO}$. This plate material shall be ultrasonically inspected for minimum acceptable thickness, laminations, and indications in accordance with ASME Section V, Article 23, SE-797, SA-578, and supplementary requirements S3 and S4.

Scanning shall be along continuous parallel paths, parallel to the major plate axis, on 3 inch or smaller centers. This inspection shall be performed on the MCO shell after rolling and welding operations are performed. (Note: See section on welding for additional inspection requirements on welds). 
Circumferential measurements shall be measured clockwise (while looking down on the $\mathrm{MCO}$, and zero inches being the centerline of the longitudinal weld seam). Longitudinal measurements shall be measured from bottom to top of the MCO shell (zero inches being the bottom of the shell).

The ultrasonic inspection documentation data shall be in tabular format. The table shall indicate where each measurement was taken. An example of the tabular format shall be submitted by the Seller to the Buyer for approval prior to any measurements-of-record. The results of all UT inspections shall be made part of the final document package.

\subsubsection{Ultrasonic Inspection of Forgings}

The Seller shall develop and perform a procedure for ultrasonic testing of the forged components (base plate, shield plug, locking ring, and canister cover) that are used to fabricate the MCO. The forged components shall be ultrasonically inspected for minimum acceptable thickness, laminations, and indications in accordance with ASME Section V, Article 23, SE-797, SA-578, and supplementary requirements S3 and S4. (Alternatively, ASME Section III, Subsection 2542 Ultrasonic Examination of Forgings may be used). Ultrasonic testing of the forged component metal shall be performed after completion of all forging, heat treating, and initial profile machining. Note: It is permissible to perform the ultrasonic inspection before completion of final machining to finished dimensions to confirm material integrity only. Scanning shall be along continuous parallel paths on 3-inch or smaller centers. At a minimum, the scanning with normal beam method shall be performed in two directions (parallel and perpendicular to the major axis of the component). The Seller may, if desired, supplement the normal beam UT scans with angle beam scans. The ultrasonic inspection documentation data shall be in tabular format. The table shall indicate where each measurement was taken. An example of the tabular format shall be submitted by the Seller to the Buyer for approval prior to any measurements-of-record. The results of all UT inspections shall be made part of the final document package.

\subsubsection{Visual Inspections}

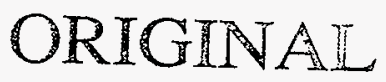

The Seller shall develop and implement a procedure to visually inspect all components of the MCO.

The Seller's procedure is to pay special attention to the threads and sealing surfaces (buttress threads on the locking ring and vessel collar, sealing surfaces of the flange covers, shield plug process ports, shield plug seal area, etc.). Inspection of mill finished, ground, or machined surface finishes shall be in accordance with an approved comparison sample/standard, and shall confirm that design drawing requirements are met.

Where visual inspection of the sealing surfaces reveals any scratches, the component shall be declared nonconforming. The part shall be dispositioned for rework or rejection. The Seller is to maintain a log of all visual inspections. 


\subsection{Helium Leak Tests}

\subsubsection{Helium Leak Test for MCO}

Upon completion of the $\mathrm{MCO}$ shell, the MCO shall be helium mass spectrometer leak tested in accordance with ANSI N14.5 meeting the fabrication test requirements and ASME Code, Section V, Article 10, Hood Method (hood to be covering the top of the installed shield plug, locking ring, and process port flange covers). The leak test shall include the metal seals for the first unit of each production run. Units following the first unit may be tested with elastomeric seals or equivalent that will not damage the seal surface for production use of the metal seals in the field assembly. Each $\mathrm{MCO}$ is to be tested at a minimum differential pressure of 15 psi using helium gas at a $99.9 \%$ minimum purity. The maximum acceptable leakage is $1 \times 10^{-7} \mathrm{std} . \mathrm{cm}^{3} / \mathrm{sec}$. The leak test shall be performed after the pressure test (Section 7.4.1).

The Seller shall provide the Buyer with written procedures for the leak test for approval prior to the start of testing. The results of each leak test shall be supplied to the Buyer.

\subsection{Performance Tests}

The Seller shall complete the following functional performance tests for the MCO:

\subsubsection{Shell - Basket Gauge Test - Test 1}

Following fabrication of the shell, the Seller shall perform a gauge test using the fuel assembly basket insertion gauge indicated in Section 7.6. The test shall consist of passing the gauge through the entire length of the $\mathrm{MCO}$ shell interior with a resistance force of 50 pounds or less to verify the clear opening of the MCO shell. Lubrication of the gauge or other methods of reducing friction are not allowed. The Seller shall fabricate the gauge and provide written certification that it meets the specified dimensional requirements.

\subsubsection{Diameter and Length Test - Test 2}

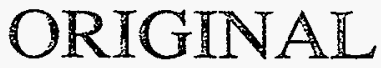

Following fabrication of the MCO shell assembly, the Seller shall perform a test to dimensionally verify that the outside diameter and length are within the specified acceptance limits.

The MCO outside dimensions shall be verified to ensure that the $\mathrm{MCO}$ does not exceed the maximum envelope dimensions specified on the drawings. This check may be made by optical survey means, or by the construction and use of an accurate full length gauge.

\subsubsection{MCO Basket Gauge Test - Test 3}

Following complete assembly of the $\mathrm{MCO}$, and with the $\mathrm{MCO}$ oriented in the vertical position, the Seller shall repeat Test 1 to demonstrate that the fuel assembly basket insertion gauge can be passed through the entire interior cavity length of the $\mathrm{MCO}$, with a resistance force of 50 pounds or less. 
This will also demonstrate that the as-built $\mathrm{MCO}$, complies with the dimensional requirements of this Fabrication Specification. The measured resistance force to achieve passage of the gauge shall be recorded and included in the MCO test report. The performance of this test shall be designated as a fabrication hold point in the Seller's manufacturing plan.

\subsubsection{Test 4}

\subsubsection{Shield Plug and Process Tube Insertion Test}

Following completion of the MCO fabrication, the Seller shall perform a test to verify fitup of the shield plug. With the MCO oriented in the vertical position and the shield plug suspended at its center grapple point, the shield plug and long Process Tube shall be oriented as required, and lowered into place inside the MCO shell. No mechanical assistance or lubricating materials are permitted. At the Buyer's option, this shield plug placement test shall be repeated if difficulty in placement of the shield plug is encountered.

\subsubsection{Shield Plug Radial Gap Test}

Following placement of the shield plug within the MCO shell assembly, the fit of the shield plug shall be verified by testing the minimum and maximum radial gap between the shield plug and the MCO shell assembly as shown on the Design Drawings. The minimum gap shall be zero. The maximum gap shall be $1 / 16^{\prime \prime}$ at any location. Any locations which cannot meet the minimum or maximum gap gauge test shall be brought into conformance by the Seller and the test indicated in 7.3.4.1 repeated. The performance of this test shall be designated as a fabrication hold point in the Seller's manufacturing plan.

\subsubsection{Locking Ring Fitup Test}

The completed MCO shall be fully assembled to verify fit up of shield plug, process ports, locking ring, bolts, etc. Metal seals shall not be installed during test. Care shall be taken not to damage seal surfaces.

\subsubsection{MCO Empty Weight Test - Test 5}

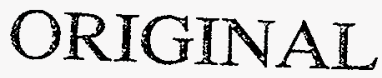

Following completion of the MCO fabrication, the Seller shall weigh the MCO components and record the weights. The dry weight of the following components shall be supplied to the Buyer.

- Fully assembled MCO less the shield plug.

- Shield Plug assembly.

- $\quad$ Locking Ring

- Canister Cover

- Process Tube 
Prior to performing the $\mathrm{MCO}$ weight tests, the calibration of the weighing device shall be verified. Calibration records for the weighing device shall be made available to the Buyer.

\subsubsection{Final MCO OD and Length Test - Test 6}

Following completion of MCO fabrication, the Seller shall repeat Diameter and Length Test to verify the inside length, and outside dimensions of the MCO. The performance of this test shall be designated as a fabrication hold point in the Seller's manufacturing plan.

\subsection{Pressure Test}

\subsubsection{Pressure Test for MCO Shell Assembly}

Upon completion of the $\mathrm{MCO}$, the assembly shall be tested in accordance with either Sub-Article NB-6200 Hydrostatic Tests or Sub-Article NB-6300 Pneumatic Tests of ASME Section III. The test shall be performed by internally pressurizing the $\mathrm{MCO}$ shell cavity to a test pressure of not less than 187.5 psig (i.e., $1.25 \mathrm{x}$ Design Pressure) for a hydrostatic test, or not less than 180 psig (i.e., $1.2 \mathrm{x}$ Design Pressure) for a pneumatic test. Elastomeric seals may be used instead of metallic seals

The Seller shall provide the Buyer with written procedures for the pressure test for approval prior to the start of fabrication. A written report documenting the results of the pressure test shall be supplied to the Buyer. The performance of this test shall be designated as a fabrication hold point in the Seller's manufacturing plan.

\subsection{Internal HEPA Filter Testing}

Perform a filter aerosol challenge to test the integrity of the filter manifold welds, filters, and flow path after the filter/manifold and protective cover plate installation on the shield plug. The final aerosol challenge tests shall be conducted in accordance with ASME N510-1989 "Testing of Nuclear Air Treatment Systems." Pretests may be performed prior to guard plate installation to permit weld repairs. The filter shall be cleaned with alcohol or other aerosol cleaning agent after each test. The documented filtering efficiency of the each shield plug and filter system shall be $99.97 \%$ DOP equivalent or better with an air flow rate of 35 ACFM. Test instrumentation shall be NIST traceable. Test report documentation of the final test (after protective shield plug guard plate installation) shall accompany each shield plug assembly and shall include, at a minimum, the specific shield plug part number, air flow rates, differential pressure across the assembly, test instrumentation NIST identification and calibration expiration dates, and the aerosol percent penetration of the shield plug assembly.

\subsection{Test Gauge Calibration}

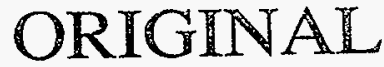

The test gauges shall be calibrated in accordance with the Seller's calibration procedures before and after each test. 
Table 7-1

TEST GAUGES

\begin{tabular}{|l|r|r|r|}
\hline \multicolumn{1}{|c|}{ Name } & \multicolumn{1}{c|}{ Outside Diameter } & Inside Diameter & Length \\
\hline MCO External 1* & $\mathrm{n} / \mathrm{a}$ & $25.310^{\prime \prime}$ & $158^{\prime \prime}$ \\
\hline MCO External 2* $^{*}$ & $\mathrm{n} / \mathrm{a}$ & $24.120^{\prime \prime}$ & $4.03^{\prime \prime}$ \\
\hline MCO External 3* $^{*}$ & $\mathrm{n} / \mathrm{a}$ & $24.940^{\prime \prime}$ & $149^{\prime \prime}$ \\
\hline MCO Internal $^{*}$ & $22.750^{\prime \prime}$ & $\mathrm{n} / \mathrm{a}$ & $28^{\prime \prime}$ \\
\hline Basket Envelope & $\mathrm{n} / \mathrm{a}$ & $22.700^{\prime \prime}$ & $28^{\prime \prime}$ \\
\hline Fuel Rack Hole & $2.570^{\prime \prime}$ & $\mathrm{n} / \mathrm{a}$ & $2.5^{\prime \prime}$ \\
\hline
\end{tabular}

"MCO external test gages may be integrated into a single gage at the manufactures discretion, with the buyer's approval of the gage design.

"Gage Outside diameter is 0.050 " larger than Basket Envelope gage inside diameter to ensure adequate clearance between actual baskets and the $\mathrm{MCO}$ interior shell.

\section{Notes:}

1. Gauges may be fabricated from steel plate or a rolled section. The minimum wall thickness of the gauges is 0.25 inches.

2. It is assumed that machining of the gauges will be required to achieve adequate dimensional control.

3. Sufficient care shall be taken to safeguard the gauges from damage when not it use. The gauges shall be dimensionally verified prior to each use. The gauges shall become the property of the Buyer at completion of the purchase contract.

4. If other than 304L (including 304 tools) stainless steel gages are used, all contact surfaces with the $\mathrm{MCO}$ components shall require cleaning and inspection to verify no contamination.

5. All listed dimensions of the gages are listed at the project's reference temperature, $72^{\circ} \mathrm{F}$.

6. If other than 304L stainless steel gages are used, then the test procedure must record and compensate for testing temperatures.

\subsection{REQUIRED ACCESSORIES}

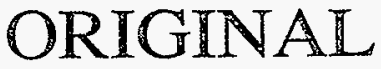

The bills of materials provided on the Design Drawings include a small number of items which are accessories for the MCO. Small parts shall be individually bagged in clear plastic. All accessories shall be identified by a metal tag securely attached to each part, or bag, and shall include the following information: 
A. Purchase Order number and Serial number (or Lot number). The serial number or lot number shall be traceable through the accompanying data package to a heat number where applicable.

B. Specific accessory part name, vendor, and part number.

C. Drawing reference, revision number, and part item number.

\subsection{QUALITY ASSURANCE REQUIREMENTS}

The Seller is required to have an acceptable Quality Assurance Program which shall be in effect throughout the fabrication, assembly, and testing of the MCO. This program shall be in accordance with section 3.6.2 of the Statement of Work. The Quality Assurance Program must be accepted by the Buyer prior to initiation of procurement of materials and fabrication.

The Seller's Quality Assurance Program shall apply to all components except as discussed in Section 9.1.3.

\subsection{Procurement of Materials}

The $\mathrm{MCO}$ are designated to be fabricated with a graded quality assurance program. The components are classified into three categories of safety significant classes (SSC). They are Safety Class (SC), Safety Significant (SS), and General Service (GS). The components of the MCO are classified in Table 9-1 in accordance with WHC-CM-4-4C, Safety Analysis Manual.

All materials shall conform to ASME specifications. Certified Material Test Reports (CMTR's) shall be provided for each heat (lot) used, and included in the final document package. Any discrepancies from the ASME specification requirements must be resolved before the material in question may be used.

Certified Material Test Reports shall, at a minimum, include the following test data: chemical analysis, yield strength, ultimate strength, elongation, and reduction of area.

The requirements for the procurement of the different quality categories, for the components on the Design Drawings, are listed in Table 9-1.

\subsubsection{Procurement of ASME Code Category "SC" Materials}

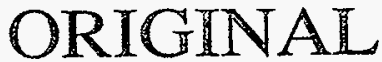

Category SC materials shall be procured from a supplier/manufacturer whose QA Program has been surveyed and/or audited by the seller to assure the program meets the applicable requirements of 10CFR71 Subpart H. QA programs meeting the requirements ASME Section III, NCA-3800, or the applicable requirements of NQA-1 are an acceptable basis for these surveys/audits. 
HNF-SD-SNF-DR-003, Rev. 0

Appendix 19

\subsubsection{Procurement of ASME Code Category "SS" Materials}

These requirements address all category "SS" materials. The four acceptable methods for the procurement of these materials are:

A. Procure material from a supplier/manufacturer holding a current ASME Section III Quality System Certificate (Materials).

B. Procure material from a supplier/manufacturer that has been surveyed and audited in accordance with the requirements of 10CFR71, Subpart H.

C. Procure materials from any supplier/manufacturer and perform all the examinations and tests required by the material specification. The Seller shall prepare and supply a CMTR stating that the material meets all the requirements of the specification.

D. Procure material from an ISO 9000 certificate holder.

\subsubsection{Procurement of Category "GS" Materials}

Category "GS" materials shall be procured from any source. A Certificate of Conformance shall be provided for all materials and items.

\subsection{Quality Control}

\subsubsection{Examination Point Program}

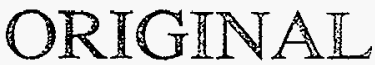

An examination point program shall be prepared and included in the Seller's manufacturing plan and submitted for approval in accordance with Section 11. This examination point program shall include a description of all examination points and hold points.

\subsubsection{Subcontractor QC Procedures}

The Seller's and the Seller's subcontractor QC procedures shall include the use of fabrication travelers or other process control documents. Fabrication travelers shall reference or describe the procedures used in accomplishing the tasks, the examination, the test requirements and any examination hold points. Fabrication travelers shall include hold and witness points for review by the Buyer.

\subsubsection{Dimension Control}

All dimensions, as indicated on the referenced Design Drawings, shall be measured and documented in accordance with the Seller's examination procedures. 


\subsubsection{Access to Facilities for QC}

The Buyer and the Owner, or the Owner's authorized agent shall have full access to the Seller's or the Seller's subcontractor's facilities for reviewing progress and determining acceptability of quality control activities.

\subsubsection{Access to Facilities for QA}

The Buyer and the Owner, or the Owner's authorized agent shall have full access to the Selier's or Seller's subcontractor's facilities for auditing the implementation of the Seller's Quality Assurance Program and for performing QC Surveillance of the MCO. Any findings resulting from audit or surveillance of the Seller's or subcontractor's facilities shall be addressed and promptly corrected to the Buyer's, Owner's, or the Owner's authorized agent's satisfaction.

\subsubsection{Nonconformance}

Nonconformances with purchase documents, drawings, approved procedures, or material requirements dispositioned as "Repair" or "Use-As-Is" shall be submitted to the Buyer for review and approval prior to implementation. The Seller shall provide a suggested technical justification for, and a recommended disposition, when submitting the Nonconformance to the Buyer. The use of electronic transmission devices, such as FAX or e-mail are acceptable methods for transmittal of nonconformance documents where time is critical, provided the transmission is legible. A hard copy must follow.

Any work that is done to a piece or assembly after the normal processing/fabrication steps are complete is considered rework. (This includes the use of filler material added to the MCO in order to rework surface defects and meet specifications.) The Seller shall keep a record (in the form of a map) of all locations where rework has been performed on an MCO. Note: Defects such as small pits or scratches less than 1/32" deep do not require rework (except for sealing surfaces). The seller shall report any nonconformances discovered after delivery of the MCO's in writing to the Buyer within 15 working days of discovery.

The accepted Nonconformance disposition shall include a technical justification provided by the Buyer.

\subsection{Quality Requirements for Shipping Release}

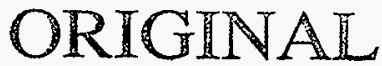

The MCO shall not be shipped until the following requirements are met:

A. All tests and examinations have been performed.

B. The MCO final documentation package is complete and approved by the Buyer's quality representative. 
C. The Seller shall notify the Buyer two (2) weeks before his intended shipping date and allow the Buyer sufficient time prior to shipment to review the documentation package as described in Section 11.3. The documentation package shall be complete and final before it is submitted for review, including documentation of the final performance tests. The Buyer reserves the right to witness repetition of any or all of the final performance tests 3 through 8 , and the pressure test, after the $\mathrm{MCO}$ and its documentation package have been completed.

\subsubsection{Certificate of Compliance}

With the final documentation package, the Seller shall submit to the Buyer, a Certificate of Compliance to this Specification and the Design Drawings. As a minimum, the Certificate shall include, but not be limited to the following information:

A. Purchase Order Number.

B. Procurement Specification and Design Drawing numbers, including any approved changes, and Nonconformances applicable to the equipment.

C. A certificate by the person who is responsible for the Seller's quality assurance function.

D. Provisions for the signature of the Buyer quality representative. The Buyer's signature is to indicate an agreement that the equipment and its documentation is ready for shipment, and does not constitute acceptance by the Buyer.

Table 9-1

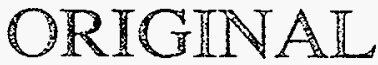

MCO MATERIAL QUALITY CATEGORY DESIGNATION

\begin{tabular}{|l|l|l|l|}
\hline $\begin{array}{c}\text { NOMENCLATURE/ } \\
\text { DESCRIPTION }\end{array}$ & CODE CRITERIA & \multicolumn{1}{|c|}{$\begin{array}{l}\text { SCC } \\
\text { DESIGNATION }\end{array}$} & \multicolumn{1}{|c|}{ QA CONDITION } \\
\hline MCO SHELL & SUBSECTION NB & SC & IMPORTANT TO SAFETY \\
\hline LOCKING RING & SUBSECTION NB & SC & IMPORTANT TO SAFETY \\
\hline $\begin{array}{l}\text { MCO SHIELD } \\
\text { PLUG }\end{array}$ & SUBSECTION NB & SC & IMPORTANT TO SAFETY \\
\hline $\begin{array}{l}\text { MCO BOTTOM ASS'Y } \\
\text { FORGING }\end{array}$ & SUBSECTION NB & SC & IMPORTANT TO SAFETY \\
\hline $\begin{array}{l}\text { MCO COLLAR } \\
\text { FORGNG }\end{array}$ & SUBSECTION NB & SC & IMPORTANT TO SAFETY \\
\hline MK1A & SUBSECTION NG & SC & IMPORTANT TO SAFETY \\
\hline MK1A SCRAP & SUBSECTION NG & SC & IMPORTANT TO SAFETY \\
\hline MKIV & NONCODE (ASTM) & GS & NOT IMPORTANT TO SAFETY \\
\hline
\end{tabular}


HNF-SD-SNF-DR-003, Rev. 0

Appendix 19

\begin{tabular}{|c|c|c|c|}
\hline $\begin{array}{l}\text { NOMENCLATURE/ } \\
\text { DESCRIPTION }\end{array}$ & CODE CRITERIA & $\begin{array}{c}\text { SCC } \\
\text { DESIGNATION }\end{array}$ & QA CONDITION \\
\hline MKIV SCRAP & NONCODE (ASTM) & GS & NOT IMPORTANT TO SAFETY \\
\hline PROCESS VALVES & SUBSECTION NB & SS & IMPORTANT TO SAFETY \\
\hline RUPTURE DISK & SUBSECTION NB & $\mathrm{SC}$ & IMPORTANT TO SAFETY \\
\hline $\begin{array}{l}\text { HEPA FILTER } \\
\text { EXTERNAL }\end{array}$ & $\begin{array}{l}\text { NONCODE, } \\
\text { COMMERCIAL GRADE }\end{array}$ & ss & NOT IMPORTANT TO SAFETY \\
\hline $\begin{array}{l}\text { BLIND FLANGES \& } \\
\text { BOLTS }\end{array}$ & SUBSECTION NB & SS & IMPORTANT TO SAFETY \\
\hline GASKETS & $\begin{array}{l}\text { NONCODE, } \\
\text { COMMERCIAL GRADE }\end{array}$ & SS & IMPORTANT TO SAFETY \\
\hline $\begin{array}{l}\text { CONDITIONING } \\
\text { CHAMBER FILTER } \\
\text { INTERNAL HEPA } \\
\text { FILTER }\end{array}$ & $\begin{array}{l}\text { NONCODE, } \\
\text { COMMERCIAL GRADE }\end{array}$ & SS & NOT IMPORTANT TO SAFETY \\
\hline LONG PROCESS TUBE & $\begin{array}{l}\text { NONCODE, } \\
\text { COMMERCIAL GRADE }\end{array}$ & SS & NOT IMPORTANT TO SAFETY \\
\hline $\begin{array}{l}\text { MCO BOTTOM ASS'Y } \\
\text { FORGING SPACER } \\
\text { PLATES }\end{array}$ & SUBSECTION NG & $\mathrm{SC}$ & IMPORTANT TO SAFETY \\
\hline LOCATOR CONE & SUBSECTION NG & $\mathrm{SC}$ & IMPORTANT TO SAFETY \\
\hline $\begin{array}{l}\text { PROCESS RELIEF } \\
\text { VALVE }\end{array}$ & $\begin{array}{l}\text { NONCODE } \\
\text { COMMERCIAL GRADE }\end{array}$ & SS & NOT IMPORTANT TO SAFETY \\
\hline $\begin{array}{l}\text { SHOVE PROCESS } \\
\text { TUBE }\end{array}$ & NONCODE (ASTM) & ss & NOT IMPORTANT TO SAFETY \\
\hline $\begin{array}{l}2 \text { MM PROCESS TUBE } \\
\text { SCREENS }\end{array}$ & NONCODE & SS & NOT IMPORTANT TO SAFETY \\
\hline ORIFICE PLATES & NONCODE & SS & NOT IMPORTANT TO SAFETY \\
\hline
\end{tabular}

\subsection{PACKAGING AND SHIPPING REQUIREMENTS}

\subsection{General Requirements}

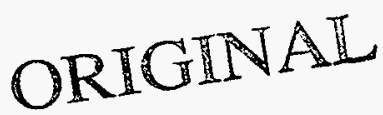

\subsubsection{Applicable ASME/ANSI Guidelines}

All packaging and shipping shall be in accordance with ASME NQA-1-1994 Edition, Subpart 2.2, "Quality Assurance Requirements for Packaging, Shipping, Receiving, Storage, and Handling of Items for Nuclear Power Plants." All packaging and shipping shall conform to Level C requirements or better. The Seller shall provide a packaging plan with details by component. 


\subsubsection{Shipping Information}

On the date of the MCO shipment, the Seller shall inform the Buyer of the following: the carrier bill of lading number, the routing and destination instructions, and a list of the items being shipped.

\subsubsection{Seller Liability For Shipping}

The Seller shall ensure that the as-fabricated, as-tested condition of the MCO is maintained, and that distortion or creep during shipment is prevented. Any damage or distortion of the $\mathrm{MCO}$ as a result of improper packaging, in transit handling, or shipping, shall be repaired at the expense of the Seller.

The MCO shall be packaged and shipped in such a manner that all components are present so Test 4 or 6, Section 7.3.4 or 7.3.6, respectively can be satisfactorily repeated upon receipt by the Buyer.

\subsection{Detailed Requirements}

\subsubsection{MCO, Locking Ring, and Shield Plug Preparation for Shipping}

The completed MCO shell shall be shipped with the locking ring (including jacking bolts) installed in the vessel collar. Before installing the locking ring, the surfaces of the jacking bolts shall be prelubed with a uniform film of Nuclear Grade (low halogen content) lubricant such as Nickel NeverSeez compound. Special care shall be taken to ensure that lube applicator brush bristles (or other contaminants) are not deposited on the threads. The locking ring jack bolts shall be installed fingertight, to full depth of thread. The locking ring and the canister collar buttress thread surfaces shall be lubricated with Neolube (graphite alcohol).

The shipping arrangement or palletization of the shield plug shall ensure that the HEPA filter manifold and basket stabilizer extension are protected during shipment. Rupture disks and relief valves shall be installed in the shield plug, and torqued to final values. Process valve plugs (without their threaded metal o-ring seal) shall be threaded in place finger-tight. Do not over tighten the process valve plugs to avoid galling the sealing surface. Process port flange covers (without their metal o-ring seals) shall be installed and the flange bolts tightened finger-tight. Flange cover bolt threads and process valve plug threads shall be pre-lubed with a uniform film of Nuclear Grade (low halogen content) lubricant such as Nickel Never-Seez compound. Special care shall be taken to ensure that lube applicator brush bristles (or other contaminants) are not deposited on the threads.

\subsubsection{Packaging, Segregation, and Labeling}

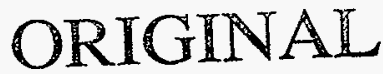

MCO components shall be packaged/segregated for shipment and storage according to the requirements specified in the following table, in addition to the requirements of NQA-1. Components on different rows of the table may not be packaged or crated together, with the exception of the locking ring ( $\mathrm{lb}$ ), which is to be shipped within the MCO shell as specified. Packages containing MCO vessels, shield plugs, process tubes, and Canister covers shall be packaged/palletized in a nesting manner so that shells remain stable when or if banding is removed, and suitable for forklift 
handling. Serial numbers of components shall be included in the outside identification marking, and component identification/serial numbers shall be clearly visible through overwrap.

\begin{tabular}{|c|c|}
\hline MCO Component & Component-specific Requirements \\
\hline 1a. MCO Shell & $\begin{array}{l}\text {-Two shells per package, side by side horizontally at the same level in the package. } \\
\text { Packages to be stackable up to three to four tiers deep. Shells should nest on } \\
\text { packaging to promote stability even after any banding is removed. Packages not to } \\
\text { exceed dimensions of } 36^{\prime \prime} \times 53 \text { "x } 168^{\prime \prime} \text {. }\end{array}$ \\
\hline 1b. Locking Ring & -Installed in the MCO shell at the factory; see Section 10.2.1. \\
\hline 2. Shield Plug & $\begin{array}{l}\text {-Each shield plug assembly to be shipped separately on a holder/pallet per constraints } \\
\text { specified in Section } 10.2 .1 \text {. Expected weight of shield plug assembly is approximately } \\
1657 \mathrm{lb} \text {, so a heavy duty pallet will be required. }\end{array}$ \\
\hline 3. Process Tube & $\begin{array}{l}\text {-Manufacturer may package multiple process tubes per package, but the total package } \\
\text { weight and dimensions may not exceed that of the MCO shell package described } \\
\text { above. Recommend crating of } 20 \text { tubes per } 12 \text { "x } 12 \text { "x } 156 \text { " package (crates will not be } \\
\text { stacked). }\end{array}$ \\
\hline 4. MCO Cap & -Packaged/shipped on pallets (two caps per pallet). \\
\hline $\begin{array}{l}\text { 5. Mark IA Basket } \\
\text { Mark IA Scrap Basket } \\
\text { Mark IV Basket } \\
\text { Mark IV Scrap Basket }\end{array}$ & - Loaded on pallets, with a maximum of two baskets per pallet. \\
\hline $\begin{array}{l}\text { 6. } 24 \text { " diameter shield } \\
\text { plug seal - } \\
\text { "helicoflex" seal }\end{array}$ & $\begin{array}{l}\text { Individually wrapped and packed in crates in conveniently sized lots. Recommend } \\
\text { crating in lots of } 20,24^{\prime \prime} \times 30^{\prime \prime} \times 30^{\prime \prime} \text { crate size maximum. }\end{array}$ \\
\hline 7. Small parts & $\begin{array}{l}\text { Wrapped/packaged as per discussion given in Section } 10.2 .3 \text { and packed in crates in } \\
\text { conveniently sized lots. These parts may be packaged together provided that the } \\
\text { outermost container identified all items and quantities contained within. }\end{array}$ \\
\hline
\end{tabular}

\subsubsection{Small Parts}

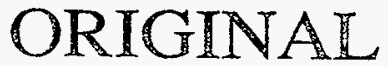

Small parts (such as flange cover seals, process plug seals, etc.) shall be bagged, boxed, bubble-packed or otherwise protected within the assembly packaging to protect against damage in transit, handling, and storage. Small parts packaging shall be clearly labeled with all relevant component identification information - part name, part number, drawing number, etc.

\subsubsection{Packaging and Shipping Procedures}

The Seller shall provide the Buyer with the written procedures (including drawings and engineering sketches as appropriate) for packaging, labeling, and shipping of the MCO assembly. The procedures shall include package lift point provisions and lifting weight. Seller shall be required to obtain Buyer approval of the packaging, labeling, and shipping procedures before shipment of MCO components. 


\subsection{Delivery}

Truck unloading will be the responsibility of the Owner. The truck may be unloaded within the Owner's protected area at a location designated by the Owner. At least twenty-four (24) hours notice of truck arrival shall be given to the Owner's receiving representative. In addition, the Seller shall comply with the specific delivery times mandated by Richland Operations.

\subsection{DOCUMENT AND RECORD SUBMITTAL REQUIREMENTS}

This section contains the requirements for document and record submittals for the MCO. Table 11-2 summarizes the required documents to be submitted for review and approval. Table 11-3 summarizes the required records to be submitted at the time of shipment.

\subsection{Documents}

\subsubsection{Shop Drawings}

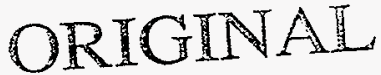

The Seller shall prepare shop drawings as necessary for normal shop practice for all assembly, subassembly, and piece parts associated with the MCO. The Seller shall prepare and submit one reproducible mylar and two prints of all shop drawings to the Buyer. If shop drawings are prepared using a computer-aided design (CAD) system, electronic copies of the drawing files shall also be submitted to the Buyer (AutoCAD Release 12 files are preferred).

The shop drawings are to be full-size and legible with uniform background density suitable for microfilming and subsequent reproduction from microfilm.

The shop drawings will be reviewed and, if satisfactory, will be approved. Drawings must be approved by the Buyer before fabrication begins. If not satisfactory, the Seller will be notified of the items requiring further explanation or correction; after which, one reproducible mylar and two prints of the corrected drawings and CAD files shall again be submitted for approval. The Seller shall appropriately note any changes by dated revisions on the drawing.

All shop drawings must be checked for accuracy prior to submission for approval. The approval of shop drawings shall not be construed as a complete check. Approval of shop drawings will not relieve the Seller of the responsibility for any error which may exist on the shop drawings. Approval of the shop drawings does not relieve the Seller of the responsibility for meeting the Buyer's design drawing requirements which are part of this specification.

If the Seller uses sub-contractors or sub-suppliers, the Seller is responsible for assuring that the subsupplier uses only Buyer-approved drawings for finished pieces, and that the sub-supplier meets all applicable specification requirements. 


\subsubsection{Drawing Standards}

Weld symbols shall be in accordance with the requirements of AWS A2.4.

The following Drafting Lettering Standards shall apply, such as supplemented by ANSI Y14.5 series:

A. Minimum character height (A, B, C, size drawings): 0.125 in. (1/8");

B. Minimum character height (D and E size drawings): 0.156 in. (5/32");

C. Minimum spacing between lines of characters: height of characters;

D. Machine and guide generated characters: 12 point size minimum;

E. Density of characters and lines: dense, sharp, and uniform.

\subsection{Examination and Test Procedures}

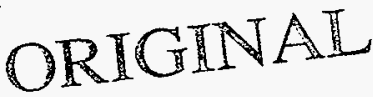

Examination and test procedures shall include identification of each characteristic or attribute evaluated, the measuring and test equipment used, the examination/testing set-up for each characteristic/attribute evaluated and other requirements as required by Code, Standard and Contract.

\subsection{Records}

The Seller shall submit for approval one sample document package for a complete $\mathrm{MCO}$ and all components to the Buyer. This sample package is to show the format for each required document, and the method of binding the documents. Buyer approval of sample document package is required prior to MCO fabrication.

The Seller shall assemble and submit to the Buyer, no later than one (1) to two (2) weeks prior to the MCO shipping date, a copy of the final records package. After the final records package has been approved by the Buyer's QA representative, two (2) copies shall be prepared. The original copy of the final document package shall be provided to the Buyer and the second copy shall be shipped with the MCO.

\subsubsection{General Requirements For Document Packages}

Each Document Package shall comply with the following general requirements:

1. Each package shall have a Table of Contents.

2. Each page of the document package is to be consecutively numbered and marked with the unique MCO serial number. 
3. All records must be legible, including signatures, (signature must be legible and or typed/printed) inspection stamps, page numbers, etc. Copies of records like Certified Material Test Reports will not be acceptable if legibility is in question.

4. Each piece of the MCO (shield plug, vessel, locking ring, process tube, etc) shall be identified with a serial number so traceability to inspection results can be maintained.

5. Inspection records shall include M\&TE traceability, M\&TE serial number, and calibration due date.

6. The Seller shall sign the Conformance Statement before presenting the items and document package to the Buyer for final inspection prior to shipment.

7. All recorded information shall be typed or recorded neatly in black ink.

8. Corrections may be made using a single line through the error, making the correction, and providing name and date. No correction fluid or tapes may be used on submitted original records.

9. Unacceptable records found upon receipt by the Buyer shall be replaced by the Seller at the Seller's expense in an expedited manner.

10. The document package shall include, but not be limited to the items identified in Tables 11-1, 11-2, and 11-3.

11. The Seller shall prepare and submit for approval by the Buyer, an Index of Records. The Index of records shall include the records identified in Table 11-3, as well as any documents used by the Seller's QA Program to trace the record to the equipment.

12. All test reports and test results shall be compared to the acceptance criteria. These reports shall contain the signature of the authorized representative or the agency performing the tests. The reports are for the Buyer review and acceptance, prior to release for shipment.

13. Functional test results shall include signed-off copies including signatures on these reports by the authorized representatives or by the agency personnel performing the tests.

14. Certified material test reports with actual chemical and physical properties for each heat number for all material (i.e., piece traceability shall be provided.). Physical properties test data shall include yield strength, ultimate strength, reduction of area, and elongation.

15. Certification of compliance that each non-evidence producing form of NDE (i.e., UT, and PT) has been by specified requirements and ASNT qualified operators. 
16. A complete radiography history including exposure diagram, reader sheet, record of defects, record of repairs, and final cleared exposure record shall be submitted to the Buyer. Film and reader sheets should be marked or noted to show any condition other than normal (i.e., surface conditions or defects within acceptance standards, etc.) and marked to show the level qualifications of the reader. Final film shall be sent to the Buyer.

17. Records of all repairs as required by Section III of the ASME Code.

18. Record of all nonconformances affecting the Seller's approved drawings and specifications, if any, along with the Buyer's supplied technical justification for acceptance.

19. Leak testing shall be performed and documented per ANSI N14.5.

20. Following satisfactory completion of all examinations and tests, the Seller shall prepare a complete set of as-built dimension documentation. This may be done by as-built drawings or check list with recorded dimensions. All dimensions shall be verified to be within the Design Drawing tolerances specified. Critical dimensions, as specified on Table 11-1 shall be measured and recorded on the as-built drawings or on separate examination sheets. As-built drawings, if accompanied by dimensional examination documents recording critical dimensions, may consist of the final revision of the shop drawing depicting the as-built condition, including nonconformances (i.e., use-as-is and repair). All as-built shop drawings shall be certified as to correctness.

21. Records of personnel qualification such as welding, NDE, etc.

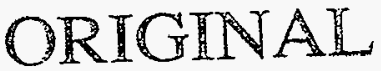

22. Records of the calibration of measuring and testing equipment used for acceptance.

23. Copies of all approved MCO fabrication instructions, procedures, and drawings.

24. Pressure testing shall be performed and documented per ASME Section III, Division 1, Subsection NB, Article 6000 .

Table 11-1

AS-BUILT DIMENSION REPORTS

\begin{tabular}{|l|l|}
\hline \multicolumn{1}{|c|}{ PART } & \multicolumn{1}{c|}{ Corresponding Critical Dimension Table } \\
\hline MCO Shell Assembly & 6.3.6-1, MCO Shell Critical Dimensions \\
\hline Process Tube & 6.3.6-2, Process Tube Critical Dimensions \\
\hline Shield Plug & $6.3 .6-3$, Shield Plug Critical Dimensions \\
\hline Locking \& Lifting Ring & $6.3 .6-4$, Locking \& Lifting Ring Critical Dimensions \\
\hline Canister Cover & $6.3 .6-5$, Canister Cover Critical Dimensions \\
\hline
\end{tabular}




\begin{tabular}{|l|l|}
\hline \multicolumn{1}{|c|}{ PART } & \multicolumn{1}{|c|}{ Corresponding Critical Dimension Table } \\
\hline Mark IA Storage & $6.3 .6-6$, Mk IA Storage Basket Critical Dimensions \\
\hline Mark IA Scrap & $6.3 .6-7$, MK IA Scrap Basket Critical Dimensions \\
\hline Mark IV Storage & $6.3 .6-8$, MK IV Storage Basket Critical Dimensions \\
\hline Mark IV Scrap & $6.3 .6-9$, MK IV Scrap Basket Critical Dimensions \\
\hline
\end{tabular}

Table 11-2

DOCUMENT SUBMITTAL LIST

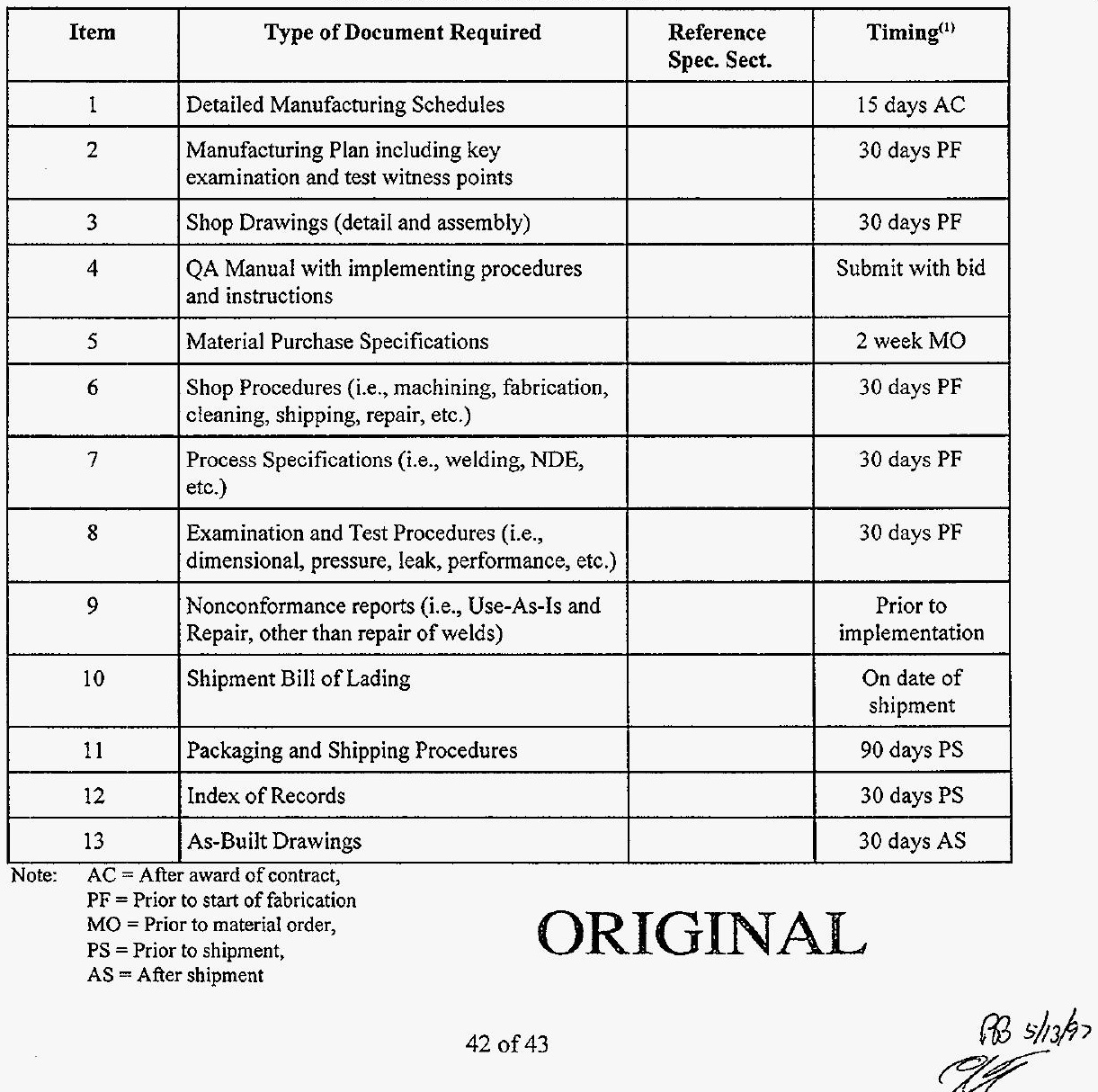


Table 11-3

RECORDS SUBMITTAL LIST

\begin{tabular}{|c|c|c|}
\hline Item & Type of Document Required & Reference Spec. Sect. \\
\hline 1 & Index of records & \\
\hline 2 & $\begin{array}{l}\text { Either Certified Material Test Reports or } \\
\text { Certificates of Compliance, as required by the } \\
\text { Specification }\end{array}$ & \\
\hline 3 & Filler Metal Certification & \\
\hline 4 & Repair Records & \\
\hline 5 & $\begin{array}{l}\text { Examination Records and Reports, including } \\
\text { Receiving Examination and Source Examination }\end{array}$ & \\
\hline 6 & Travelers and/or Checklists & \\
\hline 7 & Nonconformance Reports (Use-As-Is and Repair) & \\
\hline 8 & Performance Test Reports & \\
\hline 9 & Dimensional Examination Reports & \\
\hline 10 & Weight Certificates & \\
\hline 11 & 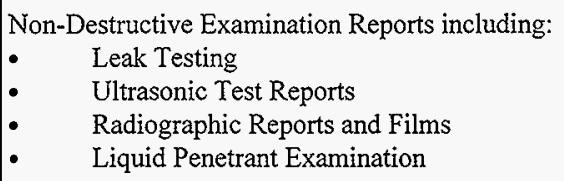 & \\
\hline 12 & Certificate of Compliance & \\
\hline 13 & As-Built Documents (including shop drawings) & \\
\hline 14 & Personnel Qualification Records & \\
\hline 15 & $\begin{array}{l}\text { Examination and Test Equipment Calibration } \\
\text { Reports }\end{array}$ & \\
\hline 16 & $\begin{array}{l}\text { Copies of All Approved MCO Fabrication } \\
\text { Instructions and Procedures }\end{array}$ & \\
\hline 17 & Pressure Test Report & \\
\hline
\end{tabular}




\section{MULTI-CANISTER OVERPACK}

FABRICATION SPECIFICATION

APPENDICES

A19-A-i 
HNF-SD-SNF-DR-003, Rev. 0

Appendix 19

\section{APPENDIX 1}

(Intentionally left Blank) 
HNF-SD-SNF-DR-003, Rev. 0

Appendix 19

\section{APPENDIX 2}

MCO INTERNAL HEPA FILTER SPECIFICATION (DESH) 


\section{MCO INTERNAL HEPA FILTER SPECIFICATION}

\subsection{INTRODUCTION}

\subsection{BACKGROUND}

The MCO internal filter is required for radioactive contaminant filtration inside the MCO to protect process equipment from contamination. The filter(s) will be filtering a saturated steam atmosphere at steam flows of 35 cubic feet per minute (cfm) at temperatures not to exceed $375^{\circ} \mathrm{C}$ inside a vacuum chamber. The filter(s) shall provide HEPA removal efficiency of 0.3 micron $(\mu \mathrm{m})$ particles and have a 40 gram loading capacity with 10 inches water column differential pressure at $35 \mathrm{~cm}$ air flow. The filter(s) shall be sized such that 3.5 inches water column differential pressure at $35 \mathrm{cfm}$ air flow clean. The filter(s) shall be capable of regeneration by back flow of gas or liquid, and be moisture repellant to saturated steam. The filter/manifold(s) shall not be larger than the envelop described in drawing H-2-828049 and be no larger than 2.6 inches in diameter (see drawing for conceptual filter arrangement scheme). The filter/manifold(s) shall be constructed of all 316 stainless steel. The filter structure shall also withstand a $100 \mathrm{~g}$ drop and maintain a minimum of $50 \%$ of flow and filter loading capacity but HEPA efficiency is not required to be maintained after the drop.

\subsection{REQUIREMENTS}

\subsection{FILTER DOCUMENTATION REQUIREMENTS}

The bidders shall provide documentation and/or test results for the following requirements equivalent to or superseding the documents described below with their proposals:

\subsubsection{Filter Airflow Resistance}

Test reports or flow versus differential pressure curves for a clean airflow (Helium, steam) to meet 3.5 inches water column at $35 \mathrm{ACFM}$ air.

\subsubsection{Filter Removal Efficiency}

Test reports documenting DOP, or other approved test material, $0.3 \mu \mathrm{m}$ particulate removal efficiency of $99.97 \%$.

\subsubsection{Filter Loading Capacity}

Test reports documenting filter loading capacity in accordance with standard SAE J726 or ASHRAE 52-76 using ASHRAE fine test dust.

\subsubsection{Filter Moisture Repellency}

Test report shall document filter efficiency and differential pressure performance while subjected to water spray during ASHRAE particulate loading per Section 2.2.3. 


\subsubsection{Filter Regenerability}

Test reports documenting filter regeneration (minimally $70 \%$ clean differential pressure, loading capacity recovery, and $99.97 \%$ particulate removal efficiency after regeneration) per ASHRAE 5276.

\subsubsection{Filter Rough Handling}

Certificate of Conformance, test reports, or engineering calculations shall be provided confirming the filter will withstand a $100 \mathrm{~g}$ drop or 100 times the filter(s) weight in bending and maintain $50 \%$ of original rated flow and $50 \%$ of filter loading capacity after the drop. The filter shall sustain no visible integrity loss (cracks or punctures in the filter media, endcaps, or manifold hardware.)

\subsubsection{Filter Heating Resistance}

Test reports documenting resistance to heated air and maintain particulate removal efficiency during 240 hours of $375^{\circ} \mathrm{C}$ per standards UL-586 and MIL-F51068.

\subsection{FILTER DESIGN REQUIREMENTS}

\subsubsection{Filter Airflow Resistance .}

The filter(s) shall be sized for a clean airflow (Helium, steam) of 3.5 inches water column at 35 ACFM air.

\subsubsection{Filter Removal Efficiency}

The filter(s) shall be designed to withstand a DOP challenge, or other approved test material, of 0.3 $\mu \mathrm{m}$ particulate and remove $99.97 \%$ of the particulate.

\subsubsection{Filter Loading Capacity and Surface Area Maximization}

The filter(s) shall provide maximum resistance of 10 inches water column when loaded with 40 grams of ASHRAE fine test dust at 35 ACFM air in accordance with standard SAE J726 or ASHRAE 52-76. The filter(s) shall also be designed to provide a maximum surface area square footage.

\subsubsection{Filter Moisture Repellency}

The filter(s) shall provide maximum repellency of 10 inches water column when the filter is challenged with supersaturated steam containing a minimum 1 gram of entrained water per I cubic foot of air at 35 ACFM.

\subsubsection{Filter Regenerability}

The filter(s) shall be capable of reverse back pulsing with gas to regain minimally $70 \%$ loading 
capacity as specified in Section 2.2.2 and differential pressure and $99.97 \%$ particulate filtration per ASHRAE 52-76. The gas reverse back pulse shall consist of rapid application of reverse flow of helium or argon to the filter manifold (all filters) from a reservoir with a maximum size of 5 cubic feet, at a maximum initial pressure of 100 psi through a 20 foot Jength of 1 inch ID maximum. discharge line.

\subsubsection{Filter Heating Resistance}

The filter shall be designed to with stand resistance to heated air and maintain particulate removal efficiency during 240 hours of $375^{\circ} \mathrm{C}$ per standards UL-586 and MIL-F51068.

\subsubsection{Filter Design Basis Accident Functionality}

The filter/manifold(s) shall be designed to withstand a $100 \mathrm{~g}$ drop or 100 times the filter(s) weight in bending and maintain $50 \%$ of original rated flow and $50 \%$ of filter loading capacity after the drop. The drop or force loading to the filter shall be applied midway on the filter in a radial direction normal to the longitudinal filter axis. The filter shall also be designed to physically withstand forces related to a $1035 \mathrm{kPa}(150 \mathrm{psig})$ pressure spike transient and maintain $50 \%$ flow and $50 \%$ loading capacity after the pressurized pulse. The intent of this requirement is to maintain a high removal efficiency (non-HEPA rated) by assuring no visible holes, punctures, cracks, or extensive deformations that circumvent or block the filter flow path.

\subsection{CONSTRUCTION REQUIREMENTS}

\subsubsection{Filter Media}

The filter(s) shall be all stainless steel construction--316L, or other suitable corrosion resistant materials. Filter end caps (if required) shall be constructed from $316 \mathrm{~L}$ stainless steel and $\mathrm{N} O \mathrm{~T}$ aftached to filter media by epoxies or sealants. Rolled or metallic end cap bonding such as welding or brazing may be appropriate. If weld bonding is utilized, it is suggested that low carbon base materials such as $316 \mathrm{~L}$ or other similar corrosion resistant materials be used.

\subsubsection{Filter Mounting Hardware}

The filter(s) mounting hardware (i.e. supports, manifolds, etc.) shall be constructed from $316 \mathrm{~L}$ stainless steel, other low carbon base, or corrosion resistant material (painted surfaces shall not be acceptable). The filter(s) shall be mounted to manifold in such a manner to prevent build-up of excess free water inside the manifold. The filter(s) shall be mounted near ( $+1-0.0625$ inches) the bottom of the manifold and the manifold angled such that any free water in the manifold will drain back into the filter media. The total weight of the filter and mounting hardware shall not exceto $22.7 \mathrm{~kg}(50 \mathrm{lbs})$. Refer to drawing H-2-828049 for filter envelop space, mounting suggestions, and additional construction suggestions.

\subsubsection{Filter Environmental Conditions}


The filters shall be designed and construsted to withstand the following environmental conditions:

- Design Basis Accident equal to a $100 \mathrm{~g}$ drop or 100 times the filter(s) weight in bending per design stated above;

- Temperature ranges from -20 to $375^{\circ} \mathrm{C}\left(375^{\circ} \mathrm{C}\right.$ for a time period of 240 hours);

- Internal to external accidental pressure spike transient of $1035 \mathrm{kPa}(150 \mathrm{psig})$ as described above; and

- All gas atmospheres such as Nitrogen, Hydrogen, Helium, Argon, Oxygen, air, and stezm.

\subsection{OPTIONS}

3.1 Optional bids may be submited for filters meeting the following design conditions:

Option \#1: $35 \mathrm{acfm}$ flow at $6.0^{\prime \prime}$ water column with $0.3 \mu \mathrm{m}$ efficiency

Option $\ddot{*} 2: 35$ acfm flow at $3.5^{\prime \prime}$ water column with $0.5 . \mu \mathrm{m}$ efficiency

All other specification requirements remain in force for the optional bids. 


\section{APPENDIX 3}

\section{POTENTIAL RUPTURE DISK SUPPLIERS}

Address:

Fike Metal Products

704 South 10 th Street

Blue Springs, MO 64015

Telephone:

(816) 229-3405

Fax:

(816) $228-9277$

Contact:

Jason Patterson

Arthur Forsyth Co.

(206) $283-5716$ phone

(206) 284-7269 fax

Address: $\quad$ Continental Disc Corporation 3160 West Heartland Drive

Liberty, MO 64068

Telephone:

(816) $792-1500$

Fax:

(816) $792-5447$

Contact:

Michael Pruitt 


\section{APPENDIX 4}

\section{CARBONE LORRAINE}

Product: Helicoflex Seals

Model/Part No.: H-305236 REV NC

Address: $\quad$ Helicoflex

2770 The Boulevard

P.O. Box 9889

Columbia, SC 28209

Telephone: $\quad$ (803) 783-1880

Fax: $\quad$ (803) $783-4279$

Contact: Michel LeFrançois 


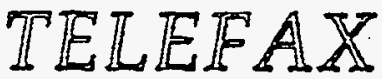

From: Mitchel LEFRANÇOIS

copy :
To: Chuck TEMUS

Compeny: VECTPA TECHNOLOGIES

Addrese;

countryistata: WA

Toletax is 2068742401

Columbla, Maren 3, 1997

\section{Subjoe:: MCO CASK SEAL / HELICOFLEX H-305236 REV NC}

Re1.:

Dear Sir,

Re. your recent contact with Gerand Anthoice, please find sketch attacbed ineluding seal description.

Please do not hesitate to call we at the number above if you bave any question.

Yours Sincercly,

Miched Lefrançois 


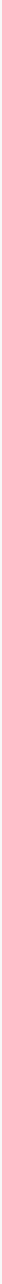




\section{APPENDIX 5}

\section{EG\&G PRESSURE SCIENCE}

Product:

Model/Part No.:

Address:

Telephone:

Fax:

Contact:
C-Seal

EG\&G Pressure Science

11642 Old Baltimore Pike

Beltsville, MA 20705-1294

(301) $937-9654$

(301) $937-7027$

Jeff Layer 
Contact Person : Jeff Layer

Phone / fax : (301) 937-9654 / (301) 937-7027

Address : EG\&G Pressure Science

11642 Old Baltimore Pike

Beltsville Md. 20705

\section{Part Number Information}

- Modified Series 80 C-Seal (Boss Size $\$ 24$ ) PSI part number $\underline{13632}$

- inch O.D. silver plated C-Seal

PSI part number $\underline{13503}$

- Series 80 C-Seal (Boss Size

PSI part number 801A91-0012 (INCO 718)

- Series 80 C-Seal (Boss Size \#4)

PSI part number 801A91-0004 (INCO 718) 
APR -10-97 THU 16:05

EG\&G

FAX NO. 3019377027

P. 04

HNF-SD-SNF-DR-003, Rev. 0

PERMISSION TO USE PAGE 23

Appendix 19

EG\&G Pressure Science grants Duke Engineering Services Hanford and Parsons permission to use page 23 of our product catalog in any reports or publications necessary.

Jeff Layer

Engineering Manager

EG\&G Pressure Science

11642 Old Baltimore Pike

Beltsville Md. 21797

Signed : Jeff Layer

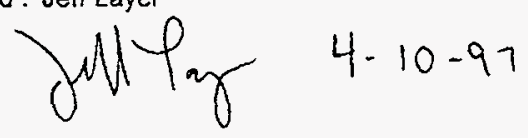

CC:

Transmitted by :

Dato.

A19-A5-3 
Type Fittings \& ANO 10050 (MS33649) Type Bosses.

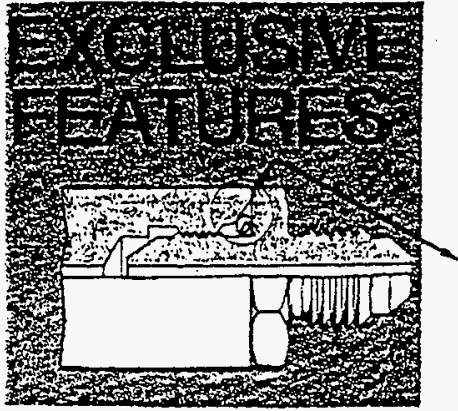

FOOLPROOF, its symmetrical and slips right on in either direction at installation.

IT'S SELF ALIGNING and installs easily regardless of attitude of the boss.

1 FOR EASY RE-USE, it stays with the fitting after the first compression.

READILY" "UNSCRENS" from the fitting when you want to discard it.
(Supersedes Series 70)

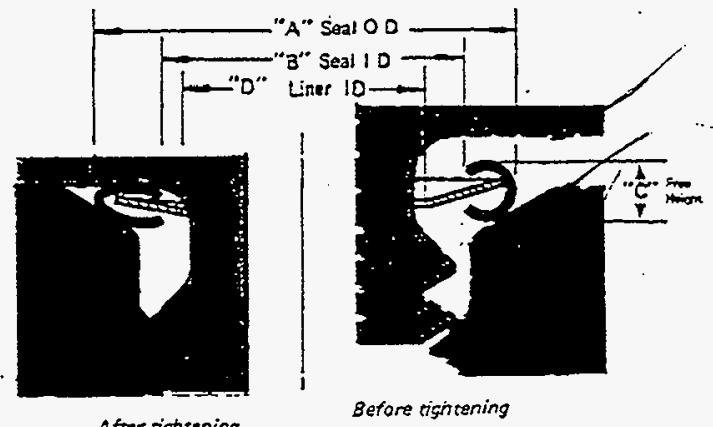

After rightening

Before tigntening
LOAD IS NOT TRANSWITTED from the fittung to the boss through the sea!.

DOES NOT REOUIRE HIGH LOAD to effec: $a$ tight seal.

SEAL IS PRESSURE ENERGIZED.

NO MARKING OR FRETTING of the boss or the fitting.

HOW TO SPSCIFY:

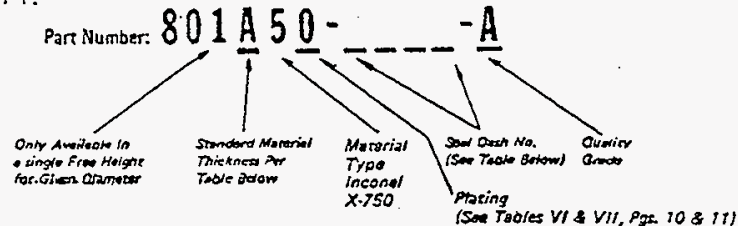

\begin{tabular}{|c|c|c|c|c|c|c|}
\hline $\begin{array}{c}\text { Boss } \\
\$ 125 \times 0\end{array}$ & $\begin{array}{c}\text { SEAL } \\
\text { OASHNQ }\end{array}$ & SEAL & SEAL 1.0 & $\begin{array}{c}\text { SEAL } \\
\text { FREE HEIGHTा } \\
\text { "C }\end{array}$ & $\begin{array}{l}0 \\
\text { Liner } \\
\text { t.t. }\end{array}$ & 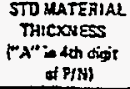 \\
\hline $\begin{array}{l}2 \\
3 \\
4 \\
5\end{array}$ & $\begin{array}{l}\infty 002 \\
\infty 003 \\
\infty 004 \\
\infty \infty 05\end{array}$ & $\begin{array}{l}.381 \\
.444 \\
.506 \\
.569\end{array}$ & $\begin{array}{l}.302 \\
.365 \\
.427 \\
.490\end{array}$ & $\begin{array}{l}.046 \\
.046 \\
.046 \\
.048\end{array}$ & $\begin{array}{l}.278 \\
.341 \\
.397 \\
.459 \\
\end{array}$ & $\begin{array}{l}.06 \\
.008 \\
.008 \\
.08\end{array}$ \\
\hline $\begin{array}{l}8 \\
7 \\
8 \\
9\end{array}$ & $\begin{array}{l}0006 \\
0007 \\
0008 \\
0009\end{array}$ & $\begin{array}{l}.831 \\
.894 \\
.819 \\
.882\end{array}$ & $\begin{array}{l}.552 \\
.615 \\
.740 \\
.803\end{array}$ & $\begin{array}{l}.046 \\
.048 \\
.048 \\
.048\end{array}$ & $\begin{array}{l}.67 \\
.579 \\
.899 \\
.761\end{array}$ & $\begin{array}{l}005 \\
006 \\
008 \\
.008\end{array}$ \\
\hline $\begin{array}{l}70 \\
11 \\
12 \\
14\end{array}$ & $\begin{array}{l}0010 \\
011 \\
\infty 12 \\
\infty 14\end{array}$ & $\begin{array}{l}.348 \\
1.100 \\
1.158 \\
1.291\end{array}$ & $\begin{array}{l}.885 \\
1.021 \\
1.051 \\
1.176\end{array}$ & $\begin{array}{l}.0 \% 6 \\
.046 \\
.082 \\
.062\end{array}$ & $\begin{array}{r}.577 \\
932 \\
.995 \\
1.120\end{array}$ & $\begin{array}{l}\text { Dos } \\
\text { D15 } \\
\text { D10 } \\
\text { D10 }\end{array}$ \\
\hline $\begin{array}{l}16 \\
10 \\
20 \\
24\end{array}$ & $\begin{array}{l}\text { कotb } \\
0018 \\
0020 \\
0024\end{array}$ & $\begin{array}{l}1.406 \\
1.593 \\
1.718 \\
1.968\end{array}$ & $\begin{array}{l}7.101 \\
1.488 \\
.1 .813 \\
1.963\end{array}$ & $\begin{array}{r}.062 \\
062 \\
.062 \\
.052\end{array}$ & $\begin{array}{l}1.245 \\
1.492 \\
1.557 \\
1.807\end{array}$ & $\begin{array}{l}\text { Dito } \\
\text { oto } \\
010 \\
010\end{array}$ \\
\hline $\begin{array}{l}28 \\
32\end{array}$ & $\begin{array}{l}0028 \\
0032\end{array}$ & $\begin{array}{l}2.343 \\
2.594\end{array}$ & $\begin{array}{l}2.238 \\
2489\end{array}$ & $\begin{array}{l}.002 \\
.052\end{array}$ & $\begin{array}{l}2182 \\
2.432\end{array}$ & D10 \\
\hline
\end{tabular}

Series 80 Saals aro groduced in only Two Guality Grades:

A - Best Seal Ling Finikn

B - Las Than Best Sod Line Finish.

Tolerencts ats the same for both gracter.

Both work at High and Low presurs,

Setect How A for critical leskapo. Claxx $B$ for low cost. 

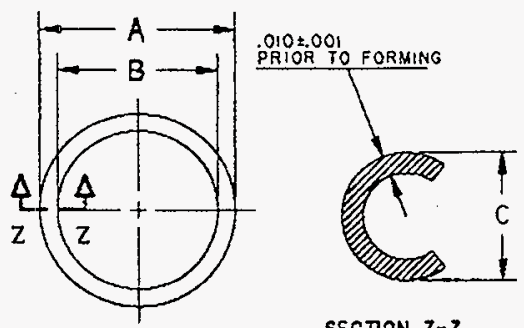

\begin{tabular}{|c|c|c|c|}
\hline \multicolumn{4}{|c|}{ REVISIONS } \\
\hline LIR & OATE & $E C O N O$. & APPROYAL \\
\hline & & & \\
\hline & & & \\
\hline & & DIMENSION & \\
\hline & $\begin{array}{l}\text { AVERAGE O.D. } \\
\text { - A- } \\
\text { SEE NOTE ¿ }\end{array}$ & $\begin{array}{l}\text { AYG. MIN, I.D. } \\
\text { - B- } \\
\text { SEE NOTE (2) }\end{array}$ & $\begin{array}{l}\text { FREE } \\
\text { HEIGHT } \\
-C-\end{array}$ \\
\hline & $2.062 \div .00$ & 1.969 & $.062 \pm .002$ \\
\hline
\end{tabular}

NoTES.

1. TIS WELOING PRIOR-TO-FORMING PERNITTEO.

(2) MAXIMUM OUT-OF-ROUNCNESS OF DIAMETERS: .016

3 INDIVIDUALLY PACKAGE DER STANDARO PRESSURESCIENCE METHOOS.

4. MARX EACH PACXAGE AS SPECIFIEO ON SALES OROER.

5. SEALING SURFACES TO CONFORM TO QCS 85345 .

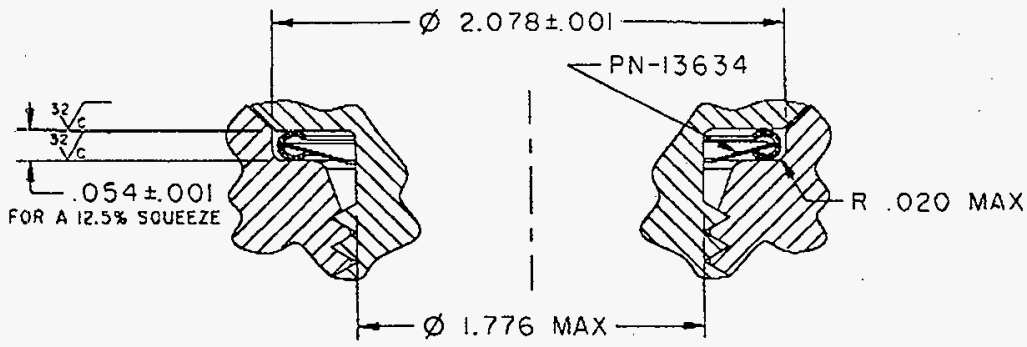

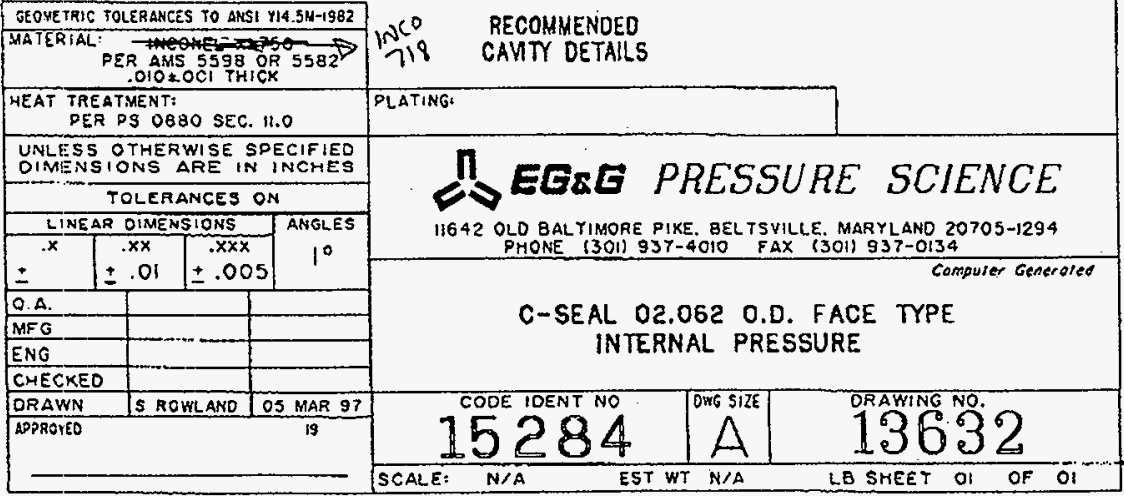




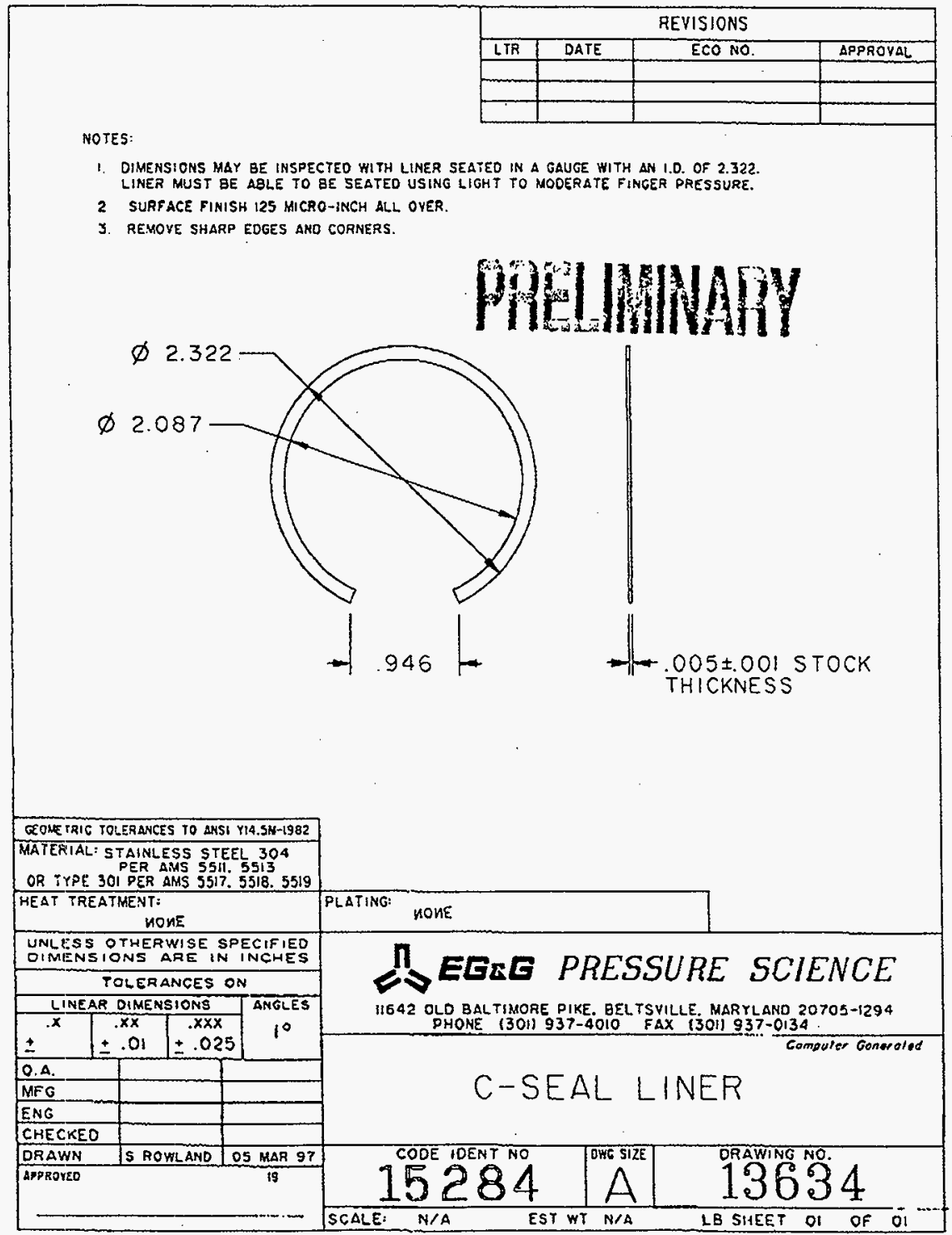




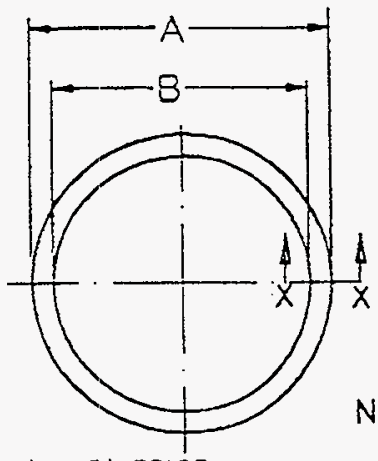

\begin{tabular}{|l|c|c|c|}
\hline \multicolumn{3}{|c|}{ REVISIONS } \\
\hline LTR & OATE & ECO NO. & APPR'L \\
\hline & & & \\
\hline & & & \\
\hline & & & \\
\hline
\end{tabular}

(.015) PRIOR

TO FORMING 72

\begin{tabular}{|c|c|c|c|}
\hline $\begin{array}{l}\text { SEAL } \\
\text { DIMENSIONS }\end{array}$ & $\begin{array}{r}\text { AVG } 0.0 . \\
-A-A \\
\text { SEE NOTE }\end{array}$ & $\begin{array}{c}\text { AVG.NIN I.D } \\
-B-A \\
\text { SEE NOTE }\end{array}$ & 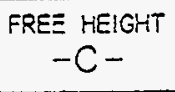 \\
\hline $\begin{array}{l}\text { BEFORE } \\
\text { PLATING }\end{array}$ & $3.520 / 3.510$ & 3.310 & $.128 / .122$ \\
\hline $\begin{array}{l}\text { AFIER } \\
\text { PLATING }\end{array}$ & $(3.519)$ & $(3.305)$ & $(.129)$ \\
\hline
\end{tabular}

NOTES:

1. REOUIRED OUT OF ROUNDNESS OF DIAMETERS : .100-.130 2. PLATING RECUIRED OVER THIS AREA AS SPECIFIEO.

3. PLATING OPTIONAL ANO MAY EE INCOMPLETE IN THIS AREA.
4. INDIVIOUALLY PACKAGE PER PSI SPECIFICATION.

4. INDIVIOUALLY PACKAGE PER PSI SPECIFICATION.
5. MARK EACH PACKAGE AS SPECIFIED ON SALES ORDER.

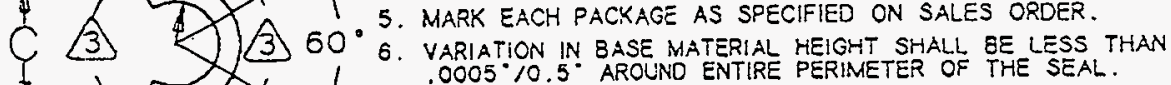

7. TIG WELDING PRIOR TO FORMING PERMITTED.

2

SECT . $x-x$

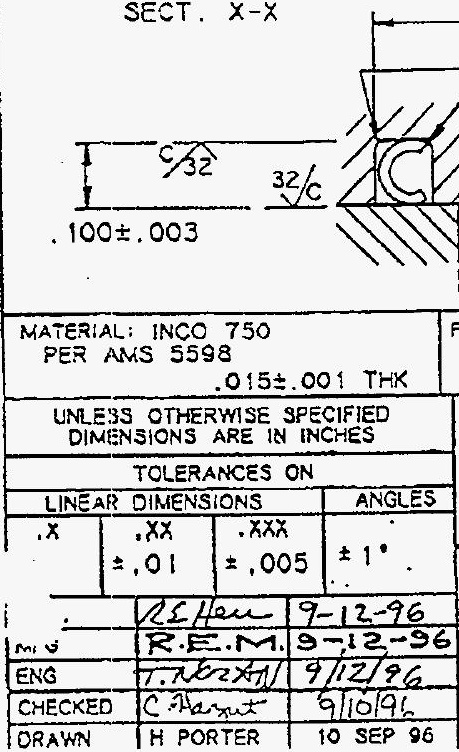

$\$ 3.555 \pm .005$ R .045 MAX.

$\mapsto \varnothing 3.250$ MAX $\rightarrow$

\section{PLATING: HEAT TREATMENT:}

SILVER PER PSA 0900 $.0015-.0025$ THK

\section{EG\&G PRESSURE SCIENCE}

11642 OLD 8ALTIMORE PIKE: BELTSVILLE, MARYLAND 20705-1294 PHONE (301) $937-4010$ FAX (301) 937-0134

$$
C-S E A L
$$

3.520 O.D. FACE TYPE. INTERNAL PRESSURE

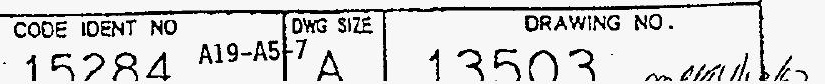




\section{APPENDIX 6 \\ JOHNSON WELL WIRE (WHEELABRATOR ENGINEERED SYSTEMS, INC.)}

Product:

Model/Part No.:

Address:

Telephone:

Fax:

Contact:
Well Wire \& Strainer

Parts are custom. Please refer to the Design Drawings for specific details.

Wheelabrator Engineered Systems, Inc.

Johnson Screens

22020 Chaparral Lane

Castro Valley, CA 94552

(510) $581-8577$

(510) 581-8622

Dan McGee 


\section{APPENDIX 7}

\section{INDUSTRIAL SCREEN}

$\begin{array}{ll}\text { Product: } & \text { Screen } \\ \text { Model/Part No.: } & \text { See Drawing } 5873 \\ \text { Address: } & \begin{array}{l}\text { Industrial Screen Products, Inc. } \\ \text { P.O. Box 10673 } \\ \text { Zephyr Cove, NV } 89448\end{array} \\ \text { Telephone: } & (800) 663-2702 \\ \text { Fax: } & (800) 663-8060 \\ \text { Contact: } & \text { Thomas Anderson }\end{array}$


$01 / 17 / 1997 \quad 11: 03$

$915-626-4375$

ISP INC

PAGE 01

HNF-SD-SNF-DR-003, Rev. 0

Appendix 19

Date: Thursday. January 16, 1997

To: Parsons / Vectra

Attn: Brent Becker / Chuck Torus

Fax: $\quad 509-946-8811$

Sub: $\quad$ MCO Screen

Attached is drawing 5873 which shows the filter nozzle that you require

A quotation for this filter nozzle and the other screen will soon follow.

Best Regards

Thomas A Anderson

A19-A7-2 


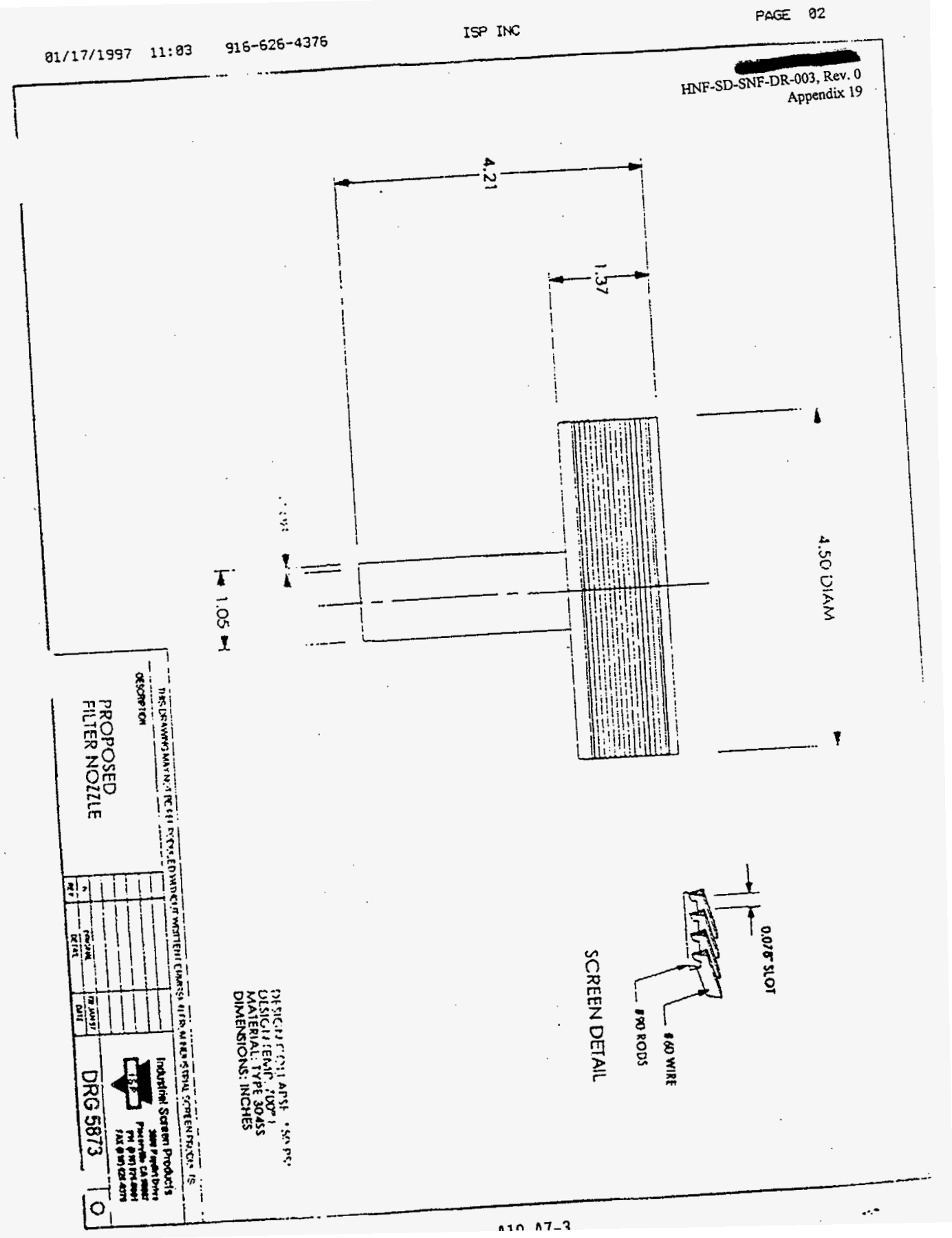




\section{APPENDIX 8}

\section{MCO CRITICAL DIMENSION SKETCHES}

NOTE: The critical dimension sketches and the critical dimension tables shall be applied in conjunction with each other to properly conform to assembled geometries, if sketches differ from design drawings, buyer will be notified immediately for resolution. 


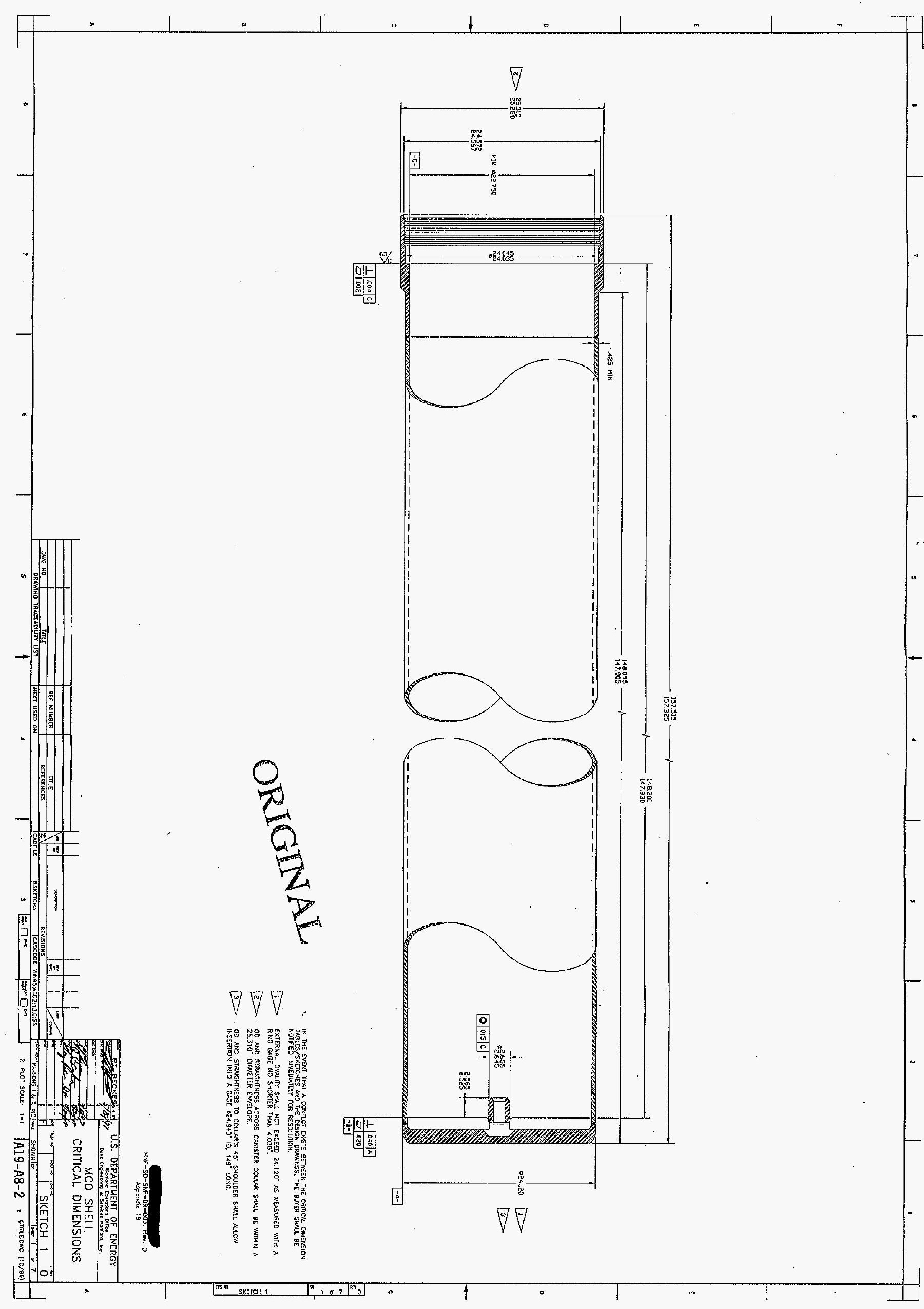




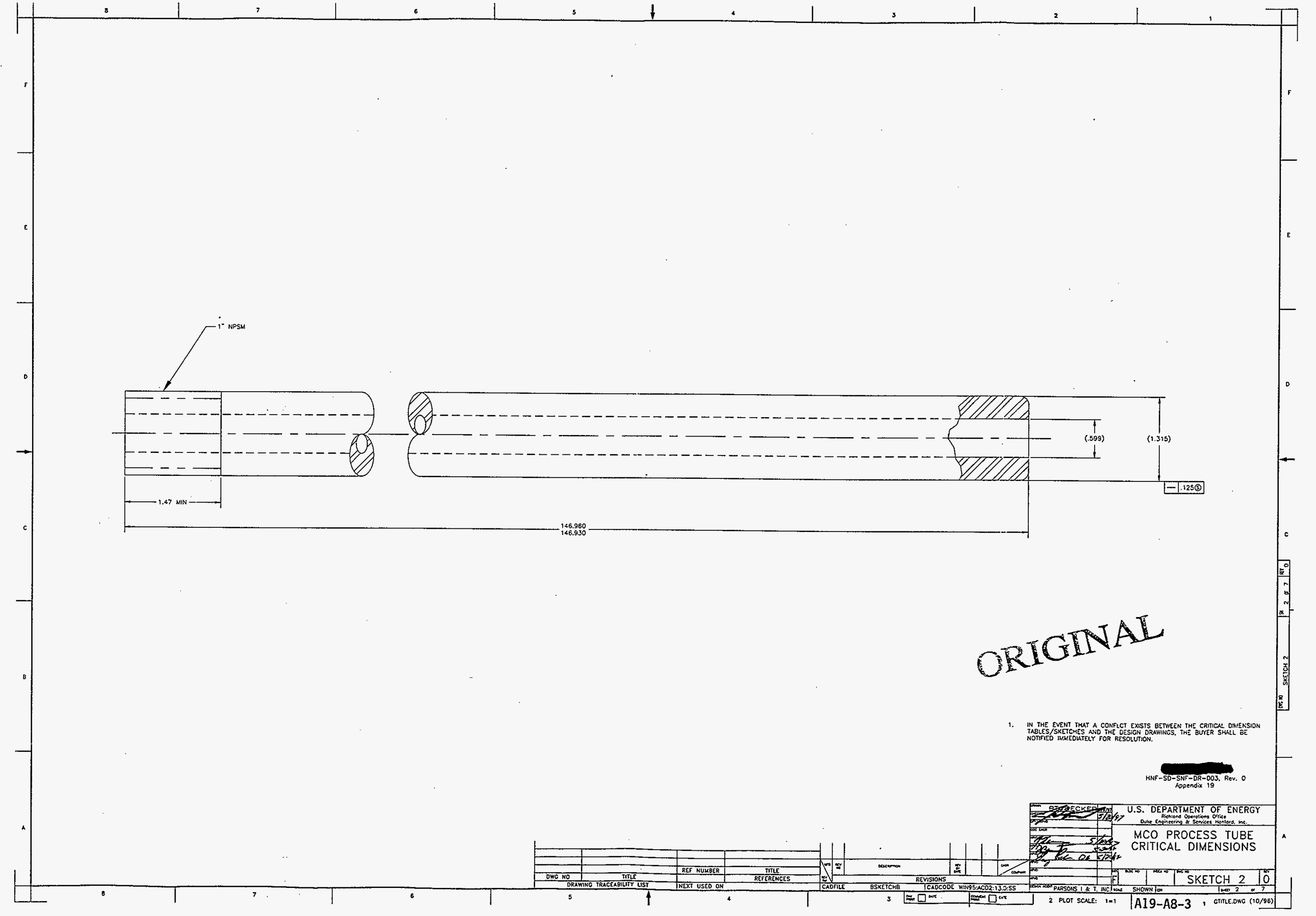




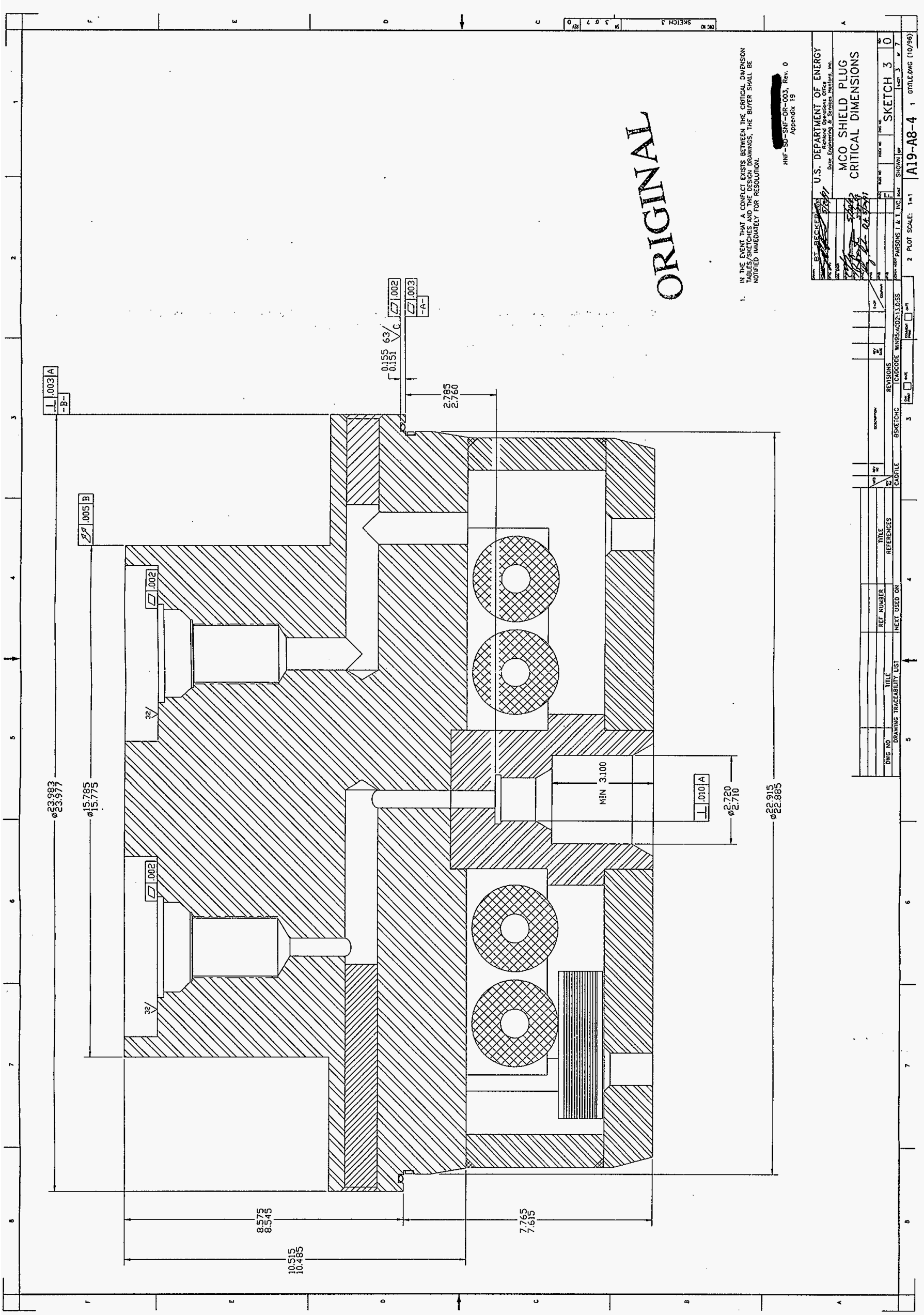




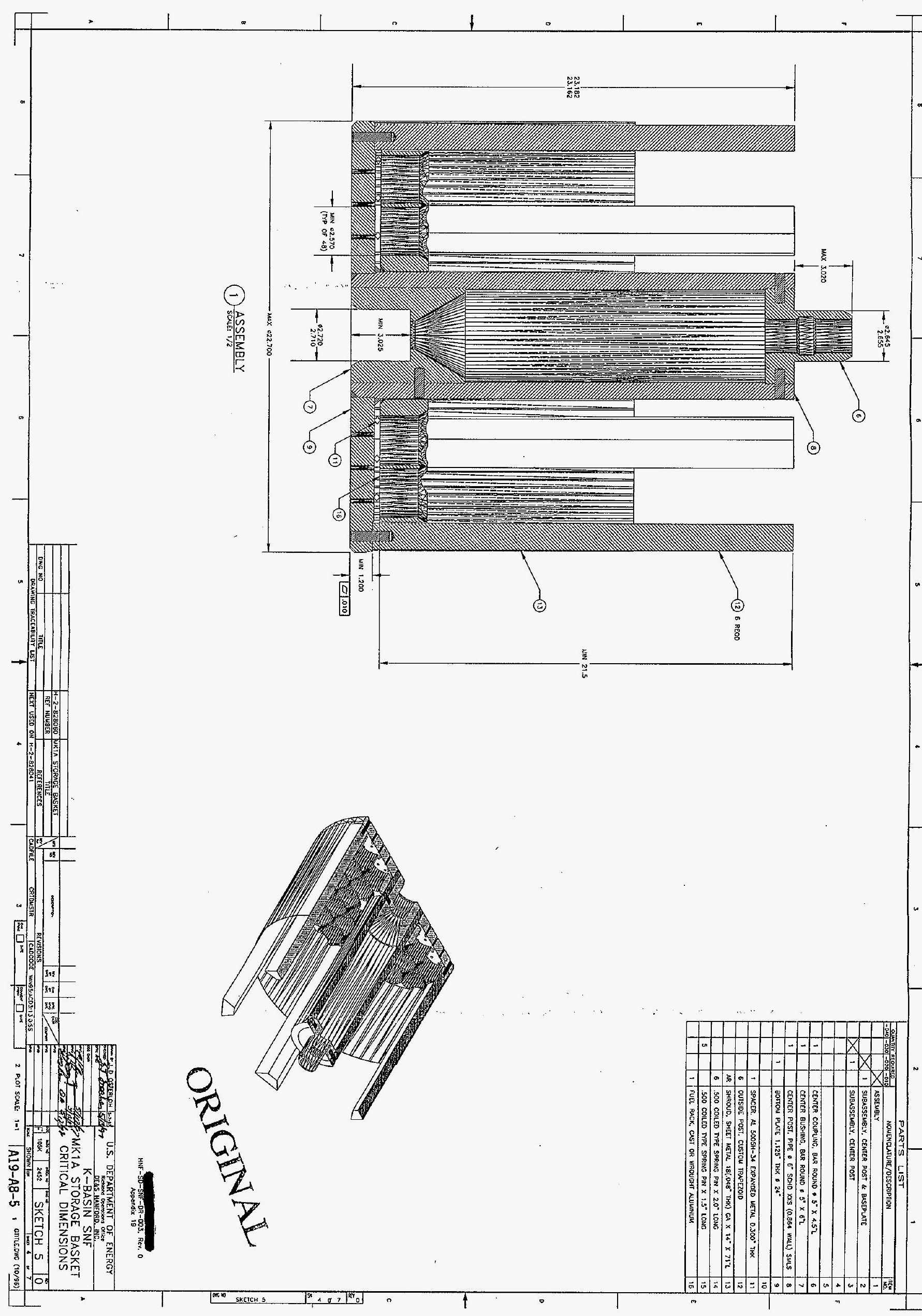




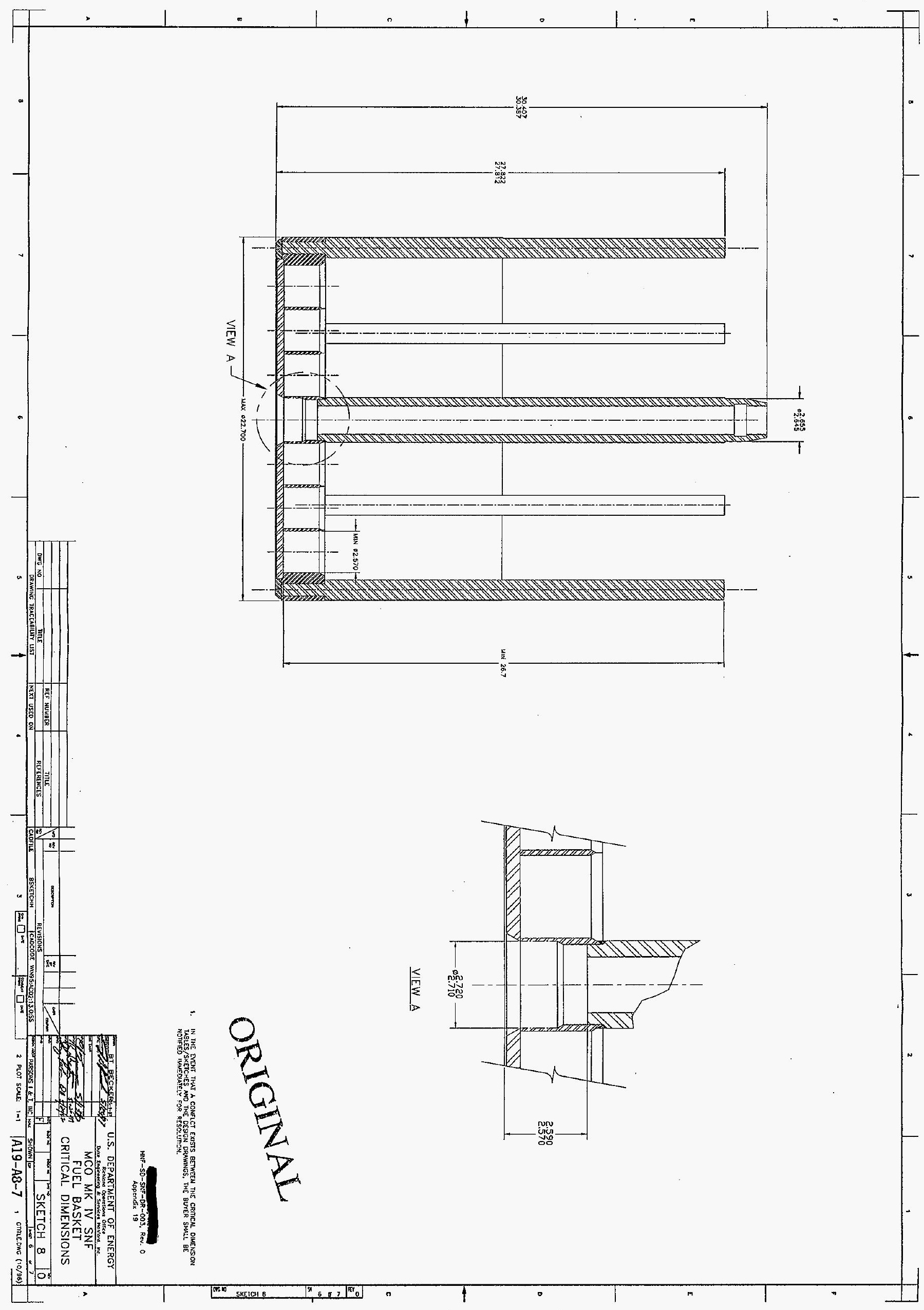




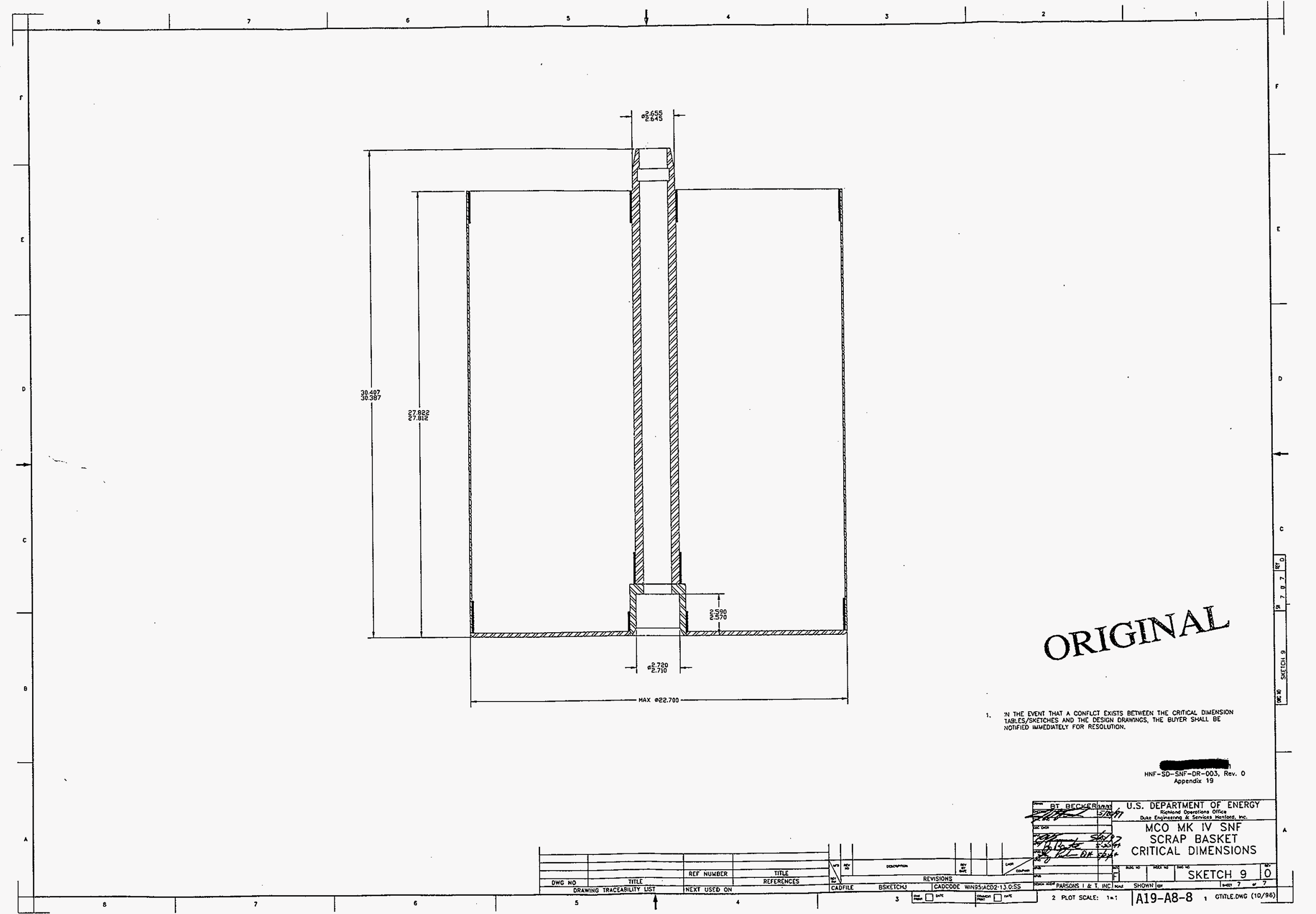




\section{DISTRIBUTION SHEET}

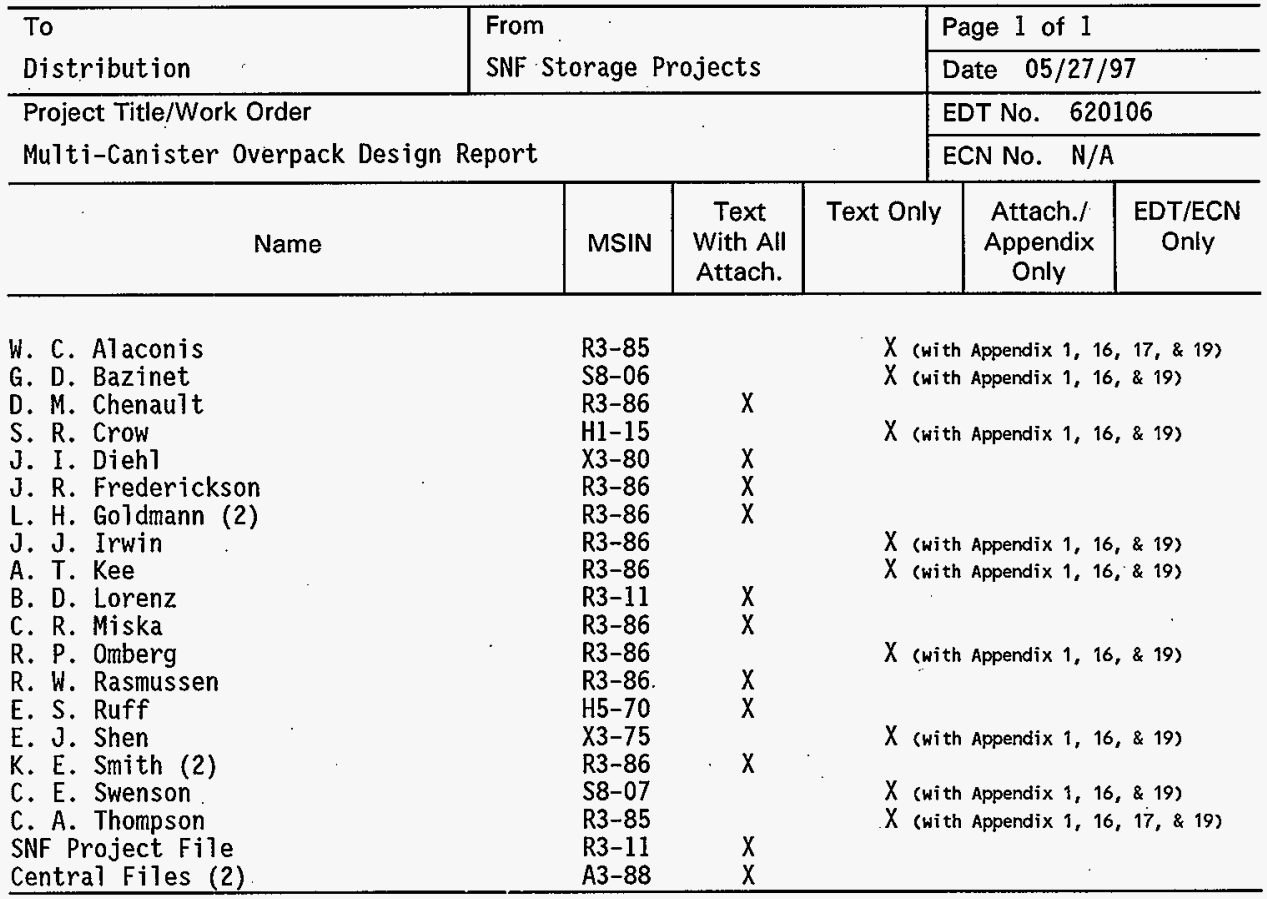

